UNITED STATES DEPARTMENT OF THE INTERIOR

Harold L. Ickes, Secretary

GEOLOGICAL SURVEY

W. C. Mendenhall, Director

Water-Supply Paper 890

GROUND-WATER RESOURCES OF THE WILLAMETTE VALLEY, OREGON

BY

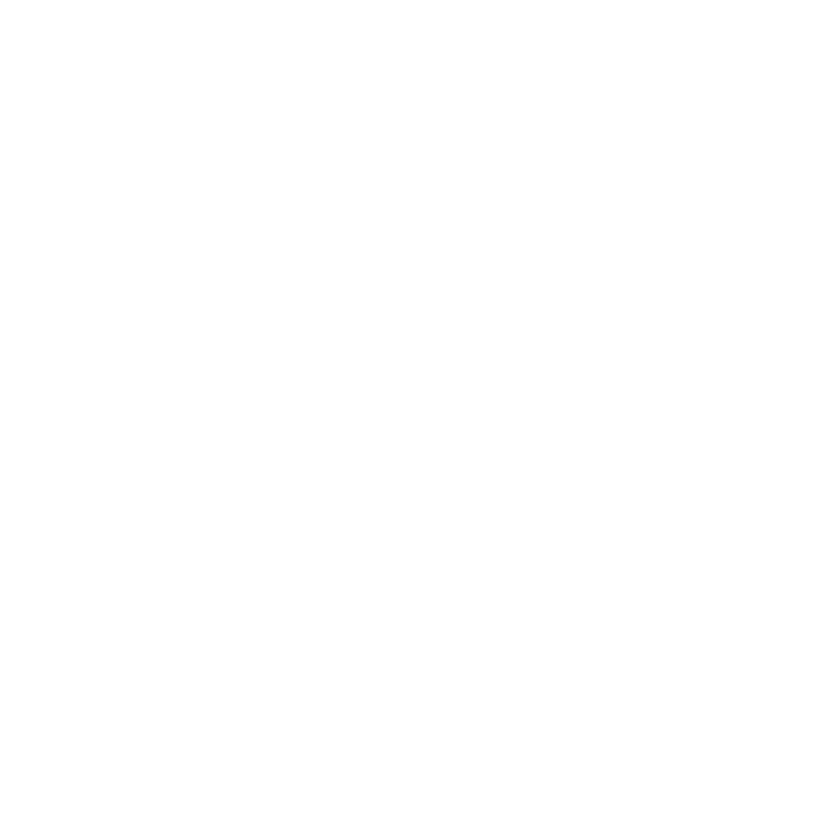

UNITED STATES

GOVERNMENT PRINTING OFFICE

WASHINGTON : 1942

For sale by the Superintendent of Documents, Washington, D. C. - . - . - - - Price 45 cents 



\section{CONTENTS}

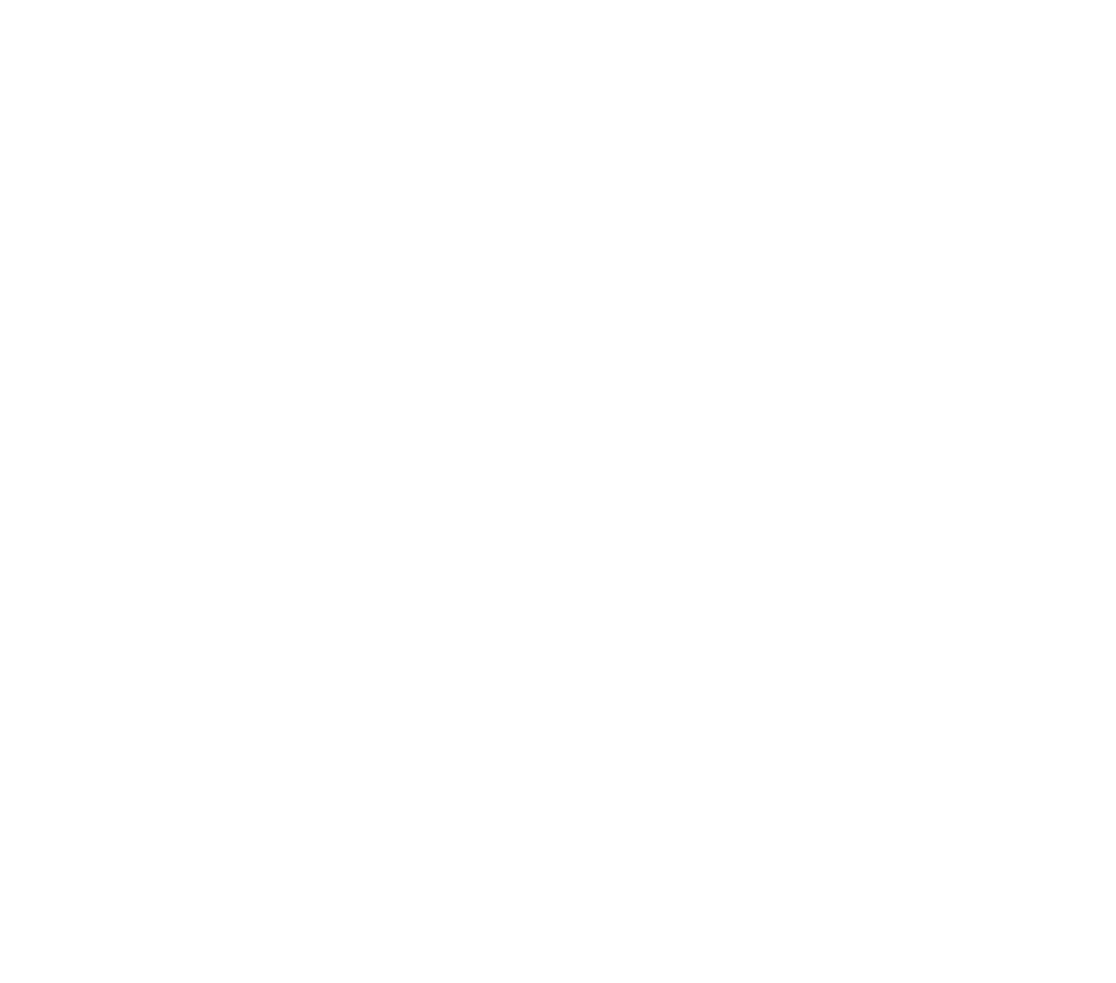

III 


\section{ILLUSTRATIONS}

Plate 1. Geologic map of the northern part of the Willamette Valley, Oregon ...... In pocket

2. Geologic map of the southern part of the Willamette Valley, Oregon............. In pocket

3. A, Three-Fingered Jack, viewed southward across Marion Lake; $B$, Three Sisters, viewed southward from McKenzie. Pass across a young lava field . . . . . . . . . . . . . . . . . . .

4. $A$, Collier Glacier, viewed from its western lateral moraine; North Sister Peak in the background; $B$, Younger alluvium of of the McKenzie River; material from lower part of well 680 .

5. Younger alluvium of the Willamette River. $A$, Gravel pit in the SE $1 / 4$ sec. 8 , T. 11 S., R. 4 W.; $B$, Pit of East Side Sand \& Gravel Co. near Corvallis, in the SW $1 / 4$ sec. 2, T. $12 \mathrm{~S}$., R. $5 \mathrm{~W}$

6. Older alluvium and related deposits. $A$, Exposure in the NW1/4 sec. 1, T. 16 S., R. 4. W.; $B$, Highway cut in the NW1/4 sec. 7, T. 4 S., R. 1 E., showing faint stratification $\ldots$

7. Map of the Willamette Valley showing thickness of younger and older alluvium, terrace deposit, and related materials...

8. $A$, Decomposed terrace deposit in highway cut in the $\mathrm{N} \frac{1}{2}$ sec. 32, T. 12 S., R. 1 W.; B, Cross-bedded sand and gravel exposed in cut along the Spokane, Portland \& Seattle Railway, 0.1 mile north of Lombard Street, Portland ................

9. Water-level fluctuations in 12 wells in the Willamette Valley and at two stations on the Willamette River, 1928-30 and 1935-36.

10. Map of the Willamette Valley showing principal subareas of the central lowland with respect to yield of ground water.......

TIGURE 1. Index map of Oregon showing drainage divide of the Willamette River, area covered by plates 1 and 2 , and areas covered by other reports relating to ground water............................

2. Maximum, average, and minimum monthly rainfall at three climatologic stations in the Willamette Valley, 1896-1935...

3. Chemical character of waters from 20 wells in the Willamette

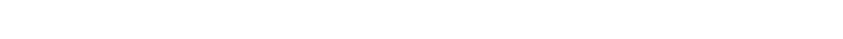




\section{TABLES}

TABLE 1. Monthly, seasonal, and yearly averages and extremes of climatic elements at Portland, Oreg., in the period ending with 1930

2. Monthly temperature, in degrees Fahrenheit, at five climatologic stations in the Willamette Valley, in the period ending with 1930

3. Maximum, average, and minimum monthly and yearly rainfall and snowfall, in inches, at five climatologic stations in the Willamette Valley, 1896-1935 ................

4. Maximum rates of precipitation, in inches, at Portland, Oreg., in the period ending with $1930 \ldots$

5. Yearly rainfall and snowfall, also surplus or deficiency with respect to 40-year average, at five climatologic stations in the Willamette Valley, 1872-1937_...................

6. Stratigraphic sections and general water-bearing properties of consolidated and semiconsolidated rocks bounding the central lowland of the Willamette River Basin...........

7. Physical properties of water-bearing materials from well 245 .

8. Approximate limits indicating the suitability of a water for

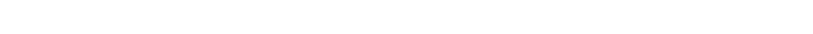

9. Chemical analyses of water from representative wells in the Willamette Valley ......................................

10. Wells in the Willamette Valley for which records of groundwater levels have been published in other water-supply

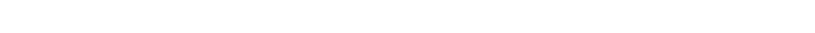

11. Records of wells in the Willamette Valley and adjacent area,

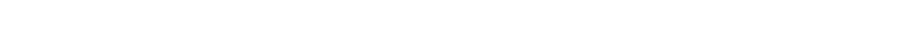

12. Materials penetrated by typical wells . . . . . . . . . . . . 130

13. Ground-water levels in observation wells, 1928-30 and 1935-36 _ $\quad 144$ 



\title{
GROUND-WATER RESOURCES OF THE WILLAMETTE VALLEY, OREGON
}

\author{
By Arthur M. Piper
}

\begin{abstract}
The drainage basin of the Willamette River lies in western Oregon between the Oregon Coast Range on the west and the Cascade Range on the east. It is about 170 miles long from north to south and covers about 11,350 square miles. The central lowland, with whose ground-water resources this report is concerned, covers nearly 3,000 square miles and ranges in altitude from 25 to 450 feet above sea level. On this lowland the average yearly rainfall is slightly less than 40 inches, but the average rainfall for the period May to August is only 4.2 inches, less than is needed to mature many crops. Thus, supplemental irrigation is profitable.
\end{abstract}

The central lowland comprises a main valley plain, remnants of at least one higher terrace with a pediment and certain other related features, and relatively narrow trenches cut into the main valley plain. These trenches are occupied by the Willamette River and its tributaries. This lowland constitutes the most extensive single agricultural area in Oregon. The area is covered with unconsolidated Quaternary deposits which, of all the rock formations in the basin, are those most likely to yield continuous large supplies of water to wells.

The bedrock formations that underlie the unconsolidated deposits of the lowland and form the foothills and mountains to the east and west include marine and nonmarine sedimentary rocks that range in age from late Eocene to Miocene (?), and fragmental and nonfragmental volcanic rocks and associated detrital rocks that range in age from Oligocene (?) to Recent. The rocks of Miocene age and older are deformed by broad shallow folds and possibly by faults of small extent and displacement.

Of the several bedrock formations, only the Yakima basalt of Miocene age yields water copiously over an extensive area; some wells in this rock yield as much as 500 gallons a minute, whereas others yield only a few gallons. Certain detrital and fragmental bedrock formations supply water copiously near Portland. The remaining bedrock formations, which together underlie most of the central lowland, are either impervious or yield water slowly or contain brackish water and brine. They supply numerous farm wells in the foothills but are not liekly to yield large supplies of fresh water from beneath the central lowland.

In general the terrace deposit and related pediment gravel are thoroughly weathered and nearly impervious. At most places they yield water slowly and are not an adequate source of water for irrigation wells.

The older alluvium and related deposits form the main lowland plain, some 1,100 square miles in extent between Eugene at the southern or upstream end and Canby at the downstream end. They rest on an eroded bedrock surface and range in thickness from a feather edge along the margins of the plain to a maximum 
of about 300 feet. Their aggregate volume is estimated as not less than $80,000,-$ 000 acre-feet, or $23 \frac{1}{2}$ cubic miles; their aggregate water-storage capacity is about $6,000,000$ acre-feet. These deposits form a plain that is divided into two nearly equal segments separated by a rock-bound narrows about 21 miles long.

The southern segment is composed of several broad alluvial fans and intervening flats. In the fans especially, the older alluvium contains many lentils and tongues of clean gravel and sand that supply as much as 500 gallons of water a minute to wells of shallow or moderate depth and of the simplest construction. Efficiently constructed wells penetrating the full thickness of the deposit very probably would yield much more copiously. The water-bearing capacity of the older alluvium is not equal in all parts of this area; with respect to potential yield, several subdivisions of the area are delimited tentatively in the full text. In this southern segment of the plain, ground-water storage increases about 500,000 acre-feet each winter, chiefly by infiltration of rain; storage declines an equal amount during the summer and autumn, chiefly by drainage into the streams. By pumping from wells, a substantial fraction of this yearly ground-water increment can doubtless be salvaged for supplemental irrigation and other uses.

At least two distinct sorts of ground-water bodies underlie the northern segment of the main plain: unconfined water that is semiperched at shallow depth beneath the "prairies" and confined water in extensive pervious zones, which in one typical well are about 70,120 , and 220 feet beneath the surface. These deeper pervious zones tend to feather out and to become less pervious toward the north and west; also, at some places they terminate by overlap against the impermeable bedrock. The semiperched water bodies are recharged by infiltration of rain on the main plain; they supply many domestic and farm wells but will not sustain large withdrawals for irrigation. The confined water presumably is derived from a catchment area to the south and east. It supplies wells that yield as much as 1,000 gallons a minute with maximum pumping lifts of about 60 feet. Doubtless it would supply several or many times its present draft, though increased withdrawal inevitably will entail some increase in lift.

Beneath the flood plains of the Willamette River and its principal tributaries, the younger alluvium is bighly pervious at most places. It supplies as much as 1,000 gallons of water a minute to wells of simple construction and would doubtless sustain a much larger aggregate withdrawal. However, through the rockbound narrows in the central part of the valley and at some other places where the alluvium is thin and rests directly on impervious bedrock, wells may not find a sufficient thickness of 'water-bearing material to yield water copiously, especially in late summer and autumn, when the ground-water level is lowest.

In chemical character the water from all the unconsolidated deposits of the Willamette Valley is satisfactory for most uses. In 31 samples of water from these deposits, the hardness ranged from about 10 to 150 parts per million, so that softening or treating to prevent the formation of scale is not necessary for some uses and not unduly costly for any use. Some waters from these deposits contain an excessive amount of iron-as much as 5.27 parts per million; water high in dissolved iron is most common in the older alluvium beneath the northern half of the main valley plain. At many places the bedrocks, especially the sedimentary rocks of marine origin, yield water of excessive hardness or so concentrated in dissolved mineral constituents as to be unsatifactory for ordinary uses.

\section{INTRODUCTION}

\section{LOCATION AND EXTENT OF THE AREA}

The drainage basin of the Willamette River, the ground-water features of whose central and lower parts are described in this report, covers about 11,350 square miles in western Oregon (fig. 1). This 


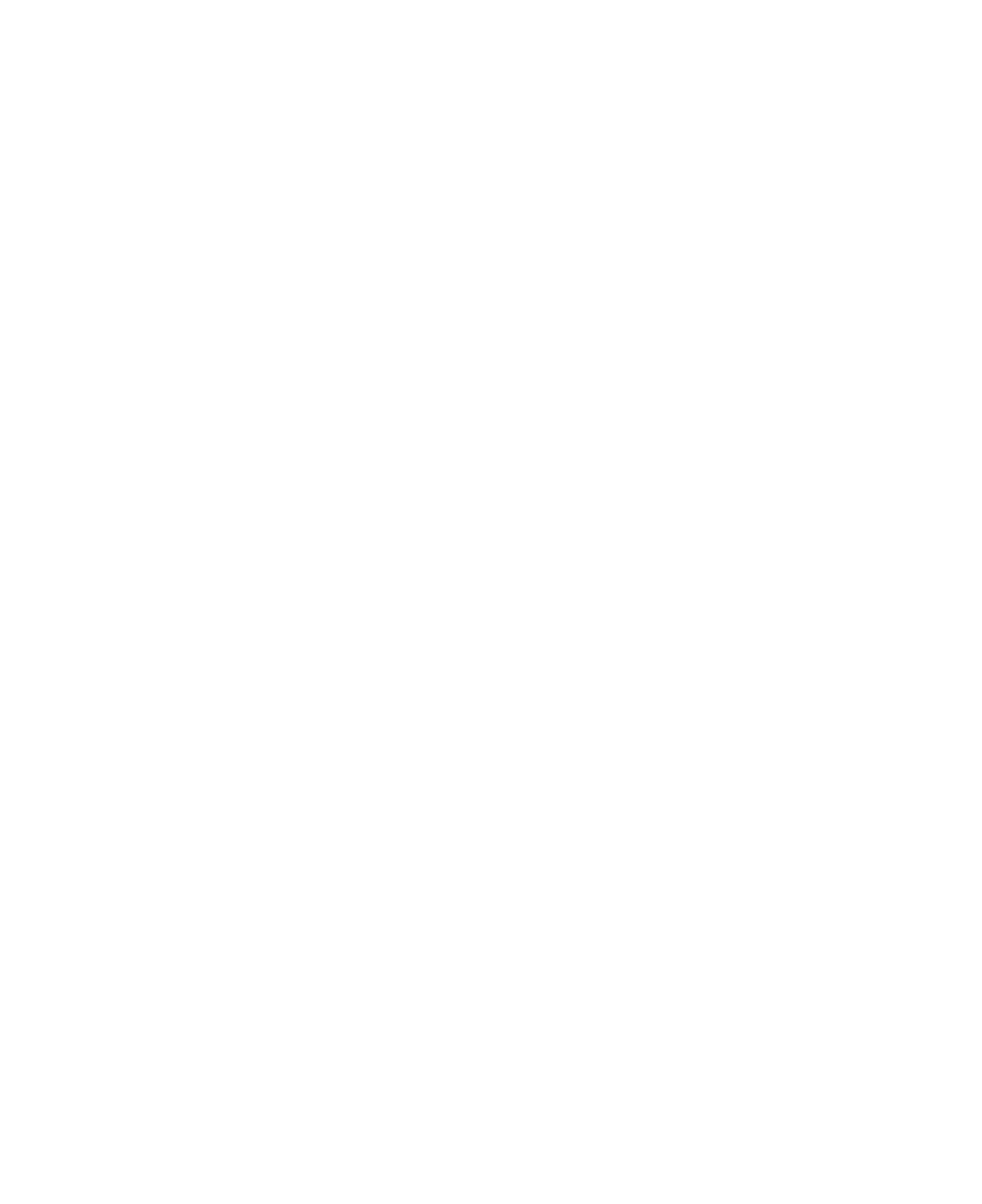


basin is approximately 170 miles long, from north to south, and as much as 100 miles wide. On the east it is bounded by the Cascade Range and on the west by the Coast Range; its northern and southern boundaries are largely in mountainous terrane of less relief. The central valley plains and adjacent terraces, known locally as the Willamette Valley, cover nearly 3,000 square miles: this part of the drainage area is represented on plates 1 and 2 .

\section{PURPOSE AND SCOPE OF THE INVESTIGATION}

The investigation whose findinge are here presented was suggested by the Department of Soils, of the Oregon Agricultural Experiment Station, and was carried on by the Geological Survey, of the United States Department of the Interior. These two agencies have shared in the cost of the field work and of the preparation of this report for publication. The primary aim of the investigation was to determine the feasibility of recovering water from wells for irrigation on the extensive fertile lowland and in this way supplementing the natural supply of soil moisture which is deficient during the greater part of the growing season in most years. (See p. 14.) Such assurance of moisture for fully maturing the growing crops would aid materially in stabilizing agriculture in the area.

Field work in the Willamette Valley was started by the writer in July 1928 and was continued during the remainder of that season and through the next. Critical hydrologic records were kept continuously until June 1930. Preparation of this report was deferred in favor of similar ground-water investigations of The Dalles region in the north-central part of Oregon, during $1930:{ }^{1}$ of the Harney Basin in the southeastern part, in $1930-32 ;^{2}$ of the Walla Walla Basin in the northeastern part, in $1933 ;{ }^{3}$ and of cooperative preliminary studies in several small districts in the State. In 1935-36 the United States Engineer Department gathered many data on ground-water levels in the Willamette Valley; this work was supervised by the writer. This report was prepared in 1937-38 through renewed cooperation between the Oregon Agricultural Experiment Station and the United States Geological Survey, utilizing all data from the field work of 1928-30 and 1935-36.

The Willamette Valley is so extensive that the time spent in the field permitted only a moderately intensive study of broad ground-water features. Briefly, this involved reconnaissance mapping of the

\footnotetext{
1 Piper, A. M., Geology and ground-water resources of The Dalles region, Oregon: U. S. Geol. Survey Water-Supply Paper 659, pp. 107-189, 1932.

${ }^{2}$ Piper, A. M., Robinson, T.W.. and Park, C. F., Jr., Geology and ground-water resources of the Harney Basin, Oregon: U. S. Geol. Survey Water-Supply Paper 841, 189 pp., 1940.

3 Piner, A. M., Robinson, T. W., and Thomas, H. E., Ground water in the Walla Walla Basin, OregonWashington: Supreme Court of the United States, October 1935, in equity, State of Washington vs. State of Oregon, Transcript of Record, stipulations and exliibits, pp. 72-142, Oct. 14, 1935.
} 
several unconsolidated deposits of Quaternary age that form the lowlands in the central part of the basin (pls. 1 and 2), keeping records of the construction and performance of typical wells, and periodically measuring the water level in a widespread net of observation wells. From these data, this report seeks to differentiate the several waterbearing zones in depth below the land surface, in lateral extent, and in water-yiclding capacity; to delimit the catchment areas from which the ground-water supply is replenished; and to evaluate the natural subdivisions of the lowland by relative capacity to sustain large withdrawals of water for irrigation and other purposes. The scope of the investigation does not afford a close estimate of the safe ground-water yield of the Willamette Valley-that is, of the quantity that could be withdrawn year after year without eventually so depleting the supply that further withdrawal at that rate would become impossible or uneconomic.

\section{ACKNOWLEDGMENTS}

Orderly progress in a regional field study such as that in the Willamette Valley results in a large measure from cooperation by many persons on whom a writer is necessarily dependent. The drillers and owners of wells in the Willamette Valley have contributed many essential records. Among his colleagues in the Geological Survey, the writer is indebted to T. W. Robinson for temporary assistance in the field during 1929 , to $\mathrm{H}$. E. Thomas for a considerable share in compiling the geologic map (pls. 1 and 2) from the writer's field sheets', and to Margaret D. Foster for chemical analyses of water samples from representative wells (table 4). T. R. Cantine, W. E. Emrick, and Arthur King, temporary employees, recorded the fluctuations in ground-water level during the greater part of the 2-year period ending June 1930.

The field work in the Willamette Valley and the preparation of this report were under the direction of O. E. Meinzer, geologist in charge of the Division of Ground Water, United States Geological Survey. To him the writer is grateful for technical counsel. Acknowledgment is due also to J. T. Jardine, director of the Oregon Agricultural Experiment Station at the time, and to W. L. Powers, head of the Department of Soils in that agency, for many courtesies which made the field work more effective.

\section{BIBLIOGRAPHY}

The geologic features of the Willamette River basin with specific reference to ground-water resources had not been studied prior to 1928, nor had the areal geology been mapped. Nevertheless, the geologic literature on the area is fairly extensive. The following 
bibliography notes the publications that are pertinent to the present investigation:

Allison, I. S., Spokane flood south of Portland, Oreg. [abstract]: Pan-Am. Geologist, vol. 57, p. 64, 1932; Geol. Soc. America Bull., vol. 43, pp. 133-134, 1932; Glacial erratics in the Willamette Valley: Geol. Soc. America Bull., vol. 46, pp. 615-632, 1935; Late Pleistocene topographic correlations on Pacific coast [abstract]: Pan-Am. Geologist, vol. 63, p. 310, 1935; Geol. Soc. America Proc., 1935, p. 333, 1936; Pleistocene alluvial stages in northwestern Oregon: Science, new ser., vol. 83, pp. 441-443, 1936.

Arnold, Ralph, and Hannibal, Harold, Marine Tertiary stratigraphy of the Pacific Coast of America: Am. Philos. Soc. Proc., vol. 52, pp. 559-605, 1913.

Bretz, J. H., Satsop formation of Oregon and Washington: Jour. Geology, vol. 25, pp. 446-458, 1917; Late Pleistocene submergence in the Columbia Valley of Oregon and Washington: Jour. Geology, vol. 27, pp. 489-506, 1919; Spokane flood beyond the channeled scablands: Jour. Geology, vol. 33, pp. 252-257, 1925; Bars of channeled scabland: Geol. Soc. America Bull., vol. 39, pp. 643-702, 1928.

Buddington, A. F., and Callagitin, Eugene, Dioritic intrumive rocks and contact metamorphism in the Cascade Range in Oregon: Am. Jour. Sci., 5th ser., vol. 31 , pp. 421-449, 1936.

Buwalda, J. P., Pleistocene and Recent topographic changes in the Pacific Coast States: Nat. Research Council Bull., No. 61, pp. 39-43, 1927.

Callaghan, Eugene, Some features of the volcanic sequence in the Cascade Range in Oregon: Am. Geophys. Union Trans. 14th Ann. Meeting, 1933, pp. 243-249; Some aspects of the geology of the Cascade Range in Oregon [abstract]: Washington Acad. Sci. Jour., vol. 24, pp. 190-191, 1934.

Callaghan, Eugene, and Buddington, A. F., Metalliferous mineral deposits of the Cascade Range in Oregon: U. S. Geol. Survey Bull. 893, pp. 7-22, 1938.

Chaney, R. W., Ecological significance of the Eagle Creek flora of the Columbia River Gorge: Jour. Geology, vol. 26, pp. 577-592, 1918; Further discussion of the ecological composition of the Eagle Creek flora [abstract]: Geol. Soc. America Bull., vol. 31, p. 222, 1920; The flora of the Eagle Creek formation: Chicago Univ., Walker Museum, Contr., vol. 2, pp. 115-181, 1920.

Chaney, R. W., and Sanborn, E. I., The Goshen flora of west-central Oregon: Carnegie Inst. Washington Pub. 439, Contr. Paleontology, 103 pp., 1933.

Condon, Thomas, Willamette Sound: Overland Monthly, vol. 7, pp. 468-473, 1871; Oregon geology: a revision of "The Two islands" (edited by E. C. McCornack), 187 pp., Portland, Oreg., J. K. Gill Co., 1910.

Coshman, J. A., and Schenck, H. G., Two foraminiferal faunules from the Oregon Tertiary: California Univ., Dept. Geol. Sci., Bull., vol. 17, pp. 305$324,1928$.

Darton, N. H., Structural materials in parts of Oregon and Washington: U. S. Geol. Survey Bull. 387, 33 pp., 1909.

Diller, J. S., A geological reconnaissance in northwestern Oregon: U. S. Geol. Survey 17th Ann. Rept., pt. 1, pp. 441-520, 1896.

Felts, W. M., Analysis of Willamette Valley fill [abstract]: Pan-Am. Geologist, vol. 64, p. 69, 1935; Geol. Soc. America Proc. 1935, p. 346, 1936.

Harrison, G. D., and Eaton, Arthur, Report on the investigation of oil and gas possibilities of western Oregon: Mineral Resources of Oregon, vol. 3, No. 1, pp. 3-37, Oregon Bur. Mines and Geology, 1920.

Hertelein, L. G., and Crickmay, C. H., A summary of the nomenclature and stratigraphy of the marine Tertiary of Oregon and Washington: Am. Philos. Soc. Proc., vol. 64, pp. 224-282, 1925. 
HodGE, E. T., Geology of Oregon Cascades [abstract]: Geol. Soc. America Bull., vol. 38, pp. 204-205, 1927; Pan-Am. Geologist, vol. 47, p. 157, 1927; Composition and structure of the Cascade Mountains in central Oregon [abstract]: Geol. Soc. America Bull., vol. 38, pp. 162-163, 1927; Pan-Am. Geologist, vol. 47, p. 157, 1927; Mount Multnomah, ancient ancestor of the Three Sisters: Oregon Univ. Pub., vol. 3, No. 2, 160 pp., 1925 [abstract]; Am. Jour. Sci., 5th ser., vol. 13, p. 270, 1927; Age of Columbia River and lower canyon [abstract]: Geol. Soc. America Bull., vol. 44, pp. 156-157, 1933; Geology of the lower Columbia River: Geol. Soc. America Bull., vol. 49, pp. 839-888. 1938.

Howe, H. VAN W., Correlation of the Empire formation, Oregon [abstract]: Geol. Soc. America Bull., vol. 32, p. 147, 1921; Faunal and stratigraphic relations of the Empire formation, Coos Bay, Oreg.: California Univ., Dept. Geol. Sci., Bull., vol. 14, pp. 85-114, 1922; Astoria, mid-Tertic type of Pacific coast: Pan-Am. Geologist, vol. 45, pp. 295-306, 1926.

McCornack, E. C., A study of Oregon Pleistocene: Oregon Univ. Bull., new ser., vol. 3, No. 5, 1906; Study of Oregon Pleistocene--the Oregon Desmostylus. skull: Oregon Univ. Bull., new ser., vol. 12, No. 2, p. 13, 1914; Contributions to the Pleistocene history of Oregon: Oregon Univ. Leaflet, Geology Bull, vol., 6, No. 3, 23 pp., 1920.

Parks, H. M., Road materials in the Willamette Valley: Oregon Bur. Mines Bull. 1, 63, pp , 1911.

Rathbun, M. J., The fossil stalk-eyed Crustacea of the Pacific slope of North America: U. S. Nat. Mus. Bull. 138, 155 pp., 1926.

Schenck, H. G., A preliminary report on the geology of the Eugene quadrangle, Lane and Linn Counties, Oreg.: Oregon Univ., unpublished thesis, 1923; Physiography of the Eugene quadrangle, Oregon [abstract]: Geol. Soc. America Bull., vol. 36, p. 203, 1925; Diatoms in western Oregon shales: Econ. Geology, vol. 22, pp. 565-568, 1927; Marine Oligocene of Oregon: California Univ., Dept. Geol. Sci., Bull., vol. 16, pp. 449-460, 1927; Stratigraphic relations of western Oregon Oligocene formations: California Univ., Dept. Geol. Sci., Bull., vol. 18, pp. 1-50, 1928; The Pittsburg Bluff fauna of the Oregon Oligocene [abstract]: Geol. Soc. America Bull., vol. 40, pp. 163-164, 1929; Cephalopods of the genus Aturia from western North America: California Univ., Dept. Geol. Sci., Bull., vol. 19, pp. 435-490, 1931; Stratigraphic and faunal relations of the Keasey formation of the Oligocene of Oregon: Geol. Soc. America Bull., vol. 44, p. 217, 1933; Nuculid bivalves of the genus Acila: Geol. Soc. America Spec. Papers, No. 4, pp. 41-44, 1936.

Schenck, H. G., and Kleinpell, R. M., Foraminifera from Gaviota formation [Calif.] [abstract]: Pan-Am. Geologist, vol. 64, p. 76, 1935; Geol. Soc. America Proc., 1935, p. 352, 1936.

Sмiтh, W. D., Petroleum possibilities of western Oregon: Econ. Geology, vol. 19, pp. 455-465, 1924.

Sмiтh, W. D., Summary of the salient features of the Oregon Cascades: Oregon Univ. Bull., new ser., vol. 14, No. 16, 1917; [abstract] Geol. Soc. America Bull., vol. 29, p. 81, 1918 .

Smith, W. D., and PACKARD, E. L., Salient features of the geology of Oregon: Oregon Univ. Bull., new ser., vol. 16, No. 7, 42 pp., 1919; Jour. Geology, vol. 27, pp. $79-120,1919$.

Thayer, T. P., Structural relation of central Willamette Valley to Cascade Mountains [abstract]: Pan-Am. Geologist, vol. 59, p. 317, 1933; Geol. Soc. America Proc. 1933, p. 315, 1934; Structure of North Santiam section of the Oregon Cascades [abstract]: Pan-Am. Geologist, vol. 61, p. 319, 1934; Geol. 
Soc. America Proc., 1934, pp. 324-325, 1935; Structure of the North Santiam River section of the Cascade Mountains in Oregon: Jour. Geology, vol. 44, pp. 701-716, 1936.

Washburne, Chester, Reconnaissance of the geology and oil prospects of northwestern Oregon: U. S. Geol. Survey Bull. 590, 108 pp., 1914.

Wells, F. G., and WAters, A. C., Quicksilver deposits of southwestern Oregon: U. S. Geol. Survey Bull. 850, pp. 5-23, 1934.

Williams, I. A., The Columbia River Gorge-its geologic history interpreted from the Columbia River Highway: Mineral Resources of Oregon, vol. 2, No. 3, 130 pp., Oregon Bur. Mines and Geology, 1916.

\section{SURFACE FEATURES OF THE WILLAMETTE VALLEY}

The Willamette River Basin has high relief and considerable diversity in its land forms. With respect to major surface features it may be divided into three parts, coextensive with parts of three physiographic sections of the United States as recognized by Fenneman. ${ }^{4}$ In order from west to east these are: (1) The Oregon Coast Range section of the Pacific Border province, which includes about 15 percent of the basin; (2) the Puget Trough section, also of the Pacific Border province, whose part in Oregon covers about 25 percent of the basin and is roughly coextensive with the central lowland plains and terraces; and (3) the Middle Cascade Mountains section of the SierraCascade province, which includes about 60 percent of the basin.

The Oregon Coast Range is a mature land surface, deeply dissected by closely spaced branching valleys. Its sinuous crest trends generally northward, is between 5 and 25 miles west of the margin of the central lowland, and in greater part is not more than about 2,500 feet above sea level, or 2,000 feet above the lowland. Above this general crest level stand several isolated peaks such as Marys Peak, which lies west of Corvallis and attains an altitude of about 4,100 feet. From the general crest level, a succession of moderately sinuous ridges descend gently eastward toward the lowland and terminate commonly in a narrow belt of foothills. With the exception of certain narrow plains along the streams, all the range has been covered with a heavy stand of coniferous timber but extensive areas have been logged over or denucied by fire. In these denuded areas many larger tracts now support a vigorous second growth, and some tracts have been cleared for farming, especially in the foothills.

The greater part of the Oregon Coast Range consists of sedimentary rocks of Tertiary age, largely shale and earthy sandstone (see pp. 19-21), deformed by broad open folds. In these rocks the higher parts of the land surface commonly are rounded in contour, with profiles convex upward; conversely, the lower slopes of the valleys are smooth but steep and commonly are concave upward. Throughout the range, however, there are many bodies of igneous rock-sheets

\footnotetext{
- Fenneman, N. M., Physiographic divisions of the United States: Assoc. Am. Geographers Annals, vol. 18, pp. 261-353, map, 1928.
} 
interbedded with the sedimentary rocks, both intrusive and extrusive; dikes of a wide range in thickness; and stocklike masses of moderate size. These bodies account for scattered areas of moderately rugged topography and sharp constrictions at numerous places along the valleys. They also form the isolated peaks that rise above the general crest of the range.

The Cascade Range is striking both in topography and in composition. Much of the range is very rugged, is topographically young, and has been sculptured by glaciers as well as by streams; it is composed almost entirely of igneous materials-nonfragmental extrusive rocks, tuff and other pyroclastic rocks in a wide range of textures, and intrusive rocks of several petrographic types. Its highest peaks rise far above timber line and so form a discontinuous alpine zone. These features characterize a belt from 40 to 60 miles wide all along the eastern side of the basin. This belt, covering nearly 7,000 square miles, is somewhat more than twice as extensive as the central lowland and four times as extensive as the eastern slope of the Coast Range.

The Cascade Range within the Willamette River Basin represents two fairly distinct land forms. The western part, adjacent to the central lowland and as much as 25 miles wide, is a belt of mature foothills and ridges in which certain older folded volcanic rocks are extensive. Here the relief fully equals or somewhat surpasses that of the Coast Range. To the east, younger volcanic rocks rise to the divide, which, for the greater part of its length, is between 4,000 and 6,500 feet above sea level. On this higher part of the range, consequent streams flow generally westward in successive impressive canyons, commonly as much as 2,500 feet deep. Some interstream flats of moderate extent preserve the initial volcanic surface, which is moderately smooth and of fairly uniform westward slope. Alined along and near the divide are several prominent volcanic peaks, commonly deeply scarred by alpine glaciers. Chief among these, in order from the north, are Mount Hood, which is 11,225 feet above sea level; Mount Jefferson, 10,485 feet; Three Fingered Jack, 7,848 feet (pl. 3, A) ; Mount Washington, 7,802 feet; the North Sister, Middle Sister, and South Sister (pl. 3, B), which are respectively 10,094 feet, 10,053 feet, and 10,354 feet above sea level; and Diamond Peak, 8,750 feet. Less prominent volcanic peaks, younger than those just cited and unscarred by glaciers, are Olallie Butte, 7,210 feet above sea level; Belknap Crater, 6,877 feet; and Black Crater, 7,260 feet. These younger peaks are about midway along the eastern enge of the basin.

The Willamette Valley, the lowland that lies between the two mountainous areas just described, comprises a main valley plain, remnants of at least one higher terrace with a pediment and certain other related features, and relatively narrow trenches which are occupied by the 
Willamette River and its tributaries. These three parts of the lowland make up the most extensive single agricultural area in Oregon. They are formed of unconsolidated deposits of Quaternary age which, of all the rock formations in the basin, are those most likely to yield large supplies of water to wells.

The main valley plain, which is coextensive with the older alluvium and related deposits shown on plates 1 and 2, has several distinctive parts, which can most readily be described in order downstream, or toward the north. The higher southern segment of the plain, which is one of two principal segments, begins at Eugene near the junction of the three principal headwater branches of the Willamette River (McKenzie River, Middle Fork, and Coast Fork) and extends northward to Albany. Disregarding its tongues that extend into the bordering mountainous areas and the younger stream trenches by which it is traversed, this segment of the plain is about 40 miles long and from 9 to 20 miles wide. It is from 360 to 450 feet above sea level at its southern end and from 225 to 300 feet at its northern end. Thus, its average northward slope is about 3.5 feet to the mile. In the main, it is formed by a succession of broad alluvial fans that spread outward from the mountain valleys to the east and that coalesce along their outer margins. On these fans the land surface slopes radially northward and westward from 6 to 11 feet to the mile. Each fan is traversed by numerous faint ephemeral drains, the radiating distributaries of streams that existed while the plain was being built. Between the fans and rather generally along the western margin of the lowland, the plain includes smooth areas that slope northward as little as 2 feet to the mile.

From Albany to Salem, a distance of 20 miles, the main valley plain is sharply constricted by outlying rock hills, to a width in places of as little as $2 \frac{1}{2}$ miles. The average northward gradient is about 2.7 feet to the mile.

At Salem the plain widens abruptly into its northern principal segment, which extends northward another 22 to 28 miles, to McMinnville, Newberg, and Canby and somewhat beyond. This segment of the plain is from 9 to 25 miles wide. It comprises several "prairies" that are remarkably flat. Near Salem the plain is about 200 feet. above sea level; in the vicinity of Canby, at its northeast corner, from 185 to 190 feet; and near McMinnville, about 155 feet. Thus the average slope, from which no extensive part of the segment deviates materially, is about 2.4 feet to the mile; the direction of slope is N. $50^{\circ}$ $\mathrm{W}$., which is across the general course of the Willamette River in that vicinity and toward an alcove in the rock hills that bound the lowland. Obviously, the surface form of this northern segment of the plain is not a product of ordinary stream aggradation. 
? 
Still farther north, the main lowland plain divides into several interlacing tongues that reunite near the southern edge of Portland, some 15 miles upstream from the mouth of the Willamette River. These tongues are commonly less than a mile wide and are of uneven grade. Apparently they are a product of stream aggradation along valleys that are now occupied in part by the Willamette River and its lower tributaries, Chehalem Creek, Tualatin River, and Clackamas River. Attached to the plexus of tongues just described and largely north of the Tualatin River is another plain of moderate extent, a topographic analog of the main lowland plain of the Willamette Valley. This plain, the most northerly of those drained by the Willamette River, is known locally as the Tualatin Valley.

Beneath the main lowland plain of the Willamette Valley, the groundwater conditions are rather diverse. On this diversity the plain is divided into 20 subareas, which are described on pages 41-54.

From 30 to 100 feet higher than the main lowland plain and scattered widely along both its eastern and western margins are remnants of a terrace, which in large part is formed by stream deposits that are delimited on the geologic map (pls. 1 and 2). Corresponding terrace remnants occur along the major tributaries of the Willamette River far upstream beyond the area represented by the map. Related to these terraces in origin are certain older alluvial surfaces, notably a dissected pediment which, in the vicinity of the Molalla River, rises. as much as 300 feet above the main lowland. Possibly related in origin is the so-called Portland delta, which occupies the angle between the Willamette and Columbia Rivers and which is analogous to a'n extensive plain still farther north, in Washington. All these terraceremnants and related surfaces have been dissected to moderate depth, and so their initial form and grade have been modified considerably.

Cut into the main lowland plain are certain trenches that ordinarily are floored with young alluvium (pls. 1 and 2); these are occupied by the Willamette River and its major tributaries. Between Eugene at. the southern end of the main lowland and Newberg at the northern end, the inner trench of the Willamette River ranges between 0.5 mile and 4 miles in width. From Eugene northward to Albany, its: floor slopes equally with the main plain but is from 5 to 15 feet lower. Farther north, owing to difference in grade, the trench deepens gradu-. ally until at Newberg its floor is about 80 feet below the main lowland plain. Characteristically the floor is gently undulating, is dotted with oxbow lakes, and is traversed by many channels that are occupied' by the river during freshets. It affords some of the most fertile land of the entire valley. Downstream from Newberg the trench becomes: steadily narrower, and near Canby, at the northeastern corner of the main plain, it passes into a rock-bound narrows that extends through. 
Oregon City to the southern edge of Portland, within 14 miles of the mouth of the river.

Similar trenches, rather widely spaced over all the main lowland plain, are occupied by the larger tributaries of the Willamette River. One trench is unique in that it is not now occupied by a single perennial stream. This trench trends northeastward across the northern segment of the main plain, from the trench of the Willamette River near Salem to that of the Pudding River near Mount Angel. It is notably shallower than the two stream-occupied trenches that it connects. Doubtless it was once occupied by an ancestral Willamette River but was abandoned by that stream early in the epoch of trenching.

\section{CLIMATE ${ }^{5}$}

The broad lowland of the Willamette River Basin has a relatively equable climate, characterized by warm dry summers and cool winters with moderately abundant rain and some snow. These characteristics are shown by table 1, which assembles averages and extremes of the principal climatic elements for Portland, at the northern end of the basin.

\footnotetext{
${ }^{6}$ Adapted to this report from a statement by E. L. Wells in U. S. Dept. Agr., Weather Bur., Climate summary of the United States, sec. 3, Western Oregon, 1936.
} 


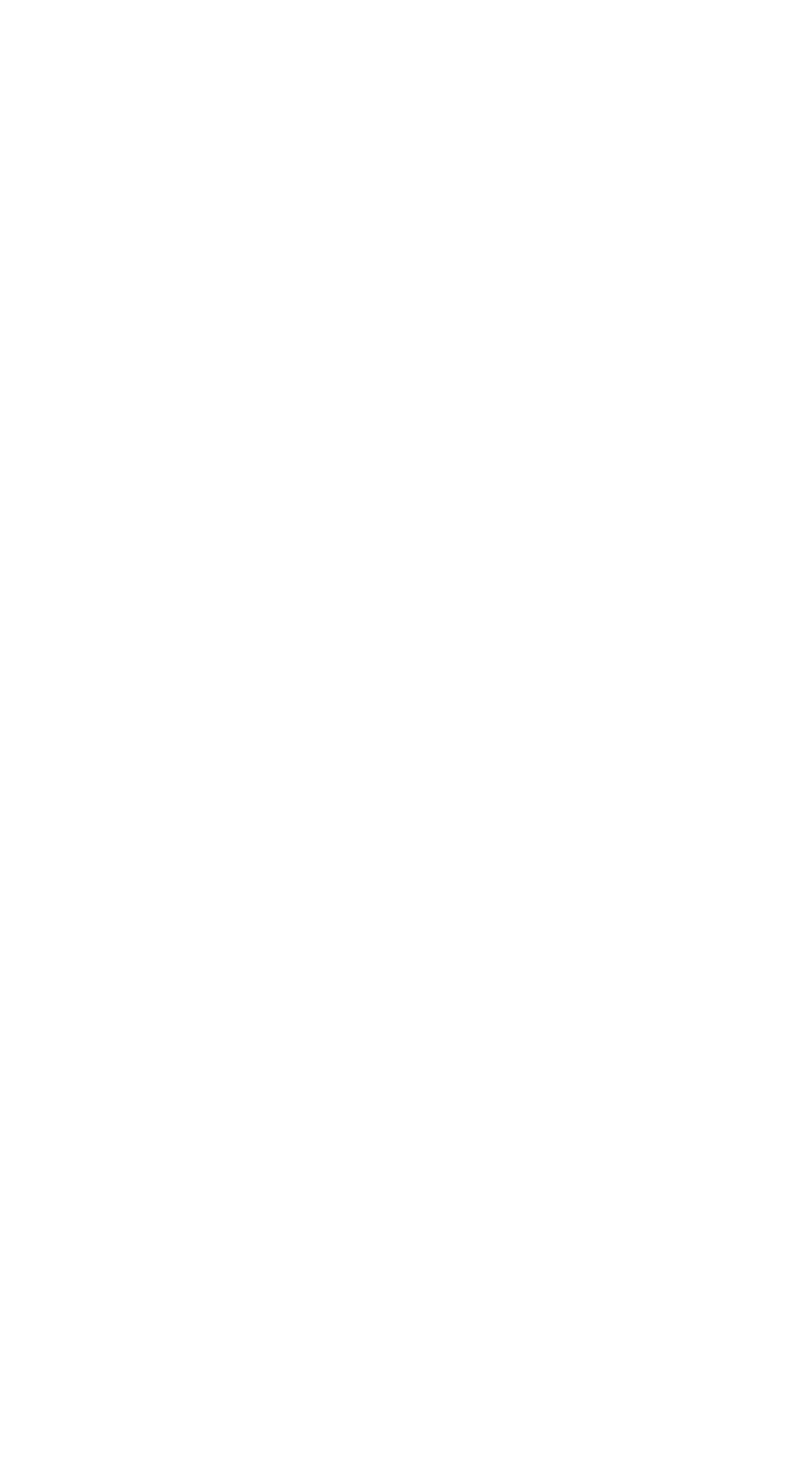


Over most of the lowland the yearly average temperature is between $51^{\circ}$ and $53^{\circ} \mathrm{F}$. The average of the highest temperatures in midsummer (July) ranges from about $78^{\circ}$ to $84^{\circ}$. Though temperatures above $100^{\circ}$ have occurred in nearly all parts of the area, they are always attended by low relative humidity and are almost alwavs followed by cool nights. Thus, the average of the lowest temperatures in July ranges between $48^{\circ}$ and $56^{\circ}$. Killing frost seldom occurs in the season when tender crops are growing. Table 2 gives monthly temperatures at five climatologic stations along the middle of the valley, from Portland at the north to Eugene at the south. (See fig. 1.)

TABLE 2.-Monthly temperature, in degrees Fahrenheit, at five climatologic stations in the Willamette Valley, in the period ending with 1930

[Data from publications of the United States Weather Bureau]

\begin{tabular}{|c|c|c|c|c|c|c|c|c|c|c|c|c|c|c|c|}
\hline & \multicolumn{3}{|c|}{ Portland } & \multicolumn{3}{|c|}{ Salem } & \multicolumn{3}{|c|}{ Albany } & \multicolumn{3}{|c|}{ Corvallis } & \multicolumn{3}{|c|}{ Eugene } \\
\hline & 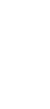 & $\begin{array}{l}0 \\
0 \\
2 \\
5 \\
0 \\
3 \\
4\end{array}$ & $\begin{array}{l}\overrightarrow{\tilde{v}_{\Delta}} \\
\stackrel{\vec{B}}{0} \\
\stackrel{\leftrightarrow}{*}\end{array}$ & 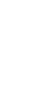 & $\begin{array}{l}0 \\
50 \\
\Xi \\
\vdots \\
\vdots \\
4\end{array}$ & $\begin{array}{l}\overrightarrow{\mathrm{w}} \\
\stackrel{2}{2} \\
\stackrel{3}{3}\end{array}$ & 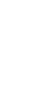 & \begin{tabular}{l}
0 \\
00 \\
0 \\
\multirow{2}{0}{} \\
0 \\
2 \\
4
\end{tabular} & \begin{tabular}{l} 
苞 \\
今゙ \\
\multirow{3}{*}{}
\end{tabular} & 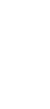 & 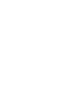 & 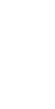 & 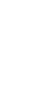 & $\begin{array}{l}0 \\
00 \\
8 \\
0 \\
5 \\
5 \\
4\end{array}$ & 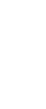 \\
\hline $\begin{array}{l}\text { Length of record } \\
\text { (years) }\end{array}$ & 57 & 59 & 57 & 38 & 38 & 38 & 36 & 47 & 36 & 41 & 41 & 41 & 36 & 39 & 36 \\
\hline Januar & 62 & 39.1 & -2 & 68 & 39.6 & -5 & 68 & 39.3 & -3 & 64 & 38.9 & -1 & 69 & 40.0 & 6 \\
\hline & 68 & 42. 2 & 7 & 68 & 42.6 & -4 & 69 & 42.4 & & 69 & 42.0 & -5 & 74 & 42.9 & 0 \\
\hline Marcl & 83 & 47.2 & 20 & 80 & 46.3 & 22 & 81 & 46.8 & 9 & 82 & 45.6 & 13 & 80 & 46.1 & 18 \\
\hline $\mathrm{Ap}$ & 93 & 51. 9 & 28 & 93 & 51. & 27 & 91 & 51.3 & 25 & 91 & 50.2 & 24 & 89 & 50.7 & 26 \\
\hline $\mathrm{M}$ & 99 & 57 & 32 & 94 & 56 & 31 & 08 & 56 & 0 & 95 & 55.1 & 0 & 91 & 55 & 30 \\
\hline Jur & 101 & 62.2 & 39 & 100 & 61. & 32 & 103 & 61. & 32 & 102 & 60.4 & 32 & 100 & 60.4 & 34 \\
\hline & 104 & 67. & 4 & 108 & 6 & 35 & 104 & & 35 & 102 & & 3 & 104 & 65.8 & 39 \\
\hline A & 98 & 66 & 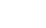 & 105 & 66 & 30 & J03 & 66 & 0 & 102 & 65.8 & 0 & 99 & 65 & 35 \\
\hline Septer & 97 & 61. & 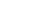 & 97 & 60. & 3 & 98 & 60. & 28 & 96 & 60.2 & 2 & 96 & 60. & 30 \\
\hline $\mathrm{Oc}$ & 86 & 54 & 3 & 89 & 53 & 23 & 86 & 52 & 25 & 85 & 52.9 & 2 & 84 & 53.3 & 25 \\
\hline Nove & 73 & 46. & 11 & 70 & 45. & 1 & 72 & 45. & & 73 & 45.2 & 1 & 75 & 46.3 & 12 \\
\hline December. & 65 & 41.2 & & 72 & 40. & - & 64 & 40.4 & -15 & 62 & 40.2 & -14 & 67 & 40.8 & -4 \\
\hline Annual.. & 104 & 53.1 & -2 & 108 & 52.6 & -6 & 104 & 52.4 & -15 & 102 & 51.8 & -14 & 104 & 52.3 & -4 \\
\hline
\end{tabular}

The precipitation on the lowland, rain for the most part, occurs largely in the winter and early spring; even in the wettest year of record at Portland, 1882, the summer rainfall was only 2.9 inches or 4.3 percent of the precipitation in that year and 7.4 percent of the average yearly precipitation. Thus in most years rainfall is not sufficient to assure full maturing of crops, and supplemental irrigation has been found profitable. Figure 2 and table 3 show this marked seasonal distribution of rainfall and snowfall, which is so characteristic of the entire Willam ette River Basin. Most of the precipitation occurs as gentle rains. The average annual number of days with 0.01 inch or more of precipitation is from about 130 to 160 . However, between May and September precipitation at excessive rates occasionally continues for an hour or less and usually between November and February it continues for a day or more. (See table 4.) Some snow falls every winter, but it seldom remains on the ground more than a few days. 


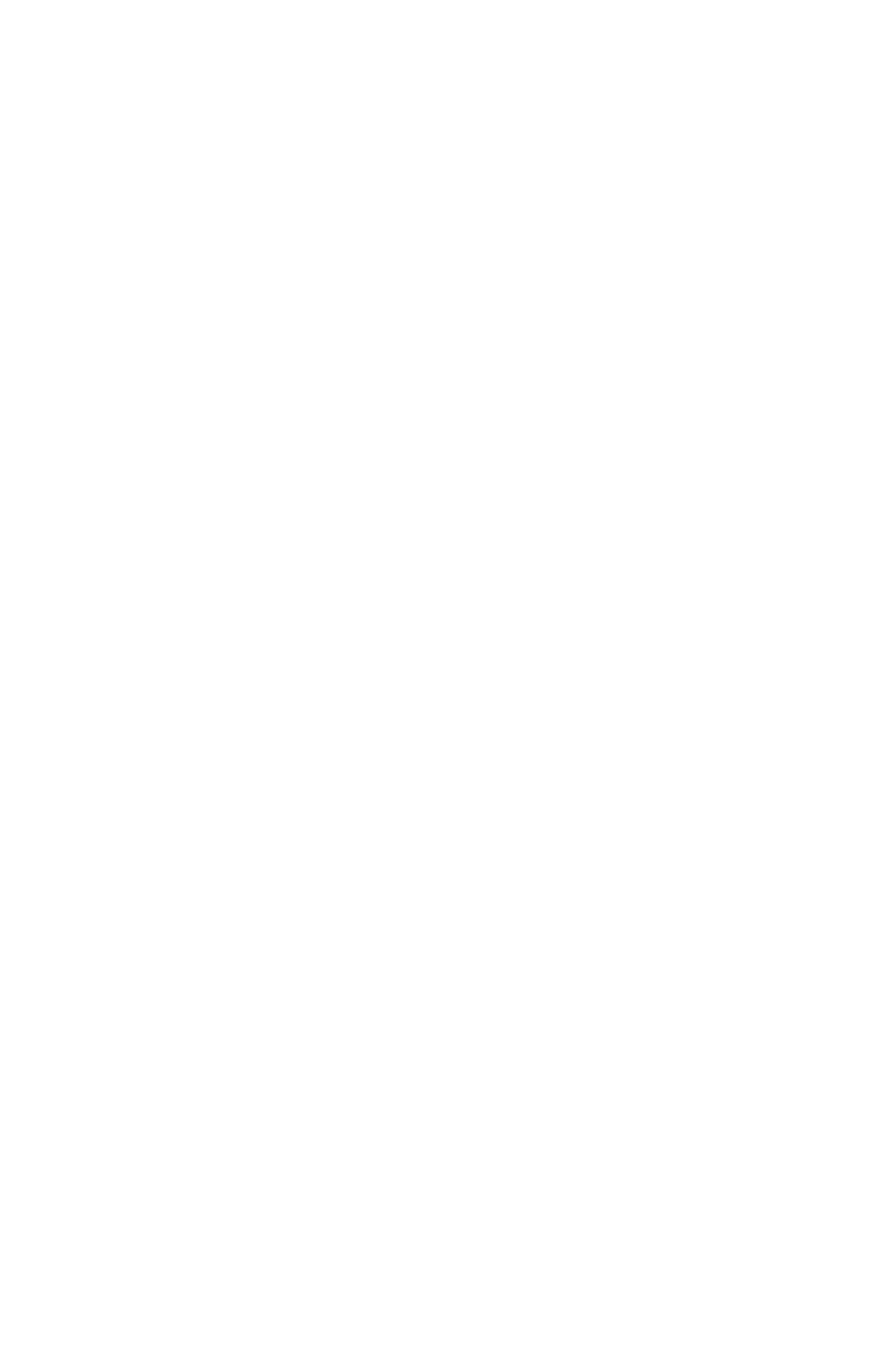

FIGURE 2.-Maximum, a verage, and minimum monthly rainfall at three climatologic stations in the Willamette Valley, 1896-1935. 
TABLE 3--Maximum, average, and minimum monthly and yearly rainfall and snowfall, in inches, at five climatologic stations in the Willamette Valley, $1896-1935$

[Data from publications of United States Weather Bureau]

\begin{tabular}{|c|c|c|c|c|c|}
\hline & Portland & Salem & Albany & Corvallis & Eugene \\
\hline $\begin{array}{l}\text { anuary: } \\
\text { Maximum } \\
\text { Average } \\
\text { Minimum }\end{array}$ & $\begin{array}{r}111.53 \\
5.87 \\
22.54\end{array}$ & $\begin{array}{r}110.17 \\
5.40 \\
1.30\end{array}$ & $\begin{array}{r}111.90 \\
6.03 \\
22.71\end{array}$ & $\begin{array}{r}13.61 \\
6.44 \\
1.99\end{array}$ & $\begin{array}{r}110.07 \\
5.47 \\
2.14\end{array}$ \\
\hline $\begin{array}{l}\text { February: } \\
\quad \text { Maximum } \\
\quad \text { A verage } \\
\quad \text { Minimum }\end{array}$ & $\begin{array}{r}111.08 \\
4.55 \\
.16\end{array}$ & $\begin{array}{r}19.47 \\
4.47 \\
.34\end{array}$ & $\begin{array}{r}12.20 \\
4.93 \\
.15\end{array}$ & $\begin{array}{r}15.23 \\
5.15 \\
.12\end{array}$ & $\begin{array}{r}12.10 \\
4.49 \\
.10\end{array}$ \\
\hline $\begin{array}{l}\text { March: } \\
\quad \text { Maximum } \\
\quad \text { Average } \\
\quad \text { Minimum }\end{array}$ & $\begin{array}{r}10.57 \\
3.94 \\
.63\end{array}$ & $\begin{array}{r}19.96 \\
3.81 \\
\quad .59\end{array}$ & $\begin{array}{r}110.44 \\
4.13 \\
\quad .58\end{array}$ & $\begin{array}{r}11.70 \\
4.16 \\
.43\end{array}$ & $\begin{array}{r}10.49 \\
3.98 \\
.40\end{array}$ \\
\hline $\begin{array}{l}\text { April: } \\
\quad \text { Maximum } \\
\text { Average } \\
\text { Minimum }\end{array}$ & $\begin{array}{r}15.36 \\
2.65 \\
.80\end{array}$ & $\begin{array}{r}16.12 \\
2.41 \\
.48\end{array}$ & $\begin{array}{r}15.55 \\
2.50 \\
.56\end{array}$ & $\begin{array}{r}16.98 \\
2.46 \\
.26\end{array}$ & $\begin{array}{r}15.89 \\
2.60 \\
.43\end{array}$ \\
\hline $\begin{array}{l}\text { May: } \\
\text { Maximum } \\
\text { Average } \\
\text { Minimum }\end{array}$ & $\begin{array}{r}14.66 \\
1.95 \\
.45\end{array}$ & $\begin{array}{r}15.54 \\
1.92 \\
.25\end{array}$ & $\begin{array}{r}5.81 \\
2.08 \\
.34\end{array}$ & $\begin{array}{r}5.71 \\
1.78 \\
.19\end{array}$ & $\begin{array}{r}4.76 \\
2.32 \\
.19\end{array}$ \\
\hline $\begin{array}{l}\text { June: } \\
\quad \text { Maximum } \\
\text { Average } \\
\text { Minimum }\end{array}$ & $\begin{array}{r}14.24 \\
1.40 \\
.07\end{array}$ & $\begin{array}{r}13.48 \\
1.18 \\
.00\end{array}$ & $\begin{array}{r}13.44 \\
1.25 \\
.02\end{array}$ & $\begin{array}{r}13.35 \\
1.12 \\
.00\end{array}$ & $\begin{array}{r}14.31 \\
1.48 \\
.02\end{array}$ \\
\hline $\begin{array}{l}\text { July: } \\
\text { Maximum } \\
\text { Average } \\
\text { Minimum }\end{array}$ & $\begin{array}{r}2.55 \\
.52 \\
.00\end{array}$ & $\begin{array}{r}2.72 \\
.39 \\
.00\end{array}$ & $\begin{array}{r}3.31 \\
.42 \\
.00\end{array}$ & $\begin{array}{r}2.34 \\
.31 \\
.00\end{array}$ & $\begin{array}{r}3.38 \\
.36 \\
.00\end{array}$ \\
\hline $\begin{array}{l}\text { August: } \\
\text { Maximum } \\
\text { Average } \\
\text { Minimum }\end{array}$ & $\begin{array}{r}3.39 \\
.64 \\
\text { Trace }\end{array}$ & $\begin{array}{r}2.91 \\
.45 \\
.00\end{array}$ & $\begin{array}{r}3.20 \\
.56 \\
. .00\end{array}$ & $\begin{array}{r}2.76 \\
.46 \\
.00\end{array}$ & $\begin{array}{r}3.14 \\
.49 \\
.00\end{array}$ \\
\hline $\begin{array}{l}\text { September: } \\
\text { Maximum } \\
\text { Average...... } \\
\text { Minimum }\end{array}$ & $\begin{array}{l}5.52 \\
1.88 \\
2.11\end{array}$ & $\begin{array}{r}4.84 \\
1.71 \\
.01\end{array}$ & $\begin{array}{r}\mathrm{t} 5.02 \\
1.77 \\
.01\end{array}$ & $\begin{array}{r}5.40 \\
1.64 \\
\text { Trace }\end{array}$ & $\begin{array}{r}5.21 \\
\text { l. } 92 \\
.12\end{array}$ \\
\hline $\begin{array}{l}\text { October: } \\
\text { Maximum } \\
\text { A verage......... } \\
\text { Minimum. }\end{array}$ & $\begin{array}{r}15.50 \\
2.82 \\
2.02\end{array}$ & $\begin{array}{r}8.31 \\
2.94 \\
.00\end{array}$ & $\begin{array}{r}16.67 \\
2.93 \\
.03\end{array}$ & $\begin{array}{r}7.60 \\
2.94 \\
\text { Trace }\end{array}$ & $\begin{array}{r}7.77 \\
2.75 \\
2.04\end{array}$ \\
\hline $\begin{array}{l}\text { November: } \\
\text { Maximum } \\
\text { Average } \\
\text { Minimum }\end{array}$ & $\begin{array}{r}13.12 \\
6.54 \\
2.67\end{array}$ & $\begin{array}{r}16.99 \\
6.21 \\
2.63\end{array}$ & $\begin{array}{r}12.50 \\
6.92 \\
2.53\end{array}$ & $\begin{array}{r}16.69 \\
7.09 \\
2.30\end{array}$ & $\begin{array}{r}11.86 \\
5.96 \\
2.80\end{array}$ \\
\hline $\begin{array}{l}\text { December: } \\
\text { Maximum } \\
\text { Average } \\
\text { Minimum }\end{array}$ & $\begin{array}{r}17.54 \\
6.50 \\
22.56\end{array}$ & $\begin{array}{r}17.54 \\
6.33 \\
1.95\end{array}$ & $\begin{array}{r}113.84 \\
6.38 \\
1.91\end{array}$ & $\begin{array}{r}14.15 \\
6.85 \\
2.33\end{array}$ & $\begin{array}{r}13.38 \\
5.77 \\
2.01\end{array}$ \\
\hline $\begin{array}{l}\text { Annual } \\
\text { Maximum } \\
\text { Average } \\
\text { Minimum }\end{array}$ & $\begin{array}{r}152.85 \\
339.26 \\
\quad 26.11\end{array}$ & $\begin{array}{r}154.93 \\
37.22 \\
24.56\end{array}$ & $\begin{array}{r}157.15 \\
39.90 \\
26.67\end{array}$ & $\begin{array}{r}157.76 \\
40.40 \\
23.68\end{array}$ & $\begin{array}{r}51.50 \\
37.59 \\
26.39\end{array}$ \\
\hline
\end{tabular}

1 Greater in 1 or more additional years of record.

2 Less in 1 or more additional vears of record.

3 For the 66-year period $1872-1937$ the yearly a verage is 42.24 inches. 
TABLE 4.-Maximum rates of precipitation, in inches, at Portland, Oreg., in the period ending with 1930

[From publication of United States Weather Bureau]

\begin{tabular}{|c|c|c|c|c|c|c|c|c|c|c|c|c|c|c|}
\hline Duration & 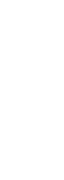 & 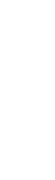 & 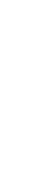 & 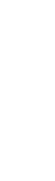 & $\frac{\overline{\mathbf{z}}}{4}$ & $\sum^{\vec{\sigma}}$ & $\stackrel{\Phi}{\Xi}$ & $\stackrel{\overrightarrow{3}}{\overrightarrow{3}}$ & 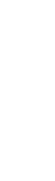 & 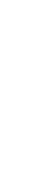 & $\begin{array}{l}\stackrel{\Xi}{0} \\
\stackrel{0}{0} \\
\stackrel{0}{0} \\
0\end{array}$ & 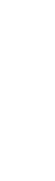 & 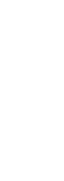 & 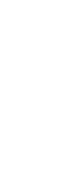 \\
\hline $\begin{array}{l}5 \text { minutes } \\
10 \text { minutes } \\
15 \text { minutes } \\
30 \text { minutes } \\
1 \text { hour } \\
2 \text { hours. } \\
24 \text { hours } \\
72 \text { hours } \\
\end{array}$ & $\begin{array}{l}41 \\
41 \\
41 \\
41 \\
41 \\
41 \\
59 \\
59\end{array}$ & $\begin{array}{r}0.14 \\
.20 \\
.23 \\
.27 \\
.50 \\
.92 \\
6.86 \\
7.74\end{array}$ & $\begin{array}{r}0.13 \\
.18 \\
.20 \\
.24 \\
.32 \\
.50 \\
3.81 \\
7.39\end{array}$ & $\begin{array}{r}0.10 \\
.17 \\
.20 \\
.28 \\
.46 \\
.64 \\
2.44 \\
5.49\end{array}$ & $\begin{array}{r}0.17 \\
.26 \\
.34 \\
.35 \\
.43 \\
.55 \\
1.92 \\
2.16\end{array}$ & $\begin{array}{r}0.21 \\
.37 \\
.37 \\
.37 \\
.59 \\
.59 \\
1.83 \\
2.75\end{array}$ & $\begin{array}{r}0.28 \\
.46 \\
.64 \\
1.06 \\
1.31 \\
1.74 \\
2.07 \\
2.22\end{array}$ & $\begin{array}{r}0.26 \\
.31 \\
.39 \\
.69 \\
.82 \\
.93 \\
1.35 \\
2.12\end{array}$ & $\begin{array}{r}0.40 \\
.70 \\
.83 \\
1.10 \\
1.25 \\
1.25 \\
1.75 \\
1.77\end{array}$ & $\begin{array}{r}0.28 \\
.53 \\
.61 \\
.69 \\
.87 \\
1.09 \\
2.88 \\
3.28\end{array}$ & $\begin{array}{r}0.21 \\
.22 \\
.23 \\
.32 \\
.49 \\
.64 \\
2.96 \\
5.42\end{array}$ & $\begin{array}{r}0.14 \\
.23 \\
.23 \\
.28 \\
.49 \\
.84 \\
4.43 \\
6.71\end{array}$ & $\begin{array}{r}0.14 \\
.18 \\
.20 \\
.28 \\
.42 \\
.64 \\
7.66 \\
10.81\end{array}$ & $\begin{array}{r}0.40 \\
.70 \\
.83 \\
1.10 \\
1.31 \\
1.74 \\
7.66 \\
10.81\end{array}$ \\
\hline
\end{tabular}

The average yearly precipitation is about 40 inches over most of the lowland, but in the foothills to the west and east it increases to about 70 inches. Table 3 shows the monthly averages and yearly averages for a common 40-year period, from 1896 to 1935, for the five climatologic stations that lie along the middle of the valley. With table 5, it shows further that during the 40-year period the yearly precipitation has ranged from about 60 to about 145 percent of the average. At Portland, whose record of yearly precipitation began in 1872 , the greatest yearly precipitation yet recorded is even greater, 171 percent of the 40-year average.

TABLE 5.-Yearly rainfall and snowfall, in inches, and surplus (+) or deficiency (-) with respect to 40-year average, at five climatologic stations in the Willamette Valley, 1872-1937

[Data from publications of United States Weather Bureau]

\begin{tabular}{|c|c|c|c|c|c|c|c|c|c|c|}
\hline \multirow[b]{2}{*}{ Year } & \multicolumn{2}{|c|}{ Portland } & \multicolumn{2}{|c|}{ Salem } & \multicolumn{2}{|c|}{ Albany } & \multicolumn{2}{|c|}{ Corvallis } & \multicolumn{2}{|c|}{ Eugene } \\
\hline & $\begin{array}{l}\text { Rain- } \\
\text { fall }\end{array}$ & $\begin{array}{l}\text { Sur- } \\
\text { plus or } \\
\text { defi- } \\
\text { ciency }\end{array}$ & $\begin{array}{l}\text { Rain- } \\
\text { fall }\end{array}$ & $\begin{array}{l}\text { Sur- } \\
\text { plus or } \\
\text { defi- } \\
\text { ciency }\end{array}$ & $\begin{array}{l}\text { Rain- } \\
\text { fall }\end{array}$ & $\begin{array}{l}\text { Sur- } \\
\text { plus or } \\
\text { defi- } \\
\text { ciency }\end{array}$ & $\begin{array}{l}\text { Rain- } \\
\text { fall }\end{array}$ & $\begin{array}{c}\text { Sur- } \\
\text { plus or } \\
\text { defi- } \\
\text { ciency }\end{array}$ & $\begin{array}{l}\text { Rain- } \\
\text { fall }\end{array}$ & $\begin{array}{l}\text { Sur- } \\
\text { plus or } \\
\text { defi- } \\
\text { ciency }\end{array}$ \\
\hline A verage 1 & 39.26 & $-\cdots$ & 37.22 & $\ldots$ & 39.90 & $\ldots$ & 40.40 & - & 37.59 & $\ldots$ \\
\hline $1872 \ldots$ & $\begin{array}{l}46.90 \\
50.52 \\
46.17 \\
60.10\end{array}$ & $\begin{array}{r}+7.64 \\
+11.26 \\
+6.91 \\
+20.84\end{array}$ & $-\cdots$ & - & & & & 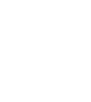 & - & 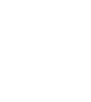 \\
\hline $\begin{array}{l}1876 \ldots \ldots \\
1877 \\
1878 \\
1879 \\
1880\end{array}$ & $\begin{array}{l}54.94 \\
58.30 \\
47.70 \\
62.22 \\
51.87\end{array}$ & $\begin{array}{r}+15.68 \\
+19.04 \\
+8.44 \\
+22.96 \\
+12.61\end{array}$ & & $-\cdots$ & $\begin{array}{r}0.14 \\
41.57\end{array}$ & $\begin{array}{r}+10.24 \\
+1.67\end{array}$ & $\begin{array}{l}\cdots \\
\cdots- \\
\cdots- \\
-\cdots\end{array}$ & $\mid \begin{array}{ll}-\cdots \\
-\cdots \\
-\cdots \\
-\cdots\end{array}$ & $\begin{array}{l}\cdots \\
-- \\
\cdots \\
\cdots\end{array}$ & $\mid \begin{array}{ll}-\cdots \\
-\cdots \\
-\cdots \\
-\cdots \\
-\cdots\end{array}$ \\
\hline $\begin{array}{l}1881 \\
1882 \\
1883 \\
1884 \\
1885\end{array}$ & $\begin{array}{l}57.05 \\
67.24 \\
51.45 \\
38.31 \\
39.59\end{array}$ & $\begin{array}{r}+17.79 \\
+27.98 \\
+12.19 \\
-.95 \\
+.33\end{array}$ & $-\cdots$ & 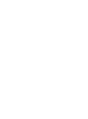 & $\begin{array}{l}59.59 \\
45.96 \\
39.39 \\
45.22 \\
36.93\end{array}$ & $\begin{array}{r}+19.69 \\
+6.06 \\
-.51 \\
+5.32 \\
-2.97\end{array}$ & $-\cdots$ & $\begin{array}{ll}-- \\
-- \\
-- \\
- \\
-\end{array}$ & - & $\begin{array}{ll}--1 \\
-\cdots \\
-\cdots\end{array}$ \\
\hline
\end{tabular}

11896 to 1935 , inclusive. 
TABLE 5.-Yearly rainfall and snowfall, in inches, and surplus (+) or deficiency

(-) with respect to 40 -year average, at five climatologic stations in the Willamette

Valley, 1872-1937-Continued

\begin{tabular}{|c|c|c|c|c|c|c|c|c|c|c|}
\hline \multirow[b]{2}{*}{ Year } & \multicolumn{2}{|c|}{ Portlànd } & \multicolumn{2}{|c|}{ Salem } & \multicolumn{2}{|c|}{ Albany } & \multicolumn{2}{|c|}{ Corvallis } & \multicolumn{2}{|c|}{ Eugene } \\
\hline & $\begin{array}{c}\text { Rain- } \\
\text { fall }\end{array}$ & $\begin{array}{c}\text { Sur- } \\
\text { plus or } \\
\text { def- } \\
\text { ciency }\end{array}$ & $\begin{array}{l}\text { Rain- } \\
\text { fall }\end{array}$ & $\begin{array}{l}\text { Sur- } \\
\text { plus or } \\
\text { defi- } \\
\text { ciency }\end{array}$ & $\begin{array}{c}\text { Rain- } \\
\text { fall }\end{array}$ & $\begin{array}{c}\text { Sur- } \\
\text { plus or } \\
\text { defi- } \\
\text { ciency }\end{array}$ & $\begin{array}{c}\text { Rain- } \\
\text { fall }\end{array}$ & $\begin{array}{l}\text { Sur- } \\
\text { plus or } \\
\text { defi- } \\
\text { ciency }\end{array}$ & $\begin{array}{c}\text { Rain- } \\
\text { fall }\end{array}$ & $\begin{array}{c}\text { Sur- } \\
\text { plus or } \\
\text { defi- } \\
\text { ciency }\end{array}$ \\
\hline $\begin{array}{l}1886 \\
1887 . \\
1888 \\
1899 \\
1890\end{array}$ & $\begin{array}{l}38.76 \\
54.17 \\
38.76 \\
31.76 \\
40.38\end{array}$ & $\begin{array}{r}-.50 \\
+14.91 \\
-.50 \\
-7.50 \\
+1.12\end{array}$ & & & $\begin{array}{l}38.38 \\
57.62 \\
35.91 \\
33.84 \\
40.02\end{array}$ & $\begin{array}{r}-1.52 \\
+17.72 \\
-3.99 \\
-6.06 \\
+.12\end{array}$ & 34.82 & -5.58 & & \\
\hline $\begin{array}{l}1891 \ldots \\
1892 . \\
1893 \ldots \\
1894 \ldots \\
1895\end{array}$ & $\begin{array}{l}47.41 \\
33.58 \\
39.03 \\
39.32 \\
30.76\end{array}$ & $\begin{array}{r}+8.15 \\
-5.68 \\
-.23 \\
+.06 \\
-8.50\end{array}$ & $\begin{array}{l}49.08 \\
48.62 \\
47.13\end{array}$ & $\begin{array}{r}+11.86 \\
+11.40 \\
+9.91\end{array}$ & $\begin{array}{l}53.39 \\
37.93 \\
46.07 \\
49.86 \\
47.48\end{array}$ & $\begin{array}{r}+13.49 \\
-1.97 \\
+6.17 \\
+9.96 \\
+7.58\end{array}$ & $\begin{array}{l}44.99 \\
31.88 \\
43.04 \\
46.44 \\
43.74\end{array}$ & $\begin{array}{l}+4.59 \\
-8.52 \\
+2.64 \\
+6.04 \\
+3.34\end{array}$ & $\begin{array}{r}44.76 \\
29.25 \\
42.02 \\
37.03\end{array}$ & $\begin{array}{r}+7.17 \\
-8.34 \\
+4.43 \\
-.56\end{array}$ \\
\hline $\begin{array}{l}1896 \ldots \\
1897 \ldots \\
1898 \ldots \\
1899 \ldots \\
1900 \ldots\end{array}$ & $\begin{array}{l}44.13 \\
43.01 \\
33.90 \\
42.21 \\
38.22\end{array}$ & $\begin{array}{l}+4.87 \\
+3.75 \\
-5.36 \\
+2.95 \\
-1.04\end{array}$ & $\begin{array}{l}54.93 \\
46.53 \\
34.19 \\
48.12 \\
37.22\end{array}$ & $\begin{array}{r}+17.71 \\
+9.31 \\
-3.03 \\
+10.90 \\
.00\end{array}$ & $\begin{array}{l}57.15 \\
50.19 \\
36.22 \\
46.99 \\
38.88\end{array}$ & $\begin{array}{r}+17.25 \\
+10.29 \\
-3.68 \\
+7.09 \\
-1.02\end{array}$ & $\begin{array}{l}57.76 \\
43.61 \\
35.02 \\
46.69 \\
38.49\end{array}$ & $\begin{array}{r}+17.36 \\
+3.21 \\
-5.38 \\
+9.29 \\
-1.91\end{array}$ & $\begin{array}{l}48.47 \\
40.55 \\
27.16 \\
41.28 \\
40.89\end{array}$ & $\begin{array}{r}+10.88 \\
+2.96 \\
-10.43 \\
+3.69 \\
+3.30\end{array}$ \\
\hline $\begin{array}{l}1901 \ldots \\
1902 \\
1903 \\
1904 \\
1905 \\
\end{array}$ & $\begin{array}{l}41.05 \\
50.15 \\
35.62 \\
46.37 \\
34.10\end{array}$ & $\begin{array}{r}+1.79 \\
+10.89 \\
-3.64 \\
+7.11 \\
-5.16\end{array}$ & $\begin{array}{l}37.42 \\
42.55 \\
24.56 \\
42.08 \\
28.49\end{array}$ & $\begin{array}{r}+.20 \\
+5.33 \\
-12.66 \\
+4.86 \\
-8.73\end{array}$ & $\begin{array}{l}38.89 \\
52.94 \\
33.65 \\
49.41 \\
30.19\end{array}$ & $\begin{array}{r}-1.01 \\
+13.04 \\
-6.25 \\
+9.51 \\
-9.71\end{array}$ & $\begin{array}{l}41.36 \\
54.02 \\
34.13 \\
56.21 \\
34.39\end{array}$ & $\begin{array}{r}+.96 \\
+13.62 \\
-6.27 \\
+15.81 \\
-6.01\end{array}$ & $\begin{array}{l}40.01 \\
51.50 \\
34.21 \\
50.71 \\
29.95\end{array}$ & $\begin{array}{r}+2.42 \\
+13.91 \\
-3.38 \\
+13.12 \\
-7.64\end{array}$ \\
\hline $\begin{array}{l}1906 \ldots \\
1907 . \\
1908- \\
1909 \\
1910 \\
\end{array}$ & $\begin{array}{l}43.29 \\
42.89 \\
34.37 \\
43.75 \\
38.65\end{array}$ & $\begin{array}{r}+4.03 \\
+3.63 \\
-4.89 \\
+4.49 \\
-.61\end{array}$ & $\begin{array}{l}34.01 \\
37.10 \\
26.76 \\
39.59 \\
31.89\end{array}$ & $\begin{array}{r}-3.21 \\
-.12 \\
-10.46 \\
+2.37 \\
-5.33\end{array}$ & $\begin{array}{l}40.72 \\
44.44 \\
28.54 \\
43.70 \\
38.30\end{array}$ & $\begin{array}{r}+.82 \\
+4.54 \\
-11.36 \\
+3.80 \\
-1.60\end{array}$ & $\begin{array}{l}45.71 \\
50.23 \\
33.29 \\
50.31 \\
37.64\end{array}$ & $\begin{array}{l}+5.31 \\
+9.83 \\
-7.11 \\
+9.91 \\
-2.76\end{array}$ & $\begin{array}{l}37.55 \\
46.27 \\
27.22 \\
39.16 \\
33.20\end{array}$ & $\begin{array}{r}-.04 \\
+8.68 \\
-10.37 \\
+1.57 \\
-4.39\end{array}$ \\
\hline $\begin{array}{l}1911 \ldots \\
1912 \\
1913 \\
1914 \ldots \\
1915 \ldots\end{array}$ & $\begin{array}{l}33.28 \\
43.47 \\
36.30 \\
36.67 \\
41.30\end{array}$ & $\begin{array}{l}-5.98 \\
+4.21 \\
-2.96 \\
-2.59 \\
+2.04\end{array}$ & $\begin{array}{l}27.55 \\
35.72 \\
31.75 \\
35.87 \\
32.55\end{array}$ & $\begin{array}{l}-9.67 \\
-1.50 \\
-5.47 \\
-1.35 \\
-4.67\end{array}$ & $\begin{array}{l}34.11 \\
46.68 \\
35.37 \\
44.24 \\
40.12\end{array}$ & $\begin{array}{r}-5.79 \\
+6.78 \\
-4.53 \\
+4.34 \\
+.22\end{array}$ & $\begin{array}{l}38.46 \\
46.81 \\
35.76 \\
44.13 \\
36.82\end{array}$ & $\begin{array}{l}-1.94 \\
+6.41 \\
-4.64 \\
+3.73 \\
-3.58\end{array}$ & $\begin{array}{l}30.21 \\
43.58 \\
31.66 \\
35.22 \\
39.83\end{array}$ & $\begin{array}{l}-7.38 \\
+5.99 \\
-5.93 \\
-2.37 \\
+2.24\end{array}$ \\
\hline $\begin{array}{l}1916 \ldots \\
1917 . \\
1918 \ldots \\
1919 \ldots \\
1920 \ldots\end{array}$ & $\begin{array}{l}45.77 \\
40.50 \\
31.50 \\
45.70 \\
41.17\end{array}$ & $\begin{array}{l}+6.51 \\
+1.24 \\
+7.76 \\
+6.44 \\
+1.91\end{array}$ & $\begin{array}{l}45.76 \\
32.82 \\
27.92 \\
41.01 \\
40.78\end{array}$ & $\begin{array}{l}+8.54 \\
-4.40 \\
-9.30 \\
+3.79 \\
+3.56\end{array}$ & $\begin{array}{l}47.74 \\
39.50 \\
34.31 \\
52.85 \\
38.59\end{array}$ & $\begin{array}{r}+7.84 \\
-.40 \\
-5.59 \\
+12.95 \\
-1.31\end{array}$ & $\begin{array}{l}46.65 \\
39.13 \\
33.52 \\
50.57 \\
37.12\end{array}$ & $\begin{array}{r}+6.25 \\
-1.27 \\
-6.88 \\
+10.17 \\
-3.28\end{array}$ & $\begin{array}{l}48.18 \\
41.17 \\
31.40 \\
46.27 \\
40.67\end{array}$ & $\begin{array}{r}+10.59 \\
+3.58 \\
-6.19 \\
+8.68 \\
+3.08\end{array}$ \\
\hline $\begin{array}{l}1921 \\
1922 \\
1923 \\
1924 \\
1925 \ldots\end{array}$ & $\begin{array}{l}43.21 \\
38.76 \\
32.81 \\
31.22 \\
31.36\end{array}$ & $\begin{array}{r}+3.95 \\
-.50 \\
-6.45 \\
-8.04 \\
-7.90\end{array}$ & $\begin{array}{l}43.12 \\
38.03 \\
33.53 \\
33.15 \\
33.32\end{array}$ & $\begin{array}{r}+5.90 \\
+.81 \\
-3.69 \\
-4.07 \\
-3.90\end{array}$ & $\begin{array}{l}44.59 \\
38.99 \\
36.00 \\
33.72 \\
34.18\end{array}$ & $\begin{array}{r}+4.69 \\
-.91 \\
-3.90 \\
-6.18 \\
-5.72\end{array}$ & $\begin{array}{l}41.05 \\
38.45 \\
37.07 \\
37.87 \\
34.07\end{array}$ & $\begin{array}{r}+.65 \\
-1.95 \\
-3.33 \\
-2.53 \\
-6.33\end{array}$ & $\begin{array}{l}36.60 \\
37.06 \\
33.05 \\
35.88 \\
33.70\end{array}$ & $\begin{array}{r}-.99 \\
-.53 \\
-4.54 \\
-1.71 \\
-3.89\end{array}$ \\
\hline $\begin{array}{l}1926 \\
1927 \\
1928 \\
1929 \\
1930\end{array}$ & $\begin{array}{l}41.17 \\
45.78 \\
34.69 \\
26.11 \\
27.16\end{array}$ & $\begin{array}{r}+1.91 \\
+6.52 \\
-4.57 \\
-13.15 \\
-12.10\end{array}$ & $\begin{array}{l}39.17 \\
43.43 \\
38.03 \\
26.40 \\
30.05\end{array}$ & $\begin{array}{r}+1.95 \\
+6.21 \\
+.81 \\
-10.82 \\
-7.17\end{array}$ & $\begin{array}{l}37.87 \\
44.60 \\
37.97 \\
27.03 \\
26.67\end{array}$ & $\begin{array}{r}-2.03 \\
+4.70 \\
-1.93 \\
-12.87 \\
-13.23\end{array}$ & $\begin{array}{l}40.04 \\
47.87 \\
39.87 \\
24.45 \\
23.68\end{array}$ & $\begin{array}{r}-.36 \\
+7.47 \\
-.53 \\
-15.95 \\
-16.72\end{array}$ & $\begin{array}{l}41.16 \\
43.58 \\
34.91 \\
32.10 \\
26.39\end{array}$ & $\begin{array}{r}+3.57 \\
+5.99 \\
-2.68 \\
-5.49 \\
-11.20\end{array}$ \\
\hline $\begin{array}{l}1931 \ldots \ldots \\
1932 \\
1933 \\
1934 \\
1935\end{array}$ & $\begin{array}{l}42.68 \\
39.98 \\
52.85 \\
45.98 \\
29.19\end{array}$ & $\begin{array}{r}+3.42 \\
+.72 \\
+13.59 \\
+6.72 \\
-10.07\end{array}$ & $\begin{array}{l}48.64 \\
42.06 \\
52.56 \\
39.41 \\
30.90\end{array}$ & $\begin{array}{r}+11.42 \\
+4.84 \\
+15.34 \\
+2.19 \\
-6.32\end{array}$ & $\begin{array}{l}41.89 \\
40.30 \\
43.20 \\
38.10 \\
27.77\end{array}$ & $\begin{array}{r}+1.99 \\
+.40 \\
+3.30 \\
-1.80 \\
-12.13\end{array}$ & $\begin{array}{l}39.13 \\
36.94 \\
42.59 \\
35.42 \\
26.35\end{array}$ & $\begin{array}{r}-1.27 \\
-3.46 \\
+2.19 \\
-4.98 \\
-14.05\end{array}$ & $\begin{array}{l}36.78 \\
36.22 \\
36.01 \\
34.99 \\
28.91\end{array}$ & $\begin{array}{l}-.81 \\
-1.37 \\
-1.58 \\
-2.60 \\
-8.68\end{array}$ \\
\hline $\begin{array}{l}1936 \\
1937\end{array}$ & $\begin{array}{l}34.29 \\
56.85\end{array}$ & $\begin{array}{r}-4.97 \\
+17.59\end{array}$ & $\begin{array}{l}33.61 \\
63.50\end{array}$ & $\begin{array}{r}-3.61 \\
+26.28\end{array}$ & $\begin{array}{l}32.23 \\
57.44\end{array}$ & $\begin{array}{r}-7.67 \\
+17.54\end{array}$ & $\begin{array}{l}32.11 \\
58.06\end{array}$ & $\begin{array}{r}-8.29 \\
+17.66\end{array}$ & $\begin{array}{l}34.02 \\
55.21\end{array}$ & $\begin{array}{r}-3.57 \\
+17.62\end{array}$ \\
\hline
\end{tabular}


The prevailing wind is from the north and northwest in summer and from the south and southwest in winter, but the variation in wind direction is moderate. Winds from the south and southwest attain the greatest velocities but are seldom destructive. In and near the mouth of the Columbia Gorge east winds prevail at times; these are cold in winter and warm at other times.

The climatic features of the lowland and adjacent foothills, just described, do not apply to the mountainous terrane on either side. Thus, to the east, the extensive west slope of the Cascade Range is, on the average, appreciably cooler than the lowland, though the extremes of temperature are not materially lower. There the average yearly temperature ranges from about $44^{\circ}$ to $51^{\circ} \mathrm{F}$. On this mountain slope the yearly precipitation is generally more than 50 inches, and some small areas near Mount Hood receive more than 100 inches. In some winters a large part of the precipitation is rain, but considerable snow falls at times; the average yearly snowfall at the several stations ranges from about 14 inches to 300 inches or more. Most of the higher part of the range probably has an average yearly temperature of less than $42^{\circ}$; some of the highest peaks probably less than $32^{\circ}$. Nevertheless, the highest temperatures are greater than those along the Pacific coast, to the west, and the lowest temperatures are not so low as at some localities on the high semiarid plains to the east. Snow accumulates to great depths, the average at recording stations being from 200 to 300 inches and more. Permanent snow fields and alpine glaciers (see pl. 4, A) exist on several of the highest peaks.

Across the valley, on the lower and less extensive east slope of the Coast Range, there is much variation in climate. The average yearly temperature is generally less than $50^{\circ}$ and the average yearly precipitation is 50 inches or more. In winter there is considerable freezing weather, and at some places snow accumulates to a considerable depth.

\section{GEOLOGY AND HYDROLOGY OF THE CONSOLIDATED AND SEMICONSOLIDATED ROCKS}

GENERAL CHARACTER, SUCCESSION, AND AGE OF THE ROCKS

The rocks that form the foothills and mountains on both sides of the central Willamette lowland and that pass beneath the unconsolidated deposits of the lowland include fine-grained marine and terrestrial sedimentary rocks, fragmental and nonfragmental igneous rocks, and some coarse-grained terrestrial deposits. The marine sedimentary rocks are chiefly shale and earthy sandstone of Eocene, Oligocene, and Miocene (?) age. These make up the greater part of the Coast Range west of the lowland, where they are associated with basaltic extrusive rocks of Miocene (?) age and with intrusive rocks. This 
succession of marine sedimentary rocks and igneous rocks extends eastward beneath nearly all the lowland and, at some places, into the lowest foothills of the Cascade Range. At the very southern end of the central lowland, the sedimentary rocks of latest Eocene and Oligocene age are partly marine and partly nonmarine in origin, as though the seashore of that time oscillated repeatedly across that area. Fragmental and nonfragmental volcanic rocks form nearly all the Cascade Range except the lowest part of the most westerly foothills. These rocks are the products of volcanic activity that began in the Oligocene (?) epoch, continued itermittently during the remainder of the Tertiary and far into the Pleistocene, then waned in latest Pleistocene or Recent time. The rocks are largely basalt or andesite and include both nonfragmental flow rocks and fragmental rocks that range from unstratified or poorly stratified coarse agglomerate to finegrained. well-stratified tuff. The coarse-grained terrestrial deposits occur in the northeastern part of the Willamette Valley, in the vicinity of Portland. They are underlain and overlain by volcanic rocks and are of Pliocene or early Pleistocene age.

The accompanying table shows the character, stratigraphic sequence, and general water-bearing properties of the rock formations that occur aiong the margin of the central Willamette lowland. These several formations were not mapped separately but are included under one symbol on plates 1 and 2 .

\section{STRUCTURE}

The structure of the rocks that surround the central lowland of the Willamette River Basin was not mapped by the writer. No horizon markers can be traced over extensive areas, and dense forest and brush and a thick mantle of nondescript detritus cover most of the basin except the highest part of the Cascade Range. Nevertheless, some general features of the structure can be summarized. Probably late in the Miocene epoch the region was gently compressed and its rocks were thrown into broad open folds, seemingly with few or no normal faults of consequence and, so far as is now known, with no close or overturned folds or thrust faults. As is pointed out by Callaghan and Buddington, ${ }^{6}$ with citations to earlier workers, the most conspicuous structural feature of the rocks adjacent to the central lowland is the gentle eastward or northeastward dip, commonly about $10^{\circ}$, that prevails south of the Santiam River. To the north, along the east flank of the lowland, the general dip changes to the northwest and maintains that direction nearly or quite to the Columbia River. West of the lowland, in the flank of the Coast Range, the prevailing dip continues generally eastward. These structural features coordi-

\footnotetext{
6 Callagh, Eugene; apd Buddington, A. F., Metalliferous mineral deposits of the Cascade Range in Oregon: U. S. Geol. Survey Bull. 893, pp. 18-20, 1938.
} 
nate with the western two of four broad folds that have been traced by Thayer ${ }^{7}$ in the region east of Salem; in order from the west, these two folds are the Willamette syncline and the Mehama anticline. The projected axes of these folds diverge northward from a common point near Eugene; the syncline trends about N. $15^{\circ} \mathrm{E}$. through the Stayton Basin to Portland, whereas the anticline trends about N. $30^{\circ}$ E. toward Wyeth, which is on the Columbia River some 40 miles east of Portland. In the region between the Santiam and Clackamas Rivers, both axes plunge gradually northward. Thus, at the southern end of the lowland the two folds begin as minor flexures on the general monocline near Eugene; toward the north they gradually broaden and increase in amplitude.

Immediately west of Portland the Tualatin Valley discloses structural features seemingly unlike any others now known in the regiona relatively narrow anticline and a sharply folded syncline that plunge about S. $45^{\circ} \mathrm{E}$. across the axies of the broad regional folds just described. The syncline may be faulted. The form of these structural features is not known in detail, especially in the vicinity of Oregon City, where they meet the Willamette syncline, described by Thayer. In general, the Tualatin Valley appears to have formed along the anticlinal axis, where erosion breached the resistant basalt of Miocene age and then planed rather widely into the underlying shale.

All the structural features so far described are tentatively ascribed to the one period of deformation late in the Miocéne epoch. However, the region probably has not been wholly stable in all subsequent time, for during the Pleistocene or glacial epoch much of the Pacific coast region in Oregon and Washington has oscillated through a vertical range of 1,000 feet or more with respect to present sea level. Some features of the Willamette River Basin suggest that the region was again deformed just before the bulk of the unconsolidated valley fill was deposited, possibly between the two periods of sedimentation that are represented by the terrace deposits and the older alluvium. (See pp. 28,32.) However, this late deformation cannot now be demonstrated with assurance.

7 Thayer, T. P., Structure of the North Santiam River section of the Cascade Mountains in Oregon: Jour. Geology, vol. 44, pp. 701-716, 1936. 
TABLE 6.-Stratigraphic sections añd generial water-bearing properties of consolidated IStratigraphy largely after Hodge $(1933,1938)$, Schenok

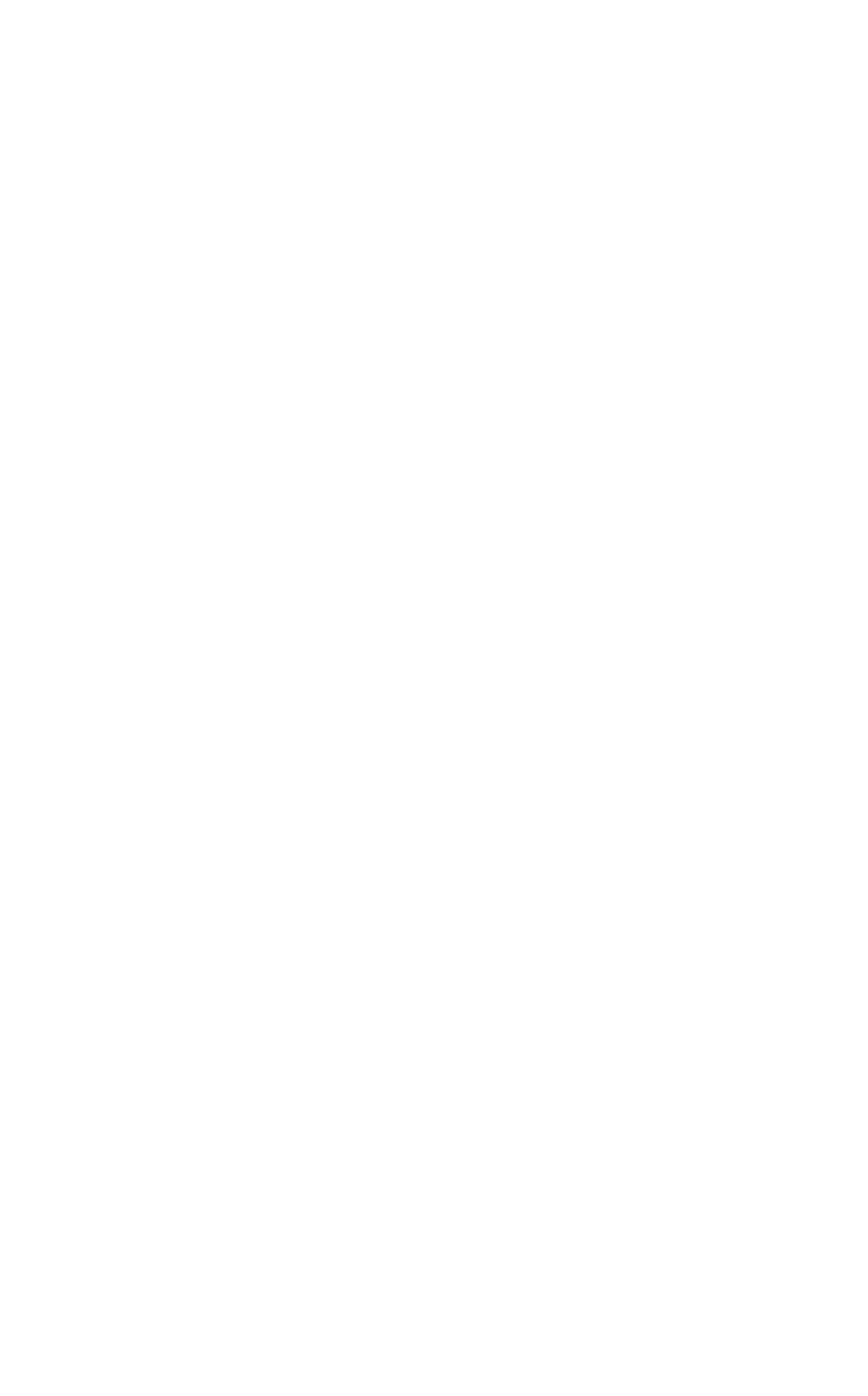


and semiconsolidated rocks bounding the central lowland of the Willamette River Basin (1927, 1933, 1936), W. D. Smith (1924), and Thayer (1933)]

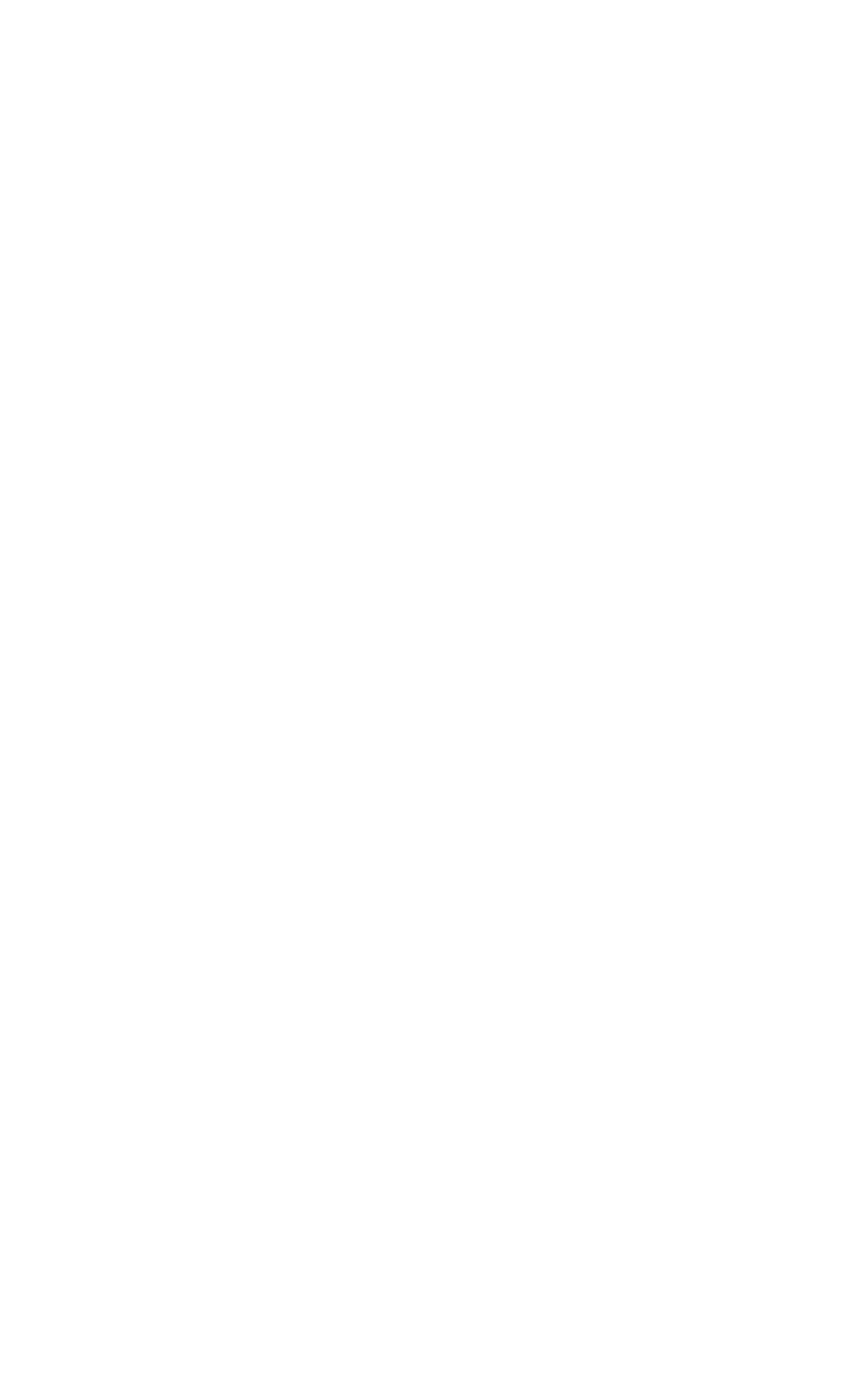




\section{WATER-BEARING PROPERTIES}

The youngest or uppermost consolidated and semiconsolidated rocks - the Cascade, Rhododendron, and Troutdale formations of Hodge--border and underlie only the northernmost part of the central lowland, from Portland southward to Oregon City and beneath the Molalla pediment. The Cascade and Rhododendron formations, which are volcanic, form outliers in east Portland, such as Rocky Butte, part of Mount Tabor, and Kelly Butte; a few miles to the south, they also form Mount Scott and the contiguous volcanic terrane that lies between Johnson Creek and the Clackamas River. Little is known regarding the water-bearing properties of these two formations. The flow rocks and accompanying scoria yield some water from discontinuous pervious zones, but at most places not copiously. However, must of the agglomerate and tuff is derise and nearly impervious. Much of the detrital Troutdale formation of Hodge is composed of unassorted particles and is sufficiently consolidated to be nearly impervious. In some coarse-grained beds, however, the particles are somewhat assorted as to size, and so the beds are perhaps moderately pervious. 'This formation crops out along the west side of the Willamette River in Portland and probably underlies much of the "delta" that extends eastward from the Willamette! River to and beyond the edge of the area that is shown on plate 1 . It may include part of the "cemented gravel" and certain of the lower water-bearing zones that are penetrated by the deeper wells in and near Portland. However, the data now at hand are not sufficient to show the depth, thickness, and extent of its water-bearing zones.

Of the several rock formations that surround and underlie the principal two segments of the central lowland, only one-the basalt of Miocene age (Yakima basalt and Stayton lavas of Thayer)-yields water freely over an extensive area. However, this formation is far from uniform in its water-yielding capacity. The water occurs under! two distinct conditions: First, at shallow or moderate depth from zones that have become pervious through weathering, at some places as much as 100 feet beneath the land surface; and second, from scoria-' ceous or fractured zones and granular partings at depth in the un-' weathered rock. Water in the zone of weathering is commonly. perched high above the lowland plains and affords domestic and farm supplies from small springs and wells on outlying ridges and peaks, such as the Chehalem Mountains along the south side of the Tualatin Valley in Tps. 1 and 2 S., Rs. 2 and 3 W., Saxton, Cooper, and Bull Mountains, in the southeastern part of that valley, and Petes Moun-. tain and corresponding ridges in the vicinity of Oregon City in Tps. 2 and 3 S., R. 1 E. There appear to be numerous small disconnected bodies of this perched water at various heights above the lowland, so 
that some shallow wells are either dry or are not dependable in dry years. The location and extent of these bodies of perched water cannot be traced at this time. At depth in the unweathered rock the water-bearing zones are not continuous over extensive areas and differ greatly in thickness and perviousness. For example, well 53 reached the basalt about 470 feet below the land surface and continued in that rock formation to a depth of 800 feet; its tested yield was 110 gallons a minute, and its specific capacity 1.1 gallons a minute for each foot of draw-down. On the other hand, two other wells within half a mile, Nos. 52 and 51, are not nearly so deep and yield about 500 gallons a minute each with respective specific capacities of about 7.4 and 6.4 gallons a minute for each foot of draw-down. In these three wells the water-bearing zones are below sea level and are far beneath the lowland plains. Several miles to the southeast, along the flanks of the basalt ridge that lies between the Willamette and Tualatin Rivers, wells 74 and 76 also yield several hundred gallons a minute from pervious zones far beneath the valley surface. To the west, in about the NW $1 \frac{1}{4}$ sec. 32 , T. 1 S., R. 2 W., a deep well not visited by the writer is reported to flow about 1 second foot by artesian pressure; the water is inferred to have been struck in the basalt. Not all wells, however, yield so copiously. Well 54, for example, taps the basalt below the lowland plains, but its reported ultimate capacity is only 4 gallons a minute. Drilled wells high on the basalt ridges are erratic in depth and yield. Thus, according to an unconfirmed roport, farm wells along the crest of the ridge between the Willamette and Tualatin Rivers range in depth from little more than 100 feet to as much as 300 feet. Presumably these wells were drilled only deep enough to assure dependable farm water supplies, for which purpose a yield of a few gallons a minute would be adequate. Probably the wells range so widely in depth because the pervious zones are discontinuous and because certain of the more extensive zones drain into the adjacent, valley.

To the south, in the vicinity of Salem, well 331 has a sustained yield of about $7 \frac{1}{2}$ gallons a minute from the basalt. About a mile to the southeast, on the farm of Albert Bouffleur, a well drilled into the basalt since the writer's field work is being used successfully for smallscale irrigation; this well is 8 inches in diameter and 170 feet deep and yields 285 gallons a minute. In both wells the pervious zones are in the flank of the eastward-dipping Eola Hills and slightly lower than the adjacent flood plain of the Williamette River. Still farther south, well 345 yields about 15 gallons a minute, probably from the lower part of the zone of weathering. Still fartber south, well 382 taps perched water in the basalt high above the level of the central lowland; a companion well, no. 381 , found the next water-bearing zone 265 feet lower. 
From the typical conditions just described it is clear that certain water-bearing zones in the unweathered Miocene basalt are sufficiently pervious and thick at some places to sustain withdrawals for irrigation on a moderate scale, for small public supplies, or for industrial purposes. Because these zones doubtless are discontinuous and scattered irregularly through the Miocene basalt it is not possible to foretell, for any particular place, the depth to which a well must be drilled to obtain an adequate supply of water. Neither can it be foretold whether an adequate supply exists within a practicable depth below the land surface. In general, however, the well is more likely to be successful if its site is not high above the central lowland, for then the shallower pervious zones in the basalt will not be extensively drained and the ground-water level so lowered that the pumping lift is excessive.

The two rock formations that occur next below the basalt of Miocene age - the Eugene formation and Fisher formation of Schenck and their correlatives-flank and underlie much of the central lowland from Oregon City southward to Salem and again, far to the south, near Eugene. For several tens of feet below the land surface these formations commonly are slightly pervious, owing to weathering and leaching, and so yield some water to shallow farm wells. At depth, however, they appear to be almost wholly impervious. For example, well 23, near the center of the Tualatin Valley at Hillsboro, reached the bottom of the unconsolidated material about 180 feet below the land surface and continued to a total depth of 1,619 feet, largely in "shale and hard clay." This 1,439-foot section of bedrock, which is correlated tentatively with the Pittsburg Bluff sandstone of Schenck, was reported devoid of water-bearing beds. Much farther south, at the Cottage Farm of the Oregon State Hospital, near Salem, well 385 was drilled to a depth of 1,006 feet in sedimentary rocks without finding water; at least the upper strata penetrated by this well represent the Illahe formation of Thayer. At the very southern end of the valley, near Eugene, the six wells, 722 to 727, are drilled in the Eugene or Fisher formation to depths between 225 and 3,000 feet; three of these wells are barely adequate for household supply and the remaining three, which include the deepest two, proved inadequate. Thus deep drilling to tap these bedrock formations beneath the unconsolidated deposits of the central lowland is unlikely to be successful.

In contrast with the two rock formations just described, the next formation in downward succession contains many beds that yield water. This next formation, the oldest and lowest of those that surround and underlie the central lowland, includes interbedded sandstone, sandy shale, and shale. It is approximately if not strictly correlative with the Tyee sandstone and the Burpee formation of Schenck and is of late Eocene age. This formation constitutes all 
or nearly all of the western flank of the central lowland southward from Dallas, in Tps. 7 to $18 \mathrm{~S}$. Its water-bearing zones are the discontinuous and lenticular beds of sandstone and sandy shale, which yield water slowly to numerous farm wells among the foothills. So far as is known, however, none of these wells yields more than about 10 gallons a minute. Several wells, for example well 533 near Corvallis, have struck brackish water and brine in the formation, in some places less than 100 feet below the level of the lowland plain. (See p. 64.) Accordingly, as is true of the younger sedimentary rocks, these rocks of late Eocene age are not likely to yield copious supplies of fresh water if tapped by deep wells passing through the unconsolidated deposits of the central lowland.

\section{GEOLOGY AND HYDROLOGY OF THE UNCONSOLIDATED DEPOSITS}

\section{GENERAL CHARACTER, EXTENT, AND THICKNESS OF THE DEPOSITS}

\section{YOUNGER ALLUVIUM}

The unconsolidated deposits that form the central lowland of the Willamette River Basin contain the materials most likely to sustain large withdrawalš of water from wells. Of these deposits the youngest underlies or forms the flood plains of the present streams and is shown on plates 1 and 2 as younger alluvium.

Along the Willamette River below Eugene, this youngest alluvial. or stream-laid deposit is composed of poorly or rudely assorted gravel and sand with a small proportion of silt. Upstream, to the south, it consists largely of coarse gravel and cobbles as much as 6 inches in diameter (pl. $4, B$ ), and some sandy beds or zones. Downstream the deposit becomes progressively finer and better assorted and at numerous places is rudely stratified and cross-bedded (pl. 5), but even at the northern end of the valley, as near Newberg, it contains a fairly large proportion of pebbles as much as 2 inches in diameter. In this deposit the beds of large water-yielding capacity are tongues of clean sand or gravel; one or more such tongues have been penetrated by wells at most places on the flood plain, though their depth and thickness at any particular place cannot be foretold. At some places the deposit contains many fine particles so thoroughly interspersed with. the sand and gravel that water is yielded slowly to wells; some such places are in the bottoms of ephemeral drains and channels that are occupied by slack water when the river is in freshet. It appears that here much of the silt has been brought in after the gravel and sand has been carried downward into the deposit as the silt-laden backwater seeped away. All the materials of the younger alluvium, even the finest sand and silt, appear to be quite fresh; from this and from the 
relative coarseness of the materials, it is judged that over most of the flood plain water can penetrate easily from the land surface to the water table.

Along the main branch of the Willamette River, the alluvial deposit just described forms a tongue that is from half a mile to 4 miles wide and is coextensive with the flood plain. Its thickness ranges from a feather edge along cither margin of the flood plain to a few tens of feet; the greatest known thickness, in well 463 , near Corvallis, ${ }^{8}$ is 42 feet. Branching from it, tongues of similar material extend eastward along those tributary streams that rise high in the Cascade Mountains and that reach the central lowland with relatively steep gradients. In order northward these tributaries are the Middle Fork, McKenzie River, the two branches of the Santiam River, and the Molalla River.

In contrast with the deposits just described, the younger alluvium is of decidedly different character along the smaller tributaries that enter the Willamette River from the east and along all the tributaries that enter from the west. Along the smaller tributaries from the east-such as Muddy Creek and the Calapooya River to the south and the Pudding River to the north-young alluvial deposits are formed largely of fine-grained materials derived from the lowland. At most places, therefore, they do not transmit water freely and do not promise wells of large capacity. The tributaries that enter the Willamette River from the west reach the lowland with a very slight gradient. Thus, their young alluvial deposits contain little clean sand and gravel within the area that is shown on plates 1 and 2 and so generally do not yield water freely. These streams, in order from the south, include the Long Tom, Muddy, Luckiamute, Yamhill, and Tualatin Rivers.

Three tongues of young alluvium delimited on plate 1 are somewhat higher than the flood plains of present streams. The largest of these is the so-called Lake Labish channel, which trends northeastward from the flood plain of the Willamette River just north of Salem to the plain of Pudding River near the town of Mount Angel. The two smaller tongues occur to the east, directly across Pudding River; they diverge from the present valley of Abiqua Creek about 3 miles southeast of Mount Angel. All three tongues floor abandoned streamways. All three appear to be composed of fine-grained materials, are probably thin, and are judged to be low in water-yielding capacity.

\section{OLDER ALLUVIUM AND RELATED DEPOSITS}

The materials that form and lie immediately beneath the main lowland plain of the Willamette River Basin are shown on plate 1 as older alluvium and related deposits. At least two distinctive sorts

\footnotetext{
8 Descriptions of wells appear in tables 11 and 12; locations are shown on plates 1 and 2.
} 
of material are included: comparatively coarse alluvial or stream-laid material beneath all the main plain southward from the rock-bound narrows at Salem; distinctly finer material northward from Salem, of which that lying immediately beneath the land surface appears not to have been deposited by vigorous streams. The principal characteristics of the material in these two parts of the valley are described below. The mode of occurrence of the ground-water and the yield from the deposits are treated on pages 34 to 54 .

As has been stated, the main plain in the southern segment of the valley comprises several broad alluvial fans and intervening flats of very gentle slope. It covers about 400,000 acres. To a depth of at least 50 feet below the land surface, the central and higher parts of the fans appear to be composed largely of tongues and lentils of gravel and sand interspersed with unassorted mixtures of sand and silt (pl. 6, A). The better-assorted bodies of gravel and sand serve as arteries whích, though perhaps devious, appear to be rather thoroughly interconnected, thus allowing free movement of ground water. One or more ground-water arteries of this sort are penetrated by numerous wells. Toward the lower margins of the alluvial fans the coarser alluvial deposits just described appear to grade into and interfinger with poorly assorted or unassorted mixtures of sand, silt, and pebbles which in turn, beneath the flats that intervene between alluvial fans, grade into heterogeneous material that is largely earthy. These finer alluvial materials yield water slowly.

Little is known of the character of the lower or deeper part of the older alluvium in this part of the valley. Near the center of the area the deposit, according to the driller's record of well 637 (table 12), contains much "clay" and "mud" below a depth of 60 feet and especially below a depth of 145 feet. Unfortunately that record, the only one available for the purpose, does not show whether the claylike material occurs in distinct beds that alternate with layers of sand and gravel or whether fine and coarse particles are commingled throughout the lower part of the deposit. That single record does not preclude the possibility that elsewhere in the area the deeper part of the older alluvium may be composed largely of gravel and sand, such as predominate in the higher parts of the alluvial fans that form the land surface. Neither does the single record warrant a judgment as to the water-yielding capacity of the deeper alluvial materials.

So far as is indicated by data now available, the older alluvium in the southern segment of the valley is somewhat finer and less thoroughly assorted than the younger alluvium of the same area. Although its upper part, at least, contains fairly extensive arteries that yield water quite freely, the deposit as a whole is probably less pervious than the younger alluvium. In the soil zone, the finer particles of 
the older alluvium at some places have been disintegrated by weathering. Consequently infiltration of water from the land surface to the water table doubtless is somewhat impeded here and there; however, infiltration probably is nearly as free as through the younger alluvium except near the outer margins of the alluvial fans and over the intervening flats.

Because the older alluvium was deposited over an eroded bedrock surface, its thickness must range from a feather edge along the outer margin of the alluvial plain to a maximum over the lowest point on the bedrock floor. Geologic features of the valley suggest that the bedrock floor had moderate local relief. Consequently the thickness of the alluvial deposit may vary decidedly, even within small areas. Few determinations of the thickness in the southern half of the valley are available (see pl. 7). These few, which are drawn wholly from driller's records of wells, show fairly well the thickness and the altitude of the underlying bedrock floor near the upstream end of the rockbound narrows that extends from Albany to Salem and at several places along the margin of the alluvial plain. Only three records afford any data for the central part of the plain; of these, only the record for well 637, at Junction City, is at all detailed. At this place, the alluvial deposits appear to be not less than 200 feet nor more than 233 feet thick; the thickness cannot be fixed precisely, owing to the uncertain driller's classification of fine-textured materials low in the alluvial deposit in distinction from the bedrock, which is largely shale. That the thickness found at Junction City is about the greatest in all the southern segment of the valley is suggested by abandoned well, 610 , which is reported to have been drilled 300 feet deep and to have encountered strong salt water, doubtless in bedrock. In the well at Junction City the bedrock floor underneath the alluvium appears to be not more than 120 feet nor less than about 90 feet above sea level-that is, at least 40 feet lower than at the rock-bound narrows to the north, near Albany. This feature suggests that the floor on which the alluvium rests, although shaped primarily by erosion, may have been deformed by movements of the earth's crust before alluviation began. If so, the thickness of the alluvium may vary in a most irregular fashion.

A conservative estimate of the average thickness of the alluvial deposits that underlie the southern half of the main valley plain, largely older alluvium, appears to be about 70 feet. If so, these unconsolidated materials would have a total volume of about $30,000,-$ 000 acre-feet and would have the capacity to store about $2,500,000$ acre-feet of ground water. (See p. 40.)

As has been stated, the materials that lie immediately beneath the main lowland plain northward from Salem to the vicinities of Newberg and Canby are distinct from those to the south, just described. With 
the exception of a few gravel fans or tongues along the margin of the plain, as along the lower reaches of the Yamhill and Molalla Rivers, only fine sediments (largely medium sand to silt) crop out or have been exposed by highway cuts. These are the sediments whose composition has been treated briefly by Felts. ${ }^{9}$ At most places they are rudely stratified in beds that average about a foot in thickness (pl. 6, B); at a few places they include streaks of coarse sand. Even though finegrained, they are appreciably pervious in part, for they contain at least one extensive body of semiperched water so high above the major streams that recharge can take place only by infiltration from rain falling on the highest parts of the main lowland plain. That semiperched body supplies many domestic wells but probably will not yield water in large quantities, such as for irrigation. At well 245, which is about 0.7 mile east of Gervais and which is believed to afford a typical section of the materials that underlie the northern half of the main lowland plain, these fine sediments extend to a depth of 68 feet beneath the land surface. From the base of the fine sediments to the bottom of the well, at a depth of 252 feet, beds of clay and silt make up slightly less than half the thickness, and beds of pervious sand and gravel the remainder. Most of the pervious beds occur in four zones whose tops are $68,108,146$, and 218 feet beneath the land surface. Apparently the well did not reach the underlying bedrock, so that the character of the deepest unconsolidated materials in the vicinity is not known.

TABLE 7.-Physical properties of water-bearing materials from well 245

[V. C. Fishel, analyst]

\begin{tabular}{|c|c|c|}
\hline (feet & $\begin{array}{l}\text { Sample } 1 \\
230\end{array}$ & $\begin{array}{c}\text { Sample } 2 \\
241\end{array}$ \\
\hline t specific gravity, laboratory packi & 1. 67 & 1. 57 \\
\hline rosity (percent), laboratory packing & 38. 3 & 41.3 \\
\hline ient of permeability & 695 & 1,050 \\
\hline t by weight): & & \\
\hline Gravel, more than $2.0_{\ldots}$ & 20. 2 & 1. 9 \\
\hline Fine gravel, 2.0-1.0 & 9. 7 & 8. 9 \\
\hline $1,1.0-0.5$ & 20. 1 & 24. 0 \\
\hline $0.5-0.25$ & 34.0 & 51.1 \\
\hline (6. & 11. 3 & 8. 5 \\
\hline sand, $0.125-0.062$ & 1. 6 & 1. 5 \\
\hline Silt, $0.062-0.005$ & 1. 9 & 2. 7 \\
\hline Clay, less than 0.005 & 1. 3 & 1.5 \\
\hline
\end{tabular}

About the same succession of impervious and pervious beds is shown by the records of several other wells to the west and north, to the Willamette River and beyond. The pervious beds of sand and gravel supply several wells such as Nos. 245, 247, 248, 252, 262, and 263,

- Felts, W. M., Analysis of Willamette Valley fill [abstract]: Geol. Soc. America Proc. 1935, p. 346, 1936. 
which are heavily pumped; also wells 139,153 , and 159 , which are not pumped severely.

In the area from Salem northward to Newberg and Canby, as in the area to the south, the older alluvium and related deposits rest on a bedrock floor and, along the margin of the main valley plain, lap over the bedrock and feather out against it. Thus, the pervious zones in these deposits terminate against the bedrock at a distance from the outer margin of the plain, especially beneath tongues of the plain that extend far into the foothills. Obviously, therefore, the pervious zones are less extensive than the plain, and the deepest zone is the least extensive of all. However, even the deepest zone is believed to extend over half the area or more.

Over a large part of this area the older alluvium and related deposits appear to be more than 200 feet thick (see pl. 7); the greatest known thickness, 296 feet, is at well 221 , in sec. 8 , T. 5 S., R. 3 W., about 12 miles west of Woodburn. There the bedrock floor is about 140 feet below sea level. Obviously that floor has been depressed with respect to sea level; if it has been materially warped or faulted as well as depressed, most of that deformation must have taken place before the main valley plain was constructed, for the plain is remarkably flat (see p. 10).

If the average thickness of the older alluvium and related deposits in this northern segment of the main lowland plain is taken as 150 feet, an estimate which appears to be conservative, the total volume of the deposits would be about $50,000,000$ acre-feet and the water-storage capacity about $3,500,000$ acre-feet.

Still farther north, the Tualatin Valley is a topographic analog of the segment of the main lowland plain whose older unconsolidated deposits have just been described. That valley is floored by an unconsolidated deposit some 200 feet thick which in its upper part is quite similar to that of the main lowland plain. However, in its lower part the beds of pervious sand and gravel appear to be neither thick nor very extensive. To the east, along the Willamette River in the vicinity of Portland, there are correlative unconsolidated materials in part of similar texture and composition. Neither in the Tualatin Valley nor in the vicinity of Portland, however, has the deposit equivalent to the older alluvium been discriminated from younger and older unconsolidated deposits with which it is complexly associated.

TERRACE DEPOSIT AND RELATED ALLUVIAL MATERIALS

Along the outer margin of the main lowland plain, commonly from 30 to 100 feet above the plain and throughout all the length of the Willamette Valley, there are scattered though conspicuous remnants of a terrace formed by a coarse unassorted stream deposit (see pls. 1 and 2). At most places the deposit appears to be no more 
than a few tens of feet thick and commonly rests on a bedrock shelf that is higher than the main lowland plain, especially in the northern half of the valley. Consequently much of the deposit is so thoroughly drained that it contains little ground water. However, certain of the remnant deposits in the southern half of the valley-such as Sand Ridge, in secs. 24 and 25, T. 12 S., R. 3 W., and Fern Ridge, in the northeastern part of T. 17 S., R. 5 W.-extend to an unknown depth below the main lowland plain and so into the regional body of ground water.

Everywhere this terrace deposit is weathered rather severely, but especially in the southern half of the valley. There. at least the upper 20 feet of the finer matrix of the deposit is thoroughly decomposed to a compact clay and even the largest cobbles are so much disintegrated and decomposed that they can be cut through by a single sharp stroke with pick or shovel. (See pl. 8, A.) Material so thoroughly decomposed has little or no capacity for the infiltration or transmission of water. On the other hand, test borings made recently by the United States Engineer Department at the Fern Ridge dam site, in secs. 3 and 4 , T. 17 S., R. 5 W., indicate that there the terrace deposit is not much weathered at depth and so probably is sufficiently pervious to transmit water rather freely. This condition probably does not exist beneath most of the terrace remnants.

There are correlated tentatively with the terrace deposit two relatively extensive bodies of unconsolidated material: the gravel that veneers the Molalla pediment, which is in Tps. 4 and 5 S., Rs. 1 and 2 E., between the Molalla River on the northeast and Butte Creek on the southwest; and the Portland "delta," which lies in the angle between the Columbia and Willamette Rivers, extends eastward across R. $3 \mathrm{E}$., and is topographically correlative with an extensive plain to the north in the State of Washington.

The Molalla pediment rises gradually eastward to an altitude of about 475 feet above sea level or nearly 300 feet above the main lowland plain. The character of the materials that underlie the pediment is only imperfectly known, though a few scattered outcrops and the driller's record of well 280 (table 12) suggest that the pediment is veneered with poorly assorted gravel and sand a few tens of feet thick, beneath which compact "clay" with a few thin beds of "sand" extend to a depth of at least 300 feet. Well 188, which is on the main lowland plain just west of the Molalla pediment, also penerated "clay" and "sandy clay" between depths of 65 and 350 feet below the land surface and then entered shale. (See table 12.) Outcrops show that part of the thick mass of "clay" penetrated by these two wells and by others of the vicinity is volcanic tuff, both massive and stratified, and should be correlated with the partly consolidated Troutdale or Rhodo- 
dendron formations of Hodge, ${ }^{10}$ or both. Regardless of the precise correlation, these materials that underlie the pediment have small capacity to transmit water.

The Portland "delta" is triangular in plan and extends about 14 miles southward from the mouth of the Willamette River and about 19 miles eastward along the Columbia River, to a point $7 \frac{1}{2}$ miles beyond the eastern edge of the area represented on plate 1 . Its highest part is about 300 feet above sea level. Numerous pits and cuts show that to a depth of at least 100 feet it is composed of stratified and crossbedded sand and gravel, with a few thin partings of sandy silt. This characteristic bedding is well-shown on plate $8, B$. Obviously, the deposit was stream-laid on a gradually rising base level and was not built in a single stage by progression of a delta into deep water. The thickness of the deposit is suggested by the record of well 33 (table 12), which is reported to have penetrated little but gravel and sand to a depth of 360 feet and then alternate layers of clay, sand, and boulders to a depth of 405 feet. Certain layers of "conglomerate" between 220 and 330 feet in depth may indicate that the lower part of this zone should be ascribed to the bedrock, specifically to the Troutdale formation of Hodge. ${ }^{11}$ (See p. 23.) Below a depth of 405 feet and to a depth of 1,300 feet, the well passed largely through "marl" and shale, which very probably should be ascribed to the bedrock. Irrespective of these uncertainties in correlation, the unconsolidated "delta" deposit probably is not less than 300 feet thick in some places. A large proportion of the deposit is highly pervious and supplies numerous wells of large capacity.

\section{OCCURRENCE, SOURCE, AND DISPOSAL OF WATER IN THE UNCONSOLIDATED DEPOSITS}

FORM AND FLUCTUATIONS OF THE WATER TABLE

In the southern half of the Willamette Valley and through the rockbound narrows that extends to Salem, both the younger and the older alluvium are highly pervious almost throughout and are underlain by bedrock formations that are much less pervious. These two alluvial deposits act essentially as a single ground-water reservoir of very large capacity (see p. 32), quite analogous to a surface water reservoir. Thus, as water is added to the reservoir more of the alluvium is saturated and the ground-water level rises more or less proportionately; as water is discharged, either through natural drainage downstream or through withdrawals by pumping, some alluvium is unwatered and the groundwater level falls. Obviously, the height of the water table - the upper surface of that part of the alluvium which is saturated with water-is never quite steady but fluctuates constantly.

${ }^{10}$ Hodge, E. T., Age of Columbia River and lower canyon labstract]: Geol. Soc. America Bull., vol. 44, p. 157, 1933.

11 Idem. 


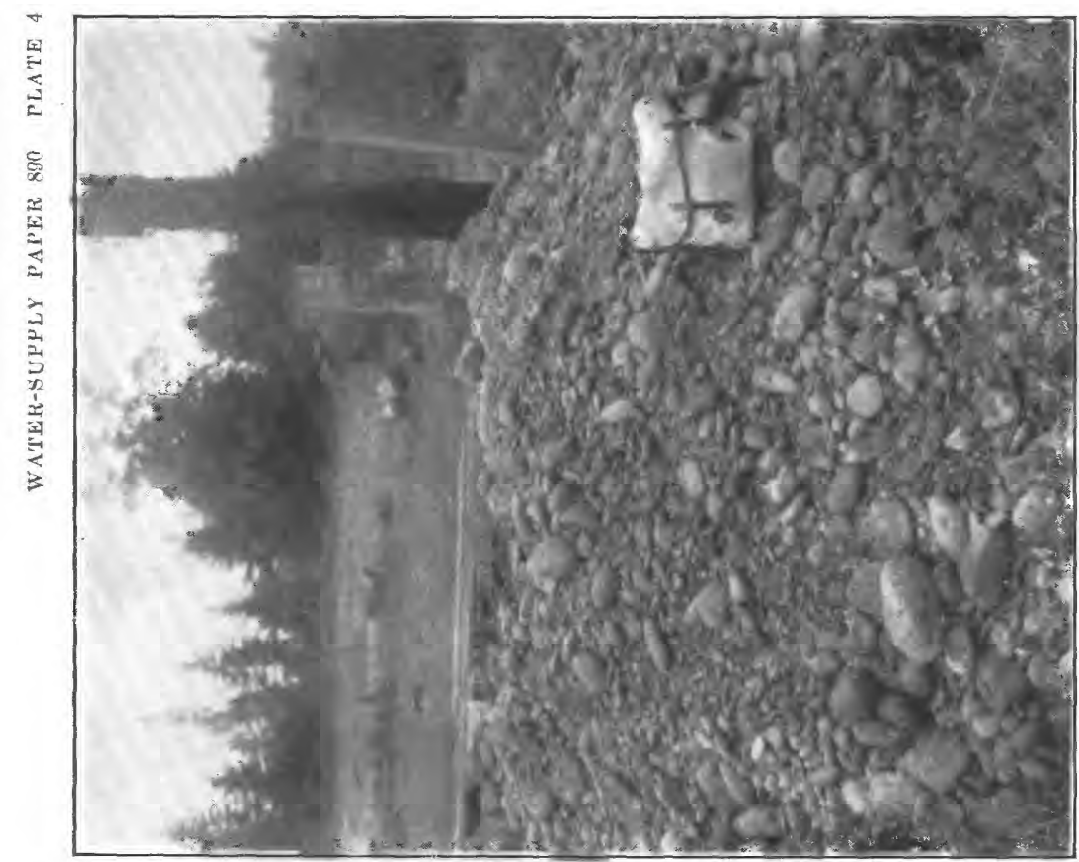

每
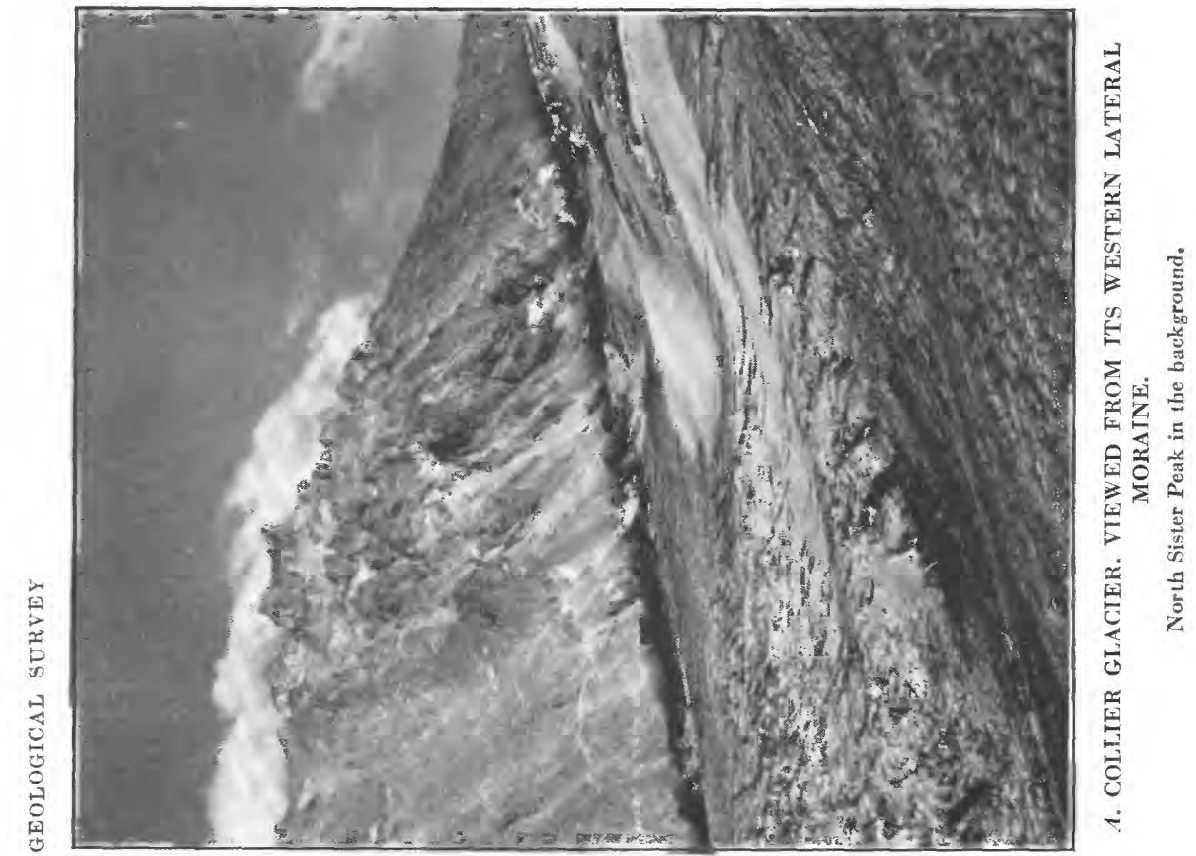


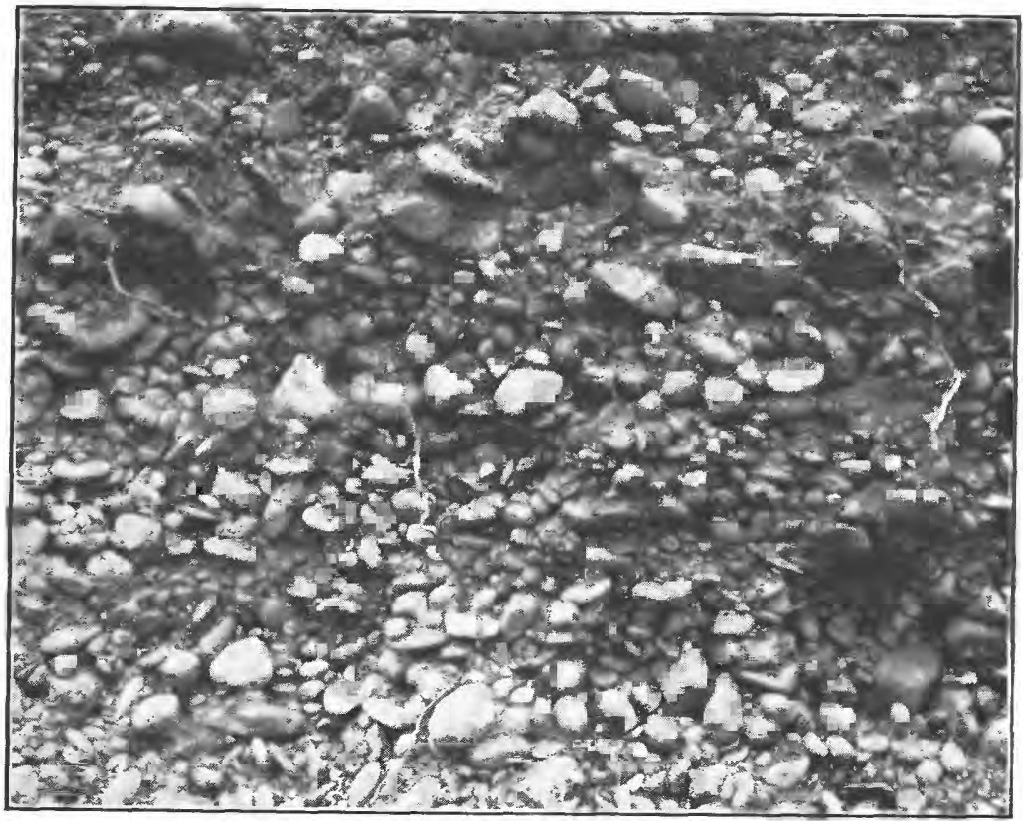

A. GRIVEL PIT IN THE SE $1 / 4$ SEC. 8 , T. 11 S., R. 4 W.

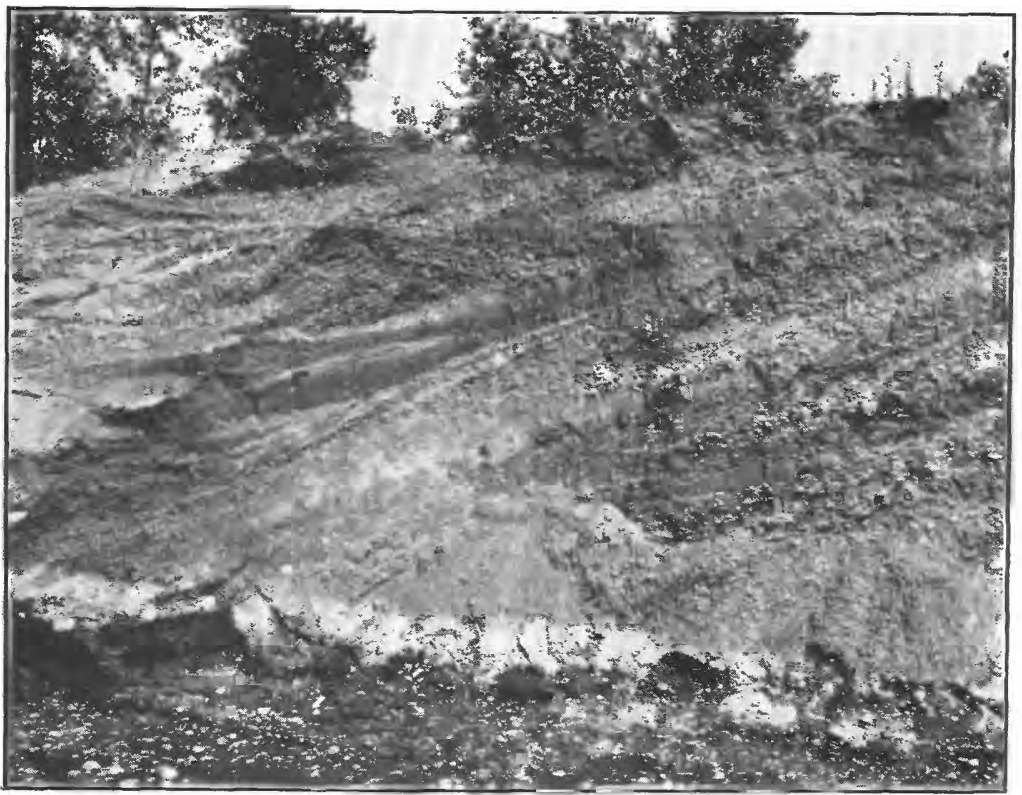

B. PIT OF EAST SIDE SAND \& GRAVEL CO. NEAR CORVALLIS.

In SW1/4 sec. 2, T. 12 S., R. 5 W.

YOUNGER ALLUVIUM OF THE WILLAMETTE RIVER. 


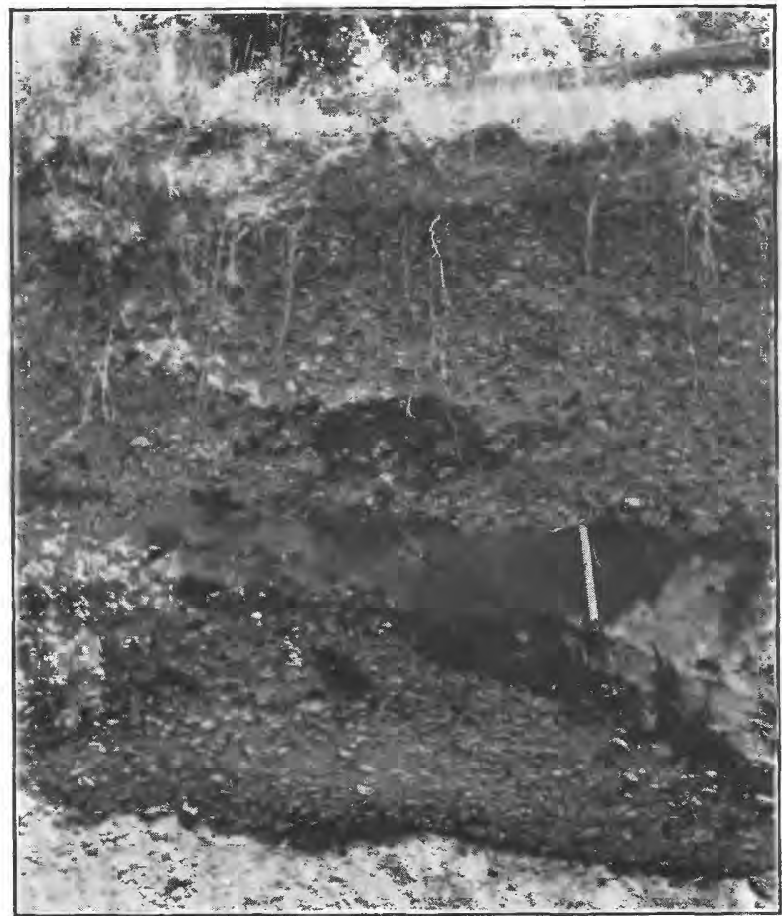

A. EXPOSLRE IN THE NW $1 / 4$ SEC. 1 , T. 16 S., R. 4 W.

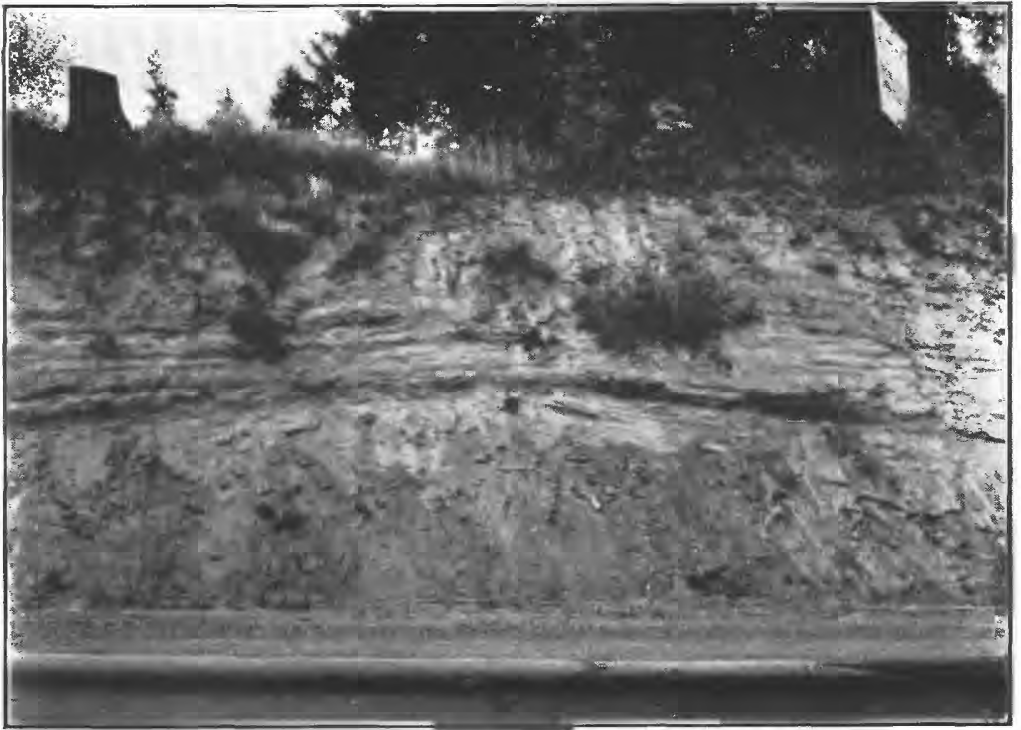

B. HIGHWAY CUT IN THE NW1/4 SEC. 7. T. 4 S., R. 1. E., SHOWING FAINT STRATIFICATION. 



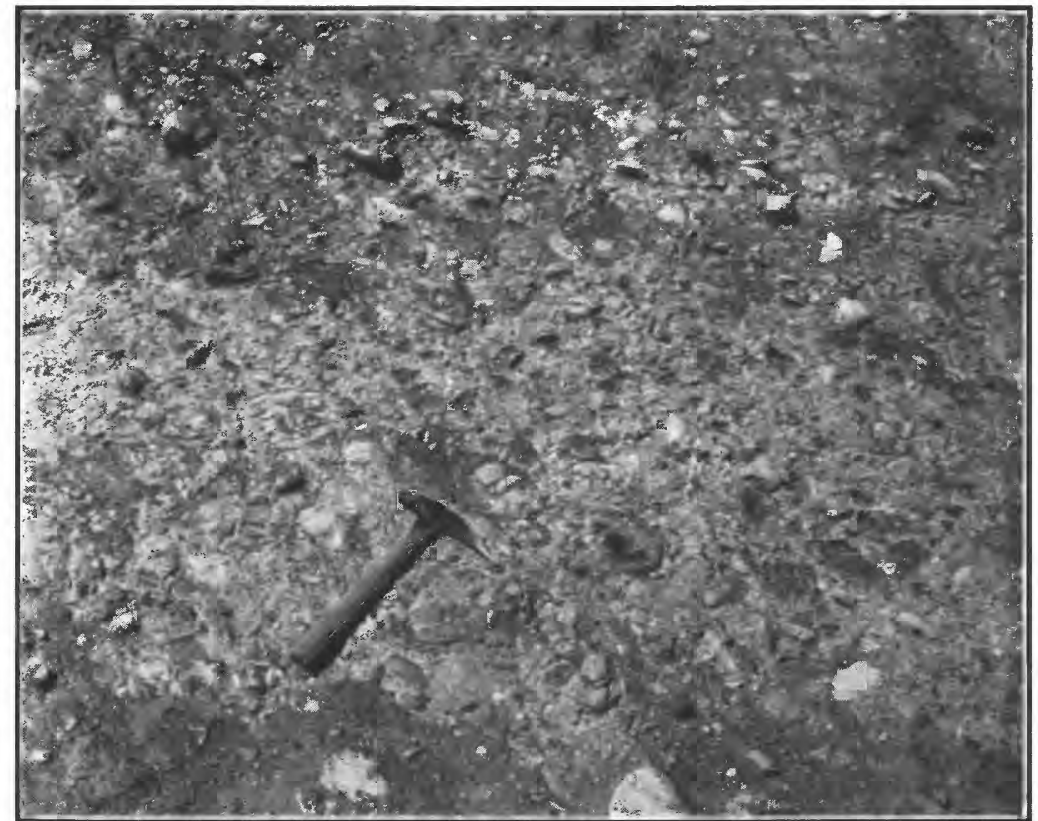

A. DECOMPOSED TERRACE DEPOSIT IN HIGHWAY CUT IN THE N1. SEC. 32 , T. 12 S., R. I W

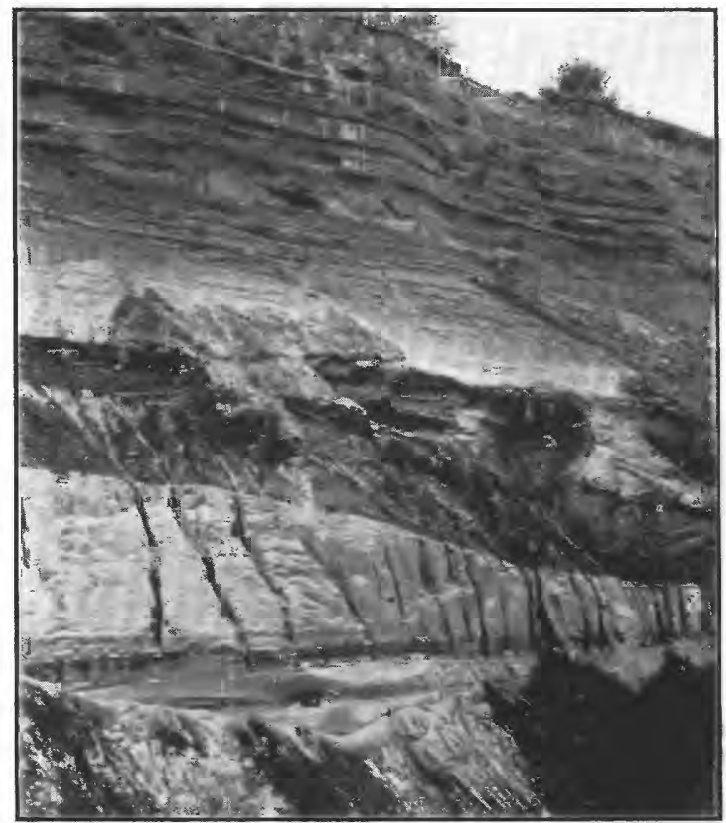

B. CROSS-BEDDED SAND AND GRAVEL EXPOSED IN CUT ALONG THE SPOKANE PORTLAND \& SEATTLE RAILWAY, 0.1 MILE NORTH OF LOMBARD STREET, PORTLAND. 

Contour lines on plates 1 and 2 show the height and general form of the water table as of November 1935, when the water table was about at its lowest stage for that water year and very little above its lowest known stage. That general form changes little as the water table rises and falls because, as indicated later, fluctuations of the ground-water level are of about the same order of magnitude and roughly synchronous over most of the valley. Beneath the broad interstream areas in the southern half of the valley, the water table is higher than the streams and slopes downward away from the foothills and northward toward and through the rock-bound narrows. Along either side of the Willamette River and the larger tributary streams, however, the water table ordinarily pitches downward to form a broad shallow trough whose bottom meets and prolongs the water surface of the stream. These troughs or valleys of the water table persist throughout most of the year but may be partly or largely obliterated during brief periods as the ground-water level rises sharply with freshets. (See p. 38.) In contrast to these troughs, the water table also rises into a number of minor ridges where relatively impervious material is not far beneath the land surface, as south of Corvallis in Tps. 12 and 13 S., R. 5 W., east of Corvallis in T. 12 S., R. 4 W., and west of Eugene in T. 17 S., R. 5 W.

In the northern half of the valley ground water occurs under conditions that are quite distinct from those just described. There, the unconsolidated deposits are not continuously pervious, and at least three separate bodies of ground water can be delimited. The first is in the tongue of younger alluvium that underlies the flood plain of the Willamette River, and the water is unconfined, as in the southern half of the valley. Distinct water-table contours for this body of unconfined water are shown on plate 1 . The second occurs beneath each of the extensive "prairies" or interstream segments of the main lowland plain. There, the shallowest ground water is also unconfined, but it is held high above river level by beds of low perviousness. These bodies of water are semiperched; contours on their respective water tables are shown on plate 1 . In general these water tables are highest beneath the central parts of the prairies and descend in all directions toward the stream trenches by which the prairies are bounded; in detail, their slopes are rather uneven. The third body, which is in the deep pervious beds that pass below the floors of the stream trenches, is confined under hydrostatic pressure. The height of the static level for this confined water is shown approximately by appropriate contour lines; over most of the area this static level is somewhat lower than the semiperched water table, but at some places it appears to be higher. It is considerably higher than the unconfined water table in the younger alluvium. 
Plate 9 shows typical water-level fluctuations in wells of the Willamette Valley; the fluctuations in numerous other wells are shown by the data on water levels, table 13 . Of the 12 wells represented on plate 9 , Nos. $421,463,553,568,636$, and 680 are in the southerm half of the valley and their graphs show fluctuations of the regional water table in comparison with fluctuations of stage in the Willamette River at Corvallis and at Salem. Of the remaining 6 wells, which are in the northern half of the valley, well 171 and probably well 318 show fluctuations of semiperched water tables; well 245 and probably well 297 show fluctuations in pressure head of the confined water; and well 148 and possibly well 196 show fluctuations of the regional water table. Characteristic features of the fluctuations, their causes, and their relation to the withdrawal of ground water for irrigation and for other purposes are discussed later.

The water-level fluctuations thus far observed in the Willamette Valley have been due primarily to natural causes; withdrawals by pumping are relatively so small that they depress the ground-water level only locally and transiently. Throughout the valley the groundwater levels are characteristically lowest in late autumn, ordinarily in the last half of October or the first half of November. The replenishment of the soil-moisture deficiency begins with the onset of the rainy season (see p. 37), and within a relatively short time, ordinarily by mid-January for a water body that is unconfined and somewhat later for a body that is confined, the water level rises to its highest stage of the year. At many wells it then oscillates through a range of a few feet during the early spring, but after April it tends to decline steadily throughout the valley until the ensuing low stage is reached. Both the maximum and the minimum yearly ranges of ground-water level thus far determined-about 28 feet at well 426 and $31 / 2$ feet at well 409 in 1935-36-have been in the southern half of the valley where virtually all the ground water is unconfined. The smallest ranges occur commonly near the outer margin of the valley plain and in foothill alcoves; the largest, beneath the open plain at a distance from the larger streams. In the younger alluvium along the principal streams, the yearly range is moderate-for example, 6 miles north of Albany at well 421, the range was 5 feet in 1928-29 and 15\%/2 feet in 1935-36;2 miles east of Corvallis at well 463 the ranges for those water years were $121 / 2$ and $131 / 2$ feet, respectively; at Lebanon in well 568, $4 \frac{1}{2}$ and $8 \frac{1}{2}$ feet; and 6 miles north of Eugene at well $680,4 \frac{1}{2}$ and $6 \frac{1}{2}$ feet. In the northern half of the valley the yearly range of ground-water level appears to be moderate at most places. The greatest range is in the unconfined semiperched water. For example, 4 miles northwest of Woodburn at well 171 the water level had a range of 15 feet in 1928-29 and of 17 feet in 1935-36. For the pressure head of the deep confined water in the same area, 
the yearly range is only about half as great; 3 miles southwest of Woodburn at well 245 it was 9 feet in 1935-36; $4 \frac{132}{2}$ miles north of Salem at well 297 it was $81 / 2$ feet in 1929-30 and 10 feet in 1935-36.

With reference to the unconfined water in the older alluvium and related deposits, including the semiperched water in the northern half of the valley, the sharp rise of ground-water level in December and January must be due almost entirely to the infiltration of rain that falls in the immediate vicinity; for even when lowest the ground-water level beneath most of the valley is higher than the streams, and the ground-water level rises so nearly simultaneously over all the valley that replenishment can scarcely be caused by extensive lateral percolation. In those 2 months the average rainfall is about 12 inches, or roughly a third of the yearly total (p. 16); it is far more than the concurrent evaporation or consumptive use and is adequate for a very substantial addition to ground-water storage in most years. The repeated rise and fall of ground-water level that has been observed at many wells in late winter and early spring is here ascribed to intermittent infiltration from successive storms with alternate partial drainage of the newly saturated material; the steady decline after April is ascribed largely to continuous unwatering of the alluvial deposits as the ground water moves laterally toward and into the streams. The relation of this unwatering to stream flow is treated on page 39. Only a negligible part of the steady decline can be caused by withdrawals from wells, which so far have been very small in proportion to the volume of ground water involved in the yearly saturation and unwatering.

The fluctuations in the static level of the water that is confined beneath the northern part of the valley, unlike those of the unconfined water, just treated, do not indicate commensurate saturation and unwatering of pervious material at that place. Rather, all the deep pervious material is constantly saturated, and the fluctuations indicate merely changes in pressure transmitted from some external source. Of necessity, the water is derived from an outlying catchment and intake area. That intake area presumably lies chiefly to the south and east. The winter increase of pressure head in the deep water-bearing beds, which lags only slightly behind the rising level of the semiperched water above, might come about in either or both of two ways: First, the weight of the water taken into storage in the semiperched zone imposing a commensurate load on the deeper aquifers through elastic deformation of the strata; ${ }^{12}$ and second, a rising water level in the remote catchment area being transmitted laterally through the aquifer as a wave of pressure head. Conversely, the decline of the pressure head in summer and autumn would be caused by relief of

12 Meinzer, O. E., Compressibility and elasticity of artesian aquifers: Econ. Geology, vol. 23, pp. 272276,1928 . 
load as the semiperched zone became unwatered, by recession of the water level in the catchment area, or both.

In the younger alluvium along the Willamette River and its principal tributaries, the fluctuations of ground-water level appear to follow rather closely the fluctuations in stream stage. (See pl. 9, well 463, and river station at Corvallis.) However, the ground-water level probably is determined more by a backwater effect of rising and falling river stage than by extensive percolation from the river into the alluvium and the reverse, for ordinarily the water table slopes downward toward the river. Thus, the yearly saturation of the younger alluvium is doubtless due in considerable part to infiltration of rain; the alluvium is unwatered by drainage to the streams, as in the older alluvium.

With respect to the withdrawal of ground water for irrigation and other uses, the natural water-level fluctuations have several practical applications. If large withdrawals are to be made from single wells at a minimum operating cost, each pumping plant should be designed to accommodate the water-level range of the particular locality. Where the alluvium is thin and is underlain by impervious rock, as along certain reaches of the flood plains (p. 44), it may be largely unwatered by midsummer, so that large withdrawals cannot be sustained. For example, the irrigation well 463 on the east farm of the Oregon Agricultural Experiment Station near Corvallis encountered impermeable shale 42 feet beneath the land surface. There, the highest static water level so far recorded was 32 feet above the shale, the lowest 18 feet. After deducting the draw-down by the pump, the saturated zone that yields water to the well is only half as thick at the low static level as at the high; the yield of water is correspondingly smaller. At many other places, as in the vicinity of Albany, the alluvium is still thinner and the capacity of wells diminishes even more as the water level recedes during the summer. Under these conditions, even greater diminution of yield would be expected if many wells were pumped heavily, with consequent interference and comulative depression of the ground-water level; in 1938 heavily pumped wells were few and widely scattered.

Should the ground-water resources in the southern half of the valley be developed extensively in the future for supplemental irrigation, the natural water-level fluctuations that have been described would doubtless be modified appreciably. In areas where large withdrawals are concentrated, the water-level decline in early summer probably would be quickened and the ultimate level in autumn would be lower. Thus, the deficiency in ground-water storage at the onset of the rainy season would be greater, and full replenishment by infiltration would be somewhat delayed. These effects of extensive withdrawals are unavoidable; in fact, they are the means by which the underground res- 
ervoir can be made to store additional water during the rainy season for later use. In this connection, the infiltration capacity of the alluvial deposits appears to be so large and the winter rainfall so much in excess of natural use, that withdrawals much greater than at present probably would be fully replaced between the seasons of pumping. (See also p. 40.)

In the northern half of the valley, heavy withdrawals from the confined water bodies in deep pervious beds would modify the natural water-level fluctuations in another manner. There, withdrawals can be increased only in proportion to the extent that the natural movement of water from the remote intake area can be accelerated. Accelerated movement is possible only if the hydraulic gradient is steepened from the area of pumping to the area of intake; in other words, the pressure head in the pumping area must fall. Thus, sustained large withdrawals probably would depress the water level somewhat more than in the southern half of the valley, and full recovery of head probably would not come about as quickly.

\section{RELA'TION OF GROUND WA'TER TO RAINFALL AND S'TREAMS}

As has been suggested by the foregoing treatment of water-level fluctuations, the bodies of ground water in the unconsolidated deposits of the Willamette Valley are integral parts of the drainage system. These bodies receive water chiefly by infiltration of rain from the lowland plains directly above or, in the case of the confined water in the northern half of the valley, from the remote intake area. From these bodies water is discharged naturally into the streams and so helps to sustain the stream flow, especially during the summer and autumn.

The general direction of ground-water movement, from points of intake to points of discharge, may be deduced from the form of the water table or other pressure-indicating surfaces, for movement must be in the direction of the steepest downward hydraulic gradient. In other words, the direction of movement must be at right angles to the contour lines on the water table or analogous surface and from higher contours toward the lower. Thus, the contour lines shown on plates 1 and 2 indicate that the unconfined water in the southern half of the valley moves inward away from the foothills and in a general northward direction until it seeps into the streams and so passes out of the basin. In the northern half of the valley, between Salem and Canby, the semiperched water tends to move radially outward from the center of the "prairie" into several perennial creeks, also to numerous small springs and seeps along the slopes or bluffs by which the prairie is surrounded. Probably some of the semiperched water is discharged through a ground-water cascade along the outer margin of the prairie. In the same area, the confined water in the deeper pervious zone appears to move generally northwestward 
away from the foothill area that lies south of Salem and Silverton. Where this water is returned to the land surface is not definitely known. Leakage upward into the younger alluvium along the Willamette River and the lower Yamhill River and thence into the streams is inferred tentatively because the pressure head appears to diminish sharply beneath the flood plains. A possible effect of withdrawals from wells is shown by the southward deflection of the lines of equal pressure head in the vicinity of Woodburn.

\section{DEPENDABILITY OF THE GROUND-WATER SUPPLY}

In an area such as the Willamette Valley the pervious materials beneath the land surface have long since been saturated to capacity and, over a term of years, as much ground water must be discharged as is received by infiltration or other means of recharge. Thus, if ground water is to be withdrawn for use year after year without ultimately depleting the initial storage, the volume withdrawn must be salvaged directly or indirectly from water that otherwise would be discharged naturally.

With respect to the unconfined water in the younger and older alluvium in the southern half of the Willamette Valley, withdrawals must be salvaged from the water that is stored intermittently in the zone of yearly saturation and unwatering. The volume of this water is large. Thus, among 63 representative water-table wells in that part of the valley, the thickness of the zone that was saturated in the water year of 1935-36 ranged from 3.8 to 28.2 feet and averaged 12.0 feet. From the high stage of that winter until the end of the next September, the zone of unwatering ranged from 3.0 to 25.3 feet in thickness and averaged 10.7 feet. If these averages from the 63 wells are applied to all the area, the volume of material saturated was about 5,300,000 acre-feet; of that subsequently unwatered, 4,700,000 acre-feet. If then the mean specific yield of the alluvial depositsthe ratio between the volume of the deposits and the volume of water that the deposits, after being saturated, will yield by gravity drainage- is taken as 10 percent, ${ }^{13}$ a percentage that is probably conservative, ground-water storage in the area increased about 530,000 acrefeet during the winter of $1935-36$ and diminished about 470,000 acrefeet during the following spring and summer. During the 4-month period November to February the rainfall was about 1 percent less than the 40-year average. Therefore the opportunity for recharge by infiltration certainly was not excessive. In contrast, the winter rainfall in 1928-29 was about 20 percent less than the 40 -year average; in $1929-30$, about 10 percent less. In these 2 years, the range in ground-water storage appears to have been little, if any, less than

13 Piper, A. M., Gale, H. S., Thomas, H. E., and Robinson, T. W., Geology and ground-water hydrology of the Mokelumne area, Calif.: U. S. Geol. Survey Water-Supply Paper 780, pp. 101-118, 1939. 
in 1935-36. Thus, ground-water recharge and discharge in 1935-36, amounting to about 500,000 acre-feet, may be assumed to have been roughly equal to the long-term average. In comparison, the total withdrawal of ground water for use in 1928-36-by numerous wells for rural household use, for a few community water systems serving a few thousand people, and for supplemental irrigation on a few thousand acres of land-is very small.

It is impracticable to salvage and use all the yearly ground-water recharge in the southern half of the valley. To do so would require that the ground-water level be sufficiently depressed by pumping to keep it below the level of the streams; thus certain thin parts of the alluvial deposits would be completely and permanently unwatered. Nevetheless, it appears wholly practicable to withdraw water at several times the rate of the 1928-36 withdrawals, and so to increase considerably the irrigated acreage and other water services. The scope of the reconnaissance on which this report is based does not permit a close estimate of the greatest practicable yearly withdrawalthe so-called safe yield, or the volume of water that could be withdrawn year after year without ultimately so depleting the supply that further withdrawals at that rate would become too costly. A reliable determination of the safe yield will be desirable if and when groundwater utilization increases several fold, but that determination would require a very intensive investigation.

No definite conclusions can be drawn at this time with respect to the safe yield of the deep water-bearing zone in the northern half of the valley, the only zone in the older unconsolidated deposit in that area that appears adequate to supply wells of large capacity. Thus far, the withdrawals for all uses have been no larger than in the southern half of the valley, if as large. Further, no cumulative decrease in the pressure head of the water is shown by the water-level data now available. Accordingly, it appears that the present withdrawals can be increased several times without causing excessive interference between wells and possibly many times before the safe yield is exceeded.

\section{SUBDIVISIONS OF THE CENTRAL LOWLAND WITH RESPECT TO YIELD OF GROUND WATER}

The central lowland of the Willamette River Basin may be divided tentatively into 20 subareas that are fairly distinct with respect to conditions of ground-water occurrence and to water-yielding capacity of the unconsolidated deposits. The position and extent of these subareas are shown on plate 10 . The locations of typical wells in the several subareas are shown on plates 1 and 2 ; descriptions and driller's records of the wells are given in tables 11 and 12 , respectively; and data on water-level fluctuations are given in table 13. The subareas 
are listed below, and the list is followed by a summary of their groundwater features.

\section{TENTATIVE SUBAREAS OF THE CENTRAL LOWLAND}

Flood plains of the principal streams, including some related segments of the main valley plain:

1. Springfield "delta," comprising the younger alluvial plains of the McKenzie and Willamette Rivers above their junction and an intervening segment of the older alluvial plain.

2. Upstream reach of the Willamette River flood plain, from the Springfield "delta" downstream nearly to Albany.

3. Middle reach of the Willamette River flood plain, through the rockbound narrows that begins about 2 miles upstream from Albany and extends to Salem, an air-line distance of 21 miles.

4. Lebanon alluvial fan, comprising the younger alluvial plains of the North Santiam and South Santiam Rivers about their junction and a. contiguous part of the older alluvial plain that extends westward to Albany and southward to Oak Creek.

5. Downstream reach of the Willamette River flood plain, from Salem to the mouth of the Pudding River.

6. Canby fan, comprising the younger alluvial plains of the Pudding River below Aurora and of the Molalla River below Molalla and the older alluvial plain between the Molalla River and the terrace about 2 miles east of Canby.

7. Younger alluvial plain of the Willamette River below Portland and of the adjacent part of the Columbia River.

Main valley plain:

8. Eugene-Junction City segment extending westward about 3 miles from the Willamette River flood plain.

9. Coburg-Harrisburg segment between the Willamette River flood plain on the west and Lake and Muddy Creeks on the east.

10. Elmira segment, including all the older plain between subarea 8 on the east and the terrace remnants or foothills on the west, in Tps. 15-17 S.

11. Brownsville-Albany segment, all the older plain between subareas 2 and 9 on the west and subarea 4 on the northeast; outliers of the Tertiary bedrock are excluded.

12. Corvallis segment, bounded on the east by subarea 2 and on the west by foothills or terrace remnants.

13. Minor segments in the vicinity of Albany, remnants of the older plain on both sides of the Willamette River in Tps. 9 and $10 \mathrm{~S}$.

14. Monmouth-Independence segment, lying west of the Willamette River flood plain in Tps. 7-9 S.

15. Stayton-Mill Creek basin, comprising the alluvial fan north of the North Santiam River in Tps. 8 and 9 S., Rs. 1 and 2 W., and the older plain from Salem eastward to the Little Pudding River and northward to the Lake Labish channel.

16. French Prairie, bounded on the west and north by the Willamette River flood plain, on the east and south by Pudding River and the Lake Labish channel.

17. Dayton Prairie and adjacent areas, all segments of the younger and older plains west or north of the Willamette River flood plain and adjacent. to it, from Salem downstream to Canby. 
18. Tualatin Valley, comprising the younger and older plains of the Tualatin River Basin but excluding numerous remnants of a higher terrace and two outliers of bedrock, one extensive.

Areas largely higher than the main valley plain:

19. Molalla pediment and related parts of the central lowland, bounded on the west by subareas 15,16 , and 6 and on the southeast by bedrock foothills; excludes the bedrock outlier Mount Angel but includes terrace remnants east of the Molalla River, one east of Canby and another around Mulino.

20. Portland "delta," including all alluvial plains in the vicinity of Portland between Kellogg Creek and the Clackamas River to the south and the Columbia River flood plain to the north.

Spring field delta (subarea 1).-Along the lower reaches of the Willamette and McKenzie Rivers, over the so-called Springfield or McKenzie River delta, both the younger and the older alluvial deposits contain many interconnected lentils and tongues of highly pervious gravel and coarse sand. One or more of these pervious members has been encountered in every well known to have been constructed in the area. Wells 675 to 684,696 , and 712 are typical of numerous irrigation wells, which together serve at least 1,500 acres of land. Most of the existing wells in this area are dug from $6^{\frac{1}{2}}$ to 25 feet deep and have simple open-bottom casings of wood or concrete tile. Their penetration, the distance they extend below the water table into saturated material, is not more than about 5 feet when the static level of the ground water is lowest nor more than about 12 feet when the water level is highest. The few drilled wells have somewhat greater penetration, but none are more than 12 inches in diameter and none have been thoroughly developed to secure the largest possible yield. Even these simple and relatively shallow wells yield from 250 to 400 gallons a minute, and several have specific capacities of 100 gallons a minute or more for each foot of draw-down. Doubtless, much larger yields could be obtained without excessive draw-down by constructing wells that are adequately screened, ample in diameter, thoroughly developed, and of greater penetration.

For wells of similar construction in the highly pervious alluvium, the specific capacity should increase with greater penetration into water-bearing beds; obviously, penetration is limited by the thickness of the alluvial deposit, which is imperfectly known (see pl. 7). The few data available suggest that the greatest thickness may' exceed 100 feet, which would afford wells with a penetration and potential capacity many times greater than any now existing. On the other hand, the bedrock floor that underlies the "delta" presumably has moderate relief, somewhat similar to that of the adjoining foothills. Accordingly, the thickness and total water-yielding capacity of the alluvium may vary greatly within small areas. 
Upstream from Springfield the alluvial deposits along the McKenzie River and the Middle Fork are inferred commonly to have a large capacity to yield water. On the other hand, the alluvial tongue that extends along the Coast Fork to the vicinity of Cottage Grove appears to be much less pervious on the average and so does not promise wells of large capacity.

Upstream and downstream reaches of the Willamette River flood plain (subareas 2 and 5).-Downstream from the Springfield "delta," except through the narrows between Albany and Salem, the tongue of younger alluvium along the Willamette Riverhas fairly uniform capacity to transmit water. Wells $653,654,538,539,550,463,464$, and 234 , in order downstream, are those most heavily pumped. All are of the simplest construction. Excluding wells 463, 464, and 234, these wells penetrate the water-bearing zone from 4 to 6 feet only, yet they yield as nuch as 525 gallons a minute, or 1.2 second-feet. Well 234 comprises three driven wells 2 inches. in diameter with a penetration of about 15 feet, yet it yields rather freely. Wells 463 and 464 are deepest; they also have the greatest penetration-18 feet below the lowest static water level-and yield 750 gallons a minute, or 1.7 second-feet. Nearly everywhere along these two reaches of the flood plain the younger alluvium is underlain by older alluvium, which also is water-bearing. (See descriptions of subareas $8,9,12$, and 16.) Thus, wells of thorough construction penetrating the full thickness of the two alluvial deposits promise yields considerably greater than those from existing wells.

Over these reaches of the flood plain, the yearly range in groundwater level is about 7 to $13 \frac{1}{2}$ feet. Thus pumping plants on wells must accommodate a moderate variation in pumping lift.

Middle reach of the Willamette River flood plain (subarea 3).Through the rock-bound narrows that extends from Albany to Salem, the younger alluvium is quite as pervious as in the reaches next upstream and downstream, just described. For example, wells 362 and 368 each yield about 1,000 gallons a minute, or 2.2 sccond-feet. Well 368 is 12 inches in diameter and penetrates water-bearing sand and gravel 19 feet in thickness; with respect to proportionate diameter and penetration, its yield is comparable to that of well 463 , to the south. Well 373 likewise has a large capacity.

This middle reach of the flood plain is treated as a distinct groundwater area only because the tongue of younger alluvium probably is not continuously underlain by the older alluvium and because at some places the younger tongue is doubtless thin and rests directly on slightly pervious bedrock, which is largely shale. Thus there is no assurance that a prospective well will encounter pervious beds of sufficient thickness to yield water copiously, especially when the 
ground-water level is lowest. The location and extent of the places of scant yield cannot be determined from the data now available.

Lebanon alluvial fan (subarea 4).-Along the lower reaches of the North Santiam and South Santiam Rivers the alluvial deposits are similar in thickness and water-yielding capacity to the deposits of the Springfield "delta," previously described. They are similar also in the yearly range of ground-water level, which is from 4 to 9 feet. Wells 562 and 566 to 568 are those pumped most heavily.

Northward from Lebanon, along the western edge of the younger alluvial tongue, the older alluvium that underlies the main valley plain also seems to be high in water-yielding capacity and in that respect can scarcely be discriminated. How far westward the older alluvium is so pervious remains unknown; somewhat arbitrarily, however, the limit is placed along Oak and First Periwinkle Creeks. Even though highly pervious, the older alluvium becomes thinner in the most westerly part of the subarea, in particular near the bedrock outlier that borders the subarea at Albany. There, extensive test drilling in wells 503 to 511 found the older alluvium from 28 to 47 feet thick and its water-bearing zone from 22 to 28 feet thick. From the results of this test drilling, the locality was judged unsuitable for a public-supply well field, because a source so shallow would not assure freedom from contamination. However, for irrigation and other uses not requiring water free from harmful bacteria, even that locality probably would sustain a moderate withdrawal of ground water.

Canby fan (subarea 6).---The younger and older alluvial deposits that have been grouped as the Canby fan doubtless contain pervious members of considerable water-yielding capacity, though little is known as to the extent and thickness of those members. The driller's records of wells 102 and 103, at Canby (table 12), shows the character of the older deposit. At that place it appears to be 281 feet thick and to a depth of 87 feet is composed largely of coarse gravel, but below that depth it is largely "clay" with a few water-bearing beds a foot or two in thickness. In water-yielding capacity the younger alluvial tongue is inferred to be similar to the corresponding tongue along the lower Willamette River. At some places the tongue is doubtless too thin to afford wells of large penetration.

Younger alluvial plain of the Columbia River (subarea 7).-Within the area that is shown on plate 1, the younger alluvial deposits of the Columbia River are composed largely of silt and rather fine sand. Shallow wells in this material encounter few beds that yield water freely, and in some wells the yield is barely adequate for individual household supplies. To satisfy the larger need for dairies and irrigation, drilled wells have been sunk to beds of coarse sand and gravel between 85 and 235 feet beneath the flood plain. These deeper 
pervious beds may belong to deposits older than the younger alluvium. Wells 1 to 3,5 to $14,16,17,34$ and 35 are typical; of these, well 34 has the largest yield-reported to be 750 gallons a minute, or 1.7 second-feet-and is pumped about 8 hours a day throughout the summer. The decper pervious beds lap out against bedrock so that some wells, such as 4 and 15, fail to develop an adequate supply of water within the alluvium.

Eugene-Junction City and Coburg-Harrisburg segments of the main valley plain (subareas 8 and 9). - In the southernmost part of the valley, for a width of 2 to 3 miles on either side of the Willamette River flood plain in Tps. 14 to 17 S., much of the older alluvium has a. fairly high water-yielding capacity. The several fire-protection wells at Junction City and the deep well recently drilled there for public supply (Nos. 636 and 637, respectively) are among the most productive. Well 637 passed entirely through the older alluvium and entered bedrock of sandy shale between 200 and 233 feet beneath the land surface. It draws water from sand and fine gravel through a 10-foot section of perforated casing from $138^{\circ}$ to 148 feet in depth; in this section of casing there are 300 perforations each $1 / 4$ inch wide and 1/4 inches long. Although not gravel-walled artificially, the well has a reported specific capacity of about 10 gallons a minute for each foot of draw-down. However, as is shown by the driller's record (table 12), it draws from only a small fraction of the total thickness of water-bearing beds and, owing to requirements of sanitation, not. at all from the most pervious beds, which are at shallow depth. For irrigation, much larger yields probably could be obtained from wells of adequate construction drawing from all pervious beds. For permanence, heavily pumped wells tapping the finer pervious beds at depth should be adequately screened, possibly also artificially gravelwalled, and thoroughly developed to arrest the movement of sand.

Not all parts of the older plain in these two subareas of the valley are underlain by pervious beds with a water-yielding capacity equal to that at Junction City. For example, wells 655 and 656 are somewhat inadequate in yield for efficient irrigation, though they are not known to have reached the bottom of the older alluvium and so to have exhausted the possibility of finding beds of high-water-yielding capacity. In general the ground-water productiveness of the two subareas probably diminishes somewhat gradually away from the Willamette River. The precise western and eastern limits of the highly productive area cannot be traced with the data now at hand; they must be felt out by further well construction and may deviate considerably from the tentative limits that have been arbitrarily defined and shown on plate 10 .

Elmira segment of the main valley plain (subarea 10). - In the extreme southwestern part of the Willamette Valley, along the Long 
Tom River in Tps. 15 to 17 S., R. 15 W., and eastward to a point about 2 miles beyond Fern Ridge, the upper part of the older alluvium is largely unassorted and contains much fine sand and silt. Accordingly, shallow wells do not yield copiously. No records of deep wells are available to indicate the water-yielding capacity of the alluvium at depth, though the capacity is inferred to be small and very probably inadequate to sustain large irrigation wells.

Brownsville-Albany segment of the main valley plain (subarea 11).Although bounded on all sides except. the eastern by subareas of large water-yielding capacity, the Brownsville-Albany segment of the older alluvial plain comprises areas that range widely in water-yielding capacity. However, the data now available do not permit close discrimination of those areas. In the central part of the segment, southward from the vicinity of Tangent to Halsey and thence eastward toward Brownsville, there are many dwellings and farms amply supplied with water from the older alluvium by driven or drilled wells less than 4 inches in diameter and from 20 to 70 feet deep. Wells 551 to 553,584 to $586,590,605,606$, and 620 are typical. The older alluvium promises relatively large yields to adequately constructed wells of greater diameter and depth, though the yield would probably not be uniform over all parts of the segment. The yearly range of ground-water level is not excessive, about 15 feet at the most. There are few data to show the thickness of the alluvium, but the thickness diminishes greatly along the Calapooya River near Brownsville.

In contrast with the area just described, in at least two extensive parts of the segment much of the older alluvium is rather tight and numerous wells are not wholly adequate for domestic and farm supplies. These parts are the triangle that extends eastward from Muddy and Dry Muddy Creeks to the foothills in the southern half of T. $14 \mathrm{~S}$. and across all of T. $15 \mathrm{~S}$. and an ill-defined belt $1 \frac{11}{2}$ to 5 miles wide along the east edge of the segment in Tps. 11 and $12 \mathrm{~S}$. and the northern two-thirds of T. $13 \mathrm{~S}$.

Along the west edge of the segment in Tps. 11-14 S., from Albany southward about to Lake Creek, the older alluvium is inferred to be underlain at relatively shallow depth by a ridge of bedrock shale whose highest parts rise slightly above the alluvial plain in scattered outliers. (See pl. 1.) Although the older alluvium there appears to be moderately or highly pervious, it may prove too thin at some places to sustain large withdrawals of water, as at wells 466,467 , and 473 to 482 , which are near the most northerly bedrock outlier, near Albany. Analogous conditions doubtless exist not only near the other outliers but possibly some distance from them as well. In this western part of the segment the yearly range in water level is large $-21 \frac{1}{2}$ feet at well 486 in $1935-36$. 
Corvallis segment of the main valley plain (subarea 12).-Beneath that part of the main valley plain which lies west of the Willamette River near Corvallis, in Tps. 11-14 S., much of the older alluvium is judged to be moderately pervious, though at some places it is probably too thin for sustained large withdrawals. Most of the wells are less than 4 inches in diameter; they were driven or drilled to depths of 25 to 45 feet and are not heavily pumped. Wells 460 and 573 are the two of greatest known capacity in the area; they suggest the feasibility of constructing wells for moderately large yield. In this area the yearly range in water level is about as large as in any part of the Willamette Valley. In 1935-36 the range was 27 feet at well 459, 21 feet at well 540, and 17 feet at wells 574 and 575 . In selecting pumps for wells in this area, therefore, special consideration should be given to this hydrologic feature.

Monmouth-Independence segment and minor segments of the main valley plain near Albany (subareas 13 and 14).-Beneath the discontinuous segments of the main valley plain that lie on both sides of the Williamette River in the narrows between Albany and Salem, the older alluvium is somewhat diverse in texture and in capacity to yield water. There much of the alluvium is quite unsorted and tight so that it yields water slowly, as at well 376 near Monmouth and well 441 near Albany. At Independence, on the other hand, well 372 encountered gravel that appears to have yielded water somewhat freely. Commonly in these areas the older alluvium is moderately thin and at no place nearly so pervious as the younger alluvium of the adjacent flood plain. Owing to the diverse conditions just described, which are known only superficially from the data now available, it seems unlikely that these scattered areas will sustain large withdrawals of water from many wells.

Stayton-Mill Creek basin (subarea 15). - The older alluvial fan that extends westward from Stayton, in Tps. 8 and 9 S., Rs. 1 and 2 W., is composed largely of coarse gravel, which is saturated with water to within 15 feet of the land surface, or less. Some of the gravel presumably is well assorted and highly pervious. The thickness of the alluvial deposit is estimated to be about 100 feet. The existing wells are of the simplest construction and ordinarily not more than 25 feet deep; they do not afford a measure of the ultimate water-yielding capacity of the deposit. In general, however, conditions appear favorable for wells of moderate or large yield, if the wells are adequate in diameter, penetration, and type of construction.

To the north, in the vicinity of Salem, ground-water conditions along Mill Creek and eastward to the Little Pudding River are rather well known from the records of existing wells. Along the western edge of the area, in Salem, the alluvium is relatively thin and yields no more than 100 gallons of water a minute to wells such as Nos. 
$331 \frac{11}{12}, 331 \frac{31}{4}, 340$, and 341 . A short distance to the east, wells 333 to 335 pass through water-bearing beds in the older alluvium but are tightly cased, probably into the underlying bedrock. Still farther east, several wells draw rather heavily from the older alluvium. Wells $297 \frac{1}{2}, 338,338 \frac{1}{2}, 360$, and 384 are representative; these have reported yields of 150 to 500 gallons a minute and reported specific capacities from 1.4 to 16.5 gallons a minute for each foot of draw-down. Driller's records (table 12) indicate that this wide range in specific capacity is due to beds of "cemented gravel," "hardpan," or "packed sand" of considerable aggregate thickness in certain wells. These beds, probably unassorted mixtures of pebbles and sand in an earthy or clayey matrix, would naturally have small capacity to transmit water. Probably, however, no one bed of this sort underlies all the area, and the water-bearing beds are interconnected to some extent Under such conditions wells of similạr size and construction may differ considerably in specific capacity, but the area as a whole probably will sustain a considerable aggregate withdrawal of water.

To the east, this northern part of the Stayton-Mill Creek basin abuts on an older terrane whose capacity to yield ground water seems in general to be very much less (see pp. 53-54). The eastward extent of the area of greater potential yield is not indicated by features exposed on the land surface and is not defined by the records of wells now available. The limit that has been set somewhat arbitrarily, the Little Pudding River, may ultimately prove to be considerably in error.

French Prairie (subarea 16).-With respect to the occurrence and potential yield of ground water, French Prairie, the most extensive segment of the main valley plain north of Salem, is quite distinct from any other subarea of the lowland plains thus far treated. In general, two bodies of ground-water exist. The first is semiperched water that supplies numerous shallow farm wells from the fine-grained upper part of the unconsolidated deposit (p.31). It is yielded much too slowly to sustain any large continual draft, such as for irrigation. The second is water confined under head in beds of clean sand and gravel in the lower part of the deposit. It is now withdrawn in moderate volume for public and private supplies and for irrigation and would doubtless sustain a much greater withdrawal.

In the central part of the area, at irrigation well 245 near Gervais, the confined water has been found in three previous zones that are thick and presumably extensive, also in several thin zones that may not be extensive. The driller's record (p. 138) shows the character and thickness of these zones; the physical properties of samples from the lowest zone are shown on page 31 . Only one well in the area, No. 168 , is deeper, and for it no complete driller's record is available. Briefly, the three principal pervious zones penetrated by well 245 are 
at depths of 68 feet, 117 feet, and 218 feet below the land surface. The upper zone consists of a 13-foot bed of sand and fine gravel; the middle zone is a 45 -foot bed of gravel and sand with a 3 -foot interbed of pebbly clay; and the lower zone consists of $25 \frac{1}{2}$ feet of gravel and sand with one thin clay interbed, cemented in part. The well was tightly cased through the upper zone and was reduced from 18 to 6 inches in diameter through the lower zone, so it draws most of its water from the middle pervious zone. From April to August 1931 the well was pumped about 525 hours, its yield ranged between 875 and 940 gallons a minute, or 1.9 to 2.1 second-feet, and its greatest draw-down and pumping lift were about 49 feet and 69 feet, respectively. The specific capacity of the well is moderate, about 18 gallons a minute for each foot of draw-down.

The extent of these three water-bearing zones can be traced only approximately. The data available from wells suggest that all three zones tend to become thinner and finer-textured toward the north and west. Possibly the third or lowest zone extends the farthest in those directions. In altitude above sea level the first or upper zone in well 245 corresponds to the coarse gravel that supplies well $2971 \%$, about 9 miles to the southwest and just beyond the edge of French Prairie; to the uppermost pervious zone in well 247 , two-thirds of a mile to the west, and in wells $261 \frac{1}{2}$ to 263 at Woodburn, 3 miles to the northeast; and to the water-bearing zone at the bottom of well 169 , about 7 miles to the northwest. Records of wells still farther north do not report pervious material at that altitude. Pervious strata about equal in altitude to the second or middle water-bearing zone are penetrated by all known wells of adequate depth as far eastward as wells 259 and 301 , about 2 miles beyond the prairie, southward to well 299, and northward to well 164. Still farther north and west, corresponding pervious material apparently was not found in well 176 at Aurora, near the northeast corner of the prairie; in wells 159 and 160 , just south of the Willamette River; nor in well 153, beyond the prairie to the west. Accordingly, this second zone seems to underlie all or nearly all the southern two-thirds of the prairie, locally to extend eastward somewhat beyond the prairie, but to feather out toward the north and west near the Willamette River. Beyond the supposed feather edge, wells of adequate depth find water at still lower horizons that are equivalent, at least in part, to the lowest pervious zone of the well near Gervais. These remote wells include Nos. 176, 159, and 153, to which reference has just been made.

The moderate specific capacity of the irrigation well near Gervais-18 gallons a minute for each foot of draw-down-indicates that the water is drawn from material which is moderately but not extremely pervious. As has been stated, most of the draft is from the second zone of confined water but, so far as can be judged from their textures, 


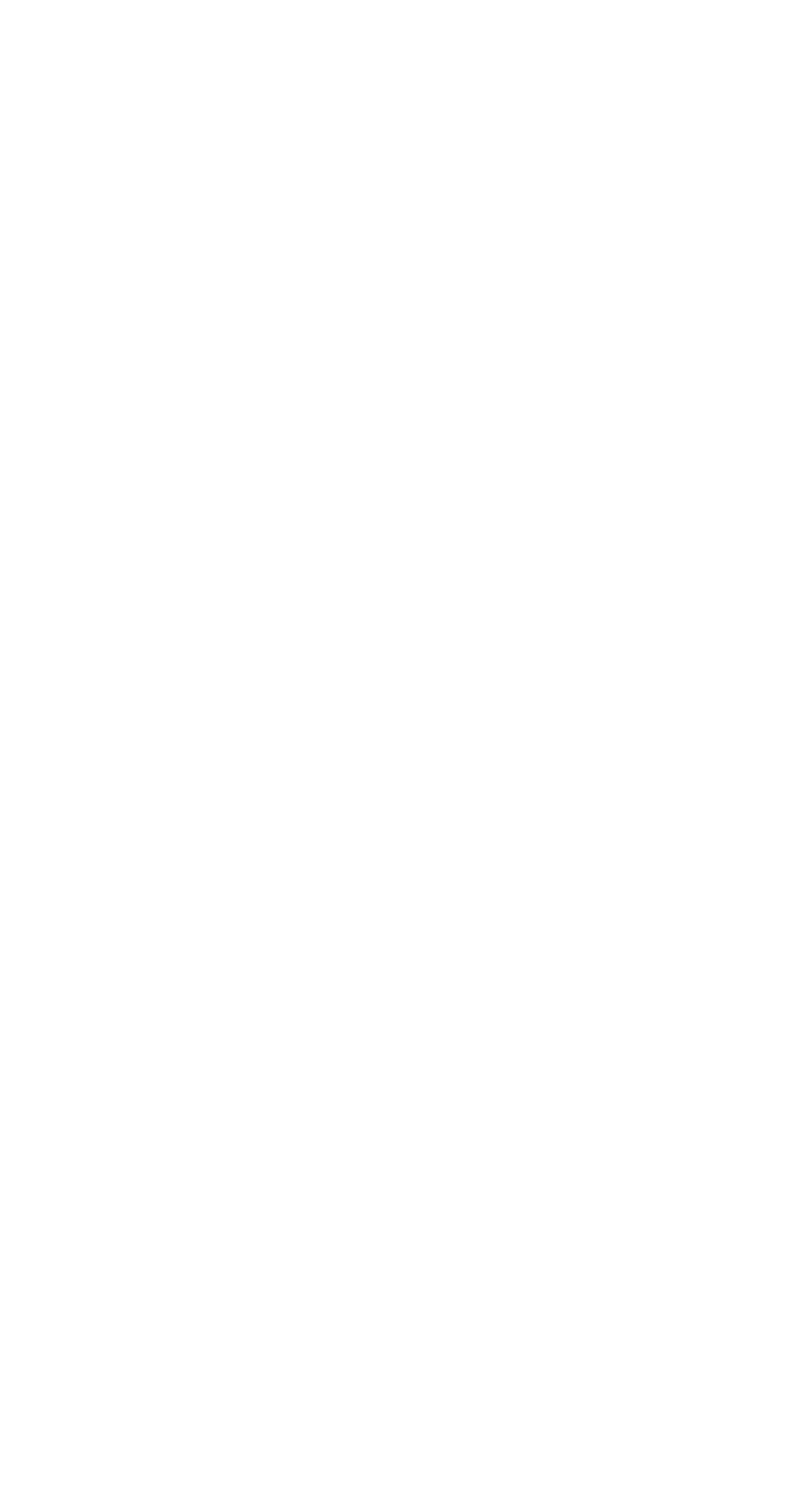




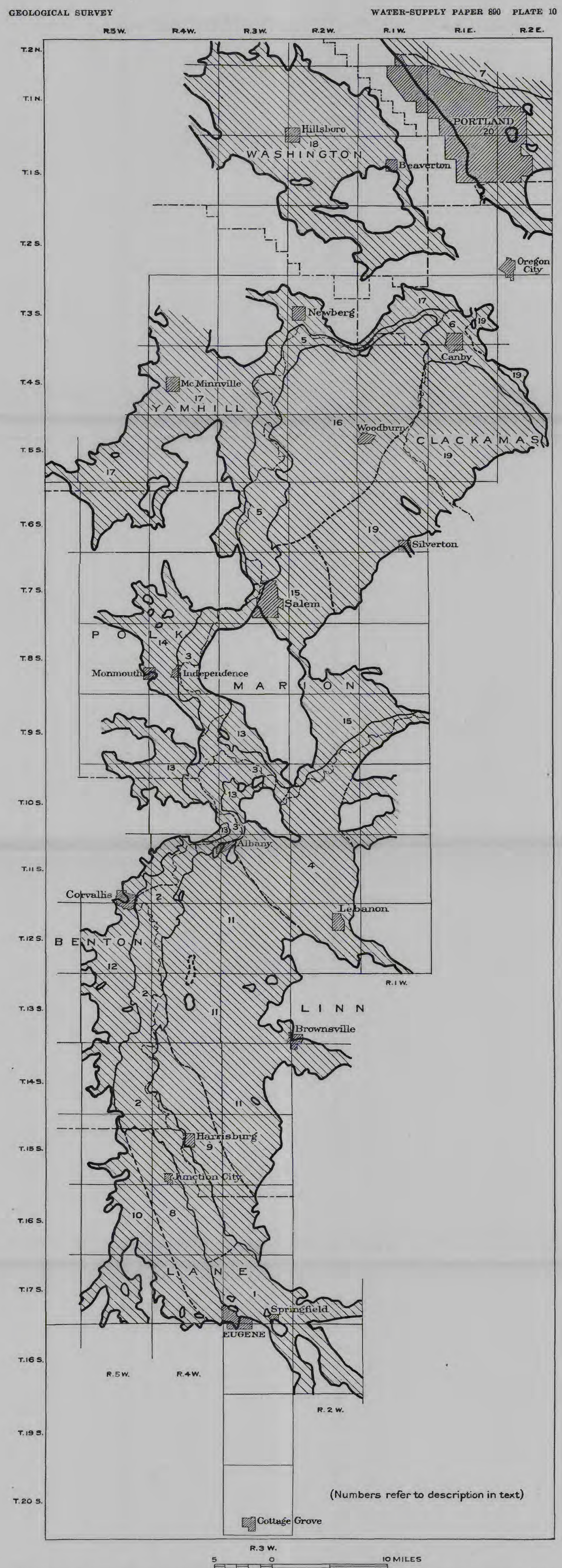

MAP OF THE WILLAMETTE VALLEY SHOWING PRINCIPAL SUBAREAS OF THE CENTRAL LOWLAND WITH RESPECT TO YIELD OF GROUND WATER. 

all three water-bearing zones are about equally pervious at that place. To the extent that each zone tends to become thinner and finertextured toward the north and west, its perviousness would tend to decrease somewhat in those directions and its total water-yielding capacity to diminish even more. As more wells are constructed to draw heavily from these zones, an appreciable decline in pressure head of the water will follow inevitably. As has been suggested, however, it is believed that the present yearly withdrawal can be increased very materially without diminishing the pressure head excessively, that is, without surpassing the safe yield. But the precise magnitude of the safe yield is not determinable from data now available.

To assure long life, wells that draw heavily from the deeper pervious zones beneath French Prairie must be adequately screened and thoroughly developed. In these respects, well 245 near Gervais has proved somewhat deficient in construction. The casing in that well was perforated in place and no gravel was placed around the casing to form an artificial envelope. In service, a considerable amount of sand has been discharged with the water, and the beds above the water-bearing zone have subsided, even up to the land surface. Under such conditions, combined with continuous heavy draft, the overlying beds may cave suddenly and in such volume as to retard the movement of water toward a well or even to destroy a well by crushing the casing. In future this hazard may be abated by determining the sizes of the grains in the water-bearing materials-through sieve analyses of an adequate set of samples-and choosing a well screen with ports or openings of suitable width. After a suitable screen has been placed, the newly" constructed well may be "rawhided" or otherwise developed. If the well is constructed by perforating the casing in place, which may produce openings too wide to restrain the sand properly, screened gravel of suitable size may be inserted around the casing during the development until a stable envelope of coarse grains is formed. In this type of construction, the gravel envelope commonly is extended above the water-bearing zone or even to the land surface, thereby forming a reservoir of highly pervious material to replace any sand that might otherwise be drawn through the perforated casing after the well is in use.

Dayton Prairie and adjacent areas (subarea 17).-Topographically and genetically, Dayton Prairie and the adjacent parts of the main lowland plain west and north of the Willamette River are strictly analogous to French Prairie, but in spite of common genesis the two areas are unlike in the relative extent of their principal water-bearing strata. In part, this difference is determined by the form of the common bedrock floor that underlies all the lowland plains. In general the bedrock is impervious shale and earthy sandstone. The older unconsolidated deposit, which forms the prairies and contains 
the three water-bearing zones of the French Prairie area, rests on the impervious bedrock and along the margin of the main lowland plain, thins to a feather edge as it laps over the sloping bedrock surface. Thus, along that margin, there is a belt in which the water-bearing zones are cut off successively by overlap on the bedrock; obviously, the deepest pervious zone is cut off farthest from the margin of the plain. With respect to Dayton Prairie, which lies in an alcove extending deep into the foothills of the Coast Range, the belt of overlap appears to be rather wide at many places, except possibly in R. $3 \mathrm{~W}$. (See pl. 7.)

Of 42 drilled wells on Dayton Prairie for which adequate records are available, one-third passed through the unconsolidated deposit without finding sufficient water for individual household supplies. Of the remaining wells, 7 found water at about the same altitude above sea level as the upper or first zone of confined water beneath French Prairie. These occur northeast of McMinnville in secs. 1, 2, 11, and 12, T. 4 N., R. 4 W.; southwest of McMinnville in secs. 1 and 12, T. 4 N., R. 5 W.; and far to the east in sec. 8 , T. 5 N., R. 3 W. At the altitude of the second zone 17 wells found water; these are widely scattered over all the Dayton Prairie area. None of these wells found the pervious zones in the unconsolidated deposit to be as thick or as pervious as in the area east of the Willamette River; only threewells 125,194 (?), and 214-have specific capacities as large as 1 gallon a minute for 1 foot of draw-down.

In general, it is inferred that the upper two zones of confined water which occur beneath French Prairie- that is, east of the Willamette River-extend discontinuously beneath the river flood plain and far into the Dayton Prairie area; also that there the zones thin markedly and finger out irregularly by gradation into nonpervious material and by overlap on the bedrock. Where these zones are thickest and of coarsest texture, wells of adequate construction might yield sufficient water for irrigation on a modest scale. However, no specific areas so productive of water can be delimited from data now available; if proved by future drilling, their extent must be felt out by cautious exploration.

Only three wells of the Dayton Prairie area-wells 139, 140, and 153-are known to have found water in the unconsolidated deposit at an altitude corresponding to that of the third zone beneath French Prairie. All three are less than a mile west of the Willamette River. Probably this deep pervious zone does not extend far westward beneath Dayton Prairie but wedges out by overlap on the bedrock in R. $3 \mathrm{~W}$.

The Dayton Prairie area includes the tongues of younger alluvium along the two forks of the Yambill River. Like the younger alluvium along the Willamette River, these tongues doubtless hold unconfined 
ground water. To a considerable extent, however, they appear to be composed of material too fine or too poorly sorted to be extremely pervious, and so probably will yield water only moderately or slowly. Information now at hand does not indicate whether these tongues are so thick that wells can have sufficient penetration to yield adequate irrigation supplies.

Tualatin Valley (subarea 18).- So far as known to the writer, the unconsolidated deposits over most of the Tualatin Valley are of relatively fine texture and of small or moderate water-yielding capacity. Along the Tualatin River and lower Gales Creek in the vicinity of Forest Grove, the younger alluvium contains coarse sand and gravel and might yield water rather freely. However, data from wells are not at hand to indicate either the water-yielding capacity or the thickness of the deposit. The deposit that underlies the main valley plain, like the corresponding deposit beneath French Prairie to the south, is fine and rather tight to a considerable depth below the land surface. Locally at least, the lower part of the deposit yields water, as in well 23 at Hillsboro. That well passed through water-bearing sand from 135 to 180 feet beneath the land surface and then entered impervious bedrock, largely soft shale. During construction, a driller's test indicated a yield of 75 gallons a minute with a draw-down of 35 feet; thus, the specific capacity was rather small, slightly more than 2 gallons a minute for each foot of draw-down. It is reported that another well has been sunk there through the same zone and, after thorough development, it yielded more copiously. It is presumed that the sand is stream-laid and therefore would tend to be coarser and more pervious toward the west. With adequately constructed wells it might yield water sufficient for irrigation. However, the deposit seems not to contain pervious beds beneath all the valley plain, for numerous farm wells, such as well 20 , have been sunk far into the bedrock to obtain dependable supplies.

Molalla pediment and related parts of the central lowland (subarea 19). -Along the eastern edge of the central lowland from Salem northward to Canby there is a gently rolling belt between 4 and 9 miles wide that rises eastward from the main valley plain. Toward the north it includes a conspicuous alluvial slope, or pediment, whose highest part is at Molalla (p. 11). The northern half of this belt, if not all of it, is underlain by partly consolidated bedrock (p. 54), which is older than the main valley plain and to the west extends beneath that plain.

The Molalla pediment is veneered with poorly assorted coarse sand and gravel that supplies numerous farm wells that were dug from 20 to 30 feet deep. However, this veneer is thin and is cut entirely through by many creek valleys and gullies, so that it would not sustain a continual large withdrawal of water. Beneath this veneer, the ma- 
terial that underlies the pediment is largely "clay" and "shale" and includes only a few water-bearing beds. (See driller's records for wells 188 and 281 , table 12.) These beds have small capacity to yield water. Wells 271,281 , and 282 yield only a small fraction of a gallon a minute for each foot of draw-down. Well 188, about 2 miles west of the edge of the pediment, passes through the feather edge of the deposit that forms the main valley plain and extends far down into material that is inferred to be partly consolidated bedrock. Even though the principal water-bearing bed in that well occurs in the over-lapping younger deposit, the specific capacity is only about 0.4 gallon a minute for each foot of draw-down. Material so slightly pervious as the water-bearing beds penetrated by these wells certainly will not sustain continual withdrawals of more than a few gallons or tens of gallons a minute from single wells. Accordingly they do not seem adequate for efficient irrigation wells.

Conditions such as those just described may prevail in all the relatively high and rolling lowland terrane beyond the Molalla pediment to the south - that is, from Butte Creek to the vicinity of Mount Angel and again from Silverton about to the Little Pudding River. In these areas, the old terrane, which is not highly productive of water, abuts against the French Prairie subarea on the west and the Mill Creek subarea on the south, both of which have relatively large capacity to yield water. The boundary between the older nonproductive deposit and the overlapping productive beds to the west and south cannot now be traced precisely but has been fixed somewhat arbitrarily (p. 42). It can be felt out by cautious further drilling.

Portland "delta" (subarea 20).--At the land surface, the area of the so-called Portland delta includes several distinct unconsolidated stream deposits of Quaternary age. At depth the area is underlain, at least in part, by semiconsolidated deposits of early Quaternary or latest Tertiary age, chiefly by the Troutdale formation of Hodge (p. 23). During the reconnaissance that forms the basis of this report, only cursory attention was given to the character, extent, and stratigraphic relations of these deposits; also, records were obtained from only a few typical wells (Nos. 26-33, 36-39, 55-60, and 62-67). Although these data indicate several distinct water-bearing zones, they are not adequate to discriminate the zones sharply with respect to depth beneath the land surface, lateral extent, and relative pressure head of the water. In reported yield, the typical wells range between 50 and 1,000 gallons a minute; in reported specific capacity, commonly from 10 to about 33 gallons a minute for each foot of draw-down. It is judged that the area will sustain a much greater aggregate withdrawal and will readily afford wells of eapacities adequate for irrigation and for many industrial purposes. 


\section{FLOWING WELLS}

Certain pervious beds in both the unconsolidated deposits and underlying consolidated rocks of the Willamette Valley contain water under sufficient pressure head to rise above the land surface and so to yield flowing wells. However, the known areas of artesian flow are few, small, and scattered; none yield copiously by artesian head alone. Further, some of the flowing wells in the consolidated rocks yield salt water.

With one exception, the flowing wells known to yield water from the unconsolidated deposits are in the northern half of the lowland, between Salem and Canby, and on the flanks or floors of the larger stream trenches. These wells are Nos. 176,233, and 268; all three are inferred to draw water from deposits related to the older alluvium. All have small pressure heads at the land surface and so flow but slightly; however, it is not known whether the wells are so cased that none of the artesian head is dissipated by leakage into nonartesian aquifers. Corresponding areas of artesian flow doubtless extend only to a moderate height above the floors of the several stream trenches. Their extent along the trenches is suggested by the contours of pressure head for tightly cased deep wells (pl. 1). This head appears adequate to yield flowing wells over much of the Willamette River flood plain from the vicinity of Salem northward about to Newberg, in Tps. 4 to $6 \mathrm{~S}$.; on the lower flanks and floor of the Pudding River and Butte Creek trenches downstream from the vicinity of Monitor, in Tps. 4 and $5 \mathrm{~S}$.; and, possibly, in some other low areas such as the Lake Labish trench. The areas of artesian flow terminate northward as the pervious zones feather out (see p. 50) and southward as the pervious zones lap against older and tighter material (see p. 54).

The one well in the southern half of the lowland known to flow from the older alluvium, No. 566, is near the apex of the Lebanon alluvial fan but low on the flank of a shallow drain. In that well the artesian flow is variable and commonly ceases in late summer when the pressure head is least. If the artesian head is the result of the water-bearing zone fingering into less pervious material, as seems probable, the potential area of flow is not extensive.

Wells that flow by artesian pressure from the consolidated rocks are scattered widely the full length of the Willamette Valley and derive water from numerous stratigraphic zones. Apparently there are numerous areas of artesian flow, but none are extensive and none of the wells flow very copiously. In and near Portland the west flank of the Willamette syncline and the southeastward-plunging anticline that underlies the Tualatin Valley together afford an artesian slope. The uppermost bedrock formation of that slope, the Yakima basalt, is the probable source of water for flowing wells 26,51 , and 74 . How- 
ever, water-bearing zones in that formation are discontinuous and appear to have distinct pressure heads; also, the land surface on that formation has a relief of about 1,000 feet. Thus, flowing wells can be obtained only in small areas that are indeterminate in extent and location. For example, well 51 flows rather copiously, yet well 53 does not flow, although it is only half a mile to the east, and is 400 feet deeper and some 20 feet below the reported static water level of well 51. About $3 \frac{1}{2}$ miles southeast of flowing well 74 , at a point seemingly lower on the artesian slope and lower in altitude of land surface, another well--No. 76-is considerably deeper and yet does not flow. However, neither well is tightly cased through shallow pervious zones, so that some pressure head may be dissipated in either well or both. West of these flowing wells are wells $18,20,44$, and 45 which flow slightly from sedimentary rocks in the same artesian slope. These rocks are tentatively inferred to lie stratigraphically beneath the basalt, which has been breached by erosion along the axis of the plunging anticline. It seems unlikely that these rocks will yield flowing wells over an extensive part of the Tualatin Valley plain, for in general they contain only a few discontinuous pervious zones. (See p. 26.)

Southwest of the Tualatin Valley, about 5 miles northeast of McMinnville, well 110 flows a trickle, presumably from sedimentary rocks below the Yakima basalt. This well yields "mineral water" from an unknown depth below the land surface. Its pressure lead is so small that similar flowing wells could be obtained only within a very narrow belt along the footslope of the bedrock hills.

Still farther south, along and near the west edge of the central lowland, beds of sandstone and sandy shale in the Eocene rocks yield or have yielded water under sufficient pressure head to flow at wells $287,457,577,593,597$, and 610 . In general, these rocks form an artesian slope that dips gently eastward beneath the central lowland. However, they are relatively impervious, and the pressure head of their water is not adequate for flowing wells high above the lowland plain; so large yields by artesian pressure are unlikely. Wells 287 and 610 are reported to have yielded salt water, well 610 from a depth of only 300 feet beneath the main lowland plain.

Flowing well 564, near Lebanon, taps volcanic rock, possibly correlative to the Yakima basalt, very near the west margin of the lowland. Flowing well 713, at Eugene, finds its water in igneous rock associated with the Eugene formation, of Oligocene age. At neither place is the extent of the area of artesian flow known.

\section{CHEMICAL CHARACTER OF THE GROUND WATER}

Table 9 shows the chemical character of water samples from 35 wells in the Willamette Valley. Of these, 28 samples were taken from wells in the unweathered younger or older alluvium, 3 from wells 
in the weathered terrace deposit, and 4 from wells that penetrate bedrock. Figure 3 shows graphically the results of analyses of the waters from 20 of the wells. These chemical analyses show the mineral content of the water, but they show little with respect to the sanitary quality. Thus, from the analyses the suitability of the waters for the common industrial or commercial uses and for irrigation can be determined, but not their suitability for human consumption.

Waters that contain less than 500 parts per million of dissolved solids ordinarily are suitable for domestic purposes and for many industrial and commercial uses, except for the inconveniences that may be caused by their hardness. Most but not all waters that contain more than 1,000 parts per million are likely to have a perceptible taste and to be unsatisfactory for ordinary uses owing to an excessive amount of some one or several constituents. According to these limits, all waters in the unconsolidated deposits of the Willamette Valley appear to be quite satisfactory for most ordinary uses; but certain waters from the consolidated rocks are unsatisfactory. (See p. 64.)

With respect to ordinary uses, the chemical suitability of the ground waters of the Willamette Valley depends largely on the content of calcium and magnesium, the constituents that cause essentially all the hardness of a water. A hard water, of course, requires more s oap to be used in laundering; it forms scale in steam boilers, hot-water tanks, and other receptacles; and it must be treated to render it suitable for some common industrial-chemical processes. Water with hardness less than 50 is commonly regarded as soft, and requires no treatment to condition it for many ordinary purposes. ${ }^{14}$ The limits of hardness below which the cost of treatment to soften a water is not justified are about as follows: 50 parts per million for the smaller laundries or other industries that use considerable soap, much less than 50 parts per million for the larger laundries and for dyeing and bleaching works; 100 parts per million for supply to small boilers and very much less for supply to large modern high-pressure boilers, to prevent the formation of scale; and 200 parts per million or less for domestic uses. In the treatment of municipal supplies, the present tendency is to furnish water with a residual hardness of about 80 parts per million. So far as is shown by the analyses in table 9 , the hardness of the water in unconsolidated deposits of the Willamette Valley is commonly between 50 and 150 parts per million, though in one sample (No. 690) the hardness was only 9.5 parts per million. Thus, water from these deposits need not be softened for many uses. On the other hand, the water in the consolidated sedimentary rocks is commonly very hard.

\footnotetext{
14 Much of this general discussion of the chemical character of water is adapted from Collins, W. D., and Howard, C. S., Chemical character of waters of Florida: U. S. Geol. Survey Water-Supply Paper 596, pp. 181-186, 1928.
} 


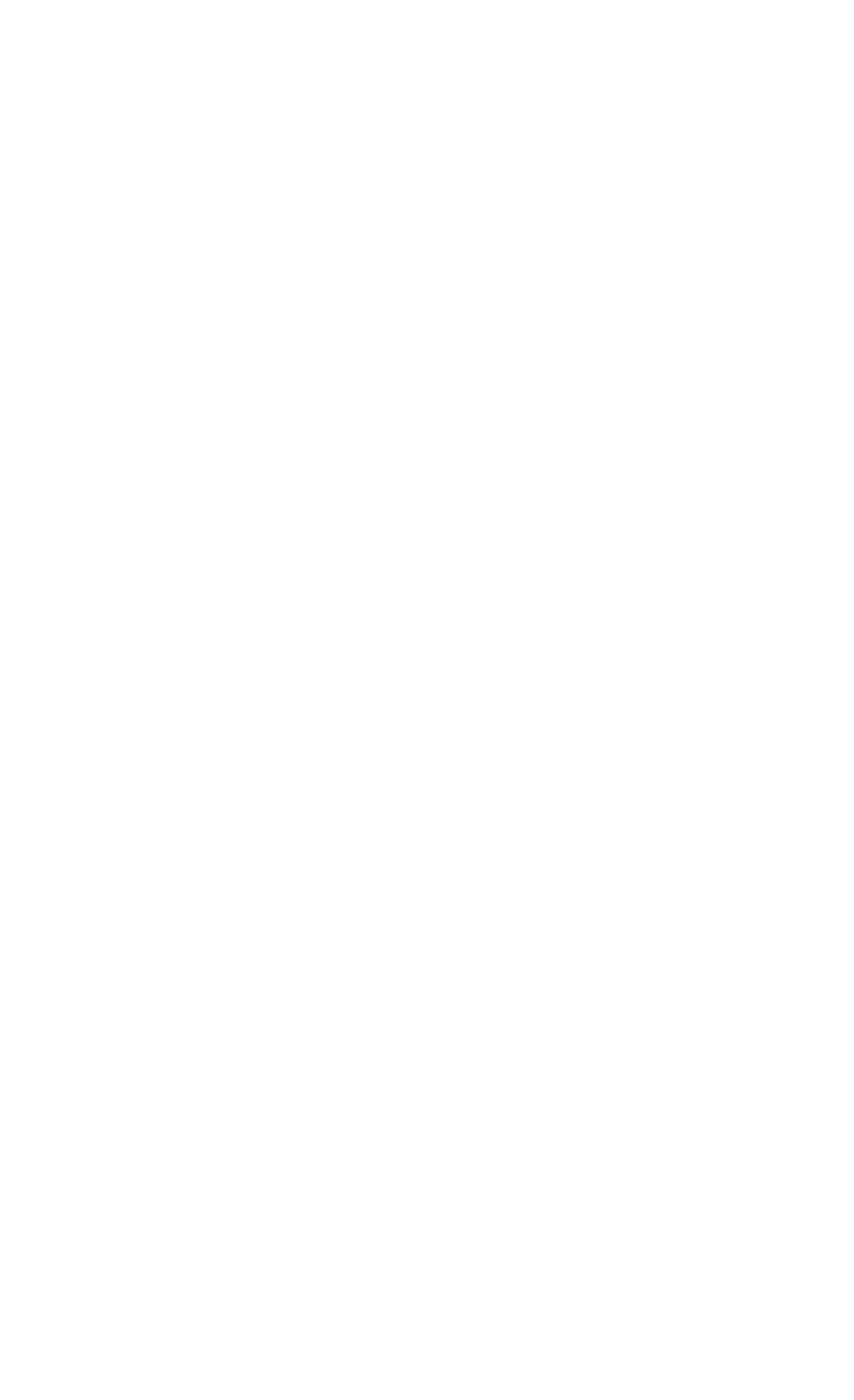


In irrigation, the chemical character of the water used is critical both with respect to the tolerance of the crop plant toward the concentration of salts in the root zone and with respect to maintenance of good tilth and suitable permeability in the soil. Other critical factors include the quantity of water applied to the land, rainfail, chemical and physical characteristics of the soil, and drainage. Scofield ${ }^{15}$ has proposed limits for the concentration of certain constituents by which to judge whether the water is satisfactory for irrigation. These limits are shown by the following table:

TABLE 8.-Approximate limits indicating the suitability of a water for irrigation

[Parts per million except as indicated]

\begin{tabular}{|c|c|c|}
\hline Constituent & Satisfactory water & Unsatisfactory water \\
\hline $\begin{array}{l}\text { Total dissolved solids } \\
\text { Sulphate, SO } \\
\text { Chloride, C] } \\
\text { Percent sodium } 1\end{array}$ & $\begin{array}{l}\text { Less than } 700 \\
192 \\
502\end{array}$ & $\begin{array}{l}\text { More than } 2,000 \text {. } \\
480 \text {. } \\
355 . \\
60 .\end{array}$ \\
\hline
\end{tabular}

1 Percent sodium $=\frac{100 \mathrm{Na}}{\mathrm{Na}+\mathrm{Ca}+\mathrm{Mg}}$, in which the three chemical constituents are expressed in equivalents in parts per million.

According to Scofield a water classified by these limits as satisfactory for irrigation is unlikely to cause root-zone conditions intolerable to ordinary crop plants or to impair seriously the physical condition of the soil. A water classified as unsatisfactory in one of the four constituents may prove intolerable to crop plants or may tend to render the soil impermeable and of such consistency that good tilth is difficult to maintain. A water whose concentration of sulphate or chloride is greater than the satisfactory limit but less than the unsatisfactory limit may be used successfully for irrigating tolerant plants if the soil is adequately drained and if the water is applied so copiously that the root zone is continually leached. By these limits, all water in the unconsolidated deposits of the Willamette Valley is highly satisfactory for irrigation and only certain waters from the consolidated rocks are unsatisfactory.

If a water contains much more than 0.1 part per million of iron, the excess iron commonly separates out as a reddish-brown sludge when the water is allowed to stand; also, it may stain cooking utensils and fabrics that are laundered. For some industrial-chemical processes, such as the manufacture of paper or rayon products, the upper limit of dissolved iron that can be tolerated in the water is even less than 0.1 part per million. Of the 31 samples of water from wells in the unconsolidated deposits of the Willamette Valley, only 13 contained 0.1 part or less per million of dissolved iron; all these were from

${ }_{15}$ Scofield, C. S., South Coastal Basin investigation, quality of irrigation waters: California Dept. Public Works, Water Resources Div., Bull. 40, pp. 21-24, 1933.

$408526-42--5$ 
TABLE 9.-Chemical analyses of water from

\begin{tabular}{|c|c|c|c|}
\hline No. & Description & $\begin{array}{l}\text { Date of } \\
\text { sample }\end{array}$ & $\begin{array}{l}\text { Tem- } \\
\text { pera- } \\
\text { ture } \\
\left({ }^{\circ} \mathrm{F} .\right)\end{array}$ \\
\hline 101 & $\begin{array}{l}\text { Canby, } 21 / 2 \text { miles northwest of; in the NW1/4SW1/4 sec. 30, T. 3 S., R. 1 E. } \\
\text { Bored well } 10 \text { inches in diameter and } 57 \text { feet deep. Isaac A. Miley } \\
\text { estate. Water from valley fill. }\end{array}$ & Oct. 10,1928 & 52 \\
\hline 102 & $\begin{array}{l}\text { Canby, municipal supply: in the } \mathrm{NW}_{1 / 4} \mathrm{SW}^{1 / 4} \text { sec. } 33, \mathrm{~T}, 3 \mathrm{~S} ., \mathrm{R} .1 \mathrm{E} \text {. } \\
\text { Drilled well } 8 \text { inches in diameter and } 107 \text { feet deep (No. 1). Water } \\
\text { from gravel (valley fill). }\end{array}$ & ......do_.... & 53 \\
\hline 103 & $\begin{array}{l}\text { Canby, municipal supply; in the } \mathrm{NW}^{1} / 4 \mathrm{SW}^{1} / 4 \mathrm{sec} \text {. } 33 \text {, T. } 3 \mathrm{~S} . \text { R. } 1 \mathrm{E} \text {. } \\
\text { Drilled well } 8 \text { inches in diameter and } 652 \text { feet deep (No. } 2) \text {. Water } \\
\text { from "fine sand" (volcanic tuff?). }\end{array}$ & .... do do & 60 \\
\hline 133 & $\begin{array}{l}\text { McMinnville, } 3 \text { miles east of: in the SW1/4SW1/4 sec. } 24, \mathrm{~T} .4 \mathrm{~S} ., \mathrm{R}_{.} 4 \mathrm{~W} \text {. } \\
\text { Bored well } 12 \text { inches in diameter and } 41 \text { feet deep. A J. Mott. Water } \\
\text { from valley fill. }\end{array}$ & Oct. 15,1928 & 52 \\
\hline $1451 / 2$ & $\begin{array}{l}\text { Dayton, } 31 / 4 \text { miles southeast of; in the NW1/4SE1/4 sec. } 26, \mathrm{~T} .4 \mathrm{~S} ., \mathrm{R} .3 \mathrm{~W} \text {. } \\
\text { Drilled well } 6 \text { inches in diameter and } 40 \text { feet deep. R. II. Wood. } \\
\text { Water from sand and gravel (young alluvium). }\end{array}$ & Oct. 13,1928 & 52 \\
\hline 164 & $\begin{array}{l}\text { St. Paul, } 1 \text { mile east of; in the } \mathrm{SW} 1 / 4 \mathrm{NE} 1 / 4 \text { sec. } 20, \mathrm{~T} .4 \mathrm{~S} ., \mathrm{R} .2 \mathrm{~W} . \\
\text { Drilled well } 6 \text { inches in diameter and } 147 \text { feet deep. Wm. Gooding. } \\
\text { Water from valley fill. }\end{array}$ & Oct. 10,1928 & 52 \\
\hline 171 & $\begin{array}{l}\text { St. Paul, } 4 \text { miles southeast of; in the SE1/4SE1/4 sec. 34, T. } 4 \mathrm{~S} ., \mathrm{R}^{2} \mathrm{~W} \text {. } \\
\text { Dug well } 18 \text { inches in diameter and } 20 \text { feet deep. Johnson School. } \\
\text { Water from valley fill. }\end{array}$ & . do do....... & 53 \\
\hline 182 & $\begin{array}{l}\text { Hubbard, municipal supply; in the } \mathrm{SW} 1 / 4 \mathrm{NW} 1 / 4 \mathrm{sec} .34, \mathrm{~T} .4 \mathrm{~S} ., \mathrm{R} .1 \mathrm{~W} . \\
\text { Drilled well } 8 \text { inches in diameter and } 130 \text { feet deep. Water irom valley } \\
\text { fill. Known locally as "sulphur water." }\end{array}$ & ..... do do & 54 \\
\hline 203 & $\begin{array}{l}\text { Sheridan, } 31 / 2 \text { miles east of; in the NE1/4NE1/4 sec. } 32, T \text {, } 5 \mathrm{~S} ., \mathrm{R}^{5} \mathrm{~W} \text {. } \\
\text { Dug well } 4 \text { feet in diameter and } 32 \text { feet deep. Wm. Piller. Water } \\
\text { from valley fill. }\end{array}$ & Oct. 15,1928 & 52 \\
\hline 228 & $\begin{array}{l}\text { Gervais, } 5 \text { miles northwest of; in the } \mathrm{SE} 1 / 4 \mathrm{SW} 1 / 4 \mathrm{sec} .13, \mathrm{~T} .5 \mathrm{~S} ., \mathrm{R} .3 \mathrm{~W} \text {. } \\
\text { Dug well } 2 \text { feet in diameter and } 291 / 2 \text { feet deep. S. F. Parker. Water } \\
\text { from valley fill. }\end{array}$ & Oct. 10,1928 & 50 \\
\hline 247 & 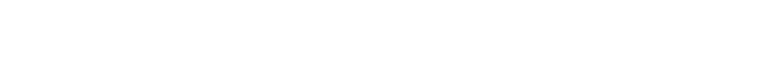 & Oct. 20,1928 & 56 \\
\hline 274 & $\begin{array}{l}\text { Molalla, } 31 / 2 \text { miles west of; in the NE1/4SW1/4 sec. } 11, \text { T. } 5 \mathrm{~S} ., \text { R. } 1 \mathrm{E} \text {. } \\
\text { Dug well } 4 \text { feet in diameter and } 27 \text { feet deep. District school. Water } \\
\text { from terrace deposit. }\end{array}$ & Oct. 10,1928 & 52 \\
\hline 294 & $\begin{array}{l}\text { Salem, } 61 / 2 \text { miles northwest of; in the } \mathrm{SE} 1 / 4 \mathrm{SE} 1 / 4 \mathrm{sec} .28, \mathrm{~T} .6 \mathrm{~S} \text {., R. } 3 \mathrm{~W} \text {. } \\
\text { Driven well } 11 / 4 \text { inches in diameter and } 31 \text { feet deep. J. J. Burton. } \\
\text { Water from valley fill. }\end{array}$ & Oct. 13,1928 & 52 \\
\hline 300 & $\begin{array}{l}\text { Gervais, } 41 / 2 \text { miles southeast of; in the SW1/4SE1/4 sec. } 13 \text {, T. } 6 \text { S., R. } 2 \text { W. } \\
\text { Dug well 31/2 feet in diameter and } 21 \text { feet deep. R. O. Dunn. Water } \\
\text { from "quicksand" (valley fill). }\end{array}$ & do & 52 \\
\hline 333 & $\begin{array}{l}\text { Salem, Madison and } 14 \text { th Streets; in the } \mathrm{NW}^{1} / 4 \mathrm{NE}^{1 / 4} \mathrm{sec} .23, \mathrm{~T} .7 \mathrm{~S} ., \mathrm{R} \text {. } \\
3 \mathrm{~W} \text {. Drilled well } 10 \text { inches in diameter and } 2481 / 2 \text { feet deep. Oregon } \\
\text { Linen Mills, Inc. Water from basalt (?). }\end{array}$ & -8 & 56 \\
\hline 342 & $\begin{array}{l}\text { West Salem, municipal supply; in the } \mathrm{NW}^{1} / 4 \mathrm{NE} 1 / 4 \text { sec. } 28, \mathrm{~T} .7 \mathrm{~S} ., \mathrm{R} .3 \\
\text { W. Dug well } 14 \text { feet in diameter and } 34 \text { feet deep. Water from } \\
\text { young alluvium. }\end{array}$ & do & 54 \\
\hline 350 & $\begin{array}{l}\text { Silverton, } 5 \text { miles southwest of; in the } \mathrm{SE} 1 / 4 \mathrm{SE} 1 / 4 \mathrm{sec} .12 \text {, T. } 7 \mathrm{~S} ., \mathrm{R} .2 \mathrm{~W} \text {. } \\
\text { Dug well } 47 \text { feet deep. R. J. Janz. Water from gravel (old allu- } \\
\text { vium). }\end{array}$ & -do & 52 \\
\hline 373 & $\begin{array}{l}\text { Independence, municipal supply; in the } \mathrm{SE} 1 / 4 \mathrm{NW} 1 / 4 \text { sec. } 28 \text {, T. } 8 \mathrm{~S} ., \mathrm{R} .4 \\
\text { W. Gang of three drilled wells } 8 \text { inches in diameter and about } 45 \text { feet } \\
\text { deep. Mountain States Power Co., Albany, Oreg. Water from } \\
\text { young alluvium. }\end{array}$ & -do_.. & 54 \\
\hline 465 & $\begin{array}{l}\text { Corvallis, } 1 \text { mile east of; in the SW1/4SE1/4 sec. } 36, T \text {, } 11 \mathrm{~S} ., \text { R. } 5 \mathrm{~W} \text {. } \\
\text { Driven well } 11 / 4 \text { inches in diameter and } 28 \text { feet deep. East farm of } \\
\text { Oregon State Agricultural College. Water from old (?) alluvium. }\end{array}$ & ....do. & 52 \\
\hline 490 & $\begin{array}{l}\text { Albany, } 51 / 2 \text { miles southwest of; in the NW1/4SE1/4 sec. 33, T. 11 S., R. } 4 \\
\text { W. Driled well } 4 \text { inches in diameter and } 295 \text { feet deep. P. A. Good- } \\
\text { win. Water from shale (bedrock of Tertiary age). }\end{array}$ & Oct. 12,1928 & 54 \\
\hline $\begin{array}{l}494- \\
498\end{array}$ & $\begin{array}{l}\text { Albany, } 3 \text { miles northeast of; in the NE1/4NW1/4 sec. } 4, \text { T. } 11 \mathrm{~S} ., \text { R. } 3 \mathrm{~W} \\
\text { Driven well } 11 / 4 \text { inches in diameter and } 41 \text { feet deep. Albany Floral } \\
\text { Co. Water from gravel (old alluvium). }\end{array}$ & do. & \\
\hline 519 & $\begin{array}{l}\text { Lebanon, } 5 \text { miles northeast of; in the NW1/4NE1/4 sec. } 24, \mathrm{~T}, 11 \mathrm{~S} ., \mathrm{R} .2 \mathrm{~W} \text {. } \\
\text { Drilled well } 4 \text { inches in diameter and } 73 \text { feet deep. Griggs School, } \\
\text { district } 4 \text {. Water from terrace deposit (?). }\end{array}$ & .....do & 54 \\
\hline 530 & $\begin{array}{l}\text { Corvallis, } 1 \text { mile south of; in the } \mathrm{SE} 1 / 4 \mathrm{SW} 1 / 4 \mathrm{sec} .2 \text {, T. } 12 \mathrm{~S} \text {., R. } 5 \mathrm{~W} \text {. } \\
\text { Drilled well } 3 \text { inches in diameter and } 32 \text { feet deep. Shell Co. of Cali- } \\
\text { fornia. Water from gravel (old ? alluvium). }\end{array}$ & .....do.. & 54 \\
\hline
\end{tabular}


[Margaret D. Foster, analyst]

\begin{tabular}{|c|c|c|c|c|c|c|c|c|c|c|c|c|c|}
\hline \multicolumn{10}{|c|}{ Analyses (parts per million) } & \multicolumn{4}{|l|}{ - } \\
\hline $\begin{array}{l}\text { Total } \\
\text { dis- } \\
\text { solved } \\
\text { solids } \\
\text { at } 180^{\circ} \\
\text { C. }\end{array}$ & $\begin{array}{l}\text { Silica } \\
\left(\mathrm{SiO}_{2}\right)\end{array}$ & $\begin{array}{l}\text { Iron } \\
(\mathrm{Fe})^{1}\end{array}$ & $\begin{array}{l}\text { Cal- } \\
\text { cium } \\
\text { (Ca) }\end{array}$ & $\begin{array}{c}\text { Mag- } \\
\text { ne- } \\
\text { sium } \\
(\mathrm{Mg})\end{array}$ & $\begin{array}{c}\text { So- } \\
\text { dium } \\
\text { (Na) }\end{array}$ & $\begin{array}{c}\text { Po- } \\
\text { tas- } \\
\text { sium } \\
(\mathbf{K})\end{array}$ & $\begin{array}{c}\text { Bicar- } \\
\text { bonate } \\
\left(\mathrm{HCO}_{3}\right)\end{array}$ & $\begin{array}{c}\text { Sul- } \\
\text { phate } \\
\left(\mathrm{SO}_{4}\right)\end{array}$ & $\begin{array}{l}\text { Chlo- } \\
\text { ride } \\
\text { (Cl) }\end{array}$ & $\begin{array}{c}\mathrm{Ni}- \\
\text { trate } \\
\left(\mathrm{NO}_{3}\right)\end{array}$ & $\begin{array}{c}\text { Total } \\
\text { hard- } \\
\text { ness as } \\
\mathrm{CaCO}_{3}^{2}\end{array}$ & $\begin{array}{c}\text { Per- } \\
\text { cent } \\
\text { so- } \\
\text { dium } 3\end{array}$ & No. \\
\hline 112 & 60 & 0.56 & 8.7 & 7.0 & 4.1 & 1.1 & 62 & 5.4 & 1.8 & 0.05 & 50 & 17.0 & 101 \\
\hline 163 & 41 & .19 & 24 & 11 & 7.4 & 1.8 & 132 & 5.0 & 4.0 & 2.4 & 105 & 14.9 & 102 \\
\hline 326 & 45 & .21 & 11 & 5.5 & 93 & 2.9 & 258 & 4.1 & 29 & .96 & 50 & 80.4 & 103 \\
\hline 197 & 21 & .23 & 40 & 10 & 12 & 1.4 & 160 & 17 & 10 & 3.8 & 141 & 16.5 & 133 \\
\hline 132 & 42 & .37 & 15 & 8.0 & 7.2 & 1.3 & 88 & 5.5 & 2.6 & 5. 3 & 70 & 19.8 & $1451 / 2$ \\
\hline 179 & 43 & 5. 27 & 27 & 11 & 12 & 1.7 & 165 & 3. 3 & 2.5 & .10 & 113 & 20.1 & 164 \\
\hline 173 & 28 & .15 & 41 & 4.4 & 9.0 & 1.2 & 159 & 5.8 & 3.0 & 1.0 & 121 & 14.9 & 171 \\
\hline 123 & 41 & .50 & 17 & 6.3 & 5.6 & 1.4 & 88 & 4.9 & 2.6 & .45 & 68 & 17.0 & 182 \\
\hline 188 & 39 & .37 & 29 & 11 & 14 & 1.0 & 146 & 4.3 & 10 & 7.8 & 118 & 21.2 & 203 \\
\hline 198 & 19 & .46 & 17 & 6.1 & 26 & 6.0 & 57 & 12 & 26 & 43 & 68 & 48.7 & 228 \\
\hline 204 & 48 & 1. 17 & 25 & 18 & 15 & 1.7 & 193 & 2.9 & 3.5 & 1.1 & 136 & 20.3 & 247 \\
\hline 104 & 41 & .19 & 12 & 2.1 & 5.9 & 1.0 & 48 & 4. 2 & 2.4 & 5.2 & 39 & 26.8 & 274 \\
\hline 129 & 42 & .15 & 16 & 10 & 3.5 & .4 & 92 & 5.8 & 5.0 & 4.0 & 81 & 9. 1 & 294 \\
\hline 134 & 59 & .23 & 12 & 5.9 & 6.7 & 1.0 & 63 & 4.2 & 5.8 & 4. 2 & 54 & 22.6 & 300 \\
\hline 146 & 46 & .02 & 17 & 12 & 8.7 & 1.2 & 130 & 3.5 & 3.0 & .0 & 92 & 18.2 & 333 \\
\hline 156 & 57 & .10 & 18 & 10 & 9.1 & .7 & 109 & 5.3 & 5.5 & 2.8 & 86 & 19.4 & 342 \\
\hline 114 & 34 & 2.13 & 11 & 4.5 & 7.6 & .6 & 35 & 4.8 & 11 & 15 & 46 & 27.3 & 350 \\
\hline 188 & 44 & .12 & 24 & 15 & 13 & 1.3 & 145 & 8.2 & 12 & 3.6 & 122 & 19.7 & 373 \\
\hline 113 & 41 & .02 & 12 & 8. 6 & 5.2 & .7 & 80 & 3.5 & 3.2 & 2.3 & 65 & 15.7 & 465 \\
\hline 4,967 & .15 & 1.03 & 324 & 62 & 1,450 & 14 & 100 & 1.9 & 2,956 & .0 & 1,064 & 74.9 & 490 \\
\hline 116 & 38 & .05 & 12 & 6.9 & 5.7 & 1.6 & 71 & 4.4 & 2.4 & 6.7 & 58 & 19.8 & $\left\{\begin{array}{l}49 \div- \\
498\end{array}\right.$ \\
\hline 150 & 25 & .93 & 7.1 & 3.5 & 38 & 2. 2 & 132 & 3.0 & 5.5 & 1. 0 & 32 & 72.7 & 519 \\
\hline 230 & 45 & .04 & 34 & 14 & 22 & 1.6 & 182 & 2.7 & 28 & .10 & 142 & 25.9 & 530 \\
\hline
\end{tabular}

1 Includes iron precipitated at time of analysis.

3 After Scofield; see explanation in text. 
TABLE 9.-Chemical analyses of water from

\begin{tabular}{|c|c|c|c|}
\hline No. & Description & $\begin{array}{l}\text { Date of } \\
\text { sample }\end{array}$ & $\begin{array}{l}\text { Tem- } \\
\text { pera- } \\
\text { ture } \\
\left({ }^{\circ} \text { F.) }\right.\end{array}$ \\
\hline 533 & $\begin{array}{l}\text { Corvallis, } 21 / 2 \text { miles south of; in the } \mathrm{SE} 1 / 4 \mathrm{SE} 1 / 4 \mathrm{sec} .10, \mathrm{~T} .12 \mathrm{~S} ., \mathrm{R} .5 \mathrm{~W} . \\
\text { Drilled well } 3 \text { inches in diameter and } 122 \text { feet deep. Mrs. A. Sims. } \\
\text { Water from shale (bedrock of Tertiary age). }\end{array}$ & Oct. 12,1928 & 53 \\
\hline 551 & $\begin{array}{l}\text { Tangent, municipal supply; in the SW1 } 1 \text { NW1/4 sec. } 7 \text {, T. } 12 \mathrm{~S} \text {, R. } 3 \mathrm{~W} \text {. } \\
\text { Gang of five driven wells } 11 / 4 \text { inches in diameter and } 45 \text { feet deep. } \\
\text { Water from gravel (old alluvium). }\end{array}$ & Apr. 18,1929 & 51 \\
\hline 566 & $\begin{array}{l}\text { Lebanon, in the SW1/4SW1/4 sec. } 11, \text { T. } 12 \mathrm{~S}, \text { R. } 2 \mathrm{~W} \text {. Drilled well } 6 \\
\text { inches in diameter and } 115 \text { feet deep. Mountain States Power Co. } \\
\text { Water from gravel (old ? alluvium). }\end{array}$ & Oct. 12,1928 & 54 \\
\hline 580 & $\begin{array}{l}\text { Shedd, } 5 \text { miles west of; in the } \mathrm{SE} 1 / 4 \mathrm{NW} 1 / 4 \text { sec. } 5, \mathrm{~T} .13 \mathrm{~S} ., \mathrm{R} .4 \mathrm{~W} \text {. Driven } \\
\text { well } 11 / 4 \text { inches in diameter and } 31 \text { feet deep. Gustav Hahn. Water } \\
\text { from gravel (young alluvium). }\end{array}$ & Oct. 10,1928 & 54 \\
\hline 595 & $\begin{array}{l}\text { Monroe, } 4 \text { miles north of in the } \mathrm{SW} 1 / 4 \mathrm{SW} 1 / 4 \mathrm{sec} .3 \text {, } \mathrm{T} .14 \mathrm{~S} \text {., R. } 5 \mathrm{~W} \text {. } \\
\text { Driven well } 11 / 4 \text { inches in diameter and } 34 \text { feet deep. McFarland }\end{array}$ & Oct. 12,1928 & 50 \\
\hline 606 & $\begin{array}{l}\text { Halsey, municipal supply; in the SE1/4NE1/4 sec. } 1, \text { T. } 14 \mathrm{~S} \text {., R. } 4 \mathrm{~W} \text {. } \\
\text { Dug well } 10 \text { feet in diameter and } 28 \text { feet deep with two drilled wells } 4 \\
\text { inches in diameter in bottom, plugged at total depth of } 72 \text { feet. Citi- } \\
\text { zens Light \& Water Co. Water mainly rom old alluvium (some } \\
\text { saline water from bedrock probably passes the plug). }\end{array}$ & Apr. 18,1929 & 52 \\
\hline 631 & $\begin{array}{l}\text { Harrisburg, municipal supply; in the NE1/4NE1/4 sec. } 16 \text {, T. 15 S., R. } 4 \\
\text { W. Dug well } 10 \text { feet in diamter and } 30 \text { feet deep with six 3-inch drilled } \\
\text { wells in bottom, total depth of } 60 \text { feet. H. F. Meersdorf. Water } \\
\text { from old alluvium. }\end{array}$ & Oct. 12,1928 & 55 \\
\hline 638 & $\begin{array}{l}\text { Junction City, municipal supply; in the } S E 1 / 4 \mathrm{SW}_{1} / 4 \text { sec. } 32, \mathrm{~T} .15 \mathrm{~S} . \text { R. } 4 \\
\text { W. Drilled well } 4 \text { inches in diameter and } 189 \text { feet deep. J. H. Miller } \\
\text { \& Sons. Water from old alluvium (?). }\end{array}$ & $\ldots$ do & 54 \\
\hline 653 & $\begin{array}{l}\text { Coburg, } 41 / 2 \text { miles northwest of; in the NE } 1 / 4 \mathrm{NE}^{1 / 4} \text { sec. } 14, \mathrm{~T} .16 \mathrm{~S} ., \mathrm{R} .4 \\
\text { W. Driven well } 11 / 2 \text { inches in diameter and } 16 \text { feet deep. R. W. Har- } \\
\text { rington. Water from young alluvium. }\end{array}$ & .... do & 58 \\
\hline 690 & 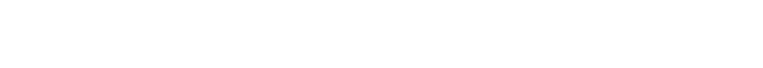 & ..... do. & 52 \\
\hline 695 & $\begin{array}{l}\text { Eugene, } 61 / 2 \text { miles northwest of; in the NW1,4 SW1 } 1 / 4 \text { sec. } 8 \text {, T. } 17 \mathrm{~S} ., \mathrm{R}^{4} 4 \\
\text { W. Driven well } 11 / 4 \text { inches in diameter and } 25 \text { feet deep. Clear Lake } \\
\text { School. Water from old alluvium. }\end{array}$ & Apr. 18,1929 & 53 \\
\hline 711 & $\begin{array}{l}\text { Eugene, } 2 \text { miles east of; in the SW1/4 SE } 1 / 4 \text { sec. } 28, \text { T. } 17 \text { S., R. } 3 \text { W. Dug } \\
\text { well } 3 \text { feet in diameter and } 161 / 2 \text { feet deep. Chose Gardens. Water } \\
\text { from old alluvium. }\end{array}$ & Oct. 12,1928 & 54 \\
\hline
\end{tabular}


representative wells in the Willamette Valley-Continued

\begin{tabular}{|c|c|c|c|c|c|c|c|c|c|c|c|c|c|}
\hline \multicolumn{14}{|c|}{ Analyses (parts per million) } \\
\hline \begin{tabular}{|c} 
Total \\
dis- \\
solved \\
solidis \\
at $180^{\circ}$ \\
C.
\end{tabular} & $\begin{array}{l}\text { Silica } \\
\left(\mathrm{SiO}_{2}\right)\end{array}$ & $\begin{array}{l}\text { Iron } \\
(\mathrm{Fe})\end{array}$ & $\begin{array}{l}\text { Cal- } \\
\text { cium } \\
\text { (Ca) }\end{array}$ & $\begin{array}{c}\text { Mag- } \\
\text { ne- } \\
\text { sium } \\
(\mathrm{Mg})\end{array}$ & $\begin{array}{c}\text { So- } \\
\text { dium } \\
\text { (Na) }\end{array}$ & $\begin{array}{c}\text { Po- } \\
\text { tas- } \\
\text { sium } \\
(\mathbf{K})\end{array}$ & $\begin{array}{c}\text { Bicar- } \\
\text { bonate } \\
\left(\mathrm{HCO}_{3}\right)\end{array}$ & $\begin{array}{c}\text { Sul- } \\
\text { phate } \\
\left(\mathrm{SO}_{4}\right)\end{array}$ & $\mid \begin{array}{c}\text { Chlo- } \\
\text { ride } \\
\text { (Cl) }\end{array}$ & $\begin{array}{c}\mathrm{Ni}- \\
\text { trate } \\
\left(\mathrm{NO}_{3}\right)\end{array}$ & $\begin{array}{c}\text { Total } \\
\text { hard- } \\
\text { ness as } \\
\mathrm{CaCO}_{3}\end{array}$ & $\begin{array}{c}\text { Per- } \\
\text { cent } \\
\text { so- } \\
\text { dium }\end{array}$ & No. \\
\hline 18,102 & 19 & 0.86 & 4,630 & 22 & 2,025 & 18 & 20 & 3.5 & 11,381 & 0.0 & 11,665 & 27.5 & 533 \\
\hline 204 & 35 & .08 & 31 & 18 & 14 & 1.1 & 193 & 2.1 & 13 & 3.8 & 151 & 17.4 & 551 \\
\hline 98 & 36 & .03 & 12 & 5.1 & 6.3 & 1.4 & 74 & 3.5 & 2.0 & .25 & 51 & 23.3 & 566 \\
\hline 197 & 49 & .09 & 26 & 15 & 7.0 & 2.2 & 134 & 4.7 & 9.0 & 16 & 126 & 12.5 & 580 \\
\hline 220 & 52 & .03 & 27 & 16 & 21 & 1.2 & 168 & 3.5 & 28 & .73 & 133 & 26.2 & 595 \\
\hline 502 & 45 & .03 & 74 & 23 & 55 & 1.9 & 211 & 2.1 & 153 & 9.0 & 279 & 30.4 & 606 \\
\hline 207 & 49 & .18 & 24 & 16 & 7.0 & 1.8 & 110 & 10 & 22 & 10 & 126 & 12.2 & 631 \\
\hline 168 & 40 & .38 & 15 & 10 & 24 & 1.2 & 100 & 2.7 & 32 & .05 & 78 & 40.6 & 638 \\
\hline 56 & 26 & .06 & 5.0 & 2.9 & 3.2 & 1.5 & 33 & 3.8 & 1.8 & .20 & 24 & 26. 7 & 653 \\
\hline 26 & 8.8 & . 16 & 2.0 & 1.1 & 2.2 & .5 & 5.0 & 3.4 & 2.2 & 4.0 & 9.5 & 36.3 & 690 \\
\hline 109 & 51 & .02 & 9.0 & 5.1 & 5.5 & .4 & 58 & 2.8 & 2.2 & 2.7 & 43 & 22.3 & 695 \\
\hline 128 & 43 & .06 & 15 & 9.0 & 4.0 & 1.4 & 84 & 5.9 & 2.7 & 4.6 & 74 & 12.3 & 711 \\
\hline
\end{tabular}


the southern half of the valley, between Salem and Eugene. All the samples of water from the unconsolidated materials in the northern half of the valley contained more than 0.1 part per million of iron; the greatest quantity, 5.27 parts per million, was from well 164 , which is 7 miles northwest of Woodburn. The general data on wells in the vicinity of Portland indicate that several yield water containing an excessive quantity of iron; these include wells $29,30,47,52$, and 77. Other wells in the same area doubtless yield water with an iron content so large as to be troublesome for some uses. From most waters, the excess iron may be removed by simple aeration and filtration; some waters, however, must be treated with lime or certain other substances to assist in precipitating the excess iron.

Reference has been made before to the salty to brackish water in the consolidated sedimentary rocks. The analyses of samples from wells 490 and 533 show that these waters are chemically unfit for many ordinary uses. The sample from well 606 doubtless represents a water of mixed origin-nonsaline water from the older alluvium with a small admixture of saline water from the underlying sedimentary rock. Saline water is reported to have been struck in numerous other wells that are widely scattered in the region. These include wells 4,5 , and 15 , which entered bedrock, presumably sedimentary rock of marine origin, beneath the flood-plain deposit of the Columbia River downstream from Portland; wells 112, 123 to 125, 142, 200, 218, and 287, in the basin of the South Yamhill River near McMinnville, in Tps. 4 to 6 S., Rs. 4 and 5 W.; wells $375,391,469,533$, and 537, along the west flank of the central lowland from Monmouth southward to Corvallis and somewhat beyond, in Tps. 8 to 12 S., R. 4 W.; wells 490 and 610 , on the lowland east of the Willamette River, in R. 4 W., Tps. 11 and 14 S., respectively; and well 641 , along the east flank of the lowland in T. 15 S., R. 3 W. These general data suggest that wells much more than 100 feet deep at any place along the flank of the western foothills, or wells in the western half of the central lowland that pass through the unconsolidated deposits and penetrate the sedimentary rocks more than a few tens of feet, may strike salty water unfit for most uses, certainly unfit for domestic supplies or for irrigation. 
WELL RECORDS

TABLE 10.-Wells in the Willamette Valley for which records of ground-water level have been published in other water-supply papers ${ }^{1}$

\begin{tabular}{|c|c|c|c|c|c|}
\hline $\begin{array}{c}\text { Number } \\
\text { of well in } \\
\text { this re- } \\
\text { port }\end{array}$ & $\begin{array}{c}\text { Number of } \\
\text { well in other } \\
\text { published } \\
\text { water-supply } \\
\text { papers }\end{array}$ & Owner & $\begin{array}{l}\text { Number } \\
\text { of well in } \\
\text { this re- } \\
\text { port }\end{array}$ & $\begin{array}{c}\text { Number of } \\
\text { well in other } \\
\text { published } \\
\text { water-supply } \\
\text { papers }\end{array}$ & Owner \\
\hline $\begin{array}{l}158 \ldots \\
171 \ldots \ldots \\
196 \ldots \ldots \\
297 \ldots \ldots \\
318 \ldots \ldots \\
421 \ldots \ldots \\
463\end{array}$ & $\begin{array}{l}8 \mathrm{H}-4 \mathrm{cl} \\
8 \mathrm{H}-34 \mathrm{rl} \ldots \\
9 \mathrm{E}-13 \mathrm{bl} \\
10 \mathrm{G}-33 \mathrm{r} 1 \ldots \\
10 \mathrm{~K}-7 \mathrm{ml} \\
14 \mathrm{~F}-12 \mathrm{f1} \\
15 \mathrm{E}-36 \mathrm{q} 1\end{array}$ & $\begin{array}{l}\text { W. J. Gering. } \\
\text { Johnson School. } \\
\text { George Fuller. } \\
\text { Gideon E. Stolz. } \\
\text { Fred Lucht. } \\
\text { Henry Hoefer. } \\
\text { Oregon Agricultural Ex- } \\
\text { periment Station. }\end{array}$ & $\begin{array}{l}553 \ldots \\
568 \ldots \\
590 \ldots \\
636 \ldots \ldots \\
680 \ldots \ldots\end{array}$ & $\begin{array}{l}16 \mathrm{G}-9 \mathrm{r} 1 \\
16 \mathrm{H}-14 \mathrm{cl} \\
17 \mathrm{G}-34 \mathrm{nl} \\
19 \mathrm{~F}-32 \mathrm{ml} \\
20 \mathrm{G}-32 \mathrm{~g} 3\end{array}$ & $\begin{array}{l}\text { J. H. Swatzka. } \\
\text { Ray Fisher. } \\
\text { Keeney School. } \\
\text { Junction City. } \\
\text { Leo Sidwell. }\end{array}$ \\
\hline
\end{tabular}

1 U. S. Geol. Survey Water-Supply Papers 777, pp. 145-149, 1936; 817, pp. 256-259, 1937. 


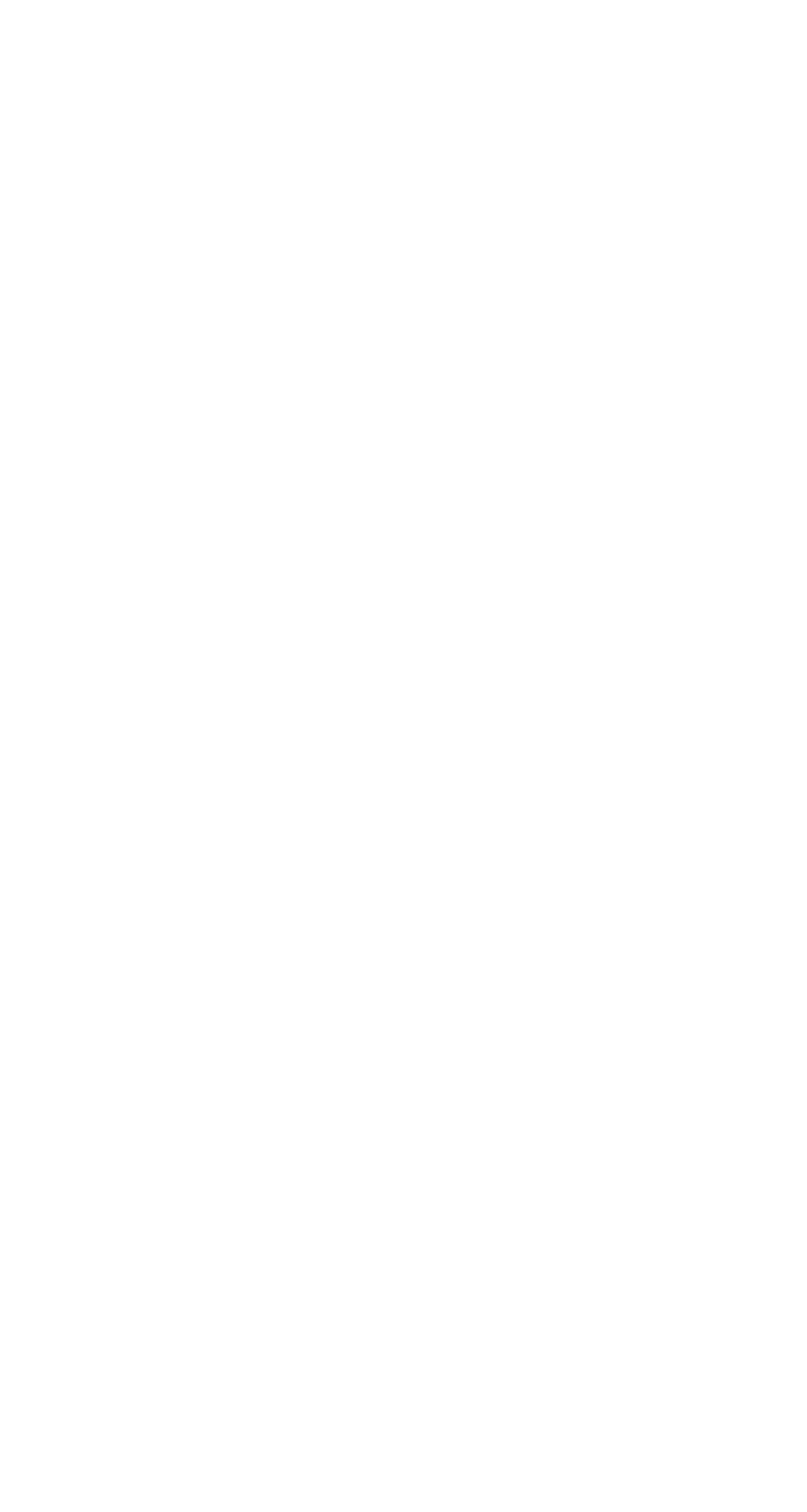


WELL RECORDS

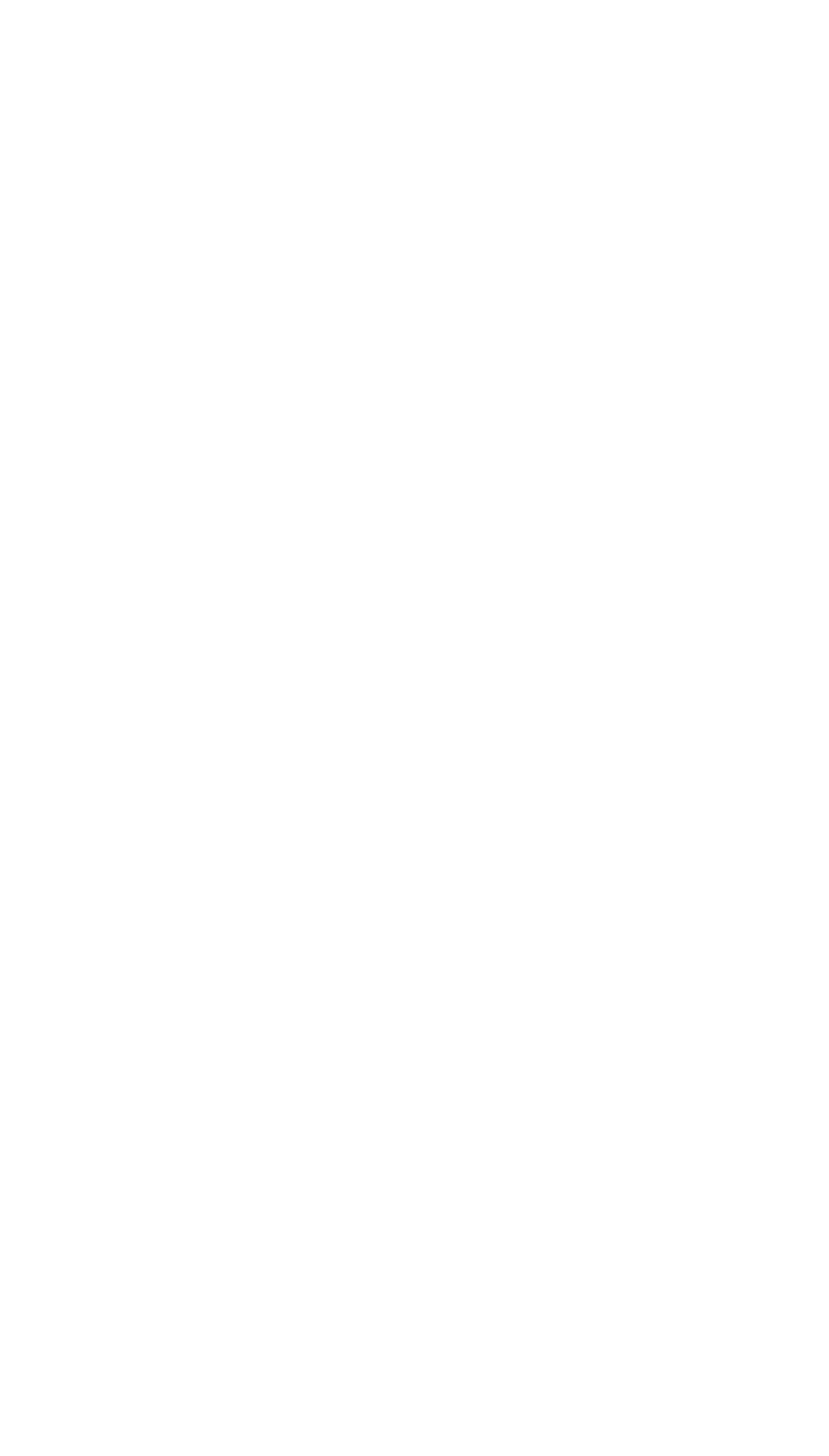




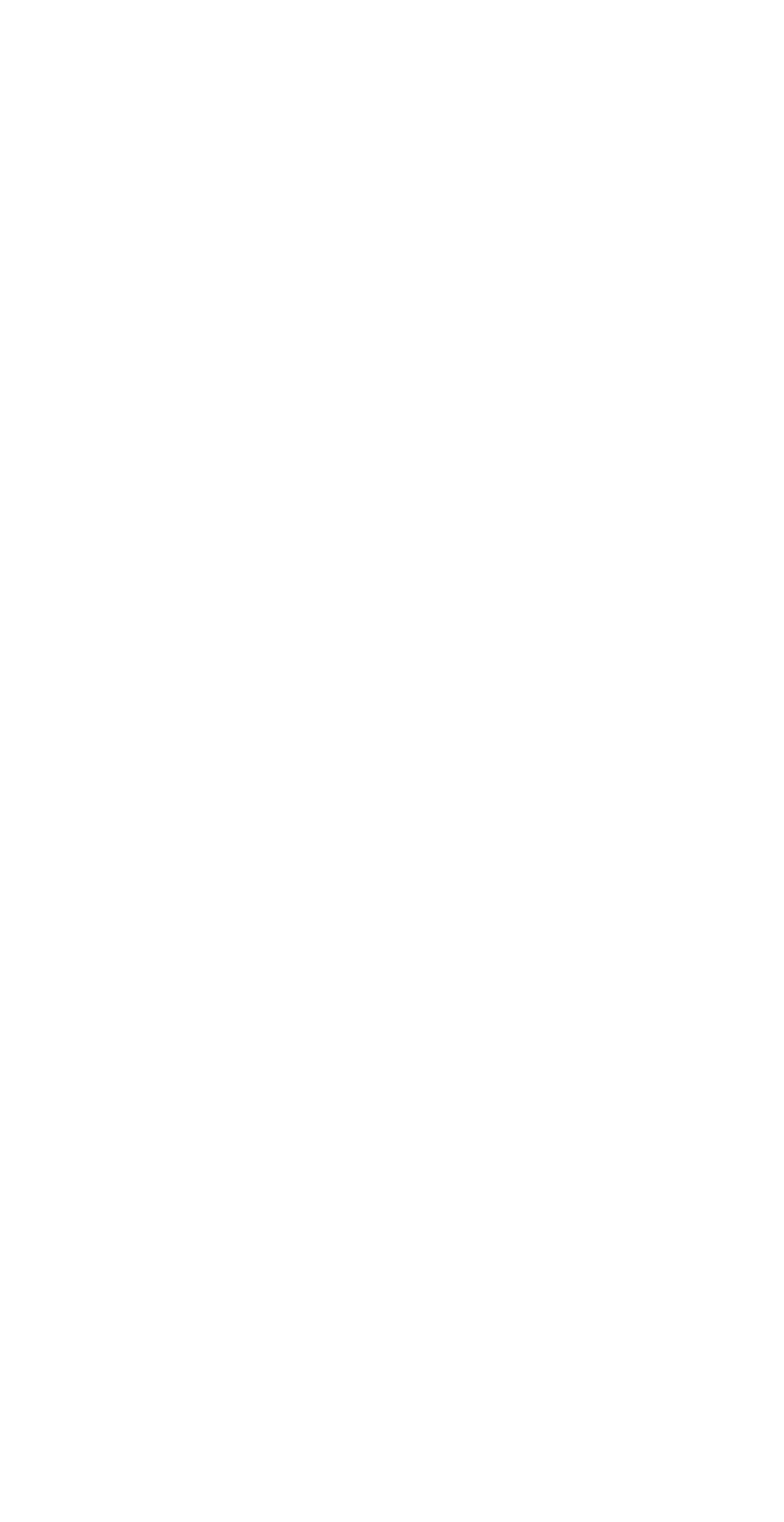




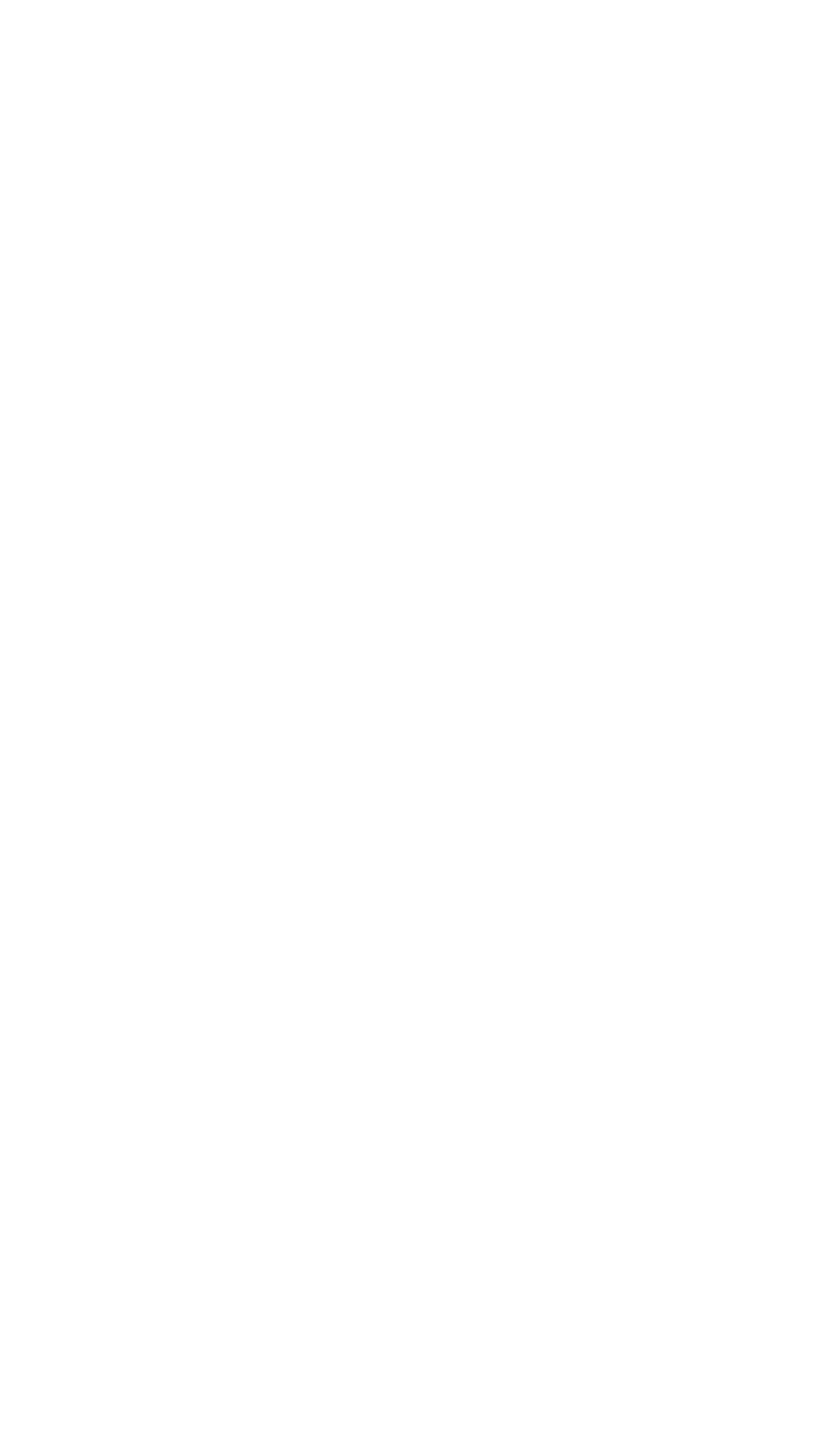




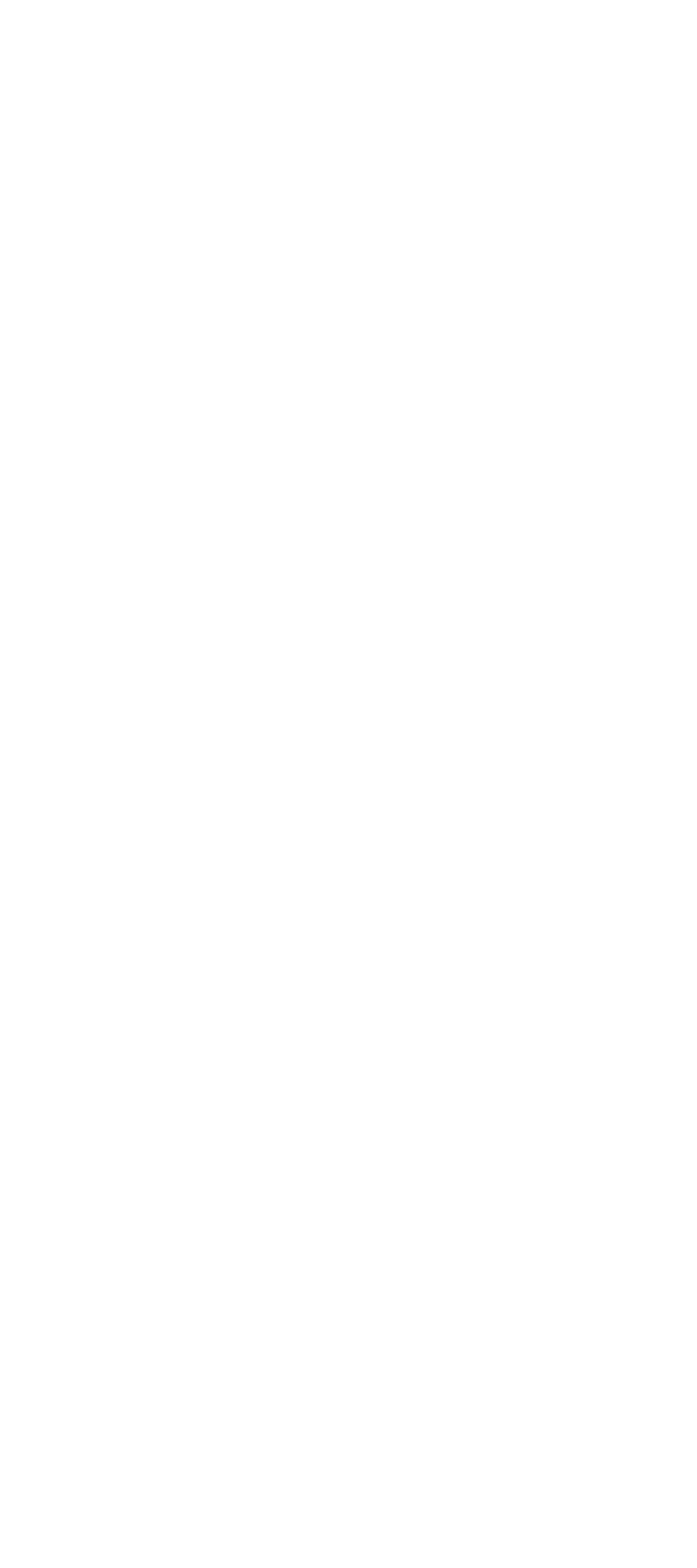




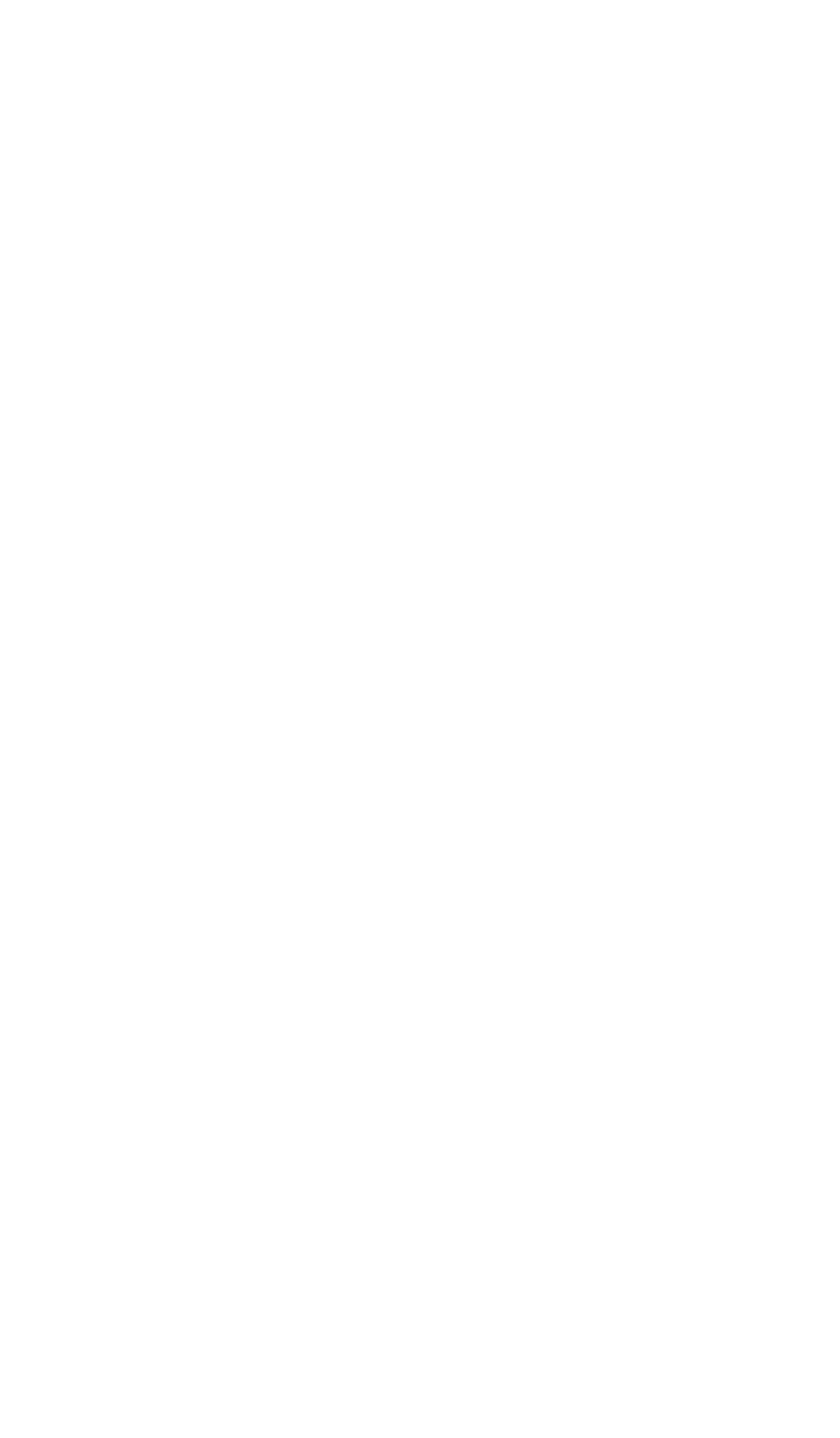




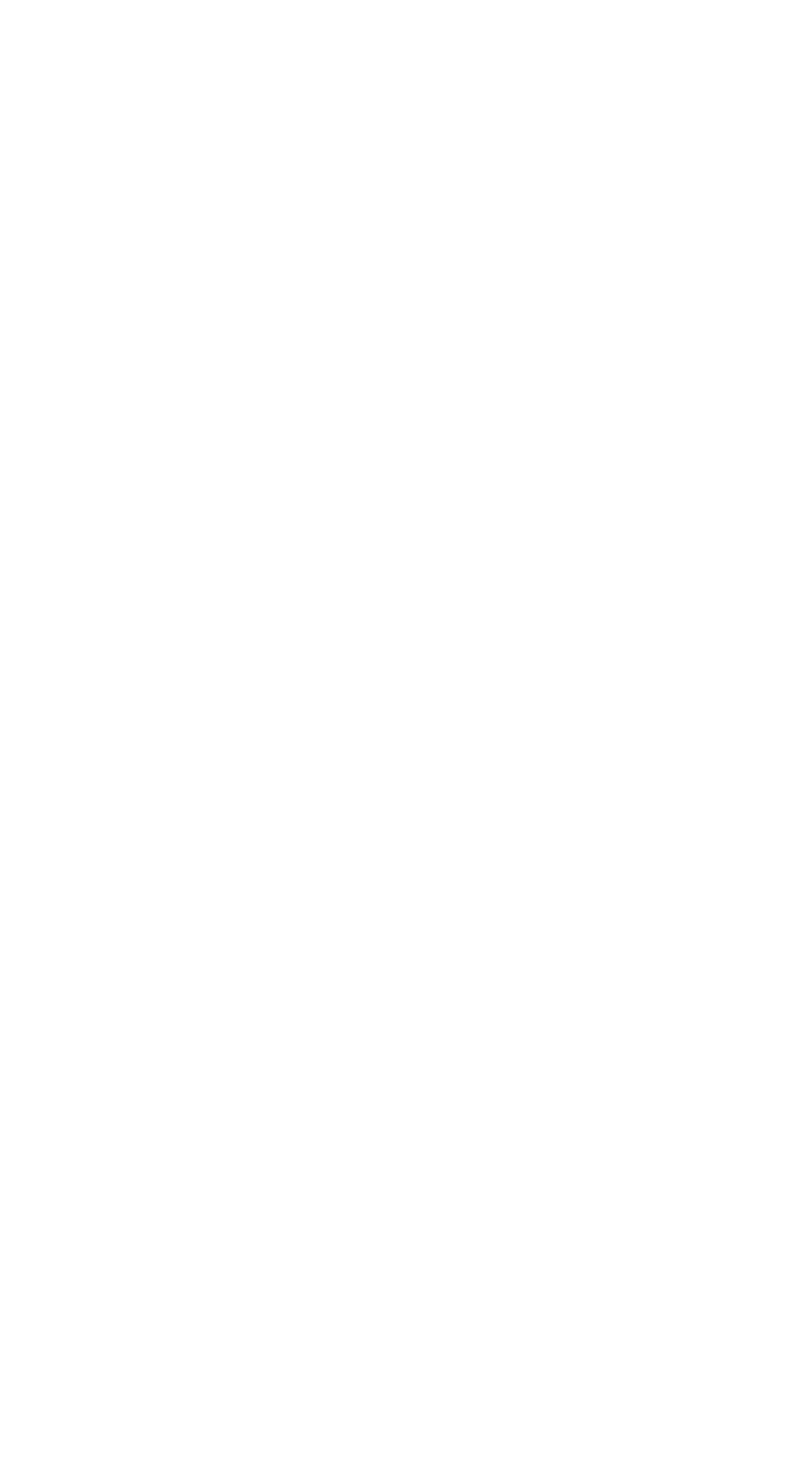




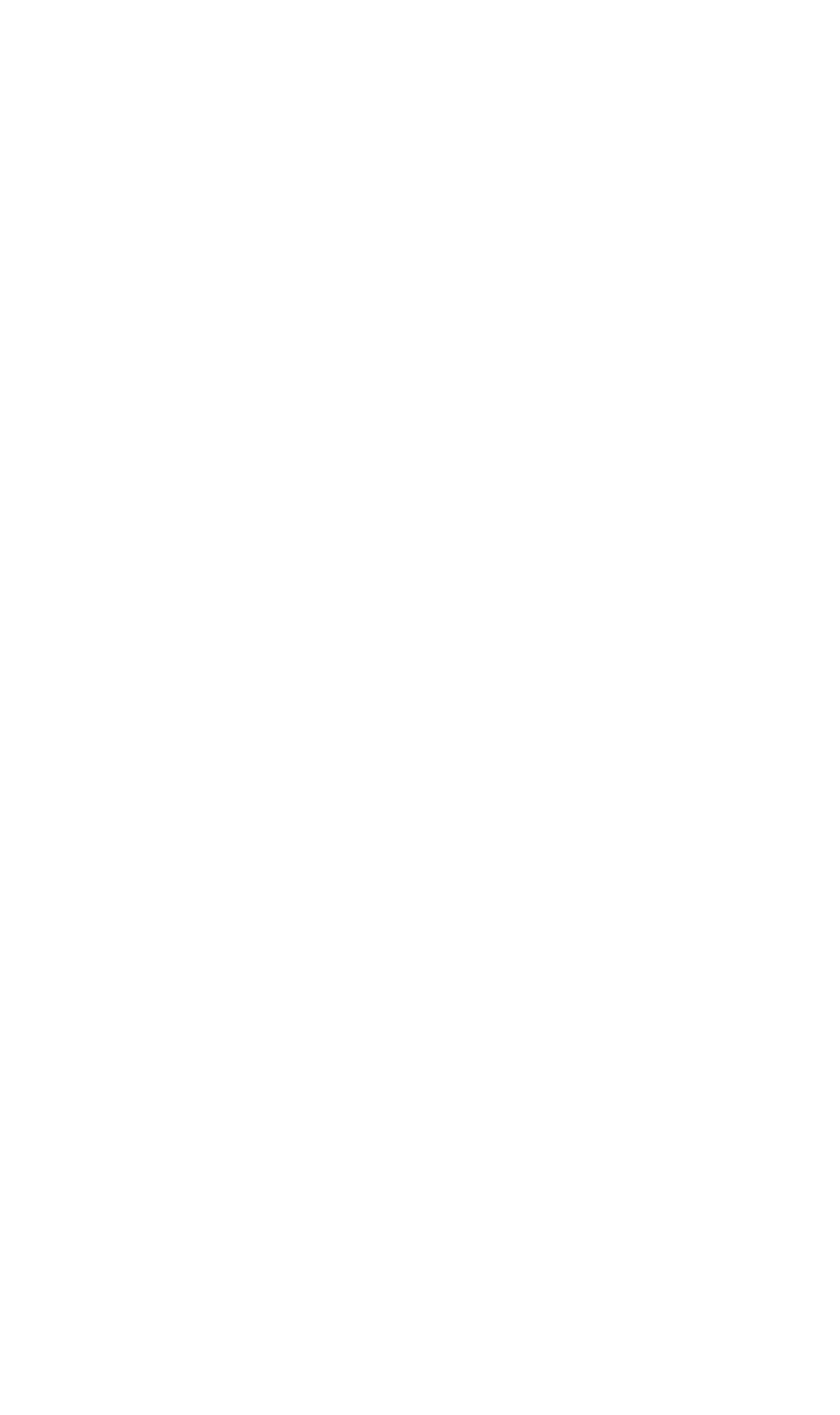




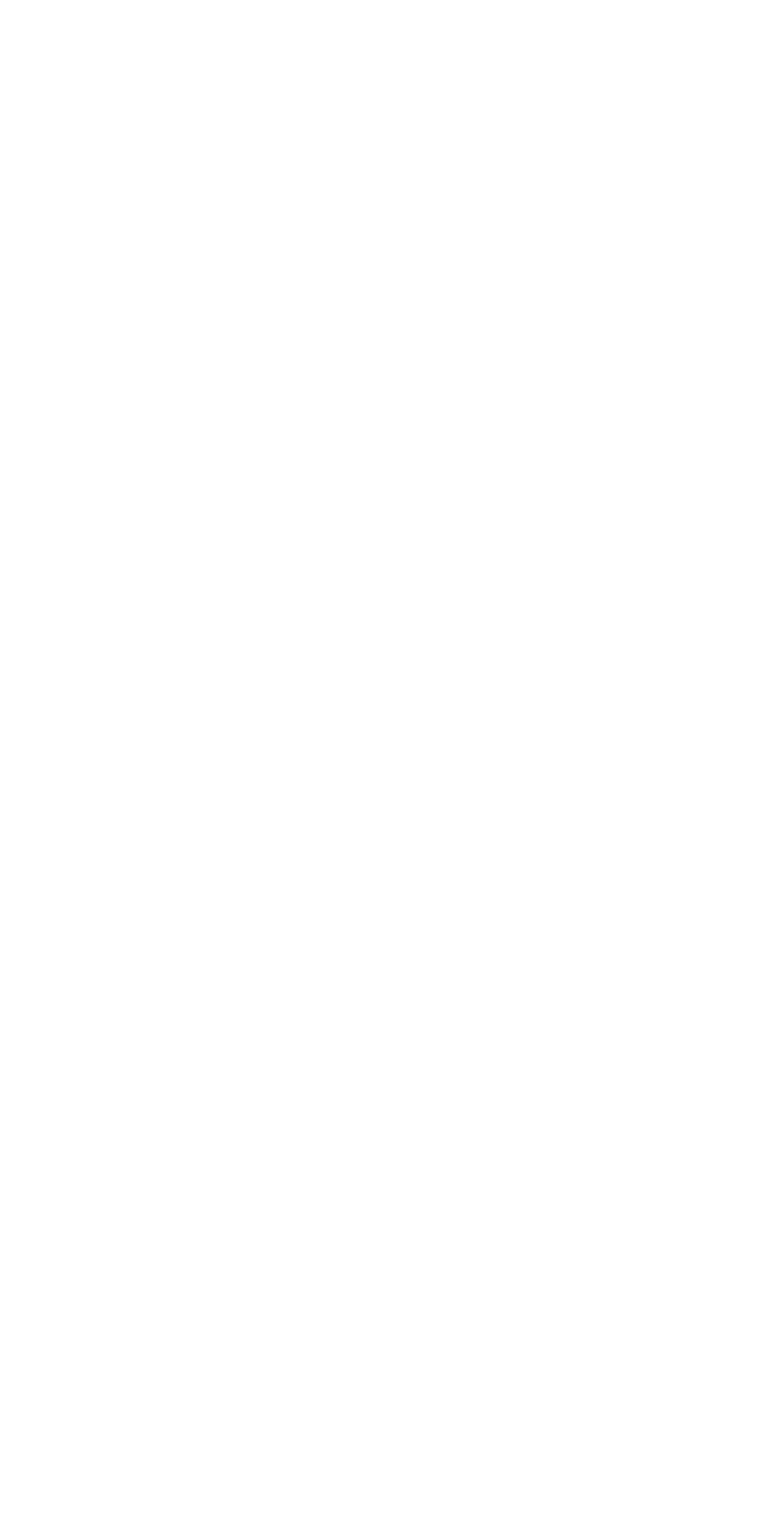




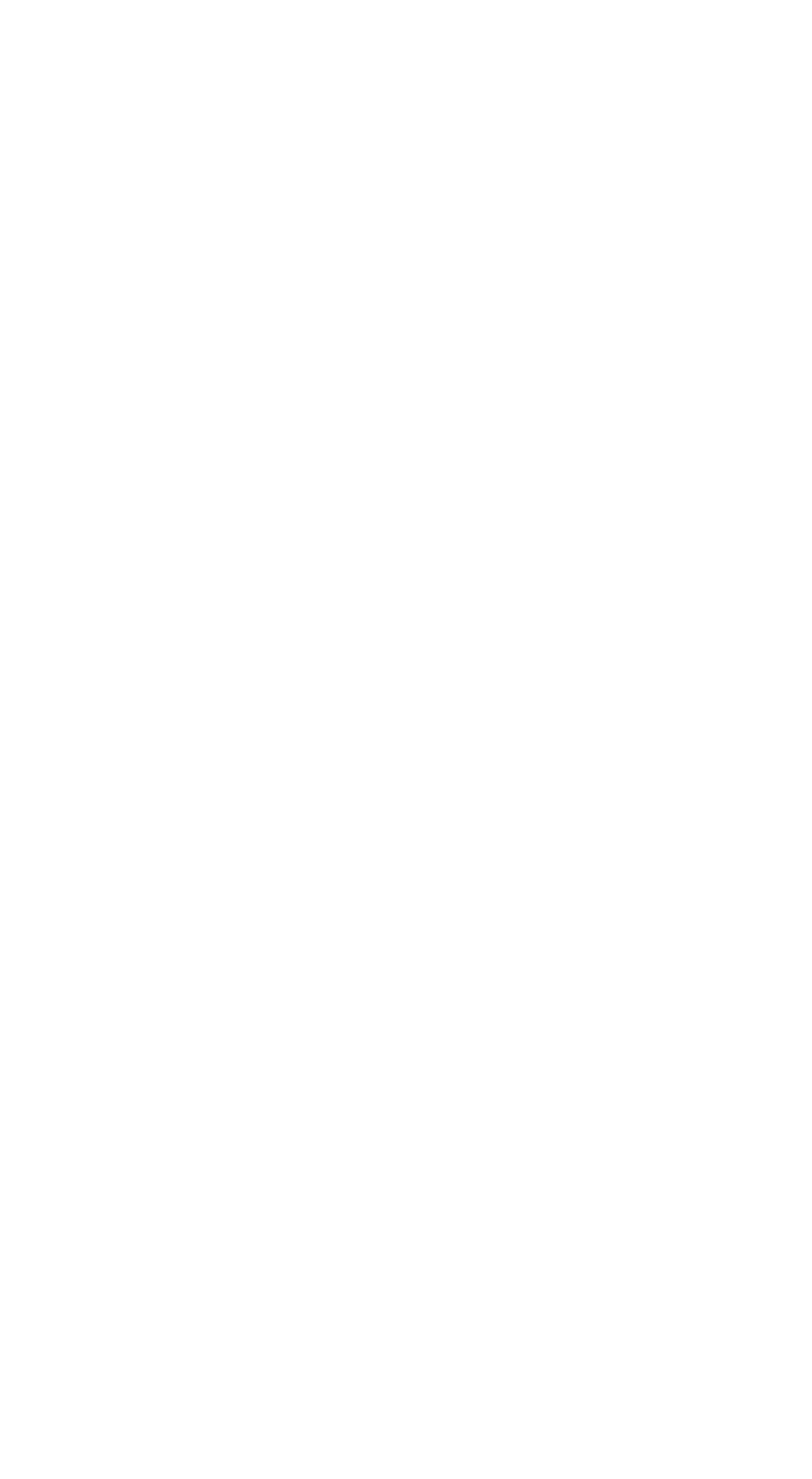




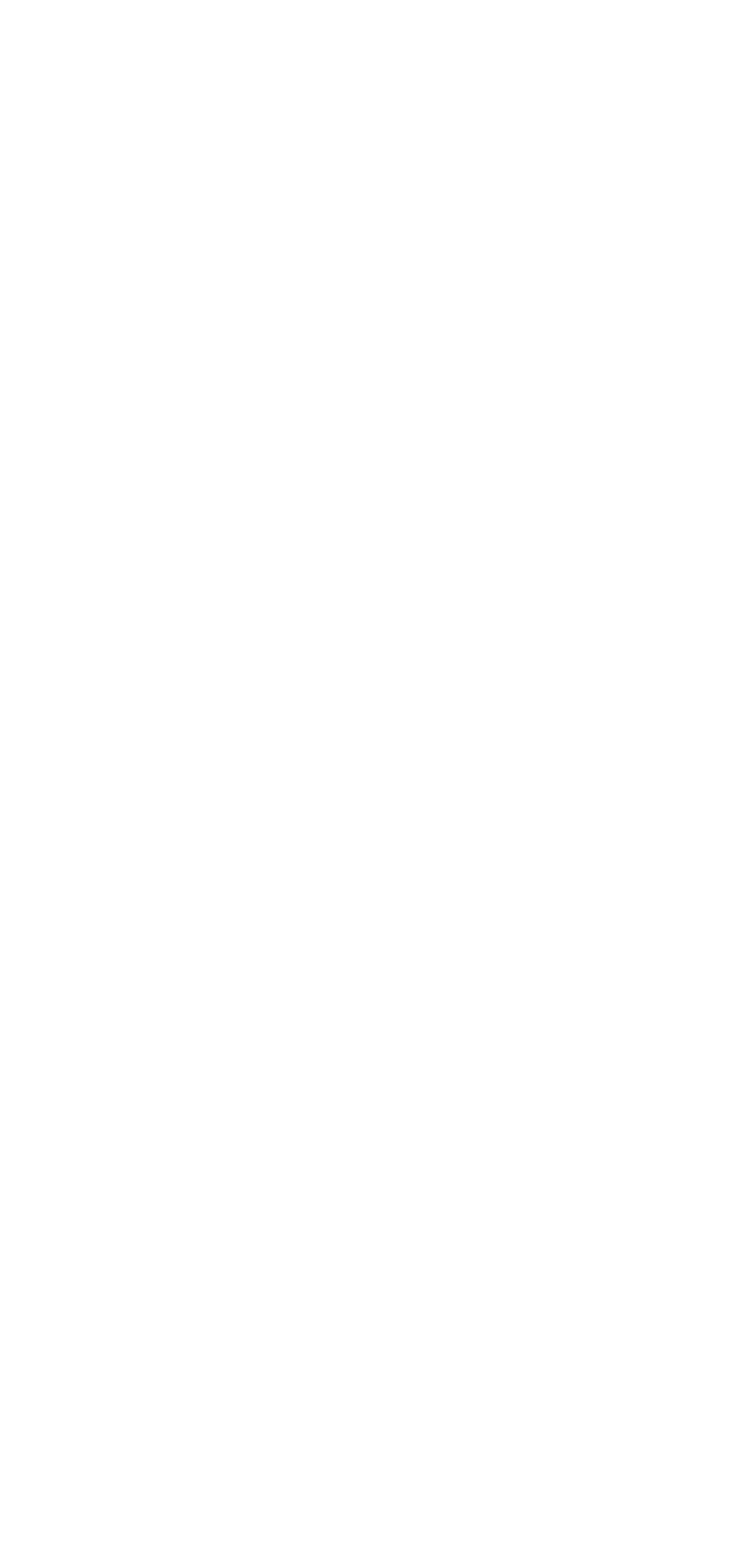




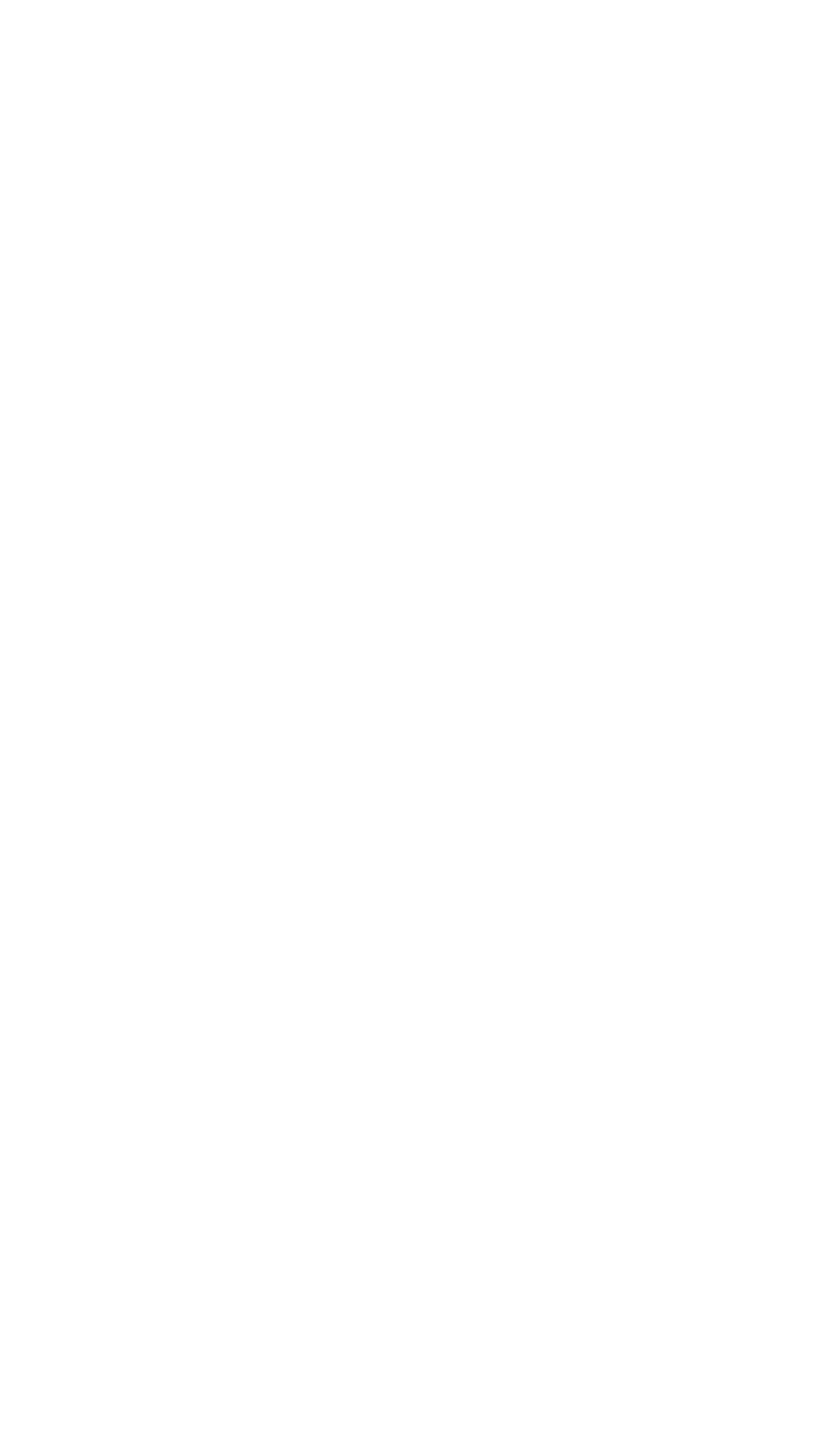




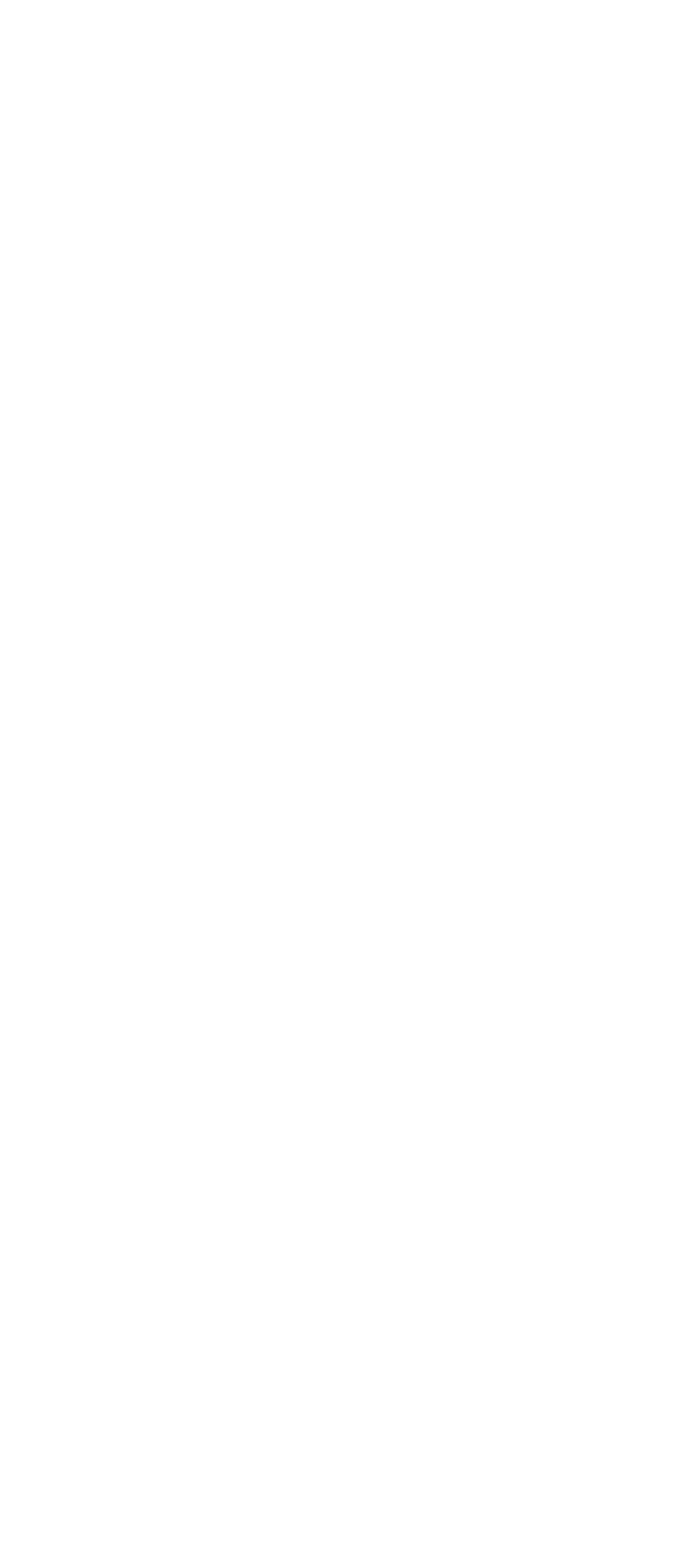




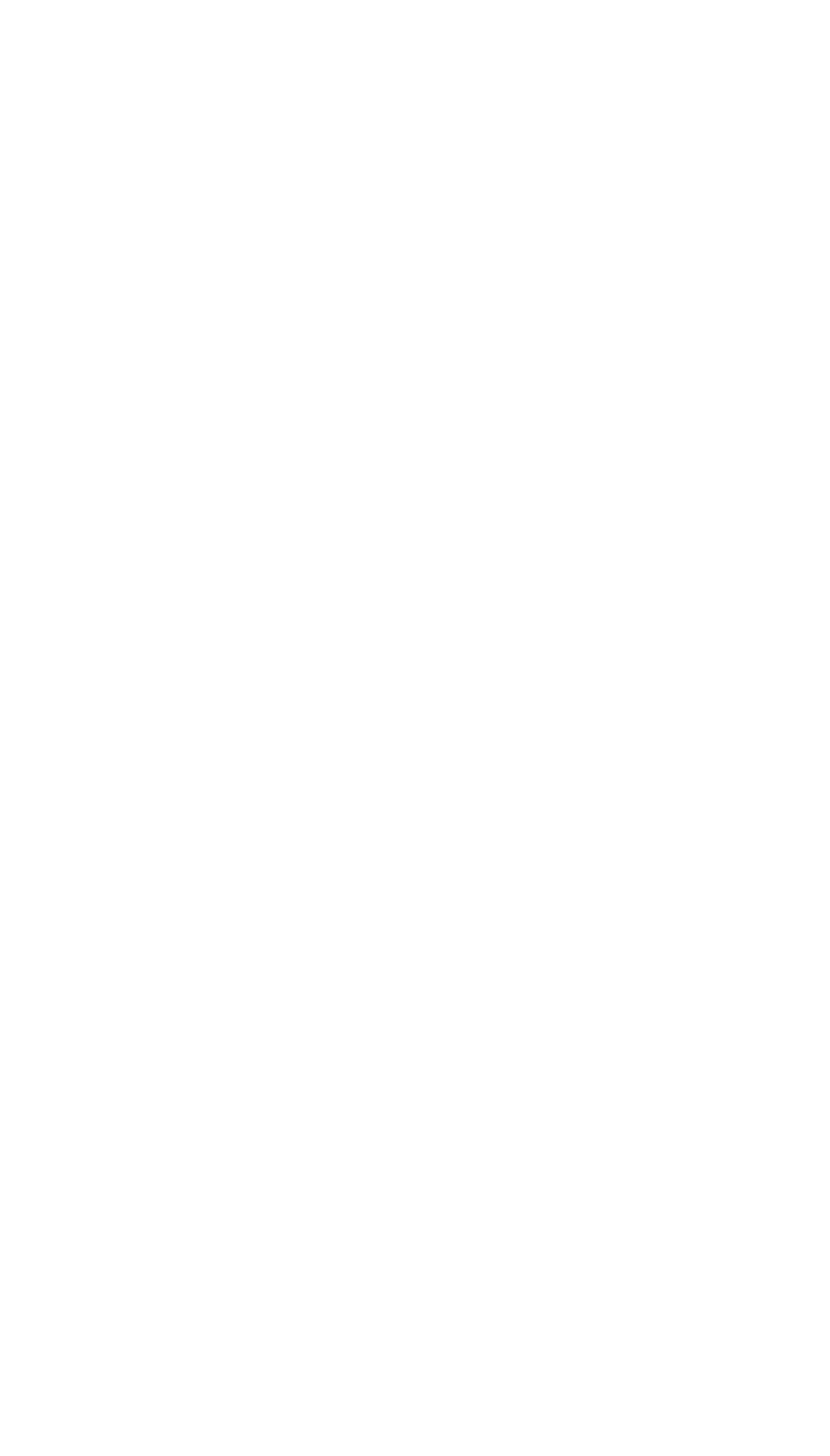




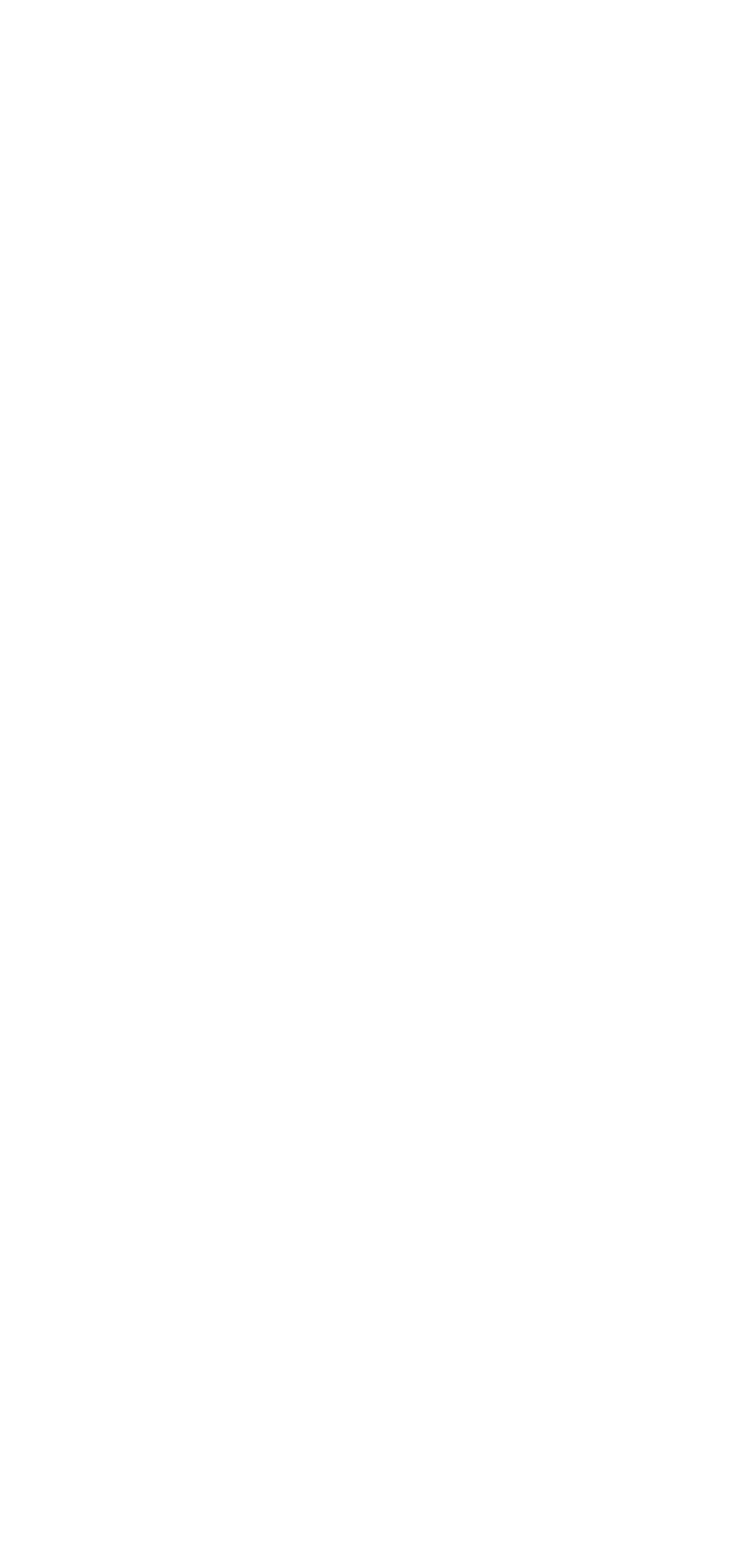




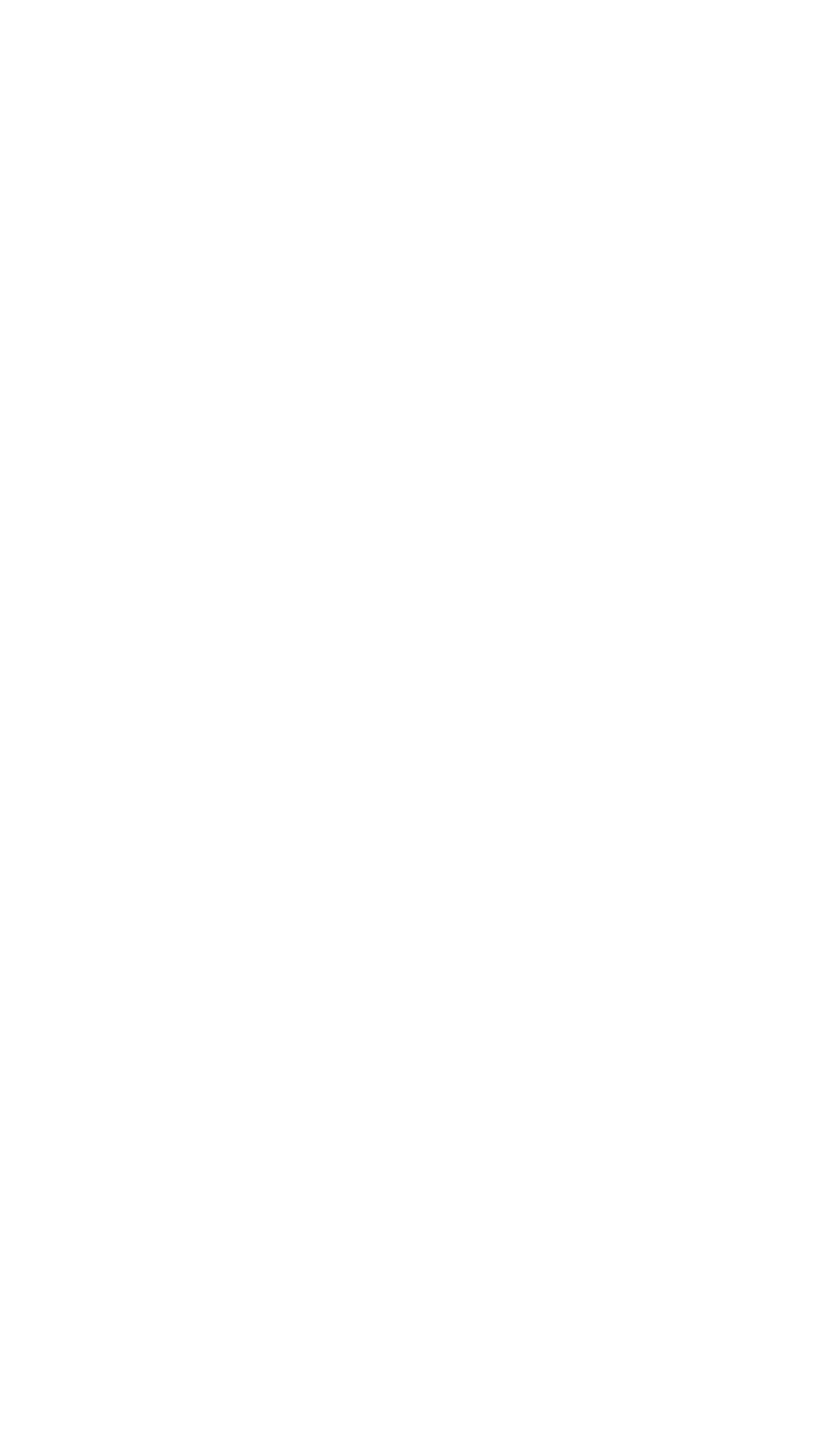




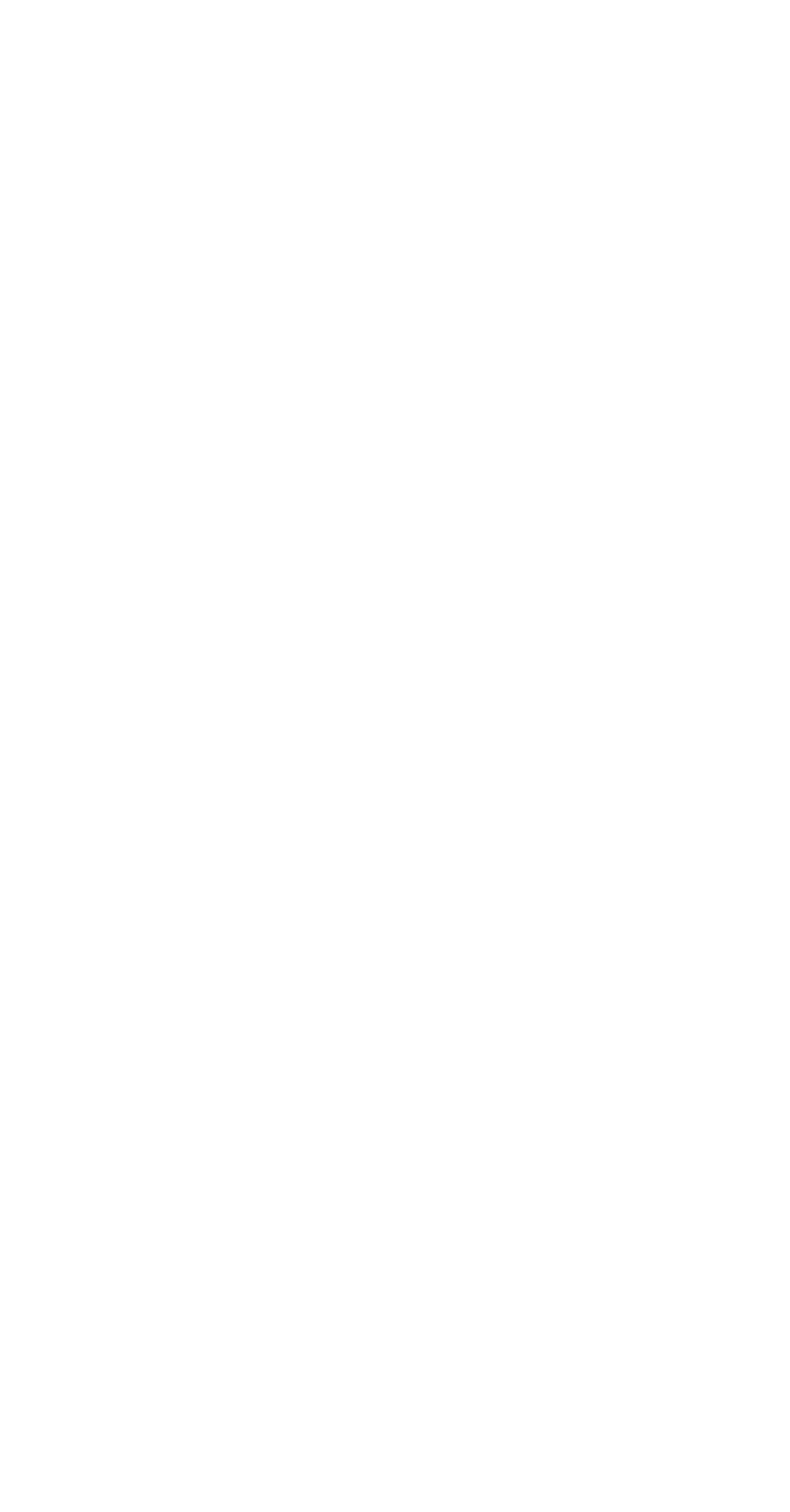




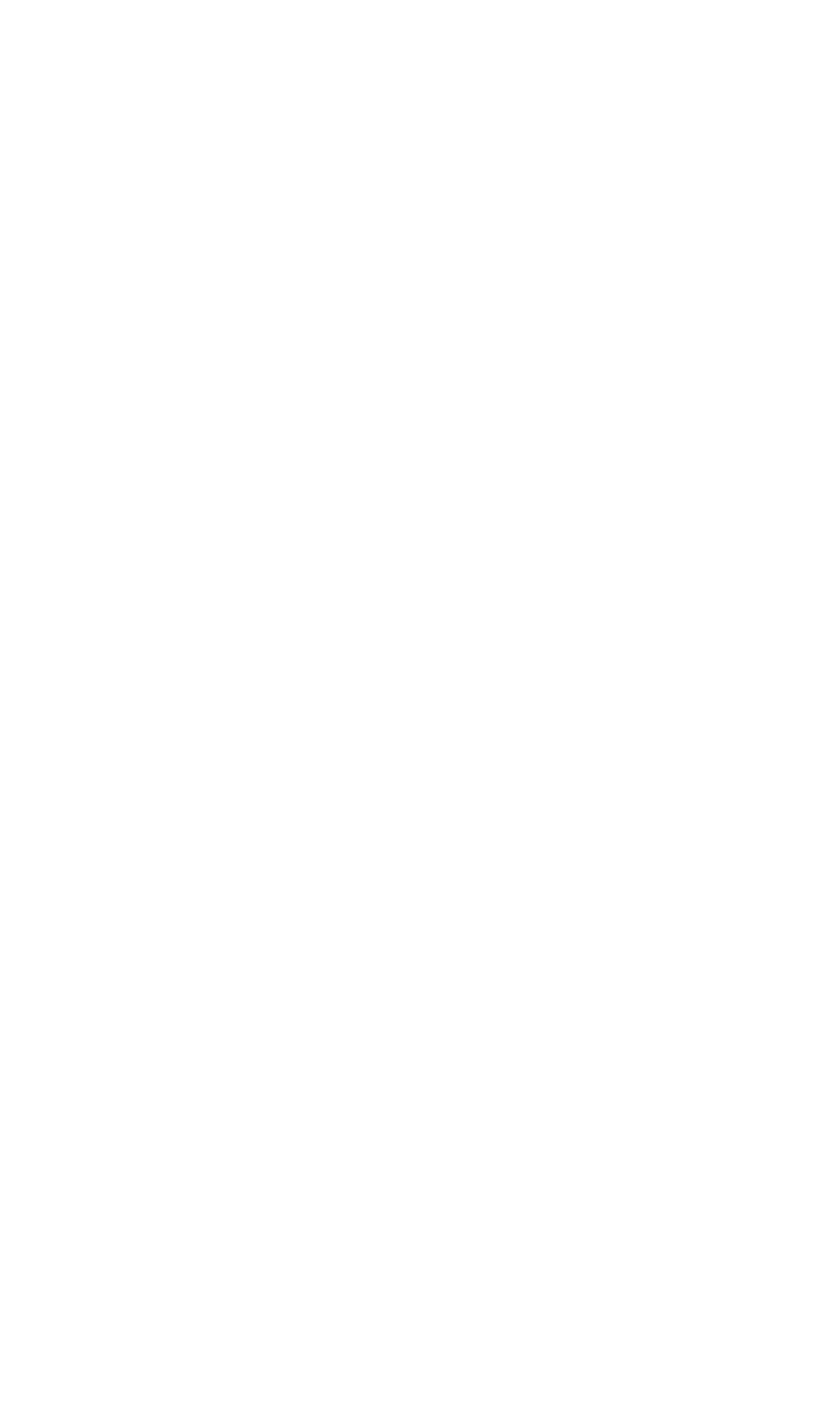




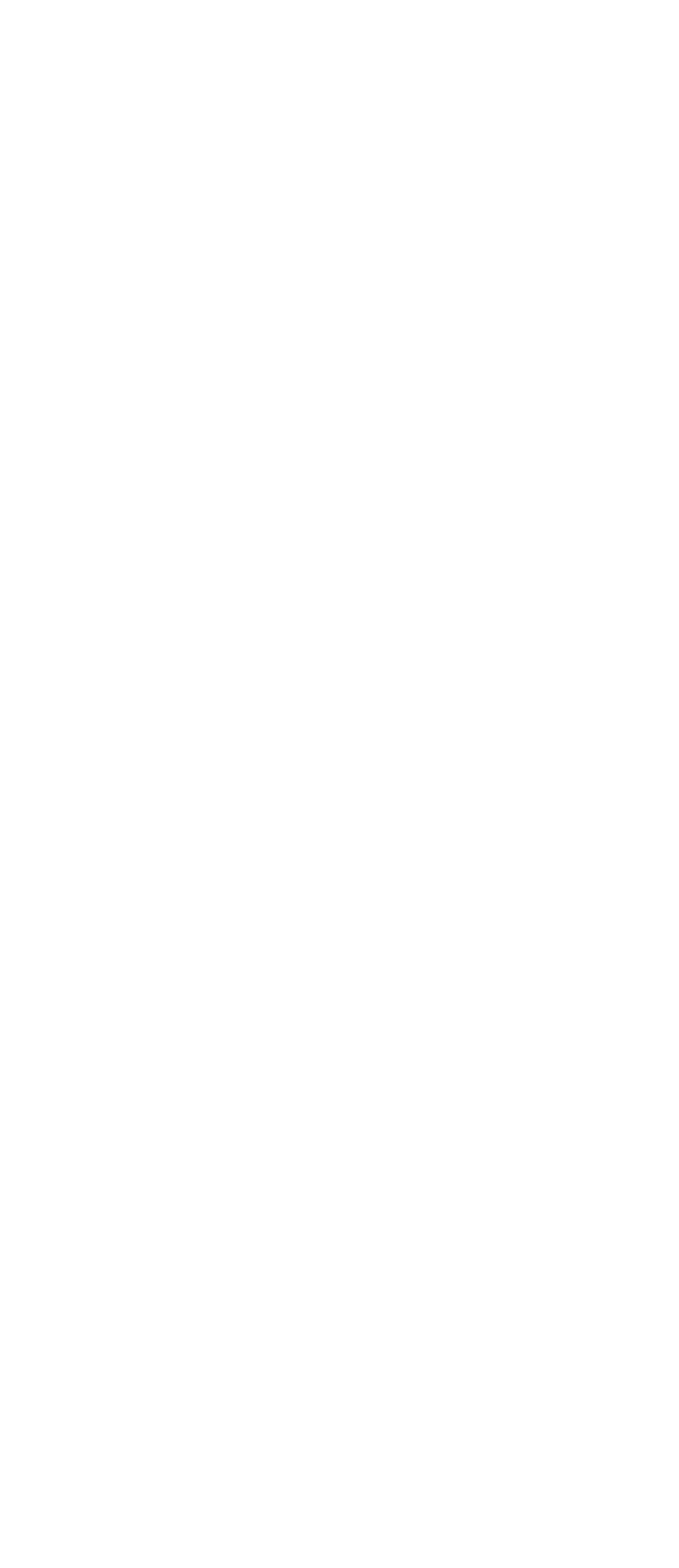




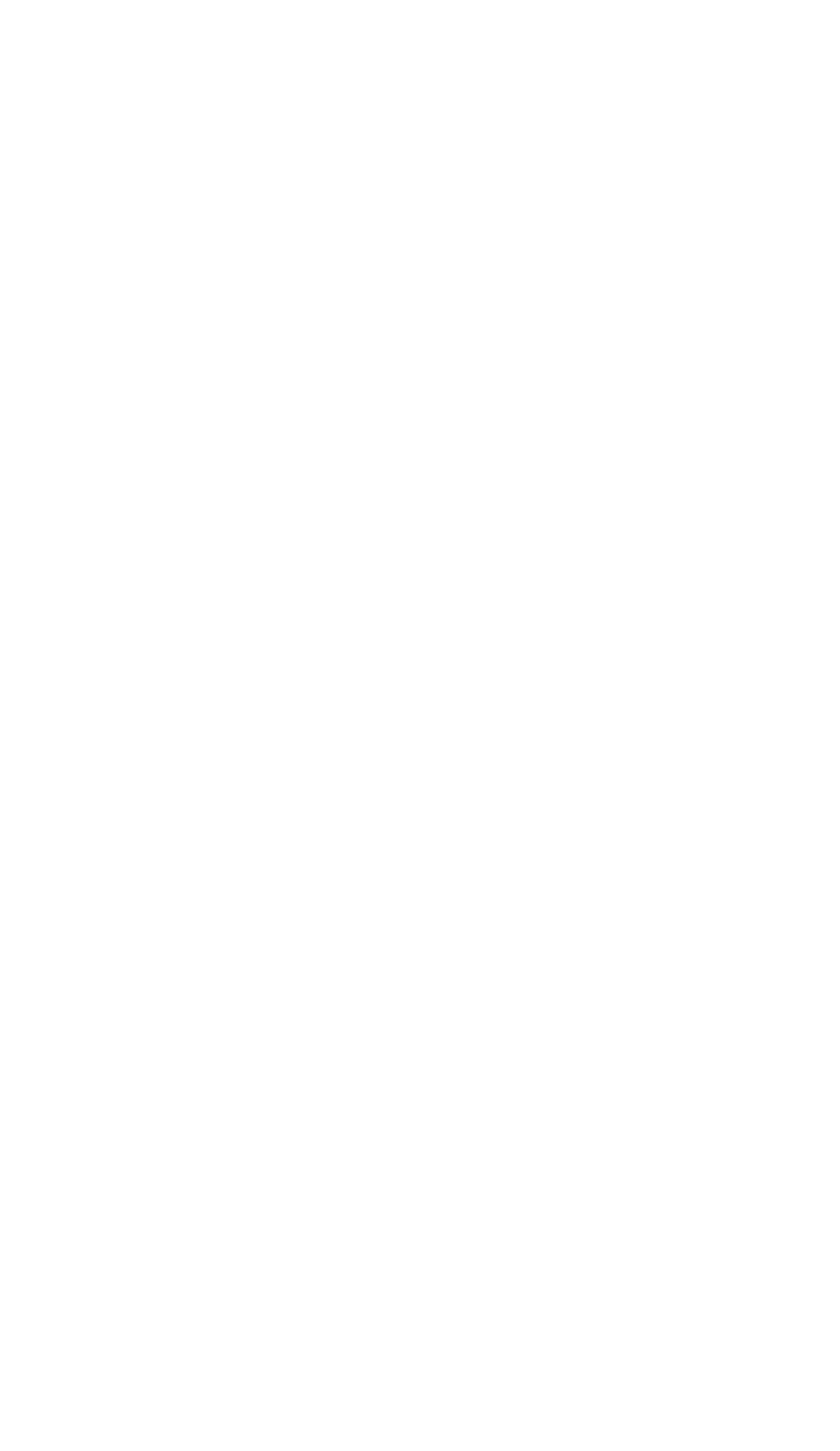




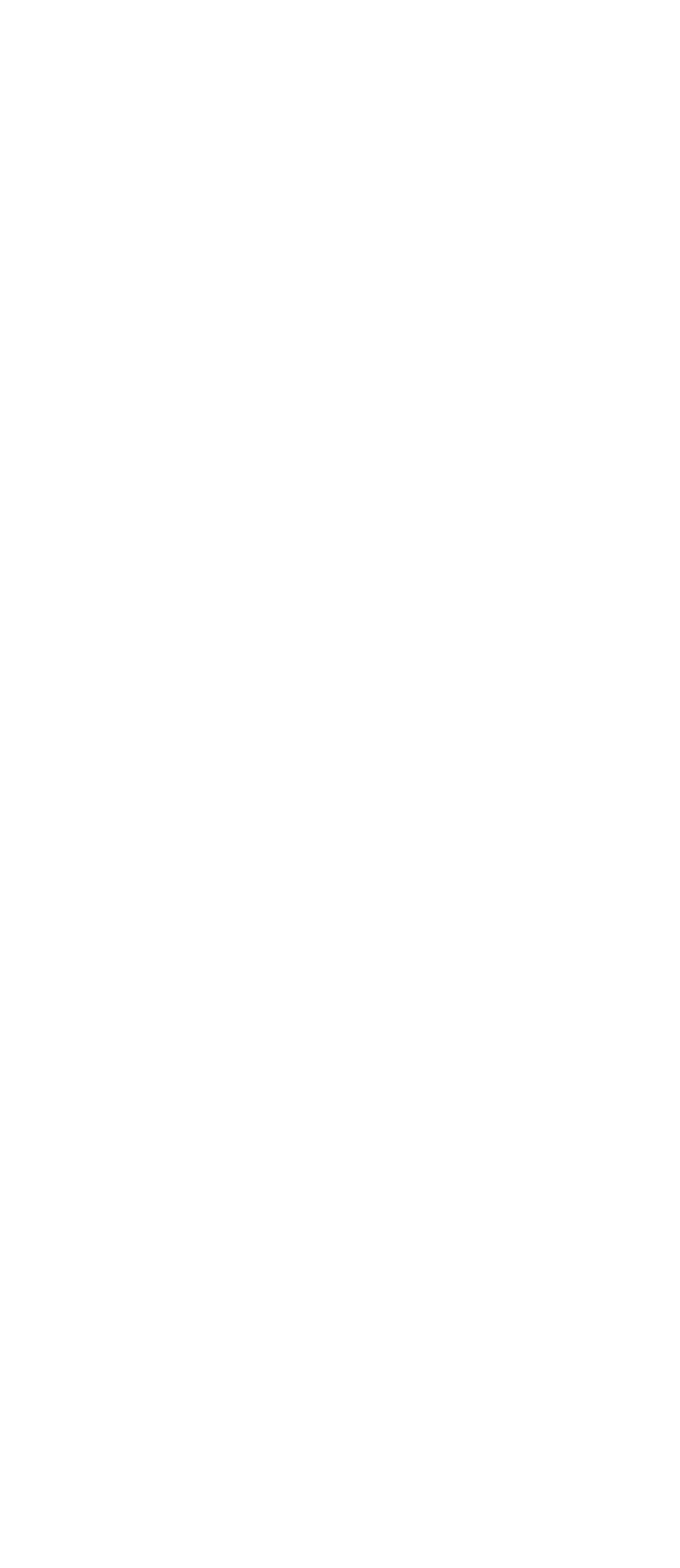


WELL RECORDS

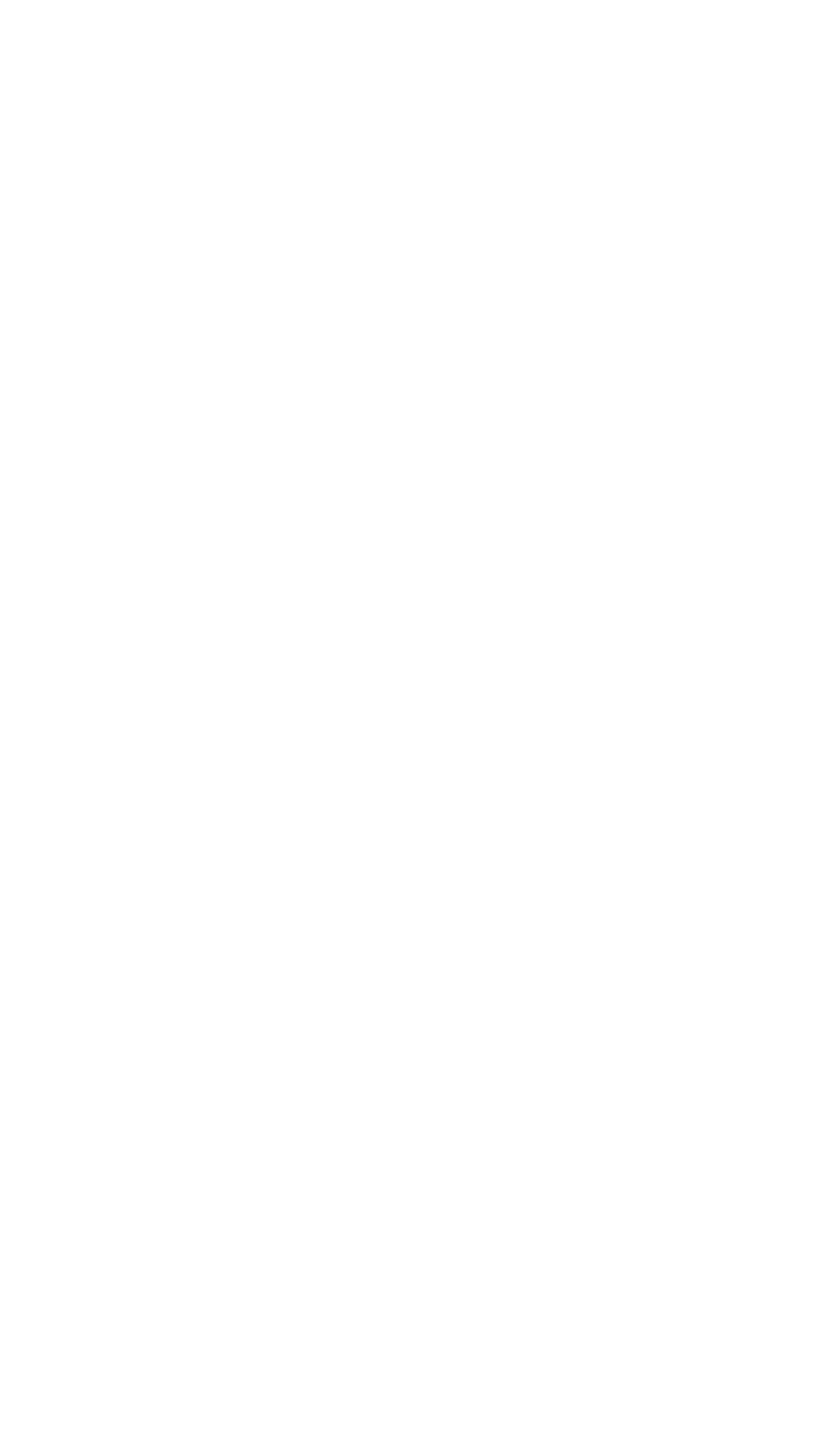




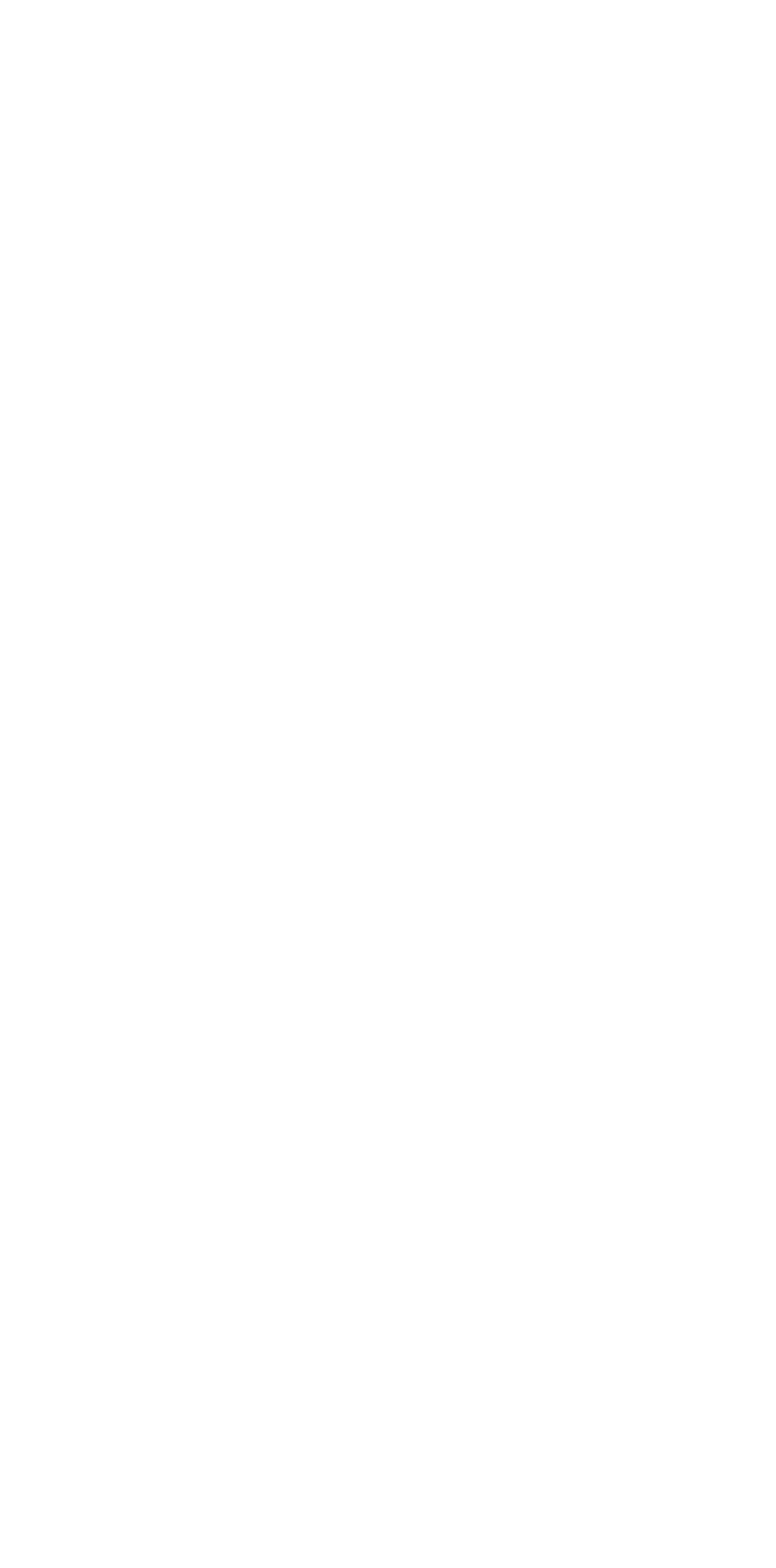




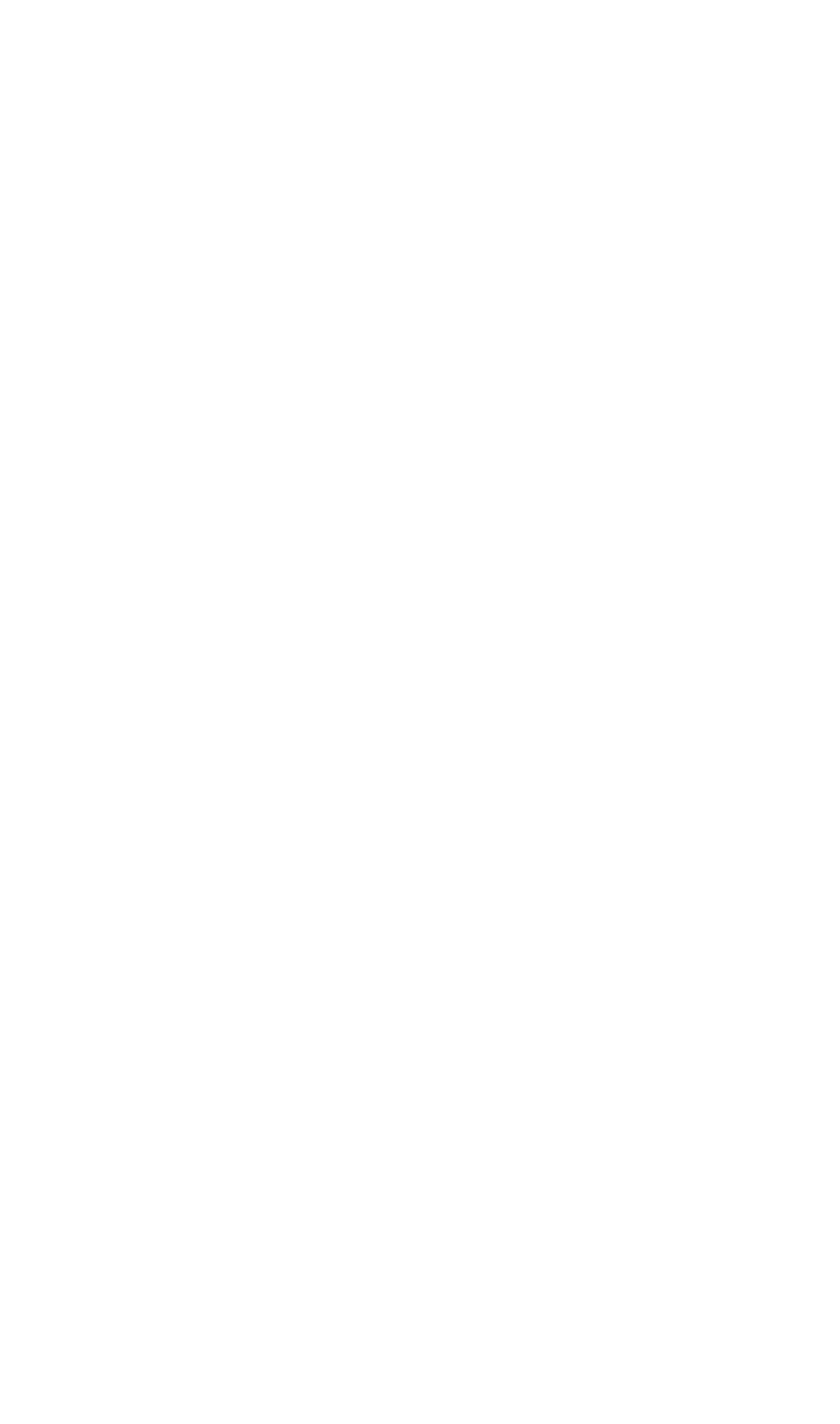




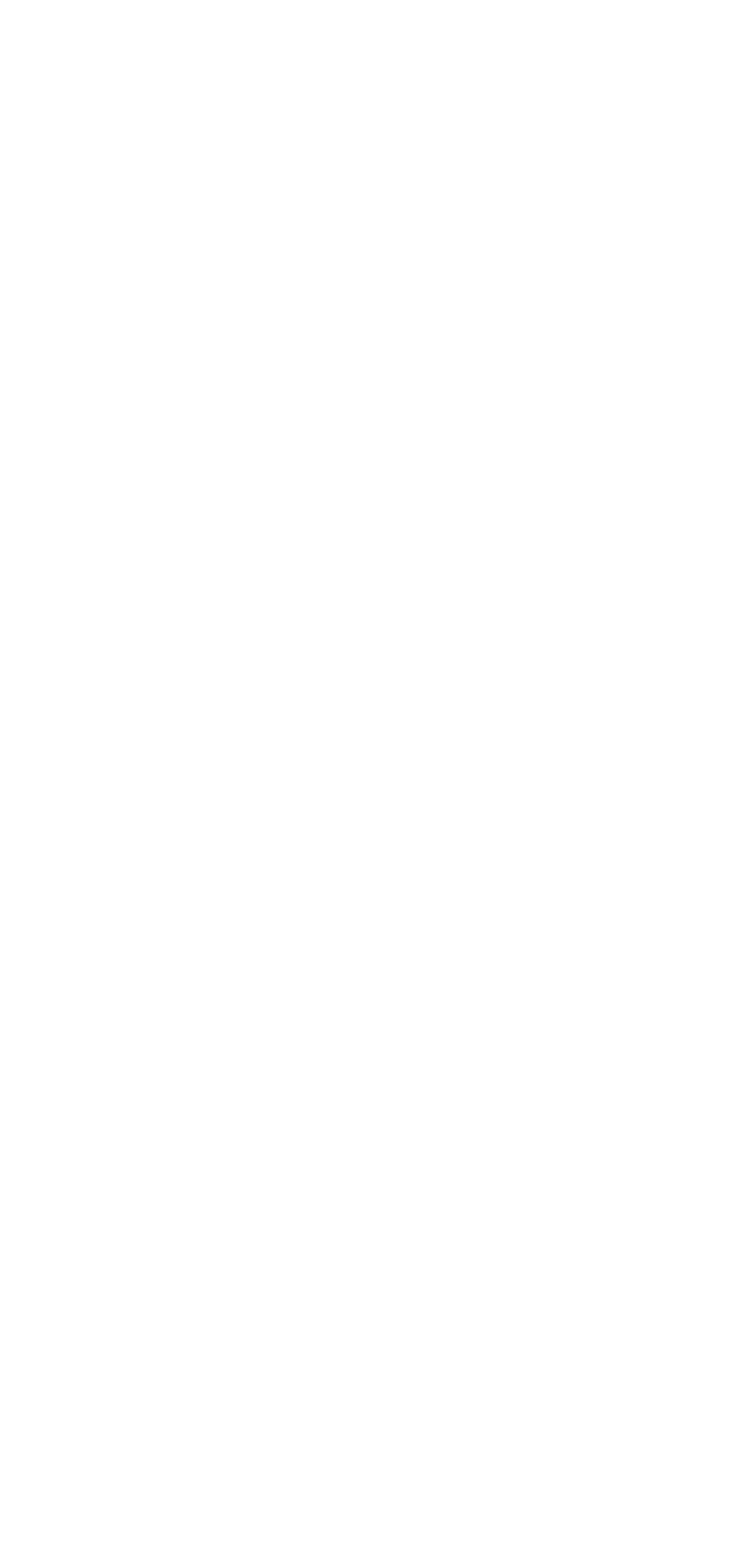




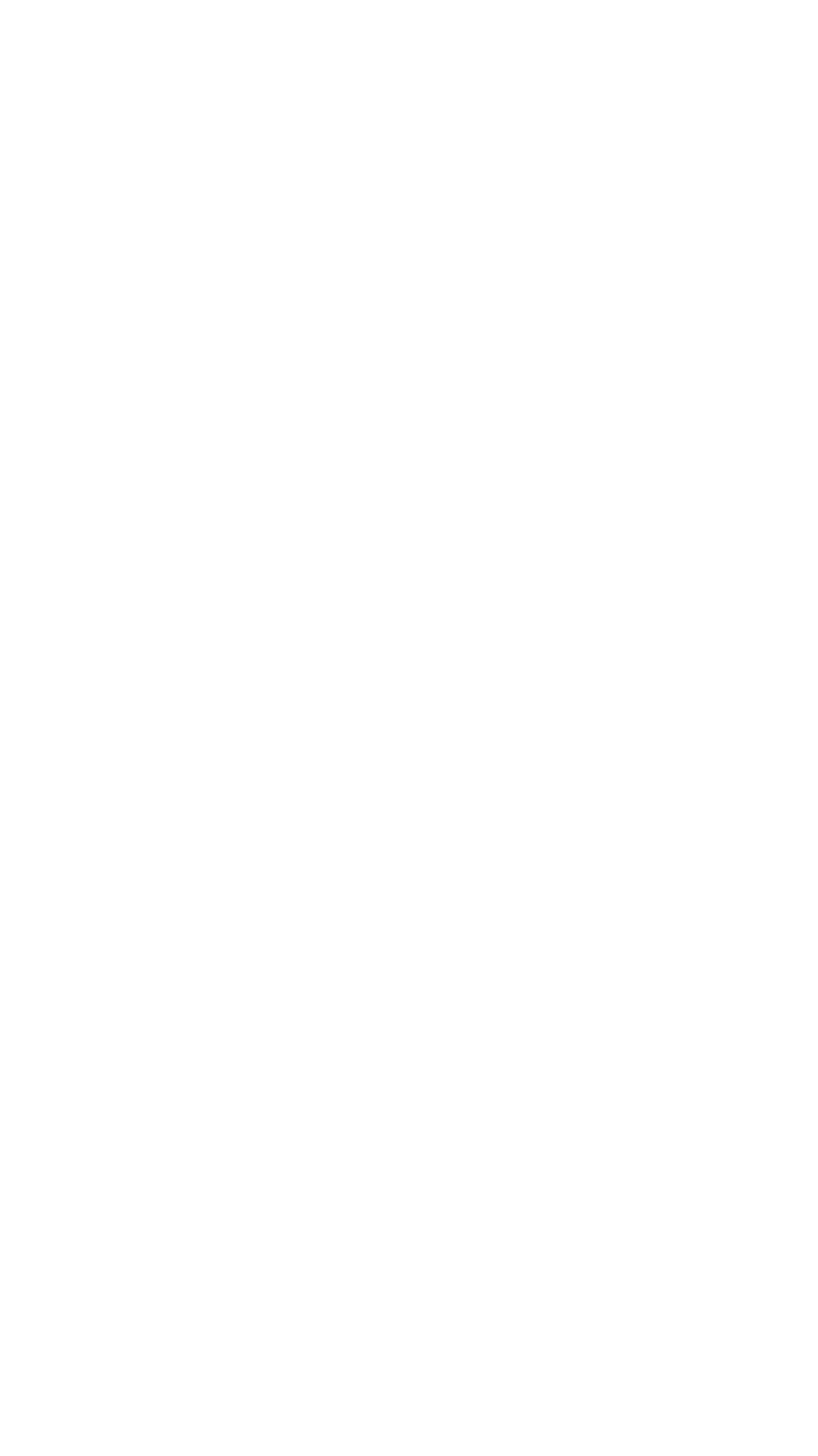




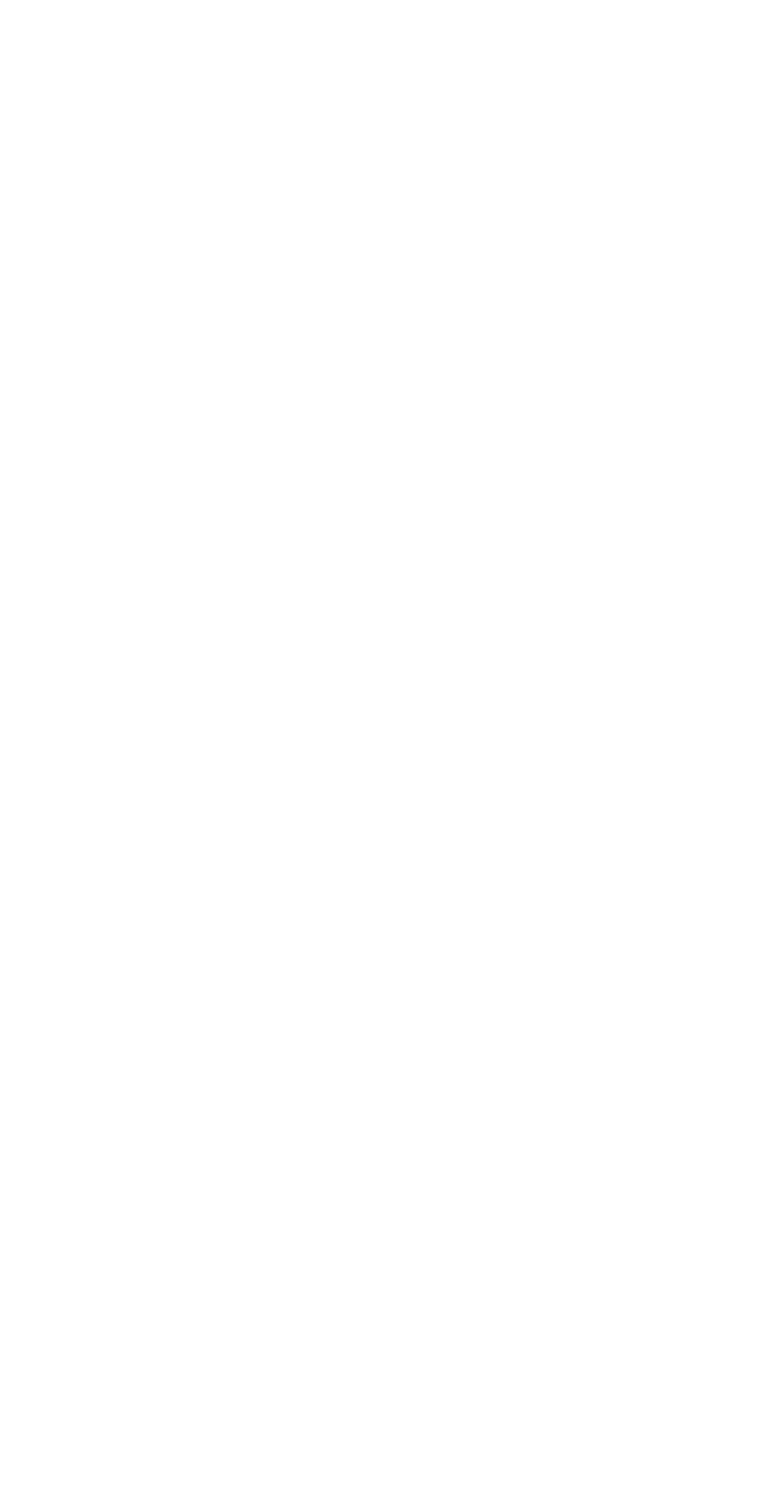




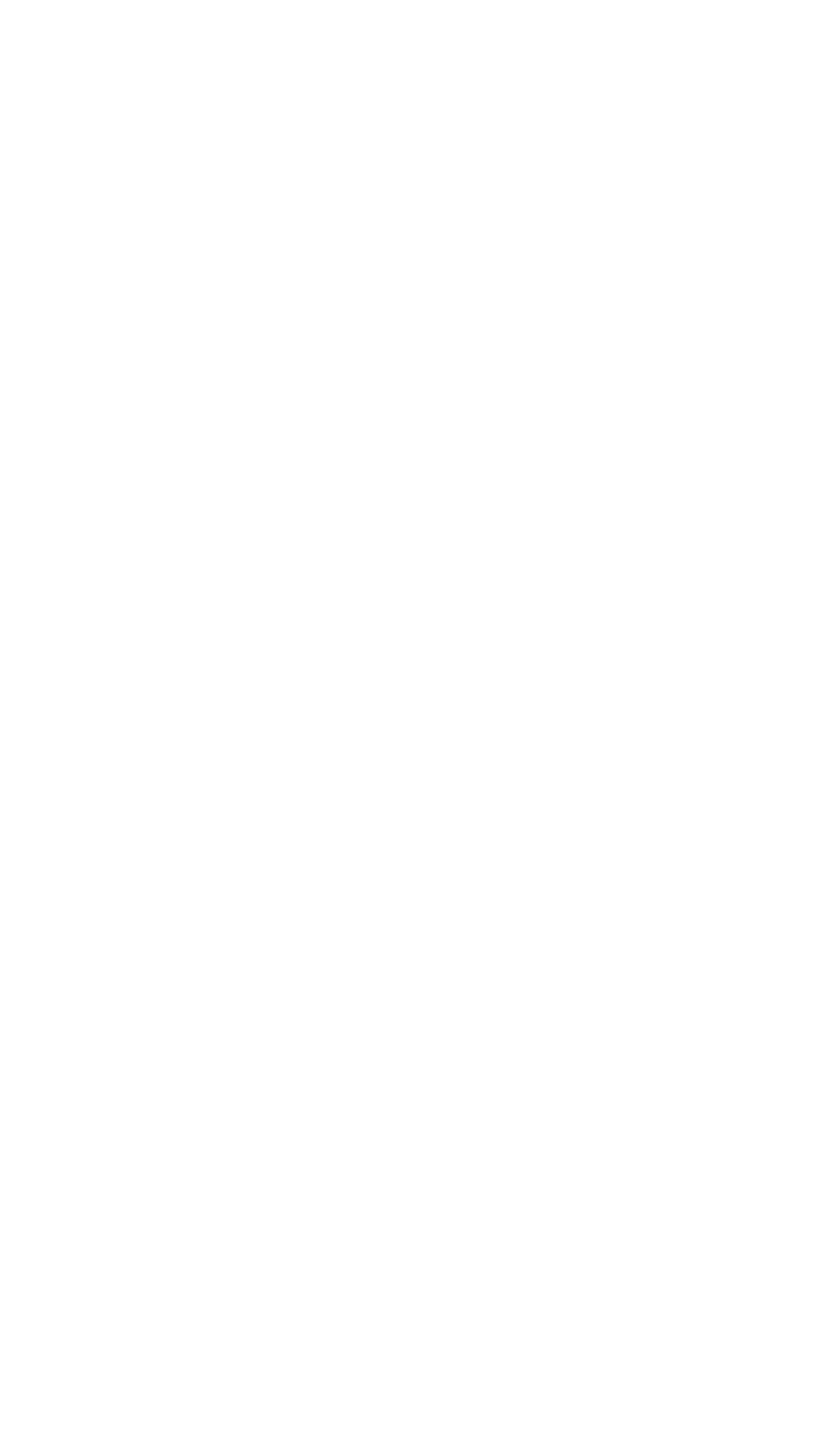




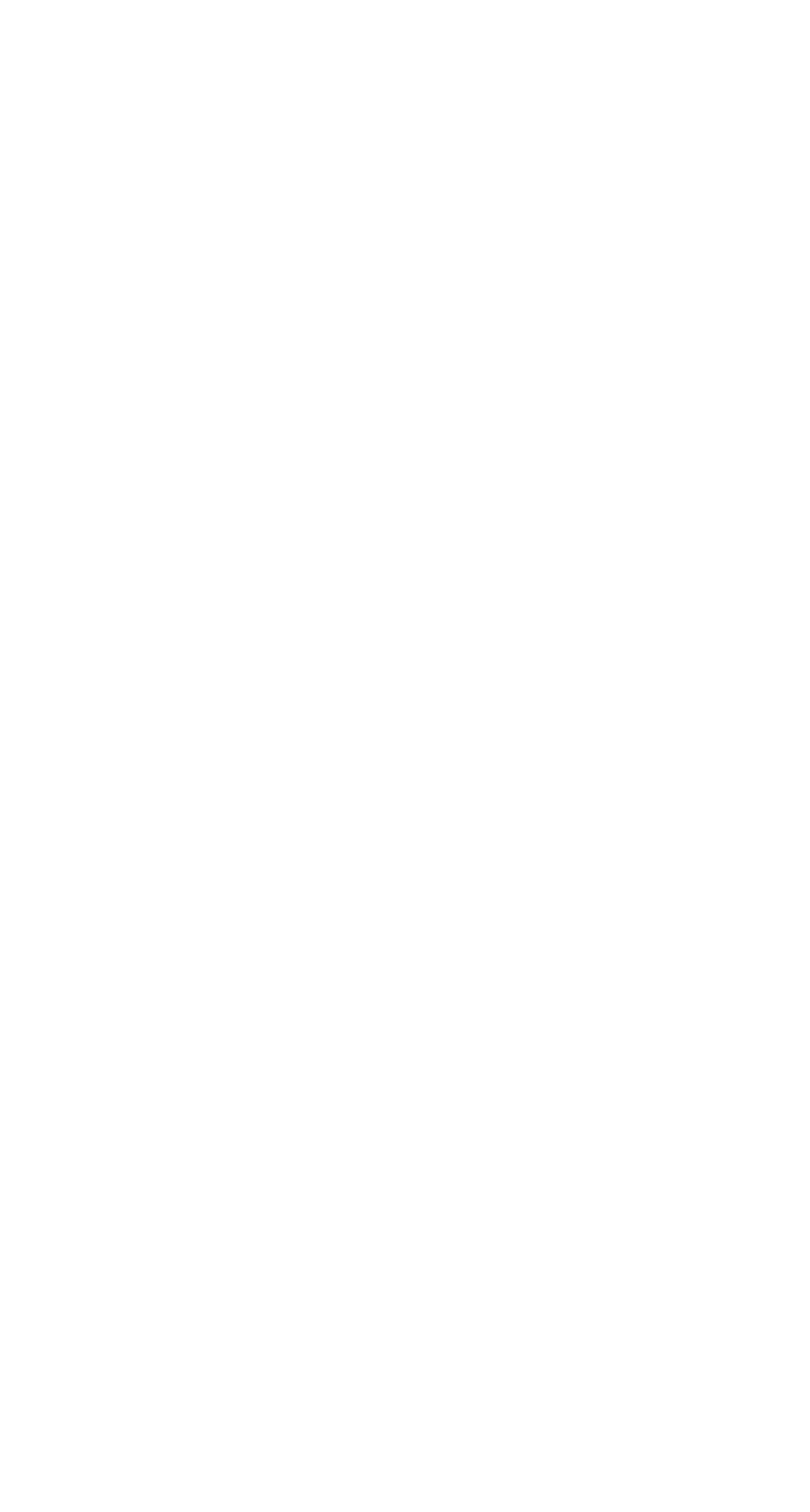




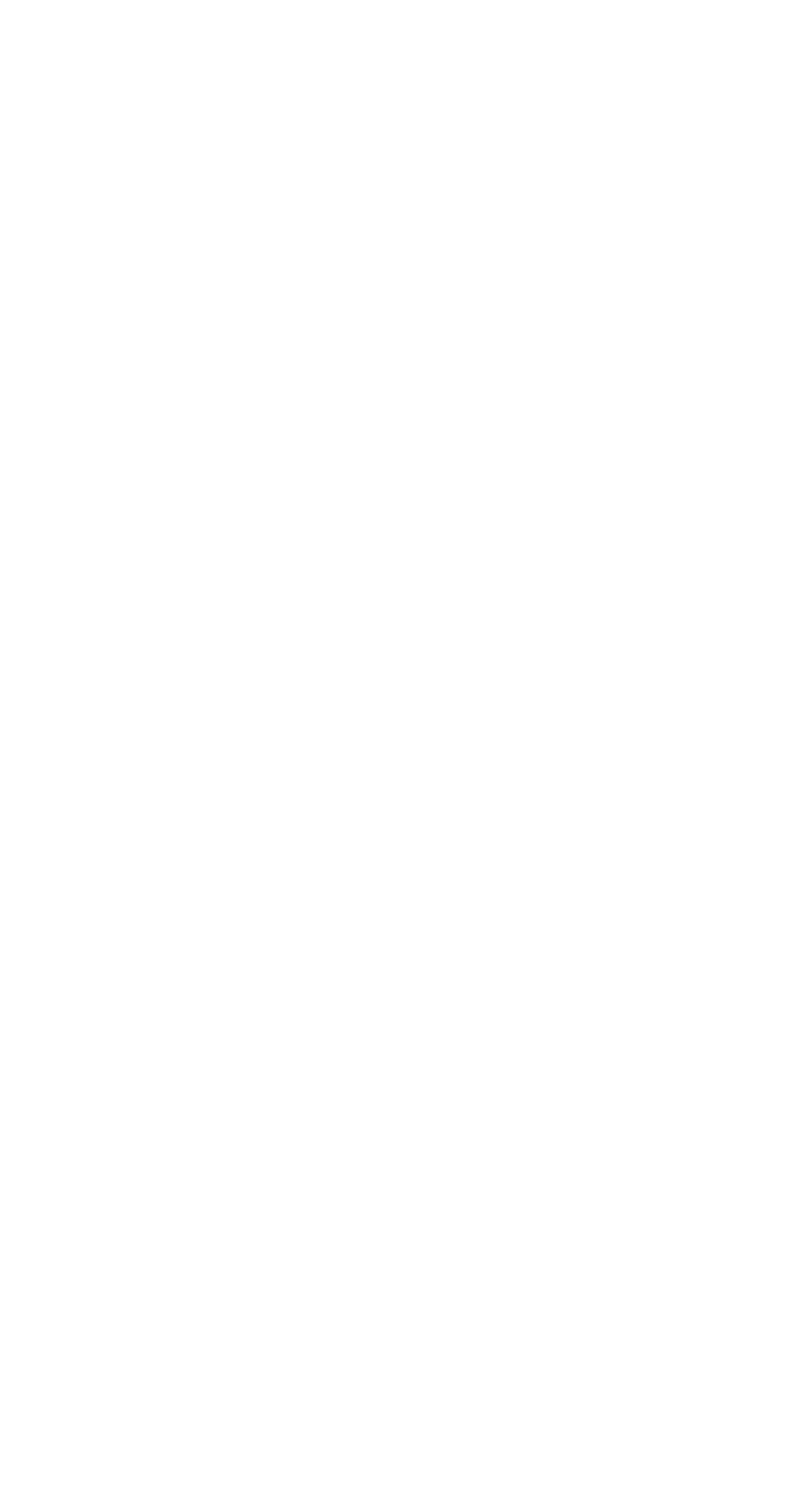


GROUND WATER OF WILLAMETTE VALLEY, OREG.

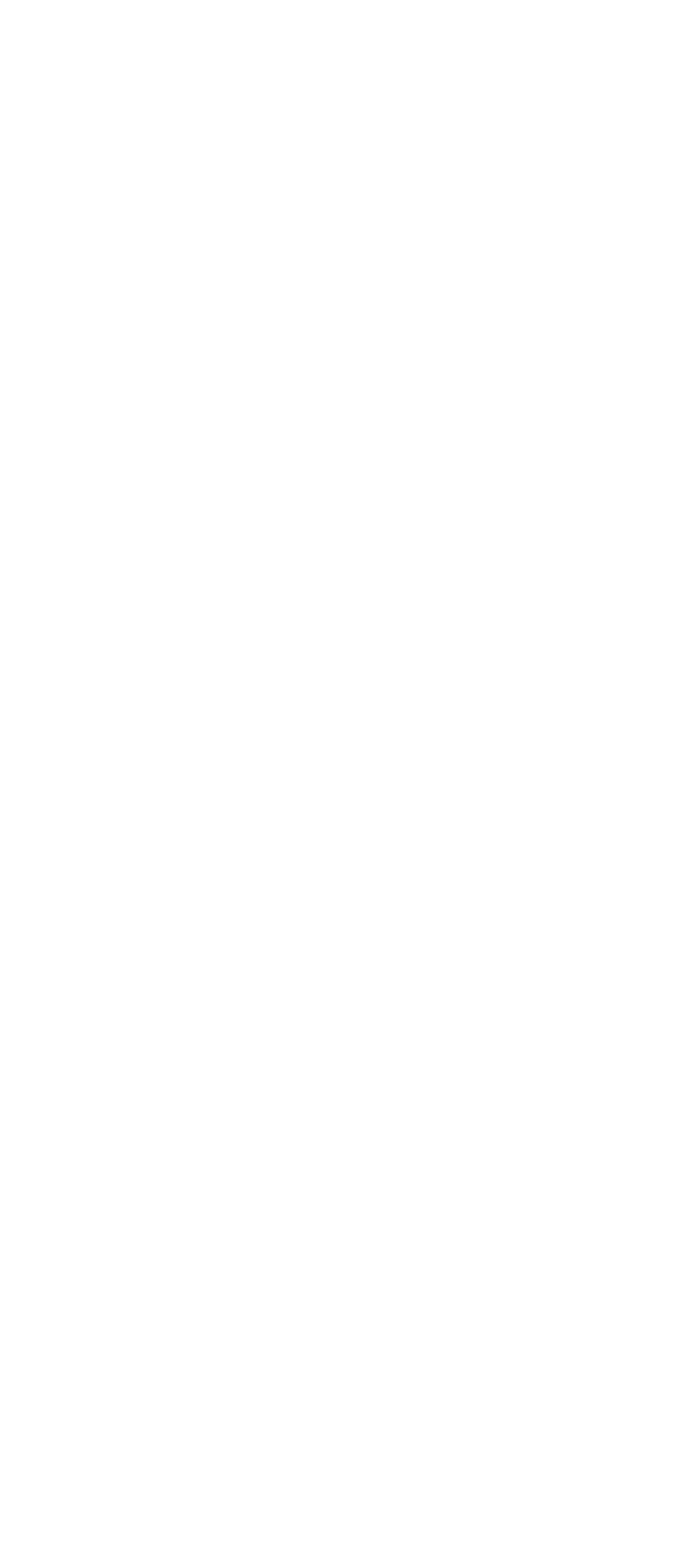




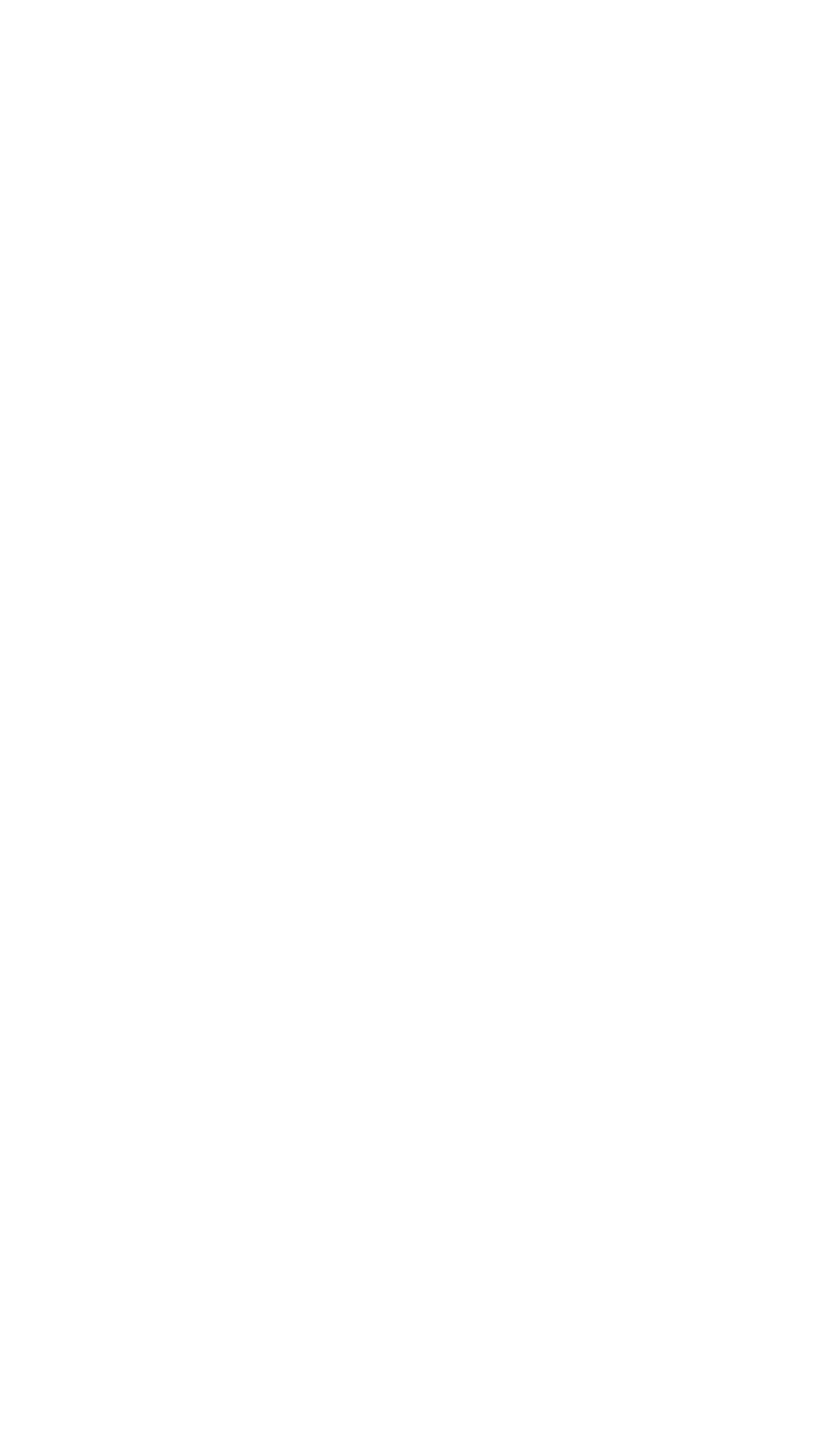




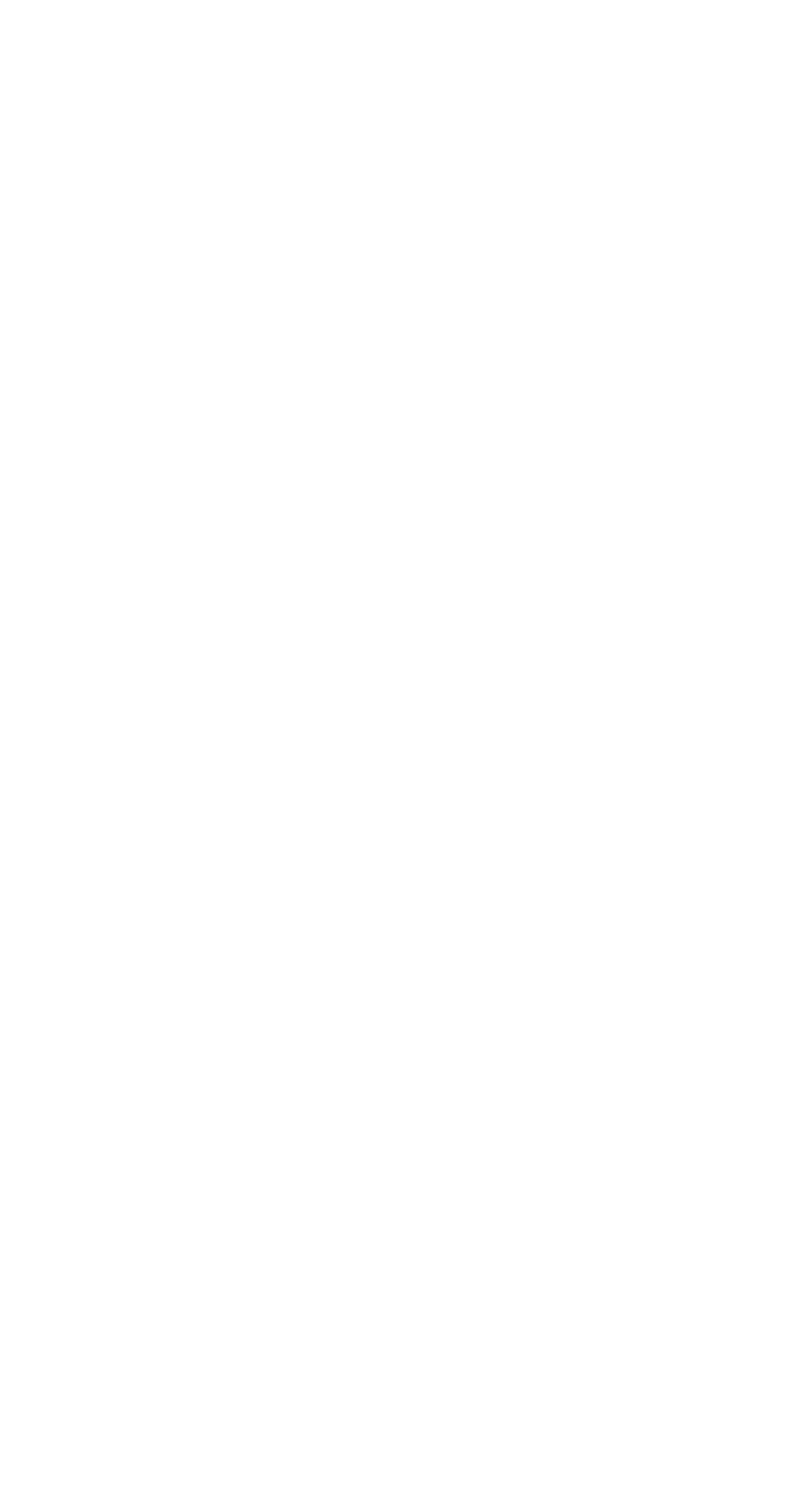




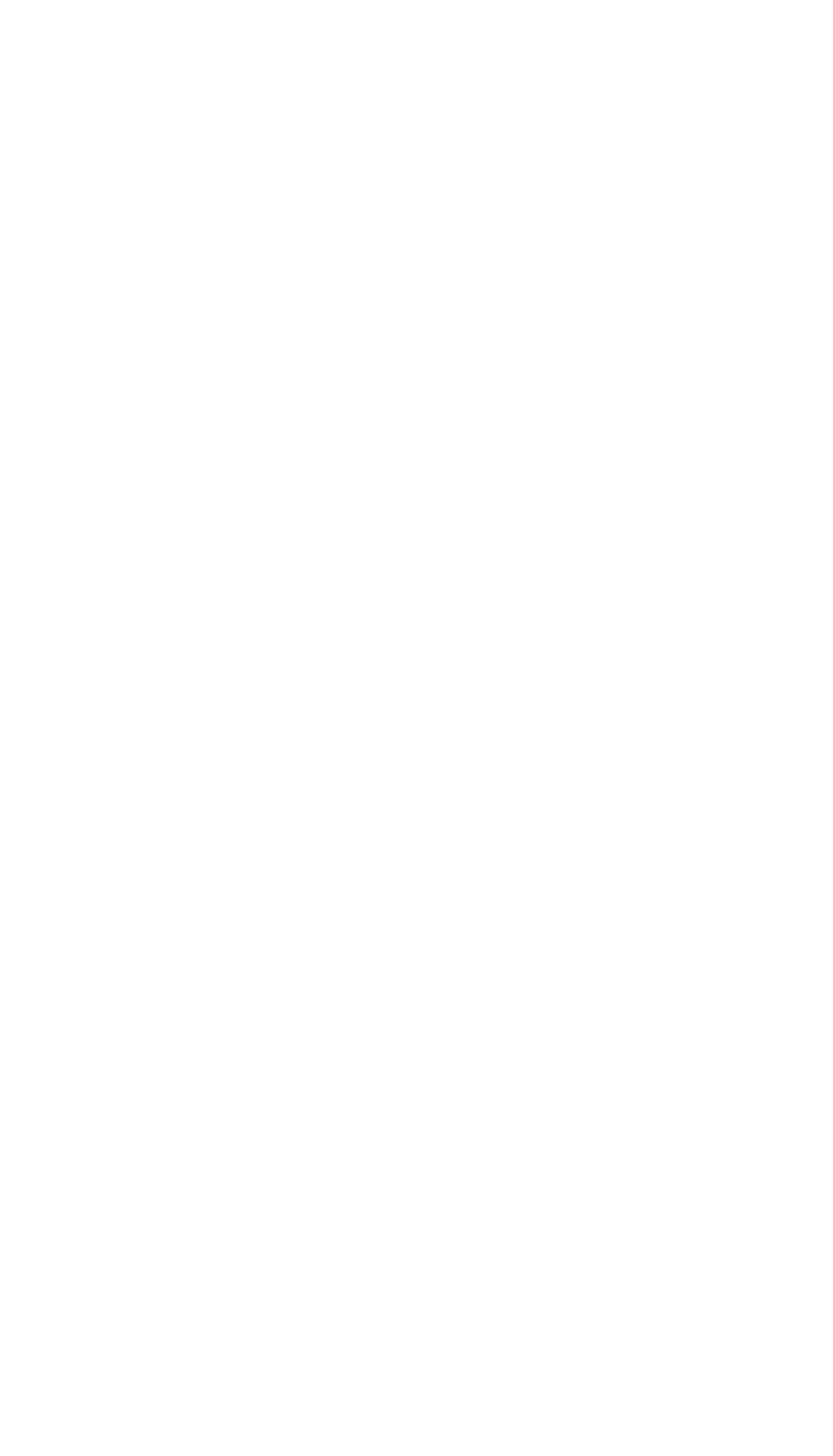


100 GROUND WATER OF WILLAMETTE VALLEY, OREG.

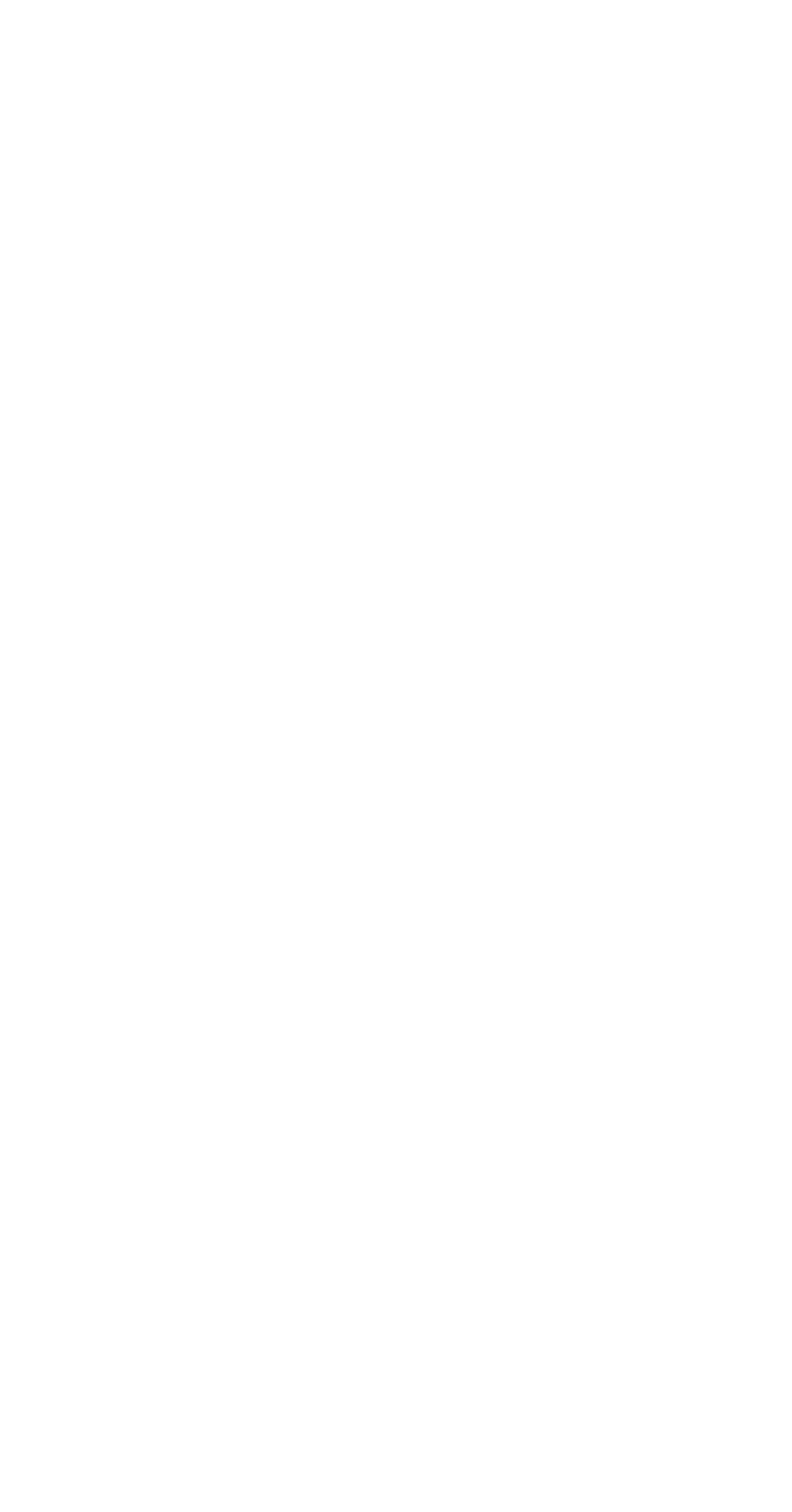




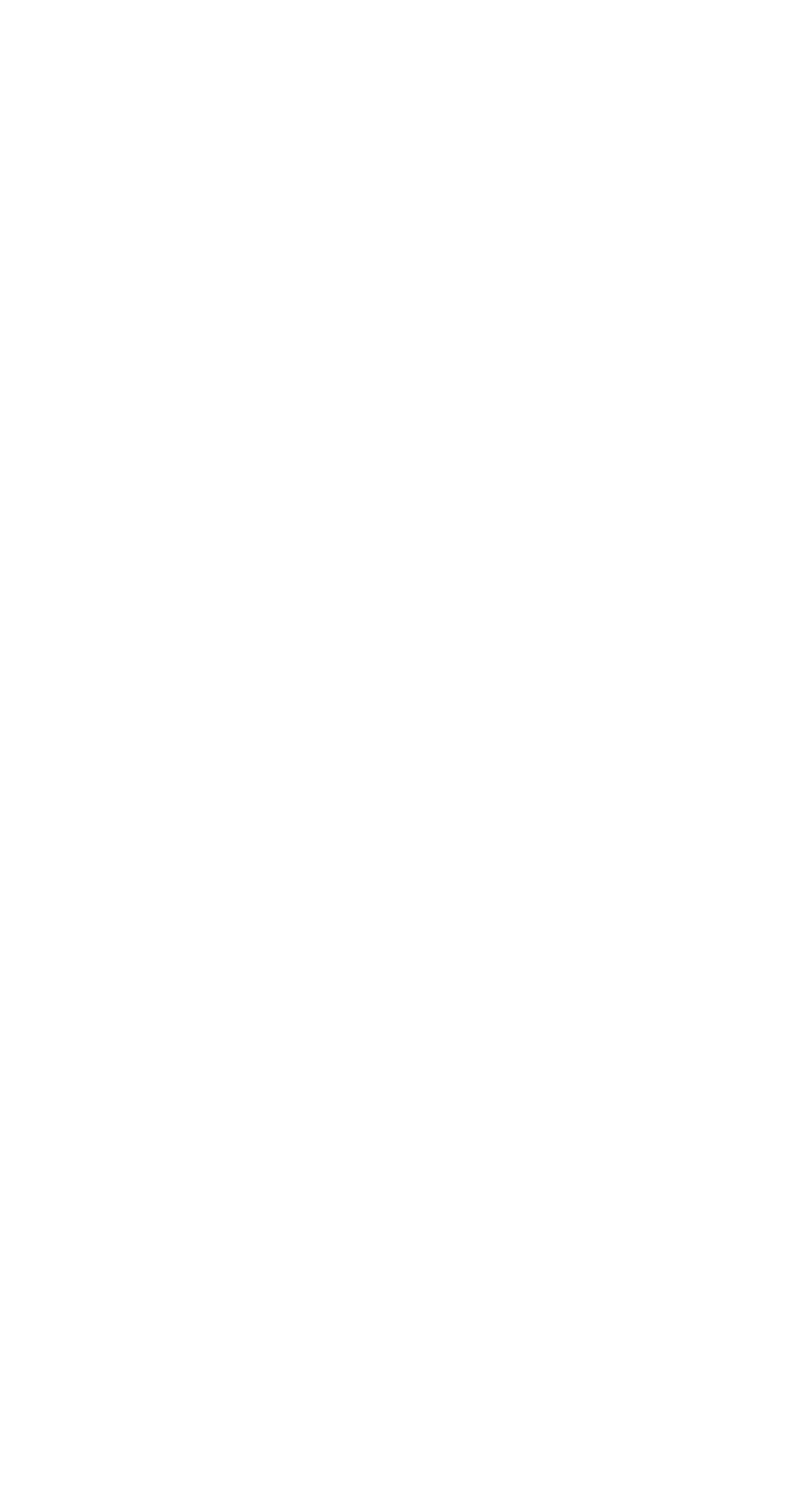


102 GROUND WATER OF WILLAMETTE VALLEY, OREG.

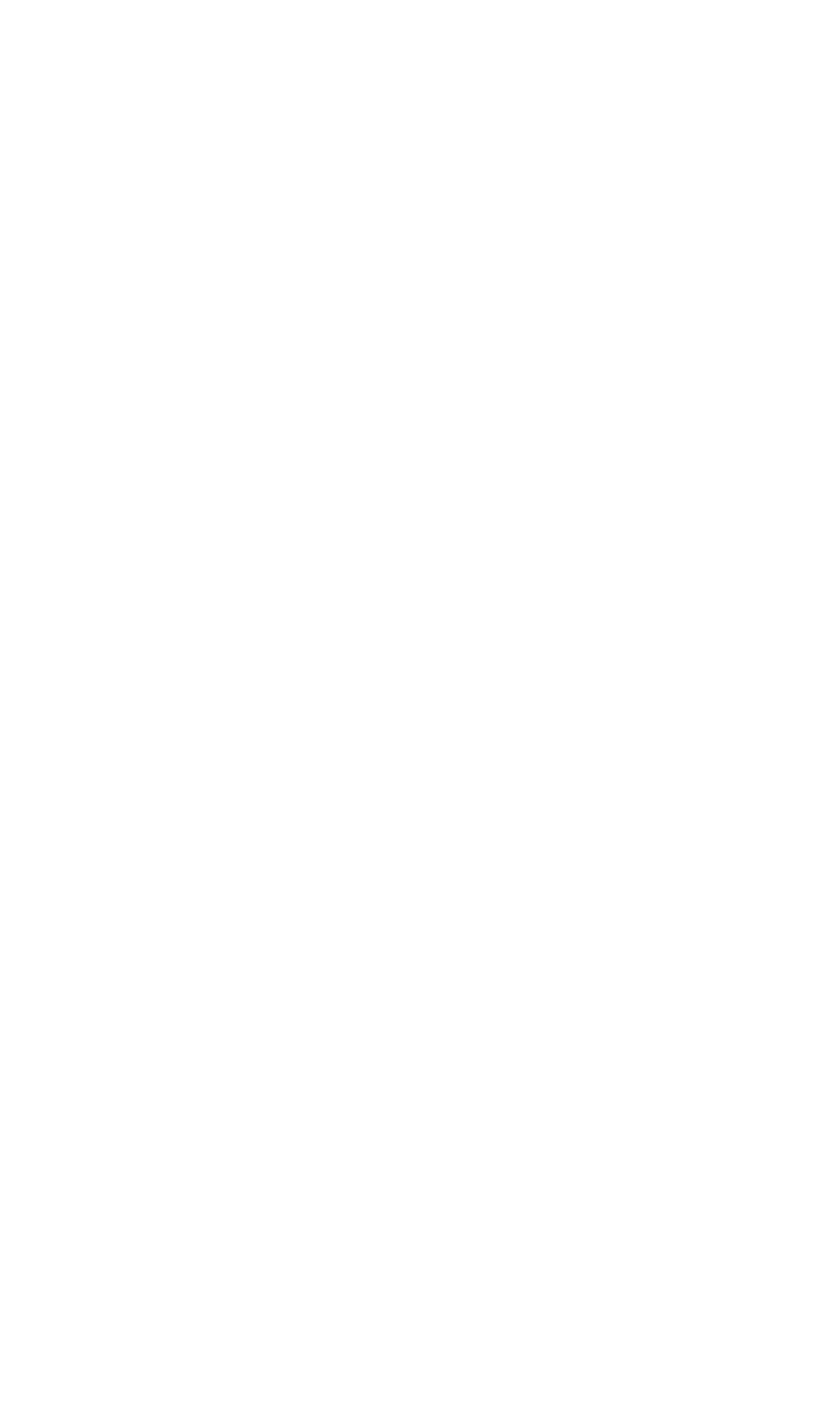




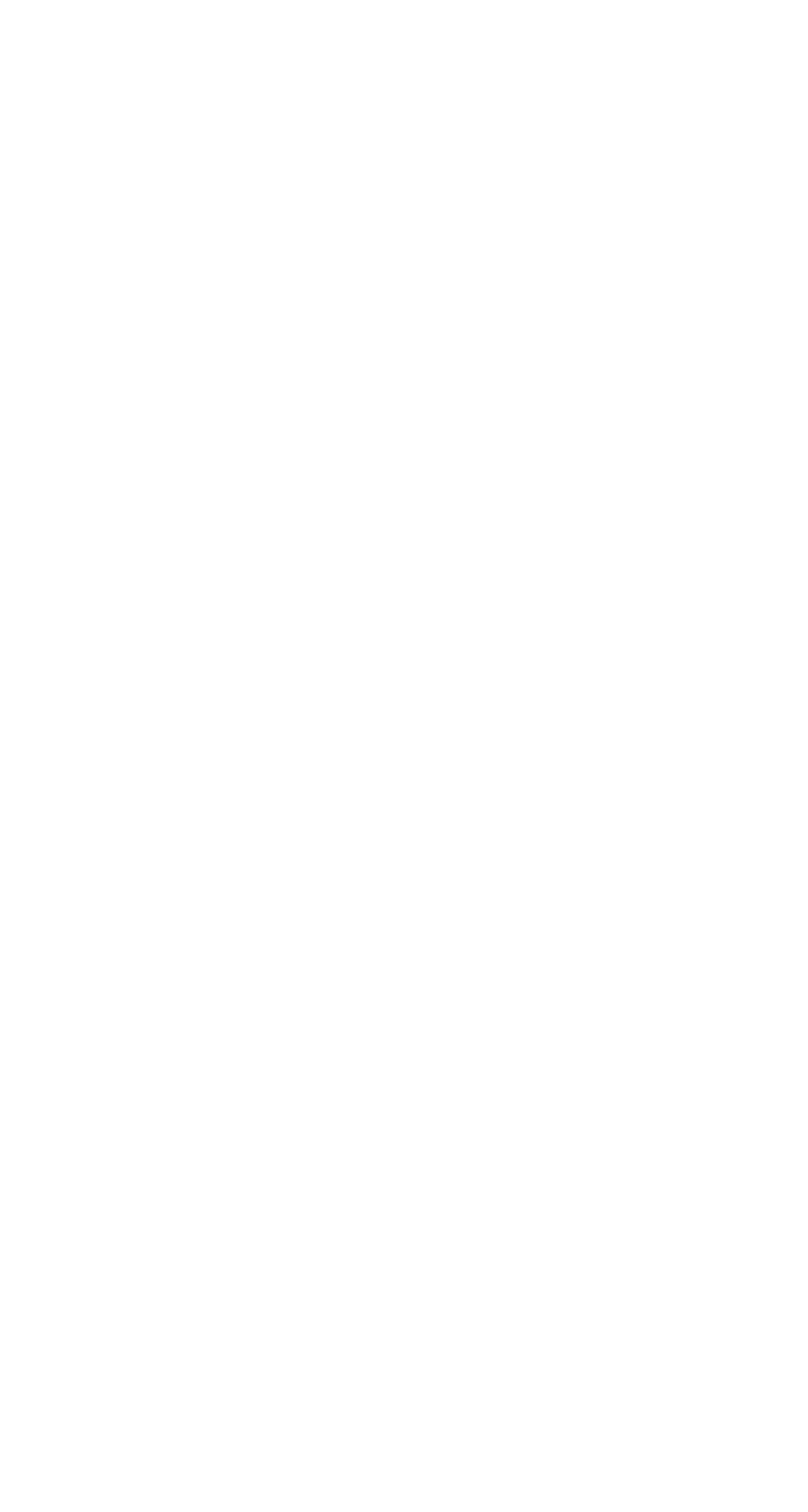




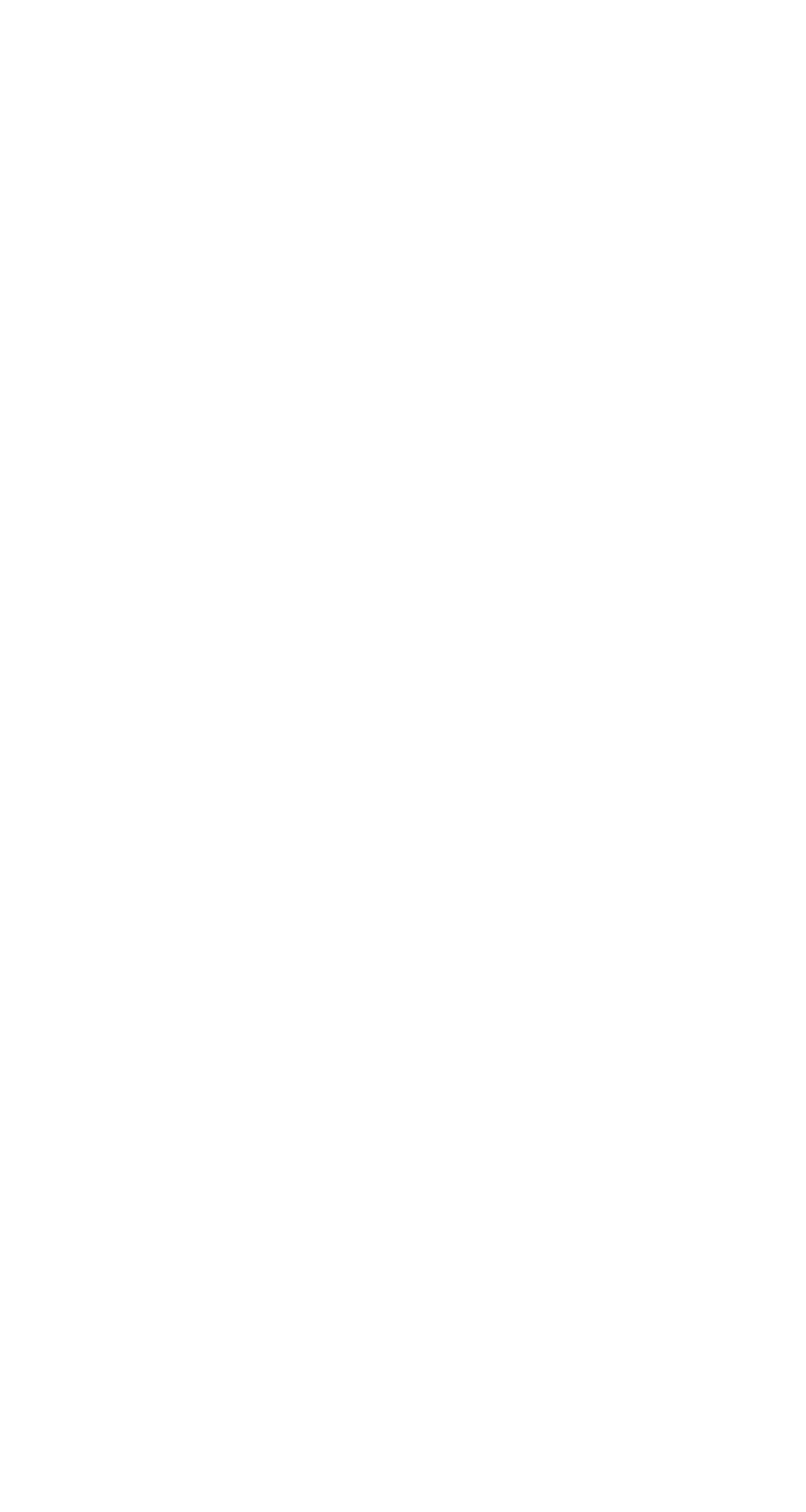




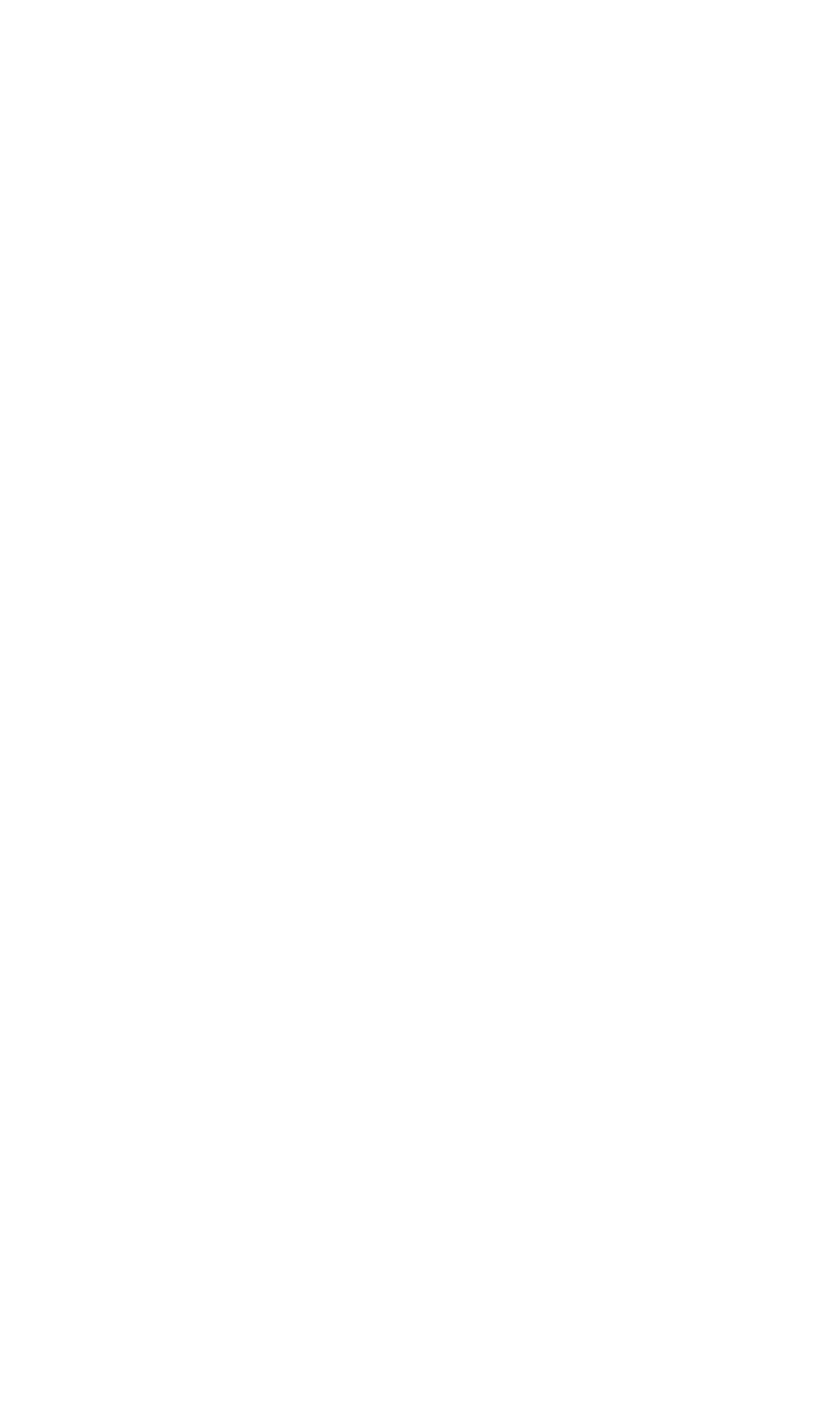




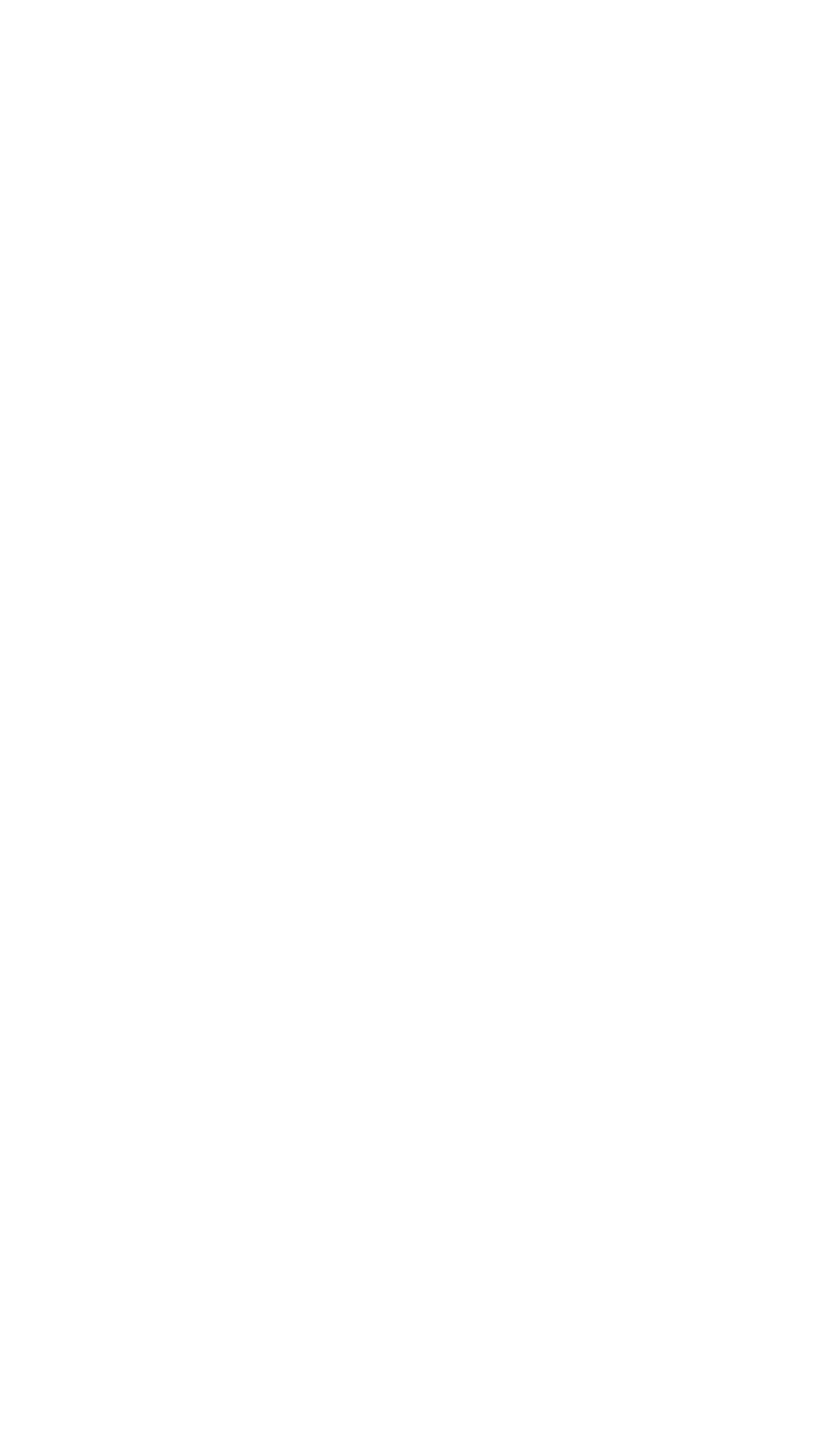


WELL RECORDS

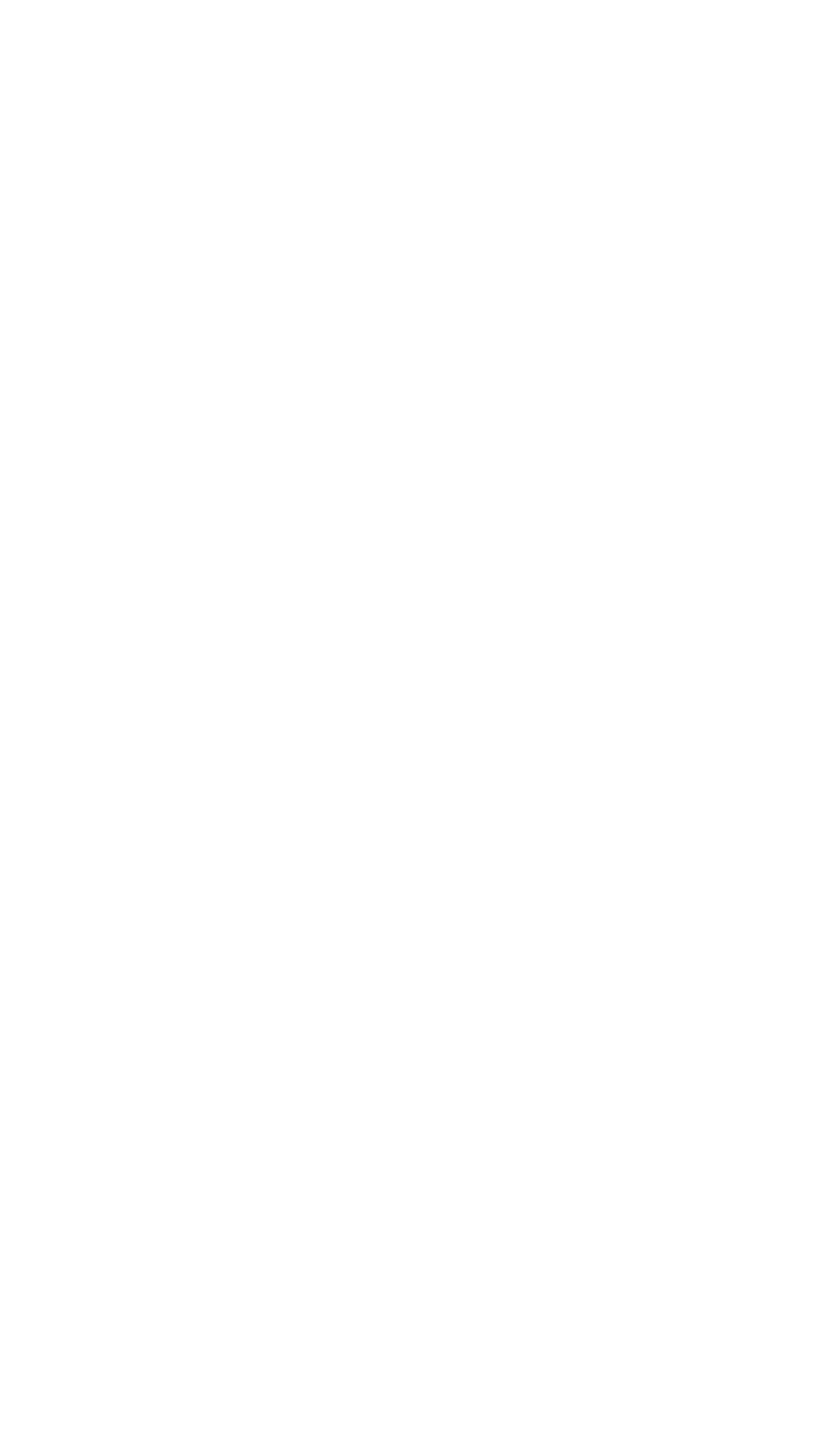




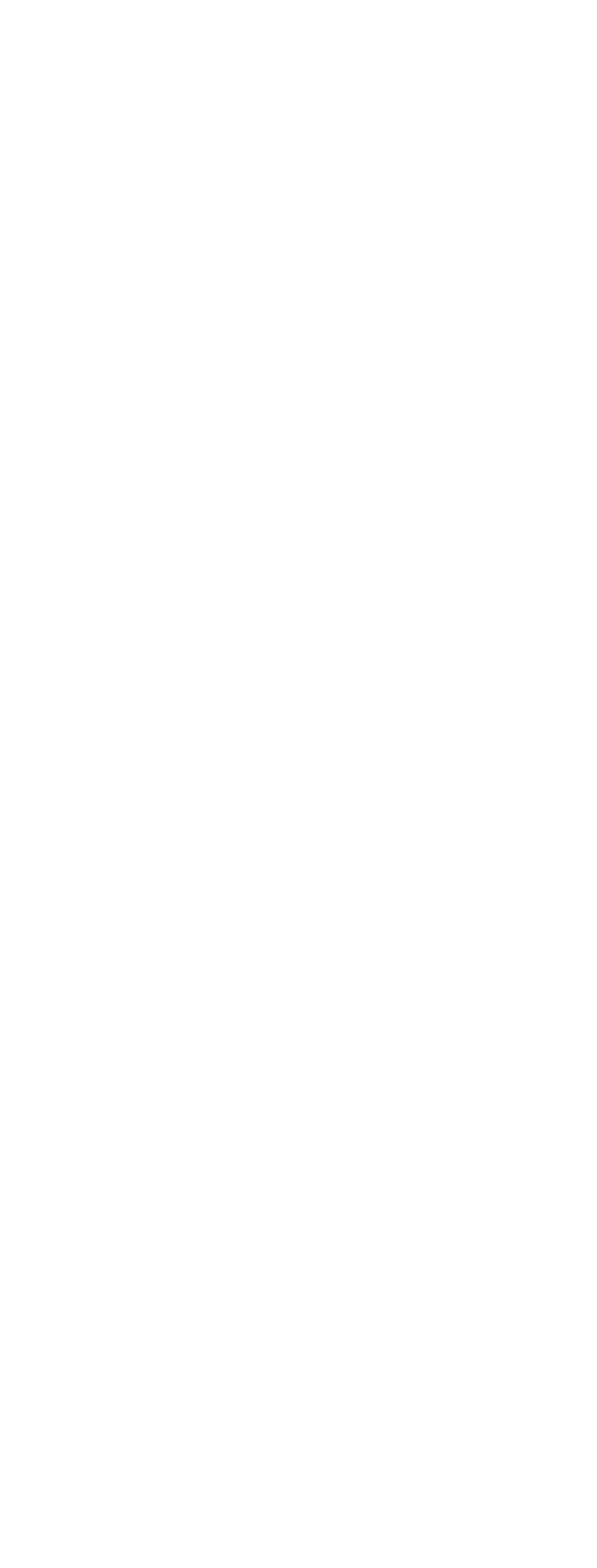




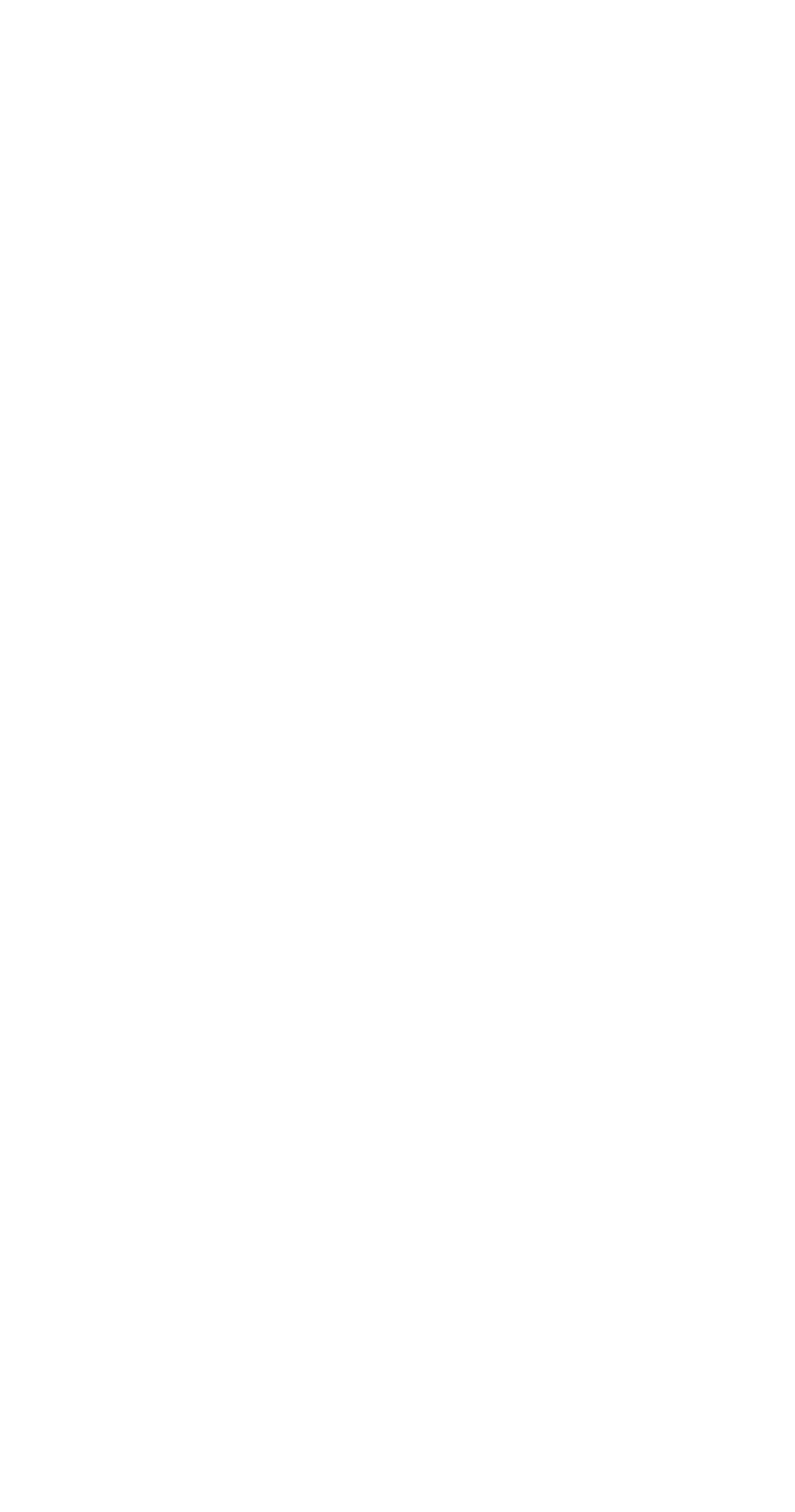


110 GROUND WATER OF WILLAMETTE VALLEY, OREG.

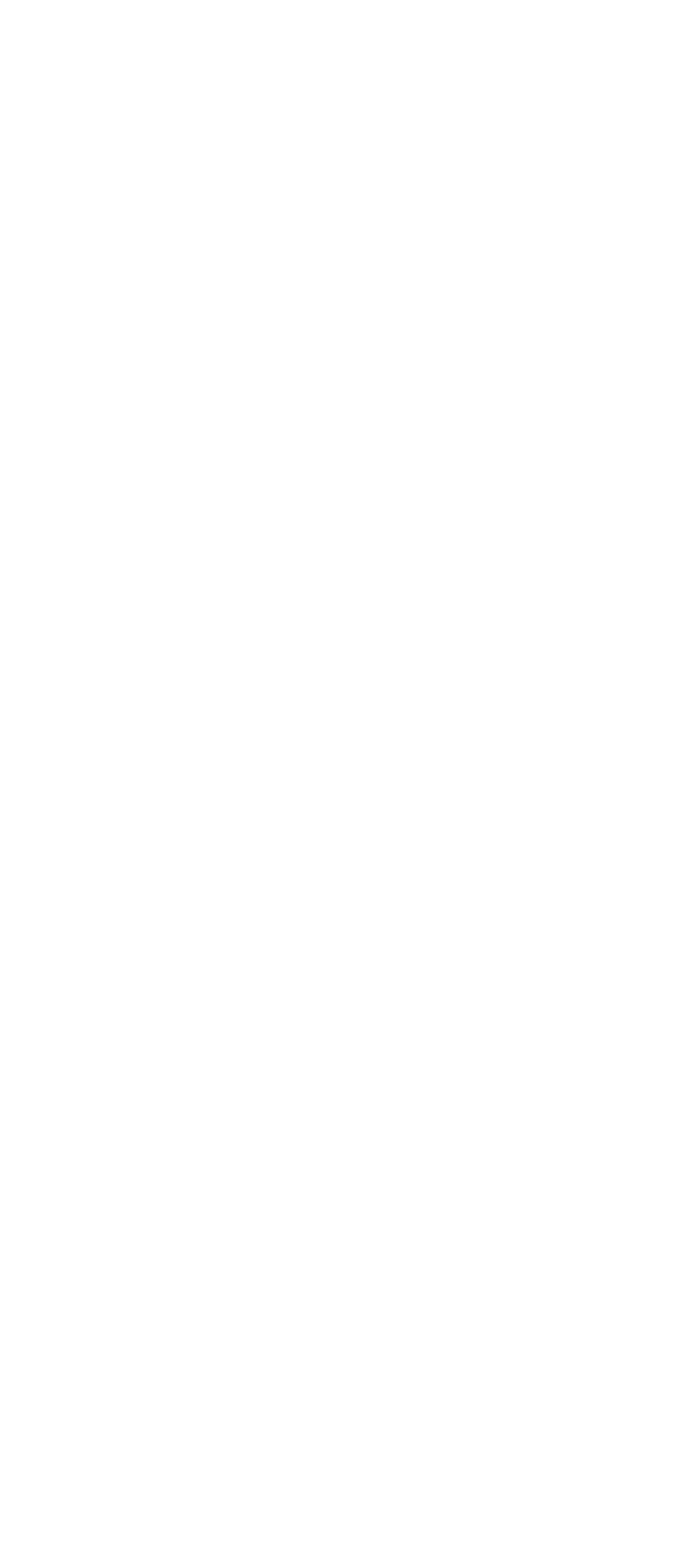




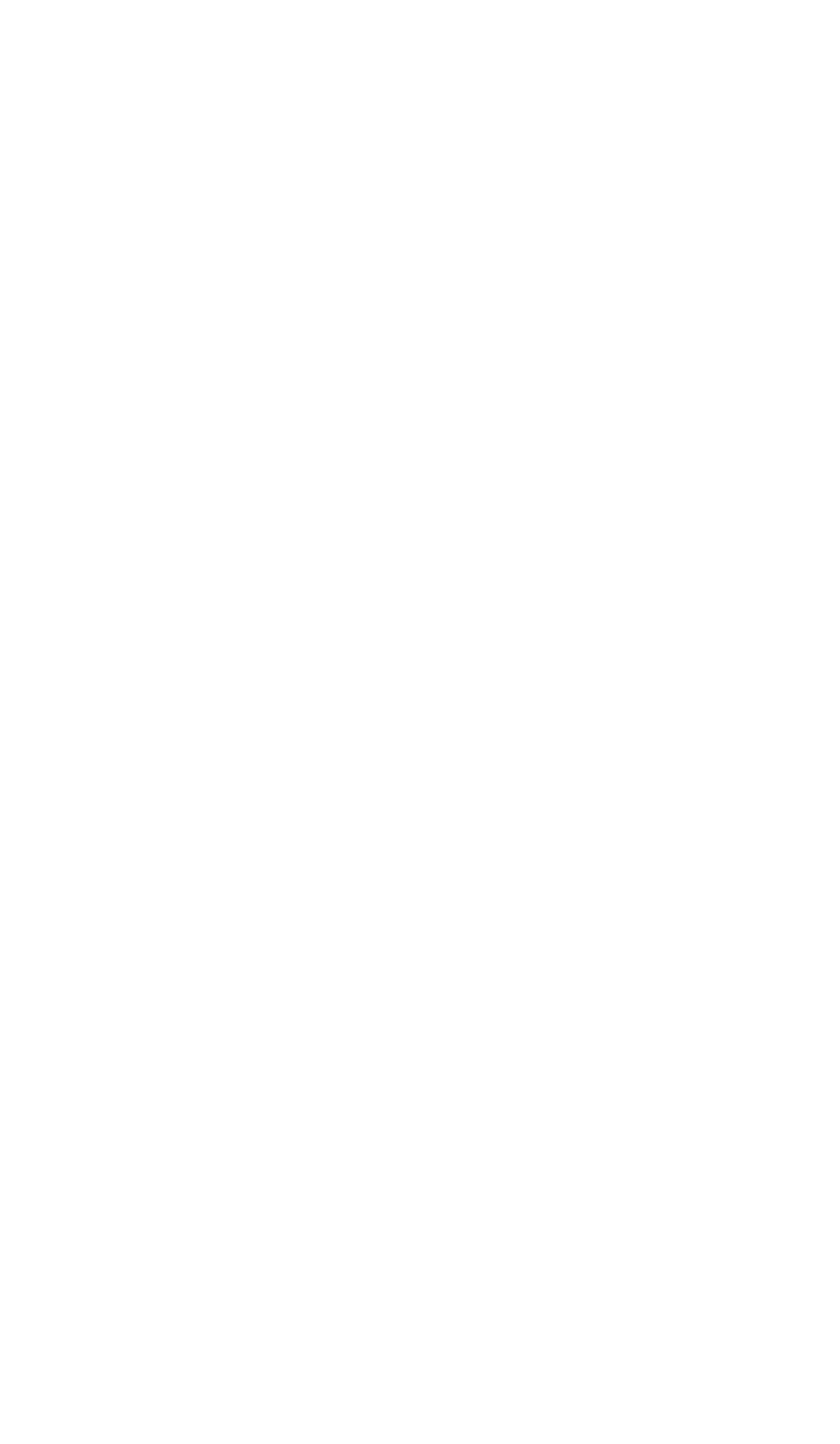




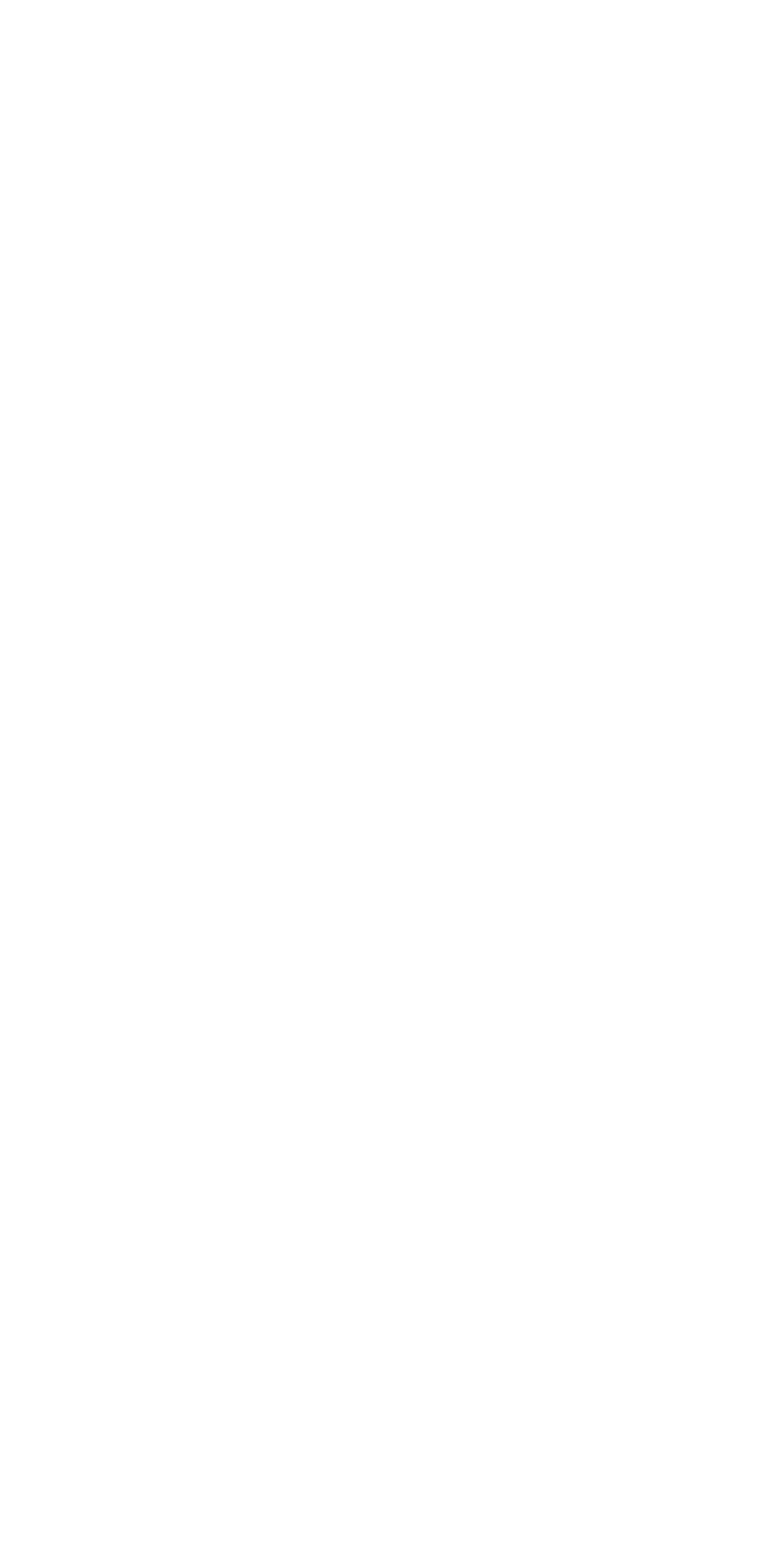




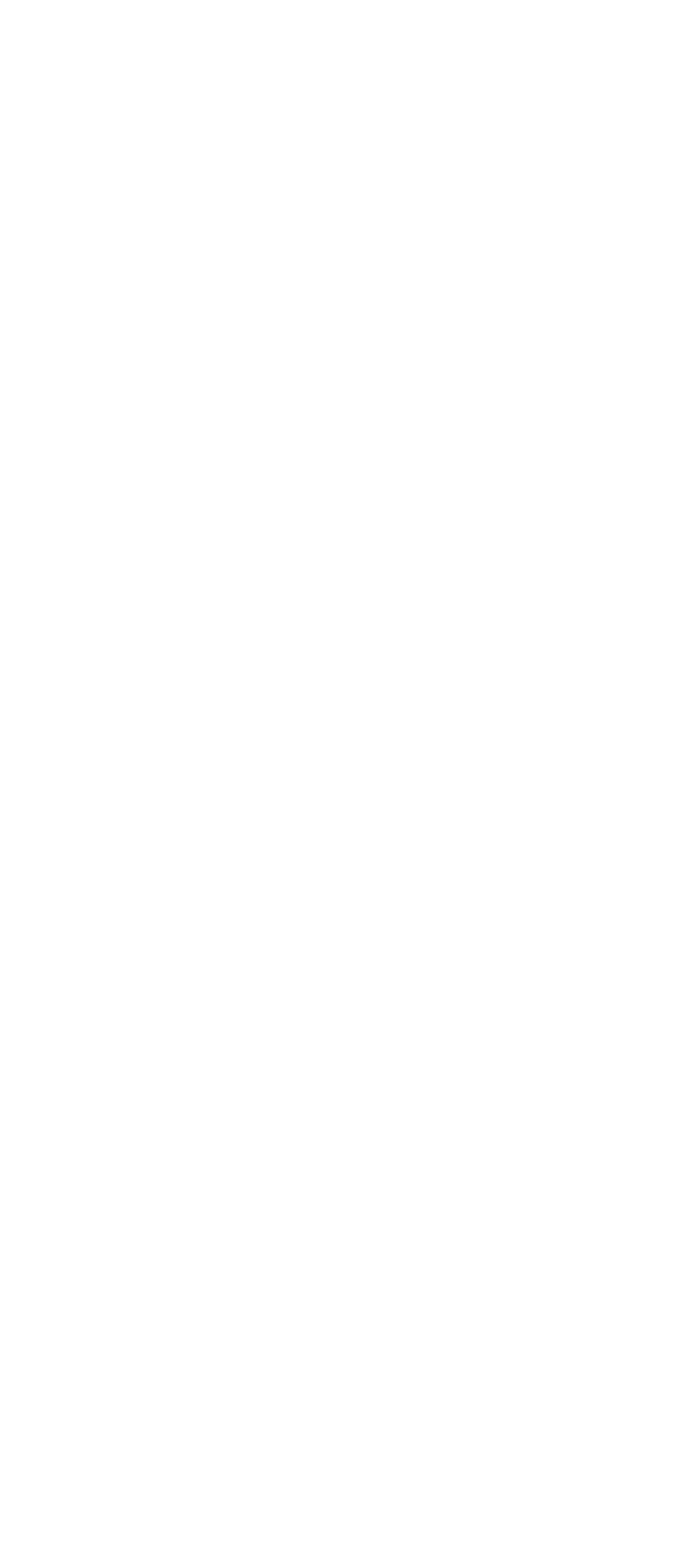




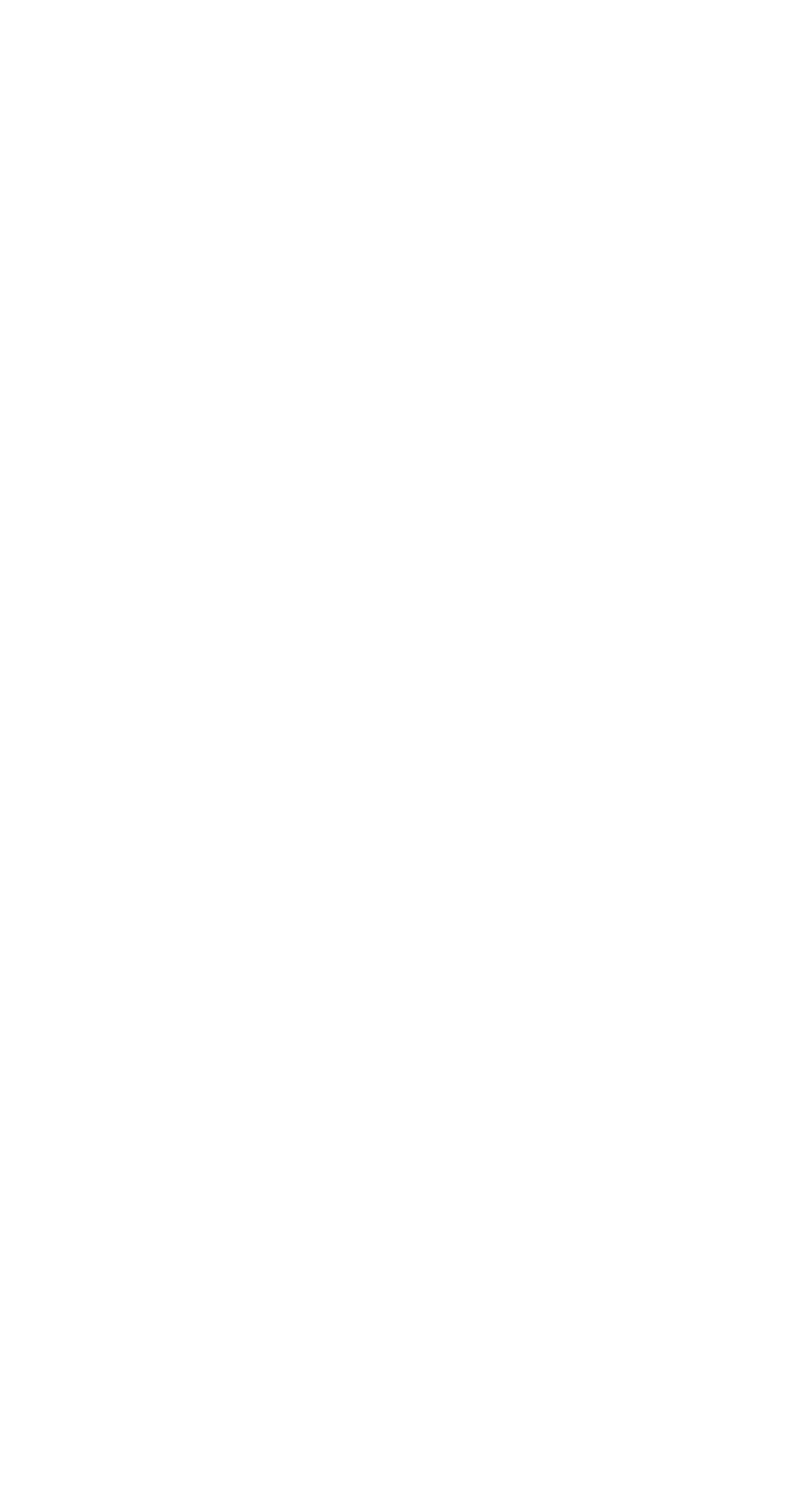


WELL RECORDS

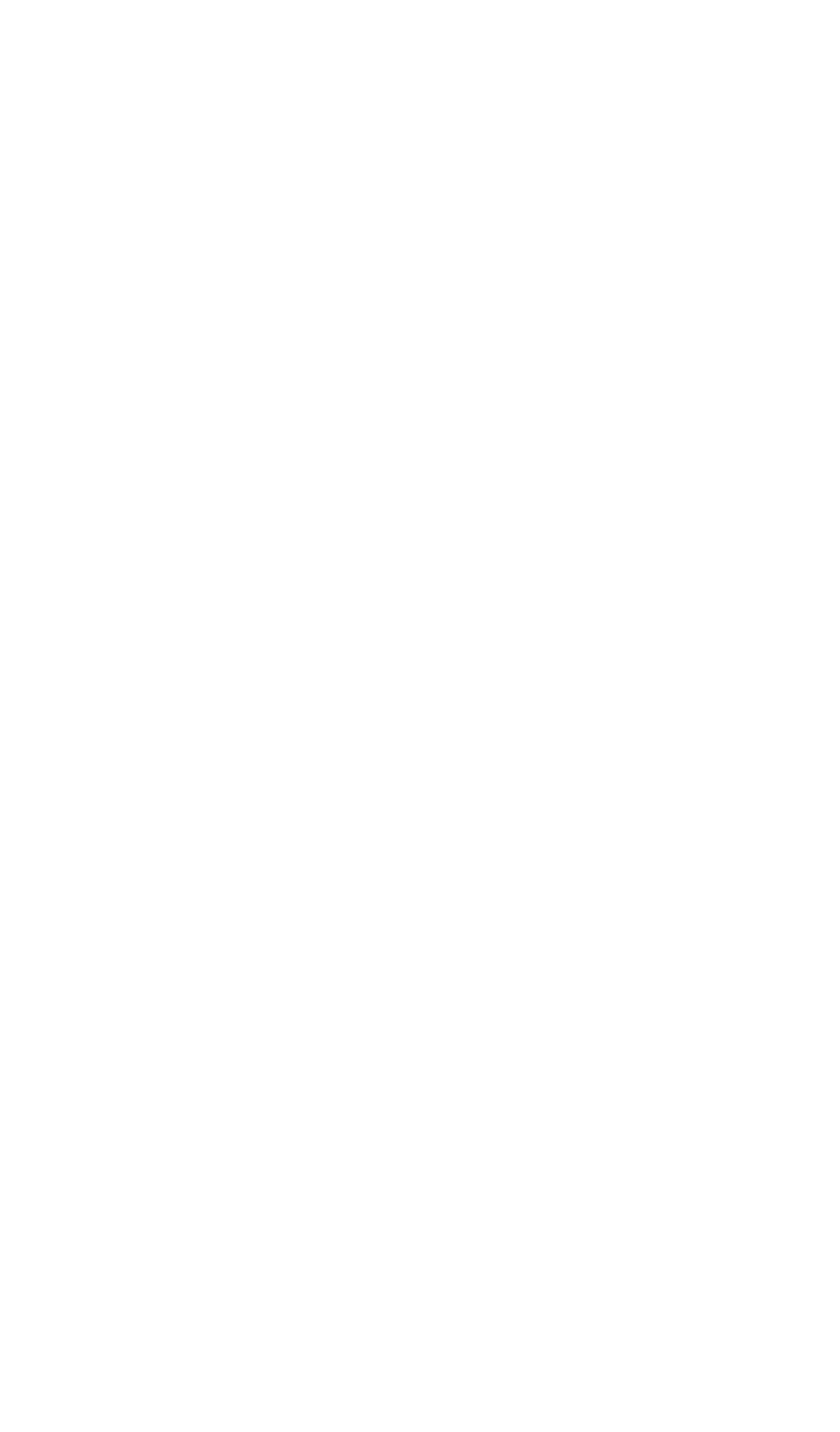




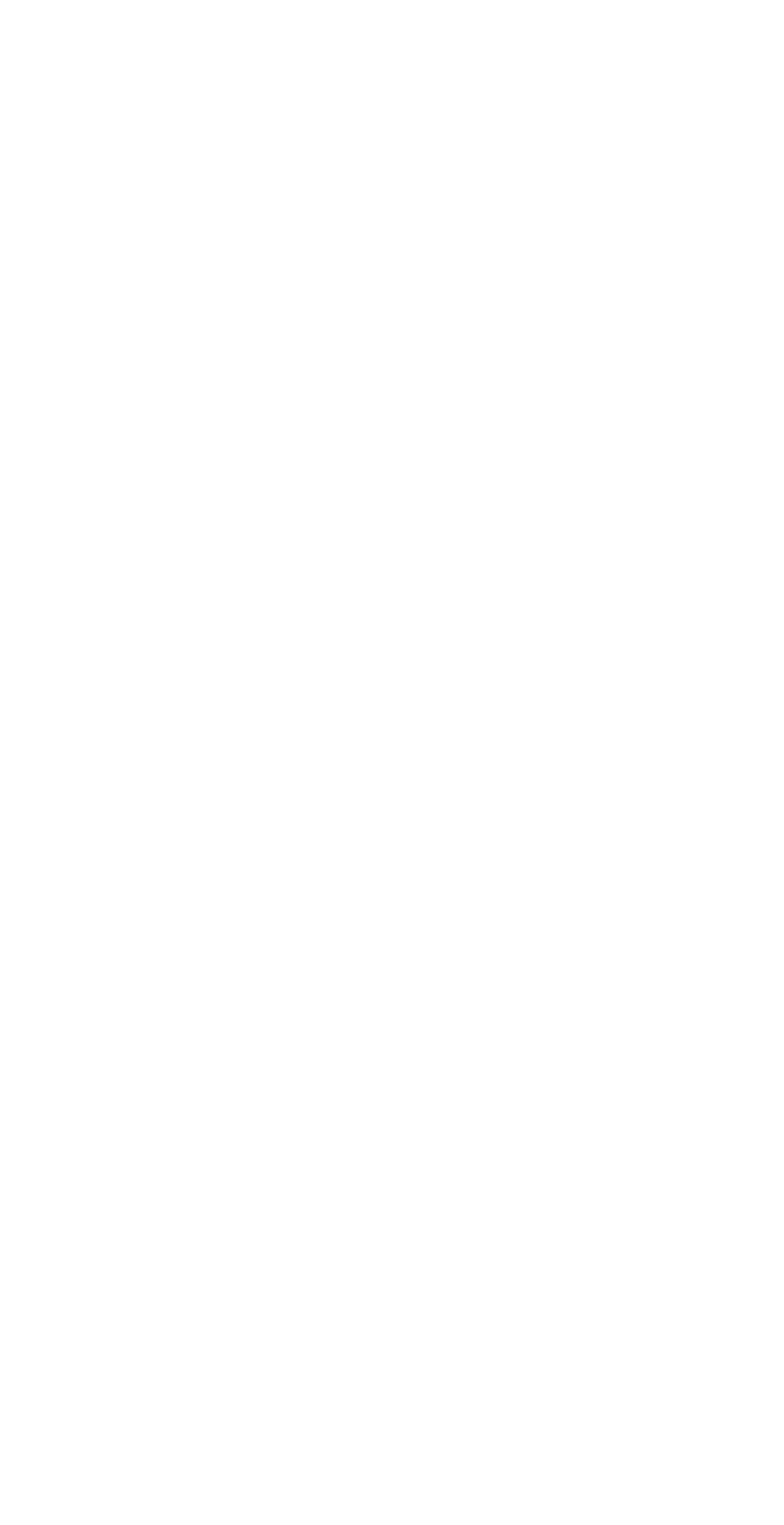




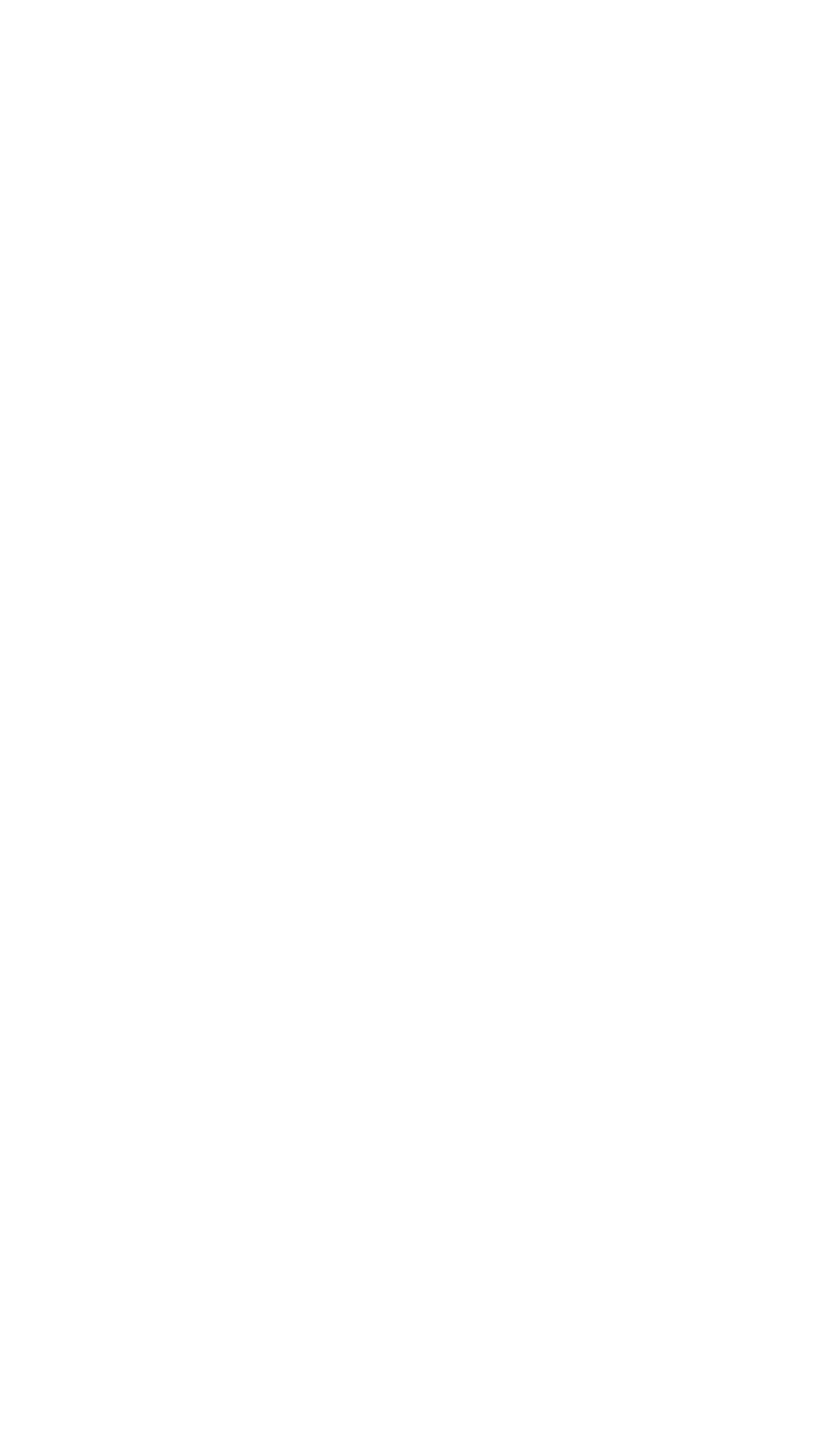


118 GROUND WATER OF WILLAMETTE VALLEY, OREG.

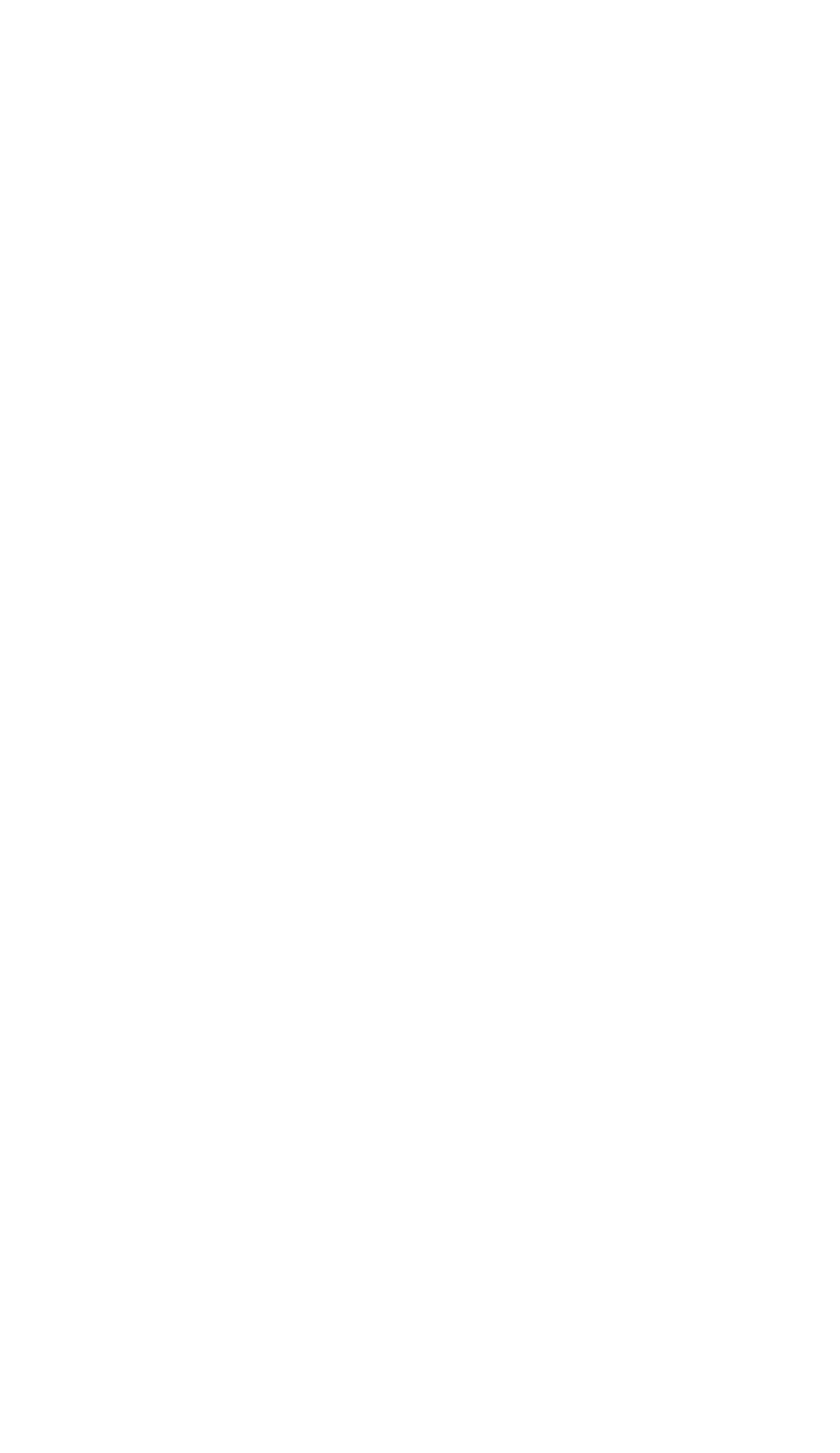




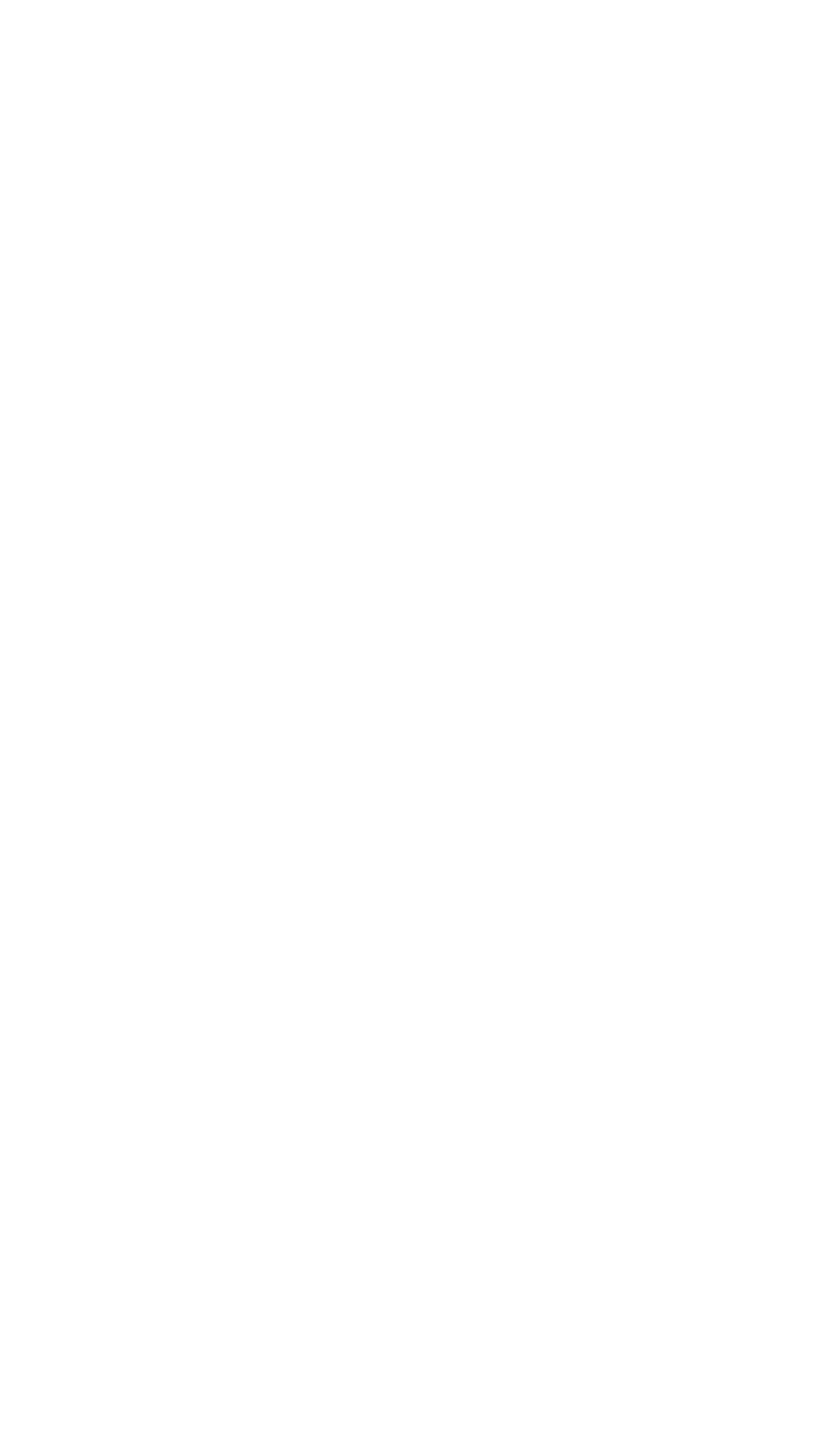




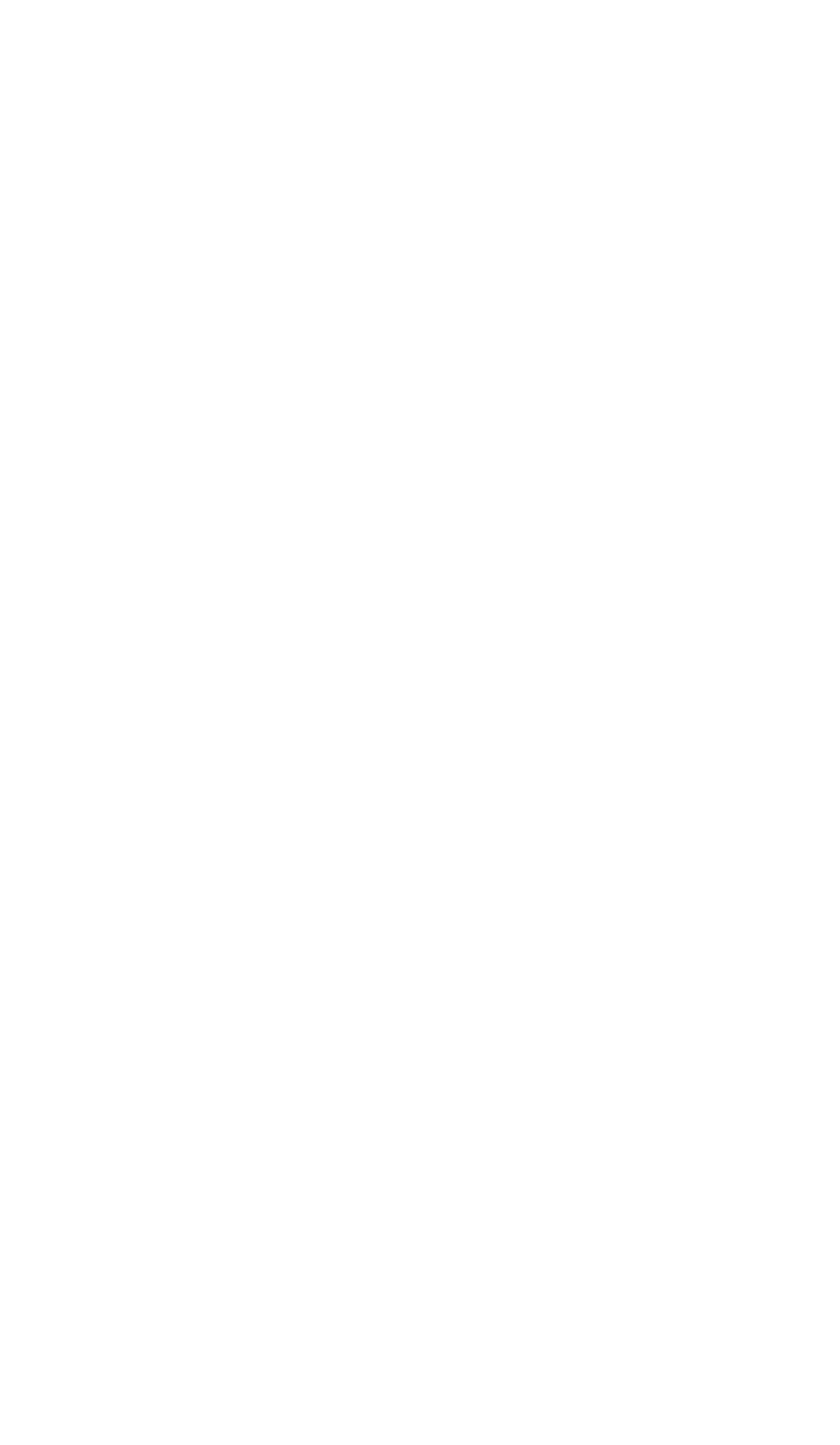


WELL RECORDS

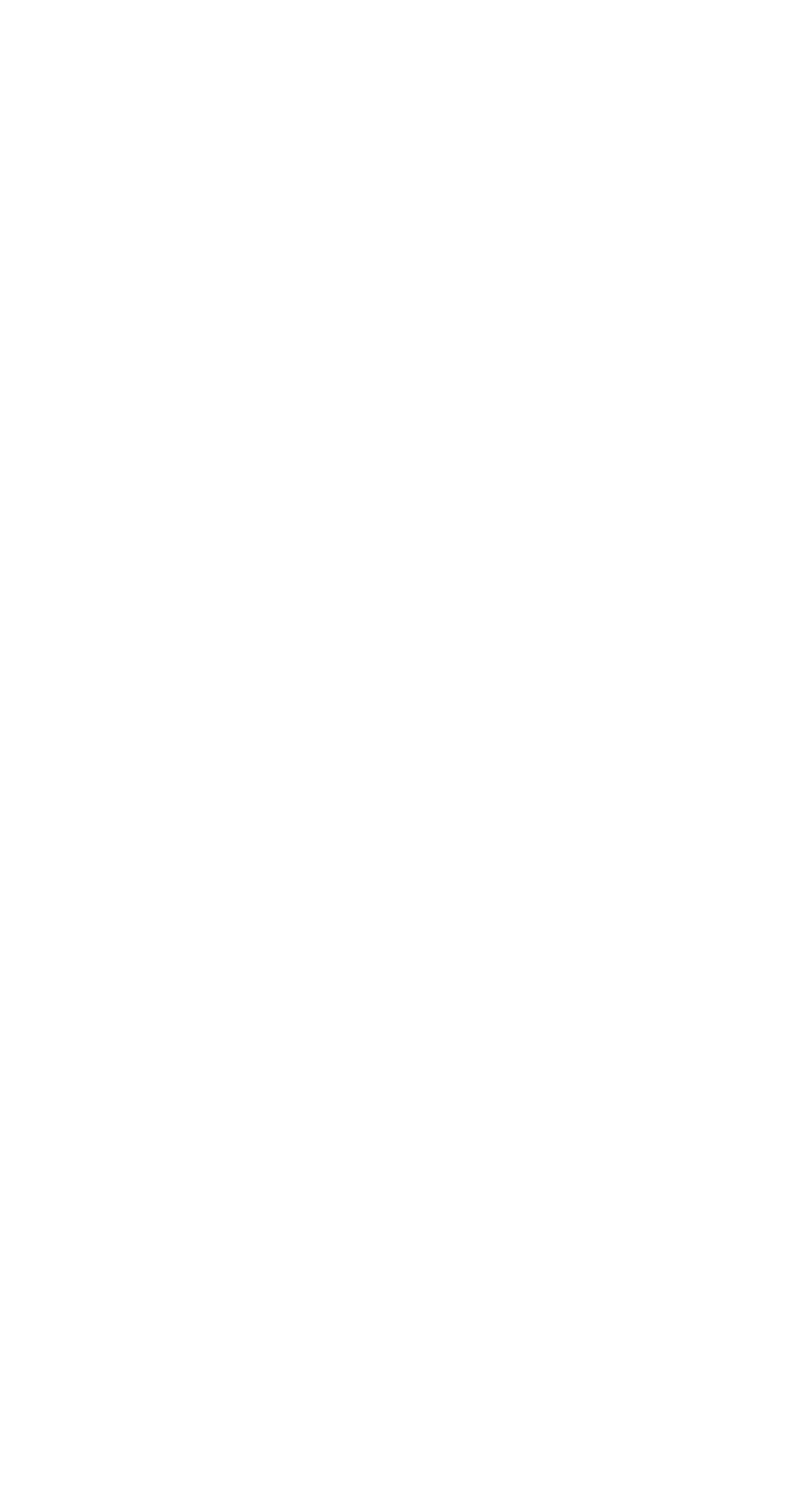




\begin{tabular}{|c|c|c|c|c|}
\hline 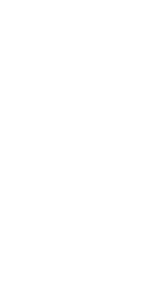 & 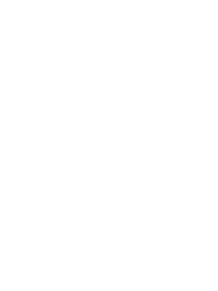 & 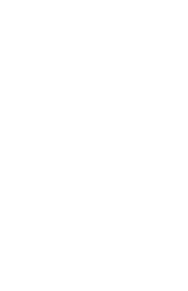 & 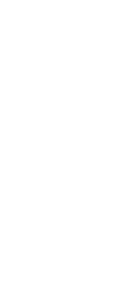 & 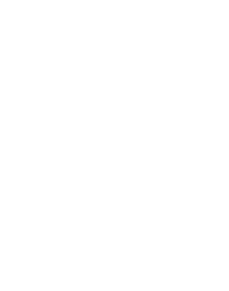 \\
\hline 曾鸣 & 踝 & & & \\
\hline 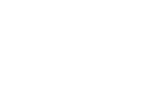 & 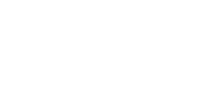 & 是 & & \\
\hline 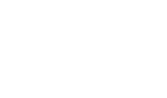 & $\exists$ & $\infty \infty=$ & 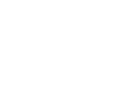 & \\
\hline 崂 & 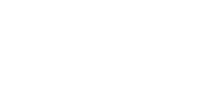 & 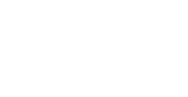 & 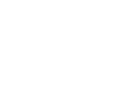 & आ \\
\hline 象 & 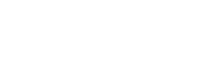 & 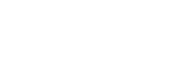 & คีอีี & 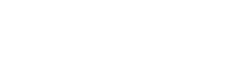 \\
\hline 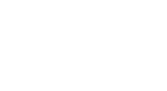 & 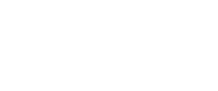 & 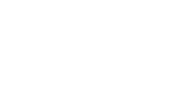 & ๙ & 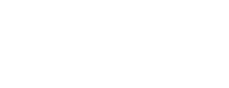 \\
\hline 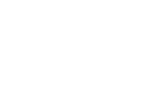 & 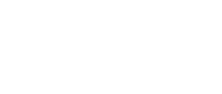 & $\Rightarrow>P D>D$ & $\Rightarrow$ & $\Leftrightarrow>F$ \\
\hline 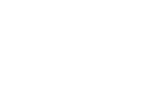 & 眖 & 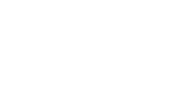 & & 离 \\
\hline 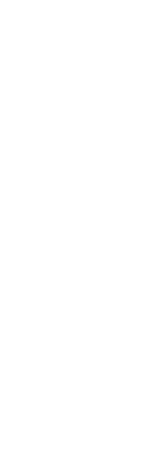 & 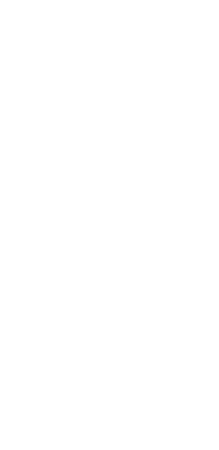 & 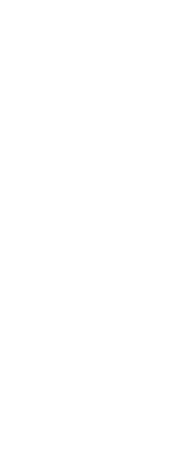 & 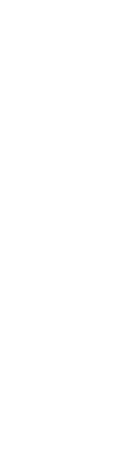 & 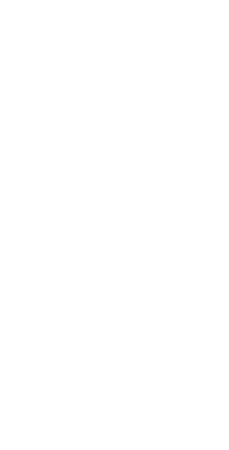 \\
\hline 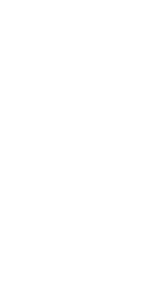 & 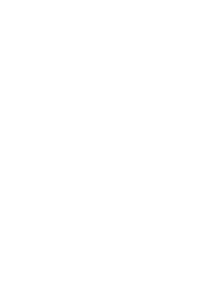 & 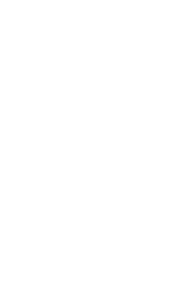 & 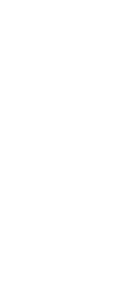 & 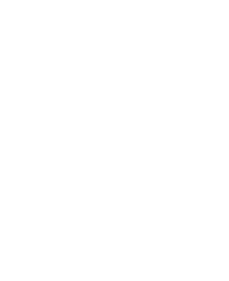 \\
\hline$\dot{0} \dot{8} \frac{5}{2}$ & 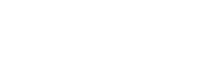 & 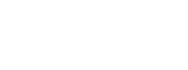 & 융영용용 & 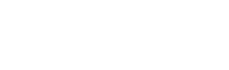 \\
\hline
\end{tabular}




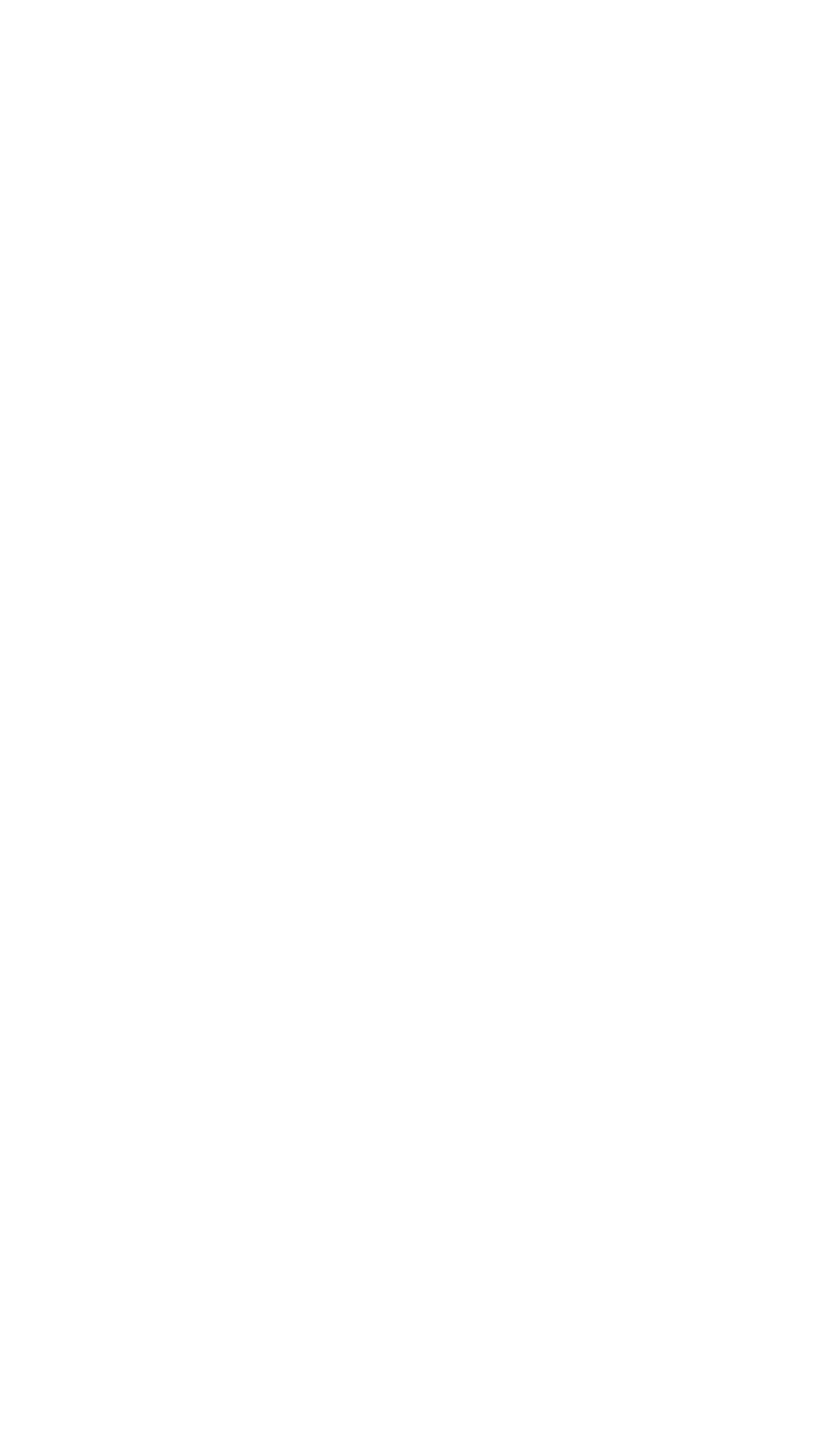


GROU'ND WATER OF WILLAMETTE VALLEY, OREG.

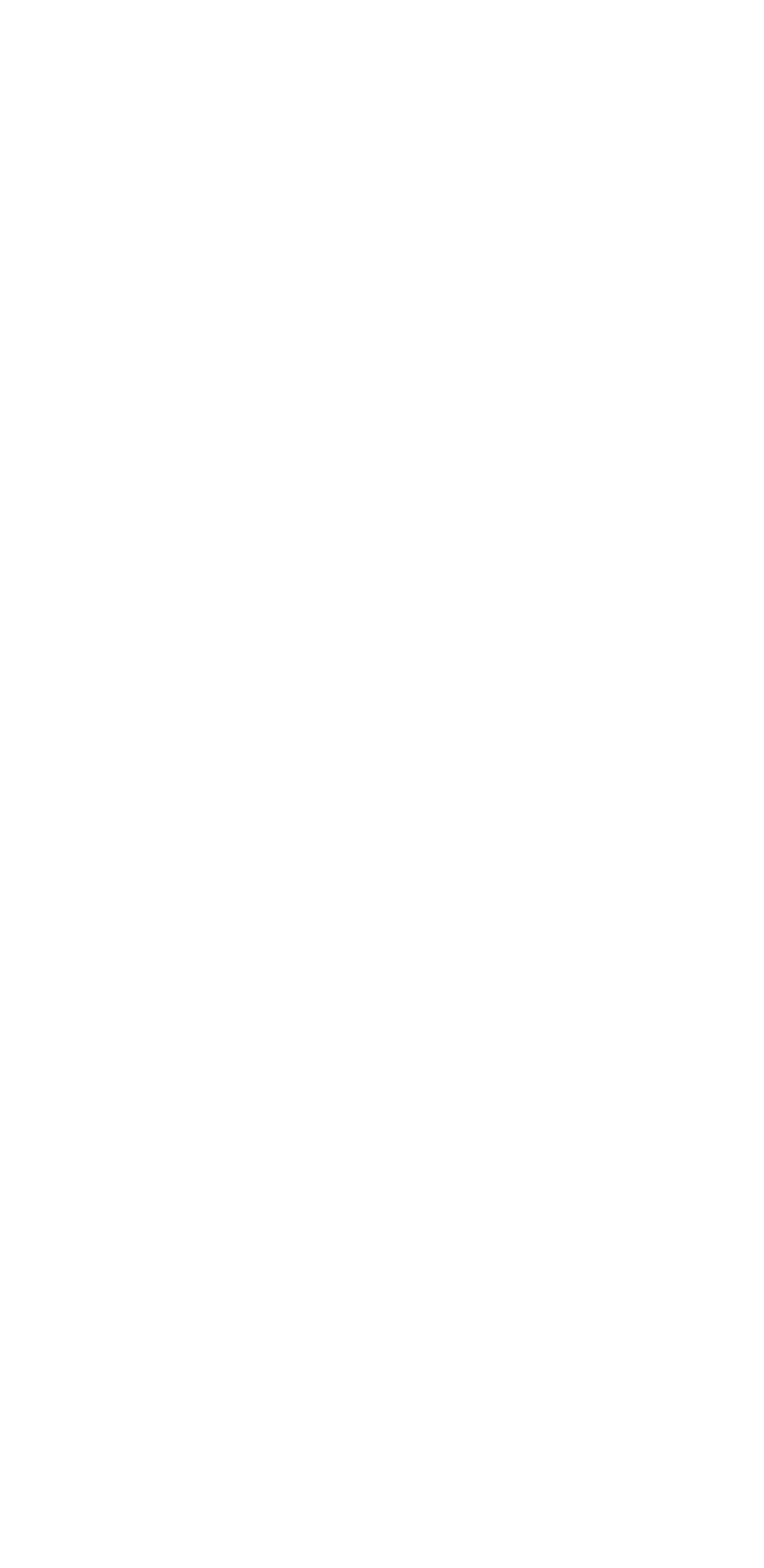




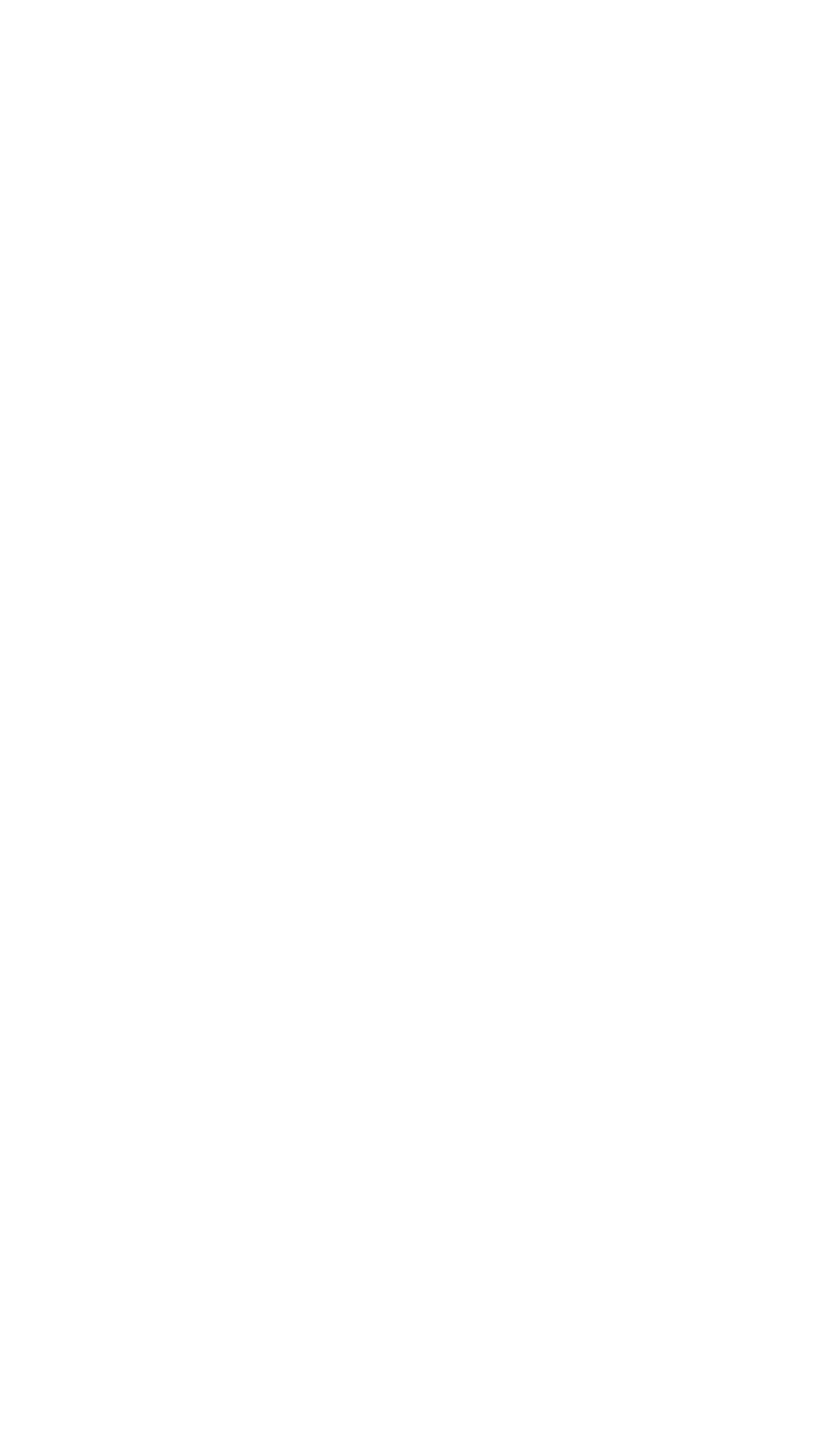




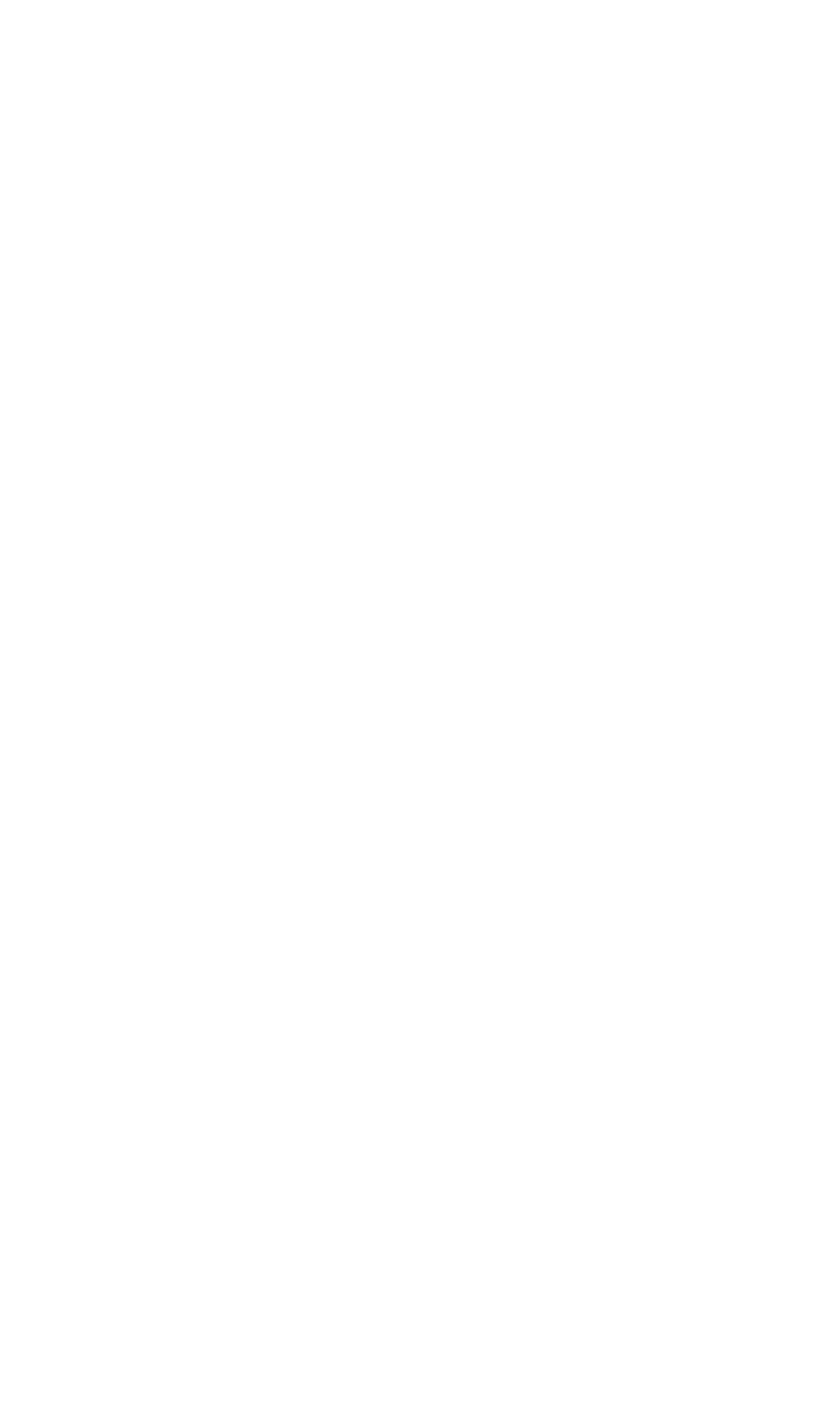




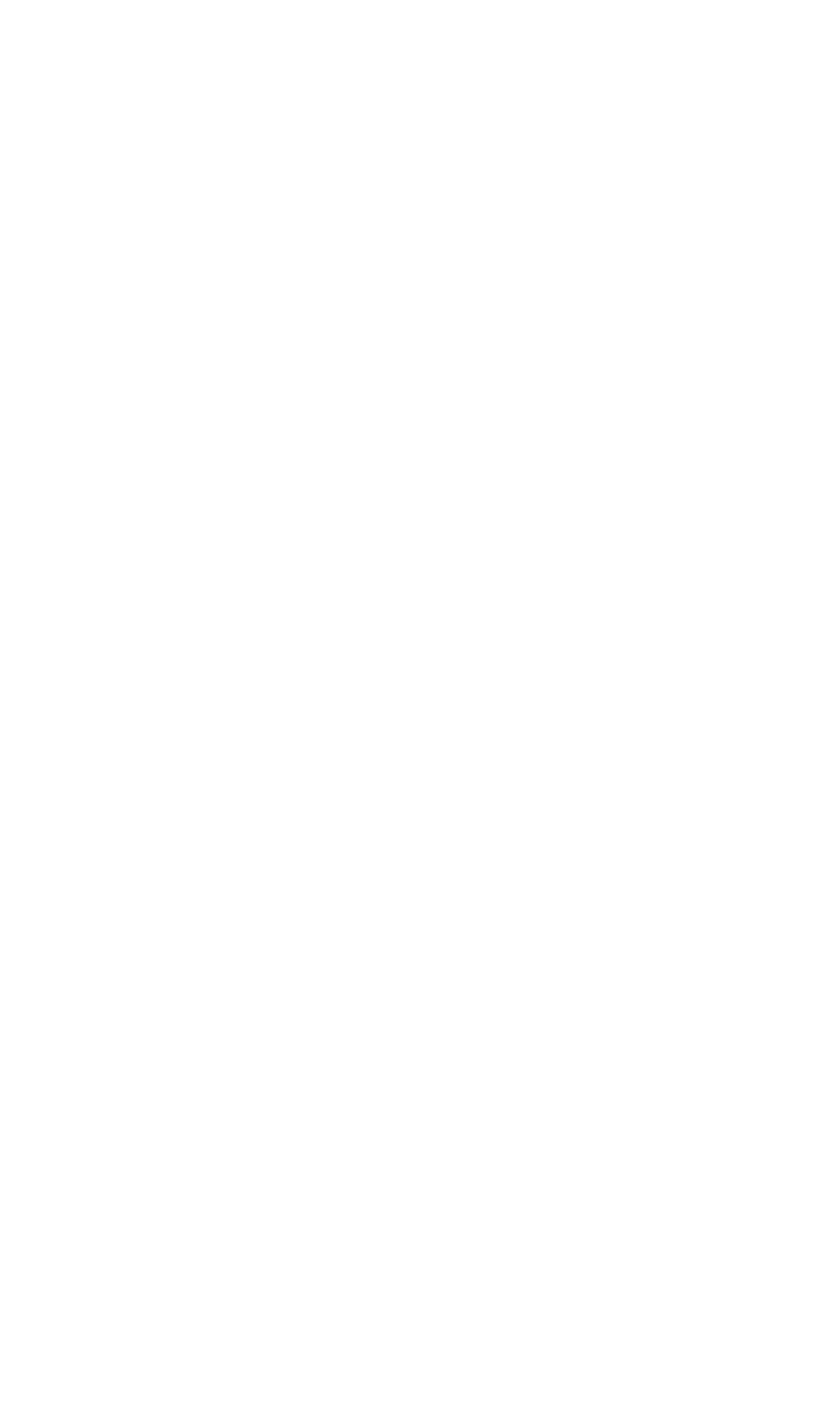


GROUND WATER OF WILLAMETTE' VALLEY, OREG.

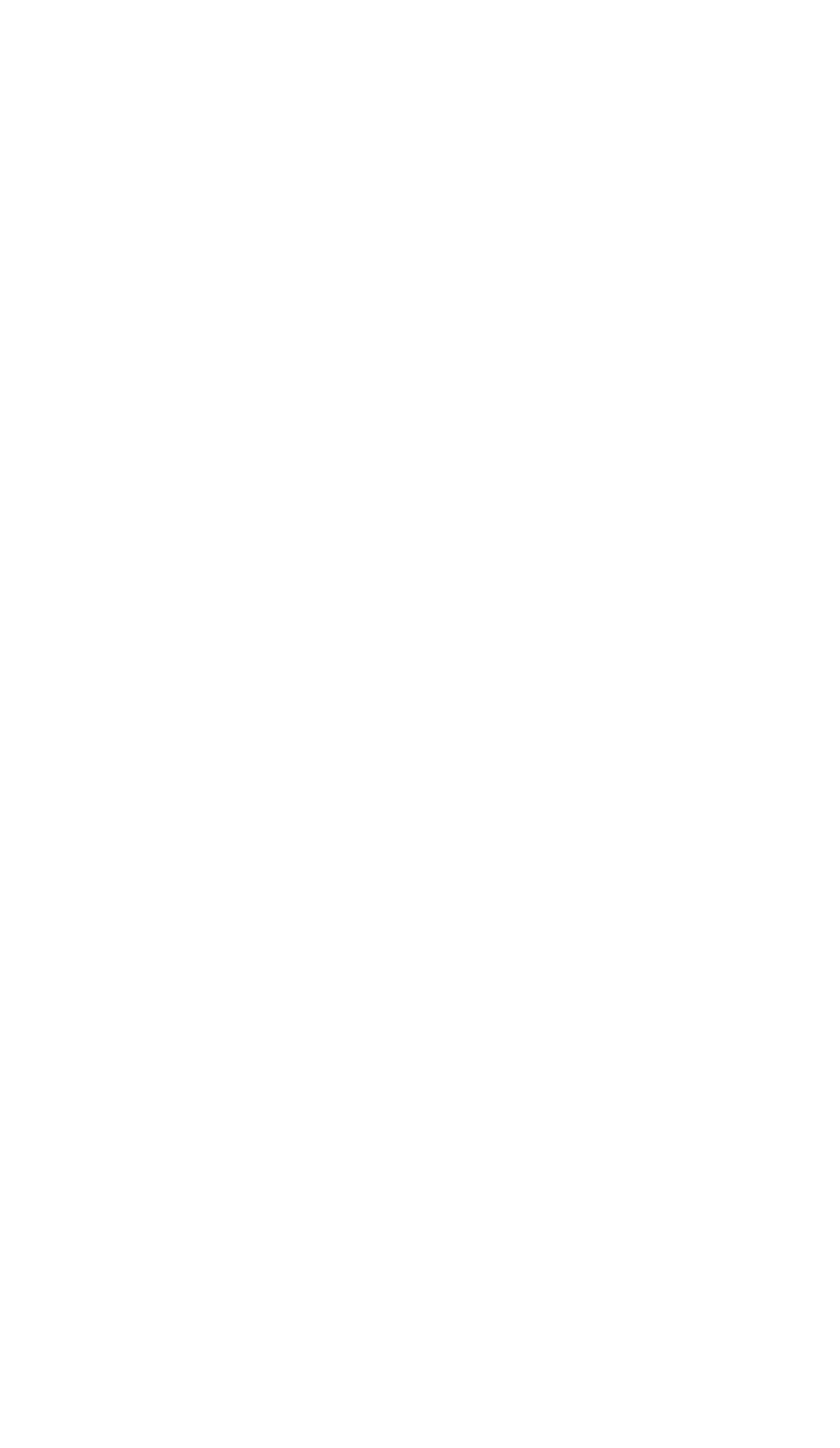


WELL RECORDS

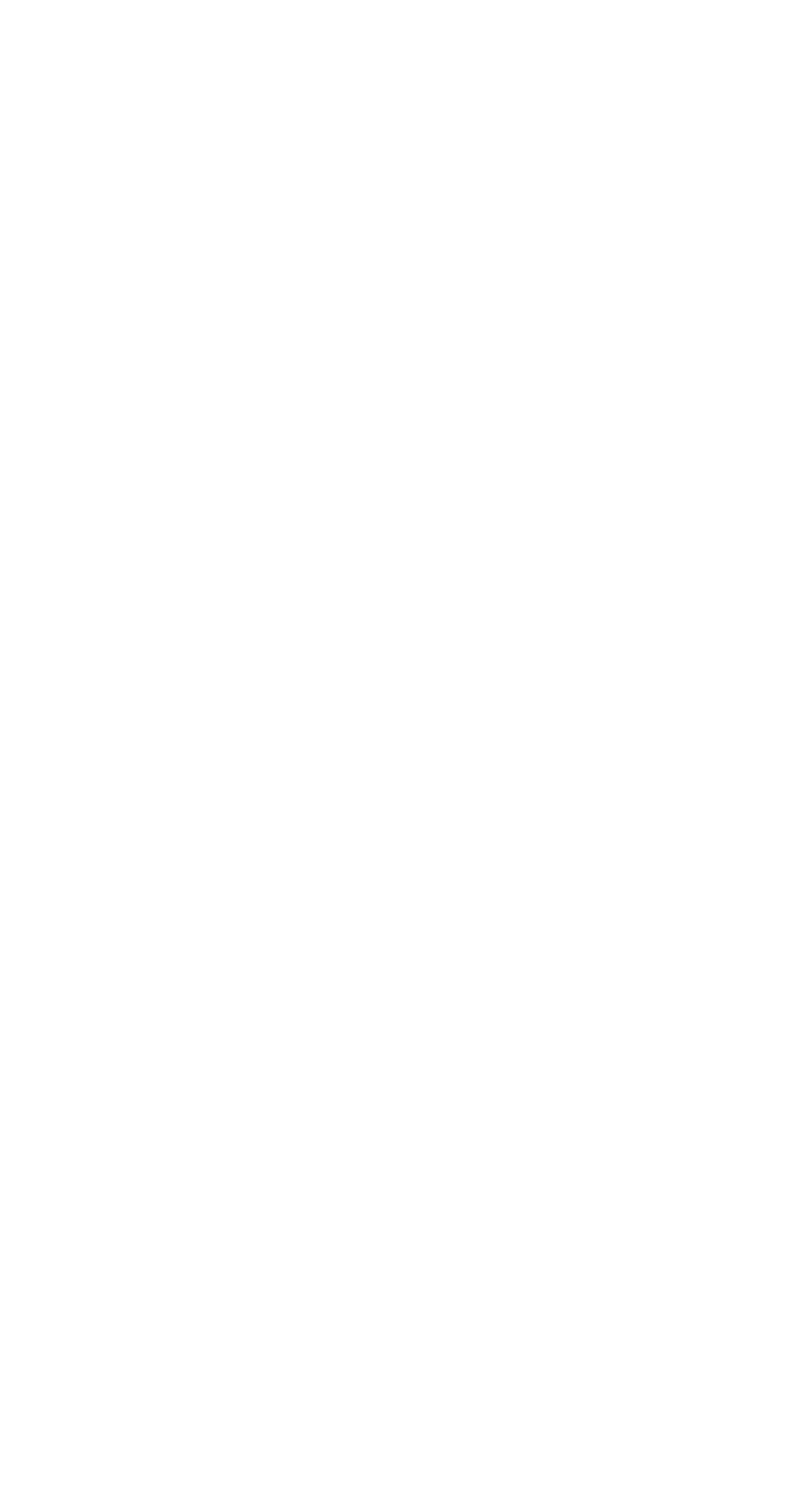




\section{TABLE 12.-Materials penetrated by typical wells}

[Based largely on drillers' records. Stratigraphic correlations, by A. M. Piper, are tentative]

\section{1. ${ }^{1}$ Scappoose Dike District}

[Drilled by O. E. Jannsen in 1928]

Clay and gravel

Sand and gravel $\ldots \ldots \ldots$

Sand

Sand and pea gravel

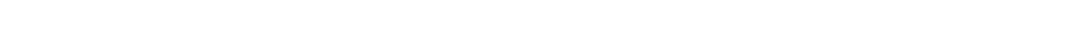

Sand and pea gravel $\ldots \ldots \ldots$ (4

Sand

Gravel, cemented................... 17

\section{Owner unknown}

[Drilled by R. J. Strasser Drilling Co. in 1931. Deepest well on Sauvie Island]

Aluvium:

Silt and sand

Thickness Depth

(feet)

Unclassified:

"Sandstone" . .

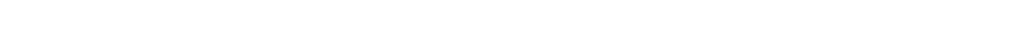

Sedimentary rocks of Tertiary age, undifferentiated:

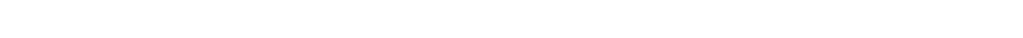

Sand, fine, lower half packed.....

Clay, variegated

Sandstone_._.

Rock, central part soft

22. Masonic and Eastern Star Home

[Drilled by A..M. Jannsen Drilling Co. in 1929]

Alluvium:

Soil, clayey _...

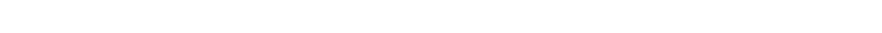

Clay, sandy

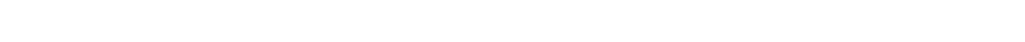

Clay, sandy

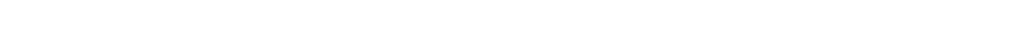

Fine gravel, water-bearing $\ldots \ldots \ldots 6$

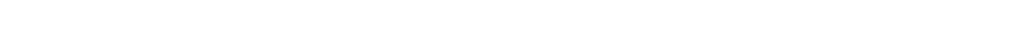

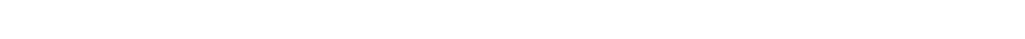

Clay

27. Terminal Ice \& Cold Storage Co.

[Drilled by O. E. Jannsen in 1914]

Alluvium:

Unknown

Gravel, loose

\section{Unclassified:}

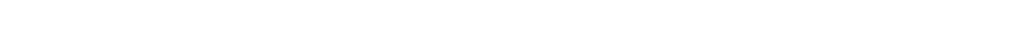

Sand and blue clay, compact._._.

1 Numbers of wells in this table correspond with the numbers used in table 11. 
TABLE 12.-Materials penetrated by typical wells-Continued

27. Terminal Ice \& Cold Storage Co.-Continued

Troutdale formation of Hodge (?):

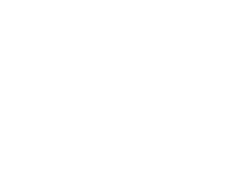

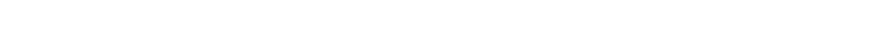

Gravel, cemented, and sand

Gravel, cemented, and clay ..................... 10

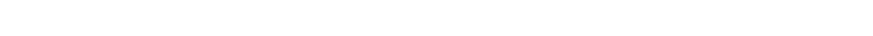

385

Yakima basalt (?):

Basalt; soft porous zones at $4161 \frac{1}{2}, 517-525$, and $540 \pm-5511 / 2$

feet; water chiefly at 478 feet but also at 465 feet ....... $166^{2} / 3$

31. Corvallis Creamery Co.

[Drilled by O. E. Jannsen Drilling Co.]

Alluvium:

\begin{tabular}{|c|c|}
\hline $\begin{array}{c}\text { Thickness } \\
\text { (feet) }\end{array}$ & $\begin{array}{c}\text { Depth } \\
\text { (feet) }\end{array}$ \\
\hline 25 & 25 \\
\hline 10 & 35 \\
\hline 19 & 54 \\
\hline 3 & 57 \\
\hline 63 & 120 \\
\hline 10 & 130 \\
\hline 30 & 160 \\
\hline 12 & 172 \\
\hline 39 & 211 \\
\hline 5 & 216 \\
\hline 38 & 254 \\
\hline 9 & 263 \\
\hline
\end{tabular}

Clay

Sand and gravel, cemented, upper part water-bearing

Gravel, cemented

Sand and gravel, water-bearing

Unclassified:

Gravel, cemented

Gravel, loosely cemented, water-bearing

Gravel, cemented, interstratified with clay . . . . . . . . . . . 30

Clay and gravel, water-bearing in part . . . . . . . . 12

"Shale," blue and brown, lower part sandy

"Shale," blue sandy; cemented sand _._.

263

Gravel, cemented

32. Loyd Corporation, Inc.

[Drilled by A. M. Jannsen Drilling Co, in 1930-31]

Alluvium:

Sand, white, packed $\ldots \ldots \ldots 77$

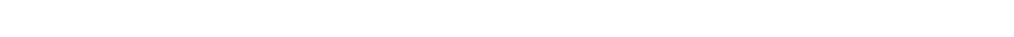

Gravel, cobbles, and boulders, cemented_............ 99145

Fine sand (?), in part clayey; cobbles in one 3-foot zone _-- $30 \quad 30175$

Clay, sandy in part

Troutdale formation of Hodge (?):

"Sandrock," soft . . .

Sand, fine; packed in lower part .

Sand .................

Clay, blue, and gravel.

Clay, blue.....

Sand, fine.

Sand and clay

Sand, fine at top, coarsens toward bottom

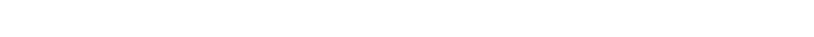

Sand _..._._.

Clay, sandy

Sand 
TABLE 12.-Materials penetrated by typical wells-Continued

33. So-called Ladd well

[Drilled about 1885 and long ago abandoned. Record from photostat copy in files of Surveys Division

Aluvium: City of Portland]

Earth, clayey sand, and "drift" sand

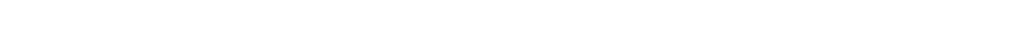

Sand, with boulders of basalt and "granite"

Boulders and gravel with layers of sand, water-bearing---- $40 \quad 40$

Gravel and sand................................. $20 \quad 200$

Boulders, cobbles, and pebbles_._.

Gravel and coarse sand, with layers of sand and "con-

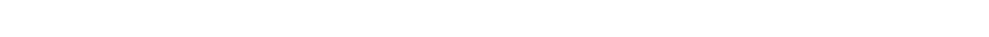

Sand and fine basaltic gravel_.._._.

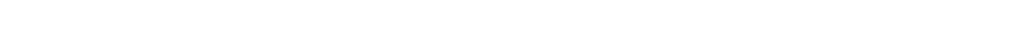

Clay, sand, and "granite" boulders in alternate layers_._- $45 \quad 45$

Troutdale formation of Hodge (?):

"Marl" and compact clay, in part sandy; fossil wood and

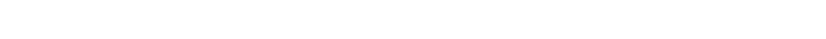

Sand, fine and coarse, with gravel_._._._._._._. $10 \quad 730$

"Marl" and compact shale, with gravel and layers of soft fine "sandstone"

Clayey shale with layers of fine sand and plentiful fossil wood and plants

Fine micaceous sand, white and compact; coarse basaltic

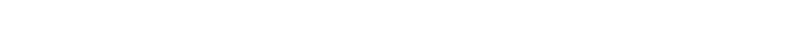

Conglomerate, shale, and "marl," with iron-stained sand and fossil plants................................. $120 \quad 1,200$

Coarse sand and gravel, basaltic $\ldots \ldots \ldots \ldots \ldots \ldots$

"Marl," fine and sandy; soft shale with iron-stained gravel and fossil plants............................. $50 \quad 1,300$

Yakima basalt (?):

"Granite" . . .

44. United States Resettlement Administration, Yamhill Farms, unit 85

Aluvium:

[Drilled by $\mathbf{P}$. Steinman in 1937]

Clay, yellow . .

Sandy clay, blue.... $77 \quad 95$

Sedimentary rocks of Tertiary age (?), undifferentiated:

Clay, alternately blue and yellow............... $138 \quad 233$

Clay, red, with fragmental rock; water-bearing......... $4 \quad 4237$

Clay, yellow and red, water-bearing

"Hardpan" _._._._._._.

"Solid rock", _... 
TABLE 12.-Materials penetrated by typical wells-Continued

53. Aaron Frank, well 2

[Drilled by A. M. Jannsen Drilling Co. in 1938. Record below 460 feet in depth from cuttings]

Unclassified:

Clay, sticky

$\begin{array}{cc}\begin{array}{c}\text { Thickness } \\ \text { (feet) }\end{array} & \begin{array}{c}\text { Depth } \\ \text { (feet) }\end{array} \\ 460 & 460 \\ 10 & 470 \\ & \\ 145 & 615 \\ 25 & 640 \\ & \\ 5 & 645 \\ 155 & 800\end{array}$

Yakima (?) basalt:

Basalt, dense, microcrystalline in part

Basalt, vesicular, slightly iron-stained in upper 3 feet.....

Sand and grit, tuffaceous (?) and iron-stained; water-

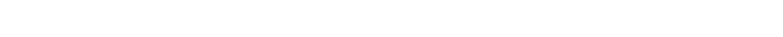

Basalt, fine-grained, dense

58. Northwestern Ice \& Cold Storage Co.

Fill and aluvium:

[Drilled by R. J. Strasser in 1928]

Fill and "muck".

Clay and "muck"

$33 \quad 33$

Clay and "muck" - .

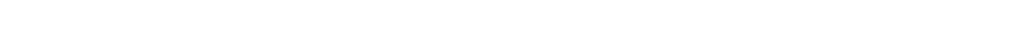

Gravel, some cobbles and boulders

Sand ...

Gravel, cemented

Troutdale formation of Hodge (?):

Gravel and "shale" .............. 15

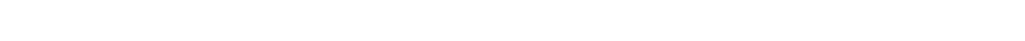

Clay, blue, with some yellow sand..._.

Sand, yellow, and clay

Sand, clean.......... $10 \quad 265$

62. Libby, McNeill \& Libby

[Drilled by R. J. Strasser in 1929. Driller's record with interpretations from representative cuttings]

Alluvium:

Fill and sandy clay

$\begin{array}{cr}\begin{array}{c}\text { Thickness } \\ \text { (feet) }\end{array} & \begin{array}{c}\text { Depth } \\ \text { (feet) }\end{array} \\ 18 & 18 \\ 7 & 25 \\ 13 & 38 \\ 24 & 62 \\ 45 & 107 \\ 3 & 110\end{array}$

Unclassified:

Sand and pebbles, largely basaltic ..................

Gravel, particles less than 2 inches in diameter.........

Clay, drab; lower part sandy ... . . . . .

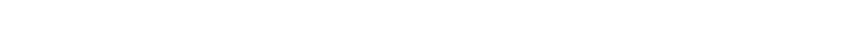

Clay, yellowish drab.

110

Gravel, cemented (tuffaceous (?) conglomerate, basaltic grit and abundant quartz grains in matrix) ............

Gravel, clay, and sand (tuffaceous (?) conglomerate, pebbles largely basaltic but some of quartzite and other rocks of distant origin; matrix contains plentiful basaltic grit, quartz grains, and some mica...........................

Troutdale formation of Hodge (?):

Clay, sandy, blue and yellow (similar to matrix of overlying conglomerate; a few pebbles, all basaltic in cuttings) ....-

Gravel, cemented, with hard sandy matrix; contains a few cobbles (pebbles basaltic; a little mica in matrix) .......-

Gravel, cemented, matrix clayey 
TABLE 12.-Materials penetrated by typical wells-Continued

\section{Rockwood Water District}

[Well 1, drilled by Ziesing]

Alluvium:

Clay $\begin{array}{cc}\text { Thickness } & \text { Depth } \\ \text { (feet) } & \text { (feet) }\end{array}$

66

Sand and gravel, water-bearing $\ldots \ldots \ldots$

Troutdale formation of Hodge (?):

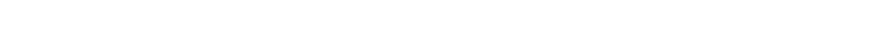

Clay, yellow, blue, and brown

75. H. A. Roberts

[Drilled by O. E. Jannsen Drilling Co. in 1928]

Alluvium:

Clay

Gravel

"Shale," clayey at top

Sand, coarse in part, water-bearing .............. 15

Clay, greenish

Troutdale formation of Hodge (?):

"Shale," sandy

Sand, cemented

Yakima (?) basalt:

Bedrock

77. A. L. Alexander

[Drilled by O. E. Jannsen Drilling Co. in 1927]

Alluvium:

Soil and clay

Gravel, cemented

Sand

Gravel, cemented, and sand in alternate layers 4 to 10 feet thick

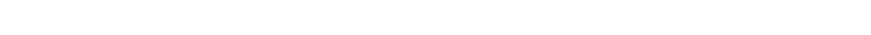

Gravel, cemented

78. F. L. Williams

[Drilled by O. E. Jannsen Drilling Co.]

Alluvium:

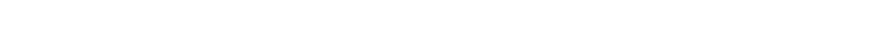

Sand and gravel

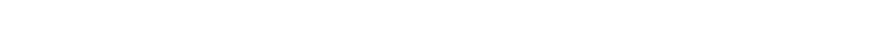

Sand

Gravel, cemented .

Troutdale formation of Hodge (?):

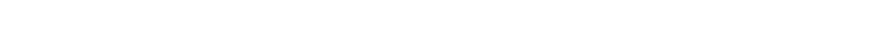

$\begin{array}{llll}\text { "Shale," brown and blue, grading to sand at bottom ..... } & 56 & 128\end{array}$

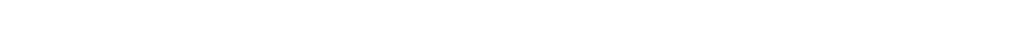

Sand, water-bearing

Sand and "shale," blue _... _._. _._.

"Shale," blue and green _... 
TABLE 12.-Materials penetrated by typical wells-Continued

79. Mrs. Beebe

[Drilled by O. E. Jannsen Drilling Co. in 1929]

Alluvium:

$\begin{array}{cc}\text { Thickness } & \text { Depth } \\ \text { (feet) } & \text { (feet) }\end{array}$

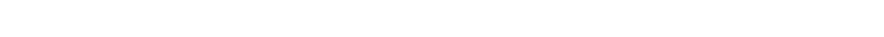

Gravel, slightly cemented; some boulders .............. 19

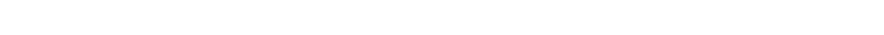

"Sandstone," yellow .

Clay, sandy .......... 10

Troutdale formation of Hodge (?):

Shale, blue, in part sandy ........ 154

Sand, water-bearing.

102. City of Canby

[Drilled by Peter Hornig in 1912]

Alluvium:

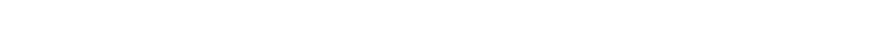

Depth (feet)

Coarse gravel and boulders, water-bearing below a depth of 40 feet. . . . . . . . . . . 62

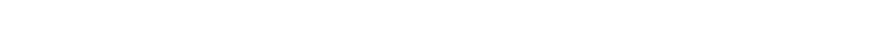

Boulders, loose . . . . . . 12

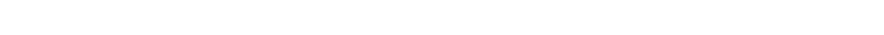

Gravel (?), loose, water-bearing .................... 2

Clay, blue, no sand or pebbles . . . .

Gravel and pebbles, loose, water-bearing ............ 2

Clay, blue.

103. City of Canby

[Drilled by Peter Hornig ln 1921]

Alluvium:

Gravel and boulders in large part .................. 87

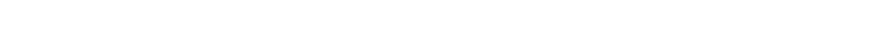

Gravel and coorse sand, water-bearing

Clay, blue, no sand or pebbles. . . .

107

Sand, fine and clean ...................................

280

Rocks of Tertiary age, undifferentiated:

Clay, red . . . . . . . . . 229

"Asphalt" .

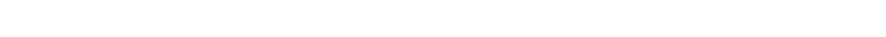

Fine sand, gray, and clay, blue, in alternate beds several feet thick; water-bearing ...................... 127

"Sandstone, gray, not water-bearing ............... 1

\section{J. C. Mattoon}

[Drilled by O. E. Jannsen Drilling Co.]

Soil and clay

ay

$8 \quad 8$

Clay, yellow . . . . .

Sandstone, yellow, and "soapstone", yellow and blue ........ 52

Sandstone, brown and yellow

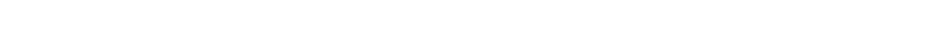

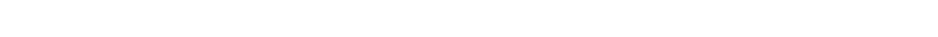

Sandstone, yellow, and shale, blue.................... 25

Sandy shale, blue and gray, water-bearing $\ldots \ldots \ldots \ldots$ 
TABLE 12.-Materials penetrated by typicals wells-Continued

118. United States Reset tlement Administration, Yamhill Farms, unit 68

[Drilled by A. M. Jannsen Drilling Co. in 1937]

Alluvium:

Soil and "hardpan"

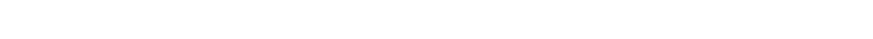

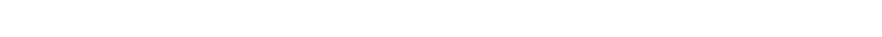

Sand, blue, and pea gravel, water-bearing ............ 2

Sand, coarse and blue, with wood and bark . . . . . . . . . 12

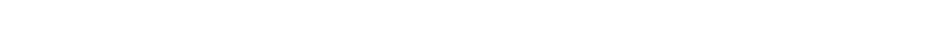

Sedimentary rocks of Tertiary age, undifferentiated:

Sandstone . . . . . . . . . . . . . . . . . . . . . . . . 6

Shale, sandy, gray, alternately hard and soft $\ldots \ldots \ldots \ldots . . .91$

$\begin{array}{cr}\begin{array}{c}\text { Thickness } \\ \text { (feet) }\end{array} & \begin{array}{r}\text { Depth } \\ \text { (feet) }\end{array} \\ 3 & 3 \\ 62 & 65 \\ 13 & 78 \\ 2 & 80 \\ 12 & 92 \\ 23 & 115 \\ & - \\ 6 & 121 \\ 91 & 212\end{array}$

139. United States Resettlement Administration, Yamhill Farms, unit 16

[Drilled by A. M. Jannsen Drilling Co. in 1937]

Alluvium:

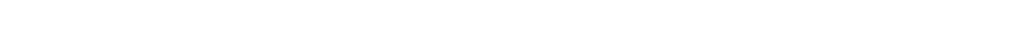

Clay, yellow . . .

Fine sand; yellow, gray, brown, and pink _........... 75

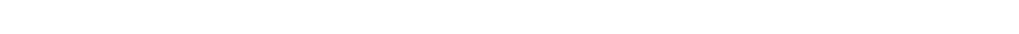

Rock, soft. . . . .

Sand and fine gravel, water-bearing ................. 1

Sedimentary rocks of Tertiary age, undifferentiated:

Shale, gray . . . .

183

143. United States Resettlement Administration, Yamhill Farms, unit 19

[Drilled by A. M. Jannsen Drilling Co. in 1937]

Alluvium:

Thickness (feet)'

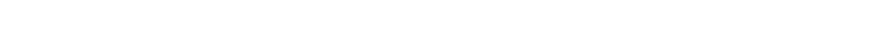

Sand, yellow and blue, hard at bottom ........... 40

Coarse sand, water-bearing . . . . .

Sand, blue................ 20

Clay, yellow, and sand . . .

Sandstone and clay . . . . . . . . . . . .

Sedimentary rocks of Tertiary age, undifferentiated:

Rock, topmost foot porous and water-bearing

153. Pleasantdale School

[Drilled by H. E. Evans]

Alluvium:

Soil and clay

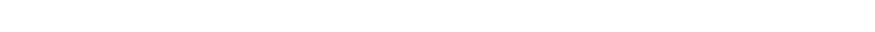

Sand, packed, not water-bearing

Clay, blue ... . . . . . . . 126

Gravel, sand, and greenish-blue clay; coarse members water-bearing

Gravel, water-bearing. 
TABLE 12.-Materials penetrated by typical wells-Continued

\section{William Gooding}

[Drilled by Peter Hornig. Record interpreted from samples of cuttings]

Alluvium:

Thickness (feet)

Soil and subsoil. 20

"Quicksand" (very fine sand and silt)

92

Sand, medium and coarse (basaltic)

Coarse sand and pebbles (basaltic)

[Drilled in 1931. Record interpreted from samples of cuttings]

Alluvium:

Soil.

Sand, dark gray (basaltic, poorly rounded grains, pebbles as much as 4 millimeters in diameter.............. 18

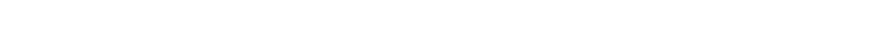

Clay, blue (fine sand or silt with basaltic grit)

Sand, blue, and gravel; coarser toward bottom (basaltic, grains poorly rounded, 1 to 20 millimeters in diameter); water-bearing

Troutdale or Rhododendron formation of Hodge, or both (?):

Clay and sandy clay, blue (nondescript mixture of particles

. from silt to coarse sand, some of quartz and mica, many waterworn; tuffaceous?) _........................... 184

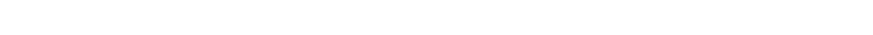

Clay, sandy, a few pebbles (from silt to medium sand; grains angular; largely basaltic though feldspar and quartz are abundant; tuffaceous?)

Sand, very fine (medium sand; grains angular to partly rounded, one-half of quartz, feldspar, muscovite, or

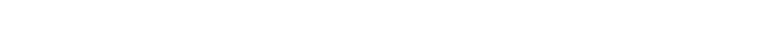

Sand, very fine, with a little clay .

Coarse sand (largest grains $2 \frac{1}{2}$ millimeters in diameter, well-rounded and assorted; include basaltic clinker, quartz, and feldspar)

Clay (silt and fine sand, nonlaminated, abundant mica, tuffaceous?) _.

Shale, soft (with fine to coarse sand, rude laminae, carbonized wood in coarser layers, moderately consolidated) ....

206. United States Resettlement Administration, Yamhill Farms, unit 6

[Drilled in 1937]

Alluvium:

Soil

Clay, yellow and blue............. 66

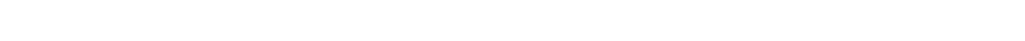

Sand, yellow, and gravel._. 38

Clay, yellow, and sand.

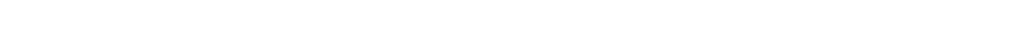

Gravel and coarse sand, water-bearing $\ldots \ldots \ldots$ 


\section{TABLE 12.-Materials penetrated by typical wells-Continued}

\section{United States Resettlement Administration, Yamhill Farms, unit 24}

[Drilled by Steinman Bros. Drilling Co. in 1937]

Alluvium:

Thickness Depth

(feet) (feet)

Soil

Clay, yellow

Silt

Clay, blue

Sand, fine, water-bearing . . . . . .

Unclassified:

Clay, blue and gray, interlaminated with silt........... 115

Clay, yellow and brown. ........................ 90

Clay, sandy, brown

Sedimentary rocks of Tertiary age:

Shale, gray, caving

\section{Agricultural Research Corporation (Sam H. Brown)}

Applied by A. M. Jannsen Drilling Co. in 1930. Record interpreted from samples of cuttings. (See physical properties of water-bearing materials, p. 31)]

Allıvium:

Thickness Depth (feet) (feet)

Loam 2

Clay, yellow and blue, with streaks of pea gravel at $18^{1 / 2}$ feet and of fine sand below 38 feet (sparse mica)

Clay grading downward into sand.

Sand and gravel, fine (unassorted fine sand to pebbles half an inch in diameter; largely basaltic though quartz and feldspar abundant); water-bearing

Clay; gray, blue, and brown; in part sandy and pebbly....

Silt, dark bluish gray (very fine sand to medium sand; quartz grains abundant)

and, fine and black

Sand and fine gravel, somewhat cemented (pebbles all from

basic volcanic rocks)

Gravel, coarse, and sand (grains fairly well rounded and somewhat assorted; pebbles an inch in diameter are abundant; largest are $2 \frac{1}{2}$ inches long); water-bearing ...

Clay, blue and pebbly

Sand, black (fine to coarse)

Clay, green and sticky ................................

Sand, black and loose (medium to very coarse, fairly well assorted; feldspar and quartz abundant) $\ldots \ldots \ldots$

Clay or shale, green, streak of peat at top_._._.

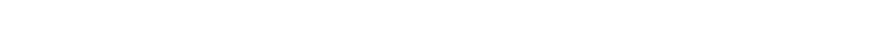

Clay, blue, watertight

Gravel and sand, streak of clay at 227 feet, slightly cemented in part (largely basaltic; above 224 feet, poorly assorted very fine sand to pebbles; moderately assorted below 230 feet); water-bearing.

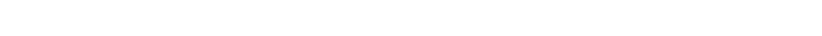


TABLE 12.-Materials penetrated by typical wells-Continued 247. City of Gervais

[Drilled by A. M. Jannsen Drilling Co. in 1928

Alluvium:

Thickness Depth $\begin{array}{ll}\text { (feet) } & \text { (feet) } \\ \text { fepth } & \\ 20 & \end{array}$

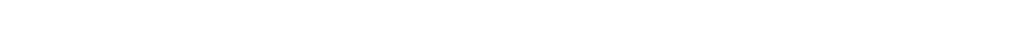

Sand, water-bearing .............................. 22

Clay, blue, and fine sand . . . . .

Sand, black, water-bearing _._.

Clay, blue and sticky _..._.

Sand, black, water-bearing _........................ 17

Gravel and coarse sand, black, water-bearing _......... 6

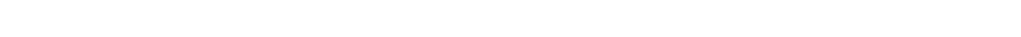

256. Oregon State Training School

[Drilled by George E. Scott in 1924]

Alluvium:

Thickness Depth (feet) (feet)

Clav . . . . . .

Sand, fine, dark, gravelly in part _............... $93 \quad 116$

Gravel, water-bearing

Sand, dark

Clay, blue .................................... 11

Sand, dark ...

Gravel, "dirty" .

Gravel, water-bearing $\ldots \ldots 15$

Clay, blue...... 15

262. City of Woodburn

[Drilled by George E. Scott in 1925]

Alluvium:

Clay

Sand, fine, water-bearing $\ldots \ldots \ldots$

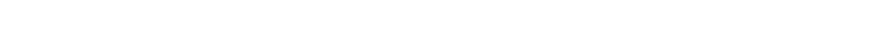

Gravel, coarse, and sand.

Clay, blue and soft

Gravel, coarse, and sand; water-bearing

Clay, blue ......... 44185

263. Southern Pacific Railroad Co., Woodburn station

[Drilled by George E. Scott]

Alluvium:

Clay, yellow $\ldots \ldots$

Sand, black $\ldots \ldots \ldots 51$

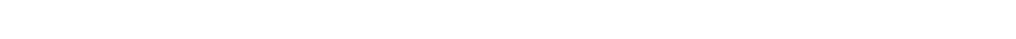

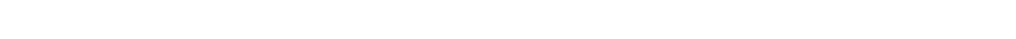

Clay, blue, and sand _._.

Gravel, coarse, black, water-bearing $\ldots \ldots \ldots \ldots$ 
TABLE 12.-Materials penetrated by typical wells-Continued

280. Eastern-Western Lumber Co.

[Drilled by O. E. Jannsen Drilling Co. in 1927]

Pediment deposit:

Thickness Depth

(feet)

Boulders........ 15

$15 \quad 15$

Clay _. 20

Clay, sandy _... 25

Troutdale or Rhododendron formation of Hodge, or both (?):

Clay, blue........... 100

"Shale"

Sand, water-bearing

"Shale"

"Sandstone," streak of "shale" at 265 feet, another of cemented gravel at bottom; thin water-bearing zones at

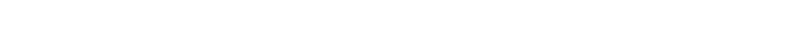

"Shale",

Sand

3321/2. Public Works Engineering Corporation

[Record interpreted [ rom samples of cuttings]

Younger and older alluvium:

Sand, gravel, and pebbles as much as $1 \frac{1}{2}$ inches in diameter; lower half free from fine and medium sand; water-bearing below depth of 21 feet

Coarse sand and pebbles, cemented with fine sand and silt; not water-bearing.

Coarse sand to pebbles half an inch in diameter, loose, waterbearing

Illahe formation of Thayer (?):

Sandstone, fine-grained and earthy, weathered yellowish drab; largely of quartz grains but some basaltic grit; yields a little water

Clay "gumbo," blue (with fine sand, largely quartz but some basaltic grit, sparse mica, tuffaceous ?); not water-bearingClay shale, blue and sandy (unassorted and unstratified, grain sizes from fine to coarse sand, tuffaceous (?)); yields a little water from several zones...................

Blue shale (with mixture of fine to medium sand and some grit, moderately indurated, basaltic grains and quartz grains about equally abundant, tuffaceous ?); not waterbearing 
TABLE 12.-Materials penetrated by typical wells-Continued 333. Oregon Linen Mills, Inc.

[Drilled by Harvey Evans]

Older alluvium:

Thickness Depth (feet) (feet)

Soil and clay

Clay and boulder "hardpan"............

Sand, brown and packed, not water-bearing _._._. $30 \quad 38$

Sand and gravel, water-bearing

Clay and gravel "hardpan"

Sand and gravel, yields a little water._._.

Unclassified:

Gravel, cemented.

Sand and gravel, packed.

Yakima basalt (?):

"Medium blue stone," water-bearing _...... $601 / 2$

$248 \frac{1}{2}$

334. Public Works Engineering Corporation

[Drilled by Ferris \& Gardner. Record interpreted from samples of cuttings]

Older alluvium:

Thickness (feet)

Clay, water at base 20

Pebbly clay (basaltic pebbles) 3

Gravel, loose (basaltic, coarse sand to pebbles an inch in diameter), water-bearing ..... 5

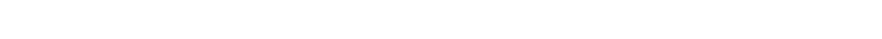

Gravel, coarse (basaltic pebbles, fine matrix in several zones),

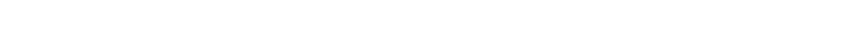

Unclassified:

Clay, gravel, and boulders (bloulder clay ?); not water-bear-

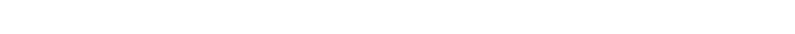

Gravel_...

Clay, gravel, and boulders (samples show pronounced iron stain below 230 feet)

Yakima (?) basalt:

Rock (weathered basalt)

336. A. W. Stryker

Alluvium:

[Drilled by West in 1929]

Thickness Depth

$45 \quad 45$

Soil and clay, blue in lower part.

Sand and gravel, water-bearing

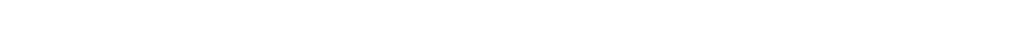

Sand, loose, and gravel; water-bearing

Gravel, cemented with clay _..._.

Gravel, loose, water-bearing .... 
TABLF 12.-Materials penetrated by typical wells—Continued

338. Oregon State Hospital, well 3

[Drilled by O. E. Jannsen Drilling Co. in 1925]

Alluvium:

Clay and sand .................................. 35

$35 \quad 35$

Gravel, cemented.................................... 22

Gravel and cobbles, some clay in lower half; not water-

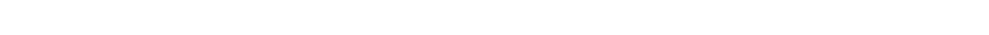

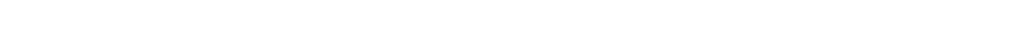

Gravel and sand, with clay matrix._._._._.......... 21

Gravel and sand, water-bearing._.................. $16 \quad 111$

Clay and gravel ..................................... 11/2 1121/2

3381/2. Oregon State Penitentiary

[Drilled by A. M. Jannsen Drilling Co.]

Alluvium:

Soil and clay

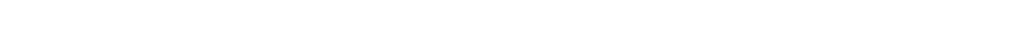

Sand and gravel, yields a little water.... $\ldots \ldots \ldots \ldots \ldots 26$

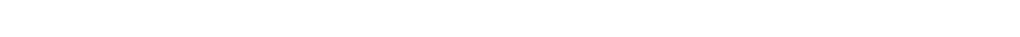

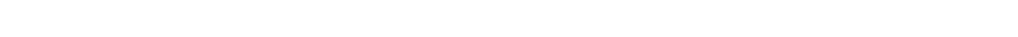

Gravel, cemented $\ldots \ldots \ldots$

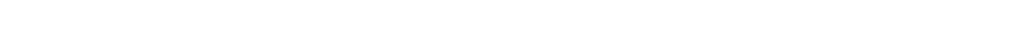

Gravel, water-bearing

Gravel, cemented................................ $20 \quad 122$

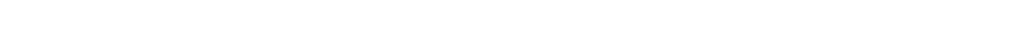

Clay, yellow and red ... $\ldots \ldots \ldots \ldots$

Rocks of Tertiary age, undifferentiated:

Bedrock ................................... 1160

385. Cottage Farm, Oregon State Hospital

[Drilled by O. E. Jannsen Drilling Co. in 1925]

Unclassified:

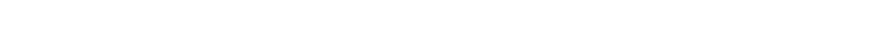

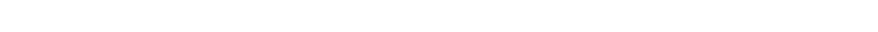

Illahe formation of Thayer:

Shale, blue and gray $\ldots \ldots \ldots 5$

Shale and sandstone, interbedded . . . . . . . . . . . 121

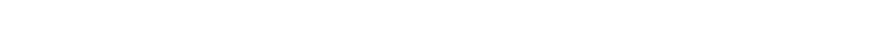

Sandstone, yields a little water at about 450 feet_....... $190 \quad 515$

Shale, with a few thin layers of sandstone............ $490 \quad 1,005$

435. Conser

Alluvium:

[Drilled by C. E. Gordonier]

Soil

Clay, yellow . . . . .

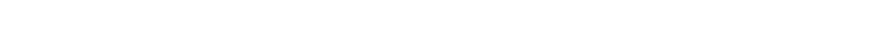

$7-32$

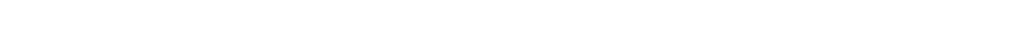

Sedimentary rocks of Tertiary age, undifferentiated:

Sandstone, blue . . .

Sandstone, yields "salt" water................... 191

Sandstone, blue; water of poor quality at base $\ldots \ldots \ldots \ldots 69$ 
TABLE 12.-Materials penetrated by typical wells-Continued

526. Bingenheimer

Alluvium:

[Drilled by H. E. Evans]

Soil

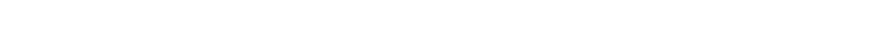

Thickness Depth

$3 \quad 3$

Gravel (?), water-bearing $\ldots \ldots \ldots$

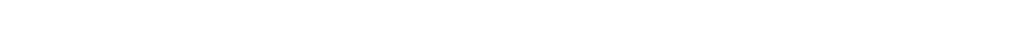

Rocks of Tertiary age, undifferentiated:

Clay, blue and red

Coarse gravel and cobbles, no sand; water-bearing _...... $17 \quad 193$

\section{Junction City}

Alluvium:

[Drilled in 1938]

\begin{tabular}{|c|c|}
\hline $\begin{array}{c}\text { Thickness } \\
\text { (feet) }\end{array}$ & $\begin{array}{c}\text { Depth } \\
\text { (feet) }\end{array}$ \\
\hline 2 & 2 \\
\hline 6 & 8 \\
\hline 7 & 15 \\
\hline 16 & 31 \\
\hline 7 & 38 \\
\hline 22 & 60 \\
\hline 8 & 68 \\
\hline 132 & 200 \\
\hline 33 & 233 \\
\hline 47 & 280 \\
\hline 70 & 350 \\
\hline 17 & 367 \\
\hline 63 & 430 \\
\hline 10 & 440 \\
\hline 10 & 450 \\
\hline
\end{tabular}

Soil $\ldots \ldots \ldots 2$

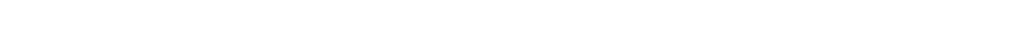

Clay and gravel $\ldots$

Gravel and sand, clayey at top and bottom, water-bearing - $16 \quad 31$

Clay, brown and blue .......................... 78

Sand, coarse, and soft brown clay, water-bearing _....... $22 \quad 60$

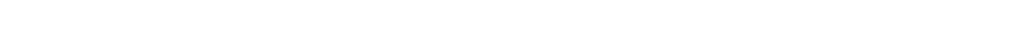

Sand, fine gravel, and blue-gray clay, water-bearing _..... $132 \quad 200$

Sedimentary rocks of Tertiary age, undifferentiated:

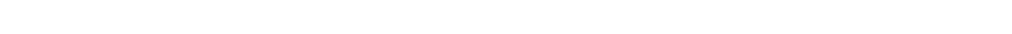

Shale, blue-gray and brown $\ldots \ldots \ldots$

Sandstone, blue-gray, soft; yields a little water _......... $70 \quad 350$

"Cement gravel" (agglomerate or conglomerate ?) _....... $17 \quad 367$

Shale, brownish-gray, lower part sandy _._._._._._._. $63 \quad 430$

"Conglomerate," very light gray, hard ............. $10 \quad 440$

Shale, brownish-gray .................. 10

701. Bethel School

Alluvium:

[Drilled by A. F. Moody]

Thickness Depth

Soil_...

Gravel.

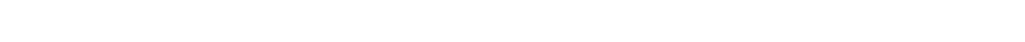

Sand and fine gravel._._.

Gravel, cemented:-...

Sand

Gravel, cemented; water-bearing ................. 2 106

722. M. E. Furrow

[Drilled by A. F. Moody]

Rocks of Tertiary age, undifferentiated:

Basalt.

"Sandrock," brown, very hard $\ldots$

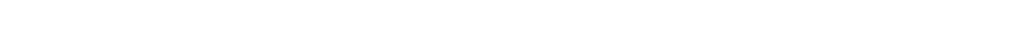

Rock, white and very soft (pumice ?) _._._._._._. $25 \quad 347$

Sandstone, water-bearing . 


\section{TABLE 13.-Ground-water levels in observation wells, 1928-30 and 1935-96 1}

87. Frank H. Newland. SW1/4SE1// sec. 32, T. 3 S., R. 4 W. Domestic well, dug $31 / 2$ feet in diameter and 39 feet deep, brick curb. Measuring points: Top of pump-house sill, 157.88 feet above mean sea level; and top of concrete wall, 0.7 foot above land surface and 157.98 feet ${ }^{2}$ above sea level.

\begin{tabular}{|c|c|c|c|c|c|c|c|}
\hline Date & $\begin{array}{l}\text { Water } \\
\text { level } \\
\text { (feet) }\end{array}$ & Date & $\begin{array}{l}\text { Water } \\
\text { level } \\
\text { (feet) }\end{array}$ & Date & $\begin{array}{l}\text { Water } \\
\text { level } \\
\text { (feet) }\end{array}$ & Date & $\begin{array}{l}\text { Water } \\
\text { level } \\
\text { (feet) }\end{array}$ \\
\hline 1928 & & 1936 & & 1936 & & 1936 & \\
\hline Sept. 25 & 138.5 & Mar. 30 & 152.42 & June 1 . & 148. 94 & Aug. 3. & 143.89 \\
\hline 1936 & & $\begin{array}{l}\text { Apr. } 6 \\
\text { Apr. } 13\end{array}$ & $\begin{array}{l}150.86 \\
149.43\end{array}$ & June 15 & $\begin{array}{l}147.81 \\
148.26\end{array}$ & $\begin{array}{l}\text { Aug. 10. } \\
\text { Aug. } 17 .-\end{array}$ & $\begin{array}{l}142.07 \\
141.92\end{array}$ \\
\hline & & Apr. 20 & 149.39 & June 22 & 148. 21 & Aug. 24 & 141.71 \\
\hline Feb. 19 & 150.68 & Apr. 27.- & 149.70 & June $29 \ldots$ & 146.70 & Aug. 31 & 141.69 \\
\hline Feb. 24 & 153.51 & May 4 & 149.79 & July 6 . & & Sept. 8 & 139.67 \\
\hline $\begin{array}{l}\text { Mar. } 2 \\
\text { Mar. } 9\end{array}$ & 153.59 & May 11 & 149.44 & July 13 & 145. 66 & Sept. 14 & $\begin{array}{l}140.46 \\
137.88\end{array}$ \\
\hline Mar. 16 & $\begin{array}{l}150.90 \\
150.77\end{array}$ & May 25 & $\begin{array}{l}149.41 \\
148.92\end{array}$ & $\begin{array}{l}\text { July } 20 \ldots \\
\text { July } 27 \ldots\end{array}$ & $\begin{array}{l}144.60 \\
144.39\end{array}$ & Sept. 28 & $\begin{array}{l}101.00 \\
137.62\end{array}$ \\
\hline Mar. 23 & 150.93 & & & & & & \\
\hline
\end{tabular}

1 Ground-water levels in 1928-30, by U. S. Geological Survey; in 1935-36, by U. S. Engineer Department.

2 Leveling by U. S. Engineer Department.

100. Pietro Presutti. SW1/4NW1/4 sec. 30, T. 2 S., R. 1 E. Domestic well, drilled 6 inches in diameter and 145 feet deep, standard steel casing. Substitute for well 101, which was destroyed between 1930 and 1935. Measuring point is bottom of plugged hole in base of pump, 0.4 foot above land surface and 159.28 feet ${ }^{2}$ above mean sea level.

\begin{tabular}{|c|c|c|c|c|c|c|c|}
\hline Date & $\begin{array}{l}\text { Water } \\
\text { level } \\
\text { (feet) }\end{array}$ & Date & $\begin{array}{l}\text { Water } \\
\text { level } \\
\text { (feet) }\end{array}$ & Date & $\begin{array}{l}\text { Water } \\
\text { level } \\
\text { (feet) }\end{array}$ & Date & $\begin{array}{l}\text { Water } \\
\text { level } \\
\text { (feet) }\end{array}$ \\
\hline 1935 & & 1936 & & 1936 & & 1936 & \\
\hline $\begin{array}{l}\text { Oct. } 18 \\
\text { Oct. } 23 \\
\text { Oct. } 28 \\
\text { Nov. } 4 \\
\text { Nov. } 11 \\
\text { Nov. } 18 \\
\text { Dec. } 2 \\
\text { Dec. } 9 \\
\text { Dec. } 16 \\
\text { Dec. } 23 \\
\text { Dec. } 30 \\
\quad 1936 \\
\text { Jan. } 6 \\
\text { Jan. } 13\end{array}$ & \begin{tabular}{|l}
110.20 \\
110.28 \\
109.54 \\
110.19 \\
110.60 \\
110.58 \\
109.60 \\
109.79 \\
106.09 \\
110.68 \\
110.93 \\
\\
\\
113.28 \\
3154.44
\end{tabular} & $\begin{array}{l}\text { Jan. 20 } \\
\text { Jan. 27 } \\
\text { Feb. } 3 \\
\text { Feb. 10 } \\
\text { Feb. 17 } \\
\text { Feb. 24 } \\
\text { Mar. 2 } \\
\text { Mar. } 9 \\
\text { Mar. 16 } \\
\text { Mar. 23 } \\
\text { Mar. 30 } \\
\text { Apr. } 6 \\
\text { A pr. } 13 \\
\end{array}$ & $\begin{array}{r}3155.11 \\
108.93 \\
109.07 \\
109.13 \\
109.13 \\
109.63 \\
109.96 \\
108.83 \\
109.13 \\
109.00 \\
108.96 \\
109.01 \\
109.56\end{array}$ & $\begin{array}{l}\text { Apr. 21 } \\
\text { Apr. 27 } \\
\text { May 4 } \\
\text { May 11 } \\
\text { May 18 } \\
\text { May 25 } \\
\text { June 1. } \\
\text { June } 8 \\
\text { June 15 } \\
\text { June 22 } \\
\text { June 29 } \\
\text { July } 8\end{array}$ & $\begin{array}{r}109.90 \\
108.38 \\
108.06 \\
109.12 \\
4104.71 \\
108.72 \\
107.51 \\
108.46 \\
108.46 \\
108.15 \\
107.64 \\
107.81\end{array}$ & \begin{tabular}{|} 
July 13 \\
July 20 \\
July 28 \\
Aug. 3 \\
Aug. 10 \\
Aug. 17. \\
Aug. 24 \\
Aug. 31 \\
Sept. 8 \\
Sept. 14 \\
Sept. 21 \\
Sept. 28 \\
\end{tabular} & $\begin{array}{l}107.99 \\
107.73 \\
107.21 \\
108.14 \\
108.18 \\
107.67 \\
107.70 \\
107.53 \\
102.39 \\
106.08 \\
106.00 \\
105.81\end{array}$ \\
\hline
\end{tabular}

2 Leveling by U. S. Engineer Department.

3 Water probably entering well above static level.

4 Water level depressed by inordinately large withdrawals.

101. Isaac A. Miley estate. NW1/4SW1/4 sec. 30, T. 3 S., R. 1 E. Domestic well, bored 10 inches in diameter and 57 feet deep. Measuring point is top of casing, 0.4 foot above land surface and 157.5 feet above sea level (altimeter).

\begin{tabular}{|c|c|c|c|c|c|c|c|}
\hline Date & $\begin{array}{l}\text { Water } \\
\text { level } \\
\text { (feet) }\end{array}$ & Date & $\begin{array}{l}\text { Water } \\
\text { level } \\
\text { (feet) }\end{array}$ & Date & $\begin{array}{l}\text { Water } \\
\text { level } \\
\text { (feet) }\end{array}$ & Date & $\begin{array}{l}\text { Water } \\
\text { level } \\
\text { (feet) }\end{array}$ \\
\hline 1928 & & 1929 & & 1929 & & 1930 & \\
\hline $\begin{array}{l}\text { Sept. } 27 \\
\text { Oct. } 10 \\
\text { Dec. } 17\end{array}$ & $\begin{array}{l}108.2 \\
106.3 \\
126.7\end{array}$ & $\begin{array}{l}\text { Feb. } 3 \\
\text { Mar. } 23 \\
\text { May } 12 \\
\text { June } 7 \\
\end{array}$ & $\begin{array}{l}133.4 \\
132.45 \\
120.85 \\
107.10\end{array}$ & $\begin{array}{l}\text { July } 3 . \\
\text { Aug. } 5 \\
\text { Sept. } 24 \\
\text { Oct. } 27 \\
\text { Nov. } 29 \\
\end{array}$ & $\begin{array}{l}105.90 \\
105.45 \\
102.05 \\
106.65 \\
106.45\end{array}$ & $\begin{array}{l}\text { Feb. } 2 \\
\text { Mar. } 2 \\
\text { Mar. } 30 \\
\text { Apr. 27 } \\
\text { July } 22 \\
\end{array}$ & $\begin{array}{l}106.70 \\
105.60 \\
105.50 \\
105.25 \\
103.22\end{array}$ \\
\hline
\end{tabular}


TABLE 13.-Ground-water levels in observation wells, 1928-30 and 1985-36-Con.

126. O. B. Belieu. NW1/4NE1/4sec. 12, T. $4 \mathrm{~S}$., R. 4 W. Domestic and stock well, dug 4 feet in diameter and 27.7 feet deep, brick curb. Measuring point is top of 3 -inch plank deck, through bored hole 10 inches southeast of pump, 170.14 feet 2 above mean sea level.

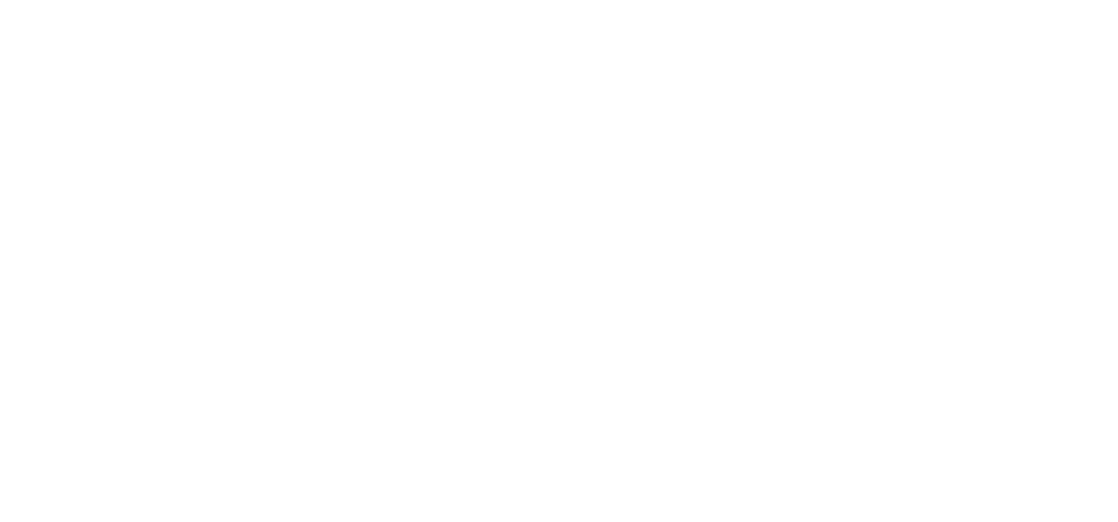

2 Leveling by U. S. Engineer Department.

5 Pump operating in well.

133. J. J. Kneeland, renter; Fred Allison, owner (formerly owned by A. J. Mott). SW1/4SW1/4 sec. 24, T. 4 S., R. 4 W. Domestic and stock well, bored 12 inches in diameter and 41 feet deep, in shed at northeast corner of residence. Copper nail with washer stamped 4-4-5 in wood pump base Measuring point is top of concrete-tile casing, 0.4 foot above land surface and 152 feet above mean sea level (hand level).

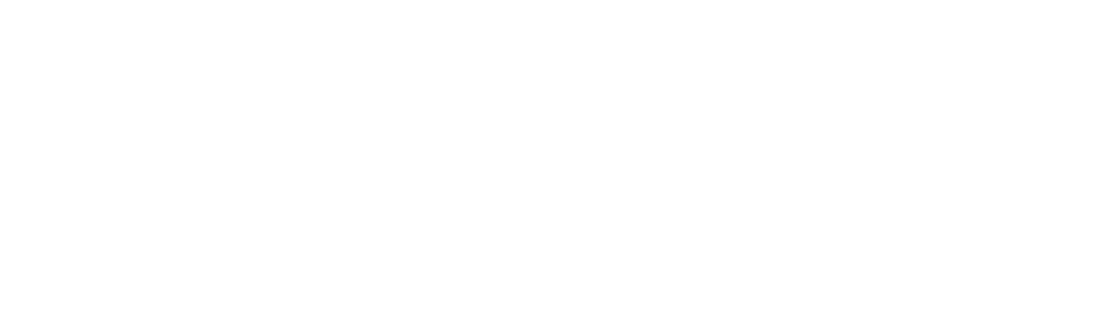

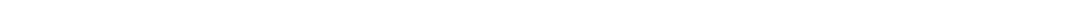
27 feet deep, concrete-tile casing. Substitute for well 133, which was inaccessible for water-level measurements in 1935-36. Measuring point is top of concrete collar of casing, 2.0 feet above land surface and 157.69 feet ${ }^{2}$ above mean sea level.

\begin{tabular}{|c|c|c|c|c|c|c|c|}
\hline Date & $\begin{array}{l}\text { Water } \\
\text { level } \\
\text { (feet) }\end{array}$ & Date & $\begin{array}{l}\text { Water } \\
\text { level } \\
\text { (feet) }\end{array}$ & Date & $\begin{array}{l}\text { Water } \\
\text { level } \\
\text { (feet) }\end{array}$ & Date & $\begin{array}{l}\text { Water } \\
\text { level } \\
\text { (feet) }\end{array}$ \\
\hline 1936 & & 1936 & & 1936 & & 1936 & \\
\hline $\begin{array}{l}\text { Feb. } 3 \\
\text { Feb. } 10 \\
\text { Feb. } 19 \\
\text { Feb. } 24 \\
\text { Mar. } 2 \\
\text { Mar. } 9 \\
\text { Mar. } 16 \\
\text { Mar. } 23 \\
\text { Mar. } 30\end{array}$ & $\begin{array}{l}152.52 \\
152.61 \\
152.56 \\
154.05 \\
154.50 \\
153.11 \\
153.01 \\
152.87 \\
153.96\end{array}$ & $\begin{array}{l}\text { Apr. } 6 \\
\text { Apr. } 13 \\
\text { Apr. } 20 \\
\text { Apr. } 27 \\
\text { May } 4 \\
\text { May } 11 \\
\text { May 18 } \\
\text { May } 25 \\
\text { June } 1\end{array}$ & $\begin{array}{l}153.14 \\
152.80 \\
152.71 \\
151.64 \\
151.00 \\
150.83 \\
150.51 \\
150.15 \\
150.10\end{array}$ & $\begin{array}{l}\text { June } 8 \\
\text { June } 15 \\
\text { June } 22 \\
\text { June } 29 \\
\text { July } 6 \\
\text { July } 13 \\
\text { July } 20 \\
\text { July } 27 \\
\text { Aug. } 3\end{array}$ & $\begin{array}{l}148.60 \\
148.28 \\
147.69 \\
147.50 \\
147.43 \\
145.37 \\
145.12 \\
145.03 \\
144.62\end{array}$ & $\begin{array}{l}\text { Aug. } 10 \\
\text { Aug. } 17 \\
\text { Aug. } 24 \\
\text { Aug. } 31 \\
\text { Sept. } 8 \\
\text { Sept. } 14 \\
\text { Sept. } 21 \\
\text { Sept. } 28 \\
\end{array}$ & $\begin{array}{l}142.65 \\
141.80 \\
141.47 \\
141.28 \\
140.66 \\
140.43 \\
139.99 \\
138.77\end{array}$ \\
\hline
\end{tabular}

2 Leveling by U. S. Engineer Department. 
TABLE 13.-Ground-water levels in observation wells, 1928-30 and 1935-36-Con.

136. W. J. Odell. SW $\frac{1}{4} \mathrm{SE} \frac{1}{4}$ sec. 29, T, 4 S., R. 4 W. Domestic and stock well, dug 4 feet in diameter and 30.6 feet deep, brick curb. Measuring point is top of brick curb, 0.6 foot above land surface and 141.88 feet ${ }^{2}$ above mean sea level.

\begin{tabular}{|c|c|c|c|c|c|c|c|}
\hline Date & $\begin{array}{l}\text { Water } \\
\text { level } \\
\text { (feet) }\end{array}$ & Date & $\begin{array}{l}\text { Water } \\
\text { level } \\
\text { (feet) }\end{array}$ & Date & $\begin{array}{l}\text { Water } \\
\text { level } \\
\text { (feet) }\end{array}$ & Date & $\begin{array}{c}\text { Water } \\
\text { level } \\
\text { (feet) }\end{array}$ \\
\hline 1935 & & 1936 & & 1936 & & 1936 & \\
\hline Oct. 30 & 119.95 & Jan. 13 & 139.61 & Apr. 14 & 133.13 & July 14 & 125.49 \\
\hline Nov. 4 & 120.43 & Jan. 18 & 138.51 & Apr. 21 & 133.11 & July 21 & 123.85 \\
\hline Nov. 11 & 114.63 & Jan. 27 & 137.03 & Apr. 28 & 129.77 & July 28 & 123.47 \\
\hline Nov. 18 & 121.02 & Feb. 3 & 134,75 & May 5 & 128.80 & Aug. 4 & 122.95 \\
\hline Nov. 26 & 120,84 & Feb. 10 & 135.12 & May 12 & 129. 19 & Aug. 11 & 4116.96 \\
\hline Dec. 2 . & 121.03 & Feb. 19 & 135. 06 & May 19 & 123.27 & Aug. 18 & 4116.57 \\
\hline Dec. 9 - & 121.44 & Feb. 24 & 137. 90 & May 26 & 129.71 & Aug. 25 & 4115.46 \\
\hline Dec. 16 & 121.47 & Mar. 2 & 137.61 & June 1 & 129.84 & Sept. 1 . & 4115.56 \\
\hline Dec. 23 & 121.45 & Mar. 9 & 135,45 & June 8 & 129.57 & Sept. 9 & 119.81 \\
\hline Dec. 30 & 131.55 & Mar. $17_{-}$ & 134. 30 & June 16 & 129. 19 & Sept. 15 & 118. 36 \\
\hline 1936 & & Mar. 24 & 133.62 & June 23 & 128.44 & Sept, 22 & 120.79 \\
\hline 1936 & & Mar. 31 & 136.19 & June 30 & 127.45 & Sept. 29 & 119.52 \\
\hline Jan. 6 & 130.85 & Apr. 7 & 136.40 & July 7 & 126.00 & & \\
\hline
\end{tabular}

${ }^{2}$ Leveling by U. S. Engineer Department.

W' Water level depressed by inordinately large withdrawals.

147. W. E. Grabenhorst. NW 1 NE⿺ sec. 28, T. 4 N., R. 3 W. Domestic and stock well, dug $31 / 2$ fee in diameter and 23 feet deep, brick curb. Measuring points: Top of 2-inch plank deck, through bored hole 10 inches north of pump, 1.2 teet above land surface and 163.94 feet ${ }^{2}$ above mean sea level; and beginning July $6,1936,0.6$ foot above plank deck.

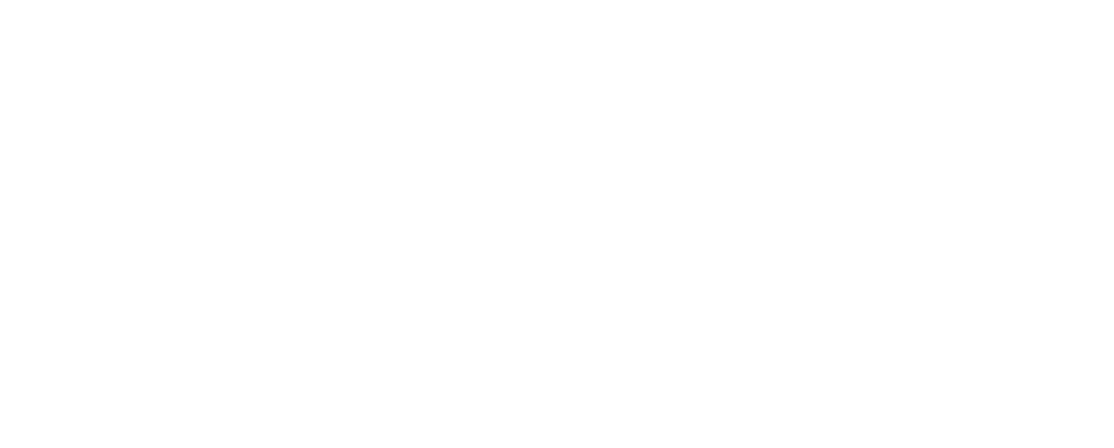

2 Leveling by U. S. Engineer Department.

158. W. J. Gering, formerly Basil Gering. NE $\frac{1}{4} \mathrm{NW}_{\frac{1}{4}}$ sec. 4, T. 4 S., R. 2 W. Domestic and stock well, dug 30 inches in diameter and 23 feet deep, 30 feet southwest of tank tower. Measuring point is top of concrete-tile casing, east side, 1.8 feet above land surface and 125.37 feet ${ }^{2}$ above mean sea level.

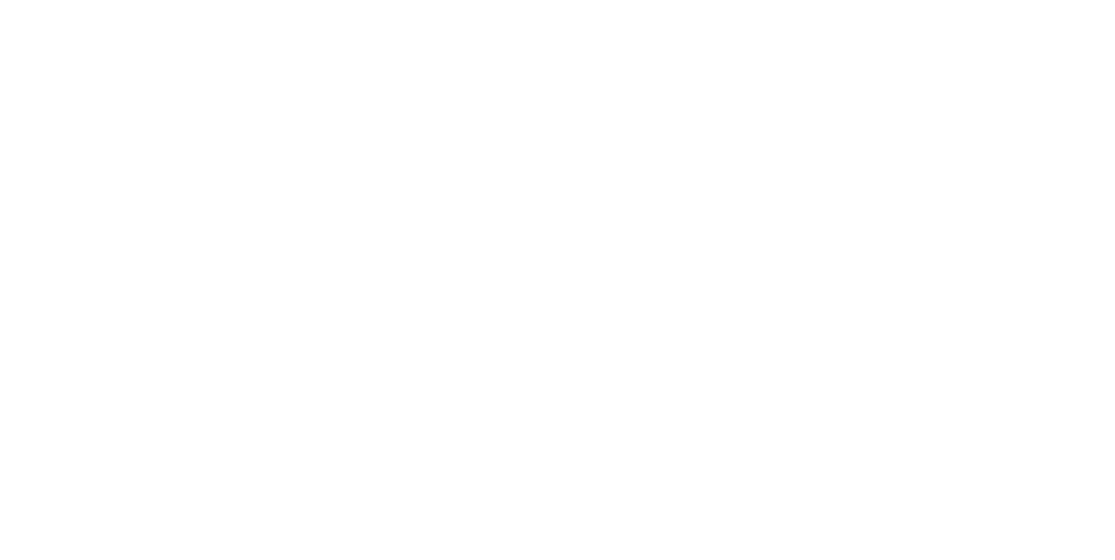

\footnotetext{
2 Leveling by U. S. Engineer Department.
} 
TABLE 13.-Ground-water levels in observation wells, 1928-30 and 1935-36-Con.

161. Clara E. Case. SE $\frac{1}{4} \mathrm{NW}_{\frac{1}{4}}$ sec. 13, T. 4 S., R. 2 W. Domestic well, dug 28 feet deep, brick curb. Measuring point is pump-house floor at south edge of trap door, about 2 feet above land surface and 177.76 feet ${ }^{2}$ above mean sea level.

\begin{tabular}{|c|c|c|c|c|c|c|c|}
\hline Date & $\begin{array}{l}\text { Water } \\
\text { level } \\
\text { (feet) }\end{array}$ & Date & $\begin{array}{l}\text { Water } \\
\text { level } \\
\text { (feet) }\end{array}$ & Date & $\begin{array}{l}\text { Water } \\
\text { level } \\
\text { (feet) }\end{array}$ & Date & $\begin{array}{l}\text { Water } \\
\text { level } \\
\text { (feet) }\end{array}$ \\
\hline 1935 & & 1936 & & 1936 & & 1036 & \\
\hline Oet. 9 & 156.30 & Jan. 6 & 154. 71 & Apr. 6 . & 160.80 & July 6 & 156.93 \\
\hline Oct. 15 & 156. 21 & Jan. 13 & 157.59 & Apr. 13 & 160.84 & July 13 & 156.79 \\
\hline Oct. 23 . & 156.11 & Jan. 20 & 158. 49 & Apr. 20 & 160.79 & July 20 & 157.32 \\
\hline Oct. 28 & 156. 28 & Jan. 27 & 157.78 & Apr. 27 & 159.47 & July 27 . & 157.04 \\
\hline Nov. 4 & 155.07 & Feb. 3 & 157.74 & May 4 & 159.24 & Aug. 3 & 156.88 \\
\hline Nov. 11 & 155.22 & Feb. 10 & 157.18 & May 11 & 159.23 & Aug. 10 & 156.79 \\
\hline Nov. 18 & 154.74 & Feb. 19 & 161.82 & May 18 & 159. 10 & Aug. 17- & 156.37 \\
\hline Dec. 3 & 155. 07 & Feb. 24 & 159.66 & May 25 & 159.06 & Aug. 24 & 156. 28 \\
\hline Dec. 9. & 154.09 & Mar. 2 & 160.17 & June 1 . & 158.81 & Aug. 31 & 156.05 \\
\hline Dec. 16 & 155,12 & Mar. 9 & 160.26 & June 8 & 158. 64 & Sept. 8 & 155.83 \\
\hline Dec. 23 & 154.08 & Mar. 16 & 159. 9 & June 15 & 158. 37 & Sept. 14 & 156.77 \\
\hline Dec. 30 . & 153.67 & Mar. 30 & $\begin{array}{l}100.12 \\
161.36\end{array}$ & June 29 & $\begin{array}{l}100.10 \\
157.85\end{array}$ & Sept. 28 & 158.00 \\
\hline
\end{tabular}

${ }^{2}$ Leveling by U. S. Engineer Department.

164. Wm. Gooding. SW $1 \mathrm{NE}_{\frac{1}{2}} \mathrm{sec}, 20, \mathrm{~T}, 4 \mathrm{~S}, \mathrm{R}, 2 \mathrm{~W}$. Domestic and stock well, drilled 6 inches in diameter and 147 feet deep, 75 feet northeast of dwelling. Casing stamped 4-2-1 on east side. Measuring point is top of casing, 0.8 foot above land surface and 171 feet above mean sea level (altimeter). On September 26,1928 , the water level was 151.9 feet; on October $20,1928,151.2$ feet; and on December 17, 1928, 152 feet.

165. Ann M. Kaufman. NWisE: sec. 21, T. 4 S., R. 2 W. Domestic well, dug 29 feet deep, brick curb. Measuring point is top of 2 -inch plank deck, about 0.8 foot above land surface and 170.72 feet ${ }^{2}$ above mean sea level.

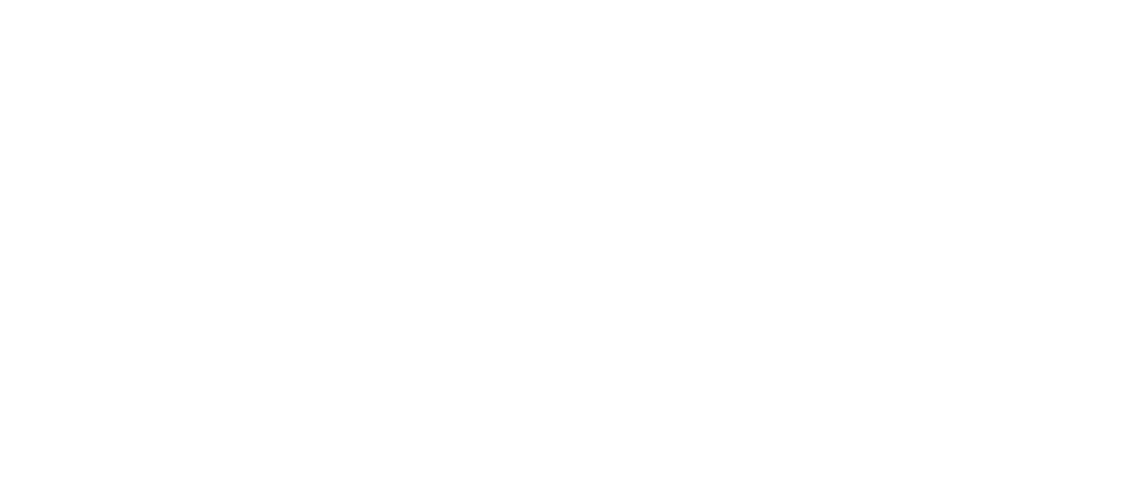

Leveling by U. S. Engineer Department. 
TABLE 13.-Ground-water levels in observation wells, 1928-30 and 1935-36-Con.

169. Theodore Boutin. NW1/4SW1/4 sec. 31, T. 4 S., R. 2 W. Domestic and stock well, drilled 10 inches in diameter and 74 feet deep, in pump shed 60 feet southwest of deep petroleum test well. Measuring point is top of 10-inch steel casing, level with land surface and 171.08 feet 2 above mean sea level.

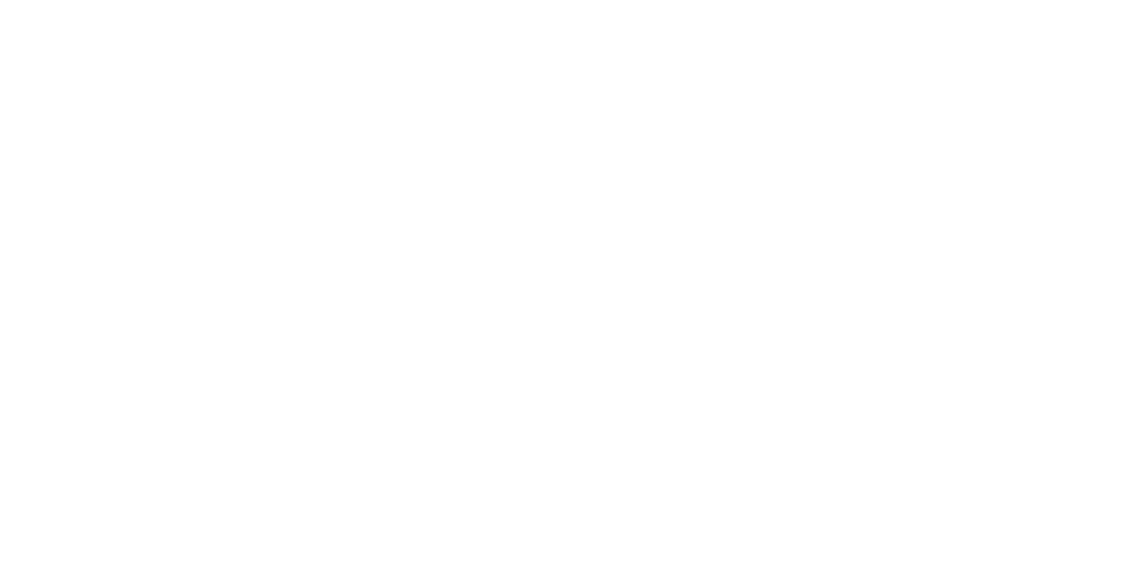

- 2 Leveling by U. S. Engineer Department.

- Pump operating in well a short time prior to measurement.

171. Johnson School. SE1/4SE1/4 sec. 34, T. 4 S., R. 2 W. Domestic well, dug 18 inches in diameter and 20 feet deep, in school yard. Copper nail with washer stamped 4-2-7 in west side of plank well cover. Measuring point is top of concrete-tile casing at copper nail with washer, 1.8 feet above land surface and 184.66 feet? above mean sea level.

\begin{tabular}{|c|c|c|c|c|c|c|c|}
\hline Date & $\begin{array}{l}\text { Water } \\
\text { level } \\
\text { (feet) }\end{array}$ & Date & $\begin{array}{l}\text { Water } \\
\text { level } \\
\text { (feet) }\end{array}$ & Date & $\begin{array}{l}\text { Water } \\
\text { level } \\
\text { (feet) }\end{array}$ & Date & $\begin{array}{l}\text { Water } \\
\text { level } \\
\text { (feet) }\end{array}$ \\
\hline 1928 & & 1930 & & 1936 & & 1936 & \\
\hline Sept. 26 & 156.8 & Mar. 2 & 171.21 & $\operatorname{Jan} 6$ & 158.06 & May 25 & 164.59 \\
\hline Oct. 20 . & 156.2 & Mar. 30 & 169. 91 & Jan. 13 & 172. 06 & June 1 & 163. 66 \\
\hline Dec. 17 & 159. 4 & A pr. 27 & 167.11 & Jan. 20 & $\begin{array}{l}171.90 \\
171.98\end{array}$ & June 8 & 162.84 \\
\hline 1929 & & July 22 . & 159.35 & Jan. 27... & $\begin{array}{l}171.01 \\
171.13\end{array}$ & June 15 & 161.93 \\
\hline & & 1935 & $\begin{array}{l}155.29 \\
150\end{array}$ & Feb. 10 & 169. 77 & June 29 & 160.73 \\
\hline Feb. 3 & 171.8 & & 155. 31 & Feb. 19 & 169. 21 & July 6 . & 159.75 \\
\hline Mar. 23 & 169. 21 & Oct. 3 . & 155. 39 & Feb. 24 & 171. 90 & Jnly 13. & 159. 35 \\
\hline May 12 & 168. 26 & Oct. 10 & 155.09 & Mar. 2 & 171. 29 & July 20 . & 158. 77 \\
\hline June 7 & 164. 56 & Oet. 15 & 154.86 & Mar. 9 & 170.48 & Suly 27 - & 158.59 \\
\hline July 3 & 161.1 & Oct 23 & & Mar 16 & 170.15 & Ang 3 & 157.94 \\
\hline Aug. 5 . & 158.11 & Oct. 28 & & Mar. 23 & 169.73 & Aug 10 & 157.80 \\
\hline Sept. 24 & 156. 36 & Nov. 4 & & Mar. 30 & 171. 25 & Aug. 17 & 157. 32 \\
\hline Oct. 27 . & 155.71 & Nov. 11 & 155.01 & Apr. 6 & 170.48 & Ang. 24 . & 157.14 \\
\hline Nov. 29 & 155.11 & Now, 18 & 155. 09 & Apr. 13. & 169.60 & Aug. 31 . & 156.83 \\
\hline Dec. 30 . & 162.66 & Dec. 2 & 155.44 & Apr. 20 & 168.23 & Sept. 8 & $\begin{array}{l}156.62 \\
\text {. }\end{array}$ \\
\hline & & Dec. 9 & 155. 05 & Apr. 27 & 167.72 & Sept. 14 & 156.80 \\
\hline 1930 & & Dec. 16 & 155.99 & May 4 & 166. 29 & Sept. 21 & 156.43 \\
\hline & & Dec. 23 & 156.42 & May 11 & 166.02 & Sept. 28 & 156.25 \\
\hline Feb. 2 & 171.26 & Dec. 30 & 159.05 & May 18 & 166.00 & & \\
\hline
\end{tabular}

Leveling by U. s. Engineer Department. 
TABLE 13.-Ground-water levels in observation wells, 1928-30 and 1935-36-Con.

172. J. W. Keil (formerly D. A. Keil). NE1/4NW1/4 sec. 2, T. 4 S., R. 1 W. Domestic well, bored 10 inches in diameter and 25.5 feet deep, at rear porch at northeast corner of dwelling, concrete-tile and stovepipe casings. Stamped 4-1-2 on pump discharge pipe just below spigot. Measuring points: Prior to May 1929, top of stovepipe casing, 0.7 foot above land surface and 187.4 feet above mean sea level; June 7 and July 3, 1939, top of casing (cut-oft), 187.3 feet above mean sea level; beginning Aug. 5, 1929, top of pumpbase flange marked with white paint, 1.0 foot above land surface and 187.69 feet $^{2}$ above mean sea level.

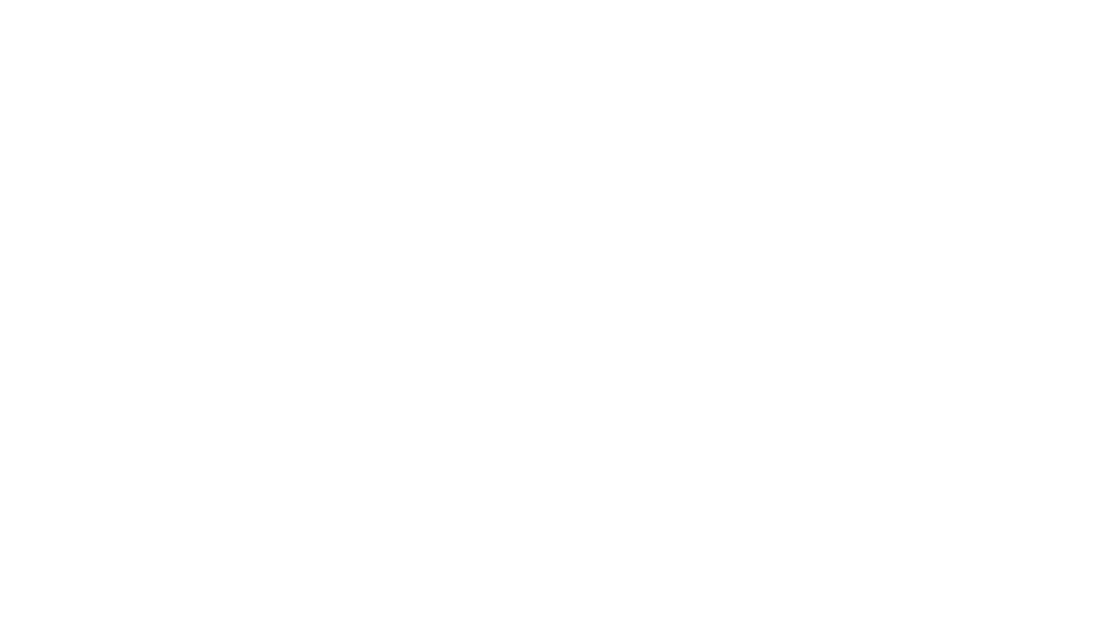

Leveling by U. S. Engineer Department.

174. A. W. Geisey. NE 34 SW1/4 sec. 9, T. 4 S., R. 1 W. Stock well, dug 7 feet in diameter and 25 feet deep, concrete curb. Measuring point is top of plank deck, through crack, 1.0 foot above land surface and 191.21 feet ${ }^{2}$ above mean sea level.

\begin{tabular}{|c|c|c|c|c|c|c|c|}
\hline Date & $\begin{array}{l}\text { Water } \\
\text { level } \\
\text { (feet) }\end{array}$ & Date & $\begin{array}{l}\text { Water } \\
\text { level } \\
\text { (feet) }\end{array}$ & Date & $\begin{array}{l}\text { Water } \\
\text { level } \\
\text { (feet) }\end{array}$ & Date & $\begin{array}{l}\text { Water } \\
\text { level } \\
\text { (feet) }\end{array}$ \\
\hline 1935 & & 1936 & & 1936 & & 1936 & \\
\hline $\begin{array}{l}\text { Oct. } 9 \\
\text { Oct. } 15 \\
\text { Oct. } 23 \\
\text { Oct. } 28 \\
\text { Nov. } 4 \\
\text { Nov. } 11 \\
\text { Nov. } 18 \\
\text { Dec. } 3 \\
\text { Dec. } 9 \\
\text { Dec. } 16 \\
\text { Dec. } 23 \\
\text { Dec. } 30\end{array}$ & $\begin{array}{l}174.31 \\
171.74 \\
175.63 \\
174.12 \\
173.78 \\
173.94 \\
173.76 \\
173.61 \\
174.03 \\
176.51 \\
176.26 \\
181.71\end{array}$ & $\begin{array}{l}\text { Jan. } 6 \\
\text { Jan. } 13 \\
\text { Jan. } 20 \\
\text { Jan. } 27 \\
\text { Feb. } 3 \\
\text { Feb. } 10 \\
\text { Feb. } 17 \\
\text { Feb. } 24 \\
\text { Mar. } 2 \\
\text { Mar. } 9 \\
\text { Mar. } 16 \\
\text { Mar. } 23 \\
\text { Mar. } 30\end{array}$ & $\begin{array}{l}188.63 \\
188.82 \\
189.72 \\
187.52 \\
187.09 \\
186.14 \\
185.86 \\
188.36 \\
187.93 \\
188.05 \\
186.79 \\
186.56 \\
188.09\end{array}$ & $\begin{array}{l}\text { Apr. } 6 \\
\text { Apr. 13 } \\
\text { Apr. 20 } \\
\text { Apr. } 27 \\
\text { May } 4 \\
\text { May 11 } \\
\text { May 18 } \\
\text { May } 25 \\
\text { June } 1 \\
\text { June } 8 \\
\text { June } 15 \\
\text { June } 22 \\
\text { June } 29 \\
\text { Jun }\end{array}$ & $\begin{array}{l}187.96 \\
187.67 \\
185.51 \\
184.93 \\
184.54 \\
183.95 \\
182.78 \\
183.00 \\
181.89 \\
181.19 \\
180.13 \\
178.97 \\
178.17\end{array}$ & $\begin{array}{l}\text { July } 8 \\
\text { July 13 } 13 \\
\text { July 20 } 28 \\
\text { July } 28 \\
\text { Aug. } 3 \\
\text { Aug. 10 } \\
\text { Aug. 17 } \\
\text { Aug. 24 } \\
\text { Aug. 31. } \\
\text { Sept. 8 } \\
\text { Sept. 21 } \\
\text { Sept. 28 } \\
\end{array}$ & $\begin{array}{l}177.23 \\
176.23 \\
176.69 \\
176.23 \\
176.13 \\
176.24 \\
175.26 \\
174.48 \\
175.29 \\
175.09 \\
174.96 \\
174.82\end{array}$ \\
\hline
\end{tabular}

${ }^{2}$ Leveling by U. S. Engineer Department. 
TABLE 13.-Ground-water levels in observation: wells, 1928-30 and 1935-36-Con.

184. Harry Cochran. SW1/4SE1/4 sec. 4, T. 4 S. R. 1. E. Domestic well, dug 4 feet square and 67 feet deep, timber curb. Measuring point is top of plank curb. at middle of west side, 1.5 feet above land surface and 177.80 feet $^{2}$ above mean sea level.

\begin{tabular}{|c|c|c|c|c|c|c|c|}
\hline Date & $\begin{array}{l}\text { Water } \\
\text { level } \\
\text { (feet) }\end{array}$ & Date & $\begin{array}{l}\text { Water } \\
\text { level } \\
\text { (feet) }\end{array}$ & Date & $\begin{array}{l}\text { Water } \\
\text { level } \\
\text { (feet) }\end{array}$ & Date & $\begin{array}{l}\text { Water } \\
\text { level } \\
\text { (feet) }\end{array}$ \\
\hline 1935 & & 1936 & & 1936 & & 1936 & \\
\hline $\begin{array}{l}\text { Oct. } 9 \\
\text { Oct. } 15 \\
\text { Oct. } 23 \\
\text { Oct. } 28 \\
\text { Nov. } 4 \\
\text { Nov. } 11 \\
\text { Nov. } 18 \\
\text { Dec. } 2 \\
\text { Dec. } 9 \\
\text { Dec. } 16 \\
\text { Dec. } 23 \\
\text { Dec. } 30\end{array}$ & $\begin{array}{l}112.14 \\
112.00 \\
112.82 \\
112.85 \\
111.35 \\
111.30 \\
111.25 \\
111.00 \\
111.10 \\
111.10 \\
111.30 \\
111.30\end{array}$ & $\begin{array}{l}\text { Jan. } 6 \\
\text { Jan. } 13 \\
\text { Jan. } 20 \\
\text { Jan. } 27 \\
\text { Feb. } 3 \\
\text { Feb. } 10 \\
\text { Feb. } 17 \\
\text { Feb. } 24 \\
\text { Mar. } 2 \\
\text { Mar. } 9 \\
\text { Mar. } 16 \\
\text { Mar. } 23 \\
\text { Mar. } 30\end{array}$ & $\begin{array}{l}111.30 \\
111.30 \\
113.30 \\
114.55 \\
114.98 \\
115.15 \\
115.50 \\
115.95 \\
115.51 \\
115.55 \\
116.06 \\
115.77 \\
116.07\end{array}$ & $\begin{array}{l}\text { Apr. 6. } \\
\text { Apr. } 13 \\
\text { Apr. 20 } \\
\text { Apr. 27 } \\
\text { May } 4 \\
\text { May } 11 \\
\text { May } 18 \\
\text { May } 25 \\
\text { June } 1 \\
\text { June } 22 \\
\text { June } 29 \\
\text { July } 8\end{array}$ & $\begin{array}{l}116.67 \\
116.82 \\
115.69 \\
115.48 \\
115.43 \\
114.18 \\
115.05 \\
114.85 \\
114.60 \\
113.95 \\
113.65 \\
113.52\end{array}$ & $\begin{array}{l}\text { July } 13 \\
\text { July } 20 \\
\text { July } 28 \\
\text { Aug. } 3 \\
\text { Aug. 10 } \\
\text { Aug. 17 } \\
\text { Aug. } 24 \\
\text { Aug. } 31 \\
\text { Sept. } 8 \\
\text { Sept. 14 } \\
\text { Sept. 21 } \\
\text { Sept. 28. }\end{array}$ & $\begin{array}{l}113.40 \\
112.58 \\
112.48 \\
112.38 \\
112.13 \\
111.93 \\
111.81 \\
111.76 \\
111.61 \\
111.38 \\
111.33 \\
111.28\end{array}$ \\
\hline
\end{tabular}

${ }^{2}$ Lereling by U. S. Engineer Department.

193. Roy Wisecarver. SW 14 NE1/4 sec. 1 , T. 5 S., R. 5 W. Domestic and stock well, dug 4 feet in diameter and 39 feet deep, brick curb. Measuring point is concrete floor of pump house, 0.3 foot above land sirface and 148.43 feet ${ }^{2}$ above mean sea level.

\begin{tabular}{|c|c|c|c|c|c|c|c|}
\hline Date & $\begin{array}{l}\text { Water } \\
\text { level } \\
\text { (feet) }\end{array}$ & Date & $\begin{array}{l}\text { Water } \\
\text { level } \\
\text { (feet) }\end{array}$ & Date & $\begin{array}{l}\text { Water } \\
\text { level } \\
\text { (feet) }\end{array}$ & Date & $\begin{array}{l}\text { Water } \\
\text { level } \\
\text { (feet) }\end{array}$ \\
\hline 1936 & & 1936 & & 1936 & & 1936 & \\
\hline $\begin{array}{l}\text { Mar. 2 } \\
\text { Mar. } 9 \\
\text { Mar. 16 } \\
\text { Mar. 23 } \\
\text { Mar. } 30 \\
\text { Apr. } 6 \\
\text { Apr. } 13 \\
\text { Apr. } 20\end{array}$ & $\begin{array}{l}146.06 \\
144.31 \\
144.06 \\
142.50 \\
144.67 \\
143.02 \\
142.97 \\
140.03\end{array}$ & $\begin{array}{l}\text { Apr. 24 } \\
\text { Apr. } 27 \\
\text { May } 4 \\
\text { May } 11 \\
\text { May } 18 \\
\text { May } 25 \\
\text { June } 1 \\
\text { June } 8\end{array}$ & $\begin{array}{l}139.93 \\
139.00 \\
137.71 \\
135.82 \\
136.43 \\
136.58 \\
136.71 \\
135.49\end{array}$ & $\begin{array}{l}\text { June } 15 \\
\text { June } 22 \\
\text { June } 29 \\
\text { July } 6 \\
\text { July } 13 \\
\text { July } 20 \\
\text { July } 27 \\
\text { Aug. } 3\end{array}$ & $\begin{array}{l}135.09 \\
134.59 \\
134.16 \\
134.10 \\
133.12 \\
131.94 \\
131.86 \\
130.62\end{array}$ & $\begin{array}{l}\text { Aug. 10 } \\
\text { Aug. 17 } \\
\text { Aug. 24 } \\
\text { Aug. } 31 \\
\text { Sept. } 8 \\
\text { Sept. } 14 \\
\text { Sept. } 21 \\
\text { Sept. } 28\end{array}$ & $\begin{array}{r}130.11 \\
129.84 \\
129.70 \\
129.15 \\
+126.43 \\
126.92 \\
125.27 \\
125.57\end{array}$ \\
\hline
\end{tabular}

${ }^{2}$ Leveling by U. S. Engineer Department.

4 Water level depressed by inordinately large withdrawals.

196. George Fuller. NW1/4NE1/4 sec. 13, T. 5 S., R. 5. W. Domestic and stock well, drilled 6 inches in diameter and 64 feet deep, in pump house 45 feet northwest of residence. Copper nail with washer stamped 5-5-2 in wood clamp that supports suction pipe. Measuring point is top of casing, 0.5 foot above land surface and 151.59 feet ${ }^{2}$ above mean sea level.

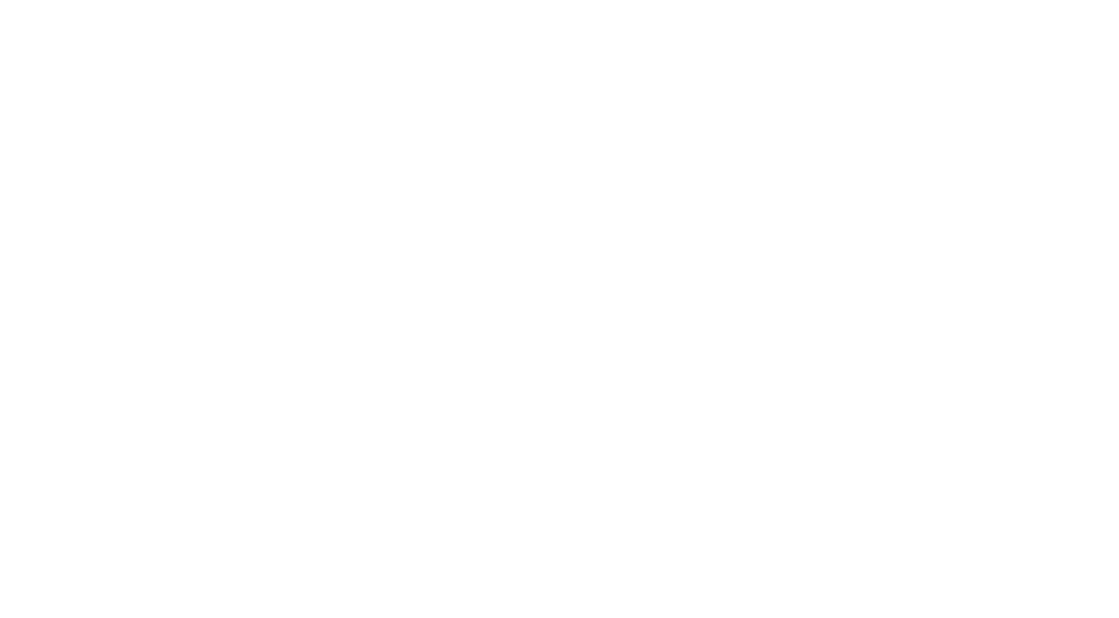

${ }^{2}$ Leveling by U. S. Engineer Department.

4 Water level depressed by inordinately large withdrawals.

$\checkmark$ Pump operating in well. 
TABLE 13.-Ground-water levels in observation wells, 1928-30 and 1935-36-Con.

203. William Piller. NE1/4NE1/4 sec. 32, T. 5 S., R. 5 W. Stock well, dug $41 / 2$ feet in diameter and 32 feet deep, masonry curb. Copper nail with washer stamped 5-5-1 in plank deck at north side of pump. Measuring point is top of 2-inch plank deck, 1.0 foot above land surface and 209.74 feet above mean sea level.

\begin{tabular}{|c|c|c|c|c|c|c|c|}
\hline Date & $\begin{array}{l}\text { Water } \\
\text { level } \\
\text { (feet) }\end{array}$ & Date & $\begin{array}{l}\text { Water } \\
\text { level } \\
\text { (feet) }\end{array}$ & Date & $\begin{array}{l}\text { Water } \\
\text { level } \\
\text { (feet) }\end{array}$ & Date & $\begin{array}{l}\text { Water } \\
\text { level } \\
\text { (feet) }\end{array}$ \\
\hline 1928 & \multirow{4}{*}{189.5} & 1936 & & 1936 & \multirow{9}{*}{$\begin{array}{r}197.65 \\
197.70 \\
193.90 \\
193.48 \\
193.39 \\
193.05 \\
189.34 \\
5188.72 \\
188.36\end{array}$} & 1936 & \multirow{9}{*}{$\begin{array}{r}187.33 \\
186.31 \\
186.17 \\
184.77 \\
185.05 \\
184.58 \\
184.42 \\
5184.27 \\
184.27\end{array}$} \\
\hline Sept. 24 & & Mar. 23 & 203. 35 & May 25 & & Aug. 3 & \\
\hline 1936 & & $\begin{array}{l}\text { Mar. } 30 \\
\text { Apr. } 6\end{array}$ & $\begin{array}{l}202.83 \\
203.57\end{array}$ & $\begin{array}{l}\text { June } 1 \\
\text { June } 15\end{array}$ & & $\begin{array}{l}\text { Aug. 10- } \\
\text { Aug. 17 }\end{array}$ & \\
\hline & & Apr. 13 & 202.17 & June 22 & & Aug. 24 & \\
\hline Feb. 19 & 203.63 & Apr. 20 . & 202.14 & June 29 & & Aug. 31 & \\
\hline Feb. 24 & 206.72 & Apr. 27 & 200.39 & July 6. & & Sept. 8 - & \\
\hline Mar. 2 & 206.53 & May 4 & 198.02 & July 13 & & Sept. 14 & \\
\hline Mar. 9 & 204.70 & May 11. & 199.01 & July 20 . & & Sept. 21 & \\
\hline Mar. 16 & 204.78 & May 18 & 197.93 & July 27 & & Sept. 28 & \\
\hline
\end{tabular}

6 Pump operating in well.

210. Glenn Patty. NE 14 NE 14 sec. 10, T. 5 S., R. 4 W. Domestic and stock well dug, 3 feet in diameter and 38.6 feet deep, brick curb. Measuring point is top of brick curb, at west side, 2.2 feet above land surface and 158.62 feet 2 above mean sea level.

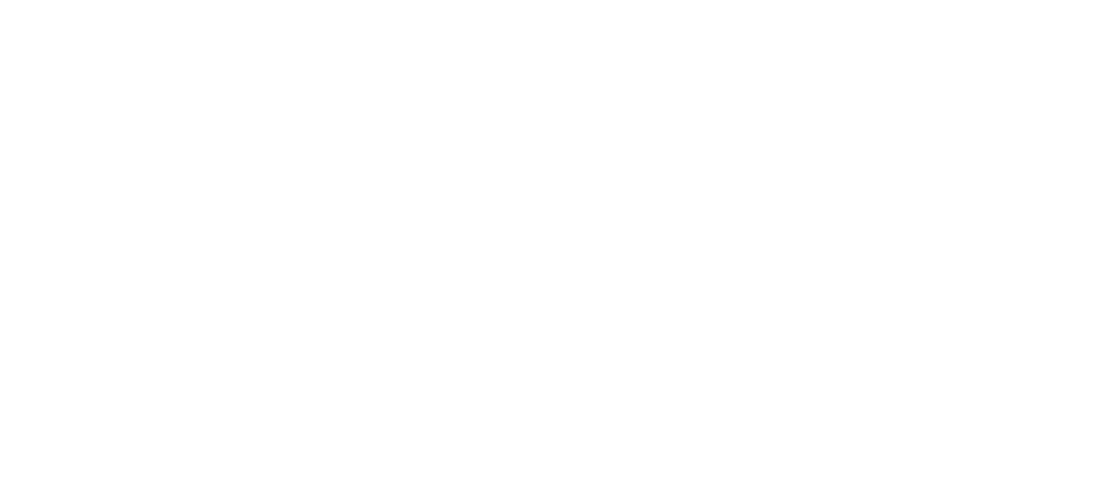

2 Leveling by U.S. Engineer Department.

225. Eunice Hibbs. NW1/4SE 14 sec. 9 , T. 5 S., R. 3 W. Domestic well, dug 4 feet in diameter and 32 feet deep, brick curb with concrete collar. Measuring point is top of 2-inch plank deck, through bored hole 8 inches northeast of pump, 0.7 foot above land surface and 163.53 feet ${ }^{2}$ above mean sea level.

\begin{tabular}{|c|c|c|c|c|c|c|c|}
\hline Date & $\begin{array}{l}\text { Water } \\
\text { level } \\
\text { (feet) }\end{array}$ & Date & $\begin{array}{l}\text { Water } \\
\text { level } \\
\text { (feet) }\end{array}$ & Date & $\begin{array}{l}\text { Water } \\
\text { level } \\
\text { (feet) }\end{array}$ & Date & $\begin{array}{l}\text { Water } \\
\text { level } \\
\text { (feet) }\end{array}$ \\
\hline 1935 & & 1936 & & 1936 & & 1936 & \\
\hline $\begin{array}{l}\text { Oct. } 31 \\
\text { Nov. } 5 \\
\text { Nov. } 11 \\
\text { Nov. } 18 \\
\text { Nov. } 26 \\
\text { Dec. } 3 \\
\text { Dec. } 9 \\
\text { Dec. } 16 \\
\text { Dec. } 23 \\
\text { Dec. } 30 \\
\quad 1936 \\
\text { Jan. } 6\end{array}$ & $\begin{array}{l}140.57 \\
140.93 \\
140.38 \\
140.18 \\
140.22 \\
141.85 \\
142.17 \\
142.26 \\
140.83 \\
144.17 \\
\\
145.32\end{array}$ & $\begin{array}{l}\text { Jan. 13 } \\
\text { Jan. 20 } \\
\text { Jan. 27 } \\
\text { Feb. 3 } \\
\text { Feb. 10 } \\
\text { Feb. 19 } \\
\text { Feb. 24 } \\
\text { Mar. 2 } \\
\text { Mar. } 9 \\
\text { Mar. 16 } \\
\text { Mar. 23 } \\
\text { Mar. 30 } \\
\text { Apr. 6. }\end{array}$ & $\begin{array}{l}160.47 \\
160.87 \\
157.46 \\
159.43 \\
160.22 \\
160.26 \\
161.45 \\
161.01 \\
160.46 \\
160.26 \\
159.39 \\
160.46 \\
160.40\end{array}$ & $\begin{array}{l}\text { Apr. } 13 \\
\text { Apr. } 20 \\
\text { Apr. } 27 \\
\text { May } 4 \\
\text { May } 11 \\
\text { May } 18 \\
\text { May } 25 \\
\text { June } 1 \\
\text { June } 8 \\
\text { June } 15 \\
\text { June } 22 \\
\text { June } 29 \\
\text { July } 6\end{array}$ & $\begin{array}{l}158.89 \\
158.64 \\
156.85 \\
155.78 \\
155.76 \\
153.74 \\
152.79 \\
151.49 \\
150.50 \\
150.36 \\
149.01 \\
147.60 \\
147.15\end{array}$ & $\begin{array}{l}\text { July } 13 \\
\text { July } 20 \\
\text { July } 27 \\
\text { Aug. } 3 \\
\text { Aug. } 10 \\
\text { Aug. } 17 \\
\text { Aug. } 24 \\
\text { Aug. } 31 \\
\text { Sept. } 8 \\
\text { Sept. } 14 \\
\text { Sept. } 21 \\
\text { Sept. } 28 \\
\end{array}$ & $\begin{array}{l}146.48 \\
146.12 \\
145.76 \\
145.04 \\
143.89 \\
143.80 \\
142.87 \\
142.46 \\
142.27 \\
141.96 \\
141.73 \\
141.40\end{array}$ \\
\hline
\end{tabular}

2 Leveling by U. S. Engineer Department. 
TABLE 13.-Ground-water levels in observation wells, 1928-30 and 1935-36-Con.

228. C. Anderson (formerly S. F. Parker). SE1/4SW1/4 sec. 13, T. 5 S., R. 3 W. Domestic well, dug 36 inches in diameter and 29.5 feet deep, in yard at northeast corner of residence. Measuring points: Top of box curb at center of west side, copper nail with washer, 1.8 feet above land surface and 177.4 feet above mean sea level; and in 1935-36, top of 2-inch plank platform, 0.8 foot above land surface and 176.40 feet 2 above mean sea level.

\begin{tabular}{|c|c|c|c|c|c|c|c|}
\hline Date & $\begin{array}{l}\text { Water } \\
\text { level } \\
\text { (feet) }\end{array}$ & Date & $\begin{array}{l}\text { Water } \\
\text { level } \\
\text { (feet) }\end{array}$ & Date & $\begin{array}{l}\text { Water } \\
\text { level } \\
\text { (feet) }\end{array}$ & Date & $\begin{array}{l}\text { Water } \\
\text { level } \\
\text { (feet) }\end{array}$ \\
\hline 1928 & & 1930 & & 1936 & & $1936^{\circ}$ & \\
\hline Sept. 20 & 149.9 & Mar. 30 & 156.25 & $\operatorname{Jan} .6$ & 153.88 & May 25 & 153.11 \\
\hline Oct. 20 & 149.4 & Apr. 27 & 153.60 & Jan. 27 & 173.32 & June $\mathbf{1}$. & 152.50 \\
\hline Dec. 16 & 148.5 & July 22 & 148.74 & Feb. 3 & 173.15 & June 8 & 152. 50 \\
\hline 1929 & & 1935 & & Feb. 19 & 161.05 & June 22 & 151.11 \\
\hline Fel & & Oct & & Feb. 24 & 173.34 & June $29 \ldots$ & 150.83 \\
\hline Mar & $\begin{array}{l}156.4 \\
154.2\end{array}$ & $\begin{array}{l}\text { Uct. } 3- \\
\text { Oct. } 11\end{array}$ & $\begin{array}{l}149.85 \\
149.75\end{array}$ & $\begin{array}{l}\text { Mar. } 2 \\
\text { Mar. } 9\end{array}$ & $\begin{array}{l}173.21 \\
170.94\end{array}$ & July 6 & $\begin{array}{l}151.20 \\
150.61\end{array}$ \\
\hline May 12 & 156.2 & Oct. 15 & 149.82 & Mar. 16 & 171.08 & July 20 & 150.87 \\
\hline Jun & 152.60 & Oct. 23 & 149.90 & Mar. 23 & 164. & July 27 . & 150.51 \\
\hline July 3 - & 151.15 & Oct. 28 & 149.63 & Mar. 27 . & 165.35 & Aug. 3 . & 150.67 \\
\hline & 150.05 & Nov. 4 & 149.17 & Mar. 30 & 166.83 & Aug. 10 & 150.45 \\
\hline Sep & 149. 40 & Nov. 11 & 149.58 & A pr. 6 & 163.82 & Aug. 17. & 150.48 \\
\hline Oct. 27 & 148. 90 & Nov. 18 & 149.21 & Apr. 13 & 161.34 & Aug. 24 & 150.46 \\
\hline Nov. 29 & 147. 30 & Dec. 2 & 148.87 & A pr. 20 & 161.29 & Ang. 31 & 150.13 \\
\hline Dec. 30 & 146.70 & Dec. 9 & 148.92 & Apr. 27 . & 156.70 & Sept. 8 & 149. 75 \\
\hline 1930 & & Dec & 148. 71 & May 4 & 155.06 & Sept. 14 & 149.85 \\
\hline Feb. 2 & 148.40 & Dec. 30. & $\begin{array}{l}148.41 \\
149.06\end{array}$ & May 18 & $\begin{array}{l}154.25 \\
153.50\end{array}$ & $\begin{array}{l}\text { Sept. } 21 \\
\text { Sept. } 28\end{array}$ & $\begin{array}{l}149.90 \\
149.77\end{array}$ \\
\hline
\end{tabular}

2 Leveling by U. s. Engineer Department.

235. Cora M. Nash. NE1/4NE 1/4 sec. 32, T. 5 S., R. 3 W. Unused well, dug $31 / 2$ feet in diameter and 27.4 feet deep, brick curb. Measuring point is top of concrete well cover, through 1 -inch hole 10 inches north of pump, 0.5 foot above land surface and 161.63 feet ${ }^{2}$ above mean sea level.

\begin{tabular}{|c|c|c|c|c|c|c|c|}
\hline Date & $\begin{array}{l}\text { Water } \\
\text { level } \\
\text { (feet) }\end{array}$ & Date & $\begin{array}{l}\text { Water } \\
\text { level } \\
\text { (feet) }\end{array}$ & Date & $\begin{array}{l}\text { Water } \\
\text { level } \\
\text { (feet) }\end{array}$ & Date & $\begin{array}{l}\text { Water } \\
\text { level } \\
\text { (feet) }\end{array}$ \\
\hline 1935 & & 1936 & & 1936 & & 1936 & \\
\hline $\begin{array}{l}\text { Oct. } 31 \\
\text { Nov. } 5\end{array}$ & $\begin{array}{l}142.95 \\
142.93\end{array}$ & Jan. 13 & 160.46 & Apr. 14 & 158.89 & July 15 & 150.84 \\
\hline Nov. 11 & 142. 66 & Jan. 20 & 160.00 & A pr. 21 & 159.00 & July 21 & 150.77 \\
\hline Nov. 18 & 142. 78 & Jan. 27 & 157.92 & A pr. 28 & 157.96 & July 28 . & 150.47 \\
\hline Nov. 26 & 142.61 & Feb. 3 & 159.54 & May 5 & 157.54 & Aug. 4 & 150.15 \\
\hline Dec. & 142.48 & Feb. 10 . & 159.01 & May 12 & 157.53 & Aug. 11 . & ${ }^{7} 147.77$ \\
\hline Dec. 9 & 142.52 & Feb. 19 & 158. 65 & May 18 & 157.51 & Aug. 18 & 147.54 \\
\hline Dec. 16 . & 142.44 & Feb. 25. & 160.44 & May 25_ & 156.26 & Aug. 25 & 146.63 \\
\hline Dec. 23 . & 148.48 & Mar. 2 & 159.74 & June 1. & 156.34 & Sept. 1 & 145.92 \\
\hline Dec. 28 . & 148.50 & Mar. 9. & 158.48 & June 8. & 154.04 & Sept. 9 & 145.54 \\
\hline Dec. 30 & 158.39 & Mar. 17 & 159.85 & June 16 & 153.52 & Sept. 1 & 145. 26 \\
\hline 1936 & & Mar. 23 & 159.05 & June 23 & 152.36 & Sept. 22. - & 144. 20 \\
\hline Jan. $6_{-}$ & 158.67 & Apr. 7. & 159.95 & July 7 & 151. 54 & sept. 29 & \\
\hline
\end{tabular}

2 Leveling by U. S. Engineer Department.

7 Adjacent land being irrigated. 
TABLE 13.-Ground-water levels in observation wells, 1928-30 and 1935-36-Con.

243. Ernest Andres. SW $1 / 4$ NE1/4 sec. 20, T. 5 S., R. 2 W. Domestic and stock well, dug 30 inches in diameter and 23 feet deep, concrete-tile casing. Measuring point is top of plank deck, through hole for discharge pipe, 0.5 foot above land surface and 177.16 feet ${ }^{2}$ above mean sea level.

\begin{tabular}{|c|c|c|c|c|c|c|c|}
\hline Date & $\begin{array}{l}\text { Water } \\
\text { level } \\
\text { (feet) }\end{array}$ & Date & $\begin{array}{l}\text { Water } \\
\text { level } \\
\text { (feet) }\end{array}$ & Date & $\begin{array}{l}\text { Water } \\
\text { level } \\
\text { (feet) }\end{array}$ & Date & $\begin{array}{l}\text { Water } \\
\text { level } \\
\text { (feet) }\end{array}$ \\
\hline 1935 & & 1936 & & 1936 & & 1936 & \\
\hline $\begin{array}{l}\text { Oct. } 9 \\
\text { Oct. } 15 \\
\text { Oct. } 24 \\
\text { Oct. } 28 \\
\text { Nov. } 4 \\
\text { Nov. } 11 \\
\text { Nov. } 18 \\
\text { Dec. } 2 \\
\text { Dec. } 9 \\
\text { Dec. } 16 \\
\text { Dec. } 23 \\
\text { Dec. } 30\end{array}$ & $\begin{array}{l}155.75 \\
156.91 \\
156.86 \\
156.86 \\
156.78 \\
156.66 \\
156.51 \\
156.58 \\
156.94 \\
158.31 \\
158.08 \\
159.87\end{array}$ & $\begin{array}{l}\text { Jan. } 6 \\
\text { Jan. } 27 \\
\text { Feb. } 3 \\
\text { Feb. } 10 \\
\text { Feb. } 19 \\
\text { Feb. } 24 \\
\text { Mar. } 2 \\
\text { Mar. } 9 \\
\text { Mar. } 16 \\
\text { Mar. 23 } \\
\text { Mar. } 30 \\
\text { Apr. } 6\end{array}$ & $\begin{array}{l}166.44 \\
171.14 \\
171.19 \\
169.42 \\
170.50 \\
175.28 \\
174.92 \\
174.15 \\
173.25 \\
172.99 \\
174.18 \\
173.42\end{array}$ & $\begin{array}{l}\text { Apr. 13 } \\
\text { Apr. } 20 \\
\text { Apr. } 27 \\
\text { May 4 } \\
\text { May } 11 \\
\text { May } 18 \\
\text { May 25 } \\
\text { June } 1 \\
\text { June } 8 \\
\text { June } 15 \\
\text { June } 22 \\
\text { June } 29 \\
\end{array}$ & $\begin{array}{l}172.85 \\
172.79 \\
171.31 \\
170.41 \\
169.97 \\
169.69 \\
168.18 \\
167.30 \\
165.90 \\
165.18 \\
164.20 \\
164.09\end{array}$ & 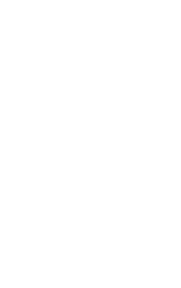 & $\begin{array}{l}162.76 \\
161.88 \\
161.31 \\
161.22 \\
160.23 \\
159.97 \\
158.20 \\
158.09 \\
157.70 \\
157.69 \\
158.80 \\
157.55 \\
157.33\end{array}$ \\
\hline
\end{tabular}

2 Leveling by U. S. Engineer Department.

245. Agricultural Research Corporation (Sam H. Brown). NW1/4SW1/4 sec. 25, T. 5 S., R. 2 W. Irrigation well, drilled; 18 -inch double stoveripe easing to depth of 155 feet, perforated from 117 to 147 feet; 6 -inch standard casing from 150 to 252 feet, perforated from 215 to 245 feet. In pump house northeast of residence. Measuring points: Drilline platform, about 0.5 foot above land surface, and 181 feet above mean sea level (by Oregon Arricultural Experiment Station); beginning April 10, 1930, upper face of pump-base flange at drilled hole, 1.0 foot above land surface and 181.31 feet 2 above mean sea level.

\begin{tabular}{|c|c|c|c|c|c|}
\hline Date & $\begin{array}{l}\text { Water } \\
\text { leve! } \\
\text { (feet) }\end{array}$ & Date & $\begin{array}{l}\text { Water } \\
\text { level } \\
\text { (feet) }\end{array}$ & Date & $\begin{array}{l}\text { Water } \\
\text { level } \\
\text { (feet) }\end{array}$ \\
\hline 1930 & & 1930 & & 1936 & \\
\hline Mar. 5 & 158.1 & Aug. 28 & 13155.5 & Mar. 30 & 166.14 \\
\hline Mar. $10 \ldots$ & 162.6 & Aug. 29 . & 13155.5 & Apr. $6 \ldots$ & 166. 20 \\
\hline Mar. 11- & 9162.2 & & & Apr. 13 & 166.11 \\
\hline Mar. 13, 1:30 p. m.... & 163.4 & 1935 & & Apr. 20 & 166.37 \\
\hline Mar. 13,5 p. m & 10117.2 & Oct. 10 & 158.01 & Apr. 27 & 164.79 \\
\hline Mar. 14,8 a. $\mathrm{m}$ & 162.9 & Oct. 16 & 157.86 & May 4 . & 164.43 \\
\hline Mar. $14,1: 30$ p. m & 11111.0 & Oct. 24 & 157.79 & May 11. & 163.92 \\
\hline Mar. 31................ & 164.7 & Oct. 28 & 157.79 & May 18 & 163.67 \\
\hline Apr. $10,3: 35$ p. m & 161.0 & Nov. 4 & 157.51 & May 25 . & 163.2 \\
\hline Apr. 10,4 p. m & 12124.65 & Nov. 11 & 157.55 & June 1 . & 162.7 \\
\hline Apr. $10,4: 45$ p. m & 12122.9 & Nov. 18 & 157.46 & June 8. & 162.52 \\
\hline Apr. $10,4: 55$ p. m.... & 12120.85 & Dec. 3. & 157.41 & June $15 \ldots$ & 162.42 \\
\hline Apr. $10,5: 17$ p. m. & 12117.7 & Dec. 9 & 157.47 & June 22 & 161.28 \\
\hline Apr. $10,5: 45$ p. m & 12115.8 & Dec. 17 & 157.74 & June 29 . & 160.09 \\
\hline Apr. $10,6: 04$ F. m & 12114.7 & Dec. 23 . & 158.04 & Julv 8 & 160.75 \\
\hline Apr. $10,6: 15$ p. $\mathrm{m}$ & 12114.1 & Dec. 31 & 158. 36 & July 13 & 160. 71 \\
\hline Apr. $10,6: 20$ p. m & 12114.0 & & & July 20 . & 159.99 \\
\hline Apr. 11 & 157. 9 & 1936 & & July 28 . & 159.8 \\
\hline Aug. $8,1 \mathrm{p}$ & 13148.3 & Jan. $6 \ldots . . . .$. & 160.15 & Aug. 3 & 159. 2 \\
\hline Aug. $8 \ldots$ & 6 116.3 & Jan. 13.... & 162.17 & Aug. 10 & 159.07 \\
\hline Aug. 12 . & 156. 2 & Jan. 20 & 162.79 & Aug. 17 & 158.54 \\
\hline Aug. 14 & 13155.20 & Jan. 27 & 164. 36 & Aug. 24 & 158.42 \\
\hline Aug. 15 & 13155.20 & Feb. 3 & 166. 11 & Aug. 31 & 158.39 \\
\hline Aug. 21, 10 & 157.5 & Feb. 10 & 166.39 & Ser.t. 8 & 158. 24 \\
\hline Aug. $21,11:$ & 8 119.3 & Feb. 17 . & 165. 73 & Sept. 14 & 158.11 \\
\hline Aug. $21,1: 35$ p. m & 6112.8 & Feb. 24 & 166.08 & Septl 21 & 157.98 \\
\hline Aug. $21,2: 45$ p. m & 5112.1 & Mar. 2.. & 165.88 & Sept. $28 \ldots$ & 157. 78 \\
\hline Aug. 27, 1:20 p. m..... & 157.83 & Mar. 9 & 166.09 & & \\
\hline Aug. $27,4: 15$ p. m & 5 120.87 & Mar. 16 & 165.53 & & \\
\hline Aug. 27,8 p. m $\ldots$ & ${ }^{6} 119.71$ & Mar. 23... & 165.61 & & \\
\hline
\end{tabular}

2 Leveling by U. S. Engineer Department.

5 Pump operating in well.

8 Outer 18-inch casing not perforated; inner 6-inch casing perforated and cut off at depth of 150 feet on

March 4.

8 Outer 18-inch casing perforated March 5-6, well being developed by intermittent pumping beginning March 8.

10 Pumping level after withdrawal at rate of 1.8 second-feet for $31 / 2$ hours.

11 Pumping level after withdrawal at rate of 1.95 second-feet for $51 / 6$ hours.

12 Water level during pumping test from $3: 35$ to $6: 20 \mathrm{p}$. m.; withdrawal began at 2.09 second-feet but declined gradually to 1.82 second-feet.

13 Pump idle but water level depressed by pumping within 24 hours or less. 
TABLE 13.-Ground-water levels in observation wells, 1928-30 and 1935-36-Con.

250. Joseph Kuhn. NW1/4SE1/4 sec. 26 , T. 5 S., R. 2 W. Domestic well, dug $41 / 2$ feet square and 19 feet deep, brick curb. Measuring point is edge of 2 -inch plank, 0.5 foot above land surface and 181.47 feet ${ }^{2}$ above mean sea level.

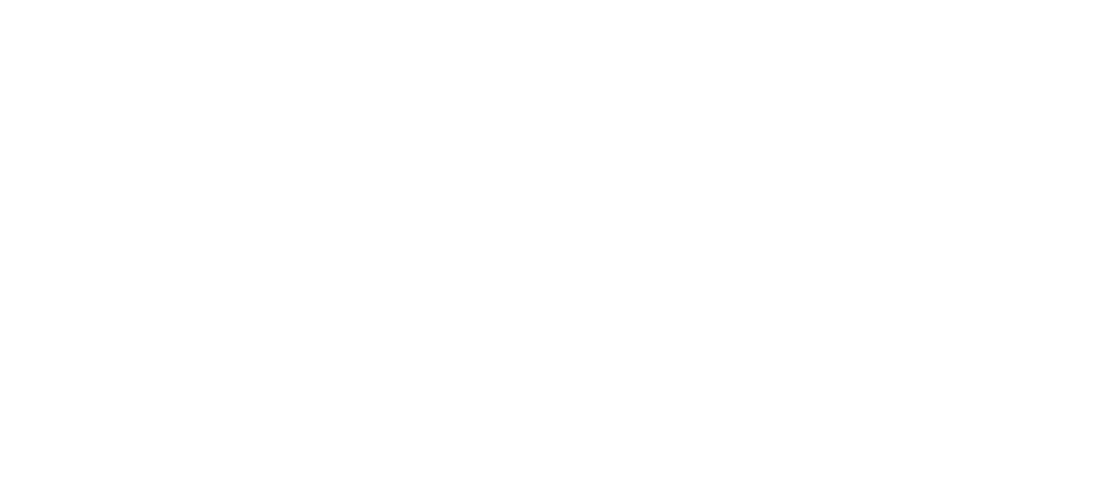

2 Leveling by U. S. Engineer Department.

254. J. P. Landen. SE1/4 NE1/4 sec. 8, T. 5 S., R. 1. W. Domestic well, dug 30 inches in diameter and 18 feet deep, culvert-pipe casing with concrete collar. Measuring point is top of concrete collar at south side, 2.8 feet above land surface and 183.95 feet ${ }^{2}$ above mean sea level.

\begin{tabular}{|c|c|c|c|c|c|c|c|}
\hline Date & $\begin{array}{l}\text { Water } \\
\text { level } \\
\text { (feet) }\end{array}$ & Date & $\begin{array}{l}\text { Water } \\
\text { level } \\
\text { (feet) }\end{array}$ & Date & $\begin{array}{l}\text { Water } \\
\text { level } \\
\text { (feet) }\end{array}$ & Date & $\begin{array}{l}\text { Water } \\
\text { level } \\
\text { (feet) }\end{array}$ \\
\hline 1935 & & 1936 & & 1936 & & 1936 & \\
\hline $\begin{array}{l}\text { Oct. } 10 \\
\text { Oct. } 15 \\
\text { Oct. } 24 \\
\text { Oct. } 28 \\
\text { Nov. } 4 \\
\text { Nov. } 11 \\
\text { Nov. } 18 \\
\text { Dec. } 3 \\
\text { Dec. } 9 \\
\text { Dec. } 16 \\
\text { Dec. } 23 \\
\text { Dec. } 30\end{array}$ & $\begin{array}{l}168.25 \\
168.48 \\
168.55 \\
168.23 \\
168.27 \\
168.13 \\
168.21 \\
167.88 \\
167.97 \\
168.16 \\
168.01 \\
168.34\end{array}$ & $\begin{array}{l}\text { Jan. } 6 \\
\text { Jan. } 13 \\
\text { Jan. } 20 \\
\text { Jan. } 27 \\
\text { Feb. } 3 \\
\text { Feb. } 7 \\
\text { Feb. } 10 \\
\text { Feb. } 17 \\
\text { Feb. } 24 \\
\text { Feb. 28 } \\
\text { Mar. } 2 \\
\text { Mar } 9 \\
\text { Mar } 16 \\
\text { Mar. } 23 \\
\end{array}$ & $\begin{array}{l}171.47 \\
173.67 \\
175.28 \\
174.10 \\
174.47 \\
174.84 \\
175.37 \\
175.67 \\
175.53 \\
177.56 \\
177.74 \\
177.81 \\
177.95 \\
178.68\end{array}$ & $\begin{array}{l}\text { Mar. 30 } \\
\text { Apr. } 6 \\
\text { Apr. 13 } \\
\text { Apr. 20 } \\
\text { Apr. 27 } \\
\text { May } 4 \\
\text { May } 11 \\
\text { May } 18 \\
\text { May 25 } \\
\text { June 1 } 1 \\
\text { June } 8 \\
\text { June } 15 \\
\text { June } 22 \\
\text { June } 29\end{array}$ & $\begin{array}{l}177.95 \\
177.73 \\
177.33 \\
176.37 \\
175.73 \\
175.02 \\
174.90 \\
174.60 \\
173.73 \\
172.64 \\
172.26 \\
172.15 \\
171.39 \\
171.66\end{array}$ & $\begin{array}{l}\text { July } 8 \\
\text { July } 13 \\
\text { July } 20 \\
\text { July } 28 \\
\text { Aug. } 3 \\
\text { Aug. 10 } \\
\text { Aug. 17 } \\
\text { Aug. } 24 \\
\text { Aug. } 31 \\
\text { Sept. } 8 \\
\text { Sept. 14 } \\
\text { Sept. } 21 \\
\text { Sept. } 28 \\
\end{array}$ & $\begin{array}{l}170.27 \\
170.78 \\
170.51 \\
170.32 \\
170.13 \\
169.25 \\
169.85 \\
169.64 \\
169.43 \\
169.30 \\
169.63 \\
169.33 \\
169.20\end{array}$ \\
\hline
\end{tabular}

${ }^{2}$ Leveling by U. S. Engineer Department.

258. Prudential Insurance Co. (formerly B. F. Shrock). NE1/4NW1/4 sec. 13, T. 5 S., R. 1 W. Unused well, dug 36 inches in diameter and 29 feet deep, at northwest corner of residence. Well receives run-sf f:om roof of residence, so that water level is not reliable during rainy season. Measuring points: Top of brick curb, south side, 0.5 foot above land surface and 196.42 feet above mean sea level; beginning Oct.jber 10,1935 , top face of concrete sidewalk over well, through hand hole, 196.02 feet $^{2}$ above mean sea level.

\begin{tabular}{|c|c|c|c|c|c|c|c|}
\hline Date & $\begin{array}{l}\text { Water } \\
\text { level } \\
\text { (feet) }\end{array}$ & Date & $\begin{array}{l}\text { Water } \\
\text { level } \\
\text { (feet) }\end{array}$ & Date & $\begin{array}{l}\text { Water } \\
\text { level } \\
\text { (feet) }\end{array}$ & Date & $\begin{array}{l}\text { Water } \\
\text { level } \\
\text { (feet) }\end{array}$ \\
\hline 1928 & & 1929 & & 1935 & & 1935 & \\
\hline Sept. 26 & 168.5 & Sept. 24 & 168.6 & Oct. 3 - & 169.01 & Dec. 31 & 169.45 \\
\hline Oct. 20 & 167.97 & Oct. $26 \ldots$ & 168.17 & Oct. 10 & 168. 62 & 1936 & \\
\hline Dec. 17 & 168.7 & $\begin{array}{l}\text { Nov. } 29 \\
\text { Dec. } 30\end{array}$ & $\begin{array}{l}167.32 \\
169.32\end{array}$ & $\begin{array}{l}\text { Uct. } 16 \\
\text { Oct. } 24\end{array}$ & $\begin{array}{l}168.53 \\
168.52\end{array}$ & 1936 & \\
\hline 1929 & & 1930 & & $\begin{array}{l}\text { Oct. } 28 \\
\text { Nov. } 4\end{array}$ & $\begin{array}{l}169.00 \\
168.02\end{array}$ & $\begin{array}{l}\text { Jan. } 6 \\
\text { Jan. } 13\end{array}$ & $\begin{array}{l}177.13 \\
182.03\end{array}$ \\
\hline Feb. 3 . & 173.3 & & & Nov. 11 & 169.74 & Jan. $20_{-}$ & 176.63 \\
\hline Mar. 23 & 172.07 & Feb. 2 & 172.37 & Nov. 18 & 168.12 & Jan. 27 & 172.34 \\
\hline May 12 & 176.1 & Mar. 2 & 176.17 & Dec. 3 . & 166.82 & & \\
\hline June 7 - & 173.7 & Mar. 30 & 175.77 & Dec. 9 & 167.94 & & \\
\hline July 3 . & 171.4 & Apr. 27 & 173.62 & Dec. 17 & 167.72 & & \\
\hline Aug. 5 & 169.9 & July 25 & 170.57 & Dec. 23 . & 167.56 & & \\
\hline
\end{tabular}

2 Leveling by U. S. Engineer Department. 
TABLE 13.-Ground-water levels in observation wells, 1928-30 and 1985-36-Con.

259. Prudential Insurance Co. (formerly B. F. Shrock). NE1/4NW1/4 sec. 13, T. 5 S., R. 1 W. Domestic and stock well, drilled 4 inches in diameter and 137 feet deep, in pump bouse 15 feet west of well 258 . Measuring point is top of 4 -inch casing, 0.5 foot above land surface and 196.24 feet 2 above mean sea level.

\begin{tabular}{|c|c|c|c|c|c|c|c|}
\hline Date & $\begin{array}{l}\text { Water } \\
\text { level } \\
\text { (feet) }\end{array}$ & Date & $\begin{array}{l}\text { Water } \\
\text { level } \\
\text { (feet) }\end{array}$ & Date & $\begin{array}{l}\text { Water } \\
\text { level } \\
\text { (feet) }\end{array}$ & Date & $\begin{array}{l}\text { Water } \\
\text { level } \\
\text { (feet) }\end{array}$ \\
\hline 1929 & & 1935 & & 1935 & & 1936 & \\
\hline $\begin{array}{l}\text { Sept. } 24 \\
\text { Oct. } 26 \\
\text { Nov. } 29 \\
\text { Dec. } 30 \\
\qquad 1930 \\
\text { Feb. } 2 \\
\text { Mar. } 2 \\
\text { Mar. } 30 \\
\text { Apr. } 27 \\
\text { July } 25\end{array}$ & \begin{tabular}{|c|}
157.5 \\
6157.04 \\
155.74 \\
159.44 \\
\end{tabular} & $\begin{array}{l}\text { Oct. } 3 \\
\text { Oct. } 10 \\
\text { Oct. } 16 \\
\text { Oct. } 24 \\
\text { Oct. } 28 \\
\text { Nov. } 4 \\
\text { Nov. } 11 \\
\text { Nov. } 18 \\
\text { Dec. } 3 \\
\text { Dec. } 9 \\
\text { Dec. } 17\end{array}$ & $\begin{array}{l}157.55 \\
157.46 \\
157.41 \\
157.44 \\
156.74 \\
157.44 \\
157.16 \\
157.24 \\
156.62 \\
157.64 \\
157.44\end{array}$ & $\begin{array}{l}\text { Dec. } 23 \\
\text { Dec. } 31 \\
\qquad 1936 \\
\text { Jan. } 6 \\
\text { Jan. } 13 \\
\text { Feb. } 10 \\
\text { Mar. } 9 \\
\text { Mar. } 16 \\
\text { Apr. } 13 \\
\end{array}$ & $\begin{array}{l}157.18 \\
157.56 \\
\\
159.87 \\
164.87 \\
160.29 \\
158.82 \\
162.77 \\
162.44\end{array}$ & $\begin{array}{l}\text { May } 25 \\
\text { July } 13 \\
\text { Aug. } 23 \\
\text { Sept. } 14 \\
\text { Sept. } 21 \\
\text { Sept. 28........... }\end{array}$ & $\begin{array}{l}160.79 \\
160.04 \\
158.26 \\
156.86 \\
156.84 \\
156.62\end{array}$ \\
\hline
\end{tabular}

2 Leveling by U. S. Engineer Department.

o Pump operating in well a short time prior to measurement.

260. Prudential Insurance Co. NE1/4NW1/4 sec. 13, T. 5 S., R. 1 W. Unused well, dug 4 feet in diameter and 27 feet deep, brick curb. Beginning Jan. 20, 1936, a substitute for well 258, which is ab out 650 feet to the south. Measuring point is top of brick curb, level with land surface and 193.87 feet 2 above mean sea level

\begin{tabular}{|c|c|c|c|c|c|c|c|}
\hline Date & $\begin{array}{l}\text { Water } \\
\text { level } \\
\text { (feet) }\end{array}$ & Date & $\begin{array}{l}\text { Water } \\
\text { level } \\
\text { (feet) }\end{array}$ & Date & $\begin{array}{l}\text { Water } \\
\text { level } \\
\text { (feet) }\end{array}$ & Date & $\begin{array}{l}\text { Water } \\
\text { level } \\
\text { (feet) }\end{array}$ \\
\hline 1936 & & 1936 & & 1936 & & 1936 & \\
\hline Jan. 20 & 172.47 & Mar. 9 & 178.13 & May 18 & 173.90 & July 28 . & 169.74 \\
\hline Jan. 27 & 179. 74 & Mar. 16 & $\begin{array}{l}177.92 \\
\end{array}$ & May 25 & 173.53 & Aug. 3 & 169. 54 \\
\hline Jan. 31 & 178.65 & Mar. 23 & 177.70 & June 1 & 172.81 & Aug. 10 & / 169.40 \\
\hline Feb. 3. & 175. 99 & Mar. 30 & 177.97 & June 8 & 172.37 & Aug. 17 & 169.12 \\
\hline Feb. 7 . & 177. 39 & Apr. 6 & 177.79 & June 15 & 171.98 & Aug. 24 & 168.90 \\
\hline Feb. 10 & 175. 13 & Apr. 13 & 177.57 & June 22 & 171.43 & Aug. 31 & 169.85 \\
\hline Feb. 1 & 175. 19 & Apr. 20 & 176.70 & June 29 & 171.10 & Sept. 8 & 169. 60 \\
\hline Feb. 2 & 177. 12 & Apr. 27 & 176.18 & July 8 . & 170.89 & Sept. 14 & 168. 27 \\
\hline Feb. 2 & 177.53 & May 4 & 174.82 & July 13 & 170.23 & Sept. 21 & 168.10 \\
\hline Mar. & 177. 97 & May 11 & 174.22 & July 20 & 170.03 & Sept. 28 & 167.96 \\
\hline
\end{tabular}

2 Leveling by U. S. Engineer Department.

283. I. O. O. F. Hall. SE1/4NE1/4 sec. 8 , T. 6 S., R. 5 W. Domestic well, dug 36 inches in diameter and 27 feet deep, brick curb. Measuring points: Top of box curb, 3.1 feet above land surface and 184.10 feet above mean sea level; beginning February 1936, top of 2-inch plank cover, through bored hole at center, 1.0 foot above land surface and 181.94 feet $^{2}$ above mean sea level.

\begin{tabular}{|c|c|c|c|c|c|c|c|}
\hline Date & $\begin{array}{l}\text { Water } \\
\text { level } \\
\text { (feet) }\end{array}$ & Date & $\begin{array}{l}\text { Water } \\
\text { level } \\
\text { (feet) }\end{array}$ & Date & $\begin{array}{l}\text { Water } \\
\text { level } \\
\text { (feet) }\end{array}$ & Date & $\begin{array}{l}\text { Water } \\
\text { level } \\
\text { (feet) }\end{array}$ \\
\hline 1928 & \multirow{4}{*}{163.8} & 1936 & & 1936 & & 1936 & \multirow{9}{*}{$\begin{array}{l}168.1 \\
167.7 \\
167.2 \\
167.21 \\
166.97 \\
164.71 \\
164.8 \\
164.02 \\
163.8\end{array}$} \\
\hline Sept. 24 & & Mar. 30. & 179.15 & June 1. & 177.03 & Aug. 3 & \\
\hline 1936 & & $\begin{array}{l}\text { Apr. } 6 \\
\text { Apr. } 13\end{array}$ & $\begin{array}{l}179.37 \\
178.65\end{array}$ & June 15 & $\begin{array}{l}176.21 \\
174.91\end{array}$ & $\begin{array}{l}\text { Aug. 10- } \\
\text { Aug. 17. }\end{array}$ & \\
\hline & & Apr. 20 & 178.24 & June 22-- & 174. 18 & Aug. 24 & \\
\hline Feb. 24 & 179. 40 & Apr. 27. & 178.01 & June $29 \ldots$ & 172.75 & Aug. 31 & \\
\hline Mar. 2 & 179. 10 & May 4 & 177.76 & July 6 .. & 172.11 & Sept. 8 & \\
\hline Mar. 9 & 178.94 & May 11 & 177.70 & July 13 & 170.61 & Sept. 14 & \\
\hline Mar. 16 & 179.75 & May 18 & 177.43 & July 20 & 170.06 & Sept. 21 & \\
\hline Mar. 23 & 178.80 & May 25 & 177.70 & July 27. & 169.87 & Sept. 28 & \\
\hline
\end{tabular}

2 Leveling by U. S. Engineer Department. 
TABLE 13.-Ground-water levels in observation wells, 1928-30 and 1935-36-Con.

284. Frank M. McCann. SW1/4NW1/4 sec. 11, T. 6 S., R. 5 W. Stock well, bored 6 inches in diameter and 29 feet deep, concrete-tile casing. Measuring points: Top of casing, 1.9 feet above land surface and 187.18 feet above mean sea level; beginning February 1936, top of casing (cut-off), 0.8 foot above land surface and 186.39 feet 2 above mean sea level.

\begin{tabular}{|c|c|c|c|c|c|c|c|}
\hline Date & $\begin{array}{l}\text { Water } \\
\text { level } \\
\text { (feet) }\end{array}$ & Date & $\begin{array}{l}\text { Water } \\
\text { level } \\
\text { (feet) }\end{array}$ & Date & $\begin{array}{l}\text { Water } \\
\text { level } \\
\text { (feet) }\end{array}$ & Date & $\begin{array}{l}\text { Water } \\
\text { level } \\
\text { (feet) }\end{array}$ \\
\hline 1928 & \multirow{3}{*}{169.8} & 1936 & \multirow{8}{*}{$\begin{array}{l}184.08 \\
184.17 \\
182.95 \\
182.91 \\
182.17 \\
181.87 \\
180.15 \\
181.07 \\
180.51\end{array}$} & 1936 & \multirow{8}{*}{$\begin{array}{l}180.82 \\
179.36 \\
179.16 \\
178.26 \\
177.47 \\
177.02 \\
175.61 \\
175.03 \\
174.90\end{array}$} & \multirow{8}{*}{ 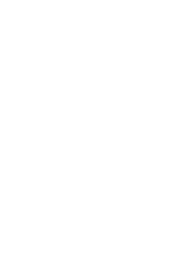 } & \multirow{8}{*}{$\begin{array}{l}174.45 \\
172.87 \\
172.76 \\
172.32 \\
172.00 \\
169.32 \\
169.18 \\
169.62 \\
169.30\end{array}$} \\
\hline Sept. 24. & & Mar. 30 & & June 1 & & & \\
\hline 1936 & & $\begin{array}{l}\text { Apr. } \\
\text { Apr. } 13\end{array}$ & & June 15. & & & \\
\hline & & Apr. 20 & & June 22-- & & & \\
\hline Feb. 19 & 177.75 & Apr. 27 & & June 29... & & & \\
\hline $\begin{array}{l}\text { Feb. 24 - } \\
\text { Mar. } 2\end{array}$ & $\begin{array}{l}185.34 \\
185.82\end{array}$ & May 4 & & July $6 \ldots$ & & & \\
\hline Mar. 9 & $\begin{array}{l}103.82 \\
183.99\end{array}$ & May $18 .$. & & July 20. & & & \\
\hline $\begin{array}{l}\text { Mar. } 16 \\
\text { Mar. } 23\end{array}$ & $\begin{array}{l}183.97 \\
183.35\end{array}$ & May $25 .$. & & July 27 & & & \\
\hline
\end{tabular}

2 Leveling by U. S. Engineer Department.

291. Robert Cole. NE1/4SE1/4 sec. 11, T. 6 S., R. 3 W. Domestic and stock well, dug 3 feet in diameter and 22.5 feet deep, brick curb. Measuring point is top of 4 -inch plank deck, through bored hole, 0.9 foot above land surface and 122.76 feet ${ }^{2}$ above mean sea level.

\begin{tabular}{|c|c|c|c|c|c|c|c|}
\hline Date & $\begin{array}{l}\text { Water } \\
\text { level } \\
\text { (feet) }\end{array}$ & Date & $\begin{array}{l}\text { Water } \\
\text { level } \\
\text { (feet) }\end{array}$ & Date & $\begin{array}{l}\text { Water } \\
\text { level } \\
\text { (feet) }\end{array}$ & Date & $\begin{array}{l}\text { Water } \\
\text { level } \\
\text { (feet) }\end{array}$ \\
\hline 1935 & & 1936 & & 1936 & & 1936 & \\
\hline Nov. 5 & 100.69 & Jan. 21 & 106.44 & Apr. 14 & 102.18 & July 14 & 100.57 \\
\hline Nov. 12 & 99.56 & Jan. 28 & $\begin{array}{l}104.76 \\
104.74\end{array}$ & Apr. 20 & 101.57 & July 21 & 100.50 \\
\hline Nov. 19 & 100.08 & Feb. 4 & 103.97 & Apr. 28 & 101.50 & July 28 & 100.33 \\
\hline Dec. 3 & 99.58 & Feb. 11. & 103.38 & May 5 & 100.83 & Aug. 4 & 100.29 \\
\hline Dec. 10 & 99.78 & Feb. 19 & 102.74 & May 12 & 100.49 & Aug. 11. & 100.22 \\
\hline Dec. 17 - & 99.78 & Feb. 24 & 103.14 & May 19 & 101.18 & Aug. 18 . & 100.21 \\
\hline Dec. 24 & 99.97 & Mar. 2 & 103.17 & May 26 & 101.11 & Aug. 24 & 99.82 \\
\hline Dec. 31 & 100.52 & Mar. 9 & 102.90 & June 2 & 100.52 & Sept. 1 & 99.89 \\
\hline 1936 & & $\begin{array}{l}\text { Mar. } 16 \\
\text { Mar. } 24\end{array}$ & $\begin{array}{l}102.58 \\
103.43\end{array}$ & $\begin{array}{l}\text { June } 9 \\
\text { June } 15\end{array}$ & $\begin{array}{l}100.97 \\
101.50\end{array}$ & $\begin{array}{l}\text { Sept. } 9 \\
\text { Sept. } 15\end{array}$ & $\begin{array}{l}100.75 \\
100.68\end{array}$ \\
\hline & & Mar. 31 & 102. 09 & June 22 & 100.95 & Sept. 22 & 100.57 \\
\hline Jan. 7. - & 101.04 & Apr. 7 . & 102.50 & July 9 & 100.64 & Sept. 29 & 99.97 \\
\hline
\end{tabular}

2 Leveling by U. S. Engineer Department.

293. Nick Valiech. SE14NW14 sec. 23 , T. 6 S., R. 3 W. Unused well, dug 3 feet in diameter and 75.3 feet deep. Measuring point is top of concrete deck, at east side of $5 \frac{1}{2}$-inch hole, 0.4 foot above land surface and 181.41 feet 2 above mean sea level.

\begin{tabular}{|c|c|c|c|c|c|c|c|}
\hline Date & $\begin{array}{l}\text { Water } \\
\text { level } \\
\text { (feet) }\end{array}$ & Date & $\begin{array}{l}\text { Water } \\
\text { level } \\
\text { (feet) }\end{array}$ & Date & $\begin{array}{l}\text { Water } \\
\text { level } \\
\text { (feet) }\end{array}$ & Date & $\begin{array}{l}\text { Water } \\
\text { level } \\
\text { (feet) }\end{array}$ \\
\hline 1935 & & 1936 & & 1936 & & 1936 & \\
\hline $\begin{array}{l}\text { Nov. } 5 \\
\text { Nov. } 12 \\
\text { Nov. } 19 \\
\text { Dec. } 3 \\
\text { Dec. } 10 \\
\text { Dec. } 17 \\
\text { Dec. } 24 \\
\text { Dec. } 31 \\
\quad 1936 \\
\text { Jan. } 7 \\
\text { Jan. } 14 \\
\end{array}$ & $\begin{array}{l}110.76 \\
110.74 \\
110.69 \\
110.51 \\
110.69 \\
110.81 \\
111.03 \\
111.30\end{array}$ & $\begin{array}{l}\text { Jan. } 21 \\
\text { Jan. } 28 \\
\text { Feb. } 4 \\
\text { Feb. } 11 \\
\text { Feb. } 19 \\
\text { Feb. } 24 \\
\text { Mar. } 3 \\
\text { Mar. } 9 \\
\text { Mar. } 16 \\
\text { Mar. } 24 \\
\text { Mar. } 31 \\
\text { Apr. } 7 \\
\text { Apr. } 14\end{array}$ & $\begin{array}{l}117.56 \\
113.89 \\
112.86 \\
113.51 \\
114.01 \\
112.47 \\
113.75 \\
113.16 \\
111.99 \\
111.68 \\
112.08 \\
112.26 \\
111.44\end{array}$ & $\begin{array}{l}\text { Apr. } 20 \\
\text { Apr. } 28 \\
\text { May } 5 \\
\text { May } 12 \\
\text { May } 19 \\
\text { May } 26 \\
\text { June } 2 \\
\text { June } 9 \\
\text { June } 16 \\
\text { June } 23 \\
\text { June } 30 \\
\text { July } 9 \\
\text { July } 14\end{array}$ & $\begin{array}{l}111.26 \\
111.12 \\
110.30 \\
110.19 \\
111.94 \\
111.06 \\
110.28 \\
110.91 \\
110.93 \\
109.90 \\
109.72 \\
110.41 \\
110.51\end{array}$ & $\begin{array}{l}\text { July } 21 \\
\text { July } 28 \\
\text { Aug. } 4 \\
\text { Aug. 11 } \\
\text { Aug. } 18 \\
\text { Aug. } 25 \\
\text { Sept. 1 } \\
\text { Sept. } 9 \\
\text { Sept. } 15 \\
\text { Sept. } 22 \\
\text { Sept. } 29 \\
\end{array}$ & $\begin{array}{l}110.69 \\
110.71 \\
110.69 \\
110.71 \\
110.61 \\
110.54 \\
109.32 \\
109.41 \\
109.27 \\
109.21 \\
108.03\end{array}$ \\
\hline
\end{tabular}

2 Leveling by U. S. Engineer Department. 
TABLE 13.-Ground-water levels in observation wells, 1928-30 and 1985-36-Con.

295. R. J. Hackett. NE1/4SW1/4 sec. 29, T. 6 S., R. 3 W. Domestic and stock well, dug 5 feet in diameter and 41.6 feet deep, brick curb. Measuring point is top of 2-inch plank deck, about 2 feet south and 10 inches west of pump, 1.2 feet above land surface and 174.08 feet ${ }^{2}$ above mean sea level.

\begin{tabular}{|c|c|c|c|c|c|c|c|}
\hline Date & $\begin{array}{l}\text { Water } \\
\text { level } \\
\text { (feet) }\end{array}$ & Date & $\begin{array}{l}\text { Water } \\
\text { level } \\
\text { (feet) }\end{array}$ & Date & $\begin{array}{l}\text { Water } \\
\text { level } \\
\text { (feet) }\end{array}$ & Date & $\begin{array}{l}\text { Water } \\
\text { level } \\
\text { (feet) }\end{array}$ \\
\hline 1935 & & 1936 & & 1936 & & 1936 & \\
\hline $\begin{array}{l}\text { Oct. } 31 \\
\text { Nov. } 5 \\
\text { Nov. } 11 \\
\text { Nov. } 18 \\
\text { Nov. } 26 \\
\text { Dec. } 3 \\
\text { Dec. } 9 \\
\text { Dec. } 16 \\
\text { Dec. } 23 \\
\text { Dec. } 28 \\
\qquad 1936 \\
\text { Jan. } 4\end{array}$ & $\begin{array}{l}144.58 \\
143.83 \\
142.53 \\
144.95 \\
143.74 \\
145.03 \\
146.20 \\
145.04 \\
146.18 \\
146.41\end{array}$ & $\begin{array}{l}\text { Jan. } 6 \\
\text { Jan. } 13 \\
\text { Jan. } 18 \\
\text { Jan. } 27 \\
\text { Feb. } 3 \\
\text { Feb. } 10 \\
\text { Feb. 20 } \\
\text { Feb. } 25 \\
\text { Mar. } 3 \\
\text { Mar. } 10 \\
\text { Mar. 17 } \\
\text { Mar. 24 } \\
\text { Mar. 31 } \\
\text { Apr. } 7\end{array}$ & $\begin{array}{l}149.55 \\
170.61 \\
167.95 \\
158.53 \\
158.90 \\
161.52 \\
162.05 \\
167.94 \\
164.89 \\
164.92 \\
157.14 \\
157.01 \\
165.08 \\
165.32\end{array}$ & $\begin{array}{l}\text { Apr. } 14 \\
\text { Apr. 21 } \\
\text { Apr. 24 } \\
\text { Apr. 28 } \\
\text { May } 5 \\
\text { May } 12 \\
\text { May } 19 \\
\text { May } 26 \\
\text { June } 1 \\
\text { June } 9 \\
\text { June } 16 \\
\text { June } 23 \\
\text { June } 30 \\
\text { July } 7\end{array}$ & $\begin{array}{l}165.34 \\
5158.63 \\
5158.61 \\
5154.84 \\
153.38 \\
153.40 \\
151.94 \\
150.44 \\
151.49 \\
148.26 \\
147.20 \\
148.30 \\
145.69 \\
145.10\end{array}$ & $\begin{array}{l}\text { July } 14 \\
\text { July } 21 \\
\text { July } 28 \\
\text { Aug. } 4 \\
\text { Aug. } 11 \\
\text { Aug. } 18 \\
\text { Aug. } 25 \\
\text { Sept. } 1 \\
\text { Sept. } 9 \\
\text { Sept. } 15 \\
\text { Sept. } 22 \\
\text { Sept. } 29\end{array}$ & $\begin{array}{l}144.88 \\
144.49 \\
142.99 \\
142.26 \\
144.46 \\
3142.99 \\
145.59 \\
145.05 \\
143.97 \\
141.22 \\
144.48 \\
143.15\end{array}$ \\
\hline
\end{tabular}

2 Leveling by U. S. Engineer Department.

5 Pump operating in well.

297. Gideon E. Stolz. SE1/4 SE1/4 sec. 33, T. $6 \mathrm{~S}$., R. $3 \mathrm{~W}$. Irrigation well, drilled 8 inches in diameter and 57 feet deep, in shed west of residence, standard steel casing and concrete-lined pit. Measuring points: Top of casing in pit, 119.56 feet above mean sea level; beginning November 12, 1935, top of timber, through 1-inch bored hole at north edge of pump base, 1.0 foot above land surface and 134.14 feet above mean sea level. Reference bench mark, top of concrete pit curb at northwest corner, 13.70 feet above top of casing and 133.02 feet above mean sea level. Water-stage recorder operated on well from August 3, 1929, to July $24,1930$.

\begin{tabular}{|c|c|c|c|c|c|c|c|}
\hline Date & $\begin{array}{l}\text { Water } \\
\text { level } \\
\text { (feet) }\end{array}$ & Date & $\begin{array}{l}\text { Water } \\
\text { level } \\
\text { (feet) }\end{array}$ & Date & $\begin{array}{l}\text { Water } \\
\text { level } \\
\text { (feet) }\end{array}$ & Date & $\begin{array}{l}\text { Water } \\
\text { level } \\
\text { (feet) }\end{array}$ \\
\hline 1929 & & 1930 & & 1936 & & 1936 & \\
\hline July 3. & 109.26 & Mar. 30 & 111.31 & Jan. 7 & 109.52 & June 2. & 110.91 \\
\hline Aug. 3 & 107.26 & Apr. 27 & 109. 71 & Jan. 14 & 113.01 & June 9 & 110.61 \\
\hline Sept. 3 & 106.01 & May 29 & 109.16 & Jan. 21 & 115. 16 & June 16 & 110. 10 \\
\hline Sept. 24 & 105.47 & July 24 & 106.75 & Jan. 28 & 114.01 & June 23 & 109.58 \\
\hline Oct. 23 . & 105.07 & & & Feb. 4 & 114. 17 & June 30 & 109.40 \\
\hline Oct. 26 & 105.06 & 1935 & & Feb. 11 & 113. 49 & July 9 & 108.95 \\
\hline Nov. 29 & 104.81 & & & Feb. 19 & 113. 70 & July 14 & 108.55 \\
\hline Dec. 13 & 104.93 & Oct. 4 & 104.74 & Feb. 24 & 113. 32 & July 21 . & 109.17 \\
\hline Dec. 21 . & 107.71 & Oct. 11 & 104.89 & Mar. 3 & 114.39 & July 28 & 107. 76 \\
\hline Dec. 30 & 109.26 & Oct. 16 & 104.86 & Mar. 9 & 114.55 & Aug. 4 & 107.87 \\
\hline & & Oct. 24 . & 104.91 & Mar. 16 & 113. 78 & Aug. 11 & 107.55 \\
\hline 1930 & & Oct. 29 & 104.98 & Mar. 24 & 113.53 & Aug. 18 & 106.74 \\
\hline & & Nov. 5 & 105.39 & Mar. 31 & 112.82 & Aug. 25 & 106. 67 \\
\hline Jan. 12. & 109.16 & Nov. 12 & 105.46 & Apr. 7 & 112.93 & Sept. 1 & 106. 26 \\
\hline Jan. 25 & 108.66 & Nov. 19 & 106.54 & Apr. 14 & 113. 27 & Sept. 9 & 106.24 \\
\hline Feb. 2 & 108. 71 & Dec. 3 & 106.42 & Apr. 21 & 113.02 & Sept. 15 & 106.14 \\
\hline Feb. 8 & 101.39 & Dec. 10 . & 106.15 & Apr. 28 & 112.17 & Sept. 22 & 106.00 \\
\hline Feb. 16 & 112.11 & Dec. 17 . & 106.84 & May 5 & 112.41 & Sept. 29 & 105.78 \\
\hline Feb. 23 & 112.86 & Dec. 24 & 106.97 & May 12 & 4110.81 & & \\
\hline Mar. 2 & 113. 22 & Dec. 31.. & 106.92 & May 19 & 112.17 & & \\
\hline Mar. 19 & 111.79 & & & May 26........ & 4111.38 & & \\
\hline
\end{tabular}

- Water level depressed by inordinately large withdrawals. 
TABLE 13.-Ground-water levels in observation wells, 1928-30 and 1935-36-Con.

302. R. J. Galagher (formerly J. E. Boyce). SE1/4NE1/4 sec. 17, T. 6 S., R. 2 W. Domestic well, dug 24 inches in diameter and 21 feet deep, concrete-tile casing. Measuring point is top of casing, at east side, 0.3 foot below land surface and 178.84 feet " above mean sea level.

\begin{tabular}{|c|c|c|c|c|c|c|c|}
\hline Date & $\begin{array}{l}\text { Water } \\
\text { level } \\
\text { (feet) }\end{array}$ & Date & $\begin{array}{l}\text { Water } \\
\text { level } \\
\text { (feet) }\end{array}$ & Date & $\begin{array}{l}\text { Water } \\
\text { level } \\
\text { (feet) }\end{array}$ & Date & $\begin{array}{l}\text { Water } \\
\text { level } \\
\text { (feet) }\end{array}$ \\
\hline 1935 & & 1936 & & 1936 & & 1936 & \\
\hline $\begin{array}{l}\text { Oct. } 10 \\
\text { Oct. } 16 \\
\text { Oct. } 24 \\
\text { Oct. } 29 \\
\text { Nov. } 5 \\
\text { Nov. } 12 \\
\text { Nov. } 19 \\
\text { Dec. } 3 \\
\text { Dec. } 9 \\
\text { Dec. } 17 \\
\text { Dec. } 23 \\
\text { Dec. } 31\end{array}$ & $\begin{array}{l}163.19 \\
164.37 \\
163.18 \\
164.22 \\
162.45 \\
162.69 \\
162.60 \\
161.59 \\
164.57 \\
162.52 \\
162.62 \\
163.26\end{array}$ & $\begin{array}{l}\text { Jan. } 6 \\
\text { Jan. } 13 \\
\text { Jan. } 20 \\
\text { Jan. } 24 \\
\text { Jan. } 27 \\
\text { Feb. } 3 \\
\text { Feb. } 10 \\
\text { Feb. } 17 \\
\text { Feb. } 24 \\
\text { Mar. } 2 \\
\text { Mar. } 9 \\
\text { Mar. } 16\end{array}$ & $\begin{array}{l}166.87 \\
177.51 \\
177.27 \\
176.76 \\
174.17 \\
173.26 \\
173.21 \\
173.09 \\
177.61 \\
177.34 \\
176.46 \\
176.09\end{array}$ & $\begin{array}{l}\text { Mar. 23 } \\
\text { Mar. } 30 \\
\text { Apr. } 6 \\
\text { Apr. } 14 \\
\text { Apr. 20 } \\
\text { Apr. 27 } \\
\text { May } 4 \\
\text { May } 11 \\
\text { May } 18 \\
\text { May } 25 \\
\text { June } 1 \\
\text { June } 8 \\
\text { June } 15\end{array}$ & $\begin{array}{l}176.41 \\
177.03 \\
175.63 \\
176.63 \\
175.52 \\
172.00 \\
170.90 \\
172.19 \\
169.28 \\
5168.17 \\
167.30 \\
165.89 \\
\text { 5 } 163.74\end{array}$ & $\begin{array}{l}\text { June } 22 \\
\text { July } 8 \\
\text { July } 13 \\
\text { July } 28 \\
\text { Aug. } 3 \\
\text { Aug. } 10 \\
\text { Aug. } 17 \\
\text { Aug. } 24 \\
\text { Aug. } 31 \\
\text { Sept. } 8 \\
\text { Sept. } 14 \\
\text { Sept. } 21 \\
\text { Sept. } 28\end{array}$ & $\begin{array}{l}166.74 \\
165.38 \\
165.02 \\
165.24 \\
164.24 \\
164.01 \\
164.41 \\
164.21 \\
163.80 \\
163.63 \\
163.52 \\
163.23 \\
163.02\end{array}$ \\
\hline
\end{tabular}

2 Leveling by U. S. Engineer Department.

$\checkmark$ Pump operating in well.

303. Carl Aspinwall. NE1/4SW1/4 sec. 17, T. 6 S., R. 2 W. Domestic well, dug 36 inches in diameter and 16 feet deep, in yard 45 feet west of southwest corner of post office and store. Copper nail with washer stamped 6-2-4 in plank well cover. Measuring point is top of plank well cover, at copper nail with washer, 180.5 feet above mean sea level (hand level).

\begin{tabular}{|c|c|c|c|c|c|c|c|}
\hline Date & $\begin{array}{l}\text { Water } \\
\text { level } \\
\text { (feet) }\end{array}$ & Date & $\begin{array}{l}\text { Water } \\
\text { level } \\
\text { (feet) }\end{array}$ & Date & $\begin{array}{l}\text { Water } \\
\text { level } \\
\text { (feet) }\end{array}$ & Date & $\begin{array}{l}\text { Water } \\
\text { level } \\
\text { (feet) }\end{array}$ \\
\hline 1928 & & 1928 & & 1929 & & 1929 & \\
\hline $\begin{array}{l}\text { Bept. } 21 \\
\text { Oct. } 20\end{array}$ & $\begin{array}{l}166.9 \\
166.4\end{array}$ & Dec. $17 .$. & 169.1 & $\begin{array}{l}\text { Feb. } 3 \\
\text { Mar. } 23 \\
\text { May } 12\end{array}$ & $\begin{array}{r}179.0 \\
178.5 \\
177.45\end{array}$ & $\begin{array}{l}\text { June } 7 \ldots \\
\text { July } 3 \ldots\end{array}$ & $\begin{array}{l}173.70 \\
169.48\end{array}$ \\
\hline
\end{tabular}

305. Nels C. Johnson. NW1/4SE1/4 sec. 24, T. $6 \mathrm{~S} .$, R. 2 W. Stock well, dug 3 feet in diameter and 20 feet deep, brick curb. Measuring point is top of 2 -inch wood deck, through bored hole, 0.5 foot above land surface and 201.66 feet 2 above mean sea level.

\begin{tabular}{|c|c|c|c|c|c|c|c|}
\hline Date & $\begin{array}{l}\text { Water } \\
\text { level } \\
\text { (feet) }\end{array}$ & Date & $\begin{array}{l}\text { Water } \\
\text { level } \\
\text { (feet) }\end{array}$ & Date & $\begin{array}{l}\text { Water } \\
\text { level } \\
\text { (feet) }\end{array}$ & Date & $\begin{array}{l}\text { Water } \\
\text { level } \\
\text { (feet) }\end{array}$ \\
\hline 1935 & & 1936 & & 1936 & & 1936 & \\
\hline $\begin{array}{l}\text { Oct. } 10 \\
\text { Oct. } 16 \\
\text { Oct. } 24 \\
\text { Oct. } 29 \\
\text { Nov. } 5 \\
\text { Nov. } 12 \\
\text { Nov. } 19 \\
\text { Dec. } 3 \\
\text { Dec. } 10 \\
\text { Dec. } 23 \\
\text { Dec. } 28 \\
\text { Dec. } 31\end{array}$ & $\begin{array}{l}184.16 \\
184.21 \\
184.04 \\
183.81 \\
183.82 \\
183.96 \\
184.08 \\
183.52 \\
186.44 \\
185.91 \\
186.39 \\
188.36\end{array}$ & $\begin{array}{l}\text { Jan. } 7 \\
\text { Jan. 14 } \\
\text { Jan. } 21 \\
\text { Jan. } 28 \\
\text { Feb. } 4 \\
\text { Feb. 11 } \\
\text { Feb. 19 } \\
\text { Feb. 24 } \\
\text { Mar. } 3 \\
\text { Mar. } 9 \\
\text { Mar. } 16 \\
\text { Mar. 23 } \\
\text { Mar. } 30 \\
\end{array}$ & $\begin{array}{l}195.78 \\
200.28 \\
200.03 \\
198.56 \\
198.71 \\
198.81 \\
198.12 \\
200.13 \\
199.36 \\
199.05 \\
199.14 \\
198.97 \\
200.08\end{array}$ & $\begin{array}{l}\text { Apr. } 6 \\
\text { Apr. } 13 \\
\text { Appr. 20 } \\
\text { Apr. 27 } \\
\text { May } 4 \\
\text { May } 11 \\
\text { May } 18 \\
\text { May } 25 \\
\text { June } 1 \\
\text { June } 8 \\
\text { June } 15 \\
\text { June } 22 \\
\text { June } 29 \\
\end{array}$ & $\begin{array}{l}201.66 \\
198.66 \\
198.08 \\
197.68 \\
197.28 \\
196.55 \\
196.09 \\
195.62 \\
194.44 \\
194.27 \\
193.64 \\
192.21 \\
191.47\end{array}$ & $\begin{array}{l}\text { July } 8 \\
\text { July } 13 \\
\text { July } 20 \\
\text { July } 28 \\
\text { Aug. } 3 \\
\text { Aug. } 10 \\
\text { Aug. 17} \\
\text { Aug. } 24 \\
\text { Aug. } 31 \\
\text { Sept. } 8 \\
\text { Sept. 14 } \\
\text { Sept } 21 \\
\text { Sept. } 28 \\
\end{array}$ & $\begin{array}{l}188.85 \\
188.09 \\
187.38 \\
187.26 \\
186.63 \\
186.27 \\
185.63 \\
185.29 \\
185.07 \\
184.99 \\
185.22 \\
184.47 \\
184.25\end{array}$ \\
\hline
\end{tabular}

Leveling by U. s. Engineer Department. 
TABLE 13.-Ground-water levels in observation wells, 1928-30 and 1995-36-Con.

318. Fred Lucht. NW1/4SW1/4 sec. 1, T. 6 S., R. 1 E. Stock well, dug 42 inches in diameter and 21 feet deep, in yard 60 feet west of northwest corner of barn. Copper nail with washer stamped 6-1-1 in plank well cover. Measuring point is top of plank well cover at copper nail with washer, 1.0 foot above land surface and 261.38 feet above mean sea level.

\begin{tabular}{|c|c|c|c|c|c|c|c|}
\hline Date & $\begin{array}{l}\text { Water } \\
\text { level } \\
\text { (feet) }\end{array}$ & Date & $\begin{array}{l}\text { Water } \\
\text { level } \\
\text { (feet) }\end{array}$ & Date & $\begin{array}{l}\text { Water } \\
\text { level } \\
\text { (feet) }\end{array}$ & Date & $\begin{array}{l}\text { Water } \\
\text { level } \\
\text { (feet) }\end{array}$ \\
\hline 1928 & \multirow{5}{*}{$\begin{array}{l}248.8 \\
248.53\end{array}$} & 1935 & & 1936 & \multirow{19}{*}{$\begin{array}{l}259.35 \\
258.61 \\
258.40 \\
257.76 \\
258.21 \\
258.06 \\
258.21 \\
260.03 \\
259.62 \\
259.29 \\
258.31 \\
258.53 \\
257.77 \\
259.73 \\
259.79 \\
257.54 \\
256.66 \\
256.23 \\
255.41 \\
255.18\end{array}$} & 1936 & \multirow{19}{*}{$\begin{array}{r}254.91 \\
254.32 \\
253.14 \\
253.90 \\
254.58 \\
252.89 \\
252.34 \\
251.57 \\
251.35 \\
251.04 \\
250.53 \\
249.93 \\
249.70 \\
249.04 \\
248.65 \\
247.10 \\
4246.79 \\
247.88 \\
247.76 \\
247.33\end{array}$} \\
\hline Sept. 21 & & Oct. 3. & 245.42 & Jan. 24 & & May 18 & \\
\hline Oet. 20 & & Oct. 11 & 247.06 & Jan. 27 & & May 25 & \\
\hline 1929 & & Oct. 16 . & $\begin{array}{l}247.70 \\
246.18\end{array}$ & Jan. 31 & & June 1- & \\
\hline & & Oct. 28 & $\begin{array}{l}240.10 \\
246.33\end{array}$ & Feb. 7 & & June 15 . & \\
\hline July 3 . & 252.23 & Nov. 4 & 245.11 & Feb. 10 & & June 22 & \\
\hline Aug. 5 & 249.93 & Nov. 11 & 245.08 & Feb. 17 & & June 29 & \\
\hline Sept. 24 & 247.28 & Nov. 18 & 249.74 & Feb. 24 & & July 8 . & \\
\hline Oct. 26 . & 245.78 & Dec. 3 . & 246.57 & Feb. 28 & & July 13 & \\
\hline Nov. 29 & 245.13 & Dec. 9 & 252.34 & Mar. 2 & & July 20 . & \\
\hline Dec. 30 & 258.18 & Dec. 17 . & 253.70 & Mar. 9 & & July 28. & \\
\hline \multirow{2}{*}{1930} & \multirow{8}{*}{$\begin{array}{l}260.13 \\
259.13 \\
257.78 \\
256.53 \\
250.69\end{array}$} & Dec. 23- & $\begin{array}{l}252.26 \\
252.97\end{array}$ & Mar. 16 & & Aug. 3 & \\
\hline & & Dec. 31 . & 258.20 & Mar. 30 & & Aug. 17. & \\
\hline Feb. 2 & & & & Apr. 6 . & & Aug. 24 & \\
\hline & & 19 & & Apr. 13 & & Aug. 31. & \\
\hline Mar. 30 & & Jan. 6 & 260.10 & Apr. 20 & & Sept. 8 & \\
\hline $\mathrm{Apr}$ & & Jan, 10 & 259.50 & $\mathrm{Ap}$ & & Sept. 14 & \\
\hline July 25 & & Jan. 13 & 260.06 & May 4 & & Sept. 21. & \\
\hline & & Jan. 20 & 259.58 & May 11 & & Sept. & \\
\hline
\end{tabular}

- Water level depressed by inordinately large withdrawals.

321. J. J. Neufeld. SW1/4NW14 sec. 8, T. 7 S., R. 4 W. Domestic and stock well, dug 4 feet in diameter and 13 feet deep, brick curb. Measuring point is top of cover of well housing, at northwest side, 2.6 feet above land surface and 186.54 feet $^{2}$ above mean sea level.

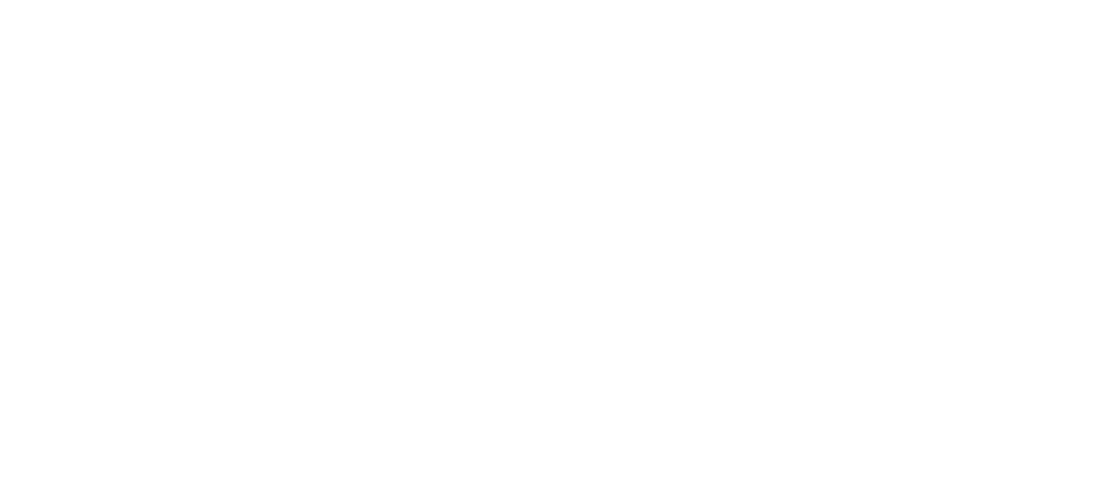

Leveling by U. S. Engineer Department.

14 Well dry. 
TABLE 13.-Ground-water levels in observation wells, 1928-30 and 1935-36-Con.

323. Wm. C. Palmer. NE1/4SW14 sec. 19, T. $7 \mathrm{~S} .$, R. $4 \mathrm{~W}$. Stock well, bored 4 inches in diameter and 21.1 feet deep, concrete-tile casing. About 235 feet north of well 322, in which the ground-water level was measured once in 1928 and again in 1935 . Measuring point is top of casing, level with land surface and 186.77 feet ${ }^{2}$ above mean sea level.

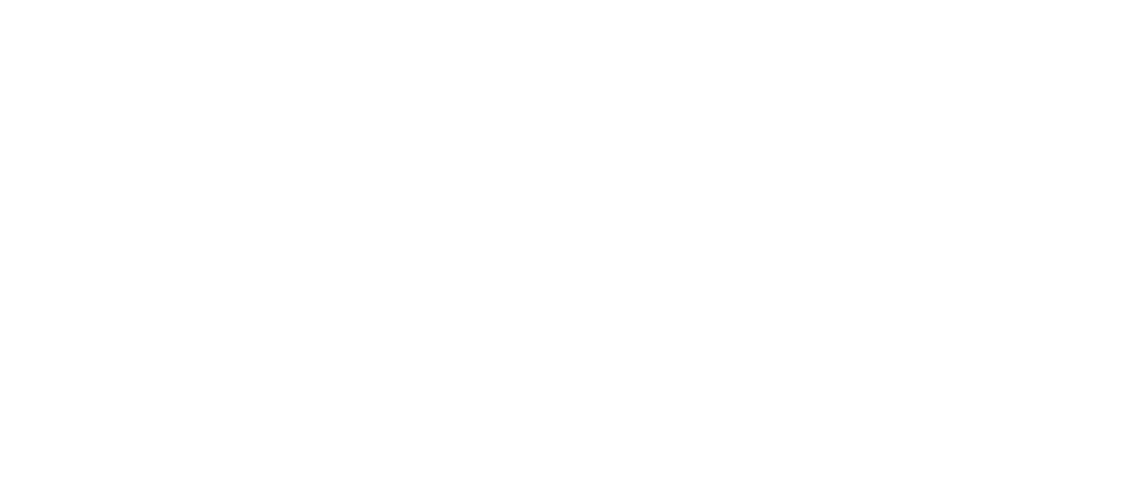

${ }^{2}$ Leveling by U. S. Engineer Department.

5 Pump operating in well.

327. Lauren Stettler. NW14SE1/4 sec. 1, T. 7 S., R. 3 W. Domestic and stock well, dug 3 feet in diameter and 19.6 feet deep, brick curb. Measuring point is top of brick curb, at east side, 1.0 foot above land surface and 152.85 feet ${ }^{2}$ above mean sea level.

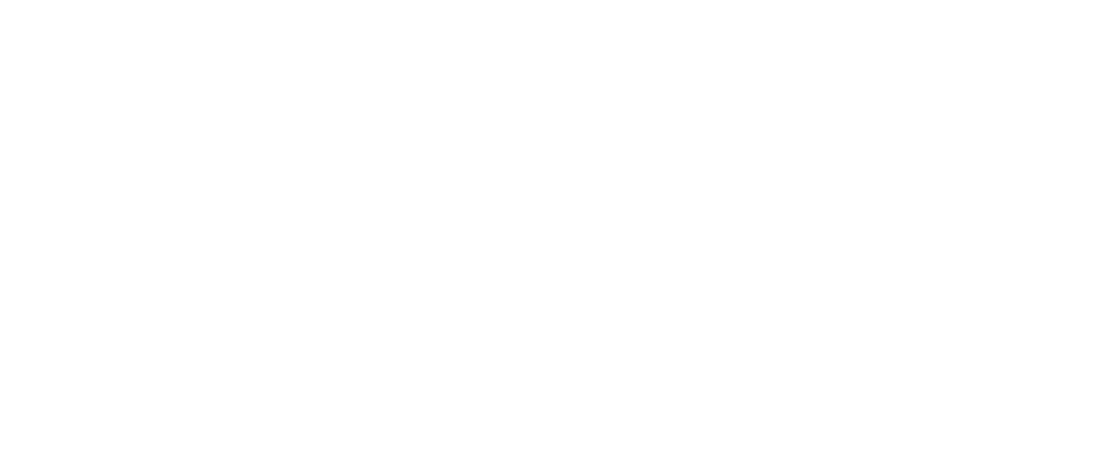

2 Leveling by U. S. Engineer Department.

4 Water level depressed by inordinately large withdrawals.

328. John C. Jackson. NE1/4NW1/4 sec. 13, T. $7 \mathrm{~S}$., R. $3 \mathrm{~W}$. Unused well, dug $31 / 2$ feet in diameter and 35.5 feet deep, brick curb. Measuring point is top of brick curb, at south side, level with land surface and 167.30 feet 2 above sea level.

\begin{tabular}{|c|c|c|c|c|c|c|c|}
\hline Date & $\begin{array}{l}\text { Water } \\
\text { level } \\
\text { (feet) }\end{array}$ & Date & $\begin{array}{l}\text { Water } \\
\text { level } \\
\text { (feet) }\end{array}$ & Date & $\begin{array}{l}\text { Water } \\
\text { level } \\
\text { (feet) }\end{array}$ & Date & $\begin{array}{l}\text { Water } \\
\text { level } \\
\text { (feet) }\end{array}$ \\
\hline 1935 & & 1936 & & 1936 & & 1936 & \\
\hline $\begin{array}{l}\text { Nov. } 5 \\
\text { Nov. } 12 \\
\text { Nov. } 19 \\
\text { Dec. } 3 \\
\text { Dec. } 10 \\
\text { Dec. } 17 \\
\text { Dec. } 24 \\
\text { Dec. } 31 \\
\qquad 1936 \\
\text { Jan. } 7 \\
\text { Jan. } 14\end{array}$ & $\begin{array}{l}132.21 \\
132.15 \\
132.20 \\
132.00 \\
132.00 \\
132.20 \\
132.45 \\
132.30\end{array}$ & $\begin{array}{l}\text { Jan. } 21 \\
\text { Jan. } 28 \\
\text { Jan. } 31 \\
\text { Feb. } 4 \\
\text { Feb. } 11 \\
\text { Feb. 19 } \\
\text { Feb. 25. } \\
\text { Mar. } 3 \\
\text { Mar. } 10 \\
\text { Mar. 17 } \\
\text { Mar. 24 } \\
\text { Mar. 31 } \\
\text { A pr. } 7\end{array}$ & $\begin{array}{l}133.85 \\
134.15 \\
134.87 \\
134.30 \\
134.15 \\
134.44 \\
134.82 \\
135.20 \\
134.50 \\
135.60 \\
136.08 \\
135.65 \\
135.86\end{array}$ & $\begin{array}{l}\text { Apr. } 14 \\
\text { Apr. } 21 \\
\text { Apr. } 28 \\
\text { May } 5 \\
\text { May } 12 \\
\text { May } 19 \\
\text { May } 26 \\
\text { June } 2 \\
\text { June } 9 \\
\text { June } 16 \\
\text { June } 23 \\
\text { June } 30 \\
\text { July } 9\end{array}$ & $\begin{array}{l}135.78 \\
134.88 \\
135.37 \\
134.70 \\
134.00 \\
134.22 \\
134.10 \\
134.40 \\
134.76 \\
134.66 \\
134.52 \\
133.93 \\
134.97\end{array}$ & $\begin{array}{l}\text { July } 14 \\
\text { July } 21 \\
\text { July } 28 \\
\text { Aug. } 4 \\
\text { Aug. 11 } \\
\text { Aug. 18} \\
\text { Aug. } 25 \\
\text { Sept. } 1 \\
\text { Sept. } 9 \\
\text { Sept. } 15 \\
\text { Sept. } 22 \\
\text { Sept. } 29\end{array}$ & $\begin{array}{l}133.91 \\
133.70 \\
135.45 \\
133.25 \\
133.30 \\
133.00 \\
132.80 \\
132.45 \\
132.37 \\
132.34 \\
132.09 \\
132.30\end{array}$ \\
\hline
\end{tabular}

2 Leveling by U. s. Engineer Department. 
TABLE 13.-Ground-water levels in observation wells, 1928-80 and 1985-36-Con.

346. E. O. Armann. NE1/4SW1/4 sec. 35, T. 7 S., R. 3 W. Domestic well, drilled 6 inches in diameter and 28.0 feet deep, tile casing. Measuring point is top of casing, at south side, level with land surface and 179.40 feet 2 above mean sea level.

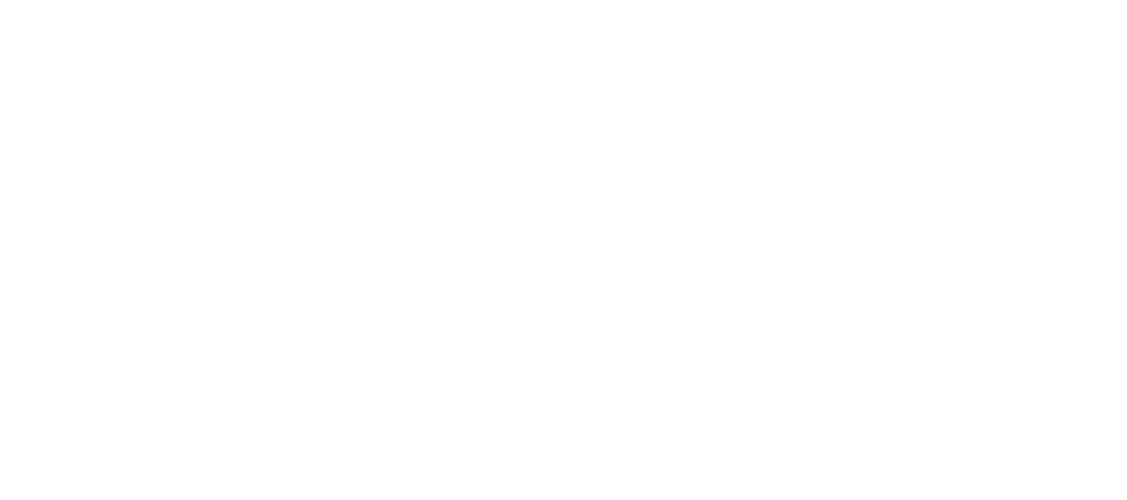

2 Leveling by U. S. Engineer Department.

4 Water level depressed by inordinately large withdrawals.

347. D. A. Steffen. SE1/4SW1/4 sec. 1, T. 7 S., R. 2 W. Domestic well, dug 6 feet in diameter and 33 feet deep, brick curb. Measuring point is top of pump platform, 0.5 foot above land surface and 199.41 feet 2 above sea level.

\begin{tabular}{|c|c|c|c|c|c|c|c|}
\hline Date & $\begin{array}{l}\text { Water } \\
\text { level } \\
\text { (feet) }\end{array}$ & Date & $\begin{array}{l}\text { Water } \\
\text { level } \\
\text { (feet) }\end{array}$ & Date & $\begin{array}{l}\text { Water } \\
\text { level } \\
\text { (feet) }\end{array}$ & Date & $\begin{array}{l}\text { Water } \\
\text { level } \\
\text { (feet) }\end{array}$ \\
\hline 1935 & & 1935 & & 1936 & & 1936 & \\
\hline $\begin{array}{l}\text { Oct. } 10 \\
\text { Oct. } 16 \\
\text { Oct. } 24 \\
\text { Oct. } 29 \\
\text { Nov. } 5 \\
\text { Nov. } 19 \\
\text { Nov. } 17\end{array}$ & $\begin{array}{l}178.51 \\
177.06 \\
176.61 \\
177.09 \\
177.11 \\
176.71 \\
178.56\end{array}$ & $\begin{array}{l}\text { Dec. } 23 \\
\text { Dec. } 31 \ldots \\
\qquad 1936 \\
\text { Jan. } 7 \ldots \ldots \\
\text { Jan. } 14\end{array}$ & $\begin{array}{l}178.70 \\
178.83 \\
\\
\\
191.50 \\
197.17\end{array}$ & $\begin{array}{l}\text { Jan. } 21 \\
\text { Feb. } 4 \\
\text { Feb. } 11 \\
\text { Feb. } 19 \\
\text { Feb. 25 } \\
\text { Mar. } 2 \\
\text { Mar. } 9\end{array}$ & $\begin{array}{l}196.52 \\
195.09 \\
194.79 \\
194.63 \\
196.94 \\
196.50 \\
196.38\end{array}$ & $\begin{array}{l}\text { Mar. 16 } \\
\text { Mar. } 23\end{array}$ & $\begin{array}{l}195.97 \\
(15)\end{array}$ \\
\hline
\end{tabular}

${ }^{2}$ Leveling by U. S. Engineer Department.

15 Discontinued as observation well.

350. Oscar Meyer (formerly R. J. Janz). SE1/4SE 14 sec. 12, 'T. 7 S., R. 2 W. Domestic well, dug 8 feet in diameter and 46.2 feet deep, dry masonry curb. Measuring points: Top of porch floor at northwest corner of trap door, 1.5 feet above land surface and 225.67 feet 2 above mean sea level; 224.70 feet 2 above mean sea level.

\begin{tabular}{|c|c|c|c|c|c|c|c|}
\hline Date & $\begin{array}{l}\text { Water } \\
\text { level } \\
\text { (foet) }\end{array}$ & Date & $\begin{array}{l}\text { Water } \\
\text { level } \\
\text { (feet) }\end{array}$ & Date & $\begin{array}{l}\text { Water } \\
\text { level } \\
\text { (feet) }\end{array}$ & Date & $\begin{array}{l}\text { Water } \\
\text { level } \\
\text { (feet) }\end{array}$ \\
\hline 1928 & & 1036 & & 1936 & & 1936 & \\
\hline Sept. 13. & 182.9 & Jan. 21 & 219.04 & Apr. 13 & 211. 39 & July 13 & 184. 29 \\
\hline 1935 & & $\begin{array}{l}\text { Jan. } 28 \\
\text { Fcb. } 4\end{array}$ & $\begin{array}{r}216.12 \\
4212.32\end{array}$ & $\begin{array}{l}\text { Apr. 20- } \\
\text { Apr. } 27 .\end{array}$ & $\begin{array}{l}210: 40 \\
210.15\end{array}$ & $\begin{array}{l}\text { July 28.. } \\
\text { Aug. } 3 . . .\end{array}$ & $\begin{array}{l}183.65 \\
181.81\end{array}$ \\
\hline & & Feb. 11 & 212. 90 & May 4 & 206.19 & Aug. 10. & 181.48 \\
\hline $\begin{array}{l}\text { Dec. } 16 \\
\text { Dec. } 23\end{array}$ & $\begin{array}{l}179.32 \\
179.29\end{array}$ & $\begin{array}{l}\text { Feb. } 19 \\
\text { Feb. } 25\end{array}$ & $\begin{array}{l}213.47 \\
219.59\end{array}$ & $\begin{array}{l}\text { May } 11 \\
\text { May } 18\end{array}$ & $\begin{array}{l}205.63 \\
202.09\end{array}$ & $\begin{array}{l}\text { Aug. 17 } \\
\text { Aug. 31 }\end{array}$ & $\begin{array}{l}181.98 \\
181.08\end{array}$ \\
\hline Dec. 31 & 179. 53 & Mar. 3 & 218. 39 & June 1.. & 196. 21 & Sept. 8 & 181.15 \\
\hline 1936 & & $\begin{array}{l}\text { Mar. } 9 \\
\text { Mar. } 16\end{array}$ & $\begin{array}{l}217.82 \\
214.14\end{array}$ & $\begin{array}{l}\text { June } 8 \ldots \\
\text { June } 15 .-\end{array}$ & $\begin{array}{l}196.09 \\
193.63\end{array}$ & $\begin{array}{l}\text { Sept. 14 } \\
\text { Sept. 21. }\end{array}$ & $\begin{array}{l}181.17 \\
180.89\end{array}$ \\
\hline $\begin{array}{l}\text { Jan. } 7 \\
\text { Jan. } 14 \ldots \ldots\end{array}$ & $\begin{array}{l}\text { 199. } 59 \\
220.92\end{array}$ & $\begin{array}{l}\text { Mar. } 23 \\
\text { Mar. } 30 \\
\text { Apr. } 6\end{array}$ & $\begin{array}{l}214.20 \\
215.10 \\
215.10\end{array}$ & $\begin{array}{l}\text { June 22.- } \\
\text { June 29.- } \\
\text { July } 8 . .\end{array}$ & $\begin{array}{l}192.81 \\
191.83 \\
185.39\end{array}$ & Sept. 28 & 179.63 \\
\hline
\end{tabular}

2 Leveling by U. S. Engineer Department.

4 Water level depressed inordinately by large withdrawals. 
TABLE 13.-Ground-water levels in observation wells, 1928-30 and 1935-36-Con.

351. Cole (formerly Paul Zirkel). NW1/4NW1/4 sec. 15, T. $7 \mathrm{~S} ., \mathrm{R} .2 \mathrm{~W}$. Domestic and stock well, dug 4 feet square and 24 feet deep with brick curb, bored to total depth of about 32 feet. Measuring point is top of 2-inch plank deck, through bored hole, 0.3 foot above land surface and 191.60 feet ${ }^{2}$ above mean sea level.

\begin{tabular}{|c|c|c|c|c|c|c|c|}
\hline Date & $\begin{array}{l}\text { Water } \\
\text { level } \\
\text { (feet) }\end{array}$ & Date & $\begin{array}{l}\text { Water } \\
\text { level } \\
\text { (feet) }\end{array}$ & Date & $\begin{array}{l}\text { Water } \\
\text { level } \\
\text { (feet) }\end{array}$ & Date & $\begin{array}{l}\text { Water } \\
\text { level } \\
\text { (feet) }\end{array}$ \\
\hline 1928 & & 1935 & & 1936 & & 1936 & \\
\hline $\begin{array}{l}\text { Sept. } 14 \\
1929 \\
\text { July } 3 \\
\text { Aug. } 5 \\
\text { Sept. } 24 \\
\text { Oct. } 26 \\
\text { Nov. } 29 \\
\text { Dec. } 30 \\
\qquad 1930 \\
\text { Feb. } 2 \\
\text { Mar. } 2 \\
\text { Mar. } 30 \\
\text { Apr. } 27 \\
\text { July } 25 \\
\end{array}$ & $\begin{array}{l}170.9 \\
169.65 \\
168.68 \\
168.20 \\
167.95 \\
168.75\end{array}$ & $\begin{array}{l}\text { Oct. } 4 \\
\text { Oct. } 11 \\
\text { Oct. } 16 \\
\text { Oct. } 24 \\
\text { Oct. } 29 \\
\text { Nov. } 5 \\
\text { Nov. } 12 \\
\text { Nov. } 19 \\
\text { Dec. } 3 \\
\text { Dec. } 10 \\
\text { Dec. } 17 \\
\text { Dec. } 23 \\
\text { Dec. } 31 \\
\qquad 1936 \\
\text { Jan. } 7 \\
\text { Jan. } 14\end{array}$ & $\begin{array}{l}169.03 \\
168.96 \\
168.94 \\
168.46 \\
168.52 \\
168.33 \\
168.20 \\
168.23 \\
168.16 \\
168.22 \\
168.15 \\
168.81 \\
169.24\end{array}$ & $\begin{array}{l}\text { Jan. } 21 \\
\text { Jan. } 28 \\
\text { Feb. } 4 \\
\text { Feb. } 11 \\
\text { Feb. } 19 \\
\text { Feb. } 25 \\
\text { Mar. } 2 \\
\text { Mar. } 9 \\
\text { Mar. } 16 \\
\text { Mar. } 23 \\
\text { Mar. } 30 \\
\text { Apr. } 6 \\
\text { Apr. } 13 \\
\text { Apr. } 20 \\
\text { Apr. } 27 \\
\text { May } 4 \\
\text { May } 11 \\
\text { May } 18 \\
\end{array}$ & $\begin{array}{l}171.12 \\
171.75 \\
171.83 \\
171.28 \\
172.02 \\
172.72 \\
173.18 \\
173.37 \\
173.62 \\
173.02 \\
173.69 \\
173.60 \\
173.44 \\
173.48 \\
173.30 \\
173.30 \\
172.12 \\
172.13\end{array}$ & $\begin{array}{l}\text { May } 25 \\
\text { June } 1 \\
\text { June } 8 \\
\text { June } 15 \\
\text { June } 22 \\
\text { June } 29 \\
\text { July } 8 \\
\text { July } 13 \\
\text { July } 20 \\
\text { July } 28 \\
\text { Aug. } 3 \\
\text { Aug. } 10 \\
\text { Aug. } 17 \\
\text { Aug. } 24 \\
\text { Aug. } 31 \\
\text { Sept. } 8 \\
\text { Sept. } 14 \\
\text { Sept. 21 } \\
\text { Sept. } 28 \\
\text { Sep. }\end{array}$ & $\begin{array}{l}171.62 \\
171.00 \\
170.91 \\
171.85 \\
171.65 \\
170.48 \\
170.86 \\
170.61 \\
168.70 \\
170.15 \\
170.17 \\
169.85 \\
169.67 \\
169.59 \\
168.48 \\
169.14 \\
169.02 \\
169.09 \\
168.99\end{array}$ \\
\hline
\end{tabular}

2 Leveling by U. S. Engineer Department.

352. Fred Scharf. NE1/4NW1/4 sec. 18, T. 7 S., R. 2 W. Stock well, bored 6 inches in diameter and 38 feet decp, in yard northwest of residence. Copper nail with washer stamped 7-2-4 in well cover. Measuring point, top of wooden well cover at copper nail with washer, 1.0 foot above land surface and 182 feet above mean sea level (altimeter).

\begin{tabular}{|c|c|c|c|}
\hline Date & $\begin{array}{l}\text { Water } \\
\text { level } \\
\text { (feet) }\end{array}$ & Date & $\begin{array}{l}\text { Water } \\
\text { level } \\
\text { (feet) }\end{array}$ \\
\hline $\begin{array}{l}\text { Sept. } 14 \\
\text { Oct. } 20 \\
\text { Dec. } 16\end{array}$ & $\begin{array}{l}164.5 \\
162.6 \\
172.2\end{array}$ & $\begin{array}{l}\text { Feb. } 3 \ldots \\
\text { Mar. } 23, \ldots 29 \\
\text { May } 12-\ldots\end{array}$ & $\begin{array}{l}179.2 \\
179.7 \\
176.6\end{array}$ \\
\hline
\end{tabular}

355. J. Grant. SW1/4SE 14 sec. 28, T. 7 S., R. 2 W. Domestic well, dug 3 feet in diameter and 35 feet deep, brick curb. Measuring point, top of 2 -inch plank deck, through bored hole, 0.6 foot above land surface and 229.70 feet 2 above mean sea level.

\begin{tabular}{|c|c|c|c|c|c|c|c|}
\hline Date & $\begin{array}{l}\text { Water } \\
\text { level } \\
\text { (feet) }\end{array}$ & Date & $\begin{array}{l}\text { Water } \\
\text { level } \\
\text { (feet) }\end{array}$ & Date & $\begin{array}{l}\text { Water } \\
\text { level } \\
\text { (feet) }\end{array}$ & Date & $\begin{array}{l}\text { Water } \\
\text { level } \\
\text { (feet) }\end{array}$ \\
\hline 1935 & & 1936 & & 1936 & & 1936 & \\
\hline $\begin{array}{l}\text { Oct. } 10 \\
\text { Oct. } 16 \\
\text { Oct. } 24 \\
\text { Oct. } 29 \\
\text { Nov. } 5 \\
\text { Nov. } 12 \\
\text { Nov. } 19 \\
\text { Dec. } 3 \\
\text { Dec. } 10 \\
\text { Dec. } 17 \\
\text { Dec. } 24 \\
\text { Dec. } 31\end{array}$ & $\begin{array}{l}198.10 \\
198.31 \\
198.15 \\
197.21 \\
196.92 \\
197.20 \\
197.75 \\
199.01 \\
20000 \\
200.60 \\
200.73 \\
201.22\end{array}$ & $\begin{array}{l}\text { Jan. } 7 \\
\text { Jan. } 14 \\
\text { Jan. } 21 \\
\text { Jan. } 28 \\
\text { Feb. } 4 \\
\text { Feb. 11 } \\
\text { Feb. 19. } \\
\text { Feb. 25 } \\
\text { Mar. } 3 \\
\text { Mar. 10 } \\
\text { Mar. 17 } \\
\text { Mar. 24 } \\
\text { Mar. } 31 \\
\end{array}$ & $\begin{array}{l}212.92 \\
224.18 \\
223.98 \\
218.91 \\
218.06 \\
217.82 \\
219.15 \\
220.68 \\
222.25 \\
222.44 \\
220.40 \\
219.98 \\
223.95\end{array}$ & $\begin{array}{l}\text { Apr. } 7 \\
\text { Apr. } 14 \\
\text { Apr. } 21 \\
\text { Apr. } 28 \\
\text { May } 5 \\
\text { May } 12 \\
\text { May } 19 \\
\text { May 26 } \\
\text { June } 2 \\
\text { June } 9 \\
\text { June } 16 \\
\text { June } 23 \\
\text { June } 30\end{array}$ & $\begin{array}{l}224.00 \\
220.84 \\
219.45 \\
218.88 \\
218.93 \\
218.37 \\
217.98 \\
218.48 \\
217.52 \\
216.74 \\
216.98 \\
214.95 \\
214.13\end{array}$ & $\begin{array}{l}\text { July } 9 \\
\text { July } 14 \\
\text { July } 21 \\
\text { July } 28 \\
\text { Aug. } 4 \\
\text { Aug. 11 } \\
\text { Aug. 18 } \\
\text { Aug. } 25 \\
\text { Sept. } 1 \\
\text { Sept. } 9 \\
\text { Sept. 15 } \\
\text { Sept. } 22 \\
\text { Sept. } 29 \\
\end{array}$ & $\begin{array}{l}213.82 \\
208.51 \\
206.55 \\
205.72 \\
205.86 \\
205.51 \\
202.85 \\
203.30 \\
202.24 \\
202.31 \\
202.23 \\
202.11 \\
201.95\end{array}$ \\
\hline
\end{tabular}

'Leveling by U. S. Engineer Department. 
TABLE 13.-Ground-water levels in observation wells, 1928-30 and 1935-36-Con.

365. Clow. NW $1 / 4$ NW 14 sec. 7, T. 8 S., R. 4 W. Stock well, dug 3 feet in diameter and 15.3 feet deep brick curb. Measuring point, top of 2 -inch plank deck at west side of pump pipe, 0.6 foot above land surface and 208.4 feet 2 above mean sea level.

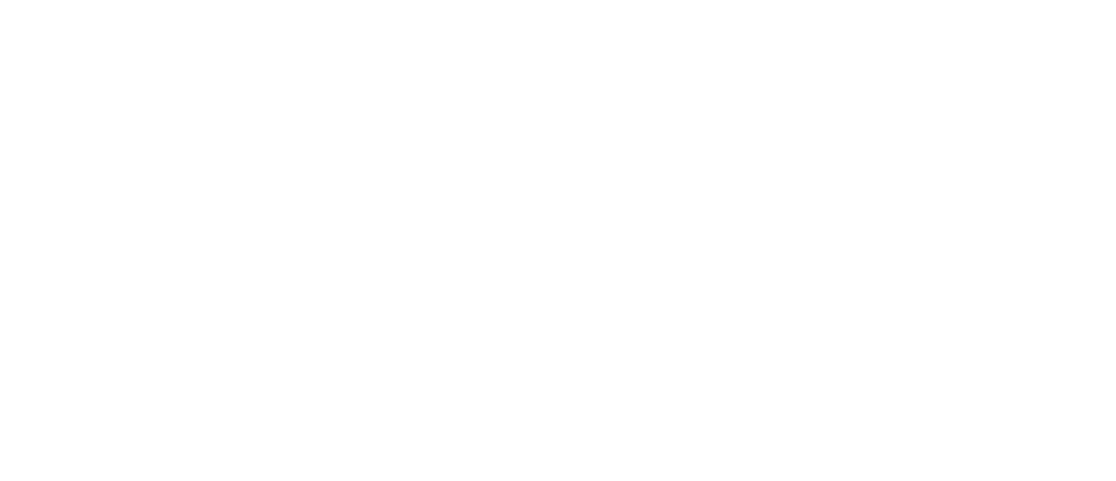

2 Leveling by U. S. Engineer Department.

366. Park Franks. NE1/4NE1/4 sec. 9, T. 8 S., R. 4 W. Stock well, dug 6 inches in diameter and 30 feet deep, in pasture 60 feet east of gate. Destroyed in March 1929. Measuring point is top of tile casing, 0.4 foot above land surface and 165.5 feet above mean sea level (hand level).

\begin{tabular}{|c|c|c|c|}
\hline Date & $\begin{array}{l}\text { Water } \\
\text { level } \\
\text { (feet) }\end{array}$ & Date & $\begin{array}{l}\text { Water } \\
\text { level } \\
\text { (feet) }\end{array}$ \\
\hline $\begin{array}{l}\text { Sept. } 11 \\
\text { Oct. } 22 \\
\text { Dec. } 1628 \\
\end{array}$ & $\begin{array}{l}140.6 \\
140.05 \\
145.70\end{array}$ & Feb. 3 1929 & 148.85 \\
\hline
\end{tabular}

367. E. Lauderback. NE1/4NE1/4 sec. 9, T. 8 S., R. 4 W. Domestic and stock well, bored 4 inches in diameter and 27 feet deep, stovepipe casing. Measuring point is top of casing, 2.0 feet above land surface and 167.34 feet ${ }^{2}$ above mean sea level.

\begin{tabular}{|c|c|c|c|c|c|c|c|}
\hline Date & $\begin{array}{l}\text { Water } \\
\text { level } \\
\text { (feet) }\end{array}$ & Date & $\begin{array}{l}\text { Water } \\
\text { level } \\
\text { (feet) }\end{array}$ & Date & $\begin{array}{l}\text { Water } \\
\text { level } \\
\text { (feet) }\end{array}$ & Date & $\begin{array}{l}\text { Water } \\
\text { level } \\
\text { (feet) }\end{array}$ \\
\hline 1935 & & 1936 & & 1936 & & 1936 & \\
\hline Oct. 28 & 140.30 & Jan. 8 . & 145.07 & Apr. 7 . & 147.92 & July 7. & 141.81 \\
\hline Nov. 6 . & $\begin{array}{l}140.30 \\
140.14\end{array}$ & Jan. 14 & $\begin{array}{l}140.07 \\
145.55\end{array}$ & Apr. 14 & $\begin{array}{l}47.92 \\
147.96\end{array}$ & July 14 & $\begin{array}{l}1+1.01 \\
141.58\end{array}$ \\
\hline Nov. 12 & 140.14 & Jan. 21 & 147.90 & A pr. 21 & 147.21 & July 21 & 142.40 \\
\hline Nov. 19 & 139. 96 & Jan. 28 & 147.48 & A pr. 28 & $\begin{array}{l}6 \\
6\end{array}$ & July 28 & 4140.78 \\
\hline Nov. 27 & 139. 95 & Feb. 4 & 146. 53 & May 5 & 145.27 & Aug. 4 & 140.72 \\
\hline Dec. 3 & 140.06 & Feb. 11 & 146.75 & May 12 & 144.49 & Aug. 11 & 140.63 \\
\hline Dec. 10 & 139.92 & Fob. 20 & 117.83 & May 19 & 144.40 & Aug. 18 & 144.90 \\
\hline Dec. 17 & 139.91 & Feb. 25- & 150.32 & May 26. & 143. 58 & Aug. 25- & 141. 94 \\
\hline Dec. 24 & 138.44 & Mar. 3 & 150.07 & June 2 & 142.96 & Sept. 1 & 112.18 \\
\hline \multirow[t]{4}{*}{ Dec. 31} & 144. 23 & Mar. 10 & 149.14 & June 9 . & 141. 46 & Sept. 9 & 141.34 \\
\hline & & Mar. 17 & 147.87 & June 16 & 142.15 & Sept. 15_ & 141.40 \\
\hline & & Mar. 24 & 147. 11 & June 23 & 142.02 & Sept. 22 & 141.96 \\
\hline & & Mar. 31. & 148.12 & June 30 & 142.86 & Sept. 29 & 141.93 \\
\hline
\end{tabular}

2 Leveling by U. S. Engineer Department.

4 Water level depressed by inordinately large withdra wals.

5 Pump, operating in well. 
TABLE 13.-Ground-water levels in observation wells, 1928-30 and 1935-36-Con.

370. O. L. Foster. NE1/4SW1/4 sec. 21, T. $8 \mathrm{~S} .$, R. 4 W. Unused well, drilled 3 inches in diameter and 33.5 feet deep in yard south of residence. Casing stamped 8-4-2 on east side. Measuring point is top of casing at highest point, 0.9 foot above land surface and 160.5 feet above mean sea level (altimeter). On September 11, 1928, the water level was 133.55 feet; July 11, 1929, 135.50 feet; August 3, 1929, 134.51 feet.

374. J. W. Kelley estate. NW 1/4 NE $1 / 4$ sec. 29 T. 8 S., R. 4 W. Unused well, dug 3 feet in diameter and 27.5 feet deep, brick curb. Measuring point is top of iron bar spanning well, at south side, level with land surface and 174.03 feet ${ }^{2}$ above mean sea level.

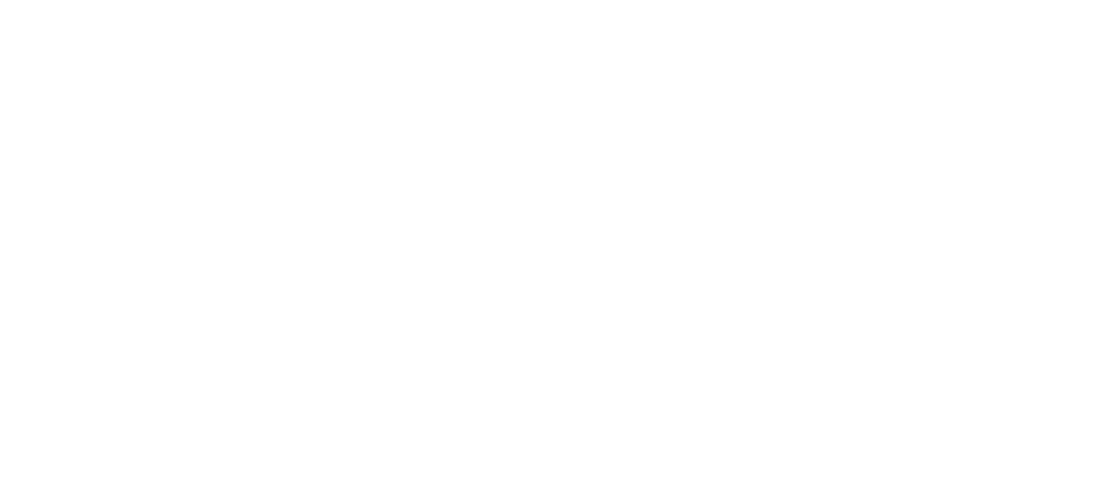

2 Leveling by U. S. Engineer Department.

377. P. O. Burbank. NW1/4NW1/4 sec. 31, T. 8 S., R. 4 W. Domestic well, dug $31 / 2$ feet in diameter and 25.5 feet deep, brick curb with concrete collar. Measuring point is top of 2-by 4-inch timber frame, north side of well housing, 7.8 feet a bove land surface and 218.68 feet 2 a bove mean sea level.

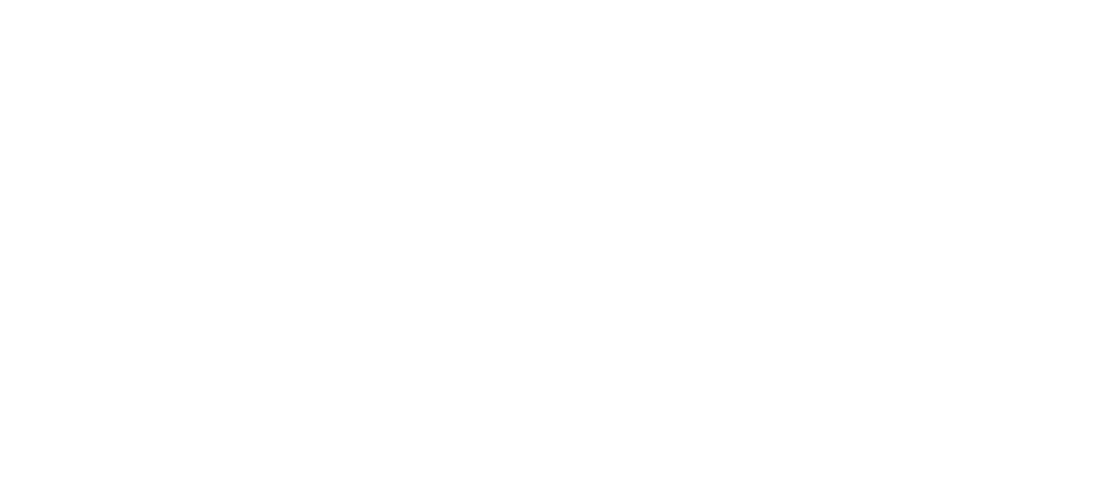

' Leveling by U. S. Engineer Department. 
TABLE 13.-Ground-water levels in observation wells, 1928-30 and 1935-36-Con.

394. Sloper Bros. SE1/4NE1/4 sec. 11, T. 9 S., R. 4 W. Unused well, drilled 8 inches in diameterand 32 feet deep, standard steel casing, in field 750 feet northeast of American Bottom School. Copper nail with wa sher stamped 9-4-4 in wood casing cap. Measuring point is top of casing flange, 1.0 foot above land surface and 157.93 feet 2 a bove mean sea level.

\begin{tabular}{|c|c|c|c|c|c|c|c|}
\hline Date & $\begin{array}{l}\text { Water } \\
\text { level } \\
\text { (feet) }\end{array}$ & Date & $\begin{array}{l}\text { Water } \\
\text { level } \\
\text { (feet) }\end{array}$ & Date & $\begin{array}{l}\text { Water } \\
\text { level } \\
\text { (feet) }\end{array}$ & Date & $\begin{array}{l}\text { Water } \\
\text { level } \\
\text { (feet) }\end{array}$ \\
\hline 1928 & \multirow{5}{*}{141.4} & 1935 & \multirow{19}{*}{$\begin{array}{l}140.39 \\
140.37 \\
140.50 \\
140.54 \\
140.45 \\
140.33 \\
140.38 \\
140.91 \\
141.15 \\
141.09 \\
141.06 \\
141.76 \\
141.73 \\
142.19\end{array}$} & 1936 & & 1936 & \multirow{19}{*}{$\begin{array}{l}145.91 \\
145.95 \\
147.00 \\
144.57 \\
144.72 \\
143.95 \\
143.54 \\
143.10 \\
142.84 \\
142.34 \\
141.95 \\
141.67 \\
141.45 \\
141.17 \\
140.00 \\
140.82 \\
140.52 \\
140.61 \\
140.44\end{array}$} \\
\hline \multirow[t]{4}{*}{ Sept. $15 \ldots$} & & Oct. 5. & & Jan. 8. & 143.40 & May 26 & \\
\hline & & Oct. 12 & & Jan. 21 & 153.08 & June $2 \ldots$ & \\
\hline & & Oct. 18 & & Jan. 28 & 151. 49 & June 9 & \\
\hline & & Oet. 21 & & Feb. 4 & 149.21 & June 16 & \\
\hline Sept. 26. & 141. 18 & Oct. 28 & & Feb. 11 & 149.64 & June 23 , & \\
\hline Oct. 27 & 140.88 & Nov. 6 & & Feb. 20 . & 148.82 & June 30 & \\
\hline Nov. 29 & 140.68 & Nov. 12 & & Feb. 25 & 153.42 & July 7 & \\
\hline Dec. 30 & 147.23 & Nov. 19 & & Mar. 3 & 150.04 & July 14 & \\
\hline 1030 & & Nov. $27 \ldots$ & & Mar. 10 & 149.40 & July $21 \ldots \ldots$ & \\
\hline 1930 & & $\begin{array}{l}\text { Dec. } 3 \\
\text { Dec. } 10\end{array}$ & & $\begin{array}{l}\text { Mar. } 17 \\
\text { Mar. } 24\end{array}$ & $\begin{array}{l}147.96 \\
147.17\end{array}$ & $\begin{array}{l}\text { July } 28 \\
\text { Aug. } 4\end{array}$ & \\
\hline Feb. 2 . & 146.08 & Dec. 17 & & Mar. 31 & 147.22 & Aug. 11 & \\
\hline Mar. 2 & 150.38 & Dec. 24 & & Apr. 7 & 147.39 & Aug. 18. & \\
\hline Mar. 30. & 146.48 & Dec. 31 & & Apr. 14 & 146.54 & Aug. 25 & \\
\hline Apr. 27 & 144.83 & & & Apr. 21 & 146.51 & Sept. 1 & \\
\hline May 27 & 144.68 & & & A pr, 28 & 146.50 & Sept. 9 & \\
\hline July 23.. & 142.30 & & & May $5 \ldots$ & 146.09 & Sept. $15 \ldots$ & \\
\hline & & & & May $12 \ldots$ & 146.17 & Sept. 22 & \\
\hline & & & & May 19 & 146.09 & Sept. $29 \ldots$ & \\
\hline
\end{tabular}

${ }^{2}$ Leveling by U. S. Engineer Department.

396. Susan Nash. SW1/4SW1/4 sec. 23 , T. 9 S., R. 4 W. Domestic well, dug 30.9 feet deep. Measuring point is top of well deck, through bored hole at east side, 1.0 foot above land surface and 239.68 feet ${ }^{2}$ above mean sea level.

\begin{tabular}{|c|c|c|c|c|c|c|c|}
\hline Date & $\begin{array}{l}\text { Water } \\
\text { level } \\
\text { (feet) }\end{array}$ & Date & $\begin{array}{l}\text { Water } \\
\text { level } \\
\text { (feet) }\end{array}$ & Date & $\begin{array}{l}\text { Water } \\
\text { level } \\
\text { (feet) }\end{array}$ & Date & $\begin{array}{l}\text { Water } \\
\text { level } \\
\text { (feet) }\end{array}$ \\
\hline \begin{tabular}{l}
\multicolumn{1}{|c|}{1935} \\
Oct. 25 \\
Oct. 28 \\
Nov. 6 \\
Nov. 12 \\
Nov. 19 \\
Nov. 27 \\
Dec. 3 \\
Dec. 10 \\
Dec. 17 \\
Dec. 24 \\
Dec. 31
\end{tabular} & $\begin{array}{l}213.78 \\
214.03 \\
214.12 \\
213.73 \\
213.66 \\
212.87 \\
212.49 \\
213.16 \\
212.77 \\
213.41 \\
221.28\end{array}$ & \begin{tabular}{l}
\multicolumn{1}{l}{1936} \\
Jan. 8 \\
Jan. 14 \\
Jan. 21 \\
Jan. 28 \\
Feb. 4 \\
Feb. 11 \\
Feb. 20 \\
Feb. 25 \\
Mar. 3 \\
Mar. 10. \\
Mar. 17 \\
Mar. 24. \\
Mar. 31
\end{tabular} & $\begin{array}{l}222.59 \\
229.11 \\
237.79 \\
237.11 \\
236.52 \\
236.62 \\
236.42 \\
238.40 \\
237.39 \\
236.97 \\
236.46 \\
236.07 \\
237.47\end{array}$ & $\begin{array}{l}1936 \\
\text { Apr. } 7 \\
\text { Apr. } 14 \ldots \\
\text { Apr. } 21 \\
\text { Apr. } 28 \\
\text { May } 5 \\
\text { May } 12 \\
\text { May } 19 \\
\text { May } 26 \ldots \ldots \\
\text { June } 2 \\
\text { June } 9 \\
\text { June } 16 \ldots \\
\text { June } 23 \\
\text { June } 30\end{array}$ & $\begin{array}{l}237.50 \\
236.36 \\
235.29 \\
234.61 \\
234.24 \\
234.71 \\
233.44 \\
233.51 \\
233.60 \\
231.69 \\
230.95 \\
230.37 \\
228.37\end{array}$ & $\begin{array}{l}1936 \\
\text { July } 7 \\
\text { July } 14 \\
\text { July } 21 \\
\text { July } 28 \\
\text { Aug. } 4 \\
\text { Aug. } 11 \\
\text { Aug. } 18 \\
\text { Aug. 25 } \\
\text { Sept. } 1 \\
\text { Sept. } 9 \\
\text { Sept. } 15 \\
\text { Sept. } 22 \\
\text { Sept. } 29\end{array}$ & $\begin{array}{r}227.09 \\
4224.65 \\
224.40 \\
4220.50 \\
220.39 \\
+218.29 \\
218.16 \\
217.48 \\
217.26 \\
215.77 \\
215.84 \\
215.56 \\
215.21\end{array}$ \\
\hline
\end{tabular}

2 Leveling by U. S. Engineer Department

4 Water level depressed by inordinately large withdrawals.

404. Addie Harman. NW1/4SE1/4 sec. 27 , T. 9 S., R. 4 W. Domestic and stock, well dug 3 feet in diameter and 30.8 feet deep, concrete-tile casing. Measuring point is top of 2-inch plank deck, through bored hole near southeast side of hand pump, 0.5 foot above land surface and 216.76 feet 2 above mean sea level.

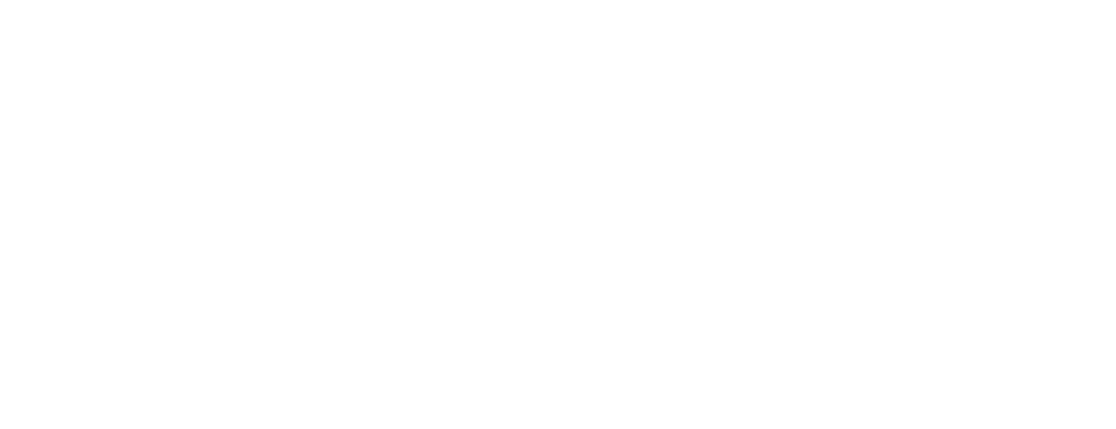

2 Leveling by U. S. Engineer Department.

4 Water level depressed by inordinately large withdrawals. 
TABLE 13.-Ground-water levels in observation wells, 1928-30 and 1985-36-Con.

405. Alfred Flickinger. NE1/4SW1/4 sec. 31, T. 9 S., R. 4 W. Domestic and stock well, bored 4 mches in diameter and 28.9 feet deep, concrete-tile casing. Measuring point is top of 2 -inch plank cover, level with land surface and 207.61 feet 2 above mean sea level.

\begin{tabular}{|c|c|c|c|c|c|c|c|}
\hline Date & $\begin{array}{l}\text { Water } \\
\text { level } \\
\text { (feet) }\end{array}$ & Date & $\begin{array}{l}\text { Water } \\
\text { level } \\
\text { (feet) }\end{array}$ & Date & $\begin{array}{l}\text { Water } \\
\text { level } \\
\text { (feet) }\end{array}$ & Date & $\begin{array}{l}\text { Water } \\
\text { level } \\
\text { (feet) }\end{array}$ \\
\hline $\begin{array}{l}\quad 1935 \\
\text { Oct. } 30 \\
\text { Nov. } 6 \\
\text { Nov. } 12 \\
\text { Nov. } 19 \\
\text { Nov. } 29 \\
\text { Dec. } 4 \\
\text { Dee. } 11 \\
\text { Dee. } 18 \\
\text { Dec. } 24 \\
\text { Dec. } 31\end{array}$ & $\begin{array}{l}194.58 \\
194.86 \\
195.01 \\
195.09 \\
194.91 \\
195.10 \\
198.57 \\
199.48 \\
199.22 \\
201.14\end{array}$ & $\begin{array}{l}\text { Jan. } 1936 \\
\text { Jan. } 14 \\
\text { Jan. } 21 \\
\text { Jan. } 28 \\
\text { Feb. 4 } \\
\text { Feb. 11 } \\
\text { Feb. 20 } \\
\text { Feb. } 26 \\
\text { Mar. } 3 \\
\text { Mar. 10 } \\
\text { Mar. } 17 \\
\text { Mar. 24 } \\
\text { Apr. 1 }\end{array}$ & $\begin{array}{l}202.32 \\
204.97 \\
206.08 \\
205.79 \\
205.54 \\
204.64 \\
204.02 \\
207.21 \\
207.18 \\
206.32 \\
205.13 \\
205.75 \\
206.00\end{array}$ & $\begin{array}{l}\quad 1936 \\
\text { Apr. } 8 \\
\text { Apr. } 15 \\
\text { Apr. } 22 \\
\text { Apr. } 29 \\
\text { May } 6 \\
\text { May } 12 \\
\text { May } 20 \\
\text { May } 27 \\
\text { June } 3 \\
\text { June } 10 \\
\text { June } 17 \\
\text { June 24 } \\
\text { July } 1 \\
\end{array}$ & $\begin{array}{l}205.98 \\
205.80 \\
205.83 \\
205.80 \\
205.04 \\
205.01 \\
205.03 \\
204.55 \\
204.43 \\
204.37 \\
203.54 \\
203.47 \\
201.77\end{array}$ & $\begin{array}{l}\quad 1936 \\
\text { July } 8 \\
\text { July } 15 \\
\text { July } 22 \\
\text { July } 29 \\
\text { Aug. } 5 \\
\text { Aug. } 12 \\
\text { Aug. } 19 \\
\text { Aug. } 26 \\
\text { Sept. } 2 \\
\text { Sept. } 10 \\
\text { Sept. } 16 \\
\text { Sept. } 23 \\
\text { Sept. } 30\end{array}$ & $\begin{array}{l}202.06 \\
201.20 \\
200.29 \\
199.64 \\
199.60 \\
198.42 \\
198.37 \\
196.21 \\
195.98 \\
195.83 \\
195.54 \\
193.52 \\
193.34\end{array}$ \\
\hline
\end{tabular}

2 Leveling by U. s. Engineer Department.

409. C. A. Bear. NE1/4NW1/4 sec. 4, T. 9 S., R. 2 W. Domestic well, driven $11 / 4$ inches in diameter and 29 feet deep, standard pipe casing. Measuring point is top of $1 \frac{1}{4}$-inch standpipe, 3.0 feet above land surface and 308.14 feet 2 above mean sea level.

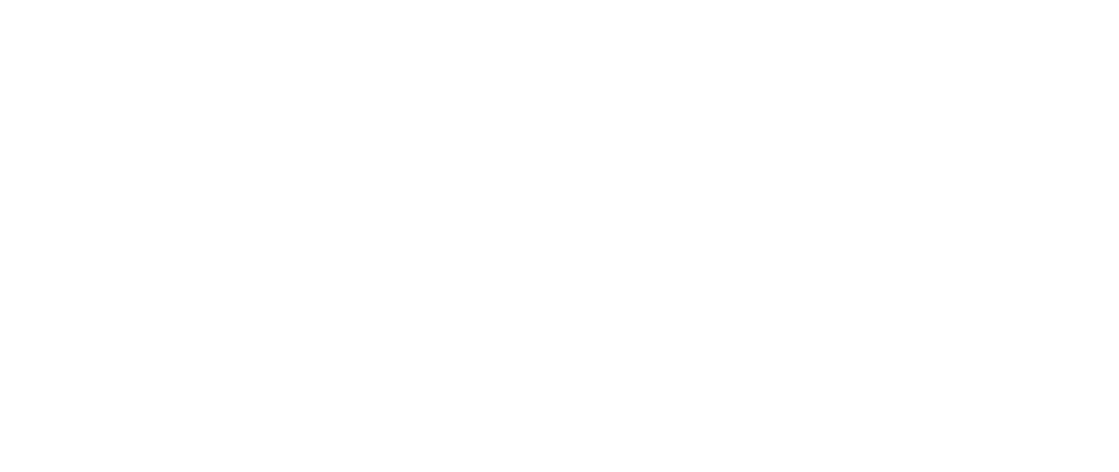

2 Leveling by U. S. Engineer Department.

412. George K. Miller. SE $1 / 4$ NE $1 / 4$ sec. 26, T. 9 S., R. 2 W. Domestic well, driven $11 / 2$ inches in diameter and 13.3 feet deep, standard pipe casing. Measuring point is top of $11 / 2$-inch standpipe, 2.8 feet above land surface and 350.63 feet 2 above mean sea level.

\begin{tabular}{|c|c|c|c|c|c|c|c|}
\hline Date & $\begin{array}{l}\text { Water } \\
\text { level } \\
\text { (feet) }\end{array}$ & Date & $\begin{array}{l}\text { Water } \\
\text { level } \\
\text { (feet) }\end{array}$ & Date & $\begin{array}{l}\text { Water } \\
\text { level } \\
\text { (feet) }\end{array}$ & Date & $\begin{array}{l}\text { Water } \\
\text { level } \\
\text { (feet) }\end{array}$ \\
\hline 1935 & & 1935 & & 1936 & & 1936 & \\
\hline $\begin{array}{l}\text { Oct. } 10 \\
\text { Oct. } 16 \\
\text { Oct. } 24 \\
\text { Oct. } 28 \\
\text { Nov. } 5 \\
\text { Nov. } 12 \\
\end{array}$ & $\begin{array}{l}337.93 \\
338.11 \\
338.05 \\
338.05 \\
338.13 \\
338.11\end{array}$ & $\begin{array}{l}\text { Nov. } 19 \\
\text { Dec. } 4 \\
\text { Dec. } 10 \\
\text { Dec. } 18 \\
\text { Dec. } 24 \\
\text { Dec. } 31\end{array}$ & $\begin{array}{l}338.27 \\
338.44 \\
338,38 \\
338.44 \\
338.76 \\
339.06\end{array}$ & $\begin{array}{l}\text { Jan. } 7 \\
\text { Jan. } 14 \\
\text { Jan. } 21 \\
\text { Jan. } 28 \\
\text { Feb. 4 }\end{array}$ & $\begin{array}{l}341.38 \\
343.73 \\
343.35 \\
342.47 \\
341.83\end{array}$ & $\begin{array}{l}\text { Feb. } 11 \\
\text { Feb. } 19 \\
\text { Feb. } 25 \\
\text { Mar. } 3 \\
\text { Mar. } 10\end{array}$ & $\begin{array}{l}341.47 \\
341.28 \\
343.86 \\
340.85 \\
339.67\end{array}$ \\
\hline
\end{tabular}

2 Leveling by U. S. Engineer Department. 
TABLE 13.-Ground-water levels in observation wells, 1928-30 and 1935-36-Con.

414. Government Land Bank (Cliff Donner, lessor). NW1/4NE1/4 sec. 34 . T. 9 S., R. 2 W. Stock well, dug 8 feet square and 18.8 feet deep. Established as observation well in lieu of well 412 . Measuring point is top of 2-inch plank deck, through crack near draft pipe, 0.4 foot above land surface and 325.14 feet 2 above mean sea level.

\begin{tabular}{|c|c|c|c|c|c|c|c|}
\hline Date & $\begin{array}{c}\text { Water } \\
\text { level } \\
\text { (feet) }\end{array}$ & Date & $\begin{array}{l}\text { Water } \\
\text { level } \\
\text { (feet) }\end{array}$ & Date & $\begin{array}{l}\text { Water } \\
\text { level } \\
\text { (feet) }\end{array}$ & Date & $\begin{array}{l}\text { Water } \\
\text { level } \\
\text { (feet) }\end{array}$ \\
\hline 1936 & & 1936 & & 1936 & & 1936 & \multirow{10}{*}{$\begin{array}{l}309.86 \\
309.10 \\
308.96 \\
309.02 \\
310.32 \\
310.17 \\
309.81 \\
\mathbf{3 1 0 . 2 3}\end{array}$} \\
\hline Feb. 19 & 315. 19 & Apr. 21. & 314.40 & June 16 & 311.59 & Aug. 11 & \\
\hline Feb. 25 & 318.14 & Apr, 28 & 313.36 & June 23 & 311.39 & Aug. 18 & \\
\hline Mar. 3. & 317.75 & May 5 & 312.81 & June 30 & 311.05 & Aug. 25 & \\
\hline Mar. 10 & 316.24 & May 12 & 312.60 & July 9. & 311.20 & Sept. 1 & \\
\hline Mar. 17 & 315.24 & May 19 & 312.33 & July 14 & 310.90 & Sept. 9 & \\
\hline Mar. 24 & 314.64 & May 26 & 312.42 & July 21 & 310.71 & Sept. 15 & \\
\hline Mar. 31 & 315.00 & June 2. & 311.76 & July 28 & 310.34 & Sept. 22 & \\
\hline Apr. 7 & 315.04 & June 9. & 311.90 & Aug. 4 & 310.20 & Sept. 29 & \\
\hline Apr. 14 & 314.72 & & & & & & \\
\hline
\end{tabular}

${ }^{2}$ Leveling by U. S. Engineer Department.

415. Mrs. G. M. H. Brewer. NE1/4NE1/4 sec. 7, T. 9 S., R. 1 W. Unused well, dug 16 feet deep, backfilled around standpipe. Measuring point is top of pump coupling on standpipe, 3.6 feet above land surface and 399.91 feet $^{2}$ above mean sea level.

\begin{tabular}{|c|c|c|c|c|c|c|c|}
\hline Date & $\begin{array}{l}\text { Water } \\
\text { level } \\
\text { (feet) }\end{array}$ & Date & $\begin{array}{l}\text { Water } \\
\text { level } \\
\text { (feet) }\end{array}$ & Date & $\begin{array}{l}\text { Water } \\
\text { level } \\
\text { (feet) }\end{array}$ & Date & $\begin{array}{l}\text { Water } \\
\text { level } \\
\text { (feet) }\end{array}$ \\
\hline 1928 & & 1933 & \multirow{10}{*}{$\begin{array}{l}394.32 \\
393.22 \\
392.64 \\
392.75 \\
393.69 \\
394.26 \\
393.66 \\
393.59 \\
393.17 \\
393.83 \\
393.49 \\
393.72\end{array}$} & 1936 & \multirow{10}{*}{$\begin{array}{l}392.55 \\
391.98 \\
391.91 \\
391.64 \\
392.03 \\
392.35 \\
392.23 \\
391.69 \\
391.25 \\
391.75 \\
391.67 \\
390.89\end{array}$} & 1936 & \multirow{10}{*}{$\begin{array}{l}390.97 \\
390.74 \\
390.43 \\
390.74 \\
390.41 \\
390.97 \\
391.09 \\
390.87 \\
390.94 \\
390.84 \\
391.05 \\
390.88\end{array}$} \\
\hline Aug. 31 & 392.16 & Jan. 21.. & & Apr. 14. & & July 14 & \\
\hline Oct. 20 & 389.2 & Jan. 28 & & Apr. 21 & & July 21 . & \\
\hline 1935 & & Feb. 11 & & $\begin{array}{l}\text { Apr. } 28 . \\
\text { May } 5 .\end{array}$ & & $\begin{array}{l}\text { July } 28 \text { - } \\
\text { Aug. } 4\end{array}$ & \\
\hline & & Feb. 19 & & May 12 & & Aug. 11 & \\
\hline $\begin{array}{l}\text { Dec. } 16 \\
\text { Dec } 24\end{array}$ & 392.62 & Feb. 25 & & May $19 \ldots$ & & Aug. 18.: & \\
\hline $\begin{array}{l}\text { Dec. } 24 \\
\text { Dec. } 31\end{array}$ & $\begin{array}{l}392.24 \\
393.84\end{array}$ & Mar. 10. & & June $2 \ldots$ & & $\begin{array}{l}\text { Aug. 25 } \\
\text { Sept. 1... }\end{array}$ & \\
\hline 1936 & & $\begin{array}{l}\text { Mar. } 17 \\
\text { Mar. } 24\end{array}$ & & $\begin{array}{l}\text { June } 9 \\
\text { June } 16 .\end{array}$ & & $\begin{array}{l}\text { Sept. } 9 \\
\text { Sept. } 15\end{array}$ & \\
\hline & & Mar, 31 & & June 23 & & Sept. 22 & \\
\hline $\begin{array}{l}\text { Jan. } 7 \\
\text { Jan. } 14\end{array}$ & $\begin{array}{l}394.85 \\
394.71\end{array}$ & & & July $9 . \ldots$ & & & \\
\hline
\end{tabular}

${ }^{2}$ Leveling by U. S. Engineer Department.

421. Fenry Hoefer. SE1/4NW1/4 sec. 12, T, $10 \mathrm{~S}$., R, 4 W. Unused well, dug 24 inches in diameter and 24.5 feet deep, concrete-tile casing, in yard southwest of residence. Measuring point is top of casing, 1.7 feet above land surface and 187.44 feet 2 above mean sea level.

\begin{tabular}{|c|c|c|c|c|c|c|c|}
\hline Date & $\begin{array}{l}\text { Water } \\
\text { level } \\
\text { (feet) }\end{array}$ & Date & $\begin{array}{l}\text { Water } \\
\text { level } \\
\text { (feet) }\end{array}$ & Date & $\begin{array}{l}\text { Water } \\
\text { level } \\
\text { (feet) }\end{array}$ & Date & $\begin{array}{l}\text { Water } \\
\text { level } \\
\text { (feet) }\end{array}$ \\
\hline 1928 & & 1930 & & 1936 & & 1936 & \\
\hline Aug. 1. & 165.64 & Apr. 27. & 167.24 & Jan. $10_{-}$ & 175.84 & June 2 & 166.94 \\
\hline Sept. 7 & 164.49 & May 29 . & 166.79 & Jan. 14 & 17S. 12 & June 9 & 166.81 \\
\hline Oct. $20_{-}$ & 163.8 & July 24 & 165.55 & Jan. 21 & 171.79 & June 16 & 166.73 \\
\hline Dec. 17 & 166. 2 & & & Jan. 24 & 171. 09 & June 23 & 166.62 \\
\hline & & 1935 & & Jan. 28 & 170. 09 & June $30 \ldots$ & 166. 33 \\
\hline 1929 & & Oct, 4 & 163.44 & $\begin{array}{l}\text { Jan. } 31 \\
\text { Feb } 4\end{array}$ & $\begin{array}{l}\text { I71. } 59 \\
168.71\end{array}$ & $\begin{array}{l}\text { July } 9 \\
\text { July } 14\end{array}$ & $\begin{array}{l}166.30 \\
166.29\end{array}$ \\
\hline Feb. 23 & 168.0 & Oct. 11 & 163.24 & Feb. 12 & 168.89 & July 21 & 166. 79 \\
\hline Mar. 19 & 167.3 & Oct. 17 & 163.24 & Feb. 20 & 170.03 & July $28 \ldots$ & 165.63 \\
\hline May 12 & 167.7 & Oct. 25 & I 63.24 & Feb. 25 & 170.32 & Aug. 4 & 165.34 \\
\hline June $7 \ldots$ & 167.14 & Oct. $28 \ldots$ & 163.24 & Mar. 3 & 170.57 & Aug. $11 \ldots$ & 164.64 \\
\hline July $3 \ldots$ & 166.64 & Nov. 5 & 163.04 & Mar. 10 & 171.12 & Aug. 18 & 165.09 \\
\hline Aug. $3 \ldots$ & 165.79 & Nov. 12 & 163.14 & Mar. 17 & 171.78 & Aug. 25 & 164.80 \\
\hline Sept. 24 & 164.19 & Nov. 20. & 163.14 & Mar. 24 & 167.89 & Sept. 1 & 164.73 \\
\hline Oct. 26 & 163. 64 & Dec. 4 & 163.44 & Mar. 31 & 168.04 & Sept. 9. & 164.48 \\
\hline Nov. 28 & 163. 29 & Dec. 10 & 162.54 & Apr. 7 & 167.69 & Sept. 15 & 164. 69 \\
\hline Dec. 29 & 167.74 & Dec. 18 & 162.69 & Apr. 14 & 167.79 & Sept. 22 & 163.91 \\
\hline 1930 & & & 164 & $\begin{array}{l}\text { Apr. } 21 \\
\text { Apr. } 28 \ldots\end{array}$ & $\begin{array}{l}167.33 \\
167.49\end{array}$ & sept. & 163.68 \\
\hline & & 1936 & & May 5 & 167.39 & & \\
\hline $\mathrm{Fe}$ & 167.94 & & & May 12 & I $66.6 \mathrm{I}$ & & \\
\hline$\ldots$ & 169.04 & Jan. 1 & 166.29 & May 19 & 167.42 & & \\
\hline $\mathrm{Ma}$ & 166.79 & Jan. 7 & 172.34 & May 26 & 167.71 & & \\
\hline
\end{tabular}

${ }^{2}$ Leveling by U. S. Engineer Department. 
TABLE 13.-Ground-water levels in observation wells, 1928-30 and 1935-38-Con.

422. A. E. Holmes. NE1/4NE1/4 sec. 15, T. $10 \mathrm{~S}$, R. 4 W. Stock well, driven 23 feet deep. Measuring point is top of draft pipe (after unscrewing pump, nipple, and coupling), 2.5 feet above land surface and 189.34 feet $^{2}$ above mean sea level.

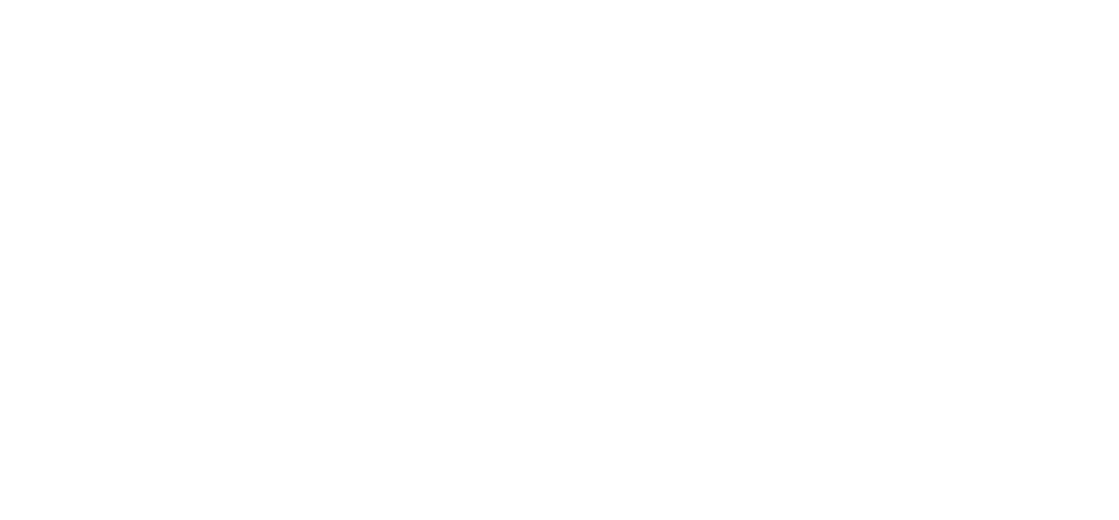

2 Leveling by U. S. Engineer Department.

425. Southern Pacific Railroad (Wellsdale). NW1/4SW1/4 sec. 17, T. 10 S., R. 4 W. Unused well, dug 6 feet in diameter and 28.5 feet deep, octagonal timber curb. Measuring point is top of 3 -inch plank deck, 1.3 feet a bove land surface and 217.15 feet ${ }^{2}$ a bove mean sea level.

\begin{tabular}{|c|c|c|c|c|c|c|c|}
\hline Date & $\begin{array}{l}\text { Water } \\
\text { level } \\
\text { (feet) }\end{array}$ & Date & $\begin{array}{l}\text { Water } \\
\text { level } \\
\text { (feet) }\end{array}$ & Date & $\begin{array}{l}\text { Water } \\
\text { level } \\
\text { (feet) }\end{array}$ & Date & $\begin{array}{l}\text { Water } \\
\text { level } \\
\text { (feet) }\end{array}$ \\
\hline 1935 & & 1936 & & 1936 & & 1936 & \\
\hline Oct. 24 & 198.18 & Jan. 14 & 214.94 & Apr. 15 & 213.88 & July 15 & 208.48 \\
\hline Oct. 29 & 198.06 & Jan. 21 & 215.01 & Apr. 22 & 212.66 & July 22 & 207.82 \\
\hline Nov. 6 & 197.95 & Jan. 28. & 213.98 & Apr. 29 & 212.58 & July 29. & 206.69 \\
\hline Nov. 12 . & 198. 09 & Feb. 4 & 214.68 & May 6.- & 212.28 & Aug. 5 & 206. 32 \\
\hline Nov. 19 & 198. 23 & Feb. 11 & 213.88 & May 13 & 212.31 & Aug. 12 & 204.77 \\
\hline Nov, 27 & 198.10 & Feb. 20 & 213.52 & May 20 . & 212. 63 & Aug. 19 & 204.43 \\
\hline Dec. 4 & 197.98 & Feb. 26 & 214.79 & May 27. & 211.87 & Aug. 26 & 203.08 \\
\hline Dec. 10 & 198.81 & Mar. 3 & $\begin{array}{l}213.98 \\
213.98\end{array}$ & June 3 & $\begin{array}{l}211.39 \\
219\end{array}$ & Sept. 2 & 202.93 \\
\hline Dec. 17 & 200.12 & Mar. 10 & 213.94 & June 10 & 210.97 & Sept. 10. & 201.68 \\
\hline Dec. 24 & 200.36 & Mar. 17_ & 213.72 & June 17 & 210.61 & Sept. 15 . & 201.36 \\
\hline Dec. 31 & 211. 39 & Mar. 24 & 213.33 & June 24 & 210.16 & Sept. 23 & 200.83 \\
\hline 1936 & & Apr. 1. & 213.29 & July 1- - - & 209.65 & Sept. 30 & 200.66 \\
\hline Jan. 8 . & 214.49 & Apr. 8 & 213. 33 & July 8... & 209.13 & & \\
\hline
\end{tabular}

${ }^{2}$ Leveling by U. S. Engineer Department.

426. L. C. Williamson. SE $1 / 4$ NE $1 / 4$ sec. 18 , T. 10 S.. R. 4 W. Stock well, dug 4 feet in diameter and 28.8 feet deep, brick curb. Measuring point is top of brick curb, at south side, 0.3 foot above land surface and 218.44 feet ${ }^{2}$ a bove mean sea level.

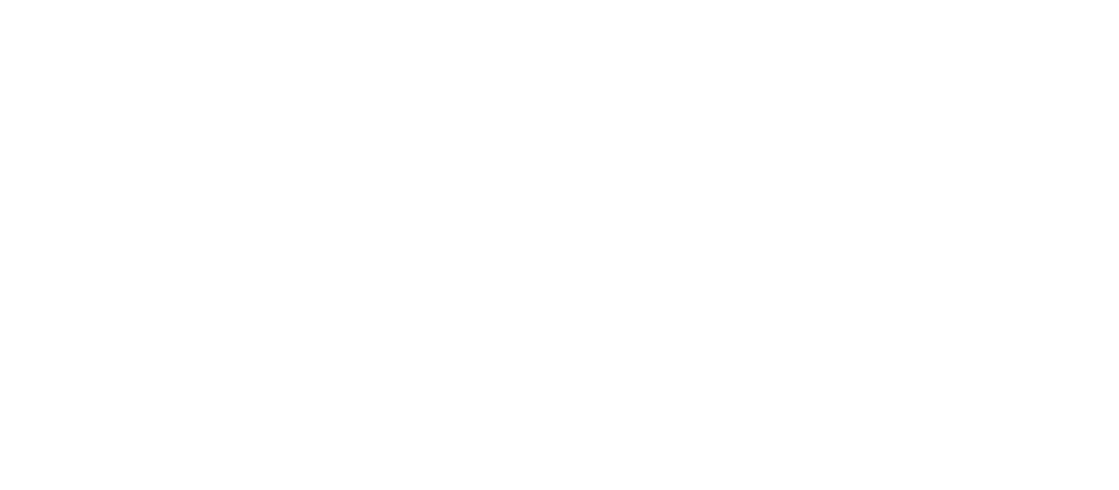

${ }^{2}$ Leveling by U. S. Engineer Department.

s Pump operating in well. 
TABLE 13.-Ground-water levels in observation wells, 1928-30 and 1935-36-Con.

433. C. B. Harnich. NE $1 / 4$ NE1/4 sec. 8, T. $10 \mathrm{~S} .$, R. 3 W. Stock well, driven $11 / 2$ in ches in diameter and 17.7 feet deep. Measuring point is bottom of pump flange, 3.0 feet above land surface and 199.52 feet ${ }^{2}$ above mean sea level.

\begin{tabular}{|c|c|c|c|c|c|c|c|}
\hline Date & $\begin{array}{l}\text { Water } \\
\text { level } \\
\text { (feet) }\end{array}$ & Date & $\begin{array}{l}\text { Water } \\
\text { level } \\
\text { (feet) }\end{array}$ & Date & $\begin{array}{l}\text { Water } \\
\text { level } \\
\text { (feet) }\end{array}$ & Date & $\begin{array}{l}\text { Water } \\
\text { level } \\
\text { (feet) }\end{array}$ \\
\hline 1936 & & 1936 & & 1936 & & 1936 & \\
\hline $\begin{array}{l}\text { Apr. } 27 \\
\text { May } 5 \\
\text { May } 12 \\
\text { May } 19 \\
\text { May } 26 \\
\text { June } 2\end{array}$ & $\begin{array}{l}184.24 \\
185.28 \\
184.35 \\
184.88 \\
184.32 \\
183.55\end{array}$ & $\begin{array}{l}\text { June } 9 \\
\text { June } 16 \\
\text { June } 23 \\
\text { June } 30 \\
\text { July } 9 \\
\text { July } 14\end{array}$ & $\begin{array}{l}184.91 \\
184.84 \\
184.78 \\
184.25 \\
184.33 \\
184.23\end{array}$ & $\begin{array}{l}\text { July } 21 . \ldots \\
\text { July } 28 \\
\text { Aug. } 4 \\
\text { Aug. } 11 \\
\text { Aug. } 18 \\
\text { Aug. } 25\end{array}$ & $\begin{array}{l}\mathbf{1 8 3 . 7 8} \\
\text { 183. } 67 \\
183.79 \\
182.69 \\
183.32 \\
\mathbf{1 8 3 . 4 4}\end{array}$ & $\begin{array}{l}\text { Sept. } 1 \\
\text { Sept. } 9 \\
\text { Sept. 15 } \\
\text { Sept. } 22\end{array}$ & $\begin{array}{l}183.33 \\
182.83 \\
182.73 \\
182.33\end{array}$ \\
\hline
\end{tabular}

${ }^{2}$ Leveling by U. S. Engineer Department.

438. J. G. Gourley. NW1/4SE1/4 sec. 21 , T. $10 \mathrm{~S}$., R. 3 W. Stock well, dug 6 feet in diameter and 17.9 feet deep, dry-masonry curb. Measuring point is top of curb, at base of pump, 0.3 foot above land surface and 242.53 feet ${ }^{2}$ a bove mean sea level.

\begin{tabular}{|c|c|c|c|c|c|c|c|}
\hline Date & $\begin{array}{l}\text { Water } \\
\text { level } \\
\text { (feet) }\end{array}$ & Date & $\begin{array}{l}\text { Water } \\
\text { level } \\
\text { (feet) }\end{array}$ & Date & $\begin{array}{l}\text { Water } \\
\text { level } \\
\text { (feet) }\end{array}$ & Date & $\begin{array}{l}\text { Water } \\
\text { level } \\
\text { (feet) }\end{array}$ \\
\hline 1935 & & 1936 & & 1936 & & 1936 & \\
\hline Oct. 11 & 225.51 & Jan. 7 & 240.84 & Mar. 24 & 239.12 & June 30 . & 232.53 \\
\hline Oct. 17 - & 225.94 & Jan. $10 \ldots$ & 241.01 & Mar. 31 & 240.18 & July 9 . & 230.99 \\
\hline Oct. 29 & 226. 06 & Jan. 14 & 241.14 & Apr. 7 & 239.85 & July 21 . & 230.19 \\
\hline Nov. 5 & 225.85 & Jan. 21. & 241.08 & Apr. 14 & 239.27 & July 28 & 229.86 \\
\hline Nov. 12 & 225.93 & Jan. 24 & 240.41 & Apr. 21 . & 237.98 & Aug. 4 & 229.13 \\
\hline Nov. 20 & 226.18 & Jan. 28 & 238.49 & A pr. 28 & 236.74 & Aug. 11 . & 228.93 \\
\hline Dec. 4 . & 226.20 & Jan. 31 & 239. 71 & May 5 & 236.42 & Aug. 18. & 228.80 \\
\hline Dec. 10 & 226.79 & Feb. 4 & 236.49 & May 12 & 238.56 & Aug. 25 - & 227.47 \\
\hline Dec. 18 . & 227.44 & Feb. 12 & 236. 21 & May 19 & 239.00 & Sept. 1 & 227.28 \\
\hline Dec. 24 & 229.15 & Feb. 20 & 235.25 & May 26 & 237.76 & Sept. 9 & 226.80 \\
\hline 1936 & & $\begin{array}{l}\text { Feb. } 25 \\
\text { Mar } 3\end{array}$ & $\begin{array}{l}240.78 \\
240.18\end{array}$ & $\begin{array}{l}\text { June 2- } \\
\text { June 9- }\end{array}$ & $\begin{array}{l}236.78 \\
235,67\end{array}$ & $\begin{array}{l}\text { Sept. } 15 \\
\text { Sept. } 22\end{array}$ & $\begin{array}{l}226.93 \\
226.50\end{array}$ \\
\hline & & Mar. 10 & 239.41 & June 16 & 235.58 & Sept. 29 & 226.04 \\
\hline Jan. $1 \ldots$ & 230.06 & Mar. 17.. & 239.01 & June 23 & 234.15 & & \\
\hline
\end{tabular}

2 Leveling by U. S. Engineer Department.

448. J. E. Watkins (formerly D. Watkins). NW1/4 NE $1 / 4$ sec. 17, T. 10 S., R. 2 W. Unused well, dug 36 inches in diameter and 27 feet deep, in rear of residence. Measuring points: Top of brick curb, level with land surface and 276.31 feet ${ }^{2}$ above mean sea level; beginning December 10 , 1935, bottom of board spanning well, level with top edge of new concrete and steel collar, 276.67 feet ${ }^{2}$ above mean sea level.

\begin{tabular}{|c|c|c|c|c|c|c|c|}
\hline Date & $\begin{array}{l}\text { Water } \\
\text { level } \\
\text { (feet) }\end{array}$ & Date & $\begin{array}{l}\text { Water } \\
\text { level } \\
\text { (feet) }\end{array}$ & Date & $\begin{array}{l}\text { Water } \\
\text { level } \\
\text { (feet) }\end{array}$ & Date & $\begin{array}{l}\text { Water } \\
\text { level } \\
\text { (feet) }\end{array}$ \\
\hline 1928 & & 1930 & & 1936 & & 1936 & \\
\hline Aug. 30 & 251.6 & Apr. 27 & 252.96 & Jan. 14 & 259. 52 & June 16 & 252.37 \\
\hline Oct. 20 . & 251.6 & May 29 & 252.96 & Jan. 21 & 255.67 & June 23 & 252.22 \\
\hline Dec. 16. & 252.5 & July 24 & 251.56 & Jan. 28 & 254.87 & June 30 & 251.95 \\
\hline 1929 & & 1935 & & Feb. 12 & 254.02 & July 14 & 251.87 \\
\hline Feb. 3 & 255.9 & Oct. 4 & 250.59 & $\begin{array}{l}\text { Feb. } 19 \\
\text { Feb. } 25\end{array}$ & $\begin{array}{l}253.67 \\
254.65\end{array}$ & $\begin{array}{l}\text { July } 21 \text { - } \\
\text { July } 28 .\end{array}$ & $\begin{array}{l}252.61 \\
251.30\end{array}$ \\
\hline Mar. 19 & 254.2 & Oct. 11 & 250.66 & Mar. 3 & 253.02 & Aug. 4 & 251.27 \\
\hline May 12 & 254.0 & Oct. 17 & 250.63 & Mar. 10 & 252.57 & Aug. 11 & 251.17 \\
\hline June 7 & 253.11 & Oct. 25 & 251,29 & Mar. 17 & 252.77 & Aug. 18 . & 250.37 \\
\hline July 23 & 252.76 & Oct. 28 & 251.26 & Mar. 24 & 251.47 & Aug. 25 . & 250.37 \\
\hline Aug. 3 & 251.76 & Nov. 12 & 251.11 & Mar. 31 & 253.48 & Sept. 1 & 250.32 \\
\hline Sept. 24 & 251. 21 & NoF. 19 & 251.01 & Apr. 7.- & 253.44 & Sept. 9 & 255.89 \\
\hline Oct. 26 & 251.11 & Dec. 4 & 250.91 & Apr. 14 & 253.03 & Sept. 15 & 255.87 \\
\hline Nov. 28 & 250.96 & Dec. 10 & 251.97 & Apr. 21 & 252.78 & Sept. 19 & 251.91 \\
\hline Dec. 30 & 253.86 & Dec. 18 & 251.95 & Apr. 28 & 252,71 & Sept. 22 & 250.88 \\
\hline & & Dec. 24 & 251.73 & May 5 & 252.67 & Sept. 29 & 250.86 \\
\hline 1930 & & & & May 12 & 253.35 & & \\
\hline Feb. 2 . & 253.81 & & & $\begin{array}{l}\text { May } 19 \\
\text { May } 26\end{array}$ & $\begin{array}{l}253.22 \\
253.27\end{array}$ & & \\
\hline Mar. & 255.31 & Jan. 1 & 253.50 & June 2 & 252.57 & & \\
\hline Mar. 3 & 253.51 & Jan. 7. & 255. 30 & June 9 & 252.47 & & \\
\hline
\end{tabular}

${ }^{2}$ Leveling by U. S. Engineer Department. 
TABLE 13.-Ground-water levels in observation wells, 1928-30 and 1935-36-Con.

453. Joe Wesley (Jan Brunt Sawmill, renter). NE1/4SW1/4 sec. 18. T. 10 S., R. 1 W. Unused well, dug 6 feet square and 11.7 feet deep. Measuring point is top at southeast corner of curb, about 1 foot above land surface and 315.26 feet ${ }^{2}$ above mean sea level.

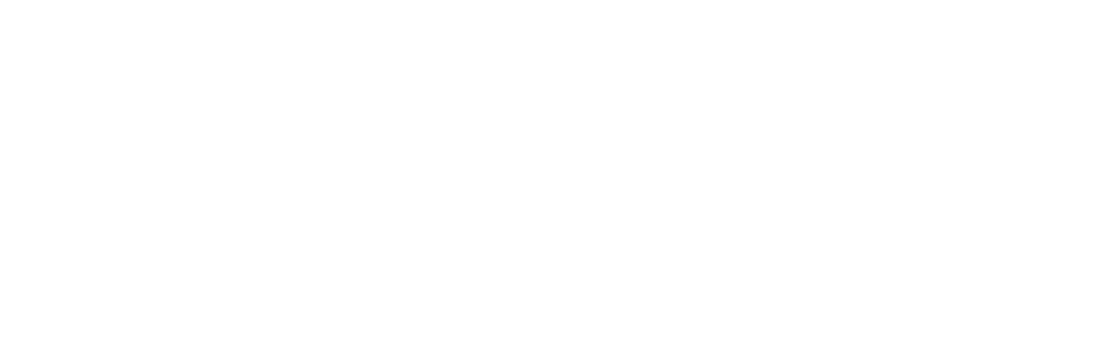

Leveling by U. s. Engineer Department.

456. Ida Locke estate (E. W. Elliott, renter). SW1/4NE1/4 sec. 13, T. $11 \mathrm{~S}$., R. 5 W. Stock well, dug $31 / 2$ feet in diameter and 25 feet deep, brick curb. Measuring point is top of 2 -inch plank deck, through opening for draft pipe, 240.34 feet 2 above mean sea level.

\begin{tabular}{|c|c|c|c|c|c|c|c|}
\hline Date & $\begin{array}{l}\text { Water } \\
\text { level } \\
\text { (feet) }\end{array}$ & Date & $\begin{array}{l}\text { Water } \\
\text { level } \\
\text { (feet) }\end{array}$ & Date & $\begin{array}{l}\text { Water } \\
\text { level } \\
\text { (feet) }\end{array}$ & Date & $\begin{array}{l}\text { Water } \\
\text { level } \\
\text { (feet) }\end{array}$ \\
\hline 1935 & & 1936 & & 1936 & & 1936 & \\
\hline Oct. 24 & 219.12 & Jan. 21 & 238. 49 & May 6 & 235.90 & Aug. 26 . & 225.81 \\
\hline Oet. 29 & 219.53 & Jan. 28 & 238.49 & May 13 & 235.85 & Sept. 2 & 225.26 \\
\hline Nov. 6. & 218.09 & Feb. 4 & 238.35 & May 20 . & 235.80 & Sept. 10 & 224.27 \\
\hline Nov. 12 & 218.67 & Feb. 11 & 237.63 & May 27 & 232. 11 & Sept. 15 & 224.02 \\
\hline Nov. 19 & 218.19 & Feb. 20 & 237.70 & June 3 & 232.30 & Sept. 23 & 224.30 \\
\hline Nov. 29 & 219.47 & Feb. 26 & 238.17 & June 10. & 231.91 & Sept. 30 & 223.91 \\
\hline Dec. 4 & 219.95 & Mar. 3 & 238.31 & June 17 - & 231. 32 & & \\
\hline Dec. 11 & 219.91 & Mar. 10 & 237.87 & June 24 & 231. 37 & & \\
\hline Dec. 18 & 219.75 & Mar. 17.- & 238.01 & July $1 \ldots$ & 230.18 & & \\
\hline Dec. 24 & 219.77 & Mar. 24 & 235.05 & July 8 & 229.90 & & \\
\hline Dec. 31 & 223.06 & Mar. 27 & 235.16 & July 15 & 229.67 & & \\
\hline 1936 & & Apr. 1 & 236.13 & July $23 \ldots$ & 228.56 & & \\
\hline & & $\begin{array}{l}\text { Apr. } 8 \\
\text { Apr. } 15\end{array}$ & $\begin{array}{l}236.01 \\
234.59\end{array}$ & $\begin{array}{l}\text { July } 29 \ldots \\
\text { Aug. } 5 \ldots\end{array}$ & $\begin{array}{l}228.26 \\
227.98\end{array}$ & & \\
\hline Jan. 8 & 223.80 & Apr. 22 & 234.62 & Aug. 12 & 227.77 & & \\
\hline Jan. 14 & 227.37 & Apr. 29 & 234.49 & Aug. 19 & 227.28 & & \\
\hline
\end{tabular}

I Leveling by U. S. Engineer Department.

459. W. J. Moyer (J. M. Pitts, renter). SE1/4SE1/4 sec. 27, T. 11 S., R. 5 W. Domestic well, driven 40.4 feet deep. Measuring point is lower valve seat of pump, 4.7 feet above land surface and 236.28 feet 2 above mean sea level.

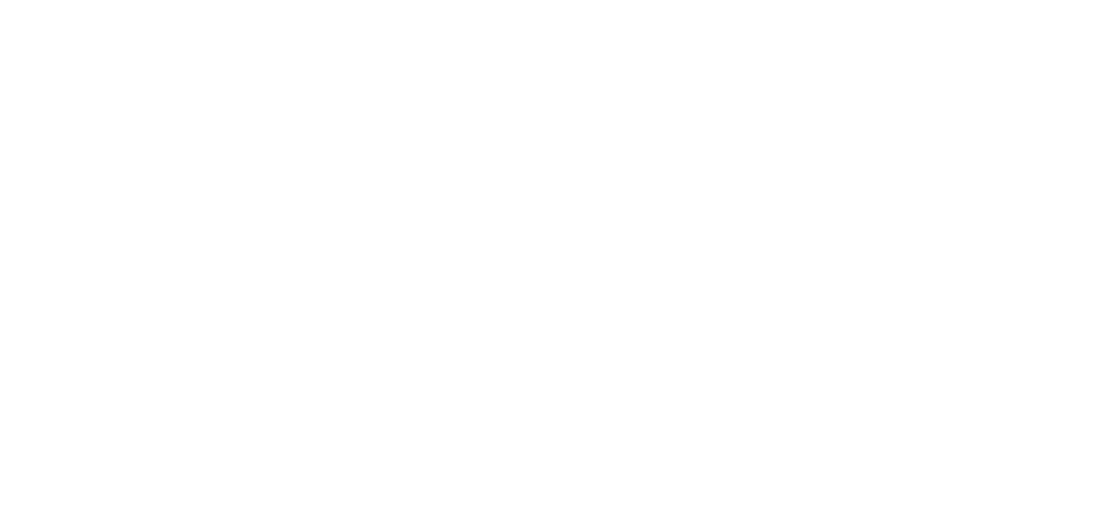

2 Leveling by U. S. Engineer Department. 
TABLE 13.-Ground-water levels in observation wells, 1928-30 and 1935-36-Con.

463. Oregon Agricultural Experiment Station, East Farm. SW $14 \mathrm{SE} 1 / 4 \mathrm{sec} .36$, T. $11 \mathrm{~S}$. R. 5 W. Irrigation well, drilled 8 inches in diameter and 42 feet deep, about 6 feet east of well 464 and in the northeast corner of a common pit. Both wells pumped by joint suction. Measuring points: July 26 and Oct. 21, 1928, top face of flange at elbow in suction pipe, 197.53 feet above mean sea level; beginning Oct. 26, 1928, top of casing (level with zero of staff gage on east wall of pit east of well), 21.1 feet below land surface and 197.17 feet above mean sea level; beginning January 22, 1936, top of 6 - by 6 -inch timber girt bolted to top of concrete pit curb, 220.97 feet above mean sea level.

\begin{tabular}{|c|c|c|c|c|c|}
\hline Date & $\begin{array}{l}\text { Water } \\
\text { level } \\
\text { (feet) }\end{array}$ & Date & $\begin{array}{c}\text { Water } \\
\text { level } \\
\text { (feet) }\end{array}$ & Date & $\begin{array}{l}\text { Water } \\
\text { level } \\
\text { (feet) }\end{array}$ \\
\hline 1928 & & 1929 & & 29 & \\
\hline July 26. & 196.21 & Feb. 14 & 199.62 & Apr. 21 & 202.67 \\
\hline Oct. 21. & 195.43 & Feb. 15 & 199.57 & Apr. 22 & 202.82 \\
\hline Oct. 26 & 195.2 & Feb. 16 & 199.57 & Apr. 23 & 202.97 \\
\hline Oct. 27 & 195.2 & Feb. 16 & 199.32 & Apr. 24 & 202.97 \\
\hline Oct. 30 & 195. 22 & Feb. 17 & 198.97 & Apr. 25 & 202.92 \\
\hline Nov. 4 & 195. 32 & Feb. 18 & 198.72 & Apr. 26 & 202.77 \\
\hline Nov. 10 & 195.47 & Feb. 19 & 198.67 & Apr. 27- & 202.62 \\
\hline Nov. 18 & 195.57 & Feb. 20 & 198. 77 & Apr. 28 & 202.47 \\
\hline Nov. 24 & 195. 67 & Feb. 21 & 198.77 & Apr. 29 & 202.37 \\
\hline Dec. 1 & 195. 82 & Feb. 22 & 198.77 & Apr. 30 & 202. 27 \\
\hline Dec. 8 & 195.93 & Feb. 23 & 198.77 & May 1. & 202.07 \\
\hline Dec. 13. & 19602 & Feb. 24 & 198.77 & May 2 & 201.92 \\
\hline Dec. $15_{-}$ & $196.6^{\prime \prime}$ & Feb. 25 & 198.77 & May 3 & 201.72 \\
\hline Dec. 18 & 196.72 & Feb. 26 & 198.87 & May 4 & 201.62 \\
\hline Dec. 19 & 196.77 & Feb. 27 & 198. 97 & May 5 & 201.57 \\
\hline Dec. 22 & 196.87 & Feb. 28 & 198.92 & May 6 & 201.47 \\
\hline Dec. 25_ & 196.97 & Mar. 1. & 198.87 & May 7 & 201.32 \\
\hline Dec. 27 & 197.17 & Mar. 2 & 198.87 & May 8 & 201.17 \\
\hline Dec. 28 & $197.4 i$ & Mar. 3 & 198.82 & May 9 & 201.07 \\
\hline Dec. 29. & 198. C2 & Mar. 4 & 198. 77 & May 10 & 200.72 \\
\hline Dec. 30 & 198.97 & Mar. 5. & 198.72 & May 11 & 200.62 \\
\hline Dec. 31 & 200.92 & Mar. 6 & 198.72 & May 12 & 200.63 \\
\hline & & $\begin{array}{l}\text { Mar. 7-- } \\
\text { Mar. 8... }\end{array}$ & $\begin{array}{l}\text { 199. } 27 \\
\text { 199.37 }\end{array}$ & $\begin{array}{l}\text { May } 13- \\
\text { May } 14\end{array}$ & $\begin{array}{l}200.47 \\
200.47\end{array}$ \\
\hline Jan. 1 & 200.92 & Mar. 9 & 199.37 & May 15. & 200.47 \\
\hline an. 2 & 201.77 & Mar. 10 & 199.17 & May 16 & 200.47 \\
\hline Jan. 3 & 202.37 & Mar. 11 & 199. 22 & May 17 & 200.42 \\
\hline & 202.47 & .12 & 199.52 & 18 & 200.42 \\
\hline Jan. 5 & 202. 67 & Mar. 13 & 199.67 & May 19 & 200.37 \\
\hline Jan. 6... & 202.97 & Mar, 14 & 199.77 & 20 & 200.32 \\
\hline JaI & 202. 97 & 15 & 199.77 & 21 & 200.27 \\
\hline Jan. 8 & 202.82 & Mar. 16 & 199. 72 & May 22 & 200.27 \\
\hline Jan. 9 & 202.07 & $\mathrm{Ma}$ & 199.62 & May 23 & 200.27 \\
\hline & 202.02 & & 198. 97 & 24 & 200.27 \\
\hline Jan. 11 & 198. 92 & 19. & 198.87 & 25 & 200.22 \\
\hline Jan. 12 & 198.67 & 20. & 198.87 & 26 & 200.17 \\
\hline & 198. $6 i$ & & 198.97 & & 200.12 \\
\hline Jan. 14 & 198. 67 & .22 & 199. 77 & M & 200.07 \\
\hline Jan. 15 & 198. 67 & 23 & 200.87 & 29 & 199.97 \\
\hline & 198. 67 & & 202.37 & & 199.87 \\
\hline Jar & 198. 67 & Mar. 25 & 202.07 & May 31 & 199.47 \\
\hline Jan. 18 & 198.67 & Mar. 26. & 201.87 & June 5 & 199. 10 \\
\hline $\mathrm{Jar}$ & 198. 67 & & 201.67 & & 199.2 \\
\hline $\operatorname{Jan} .20$ & 198.77 & Mar. 27 & 201.27 & June 7 & 199. 32 \\
\hline $\operatorname{Jan} .21$ & 199.07 & Mar. 28 & 201.52 & June 8 - & 199.4 \\
\hline & $198.9 \vec{i}$ & & 201.37 & & 199.52 \\
\hline Jan, 23 & 198.7 & Mar. 30 & 201.47 & June 10 & 199.5 \\
\hline Jan. 24 & 198.67 & Mar. 31. & 201.92 & June 11 & 199.4 \\
\hline Jar & 198.67 & & 201.32 & 12 & 199.32 \\
\hline Jan. 2 & 198.67 & Apr. 2 & 201.17 & June 13 & 199.3 \\
\hline Jan. 27 & 198. 92 & Apr. 3 & 201.42 & Jine 14 & 199.22 \\
\hline & 199.67 & & 200.82 & Jume 15 & 199.2 \\
\hline Jan. 29 & 200.67 & 4 & 200.72 & June 16 & 199.2 \\
\hline Jan. 30 & 200.67 & 5 & 200.42 & Jume 17 & 199.4 \\
\hline & 200.67 & & 200.22 & Jujle 18 & 199.47 \\
\hline & 200.77 & & 200.07 & June 19 & 199.42 \\
\hline Fel & 200.77 & A & 199.92 & June 20 & 199.4 \\
\hline $\mathrm{Fe}$ & 201.02 & & 199.87 & June 21 & 199.3 \\
\hline & 201.77 & $\mathrm{Al}_{1}$ & 199.87 & June 22 & 199.2 \\
\hline Feb. 5 . - & 201.92 & & 199.87 & June 23 & 199.1 \\
\hline Fel & 202.02 & & 199.87 & June 24 & 199.0 \\
\hline Fel & 202.02 & & 199.87 & June 25 . & 198.9 \\
\hline Fel & 202.02 & Apr. 14 & 200.12 & June $26,7: 45$ a. $\mathrm{m}$. & 198.8 \\
\hline Feb. 8 - & 201.92 & 15 & 200.37 & June $26,3: 50 \mathrm{p} . \mathrm{m}$ & 5195.8 \\
\hline Fel & 201.67 & 16. & 200.77 & June $27,8: 00$ a. $\mathrm{m}$ & 198.7 \\
\hline Feb. 10 & 201.17 & $17 \ldots$ & 202.57 & June $27,5: 00$ p. m & 6195.7 \\
\hline Fel & 200.52 & 18 & 202.87 & June $28,7: 45$ a. $\mathrm{m}$ & 198.6 \\
\hline Feb & 199. 97 & Apr. 19 & 202.87 & June $28,5: 00$ p. m & 5195.7 \\
\hline Feb. 13. & 199.72 & Apr. 20 & 202.77 & June $29,7: 45$ a. $\mathrm{m}$ & 198.5 \\
\hline
\end{tabular}

- Pump operating in well. 
TABLE 13.-Ground-water levels in observation wells, 1928-30 and 1935-36-Con.

\begin{tabular}{|c|c|c|c|c|c|}
\hline Date & $\begin{array}{l}\text { Water } \\
\text { level } \\
\text { (feet) }\end{array}$ & Date & $\begin{array}{l}\text { Water } \\
\text { level } \\
\text { (feet) }\end{array}$ & Date & $\begin{array}{l}\text { Water } \\
\text { level } \\
\text { (feet) }\end{array}$ \\
\hline 1929 & & & & 1929 & \\
\hline June $29,5: 00$ p. m & 5195.6 & Aug. 22 & 195.67 & Nov. 5 & 194. 93 \\
\hline & 198.4 & Aug. 23 . & 195. 63 & & 194. 93 \\
\hline uly $2.8 .10 \mathrm{am}$ & 198. 3 & Aug. 24. & 195.61 & Nov. 7 & 194. 93 \\
\hline July $2,8: 10$ a. $\mathrm{m}$ & 198.1 & Aug. 25- & 195.57 & Nov. 8 & 194. 92 \\
\hline $\begin{array}{l}\text { July } 2,5: 10 \text { p. m } \\
\text { July } 3,8: 00 \text { a. m }\end{array}$ & $\begin{array}{r}5195.2 \\
198.0\end{array}$ & $\begin{array}{l}\text { Aug. } 26 \\
\text { Aug. 27. }\end{array}$ & $\begin{array}{l}195.53 \\
195.49\end{array}$ & Nov. 9 & 194. 92 \\
\hline July $3,5: 10$ p. m & $\begin{array}{l}198.0 \\
5193.9\end{array}$ & Aug. 28 . & $\begin{array}{l}195.49 \\
195.47\end{array}$ & Nov. 11 & $\begin{array}{l}194.92 \\
194.93\end{array}$ \\
\hline July 4 & 197.9 & Aug. 29 . & 195.45 & Nov. 12 & 194. 94 \\
\hline July $5,7: 45$ a. m & 197.7 & Aug. 30 & 195. 43 & Nov. 13. & 194.95 \\
\hline July 5, 5:00 & S 193.7 & Aug. 31 & 195.41 & Nov. 14 & 194. 94 \\
\hline July $6,7: 55$ & 197.6 & Sept. 1 .. & 195. 39 & Nov. 15 & 194. 93 \\
\hline July $6,5: 15$ & $\begin{array}{ll}5 & 193.7\end{array}$ & Sept. $2 \ldots$ & 195.37 & Nov. 16 & 194. 92 \\
\hline July 7 & 197.4 & Sept. 3 & 195. 35 & Nov. 17 & 194.91 \\
\hline July $8,7: 30$ & 197.3 & Sept. 4 & 195. 35 & 18 & 194.91 \\
\hline July $8,4: 00$ & 5193.6 & Sept. 5 . & 195. 34 & Nov. 19 & 194.90 \\
\hline $7: 30$ & 197.3 & Sept. 6. & 195.32 & Nov. 20 & 194.89 \\
\hline $5 \cdot 30$ & 5193.6 & 7 & 195.30 & 21 & 194.88 \\
\hline July & 197.2 & 8 & 195.29 & Nov. 22 & 194.87 \\
\hline July 10,5 : & 5193.3 & 9 & 195 & Nov. 23 & 194.87 \\
\hline Jul & 197.2 & 10 & 195.23 & .24 & 194.87 \\
\hline Julj & 5193.3 & Sept. 11. & 195.23 & Nov. 25 & 194.87 \\
\hline $\mathrm{Jul}$ & 197.2 & 12 & 19 & 26 & 194. 89 \\
\hline Jul & 197.1 & 13 & 195.22 & Nov. 27 , & 194.85 \\
\hline Jul & 197.1 & Sept. 14 & 195. 22 & Nov. 27 , & 194.87 \\
\hline & 197.0 & 15 & 19 & No & 194.85 \\
\hline & 197.0 & & 195.21 & 29 . & 194.83 \\
\hline $\mathrm{Ju}$ & 196.9 & 17 & 195.20 & 30 & 194.82 \\
\hline & 196.9 & & 19 & & 19 \\
\hline & 196.9 & & 195.19 & & 194.82 \\
\hline Jul & 197.0 & .20 & 195.19 & Dec. 3 & 194.82 \\
\hline & 196.9 & & & $\mathrm{D}$ & 194.82 \\
\hline & 196 & & & & 19 \\
\hline $7: 10$ & 196.8 & 23 & 195.20 & Dec. 6 . & 194.82 \\
\hline $3: 30$ & $\begin{array}{l}5193.2 \\
\end{array}$ & 23, & 195 & De & 194.83 \\
\hline 45 & 196.8 & 24 & 195 & & 194.83 \\
\hline 4:00 p. m & $\begin{array}{l}5193.2 \\
\end{array}$ & 25 & 195.19 & 9 & 194.84 \\
\hline 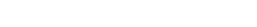 & 196.7 & 6 & 19 & De & 194 \\
\hline & & & & & 19 \\
\hline & 196. 6 & 8 & 195 & 12 & 195. \\
\hline - & 196.5 & & 19 & De & 195. \\
\hline & 196 & & & $\mathrm{D}$ & 19 \\
\hline$\pi$ & 196.4 & & 195. & 15 & 196. \\
\hline $\mathrm{J}$ & 196.3 & & 19 & $\mathrm{D}$ & 198. \\
\hline & 196 & & & & 19 \\
\hline $2,8: 15$ & 196. 36 & 4. & 195.12 & & 199.37 \\
\hline Aug. $2,5: 30$ p. m.. & 196. & 5 & 19511 & Dec. 19 & 199.97 \\
\hline & & & 19 & & 20 \\
\hline A & 196.18 & & 19509 & $\mathrm{D}$ & 210.49 \\
\hline 5 & 196. & & 19 & & 209.27 \\
\hline $6 \quad 7.50$ & & & & & 20 \\
\hline Aug. $6,12: 0$ & 5192. & & 195. & & \\
\hline $\mathrm{m}$ & 196.02 & & 195.11 & De & 208. \\
\hline A & 519 & & & & 20 \\
\hline $\mathbf{m}_{-}$ & 196.00 & 3 & & & \\
\hline $8,5: 00$ & 5192.33 & & 19 & $\mathrm{D}$ & 207.42 \\
\hline & & & & & $20 \% .27$ \\
\hline m. & 5 192.22 & Oct. 16 . & 195 & & 20 \\
\hline $\mathrm{m}$ & 195.90 & . & 195 & De & 204.47 \\
\hline $\mathrm{Au}$ & 519 & & & Dec. $31,12: 00$ & 206.17 \\
\hline & & & & & \\
\hline $\mathrm{m}$ & 195. & & 195 & 1930 & \\
\hline Al & ${ }^{5} 192$. & & & & \\
\hline & 195.83 & & 195 & Jar & 205.64 \\
\hline m & $\begin{array}{l}6 \\
6 \\
192.47\end{array}$ & & & & \\
\hline $\mathrm{m}$ & & & & Ja & 203.27 \\
\hline $\mathrm{m}$ & 5192.37 & 12.45 & 195 & & 202.77 \\
\hline $\mathrm{m}$ & 195.76 & Oct. $25,1: 40 \mathrm{p}$ & 195 & Jan. 5 & 202. 62 \\
\hline $15,5: 10$ p. m & $\begin{array}{l}5 \\
5 \\
192.22\end{array}$ & Oct. 26 . & 1950 & & 202.57 \\
\hline $\mathrm{m}$ & 195. 75 & 0 & 195.01 & & 202.47 \\
\hline $\mathrm{Au}$ & $\begin{array}{l}5 \\
192.22\end{array}$ & & 195.00 & Jan. 8 & 202.42 \\
\hline $\mathbf{A}$ & 195. 70 & Oct. 29 & 194.97 & Jan. 9 & 202.17 \\
\hline p. $\mathrm{m}_{\text {- }}$ & ${ }^{5} 192.32$ & Oet. 30 & 194.94 & Jan. 10 & 201.97 \\
\hline a. $\mathrm{m}$ & 195.70 & Oet. 31 . & 194.91 & Jan. 11 & 201. 53 \\
\hline $18,5: 15$ p. $\mathrm{m}$ & 5192.27 & & 194.91 & Jan. 12 & 201.77 \\
\hline $\mathrm{Au}$ & 195. & & 194. & Jan. 13 & 200.99 \\
\hline $\mathrm{Au}$ & 195.71 & Nov & 194.92 & Jar & 201.01 \\
\hline Aug. & 195.69 & Nov. 4 & 194. 92 & Jan. 15 & 200.61 \\
\hline
\end{tabular}

${ }^{5}$ Pump operating in well. 
TABLE 13.-Ground-water levels in observation wells, 1928-30 and 1935-36-Con.

\begin{tabular}{|c|c|c|c|c|c|}
\hline Date & $\begin{array}{l}\text { Water } \\
\text { level } \\
\text { (feet) }\end{array}$ & Date & $\begin{array}{l}\text { Water } \\
\text { level } \\
\text { (feet) }\end{array}$ & Date & $\begin{array}{l}\text { Water } \\
\text { level } \\
\text { (feet) }\end{array}$ \\
\hline 1930 & & 1930 & & 1930 & \\
\hline Jan. 16.. & 200.57 & Apr. 1 & 199.42 & June $14,8: 05$ a. $\mathrm{m}$ & 197.97 \\
\hline Jan. 17.- & 200.53 & Apr. 2 & 199. 32 & 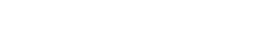 & 5192.22 \\
\hline Jan. 18 & 200.27 & 3. & 199.22 & June 15. & 197.87 \\
\hline Jan. 19 & 200.19 & n-m- & 199.12 & June $16,8: 10$ a. $m \ldots$ & 197.77 \\
\hline 20 & 200.15 & - n- & 199.07 & June $16,4: 30$ p. m $\ldots$ & 5192.22 \\
\hline 21 & 200.08 & r. $6 \ldots \ldots$ & 199.02 & June 17 & 197.57 \\
\hline$\ldots$ & 199.91 & r. $7 y_{0}$ & 199.02 & June $18,7: 30$ a. m.... & 197.51 \\
\hline 23 & 89 & - n & 198.97 & June 18, 12 & 5 193.17 \\
\hline 24 & 199.77 & 9 & & a. $m_{-}$ & .47 \\
\hline 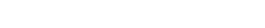 & 199.69 & $\begin{array}{l}\text { Apr. } 10 \\
\text { Apr. } 11\end{array}$ & $\begin{array}{l}198.82 \\
198.72\end{array}$ & $m \ldots$ & $\begin{array}{r}5192.67 \\
107.37\end{array}$ \\
\hline Jan. 26 & 199.57 & $\begin{array}{l}\text { Apr. } 11 \\
\text { Apr. } 12\end{array}$ & $\begin{array}{l}198.72 \\
198.62\end{array}$ & $\begin{array}{l}\text { June } 20,7: 05 \text { a. } \mathrm{m} \\
\text { June } 20,12 \mathrm{~m}\end{array}$ & $\begin{array}{r}197.37 \\
5192.92\end{array}$ \\
\hline Jan. 28 & $\begin{array}{l}199.49 \\
199.39\end{array}$ & Apr. 13 & 198.62 & June 21 & $\begin{array}{r}192.92 \\
197.17\end{array}$ \\
\hline 29. & 199.27 & 14 & 198.47 & June 22 & 197.12 \\
\hline 30 & 199.67 & $15 \ldots$ & 198.41 & June $23,7: 40$ a. m & 197.07 \\
\hline$-\ldots$ & 199.93 & 16 & 198.42 & June 23,5 p. m & 5192.22 \\
\hline Feb. 1, 12:45 p. m & 200.87 & $17 \ldots$ & 198.47 & June 24 & 196.97 \\
\hline p. $\mathrm{m}_{\ldots} \ldots$ & .82 & - & 198.42 & Jun & 196.89 \\
\hline 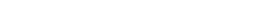 & .77 & $\ldots \ldots$ & 198.52 & June 26 & 196.87 \\
\hline 3 & 202.71 & 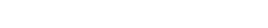 & 198. 57 & June 27 & 196. 87 \\
\hline F & 202.51 & 21 & 198.37 & June 28 & 196. 79 \\
\hline b. $5-$ & 203.57 & Apr. 22 & 19 & Jun & 196.72 \\
\hline Feb. 7 & $\begin{array}{l}203.67 \\
203.81\end{array}$ & $\begin{array}{l}\text { Apr. } 23 \\
\text { Apr. } 24\end{array}$ & $\begin{array}{l}198.37 \\
198.52\end{array}$ & June 30 & 196.67 \\
\hline 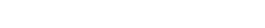 & $\begin{array}{l}200.01 \\
203.87\end{array}$ & Apr. 25 & $\begin{array}{l}198.52 \\
198.52\end{array}$ & 1935 & \\
\hline$\ldots-\ldots \ldots$ & 204. 13 & Apr. $26,1: 25$ p. m & 198.53 & Oct. 5. & 195.24 \\
\hline$-\ldots-\ldots$ & 204.37 & Apr. $26,1: 35$ p. m. & 198.47 & Oct. 12 & 195.21 \\
\hline Feb. 11 & 204.2 & Apr. 27 & 198.42 & Oet. 18 & 195.52 \\
\hline Feb. 12 & 20 & Apr. 28 & 198.42 & Oct. 21 & 195.47 \\
\hline 13. & 205.2 & Apr. 29 & 198.62 & Oct. 30 & 195. 32 \\
\hline 14 & 205.3 & Apr. 30 & 198.67 & Nov. 7 & 195.22 \\
\hline 15 & 205.6 & y 1 & 198.82 & Nov. 13 & 195.44 \\
\hline$-\ldots .$. & 205.6 & May 2 & 198.77 & Nov. 20 & 196.05 \\
\hline 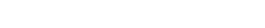 & 205.5 & May 3 & .77 & $n-n-1-n$ & 196.02 \\
\hline Feb. 18 & 205.4 & May $4 \ldots$ & 198. 72 & - n-n & 195.16 \\
\hline Feb. 19 & 205.1 & $-1-n-1-n$ & 198. 72 & Dec. 11 & 195.63 \\
\hline .20 & 204.8 & Tay $6 \ldots \ldots$ & 67 & Dec. 18 & 197.6 \\
\hline Fel & 205.1 & . & 19 & Dec. 26 & 197.4 \\
\hline $\mathbf{F}$ & 205.1 & May 8 & 19 & & \\
\hline$\ldots-$ & 205. 1 & $\begin{array}{l}\text { ay } 9-- \\
\text { ay } 10\end{array}$ & $\begin{array}{l}57 \\
57\end{array}$ & $\operatorname{Jan} 2$ & \\
\hline-1 & 205.1 & $10 \ldots$ & $\begin{array}{l}57 \\
52\end{array}$ & Jan. 2 & 197.9 \\
\hline$-\cdots$ & $\begin{array}{l}204.9 \\
204.8\end{array}$ & $11=$ & $\begin{array}{l}52 \\
47\end{array}$ & Jan. 9 & 205.7 \\
\hline $\mathrm{Fe}$ & $\begin{array}{l}204.8 \\
204.6\end{array}$ & $\begin{array}{l}\text { May } 12 \ldots \\
\text { May } 13 .\end{array}$ & $\begin{array}{l}47 \\
42\end{array}$ & $\begin{array}{l}\text { Jan. } 15 \\
\text { Jan } 22\end{array}$ & 201. 1 \\
\hline $\begin{array}{l}27 \\
.28\end{array}$ & 204.4 & May 14 & 42 & $\begin{array}{l}\text { Jan. } 22 \\
\text { Jan. } 29\end{array}$ & 208. 78 \\
\hline $\begin{array}{l}\text { Feb. } 28 \\
\text { Mar. 1, }\end{array}$ & 204.17 & May 15 & 198. 32 & Jan. 29 & $\begin{array}{l}205.27 \\
202.68\end{array}$ \\
\hline Mar. $1,12: 50$ p. m. & 204.04 & May 16 & 198.27 & Feb. 12 & $\begin{array}{l}202.68 \\
200.98\end{array}$ \\
\hline Mar. 2 . & 203.67 & May 17 & 198.27 & Feb. 21 & $\begin{array}{l}200.98 \\
200.87\end{array}$ \\
\hline Mar. 3 & 203.57 & May 18 & 198.17 & Feb. 26 & 203.81 \\
\hline Mar. 4 & 203.47 & May 19 & 198.17 & Mar. 4 & 204.69 \\
\hline Mar. 5 & 202.87 & May 20. & 198.12 & Mar. 11 & 204.07 \\
\hline 6 & 202.37 & May 21 & 17 & Mar. 18 & 201.67 \\
\hline 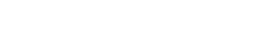 & 202.17 & May 22 & 19 & Mar. 25 & 200.67 \\
\hline Mar. 8 & 201.92 & $\ldots$ & 32 & 1 & 20047 \\
\hline Mar. 9 & $201 . f 7$ & $24 \ldots$ & 37 & A & 201. 21 \\
\hline Mar & 201.32 & - n & 47 & 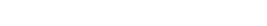 & 202.43 \\
\hline M & 201.27 & - n & 198.52 & $-\ldots$. & 200.99 \\
\hline M & 201.07 & Tay 27 & 19 & Apr. 29 & 200.69 \\
\hline $\mathbf{M}$ & 200.97 & May $28,11: 35$ a. m & 37 & May 6 & 200.66 \\
\hline - - - & 200.77 & May $28,5: 35$ p. m & 198. 34 & May 13 & 200.55 \\
\hline 15 & 200.57 & May 29. & 32 & May 20 & 200.18 \\
\hline$\ldots$. & 200.42 & y $30 \ldots$ & 19 & May 27 & 200.00 \\
\hline$-\infty$ & 200.27 & 31 & 19 & $\mathrm{Ju}$ & 199. 35 \\
\hline$-\ldots$. & 200.07 & $\ldots$ & 17 & $\ldots$ & 199. 09 \\
\hline Mar. 1 & 199.97 & June 2. & & $-\ldots$ & 198. 87 \\
\hline Mar. 20 & 199.77 & June $3 \ldots \ldots$ & 19 & ae $24 \ldots \ldots \ldots$ & 198.52 \\
\hline M & 199. & June $4 \ldots$ & 19 & July 1 & 197.97 \\
\hline$M$ & 199.57 & 5 & 198 & $\mathrm{~J}$ & 4192.86 \\
\hline $\mathbf{M}$ & 199. 72 & June 6 & 198.11 & Juiy 22 & 4 190.92 \\
\hline 24 & 200.02 & June 7 . & 198.09 & Aug. 5 & 196.21 \\
\hline$\ldots$ & 200.17 & June 8 . & 198.07 & Aug. 12 & 195. 34 \\
\hline$-\ldots$ & 200.17 & June 9 & 198.04 & Aug. 19. & 192.24 \\
\hline - & 200.12 & June $10 \ldots$ & 198.04 & t. 2 & 7180.14 \\
\hline$N$ & 199.97 & June 11,9 a. $m$ & 198. 02 & Sept. 10 & 7178.36 \\
\hline $\mathrm{A}$ & 199.77 & June 11,6 p. $\mathrm{m}$ & 5194.12 & Sept. $16 \ldots$ & 195.67 \\
\hline Mar. 30 $\ldots$ & 199.6 & June $12 \ldots$ & 198. 00 & Sept. 23 . & 5190.25 \\
\hline Mar. $31 \ldots$ & 199.57 & June 13 & 197.97 & Sept. 30 & 1188.68 \\
\hline
\end{tabular}

4 Water level depressed by inordinately large withdrawals.

5 Pump operating in well.

7 Adjacent land being irrigated. 
TABLE 13.-Ground-water levels in observation wells, 1928-30 and 1935-36 1-Con.

472. Hector Brothers estate, G. H. Hector, director. NE1/4SW1/4 sec. 4, T $11 \mathrm{~S} ., \mathrm{R} .4 \mathrm{~W}$. Unused well, dug and driven, $3 \frac{1}{2}$ feet in diameter to depth of 31 feet with brick curb; total depth 84 feet. Measuring point is top of 3-inch plank deck, south of pump, 0.8 foot above land surface and 224.83 feet $^{2}$ above mean sea level.

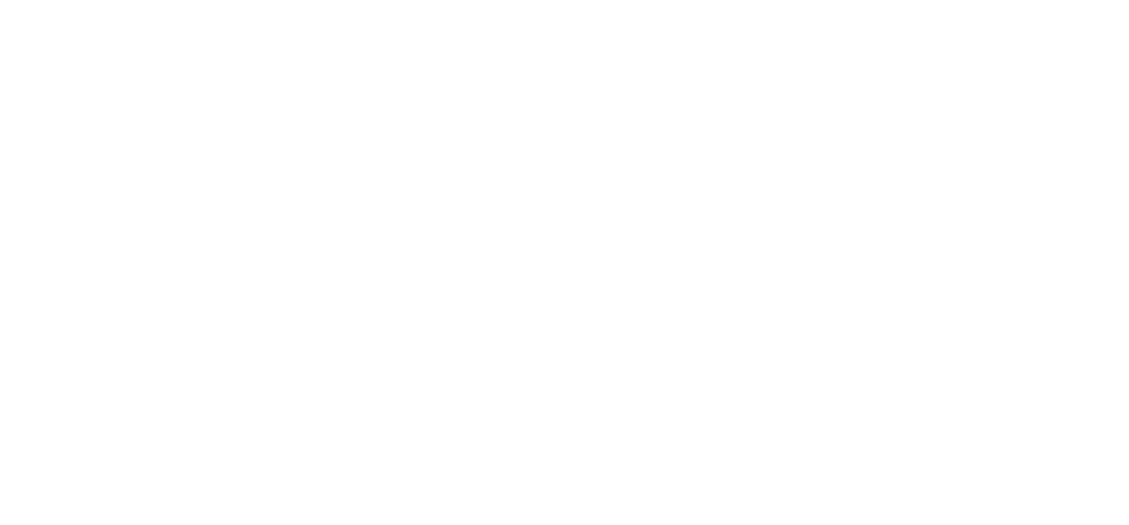

${ }^{2}$ Leveling by U. S. Engineer Department.

5 Pump operating in well.

486. Linn County Farm. SW1/4SW1/4 sec. 26 , T. 11 S., R. 4 W. Stock well, dug 6 feet in diameter and 27.3 feet deep, brick curb, in lot 75 feet south of barn. Copper nail with washer stamped 11-4-8 in well platform. Measuring point is top of 2 -inch plank deck at copper nail with washer, 0.6 foot above land surface and 221.83 feet $^{2}$ above mean sea level.

\begin{tabular}{|c|c|c|c|c|c|c|c|}
\hline Date & $\begin{array}{l}\text { Water } \\
\text { level } \\
\text { (feet) }\end{array}$ & Date & $\begin{array}{l}\text { Water } \\
\text { level } \\
\text { (feet) }\end{array}$ & Date & $\begin{array}{l}\text { Water } \\
\text { level } \\
\text { (feet) }\end{array}$ & Date & $\begin{array}{l}\text { Water } \\
\text { level } \\
\text { (feet) }\end{array}$ \\
\hline 1928 & & 1935 & & 1936 & & 1936 & \\
\hline $\begin{array}{l}\text { July } 28 \\
\qquad 1929 \\
\text { June } 6 \\
\text { July } 4 \\
\text { Aug. } 2 \\
\text { Sept. } 23 \\
\text { Oct. } 25 \\
\text { Nov. } 27 \\
\text { Dec. } 31 \\
\qquad 1930 \\
\text { Feb. } 1 \\
\text { Mar. } 1 \\
\text { Mar. } 29 \\
\text { Apr. } 26 \\
\text { May } 28 \\
\text { July } 23\end{array}$ & $\begin{array}{l} \\
215.03 \\
210.43 \\
204.7 \\
201.9 \\
200.73 \\
200.53 \\
219.38 \\
\\
\\
217.23 \\
220.08 \\
216.38 \\
216.88 \\
215.31 \\
209.82\end{array}$ & $\begin{array}{l}\text { Oct. } 4 \\
\text { Oct. } 11 \\
\text { Oct. } 17 \\
\text { Oct. } 30 \\
\text { Nov. } 6 \\
\text { Nov. } 13 \\
\text { Nov. } 20 \\
\text { Dec. } 4 \\
\text { Dec. } 11 \\
\text { Dec. } 18 \\
\text { Dec. } 26 \\
\\
\\
\text { Jan. } 19 \\
\text { Jan. } 8 \\
\text { Jan. } 15 \\
\text { Jan. } 22 \\
\text { Jan. } 29 \\
\text { Feb. } 5\end{array}$ & $\begin{array}{l}200.37 \\
200.76 \\
202.18 \\
201.99 \\
199.45 \\
200.61 \\
200.75 \\
201.46 \\
202.59 \\
203.28 \\
202.59 \\
\\
\\
204.42 \\
216.16 \\
220.14 \\
218.15 \\
216.93 \\
221.83\end{array}$ & $\begin{array}{l}\text { Feb. 12 } \\
\text { Feb. 20 } \\
\text { Feb. 26 } \\
\text { Mar. } 4 \\
\text { Mar. 11 } \\
\text { Mar. } 18 \\
\text { Mar. } 24 \\
\text { Apr. } 1 \\
\text { Apr. } 8 \\
\text { Apr. } 15 \\
\text { Apr. } 22 \\
\text { May } 6 \\
\text { May } 13 \\
\text { May } 19 \\
\text { May } 27 \\
\text { June } 3 \\
\text { June } 10 \\
\text { June } 17 \\
\text { June } 24 \\
\text { July } 1\end{array}$ & $\begin{array}{l}215.17 \\
212.34 \\
215.96 \\
220.21 \\
218.90 \\
218.40 \\
214.20 \\
220.01 \\
220.04 \\
216.11 \\
212.65 \\
220.06 \\
217.87 \\
218.90 \\
213.23 \\
215.30 \\
215.06 \\
214.44 \\
213.19 \\
209.39\end{array}$ & $\begin{array}{l}\text { July } 10 \\
\text { July } 15 \\
\text { July } 22 \\
\text { July } 29 \\
\text { Aug. } 5 \\
\text { Aug. } 12 \\
\text { Aug. } 19 \\
\text { Aug. } 26 \\
\text { Sept. } 2 \\
\text { Sept. } 10 \\
\text { Sept. } 16 \\
\text { Sept. } 23 \\
\text { Sept. } 30\end{array}$ & $\begin{array}{r}206.81 \\
206.03 \\
203.34 \\
202.48 \\
200.77 \\
5196.41 \\
201.78 \\
200.99 \\
200.79 \\
200.69 \\
200.63 \\
200.29 \\
200.02\end{array}$ \\
\hline
\end{tabular}

2 Leveling by U. S. Engineer Department.

- Pump operating in well.

491. O. H. Ehrlich. SW1/4SW1/4 sec. 35, T. 11 S., R. 4 W. Stock well, driven $11 / 4$ inches in diameter and 31 feet deep, in barnyard 150 feet south of residence. Copper nail with washer stamped 11-4-15 in plank level with land surface. Measuring point is top of coupling between casing and pump, 2.9 feet above land surface and 227.9 feet above mean sea level (interpolated).

\begin{tabular}{|c|c|c|c|c|c|}
\hline Date & $\begin{array}{l}\text { Water } \\
\text { level } \\
\text { (feet) }\end{array}$ & Date & $\begin{array}{l}\text { Water } \\
\text { level } \\
\text { (feet) }\end{array}$ & Date & $\begin{array}{l}\text { Water } \\
\text { level } \\
\text { (feet) }\end{array}$ \\
\hline \begin{tabular}{l}
\multicolumn{1}{c}{1928} \\
Aux. 13 \\
Oct. 21 \\
Dec. 16 \\
\end{tabular} & $\begin{array}{l}208.0 \\
207.3 \\
213.8\end{array}$ & $\begin{array}{l}\text { Feb. } 2 \\
\text { Mar. 27 }\end{array}$ & $\begin{array}{l}216.2 \\
214.8\end{array}$ & \begin{tabular}{|c} 
\\
May 9 \\
June 6
\end{tabular} & $\begin{array}{l}214.5 \\
211.7\end{array}$ \\
\hline
\end{tabular}


TABLE 13.-Ground-water levels in observation wells, 1928-30 and 1935-36-Con.

492. MeFarland School. SE1/4NE1/4 sec. 36, T. 11 S., R. 4 W. Domestic well, driven $11 / 2$ inches in diameter and 26 feet deep. Measuring points: In 1928, concrete pump platform, level with land surface and about 238.9 feet above mean sea level; in 1935-36, top of pump-gasket flange, 2.7 feet above land surface and 239.62 feet 2 above mean sea level.

\begin{tabular}{|c|c|c|c|c|c|c|c|}
\hline Date & $\begin{array}{l}\text { Water } \\
\text { level } \\
\text { (feet) }\end{array}$ & Date & $\begin{array}{l}\text { Water } \\
\text { level } \\
\text { (feet) }\end{array}$ & Date & $\begin{array}{l}\text { Water } \\
\text { level } \\
\text { (feet) }\end{array}$ & Date & $\begin{array}{l}\text { Water } \\
\text { level } \\
\text { (feet) }\end{array}$ \\
\hline 1928 & \multirow{3}{*}{225.9} & 1936 & & 1936 & \multirow{8}{*}{$\begin{array}{l}230.00 \\
229.85 \\
229.44 \\
228.37 \\
228.32 \\
226.84 \\
226.30 \\
225.97 \\
225.76\end{array}$} & 1936 & \multirow{8}{*}{$\begin{array}{l}224.15 \\
224.00 \\
223.17 \\
222.84 \\
222.74 \\
222.60 \\
222.70 \\
222.25 \\
222.03\end{array}$} \\
\hline Aug. 2 & & Apr. 1 . & 233. 29 & June 3 & & Aug. 5 & \\
\hline 1936 & & $\begin{array}{l}\text { Apr. } 8 \\
\text { Apr. } 15\end{array}$ & $\begin{array}{l}233.17 \\
231.80\end{array}$ & $\begin{array}{l}\text { June } 10 \\
\text { June } 17\end{array}$ & & $\begin{array}{l}\text { Aug. } 12 \\
\text { Aug. } 19\end{array}$ & \\
\hline Feb 27 & 23322 & Apr. 22 & 232.05 & June 24 & & Aug. 26 & \\
\hline Mar. 4 & $\begin{array}{l}235.22 \\
233.19\end{array}$ & $\begin{array}{l}\text { Apr. } 29 \\
\text { May } 6\end{array}$ & $\begin{array}{l}231.99 \\
231.88\end{array}$ & $\begin{array}{l}\text { July } 1 \\
\text { July } 10--\end{array}$ & & $\begin{array}{l}\text { Sept. } 2 \\
\text { Sept. } 10\end{array}$ & \\
\hline Mar. 12 & 232.87 & May 13 . & 231.55 & July 15... & & Sept. 16 & \\
\hline Mar. 18 & 232.26 & May 20 & 231. 71 & July 22. & & Sept. 23 & \\
\hline Mar. & 232.68 & May 27 & 231.70 & July 29 & & Sept. 30 & \\
\hline
\end{tabular}

2 Leveling by U. S. Engineer Department.

493. Maude Wells. NE1/4NE1/4 sec. 2, T. 11 S., R. 3 W. Stock well, dug 8 feet deep. Measuring point is top of timber cover, through bored hole, 0.8 foot above land surface and 233.35 feet 2 above mean sea level.

\begin{tabular}{|c|c|c|c|c|c|c|c|}
\hline Date & $\begin{array}{l}\text { Water } \\
\text { level } \\
\text { (feet) }\end{array}$ & Date & $\begin{array}{l}\text { Water } \\
\text { level } \\
\text { (feet) }\end{array}$ & Date & $\begin{array}{l}\text { Water } \\
\text { level } \\
\text { (feet) }\end{array}$ & Date & $\begin{array}{l}\text { Water } \\
\text { level } \\
\text { (feet) }\end{array}$ \\
\hline 1935 & & 1936 & & 1936 & & 1936 & \\
\hline $\begin{array}{l}\text { Oct. } 11 \\
\text { Oct. } 17 \\
\text { Oct. } 30 \\
\text { Nov. } 6 \\
\text { Nov. } 13 \\
\text { Nov. } 20 \\
\text { Dec. } 4 \\
\text { Dec. } 10 \\
\text { Dec. } 18 \\
\text { Dec. } 26 \\
\text { Dec. } 28 \\
\qquad 1936 \\
\text { Jan. } 1 \\
\text { Jan. } 3\end{array}$ & $\begin{array}{l}227.05 \\
226.28 \\
227.32 \\
227.07 \\
227.73 \\
227.87 \\
227.96 \\
228.97 \\
229.05 \\
231.18 \\
231.68\end{array}$ & $\begin{array}{l}\text { Jan. } 7 \\
\text { Jan. } 10 \\
\text { Jan. 14 } \\
\text { Jan. } 21 \\
\text { Jan. 24 } \\
\text { Jan. 28 } \\
\text { Jan. } 31 \\
\text { Feb. } 4 \\
\text { Feb. } 12 \\
\text { Feb. 20 } \\
\text { Feb. 25 } \\
\text { Mar. } 4 \\
\text { Mar. 10 } \\
\text { Mar. 18 } \\
\text { Mar. 25 } \\
\text { Mar. 31 }\end{array}$ & $\begin{array}{l}232.47 \\
232.57 \\
232.47 \\
232.10 \\
232.20 \\
231.87 \\
231.37 \\
232.14 \\
232.33 \\
231.57 \\
232.54 \\
232.05 \\
231.70 \\
232.10 \\
231.93 \\
231.97\end{array}$ & $\begin{array}{l}\text { Apr. } 7 \\
\text { Apr. } 14 \\
\text { Apr. } 22 \\
\text { Apr. } 28 \\
\text { May } 5 \\
\text { May } 12 \\
\text { May } 19 \\
\text { May } 26 \\
\text { June } 2 \\
\text { June } 9 \\
\text { June } 16 \\
\text { June } 23 \\
\text { June } 30 \\
\text { July } 9 \\
\text { July } 14 \\
\text { July } 21\end{array}$ & $\begin{array}{l}232.45 \\
231.64 \\
231.53 \\
231.65 \\
231.97 \\
231.08 \\
231.88 \\
231.85 \\
231.33 \\
231.22 \\
231.16 \\
230.61 \\
229.78 \\
229.38 \\
229.56 \\
228.97\end{array}$ & $\begin{array}{l}\text { July } 28 \\
\text { Aug. 4 } \\
\text { Aug. 11. } \\
\text { Aug. 18 } \\
\text { Aug. 25 } \\
\text { Sept. 1 } \\
\text { Sept. } 9 \\
\text { Sept. } 15 \\
\text { Sept. } 22 \\
\text { Sept. } 29\end{array}$ & $\begin{array}{l}228.57 \\
228.33 \\
228.21 \\
227.90 \\
227.85 \\
227.94 \\
227.82 \\
227.80 \\
227.42 \\
227.07\end{array}$ \\
\hline
\end{tabular}

2 Leveling by U. S. Engineer Department.

502. Sophie Wilson. SE1/4SW1/4 sec. 7, T. 11 S., R. 3 W. Unused well, dug 24 feet deep, backfilled around 10-inch concrete-tile casing. Measuring point is top of cover on box curb, beside draft pipe, 2.9 feet above land surface and 227.90 feet 2 above mean sea level.

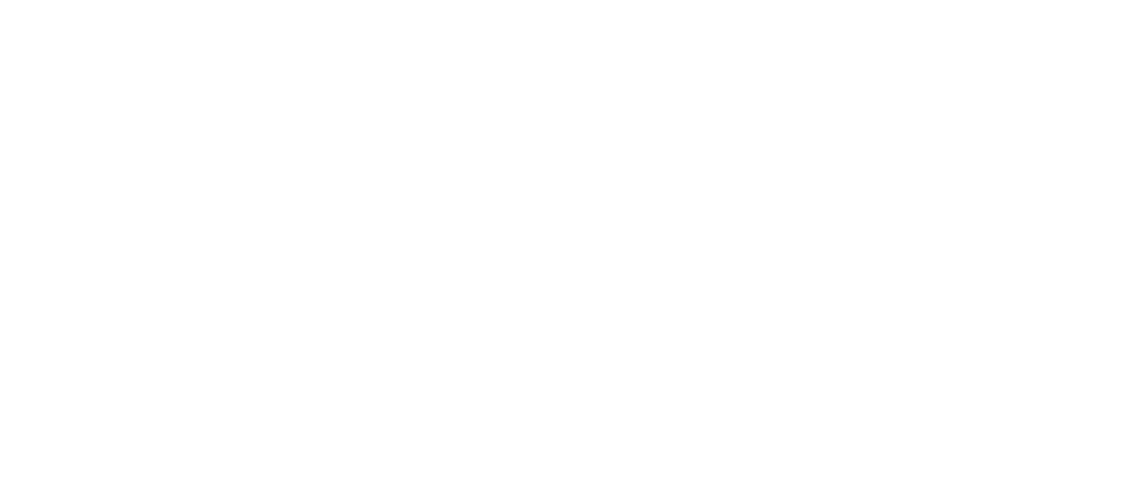

Leveling by U. S. Engineer Department. 
TABLE 13.-Ground-water levels in observation wells, 1928-30 and 1995-36-Con.

513. Leslie Cade. NE1/4NW 1/4 sec. 23, T. $11 \mathrm{~S} ., \mathrm{R}, 3 \mathrm{~W}$. Domestic well, dug 4 feet square and 22 feet deep, brick curb. Measuring point is top of brick curb, at north side, 258.81 feet $^{2}$ above mean sea level.

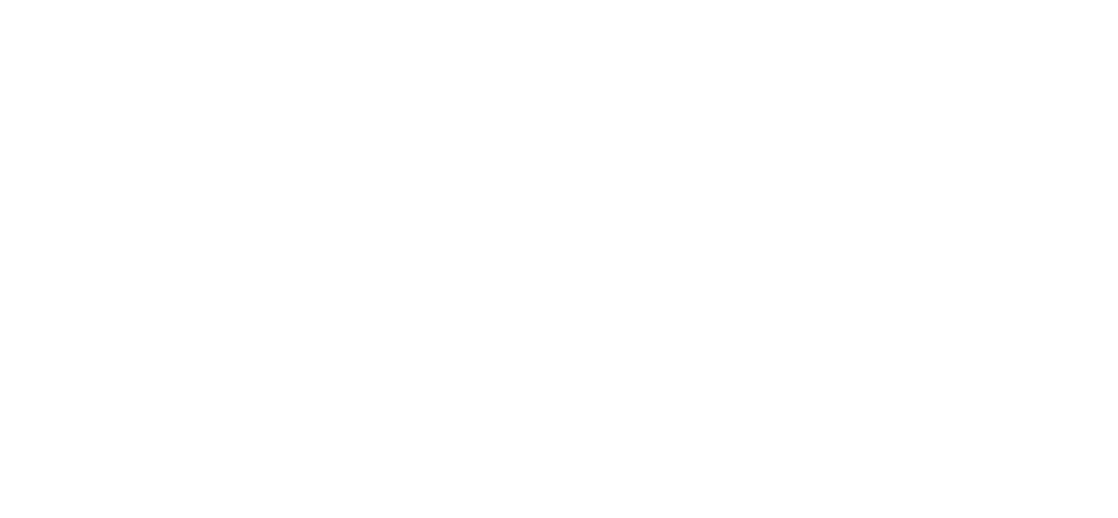

${ }^{2}$ Leveling by U. S. Engineer Department.

518. H. O. Wilson. NW $1 / 4$ NE $1 / 4$ sec. 21, T. $11 \mathrm{~S}$, R. 2 W. Irrigation well, dug 8 feet square and 15.5 feet deep. Measuring points: Top of east 6 -by 8-inch timber pump support, near southeast corner of well, a bout 1 foot above land surface and 283.23 feet ${ }^{2}$ above mean sea level; beginning July 10, 1936, new point 281.88 feet 2 above mean sea level.

\begin{tabular}{|c|c|c|c|c|c|c|c|}
\hline Date & $\begin{array}{l}\text { Water } \\
\text { Jevel } \\
\text { (feet) }\end{array}$ & Date & $\begin{array}{l}\text { Water } \\
\text { level } \\
\text { (feet) }\end{array}$ & Date & $\begin{array}{l}\text { Water } \\
\text { level } \\
\text { (feet) }\end{array}$ & Date & $\begin{array}{l}\text { Water } \\
\text { level } \\
\text { (feet) }\end{array}$ \\
\hline 1935 & & 1936 & & 1936 & & 1936 & \\
\hline $\begin{array}{l}\text { Oct. } 11 \\
\text { Oct. } 17 \\
\text { Oct. } 30 \\
\text { Nov. } 6 \\
\text { Nov. } 13 \\
\text { Nov. } 20 \\
\text { Dec. } 4 \\
\text { Dec. } 11 \\
\text { Dec. } 18 \\
\text { Dec. } 26 \\
\quad 1936 \\
\text { Jan. } 1\end{array}$ & $\begin{array}{l}274.98 \\
275.11 \\
275.08 \\
275.03 \\
275.28 \\
275.45 \\
275.55 \\
275.79 \\
276.38 \\
276.30\end{array}$ & $\begin{array}{l}\text { Jan. } 7 \\
\text { Jan. } 15 \\
\text { Jan. } 22 \\
\text { Jan. } 29 \\
\text { Feb. } 5 \\
\text { Feb. 12 } \\
\text { Feb. 20 } \\
\text { Feb. 26 } \\
\text { Mar. 4 } \\
\text { Mar. 11 } \\
\text { Mar. 18 } \\
\text { Mar. 25 } \\
\text { Apr. 15. } \\
\text { A pr. }\end{array}$ & $\begin{array}{l}277.91 \\
279.28 \\
279.13 \\
277.64 \\
277.25 \\
277.30 \\
277.23 \\
278.01 \\
277.86 \\
277.66 \\
277.28 \\
277.10 \\
277.39 \\
277.39\end{array}$ & $\begin{array}{l}\text { Apr. } 15 \\
\text { Apr. 22 } \\
\text { Apr. 29 } \\
\text { Maay } 6 \\
\text { May 13 } \\
\text { May } 20 \\
\text { May } 27 \\
\text { June } 3 \\
\text { June } 10 \\
\text { June } 17 \\
\text { June } 24 \\
\text { July } 10 \\
\text { July } 15 \\
\text { July } 22 \\
\end{array}$ & $\begin{array}{l}277.23 \\
277.11 \\
276.99 \\
277.69 \\
276.88 \\
276.85 \\
276.88 \\
276.84 \\
276.76 \\
276.67 \\
276.79 \\
276.33 \\
276.30 \\
275.98\end{array}$ & $\begin{array}{l}\text { Jaly } 29 \\
\text { Aug. } 5 \\
\text { Aug. } 12 \\
\text { Aug. } 19 \\
\text { Aug. } 26 \\
\text { Sept. } 2 \\
\text { Sept. } 10 \\
\text { Sept } 16 \\
\text { Sept. } 23\end{array}$ & $\begin{array}{l}275.60 \\
275.88 \\
275.78 \\
275.67 \\
275.63 \\
275.63 \\
275.46 \\
275.48 \\
275.44\end{array}$ \\
\hline
\end{tabular}

Leveling by U.S. Engineer Department.

527. G.W. Cummings. SW1/4SW1/4 sec. 12, T.12 S. R. $6 \mathrm{~W}$. Unused well, driven 3 inches in diameter and 27.8 feet deep. Measuring point is top of 3 -inch casing, 1.5 feet above land surface and 263.86 feet 2 above mean sea level.

\begin{tabular}{|c|c|c|c|c|c|c|c|}
\hline Date & $\begin{array}{l}\text { Water } \\
\text { level } \\
\text { (feet) }\end{array}$ & Date & $\begin{array}{l}\text { Water } \\
\text { level } \\
\text { (feet) }\end{array}$ & Date & $\begin{array}{l}\text { Water } \\
\text { level } \\
\text { (feet) }\end{array}$ & Date & $\begin{array}{l}\text { Water } \\
\text { level } \\
\text { (feet) }\end{array}$ \\
\hline 1935 & & 1936 & & 1936 & & 1936 & \\
\hline Oct. 18 & 254.39 & Jan. 4 & 258.82 & Apr. 8 & 259.59 & July 15 & 255.76 \\
\hline Oct. 23 & 254.35 & Jan. 9 & 256.86 & Apr. 15 & 257.15 & July 22 & 255.44 \\
\hline Oct. 29 & 254.46 & Jan. 15 & 257.64 & Apr. 22. & 257.20 & July 29 & 255. 36 \\
\hline Nov. 7 . & 254.41 & Jan. 21 & 256.95 & Apr. 29 & 256.78 & Aug. 5- & 255. 24 \\
\hline Nov. 13 & 254.41 & Jan. 29 & 256.89 & May 6 & 256.59 & Aug. 12 & 255.03 \\
\hline Nov. 2 & 254.58 & Feb. 5 & 256.0 & May 13 & 256.82 & Aug. 19 & 254.95 \\
\hline Nov. 2 & 254.74 & Feb. 12 & 257.30 & May 20 & 256.73 & Aug. 26. & 254.72 \\
\hline Dec. 11 & 255.25 & Feb. 21 & 257.88 & May 27 & 256.78 & Sept. 2 & 254. 59 \\
\hline Dec. 18 & 254.84 & Feb. 26 & 259.22 & June 3 & 257.57 & Sept. 10 & 254.40 \\
\hline Dec. 26 & 254.88 & Mar. 4 & 257. 49 & June 10 & 256.43 & Sept. 16. & 254.38 \\
\hline 1936 & & $\begin{array}{l}\text { Mar. } 11 \\
\text { Mar. } 18\end{array}$ & $\begin{array}{l}257.54 \\
257.38\end{array}$ & $\begin{array}{l}\text { June 17 - } \\
\text { June 24.- }\end{array}$ & $\begin{array}{l}256.22 \\
256.13\end{array}$ & $\begin{array}{l}\text { Sept. } 23 \\
\text { Sept. } 30\end{array}$ & $\begin{array}{l}254.35 \\
254.23\end{array}$ \\
\hline & & Mar. 25. & 257.17 & July $1 \ldots$ & 256.03 & & \\
\hline Jan. $2 \ldots$ & 255.62 & Apr. 1.. & 259.57 & July 8. & 255.92 & & \\
\hline
\end{tabular}

${ }^{2}$ Leveling by U. S. Engineer Department. 
TABLE 13.-Ground-water levels in observation wells, 1928-30 and 1935-36-Con-

528. Independence School, district 18. NW1/4SE1/4 sec. 25, T. 12 S., R. 6 W. Domestic well, dug 31.2 feet deep, backfilled around 6 -inch conrete-tile casing. Measuring point is top of casing, 0.5 foot above land surface and 314.36 feet ${ }^{2}$ a bove mean sea level.

\begin{tabular}{|c|c|c|c|c|c|c|c|}
\hline Date & $\begin{array}{l}\text { Water } \\
\text { level } \\
\text { (feet) }\end{array}$ & Date & $\begin{array}{l}\text { Water } \\
\text { level } \\
\text { (feet) }\end{array}$ & Date & $\begin{array}{l}\text { Water } \\
\text { level } \\
\text { (feet) }\end{array}$ & Date & $\begin{array}{l}\text { Water } \\
\text { level } \\
\text { (feet) }\end{array}$ \\
\hline 1935 & & 1936 & & 1936 & & 1936 & \\
\hline Oct. 18 . & 298.17 & Jan. 9 & 303.89 & Apr. 16 & 309.64 & July 22 . & 302.63 \\
\hline Oct. 23 & 298. 75 & Jan. 15 & 312.74 & A pr. 22 & 309.59 & July 29 . & 301.64 \\
\hline Oct. 29 & 298.31 & Jan. 16 & 311.09 & Apr. 29 & 307.60 & Aug. 5 & 301.15 \\
\hline Nov. 7 - & 298. 50 & Jan. 29 & 308.72 & May 6 & 306.90 & Aug. 12 & 301.06 \\
\hline Nov. 13 & 298.89 & Feb. 5 & 308.05 & May 13. & 306.51 & Aug. 19 & 301.23 \\
\hline Nov. 20 & 299.04 & Feb. 12 & 308.43 & May 20. & 306.07 & Aug. 26 . & 300.88 \\
\hline Dec. 4 & 299.38 & Feb. 21 & 309. 30 & May 28 & 306.16 & Sept. 2 & 300.74 \\
\hline Dec. 11 . & 299. 27 & Feb. 26 & 310.17 & June 3 .- & 307.48 & Sept. 10 & 300.43 \\
\hline Dec. 18 & 300.73 & Mar. 4 & 311.55 & June 10 & 304.52 & Sept. 16 & 300.47 \\
\hline Dec. 26 & 300.67 & Mar. 11 & 312. 18 & June 17 & 304.07 & Sept. 23 & 299. 12 \\
\hline 1936 & & Mar. 18 & 309.28 & June 24 & 303.99 & Sept. $30 \ldots$ & 298.99 \\
\hline 1930 & & Mar. 25 & 309.73 & July 1 & 303.14 & & \\
\hline Jan. 2 & 302.27 & Apr. 8 & 311. 30 & July 15. & $\begin{array}{l}505.00 \\
302.85\end{array}$ & & \\
\hline
\end{tabular}

ILeveling by U. S. Engineer Department.

540. Roy Rickard. NW1/4NW1/4 sec. 26, T. 12 S., R. 5 W. Domestic well, driven 11/4 inches in diameter and 32 feet deep. Measuring points; Top of 11/4-inch casing (after unscrewing cap), about 2 feet below surface and 236.05 feet 2 above mean sea level: beginning March 11, 1936, top of standpipe, level with land surface and 237.82 feet 2 above mean sea level.

\begin{tabular}{|c|c|c|c|c|c|c|c|}
\hline Date & $\begin{array}{l}\text { Water } \\
\text { level } \\
\text { (feet) }\end{array}$ & Date & $\begin{array}{l}\text { Water } \\
\text { level } \\
\text { (feet) }\end{array}$ & Date & $\begin{array}{l}\text { Water } \\
\text { level } \\
\text { (feet) }\end{array}$ & Date & $\begin{array}{l}\text { Water } \\
\text { level } \\
\text { (feet) }\end{array}$ \\
\hline 1928 & & 1935 & & 1936 & & 1936 & \\
\hline & & & & Feb. 26 & 217.23 & June 3.- & 211.51 \\
\hline Ang. 14 & 210.4 & Dec. 26. & 208. 39 & Mar. 11 & 217.80 & June 10 & 213. 39 \\
\hline 1935 & & - $\quad 1936$ & & $\begin{array}{l}\mathrm{Ma} \\
\mathrm{Ma}\end{array}$ & $\begin{array}{l}215.23 \\
211.36\end{array}$ & $\begin{array}{l}\text { June } 17 \ldots \\
\text { June } 24\end{array}$ & $\begin{array}{l}214.43 \\
213.31\end{array}$ \\
\hline Oct 18 & 208.55 & $\operatorname{Tan} 2$ & 210.62 & Apr. 1 & $\begin{array}{l}212.32 \\
212.38\end{array}$ & July $1 \ldots$ & $\begin{array}{l}212.60 \\
212.43\end{array}$ \\
\hline Oct. 23 & $\begin{array}{l}200.05 \\
208.53\end{array}$ & Jan. 9 & 212.91 & Apr. 15 & 212.15 & July 15 & $\begin{array}{r}212.45 \\
5210.75\end{array}$ \\
\hline Oct. 28 & 208. 35 & Jan. 15 & 228.62 & Apr. 22. & 212.01 & July 22. & 212.14 \\
\hline Nov. 7 & 208. 50 & Jan. 22 & 221.61 & Apr. 29 & 212.23 & Aug. 5 & 208.90 \\
\hline No & 207.65 & Jan. 29 & 212.46 & May 6. & 210.98 & Aug. 12 & 208.85 \\
\hline & 208.40 & Feb. 5 & 212.24 & May 13 & 211.71 & Aug. 19. & 208.83 \\
\hline Dec. 11 & 215.14 & Feb. 12 & 212.86 & May 20 & 211.30 & Sept. 2 & 210.21 \\
\hline De & 209.41 & Feb. 2 & 214.54 & May 27 & 211.39 & Sept. 16 & 209.86 \\
\hline
\end{tabular}

${ }^{2}$ Leveling by U. S. Fingineer Department.

5 Pump operating in well.

548. Trip \& Murphy. NW1/4NE1/4 sec. 22 , T. 12 s., R. 4 W. Unused well, driven $11 / 2$ inches in diameter and 27 feet deep. Measuring point is lower valve seat of pump, 3.8 feet above land surface and 244.37 feet? above mean sea level.

\begin{tabular}{|c|c|c|c|c|c|c|c|}
\hline Date & $\begin{array}{l}\text { Water } \\
\text { level } \\
\text { (feet) }\end{array}$ & Date & $\begin{array}{l}\text { Water } \\
\text { level } \\
\text { (feet) }\end{array}$ & Date & $\begin{array}{l}\text { Water } \\
\text { level } \\
\text { (feet) }\end{array}$ & Date & $\begin{array}{l}\text { Water } \\
\text { level } \\
\text { (feet) }\end{array}$ \\
\hline 1935 & & 1936 & & 1936 & & 1936 & \\
\hline Oct. 15. & 227.47 & Jan. 8 & 233.35 & Apr. 22 & 236.64 & July 29 & 232.08 \\
\hline Oct. 22 . & 227.35 & Jan. 22 & $\begin{array}{l}205.50 \\
235.19\end{array}$ & Apr. 29 & $\begin{array}{l}230.04 \\
236.26\end{array}$ & Aug. 5 & 231.52 \\
\hline Oct. 30 & 227.37 & Jan. 29 & 233.13 & May 6 & 235.76 & Aug. 12 & 231. 32 \\
\hline Nov. 6 . & 227.57 & Feb. 5 & 232.69 & May 13 & 234.40 & Aug. 19 & 231.72 \\
\hline Nov. 13 & 227.17 & Feb. 12 & 235.88 & May 20. & 235.21 & Aug. 26 . & 230.07 \\
\hline Nov. 20 & 227.22 & Feb. 20 & 234.85 & May 27. & 235. 22 & Sept. 2 & 229.94 \\
\hline Dec. 4 . & 226. 29 & Feb. 26 . & 237.69 & June 3. & 234.29 & Sept. 10 & 229.85 \\
\hline Dec. 11 & 227.60 & Mar. 4 & 237.65 & June 10 & 234.60 & Sept. 16 & 230.69 \\
\hline Dec. 18 & 228.89 & Mar. 11 & 236.65 & June 17. & 234.76 & Sept. 23 & 230.50 \\
\hline Dec. 26 & 229.21 & Mar. 18 & 236.68 & June 24 & 233.85 & Sept. 30 & 230.35 \\
\hline 1936 & & $\begin{array}{l}\text { Mar. } 25 \\
\text { A'pr. } 1 .\end{array}$ & $\begin{array}{l}236.58 \\
236.99\end{array}$ & $\begin{array}{l}\text { July } 1 . . \\
\text { July } 10 \ldots\end{array}$ & $\begin{array}{l}233.04 \\
232.68\end{array}$ & & \\
\hline & & Apr. 8 & 237.80 & July 1 & 233.17 & & \\
\hline Jan. 1. & 230.47 & Apr. 15.- & 237.01 & July 22 & 232.53 & & \\
\hline
\end{tabular}

Leveling by U. S. Engineer Department. 
TABLe 13.-Ground-water levels in observation wells, 1928-30 and 1935-36-Con.

553. J. H. Swatzka. SE14SE1/4 sec. 9, T. 12 S., R. 3 W. Domestic well, dug 4 feet in diameter and 18.7 feet deep, brick curb. Measuring points: 'Top of 2 -inch plank deck, through bored hole, 0.2 foot above land surface and 272.99 feet above mean sea level; September 6,1928 to May 28, 1930, top of instrument shelf, 274.04 feet above mean sea level. Water stage recorder operated on well from September 6,1928 , to May 28 1930.

\begin{tabular}{|c|c|c|c|c|c|c|c|}
\hline Date & $\begin{array}{l}\text { Water } \\
\text { level } \\
\text { (feet) }\end{array}$ & Date & $\begin{array}{l}\text { Water } \\
\text { level } \\
\text { (feet) }\end{array}$ & Date & $\begin{array}{l}\text { Water } \\
\text { level } \\
\text { (feet) }\end{array}$ & Date & $\begin{array}{l}\text { Water } \\
\text { level } \\
\text { (feet) }\end{array}$ \\
\hline 1928 & & 1929 & & 1935 & & 1936 & \\
\hline July 28 & 263.4 & Nov. 27 & 256.49 & Nov. 6 & 256.71 & Apr. 1 & 270.47 \\
\hline Sept. 3 & 260.26 & Dec. 31 & 270.12 & Nov. 12 & 256. 79 & Apr. 8 & 270.31 \\
\hline Sept. 6 & 260.01 & & & Nov. 20 & 256.99 & Apr. 15 & 269.59 \\
\hline Sept. 15 & 259.47 & 1930 & & Dec. 4 & 257.25 & Apr. 22 & 268.95 \\
\hline Oct. 8 & 258.23 & & & Dec. 11 & 258.77 & A pr. 29 & 268.55 \\
\hline Oct. 21 & 257.72 & Jan. 5 . & 270.64 & Dec. 18 & 261.86 & May 6 & 268. 61 \\
\hline Dec. 16 & 268.14 & Jan. 11 & 269.99 & Dec. 26 . & 262.72 & May 13 & 268.97 \\
\hline 1929 & & Jan. 18 & 269.34 & Dec. 28_- & 263.67 & May 20. & 268. 91 \\
\hline 1929 & & $\begin{array}{l}\text { Jan. } 26 \\
\text { Feb. } 1\end{array}$ & $\begin{array}{l}268.87 \\
271.14\end{array}$ & 1936 & & $\begin{array}{l}\text { May } 27 \\
\text { June } 3\end{array}$ & $\begin{array}{l}268.49 \\
267.91\end{array}$ \\
\hline Jan. 6 & 270.54 & Feb. 9 & .271 .02 & Jan. 1. & 264.82 & June 10 & 267.52 \\
\hline Jar & 270.84 & Feb. 16 & 270.71 & Jan. 3 & 03 & e 17 & 267.19 \\
\hline Feb. 2 & 271.14 & Fet & 271.29 & Jan. 8 & & e 24 & 266.69 \\
\hline Feb. 16. & 269.46 & Mar. 1 & 270.55 & Jan. 10 & 270.99 & July 1 . & 266.04 \\
\hline Mar. 7 & 269.69 & Mar. 19 & 269.31 & Jan. 15 & 271.01 & July 10 & 265. 26 \\
\hline Mar. 31 & 269.93 & Mar. 29 & 269.82 & Jan. 22 & 270.47 & July 15 & 264.80 \\
\hline Apr. 18 & 270.55 & Apr. 6 & 269.09 & Jan. 24 & 269.41 & July 22 & 265.25 \\
\hline $\mathrm{Mr}$ & 269.22 & $\mathrm{Ap}$ & 269.71 & Jan. 29 & 270 . & July 29. & 263.57 \\
\hline May 13 & 267.86 & May 28 & 268.34 & Jan. 31 & 270.37 & Aug. 5 & 262.87 \\
\hline June 3 & 267.38 & July 23 . & 264.23 & Feb. 5 & 269.64 & Aug. 12 & 262. 64 \\
\hline & 265. 92 & & & Feb. 12 & 269.71 & Aug. 19 & 263. 05 \\
\hline Jul & 265.18 & 1935 & & Feb. 20 & 269.00 & Aug. 26 & 260.88 \\
\hline $\mathrm{Au}$ & 263.80 & & & Feb. 26 & 270.03 & Sept. 2 & 260.72 \\
\hline & 260.53 & Oct. & 257. 39 & Mar. 4 & 270.51 & Sept. 10 & 260.84 \\
\hline & 260.25 & Oct. 12 & 257.18 & Mar. 11 & 269.99 & Sept. 16 & 259.19 \\
\hline Sept. 23 & 258. 91 & Oct. 17- & 257.54 & Mar. 18 & 269.79 & Sept. 23.. & 258.74 \\
\hline Oet. 25 & 257. 36 & Oct. 30. & 256.84 & Mar. 25 & 269.35 & Sept. 30 & 258. 62 \\
\hline
\end{tabular}

557. Scott Churchill. SE 1/4SW14 sec. 34, T. 12 S., R. 3 W. Domestic well, dug 23 feet deep, brick curb. Measuring point is top of 2-inch plank deck, alongside draft pipe, 1.3 feet above land surface and 268.04 feet ? above mean sea level.

\begin{tabular}{|c|c|c|c|c|c|c|c|}
\hline Date & $\begin{array}{l}\text { Water } \\
\text { level } \\
\text { (feet) }\end{array}$ & Date & $\begin{array}{l}\text { Water } \\
\text { level } \\
\text { (feet) }\end{array}$ & Date & $\begin{array}{l}\text { Water } \\
\text { level } \\
\text { (feet) }\end{array}$ & Date & $\begin{array}{l}\text { Water } \\
\text { level } \\
\text { (feet) }\end{array}$ \\
\hline 1935 & & 1936 & & 1936 & & 1936 & \\
\hline Oct. 15 & 248. 47 & Feb. 20 & 264.19 & Apr. 29.. & 262.35 & July 22 & 253.06 \\
\hline 1936 & & & & May 13 & $\begin{array}{l}262.86 \\
263.36\end{array}$ & Aug. 5 & $\begin{array}{l}251.32 \\
251.44\end{array}$ \\
\hline Jan. 8 & 265.31 & Mar. 18 & 263. 74 & May 27 & 5262.40 & Aug. 26 & 251.29 \\
\hline 15 & 265.75 & Mar. 25 & 263. & June 3 & 262 & Sept. 2 & 251.75 \\
\hline Jan. 22 & 265 & A pr. 1 & 264 . & June 10 & 6260 & Sept. 10 & 5250.62 \\
\hline Jan. 29 & 264.66 & $\mathrm{Ap}$ & 264 . & June 17 & 260 . & Sept. 16 & 250.81 \\
\hline Feb. 5 & 264.19 & Apr. 15 & 263.36 & July 10 & 255 & Sept. 23. & 249.36 \\
\hline Feb. 1 & 263.82 & Apr. 22 & 263.22 & July 15 & 255.10 & Sept. 30 & 249.11 \\
\hline
\end{tabular}

${ }^{2}$ Leveling by U.S. Engineer Department.

5 Pump operating in well.

- Pump operating in well a short time prior to measurement.

560. Cecil Ensley, renter. SW14NW14 sec. 8, T. 12 S., R. 2 W. Domestic and stock well, drilled 4 inches in diameter and 48,2 feet deep, steel casing. Measuring point is top of steel casing, 0.6 foot above land surface and 330.1 feet ${ }^{2}$ above mean sea level.

\begin{tabular}{|c|c|c|c|c|c|c|c|}
\hline Date & $\begin{array}{c}\text { Water } \\
\text { ievei } \\
\text { (feet) }\end{array}$ & Date & $\begin{array}{l}\text { Water } \\
\text { level } \\
\text { (feet) }\end{array}$ & Date & $\begin{array}{l}\text { Water } \\
\text { level } \\
\text { (feet) }\end{array}$ & Date & $\begin{array}{l}\text { Water } \\
\text { level } \\
\text { (feet) }\end{array}$ \\
\hline 1936 & & 1936 & & 1936 & & 1936 & \\
\hline $\begin{array}{l}\text { Apr. } 22 \\
\text { Apr. } 29 \\
\text { May } 6 \ldots \\
\text { May } 13 \\
\text { May } 20 \\
\text { May } 27 \\
\end{array}$ & $\begin{array}{l}323.50 \\
325.40 \\
325.57 \\
325.68 \\
325.72 \\
325.52\end{array}$ & $\begin{array}{l}\text { June } 3 \ldots \ldots \\
\text { June } 10 \ldots \ldots \\
\text { June } 17 \ldots \ldots \\
\text { June } 24 \\
\text { July } 1 . \ldots \\
\text { July } 10 \ldots \ldots\end{array}$ & $\begin{array}{l}325.31 \\
325.71 \\
324.96 \\
324.60 \\
323.84 \\
323.22\end{array}$ & $\begin{array}{l}\text { July } 15 \ldots \ldots \\
\text { July } 22, \ldots \\
\text { July } 29 \\
\text { Aug. } 5 \\
\text { Aug. } 12 \\
\text { Aug. } 19 \\
\end{array}$ & $\begin{array}{l}323.76 \\
322.72 \\
323.16 \\
322.96 \\
322.71 \\
323.08\end{array}$ & $\begin{array}{l}\text { Aug. } 26 \\
\text { Sept. } 2 \\
\text { Sept. } 10 \\
\text { Sept. } 16 \\
\text { Sept. } 23 \\
\text { Sept. } 30\end{array}$ & $\begin{array}{l}322.31 \\
322.20 \\
321.97 \\
322.13 \\
32206 \\
321.85\end{array}$ \\
\hline
\end{tabular}

${ }^{2}$ Leveling by U. S. Engineer Department. 
TABLE 13.-Ground-water levels in observation wells, 1928-30 and 1935-36-Con.

568. Ray Fisher. NE1/4NW1/4 sec. 14, T. $12 \mathrm{~S}$., R. 2 W. Irrigation well, dug 8 feet square and 19 feet deep. Measuring points: Top of pump-house sill at northwest corner of well, marked by copper nail with washer, 352.35 feet ${ }^{2}$ above mean sea level; beginning October 4, 1935, top of 6-by-6-inch timber pump support at painted arrow, about 1 foot above land surface and 353.15 feet 2 above mean sea level.

\begin{tabular}{|c|c|c|c|c|c|c|c|}
\hline Date & $\begin{array}{l}\text { Water } \\
\text { leve] } \\
\text { (feet) }\end{array}$ & Date & $\begin{array}{l}\text { Water } \\
\text { level } \\
\text { (feet) }\end{array}$ & Date & $\begin{array}{l}\text { Water } \\
\text { level } \\
\text { (feet) }\end{array}$ & Date & $\begin{array}{l}\text { Water } \\
\text { level } \\
\text { (feet) }\end{array}$ \\
\hline 1928 & \multirow{5}{*}{$\begin{array}{l}338.4 \\
339.4 \\
340.4\end{array}$} & 1930 & \multirow{7}{*}{$\begin{array}{l}341.85 \\
341.30 \\
340.30 \\
340.55 \\
338.51\end{array}$} & 1936 & \multirow{18}{*}{$\begin{array}{l}339.98 \\
346.09 \\
345.79 \\
343.20 \\
341.62 \\
342.01 \\
341.30 \\
341.47 \\
341.60 \\
341.57 \\
341.00 \\
340.50 \\
340.36 \\
340.38 \\
340.63 \\
340.40 \\
340.34 \\
340.13 \\
340.15 \\
339.23\end{array}$} & 1936 & \multirow{18}{*}{$\begin{array}{r}339.01 \\
340.01 \\
339.63 \\
339.47 \\
339.33 \\
339.33 \\
339.90 \\
338.58 \\
337.91 \\
338.15 \\
337.94 \\
337.81 \\
337.80 \\
337.46 \\
337.41 \\
5336.30 \\
336.42 \\
337.51 \\
337.31\end{array}$} \\
\hline Aug. 17. & & Mar. 1.. & & Jan. 1 . & & \multirow{17}{*}{$\begin{array}{l}\text { May } 20 \\
\text { May } 27 \\
\text { June } 3 \\
\text { June } 10 \\
\text { June } 17 \\
\text { June } 24 \\
\text { July } 9 \\
\text { July } 15 \\
\text { July } 22 \\
\text { July } 29 \\
\text { Aug. } 5 \\
\text { Aug. } 12 \\
\text { Aug. } 19 \\
\text { Aug. } 26 \\
\text { Sept. } 2 \\
\text { Sept. } 10 \\
\text { Sept. } 16 \\
\text { Sept. } 23 \\
\text { Sept. } 30\end{array}$} & \\
\hline Oct. 21 & & Mar. 29 & & Jan. 8 & & & \\
\hline Dec. 16. & & Apr. 26 & & Jan. 15 & & & \\
\hline 1929 & & $\begin{array}{l}\text { May 28. } \\
\text { Juiy } 23 .\end{array}$ & & $\begin{array}{l}\text { Jan. } 22 \\
\text { Jan. } 29\end{array}$ & & & \\
\hline Feb. 2 & 340.2 & & & $\begin{array}{l}\text { Feb. } 5 \\
\text { Feb. } 12\end{array}$ & & & \\
\hline Mar. 27 & 341.80 & 1935 & & Feb. 20 & & & \\
\hline May 9. & 341.35 & & & & & & \\
\hline & & Oct. 4 & 336.90 & Mar. 4 & & & \\
\hline & 340.05 & Oct. 12 & 336.89 & Mar. 11 & & & \\
\hline & 338. & Oct. 17 & 337.36 & Mar. 18 & & & \\
\hline Sept. 23- & 337.40 & Oct. $30 \ldots$ & 337.70 & Mar. 25 & & & \\
\hline Oct. 25 & 337.60 & Nov. 6 & 337.68 & Apr. 1 & & & \\
\hline 27 & 337.45 & Nov. 13 & 337.92 & Apr. 8. & & & \\
\hline Dec. 31 & 342.55 & Nov. 20 & 338. 01 & Apr. 15 & & & \\
\hline 1930 & & $\begin{array}{l}\text { Dec. } 4 \\
\text { Dec. } 11\end{array}$ & $\begin{array}{l}338.57 \\
338.77\end{array}$ & $\begin{array}{l}\text { Apr. } 22 \\
\text { A pr. } 29\end{array}$ & & & \\
\hline & & Dec. 18 & 339.82 & May 6 & & & \\
\hline Feb. $1 \ldots$ & 341.40 & Dec. 26 & 339.43 & May 13.- & & & \\
\hline
\end{tabular}

2 Leveling by U. S. Engineer Department.

5 Pump operating in well.

7 Adjacent land being irrigated.

571. James R. Barnes. SW $1 / 4$ NW $1 / 4$ sec. 8, T. 13 S., R. 5 W. Domestic well, dug 23 feet deep, backfilled around 12-inch concrete-tile casing. Measuring point is top of casing, 0.9 foot above land surface and 252.01 feet ${ }^{2}$ above mean sea level.

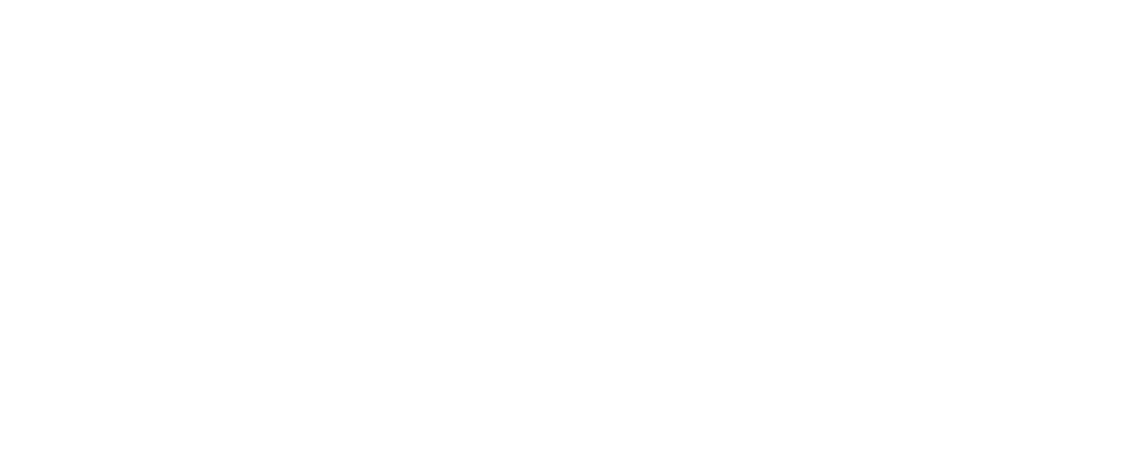

2 Leveling by U. S. Engineer Department.

- Pump operating in well a short time prior to measurement. 
TABLE 13.-Ground-water levels in observation wells, 1928-30 and 1935-36-Con.

574. L. D. Porter. SE1/4SE1/4 sec. 10, T. 13 S., R. 5 W. Stock well, dug 5 feet in diameter and 20.7 feet deep, brick curb. Measuring point is top of plank deck, at west side, 0.3 foot a bove land surface and 253.80 feet 2 a bove mean sea level.

\begin{tabular}{|c|c|c|c|c|c|c|c|}
\hline Date & $\begin{array}{l}\text { Water } \\
\text { level } \\
\text { (feet) }\end{array}$ & Date & $\begin{array}{l}\text { Water } \\
\text { level } \\
\text { (feet) }\end{array}$ & Date & $\begin{array}{l}\text { Water } \\
\text { level } \\
\text { (feet) }\end{array}$ & - Date & $\begin{array}{l}\text { Water } \\
\text { level } \\
\text { (feet) }\end{array}$ \\
\hline 1935 & & 1936 & & 1936 & & 1936 & \\
\hline Oct. 18 & 235.48 & Jan. 22 & 251.37 & May 13 & s 241.79 & Ang. 1 & (4) \\
\hline Oct. 23 . & 237.83 & Jan. 29 & 250.70 & May 20. & 239.84 & Aug. 5. & 235.12 \\
\hline Oct. 29 & 237.70 & $\mathrm{Feb} .5$ & 250.42 & May 27 & 239.89 & Aug. 8 & (4) \\
\hline Nov. 7. & 237.48 & Feb. 12 & 251.28 & June 3 & 239.33 & Aug. 12 & (4) \\
\hline Nov. 13 & 237.38 & Feb. 21 & 252.50 & June 10 & 236.61 & Aug. 15 . & (4) \\
\hline Nov. 20 & 237.26 & Feb. 26 . & 251.98 & June 17 & 238.78 & Aug. 19 . & (4) \\
\hline Nov. 29 & 236.38 & ar. 4 & 251.18 & June 24 & $\left(\begin{array}{ll}4 & 14\end{array}\right)$ & Aug. 26 & (4) \\
\hline Dec. 4 & 236.58 & Mar. 11 & 250.79 & June 26 & $(414)$ & Aug. 29 & $(4)$ \\
\hline Dec. 11 & 240.01 & Mar. 18 & 250.54 & July 1 & 236.28 & Sept. 2. & 237.93 \\
\hline Dec. 18 & 242.34 & Mar. 25... & 250.36 & July 3 . & $\left(\begin{array}{lll}4 & 14\end{array}\right)$ & Sept. 10 . & 236.89 \\
\hline Dec. 26 . & 242.37 & Apr. 1 & 251.10 & July 8 & 237.02 & Sept. 16. & 237.52 \\
\hline 1936 & & Apr. 8 & 250.89 & July 11 & 236.27 & Sept. 19 & 237. 71 \\
\hline Jan. 2 & 252.34 & Apr. 22 & $\begin{array}{r}250.49 \\
5244.41\end{array}$ & $\begin{array}{l}\text { July } 15 . \\
\text { July } 18 .\end{array}$ & $\begin{array}{l}\left(\begin{array}{ll}4 & 14\end{array}\right) \\
\left(\begin{array}{lll}4 & 14\end{array}\right)\end{array}$ & $\begin{array}{l}\text { Sept. } 23 \\
\text { Sept. } 26\end{array}$ & $\begin{array}{l}238.48 \\
237.98\end{array}$ \\
\hline Jan. 9. & 252.73 & Apr. 24 & 5244.45 & July 22 & $(4)^{\prime}$ & Sept. 30 & 237.83 \\
\hline Jan. 15 & 251.94 & Apr. 29 & 242.28 & July 25 . & (4) & & \\
\hline Jan. 18 & 251.94 & May 6 & 239.13 & July 29 & 234.5 & & \\
\hline
\end{tabular}

2 Leveling by U. S. Engineer Department.

4 Water level depressed by inordinately large withdrawals.

- Pump operating in well.

14 Well dry.

575. L. J. Porter. SE $1 / 4$ SE 144 sec. 10, T. 13 S., R. 5 W., across highway from well 574 . Unused well, dug 5 feet in diameter and 20.5 feet deep, dry-masonry curb. Measuring point is top of 2-inch plank deck, 0.6 foot above land surface and 253.52 feet ${ }^{2}$ above mean sea level.

\begin{tabular}{|c|c|c|c|c|c|c|c|}
\hline Date & $\begin{array}{l}\text { Water } \\
\text { level } \\
\text { (feet) }\end{array}$ & Date & $\begin{array}{l}\text { Water } \\
\text { level } \\
\text { (feet) }\end{array}$ & Date & $\begin{array}{l}\text { Water } \\
\text { level } \\
\text { (feet) }\end{array}$ & Date & $\begin{array}{l}\text { Water } \\
\text { level } \\
\text { (feet) }\end{array}$ \\
\hline 1935 & & 1936 & & 1936 & & 1936 & \\
\hline Oct. 18 & 237.72 & Jan. 9 & 252.09 & Apr. 8 & 251.04 & July 8 . & 240.56 \\
\hline Oct. 24 . & 235.76 & Jan. 15 & $\begin{array}{l}202.09 \\
252.66\end{array}$ & Apr. 15 & 249.59 & July 15 & 240.04 \\
\hline Oct. 29 . & 235.65 & Jan. 22 & 251.43 & Apr. 22 & 247.45 & July 22 & 240.62 \\
\hline Nov. 7. & 235.47 & Jan. 29 & 250.95 & Apr. 24 & 247.46 & July $29_{-}$ & 240.33 \\
\hline Nov. 13 & 235.40 & Feb. 5 & 250.91 & Apr. 20 & 245.78 & Aug. 5 & 240.05 \\
\hline Nov. 20 & 235.37 & Feb. 12 & 252.09 & May 6 & 244.89 & Aug. 12 & 240.03 \\
\hline Nov. 29 & 236.80 & Feb. 21 & 252.76 & May 13 & 5243.99 & Aug. 19 & 239.60 \\
\hline Dec. 4 & 236.83 & Feb. 26 . & 252.19 & May 20 & 243.22 & Aug. 26 & 239. 52 \\
\hline Dec. 11 & 240.41 & Mar. 4 & 251.05 & May 27 & 243.21 & Sept. 2 & 238.32 \\
\hline Dec. 18 & 239.60 & Mar. 11 & 250.83 & June 3 & 241.83 & Sept. 10 & 237.45 \\
\hline Dec. 26 & 240.53 & Mar. 18 & 250.68 & June 10 & 241.60 & Sept. 16 & 238.90 \\
\hline 1936 & & $\begin{array}{l}\text { Mar. 25- } \\
\text { Mar. } 27\end{array}$ & $\begin{array}{l}248.40 \\
248.12\end{array}$ & $\begin{array}{l}\text { June } 17 \\
\text { June } 24\end{array}$ & $\begin{array}{l}241.42 \\
241.25\end{array}$ & $\begin{array}{l}\text { Sept. } 23 \\
\text { Sept. } 30\end{array}$ & $\begin{array}{l}238.62 \\
238.56\end{array}$ \\
\hline Jan. 2 & 251.65 & Apr. 1 . & 251.71 & July 1 & 241.09 & & \\
\hline
\end{tabular}

${ }^{2}$ Leveling by U. S. Engineer Department.

5 Pump operating in well.

579 Harold Freeman. SE1/4 SW1/4 sec 34 , T 13 S., R. 5 W. Domestic well, driven 27.3 feet deep. Measuring point is lower valve seat of pump, 2.8 feet above land surface and 267.08 feet ${ }^{2}$ above mean sea level.

\begin{tabular}{|c|c|c|c|c|c|c|c|}
\hline Date & $\begin{array}{l}\text { Water } \\
\text { level } \\
\text { (feet) }\end{array}$ & Date & $\begin{array}{l}\text { Water } \\
\text { Ievel } \\
\text { (feet) }\end{array}$ & Date & $\begin{array}{l}\text { Water } \\
\text { level } \\
\text { (feet) }\end{array}$ & Date & $\begin{array}{l}\text { Water } \\
\text { level } \\
\text { (feet) }\end{array}$ \\
\hline 1935 & & 1936 & & 1936 & & 1936 & \\
\hline Oct. 18 & 248.36 & Feb. 12 & 256.26 & Apr. 29 & 254.57 & July 22 & 250.81 \\
\hline Oct. 24 & 248.29 & Feb. 2l & 257.00 & May 6 & 254 & July 29. & 251. 28 \\
\hline Oct. 30 & 248.23 & Feb. 26 & 257.11 & May 13 & 254.27 & Aug. 5 & 250.74 \\
\hline Nov. 7 . & 248.11 & Mar. 4 & 258.11 & May 20 & 254.55 & Aug. 12 & 249.85 \\
\hline & & & 257.05 & May 27 & 253.69 & Aug. 19 & 249.39 \\
\hline 1936 & & Mar. 18 & 258.81 & June 3 & 253.77 & Aug. 26 & 249.69 \\
\hline Jan 9 & & Mar. 25 & 255.41 & June 10 & 252.74 & Sept. 2- & 249.39 \\
\hline Jan. 15 & $\begin{array}{l}256.84 \\
258.91\end{array}$ & Mar. 27 & $\begin{array}{l}255.57 \\
255.26\end{array}$ & June $17-$ & $\begin{array}{l}252.19 \\
252.15\end{array}$ & $\begin{array}{l}\text { Sept. } 10 \\
\text { Sept. } 16\end{array}$ & $\begin{array}{l}249.20 \\
249.16\end{array}$ \\
\hline Jan, 22 . & 257.22 & Apr. 8 & 255.35 & July 1 . & 251.81 & Sept. 23 & 248.81 \\
\hline Jan. 29 & 255.18 & Apr. 15 & 254. & July 8 & 251.59 & Sept. 30 & 248.45 \\
\hline Feb. 5 & 254.27 & Apr. 22 & 252.87 & Julv 15 & 250.90 & & \\
\hline
\end{tabular}

2 Leveling by U. S. Engineer Department. 
TABLE 13.-Ground-water levels in observation wells, 1928-30 and 1935-36-Con.

581. Clingman Bros. SE 34 NW1/4 sec. 16, T. 13 S., R. 4 W. Stock well, dug 36 inches in diameter and 28 feet deep, in barnyard 20 feet southeast of pump house. Measuring points: August 13, 1928 and beginning October 4, 1935, top of brick casing level with land surface, 258.49 feet ${ }^{2}$ above mean sea level; Oct. 21, 1928 , to July 23,1930 , top of plank well cover at copper nail with washer, 0.5 foot above land surface and 259.0 feet above mean sea level.

\begin{tabular}{|c|c|c|c|c|c|c|c|}
\hline Date & $\begin{array}{l}\text { Water } \\
\text { level } \\
\text { (feet) }\end{array}$ & Date & $\begin{array}{l}\text { Water } \\
\text { level } \\
\text { (feet) }\end{array}$ & Date & $\begin{array}{l}\text { Water } \\
\text { level } \\
\text { (feet) }\end{array}$ & Date & $\begin{array}{l}\text { Water } \\
\text { level } \\
\text { (feet) }\end{array}$ \\
\hline $\begin{array}{l}\quad 1928 \\
\text { Aug. } 13 \\
\text { Oct. } 21 \\
\text { Dec. } 16 \\
\\
\text { Feb. } 2 \\
\text { Mar. } 27 \\
\text { May } 9 \\
\text { June } 6 \\
\text { July } 4 \\
\text { Aug. } 2 \\
\text { Sept. 23. } \\
\text { Oct. } 25 \\
\text { Nov. } 27 \\
\text { Dec. } 30 \\
\qquad 1930 \\
\text { Feb. } 1 \\
\text { Mar. } 1 \\
\text { Mar. } 29\end{array}$ & $\begin{array}{l}255.4 \\
251.90 \\
251.00 \\
248.65 \\
246.50 \\
244.35 \\
241.48 \\
240.45 \\
239.90 \\
252.45 \\
\\
\\
254.25 \\
253.90 \\
251.30\end{array}$ & 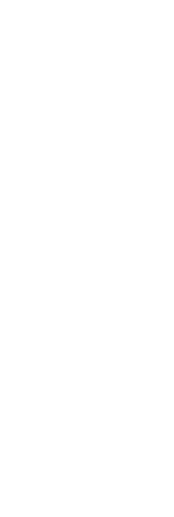 & $\begin{array}{l}250.40 \\
247.73 \\
244.15 \\
\\
240.27 \\
240.30 \\
239.92 \\
239.84 \\
239.70 \\
239.70 \\
239.79 \\
239.70 \\
243.10 \\
240.68 \\
241.01 \\
\\
243.08 \\
253.11\end{array}$ & 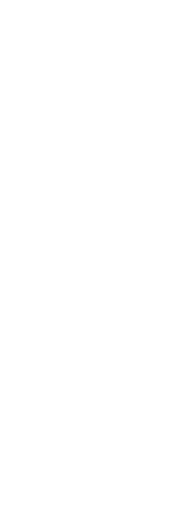 & $\begin{array}{l}256.23 \\
255.11 \\
253.87 \\
253.71 \\
252.83 \\
251.64 \\
253.46 \\
255.09 \\
253.94 \\
252.65 \\
253.11 \\
252.36 \\
252.30 \\
251.85 \\
250.32 \\
250.28 \\
248.77 \\
248.21 \\
248.43 \\
247.47 \\
246.71 \\
246.22\end{array}$ & \begin{tabular}{l}
\multicolumn{1}{c}{1936} \\
June 17 \\
June 24 \\
July 1 \\
July 10 \\
July 15 \\
July 22 \\
July 29 \\
Aug. 5 \\
Aug. 12 \\
Aug. 19 \\
Aug. 26 \\
Sept. 2 \\
Sept. 10 \\
Sept. 16 \\
Sept. 23 \\
Sept. 30
\end{tabular} & $\begin{array}{l}245.97 \\
245.59 \\
245.11 \\
244.70 \\
244.34 \\
243.78 \\
243.39 \\
242.94 \\
242.55 \\
242.20 \\
241.91 \\
241.89 \\
241.81 \\
241.77 \\
241.38 \\
241.14\end{array}$ \\
\hline
\end{tabular}

2 Leveling by U. s. Engineer Department.

589. Ida Metzger. SW14 NW1/4 sec. 30, T. 13 S., R. 3 W. Stock well, driven 24.8 feet deep. Measuring point is top of open standpipe at side of well, 1.5 feet above land surface and 274.53 feet ${ }^{2}$ above mean sea level.

\begin{tabular}{|c|c|c|c|c|c|c|c|}
\hline Date & $\begin{array}{l}\text { Water } \\
\text { level } \\
\text { (feet) }\end{array}$ & Date & $\begin{array}{l}\text { Water } \\
\text { level } \\
\text { (feet) }\end{array}$ & Dato & $\begin{array}{l}\text { Water } \\
\text { level } \\
\text { (feet) }\end{array}$ & Date & $\begin{array}{l}\text { Water } \\
\text { level } \\
\text { (feet) }\end{array}$ \\
\hline 1935 & & 1936 & & 1936 & & 1936 & \\
\hline $\begin{array}{l}\text { Oct. } 15 \\
\text { Oct. } 22 \\
\text { Oct. } 30 \\
\text { Nov. } 6 \\
\text { Nov. } 13 \\
\text { Nov. } 20 \\
\text { Dec. } 4 \\
\text { Dec. } 11 \\
\text { Dec. } 18 \\
\text { Dec. } 26 \\
\qquad 1936 \\
\text { Jan. } 1\end{array}$ & $\begin{array}{l}257.33 \\
257.30 \\
257.19 \\
257.08 \\
257.11 \\
257.15 \\
257.11 \\
258.67 \\
261.36 \\
262.11\end{array}$ & $\begin{array}{l}\text { Jan. } 8 \\
\text { Jan. } 10 \\
\text { Jan. } 15 \\
\text { Jan. } 22 \\
\text { Jan. } 24 \\
\text { Jan. } 29 \\
\text { Jan. } 31 \\
\text { Feb. } 5 \\
\text { Feb. } 13 \\
\text { Feb. } 20 \\
\text { Feb. } 26 \\
\text { Mar. } 4 \\
\text { Mar. } 11 \\
\text { Mar. } 25\end{array}$ & $\begin{array}{l}265.73 \\
266.45 \\
266.81 \\
267.05 \\
266.26 \\
266.01 \\
267.08 \\
265.72 \\
263.67 \\
264.07 \\
267.76 \\
265.51 \\
267.91 \\
267.95\end{array}$ & $\begin{array}{l}\text { Apr. 1. } \\
\text { Apr. } 8 \\
\text { Apr. 15 } \\
\text { Apr. 22 } \\
\text { Apr. 29 } \\
\text { May } 6 \\
\text { May } 13 \\
\text { May } 20 \\
\text { May } 27 \\
\text { June } 3 \\
\text { June } 10 \\
\text { June } 17 \\
\text { June } 24 \\
\text { July } 1\end{array}$ & $\begin{array}{l}268.16 \\
268.07 \\
268.00 \\
267.51 \\
267.31 \\
266.87 \\
266.01 \\
266.46 \\
267.25 \\
266.91 \\
266.45 \\
265.97 \\
266.03 \\
265.46\end{array}$ & $\begin{array}{l}\text { July } 10 \\
\text { July } 15 \\
\text { July } 22 \\
\text { July } 29 \\
\text { Aug. } 26 \\
\text { Sept. } 2 \\
\text { Sept. } 10 \\
\text { Sept. } 16 \\
\text { Sept. } 23 \\
\text { Sept. } 30\end{array}$ & $\begin{array}{l}265.20 \\
264.03 \\
263.96 \\
263.56 \\
260.64 \\
260.64 \\
260.62 \\
260.36 \\
260.14 \\
259.96\end{array}$ \\
\hline
\end{tabular}

2 Leveling by U. s. Engineer Department. 
TABLE 13.-Ground-water levels in observation wells, 1928-30 and 1935-36-Con.

590. Keeney School, district 51. SW $1 / 4$ SW1/4 sec. 34, T. 13 S., R. 3 W. Domestic well, driven $11 / 4$ inches in diameter and 18 feet deep, at east side of school near entrance. Casing stamped 13-3-5 just below pump base under pump handle. Measuring point is lower valve seat of pump, 2.5 feet above concrete pump platform and land surface and 287.5 feet ${ }^{2}$ above mean sea level.

\begin{tabular}{|c|c|c|c|c|c|c|c|}
\hline Date & $\begin{array}{l}\text { Water } \\
\text { level } \\
\text { (feet) }\end{array}$ & Date & $\begin{array}{l}\text { Water } \\
\text { level } \\
\text { (feet) }\end{array}$ & Date & $\begin{array}{l}\text { Water } \\
\text { level } \\
\text { (feet) }\end{array}$ & Date & $\begin{array}{l}\text { Water } \\
\text { level } \\
\text { (feet) }\end{array}$ \\
\hline 1928 & & 1935 & & 1936 & - & 1936 & \\
\hline Aug. 16. & 278.0 & Oet. $30_{-}$ & 277.20 & Jan. 29 & 281.93 & June 3 & 280.72 \\
\hline 1929 & & $\begin{array}{l}\text { Nov. } 6 \\
\text { Nov. } 13\end{array}$ & $\begin{array}{l}277.15 \\
277.26\end{array}$ & $\begin{array}{l}\text { Jan. } 31 \\
\text { Feb. } 5\end{array}$ & $\begin{array}{l}282.81 \\
281.98\end{array}$ & $\begin{array}{l}\text { June } 10 \\
\text { June } 17\end{array}$ & $\begin{array}{l}280.48 \\
280.71\end{array}$ \\
\hline Oct. 25 & 276.35 & Nov. 21 & 277.50 & Feb. 13 & 281.25 & June 24 & 280.18 \\
\hline Nov. 27. & 276.10 & Dec. 5 & 278.78 & Feb. 20 & 280.76 & July 1. & 279.83 \\
\hline Dec. 31 & 281.85 & Dec. 11 & 279.91 & Feb. 26. & 282.83 & July 10 & 279.65 \\
\hline 1930 & & Dec. 18 . & 281.22 & Mar. 4 & 280.68 & July 15 & $\begin{array}{l}279.44 \\
279.04\end{array}$ \\
\hline Feb. 1 & 282.50 & Dec. 28 & 281.70 & Mar. 18 & $\begin{array}{l}282.62 \\
281.77\end{array}$ & $\begin{array}{l}\text { July } 22- \\
\text { July } 29 .\end{array}$ & $\begin{array}{l}279.04 \\
278.71\end{array}$ \\
\hline Mar. 1 & 282.45 & Dec. 2 & 201.10 & Mar. 25 & 281.82 & Aug. 5 & 278.39 \\
\hline Mar. 29. & 282.25 & 1936 & & Apr. 1 & 282.33 & Aug. 12 & 278.13 \\
\hline Apr. 26 . & 282.55 & & & Apr. 8 & 282.12 & Aug. 19. & 278.53 \\
\hline 28 & 281.12 & Jan. 2 & 282.38 & Apr. 15 & 282.20 & Aug. $26 \ldots$ & 277.66 \\
\hline July 23 & 278. 79 & Jan. 3 & 282.70 & Apr. 22 & 281.36 & Sept. 2 & 277. 39 \\
\hline 1935 & & Jan. $8 \ldots$ & 283. 96 & Apr. 29 & 281.26 & $\begin{array}{l}\text { Sept. } 10 \\
\text { Sept. } 16\end{array}$ & $\begin{array}{l}277.30 \\
277.28\end{array}$ \\
\hline oct. 4 . & 276.59 & Jan. 15.- & $\begin{array}{l}284.23 \\
282.99\end{array}$ & May $13 .$. & $\begin{array}{l}280.80 \\
281.46\end{array}$ & Sept. 23 & 277.02 \\
\hline Oct. 12 & 276.57 & Jan. 22 & 282.73 & May 20 & 281.22 & Sept. $30 \ldots$. & 276.86 \\
\hline Oct. 17 & 276.48 & Jan. 24 & 282.34 & May 27. & 281.32 & & \\
\hline
\end{tabular}

2 Leveling by U. s. Engineer Department.

594. Central School, district 50. SW1/SW1/4 sec. 3, T. 14 S., R. 5 W. Unused well, 3 inches in diameter and 26 feet deep, 20 feet south of school. Stamped 14-5-1 on iron pump brace. Measuring point is lower valve seat of pump, 2.15 feet above concrete pump platform and 268.2 feet above mean sea level (interpolated).

\begin{tabular}{c|c||c|c}
\hline Date & $\begin{array}{c}\text { Water } \\
\text { level } \\
\text { (feet) }\end{array}$ & Date & $\begin{array}{c}\text { Water } \\
\text { level } \\
\text { (feet) }\end{array}$ \\
\hline 1928 & & 1929 & \\
\hline Aug. 14 & 250.8 \\
Oct. 22 & $\begin{array}{l}250.5 \\
\text { Dec. 16. Feb. 2 }\end{array}$ & & \\
\hline
\end{tabular}

596. Mrs. Thomas Harvey (formerly W. E. Thomas). SE1/4SE1/4 sec. 10, T. 14 S., R. 5 W. Stock well, driven $11 / 4$ inches in diameter and 19 feet deep, in southeast corner of field 0.3 mile east of residence. Casing stamped 14-5-5 on south side just below pump base. Measuring point is lower valve seat of pump, 2.9 feet above land surface and 270.39 feet ${ }^{2}$ above mean sea level.

\begin{tabular}{|c|c|c|c|c|c|c|c|}
\hline Date & $\begin{array}{l}\text { Water } \\
\text { level } \\
\text { (feet) }\end{array}$ & Date & $\begin{array}{l}\text { Water } \\
\text { level } \\
\text { (feet) }\end{array}$ & Date & $\begin{array}{l}\text { Water } \\
\text { leve] } \\
\text { (feet) }\end{array}$ & Date & $\begin{array}{l}\text { Water } \\
\text { level } \\
\text { (feet) }\end{array}$ \\
\hline 1929 & & 1935 & & 1936 & & 1936 & \\
\hline July 4 & 254.4 & Oct. 12 . & 252.11 & Feb. 12 & 260.60 & June 24 & 254.36 \\
\hline Aug. 2 & 253. & Oct. 18 & 252.35 & Feb. 21 & 261.15 & July 1 . & 254.05 \\
\hline Sept. 23 & 252.24 & Oct. 21 . & 252.07 & Feb. 26 & 267.70 & July 8 & 253.52 \\
\hline Oct. 26 & 251.94 & Nov. 7 & 251.99 & Mar. 4 & 261. 51 & July 15 & 254.13 \\
\hline Nov. 28 & 251.74 & Nov. 13 & 251.99 & Mar. 11 & 260.73 & July 22 & 254.05 \\
\hline 1930 & & $\begin{array}{l}\text { Nov. } 20 \\
\text { Nov } 29\end{array}$ & 252. 11 & $\begin{array}{l}\text { Mar. } 18 \\
\text { Mar. } 25\end{array}$ & $\begin{array}{l}260.10 \\
258.63\end{array}$ & $\begin{array}{l}\text { July } 29 \\
\text { Aug. } 5\end{array}$ & $\begin{array}{l}253.70 \\
253.43\end{array}$ \\
\hline & & Dec. 4 & $\begin{array}{l}252.89 \\
252.86\end{array}$ & Apr. 1 & 258. 28 & Aug. 12 & 253.07 \\
\hline Jan. 2 . & 260.14 & Dec. 11 . & 253. 75 & Apr. 8 & 256.46 & Aug. 19 . & ${ }^{4} 252.65$ \\
\hline Jan. 31 & 257.64 & Dec. 18 & 252.92 & Apr. 15 & 254.60 & Aug. 26 . & 25305 \\
\hline $\mathrm{Feb} .28$ & 261.09 & Dec. 26 & 254. 96 & Apr. 22 & 257.12 & Sept. 2 & 252.80 \\
\hline Mar. 28 & 257. 69 & & & Apr. 29 & 257.09 & Sept. 10 & 252.32 \\
\hline Apr. 25 & 255.99 & 1936 & & May 6 & 257.60 & Sept. 16 & 252.39 \\
\hline May 28 & 255.03 & & & May 13 & 258.06 & Sept. 23 & 252.05 \\
\hline July 23 . & 253.26 & Jan. 2 & 257.82 & May 20. & 258.31 & Sept. 30. & 251.58 \\
\hline 1935 & & $\begin{array}{l}\operatorname{Jan} .9 \\
\operatorname{Jan} 22\end{array}$ & $\begin{array}{l}260.25 \\
262.26\end{array}$ & $\begin{array}{l}\text { May 27 } \\
\text { June } 3\end{array}$ & $\begin{array}{l}255.60 \\
255.81\end{array}$ & & \\
\hline & & & 258.75 & June 10 & 255.21 & & \\
\hline Oct. 5 & 252.17 & Feb. 5_ & 260.25 & June 17 & 254. 98 & & \\
\hline
\end{tabular}

2 Leveling by U. S. Engineer Department.

4 Water level depressed by inordinately large withdrawals. 
TABLE 13.-Ground-water levels in observation wells, 1928-30 and 1985-36-Con.

602. W. N. Carpenter. SW $1 / 4$ NE1/4 sec. 33, T. 14 S., R. 5 W. Well drilled 3 inches in diameter and 61.3 feet deep, steel casing. Measuring point is top of 3 -inch steel casing, 1.2 feet above land surface and 287.58 feet 2 above mean sea level.

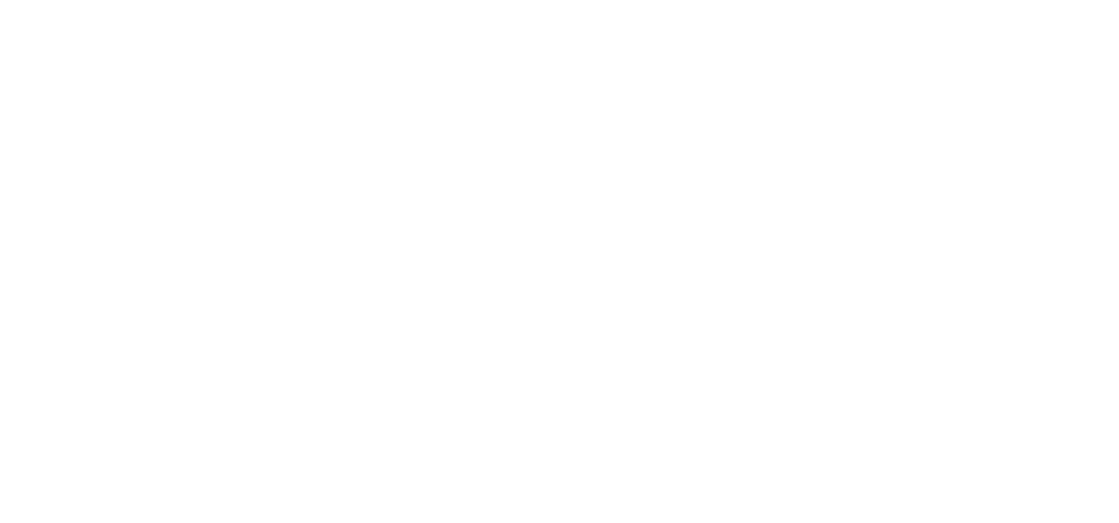

Leveling by U. S. Engineer Department.

603. R. L. George. SE $1 / 4$ NW $1 / 4$ sec. 34, T. 14 S., R. 5 W. Stock well, driven 24 feet deep. Measuring point is lower valve seat of pump, 2.4 feet above land surface and 287.65 feet 2 above mean sea level.

\begin{tabular}{|c|c|c|c|c|c|c|c|}
\hline Date & $\begin{array}{l}\text { Water } \\
\text { level } \\
\text { (feet) }\end{array}$ & Date & $\begin{array}{l}\text { Water } \\
\text { level } \\
\text { (feet) }\end{array}$ & Date & $\begin{array}{l}\text { Water } \\
\text { level } \\
\text { (feet) }\end{array}$ & Date & $\begin{array}{l}\text { Water } \\
\text { level } \\
\text { (feet) }\end{array}$ \\
\hline 1935 & & 1936 & & 1936 & & 1936 & \\
\hline $\begin{array}{l}\text { Oct. } 17 \\
\text { Oct. } 24 \\
\text { Oct. } 30 \\
\text { Nov. } 7 \\
\text { Nov. } 13 \\
\text { Nov. } 20 \\
\text { Nov. } 30 \\
\text { Dec. } 5 \\
\text { Dec. } 12 \\
\text { Dec. } 19 \\
\text { Dec. } 27 \\
\quad 1936 \\
\text { Jan. } 3\end{array}$ & $\begin{array}{l}272.28 \\
272.21 \\
272.16 \\
272.07 \\
271.97 \\
272.10 \\
272.11 \\
272.62 \\
272.97 \\
273.01 \\
273.04\end{array}$ & $\begin{array}{l}\text { Jan. } 10 \\
\text { Jan. } 16 \\
\text { Jan. } 23 \\
\text { Jan. } 30 \\
\text { Feb. } 6 \\
\text { Feb. } 13 \\
\text { Feb. } 22 \\
\text { Feb. } 27 \\
\text { Mar. } 5 \\
\text { Mar. } 12 \\
\text { Mar. } 19 \\
\text { Mar. } 26 \\
\text { Apr. } 2 \\
\text { Apr. } 9 \\
\text { Apr. } 16 \\
\end{array}$ & $\begin{array}{l}277.06 \\
285.45 \\
284.62 \\
279.68 \\
278.51 \\
277.69 \\
276.80 \\
281.84 \\
281.02 \\
280.79 \\
278.51 \\
276.97 \\
278.48 \\
278.59 \\
276.34\end{array}$ & $\begin{array}{l}\text { Apr. } 23 \\
\text { Apr. } 29 \\
\text { May } 6 \\
\text { May } 14 \\
\text { May } 21 \\
\text { May } 28 \\
\text { June } 4 \\
\text { June } 12 \\
\text { June } 18 \\
\text { June } 25 \\
\text { July } 2 \\
\text { July } 9 \\
\text { July } 15 \\
\text { July } 23 \\
\text { July } 30 \\
\end{array}$ & $\begin{array}{l}276.38 \\
276.62 \\
276.83 \\
276.37 \\
276.54 \\
276.58 \\
275.97 \\
276.21 \\
276.24 \\
275.82 \\
275.06 \\
274.87 \\
274.62 \\
274.36 \\
274.18\end{array}$ & $\begin{array}{l}\text { Aug. } 6 \\
\text { Aug. } 13 \\
\text { Aug. } 20 \\
\text { Aug. } 27 \\
\text { Sept. } 3 \\
\text { Sept. 11 } \\
\text { Sept. 17 } \\
\text { Sent. } 24 \\
\text { Oct. } 1\end{array}$ & $\begin{array}{l}273.97 \\
273.88 \\
273.59 \\
273.18 \\
272.82 \\
272.63 \\
273.06 \\
272.98 \\
272.72\end{array}$ \\
\hline
\end{tabular}

2 Leveling by U. S. Engineer Department.

604. M. L. Hewitt. SE14SW14 sec. 36, T. 14 S., R. 5 W. Stock well, driven 17.2 feet deep. Measuring point is lower valve seat of pump, 2.0 feet above land surface and 286.88 feet ${ }^{2}$ above mean sea level.

\begin{tabular}{|c|c|c|c|c|c|c|c|}
\hline Date & $\begin{array}{l}\text { Water } \\
\text { level } \\
\text { (feet) }\end{array}$ & Date & $\begin{array}{l}\text { Water } \\
\text { level } \\
\text { (feet) }\end{array}$ & Date & $\begin{array}{l}\text { Water } \\
\text { level } \\
\text { (feet) }\end{array}$ & Date & $\begin{array}{l}\text { Water } \\
\text { level } \\
\text { (feet) }\end{array}$ \\
\hline 1935 & & 1936 & & 1936 & & 1936 & \\
\hline $\begin{array}{l}\text { Nov. } 6 \\
\text { Nov. } 13 \\
\text { Nov. } 20 \\
\text { Dec. } 12 \\
\text { Dec. } 19 \\
\text { Dec. } 27 \\
\text { Dec. } 28 \\
\qquad 1936 \\
\text { Jan. } 3 \\
\text { Jan. } 10 \\
\text { Jan. } 16\end{array}$ & $\begin{array}{l}270.58 \\
270.66 \\
270.78 \\
271.17 \\
272.04 \\
272.02 \\
273.00 \\
\\
\\
273.91 \\
274.70 \\
280.75\end{array}$ & $\begin{array}{l}\text { Jan. } 23 \\
\text { Jan. } 30 \\
\text { Feb. } 6 \\
\text { Feb. } 13 \\
\text { Feb. } 22 \\
\text { Feb. } 27 \\
\text { Mar. } 5 \\
\text { Mar. } 12 \\
\text { Mar. } 19 \\
\text { Mar. } 26 \\
\text { Mar. } 27 \\
\text { Apr. } 2 \\
\text { Apr. } 9\end{array}$ & $\begin{array}{l}280.20 \\
274.75 \\
275.21 \\
275.37 \\
274.71 \\
277.23 \\
277.03 \\
281.06 \\
280.20 \\
274.82 \\
274.95 \\
274.24 \\
273.95\end{array}$ & $\begin{array}{l}\text { Apr. } 16 \\
\text { Apr. } 23 \\
\text { Apr. } 30 \\
\text { May } 7 \\
\text { May } 14 \\
\text { May } 21 \\
\text { May } 28 \\
\text { June } 4 \\
\text { June } 11 \\
\text { June } 18 \\
\text { June } 25 \\
\text { July } 2 \\
\text { July } 9\end{array}$ & $\begin{array}{l}273.43 \\
275.07 \\
274.57 \\
273.30 \\
273.75 \\
273.84 \\
273.90 \\
272.56 \\
272.34 \\
272.14 \\
272.12 \\
271.89 \\
271.52\end{array}$ & $\begin{array}{l}\text { July } 16 \ldots \\
\text { July } 23 \ldots \\
\text { July } 30 \\
\text { Aug. } 6 \\
\text { Aug. } 13 \\
\text { Aug. } 20 \\
\text { Aug. } 27 \\
\text { Sept. } 3 \\
\text { Sept. } 11 \\
\text { Sept. } 17 \\
\text { Sept. } 24 \\
\text { Oct. } 1\end{array}$ & $\begin{array}{l}271.57 \\
271.42 \\
270.84 \\
270.61 \\
272.34 \\
270.76 \\
270.41 \\
269.67 \\
269.42 \\
270.69 \\
270.15 \\
269.80\end{array}$ \\
\hline
\end{tabular}

2Leveling by U. S. Engineer Department. 
TABLe 13.-Ground-water levels in observation wells, 1928-30 and 1935-36-Con.

606. Citizens Light \& Water Co. (of Halsey). SE1/4NE1/4 sec. 1, T. 14 S., R. 4 W. Public-supply well, dug 10 feet in diameter and 28 feet deep, extended by two 4 -inch drilled holes to a total depth 72 feet, in pump house north of tank tower. Copper nail with washer stamped 14-4-6 in east end of door sill. Measuring point is base of 8- by 8-inch beam at copper nail with washer, about 7.5 feet below land surface and 274.5 feet above mean sea level (interpolated).

\begin{tabular}{|c|c|c|c|}
\hline Date & $\begin{array}{l}\text { Water } \\
\text { level } \\
\text { (feet) }\end{array}$ & Date & $\begin{array}{l}\text { Water } \\
\text { level } \\
\text { (feet) }\end{array}$ \\
\hline $\begin{array}{l}\text { Aug. } 16 \ldots 28 \\
\text { Oct. } 21 \\
\text { Dec. } 16 \\
\end{array}$ & $\begin{array}{l}264.65 \\
263.3 \\
267.79\end{array}$ & $\begin{array}{l}\text { Feb. } 2 \\
\text { Mar. } 27 \\
\text { May } 929 \\
\end{array}$ & $\begin{array}{l}270.9 \\
271.25 \\
272.3\end{array}$ \\
\hline
\end{tabular}

607. J. H. Suytor. NE1/4NW1/4 sec. 5, T. 14 S., R. 4 W. Domestic well, driven $11 / 2$ inches in diameter and 23.4 feet deep. Measuring point is lower valve seat of pump, 3.5 feet above land surface and 273.0 feet ${ }^{2}$ above mean sea level.

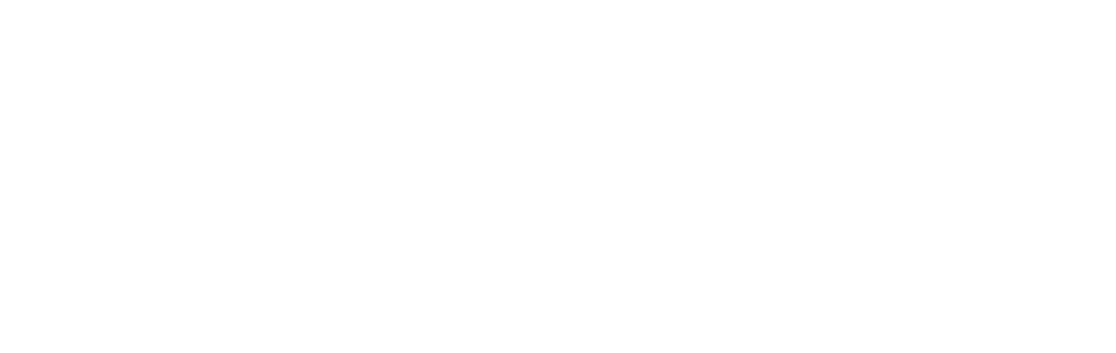

2 Leveling by U. S. Engineer Department.

608. L. O. Kroft. SE $1 / 4$ NW1/4 sec. 5, T. 14 S., R. 4 W. Unused well, drilled 6 inches in diameter and 50 feet deep, steel casing. Measuring point is top of $11 / 2$-inch standpipe, 0.2 foot above land surface and 271.87 feet ${ }^{2}$ above mean sea level.

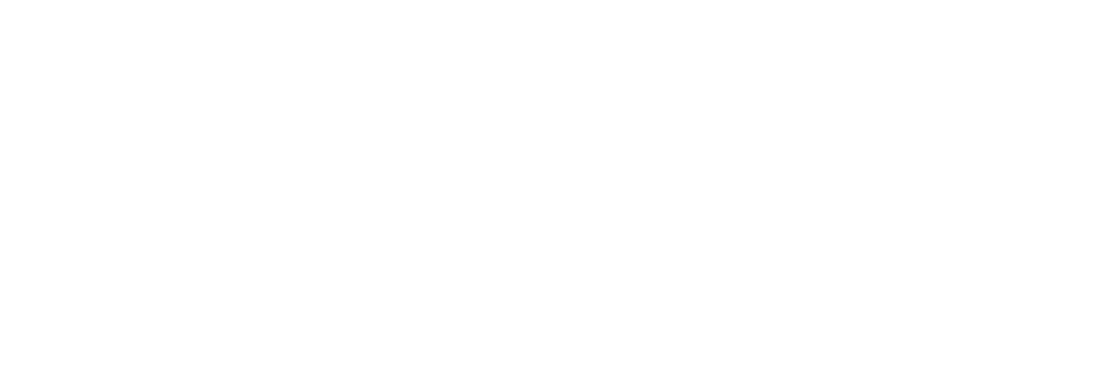

${ }^{2}$ Leveling by U. S. Engineer Department. 
TABLE 13.-Ground-water levels in observation wells, 1928-30 and 1935-36-Con.

613. B. E. Cogswell. SE1/4NW1/4 sec. 26 , T. 14 S., R. 4 W. Stoek well, dug 4 feet in diameter and 15.5 feet deep, backfilled around 6-inch tile casing, in barnyard. Copper nail with washer stamped 14-4-7 in 6- by 10-inch timber at north side of well. Measuring point is top of tile casing, painted white on west side, level with land surface and 294.56 feet ${ }^{2}$ above mean sea level.

\begin{tabular}{|c|c|c|c|c|c|c|c|}
\hline Date & $\begin{array}{l}\text { Water } \\
\text { level } \\
\text { (feet) }\end{array}$ & Date & $\begin{array}{l}\text { Water } \\
\text { level } \\
\text { (feet) }\end{array}$ & Date & $\begin{array}{l}\text { Water } \\
\text { level } \\
\text { (feet) }\end{array}$ & Date & $\begin{array}{l}\text { Water } \\
\text { level } \\
\text { (feet) }\end{array}$ \\
\hline 1928 & & 1930 & & 1936 & & 1936 & \\
\hline Aug. 18 & 283.96 & Mar. 29 & 286.16 & Jan. 8 & 286.68 & May 21 & 285.46 \\
\hline Oct. 21 . & 283.26 & Apr. 26 & 287.16 & Jan. 10 & 287.43 & May 28 & 285.43 \\
\hline Dec. 16 . & 284.81 & $\begin{array}{l}\text { May } 28 \\
\text { July } 23 .\end{array}$ & $\begin{array}{l}280.02 \\
284.04\end{array}$ & Jan. 16 & 288.38 & June 4 & $\begin{array}{l}285.09 \\
285.00\end{array}$ \\
\hline 1929 & & 1935 & & Jan. 24 & 285.94 & June 18 & 284.25 \\
\hline Feb. 2 & 292.46 & Oct. 4 & 282.65 & Feb. $6 \ldots$ & $\begin{array}{l}280.91 \\
286.09\end{array}$ & July 2 & 284.41 \\
\hline Mar. 27 & 286.26 & Oet. 12 & 282. 72 & Feb. 13 & 285,69 & July 11 & 284.32 \\
\hline May 9. & 286.36 & Oct. 17 - & 282.88 & Feb. 21- & 291.23 & July 16 & ${ }^{4} 282.49$ \\
\hline Jun & 285.56 & Oet. 31 - & 282.68 & Feb. 27 & 287.73 & July 23 & $\begin{array}{r}4282.58 \\
4\end{array}$ \\
\hline Jul & 284.66 & Nov. 7 & 282.56 & Mar. 5 & 290.29 & July 30 & 282. 79 \\
\hline $\mathbf{A u q}$ & 283.51 & Nor & 282.66 & Mar. 12 & 286.17 & Aug. 6 & 282.56 \\
\hline Sept. 23 & 283.21 & No & 282.78 & Mar. 19 & 286.75 & Aug. 12 & 282.42 \\
\hline Oet. 26 . & 282.91 & Dec. 5 . & 282.72 & Mar. 25 & 285.57 & Aug. 20 & 282.35 \\
\hline Nov. 2 & 283. 11 & Dec. 12 & 285.24 & Apr. 2. & 286.24 & Aug. 27. & 282.35 \\
\hline Dec. 31 & 285.76 & Dec. 19 & 284. 06 & Apr. 9 & 284. 59 & Sept. 3 & 282.23 \\
\hline & & Dec. 27 & 284.51 & Apr 16 & 285.75 & Sept. 11 & 282. 10 \\
\hline 1930 & & Dec. 28 & 285.74 & Apr, 23 & 285.49 & Sept. 17 & 282.06 \\
\hline & 298 & 1936 & & $\begin{array}{l}\text { Apr. } 30 \\
\text { May } 7\end{array}$ & $\begin{array}{r}285.49 \\
285.12\end{array}$ & Sept. 24_... & $\begin{array}{l}281.70 \\
281.53\end{array}$ \\
\hline & 287.31 & Jan. 2 . & 285.21 & May 14 & 285.65 & & \\
\hline
\end{tabular}

2 Leveling by U. S. Engineer Department.

4 Water level depressed by inordinately large withdrawals.

615. F. J. George. SW1/4SE1/4 sec. 28 , T. 14 S., R. 4 W. Unused driven well, 17 feet deep. Measuring point is lower valve seat of pump, 1.4 feet 2 above land surface and 294.6 feet $^{2}$ above mean sea level.

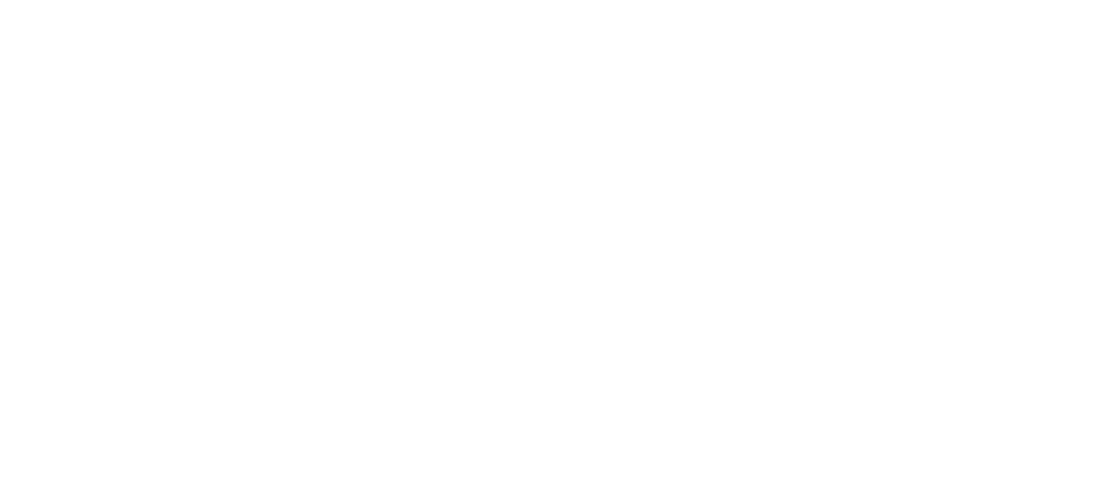

2 Leveling by U. S. Engineer Department.

616. Mrs. R. Toedtemeier. SW1/4W $1 / 4$ sec. 15, T. 14 S., R. 4 W. Unused well, dug 4 feet in diameterand 24 feet deep, in field 0.25 mile north of residence. Copper nail with washer stamped 14-3-5 in top of curb. Measuring point is lower valve seat of pump, 1.7 feet above land surface and 308.7 feet a bove mean sea level (interpolated).

\begin{tabular}{|c|c|c|c|}
\hline Date & $\begin{array}{l}\text { Water } \\
\text { level } \\
\text { (feet) }\end{array}$ & Date & $\begin{array}{l}\text { Water } \\
\text { level } \\
\text { (feet) }\end{array}$ \\
\hline $\begin{array}{l}\text { Aug. } 23 \\
\text { Aus }\end{array}$ & $\begin{array}{l}290.3 \\
290.1 \\
291.1\end{array}$ & 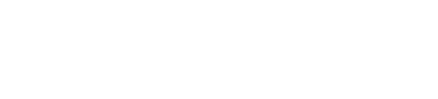 & $\begin{array}{l}302.3 \\
293.2 \\
290.2\end{array}$ \\
\hline
\end{tabular}


TABLE 13.-Ground-water levels in observation wells, 1928-30 and 1985-36-Con.

618. Charity Grange. SE1/4NE1/4 sec. 28 , T. 14 S., R. 3 W. Unused well, driven $11 / 2$ inches in diameter and 35.5 feet deep. Measuring point is lower valve seat of pump, 2.5 feet above land surface and 314.4 feet $^{2}$ above mean sea level.

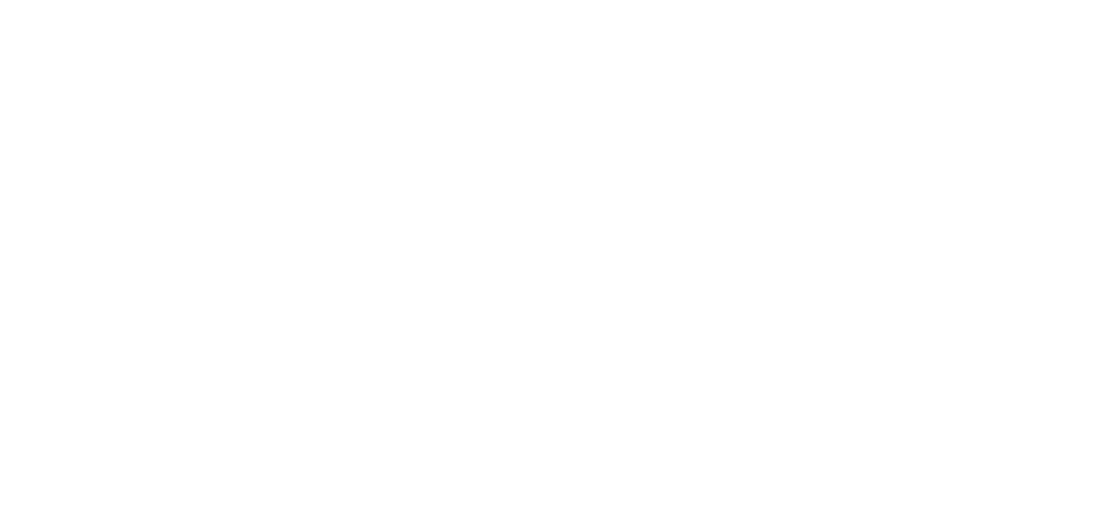

2 Leveling by U. S. Engineer Department.

621. Rosa D. Bierley (former owner, Mrs. E. Holloway). NW1/4NW1/4 sec. 7, T. 14 S., R. 2 W. Domestic well, bored 4 inches in diameter and 76 feet deep, steel lcasing. Measuring point is top of 4-inch steel casing, 0.2 foot above land surface and 332.3 feet ${ }^{2}$ above mean sea level.

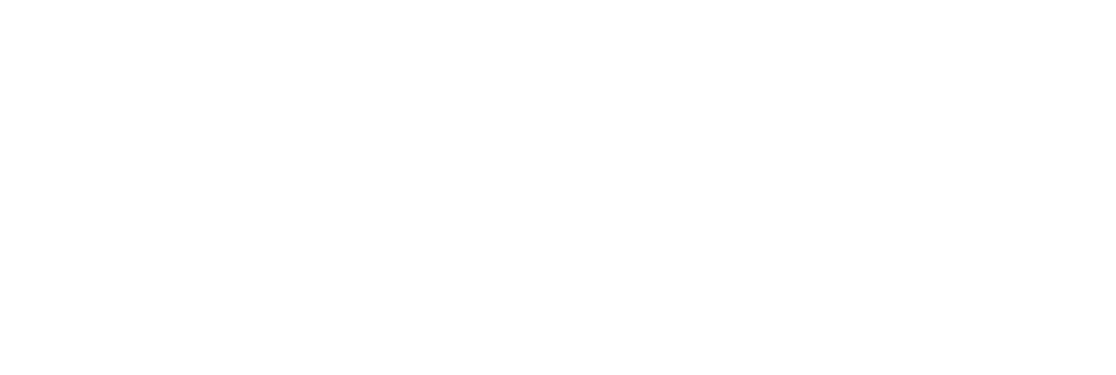

${ }^{2}$ Leveling by U. S. Engineer Department.

630. C. M. Spone. SE1/4SE $1 / 4$ sec. 9 , T. 15 S., R. 4 W. Domestic well, driven $1 \frac{1}{2}$ inches in diameterand 20.6 feet deep. Measuring point is lower valve seat of pump, 1.9 feet above land surface and 309.18 feet $^{2}$ above mean sea level.

\begin{tabular}{|c|c|c|c|c|c|c|c|}
\hline Date & $\begin{array}{l}\text { Water } \\
\text { level } \\
\text { (feet) }\end{array}$ & Date & $\begin{array}{l}\text { Water } \\
\text { level } \\
\text { (feet) }\end{array}$ & Date & $\begin{array}{l}\text { Water } \\
\text { level } \\
\text { (feet) }\end{array}$ & Date & $\begin{array}{l}\text { Water } \\
\text { level } \\
\text { (feet) }\end{array}$ \\
\hline 1935 & & 1936 & & 1936 & & 1936 & \\
\hline Oet. 17 & 292.88 & Ja11. 9 & 296.59 & Apr. 16 & 296.80 & July 23 & 294.46 \\
\hline Oct. 22 & 292.78 & Jan. 16 & 299.31 & Apr. 23 & 297.03 & July 30 . & 204.18 \\
\hline Oct. 31 & 292.76 & Jan. 23 & 298.20 & Apr. $30_{-}$ & 296.68 & Aug. 6 & 294.31 \\
\hline Nov. 7 & 292.66 & Jan. 30 & 297.34 & May 7 & 296.40 & Aug. 13 & 293.79 \\
\hline Nov. 14 & 293.01 & Feb. 6 & 296.28 & May 14 & 297.14 & Aug. 20 & 294. 38 \\
\hline Nov. 21 & 293.10 & Feb. 13 & 296.05 & May 21 . & 295.61 & Aug. 27. & 294.22 \\
\hline Dec. 5 & 293.11 & Feb. 21 & 296.99 & May 28 & 295.21 & Sept. 3 & 294.16 \\
\hline Dec. 12 & 293.37 & Feb. 27. & 298.75 & June 4 & 295.04 & Sept. 11 & 293.43 \\
\hline Dec. 19 & 293.71 & Mar. 5 & 299.31 & June $11-$ & 295.86 & Sept. 17. & 294.00 \\
\hline Dec. 27 & 293.62 & Mar. 12 & 297.43 & June 18 & 296.17 & Sept. 24 & 293.81 \\
\hline 1936 & & $\begin{array}{l}\text { Mar. } 19 \ldots \\
\text { Mar. } 26\end{array}$ & $\begin{array}{l}297.88 \\
296.61\end{array}$ & June 25 & $\begin{array}{r}295.36 \\
295.14\end{array}$ & ct. 1 & 293.59 \\
\hline & & Apr. 2 & $\begin{array}{l}290.01 \\
296.99\end{array}$ & July 11. & 294.79 & & \\
\hline Jan. 2- & 294.27 & Apr. 9 & 297.24 & July 16 & 294.61 & & \\
\hline
\end{tabular}

${ }^{2}$ Leveling by U. S. Engineer Department. 
TABLE 13.-Ground-water levels in observation wells, 1928-30 and 1935-36-Con.

631. H. F. Meersdorf. NE1/4NE1/4 sec. 16, T. 15 S., R. 4 W. Public-supply well (Harrisburg), dug 10 feet in diameterand 30 feet deep, extended by several 3 -inch driven wells to a total depth of 60 feet. Copper nail with washer stamped 15-4-2 in well curb. Measuring point is top of plank well curb at copper nail with washer, 1.0 foot below land surface and 307 feet above mean sea level (interpolated).

\begin{tabular}{|c|c|c|c|}
\hline Date & $\begin{array}{l}\text { Water } \\
\text { level } \\
\text { (feet) }\end{array}$ & Date & $\begin{array}{l}\text { Water } \\
\text { level } \\
\text { (feet) }\end{array}$ \\
\hline $\begin{array}{l}\text { Aug. } 1928 \\
\text { Oct. } 21 \\
\text { Dec. } 16\end{array}$ & $\begin{array}{l}289.6 \\
289.6 \\
290.4\end{array}$ & 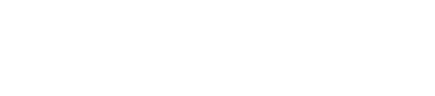 & $\begin{array}{l}294.8 \\
293.35 \\
292.85\end{array}$ \\
\hline
\end{tabular}

633. Mrs. Ingraham. SE $1 / 4 \mathrm{NW} 1 / 4$ sec. $20, \mathrm{~T} .15 \mathrm{~S}$., R. $4 \mathrm{~W}$. Unused well, driven 22.1 feet deep. Measuring point is lower valve seat of pump, 2.8 feet above land surface and 315.72 feet 2 a bove mean sea level.

\begin{tabular}{|c|c|c|c|c|c|c|c|}
\hline Date & $\begin{array}{l}\text { Water } \\
\text { leveI } \\
\text { (feet) }\end{array}$ & Date & $\begin{array}{l}\text { Water } \\
\text { Ievel } \\
\text { (feet) }\end{array}$ & Date & $\begin{array}{l}\text { Water } \\
\text { level } \\
\text { (feet) }\end{array}$ & Date & $\begin{array}{l}\text { Water } \\
\text { Ievel } \\
\text { (feet) }\end{array}$ \\
\hline 1935 & & 1936 & & 1936 & & 1936 & \\
\hline $\begin{array}{l}\text { Oct. } 17 \\
\text { Oct. } 24 \\
\text { Oct. } 30 \\
\text { Nov. } 7 \\
\text { Nov. } 13 \\
\text { Nov. } 20 \\
\text { Nov. } 30 \\
\text { Dec. } 5 \\
\text { Dec. } 12 \\
\text { Dec. } 19 \\
\text { Dec. } 27\end{array}$ & $\begin{array}{l}294.56 \\
294.65 \\
294.53 \\
294.52 \\
294.57 \\
294.79 \\
296.04 \\
294.81 \\
295.89 \\
295.55 \\
295.73\end{array}$ & $\begin{array}{l}\text { Jan. } 3 \\
\text { Jan. } 10 \\
\text { Jan. } 16 \\
\text { Jan. } 22 \\
\text { Jan. } 30 \\
\text { Feb. } 6 \\
\text { Feb. } 13 \\
\text { Feb. 27 } \\
\text { Mar. } 5 \\
\text { Mar. 12 } \\
\text { Mar. 19 } \\
\text { Mar. 26 } \\
\text { Apr. 2 }\end{array}$ & $\begin{array}{l}296.91 \\
301.08 \\
305.58 \\
304.75 \\
302.73 \\
298.65 \\
298.09 \\
300.56 \\
300.13 \\
299.71 \\
298.21 \\
298.90 \\
297.91\end{array}$ & \begin{tabular}{|l} 
Apr. 9 \\
Apr. 16 \\
Apr. 23 \\
Apr. 30 \\
May 7 \\
May 14 \\
May 21 \\
May 28 \\
June 3 \\
June 11 \\
June 18 \\
June 25 \\
July 2
\end{tabular} & $\begin{array}{l}297.68 \\
297.70 \\
297.78 \\
297.66 \\
297.74 \\
298.21 \\
297.28 \\
296.76 \\
296.58 \\
296.46 \\
296.31 \\
296.12 \\
295.83\end{array}$ & $\begin{array}{l}\text { July } 9 \\
\text { July } 16 \\
\text { July } 23 \\
\text { July } 30 \\
\text { Aug. } 6 \\
\text { Aug. 13 } \\
\text { Aug. } 20 \\
\text { Aug. } 27 \\
\text { Sept. } 3 \\
\text { Sept. } 11 \\
\text { Sept. 17 } \\
\text { Sept. } 24 \\
\text { Oct. 1. } \\
\end{array}$ & $\begin{array}{l}295.65 \\
295.46 \\
294.79 \\
293.90 \\
293.79 \\
295.08 \\
294.83 \\
294.78 \\
294.66 \\
294.40 \\
294.95 \\
294.86 \\
294.81\end{array}$ \\
\hline
\end{tabular}

2 Leveling by U. S. Engineer Department.

636. Junction City: NW1/4SW1/4 sec. 32 , T. 15 S., R. 4 W. Fire-protection well, dug 8 feet in diameter and 20 feet deep, in northwest angle of Seventh and Holly Streets. Manhole rim stamped 15-4-10 on south side. Measuring point is top of iron manhole rim at south side, level with land surface and 323.4 feet ${ }^{2}$ above mean sea level.

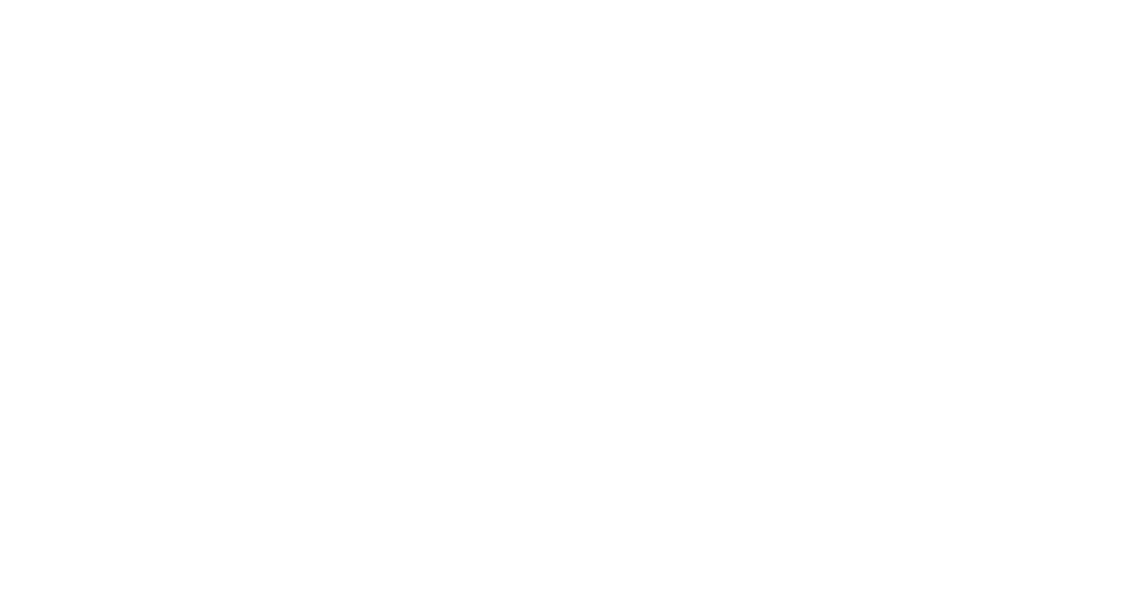

Leveling by U. S. Engineer Department. 
TABLE 13.-Ground-water levels in observation wells, 1928-30 and 1935-36-Con.

643. Doug. Wasson. SE 1/4NE1/4 sec. 11, T. 15 S., R. 3 W. Unused well, driven 115 inches in diameter and 15 feet deep. Measuring point is lower valve seat of pump, 3.4 feet above land surface and 379.47 feet 2 above a.ean sea level.

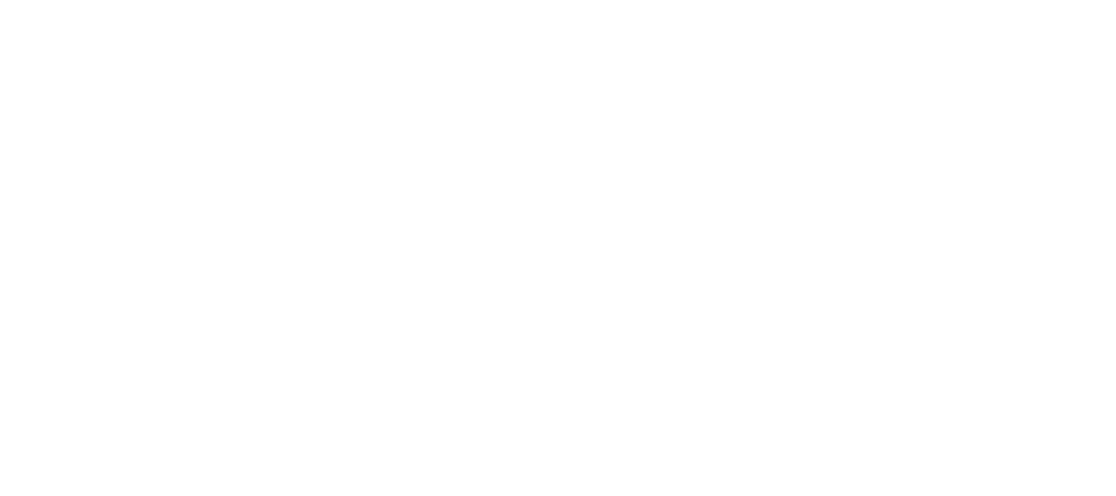

2 Leveling by U. S. Engineer Department.

Water level depressed by inordinately large withdrawals.

- Pump operating in well.

645. L. Hostetler, tenant. NW 1/4NE $1 / 4$ sec. 19, T. 15 S., R. 3 W. Stock well, dug 4 feet in diameter and 13 feet deep, at north side of barnyard. Copper nail with washer stamped 15-3-2 in wood platform. Measuring points: Top of wood pump watform at copper nail with washer, about 0.2 foot above land surface and about 326.1 feet ${ }^{2}$ above mean sea level; beginning October 5,1935 , bottom of platform, level with top of brick curb and land surface and 325.9 feet 2 above mean sea level.

\begin{tabular}{|c|c|c|c|c|c|c|c|}
\hline Date & $\begin{array}{l}\text { Water } \\
\text { level } \\
\text { (feet) }\end{array}$ & Date & $\begin{array}{l}\text { Water } \\
\text { level } \\
\text { (feet) }\end{array}$ & Date & $\begin{array}{l}\text { Wat } \backslash r \\
\text { level } \\
\text { (feet) }\end{array}$ & Date & $\begin{array}{l}\text { Water } \\
\text { level } \\
\text { (feet) }\end{array}$ \\
\hline 1928 & \multirow{3}{*}{315.0} & 1935 & & 1936 & \multirow{17}{*}{$\begin{array}{l}325.15 \\
324.69 \\
324.23 \\
324.68 \\
325.68 \\
325.45 \\
325.34 \\
324.57 \\
324.37 \\
324.23 \\
323.87 \\
323.49 \\
324.26 \\
323.69 \\
324.20 \\
324.86 \\
324.64 \\
324.23 \\
323.40\end{array}$} & \multirow{17}{*}{ 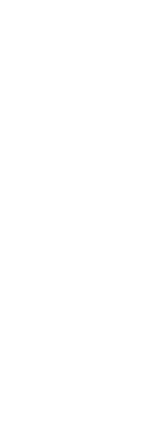 } & \multirow{17}{*}{$\begin{array}{r}323.30 \\
322.74 \\
322.09 \\
321.71 \\
320.13 \\
4317.88 \\
4316.46 \\
4315.96 \\
4314.90 \\
4314.62 \\
4314.63 \\
4314.75 \\
4315.80 \\
316.10 \\
316.36 \\
316.45 \\
316.30 \\
315.54\end{array}$} \\
\hline Aug. 18 & & Oet. 5 & 313.80 & Jan. 23 & & & \\
\hline 1929 & & $\begin{array}{l}\text { Oct. } 12 \\
\text { Oct. } 17 \text { - }\end{array}$ & $\begin{array}{l}313.80 \\
314.10\end{array}$ & $\begin{array}{l}\text { Jan. } 30- \\
\text { Feb. } 6\end{array}$ & & & \\
\hline July & 3155 & Oct. 31 & 314.68 & Feb. 13 & & & \\
\hline $\begin{array}{l}\text { July } 4 \\
\text { Aug } 2\end{array}$ & $\begin{array}{l}315.5 \\
314.4\end{array}$ & Nov. $7-$ & $\begin{array}{l}314.62 \\
314.72\end{array}$ & $\begin{array}{l}\text { Feb. } 21 \\
\text { Feb. 27 }\end{array}$ & & & \\
\hline Sept. 23 & $\begin{array}{l}313.4 \\
313.6\end{array}$ & Nov. 21 & 314.70 & Mar. 5 & & & \\
\hline Oct. 26 & (14) & Dec. 5 & 316 & Mar. 12 & & & \\
\hline Nov. 28 & (14) & Dec. 12 & 321.07 & Mar. 19 & & & \\
\hline Dec. 31 & 325.00 & Dec. 19 & 319.71 & Mar. 26. & & & \\
\hline 1930 & & Dec. 27 & 324.22 & Apr. 2 & & & \\
\hline & & & 325.66 & Apr. 16 & & & \\
\hline Feb. 1 . & 325.40 & 1936 & & Apr. 23 & & & \\
\hline Mar. 1 & $\begin{array}{l}024.80 \\
324\end{array}$ & & & & & & \\
\hline Mar. 29 & 324.10 & Jan. 2 . & 325.56 & May 7 & & & \\
\hline Apr. 26 & 324.85 & Jan. 3 & 325.60 & May 14 & & & \\
\hline May 28 & 320.50 & Jan. 9 & 325.57 & May 21 & & & \\
\hline July 23 & 314.28 & Jan. 16. & 325.68 & May 28 & & & \\
\hline
\end{tabular}

2 Ieveling by U. S. Engineer Department.

* Water level depressed by inordinately large withdrawals.

14 Well dry. 
TABLE 13.-Ground-water levels in observation wells, 1928-30 and 1935-36-Con.

649. J. W. Cleft. NE 34 SW1/4 sec. 10, T. 16 S., R. 5 W. Stock well, driven 20.3 feet deep. Measuring points: Lower valve seat of pump, 1.0 foot above land surface and 329.06 feet ${ }^{2}$ above mean sea level; begin. ning November $30,1935,1.75$ feet below valve seat and 327.31 feet ${ }^{2}$ above mean sea level.

\begin{tabular}{|c|c|c|c|c|c|c|c|}
\hline Date & $\begin{array}{l}\text { Water } \\
\text { level } \\
\text { (feet) }\end{array}$ & Date & $\begin{array}{l}\text { Water } \\
\text { level } \\
\text { (feet) }\end{array}$ & Date & $\begin{array}{l}\text { Water } \\
\text { level } \\
\text { (feet) }\end{array}$ & Date & $\begin{array}{l}\text { Water } \\
\text { level } \\
\text { (feet) }\end{array}$ \\
\hline 1935 & & 1936 & & 1936 & & 1936 & \\
\hline $\begin{array}{l}\text { Oct. } 17 \\
\text { Oct. } 24 \\
\text { Oct. } 30 \\
\text { Nov. } 7 \\
\text { Nov. } 13 \\
\text { Nov. } 20 \\
\text { Nov. } 30 \\
\text { Dee. } 5 \\
\text { Dec. } 12 \\
\text { Dec. } 19 \\
\text { Dec. } 27 \\
\end{array}$ & $\begin{array}{l}322.17 \\
321.56 \\
322.41 \\
322.46 \\
322.21 \\
323.19 \\
322.74 \\
322.67 \\
324.82 \\
324.88 \\
324.93\end{array}$ & $\begin{array}{l}\text { Jan. } 3 \\
\text { Jan. } 10 \\
\text { Jan. } 16 \\
\text { Jan. } 23 \\
\text { Jan. } 30 \\
\text { Feb. } 6 \\
\text { Feb. 13 } \\
\text { Feb. } 22 \\
\text { Feb. } 27 \\
\text { Mar. } 5 \\
\text { Mar. } 12 \\
\text { Mar. } 19 \\
\text { Mar. } 26 \\
\text { Apr. } 2\end{array}$ & $\begin{array}{l}326.47 \\
326.33 \\
326.45 \\
326.33 \\
324.09 \\
323.07 \\
326.29 \\
326.87 \\
327.38 \\
326.62 \\
325.49 \\
326.03 \\
326.03 \\
326.00\end{array}$ & $\begin{array}{l}\text { Apr. } 9 \\
\text { Apr. } 16 \\
\text { Apr. } 23 \\
\text { Apr. } 30 \\
\text { May } 7 \\
\text { May } 14 \\
\text { May } 21 \\
\text { May 28... } \\
\text { June } 4 \\
\text { June } 11 \\
\text { June } 18 \\
\text { June } 25 \\
\text { July } 2 \\
\text { July } 9\end{array}$ & $\begin{array}{l}326.04 \\
326.17 \\
325.34 \\
325.77 \\
326.07 \\
326.02 \\
326.00 \\
327.63 \\
327.57 \\
327.54 \\
327.64 \\
327.44 \\
327.40 \\
327.22\end{array}$ & $\begin{array}{l}\text { July } 16 \\
\text { July } 23 \\
\text { July } 30 \\
\text { Aug. } 6 \\
\text { Aug. } 13 \\
\text { Aug. } 20 \\
\text { Aug. } 27 \\
\text { Sept. } 3 \\
\text { Sept. } 11 \\
\text { Sept. 17 } \\
\text { Sept. } 24 \\
\text { Oct. } 1 . \\
\end{array}$ & $\begin{array}{l}325.81 \\
325.54 \\
326.34 \\
324.53 \\
322.62 \\
322.23 \\
322.94 \\
322.83 \\
322.57 \\
322.41 \\
322.23 \\
322.07\end{array}$ \\
\hline
\end{tabular}

2 Leveling by U. S. Engineer Department.

650. Owner unknown. SE1/4SW1/4 sec. 23, T. 16 S., R. 5 W. Stock well, driven $11 / 4$ inches in diameter and about 22 feet deep; at east end of barn. Casing stamped 16-5-3 on west side below pump. Measuring point is lower valve seat of pump, 2.9 feet above land surface and 342.9 feet above'mean sea level (interpolated).

\begin{tabular}{|c|c|c|c|c|c|}
\hline Date & $\begin{array}{l}\text { Water } \\
\text { level } \\
\text { (feet) }\end{array}$ & Date & $\begin{array}{l}\text { Water } \\
\text { level } \\
\text { (feet) }\end{array}$ & Date & $\begin{array}{l}\text { Water } \\
\text { level } \\
\text { (feet) }\end{array}$ \\
\hline \begin{tabular}{l}
\multicolumn{1}{c}{1928} \\
Aug. 24 \\
Oct. 22 \\
Dec. 16 \\
\end{tabular} & $\begin{array}{l}328.7 \\
327.6 \\
333.7\end{array}$ & $\begin{array}{l}\text { Feb. } 2929 \\
\text { Mar. } 27 \\
\text { May } 9\end{array}$ & $\begin{array}{l}335.7 \\
336.7 \\
334.5\end{array}$ & \begin{tabular}{|c|}
\multicolumn{2}{|c}{1929} \\
June 6 \\
July 4
\end{tabular} & $\begin{array}{l}333.3 \\
332.15\end{array}$ \\
\hline
\end{tabular}

653. R. W. Harrington. NE1/4 NE1/4 sec. 14, T, 16 S., R. 4 W. Irrigation well, dug $4 \frac{1}{2}$ feet by 6 feet across and 10.5 feet deep, at west side of driveway 150 feet south of residence. Copper nail with washer stamped 16-4-1 in well curb at south west corner. Measuring point is top of wood curb, at copper nail with washer, level with land surface and 346 feet above mean sea level (interpolated).

\begin{tabular}{|c|c|c|c|c|c|c|c|}
\hline Date & $\begin{array}{l}\text { Water } \\
\text { level } \\
\text { (feet) }\end{array}$ & Date & $\begin{array}{l}\text { Water } \\
\text { level } \\
\text { (feet) }\end{array}$ & Date & $\begin{array}{l}\text { Water } \\
\text { level } \\
\text { (feet) }\end{array}$ & Date & $\begin{array}{l}\text { Water } \\
\text { level } \\
\text { (feet) }\end{array}$ \\
\hline 1928 & & 1929 & 341.25 & 1929 & & 1929 & \\
\hline $\begin{array}{l}\text { Aug. } 22 \\
\text { Oct. } 21\end{array}$ & $\begin{array}{l}340.9 \\
339.8\end{array}$ & $\begin{array}{l}\text { Dec. } 16 \\
\text { Feb. } 2 \ldots\end{array}$ & 343.5 & $\begin{array}{l}\text { Mar. } 31 \\
\text { May } 9\end{array}$ & $\begin{array}{l}343.1 \\
342.8\end{array}$ & $\begin{array}{l}\text { June } 6 \\
\text { July } 3\end{array}$ & $\begin{array}{l}342.55 \\
341.55\end{array}$ \\
\hline
\end{tabular}

661. Celia Belleville. SW1/4NW1/4 sec. 30, T. 16 S., R. 4 W. Stock well, driven 14.2 feet deep. Measuring point is top of steel drive pipe (no pump), 2.5 feet above land surface and 348.78 feet $^{2}$ above mean sea level.

\begin{tabular}{|c|c|c|c|c|c|c|c|}
\hline Date & $\begin{array}{l}\text { Water } \\
\text { level } \\
\text { (feet) }\end{array}$ & Date & $\begin{array}{l}\text { Water } \\
\text { level } \\
\text { (feet) }\end{array}$ & Date & $\begin{array}{l}\text { Water } \\
\text { level } \\
\text { (feet) }\end{array}$ & Date & $\begin{array}{l}\text { Water } \\
\text { level } \\
\text { (feet) }\end{array}$ \\
\hline 1935 & & $\operatorname{Jan} 3^{1936}$ & 345.24 & 1936 & & 1936 & \\
\hline Oct. 17 - & 338.63 & Jan. 10 & 345.21 & Mar. 26 & 343.92 & June 11 & 342,25 \\
\hline Oct. 24 & 338.74 & Jan. 16 . & 345.17 & Apr. 2 & 344.22 & June 18 & 342.05 \\
\hline Oct. 30 & 338.76 & Jan. 23 & 345.64 & Apr. 9 & 344.12 & June 25 & 341.87 \\
\hline Nov. 8 & 338.86 & Jan. 30 & 344.21 & A.pr. 16 & 343.43 & July 2 & 341.75 \\
\hline Nov. 13 & 339.33 & Feb. 6 . & 344.74 & Apr. 23 & 343.15 & July 9 & 341.44 \\
\hline Nov. 20 & 340.36 & Feb. 13 & 344.66 & Apr. 30 & 343.20 & July 16 & 341.06 \\
\hline Nov. 30 & 341.29 & Feb. 22 & 346.64 & May 7 & 343.64 & July 23 & 340.82 \\
\hline Dec. 5 . & 341.31 & Feb. 27 & 344.90 & May 14 & 343.52 & July 30 & (4) (14) \\
\hline Dec. 12 & 343.71 & Mar. 5 & 343.97 & May 21. & 343.55 & Aug. 6 & 340.70 \\
\hline Dec. 19 & 343.67 & Mar. 12 & 344.10 & May 28 & 342.61 & Aug. 13 & 340.95 \\
\hline Dec. 27 & 343.71 & Mar. 19 & 343.97 & June 4 & 342.65 & Aug. 20 . & 340.87 \\
\hline
\end{tabular}

2 Leveling by U. S. Engineer Department.

4 Water level depressed by inordinately large withdrawals.

14 Well dry. 
TABLE 13.-Ground-water levels in observation wells, 1928-30 and 1935-36-Con.

663. A. G. Pirtle. SW1/4NE1/4 sec. 17 , T: $16 \mathrm{~S} .$, R. 3 W. Domestic well, driven $11 / 2$ inches in diameter and 20.5 feet deep. Measuring point is lower valve seat of pump, 4.2 feet above land surface and 373.8 feet above mean sea level. Record continued after March 1936 in well 664.

\begin{tabular}{|c|c|c|c|c|c|c|c|}
\hline Date & $\begin{array}{c}\text { Water } \\
\text { level } \\
\text { (feet) }\end{array}$ & Date & $\begin{array}{l}\text { Water } \\
\text { level } \\
\text { (feet) }\end{array}$ & Date & $\begin{array}{l}\text { Water } \\
\text { level } \\
\text { (feet) }\end{array}$ & Date & $\begin{array}{l}\text { Water } \\
\text { level } \\
\text { (feet) }\end{array}$ \\
\hline 1935 & & 1935 & & 1936 & & 1936 & \\
\hline Oct. 16 & 360.07 & Nov. 21 & 362.12 & $\begin{array}{l}\text { Jan. } 2 \\
\text { Jan. } 9\end{array}$ & $\begin{array}{l}368.45 \\
367.82\end{array}$ & $\begin{array}{l}\text { Feb. } 13 \\
\text { Feb. } 21\end{array}$ & $\begin{array}{l}367.47 \\
368.18\end{array}$ \\
\hline Oct. 22 & 360.10 & Dec. 5 & 361.92 & Jan. 16 & 368.17 & Mar. 5 . & 368.63 \\
\hline Oet. 31 & 360.10 & Dec. 12 & 367.05 & Jan. 23. & 367.58 & Mar, 12 & 367.87 \\
\hline Nov. 7 - & 359.99 & Dec. 19 & 367.02 & Jan. 30 & 367.36 & Mar. 19 & 367.32 \\
\hline Nov. 14 & 362.01 & Dec. 27 & 367.56 & Feb. 6 - & 366.62 & & \\
\hline
\end{tabular}

664. Millwood Stock Farm. SW1/4SE1/4 sec. 17, T. 16 S., R. 3 W. Unused well, driven 11, inches in diameter and 15.1 feet deep. Measuring point is lower valve seat of pump, painted red, 3.2 feet above land surface and 379.59 feet ${ }^{2}$ above mean sea level.

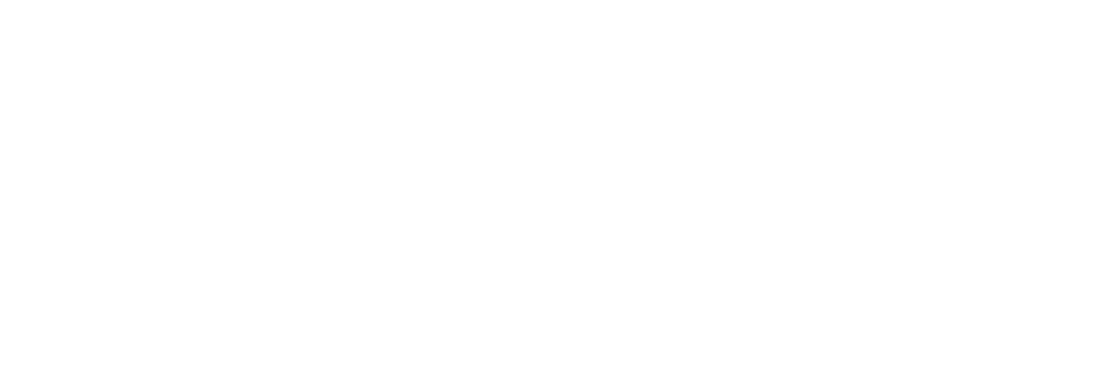

2 Leveling by the U. S. Engineer Department.

680. Leo Sidwell. SW1/4NE1/4 sec. 32, T. $16 \mathrm{~S}$, R. $3 \mathrm{~W}$. Irrigation well, dug in field 600 feet west of residence; in 1928-30, 6 feet square and 18.5 feet deep; in 1935-36, back-filled around 36-inch concrete-tile casing. Measuring points: Top of timber well curb, northwest corner, at copper nail with washer, 389.20 feet above mean sea level; from Aug. 8, 1928, to July 4, 1929, top of instrument shelf, at bored hole, 390.48 feet above mean sea level; beginning October 5, 1935, inner face at top of concrete-tile casing, marked by painted arrow, level with land surface and 388.98 feet 2 above mean sea level. Water-stage recorder operated on well from September 5,1928 , to July 4,1929 .

\begin{tabular}{|c|c|c|c|c|c|c|c|}
\hline Date & $\begin{array}{l}\text { Water } \\
\text { level } \\
\text { (feet) }\end{array}$ & Date & $\begin{array}{l}\text { Water } \\
\text { level } \\
\text { (feet) }\end{array}$ & Date & $\begin{array}{l}\text { Water } \\
\text { level } \\
\text { (feet) }\end{array}$ & Date & $\begin{array}{l}\text { Water } \\
\text { level } \\
\text { (feet) }\end{array}$ \\
\hline 1928 & & 1930 & & 1936 & & 1936 & \\
\hline Aug. 21 & 376.75 & Feb. 9 & 379.95 & Jan. 9 & 381.23 & June 18 & 376.98 \\
\hline Sept. 5 & 376.69 & Mar. 1 & 381.18 & Jan. 16 & 382.45 & June 25 & 377.72 \\
\hline Sept. 6 & 376.67 & Mar. $29_{-}$ & 379.73 & Jan. 23 & 381. 24 & July 2 . & 377.43 \\
\hline Sept. 15 & 376.66 & Apr. 26 & 379.23 & Jan. 30 & 381.75 & July 11 & 377.43 \\
\hline Oct. 8 & 376.62 & May 28. & 378.98 & Feb. 6 & 379.40 & July 16 . & 377.36 \\
\hline Oct. 21 & 376.60 & July 23 & 378.26 & Feb. 13 & 37980 & July 23 & 377.27 \\
\hline Dee. 16 & 376.88 & 1935 & & Feb. 21 & 379. 29 & July 30 & 377.08 \\
\hline 1929 & & Oct. 5 & 376.38 & $\begin{array}{l}\text { Feb. } 28 \\
\text { Mar. } 5\end{array}$ & $\begin{array}{l}350.02 \\
379.89\end{array}$ & $\begin{array}{l}\text { Aug. } 6 \\
\text { Aug. 13 }\end{array}$ & $\begin{array}{l}376.98 \\
376.88\end{array}$ \\
\hline & & oct. 12 & $\begin{array}{l}\text { (1). } \\
376.38\end{array}$ & Mar. 12 & 380.21 & Aug. 20 & 376.83 \\
\hline Jan. 6 & 379.08 & Oct. 17 & 376.66 & Mar. 19 & 379.67 & Aug. 27 & 376. 72 \\
\hline Feb. 2 . & 378.60 & Oct. 31 & 376.00 & Mar. 26 & 379.71 & Sept. 3 & 376.66 \\
\hline Feb. 16 & 378.80 & Nov. 7 & 375.98 & Apr. 2 & 380.04 & Sept. 11 & 376.43 \\
\hline Mar. 7 & 378.81 & Nov. 14 & 376.46 & Apr. 9 & 378.35 & Sept. 17 & 376.40 \\
\hline Mar. 31 & 379.20 & Nov. 21 & 376.48 & Apr. 16 & 378.16 & Sept. 21 & 376.17 \\
\hline Apr. 18 & 378. 52 & Dec. 5 & 376.60 & A pr. 23 & 378.28 & Oct. $1 \ldots$ & 376.55 \\
\hline May 9. & 379.13 & Dec. 12 & 376.76 & Apr. 30 & 378.20 & & \\
\hline June 3. & 378.25 & Dec. 19 & 377.20 & May 7 & 378.16 & & \\
\hline July 4 & 377. 67 & Dec. 27 & 377.26 & May 14 & 378.77 & & \\
\hline Sept. 23 & 377.85 & 1936 & & May 21 & 377.86 & & \\
\hline Oct. 26 & 377.73 & 1936 & & May 28 & 377.46 & & \\
\hline Nov. 18 & 377.73 & Jan. 2 & 377.46 & June 4 & 377. 11 & & \\
\hline Dec. 31 & 380.88 & Jan. $3_{-}$ & $377.9 t$ & June 11 & 377.06 & & \\
\hline
\end{tabular}

${ }^{2}$ Leveling by U. S. Engineer Department. 
TABLE 13.-Ground-water levels in observation wells, 1928-30 and 1935-36-Con.

693. Charles Frisbee, renter. SW1/4 NW1/4 sec, 3, T, 17 S., R. 4 W. Domestic and stock well, dug 19 feet deep, steel casing, 8 feet south of southeast corner of barn. Casing stamped 17-4-1-2 on south side. Measurjng point is top of 3 -inch casing, 2.6 feet above land surface and 376.77 feet ${ }^{2}$ above mean sea level.

\begin{tabular}{|c|c|c|c|c|c|c|c|}
\hline Date & $\begin{array}{l}\text { Water } \\
\text { level } \\
\text { (feet) }\end{array}$ & Date & $\begin{array}{l}\text { Water } \\
\text { level } \\
\text { (feet) }\end{array}$ & Date & $\begin{array}{l}\text { Water } \\
\text { level } \\
\text { (feet) }\end{array}$ & Date & $\begin{array}{l}\text { Water } \\
\text { level } \\
\text { (feet) }\end{array}$ \\
\hline 1929 & & 1936 & & 1936 & & 1936 & \\
\hline Sept. 23 & 362.47 & Oct. $25 \ldots \ldots$ & 361.53 & Feb. 6 & 369.39 & June 11 & 366.70 \\
\hline Oct. 26 . & 362.02 & Oct. 30 & 361.42 & Feb. 13 & 369.64 & June 18 & 366.45 \\
\hline Nov, 28 & 361.67 & Nov. 8 & 361.37 & Feb. 22 & 368. 69 & June $25 \ldots$ & 366.28 \\
\hline & & Nov. $13 \ldots$ & 361.35 & Feb. $27 \ldots$ & 369.39 & July $2 \ldots \ldots$ & 365.99 \\
\hline 1930 & & Nov. 20 & 361.61 & Mar. 5........ & 368.79 & July $9 \ldots \ldots$ & 365.78 \\
\hline & & Nov. 30 & 362.09 & Mar. 12 & 368.70 & July $16 \ldots$ & 365.19 \\
\hline Jan. 2 & 367.77 & Dec. 5 & 362.13 & Mar. $19 \ldots$ & 368.16 & July 23 & 364.88 \\
\hline Feb. 28 . & 368.67 & Dec. 12 & 363.06 & Mar. 26... & 368.09 & July 30 & 364.31 \\
\hline Mar. 28 & 368.17 & Dec. 19 & 363.09 & Apr. $2 \ldots$ & 368.11 & Aug. 6 & 364.14 \\
\hline Apr. 25. & 367.92 & Dec. 27 . & 363.14 & Apr. 9 & 368.13 & Aug. 13.. & 363.55 \\
\hline May 28 & 367.14 & & & Apr. $16 \ldots$ & 367.59 & Aug, 20 $\ldots \ldots$ & 363.06 \\
\hline July 23 & 364.56 & 1936 & & Apr. $23 \ldots$ & 367.58 & Aug. $27 \ldots \ldots$ & 363.09 \\
\hline 1935 & & Jan. 3 & 366.74 & May 7 & $\begin{array}{l}501.40 \\
367.73\end{array}$ & $\begin{array}{l}\text { Sept. } 3 \\
\text { Sept. } 11\end{array}$ & $\begin{array}{l}303.08 \\
362.94\end{array}$ \\
\hline & & Jan. 10 & 368.05 & May 14 & 367.45 & Sept. 17 & 362.49 \\
\hline Oct. 5. & $361.6 \%$ & Jan. 16 & 369.06 & Mav 21 & 367.31 & Sept. $24 \ldots \ldots$ & 362.30 \\
\hline Oct. 11 & 361.69 & Jan. 23 & 369.05 & May 28. & 367.19 & Oct. $1 \ldots$ & 361.88 \\
\hline Oct. 18 & 362.03 & Jan. 30 & 368. 13 & June 4 & 367.07 & & \\
\hline
\end{tabular}

2 Leveling by U. S. Engineer Department.

694. A. M. Rickman. SW1/4SW1/4 sec. 3, T. 17 S., R. 4 W. Irrigation well, dug 5 feet in diameter and 15 feet deep; destroyed in September 1929. Measuring point is top of plank spanning well, level with land. surface and 374 feet above mean sea level (interpolated).

\begin{tabular}{|c|c|c|c|c|c|c|c|}
\hline Date & $\begin{array}{l}\text { Water } \\
\text { level } \\
\text { (feet) }\end{array}$ & Date & $\begin{array}{l}\text { Water } \\
\text { level } \\
\text { (feet) }\end{array}$ & Date & $\begin{array}{l}\text { Water } \\
\text { level } \\
\text { (feet) }\end{array}$ & Date & $\begin{array}{l}\text { Water } \\
\text { level } \\
\text { (feet) }\end{array}$ \\
\hline 1928 & & 1928 & & 1929 & & 1929 & \\
\hline $\begin{array}{l}\text { Aug. } 24 \\
\text { Oct. } 21\end{array}$ & $\begin{array}{l}364.9 \\
364.00\end{array}$ & Dec. $16 \ldots$ & 367.9 & $\begin{array}{l}\text { Feb. } 2 \\
\text { Mar. } 27 \\
\text { May } 9\end{array}$ & $\begin{array}{l}371.8 \\
369.90 \\
369.95\end{array}$ & $\begin{array}{l}\text { June } 7 \\
\text { July 4 } \\
\text { Aug. } 2\end{array}$ & $\begin{array}{l}368.52 \\
367.05 \\
365.50\end{array}$ \\
\hline
\end{tabular}

699. John Peterson. SW 1/4SW1/4 sec. 20, T. 17 S., R. 4 W. Well driven 34.2 feet deep. Measuring point is lower valve seat of pump, 2.7 feet above land surface and 380.1 feet 2 above mean sea level.

\begin{tabular}{|c|c|c|c|c|c|c|c|}
\hline Date & $\begin{array}{l}\text { Water } \\
\text { level } \\
\text { (feet) }\end{array}$ & Date & $\begin{array}{l}\text { Water } \\
\text { level } \\
\text { (feet) }\end{array}$ & Date & $\begin{array}{l}\text { Water } \\
\text { level } \\
\text { (feet) }\end{array}$ & Date & $\begin{array}{l}\text { Water } \\
\text { level } \\
\text { (feet) }\end{array}$ \\
\hline 1935 & & 1936 & & 19.6 & & 1936 & \\
\hline $\begin{array}{l}\text { Oct. } 17 \\
\text { Oct. } 24 \\
\text { Oct. } 30 \\
\text { Nov. } 8 \\
\text { Nov. } 14 \\
\text { Nov. } 20 \\
\text { Nov. } 30 \\
\text { Dec. } 5 \\
\text { Dec. } 12 \\
\text { Dec. } 19 \\
\text { Dec. } 27 \\
\qquad 1936 \\
\text { Jan. } 3\end{array}$ & $\begin{array}{l}369.68 \\
369.74 \\
369.67 \\
369.75 \\
370.07 \\
370.55 \\
371.29 \\
371.34 \\
371.93 \\
373.62 \\
373.57\end{array}$ & $\begin{array}{l}\text { Jan. } 10 \\
\text { Jan. } 16 \\
\text { Jan. } 23 \\
\text { Jan. } 30 \\
\text { Feb. } 6 \\
\text { Feb. } 13 \\
\text { Feb. } 22 \\
\text { Feb. } 27 \\
\text { Mar. } 5 \\
\text { Mar. } 12 \\
\text { Mar. } 19 \\
\text { Mar. } 26 \\
\text { Apr. } 2 \\
\text { Apr. } 9 \\
\text { Apr. } 16\end{array}$ & $\begin{array}{l}375.25 \\
375.28 \\
375.89 \\
374.69 \\
374.52 \\
374.49 \\
377.07 \\
375.71 \\
375.49 \\
375.57 \\
375.48 \\
375.51 \\
375.19 \\
375.21 \\
374.24\end{array}$ & $\begin{array}{l}\text { Apr. } 33 \ldots \\
\text { Apr. } 30 \ldots \\
\text { May } 7 \\
\text { May } 14 \ldots \\
\text { May } 21 \ldots \\
\text { May } 28 \ldots \\
\text { June } 4 \\
\text { June } 11 \\
\text { June } 18 \\
\text { June } 25 \\
\text { July } 2 \\
\text { July } 9 \\
\text { July } 16 \ldots \\
\text { July } 23 \\
\text { July } 30\end{array}$ & $\begin{array}{l}374.04 \\
374.06 \\
374.05 \\
374.15 \\
374.56 \\
374.62 \\
374.67 \\
373.91 \\
373.07 \\
372.84 \\
373.04 \\
372.61 \\
372.46 \\
372.17 \\
371.92\end{array}$ & $\begin{array}{l}\text { Aug. 6. } \\
\text { Aug. 13 } \\
\text { Aug. } 20 \\
\text { Aug. } 27 \\
\text { Sept. } 3 \\
\text { Sept. } 11 \\
\text { Sept. } 17 \\
\text { Sept. } 24 \\
\text { Oct. } 1\end{array}$ & $\begin{array}{l}371.81 \\
371.64 \\
370.84 \\
370.62 \\
370.53 \\
370.24 \\
370.02 \\
370.03 \\
369.88\end{array}$ \\
\hline
\end{tabular}

2 Leveling by U. S. Engineer Department. 
TABLE 13.-Ground-water levels in observation wells, 1928-30 and 1935-36-Con.

702. Clara Blais. SE $1 / 4 \mathrm{NW} 1 / 4$ sec. 26, T. $17 \mathrm{~S}$., R. 4 W. Well driven 17.7 feet deep. Measuring point is lower valve seat of pump, 2.0 feet above land surface and 402.32 feet ${ }^{2}$ above mean sea level.

\begin{tabular}{|c|c|c|c|c|c|c|c|}
\hline Date & $\begin{array}{l}\text { Water } \\
\text { lpvel } \\
\text { (feet) }\end{array}$ & Date & $\begin{array}{l}\text { Water } \\
\text { level } \\
\text { (feet) }\end{array}$ & Date & $\begin{array}{l}\text { Water } \\
\text { level } \\
\text { (feet) }\end{array}$ & Date & $\begin{array}{l}\text { Water } \\
\text { level } \\
\text { (feet) }\end{array}$ \\
\hline 1935 & & 1936 & & 1936 & & 1936 & \\
\hline Oct. 17 & 398.79 & Jan. 10 & 394.11 & Apr. 16. & 396.39 & July 23 & 392.25 . \\
\hline Oct. 24 & 388.68 & Jan. 15 & $\begin{array}{l}094.11 \\
398.21\end{array}$ & Apr. 23 & 395.96 & July 30 & 391.65 \\
\hline Oct. 30 & 388.63 & Jan. 23 & 398,56 & Apr. 30 & 396.01 & Aug. 6 & 391. 44 \\
\hline Nov. 8 & 388.72 & Jan. 30 & 393.69 & May 7. & 396. 04 & Aug. 13 & 391.06 \\
\hline Nov. $14 \ldots$ & 398.64 & Feb. 6 & 397. 01 & May 14 & 394.70 & Aug. 20 & 390.91 \\
\hline Nov. 20 & 338.93 & Feb. 13 & 397.53 & May $21 \ldots$ & 395.73 & Aug. 27 & 389.33 \\
\hline Nov. $30 \ldots$ & 399.05 & Feb. 22 & 397.75 & May 28 & 395.84 & Sept. 3 & 389.24 \\
\hline Пec. 5 & 339.15 & Feb. 27 & 397.50 & June 4 & 395.91 & Sept. 11. & 388.61 \\
\hline Dec. 12 & 389.99 & Mar. 5 & 396.98 & June $11 \ldots$ & 394.81 & Sept. 17 & 388.31 \\
\hline Dec. 19 & 389.84 & Mar. 12 & 397.29 & June 18 & 394.83 & Sept. 24 & $387.93^{\circ}$ \\
\hline Dec. 27 & 389.91 & Mar. 19. & 400.41 & June $25 \ldots$ & 394,69 & Oct. $1 \ldots$ & 387.78 \\
\hline 1936 & & Mar. 2 & 397. 19 & July 2 & 394.41 & & \\
\hline Jan. 3 & 393.00 & Apr. 9 & 396. 48 & July 16 & 392.50 & & \\
\hline
\end{tabular}

${ }^{2}$ Leveling by U. S. Engineer Department.

707. George T. Morrill. NE1/4NE1/4 sec. 20, T. 17 S., R. 3 W. Domestic well, driven 115 inches in diameter and 16.7 feet deep. Measuring point is lower valve seat of pump, 4.7 feet above land surface and 427.24 feet ${ }^{2}$ above mean sea level.

\begin{tabular}{|c|c|c|c|c|c|c|c|}
\hline Date & $\begin{array}{l}\text { Water } \\
\text { level } \\
\text { (feet) }\end{array}$ & Date & $\begin{array}{l}\text { Water } \\
\text { leve] } \\
\text { (feet) }\end{array}$ & Date & $\begin{array}{l}\text { Water } \\
\text { level } \\
\text { (feet) }\end{array}$ & Date & $\begin{array}{l}\text { Water } \\
\text { level } \\
\text { (feet) }\end{array}$ \\
\hline 1935 & & 1933 & & 1936 & & 1936 & \\
\hline Oct. 15 . & 408,29 & Jan. 9 & 410.06 & Apr. 16 . & 413.96 & July 23 & 410. $92^{\prime}$ \\
\hline Oct. 22 & 409.14 & Jan. 16 & 411.47 & Apr. 23 & 412.72 & July 30 . & 410.20 , \\
\hline Oet. 31 & 40९. 09 & Jan. 23 & 411. 97 & A pr. 30 & 413. 51 & Aug. 6 & 410.12 \\
\hline Nov. 7 & 407.99 & Jan. 30 & 412. 18 & May 7 & 413.64 & Aug. 13 . & 409.60 \\
\hline Nov. 14 & 407.88 & Feb. 6 & 412.32 & May 14 & 413.77 & Aug. 20 . & 409.96 \\
\hline Nov. 21 & 407.96 & Feb. 13 & 411.83 & May 21 & 413.59 & Aug. 27 & 409.69 \\
\hline Dec. 5 & 409. 08 & Feb. 21 & 413. 16 & May 28. & 413.66 & Sept. 3 & 409.62 \\
\hline Dec. 12 & 408.56 & Feb. 28 & 413. 54 & June 4 & 413.16 & Sept. 11 & 409.46 \\
\hline Dec. 19 & 408. 03 & Mar. 5 & 414. 18 & June 11 & 413.47 & Sept. 17 & 409.70 \\
\hline Dec, 27 & 408.21 & Mar. 12 & 411. 39 & June 18 & 412.65 & Sept. 24 & 409.48 \\
\hline 1936 & & Mar. 19 & 414. 14 & June 25 & 412. 39 & Oct. 1 & $409.10^{\circ}$ \\
\hline 1936 & & Mar. 26 & 413. 60 & July 2 & 412.05 & & \\
\hline Jan. 2 . & 408.66 & Apr. $9-$ & $\begin{array}{l}414.11 \\
413.87\end{array}$ & July 16 . & 411.07 & & \\
\hline
\end{tabular}

${ }^{2}$ Leveling by U. S. Engineer Department.

712. Chase Gardens. SW $1 / 4$ SE1/4 sec. 28, T. 17 S., R. 3 W. Irrigation well, dug 4 feet square and 20 feet deep, in field 750 feet west of greenhouses. Measuring point is top of tee in discharge pipe (second tee above. pump), 1.0 foot below land surface and 430 feet above mean sea level (interpolated). Well was inaccessible * for water-level measurements in 1935-36.

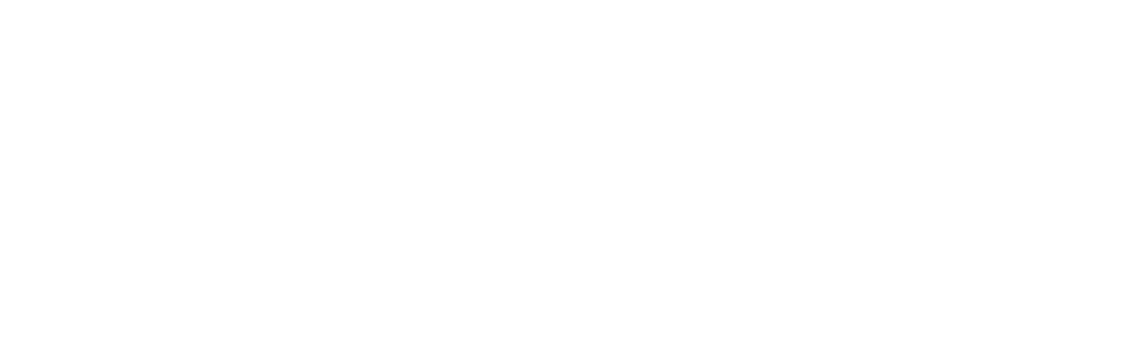




\section{INDEX}

Page

Abstract

Acknowledgments for aid

Agricultural Research Corporation, section of well of

Albany, climate at . ground water near

$14,16-18$ $44,47,48$

Alexander, A. L., section of well of ........ 134 Alluvium in the area. . ........... 27-32, pls. 1-2,4-7 Analyses of water from representative wells . _ 60-63

Beebe, Mrs., section of well of

Bethel School well, section of -

Bibliography

Bingenheimer well, section of

Brownsville, ground water near

Canby, section of well of.

Canby fan, water supply of

Cascade formation, occurrence and waterbearing properties of

Cascade Range, features of

Central lowland, subdivisions of, in relation to ground water 41-54, pls. 1-2, 7, 10

Chenical character of the ground water in the area

Climate of the area $12-19$

Coburg, ground water near

Collier Glacier, view of

Columbia River, alluvial plain of, water supply of.

Conser well, section of ..................... 142

Consolidated and semiconsolidated rocks, general character of _..... 19-20, pls. 1-2 structure of .......................... 20-23

water-bearing properties of _. . ........... 24-27

Corvallis, climate at ................ 14-18

ground water near...................... 48

Corvallis Creamery Co. well, section of ..... 131

Cottage Farm, Oregon State Hospital, section of well of . . . 142

Dayton Prairie, water supply of ... _....... 51-53

Eastern-Western Lumber Co., section of well of . ......... 140

Elmira, ground water near . ............. 46-47

Eugene, climate at ...................... 14, 16-18

ground water near.

Eugene formation, occurrence and waterbearing properties of $\ldots \ldots \ldots \ldots$

Field work in the area ....... 4-5

Fishel, V. C., analyses by ................ 31

Fisher formation, occurrence and water-

bearing properties of . . . . . . . 22,26

Flowing wells, occurrence of ......... 55-56, pl. 1

Foster. M. D., analyses by ........... . . 60-63

Frank, Aaron, well 2, section of
French Prairie, water supply of . . ......... 49-51

Furrow, M. E., section of well of .......... 143

Geologic maps of the Willamette Valley ...- pls. 1-2

(in pocket)

Geology and hydrology of the area......... 19-41,

pls. 1-2, 4-9

Gervais, city of, section of well of .......... 139

Gooding, William, section of well of $\ldots . . . \ldots . .137$

Ground water, chemical character of ..... 27. 56-64 dependability of supply of ............ 40-41

fluctuations of level of . . . . . 34-39, 144-192

for irrigation, suitability of _......... 59,64

in relation to rainfall and streams. $39-40$, pls. 1-2

previous reports on

Harrisburg, ground water near. ......... 46

Illahe formation, occurrence and water-bearing properties of .... 23

ndependence, alluvium at

ground water near.

Index map of Oregon showing areas covered by ground-water reports........... 3

Investigation, purpess and scope of _....... 4-5

Irrigation, water suitable for ......... 59,64

Junction City, ground water near......... 46

wat $r$ supply of .................. 46

Junction City well, section of . 143

Keasey shale, occurrence and water-bearing

properties of 22

Ladd well, section of . .

Lebanon alluvial fan, water supply of

Libby, McNeill \& Libby well, section of . .- 133-

Lloyd Corporation, Inc., section of well of ..... 131

Main valley plain, Brownsville-Albany seg-

ment........................ 47

Canby fan segment .................. 45

Coburg-Harrisburg sagment............. 46

Corrallis segment_.................. 48

Dayton Prairie segment_............... 5153

Elmira segment ......... $46-47$

Eugene-Junction City segment_.......... 46

French Prairie segment . . . . . . _... . . . . 49-51

Lebanon fan segment _............ 45

minor segments near Albany ............. 48

Monmouth-Independence segment..... 48

Spring.eld delta segment ..... . . . . . . . . 43-44

Stayton-Mill Creek tasin ........... . . 48-49

Tualatin Valley segment

Mark, J. C., section of well of . . ........... 13i

Masonic and Eastern Star Home well, section

of . . . 130

Materials penetrated by tycical wells . . . . . 130-143 
Mattoon, J. C., section of well of _........ $\quad \begin{array}{r}\text { Page } \\ 135\end{array}$

Mill Creek basin, ground water.

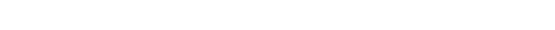
water supply of

Monmouth, ground water near................

Northwestern Ice \& Cold Storage Co., section of well of .

Oregon Coast Range, features of ... . ........ Oregon Linen Mills, Inc., section of well of .... Oregon State Hospital, well 3, section of ......

Cregon State Hospital, Cottage Farm, section of well of

Oregon State Penitentiary, section of well of.. Oregon State Training School, section of well of

Pittsburg Bluff sandstone, occurrence and water-bearing properties of . ..... 22, 26

Pleasantdale School, section of well of . . .... 136

Portland, climate at...................... 13-18

Portland "delta," features of .

water supply of

Precipitation on the area

Public Works Engineering Corporation, section of well of ................ 140, 141

Purpose and scope of the investigation. ...... 4-5

Rainfall. See Precipitation.

Rhododendron formation, occurrence and water-bearing properties of . . . . . 23, 24

Roberts, H. A., section of well of . ........ 134

Rockwood Water District well, section of .... 134

Salem, climate at

ground water near.

Salty to brackish water in consolidated sedimentary rocks

mentary rocks ................. 27,64

Scappoose Dike District well, section of ..... 130

Sedimentary rocks equivalent to Tyee and . Burpee formations, occurrence and water-bearing properties of . . . 22, 26-27

Snowfall. See Precipitation.

Southern Pacific Railroad Co., Woodburn station, section of well of ....... 139 Springfield delta, water supply of . . . . 43-44, pl. 7
Stayton, ground water near.... 48

Stayton lavas, occurrence and water-bearing properties of ................. 23, 24

Stayton-Mill Creek basin, water supply of ... 48-49 Stratigraphic sections of the rocks in the area $-22-23$,

Structure in the area ................ 20-21

Stryker, A. W., section of well of . .

Surface features of the area...... 8-12, pls. 1,2

Temperature in the area................. 14

Terminal Ice \& Cold Storage Co. well, section

of 13

Terrace deposit in the area. . . . . 32-34, pls. 1-2,8

Topography of the area.......... 8-12, pls. 1, 2

Troutdale formation, occurrence and waterbearing properties of ............. 23, 24

Tualatin Valley, water supply of .......... 53

Unconsolidated deposits, general character of . 34 , pls. 1-2, 4-8

water in . . . . . . . . . . . . 34-41, pls. 1-2, 9

United States Resettlement Administration, Yamhill Farms, unit 6, section of well of

unit 16, section of well of 137

unit 19 , section of well of ........ 136

unit 24, section of well of - 138

unit 68, section of well of 136

unit 85, section of well of ................ 132

Water. See Ground water.

Water-bearing materials, physical properties of ................ 31

Water table, form and fluctuations of ...... 34-39, pls. $1-2,9$

Wells, flowing .......... 55-56 materials penetrated by . . . . . . . 130-143

records of ......... $66-129$

water level in . . 144-192

Williams, F. L. section of well of $\ldots \ldots 134$

Willamette River, alluvial pıain of . . . 10-12, 42-53

Woodburn, eity of, section of well of ........ 139

Yakima basalt, occurrence and water-bearing properties of

$22,24-26$ 



\section{CONTENTS}

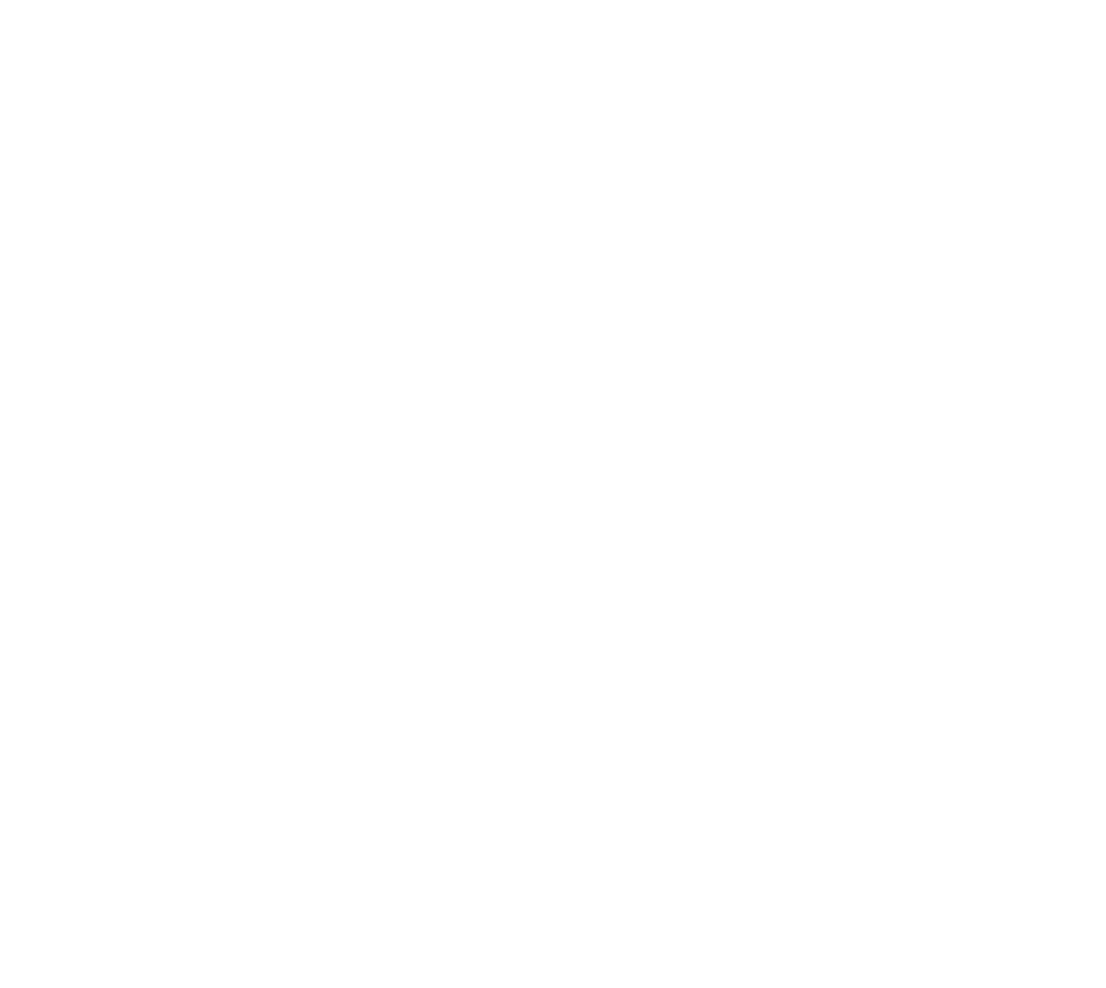

III 


\section{ILLUSTRATIONS}

Plate 1. Geologic map of the northern part of the Willamette Valley, Oregon ....... In pocket

2. Geologic map of the southern part of the Willamette Valley, Oregon............ In pocket

3. A, Three-Fingered Jack, viewed southward across Marion Lake; $B$, Three Sisters, viewed southward from McKenzie. Pass across a young lava field . . . . . . . . . . . . . . . . . . .

4. $A$, Collier Glacier, viewed from its western lateral moraine; North Sister Peak in the background; $B$, Younger alluvium of of the McKenzie River; material from lower part of well 680.

5. Younger alluvium of the Willamette River. $A$, Gravel pit in the SE $1 / 4$ sec. 8, T. 11 S., R. 4 W.; $B$, Pit of East Side Sand \& Gravel Co. near Corvallis, in the SW $1 / 4$ sec. 2, T. $12 \mathrm{~S}$., $R$. $5 \mathrm{~W}$

6. Older alluvium and related deposits. $A$, Exposure in the NW1/4 sec. 1, T. 16 S., R. 4. W.; $B$, Highway cut in the NW1/4 sec. 7, T. 4 S., R. 1 E., showing faint stratification $\ldots$

7. Map of the Willamette Valley showing thickness of younger and older alluvium, terrace deposit, and related materials...

8. $A$, Decomposed terrace deposit in highway cut in the $\mathrm{N} \frac{1}{2}$ sec. 32, T. 12 S., R. 1 W.; B, Cross-bedded sand and gravel exposed in cut along the Spokane, Portland \& Seattle Railway, 0.1 mile north of Lombard Street, Portland ................

9. Water-level fluctuations in 12 wells in the Willamette Valley and at two stations on the Willamette River, 1928-30 and 1935-36.

10. Map of the Willamette Valley showing principal subareas of the central lowland with respect to yield of ground water.......

TIGURE 1. Index map of Oregon showing drainage divide of the Willamette River, area covered by plates 1 and 2 , and areas covered by other reports relating to ground water...........................

2. Maximum, average, and minimum monthly rainfall at three climatologic stations in the Willamette Valley, 1896-1935...

3. Chemical character of waters from 20 wells in the Willamette

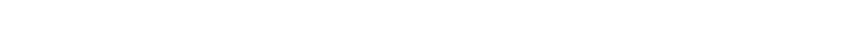




\section{TABLES}

TABLE 1. Monthly, seasonal, and yearly averages and extremes of climatic elements at Portland, Oreg., in the period ending with 1930

2. Monthly temperature, in degrees Fahrenheit, at five climatologic stations in the Willamette Valley, in the period ending with 1930

3. Maximum, average, and minimum monthly and yearly rainfall and snowfall, in inches, at five climatologic stations in the Willamette Valley, 1896-1935 . . . . . . . . . . . .

4. Maximum rates of precipitation, in inches, at Portland, Oreg., in the period ending with $1930 \ldots$

5. Yearly rainfall and snowfall, also surplus or deficiency with respect to 40-year average, at five climatologic stations in the Willamette Valley, 1872-1937_...................

6. Stratigraphic sections and general water-bearing properties of consolidated and semiconsolidated rocks bounding the central lowland of the Willamette River Basin...........

7. Physical properties of water-bearing materials from well 245.

8. Approximate limits indicating the suitability of a water for

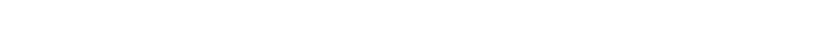

9. Chemical analyses of water from representative wells in the Willamette Valley .....................................

10. Wells in the Willamette Valley for which records of groundwater levels have been published in other water-supply

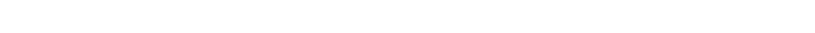

11. Records of wells in the Willamette Valley and adjacent area,

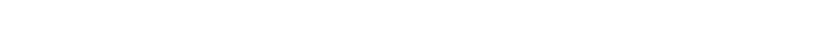

12. Materials penetrated by typical wells . . . .

13. Ground-water levels in observation wells, 1928-30 and 1935-36_ 



\title{
GROUND-WATER RESOURCES OF THE WILLAMETTE VALLEY, OREGON
}

\author{
By Arthur M. Piper
}

\begin{abstract}
The drainage basin of the Willamette River lies in western Oregon between the Oregon Coast Range on the west and the Cascade Range on the east. It is about 170 miles long from north to south and covers about 11,350 square miles. The central lowland, with whose ground-water resources this report is concerned, covers nearly 3,000 square miles and ranges in altitude from 25 to 450 feet above sea level. On this lowland the average yearly rainfall is slightly less than 40 inches, but the average rainfall for the period May to August is only 4.2 inches, less than is needed to mature many crops. Thus, supplemental irrigation is profitable.
\end{abstract}

The central lowland comprises a main valley plain, remnants of at least one higher terrace with a pediment and certain other related features, and relatively narrow trenches cut into the main valley plain. These trenches are occupied by the Willamette River and its tributaries. This lowland constitutes the most extensive single agricultural area in Oregon. The area is covered with unconsolidated Quaternary deposits which, of all the rock formations in the basin, are those most likely to yield continuous large supplies of water to wells.

The bedrock formations that underlie the unconsolidated deposits of the lowland and form the foothills and mountains to the east and west include marine and nonmarine sedimentary rocks that range in age from late Eocene to Miocene (?), and fragmental and nonfragmental volcanic rocks and associated detrital rocks that range in age from Oligocene (?) to Recent. The rocks of Miocene age and older are deformed by broad shallow folds and possibly by faults of small extent and displacement.

Of the several bedrock formations, only the Yakima basalt of Miocene age yields water copiously over an extensive area; some wells in this rock yield as much as 500 gallons a minute, whereas others yield only a few gallons. Certain detrital and fragmental bedrock formations supply water copiously near Portland. The remaining bedrock formations, which together underlie most of the central lowland, are either impervious or yield water slowly or contain brackish water and brine. They supply numerous farm wells in the foothills but are not liekly to yield large supplies of fresh water from beneath the central lowland.

In general the terrace deposit and related pediment gravel are thoroughly weathered and nearly impervious. At most places they yield water slowly and are not an adequate source of water for irrigation wells.

The older alluvium and related deposits form the main lowland plain, some 1,100 square miles in extent between Eugene at the southern or upstream end and Canby at the downstream end. They rest on an eroded bedrock surface and range in thickness from a feather edge along the margins of the plain to a maximum 
of about 300 feet. Their aggregate volume is estimated as not less than $80,000,-$ 000 acre-feet, or $23 \frac{1}{2}$ cubic miles; their aggregate water-storage capacity is about $6,000,000$ acre-feet. These deposits form a plain that is divided into two nearly equal segments separated by a rock-bound narrows about 21 miles long.

The southern segment is composed of several broad alluvial fans and intervening flats. In the fans especially, the older alluvium contains many lentils and tongues of clean gravel and sand that supply as much as 500 gallons of water a minute to wells of shallow or moderate depth and of the simplest construction. Efficiently constructed wells penetrating the full thickness of the deposit very probably would yield much more copiously. The water-bearing capacity of the older alluvium is not equal in all parts of this area; with respect to potential yield, several subdivisions of the area are delimited tentatively in the full text. In this southern segment of the plain, ground-water storage increases about 500,000 acre-feet each winter, chiefly by infiltration of rain; storage declines an equal amount during the summer and autumn, chiefly by drainage into the streams. By pumping from wells, a substantial fraction of this yearly ground-water increment can doubtless be salvaged for supplemental irrigation and other uses.

At least two distinct sorts of ground-water bodies underlie the northern segment of the main plain: unconfined water that is semiperched at shallow depth beneath the "prairies" and confined water in extensive pervious zones, which in one typical well are about 70,120 , and 220 feet beneath the surface. These deeper pervious zones tend to feather out and to become less pervious toward the north and west; also, at some places they terminate by overlap against the impermeable bedrock. The semiperched water bodies are recharged by infiltration of rain on the main plain; they supply many domestic and farm wells but will not sustain large withdrawals for irrigation. The confined water presumably is derived from a catchment area to the south and east. It supplies wells that yield as much as 1,000 gallons a minute with maximum pumping lifts of about 60 feet. Doubtless it would supply several or many times its present draft, though increased withdrawal inevitably will entail some increase in lift.

Beneath the flood plains of the Willamette River and its principal tributaries, the younger alluvium is bighly pervious at most places. It supplies as much as 1,000 gallons of water a minute to wells of simple construction and would doubtless sustain a much larger aggregate withdrawal. However, through the rockbound narrows in the central part of the valley and at some other places where the alluvium is thin and rests directly on impervious bedrock, wells may not find a sufficient thickness of 'water-bearing material to yield water copiously, especially in late summer and autumn, when the ground-water level is lowest.

In chemical character the water from all the unconsolidated deposits of the Willamette Valley is satisfactory for most uses. In 31 samples of water from these deposits, the hardness ranged from about 10 to 150 parts per million, so that softening or treating to prevent the formation of scale is not necessary for some uses and not unduly costly for any use. Some waters from these deposits contain an excessive amount of iron-as much as 5.27 parts per million; water high in dissolved iron is most common in the older alluvium beneath the northern half of the main valley plain. At many places the bedrocks, especially the sedimentary rocks of marine origin, yield water of excessive hardness or so concentrated in dissolved mineral constituents as to be unsatifactory for ordinary uses.

\section{INTRODUCTION}

\section{LOCATION AND EXTENT OF THE AREA}

The drainage basin of the Willamette River, the ground-water features of whose central and lower parts are described in this report, covers about 11,350 square miles in western Oregon (fig. 1). This 


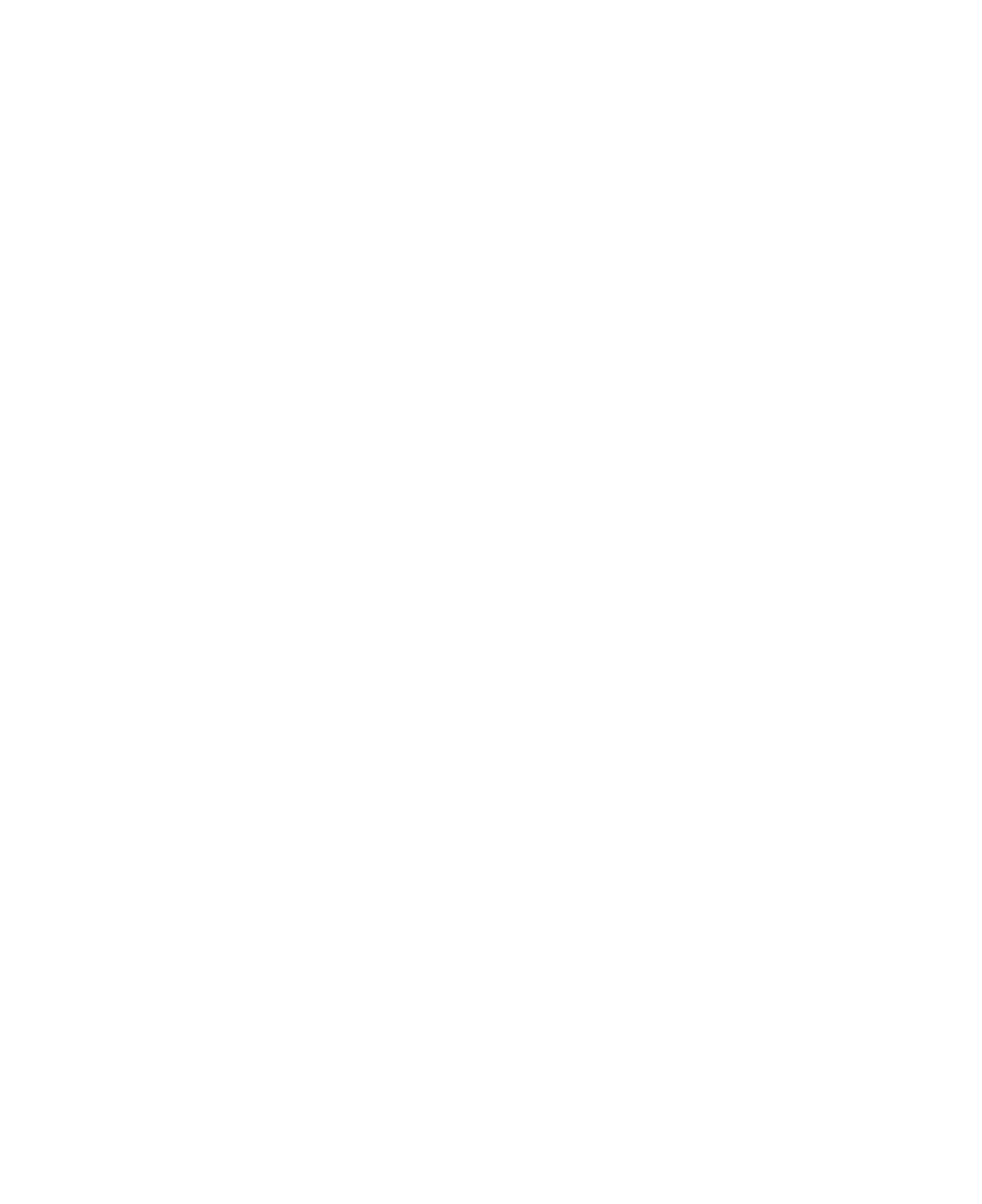


basin is approximately 170 miles long, from north to south, and as much as 100 miles wide. On the east it is bounded by the Cascade Range and on the west by the Coast Range; its northern and southern boundaries are largely in mountainous terrane of less relief. The central valley plains and adjacent terraces, known locally as the Willamette Valley, cover nearly 3,000 square miles: this part of the drainage area is represented on plates 1 and 2 .

\section{PURPOSE AND SCOPE OF THE INVESTIGATION}

The investigation whose findinge are here presented was suggested by the Department of Soils, of the Oregon Agricultural Experiment Station, and was carried on by the Geological Survey, of the United States Department of the Interior. These two agencies have shared in the cost of the field work and of the preparation of this report for publication. The primary aim of the investigation was to determine the feasibility of recovering water from wells for irrigation on the extensive fertile lowland and in this way supplementing the natural supply of soil moisture which is deficient during the greater part of the growing season in most years. (See p. 14.) Such assurance of moisture for fully maturing the growing crops would aid materially in stabilizing agriculture in the area.

Field work in the Willamette Valley was started by the writer in July 1928 and was continued during the remainder of that season and through the next. Critical hydrologic records were kept continuously until June 1930. Preparation of this report was deferred in favor of similar ground-water investigations of The Dalles region in the north-central part of Oregon, during $1930:{ }^{1}$ of the Harney Basin in the southeastern part, in $1930-32 ;^{2}$ of the Walla Walla Basin in the northeastern part, in $1933 ;{ }^{3}$ and of cooperative preliminary studies in several small districts in the State. In 1935-36 the United States Engineer Department gathered many data on ground-water levels in the Willamette Valley; this work was supervised by the writer. This report was prepared in 1937-38 through renewed cooperation between the Oregon Agricultural Experiment Station and the United States Geological Survey, utilizing all data from the field work of 1928-30 and 1935-36.

The Willamette Valley is so extensive that the time spent in the field permitted only a moderately intensive study of broad ground-water features. Briefly, this involved reconnaissance mapping of the

\footnotetext{
${ }^{1}$ Piper, A. M., Geology and ground-water resources of The Dalles region, Oregon: U. S. Geol. Survey Water-Supply Paper 659, pp. 107-189, 1932.

${ }^{2}$ Piper, A. M., Robinson, T.W.. and Park, C. F., Jr., Geology and ground-water resources of the Harney Basin, Oregon: U. S. Geol. Survey Water-Supply Paper 841, 189 pp., 1940.

3 Piner, A. M., Robinson, T. W., and Thomas, H. E., Ground water in the Walla Walla Basin, OregonWashington: Supreme Court of the United States, October 1935, in equity, State of Washington vs. State of Oregon, Transcript of Record, stipulations and exlibits, pp. 72-142, Oct. 14, 1935.
} 
several unconsolidated deposits of Quaternary age that form the lowlands in the central part of the basin (pls. 1 and 2), keeping records of the construction and performance of typical wells, and periodically measuring the water level in a widespread net of observation wells. From these data, this report seeks to differentiate the several waterbearing zones in depth below the land surface, in lateral extent, and in water-yiclding capacity; to delimit the catchment areas from which the ground-water supply is replenished; and to evaluate the natural subdivisions of the lowland by relative capacity to sustain large withdrawals of water for irrigation and other purposes. The scope of the investigation does not afford a close estimate of the safe ground-water yield of the Willamette Valley-that is, of the quantity that could be withdrawn year after year without eventually so depleting the supply that further withdrawal at that rate would become impossible or uneconomic.

\section{ACKNOWLEDGMENTS}

Orderly progress in a regional field study such as that in the Willamette Valley results in a large measure from cooperation by many persons on whom a writer is necessarily dependent. The drillers and owners of wells in the Willamette Valley have contributed many essential records. Among his colleagues in the Geological Survey, the writer is indebted to T. W. Robinson for temporary assistance in the field during 1929 , to $\mathrm{H}$. E. Thomas for a considerable share in compiling the geologic map (pls. 1 and 2) from the writer's field sheets', and to Margaret D. Foster for chemical analyses of water samples from representative wells (table 4). T. R. Cantine, W. E. Emrick, and Arthur King, temporary employees, recorded the fluctuations in ground-water level during the greater part of the 2-year period ending June 1930.

The field work in the Willamette Valley and the preparation of this report were under the direction of O. E. Meinzer, geologist in charge of the Division of Ground Water, United States Geological Survey. To him the writer is grateful for technical counsel. Acknowledgment is due also to J. T. Jardine, director of the Oregon Agricultural Experiment Station at the time, and to W. L. Powers, head of the Department of Soils in that agency, for many courtesies which made the field work more effective.

\section{BIBLIOGRAPHY}

The geologic features of the Willamette River basin with specific reference to ground-water resources had not been studied prior to 1928, nor had the areal geology been mapped. Nevertheless, the geologic literature on the area is fairly extensive. The following 
bibliography notes the publications that are pertinent to the present investigation:

Allison, I. S., Spokane flood south of Portland, Oreg. [abstract]: Pan-Am. Geologist, vol. 57, p. 64, 1932; Geol. Soc. America Bull., vol. 43, pp. 133-134, 1932; Glacial erratics in the Willamette Valley: Geol. Soc. America Bull., vol. 46, pp. 615-632, 1935; Late Pleistocene topographic correlations on Pacific coast [abstract]: Pan-Am. Geologist, vol. 63, p. 310, 1935; Geol. Soc. America Proc., 1935, p. 333, 1936; Pleistocene alluvial stages in northwestern Oregon: Science, new ser., vol. 83, pp. 441-443, 1936.

Arnold, Ralph, and Hannibal, Harold, Marine Tertiary stratigraphy of the Pacific Coast of America: Am. Philos. Soc. Proc., vol. 52, pp. 559-605, 1913.

Bretz, J. H., Satsop formation of Oregon and Washington: Jour. Geology, vol. 25, pp. 446-458, 1917; Late Pleistocene submergence in the Columbia Valley of Oregon and Washington: Jour. Geology, vol. 27, pp. 489-506, 1919; Spokane flood beyond the channeled scablands: Jour. Geology, vol. 33, pp. 252-257, 1925; Bars of channeled scabland: Geol. Soc. America Bull., vol. 39, pp. 643-702, 1928.

Buddington, A. F., and Callagitin, Eugene, Dioritic intrumive rocks and contact metamorphism in the Cascade Range in Oregon: Am. Jour. Sci., 5th ser., vol. 31 , pp. 421-449, 1936.

Buwalda, J. P., Pleistocene and Recent topographic changes in the Pacific Coast States: Nat. Research Council Bull., No. 61, pp. 39-43, 1927.

Callaghan, Eugene, Some features of the volcanic sequence in the Cascade Range in Oregon: Am. Geophys. Union Trans. 14th Ann. Meeting, 1933, pp. 243-249; Some aspects of the geology of the Cascade Range in Oregon [abstract]: Washington Acad. Sci. Jour., vol. 24, pp. 190-191, 1934.

Callaghan, Eugene, and Buddington, A. F., Metalliferous mineral deposits of the Cascade Range in Oregon: U. S. Geol. Survey Bull. 893, pp. 7-22, 1938.

Chaney, R. W., Ecological significance of the Eagle Creek flora of the Columbia River Gorge: Jour. Geology, vol. 26, pp. 577-592, 1918; Further discussion of the ecological composition of the Eagle Creek flora [abstract]: Geol. Soc. America Bull., vol. 31, p. 222, 1920; The flora of the Eagle Creek formation: Chicago Univ., Walker Museum, Contr., vol. 2, pp. 115-181, 1920.

Chaney, R. W., and Sanborn, E. I., The Goshen flora of west-central Oregon: Carnegie Inst. Washington Pub. 439, Contr. Paleontology, 103 pp., 1933.

Condon, Thomas, Willamette Sound: Overland Monthly, vol. 7, pp. 468-473, 1871; Oregon geology: a revision of "The Two islands" (edited by E. C. McCornack), 187 pp., Portland, Oreg., J. K. Gill Co., 1910.

Cushman, J. A., and Schenck, H. G., Two foraminiferal faunules from the Oregon Tertiary: California Univ., Dept. Geol. Sci., Bull., vol. 17, pp. 305$324,1928$.

Darton, N. H., Structural materials in parts of Oregon and Washington: U. S. Geol. Survey Bull. 387, 33 pp., 1909.

Diller, J. S., A geological reconnaissance in northwestern Oregon: U. S. Geol. Survey 17th Ann. Rept., pt. 1, pp. 441-520, 1896.

Felts, W. M., Analysis of Willamette Valley fill [abstract]: Pan-Am. Geologist, vol. 64, p. 69, 1935; Geol. Soc. America Proc. 1935, p. 346, 1936.

Harrison, G. D., and Eaton, Arthur, Report on the investigation of oil and gas possibilities of western Oregon: Mineral Resources of Oregon, vol. 3, No. 1, pp. 3-37, Oregon Bur. Mines and Geology, 1920.

Hertelein, L. G., and Crickmay, C. H., A summary of the nomenclature and stratigraphy of the marine Tertiary of Oregon and Washington: Am. Philos. Soc. Proc., vol. 64, pp. 224-282, 1925. 
HodGe, E. T., Geology of Oregon Cascades [abstract]: Geol. Soc. America Bull., vol. 38, pp. 204-205, 1927; Pan-Am. Geologist, vol. 47, p. 157, 1927; Composition and structure of the Cascade Mountains in central Oregon [abstract]: Geol. Soc. America Bull., vol. 38, pp. 162-163, 1927; Pan-Am. Geologist, vol. 47, p. 157, 1927; Mount Multnomah, ancient ancestor of the Three Sisters: Oregon Univ. Pub., vol. 3, No. 2, 160 pp., 1925 [abstract]; Am. Jour. Sci., 5th ser., vol. 13, p. 270, 1927; Age of Columbia River and lower canyon [abstract]: Geol. Soc. America Bull., vol. 44, pp. 156-157, 1933; Geology of the lower Columbia River: Geol. Soc. America Bull., vol. 49, pp. 839-888. 1938.

Howe, H. VAN W., Correlation of the Empire formation, Oregon [abstract]: Geol. Soc. America Bull., vol. 32, p. 147, 1921; Faunal and stratigraphic relations of the Empire formation, Coos Bay, Oreg.: California Univ., Dept. Geol. Sci., Bull., vol. 14, pp. 85-114, 1922; Astoria, mid-Tertic type of Pacific coast: Pan-Am. Geologist, vol. 45, pp. 295-306, 1926.

McCornack, E. C., A study of Oregon Pleistocene: Oregon Univ. Bull., new ser., vol. 3, No. 5, 1906; Study of Oregon Pleistocene--the Oregon Desmostylus. skull: Oregon Univ. Bull., new ser., vol. 12, No. 2, p. 13, 1914; Contributions to the Pleistocene history of Oregon: Oregon Univ. Leaflet, Geology Bull,, vol., 6, No. 3, 23 pp., 1920.

Parks, H. M., Road materials in the Willamette Valley: Oregon Bur. Mines Bull. 1, 63, pp , 1911.

Rathbun, M. J., The fossil stalk-eyed Crustacea of the Pacific slope of North America: U. S. Nat. Mus. Bull. 138, 155 pp., 1926.

Schenck, H. G., A preliminary report on the geology of the Eugene quadrangle, Lane and Linn Counties, Oreg.: Oregon Univ., unpublished thesis, 1923; Physiography of the Eugene quadrangle, Oregon [abstract]: Geol. Soc. America Bull., vol. 36, p. 203, 1925; Diatoms in western Oregon shales: Econ. Geology, vol. 22, pp. 565-568, 1927; Marine Oligocene of Oregon: California Univ., Dept. Geol. Sci., Bull., vol. 16, pp. 449-460, 1927; Stratigraphic relations of western Oregon Oligocene formations: California Univ., Dept. Geol. Sci., Bull., vol. 18, pp. 1-50, 1928; The Pittsburg Bluff fauna of the Oregon Oligocene [abstract]: Geol. Soc. America Bull., vol. 40, pp. 163-164, 1929; Cephalopods of the genus Aturia from western North America: California Univ., Dept. Geol. Sci., Bull., vol. 19, pp. 435-490, 1931; Stratigraphic and faunal relations of the Keasey formation of the Oligocene of Oregon: Geol. Soc. America Bull., vol. 44, p. 217, 1933; Nuculid bivalves of the genus Acila: Geol. Soc. America Spec. Papers, No. 4, pp. 41-44, 1936.

Schenck, H. G., and Kleinpell, R. M., Foraminifera from Gaviota formation [Calif.] [abstract]: Pan-Am. Geologist, vol. 64, p. 76, 1935; Geol. Soc. America Proc., 1935, p. 352, 1936.

Sмiтh, W. D., Petroleum possibilities of western Oregon: Econ. Geology, vol. 19, pp. 455-465, 1924.

Sмiтh, W. D., Summary of the salient features of the Oregon Cascades: Oregon Univ. Bull., new ser., vol. 14, No. 16, 1917; [abstract] Geol. Soc. America Bull., vol. 29, p. 81, 1918 .

Smith, W. D., and PACKARD, E. L., Salient features of the geology of Oregon: Oregon Univ. Bull., new ser., vol. 16, No. 7, 42 pp., 1919; Jour. Geology, vol. 27, pp. $79-120,1919$.

Thayer, T. P., Structural relation of central Willamette Valley to Cascade Mountains [abstract]: Pan-Am. Geologist, vol. 59, p. 317, 1933; Geol. Soc. America Proc. 1933, p. 315, 1934; Structure of North Santiam section of the Oregon Cascades [abstract]: Pan-Am. Geologist, vol. 61, p. 319, 1934; Geol. 
Soc. America Proc., 1934, pp. 324-325, 1935; Structure of the North Santiam River section of the Cascade Mountains in Oregon: Jour. Geology, vol. 44, pp. 701-716, 1936.

Washburne, Chester, Reconnaissance of the geology and oil prospects of northwestern Oregon: U. S. Geol. Survey Bull. 590, 108 pp., 1914.

Wells, F. G., and WAters, A. C., Quicksilver deposits of southwestern Oregon: U. S. Geol. Survey Bull. 850, pp. 5-23, 1934.

Williams, I. A., The Columbia River Gorge-its geologic history interpreted from the Columbia River Highway: Mineral Resources of Oregon, vol. 2, No. 3, 130 pp., Oregon Bur. Mines and Geology, 1916.

\section{SURFACE FEATURES OF THE WILLAMETTE VALLEY}

The Willamette River Basin has high relief and considerable diversity in its land forms. With respect to major surface features it may be divided into three parts, coextensive with parts of three physiographic sections of the United States as recognized by Fenneman. ${ }^{4}$ In order from west to east these are: (1) The Oregon Coast Range section of the Pacific Border province, which includes about 15 percent of the basin; (2) the Puget Trough section, also of the Pacific Border province, whose part in Oregon covers about 25 percent of the basin and is roughly coextensive with the central lowland plains and terraces; and (3) the Middle Cascade Mountains section of the SierraCascade province, which includes about 60 percent of the basin.

The Oregon Coast Range is a mature land surface, deeply dissected by closely spaced branching valleys. Its sinuous crest trends generally northward, is between 5 and 25 miles west of the margin of the central lowland, and in greater part is not more than about 2,500 feet above sea level, or 2,000 feet above the lowland. Above this general crest level stand several isolated peaks such as Marys Peak, which lies west of Corvallis and attains an altitude of about 4,100 feet. From the general crest level, a succession of moderately sinuous ridges descend gently eastward toward the lowland and terminate commonly in a narrow belt of foothills. With the exception of certain narrow plains along the streams, all the range has been covered with a heavy stand of coniferous timber but extensive areas have been logged over or denucied by fire. In these denuded areas many larger tracts now support a vigorous second growth, and some tracts have been cleared for farming, especially in the foothills.

The greater part of the Oregon Coast Range consists of sedimentary rocks of Tertiary age, largely shale and earthy sandstone (see pp. 19-21), deformed by broad open folds. In these rocks the higher parts of the land surface commonly are rounded in contour, with profiles convex upward; conversely, the lower slopes of the valleys are smooth but steep and commonly are concave upward. Throughout the range, however, there are many bodies of igneous rock-sheets

\footnotetext{
- Fenneman, N. M., Physiographic divisions of the United States: Assoc. Am. Geographers Annals, vol. 18, pp. 261-353, map, 1928.
} 
interbedded with the sedimentary rocks, both intrusive and extrusive; dikes of a wide range in thickness; and stocklike masses of moderate size. These bodies account for scattered areas of moderately rugged topography and sharp constrictions at numerous places along the valleys. They also form the isolated peaks that rise above the general crest of the range.

The Cascade Range is striking both in topography and in composition. Much of the range is very rugged, is topographically young, and has been sculptured by glaciers as well as by streams; it is composed almost entirely of igneous materials - nonfragmental extrusive rocks, tuff and other pyroclastic rocks in a wide range of textures, and intrusive rocks of several petrographic types. Its highest peaks rise far above timber line and so form a discontinuous alpine zone. These features characterize a belt from 40 to 60 miles wide all along the eastern side of the basin. This belt, covering nearly 7,000 square miles, is somewhat more than twice as extensive as the central lowland and four times as extensive as the eastern slope of the Coast Range.

The Cascade Range within the Willamette River Basin represents two fairly distinct land forms. The western part, adjacent to the central lowland and as much as 25 miles wide, is a belt of mature foothills and ridges in which certain older folded volcanic rocks are extensive. Here the relief fully equals or somewhat surpasses that of the Coast Range. To the east, younger volcanic rocks rise to the divide, which, for the greater part of its length, is between 4,000 and 6,500 feet above sea level. On this higher part of the range, consequent streams flow generally westward in successive impressive canyons, commonly as much as 2,500 feet deep. Some interstream flats of moderate extent preserve the initial volcanic surface, which is moderately smooth and of fairly uniform westward slope. Alined along and near the divide are several prominent volcanic peaks, commonly deeply scarred by alpine glaciers. Chief among these, in order from the north, are Mount Hood, which is 11,225 feet above sea level; Mount Jefferson, 10,485 feet; Three Fingered Jack, 7,848 feet (pl. 3, A) ; Mount Washington, 7,802 feet; the North Sister, Middle Sister, and South Sister (pl. 3, B), which are respectively 10,094 feet, 10,053 feet, and 10,354 feet above sea level; and Diamond Peak, 8,750 feet. Less prominent volcanic peaks, younger than those just cited and unscarred by glaciers, are Olallie Butte, 7,210 feet above sea level; Belknap Crater, 6,877 feet; and Black Crater, 7,260 feet. These younger peaks are about midway along the eastern enge of the basin.

The Willamette Valley, the lowland that lies between the two mountainous areas just described, comprises a main valley plain, remnants of at least one higher terrace with a pediment and certain other related features, and relatively narrow trenches which are occupied by the 
Willamette River and its tributaries. These three parts of the lowland make up the most extensive single agricultural area in Oregon. They are formed of unconsolidated deposits of Quaternary age which, of all the rock formations in the basin, are those most likely to yield large supplies of water to wells.

The main valley plain, which is coextensive with the older alluvium and related deposits shown on plates 1 and 2, has several distinctive parts, which can most readily be described in order downstream, or toward the north. The higher southern segment of the plain, which is one of two principal segments, begins at Eugene near the junction of the three principal headwater branches of the Willamette River (McKenzie River, Middle Fork, and Coast Fork) and extends northward to Albany. Disregarding its tongues that extend into the bordering mountainous areas and the younger stream trenches by which it is traversed, this segment of the plain is about 40 miles long and from 9 to 20 miles wide. It is from 360 to 450 feet above sea level at its southern end and from 225 to 300 feet at its northern end. Thus, its average northward slope is about 3.5 feet to the mile. In the main, it is formed by a succession of broad alluvial fans that spread outward from the mountain valleys to the east and that coalesce along their outer margins. On these fans the land surface slopes radially northward and westward from 6 to 11 feet to the mile. Each fan is traversed by numerous faint ephemeral drains, the radiating distributaries of streams that existed while the plain was being built. Between the fans and rather generally along the western margin of the lowland, the plain includes smooth areas that slope northward as little as 2 feet to the mile.

From Albany to Salem, a distance of 20 miles, the main valley plain is sharply constricted by outlying rock hills, to a width in places of as little as $2 \frac{1}{2}$ miles. The average northward gradient is about 2.7 feet to the mile.

At Salem the plain widens abruptly into its northern principal segment, which extends northward another 22 to 28 miles, to McMinnville, Newberg, and Canby and somewhat beyond. This segment of the plain is from 9 to 25 miles wide. It comprises several "prairies" that are remarkably flat. Near Salem the plain is about 200 feet. above sea level; in the vicinity of Canby, at its northeast corner, from 185 to 190 feet; and near McMinnville, about 155 feet. Thus the average slope, from which no extensive part of the segment deviates materially, is about 2.4 feet to the mile; the direction of slope is N. $50^{\circ}$ W., which is across the general course of the Willamette River in that vicinity and toward an alcove in the rock hills that bound the lowland. Obviously, the surface form of this northern segment of the plain is not a product of ordinary stream aggradation. 
? 
Still farther north, the main lowland plain divides into several interlacing tongues that reunite near the southern edge of Portland, some 15 miles upstream from the mouth of the Willamette River. These tongues are commonly less than a mile wide and are of uneven grade. Apparently they are a product of stream aggradation along valleys that are now occupied in part by the Willamette River and its lower tributaries, Chehalem Creek, Tualatin River, and Clackamas River. Attached to the plexus of tongues just described and largely north of the Tualatin River is another plain of moderate extent, a topographic analog of the main lowland plain of the Willamette Valley. This plain, the most northerly of those drained by the Willamette River, is known locally as the Tualatin Valley.

Beneath the main lowland plain of the Willamette Valley, the groundwater conditions are rather diverse. On this diversity the plain is divided into 20 subareas, which are described on pages 41-54.

From 30 to 100 feet higher than the main lowland plain and scattered widely along both its eastern and western margins are remnants of a terrace, which in large part is formed by stream deposits that are delimited on the geologic map (pls. 1 and 2). Corresponding terrace remnants occur along the major tributaries of the Willamette River far upstream beyond the area represented by the map. Related to these terraces in origin are certain older alluvial surfaces, notably a dissected pediment which, in the vicinity of the Molalla River, rises. as much as 300 feet above the main lowland. Possibly related in origin is the so-called Portland delta, which occupies the angle between the Willamette and Columbia Rivers and which is analogous to an extensive plain still farther north, in Washington. All these terraceremnants and related surfaces have been dissected to moderate depth, and so their initial form and grade have been modified considerably.

Cut into the main lowland plain are certain trenches that ordinarily are floored with young alluvium (pls. 1 and 2); these are occupied by the Willamette River and its major tributaries. Between Eugene at. the southern end of the main lowland and Newberg at the northern end, the inner trench of the Willamette River ranges between 0.5 mile and 4 miles in width. From Eugene northward to Albany, its: floor slopes equally with the main plain but is from 5 to 15 feet lower. Farther north, owing to difference in grade, the trench deepens gradu-. ally until at Newberg its floor is about 80 feet below the main lowland plain. Characteristically the floor is gently undulating, is dotted with oxbow lakes, and is traversed by many channels that are occupied by the river during freshets. It affords some of the most fertile land of the entire valley. Downstream from Newberg the trench becomes: steadily narrower, and near Canby, at the northeastern corner of the main plain, it passes into a rock-bound narrows that extends through. 
Oregon City to the southern edge of Portland, within 14 miles of the mouth of the river.

Similar trenches, rather widely spaced over all the main lowland plain, are occupied by the larger tributaries of the Willamette River. One trench is unique in that it is not now occupied by a single perennial stream. This trench trends northeastward across the northern segment of the main plain, from the trench of the Willamette River near Salem to that of the Pudding River near Mount Angel. It is notably shallower than the two stream-occupied trenches that it connects. Doubtless it was once occupied by an ancestral Willamette River but was abandoned by that stream early in the epoch of trenching.

\section{CLIMATE ${ }^{5}$}

The broad lowland of the Willamette River Basin has a relatively equable climate, characterized by warm dry summers and cool winters with moderately abundant rain and some snow. These characteristics are shown by table 1, which assembles averages and extremes of the principal climatic elements for Portland, at the northern end of the basin.

\footnotetext{
${ }^{6}$ Adapted to this report from a statement by E. L. Wells in U. S. Dept. Agr., Weather Bur., Climate summary of the United States, sec. 3, Western Oregon, 1936.
} 


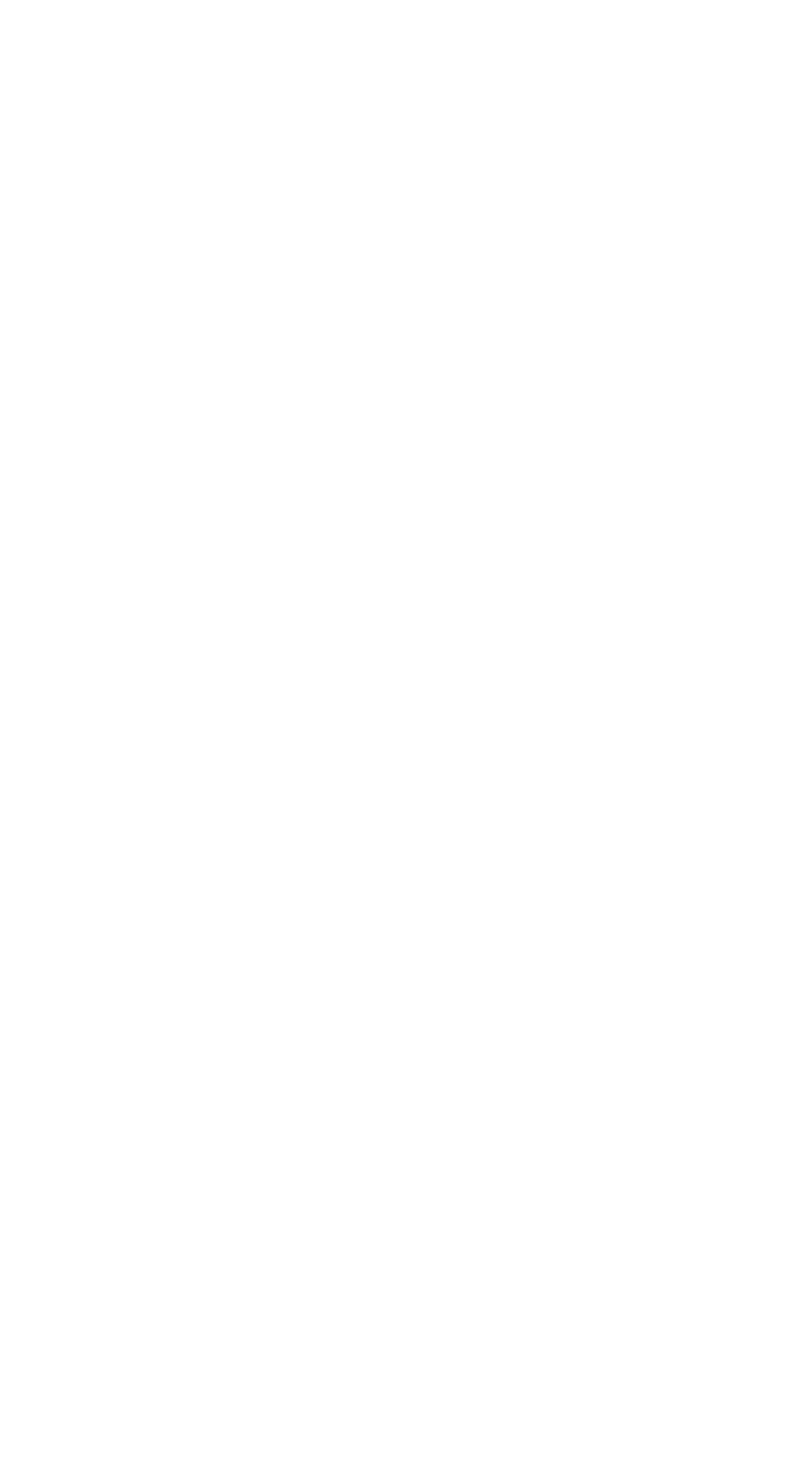


Over most of the lowland the yearly average temperature is between $51^{\circ}$ and $53^{\circ} \mathrm{F}$. The average of the highest temperatures in midsummer (July) ranges from about $78^{\circ}$ to $84^{\circ}$. Though temperatures above $100^{\circ}$ have occurred in nearly all parts of the area, they are always attended by low relative humidity and are almost alwavs followed by cool nights. Thus, the average of the lowest temperatures in July ranges between $48^{\circ}$ and $56^{\circ}$. Killing frost seldom occurs in the season when tender crops are growing. Table 2 gives monthly temperatures at five climatologic stations along the middle of the valley, from Portland at the north to Eugene at the south. (See fig. 1.)

TABLE 2.-Monthly temperature, in degrees Fahrenheit, at five climatologic stations in the Willamette Valley, in the period ending with 1930

[Data from publications of the United States Weather Bureau]

\begin{tabular}{|c|c|c|c|c|c|c|c|c|c|c|c|c|c|c|c|}
\hline & \multicolumn{3}{|c|}{ Portland } & \multicolumn{3}{|c|}{ Salem } & \multicolumn{3}{|c|}{ Albany } & \multicolumn{3}{|c|}{ Corvallis } & \multicolumn{3}{|c|}{ Eugene } \\
\hline & 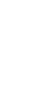 & $\begin{array}{l}0 \\
0 \\
2 \\
5 \\
0 \\
3 \\
4\end{array}$ & $\begin{array}{l}\overrightarrow{\tilde{v}_{\Delta}} \\
\stackrel{\vec{B}}{0} \\
\stackrel{\leftrightarrow}{*}\end{array}$ & 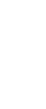 & $\begin{array}{l}0 \\
50 \\
\Xi \\
\vdots \\
\vdots \\
4\end{array}$ & $\begin{array}{l}\overrightarrow{\mathrm{w}} \\
\stackrel{2}{2} \\
\stackrel{3}{3}\end{array}$ & 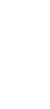 & \begin{tabular}{l}
0 \\
00 \\
0 \\
\multirow{2}{0}{} \\
0 \\
2 \\
4
\end{tabular} & \begin{tabular}{l} 
苞 \\
今゙ \\
\multirow{3}{*}{}
\end{tabular} & 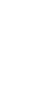 & 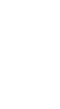 & 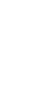 & 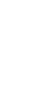 & $\begin{array}{l}0 \\
00 \\
8 \\
0 \\
5 \\
5 \\
4\end{array}$ & 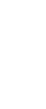 \\
\hline $\begin{array}{l}\text { Length of record } \\
\text { (years) }\end{array}$ & 57 & 59 & 57 & 38 & 38 & 38 & 36 & 47 & 36 & 41 & 41 & 41 & 36 & 39 & 36 \\
\hline Januar & 62 & 39.1 & -2 & 68 & 39.6 & -5 & 68 & 39.3 & -3 & 64 & 38.9 & -1 & 69 & 40.0 & 6 \\
\hline & 68 & 42. 2 & 7 & 68 & 42.6 & -4 & 69 & 42.4 & & 69 & 42.0 & -5 & 74 & 42.9 & 0 \\
\hline Marc & 83 & 47.2 & 20 & 80 & 46.3 & 22 & 81 & 46.8 & 9 & 82 & 45.6 & 13 & 80 & 46.1 & 18 \\
\hline $\mathrm{Ap}$ & 93 & 51. 9 & 28 & 93 & 51. & 27 & 91 & 51.3 & 25 & 91 & 50.2 & 24 & 89 & 50.7 & 26 \\
\hline $\mathrm{M}$ & 99 & 57 & 32 & 94 & 56 & 31 & 08 & 56 & 0 & 95 & & 0 & 91 & 55 & 30 \\
\hline Jur & 101 & 62.2 & 39 & 100 & 61. & 32 & 103 & 61. & 32 & 102 & 60.4 & 32 & 100 & 60.4 & 34 \\
\hline & 104 & 67. & 4 & 108 & 6 & 35 & 104 & & 35 & 102 & & 3 & 104 & 65.8 & 39 \\
\hline A & 98 & 66 & 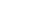 & 105 & 66 & 30 & $\mathrm{~J} 03$ & 66 & 10 & 102 & 65 & 0 & 99 & 65 & 35 \\
\hline Septer & 97 & 61. & 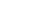 & 97 & 60. & 3 & 98 & 60. & 29 & 96 & 60.2 & 2 & 96 & 60. & 30 \\
\hline Octo & 86 & 54 & 3 & 89 & 53 & 23 & 86 & 52 & 25 & 85 & 52.9 & 2 & 84 & 53.3 & 25 \\
\hline Nove & 73 & 46. & 11 & 70 & 45. & 11 & 72 & 45. & & 73 & 45.2 & 1 & 75 & 46.3 & 12 \\
\hline December. & 65 & 41.2 & & 72 & 40.8 & - & 64 & 40. & -15 & 62 & 40.2 & -14 & 67 & 40.8 & -4 \\
\hline Annual.. & 104 & 53.1 & -2 & 108 & 52.6 & -6 & 104 & 52.4 & -15 & 102 & 51.8 & -14 & 104 & 52.3 & -4 \\
\hline
\end{tabular}

The precipitation on the lowland, rain for the most part, occurs largely in the winter and early spring; even in the wettest year of record at Portland, 1882, the summer rainfall was only 2.9 inches or 4.3 percent of the precipitation in that year and 7.4 percent of the average yearly precipitation. Thus in most years rainfall is not sufficient to assure full maturing of crops, and supplemental irrigation has been found profitable. Figure 2 and table 3 show this marked seasonal distribution of rainfall and snowfall, which is so characteristic of the entire Willam ette River Basin. Most of the precipitation occurs as gentle rains. The average annual number of days with 0.01 inch or more of precipitation is from about 130 to 160 . However, between May and September precipitation at excessive rates occasionally continues for an hour or less and usually between November and February it continues for a day or more. (See table 4.) Some snow falls every winter, but it seldom remains on the ground more than a few days. 


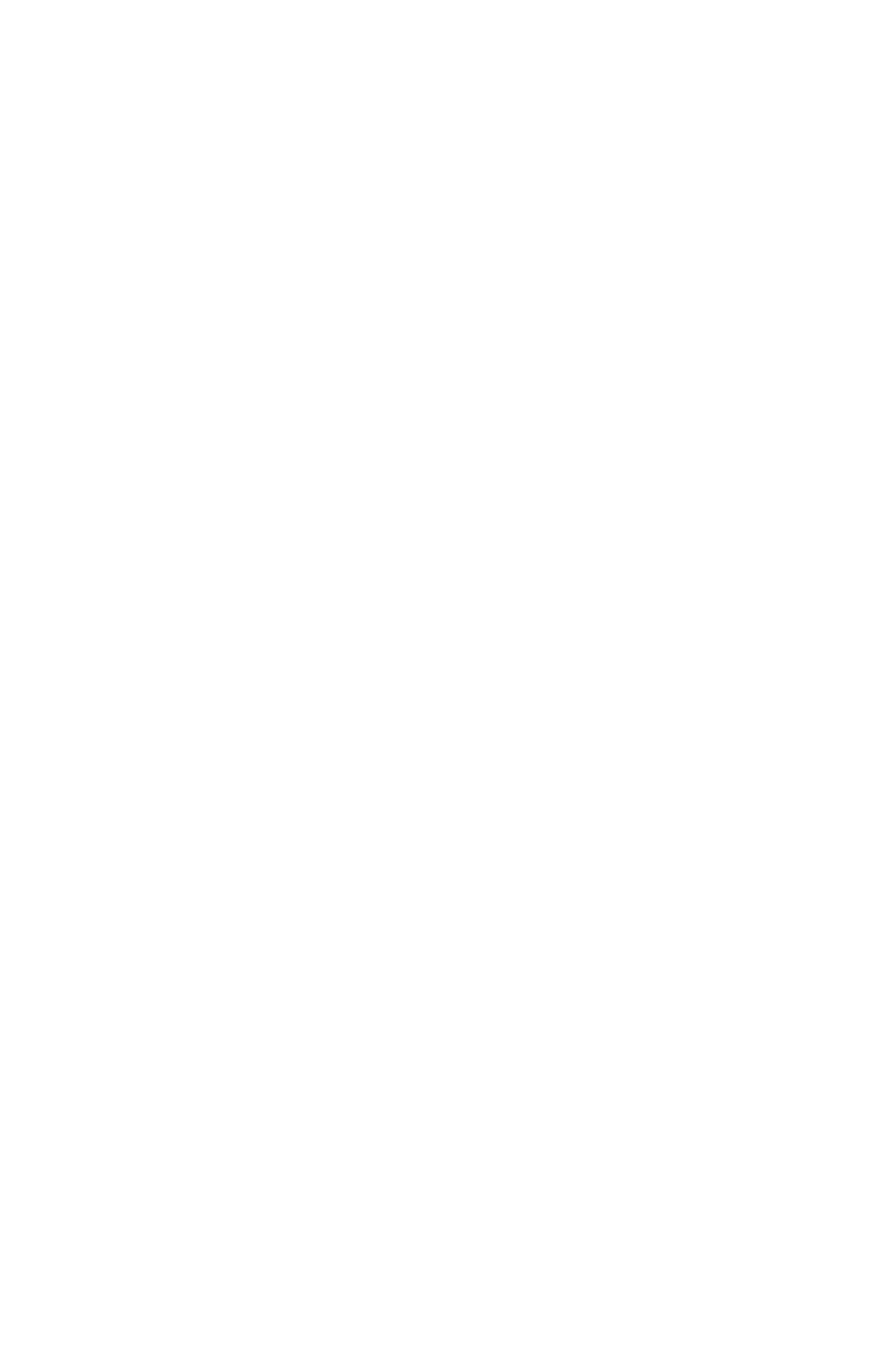

FIGURE 2.-Maximum, average, and minimum monthly rainfall at three climatologic stations in the Willamette Valley, 1896-1935. 
TABLE 3--Maximum, average, and minimum monthly and yearly rainfall and snowfall, in inches, at five climatologic stations in the Willamette Valley, $1896-1935$

[Data from publications of United States Weather Bureau]

\begin{tabular}{|c|c|c|c|c|c|}
\hline & Portland & Salem & Albany & Corvallis & Eugene \\
\hline $\begin{array}{l}\text { anuary: } \\
\text { Maximum } \\
\text { Average } \\
\text { Minimum }\end{array}$ & $\begin{array}{r}111.53 \\
5.87 \\
22.54\end{array}$ & $\begin{array}{r}110.17 \\
5.40 \\
1.30\end{array}$ & $\begin{array}{r}111.90 \\
6.03 \\
22.71\end{array}$ & $\begin{array}{r}13.61 \\
6.44 \\
1.99\end{array}$ & $\begin{array}{r}110.07 \\
5.47 \\
2.14\end{array}$ \\
\hline $\begin{array}{l}\text { February: } \\
\quad \text { Maximum } \\
\quad \text { A verage } \\
\quad \text { Minimum }\end{array}$ & $\begin{array}{r}111.08 \\
4.55 \\
.16\end{array}$ & $\begin{array}{r}19.47 \\
4.47 \\
.34\end{array}$ & $\begin{array}{r}12.20 \\
4.93 \\
.15\end{array}$ & $\begin{array}{r}15.23 \\
5.15 \\
.12\end{array}$ & $\begin{array}{r}12.10 \\
4.49 \\
.10\end{array}$ \\
\hline $\begin{array}{l}\text { March: } \\
\quad \text { Maximum } \\
\quad \text { Average } \\
\quad \text { Minimum }\end{array}$ & $\begin{array}{r}10.57 \\
3.94 \\
.63\end{array}$ & $\begin{array}{r}19.96 \\
3.81 \\
\quad .59\end{array}$ & $\begin{array}{r}110.44 \\
4.13 \\
\quad .58\end{array}$ & $\begin{array}{r}11.70 \\
4.16 \\
.43\end{array}$ & $\begin{array}{r}10.49 \\
3.98 \\
.40\end{array}$ \\
\hline $\begin{array}{l}\text { April: } \\
\quad \text { Maximum } \\
\text { Average } \\
\text { Minimum }\end{array}$ & $\begin{array}{r}15.36 \\
2.65 \\
.80\end{array}$ & $\begin{array}{r}16.12 \\
2.41 \\
.48\end{array}$ & $\begin{array}{r}15.55 \\
2.50 \\
.56\end{array}$ & $\begin{array}{r}16.98 \\
2.46 \\
.26\end{array}$ & $\begin{array}{r}15.89 \\
2.60 \\
.43\end{array}$ \\
\hline $\begin{array}{l}\text { May: } \\
\text { Maximum } \\
\text { Average } \\
\text { Minimum }\end{array}$ & $\begin{array}{r}14.66 \\
1.95 \\
.45\end{array}$ & $\begin{array}{r}15.54 \\
1.92 \\
.25\end{array}$ & $\begin{array}{r}5.81 \\
2.08 \\
.34\end{array}$ & $\begin{array}{r}5.71 \\
1.78 \\
.19\end{array}$ & $\begin{array}{r}4.76 \\
2.32 \\
.19\end{array}$ \\
\hline $\begin{array}{l}\text { June: } \\
\quad \text { Maximum } \\
\text { A verage } \\
\text { Minimum }\end{array}$ & $\begin{array}{r}14.24 \\
1.40 \\
.07\end{array}$ & $\begin{array}{r}13.48 \\
1.18 \\
.00\end{array}$ & $\begin{array}{r}13.44 \\
1.25 \\
.02\end{array}$ & $\begin{array}{r}13.35 \\
1.12 \\
.00\end{array}$ & $\begin{array}{r}14.31 \\
1.48 \\
.02\end{array}$ \\
\hline $\begin{array}{l}\text { July: } \\
\text { Maximum } \\
\text { Average } \\
\text { Minimum }\end{array}$ & $\begin{array}{r}2.55 \\
.52 \\
.00\end{array}$ & $\begin{array}{r}2.72 \\
.39 \\
.00\end{array}$ & $\begin{array}{r}3.31 \\
.42 \\
.00\end{array}$ & $\begin{array}{r}2.34 \\
.31 \\
.00\end{array}$ & $\begin{array}{r}3.38 \\
.36 \\
.00\end{array}$ \\
\hline $\begin{array}{l}\text { August: } \\
\text { Maximum } \\
\text { Average } \\
\text { Minimum }\end{array}$ & $\begin{array}{r}3.39 \\
.64 \\
\text { Trace }\end{array}$ & $\begin{array}{r}2.91 \\
.45 \\
.00\end{array}$ & $\begin{array}{r}3.20 \\
.56 \\
. .00\end{array}$ & $\begin{array}{r}2.76 \\
.46 \\
.00\end{array}$ & $\begin{array}{r}3.14 \\
.49 \\
.00\end{array}$ \\
\hline $\begin{array}{l}\text { September: } \\
\text { Maximum } \\
\text { Average...... } \\
\text { Minimum }\end{array}$ & $\begin{array}{l}5.52 \\
1.88 \\
2.11\end{array}$ & $\begin{array}{r}4.84 \\
1.71 \\
.01\end{array}$ & $\begin{array}{r}\mathrm{t} 5.02 \\
1.77 \\
.01\end{array}$ & $\begin{array}{r}5.40 \\
1.64 \\
\text { Trace }\end{array}$ & $\begin{array}{r}5.21 \\
\text { l. } 92 \\
.12\end{array}$ \\
\hline $\begin{array}{l}\text { October: } \\
\text { Maximum } \\
\text { A verage......... } \\
\text { Minimum. }\end{array}$ & $\begin{array}{r}15.50 \\
2.82 \\
2.02\end{array}$ & $\begin{array}{r}8.31 \\
2.94 \\
.00\end{array}$ & $\begin{array}{r}16.67 \\
2.93 \\
.03\end{array}$ & $\begin{array}{r}7.60 \\
2.94 \\
\text { Trace }\end{array}$ & $\begin{array}{r}7.77 \\
2.75 \\
2.04\end{array}$ \\
\hline $\begin{array}{l}\text { November: } \\
\text { Maximum } \\
\text { Alverage } \\
\text { Minimum }\end{array}$ & $\begin{array}{r}13.12 \\
6.54 \\
2.67\end{array}$ & $\begin{array}{r}16.99 \\
6.21 \\
2.63\end{array}$ & $\begin{array}{r}12.50 \\
6.92 \\
2.53\end{array}$ & $\begin{array}{r}16.69 \\
7.09 \\
2.30\end{array}$ & $\begin{array}{r}11.86 \\
5.96 \\
2.80\end{array}$ \\
\hline $\begin{array}{l}\text { December: } \\
\text { Maximum } \\
\text { Average } \\
\text { Minimum }\end{array}$ & $\begin{array}{r}17.54 \\
6.50 \\
22.56\end{array}$ & $\begin{array}{r}17.54 \\
6.33 \\
1.95\end{array}$ & $\begin{array}{r}113.84 \\
6.38 \\
1.91\end{array}$ & $\begin{array}{r}14.15 \\
6.85 \\
2.33\end{array}$ & $\begin{array}{r}13.38 \\
5.77 \\
2.01\end{array}$ \\
\hline $\begin{array}{l}\text { Annual } \\
\text { Maximum } \\
\text { Average } \\
\text { Minimum }\end{array}$ & $\begin{array}{r}152.85 \\
339.26 \\
26.11\end{array}$ & $\begin{array}{r}154.93 \\
37.22 \\
24.56\end{array}$ & $\begin{array}{r}157.15 \\
39.90 \\
26.67\end{array}$ & $\begin{array}{r}157.76 \\
40.40 \\
23.68\end{array}$ & $\begin{array}{r}51.50 \\
37.59 \\
26.39\end{array}$ \\
\hline
\end{tabular}

1 Greater in 1 or more additional years of record.

2 Less in 1 or more additional vears of record.

3 For the 66-year period $1872-1937$ the yearly a verage is 42.24 inches. 
TABLE 4.-Maximum rates of precipitation, in inches, at Portland, Oreg., in the period ending with 1930

[From publication of United States Weather Bureau]

\begin{tabular}{|c|c|c|c|c|c|c|c|c|c|c|c|c|c|c|}
\hline Duration & 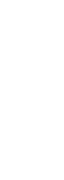 & 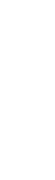 & 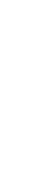 & 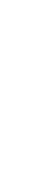 & $\frac{\overline{\mathbf{z}}}{4}$ & $\sum^{\vec{\sigma}}$ & $\stackrel{\Phi}{\Xi}$ & $\stackrel{\overrightarrow{3}}{\overrightarrow{3}}$ & 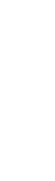 & 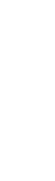 & $\begin{array}{l}\stackrel{\Xi}{0} \\
\stackrel{0}{0} \\
\stackrel{0}{0} \\
0\end{array}$ & 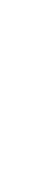 & 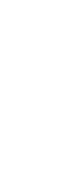 & 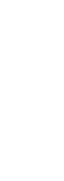 \\
\hline $\begin{array}{l}5 \text { minutes } \\
10 \text { minutes } \\
15 \text { minutes } \\
30 \text { minutes } \\
1 \text { hour } \\
2 \text { hours... } \\
24 \text { hours. } \\
72 \text { hours }\end{array}$ & $\begin{array}{l}41 \\
41 \\
41 \\
41 \\
41 \\
41 \\
59 \\
59\end{array}$ & $\begin{array}{r}0.14 \\
.20 \\
.23 \\
.27 \\
.50 \\
.92 \\
6.86 \\
7.74\end{array}$ & $\begin{array}{r}0.13 \\
.18 \\
.20 \\
.24 \\
.32 \\
.50 \\
3.81 \\
7.39\end{array}$ & $\begin{array}{r}0.10 \\
.17 \\
.20 \\
.28 \\
.46 \\
.64 \\
2.44 \\
5.49\end{array}$ & $\begin{array}{r}0.17 \\
.26 \\
.34 \\
.35 \\
.43 \\
.55 \\
1.92 \\
2.16\end{array}$ & $\begin{array}{r}0.21 \\
.37 \\
.37 \\
.37 \\
.59 \\
.59 \\
1.83 \\
2.75\end{array}$ & $\begin{array}{r}0.28 \\
.46 \\
.64 \\
1.06 \\
1.31 \\
1.74 \\
2.07 \\
2.22\end{array}$ & $\begin{array}{r}0.26 \\
.31 \\
.39 \\
.69 \\
.82 \\
.93 \\
1.35 \\
2.12\end{array}$ & $\begin{array}{r}0.40 \\
.70 \\
.83 \\
1.10 \\
1.25 \\
1.25 \\
1.75 \\
1.77\end{array}$ & $\begin{array}{r}0.28 \\
.53 \\
.61 \\
.69 \\
.87 \\
1.09 \\
2.88 \\
3.28\end{array}$ & $\begin{array}{r}0.21 \\
.22 \\
.23 \\
.32 \\
.49 \\
.64 \\
2.96 \\
5.42\end{array}$ & $\begin{array}{r}0.14 \\
.23 \\
.23 \\
.28 \\
.49 \\
.84 \\
4.43 \\
6.71\end{array}$ & $\begin{array}{r}0.14 \\
.18 \\
.20 \\
.28 \\
.42 \\
.64 \\
7.66 \\
10.81\end{array}$ & $\begin{array}{r}0.40 \\
.70 \\
.83 \\
1.10 \\
1.31 \\
1.74 \\
7.66 \\
10.81\end{array}$ \\
\hline
\end{tabular}

The average yearly precipitation is about 40 inches over most of the lowland, but in the foothills to the west and east it increases to about 70 inches. Table 3 shows the monthly averages and yearly averages for a common 40-year period, from 1896 to 1935, for the five climatologic stations that lie along the middle of the valley. With table 5, it shows further that during the 40-year period the yearly precipitation has ranged from about 60 to about 145 percent of the average. At Portland, whose record of yearly precipitation began in 1872 , the greatest yearly precipitation yet recorded is even greater, 171 percent of the 40-year average.

TABLE 5.-Yearly rainfall and snowfall, in inches, and surplus (+) or deficiency (-) with respect to 40-year average, at five climatologic stations in the Willamette Valley, 1872-1937

[Data from publications of United States Weather Bureau]

\begin{tabular}{|c|c|c|c|c|c|c|c|c|c|c|}
\hline \multirow[b]{2}{*}{ Year } & \multicolumn{2}{|c|}{ Portland } & \multicolumn{2}{|c|}{ Salem } & \multicolumn{2}{|c|}{ Albany } & \multicolumn{2}{|c|}{ Corvallis } & \multicolumn{2}{|c|}{ Eugene } \\
\hline & $\begin{array}{l}\text { Rain- } \\
\text { fall }\end{array}$ & $\begin{array}{l}\text { Sur- } \\
\text { plus or } \\
\text { defi- } \\
\text { ciency }\end{array}$ & $\begin{array}{l}\text { Rain- } \\
\text { fall }\end{array}$ & $\begin{array}{l}\text { Sur- } \\
\text { plus or } \\
\text { defi- } \\
\text { ciency }\end{array}$ & $\begin{array}{l}\text { Rain- } \\
\text { fall }\end{array}$ & $\begin{array}{l}\text { Sur- } \\
\text { plus or } \\
\text { defi- } \\
\text { ciency }\end{array}$ & $\begin{array}{l}\text { Rain- } \\
\text { fall }\end{array}$ & $\begin{array}{c}\text { Sur- } \\
\text { plus or } \\
\text { defi- } \\
\text { ciency }\end{array}$ & $\begin{array}{l}\text { Rain- } \\
\text { fall }\end{array}$ & $\begin{array}{l}\text { Sur- } \\
\text { plus or } \\
\text { defi- } \\
\text { ciency }\end{array}$ \\
\hline A verage 1 & 39.26 & $-\cdots$ & 37.22 & $\ldots$ & 39.90 & 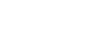 & 40.40 & - & 37.59 & $\ldots$ \\
\hline $\begin{array}{l}1872 \ldots 73 \\
1874 \\
1875\end{array}$ & $\begin{array}{l}46.90 \\
50.52 \\
46.17 \\
60.10\end{array}$ & $\begin{array}{r}+7.64 \\
+11.26 \\
+6.91 \\
+20.84\end{array}$ & $-\cdots$ & - & & & & $(--\cdots$ & 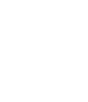 & 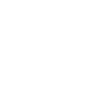 \\
\hline $\begin{array}{l}1876 \ldots \ldots \\
1877 \\
1878 \ldots 19 \\
1879\end{array}$ & $\begin{array}{l}54.94 \\
58.30 \\
47.70 \\
62.22 \\
51.87\end{array}$ & $\begin{array}{r}+15.68 \\
+19.04 \\
+8.44 \\
+22.96 \\
+12.61\end{array}$ & & $\begin{array}{l}-\cdots \\
-\cdots \\
-\cdots \\
-\cdots \\
-\cdots\end{array}$ & $\begin{array}{l}50.14 \\
41.57\end{array}$ & $\begin{array}{r}+10.24 \\
+1.67\end{array}$ & - & $\mid \begin{array}{l}-\cdots \\
-\cdots \\
-\cdots \\
-\cdots \\
-\cdots\end{array}$ & $\begin{array}{l}\cdots \\
-- \\
\cdots \\
\cdots\end{array}$ & $\mid \begin{array}{l} \\
-\cdots \\
\cdots- \\
-\cdots \\
-\cdots \\
-\cdots\end{array}$ \\
\hline $\begin{array}{l}1881 \\
1882 \\
1883 \\
1884 \\
1885\end{array}$ & $\begin{array}{l}57.05 \\
67.24 \\
51.45 \\
38.31 \\
39.59\end{array}$ & $\begin{array}{r}+17.79 \\
+27.98 \\
+12.19 \\
-.95 \\
+.33\end{array}$ & $\mid \begin{array}{l}-\cdots \\
-\cdots \\
-\cdots\end{array}$ & $\mid \begin{array}{l}-\ldots \\
-\ldots \ldots \\
-\ldots \ldots\end{array}$ & $\begin{array}{l}59.59 \\
45.96 \\
39.39 \\
45.22 \\
36.93\end{array}$ & $\begin{array}{r}+19.69 \\
+6.06 \\
-.51 \\
+5.32 \\
-2.97\end{array}$ & - & $\begin{array}{ll}-- \\
-- \\
-- \\
- \\
-\end{array}$ & - & $\begin{array}{ll}--1 \\
-1- \\
-\cdots\end{array}$ \\
\hline
\end{tabular}

11896 to 1935 , inclusive. 
TABLE 5.-Yearly rainfall and snowfall, in inches, and surplus (+) or deficiency (-) with respect to 40 -year average, at five climatologic stations in the Willamette Valley, 1872-1937-Continued

\begin{tabular}{|c|c|c|c|c|c|c|c|c|c|c|}
\hline \multirow[b]{2}{*}{ Year } & \multicolumn{2}{|c|}{ Portlànd } & \multicolumn{2}{|c|}{ Salem } & \multicolumn{2}{|c|}{ Albany } & \multicolumn{2}{|c|}{ Corvallis } & \multicolumn{2}{|c|}{ Eugene } \\
\hline & $\begin{array}{c}\text { Rain- } \\
\text { fall }\end{array}$ & $\begin{array}{c}\text { Sur- } \\
\text { plus or } \\
\text { def- } \\
\text { ciency }\end{array}$ & $\begin{array}{l}\text { Rain- } \\
\text { fall }\end{array}$ & $\begin{array}{l}\text { Sur- } \\
\text { plus or } \\
\text { defi- } \\
\text { ciency }\end{array}$ & $\begin{array}{c}\text { Rain- } \\
\text { fall }\end{array}$ & $\begin{array}{c}\text { Sur- } \\
\text { plus or } \\
\text { defi- } \\
\text { ciency }\end{array}$ & $\begin{array}{l}\text { Rain- } \\
\text { fall }\end{array}$ & $\begin{array}{c}\text { Sur- } \\
\text { plus or } \\
\text { def- } \\
\text { ciency }\end{array}$ & $\begin{array}{c}\text { Rain- } \\
\text { fall }\end{array}$ & $\begin{array}{c}\text { Sur- } \\
\text { plus or } \\
\text { defi- } \\
\text { ciency }\end{array}$ \\
\hline $\begin{array}{l}1886 \\
1887 . \\
1888 \\
1899 \\
1890\end{array}$ & $\begin{array}{l}38.76 \\
54.17 \\
38.76 \\
31.76 \\
40.38\end{array}$ & $\begin{array}{r}-.50 \\
+14.91 \\
-.50 \\
-7.50 \\
+1.12\end{array}$ & & & $\begin{array}{l}38.38 \\
57.62 \\
35.91 \\
33.84 \\
40.02\end{array}$ & $\begin{array}{r}-1.52 \\
+17.72 \\
-3.99 \\
-6.06 \\
+.12\end{array}$ & 34.82 & -5.58 & & \\
\hline $\begin{array}{l}1891 \ldots \\
1892 \ldots \\
1893 \ldots \\
1894 \ldots \\
1895\end{array}$ & $\begin{array}{l}47.41 \\
33.58 \\
39.03 \\
39.32 \\
30.76\end{array}$ & $\begin{array}{r}+8.15 \\
-5.68 \\
-.23 \\
+.06 \\
-8.50\end{array}$ & $\begin{array}{l}49.08 \\
48.62 \\
47.13\end{array}$ & $\begin{array}{r}+11.86 \\
+11.40 \\
+9.91\end{array}$ & $\begin{array}{l}53.39 \\
37.93 \\
46.07 \\
49.86 \\
47.48\end{array}$ & $\begin{array}{r}+13.49 \\
-1.97 \\
+6.17 \\
+9.96 \\
+7.58\end{array}$ & $\begin{array}{l}44.99 \\
31.88 \\
43.04 \\
46.44 \\
43.74\end{array}$ & $\begin{array}{l}+4.59 \\
+8.52 \\
+2.64 \\
+6.04 \\
+3.34\end{array}$ & $\begin{array}{r}44.76 \\
29.25 \\
42.02 \\
37.03\end{array}$ & $\begin{array}{r}+7.17 \\
-8.34 \\
+4.43 \\
-.56\end{array}$ \\
\hline $\begin{array}{l}1896 \ldots \\
1897 \ldots \\
1898 \ldots \\
1899 \\
1900 \ldots\end{array}$ & $\begin{array}{l}44.13 \\
43.01 \\
33.90 \\
42.21 \\
38.22\end{array}$ & $\begin{array}{l}+4.87 \\
+3.75 \\
-5.36 \\
+2.95 \\
-1.04\end{array}$ & $\begin{array}{l}54.93 \\
46.53 \\
34.19 \\
48.12 \\
37.22\end{array}$ & $\begin{array}{r}+17.71 \\
+9.31 \\
-3.03 \\
+10.90 \\
.00\end{array}$ & $\begin{array}{l}57.15 \\
50.19 \\
36.22 \\
46.99 \\
38.88\end{array}$ & $\begin{array}{r}+17.25 \\
+10.29 \\
-3.68 \\
+7.09 \\
-1.02\end{array}$ & $\begin{array}{l}57.76 \\
43.61 \\
35.02 \\
46.69 \\
38.49\end{array}$ & $\begin{array}{r}+17.36 \\
+3.21 \\
-5.38 \\
+9.29 \\
-1.91\end{array}$ & $\begin{array}{l}48.47 \\
40.55 \\
27.16 \\
41.28 \\
40.89\end{array}$ & $\begin{array}{r}+10.88 \\
+2.96 \\
-10.43 \\
+3.69 \\
+3.30\end{array}$ \\
\hline $\begin{array}{l}1901 \\
1902 \\
1903 \\
1904 \\
1905\end{array}$ & $\begin{array}{l}41.05 \\
50.15 \\
35.62 \\
46.37 \\
34.10\end{array}$ & $\begin{array}{r}+1.79 \\
+10.89 \\
-3.64 \\
+7.11 \\
-5.16\end{array}$ & $\begin{array}{l}37.42 \\
42.55 \\
24.56 \\
42.08 \\
28.49\end{array}$ & $\begin{array}{r}+.20 \\
+5.33 \\
-12.66 \\
+4.86 \\
-8.73\end{array}$ & $\begin{array}{l}38.89 \\
52.94 \\
33.65 \\
49.41 \\
30.19\end{array}$ & $\begin{array}{r}-1.01 \\
+13.04 \\
-6.25 \\
+9.51 \\
-9.71\end{array}$ & $\begin{array}{l}41.36 \\
54.02 \\
34.13 \\
56.21 \\
34.39\end{array}$ & $\begin{array}{r}+.96 \\
+13.62 \\
-6.27 \\
+15.81 \\
-6.01\end{array}$ & $\begin{array}{l}40.01 \\
51.50 \\
34.21 \\
50.71 \\
29.95\end{array}$ & $\begin{array}{r}+2.42 \\
+13.91 \\
-3.38 \\
+13.12 \\
-7.64\end{array}$ \\
\hline $\begin{array}{l}1906 \ldots \\
1907 . \\
1908- \\
1909 \\
1910 \\
-\ldots\end{array}$ & $\begin{array}{l}43.29 \\
42.89 \\
34.37 \\
43.75 \\
38.65\end{array}$ & $\begin{array}{r}+4.03 \\
+3.63 \\
-4.89 \\
+4.49 \\
-.61\end{array}$ & $\begin{array}{l}34.01 \\
37.10 \\
26.76 \\
39.59 \\
31.89\end{array}$ & $\begin{array}{r}-3.21 \\
-.12 \\
-10.46 \\
+2.37 \\
-5.33\end{array}$ & $\begin{array}{l}40.72 \\
44.44 \\
28.54 \\
43.70 \\
38.30\end{array}$ & $\begin{array}{r}+.82 \\
+4.54 \\
-11.36 \\
+3.80 \\
-1.60\end{array}$ & $\begin{array}{l}45.71 \\
50.23 \\
33.29 \\
50.31 \\
37.64\end{array}$ & $\begin{array}{l}+5.31 \\
+9.83 \\
-7.11 \\
+9.91 \\
-2.76\end{array}$ & $\begin{array}{l}37.55 \\
46.27 \\
27.22 \\
39.16 \\
33.20\end{array}$ & $\begin{array}{r}-.04 \\
+8.68 \\
-10.37 \\
+1.57 \\
-4.39\end{array}$ \\
\hline $\begin{array}{l}1911 \ldots \\
1912 \ldots \\
1913 \ldots \\
1914 \ldots \\
1915 \ldots\end{array}$ & $\begin{array}{l}33.28 \\
43.47 \\
36.30 \\
36.67 \\
41.30\end{array}$ & $\begin{array}{l}-5.98 \\
+4.21 \\
-2.96 \\
-2.59 \\
+2.04\end{array}$ & $\begin{array}{l}27.55 \\
35.72 \\
31.75 \\
35.87 \\
32.55\end{array}$ & $\begin{array}{l}-9.67 \\
-1.50 \\
-5.47 \\
-1.35 \\
-4.67\end{array}$ & $\begin{array}{l}34.11 \\
46.68 \\
35.37 \\
44.24 \\
40.12\end{array}$ & $\begin{array}{r}-5.79 \\
+6.78 \\
-4.53 \\
+4.34 \\
+.22\end{array}$ & $\begin{array}{l}38.46 \\
46.81 \\
35.76 \\
44.13 \\
36.82\end{array}$ & $\begin{array}{l}-1.94 \\
+6.41 \\
-4.64 \\
+3.73 \\
-3.58\end{array}$ & $\begin{array}{l}30.21 \\
43.58 \\
31.66 \\
35.22 \\
39.83\end{array}$ & $\begin{array}{l}-7.38 \\
+5.99 \\
-5.93 \\
-2.37 \\
+2.24\end{array}$ \\
\hline $\begin{array}{l}1916 \ldots \\
1917 \ldots \\
1918 \ldots \\
1919 \ldots \\
1920 \ldots\end{array}$ & $\begin{array}{l}\mathbf{4 5 . 7 7} \\
\mathbf{4 0 . 5 0} \\
31.50 \\
\mathbf{4 5 . 7 0} \\
\mathbf{4 1 . 1 7}\end{array}$ & $\begin{array}{l}+6.51 \\
+1.24 \\
+7.76 \\
+6.44 \\
+1.91\end{array}$ & $\begin{array}{l}45.76 \\
32.82 \\
27.92 \\
41.01 \\
40.78\end{array}$ & $\begin{array}{l}+8.54 \\
-4.40 \\
-9.30 \\
+3.79 \\
+3.56\end{array}$ & $\begin{array}{l}47.74 \\
39.50 \\
34.31 \\
52.85 \\
38.59\end{array}$ & $\begin{array}{r}+7.84 \\
-.40 \\
-5.59 \\
+12.95 \\
-1.31\end{array}$ & $\begin{array}{l}46.65 \\
39.13 \\
33.52 \\
50.57 \\
37.12\end{array}$ & $\begin{array}{r}+6.25 \\
-1.27 \\
-6.88 \\
+10.17 \\
-3.28\end{array}$ & $\begin{array}{l}48.18 \\
41.17 \\
31.40 \\
46.27 \\
40.67\end{array}$ & $\begin{array}{r}+10.59 \\
+3.58 \\
-6.19 \\
+8.68 \\
+3.08\end{array}$ \\
\hline $\begin{array}{l}1921 \\
1922 \\
1923 \\
1924 \\
1925\end{array}$ & $\begin{array}{l}43.21 \\
38.76 \\
32.81 \\
31.22 \\
31.36\end{array}$ & $\begin{array}{r}+3.95 \\
-.50 \\
-6.45 \\
-8.04 \\
-7.90\end{array}$ & $\begin{array}{l}43.12 \\
38.03 \\
33.53 \\
33.15 \\
33.32\end{array}$ & $\begin{array}{r}+5.90 \\
+.81 \\
-3.69 \\
-4.07 \\
-3.90\end{array}$ & $\begin{array}{l}44.59 \\
38.99 \\
36.00 \\
33.72 \\
34.18\end{array}$ & $\begin{array}{r}+4.69 \\
-.91 \\
-3.90 \\
-6.18 \\
-5.72\end{array}$ & $\begin{array}{l}41.05 \\
38.45 \\
37.07 \\
37.87 \\
34.07\end{array}$ & $\begin{array}{l}+.65 \\
-1.95 \\
-3.33 \\
-2.53 \\
-6.33\end{array}$ & $\begin{array}{l}36.60 \\
37.06 \\
33.05 \\
35.88 \\
33.70\end{array}$ & $\begin{array}{r}-.99 \\
-.53 \\
-4.54 \\
-1.71 \\
-3.89\end{array}$ \\
\hline $\begin{array}{l}1926 \ldots \\
1927- \\
1928 \\
1929 \\
1930 \\
\end{array}$ & $\begin{array}{l}41.17 \\
45.78 \\
34.69 \\
26.11 \\
27.16\end{array}$ & $\begin{array}{r}+1.91 \\
+6.52 \\
-4.57 \\
-13.15 \\
-12.10\end{array}$ & $\begin{array}{l}39.17 \\
43.43 \\
38.03 \\
26.40 \\
30.05\end{array}$ & $\begin{array}{r}+1.95 \\
+6.21 \\
+.81 \\
-10.82 \\
-7.17\end{array}$ & $\begin{array}{l}37.87 \\
44.60 \\
37.97 \\
27.03 \\
26.67\end{array}$ & $\begin{array}{r}-2.03 \\
+4.70 \\
-1.93 \\
-12.87 \\
-13.23\end{array}$ & $\begin{array}{l}40.04 \\
47.87 \\
39.87 \\
24.45 \\
23.68\end{array}$ & $\begin{array}{r}-.36 \\
+7.47 \\
-.53 \\
-15.95 \\
-16.72\end{array}$ & $\begin{array}{l}41.16 \\
43.58 \\
34.91 \\
32.10 \\
26.39\end{array}$ & $\begin{array}{r}+3.57 \\
+5.99 \\
-2.68 \\
-5.49 \\
-11.20\end{array}$ \\
\hline $\begin{array}{l}1931 \\
1932 \\
1933 \\
1934 \\
1935\end{array}$ & $\begin{array}{l}42.68 \\
39.98 \\
52.85 \\
45.98 \\
29.19\end{array}$ & $\begin{array}{r}+3.42 \\
+.72 \\
+13.59 \\
+6.72 \\
-10.07\end{array}$ & $\begin{array}{l}48.64 \\
42.06 \\
52.56 \\
39.41 \\
30.90\end{array}$ & $\begin{array}{r}+11.42 \\
+4.84 \\
+15.34 \\
+2.19 \\
-6.32\end{array}$ & $\begin{array}{l}41.89 \\
40.30 \\
43.20 \\
38.10 \\
27.77\end{array}$ & $\begin{array}{r}+1.99 \\
+.40 \\
+3.30 \\
-1.80 \\
-12.13\end{array}$ & $\begin{array}{l}39.13 \\
36.94 \\
42.59 \\
35.42 \\
26.35\end{array}$ & $\begin{array}{r}-1.27 \\
-3.46 \\
+2.19 \\
-4.98 \\
-14.05\end{array}$ & $\begin{array}{l}36.78 \\
36.22 \\
36.01 \\
34.99 \\
28.91\end{array}$ & $\begin{array}{l}-.81 \\
-1.37 \\
-1.58 \\
-2.60 \\
-8.68\end{array}$ \\
\hline $\begin{array}{l}1936 \\
1937 \ldots\end{array}$ & $\begin{array}{l}34.29 \\
56.85\end{array}$ & $\begin{array}{r}-4.97 \\
+17.59\end{array}$ & $\begin{array}{l}33.61 \\
63.50\end{array}$ & $\begin{array}{r}-3.61 \\
+26.28\end{array}$ & $\begin{array}{l}32.23 \\
57.44\end{array}$ & $\begin{array}{r}-7.67 \\
+17.54\end{array}$ & $\begin{array}{l}32.11 \\
58.06\end{array}$ & $\begin{array}{r}-8.29 \\
+17.66\end{array}$ & $\begin{array}{l}34.02 \\
55.21\end{array}$ & $\begin{array}{r}-3.57 \\
+17.62\end{array}$ \\
\hline
\end{tabular}


The prevailing wind is from the north and northwest in summer and from the south and southwest in winter, but the variation in wind direction is moderate. Winds from the south and southwest attain the greatest velocities but are seldom destructive. In and near the mouth of the Columbia Gorge east winds prevail at times; these are cold in winter and warm at other times.

The climatic features of the lowland and adjacent foothills, just described, do not apply to the mountainous terrane on either side. Thus, to the east, the extensive west slope of the Cascade Range is, on the average, appreciably cooler than the lowland, though the extremes of temperature are not materially lower. There the average yearly temperature ranges from about $44^{\circ}$ to $51^{\circ} \mathrm{F}$. On this mountain slope the yearly precipitation is generally more than 50 inches, and some small areas near Mount Hood receive more than 100 inches. In some winters a large part of the precipitation is rain, but considerable snow falls at times; the average yearly snowfall at the several stations ranges from about 14 inches to 300 inches or more. Most of the higher part of the range probably has an average yearly temperature of less than $42^{\circ}$; some of the highest peaks probably less than $32^{\circ}$. Nevertheless, the highest temperatures are greater than those along the Pacific coast, to the west, and the lowest temperatures are not so low as at some localities on the high semiarid plains to the east. Snow accumulates to great depths, the average at recording stations being from 200 to 300 inches and more. Permanent snow fields and alpine glaciers (see pl. $4, A$ ) exist on several of the highest peaks.

Across the valley, on the lower and less extensive east slope of the Coast Range, there is much variation in climate. The average yearly temperature is generally less than $50^{\circ}$ and the average yearly precipitation is 50 inches or more. In winter there is considerable freezing weather, and at some places snow accumulates to a considerable depth.

\section{GEOLOGY AND HYDROLOGY OF THE CONSOLIDATED AND SEMICONSOLIDATED ROCKS}

GENERAL CHARACTER, SUCCESSION, AND AGE OF THE ROCKS

The rocks that form the foothills and mountains on both sides of the central Willamette lowland and that pass beneath the unconsolidated deposits of the lowland include fine-grained marine and terrestrial sedimentary rocks, fragmental and nonfragmental igneous rocks, and some coarse-grained terrestrial deposits. The marine sedimentary rocks are chiefly shale and earthy sandstone of Eocene, Oligocene, and Miocene (?) age. These make up the greater part of the Coast Range west of the lowland, where they are associated with basaltic extrusive rocks of Miocene (?) age and with intrusive rocks. This 
succession of marine sedimentary rocks and igneous rocks extends eastward beneath nearly all the lowland and, at some places, into the lowest foothills of the Cascade Range. At the very southern end of the central lowland, the sedimentary rocks of latest Eocene and Oligocene age are partly marine and partly nonmarine in origin, as though the seashore of that time oscillated repeatedly across that area. Fragmental and nonfragmental volcanic rocks form nearly all the Cascade Range except the lowest part of the most westerly foothills. These rocks are the products of volcanic activity that began in the Oligocene (?) epoch, continued itermittently during the remainder of the Tertiary and far into the Pleistocene, then waned in latest Pleistocene or Recent time. The rocks are largely basalt or andesite and include both nonfragmental flow rocks and fragmental rocks that range from unstratified or poorly stratified coarse agglomerate to finegrained well-stratified tuff. The coarse-grained terrestrial deposits occur in the northeastern part of the Willamette Valley, in the vicinity of Portland. They are underlain and overlain by volcanic rocks and are of Pliocene or early Pleistocene age.

The accompanying table shows the character, stratigraphic sequence, and general water-bearing properties of the rock formations that occur aiong the margin of the central Willamette lowland. These several formations were not mapped separately but are included under one symbol on plates 1 and 2 .

\section{STRUCTURE}

The structure of the rocks that surround the central lowland of the Willamette River Basin was not mapped by the writer. No horizon markers can be traced over extensive areas, and dense forest and brush and a thick mantle of nondescript detritus cover most of the basin except the highest part of the Cascade Range. Nevertheless, some general features of the structure can be summarized. Probably late in the Miocene epoch the region was gently compressed and its rocks were thrown into broad open folds, seemingly with few or no normal faults of consequence and, so far as is now known, with no close or overturned folds or thrust faults. As is pointed out by Callaghan and Buddington, ${ }^{6}$ with citations to earlier workers, the most conspicuous structural feature of the rocks adjacent to the central lowland is the gentle eastward or northeastward dip, commonly about $10^{\circ}$, that prevails south of the Santiam River. To the north, along the east flank of the lowland, the general dip changes to the northwest and maintains that direction nearly or quite to the Columbia River. West of the lowland, in the flank of the Coast Range, the prevailing dip continues generally eastward. These structural features coordi-

\footnotetext{
${ }^{6}$ Cellaghn, Eugene; apd Buddington, A. F., Metalliferous mineral deposits of the Cascade Range in Oregon: U. S. Geol. Survey Bull. 893, pp. 18-20, 1938.
} 
nate with the western two of four broad folds that have been traced by Thayer ${ }^{7}$ in the region east of Salem; in order from the west, these two folds are the Willamette syncline and the Mehama anticline. The projected axes of these folds diverge northward from a common point near Eugene; the syncline trends about N. $15^{\circ} \mathrm{E}$. through the Stayton Basin to Portland, whereas the anticline trends about N. $30^{\circ}$ E. toward Wyeth, which is on the Columbia River some 40 miles east of Portland. In the region between the Santiam and Clackamas Rivers, both axes plunge gradually northward. Thus, at the southern end of tle lowland the two folds begin as minor flexures on the general monocline near Eugene; toward the north they gradually broaden and increase in amplitude.

Immediately west of Portland the Tualatin Valley discloses structural features seemingly unlike any others now known in the regiona relatively narrow anticline and a sharply folded syncline that plunge about $\mathrm{S} .45^{\circ} \mathrm{E}$. across the axies of the broad regional folds just described. The syncline may be faulted. The form of these structural features is not known in detail, especially in the vicinity of Oregon City, where they meet the Willamette syncline, described by Thayer. In general, the Tualatin Valley appears to have formed along the anticlinal axis, where erosion breached the resistant basalt of Miocene age and then planed rather widely into the underlying shale.

All the structural features so far described are tentatively ascribed to the one period of deformation late in the Miocéne epoch. However, the region probably has not been wholly stable in all subsequent time, for during the Pleistocene or glacial epoch much of the Pacific coast region in Oregon and Washington has oscillated through a vertical range of 1,000 feet or more with respect to present sea level. Some features of the Willamette River Basin suggest that the region was again deformed just before the bulk of the unconsolidated valley fill was deposited, possibly between the two periods of sedimentation that are represented by the terrace deposits and the older alluvium. (See pp. 28,32.) However, this late deformation cannot now be demonstrated with assurance.

7 Thayer, T. P., Structure of the North Santiam River section of the Cascade Mountains in Oregon: Jour. Geology, vol. 44, pp. 701-716, 1936. 
TABLE 6.-Stratigraphic sections añd generial water-bearing properties of consolidated IStratigraphy largely after Hodge $(1933,1938)$, Schenok

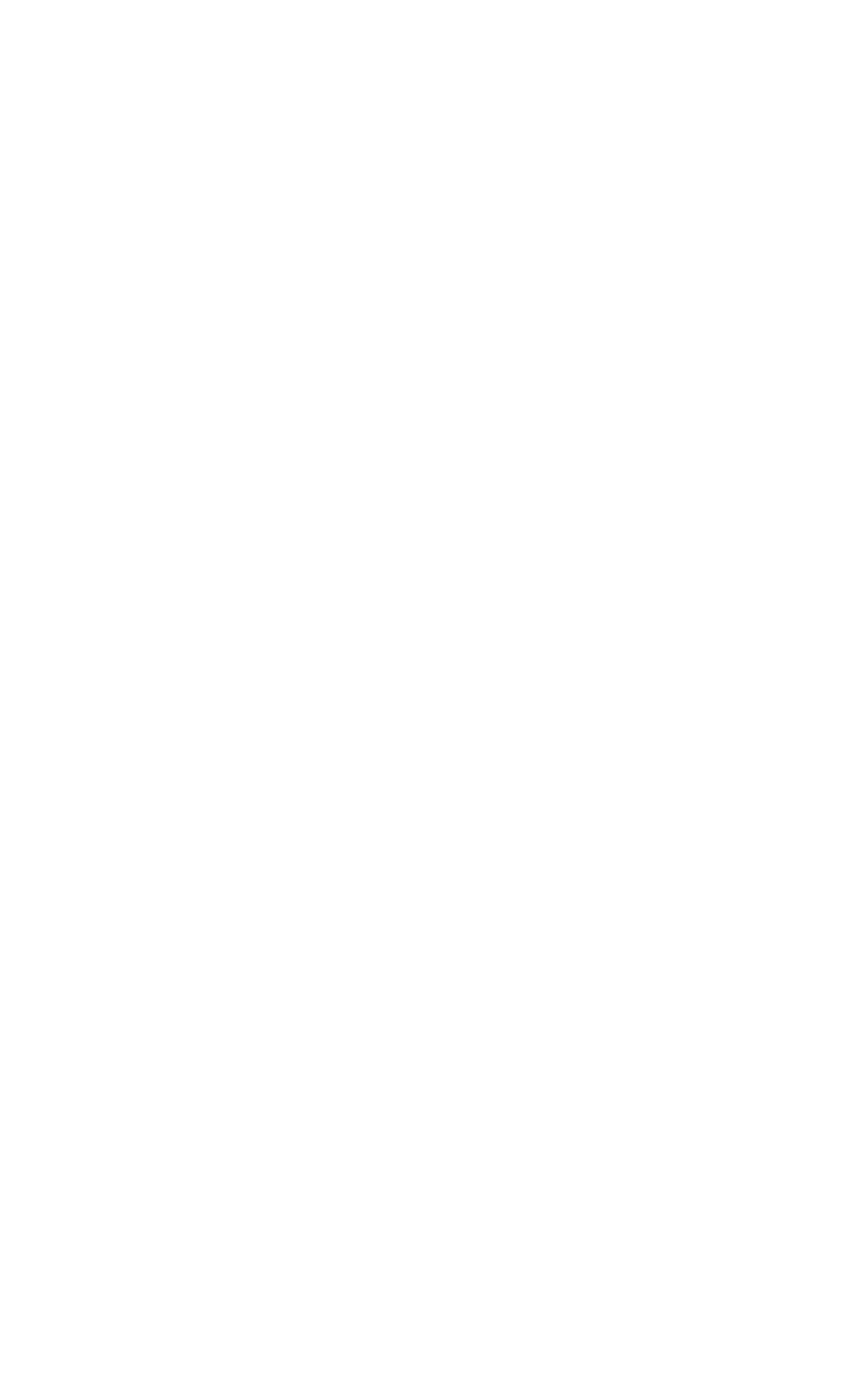


and semiconsolidated rocks bounding the central lowland of the Willamette River Basin (1927, 1933, 1936), W. D. Smith (1924), and Thayer (1933)]

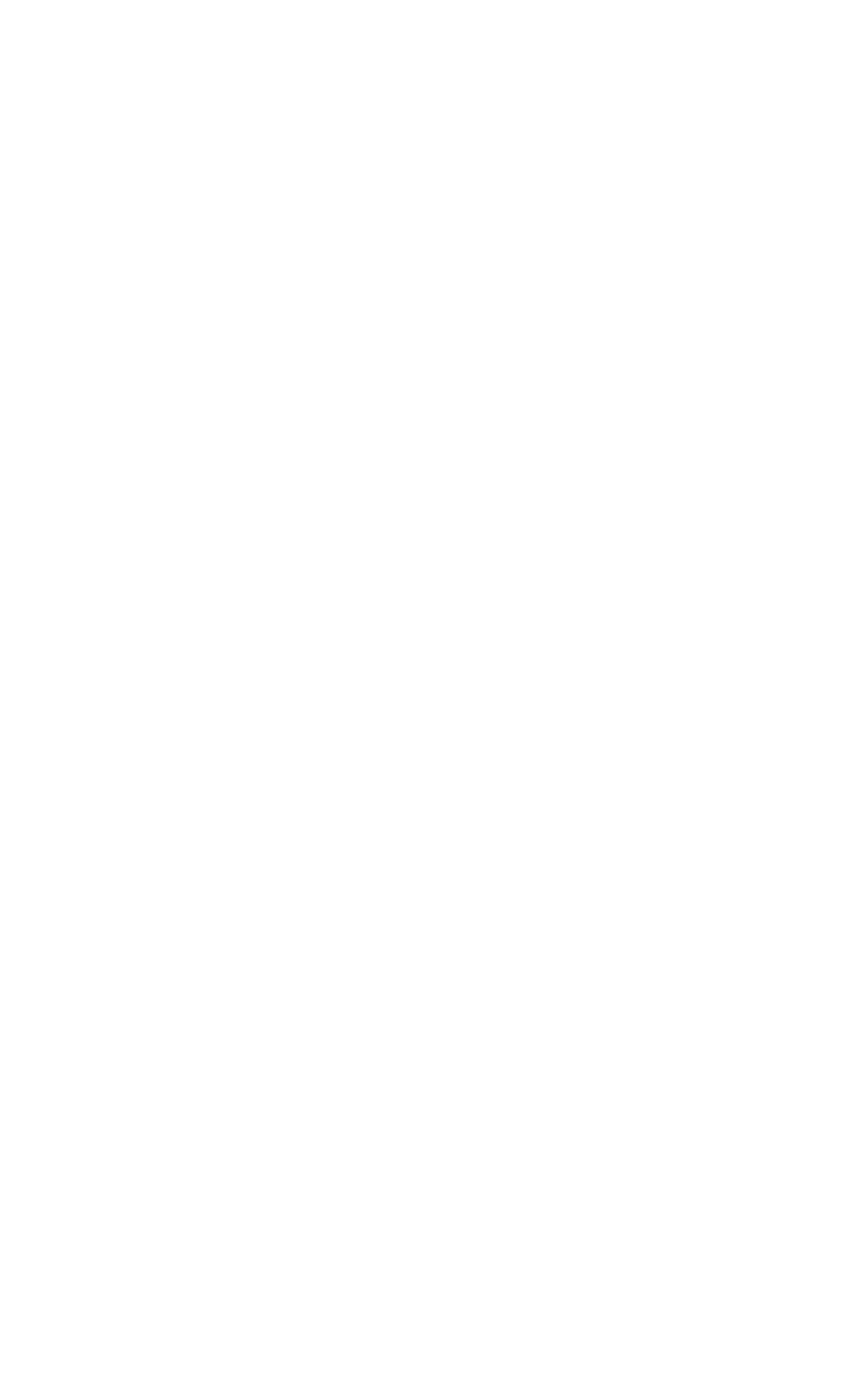




\section{WATER-BEARING PROPERTIES}

The youngest or uppermost consolidated and semiconsolidated rocks - the Cascade, Rhododendron, and Troutdale formations of Hodge--border and underlie only the northernmost part of the central lowland, from Portland southward to Oregon City and beneath the Molalla pediment. The Cascade and Rhododendron formations, which are volcanic, form outliers in east Portland, such as Rocky Butte, part of Mount Tabor, and Kelly Butte; a few miles to the south, they also form Mount Scott and the contiguous volcanic terrane that lies between Johnson Creek and the Clackamas River. Little is known regarding the water-bearing properties of these two formations. The flow rocks and accompanying scoria yield some water from discontinuous pervious zones, but at most places not copiously. However, must of the agglomerate and tuff is derise and nearly impervious. Much of the detrital Troutdale formation of Hodge is composed of unassorted particles and is sufficiently consolidated to be nearly impervious. In some coarse-grained beds, however, the particles are somewhat assorted as to size, and so the beds are perhaps moderately pervious. 'This formation crops out along the west side of the Willamette River in Portland and probably underlies much of the "delta" that extends eastward from the Willamette! River to and beyond the edge of the area that is shown on plate 1 . It may include part of the "cemented gravel" and certain of the lower water-bearing zones that are penetrated by the deeper wells in and near Portland. However, the data now at hand are not sufficient to show the depth, thickness, and extent of its water-bearing zones.

Of the several rock formations that surround and underlie the principal two segments of the central lowland, only one-the basalt of Miocene age (Yakima basalt and Stayton lavas of Thayer)-yields water freely over an extensive area. However, this formation is far from uniform in its water-yielding capacity. The water occurs under! two distinct conditions: First, at shallow or moderate depth from zones. that have become pervious through weathering, at some places as much as 100 feet beneath the land surface; and second, from scoria-' ceous or fractured zones and granular partings at depth in the un-' weathered rock. Water in the zone of weathering is commonly. perched high above the lowland plains and affords domestic and farm supplies from small springs and wells on outlying ridges and peaks, such as the Chehalem Mountains along the south side of the Tualatin Valley in Tps. 1 and 2 S., Rs. 2 and 3 W., Saxton, Cooper, and Bull Mountains, in the southeastern part of that valley, and Petes Moun-. tain and corresponding ridges in the vicinity of Oregon City in Tps. 2 and 3 S., R. 1 E. There appear to be numerous small disconnected bodies of this perched water at various heights above the lowland, so 
that some shallow wells are either dry or are not dependable in dry years. The location and extent of these bodies of perched water cannot be traced at this time. At depth in the unweathered rock the water-bearing zones are not continuous over extensive areas and differ greatly in thickness and perviousness. For example, well 53 reached the basalt about 470 feet below the land surface and continued in that rock formation to a depth of 800 feet; its tested yield was 110 gallons a minute, and its specific capacity 1.1 gallons a minute for each foot of draw-down. On the other hand, two other wells within half a mile, Nos. 52 and 51, are not nearly so deep and yield about 500 gallons a minute each with respective specific capacities of about 7.4 and 6.4 gallons a minute for each foot of draw-down. In these three wells the water-bearing zones are below sea level and are far beneath the lowland plains. Several miles to the southeast, along the flanks of the basalt ridge that lies between the Willamette and Tualatin Rivers, wells 74 and 76 also yield several hundred gallons a minute from pervious zones far beneath the valley surface. To the west, in about the NW1/4 sec. 32 , T. 1 S., R. 2 W., a deep well not visited by the writer is reported to flow about 1 second foot by artesian pressure; the water is inferred to have been struck in the basalt. Not all wells, however, yield so copiously. Well 54, for example, taps the basalt below the lowland plains, but its reported ultimate capacity is only 4 gallons a minute. Drilled wells high on the basalt ridges are erratic in depth and yield. Thus, according to an unconfirmed roport, farm wells along the crest of the ridge between the Willamette and Tualatin Rivers range in depth from little more than 100 feet to as much as 300 feet. Presumably these wells were drilled only deep enough to assure dependable farm water supplies, for which purpose a yield of a few gallons a minute would be adequate. Probably the wells range so widely in depth because the pervious zones are discontinuous and because certain of the more extensive zones drain into the adjacent, valley.

To the south, in the vicinity of Salem, well 331 has a sustained yield of about $7 \frac{1}{2}$ gallons a minute from the basalt. About a mile to the southeast, on the farm of Albert Bouffleur, a well drilled into the basalt since the writer's field work is being used successfully for smallscale irrigation; this well is 8 inches in diameter and 170 feet deep and yields 285 gallons a minute. In both wells the pervious zones are in the flank of the eastward-dipping Eola Hills and slightly lower than the adjacent flood plain of the Williamette River. Still farther south, well 345 yields about 15 gallons a minute, probably from the lower part of the zone of weathering. Still fartber south, well 382 taps perched water in the basalt high above the level of the central lowland; a companion well, no. 381 , found the next water-bearing zone 265 feet lower. 
From the typical conditions just described it is clear that certain water-bearing zones in the unweathered Miocene basalt are sufficiently pervious and thick at some places to sustain withdrawals for irrigation on a moderate scale, for small public supplies, or for industrial purposes. Because these zones doubtless are discontinuous and scattered irregularly through the Miocene basalt it is not possible to foretell, for any particular place, the depth to which a well must be drilled to obtain an adequate supply of water. Neither can it be foretold whether an adequate supply exists within a practicable depth below the land surface. In general, however, the well is more likely to be successful if its site is not high above the central lowland, for then the shallower pervious zones in the basalt will not be extensively drained and the ground-water level so lowered that the pumping lift is excessive.

The two rock formations that occur next below the basalt of Miocene age - the Eugene formation and Fisher formation of Schenck and their correlatives-flank and underlie much of the central lowland from Oregon City southward to Salem and again, far to the south, near Eugene. For several tens of feet below the land surface these formations commonly are slightly pervious, owing to weathering and leaching, and so yield some water to shallow farm wells. At depth, however, they appear to be almost wholly impervious. For example, well 23, near the center of the Tualatin Valley at Hillsboro, reached the bottom of the unconsolidated material about 180 feet below the land surface and continued to a total depth of 1,619 feet, largely in "shale and hard clay." This 1,439-foot section of bedrock, which is correlated tentatively with the Pittsburg Bluff sandstone of Schenck, was reported devoid of water-bearing beds. Much farther south, at the Cottage Farm of the Oregon State Hospital, near Salem, well 385 was drilled to a depth of 1,006 feet in sedimentary rocks without finding water; at least the upper strata penetrated by this well represent the Illahe formation of Thayer. At the very southern end of the valley, near Eugene, the six wells, 722 to 727, are drilled in the Eugene or Fisher formation to depths between 225 and 3,000 feet; three of these wells are barely adequate for household supply and the remaining three, which include the deepest two, proved inadequate. Thus deep drilling to tap these bedrock formations beneath the unconsolidated deposits of the central lowland is unlikely to be successful.

In contrast with the two rock formations just described, the next formation in downward succession contains many beds that yield water. This next formation, the oldest and lowest of those that surround and underlie the central lowland, includes interbedded sandstone, sandy shale, and shale. It is approximately if not strictly correlative with the Tyee sandstone and the Burpee formation of Schenck and is of late Eocene age. This formation constitutes all 
or nearly all of the western flank of the central lowland southward from Dallas, in Tps. 7 to $18 \mathrm{~S}$. Its water-bearing zones are the discontinuous and lenticular beds of sandstone and sandy shale, which yield water slowly to numerous farm wells among the foothills. So far as is known, however, none of these wells yields more than about 10 gallons a minute. Several wells, for example well 533 near Corvallis, have struck brackish water and brine in the formation, in some places less than 100 feet below the level of the lowland plain. (See p. 64.) Accordingly, as is true of the younger sedimentary rocks, these rocks of late Eocene age are not likely to yield copious supplies of fresh water if tapped by deep wells passing through the unconsolidated deposits of the central lowland.

\section{GEOLOGY AND HYDROLOGY OF THE UNCONSOLIDATED DEPOSITS}

\section{GENERAL CHARACTER, EXTENT, AND THICKNESS OF THE DEPOSITS}

\section{YOUNGER ALLUVIUM}

The unconsolidated deposits that form the central lowland of the Willamette River Basin contain the materials most likely to sustain large withdrawalš of water from wells. Of these deposits the youngest underlies or forms the flood plains of the present streams and is shown on plates 1 and 2 as younger alluvium.

Along the Willamette River below Eugene, this youngest alluvial. or stream-laid deposit is composed of poorly or rudely assorted gravel and sand with a small proportion of silt. Upstream, to the south, it consists largely of coarse gravel and cobbles as much as 6 inches in diameter (pl. $4, B$ ), and some sandy beds or zones. Downstream the deposit becomes progressively finer and better assorted and at numerous places is rudely stratified and cross-bedded (pl. 5), but even at the northern end of the valley, as near Newberg, it contains a fairly large proportion of pebbles as much as 2 inches in diameter. In this deposit the beds of large water-yielding capacity are tongues of clean sand or gravel; one or more such tongues have been penetrated by wells at most places on the flood plain, though their depth and thickness at any particular place cannot be foretold. At some places the deposit contains many fine particles so thoroughly interspersed with. the sand and gravel that water is yielded slowly to wells; some such places are in the bottoms of ephemeral drains and channels that are occupied by slack water when the river is in freshet. It appears that here much of the silt has been brought in after the gravel and sand has been carried downward into the deposit as the silt-laden backwater seeped away. All the materials of the younger alluvium, even the finest sand and silt, appear to be quite fresh; from this and from the 
relative coarseness of the materials, it is judged that over most of the flood plain water can penetrate easily from the land surface to the water table.

Along the main branch of the Willamette River, the alluvial deposit just described forms a tongue that is from half a mile to 4 miles wide and is coextensive with the flood plain. Its thickness ranges from a feather edge along cither margin of the flood plain to a few tens of feet; the greatest known thickness, in well 463, near Corvallis, ${ }^{8}$ is 42 feet. Branching from it, tongues of similar material extend eastward along those tributary streams that rise high in the Cascade Mountains and that reach the central lowland with relatively steep gradients. In order northward these tributaries are the Middle Fork, McKenzie River, the two branches of the Santiam River, and the Molalla River.

In contrast with the deposits just described, the younger alluvium is of decidedly different character along the smaller tributaries that enter the Willamette River from the east and along all the tributaries that enter from the west. Along the smaller tributaries from the east-such as Muddy Creek and the Calapooya River to the south and the Pudding River to the north-young alluvial deposits are formed largely of fine-grained materials derived from the lowland. At most places, therefore, they do not transmit water freely and do not promise wells of large capacity. The tributaries that enter the Willamette River from the west reach the lowland with a very slight gradient. Thus, their young alluvial deposits contain little clean sand and gravel within the area that is shown on plates 1 and 2 and so generally do not yield water freely. These streams, in order from the south, include the Long Tom, Muddy, Luckiamute, Yamhill, and Tualatin Rivers.

Three tongues of young alluvium delimited on plate 1 are somewhat higher than the flood plains of present streams. The largest of these is the so-called Lake Labish channel, which trends northeastward from the flood plain of the Willamette River just north of Salem to the plain of Pudding River near the town of Mount Angel. The two smaller tongues occur to the east, directly across Pudding River; they diverge from the present valley of Abiqua Creek about 3 miles southeast of Mount Angel. All three tongues floor abandoned streamways. All three appear to be composed of fine-grained materials, are probably thin, and are judged to be low in water-yielding capacity.

\section{OLDER ALLUVIUM AND RELATED DEPOSITS}

The materials that form and lie immediately beneath the main lowland plain of the Willamette River Basin are shown on plate 1 as older alluvium and related deposits. At least two distinctive sorts

\footnotetext{
8 Descriptions of wells appear in tables 11 and 12; locations are shown on plates 1 and 2.
} 
of material are included: comparatively coarse alluvial or stream-laid. material beneath all the main plain southward from the rock-bound narrows at Salem; distinctly finer material northward from Salem, of which that lying immediately beneath the land surface appears not to have been deposited by vigorous streams. The principal characteristics of the material in these two parts of the valley are described below. The mode of occurrence of the ground-water and the yield from the deposits are treated on pages 34 to 54 .

As has been stated, the main plain in the southern segment of the valley comprises several broad alluvial fans and intervening flats of very gentle slope. It covers about 400,000 acres. To a depth of at least 50 feet below the land surface, the central and higher parts of the fans appear to be composed largely of tongues and lentils of gravel and sand interspersed with unassorted mixtures of sand and silt (pl. 6, A). The better-assorted bodies of gravel and sand serve as arteries whích, though perhaps devious, appear to be rather thoroughly interconnected, thus allowing free movement of ground water. One or more ground-water arteries of this sort are penetrated by numerous wells. Toward the lower margins of the alluvial fans the coarser alluvial deposits just described appear to grade into and interfinger with poorly assorted or unassorted mixtures of sand, silt, and pebbles which in turn, beneath the flats that intervene between alluvial fans, grade into heterogeneous material that is largely earthy. These finer alluvial materials yield water slowly.

Little is known of the character of the lower or deeper part of the older alluvium in this part of the valley. Near the center of the area the deposit, according to the driller's record of well 637 (table 12), contains much "clay" and "mud" below a depth of 60 feet and especially below a depth of 145 feet. Unfortunately that record, the only one available for the purpose, does not show whether the claylike material occurs in distinct beds that alternate with layers of sand and gravel or whether fine and coarse particles are commingled throughout the lower part of the deposit. That single record does not preclude the possibility that elsewhere in the area the deeper part of the older alluvium may be composed largely of gravel and sand, such as predominate in the higher parts of the alluvial fans that form the land surface. Neither does the single record warrant a judgment as to the water-yielding capacity of the deeper alluvial materials.

So far as is indicated by data now available, the older alluvium in the southern segment of the valley is somewhat finer and less thoroughly assorted than the younger alluvium of the same area. Although its upper part, at least, contains fairly extensive arteries that yield water quite freely, the deposit as a whole is probably less pervious than the younger alluvium. In the soil zone, the finer particles of 
the older alluvium at some places have been disintegrated by weathering. Consequently infiltration of water from the land surface to the water table doubtless is somewhat impeded here and there; however, infiltration probably is nearly as free as through the younger alluvium except near the outer margins of the alluvial fans and over the intervening flats.

Because the older alluvium was deposited over an eroded bedrock surface, its thickness must range from a feather edge along the outer margin of the alluvial plain to a maximum over the lowest point on the bedrock floor. Geologic features of the valley suggest that the bedrock floor had moderate local relief. Consequently the thickness of the alluvial deposit may vary decidedly, even within small areas. Few determinations of the thickness in the southern half of the valley are available (see pl. 7). These few, which are drawn wholly from driller's records of wells, show fairly well the thickness and the altitude of the underlying bedrock floor near the upstream end of the rockbound narrows that extends from Albany to Salem and at several places along the margin of the alluvial plain. Only three records afford any data for the central part of the plain; of these, only the record for well 637, at Junction City, is at all detailed. At this place, the alluvial deposits appear to be not less than 200 feet nor more than 233 feet thick; the thickness cannot be fixed precisely, owing to the uncertain driller's classification of fine-textured materials low in the alluvial deposit in distinction from the bedrock, which is largely shale. That the thickness found at Junction City is about the greatest in all the southern segment of the valley is suggested by abandoned well, 610 , which is reported to have been drilled 300 feet deep and to have encountered strong salt water, doubtless in bedrock. In the well at Junction City the bedrock floor underneath the alluvium appears to be not more than 120 feet nor less than about 90 feet above sea level-that is, at least 40 feet lower than at the rock-bound narrows to the north, near Albany. This feature suggests that the floor on which the alluvium rests, although shaped primarily by erosion, may have been deformed by movements of the earth's crust before alluviation began. If so, the thickness of the alluvium may vary in a most irregular fashion.

A conservative estimate of the average thickness of the alluvial deposits that underlie the southern half of the main valley plain, largely older alluvium, appears to be about 70 feet. If so, these unconsolidated materials would have a total volume of about $30,000,-$ 000 acre-feet and would have the capacity to store about $2,500,000$ acre-feet of ground water. (See p. 40.)

As has been stated, the materials that lie immediately beneath the main lowland plain northward from Salem to the vicinities of Newberg and Canby are distinct from those to the south, just described. With 
the exception of a few gravel fans or tongues along the margin of the plain, as along the lower reaches of the Yamhill and Molalla Rivers, only fine sediments (largely medium sand to silt) crop out or have been exposed by highway cuts. These are the sediments whose composition has been treated briefly by Felts. ${ }^{9}$ At most places they are rudely stratified in beds that average about a foot in thickness (pl. 6, B); at a few places they include streaks of coarse sand. Even though finegrained, they are appreciably pervious in part, for they contain at least one extensive body of semiperched water so high above the major streams that recharge can take place only by infiltration from rain falling on the highest parts of the main lowland plain. That semiperched body supplies many domestic wells but probably will not yield water in large quantities, such as for irrigation. At well 245, which is about 0.7 mile east of Gervais and which is believed to afford a typical section of the materials that underlie the northern half of the main lowland plain, these fine sediments extend to a depth of 68 feet beneath the land surface. From the base of the fine sediments to the bottom of the well, at a depth of 252 feet, beds of clay and silt make up slightly less than half the thickness, and beds of pervious sand and gravel the remainder. Most of the pervious beds occur in four zones whose tops are $68,108,146$, and 218 feet beneath the land surface. Apparently the well did not reach the underlying bedrock, so that the character of the deepest unconsolidated materials in the vicinity is not known.

TABLE 7.-Physical properties of water-bearing materials from well 245

[V. C. Fishel, analyst]

\begin{tabular}{|c|c|c|}
\hline (feet) & $\begin{array}{l}\text { Sample } 1 \\
230\end{array}$ & $\begin{array}{c}\text { Sample } 2 \\
241\end{array}$ \\
\hline t specific gravity, laboratory pac & 1. 67 & 1. 57 \\
\hline rosity (percent), laboratory packing & 38. 3 & 41.3 \\
\hline ient of permeability & 695 & 1,050 \\
\hline by weight): & & \\
\hline Gravel, more than 2.0 & 20. 2 & 1. 9 \\
\hline Fine gravel, 2.0-1.0 & 9. 7 & 8. 9 \\
\hline $1,1.0-0.5$ & 20. 1 & 24. 0 \\
\hline $0.5-0.25$ & 34.0 & 51.1 \\
\hline (1) & 11. 3 & 8. 5 \\
\hline sand, $0.125-0.062 \ldots$ & 1. 6 & 1. 5 \\
\hline Silt, $0.062-0.005$ & 1. 9 & 2. 7 \\
\hline Clay, less than 0.005 & 1. 3 & 1.5 \\
\hline
\end{tabular}

About the same succession of impervious and pervious beds is shown by the records of several other wells to the west and north, to the Willamette River and beyond. The pervious beds of sand and gravel supply several wells such as Nos. 245, 247, 248, 252, 262, and 263,

- Felts, W. M., Analysis of Willamette Valley fill [abstract]: Geol. Soc. America Proc. 1935, p. 346, 1936. 
which are heavily pumped; also wells 139,153 , and 159 , which are not pumped severely.

In the area from Salem northward to Newberg and Canby, as in the area to the south, the older alluvium and related deposits rest on a bedrock floor and, along the margin of the main valley plain, lap over the bedrock and feather out against it. Thus, the pervious zones in these deposits terminate against the bedrock at a distance from the outer margin of the plain, especially beneath tongues of the plain that extend far into the foothills. Obviously, therefore, the pervious zones are less extensive than the plain, and the deepest zone is the least extensive of all. However, even the deepest zone is believed to extend over half the area or more.

Over a large part of this area the older alluvium and related deposits appear to be more than 200 feet thick (see pl. 7); the greatest known thickness, 296 feet, is at well 221 , in sec. 8 , T. 5 S., R. 3 W., about 12 miles west of Woodburn. There the bedrock floor is about 140 feet below sea level. Obviously that floor has been depressed with respect to sea level; if it has been materially warped or faulted as well as depressed, most of that deformation must have taken place before the main valley plain was constructed, for the plain is remarkably flat (see p. 10).

If the average thickness of the older alluvium and related deposits in this northern segment of the main lowland plain is taken as 150 feet, an estimate which appears to be conservative, the total volume of the deposits would be about $50,000,000$ acre-feet and the water-storage capacity about $3,500,000$ acre-feet.

Still farther north, the Tualatin Valley is a topographic analog of the segment of the main lowland plain whose older unconsolidated deposits have just been described. That valley is floored by an unconsolidated deposit some 200 feet thick which in its upper part is quite similar to that of the main lowland plain. However, in its lower part the beds of pervious sand and gravel appear to be neither thick nor very extensive. To the east, along the Willamette River in the vicinity of Portland, there are correlative unconsolidated materials in part of similar texture and composition. Neither in the Tualatin Valley nor in the vicinity of Portland, however, has the deposit equivalent to the older alluvium been discriminated from younger and older unconsolidated deposits with which it is complexly associated.

TERRACE DEPOSIT AND RELATED ALLUVIAL MATERIALS

Along the outer margin of the main lowland plain, commonly from 30 to 100 feet above the plain and throughout all the length of the Willamette Valley, there are scattered though conspicuous remnants of a terrace formed by a coarse unassorted stream deposit (see pls. 1 and 2). At most places the deposit appears to be no more 
than a few tens of feet thick and commonly rests on a bedrock shelf that is higher than the main lowland plain, especially in the northern half of the valley. Consequently much of the deposit is so thoroughly drained that it contains little ground water. However, certain of the remnant deposits in the southern half of the valley-such as Sand Ridge, in secs. 24 and 25, T. 12 S., R. 3 W., and Fern Ridge, in the northeastern part of T. 17 S., R. 5 W.-extend to an unknown depth below the main lowland plain and so into the regional body of ground water.

Everywhere this terrace deposit is weathered rather severely, but especially in the southern half of the valley. There. at least the upper 20 feet of the finer matrix of the deposit is thoroughly decomposed to a compact clay and even the largest cobbles are so much disintegrated and decomposed that they can be cut through by a single sharp stroke with pick or shovel. (See pl. 8, A.) Material so thoroughly decomposed has little or no capacity for the infiltration or transmission of water. On the other hand, test borings made recently by the United States Engineer Department at the Fern Ridge dam site, in secs. 3 and 4 , T. 17 S., R. 5 W., indicate that there the terrace deposit is not much weathered at depth and so probably is sufficiently pervious to transmit water rather freely. This condition probably does not exist beneath most of the terrace remnants.

There are correlated tentatively with the terrace deposit two relatively extensive bodies of unconsolidated material: the gravel that veneers the Molalla pediment, which is in Tps. 4 and 5 S., Rs. 1 and 2 E., between the Molalla River on the northeast and Butte Creek on the southwest; and the Portland "delta," which lies in the angle between the Columbia and Willamette Rivers, extends eastward across R. $3 \mathrm{E}$., and is topographically correlative with an extensive plain to the north in the State of Washington.

The Molalla pediment rises gradually eastward to an altitude of about 475 feet above sea level or nearly 300 feet above the main lowland plain. The character of the materials that underlie the pediment is only imperfectly known, though a few scattered outcrops and the driller's record of well 280 (table 12) suggest that the pediment is veneered with poorly assorted gravel and sand a few tens of feet thick, beneath which compact "clay" with a few thin beds of "sand" extend to a depth of at least 300 feet. Well 188, which is on the main lowland plain just west of the Molalla pediment, also penerated "clay" and "sandy clay" between depths of 65 and 350 feet below the land surface and then entered shale. (See table 12.) Outcrops show that part of the thick mass of "clay" penetrated by these two wells and by others of the vicinity is volcanic tuff, both massive and stratified, and should be correlated with the partly consolidated Troutdale or Rhodo- 
dendron formations of Hodge, ${ }^{10}$ or both. Regardless of the precise correlation, these materials that underlie the pediment have small capacity to transmit water.

The Portland "delta" is triangular in plan and extends about 14 miles southward from the mouth of the Willamette River and about 19 miles eastward along the Columbia River, to a point $7 \frac{1}{2}$ miles beyond the eastern edge of the area represented on plate 1 . Its highest part is about 300 feet above sea level. Numerous pits and cuts show that to a depth of at least 100 feet it is composed of stratified and crossbedded sand and gravel, with a few thin partings of sandy silt. This characteristic bedding is well-shown on plate $8, B$. Obviously, the deposit was stream-laid on a gradually rising base level and was not built in a single stage by progression of a delta into deep water. The thickness of the deposit is suggested by the record of well 33 (table 12), which is reported to have penetrated little but gravel and sand to a depth of 360 feet and then alternate layers of clay, sand, and boulders to a depth of 405 feet. Certain layers of "conglomerate" between 220 and 330 feet in depth may indicate that the lower part of this zone should be ascribed to the bedrock, specifically to the Troutdale formation of Hodge. ${ }^{11}$ (See p. 23.) Below a depth of 405 feet and to a depth of 1,300 feet, the well passed largely through "marl" and shale, which very probably should be ascribed to the bedrock. Irrespective of these uncertainties in correlation, the unconsolidated "delta" deposit probably is not less than 300 feet thick in some places. A large proportion of the deposit is highly pervious and supplies numerous wells of large capacity.

\section{OCCURRENCE, SOURCE, AND DISPOSAL OF WATER IN THE UNCONSOLIDATED DEPOSITS}

FORM AND FLUCTUATIONS OF THE WATER TABLE

In the southern half of the Willamette Valley and through the rockbound narrows that extends to Salem, both the younger and the older alluvium are highly pervious almost throughout and are underlain by bedrock formations that are much less pervious. These two alluvial deposits act essentially as a single ground-water reservoir of very large capacity (see p. 32), quite analogous to a surface water reservoir. Thus, as water is added to the reservoir more of the alluvium is saturated and the ground-water level rises more or less proportionately; as water is discharged, either through natural drainage downstream or through withdrawals by pumping, some alluvium is unwatered and the groundwater level falls. Obviously, the height of the water table - the upper surface of that part of the alluvium which is saturated with water-is never quite steady but fluctuates constantly.

${ }^{10}$ Hodge, E. T., Age of Columbia River and lower canyon labstract]: Geol. Soc. America Bull., vol. 44, p. 157, 1933.

11 Idem. 


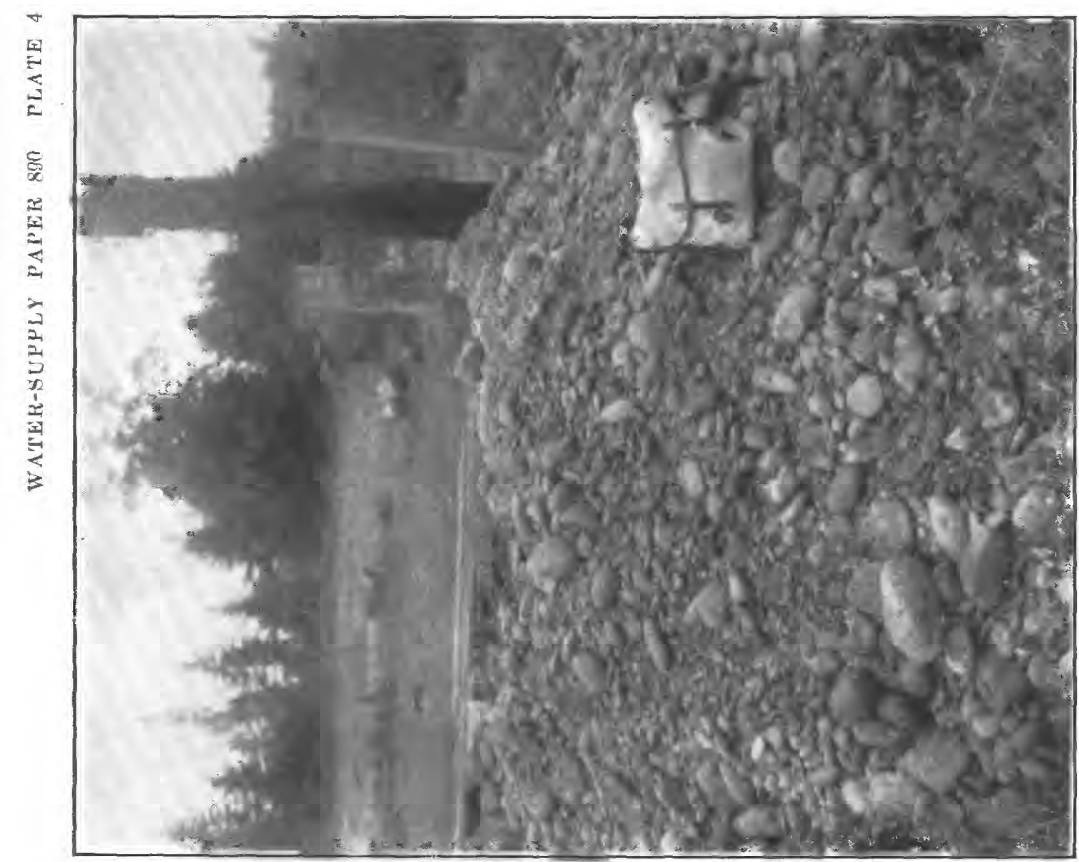

每
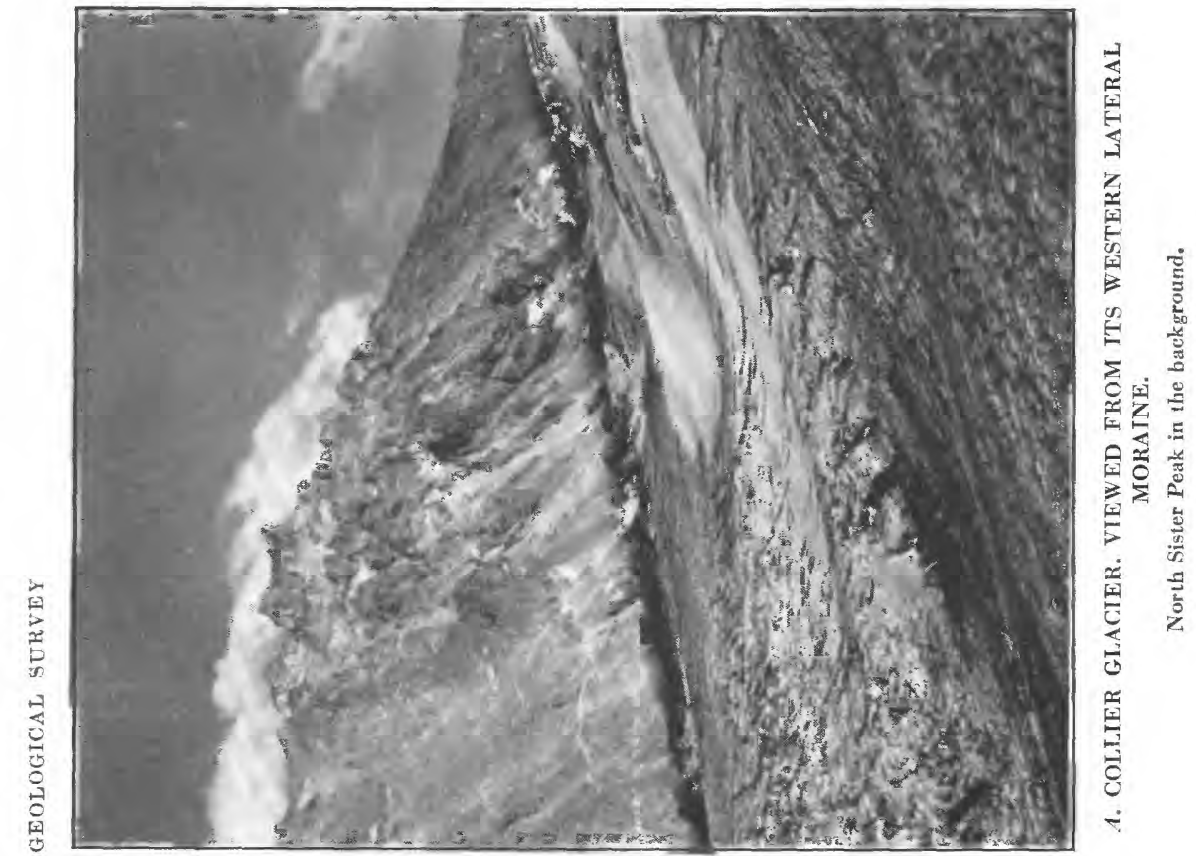


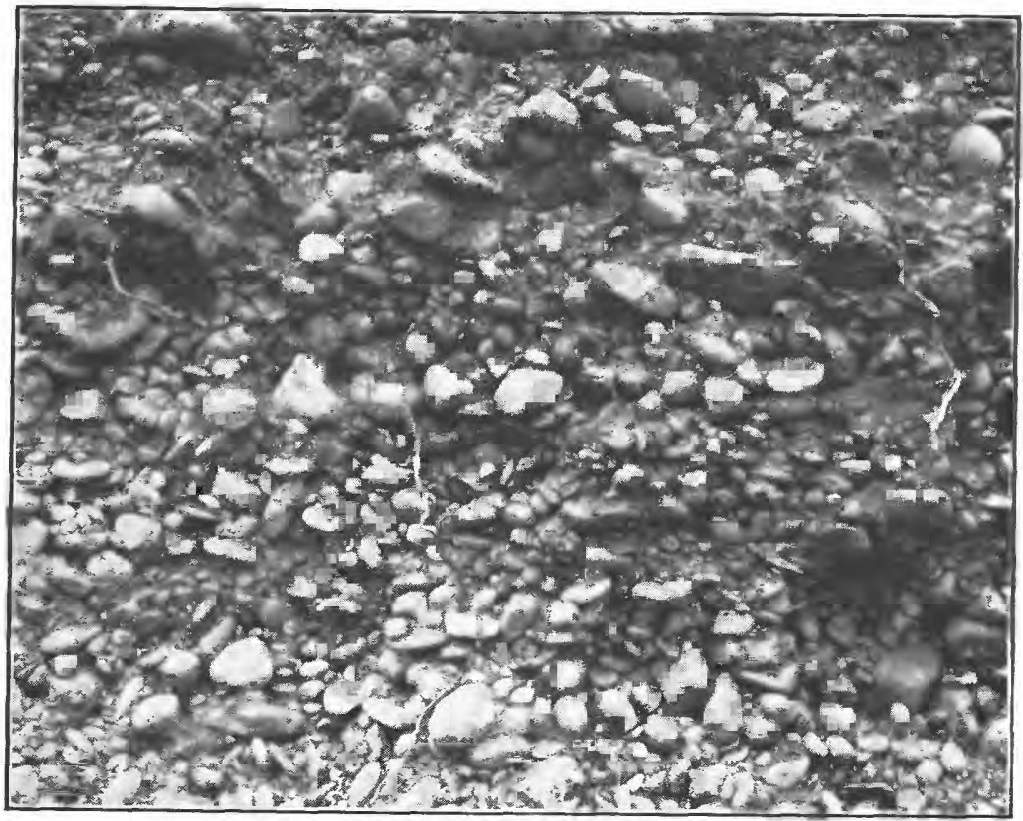

A. GRIVEL PIT IN THE SE $1 / 4$ SEC. 8 , T. 11 S., R. 4 W.

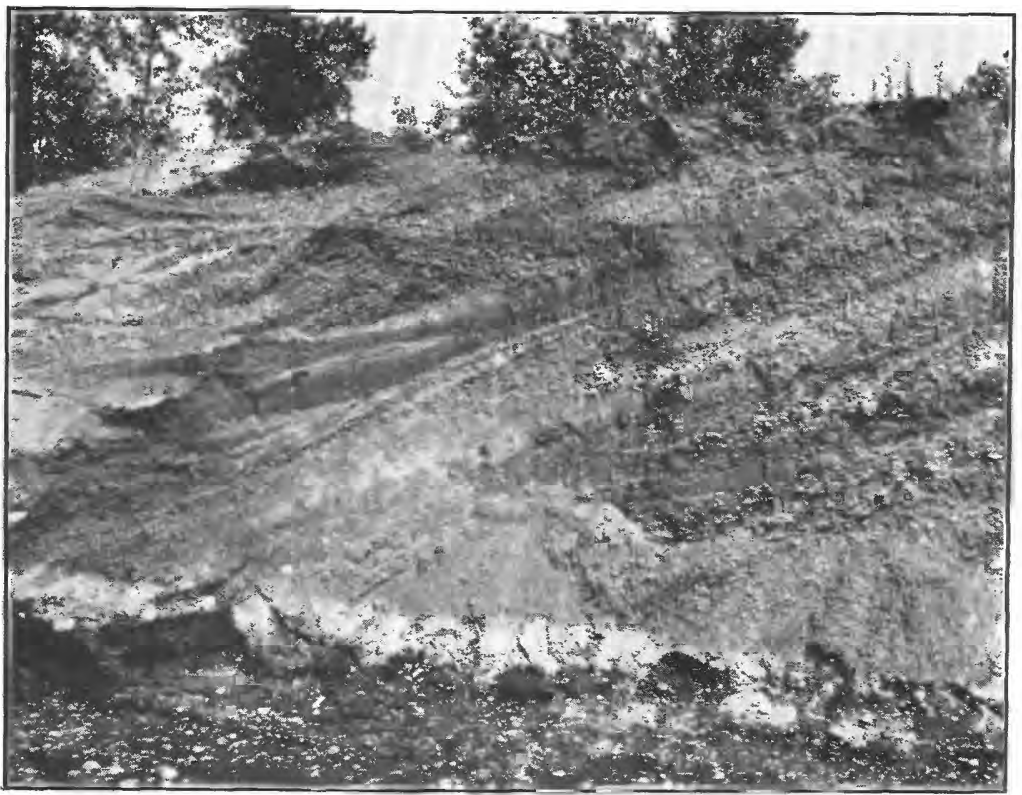

B. PIT OF EAST SIDE SAND \& GRAVEL CO. NEAR CORVALLIS.

In SW1/4 sec. 2, T. 12 S., R. 5 W.

YOUNGER ALLUVIUM OF THE WILLAMETTE RIVER. 


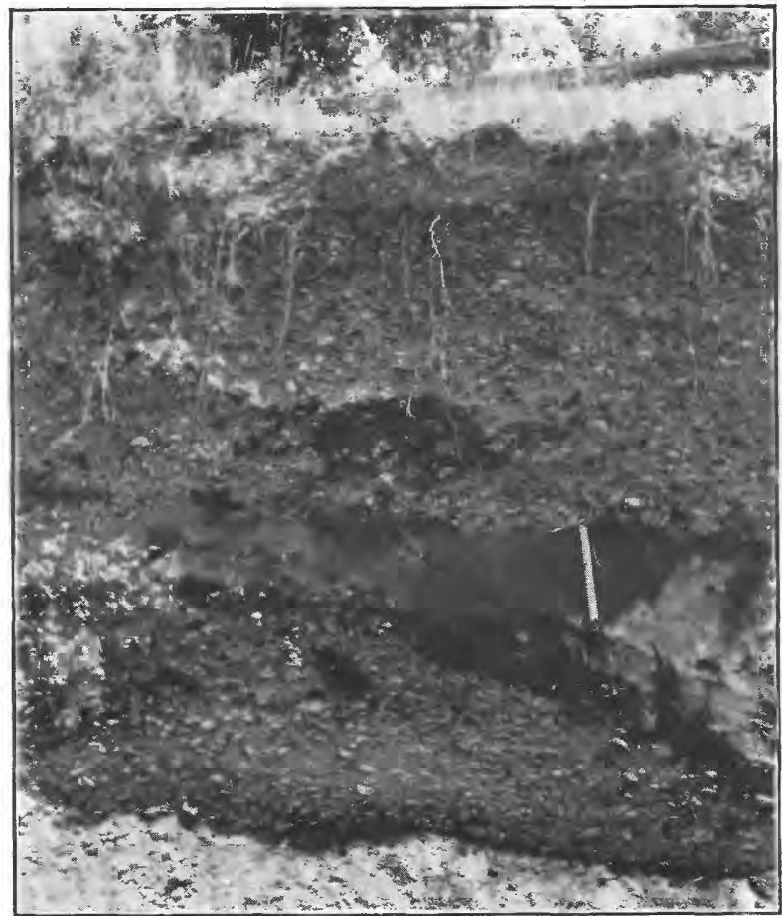

A. EXPOSLRE IN THE NW $1 / 4$ SEC. 1 , T. 16 S., R. 4 W.

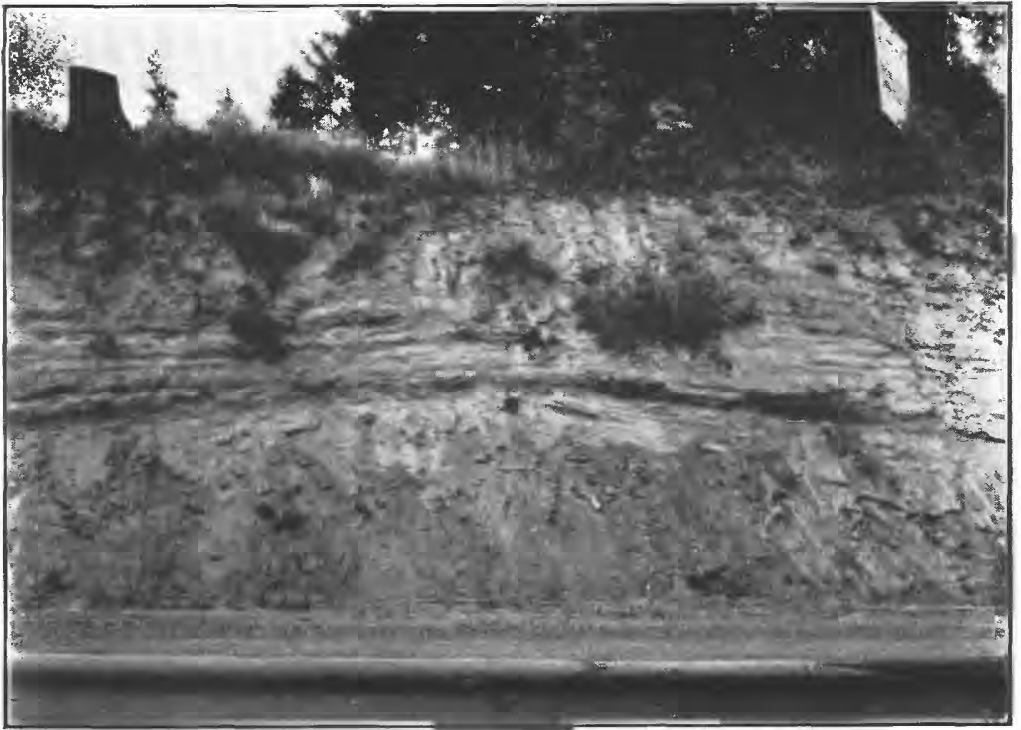

B. HIGHWAY CUT IN THE NW1/4 SEC. 7. T. 4 S., R. 1. E., SHOWING FAINT STRATIFICATION. 



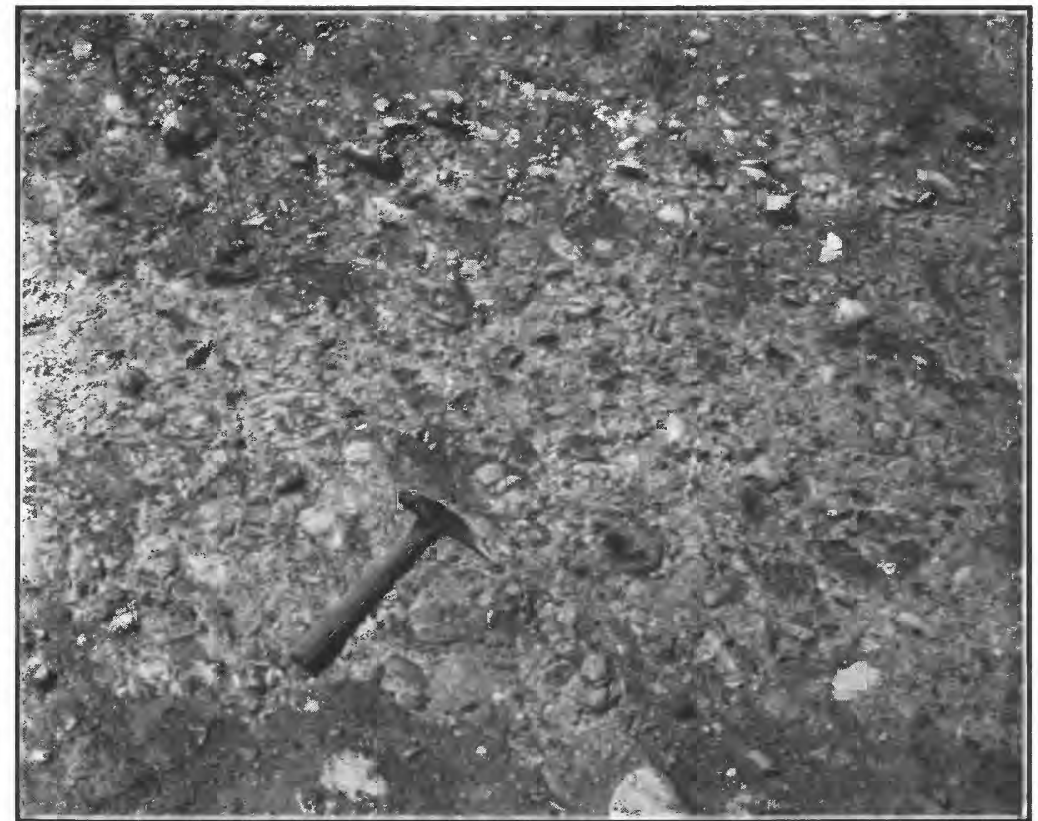

A. DECOMPOSED TERRACE DEPOSIT IN HIGHWAY CUT IN THE N1. SEC. 32 , T. 12 S., R. I W

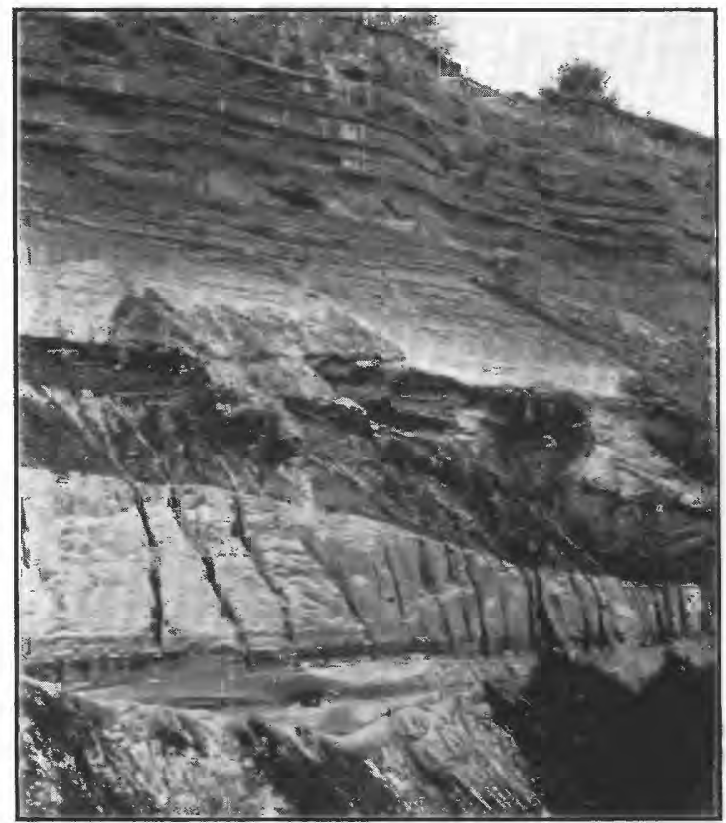

B. CROSS-BEDDED SAND AND GRAVEL EXPOSED IN CUT ALONG THE SPOKANE PORTLAND \& SEATTLE RAILWAY, 0.1 MILE NORTH OF LOMBARD STREET, PORTLAND. 

Contour lines on plates 1 and 2 show the height and general form of the water table as of November 1935, when the water table was about at its lowest stage for that water year and very little above its lowest known stage. That general form changes little as the water table rises and falls because, as indicated later, fluctuations of the ground-water level are of about the same order of magnitude and roughly synchronous over most of the valley. Beneath the broad interstream areas in the southern half of the valley, the water table is higher than the streams and slopes downward away from the foothills and northward toward and through the rock-bound narrows. Along either side of the Willamette River and the larger tributary streams, however, the water table ordinarily pitches downward to form a broad shallow trough whose bottom meets and prolongs the water surface of the stream. These troughs or valleys of the water table persist throughout most of the year but may be partly or largely obliterated during brief periods as the ground-water level rises sharply with freshets. (See p. 38.) In contrast to these troughs, the water table also rises into a number of minor ridges where relatively impervious material is not far beneath the land surface, as south of Corvallis in Tps. 12 and 13 S., R. 5 W., east of Corvallis in T. 12 S., R. 4 W., and west of Eugene in T. 17 S., R. 5 W.

In the northern half of the valley ground water occurs under conditions that are quite distinct from those just described. There, the unconsolidated deposits are not continuously pervious, and at least three separate bodies of ground water can be delimited. The first is in the tongue of younger alluvium that underlies the flood plain of the Willamette River, and the water is unconfined, as in the southern half of the valley. Distinct water-table contours for this body of unconfined water are shown on plate 1 . The second occurs beneath each of the extensive "prairies" or interstream segments of the main lowland plain. There, the shallowest ground water is also unconfined, but it is held high above river level by beds of low perviousness. These bodies of water are semiperched; contours on their respective water tables are shown on plate 1 . In general these water tables are highest beneath the central parts of the prairies and descend in all directions toward the stream trenches by which the prairies are bounded; in detail, their slopes are rather uneven. The third body, which is in the deep pervious beds that pass below the floors of the stream trenches, is confined under hydrostatic pressure. The height of the static level for this confined water is shown approximately by appropriate contour lines; over most of the area this static level is somewhat lower than the semiperched water table, but at some places it appears to be higher. It is considerably higher than the unconfined water table in the younger alluvium. 
Plate 9 shows typical water-level fluctuations in wells of the Willamette Valley; the fluctuations in numerous other wells are shown by the data on water levels, table 13 . Of the 12 wells represented on plate 9 , Nos. $421,463,553,568,636$, and 680 are in the southern half of the valley and their graphs show fluctuations of the regional water table in comparison with fluctuations of stage in the Willamette River at Corvallis and at Salem. Of the remaining 6 wells, which are in the northern half of the valley, well 171 and probably well 318 show fluctuations of semiperched water tables; well 245 and probably well 297 show fluctuations in pressure head of the confined water; and well 148 and possibly well 196 show fluctuations of the regional water table. Characteristic features of the fluctuations, their causes, and their relation to the withdrawal of ground water for irrigation and for other purposes are discussed later.

The water-level fluctuations thus far observed in the Willamette Valley have been due primarily to natural causes; withdrawals by pumping are relatively so small that they depress the ground-water level only locally and transiently. Throughout the valley the groundwater levels are characteristically lowest in late autumn, ordinarily in the last half of October or the first half of November. The replenishment of the soil-moisture deficiency begins with the onset of the rainy season (see p. 37), and within a relatively short time, ordinarily by mid-January for a water body that is unconfined and somewhat later for a body that is confined, the water level rises to its highest stage of the year. At many wells it then oscillates through a range of a few feet during the early spring, but after April it tends to decline steadily throughout the valley until the ensuing low stage is reached. Both the maximum and the minimum yearly ranges of ground-water level thus far determined-about 28 feet at well 426 and $31 / 2$ feet at well 409 in 1935-36-have been in the southern half of the valley where virtually all the ground water is unconfined. The smallest ranges occur commonly near the outer margin of the valley plain and in foothill alcoves; the largest, beneath the open plain at a distance from the larger streams. In the younger alluvium along the principal streams, the yearly range is moderate-for example, 6 miles north of Albany at well 421, the range was 5 feet in 1928-29 and 15\%/2 feet in 1935-36;2 miles east of Corvallis at well 463 the ranges for those water years were $121 / 2$ and $131 / 2$ feet, respectively; at Lebanon in well 568, $4 \frac{1}{2}$ and $8 \frac{1}{2}$ feet; and 6 miles north of Eugene at well $680,4 \frac{1}{2}$ and $6 \frac{1}{2}$ feet. In the northern half of the valley the yearly range of ground-water level appears to be moderate at most places. The greatest range is in the unconfined semiperched water. For example, 4 miles northwest of Woodburn at well 171 the water level had a range of 15 feet in 1928-29 and of 17 feet in 1935-36. For the pressure head of the deep confined water in the same area, 
the yearly range is only about half as great; 3 miles southwest of Woodburn at well 245 it was 9 feet in 1935-36; $4 \frac{132}{2}$ miles north of Salem at well 297 it was $81 / 2$ feet in 1929-30 and 10 feet in 1935-36.

With reference to the unconfined water in the older alluvium and related deposits, including the semiperched water in the northern half of the valley, the sharp rise of ground-water level in December and January must be due almost entirely to the infiltration of rain that falls in the immediate vicinity; for even when lowest the ground-water level beneath most of the valley is higher than the streams, and the ground-water level rises so nearly simultaneously over all the valley that replenishment can scarcely be caused by extensive lateral percolation. In those 2 months the average rainfall is about 12 inches, or roughly a third of the yearly total (p. 16); it is far more than the concurrent evaporation or consumptive use and is adequate for a very substantial addition to ground-water storage in most years. The repeated rise and fall of ground-water level that has been observed at many wells in late winter and early spring is here ascribed to intermittent infiltration from successive storms with alternate partial drainage of the newly saturated material; the steady decline after April is ascribed largely to continuous unwatering of the alluvial deposits as the ground water moves laterally toward and into the streams. The relation of this unwatering to stream flow is treated on page 39. Only a negligible part of the steady decline can be caused by withdrawals from wells, which so far have been very small in proportion to the volume of ground water involved in the yearly saturation and unwatering.

The fluctuations in the static level of the water that is confined beneath the northern part of the valley, unlike those of the unconfined water, just treated, do not indicate commensurate saturation and unwatering of pervious material at that place. Rather, all the deep pervious material is constantly saturated, and the fluctuations indicate merely changes in pressure transmitted from some external source. Of necessity, the water is derived from an outlying catchment and intake area. That intake area presumably lies chiefly to the south and east. The winter increase of pressure head in the deep water-bearing beds, which lags only slightly behind the rising level of the semiperched water above, might come about in either or both of two ways: First, the weight of the water taken into storage in the semiperched zone imposing a commensurate load on the deeper aquifers through elastic deformation of the strata; ${ }^{12}$ and second, a rising water level in the remote catchment area being transmitted laterally through the aquifer as a wave of pressure head. Conversely, the decline of the pressure head in summer and autumn would be caused by relief of

12 Meinzer, O. E., Compressibility and elasticity of artesian aquifers: Econ. Geology, vol. 23, pp. 272276,1928 . 
load as the semiperched zone became unwatered, by recession of the water level in the catchment area, or both.

In the younger alluvium along the Willamette River and its principal tributaries, the fluctuations of ground-water level appear to follow rather closely the fluctuations in stream stage. (See pl. 9, well 463, and river station at Corvallis.) However, the ground-water level probably is determined more by a backwater effect of rising and falling river stage than by extensive percolation from the river into the alluvium and the reverse, for ordinarily the water table slopes downward toward the river. Thus, the yearly saturation of the younger alluvium is doubtless due in considerable part to infiltration of rain; the alluvium is unwatered by drainage to the streams, as in the older alluvium.

With respect to the withdrawal of ground water for irrigation and other uses, the natural water-level fluctuations have several practical applications. If large withdrawals are to be made from single wells at a minimum operating cost, each pumping plant should be designed to accommodate the water-level range of the particular locality. Where the alluvium is thin and is underlain by impervious rock, as along certain reaches of the flood plains (p. 44), it may be largely unwatered by midsummer, so that large withdrawals cannot be sustained. For example, the irrigation well 463 on the east farm of the Oregon Agricultural Experiment Station near Corvallis encountered impermeable shale 42 feet beneath the land surface. There, the highest static water level so far recorded was 32 feet above the shale, the lowest 18 feet. After deducting the draw-down by the pump, the saturated zone that yields water to the well is only half as thick at the low static level as at the high; the yield of water is correspondingly smaller. At many other places, as in the vicinity of Albany, the alluvium is still thinner and the capacity of wells diminishes even more as the water level recedes during the summer. Under these conditions, even greater diminution of yield would be expected if many wells were pumped heavily, with consequent interference and comulative depression of the ground-water level; in 1938 heavily pumped wells were few and widely scattered.

Should the ground-water resources in the southern half of the valley be developed extensively in the future for supplemental irrigation, the natural water-level fluctuations that have been described would doubtless be modified appreciably. In areas where large withdrawals are concentrated, the water-level decline in early summer probably would be quickened and the ultimate level in autumn would be lower. Thus, the deficiency in ground-water storage at the onset of the rainy season would be greater, and full replenishment by infiltration would be somewhat delayed. These effects of extensive withdrawals are unavoidable; in fact, they are the means by which the underground res- 
ervoir can be made to store additional water during the rainy season for later use. In this connection, the infiltration capacity of the alluvial deposits appears to be so large and the winter rainfall so much in excess of natural use, that withdrawals much greater than at present probably would be fully replaced between the seasons of pumping. (See also p. 40.)

In the northern half of the valley, heavy withdrawals from the confined water bodies in deep pervious beds would modify the natural water-level fluctuations in another manner. There, withdrawals can be increased only in proportion to the extent that the natural movement of water from the remote intake area can be accelerated. Accelerated movement is possible only if the hydraulic gradient is steepened from the area of pumping to the area of intake; in other words, the pressure head in the pumping area must fall. Thus, sustained large withdrawals probably would depress the water level somewhat more than in the southern half of the valley, and full recovery of head probably would not come about as quickly.

\section{RELA'TION OF GROUND WATER TO RAINFALL AND S'TREAMS}

As has been suggested by the foregoing treatment of water-level fluctuations, the bodies of ground water in the unconsolidated deposits of the Willamette Valley are integral parts of the drainage system. These bodies receive water chiefly by infiltration of rain from the lowland plains directly above or, in the case of the confined water in the northern half of the valley, from the remote intake area. From these bodies water is discharged naturally into the streams and so helps to sustain the stream flow, especially during the summer and autumn.

The general direction of ground-water movement, from points of intake to points of discharge, may be deduced from the form of the water table or other pressure-indicating surfaces, for movement must be in the direction of the steepest downward hydraulic gradient. In other words, the direction of movement must be at right angles to the contour lines on the water table or analogous surface and from higher contours toward the lower. Thus, the contour lines shown on plates 1 and 2 indicate that the unconfined water in the southern half of the valley moves inward away from the foothills and in a general northward direction until it seeps into the streams and so passes out of the basin. In the northern half of the valley, between Salem and Canby, the semiperched water tends to move radially outward from the center of the "prairie" into several perennial creeks, also to numerous small springs and seeps along the slopes or bluffs by which the prairie is surrounded. Probably some of the semiperched water is discharged through a ground-water cascade along the outer margin of the prairie. In the same area, the confined water in the deeper pervious zone appears to move generally northwestward 
away from the foothill area that lies south of Salem and Silverton. Where this water is returned to the land surface is not definitely known. Leakage upward into the younger alluvium along the Willamette River and the lower Yamhill River and thence into the streams is inferred tentatively because the pressure head appears to diminish sharply beneath the flood plains. A possible effect of withdrawals from wells is shown by the southward deflection of the lines of equal pressure head in the vicinity of Woodburn.

\section{DEPENDABILITY OF THE GROUND-WATER SUPPLY}

In an area such as the Willamette Valley the pervious materials beneath the land surface have long since been saturated to capacity and, over a term of years, as much ground water must be discharged as is received by infiltration or other means of recharge. Thus, if ground water is to be withdrawn for use year after year without ultimately depleting the initial storage, the volume withdrawn must be salvaged directly or indirectly from water that otherwise would be discharged naturally.

With respect to the unconfined water in the younger and older alluvium in the southern half of the Willamette Valley, withdrawals must be salvaged from the water that is stored intermittently in the zone of yearly saturation and unwatering. The volume of this water is large. Thus, among 63 representative water-table wells in that part of the valley, the thickness of the zone that was saturated in the water year of 1935-36 ranged from 3.8 to 28.2 feet and averaged 12.0 feet. From the high stage of that winter until the end of the next September, the zone of unwatering ranged from 3.0 to 25.3 feet in thickness and averaged 10.7 feet. If these averages from the 63 wells are applied to all the area, the volume of material saturated was about 5,300,000 acre-feet; of that subsequently unwatered, 4,700,000 acre-feet. If then the mean specific yield of the alluvial depositsthe ratio between the volume of the deposits and the volume of water that the deposits, after being saturated, will yield by gravity drainage- is taken as 10 percent, ${ }^{13}$ a percentage that is probably conservative, ground-water storage in the area increased about 530,000 acrefeet during the winter of $1935-36$ and diminished about 470,000 acrefeet during the following spring and summer. During the 4-month period November to February the rainfall was about 1 percent less than the 40-year average. Therefore the opportunity for recharge by infiltration certainly was not excessive. In contrast, the winter rainfall in 1928-29 was about 20 percent less than the 40 -year average; in $1929-30$, about 10 percent less. In these 2 years, the range in ground-water storage appears to have been little, if any, less than

13 Piper, A. M., Gale, H. S., Thomas, H. E., and Robinson, T. W., Geology and ground-water hydrology of the Mokelumne area, Calif.: U. S. Geol. Survey Water-Supply Paper 780, pp. 101-118, 1939. 
in 1935-36. Thus, ground-water recharge and discharge in 1935-36, amounting to about 500,000 acre-feet, may be assumed to have been roughly equal to the long-term average. In comparison, the total withdrawal of ground water for use in 1928-36-by numerous wells for rural household use, for a few community water systems serving a few thousand people, and for supplemental irrigation on a few thousand acres of land-is very small.

It is impracticable to salvage and use all the yearly ground-water recharge in the southern half of the valley. To do so would require that the ground-water level be sufficiently depressed by pumping to keep it below the level of the streams; thus certain thin parts of the alluvial deposits would be completely and permanently unwatered. Nevetheless, it appears wholly practicable to withdraw water at several times the rate of the 1928-36 withdrawals, and so to increase considerably the irrigated acreage and other water services. The scope of the reconnaissance on which this report is based does not permit a close estimate of the greatest practicable yearly withdrawalthe so-called safe yield, or the volume of water that could be withdrawn year after year without ultimately so depleting the supply that further withdrawals at that rate would become too costly. A reliable determination of the safe yield will be desirable if and when groundwater utilization increases several fold, but that determination would require a very intensive investigation.

No definite conclusions can be drawn at this time with respect to the safe yield of the deep water-bearing zone in the northern half of the valley, the only zone in the older unconsolidated deposit in that area that appears adequate to supply wells of large capacity. Thus far, the withdrawals for all uses have been no larger than in the southern half of the valley, if as large. Further, no cumulative decrease in the pressure head of the water is shown by the water-level data now available. Accordingly, it appears that the present withdrawals can be increased several times without causing excessive interference between wells and possibly many times before the safe yield is exceeded.

\section{SUBDIVISIONS OF THE CENTRAL LOWLAND WITH RESPECT TO YIELD OF GROUND WATER}

The central lowland of the Willamette River Basin may be divided tentatively into 20 subareas that are fairly distinct with respect to conditions of ground-water occurrence and to water-yielding capacity of the unconsolidated deposits. The position and extent of these subareas are shown on plate 10 . The locations of typical wells in the several subareas are shown on plates 1 and 2 ; descriptions and driller's records of the wells are given in tables 11 and 12, respectively; and data on water-level fluctuations are given in table 13. The subareas 
are listed below, and the list is followed by a summary of their groundwater features.

\section{TENTATIVE SUBAREAS OF THE CENTRAL LOWLAND}

Flood plains of the principal streams, including some related segments of the main valley plain:

1. Springfield "delta," comprising the younger alluvial plains of the McKenzie and Willamette Rivers above their junction and an intervening segment of the older alluvial plain.

2. Upstream reach of the Willamette River flood plain, from the Springfield "delta" downstream nearly to Albany.

3. Middle reach of the Willamette River flood plain, through the rockbound narrows that begins about 2 miles upstream from Albany and extends to Salem, an air-line distance of 21 miles.

4. Lebanon alluvial fan, comprising the younger alluvial plains of the North Santiam and South Santiam Rivers about their junction and a. contiguous part of the older alluvial plain that extends westward to Albany and southward to Oak Creek.

5. Downstream reach of the Willamette River flood plain, from Salem to the mouth of the Pudding River.

6. Canby fan, comprising the younger alluvial plains of the Pudding River below Aurora and of the Molalla River below Molalla and the older alluvial plain between the Molalla River and the terrace about 2 miles east of Canby.

7. Younger alluvial plain of the Willamette River below Portland and of the adjacent part of the Columbia River.

Main valley plain:

8. Eugene-Junction City segment extending westward about 3 miles from the Willamette River flood plain.

9. Coburg-Harrisburg segment between the Willamette River flood plain on the west and Lake and Muddy Creeks on the east.

10. Elmira segment, including all the older plain between subarea 8 on the east and the terrace remnants or foothills on the west, in Tps. 15-17 S.

11. Brownsville-Albany segment, all the older plain between subareas 2 and 9 on the west and subarea 4 on the northeast; outliers of the Tertiary bedrock are excluded.

12. Corvallis segment, bounded on the east by subarea 2 and on the west by foothills or terrace remnants.

13. Minor segments in the vicinity of Albany, remnants of the older plain on both sides of the Willamette River in Tps. 9 and $10 \mathrm{~S}$.

14. Monmouth-Independence segment, lying west of the Willamette River flood plain in Tps. 7-9 S.

15. Stayton-Mill Creek basin, comprising the alluvial fan north of the North Santiam River in Tps. 8 and 9 S., Rs. 1 and 2 W., and the older plain from Salem eastward to the Little Pudding River and northward to the Lake Labish channel.

16. French Prairie, bounded on the west and north by the Willamette River flood plain, on the east and south by Pudding River and the Lake Labish channel.

17. Dayton Prairie and adjacent areas, all segments of the younger and older plains west or north of the Willamette River flood plain and adjacent. to it, from Salem downstream to Canby. 
18. Tualatin Valley, comprising the younger and older plains of the Tualatin River Basin but excluding numerous remnants of a higher terrace and two outliers of bedrock, one extensive.

Areas largely higher than the main valley plain:

19. Molalla pediment and related parts of the central lowland, bounded on the west by subareas 15,16 , and 6 and on the southeast by bedrock foothills; excludes the bedrock outlier Mount Angel but includes terrace remnants east of the Molalla River, one east of Canby and another around Mulino.

20. Portland "delta," including all alluvial plains in the vicinity of Portland between Kellogg Creek and the Clackamas River to the south and the Columbia River flood plain to the north.

Spring field delta (subarea 1).--Along the lower reaches of the Willamette and McKenzie Rivers, over the so-called Springfield or McKenzie River delta, both the younger and the older alluvial deposits contain many interconnected lentils and tongues of highly pervious gravel and coarse sand. One or more of these pervious members has been encountered in every well known to have been constructed in the area. Wells 675 to 684,696 , and 712 are typical of numerous irrigation wells, which together serve at least 1,500 acres of land. Most of the existing wells in this area are dug from $6^{\frac{1}{2}}$ to 25 feet deep and have simple open-bottom casings of wood or concrete tile. Their penetration, the distance they extend below the water table into saturated material, is not more than about 5 feet when the static level of the ground water is lowest nor more than about 12 feet when the water level is highest. The few drilled wells have somewhat greater penetration, but none are more than 12 inches in diameter and none have been thoroughly developed to secure the largest possible yield. Even these simple and relatively shallow wells yield from 250 to 400 gallons a minute, and several have specific capacities of 100 gallons a minute or more for each foot of draw-down. Doubtless, much larger yields could be obtained without excessive draw-down by constructing wells that are adequately screened, ample in diameter, thoroughly developed, and of greater penetration.

For wells of similar construction in the highly pervious alluvium, the specific capacity should increase with greater penetration into water-bearing beds; obviously, penetration is limited by the thickness of the alluvial deposit, which is imperfectly known (see pl. 7). The few data available suggest that the greatest thickness may' exceed 100 feet, which would afford wells with a penetration and potential capacity many times greater than any now existing. On the other hand, the bedrock floor that underlies the "delta" presumably has moderate relief, somewhat similar to that of the adjoining foothills. Accordingly, the thickness and total water-yielding capacity of the alluvium may vary greatly within small areas. 
Upstream from Springfield the alluvial deposits along the McKenzie River and the Middle Fork are inferred commonly to have a large capacity to yield water. On the other hand, the alluvial tongue that extends along the Coast Fork to the vicinity of Cottage Grove appears to be much less pervious on the average and so does not promise wells of large capacity.

Upstream and downstream reaches of the Willamette River flood plain (subareas 2 and 5).-Downstream from the Springfield "delta," except through the narrows between Albany and Salem, the tongue of younger alluvium along the Willamette Riverhas fairly uniform capacity to transmit water. Wells $653,654,538,539,550,463,464$, and 234 , in order downstream, are those most heavily pumped. All are of the simplest construction. Excluding wells 463, 464, and 234, these wells penetrate the water-bearing zone from 4 to 6 feet only, yet they yield as nuch as 525 gallons a minute, or 1.2 second-feet. Well 234 comprises three driven wells 2 inches. in diameter with a penetration of about 15 feet, yet it yields rather freely. Wells 463 and 464 are deepest; they also have the greatest penetration-18 feet below the lowest static water level-and yield 750 gallons a minute, or 1.7 second-feet. Nearly everywhere along these two reaches of the flood plain the younger alluvium is underlain by older alluvium, which also is water-bearing. (See descriptions of subareas $8,9,12$, and 16.) Thus, wells of thorough construction penetrating the full thickness of the two alluvial deposits promise yields considerably greater than those from existing wells.

Over these reaches of the flood plain, the yearly range in groundwater level is about 7 to $13 \frac{11}{2}$ feet. Thus pumping plants on wells must accommodate a moderate variation in pumping lift.

Middle reach of the Willamette River flood plain (subarea 3).Through the rock-bound narrows that extends from Albany to Salem, the younger alluvium is quite as pervious as in the reaches next upstream and downstream, just described. For example, wells 362 and 368 each yield about 1,000 gallons a minute, or 2.2 sccond-feet. Well 368 is 12 inches in diameter and penetrates water-bearing sand and gravel 19 feet in thickness; with respect to proportionate diameter and penetration, its yield is comparable to that of well 463 , to the south. Well 373 likewise has a large capacity.

This middle reach of the flood plain is treated as a distinct groundwater area only because the tongue of younger alluvium probably is not continuously underlain by the older alluvium and because at some places the younger tongue is doubtless thin and rests directly on slightly pervious bedrock, which is largely shale. Thus there is no assurance that a prospective well will encounter pervious beds of sufficient thickness to yield water copiously, especially when the 
ground-water level is lowest. The location and extent of the places of scant yield cannot be determined from the data now available.

Lebanon alluvial fan (subarea 4).-Along the lower reaches of the North Santiam and South Santiam Rivers the alluvial deposits are similar in thickness and water-yielding capacity to the deposits of the Springfield "delta," previously described. They are similar also in the yearly range of ground-water level, which is from 4 to 9 feet. Wells 562 and 566 to 568 are those pumped most heavily.

Northward from Lebanon, along the western edge of the younger alluvial tongue, the older alluvium that underlies the main valley plain also seems to be high in water-yielding capacity and in that respect can scarcely be discriminated. How far westward the older alluvium is so pervious remains unknown; somewhat arbitrarily, however, the limit is placed along Oak and First Periwinkle Creeks. Even though highly pervious, the older alluvium becomes thinner in the most westerly part of the subarea, in particular near the bedrock outlier that borders the subarea at Albany. There, extensive test drilling in wells 503 to 511 found the older alluvium from 28 to 47 feet thick and its water-bearing zone from 22 to 28 feet thick. From the results of this test drilling, the locality was judged unsuitable for a public-supply well field, because a source so shallow would not assure freedom from contamination. However, for irrigation and other uses not requiring water free from harmful bacteria, even that locality probably would sustain a moderate withdrawal of ground water.

Canby fan (subarea 6).---The younger and older alluvial deposits that have been grouped as the Canby fan doubtless contain pervious members of considerable water-yielding capacity, though little is known as to the extent and thickness of those members. The driller's records of wells 102 and 103, at Canby (table 12), shows the character of the older deposit. At that place it appears to be 281 feet thick and to a depth of 87 feet is composed largely of coarse gravel, but below that depth it is largely "clay" with a few water-bearing beds a foot or two in thickness. In water-yielding capacity the younger alluvial tongue is inferred to be similar to the corresponding tongue along the lower Willamette River. At some places the tongue is doubtless too thin to afford wells of large penetration.

Younger alluvial plain of the Columbia River (subarea 7).-Within the area that is shown on plate 1, the younger alluvial deposits of the Columbia River are composed largely of silt and rather fine sand. Shallow wells in this material encounter few beds that yield water freely, and in some wells the yield is barely adequate for individual household supplies. To satisfy the larger need for dairies and irrigation, drilled wells have been sunk to beds of coarse sand and gravel between 85 and 235 feet beneath the flood plain. These deeper 
pervious beds may belong to deposits older than the younger alluvium. Wells 1 to 3,5 to $14,16,17,34$ and 35 are typical; of these, well 34 has the largest yield-reported to be 750 gallons a minute, or 1.7 second-feet-and is pumped about 8 hours a day throughout the summer. The deeper pervious beds lap out against bedrock so that some wells, such as 4 and 15, fail to develop an adequate supply of water within the alluvium.

Eugene-Junction City and Coburg-Harrisburg segments of the main valley plain (subareas 8 and 9). - In the southernmost part of the valley, for a width of 2 to 3 miles on either side of the Willamette River flood plain in Tps. 14 to 17 S., much of the older alluvium has a. fairly high water-yielding capacity. The several fire-protection wells at Junction City and the deep well recently drilled there for public supply (Nos. 636 and 637, respectively) are among the most productive. Well 637 passed entirely through the older alluvium and entered bedrock of sandy shale between 200 and 233 feet beneath the land surface. It draws water from sand and fine gravel through a 10-foot section of perforated casing from $138^{\circ}$ to 148 feet in depth; in this section of casing there are 300 perforations each $1 / 4$ inch wide and 1/4 inches long. Although not gravel-walled artificially, the well has a reported specific capacity of about 10 gallons a minute for each foot of draw-down. However, as is shown by the driller's record (table 12), it draws from only a small fraction of the total thickness of water-bearing beds and, owing to requirements of sanitation, not. at all from the most pervious beds, which are at shallow depth. For irrigation, much larger yields probably could be obtained from wells of adequate construction drawing from all pervious beds. For permanence, heavily pumped wells tapping the finer pervious beds at depth should be adequately screened, possibly also artificially gravelwalled, and thoroughly developed to arrest the movement of sand.

Not all parts of the older plain in these two subareas of the valley are underlain by pervious beds with a water-yielding capacity equal to that at Junction City. For example, wells 655 and 656 are somewhat inadequate in yield for efficient irrigation, though they are not known to have reached the bottom of the older alluvium and so to have exhausted the possibility of finding beds of high-water-yielding capacity. In general the ground-water productiveness of the two subareas probably diminishes somewhat gradually away from the Willamette River. The precise western and eastern limits of the highly productive area cannot be traced with the data now at hand; they must be felt out by further well construction and may deviate considerably from the tentative limits that have been arbitrarily defined and shown on plate 10 .

Elmira segment of the main valley plain (subarea 10). - In the extreme southwestern part of the Willamette Valley, along the Long: 
Tom River in Tps. 15 to 17 S., R. 15 W., and eastward to a point about 2 miles beyond Fern Ridge, the upper part of the older alluvium is largely unassorted and contains much fine sand and silt. Accordingly, shallow wells do not yield copiously. No records of deep wells are available to indicate the water-yielding capacity of the alluvium at depth, though the capacity is inferred to be small and very probably inadequate to sustain large irrigation wells.

Brownsville-Albany segment of the main valley plain (subarea 11).Although bounded on all sides except. the eastern by subareas of large water-yielding capacity, the Brownsville-Albany segment of the older alluvial plain comprises areas that range widely in water-yielding capacity. However, the data now available do not permit close discrimination of those areas. In the central part of the segment, southward from the vicinity of Tangent to Halsey and thence eastward toward Brownsville, there are many dwellings and farms amply supplied with water from the older alluvium by driven or drilled wells less than 4 inches in diameter and from 20 to 70 feet deep. Wells 551 to 553,584 to $586,590,605,606$, and 620 are typical. The older alluvium promises relatively large yields to adequately constructed wells of greater diameter and depth, though the yield would probably not be uniform over all parts of the segment. The yearly range of ground-water level is not excessive, about 15 feet at the most. There are few data to show the thickness of the alluvium, but the thickness diminishes greatly along the Calapooya River near Brownsville.

In contrast with the area just described, in at least two extensive parts of the segment much of the older alluvium is rather tight and numerous wells are not wholly adequate for domestic and farm supplies. These parts are the triangle that extends eastward from Muddy and Dry Muddy Creeks to the foothills in the southern half of T. $14 \mathrm{~S}$. and across all of T. $15 \mathrm{~S}$. and an ill-defined belt $1 \frac{112}{2}$ to 5 miles wide along the east edge of the segment in Tps. 11 and $12 \mathrm{~S}$. and the northern two-thirds of T. $13 \mathrm{~S}$.

Along the west edge of the segment in Tps. 11-14 S., from Albany southward about to Lake Creek, the older alluvium is inferred to be underlain at relatively shallow depth by a ridge of bedrock shale whose highest parts rise slightly above the alluvial plain in scattered outliers. (See pl. 1.) Although the older alluvium there appears to be moderately or highly pervious, it may prove too thin at some places to sustain large withdrawals of water, as at wells 466,467 , and 473 to 482 , which are near the most northerly bedrock outlier, near Albany. Analogous conditions doubtless exist not only near the other outliers but possibly some distance from them as well. In this western part of the segment the yearly range in water level is large $-21 \frac{1}{2}$ feet at well 486 in $1935-36$. 
Corvallis segment of the main valley plain (subarea 12).-Beneath that part of the main valley plain which lies west of the Willamette River near Corvallis, in Tps. 11-14 S., much of the older alluvium is judged to be moderately pervious, though at some places it is probably too thin for sustained large withdrawals. Most of the wells are less than 4 inches in diameter; they were driven or drilled to depths of 25 to 45 feet and are not heavily pumped. Wells 460 and 573 are the two of greatest known capacity in the area; they suggest the feasibility of constructing wells for moderately large yield. In this area the yearly range in water level is about as large as in any part of the Willamette Valley. In 1935-36 the range was 27 feet at well 459, 21 feet at well 540, and 17 feet at wells 574 and 575 . In selecting pumps for wells in this area, therefore, special consideration should be given to this hydrologic feature.

Monmouth-Independence segment and minor segments of the main valley plain near Albany (subareas 13 and 14).-Beneath the discontinuous segments of the main valley plain that lie on both sides of the Williamette River in the narrows between Albany and Salem, the older alluvium is somewhat diverse in texture and in capacity to yield water. There much of the alluvium is quite unsorted and tight so that it yields water slowly, as at well 376 near Monmouth and well 441 near Albany. At Independence, on the other hand, well 372 encountered gravel that appears to have yielded water somewhat freely. Commonly in these areas the older alluvium is moderately thin and at no place nearly so pervious as the younger alluvium of the adjacent flood plain. Owing to the diverse conditions just described, which are known only superficially from the data now available, it seems unlikely that these scattered areas will sustain large withdrawals of water from many wells.

Stayton-Mill Creek basin (subarea 15). - The older alluvial fan that extends westward from Stayton, in Tps. 8 and 9 S., Rs. 1 and 2 W., is composed largely of coarse gravel, which is saturated with water to within 15 feet of the land surface, or less. Some of the gravel presumably is well assorted and highly pervious. The thickness of the alluvial deposit is estimated to be about 100 feet. The existing wells are of the simplest construction and ordinarily not more than 25 feet deep; they do not afford a measure of the ultimate water-yielding capacity of the deposit. In general, however, conditions appear favorable for wells of moderate or large yield, if the wells are adequate in diameter, penetration, and type of construction.

To the north, in the vicinity of Salem, ground-water conditions along Mill Creek and eastward to the Little Pudding River are rather well known from the records of existing wells. Along the western edge of the area, in Salem, the alluvium is relatively thin and yields no more than 100 gallons of water a minute to wells such as Nos. 
$331 \frac{11}{12}, 331 \frac{31}{4}, 340$, and 341 . A short distance to the east, wells 333 to 335 pass through water-bearing beds in the older alluvium but are tightly cased, probably into the underlying bedrock. Still farther east, several wells draw rather heavily from the older alluvium. Wells $297 \frac{1}{2}, 338,338 \frac{1}{2}, 360$, and 384 are representative; these have reported yields of 150 to 500 gallons a minute and reported specific capacities from 1.4 to 16.5 gallons a minute for each foot of draw-down. Driller's records (table 12) indicate that this wide range in specific capacity is due to beds of "cemented gravel," "hardpan," or "packed sand" of considerable aggregate thickness in certain wells. These beds, probably unassorted mixtures of pebbles and sand in an earthy or clayey matrix, would naturally have small capacity to transmit water. Probably, however, no one bed of this sort underlies all the area, and the water-bearing beds are interconnected to some extent Under such conditions wells of similar size and construction may differ considerably in specific capacity, but the area as a whole probably will sustain a considerable aggregate withdrawal of water.

To the east, this northern part of the Stayton-Mill Creek basin abuts on an older terrane whose capacity to yield ground water seems in general to be very much less (see pp. 53-54). The eastward extent of the area of greater potential yield is not indicated by features exposed on the land surface and is not defined by the records of wells now available. The limit that has been set somewhat arbitrarily, the Little Pudding River, may ultimately prove to be considerably in error.

French Prairie (subarea 16).-With respect to the occurrence and potential yield of ground water, French Prairie, the most extensive segment of the main valley plain north of Salem, is quite distinct from any other subarea of the lowland plains thus far treated. In general, two bodies of ground-water exist. The first is semiperched water that supplies numerous shallow farm wells from the fine-grained upper part of the unconsolidated deposit (p.31). It is yielded much too slowly to sustain any large continual draft, such as for irrigation. The second is water confined under head in beds of clean sand and gravel in the lower part of the deposit. It is now withdrawn in moderate volume for public and private supplies and for irrigation and would doubtless sustain a much greater withdrawal.

In the central part of the area, at irrigation well 245 near Gervais, the confined water has been found in three previous zones that are thick and presumably extensive, also in several thin zones that may not be extensive. The driller's record (p. 138) shows the character and thickness of these zones; the physical properties of samples from the lowest zone are shown on page 31 . Only one well in the area, No. 168 , is deeper, and for it no complete driller's record is available. Briefly, the three principal pervious zones penetrated by well 245 are 
at depths of 68 feet, 117 feet, and 218 feet below the land surface. The upper zone consists of a 13-foot bed of sand and fine gravel; the middle zone is a 45 -foot bed of gravel and sand with a 3 -foot interbed of pebbly clay; and the lower zone consists of $25 \frac{1}{2}$ feet of gravel and sand with one thin clay interbed, cemented in part. The well was tightly cased through the upper zone and was reduced from 18 to 6 inches in diameter through the lower zone, so it draws most of its water from the middle pervious zone. From April to August 1931 the well was pumped about 525 hours, its yield ranged between 875 and 940 gallons a minute, or 1.9 to 2.1 second-feet, and its greatest draw-down and pumping lift were about 49 feet and 69 feet, respectively. The specific capacity of the well is moderate, about 18 gallons a minute for each foot of draw-down.

The extent of these three water-bearing zones can be traced only approximately. The data available from wells suggest that all three zones tend to become thinner and finer-textured toward the north and west. Possibly the third or lowest zone extends the farthest in those directions. In altitude above sea level the first or upper zone in well 245 corresponds to the coarse gravel that supplies well $2971 \frac{1}{2}$, about 9 miles to the southwest and just beyond the edge of French Prairie; to the uppermost pervious zone in well 247 , two-thirds of a mile to the west, and in wells $261 \frac{1}{2}$ to 263 at Woodburn, 3 miles to the northeast; and to the water-bearing zone at the bottom of well 169 , about 7 miles to the northwest. Records of wells still farther north do not report pervious material at that altitude. Pervious strata about equal in altitude to the second or middle water-bearing zone are penetrated by all known wells of adequate depth as far eastward as wells 259 and 301 , about 2 miles beyond the prairie, southward to well 299, and northward to well 164. Still farther north and west, corresponding pervious material apparently was not found in well 176 at Aurora, near the northeast corner of the prairie; in wells 159 and 160 , just south of the Willamette River; nor in well 153, beyond the prairie to the west. Accordingly, this second zone seems to underlie all or nearly all the southern two-thirds of the prairie, locally to extend eastward somewhat beyond the prairie, but to feather out toward the north and west near the Willamette River. Beyond the supposed feather edge, wells of adequate depth find water at still lower horizons that are equivalent, at least in part, to the lowest pervious zone of the well near Gervais. These remote wells include Nos. 176, 159, and 153, to which reference has just been made.

The moderate specific capacity of the irrigation well near Gervais-18 gallons a minute for each foot of draw-down-indicates that the water is drawn from material which is moderately but not extremely pervious. As has been stated, most of the draft is from the second zone of confined water but, so far as can be judged from their textures, 


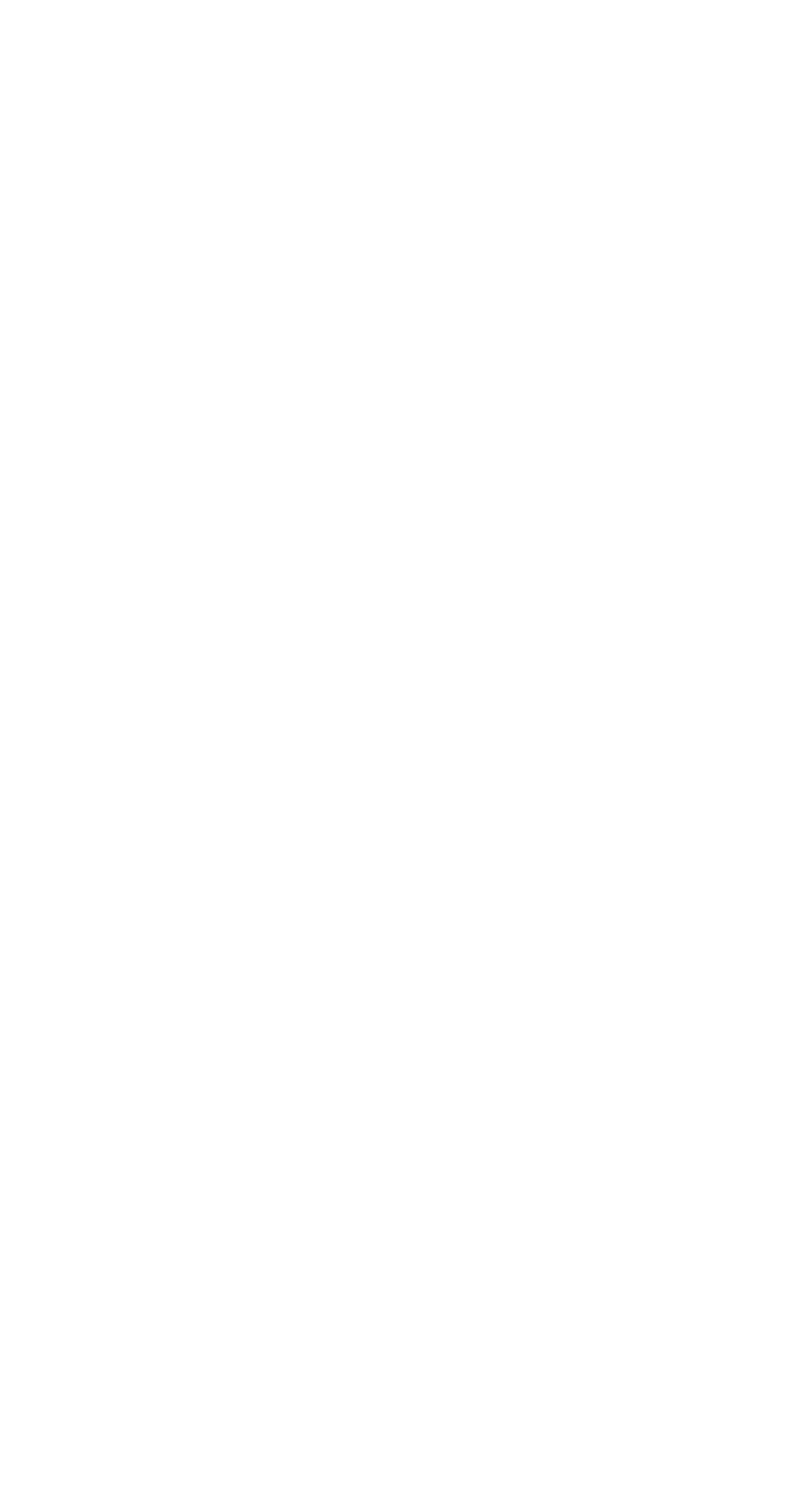



all three water-bearing zones are about equally pervious at that place. To the extent that each zone tends to become thinner and finertextured toward the north and west, its perviousness would tend to decrease somewhat in those directions and its total water-yielding capacity to diminish even more. As more wells are constructed to draw heavily from these zones, an appreciable decline in pressure head of the water will follow inevitably. As has been suggested, however, it is believed that the present yearly withdrawal can be increased very materially without diminishing the pressure head excessively, that is, without surpassing the safe yield. But the precise magnitude of the safe yield is not determinable from data now available.

To assure long life, wells that draw heavily from the deeper pervious zones beneath French Prairie must be adequately screened and thoroughly developed. In these respects, well 245 near Gervais has proved somewhat deficient in construction. The casing in that well was perforated in place and no gravel was placed around the casing to form an artificial envelope. In service, a considerable amount of sand has been discharged with the water, and the beds above the water-bearing zone have subsided, even up to the land surface. Under such conditions, combined with continuous heavy draft, the overlying beds may cave suddenly and in such volume as to retard the movement of water toward a well or even to destroy a well by crushing the casing. In future this hazard may be abated by determining the sizes of the grains in the water-bearing materials-through sieve analyses of an adequate set of samples-and choosing a well screen with ports or openings of suitable width. After a suitable screen has been placed, the newly" constructed well may be "rawhided" or otherwise developed. If the well is constructed by perforating the casing in place, which may produce openings too wide to restrain the sand properly, screened gravel of suitable size may be inserted around the casing during the development until a stable envelope of coarse grains is formed. In this type of construction, the gravel envelope commonly is extended above the water-bearing zone or even to the land surface, thereby forming a reservoir of highly pervious material to replace any sand that might otherwise be drawn through the perforated casing after the well is in use.

Dayton Prairie and adjacent areas (subarea 17).-Topographically and genetically, Dayton Prairie and the adjacent parts of the main lowland plain west and north of the Willamette River are strictly analogous to French Prairie, but in spite of common genesis the two areas are unlike in the relative extent of their principal water-bearing strata. In part, this difference is determined by the form of the common bedrock floor that underlies all the lowland plains. In general the bedrock is impervious shale and earthy sandstone. The older unconsolidated deposit, which forms the prairies and contains 
the three water-bearing zones of the French Prairie area, rests on the impervious bedrock and along the margin of the main lowland plain, thins to a feather edge as it laps over the sloping bedrock surface. Thus, along that margin, there is a belt in which the water-bearing zones are cut off successively by overlap on the bedrock; obviously, the deepest pervious zone is cut off farthest from the margin of the plain. With respect to Dayton Prairie, which lies in an alcove extending deep into the foothills of the Coast Range, the belt of overlap appears to be rather wide at many places, except possibly in R. $3 \mathrm{~W}$. (See pl. 7.)

Of 42 drilled wells on Dayton Prairie for which adequate records are available, one-third passed through the unconsolidated deposit without finding sufficient water for individual household supplies. Of the remaining wells, 7 found water at about the same altitude above sea level as the upper or first zone of confined water beneath French Prairie. These occur northeast of McMinnville in secs. 1, 2, 11, and 12, T. 4 N., R. 4 W.; southwest of McMinnville in secs. 1 and 12, T. 4 N., R. 5 W.; and far to the east in sec. 8 , T. 5 N., R. 3 W. At the altitude of the second zone 17 wells found water; these are widely scattered over all the Dayton Prairie area. None of these wells found the pervious zones in the unconsolidated deposit to be as thick or as pervious as in the area east of the Willamette River; only threewells 125,194 (?), and 214-have specific capacities as large as 1 gallon a minute for 1 foot of draw-down.

In general, it is inferred that the upper two zones of confined water which occur beneath French Prairie- that is, east of the Willamette River-extend discontinuously beneath the river flood plain and far into the Dayton Prairie area; also that there the zones thin markedly and finger out irregularly by gradation into nonpervious material and by overlap on the bedrock. Where these zones are thickest and of coarsest texture, wells of adequate construction might yield sufficient water for irrigation on a modest scale. However, no specific areas so productive of water can be delimited from data now available; if proved by future drilling, their extent must be felt out by cautious exploration.

Only three wells of the Dayton Prairie area-wells 139, 140, and 153-are known to have found water in the unconsolidated deposit at an altitude corresponding to that of the third zone beneath French Prairie. All three are less than a mile west of the Willamette River. Probably this deep pervious zone does not extend far westward beneath Dayton Prairie but wedges out by overlap on the bedrock in R. $3 \mathrm{~W}$.

The Dayton Prairie area includes the tongues of younger alluvium along the two forks of the Yambill River. Like the younger alluvium along the Willamette River, these tongues doubtless hold unconfined 
ground water. To a considerable extent, however, they appear to be composed of material too fine or too poorly sorted to be extremely pervious, and so probably will yield water only moderately or slowly. Information now at hand does not indicate whether these tongues are so thick that wells can have sufficient penetration to yield adequate irrigation supplies.

Tualatin Valley (subarea 18).- So far as known to the writer, the unconsolidated deposits over most of the Tualatin Valley are of relatively fine texture and of small or moderate water-yielding capacity. Along the Tualatin River and lower Gales Creek in the vicinity of Forest Grove, the younger alluvium contains coarse sand and gravel and might yield water rather freely. However, data from wells are not at hand to indicate either the water-yielding capacity or the thickness of the deposit. The deposit that underlies the main valley plain, like the corresponding deposit beneath French Prairie to the south, is fine and rather tight to a considerable depth below the land surface. Locally at least, the lower part of the deposit yields water, as in well 23 at Hillsboro. That well passed through water-bearing sand from 135 to 180 feet beneath the land surface and then entered impervious bedrock, largely soft shale. During construction, a driller's test indicated a yield of 75 gallons a minute with a draw-down of 35 feet; thus, the specific capacity was rather small, slightly more than 2 gallons a minute for each foot of draw-down. It is reported that another well has been sunk there through the same zone and, after thorough development, it yielded more copiously. It is presumed that the sand is stream-laid and therefore would tend to be coarser and more pervious toward the west. With adequately constructed wells it might yield water sufficient for irrigation. However, the deposit seems not to contain pervious beds beneath all the valley plain, for numerous farm wells, such as well 20 , have been sunk far into the bedrock to obtain dependable supplies.

Molalla pediment and related parts of the central lowland (subarea 19). -Along the eastern edge of the central lowland from Salem northward to Canby there is a gently rolling belt between 4 and 9 miles wide that rises eastward from the main valley plain. Toward the north it includes a conspicuous alluvial slope, or pediment, whose highest part is at Molalla (p. 11). The northern half of this belt, if not all of it, is underlain by partly consolidated bedrock (p. 54), which is older than the main valley plain and to the west extends beneath that plain.

The Molalla pediment is veneered with poorly assorted coarse sand and gravel that supplies numerous farm wells that were dug from 20 to 30 feet deep. However, this veneer is thin and is cut entirely through by many creek valleys and gullies, so that it would not sustain a continual large withdrawal of water. Beneath this veneer, the ma- 
terial that underlies the pediment is largely "clay" and "shale" and includes only a few water-bearing beds. (See driller's records for wells 188 and 281 , table 12.) These beds have small capacity to yield water. Wells 271,281 , and 282 yield only a small fraction of a gallon a minute for each foot of draw-down. Well 188, about 2 miles west of the edge of the pediment, passes through the feather edge of the deposit that forms the main valley plain and extends far down into material that is inferred to be partly consolidated bedrock. Even though the principal water-bearing bed in that well occurs in the over-lapping younger deposit, the specific capacity is only about 0.4 gallon a minute for each foot of draw-down. Material so slightly pervious as the water-bearing beds penetrated by these wells certainly will not sustain continual withdrawals of more than a few gallons or tens of gallons a minute from single wells. Accordingly they do not seem adequate for efficient irrigation wells.

Conditions such as those just described may prevail in all the relatively high and rolling lowland terrane beyond the Molalla pediment to the south - that is, from Butte Creek to the vicinity of Mount Angel and again from Silverton about to the Little Pudding River. In these areas, the old terrane, which is not highly productive of water, abuts against the French Prairie subarea on the west and the Mill Creek subarea on the south, both of which have relatively large capacity to yield water. The boundary between the older nonproductive deposit and the overlapping productive beds to the west and south cannot now be traced precisely but has been fixed somewhat arbitrarily (p. 42). It can be felt out by cautious further drilling.

Portland "delta" (subarea 20).--At the land surface, the area of the so-called Portland delta includes several distinct unconsolidated stream deposits of Quaternary age. At depth the area is underlain, at least in part, by semiconsolidated deposits of early Quaternary or latest Tertiary age, chiefly by the Troutdale formation of Hodge (p. 23). During the reconnaissance that forms the basis of this report, only cursory attention was given to the character, extent, and stratigraphic relations of these deposits; also, records were obtained from only a few typical wells (Nos. 26-33, 36-39, 55-60, and 62-67). Although these data indicate several distinct water-bearing zones, they are not adequate to discriminate the zones sharply with respect to depth beneath the land surface, lateral extent, and relative pressure head of the water. In reported yield, the typical wells range between 50 and 1,000 gallons a minute; in reported specific capacity, commonly from 10 to about 33 gallons a minute for each foot of draw-down. It is judged that the area will sustain a much greater aggregate withdrawal and will readily afford wells of eapacities adequate for irrigation and for many industrial purposes. 


\section{FLOWING WELLS}

Certain pervious beds in both the unconsolidated deposits and underlying consolidated rocks of the Willamette Valley contain water under sufficient pressure head to rise above the land surface and so to yield flowing wells. However, the known areas of artesian flow are few, small, and scattered; none yield copiously by artesian head alone. Further, some of the flowing wells in the consolidated rocks yield salt water.

With one exception, the flowing wells known to yield water from the unconsolidated deposits are in the northern half of the lowland, between Salem and Canby, and on the flanks or floors of the larger stream trenches. These wells are Nos. 176, 233, and 268; all three are inferred to draw water from deposits related to the older alluvium. All have small pressure heads at the land surface and so flow but slightly; however, it is not known whether the wells are so cased that none of the artesian head is dissipated by leakage into nonartesian aquifers. Corresponding areas of artesian flow doubtless extend only to a moderate height above the floors of the several stream trenches. Their extent along the trenches is suggested by the contours of pressure head for tightly cased deep wells (pl. 1). This head appears adequate to yield flowing wells over much of the Willamette River flood plain from the vicinity of Salem northward about to Newberg, in Tps. 4 to $6 \mathrm{~S}$.; on the lower flanks and floor of the Pudding River and Butte Creek trenches downstream from the vicinity of Monitor, in Tps. 4 and $5 \mathrm{~S}$.; and, possibly, in some other low areas such as the Lake Labish trench. The areas of artesian flow terminate northward as the pervious zones feather out (see p. 50) and southward as the pervious zones lap against older and tighter material (see p. 54).

The one well in the southern half of the lowland known to flow from the older alluvium, No. 566, is near the apex of the Lebanon alluvial fan but low on the flank of a shallow drain. In that well the artesian flow is variable and commonly ceases in late summer when the pressure head is least. If the artesian head is the result of the water-bearing zone fingering into less pervious material, as seems probable, the potential area of flow is not extensive.

Wells that flow by artesian pressure from the consolidated rocks are scattered widely the full length of the Willamette Valley and derive water from numerous stratigraphic zones. Apparently there are numerous areas of artesian flow, but none are extensive and none of the wells flow very copiously. In and near Portland the west flank of the Willamette syncline and the southeastward-plunging anticline that underlies the Tualatin Valley together afford an artesian slope. The uppermost bedrock formation of that slope, the Yakima basalt, is the probable source of water for flowing wells 26,51 , and 74 . How- 
ever, water-bearing zones in that formation are discontinuous and appear to have distinct pressure heads; also, the land surface on that formation has a relief of about 1,000 feet. Thus, flowing wells can be obtained only in small areas that are indeterminate in extent and location. For example, well 51 flows rather copiously, yet well 53 does not flow, although it is only half a mile to the east, and is 400 feet deeper and some 20 feet below the reported static water level of well 51. About $3 \frac{1}{2}$ miles southeast of flowing well 74 , at a point seemingly lower on the artesian slope and lower in altitude of land surface, another well--No. 76-is considerably deeper and yet does not flow. However, neither well is tightly cased through shallow pervious zones, so that some pressure head may be dissipated in either well or both. West of these flowing wells are wells $18,20,44$, and 45 which flow slightly from sedimentary rocks in the same artesian slope. These rocks are tentatively inferred to lie stratigraphically beneath the basalt, which has been breached by erosion along the axis of the plunging anticline. It seems unlikely that these rocks will yield flowing wells over an extensive part of the Tualatin Valley plain, for in general they contain only a few discontinuous pervious zones. (See p. 26.)

Southwest of the Tualatin Valley, about 5 miles northeast of McMinnville, well 110 flows a trickle, presumably from sedimentary rocks below the Yakima basalt. This well yields "mineral water" from an unknown depth below the land surface. Its pressure lead is so small that similar flowing wells could be obtained only within a very narrow belt along the footslope of the bedrock hills.

Still farther south, along and near the west edge of the central lowland, beds of sandstone and sandy shale in the Eocene rocks yield or have yielded water under sufficient pressure head to flow at wells $287,457,577,593,597$, and 610 . In general, these rocks form an artesian slope that dips gently eastward beneath the central lowland. However, they are relatively impervious, and the pressure head of their water is not adequate for flowing wells high above the lowland plain; so large yields by artesian pressure are unlikely. Wells 287 and 610 are reported to have yielded salt water, well 610 from a depth of only 300 feet beneath the main lowland plain.

Flowing well 564, near Lebanon, taps volcanic rock, possibly correlative to the Yakima basalt, very near the west margin of the lowland. Flowing well 713, at Eugene, finds its water in igneous rock associated with the Eugene formation, of Oligocene age. At neither place is the extent of the area of artesian flow known.

\section{CHEMICAL CHARACTER OF THE GROUND WATER}

Table 9 shows the chemical character of water samples from 35 wells in the Willamette Valley. Of these, 28 samples were taken from wells in the unweathered younger or older alluvium, 3 from wells 
in the weathered terrace deposit, and 4 from wells that penetrate bedrock. Figure 3 shows graphically the results of analyses of the waters from 20 of the wells. These chemical analyses show the mineral content of the water, but they show little with respect to the sanitary quality. Thus, from the analyses the suitability of the waters for the common industrial or commercial uses and for irrigation can be determined, but not their suitability for human consumption.

Waters that contain less than 500 parts per million of dissolved solids ordinarily are suitable for domestic purposes and for many industrial and commercial uses, except for the inconveniences that may be caused by their hardness. Most but not all waters that contain more than 1,000 parts per million are likely to have a perceptible taste and to be unsatisfactory for ordinary uses owing to an excessive amount of some one or several constituents. According to these limits, all waters in the unconsolidated deposits of the Willamette Valley appear to be quite satisfactory for most ordinary uses; but certain waters from the consolidated rocks are unsatisfactory. (See p. 64.)

With respect to ordinary uses, the chemical suitability of the ground waters of the Willamette Valley depends largely on the content of calcium and magnesium, the constituents that cause essentially all the hardness of a water. A hard water, of course, requires more $\mathrm{s}$ oap to be used in laundering; it form scale in steam boilers, hot-water tanks, and other receptacles; and it must be treated to render it suitable for some common industrial-chemical processes. Water with hardness less than 50 is commonly regarded as soft, and requires no treatment to condition it for many ordinary purposes. ${ }^{14}$ The limits of hardness below which the cost of treatment to soften a water is not justified are about as follows: 50 parts per million for the smaller laundries or other industries that use considerable soap, much less than 50 parts per million for the larger laundries and for dyeing and bleaching works; 100 parts per million for supply to small boilers and very much less for supply to large modern high-pressure boilers, to prevent the formation of scale; and 200 parts per million or less for domestic uses. In the treatment of municipal supplies, the present tendency is to furnish water with a residual hardness of about 80 parts per million. So far as is shown by the analyses in table 9 , the hardness of the water in unconsolidated deposits of the Willamette Valley is commonly between 50 and 150 parts per million, though in one sample (No. 690) the hardness was only 9.5 parts per million. Thus, water from these deposits need not be softened for many uses. On the other hand, the water in the consolidated sedimentary rocks is commonly very hard.

\footnotetext{
14 Much of this general discussion of the chemical character of water is adapted from Collins, W. D., and Howard, C. S., Chemical character of waters of Florida: U. S. Geol. Survey Water-Supply Paper 596, pp. 181-186, 1928.
} 


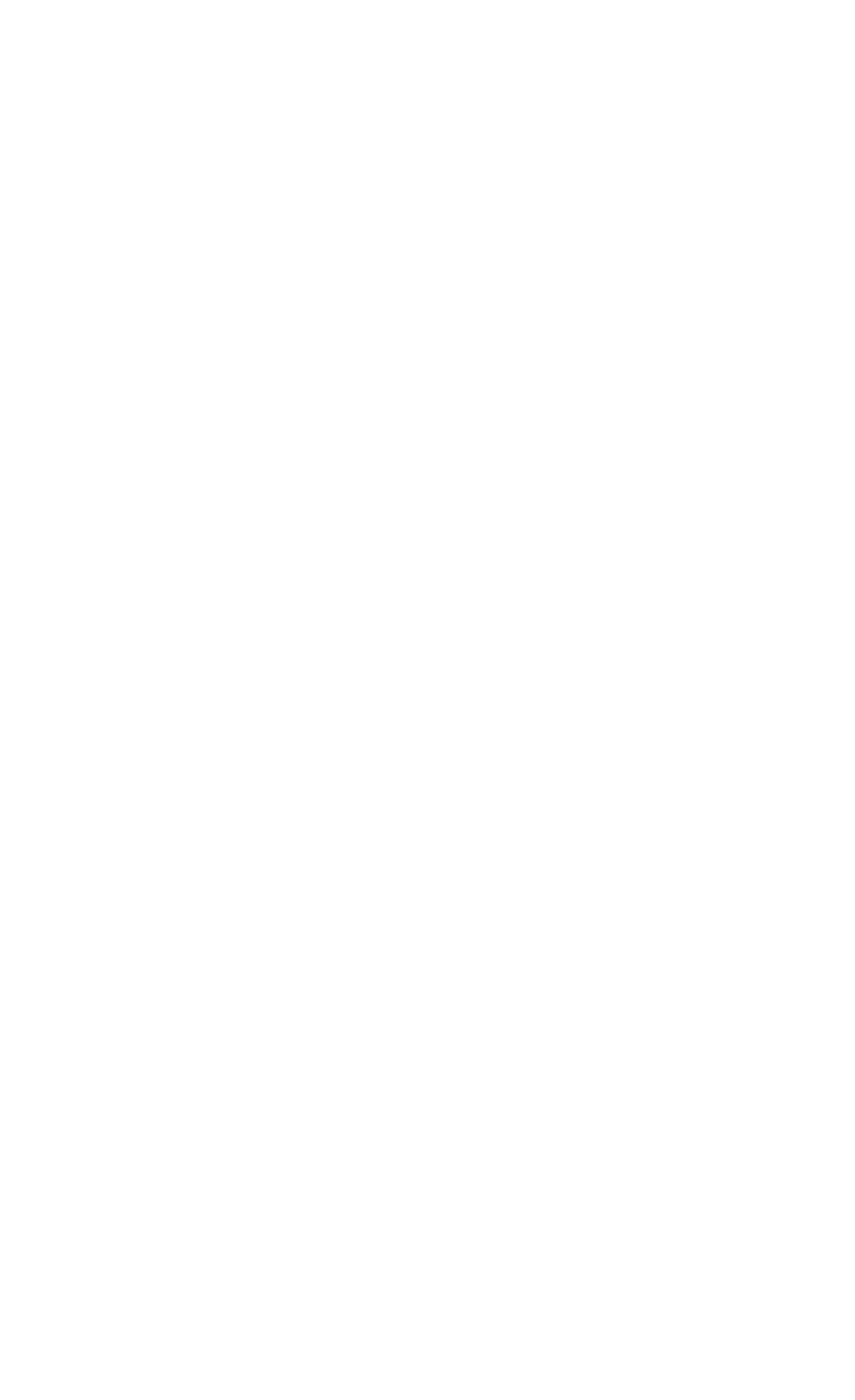


In irrigation, the chemical character of the water used is critical both with respect to the tolerance of the crop plant toward the concentration of salts in the root zone and with respect to maintenance of good tilth and suitable permeability in the soil. Other critical factors include the quantity of water applied to the land, rainfail, chemical and physical characteristics of the soil, and drainage. Scofield ${ }^{15}$ has proposed limits for the concentration of certain constituents by which to judge whether the water is satisfactory for irrigation. These limits are shown by the following table:

TABLE 8.-Approximate limits indicating the suitability of a water for irrigation

[Parts per million except as indicated]

\begin{tabular}{|c|c|c|}
\hline Constituent & Satisfactory water & Unsatisfactory water \\
\hline $\begin{array}{l}\text { Total dissolved solids } \\
\text { Sulphate, SO } \\
\text { Chloride, C] } \\
\text { Percent sodium } 1\end{array}$ & $\begin{array}{l}\text { Less than } 700 \\
192 \\
502\end{array}$ & $\begin{array}{l}\text { More than } 2,000 \text {. } \\
480 \text {. } \\
355 . \\
60 .\end{array}$ \\
\hline
\end{tabular}

1 Percent sodium $=\frac{100 \mathrm{Na}}{\mathrm{Na}+\mathrm{Ca}+\mathrm{Mg}}$, in which the three chemical constituents are expressed in equivalents in parts per million.

According to Scofield a water classified by these limits as satisfactory for irrigation is unlikely to cause root-zone conditions intolerable to ordinary crop plants or to impair seriously the physical condition of the soil. A water classified as unsatisfactory in one of the four constituents may prove intolerable to crop plants or may tend to render the soil impermeable and of such consistency that good tilth is difficult to maintain. A water whose concentration of sulphate or chloride is greater than the satisfactory limit but less than the unsatisfactory limit may be used successfully for irrigating tolerant plants if the soil is adequately drained and if the water is applied so copiously that the root zone is continually leached. By these limits, all water in the unconsolidated deposits of the Willamette Valley is highly satisfactory for irrigation and only certain waters from the consolidated rocks are unsatisfactory.

If a water contains much more than 0.1 part per million of iron, the excess iron commonly separates out as a reddish-brown sludge when the water is allowed to stand; also, it may stain cooking utensils and fabrics that are laundered. For some industrial-chemical processes, such as the manufacture of paper or rayon products, the upper limit of dissolved iron that can be tolerated in the water is even less than 0.1 part per million. Of the 31 samples of water from wells in the unconsolidated deposits of the Willamette Valley, only 13 contained 0.1 part or less per million of dissolved iron; all these were from

15 Scofield, C. S., South Coastal Basin investigation, quality of irrigation waters: California Dept. Public Works, Water Resources Div., Bull. 40, pp. 21-24, 1933.

$408526-42--5$ 
TABLE 9.-Chemical analyses of water from

\begin{tabular}{|c|c|c|c|}
\hline No. & Description & $\begin{array}{l}\text { Date of } \\
\text { sample }\end{array}$ & $\begin{array}{l}\text { Tem- } \\
\text { pera- } \\
\text { ture } \\
\left({ }^{\circ} \mathrm{F} .\right)\end{array}$ \\
\hline 101 & $\begin{array}{l}\text { Canby, } 21 / 2 \text { miles northwest of; in the NW1/4SW1/4 sec. } 30, T .3 \mathrm{~S} ., \mathrm{R} .1 \mathrm{E} \text {, } \\
\text { Bored well } 10 \text { inches in diameter and } 57 \text { feet deep. Isaac A. Miley } \\
\text { estate. Water from valley fill. }\end{array}$ & Oct. 10,1928 & 52 \\
\hline 102 & $\begin{array}{l}\text { Canby, municipal supply: in the } \mathrm{NW}_{1 / 4} \mathrm{SW}^{1 / 4} \text { sec. } 33, \mathrm{~T}, 3 \mathrm{~S} ., \mathrm{R} . \text { l } \mathrm{E} \text {. } \\
\text { Drilled well } 8 \text { inches in diameter and } 107 \text { feet deep (No. 1). Water } \\
\text { from gravel (valley fill). }\end{array}$ & ......do_.... & 53 \\
\hline 103 & 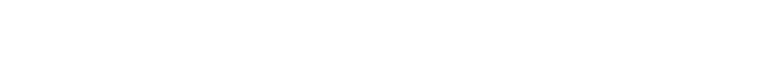 & . .... do do & 60 \\
\hline 133 & $\begin{array}{l}\text { McMinnville, } 3 \text { miles east of: in the SW1/4SW1/4 sec. } 24, \mathrm{~T} .4 \mathrm{~S} ., \mathrm{R}_{.} 4 \mathrm{~W} \text {. } \\
\text { Bored well } 12 \text { inches in diameter and } 41 \text { feet deep. A J. Mott. Water } \\
\text { from valley fill. }\end{array}$ & Oct. 15,1928 & 52 \\
\hline $1451 / 2$ & $\begin{array}{l}\text { Dayton, } 31 / 4 \text { miles southeast of; in the NW1/4SE1/4 sec. } 26, \mathrm{~T} .4 \mathrm{~S} ., \mathrm{R} .3 \mathrm{~W} \text {. } \\
\text { Drilled well } 6 \text { inches in diameter and } 40 \text { feet deep. R. II. Wood. } \\
\text { Water from sand and gravel (young alluvium). }\end{array}$ & Oct. 13,1928 & 52 \\
\hline 164 & $\begin{array}{l}\text { St. Paul, } 1 \text { mile east of; in the } \mathrm{SW} 1 / 4 \mathrm{NE}^{1 / 4} \text { sec. } 20, \mathrm{~T} .4 \mathrm{~S} ., \mathrm{R} .2 \mathrm{~W} . \\
\text { Drilled well } 6 \text { inches in diameter and } 147 \text { feet deep. Wm. Gooding. } \\
\text { Water from valley fill. }\end{array}$ & Oct. 10,1928 & 52 \\
\hline 171 & $\begin{array}{l}\text { St. Paul, } 4 \text { miles southeast of; in the SE1/4SE1/4 sec. 34, T. } 4 \mathrm{~S} ., \mathrm{R}^{2} \mathrm{~W} \text {. } \\
\text { Dug well } 18 \text { inches in diameter and } 20 \text { feet deep. Johnson School. } \\
\text { Water from valley fill. }\end{array}$ & . . do do & 53 \\
\hline 182 & $\begin{array}{l}\text { Hubbard, municipal supply; in the } \mathrm{SW} 1 / 4 \mathrm{NW} 1 / 4 \mathrm{sec} .34, \mathrm{~T} .4 \mathrm{~S} ., \mathrm{R} .1 \mathrm{~W} \text {. } \\
\text { Drilled well } 8 \text { inches in diameter and } 130 \text { feet deep. Water irom valley } \\
\text { fill. Known locally as "sulphur water." }\end{array}$ & ..... do do & 54 \\
\hline 203 & $\begin{array}{l}\text { Sheridan, } 31 / 2 \text { miles east of; in the NE1/4NE1/4 sec. } 32, T \text {, } 5 \mathrm{~S} ., \mathrm{R} .5 \mathrm{~W} \text {. } \\
\text { Dug well } 4 \text { feet in diameter and } 32 \text { feet deep. Wm. Piller. Water } \\
\text { from valley fill. }\end{array}$ & Oct. 15,1928 & 52 \\
\hline 228 & $\begin{array}{l}\text { Gervais, } 5 \text { miles northwest of; in the } \mathrm{SE} 1 / 4 \mathrm{SW} 1 / 4 \mathrm{sec} .13, \mathrm{~T} .5 \mathrm{~S} ., \mathrm{R} .3 \mathrm{~W} \text {. } \\
\text { Dug well } 2 \text { feet in diameter and } 291 / 2 \text { feet deep. S. F. Parker. Water } \\
\text { from valley fill. }\end{array}$ & Oct. 10,1928 & 50 \\
\hline 247 & 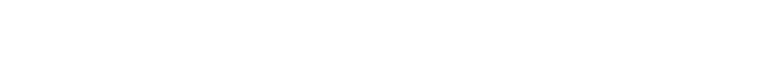 & Oct. 20,1928 & 56 \\
\hline 274 & $\begin{array}{l}\text { Molalla, } 31 / 2 \text { miles west of; in the NE1/4 SW1/4 sec. } 11, T \text { T S., R. } 1 \mathrm{E} \text {. } \\
\text { Dug well } 4 \text { feet in diameter and } 27 \text { feet deep. District school. Water } \\
\text { from terrace deposit. }\end{array}$ & Oct. 10,1928 & 52 \\
\hline 294 & $\begin{array}{l}\text { Salem, } 61 / 2 \text { miles northwest of; in the } \mathrm{SE} 1 / 4 \mathrm{SE} 1 / 4 \mathrm{sec} .28, \mathrm{~T} .6 \mathrm{~S} ., \mathrm{R} .3 \mathrm{~W} . \\
\text { Driven well } 11 / 4 \text { inches in diameter and } 31 \text { feet deep. J. J. Burton. } \\
\text { Water from valley fill. }\end{array}$ & Oct. 13,1928 & 52 \\
\hline 300 & $\begin{array}{l}\text { Gervais, } 41 / 2 \text { miles southeast of; in the SW1/4SE1/4 sec. } 13 \text {, T. } 6 \mathrm{~S} \text {., R. } 2 \mathrm{~W} \text {. } \\
\text { Dug well 31/2 feet in diameter and } 21 \text { feet deep. R. O. Dunn. Water } \\
\text { from "quicksand" (valley fill). }\end{array}$ & d & 52 \\
\hline 333 & $\begin{array}{l}\text { Salem, Madison and } 14 \text { th Streets; in the } \mathrm{NW} 1 / 4 \mathrm{NE} 1 / 4 \mathrm{sec} .23, \mathrm{~T} .7 \mathrm{~S} ., \mathrm{R} . \\
3 \mathrm{~W} \text {. Drilled well } 10 \text { inches in diameter and } 2481 / 2 \text { feet deep. Oregon } \\
\text { Linen Mills, Inc. Water from basalt (?). }\end{array}$ & -8 & 56 \\
\hline 342 & $\begin{array}{l}\text { West Salem, municipal supply; in the } \mathrm{NW}^{1} / 4 \mathrm{NE} 1 / 4 \text { sec. } 28, \mathrm{~T} .7 \mathrm{~S} ., \mathrm{R} .3 \\
\text { W. Dug well } 14 \text { feet in diameter and } 34 \text { feet deep. Water from } \\
\text { young alluvium. }\end{array}$ & do & 54 \\
\hline 350 & $\begin{array}{l}\text { Silverton, } 5 \text { miles southwest of; in the } \mathrm{SE} 1 / 4 \mathrm{SE} 1 / 4 \mathrm{sec} .12 \text {, T. } 7 \mathrm{~S} ., \mathrm{R} .2 \mathrm{~W} \text {. } \\
\text { Dug well } 47 \text { feet deep. R. J. Janz. Water from gravel (old allu- } \\
\text { vium). }\end{array}$ & -do. & 52 \\
\hline 373 & $\begin{array}{l}\text { Independence, municipal supply; in the } \mathrm{SE}^{1} / \mathrm{NW} 1 / 4 \text { sec. } 28, \mathrm{~T} .8 \mathrm{~S} ., \mathrm{R} .4 \\
\text { W. Gang of three drilled wells } 8 \text { inches in diameter and about } 45 \text { feet } \\
\text { deep. Mountain States Power Co., Albany, Oreg. Water from } \\
\text { young alluvium. }\end{array}$ & .do_.. & 54 \\
\hline 465 & $\begin{array}{l}\text { Corvallis, } 1 \text { mile east of; in the SW1/4SE1/4 sec. } 36, \mathrm{~T}, 11 \mathrm{~S}, \mathrm{R}, 5 \mathrm{~W} \text {. } \\
\text { Driven well } 11 / 4 \text { inches in diameter and } 28 \text { feet deep. East farm of } \\
\text { Oregon State Agricultural College. Water from old (?) alluvium. }\end{array}$ & ....do. & 52 \\
\hline 490 & $\begin{array}{l}\text { Albany, } 51 / 2 \text { miles southwest of; in the NW1/4SE1/4 sec. 33, T. is S., R. } 4 \\
\text { W. Drilled well } 4 \text { inches in diameter and } 295 \text { feet deep. P. A. Good- } \\
\text { win. Water from shale (bedrock of Tertiary age). }\end{array}$ & Oct. 12,1928 & 54 \\
\hline $\begin{array}{l}494- \\
498\end{array}$ & $\begin{array}{l}\text { Albany, } 3 \text { miles northeast of; in the NE1/4NW1/4 sec. } 4, \text { T. } 11 \mathrm{~S} ., \text { R. } 3 \mathrm{~W} \\
\text { Driven well } 11 / 4 \text { inches in diameter and } 41 \text { feet deep. Albany Floral } \\
\text { Co. Water from gravel (old alluvium). }\end{array}$ & . do. & \\
\hline 519 & $\begin{array}{l}\text { Lebanon, } 5 \text { miles northeast of; in the NW1/4NE1/4 sec. } 24, \mathrm{~T}, 1 \mathrm{~S} ., \mathrm{R} .2 \mathrm{~W} \text {. } \\
\text { Drilled well } 4 \text { inches in diameter and } 73 \text { feet deep. Griggs School, } \\
\text { district } 4 \text {. Water from terrace deposit (?). }\end{array}$ & .....do & 54 \\
\hline 530 & $\begin{array}{l}\text { Corvallis, } 1 \text { mile south of; in the } \mathrm{SE} 1 / 4 \mathrm{SW} 1 / 4 \mathrm{sec} .2 \text {, T. } 12 \mathrm{~S} \text {., R. } 5 \mathrm{~W} \text {. } \\
\text { Drilled well } 3 \text { inches in diameter and } 32 \text { feet deep. Shell Co. of Cali- } \\
\text { fornia. Water from gravel (old ? alluvium). }\end{array}$ & .....do.. & 54 \\
\hline
\end{tabular}


[Margaret D. Foster, analyst]

\begin{tabular}{|c|c|c|c|c|c|c|c|c|c|c|c|c|c|}
\hline \multicolumn{10}{|c|}{ Analyses (parts per million) } & \multicolumn{4}{|l|}{ - } \\
\hline $\begin{array}{l}\text { Total } \\
\text { dis- } \\
\text { solved } \\
\text { solids } \\
\text { at } 180^{\circ} \\
\text { C. }\end{array}$ & $\begin{array}{l}\text { Silica } \\
\left(\mathrm{SiO}_{2}\right)\end{array}$ & $\begin{array}{l}\text { Iron } \\
(\mathrm{Fe})^{1}\end{array}$ & $\begin{array}{c}\text { Cal- } \\
\text { cium } \\
\text { (Ca) }\end{array}$ & $\begin{array}{c}\text { Mag- } \\
\text { ne- } \\
\text { sium } \\
(\mathrm{Mg})\end{array}$ & $\begin{array}{c}\text { So- } \\
\text { dium } \\
\text { (Na) }\end{array}$ & $\begin{array}{c}\text { Po- } \\
\text { tas- } \\
\text { sium } \\
(\mathbf{K})\end{array}$ & $\begin{array}{c}\text { Bicar- } \\
\text { bonate } \\
\left(\mathrm{HCO}_{3}\right)\end{array}$ & $\begin{array}{c}\text { Sul- } \\
\text { phate } \\
\left(\mathrm{SO}_{4}\right)\end{array}$ & $\begin{array}{l}\text { Chlo- } \\
\text { ride } \\
\text { (Cl) }\end{array}$ & $\begin{array}{c}\mathrm{Ni}- \\
\text { trate } \\
\left(\mathrm{NO}_{3}\right)\end{array}$ & $\begin{array}{c}\text { Total } \\
\text { hard- } \\
\text { ness as } \\
\mathrm{CaCO}_{3}^{2}\end{array}$ & $\begin{array}{c}\text { Per- } \\
\text { cent } \\
\text { so- } \\
\text { dium } 3\end{array}$ & No. \\
\hline 112 & 60 & 0.56 & 8.7 & 7.0 & 4.1 & 1.1 & 62 & 5.4 & 1.8 & 0.05 & 50 & 17.0 & 101 \\
\hline 163 & 41 & .19 & 24 & 11 & 7.4 & 1.8 & 132 & 5.0 & 4.0 & 2.4 & 105 & 14.9 & 102 \\
\hline 326 & 45 & .21 & 11 & 5.5 & 93 & 2.9 & 258 & 4.1 & 29 & .96 & 50 & 80.4 & 103 \\
\hline 197 & 21 & .23 & 40 & 10 & 12 & 1.4 & 160 & 17 & 10 & 3.8 & 141 & 16.5 & 133 \\
\hline 132 & 42 & .37 & 15 & 8.0 & 7.2 & 1.3 & 88 & 5.5 & 2.6 & 5. 3 & 70 & 19.8 & $1451 / 2$ \\
\hline 179 & 43 & 5. 27 & 27 & 11 & 12 & 1.7 & 165 & 3. 3 & 2.5 & .10 & 113 & 20.1 & 164 \\
\hline 173 & 28 & .15 & 41 & 4.4 & 9.0 & 1.2 & 159 & 5.8 & 3.0 & 1.0 & 121 & 14.9 & 171 \\
\hline 123 & 41 & .50 & 17 & 6.3 & 5.6 & 1.4 & 88 & 4.9 & 2.6 & .45 & 68 & 17.0 & 182 \\
\hline 188 & 39 & .37 & 29 & 11 & 14 & 1.0 & 146 & 4.3 & 10 & 7.8 & 118 & 21.2 & 203 \\
\hline 198 & 19 & .46 & 17 & 6.1 & 26 & 6.0 & 57 & 12 & 26 & 43 & 68 & 48.7 & 228 \\
\hline 204 & 48 & 1. 17 & 25 & 18 & 15 & 1.7 & 193 & 2.9 & 3.5 & 1.1 & 136 & 20.3 & 247 \\
\hline 104 & 41 & .19 & 12 & 2.1 & 5.9 & 1.0 & 48 & 4. 2 & 2.4 & 5.2 & 39 & 26.8 & 274 \\
\hline 129 & 42 & .15 & 16 & 10 & 3.5 & .4 & 92 & 5.8 & 5.0 & 4.0 & 81 & 9. 1 & 294 \\
\hline 134 & 59 & .23 & 12 & 5.9 & 6.7 & 1.0 & 63 & 4.2 & 5.8 & 4. 2 & 54 & 22.6 & 300 \\
\hline 146 & 46 & .02 & 17 & 12 & 8.7 & 1.2 & 130 & 3.5 & 3.0 & .0 & 92 & 18.2 & 333 \\
\hline 156 & 57 & .10 & 18 & 10 & 9.1 & .7 & 109 & 5.3 & 5.5 & 2.8 & 86 & 19.4 & 342 \\
\hline 114 & 34 & 2.13 & 11 & 4.5 & 7.6 & .6 & 35 & 4.8 & 11 & 15 & 46 & 27.3 & 350 \\
\hline 188 & 44 & .12 & 24 & 15 & 13 & 1.3 & 145 & 8.2 & 12 & 3.6 & 122 & 19.7 & 373 \\
\hline 113 & 41 & .02 & 12 & 8. 6 & 5.2 & .7 & 80 & 3.5 & 3.2 & 2.3 & 65 & 15.7 & 465 \\
\hline 4,967 & .15 & 1.03 & 324 & 62 & 1,450 & 14 & 100 & 1.9 & 2,956 & .0 & 1,064 & 74.9 & 490 \\
\hline 116 & 38 & .05 & 12 & 6.9 & 5.7 & 1.6 & 71 & 4.4 & 2.4 & 6.7 & 58 & 19.8 & $\left\{\begin{array}{l}49 \div- \\
498\end{array}\right.$ \\
\hline 150 & 25 & .93 & 7.1 & 3.5 & 38 & 2. 2 & 132 & 3.0 & 5.5 & 1. 0 & 32 & 72.7 & 519 \\
\hline 230 & 45 & .04 & 34 & 14 & 22 & 1.6 & 182 & 2.7 & 28 & .10 & 142 & 25.9 & 530 \\
\hline
\end{tabular}

1 Includes iron precipitated at time of analysis.

3 After Scofield; see explanation in text. 
TABLE 9.-Chemical analyses of water from

\begin{tabular}{|c|c|c|c|}
\hline No. & Description & $\begin{array}{l}\text { Date of } \\
\text { sample }\end{array}$ & $\begin{array}{l}\text { Tem- } \\
\text { pera- } \\
\text { ture } \\
\left({ }^{\circ} \text { F. }\right)\end{array}$ \\
\hline 533 & $\begin{array}{l}\text { Corvallis, } 21 / 2 \text { miles south of; in the } \mathrm{SE} 1 / 4 \mathrm{SE} 1 / 4 \text { sec. } 10 \text {, T. } 12 \mathrm{~S} \text {., R. } 5 \mathrm{~W} \text {. } \\
\text { Drilled well } 3 \text { inches in diameter and } 122 \text { feet deep. Mrs. A. Sims. } \\
\text { Water from shale (bedrock of Tertiary age). }\end{array}$ & Oct. 12,1928 & 53 \\
\hline 551 & $\begin{array}{l}\text { Tangent, municipal supply; in the SW1 } 1 \text { NW1/4 sec. } 7 \text {, T. } 12 \mathrm{~S} \text {, R. } 3 \mathrm{~W} \text {. } \\
\text { Gang of five driven wells } 11 / 4 \text { inches in diameter and } 45 \text { feet deep. } \\
\text { Water from gravel (old alluvium). }\end{array}$ & Apr. 18,1929 & 51 \\
\hline 566 & $\begin{array}{l}\text { Lebanon, in the SW1/4SW1/4 sec. } 11, \text { T. } 12 \mathrm{~S}, \text { R. } 2 \mathrm{~W} \text {. Drilled well } 6 \\
\text { inches in diameter and } 115 \text { feet deep. Mountain States Power Co. } \\
\text { Water from gravel (old ? alluvium). }\end{array}$ & Oct. 12,1928 & 54 \\
\hline 580 & $\begin{array}{l}\text { Shedd, } 5 \text { miles west of; in the SE1/4NW1/4 sec. } 5, \text { T. } 13 \mathrm{~S} ., \text { R. } 4 \mathrm{~W} \text {. Driven } \\
\text { well } 11 / 4 \text { inches in diameter and } 31 \text { feet deep. Gustav Hahn. Water } \\
\text { from gravel (young alluvium). }\end{array}$ & Oct. 10,1928 & 54 \\
\hline 595 & $\begin{array}{l}\text { Monroe, } 4 \text { miles north of in the } S W 1 / 4 \mathrm{SW} 1 / 4 \text { sec. } 3 \text {, T. } 14 \mathrm{~S} \text {., R. } 5 \mathrm{~W} \text {. } \\
\text { Driven well } 11 / 4 \text { inches in diameter and } 34 \text { feet deep. McFarland }\end{array}$ & Oct. 12,1928 & 50 \\
\hline 606 & $\begin{array}{l}\text { Halsey, municipal supply; in the SE1/4NE1/4 sec. } 1 \text {, 'T. } 14 \mathrm{~S} \text {., R. } 4 \mathrm{~W} \text {. } \\
\text { Dug well } 10 \text { feet in diameter and } 28 \text { feet deep with two drilled wells } 4 \\
\text { inches in diameter in bottom, plugged at total depth of } 72 \text { feet. Citi- } \\
\text { zens Light \& Water Co. Water mainly rom old alluvium (some } \\
\text { saline water from bedrock probably passes the plug). }\end{array}$ & Apr. 18,1929 & 52 \\
\hline 631 & $\begin{array}{l}\text { Harrisburg, municipal supply; in the NE1/4NE1/4 sec. } 16 \text {, T. 15 S., R. } 4 \\
\text { W. Dug well } 10 \text { feet in diamter and } 30 \text { feet deep with six } 3 \text {-inch drilled } \\
\text { wells in bottom, total depth of } 60 \text { feet. H. F. Meersdorf. Water } \\
\text { from old alluvium. }\end{array}$ & Oct. 12,1928 & 55 \\
\hline 638 & $\begin{array}{l}\text { Junction City, municipal supply; in the } \mathrm{SE} 1 / 4 \mathrm{SW1} / 4 \text { sec. } 32, \mathrm{~T} .15 \mathrm{~S} . \text { R. } 4 \\
\text { W. Drilled well } 4 \text { inches in diameter and } 189 \text { feet deep. J. H. Miller } \\
\text { \& Sons. Water from old alluvium (?). }\end{array}$ & $\ldots$ do & 54 \\
\hline 653 & $\begin{array}{l}\text { Coburg, } 41 / 2 \text { miles northwest of; in the NE } 1 / 4 \mathrm{NE}^{1 / 4} \text { sec. } 14, \mathrm{~T} .16 \mathrm{~S} ., \mathrm{R} .4 \\
\text { W. Driven well } 11 / 2 \text { inches in diameter and } 16 \text { feet deep. R. W. Har- } \\
\text { rington. Water from young alluvium. }\end{array}$ & .... do & 58 \\
\hline 690 & $\begin{array}{l}\text { Junction City, } 7 \text { miles southewst of; in the } \mathrm{NE1} / 4 \mathrm{NW1} / 4 \text { sec. } 11, \mathrm{~T} .17 \mathrm{~S} . \text {, } \\
R .5 \mathrm{~W} \text {. Dug well } 3 \text { feet in dianieter and } 22 \text { feet deep. Karl Kruk. } \\
\text { Water from decomposed gravel (terrace deposit). }\end{array}$ & ..... do. & 52 \\
\hline 695 & 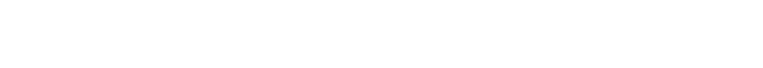 & Apr. 18,1929 & 53 \\
\hline 711 & $\begin{array}{l}\text { Eugene, } 2 \text { miles east of; in the SW1/4 SE } 1 / 4 \text { sec. } 28, \text { T. } 17 \text { S., R. } 3 \text { W. Dug } \\
\text { well } 3 \text { feet in diameter and } 161 / 2 \text { feet deep. Chose Gardens. Water } \\
\text { from old alluvium. }\end{array}$ & Oct. 12,1928 & 54 \\
\hline
\end{tabular}


representative wells in the Willamette Valley-Continued

\begin{tabular}{|c|c|c|c|c|c|c|c|c|c|c|c|c|c|}
\hline \multicolumn{14}{|c|}{ Analyses (parts per million) } \\
\hline \begin{tabular}{|c} 
Total \\
dis- \\
solved \\
solids \\
at $180^{\circ}$ \\
$\mathrm{C}$.
\end{tabular} & $\begin{array}{l}\text { Silica } \\
\left(\mathrm{SiO}_{2}\right)\end{array}$ & $\begin{array}{l}\text { Iron } \\
(\mathrm{Fe})\end{array}$ & $\begin{array}{l}\text { Cal- } \\
\text { cium } \\
\text { (Ca) }\end{array}$ & $\begin{array}{c}\text { Mag- } \\
\text { ne- } \\
\text { sium } \\
(\mathrm{Mg})\end{array}$ & $\begin{array}{c}\text { So- } \\
\text { dium } \\
\text { (Na) }\end{array}$ & $\begin{array}{c}\text { Po- } \\
\text { tas- } \\
\text { sium } \\
(\mathbf{K})\end{array}$ & $\begin{array}{c}\text { Bicar- } \\
\text { bonate } \\
\left(\mathrm{HCO}_{3}\right)\end{array}$ & $\begin{array}{c}\text { Sul- } \\
\text { phate } \\
\left(\mathrm{SO}_{4}\right)\end{array}$ & $\begin{array}{l}\text { Chlo- } \\
\text { ride } \\
\text { (Cl) }\end{array}$ & $\begin{array}{c}\mathrm{Ni}- \\
\text { trate } \\
\left(\mathrm{NO}_{3}\right)\end{array}$ & $\begin{array}{c}\text { Total } \\
\text { hard- } \\
\text { ness as } \\
\mathrm{CaCO}_{3}\end{array}$ & $\begin{array}{c}\text { Per- } \\
\text { cent } \\
\text { so- } \\
\text { dium }\end{array}$ & No. \\
\hline 18,102 & 19 & 0.86 & 4,630 & 22 & 2,025 & 18 & 20 & 3.5 & 11,381 & 0.0 & 11,665 & 27.5 & 533 \\
\hline 204 & 35 & .08 & 31 & 18 & 14 & 1.1 & 193 & 2.1 & 13 & 3.8 & 151 & 17.4 & 551 \\
\hline 98 & 36 & .03 & 12 & 5.1 & 6.3 & 1.4 & 74 & 3.5 & 2.0 & .25 & 51 & 23.3 & 566 \\
\hline 197 & 49 & .09 & 26 & 15 & 7.0 & 2.2 & 134 & 4.7 & 9.0 & 16 & 126 & 12.5 & 580 \\
\hline 220 & 52 & .03 & 27 & 16 & 21 & 1.2 & 168 & 3.5 & 28 & .73 & 133 & 26.2 & 595 \\
\hline 502 & 45 & .03 & 74 & 23 & 55 & 1.9 & 211 & 2.1 & 153 & 9.0 & 279 & 30.4 & 606 \\
\hline 207 & 49 & .18 & 24 & 16 & 7.0 & 1.8 & 110 & 10 & 22 & 10 & 126 & 12.2 & 631 \\
\hline 168 & 40 & .38 & 15 & 10 & 24 & 1.2 & 100 & 2.7 & 32 & .05 & 78 & 40.6 & 638 \\
\hline 56 & 26 & .06 & 5.0 & 2.9 & 3.2 & 1.5 & 33 & 3.8 & 1.8 & .20 & 24 & 26. 7 & 653 \\
\hline 26 & 8.8 & . 16 & 2.0 & 1.1 & 2.2 & .5 & 5. 0 & 3.4 & 2.2 & 4.0 & 9.5 & 36.3 & 690 \\
\hline 109 & 51 & .02 & 9.0 & 5.1 & 5.5 & .4 & 58 & 2.8 & 2.2 & 2.7 & 43 & 22.3 & 695 \\
\hline 128 & 43 & .06 & 15 & 9.0 & 4.0 & 1.4 & 84 & 5.9 & 2.7 & 4.6 & 74 & 12.3 & 711 \\
\hline
\end{tabular}


the southern half of the valley, between Salem and Eugene. All the samples of water from the unconsolidated materials in the northern half of the valley contained more than 0.1 part per million of iron; the greatest quantity, 5.27 parts per million, was from well 164 , which is 7 miles northwest of Woodburn. The general data on wells in the vicinity of Portland indicate that several yield water containing an excessive quantity of iron; these include wells $29,30,47,52$, and 77. Other wells in the same area doubtless yield water with an iron content so large as to be troublesome for some uses. From most waters, the excess iron may be removed by simple aeration and filtration; some waters, however, must be treated with lime or certain other substances to assist in precipitating the excess iron.

Reference has been made before to the salty to brackish water in the consolidated sedimentary rocks. The analyses of samples from wells 490 and 533 show that these waters are chemically unfit for many ordinary uses. The sample from well 606 doubtless represents a water of mixed origin-nonsaline water from the older alluvium with a small admixture of saline water from the underlying sedimentary rock. Saline water is reported to have been struck in numerous other wells that are widely scattered in the region. These include wells 4,5 , and 15 , which entered bedrock, presumably sedimentary rock of marine origin, beneath the flood-plain deposit of the Columbia River downstream from Portland; wells 112, 123 to 125, 142, 200, 218, and 287, in the basin of the South Yamhill River near McMinnville, in Tps. 4 to 6 S., Rs. 4 and 5 W.; wells $375,391,469,533$, and 537, along the west flank of the central lowland from Monmouth southward to Corvallis and somewhat beyond, in Tps. 8 to 12 S., R. 4 W.; wells 490 and 610 , on the lowland east of the Willamette River, in R. 4 W., Tps. 11 and 14 S., respectively; and well 641, along the east flank of the lowland in T. 15 S., R. 3 W. These general data suggest that wells much more than 100 feet deep at any place along the flank of the western foothills, or wells in the western half of the central lowland that pass through the unconsolidated deposits and penetrate the sedimentary rocks more than a few tens of feet, may strike salty water unfit for most uses, certainly unfit for domestic supplies or for irrigation. 
WELL RECORDS

TABLE 10.-Wells in the Willamette Valley for which records of ground-water level have been published in other water-supply papers ${ }^{1}$

\begin{tabular}{|c|c|c|c|c|c|}
\hline $\begin{array}{c}\text { Number } \\
\text { of well in } \\
\text { this re- } \\
\text { port }\end{array}$ & $\begin{array}{c}\text { Number of } \\
\text { well in other } \\
\text { published } \\
\text { water-supply } \\
\text { papers }\end{array}$ & Owner & $\begin{array}{l}\text { Number } \\
\text { of well in } \\
\text { this re- } \\
\text { port }\end{array}$ & $\begin{array}{c}\text { Number of } \\
\text { well in other } \\
\text { published } \\
\text { water-supply } \\
\text { papers }\end{array}$ & Owner \\
\hline $\begin{array}{l}158 \ldots \\
171 \ldots \ldots \\
196 \ldots \ldots \\
297 \ldots \ldots \\
318 \ldots \ldots \\
421 \ldots \ldots \\
463 \ldots \ldots\end{array}$ & $\begin{array}{l}8 \mathrm{H}-4 \mathrm{cl} \\
8 \mathrm{H}-34 \mathrm{rl} \ldots \\
9 \mathrm{E}-13 \mathrm{bl} \\
10 \mathrm{G}-33 \mathrm{r} 1 \ldots \\
10 \mathrm{~K}-7 \mathrm{ml} \\
14 \mathrm{~F}-12 \mathrm{f1} \\
15 \mathrm{E}-36 \mathrm{q} 1\end{array}$ & $\begin{array}{l}\text { W. J. Gering. } \\
\text { Johnson School. } \\
\text { George Fuller. } \\
\text { Gideon E. Stolz. } \\
\text { Fred Lucht. } \\
\text { Henry Hoefer. } \\
\text { Oregon Agricultural Ex- } \\
\text { periment Station. }\end{array}$ & $\begin{array}{l}553 \ldots \\
568 \ldots \\
590 \ldots \\
636 \ldots \ldots \\
680 \ldots \ldots\end{array}$ & $\begin{array}{l}16 \mathrm{G}-9 \mathrm{r} 1 \\
16 \mathrm{H}-14 \mathrm{cl} \\
17 \mathrm{G}-34 \mathrm{n1} \\
19 \mathrm{~F}-32 \mathrm{ml} \\
20 \mathrm{G}-32 \mathrm{~g} 3\end{array}$ & $\begin{array}{l}\text { J. H. Swatzka. } \\
\text { Ray Fisher. } \\
\text { Keeney School. } \\
\text { Junction City. } \\
\text { Leo Sidwell. }\end{array}$ \\
\hline
\end{tabular}

1 U. S. Geol. Survey Water-Supply Papers 777, pp. 145-149, 1936; 817, pp. 256-259, 1937. 


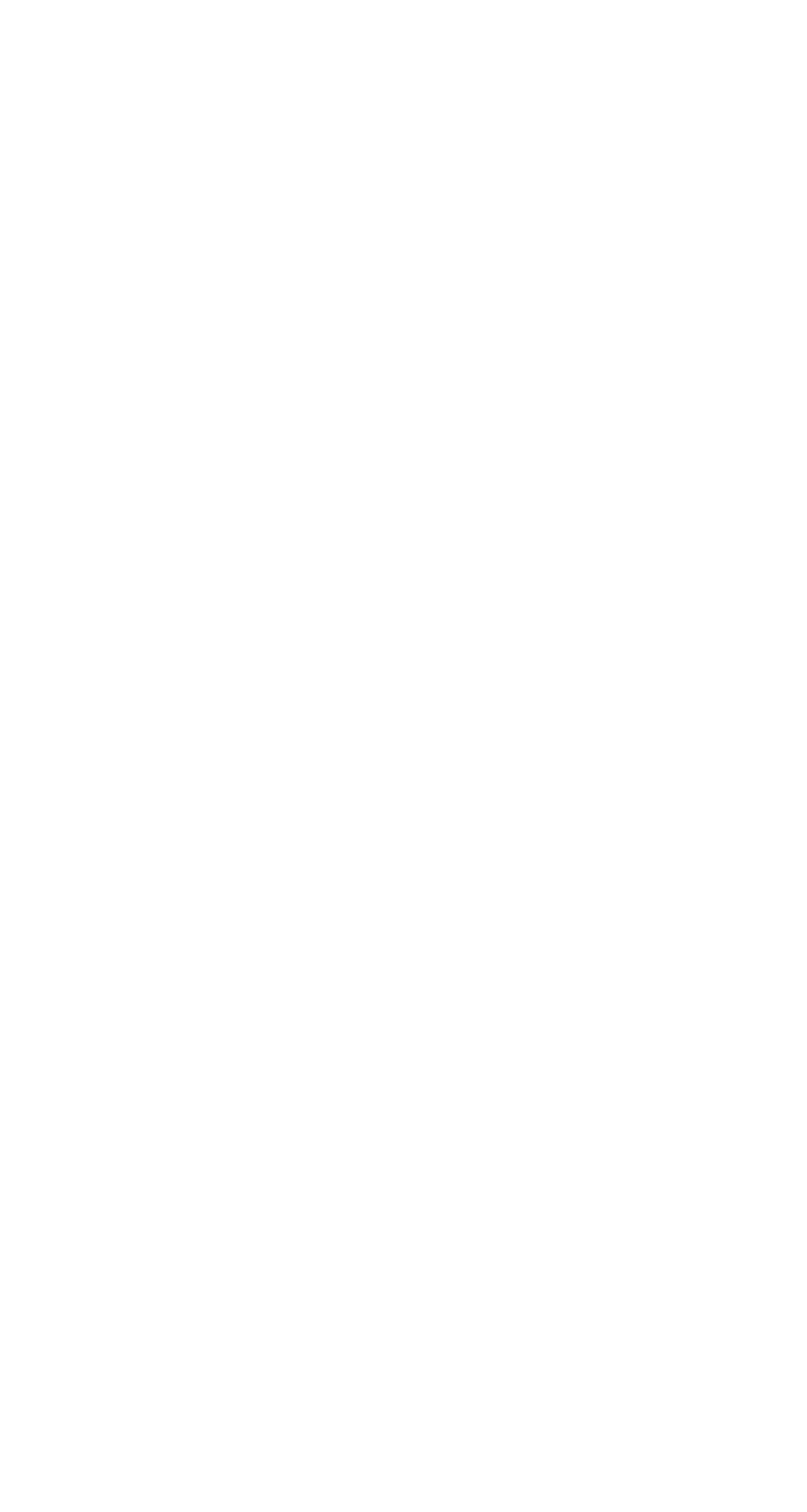


WELL RECORDS

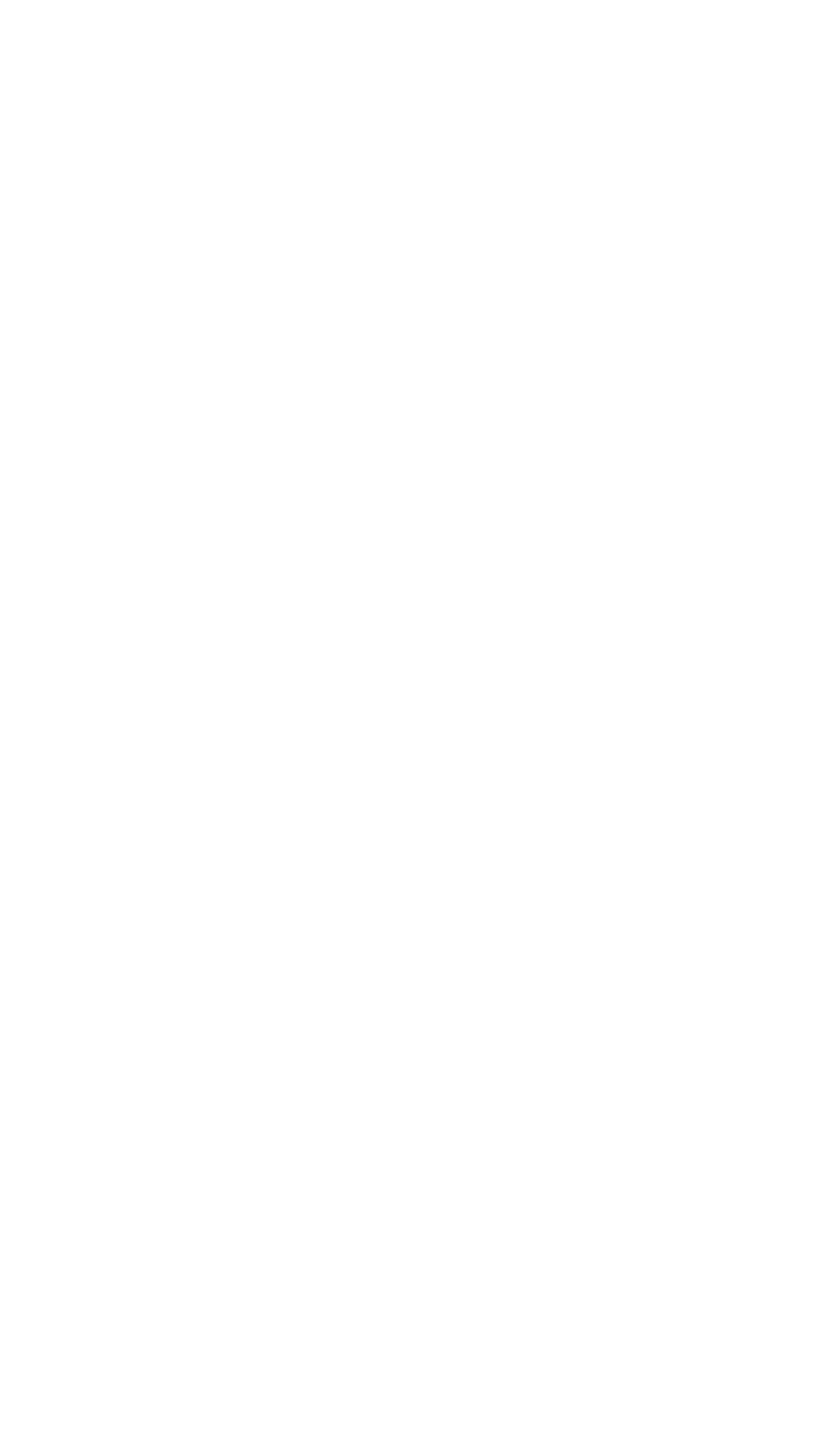




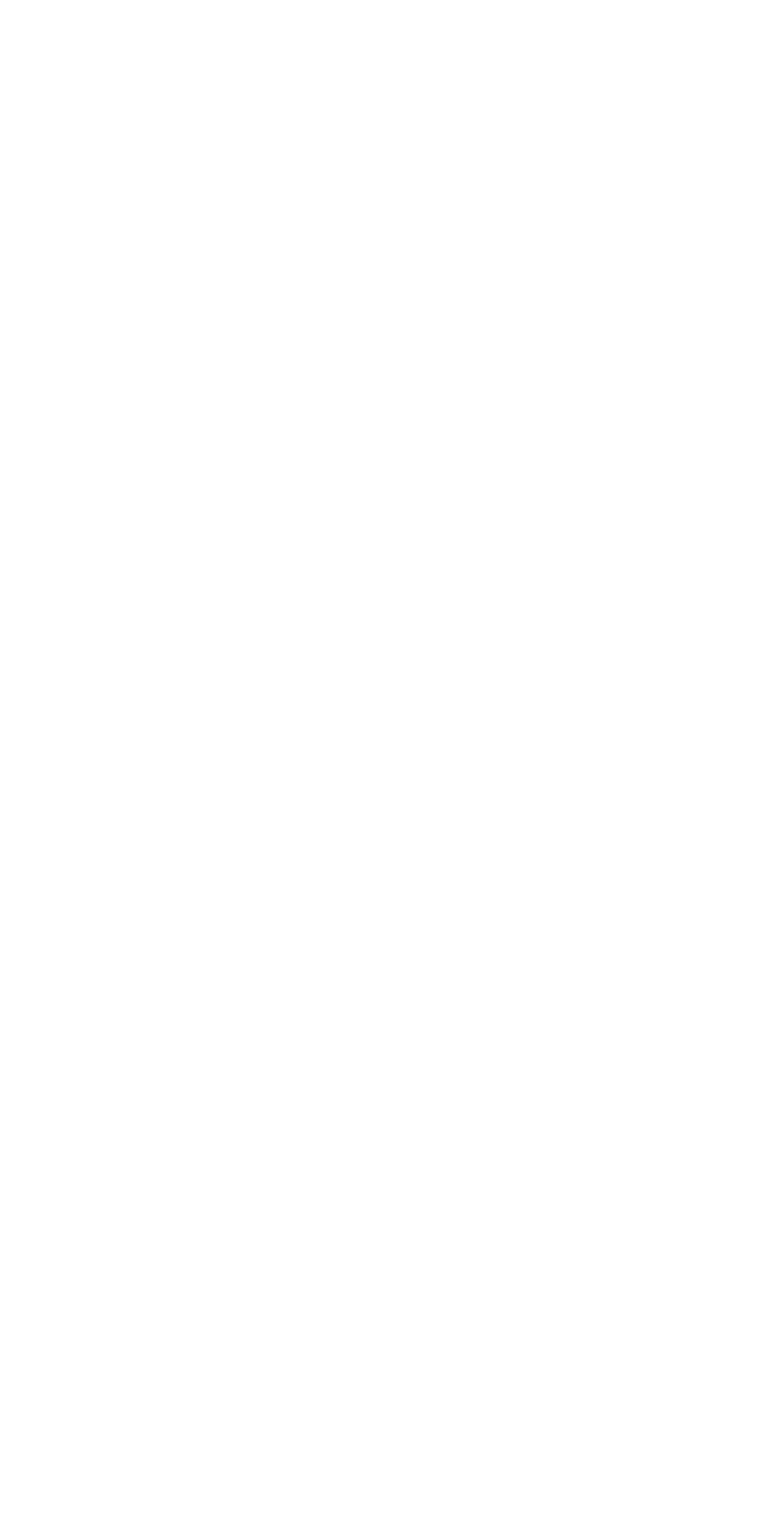




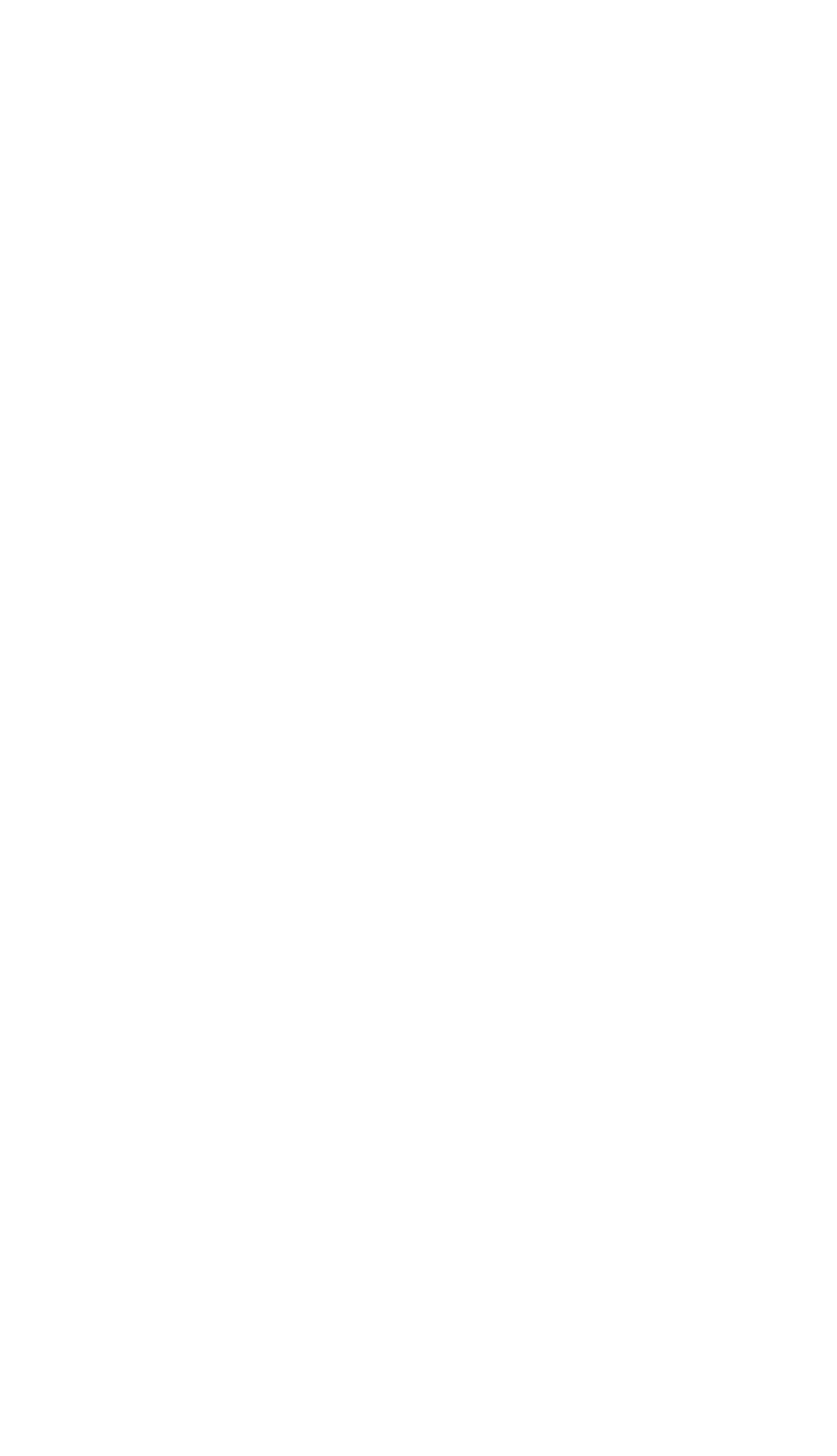




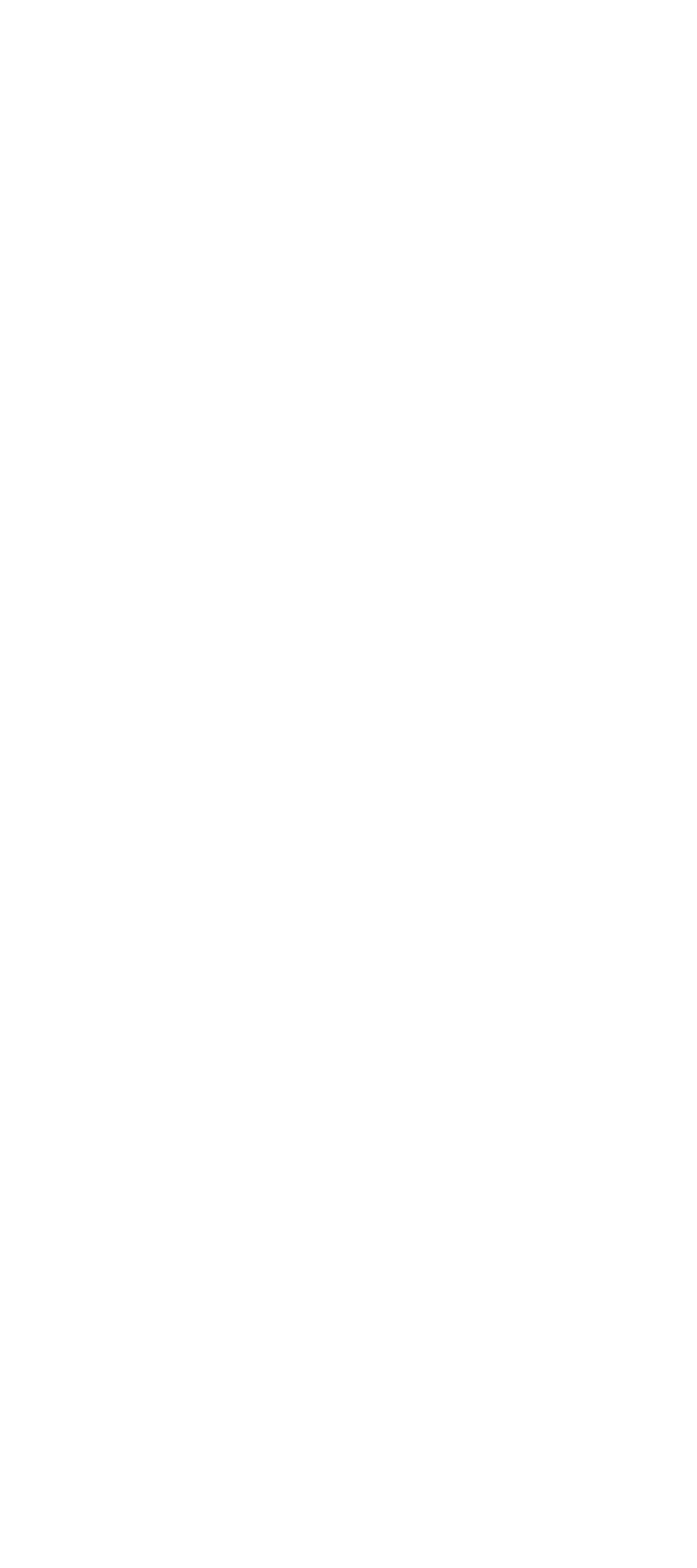




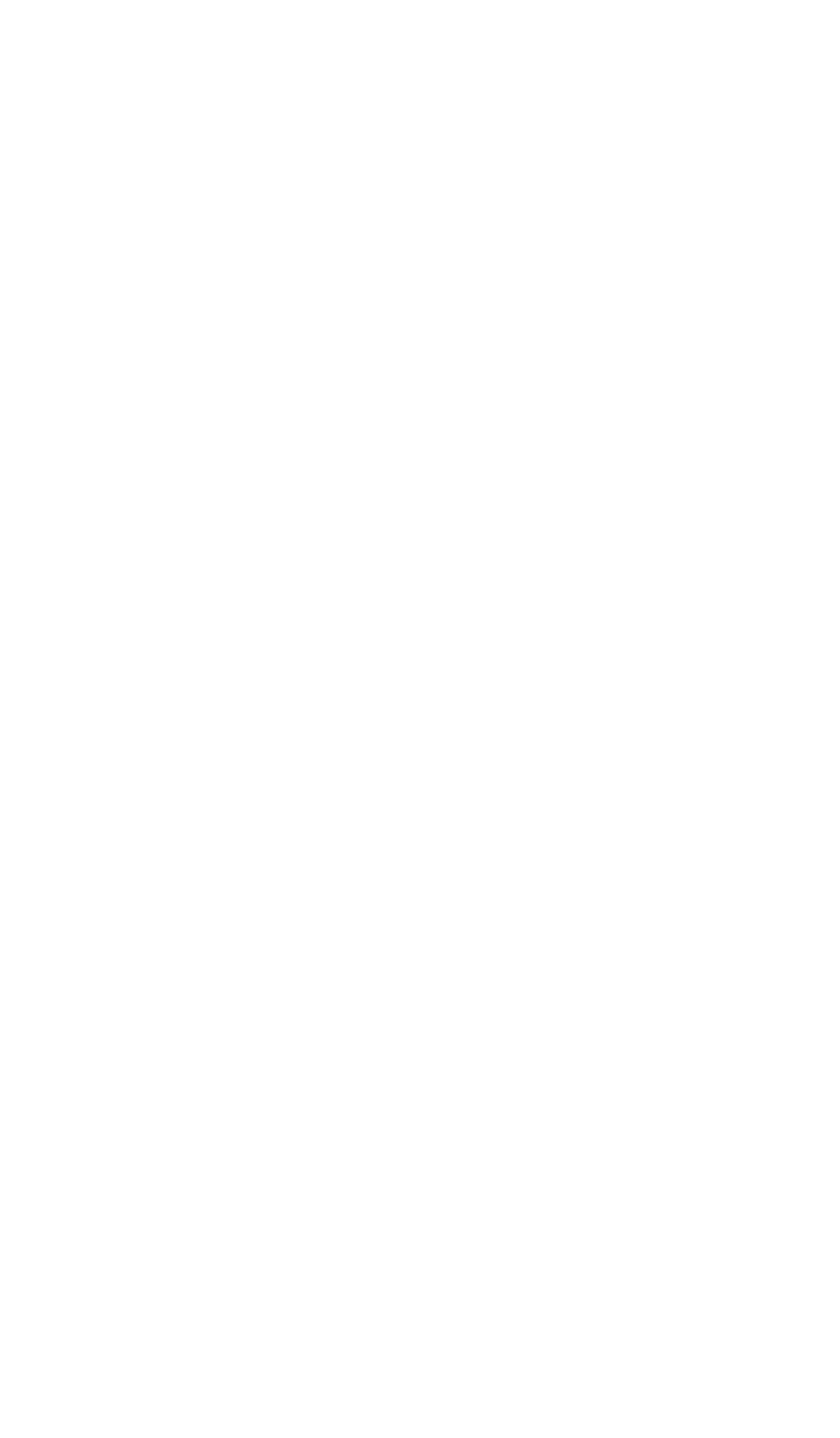




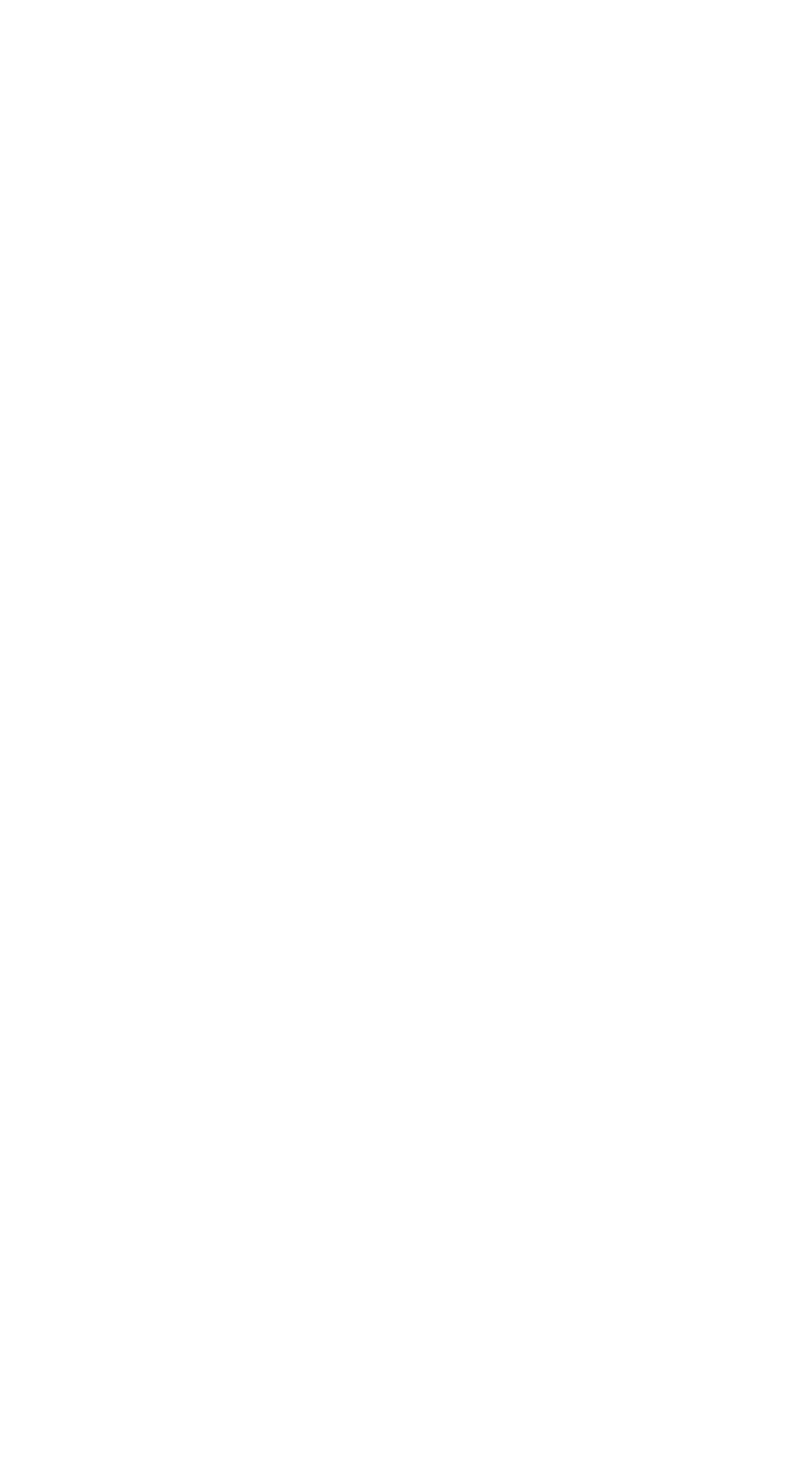




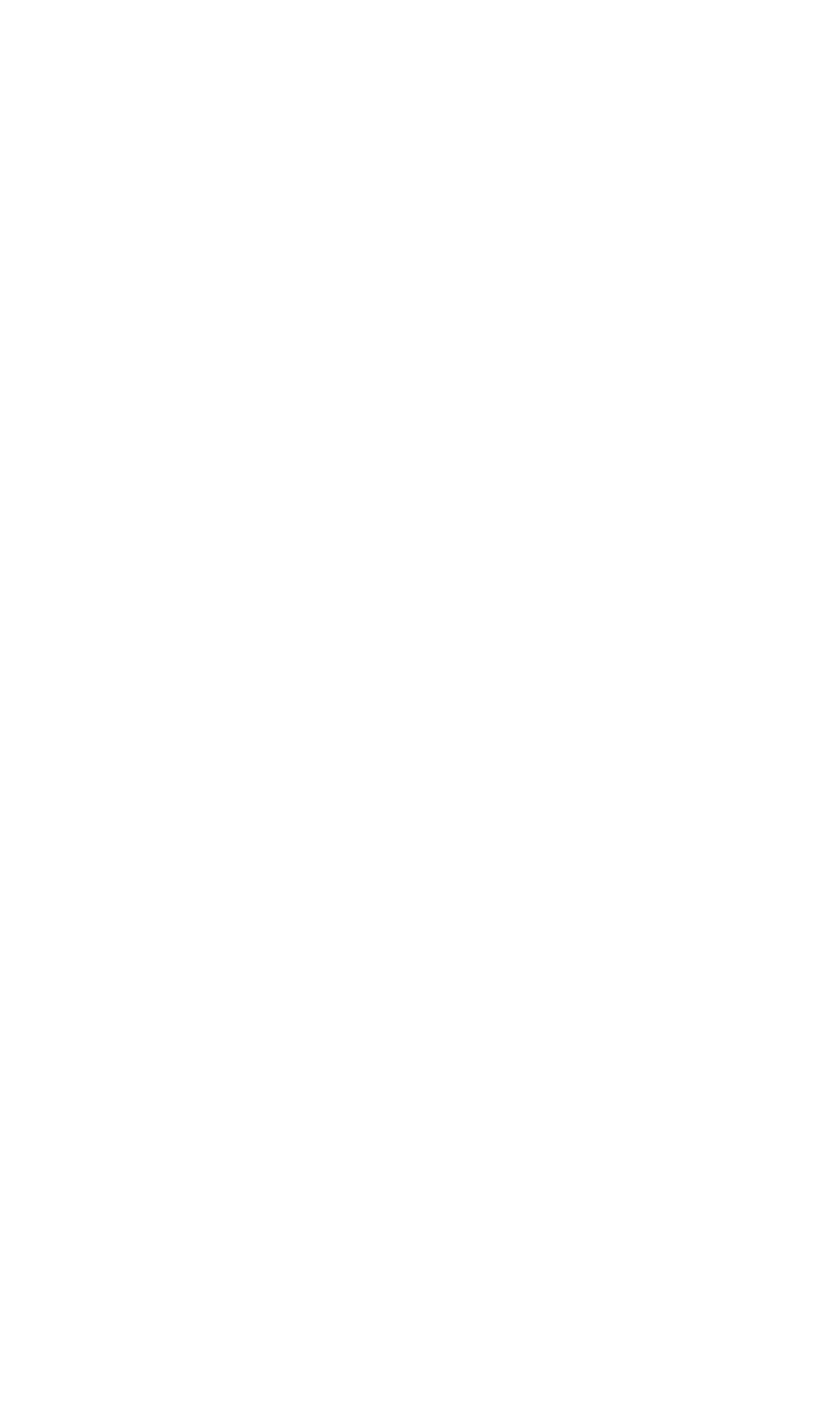




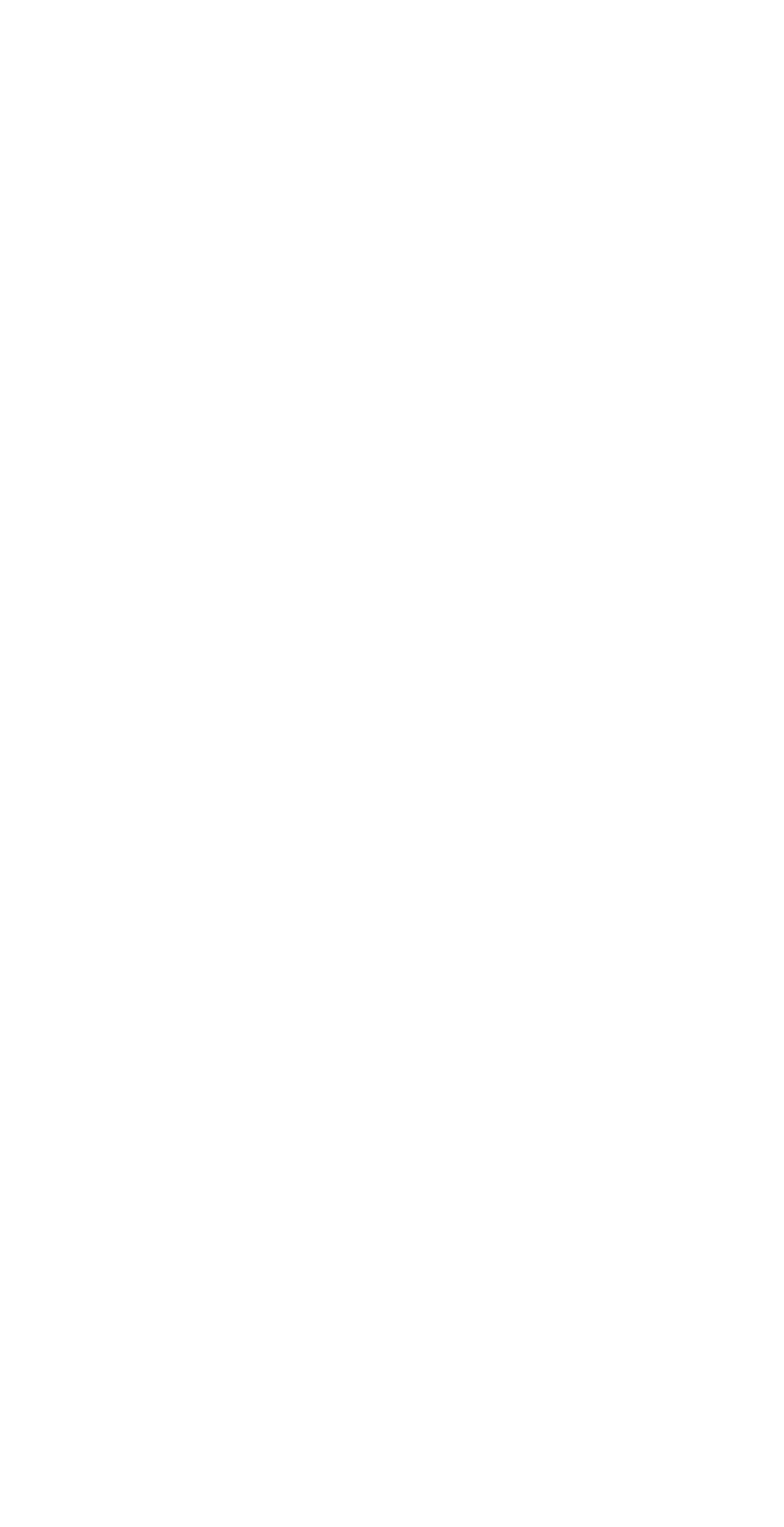




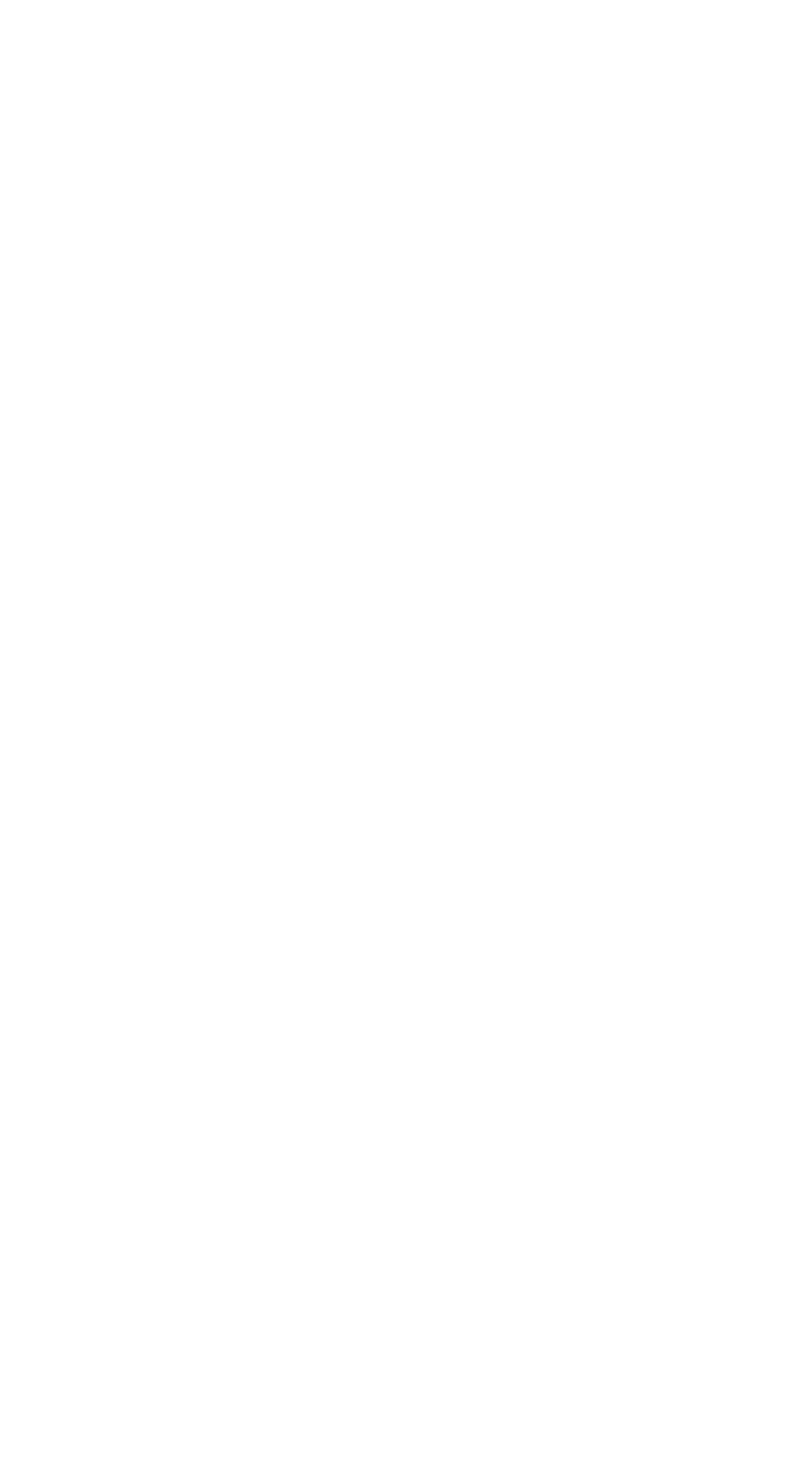




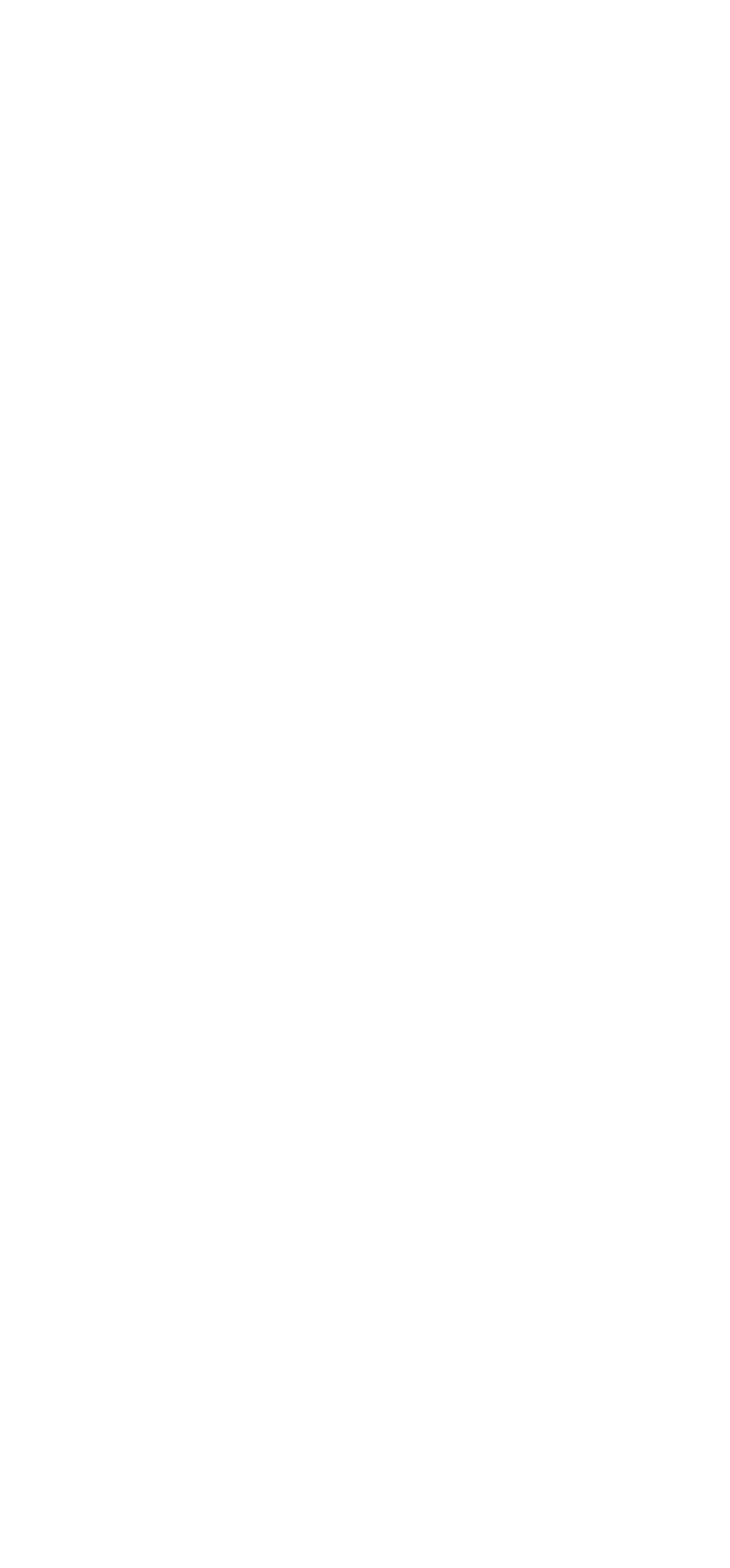




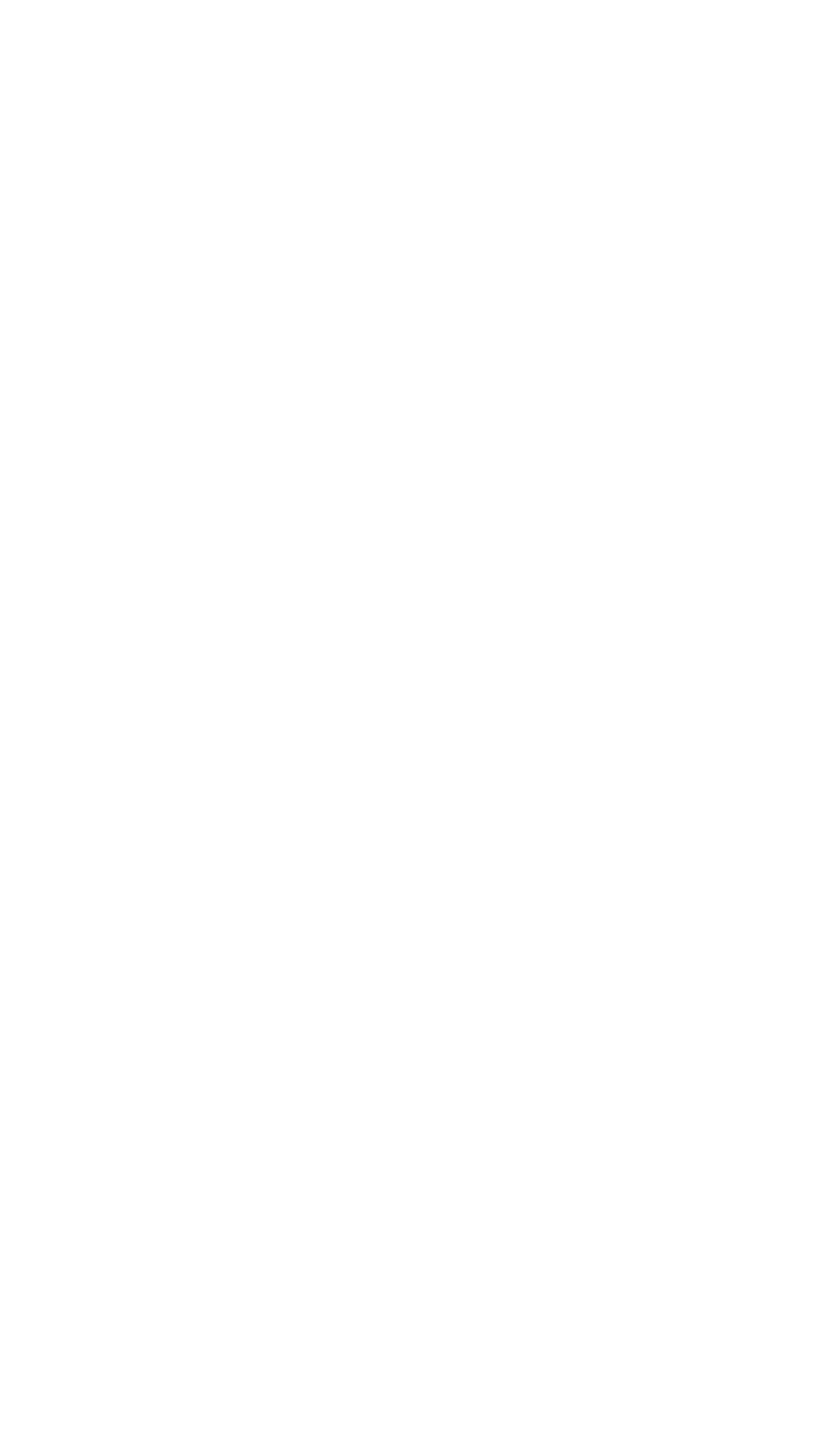




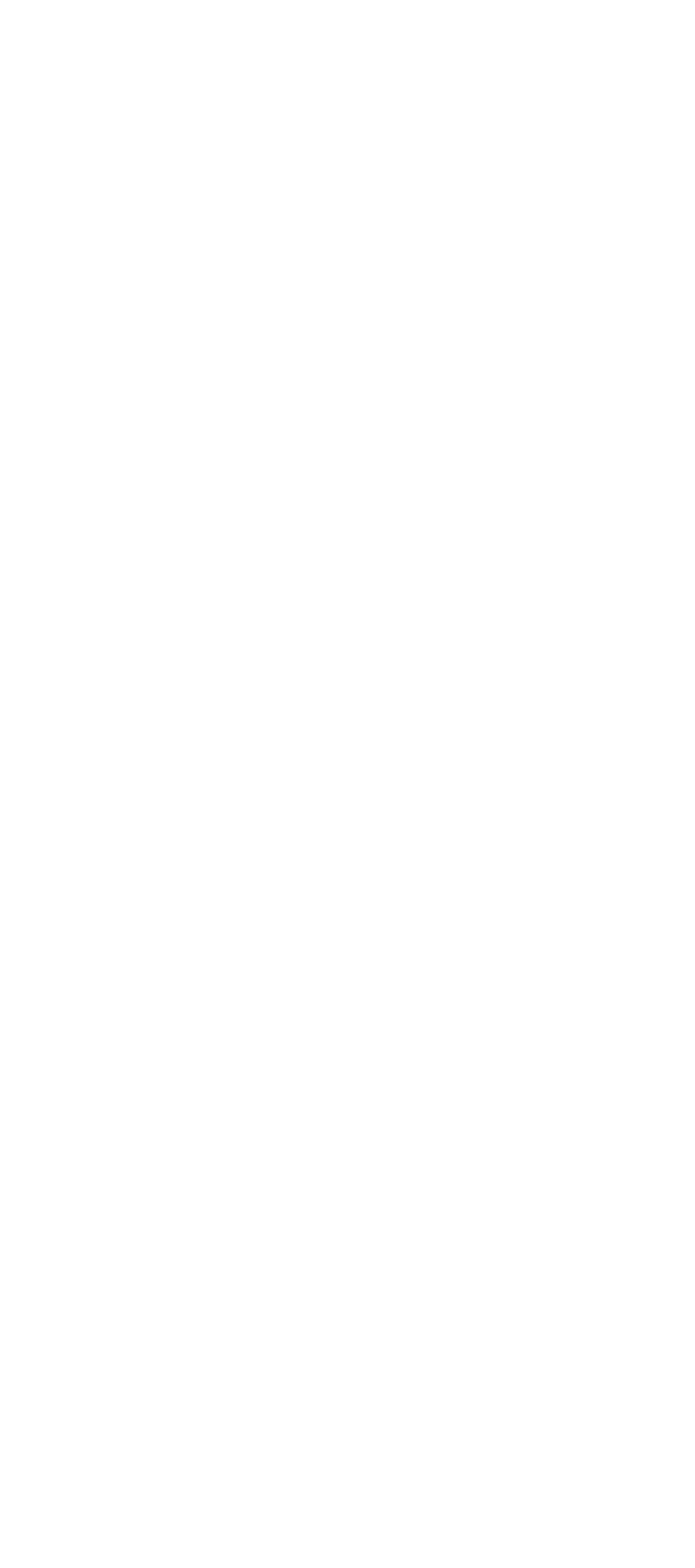




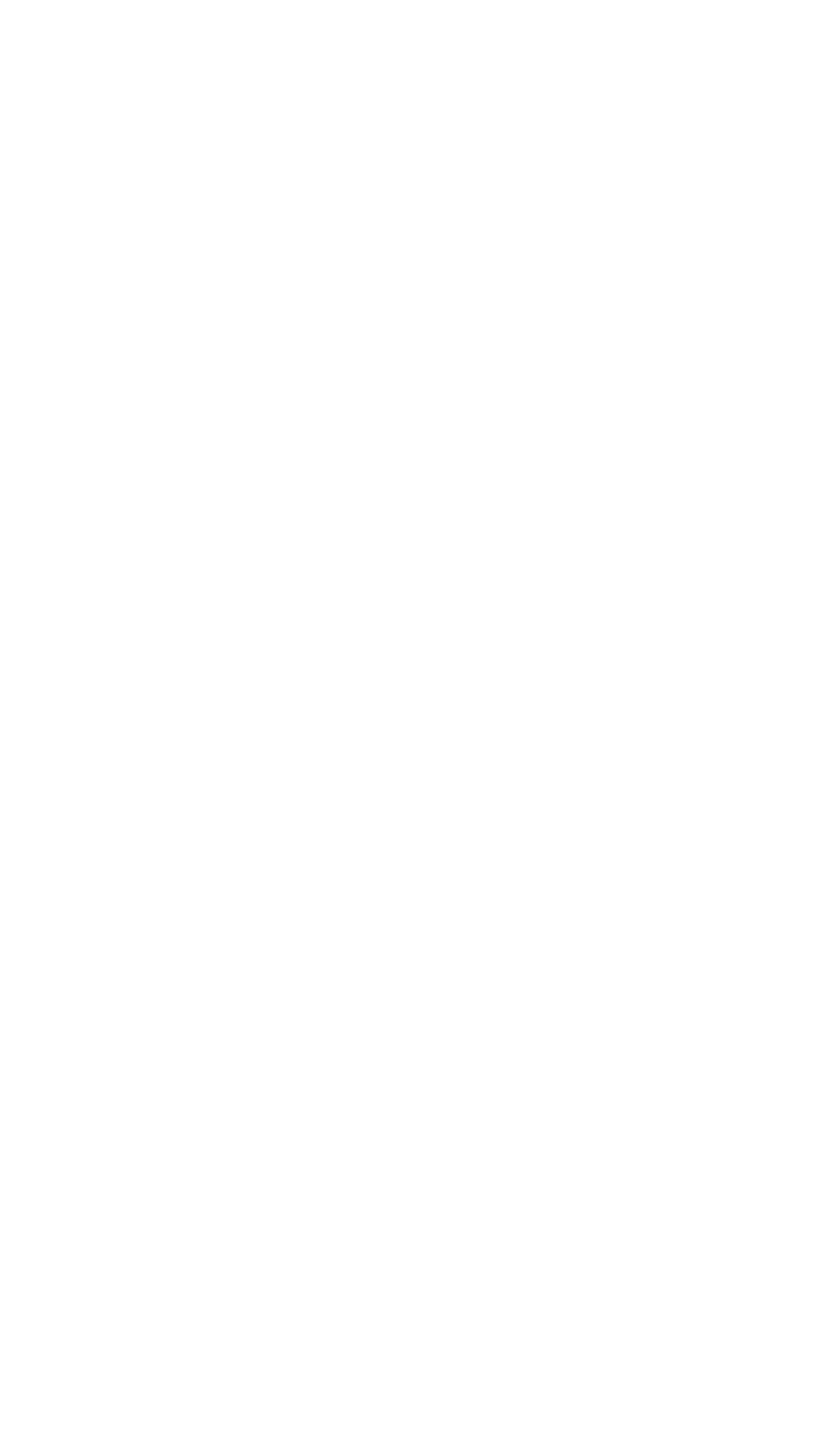




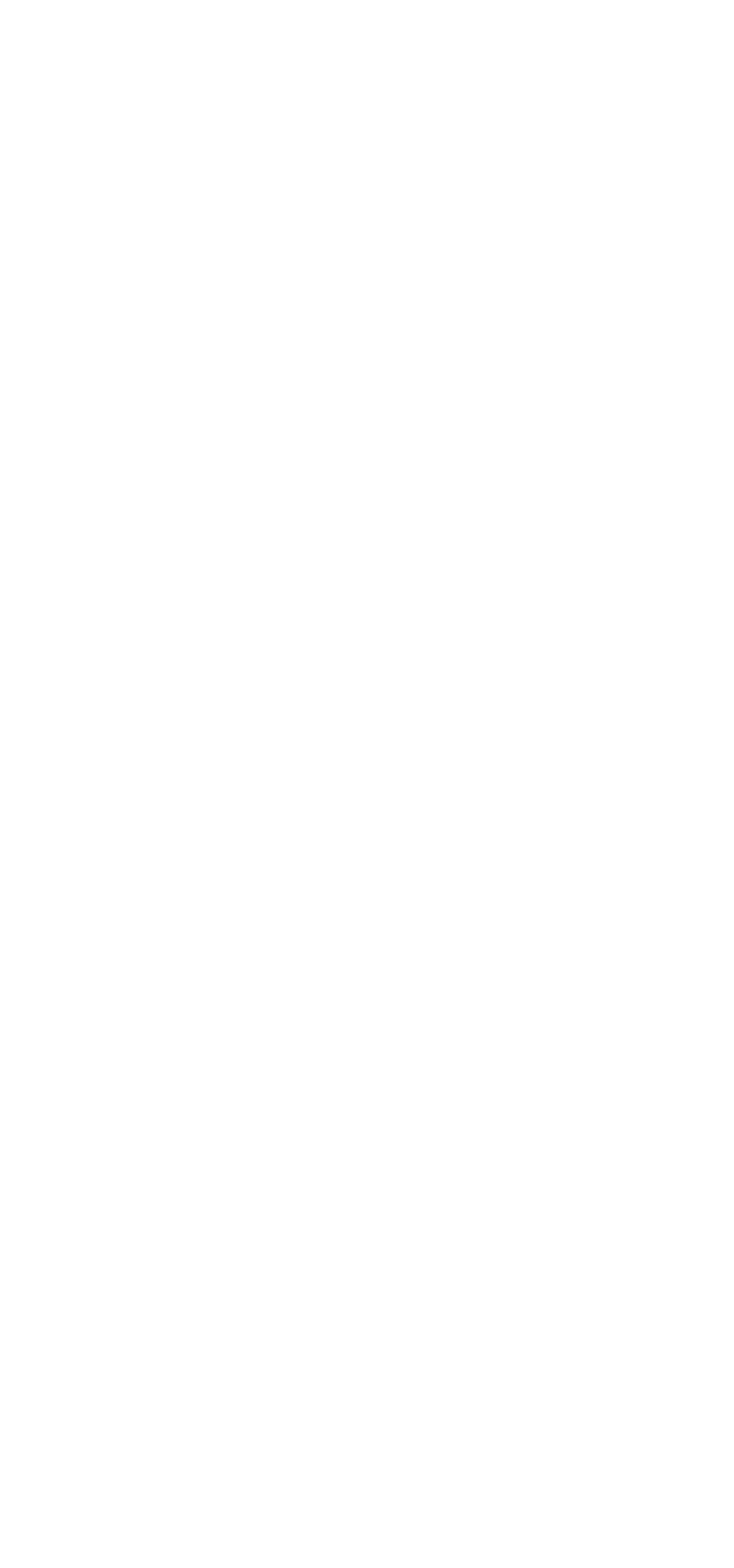




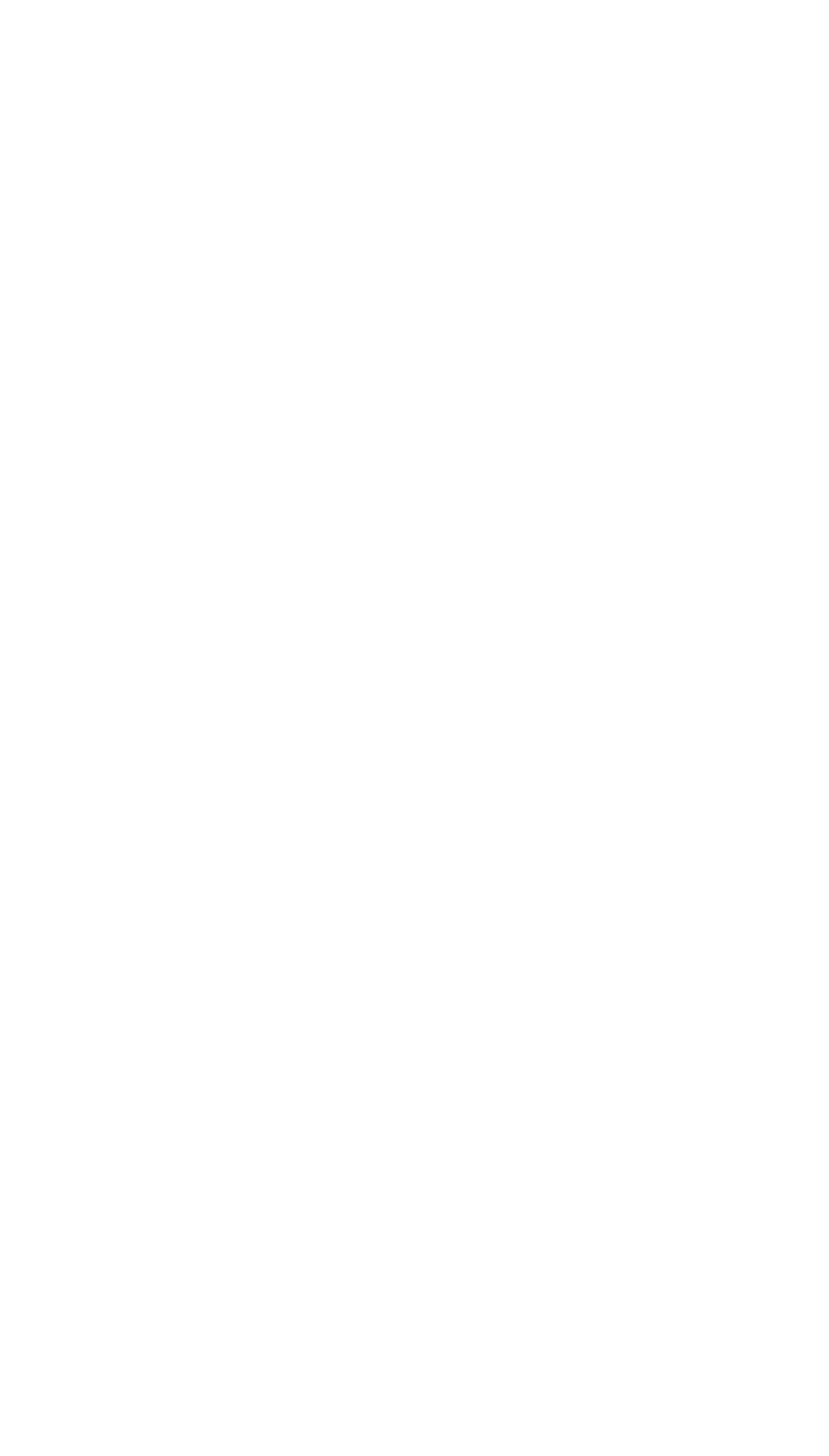




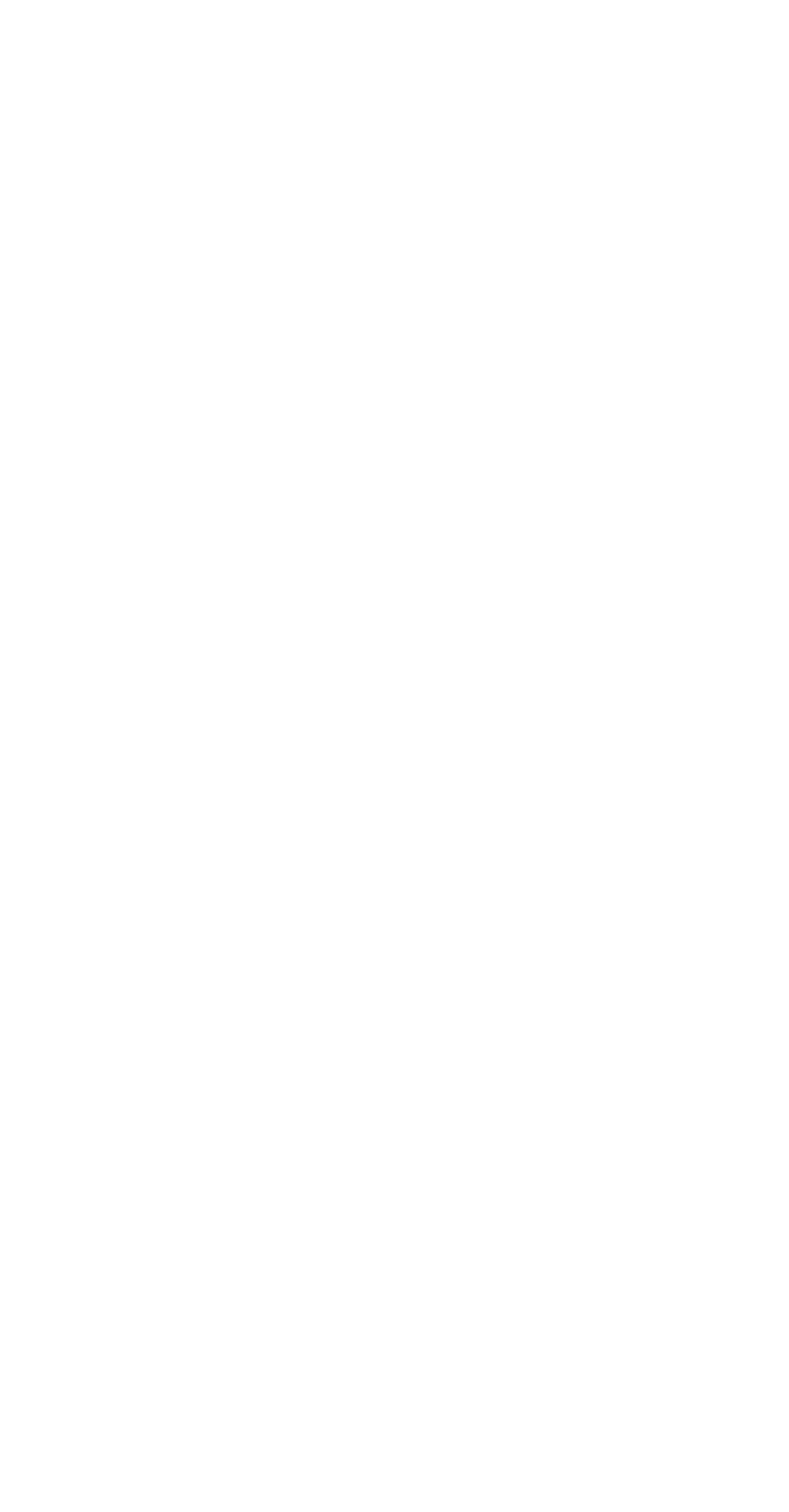




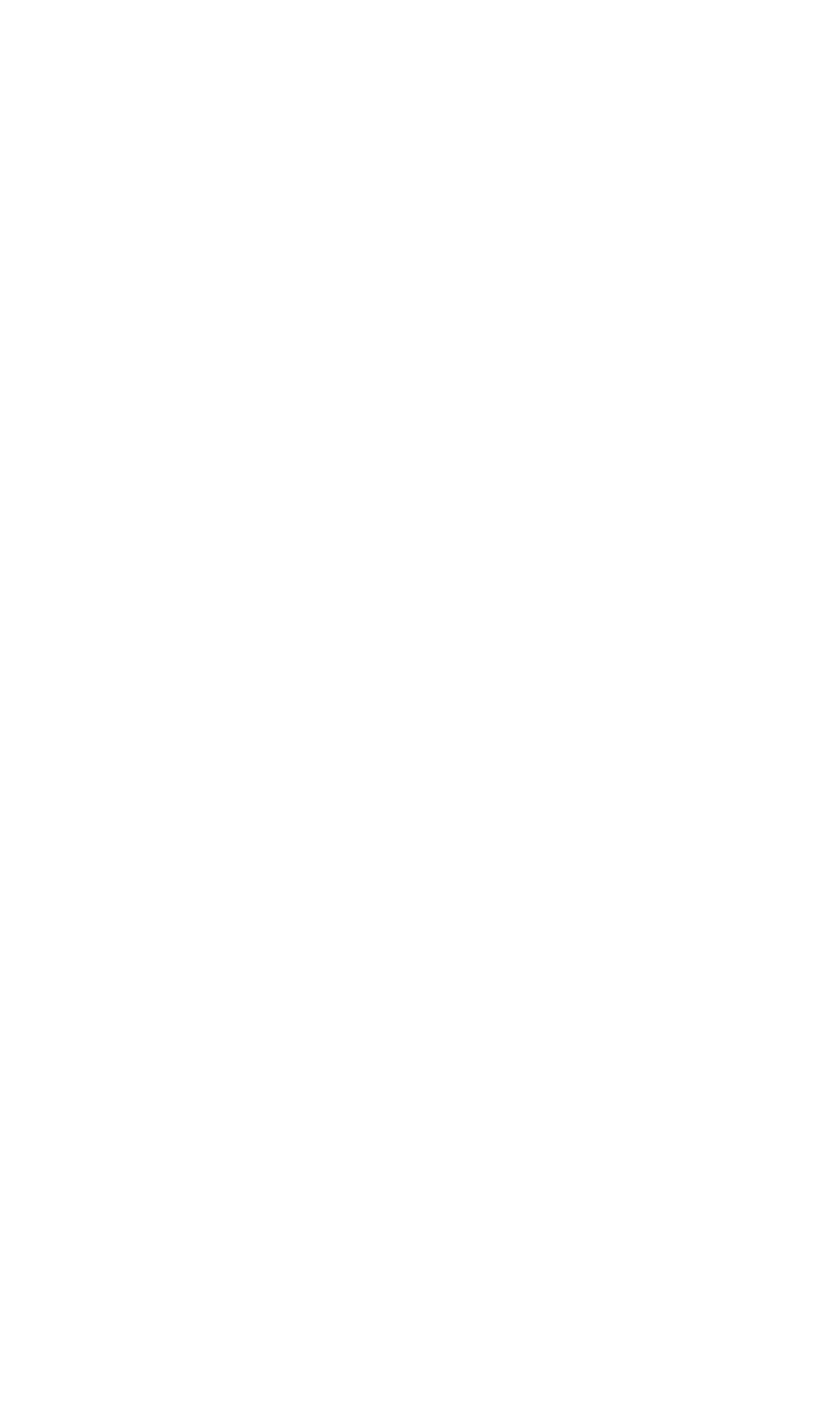




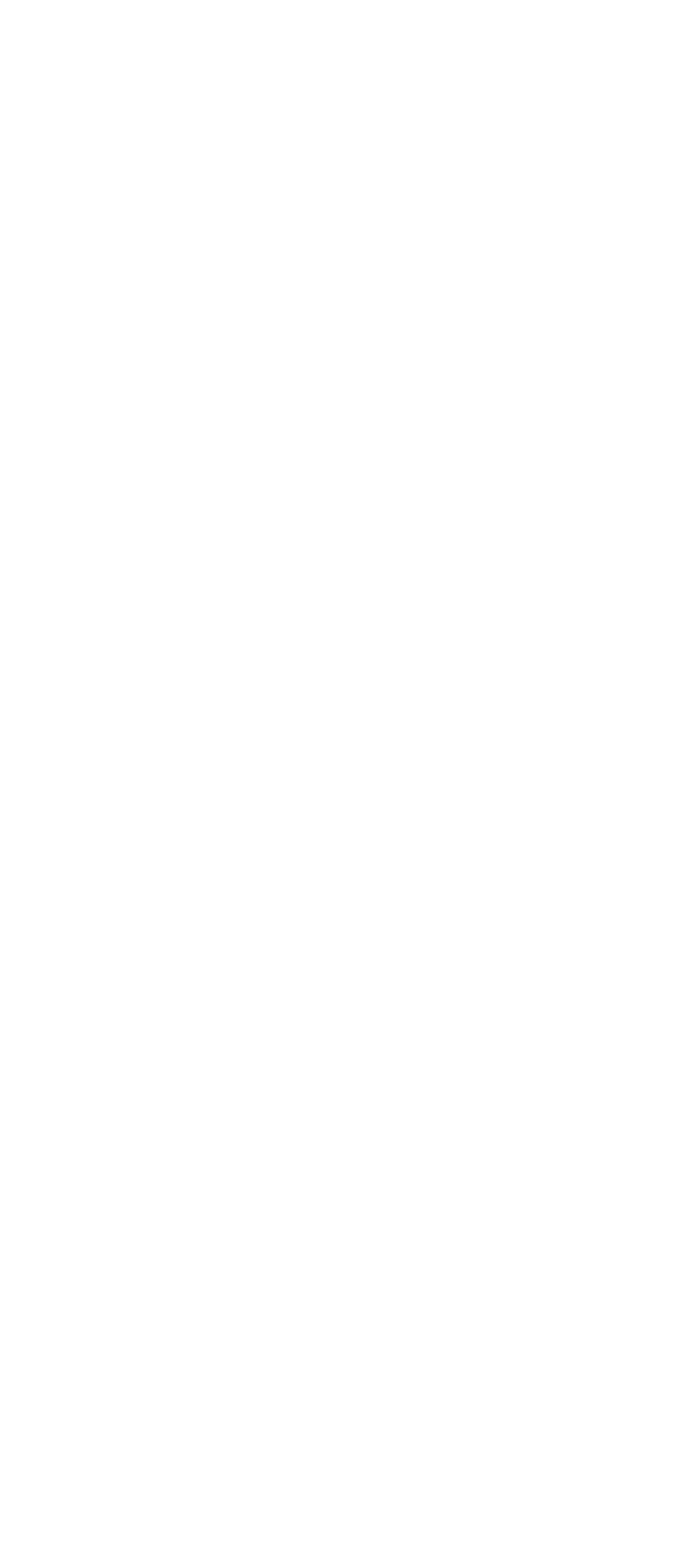




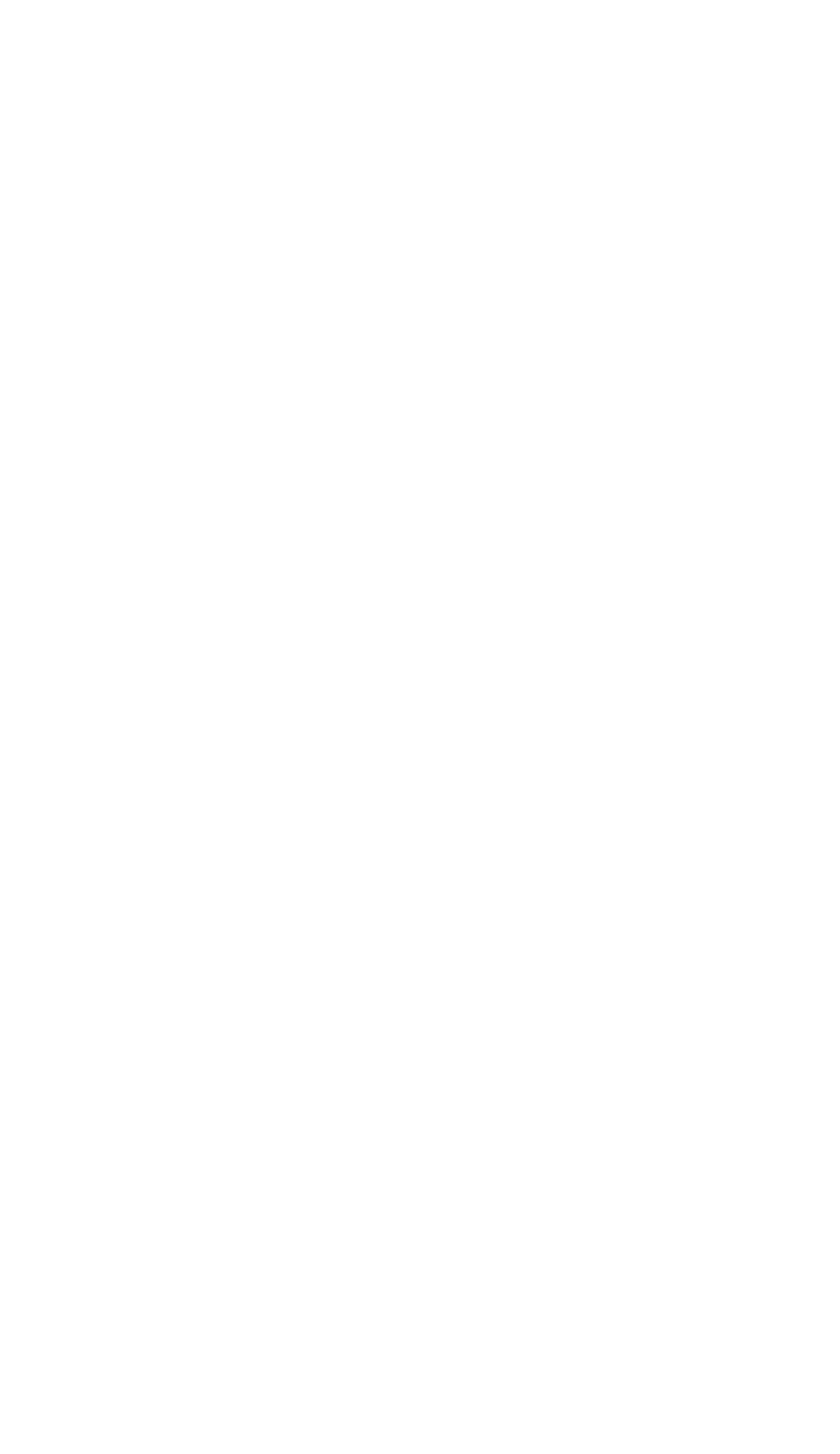




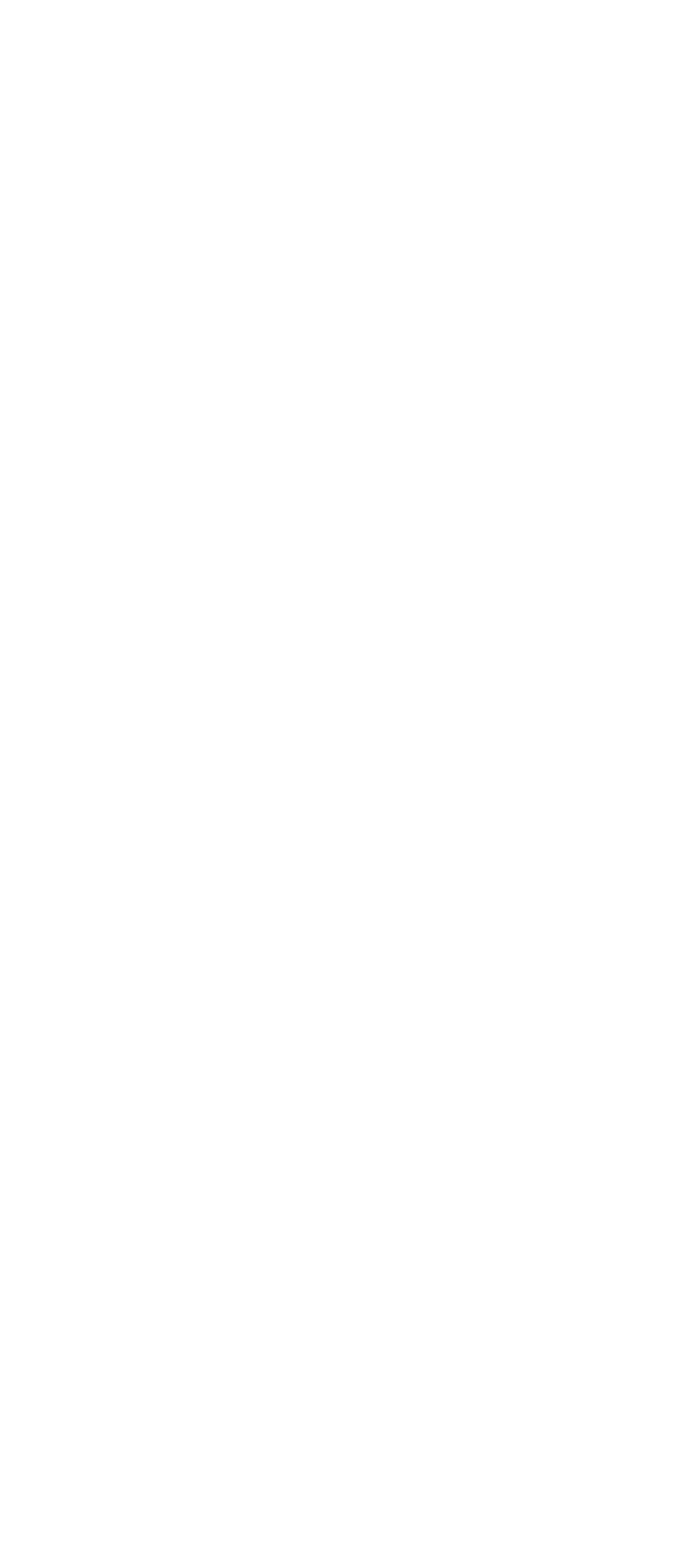


WELL RECORDS

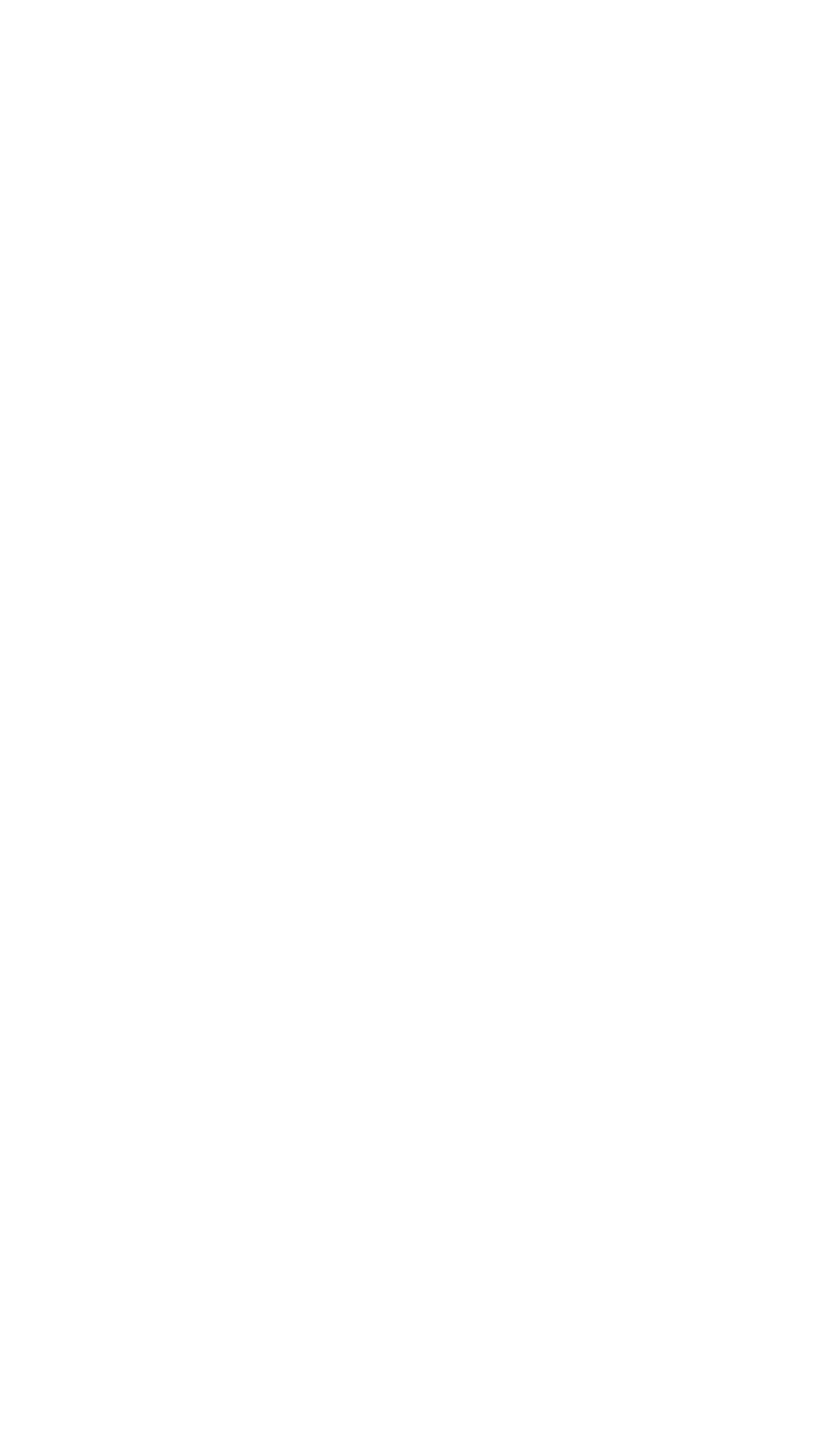




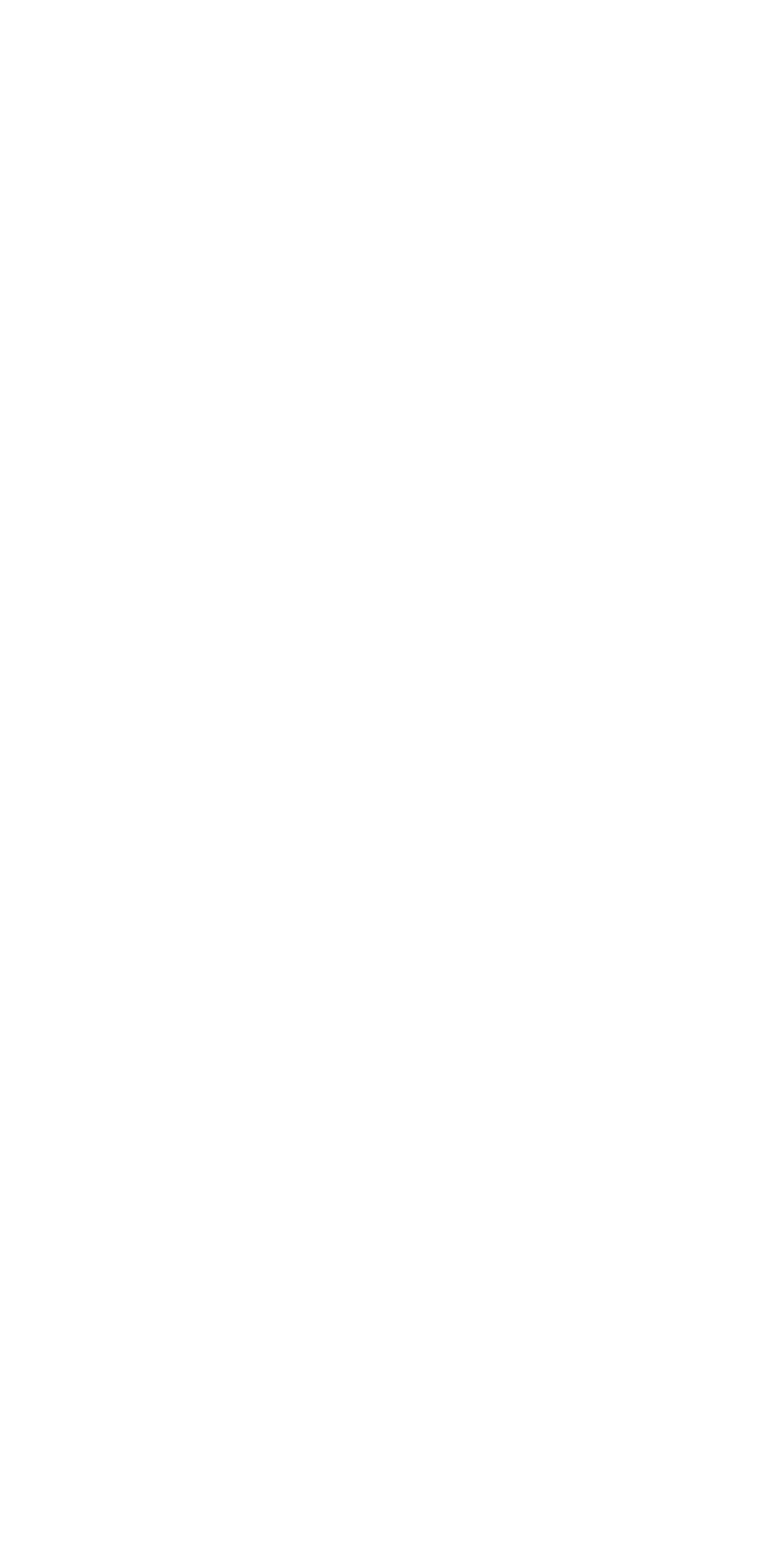




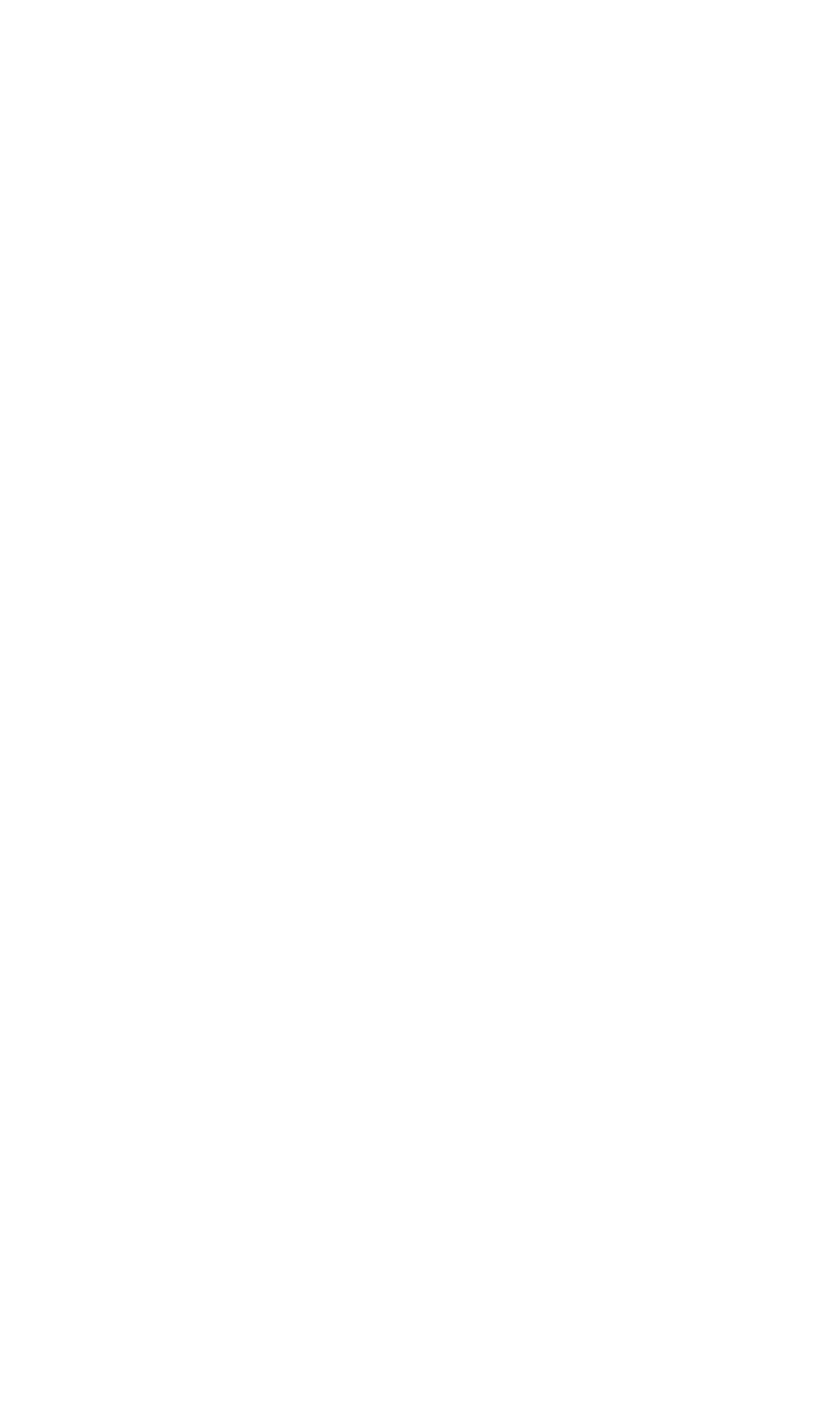




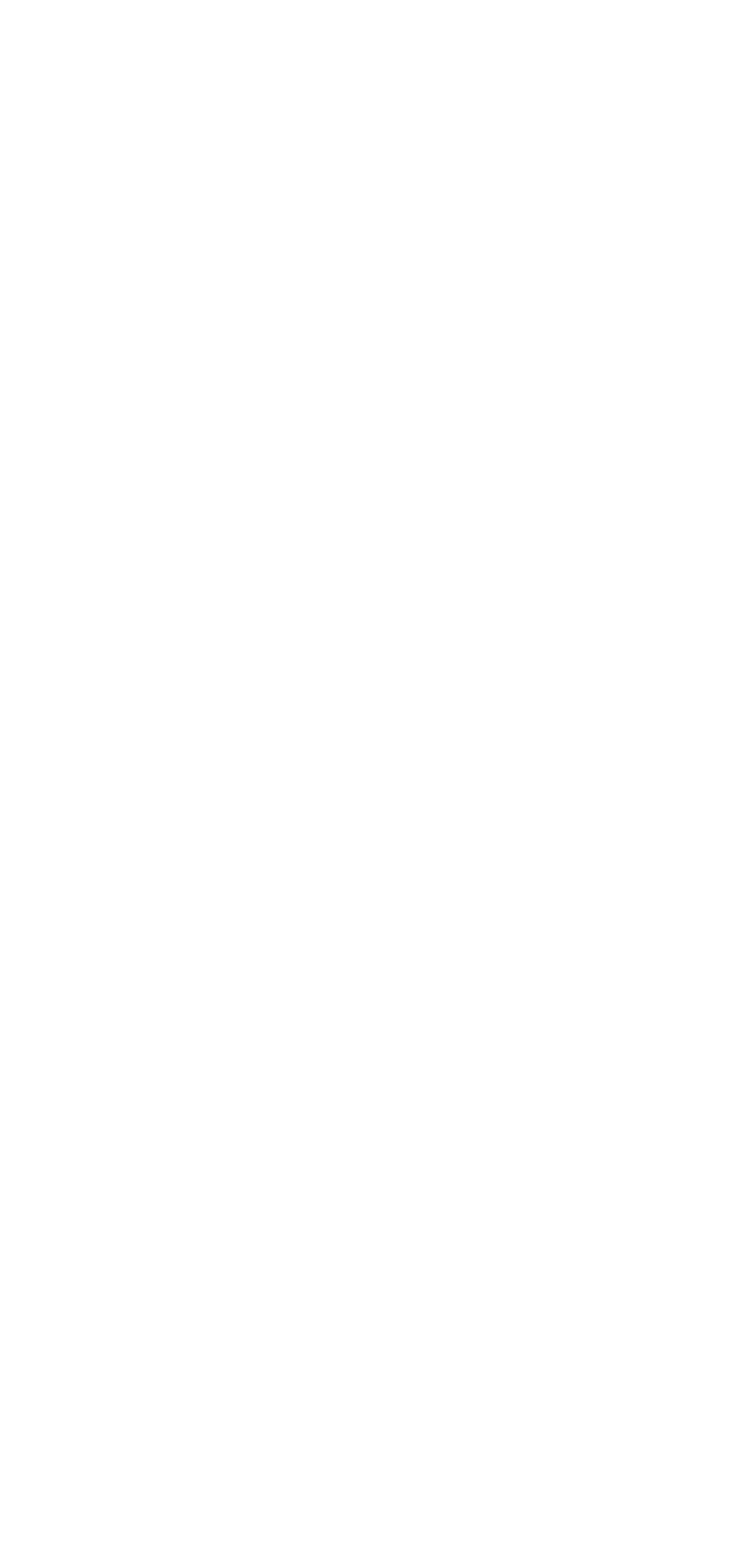




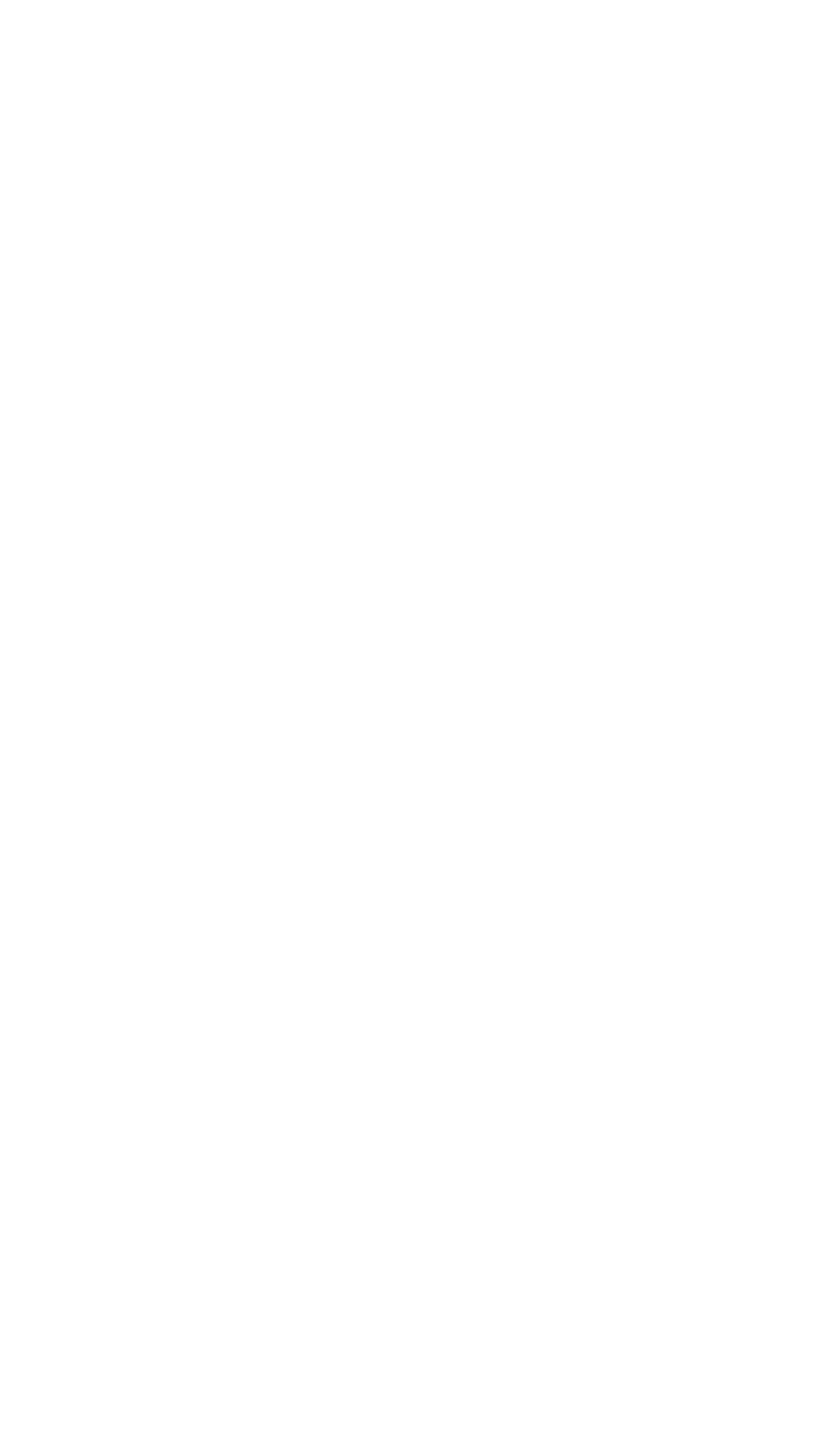




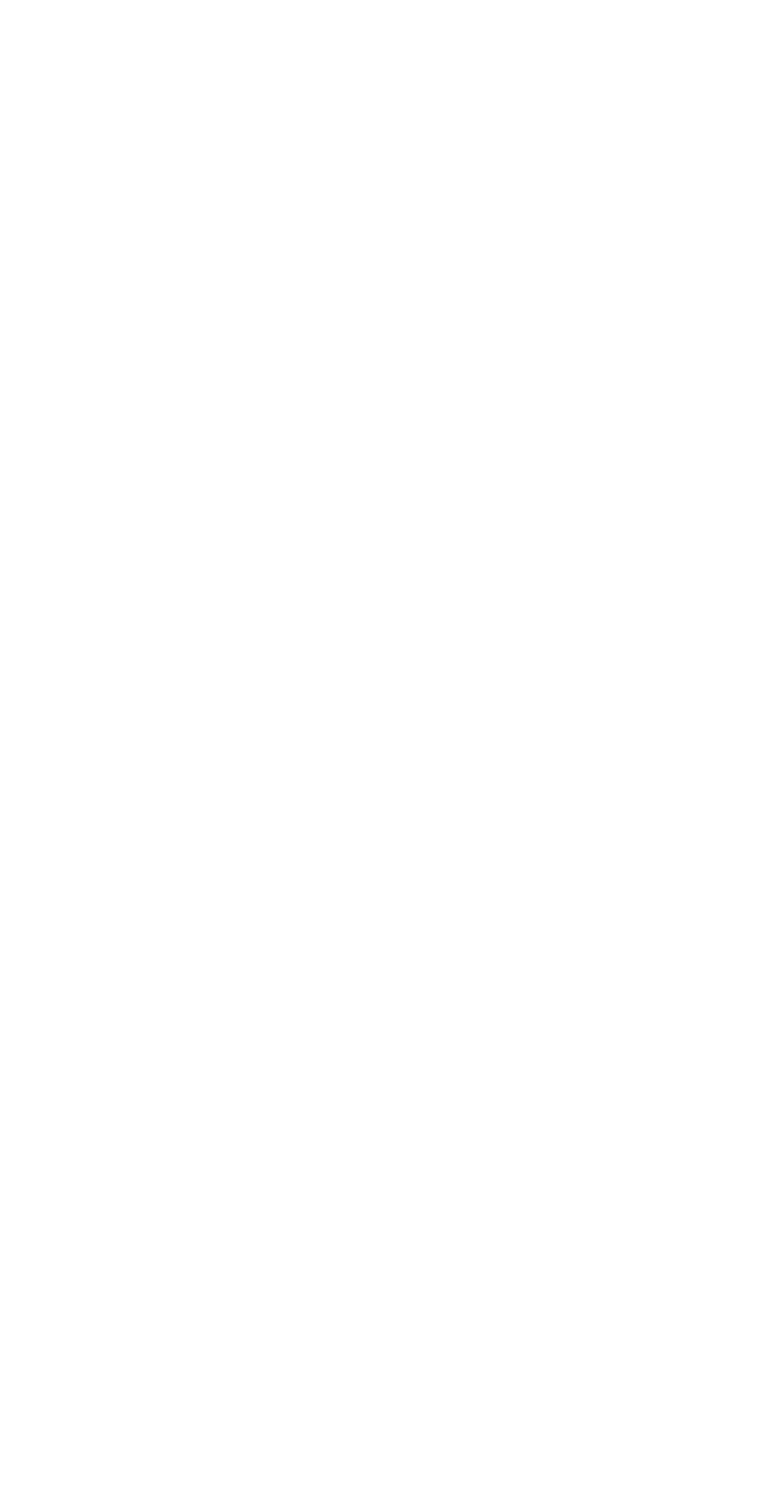




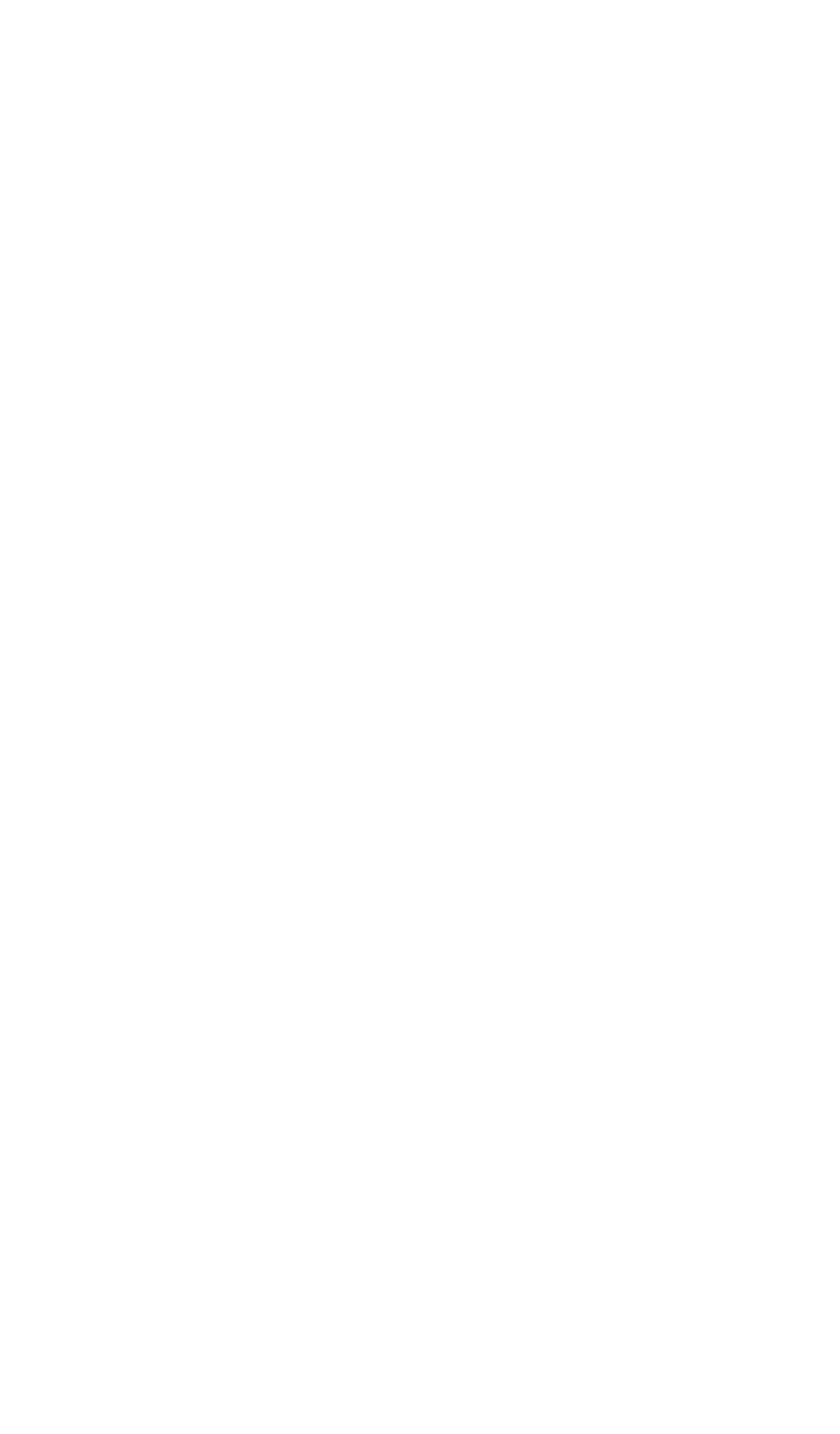




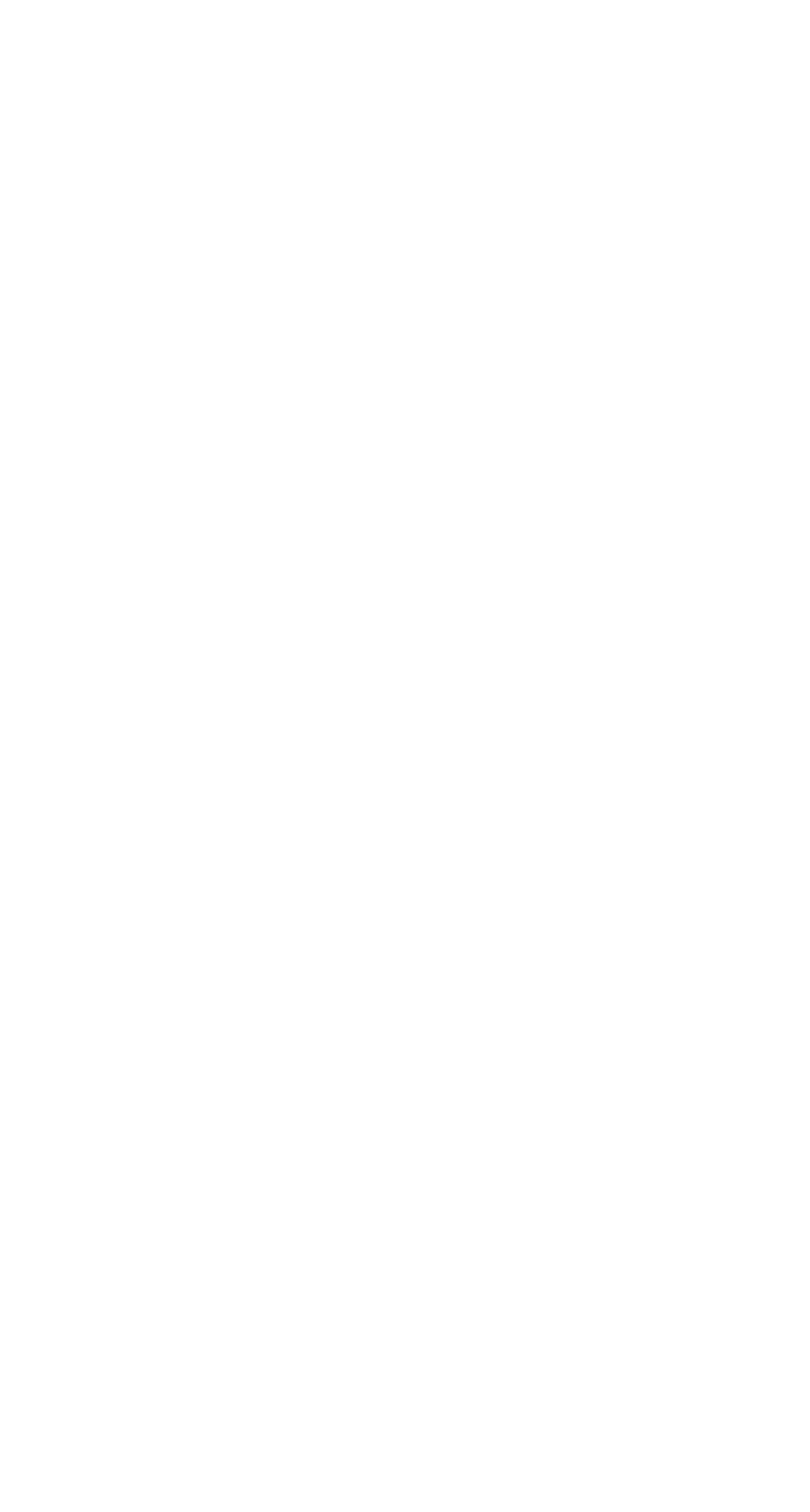




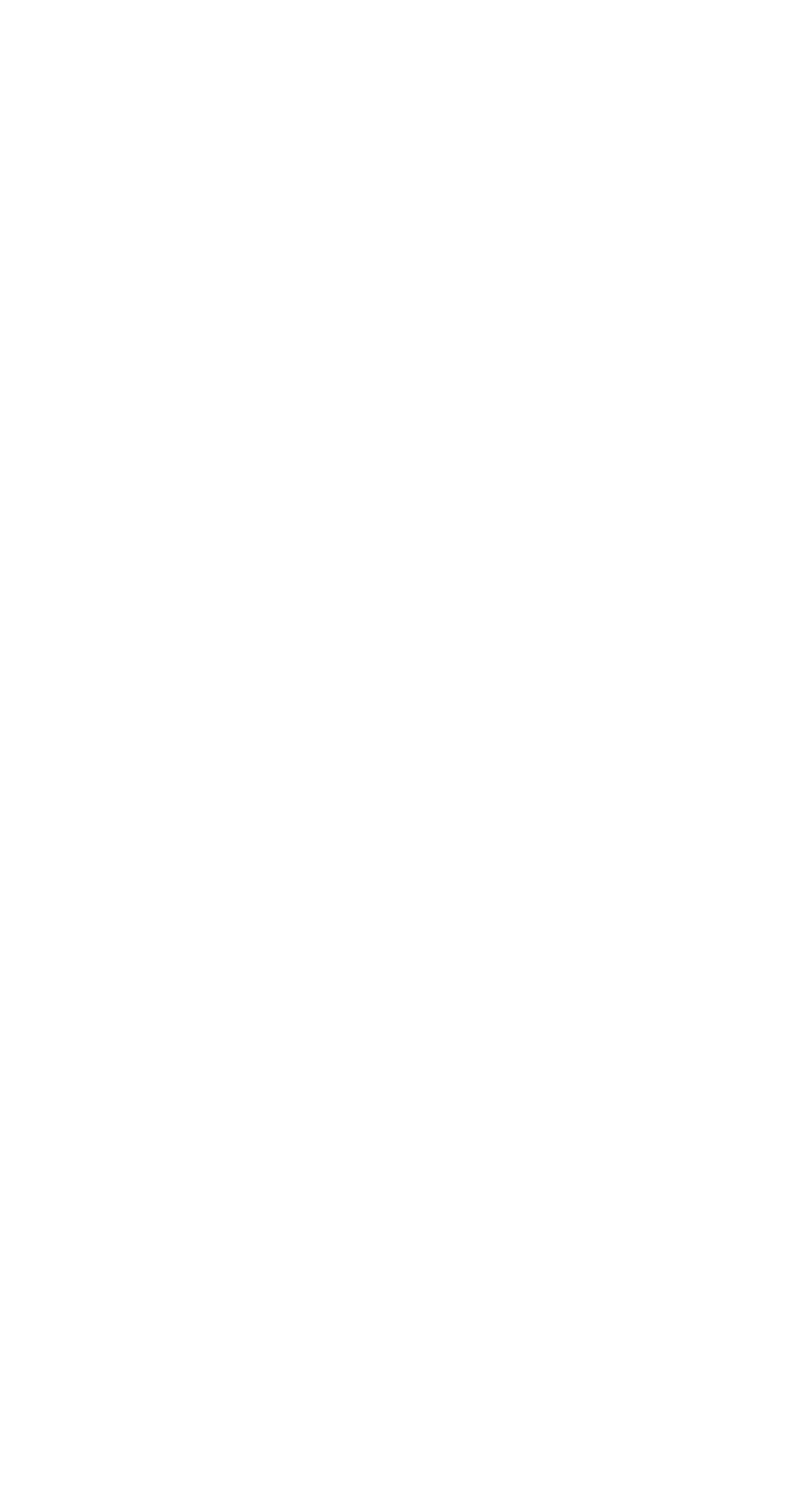


GROUND WATER OF WILLAMETTE VALLEY, OREG.

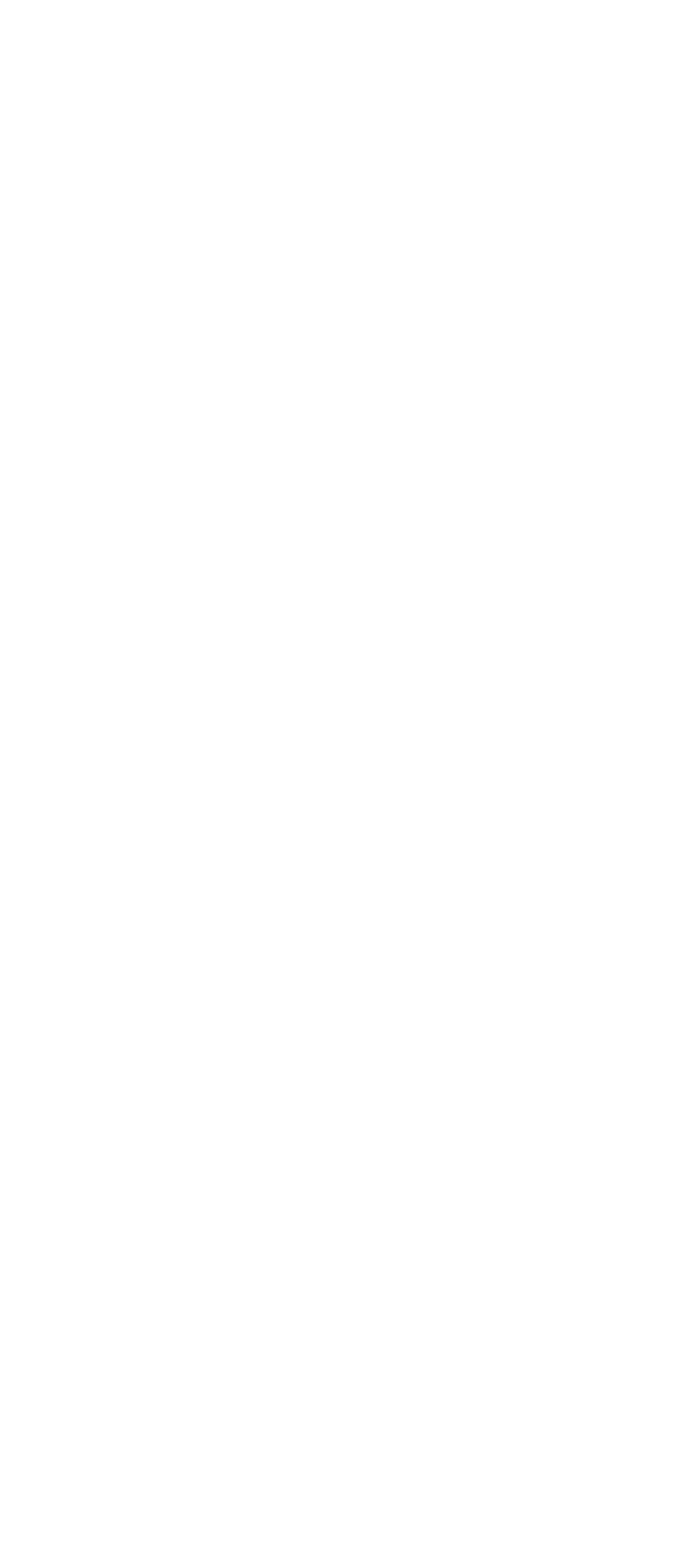




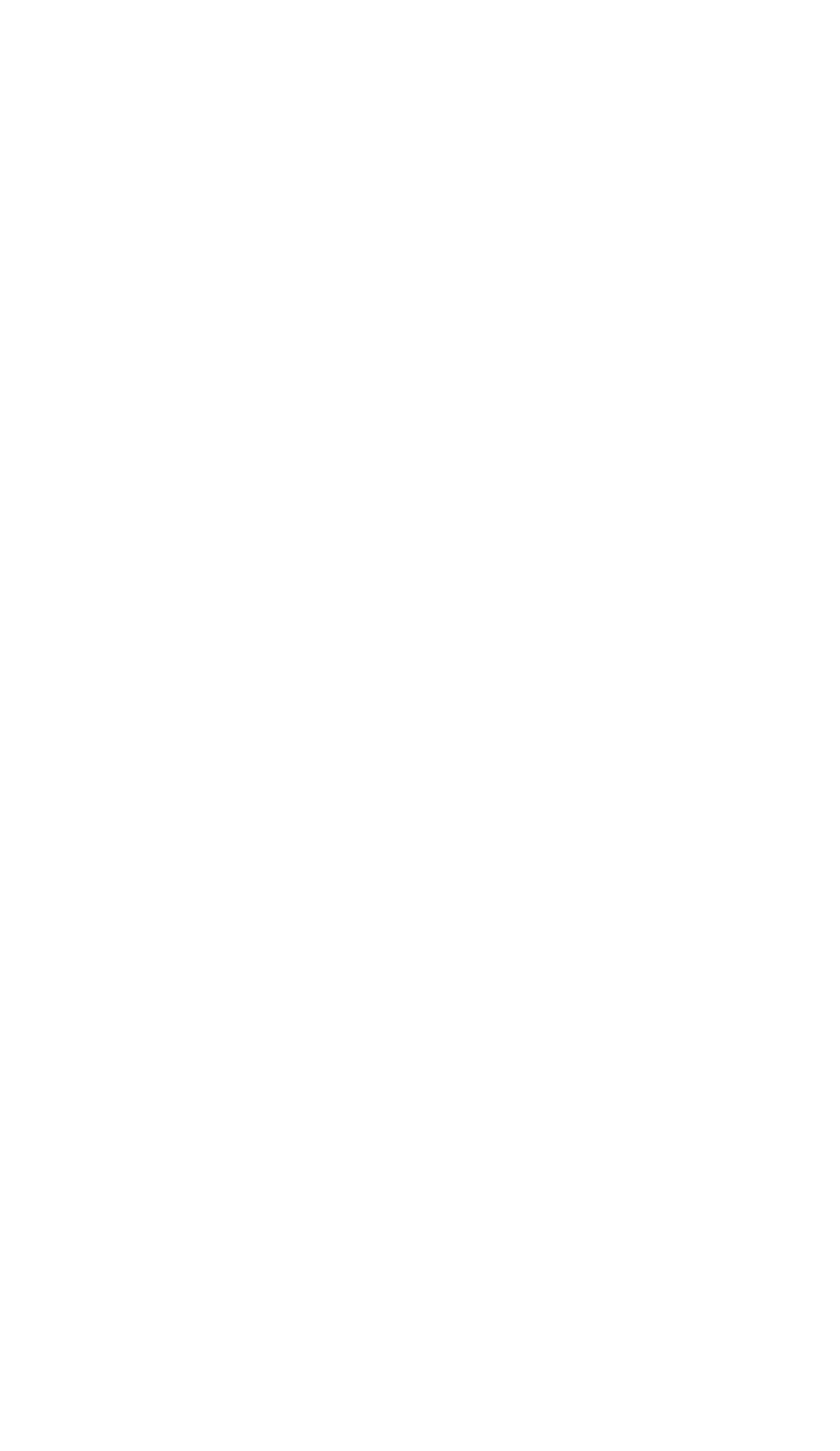




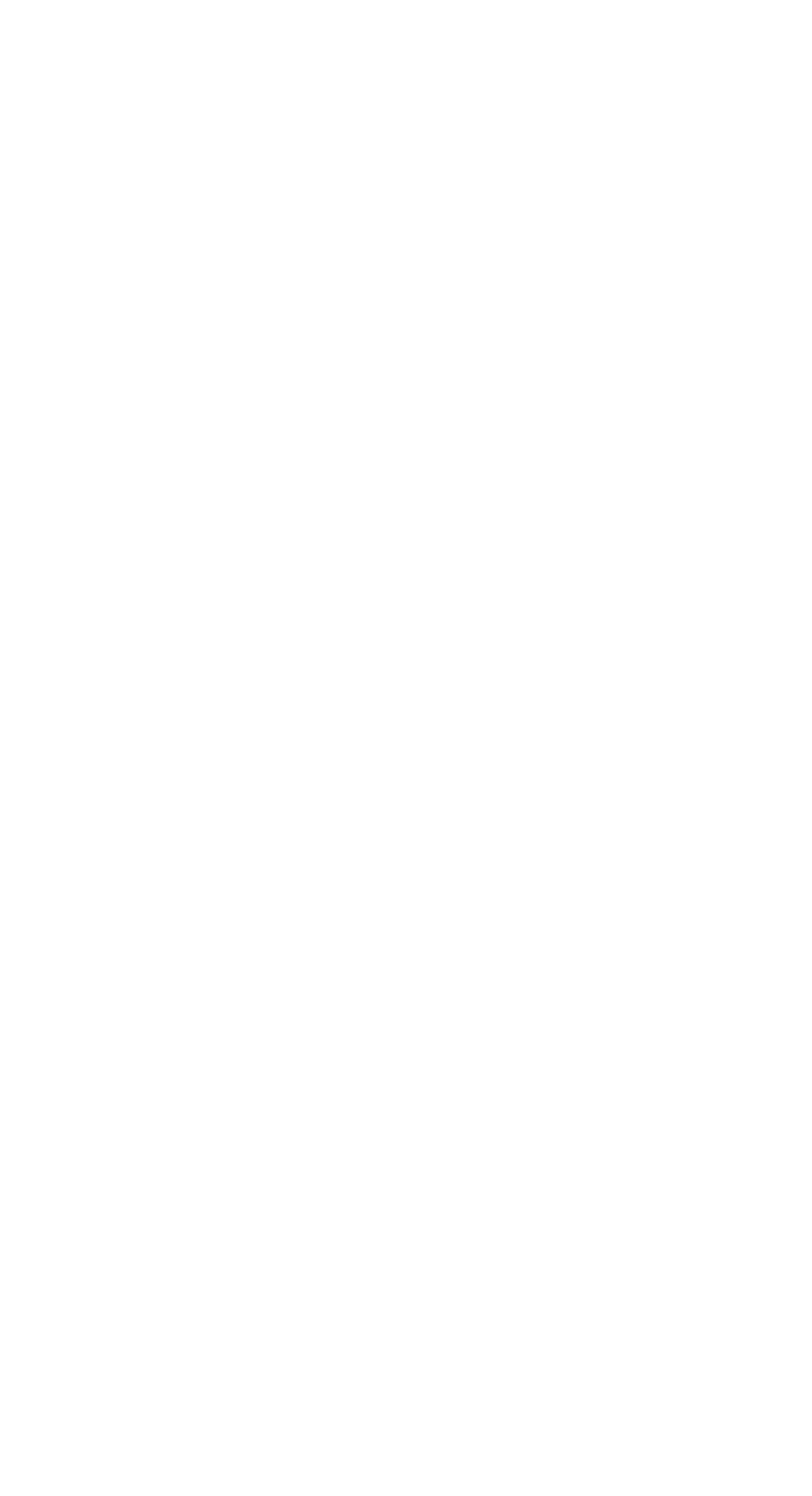




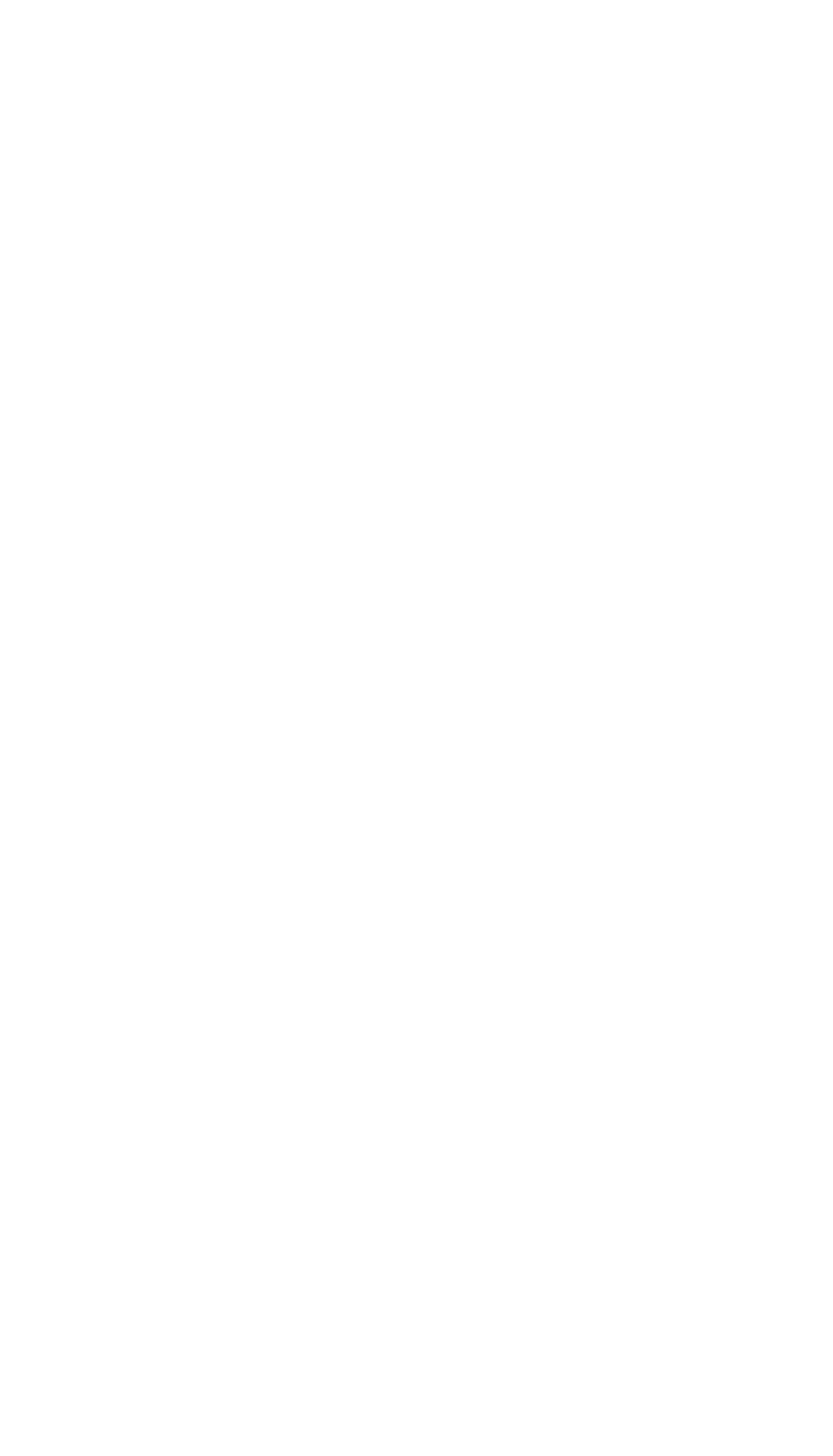


100 GROUND WATER OF WILLAMETTE VALLEY, OREG.

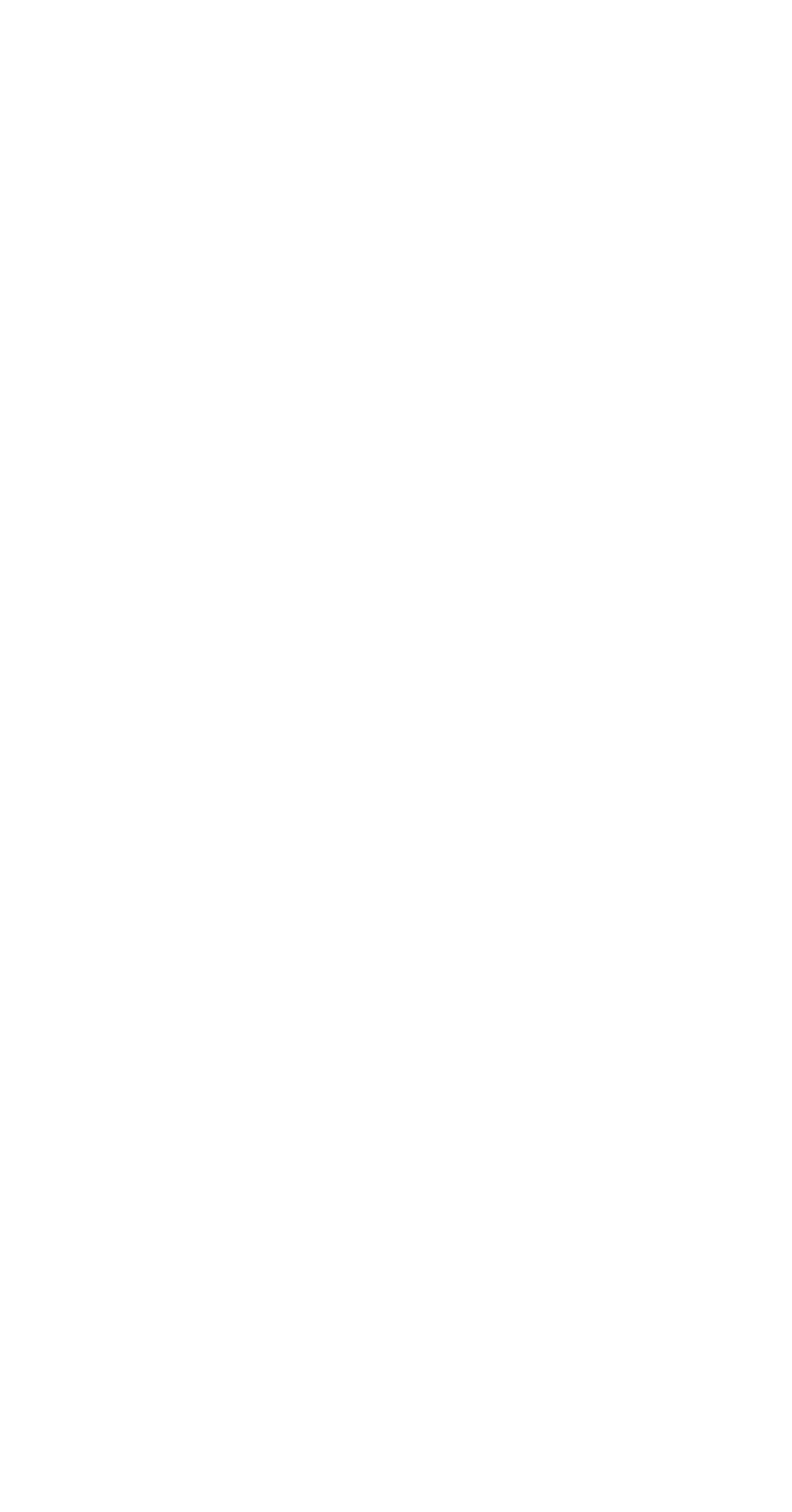




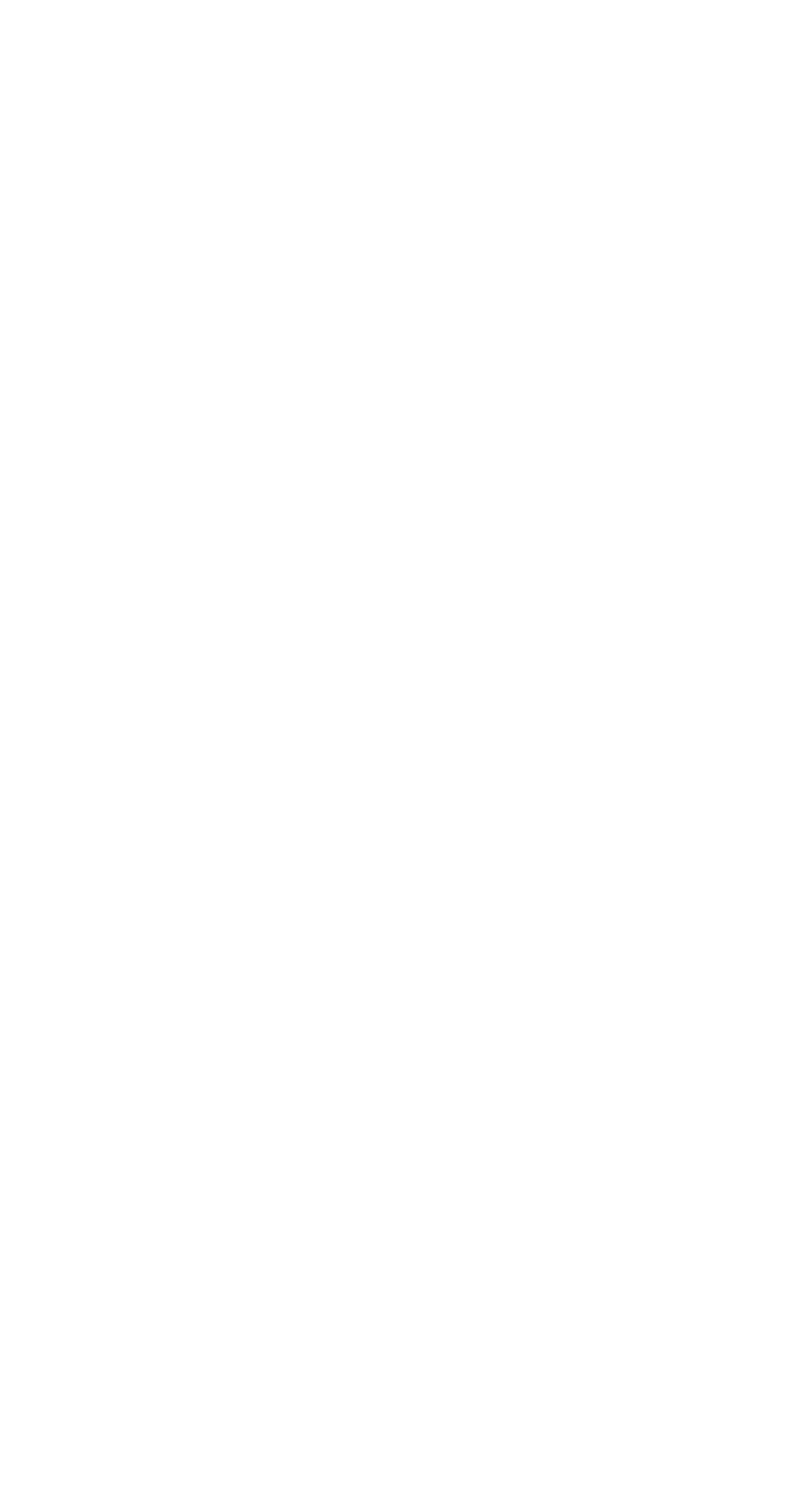


102 GROUND WATER OF WILLAMETTE VALLEY, OREG.

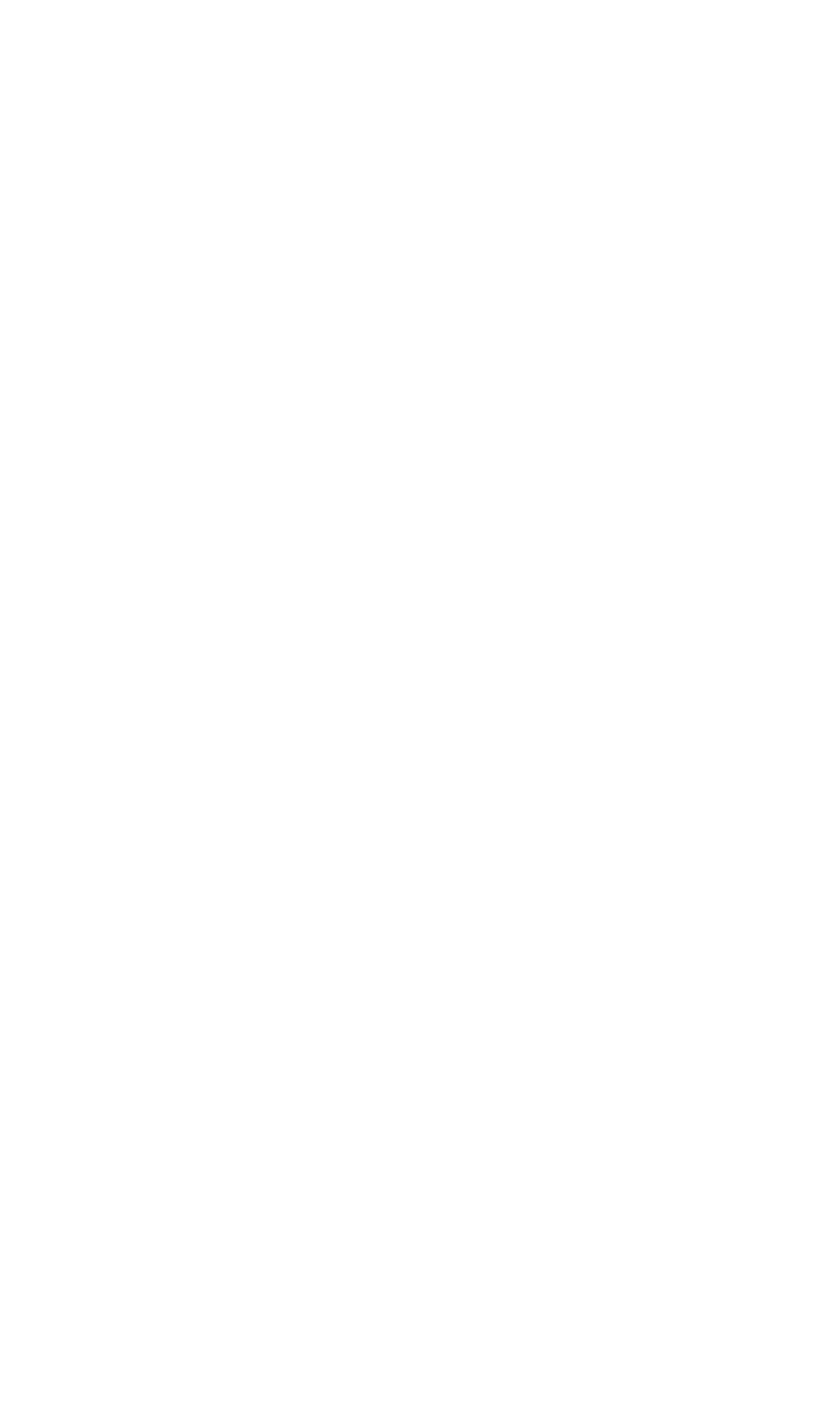




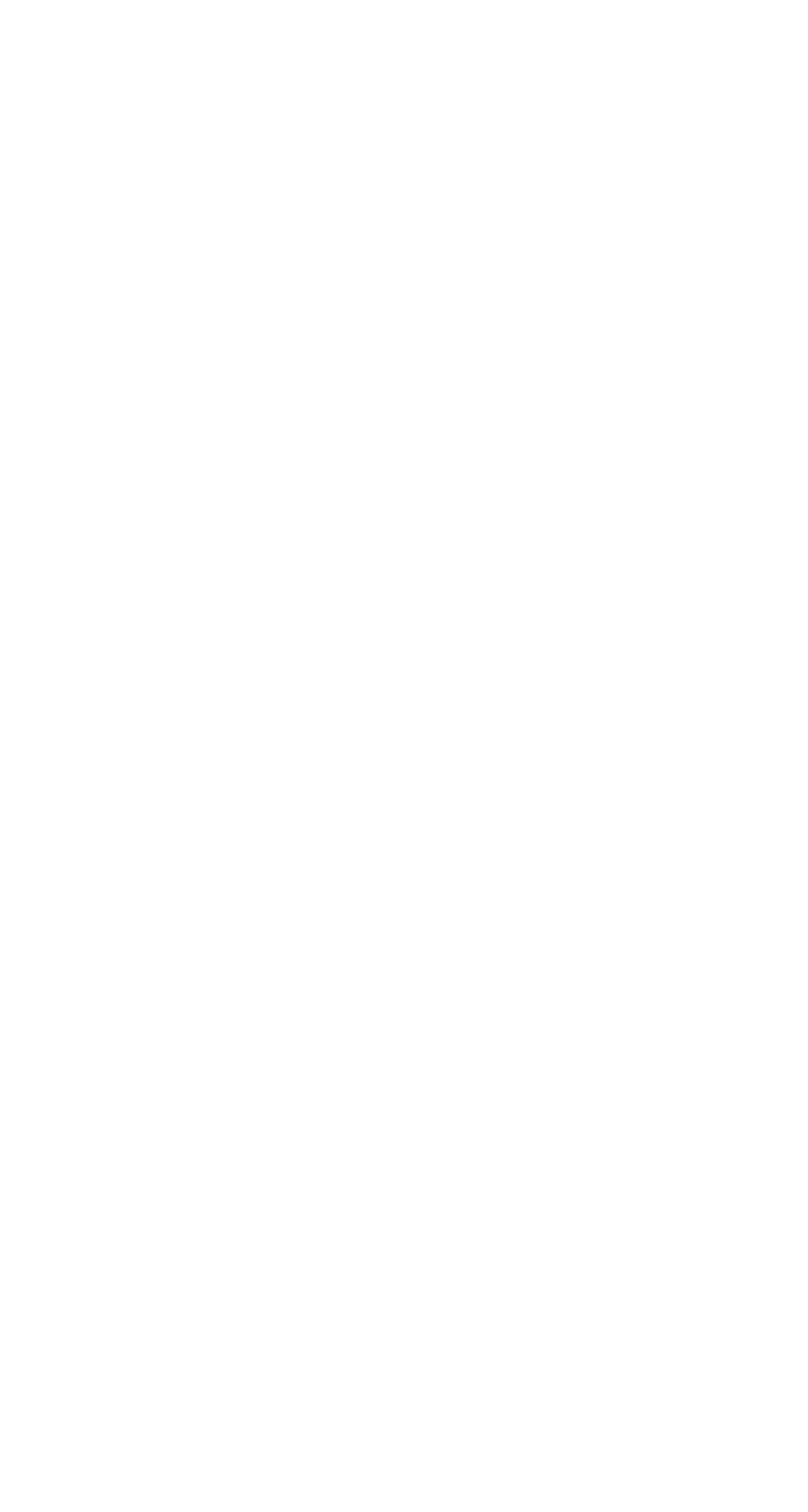




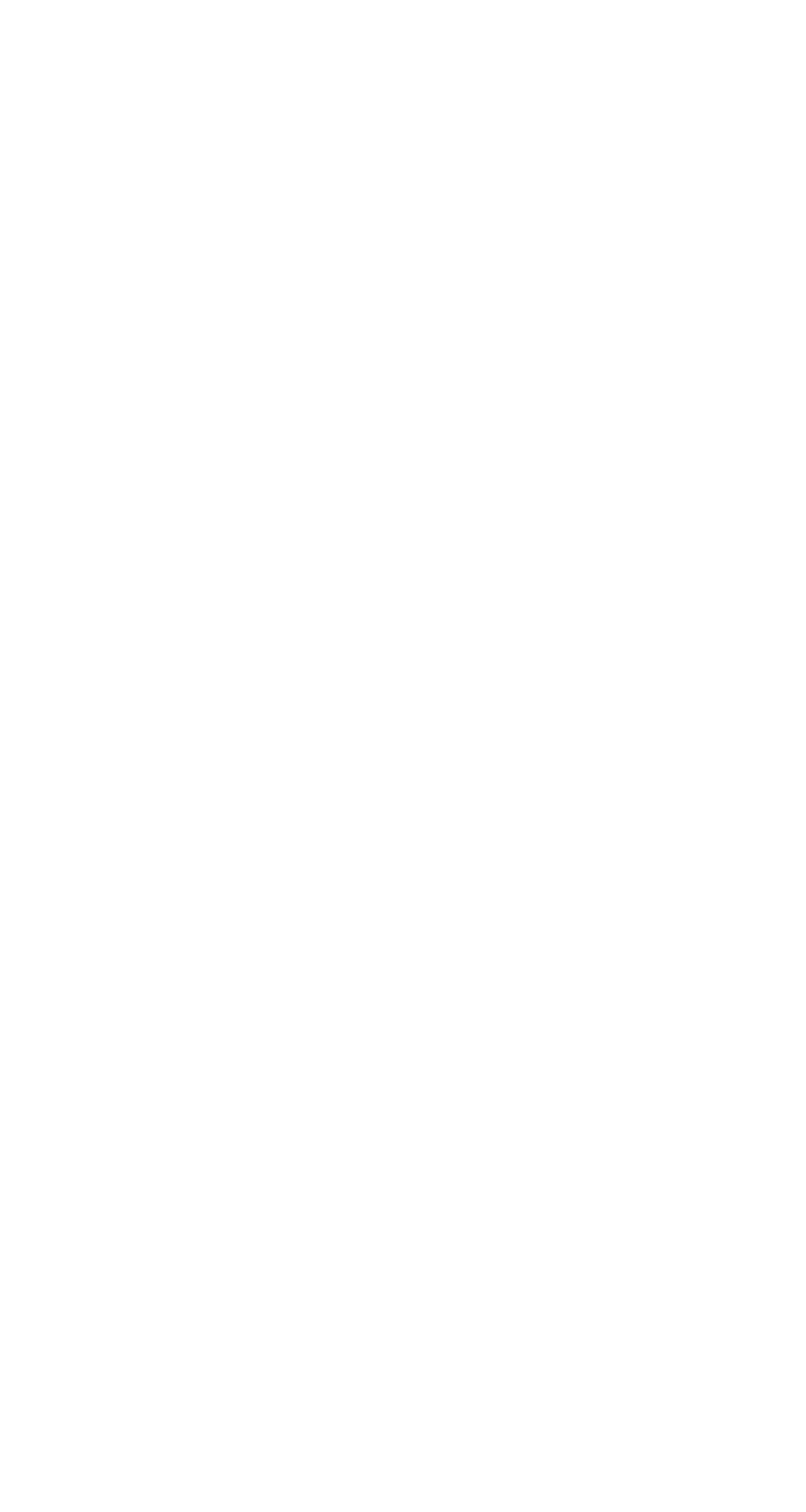




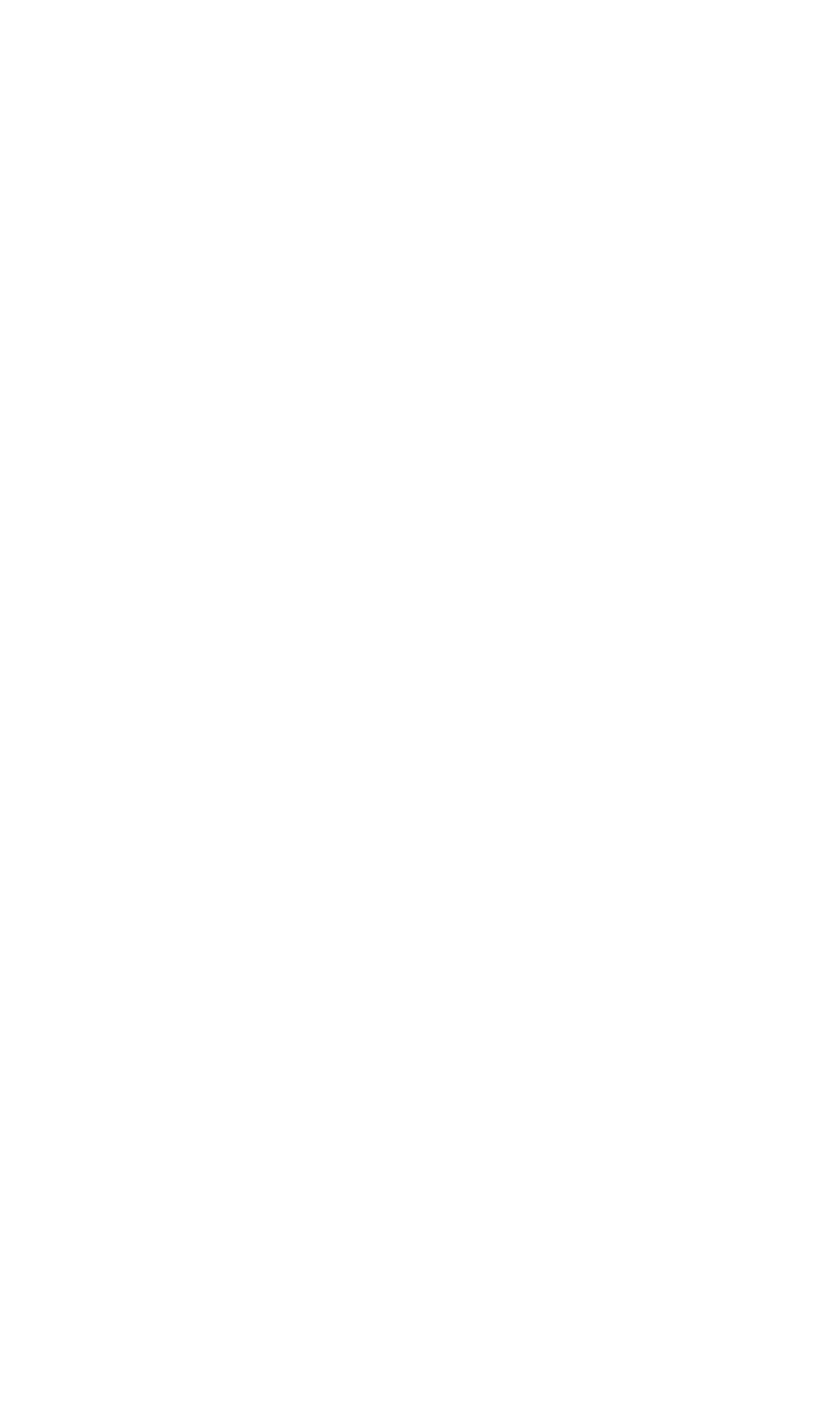




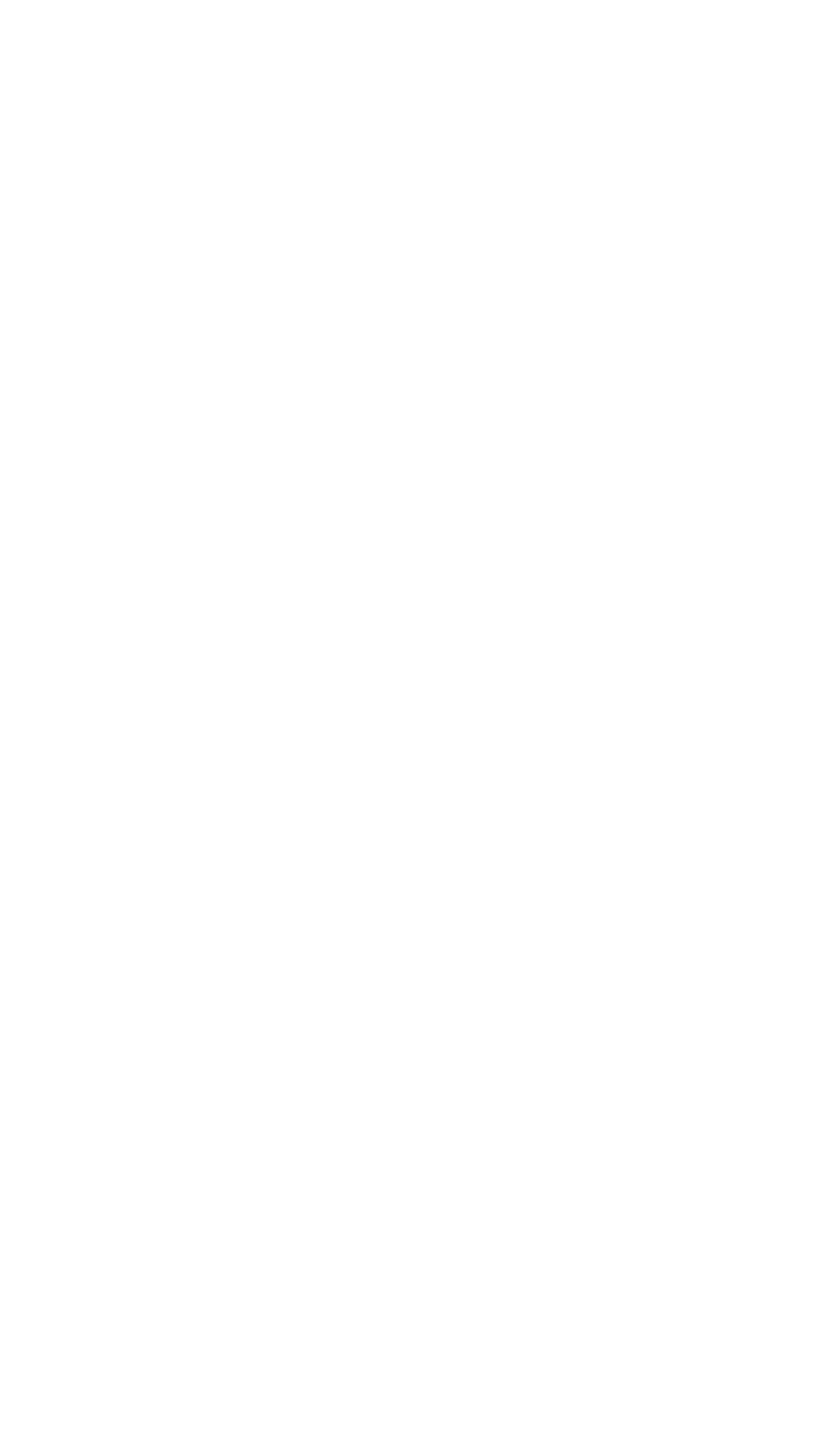


WELL RECORDS

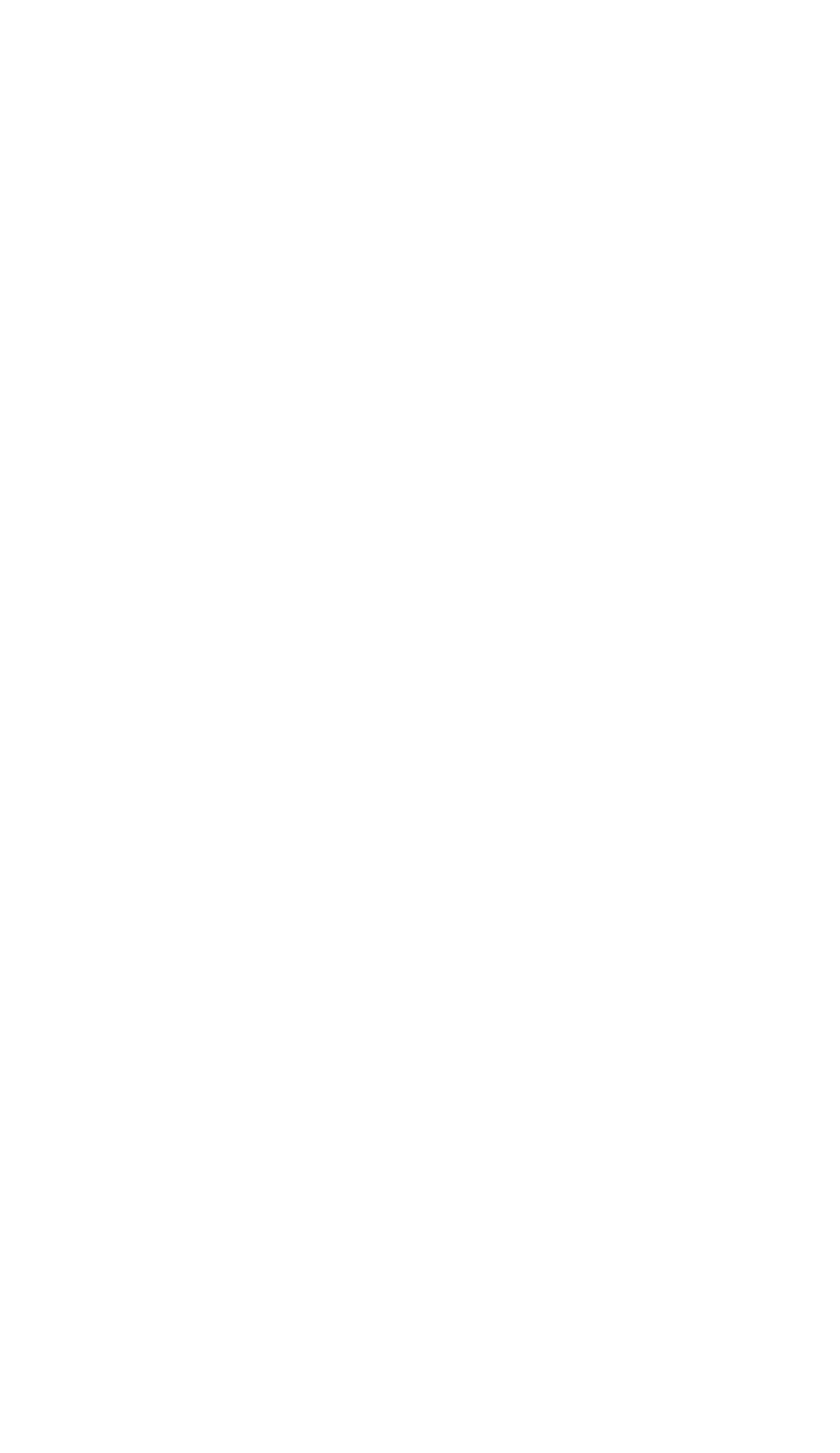




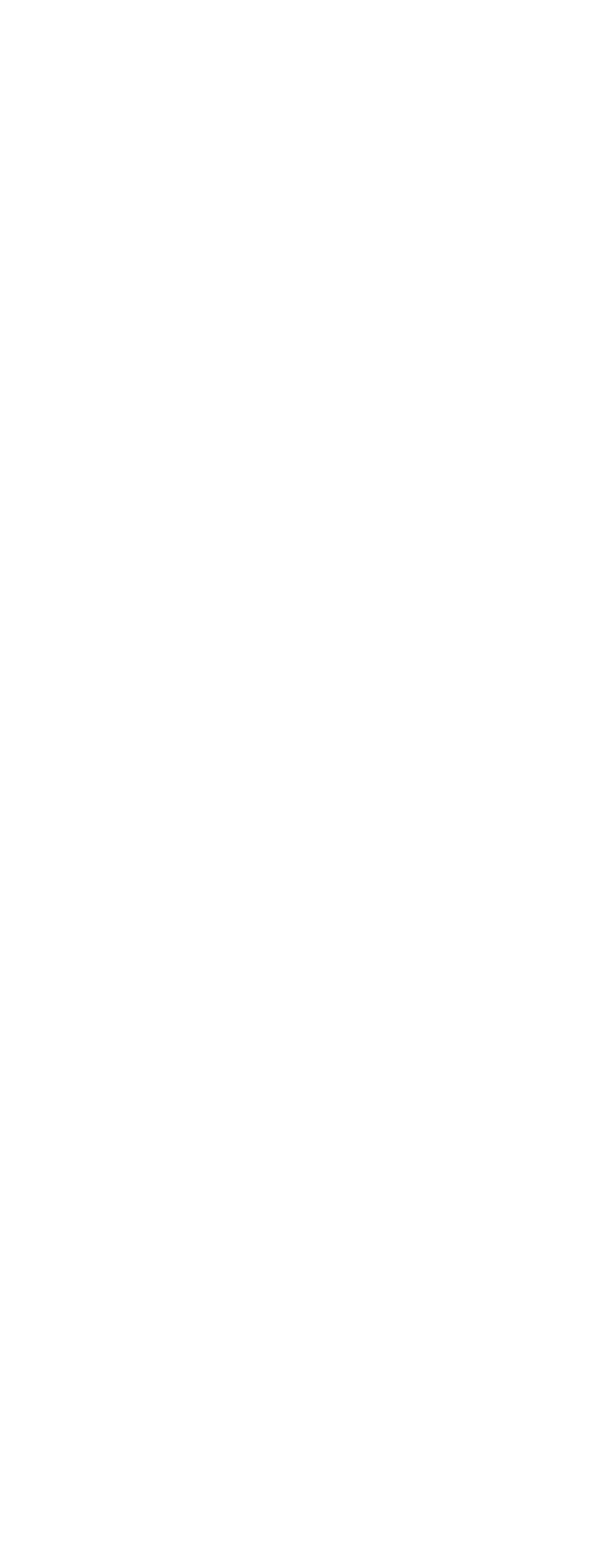




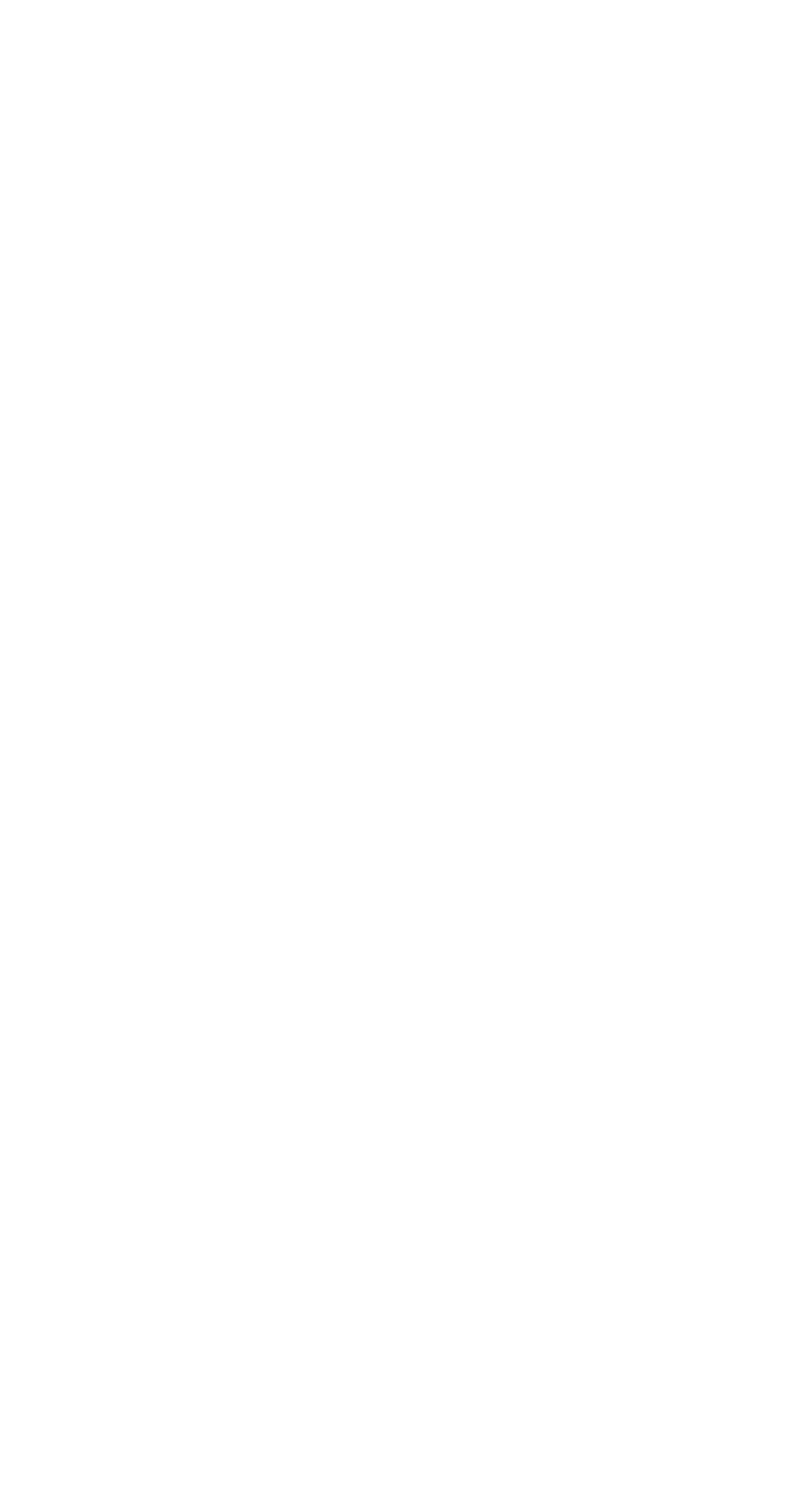


110 GROUND WATER OF WILLAMETTE VALLEY, OREG.

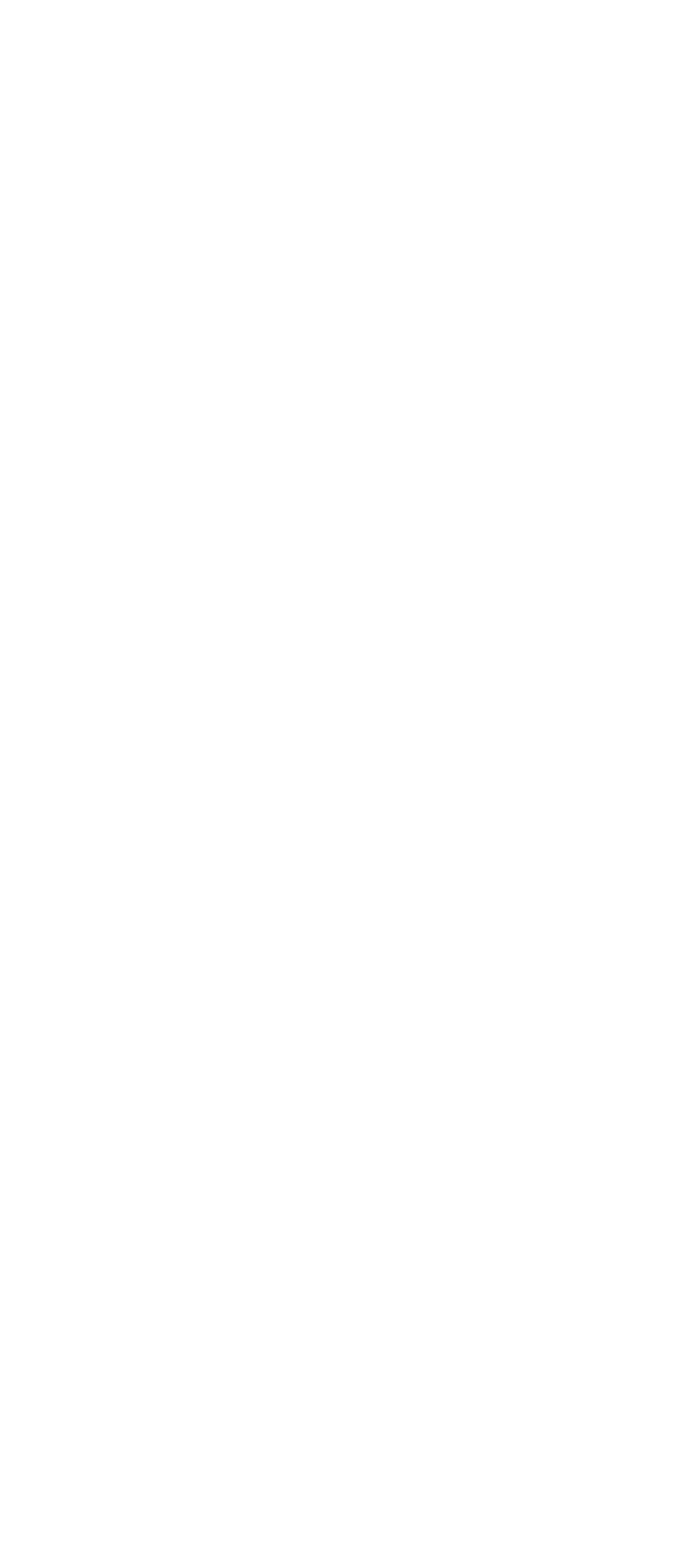




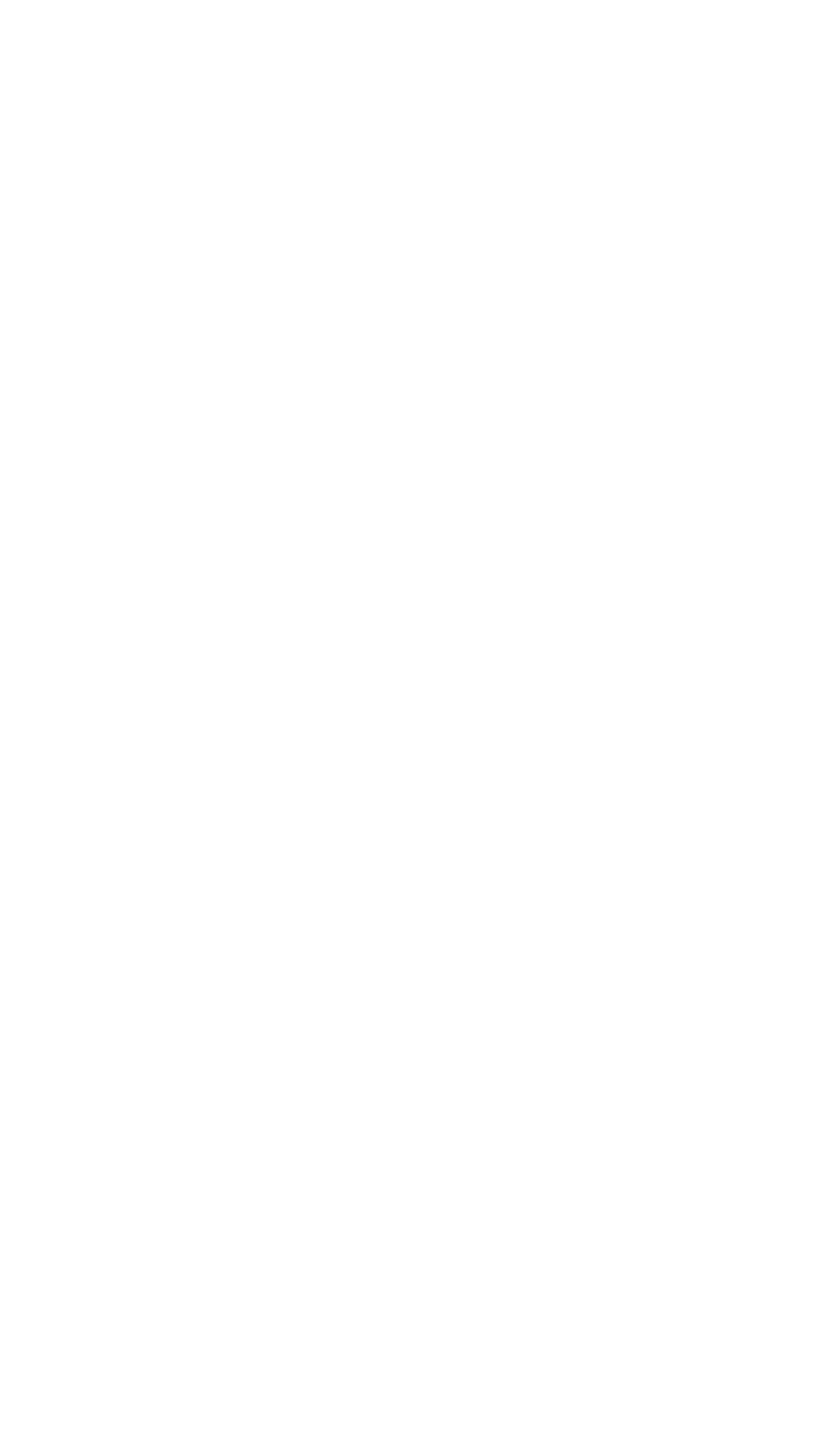




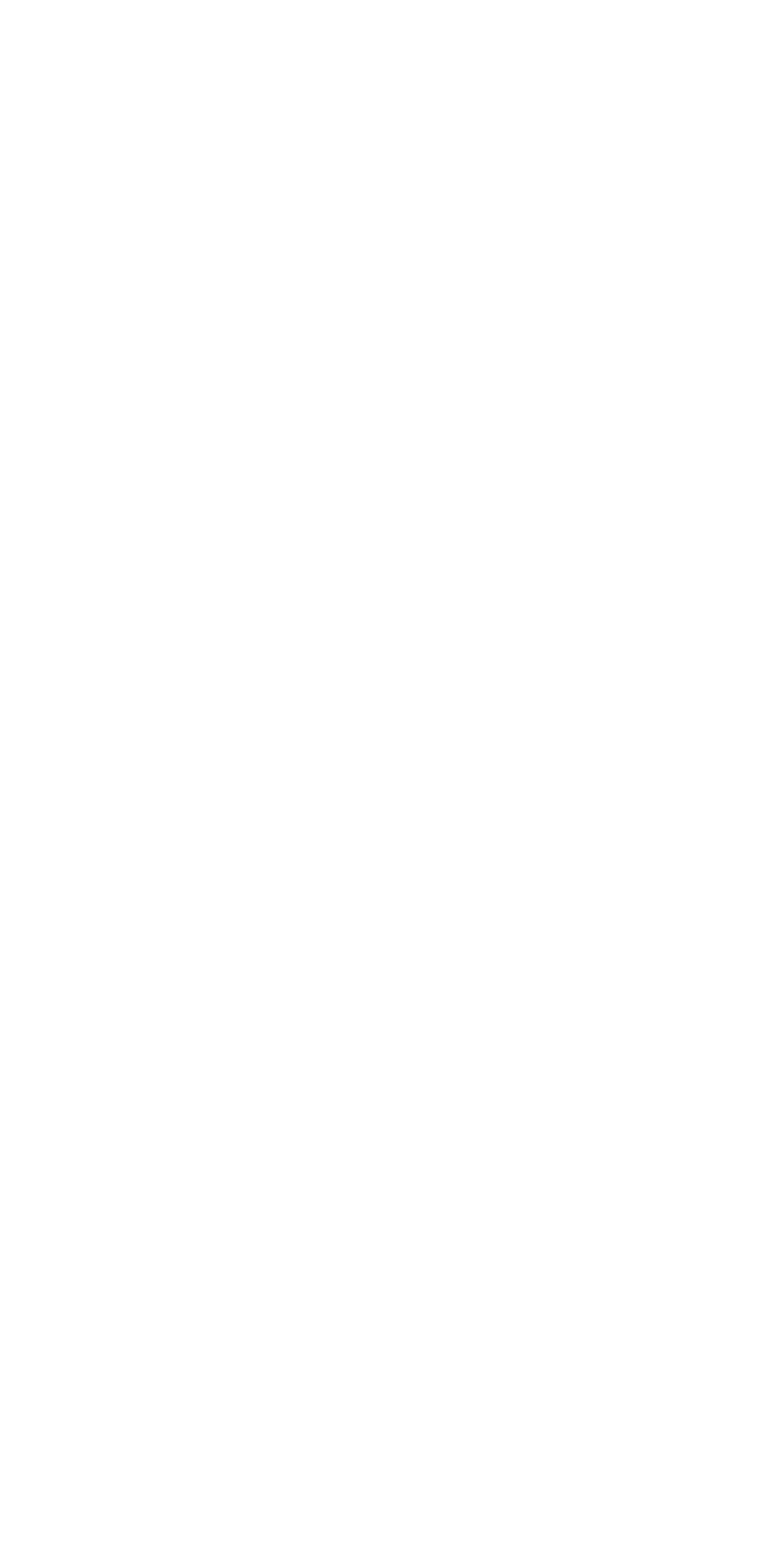




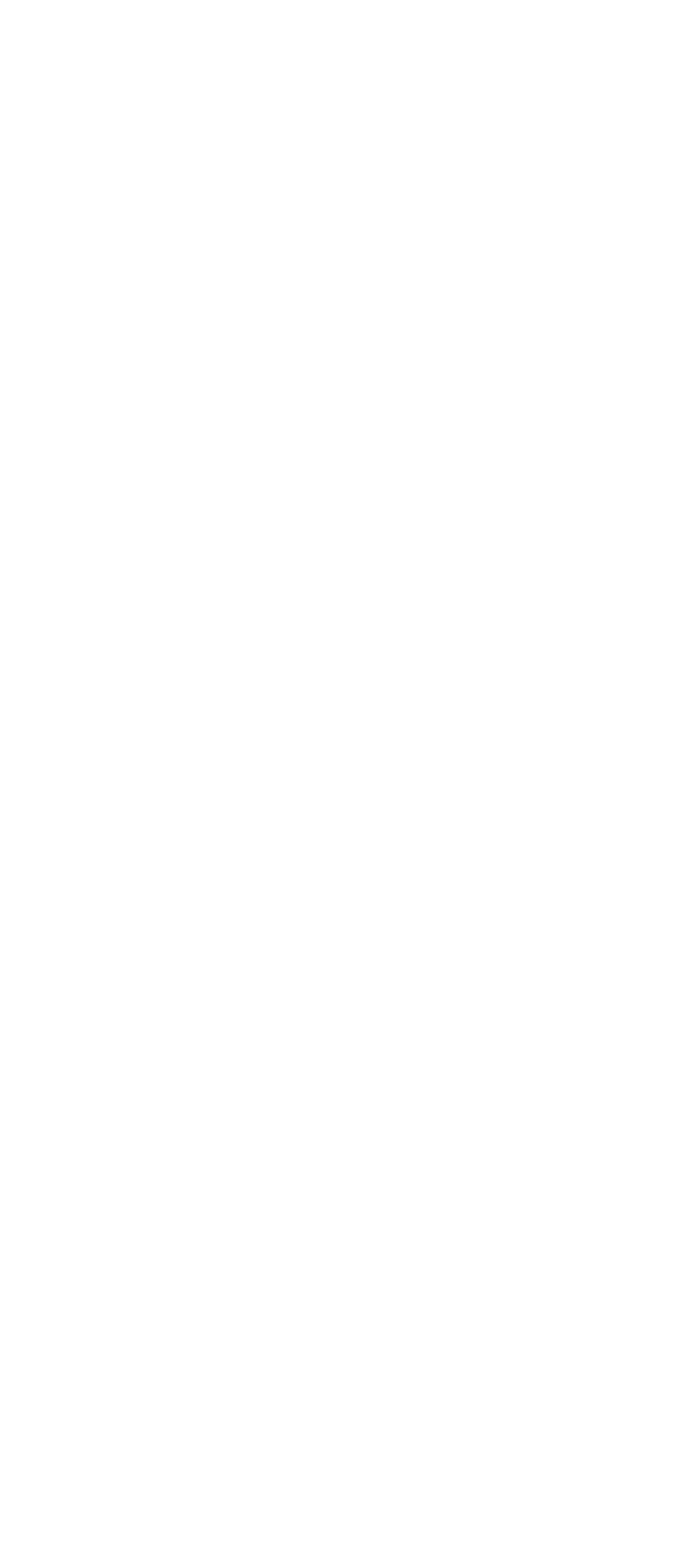




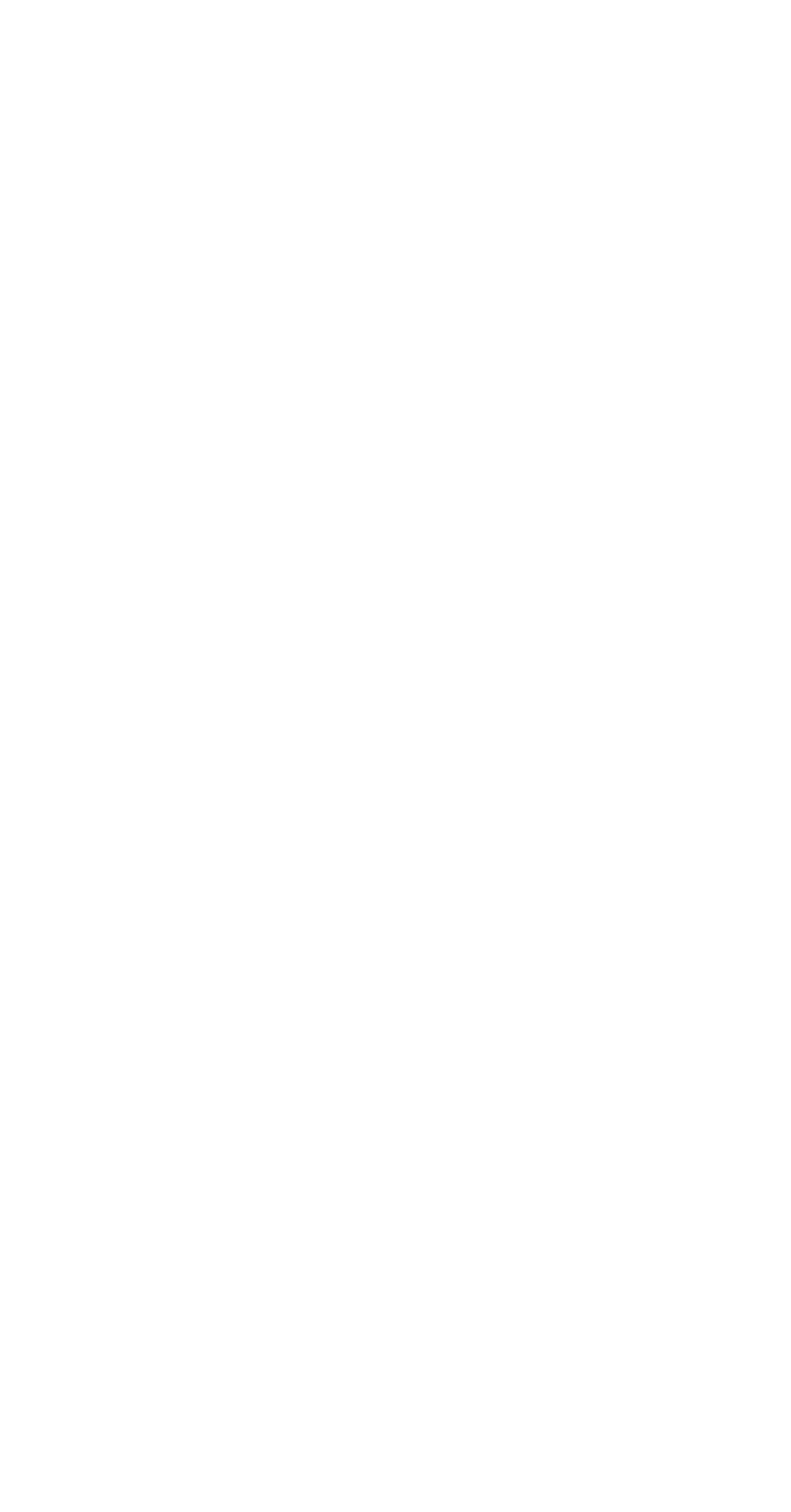


WELL RECORDS

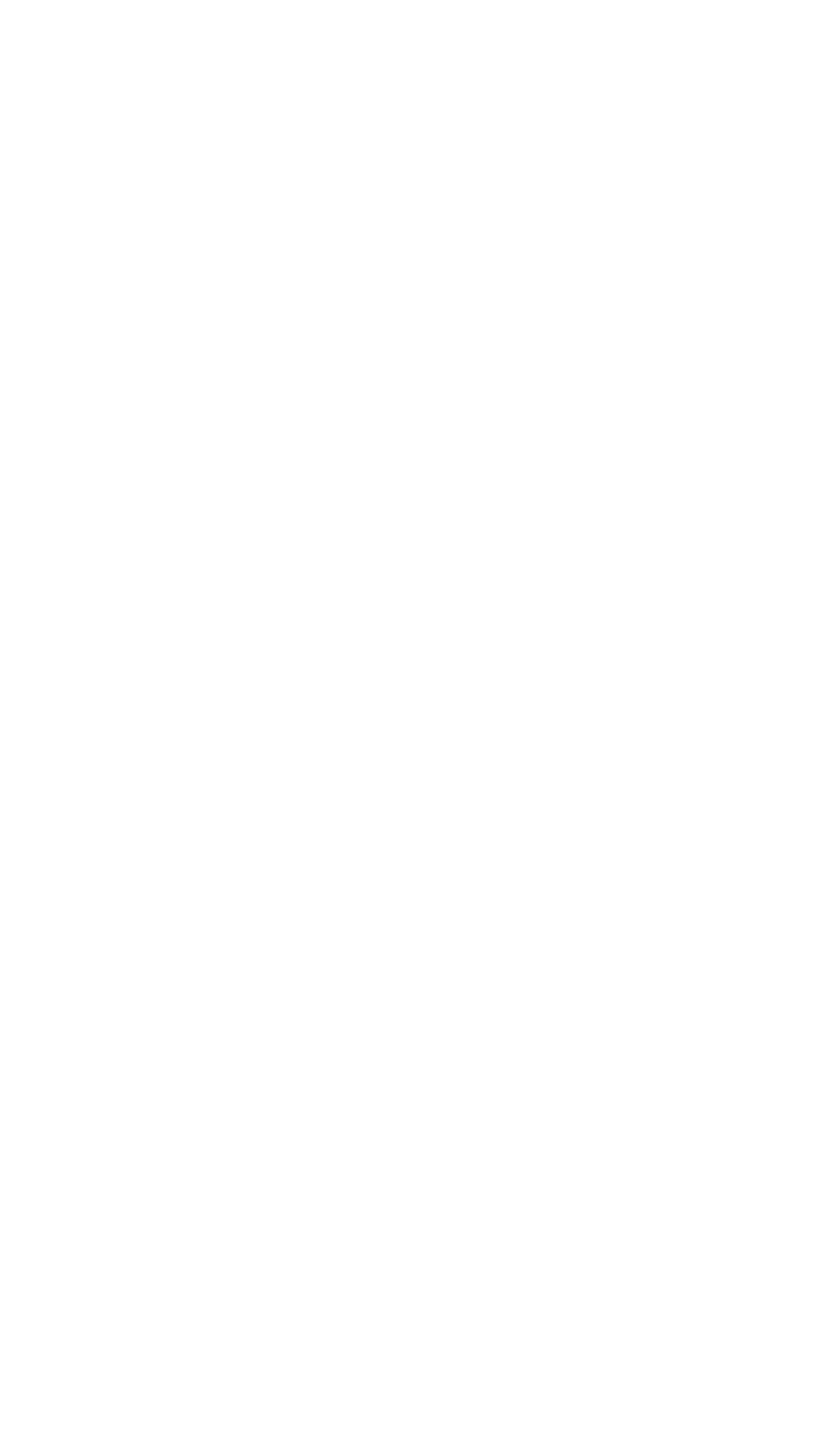




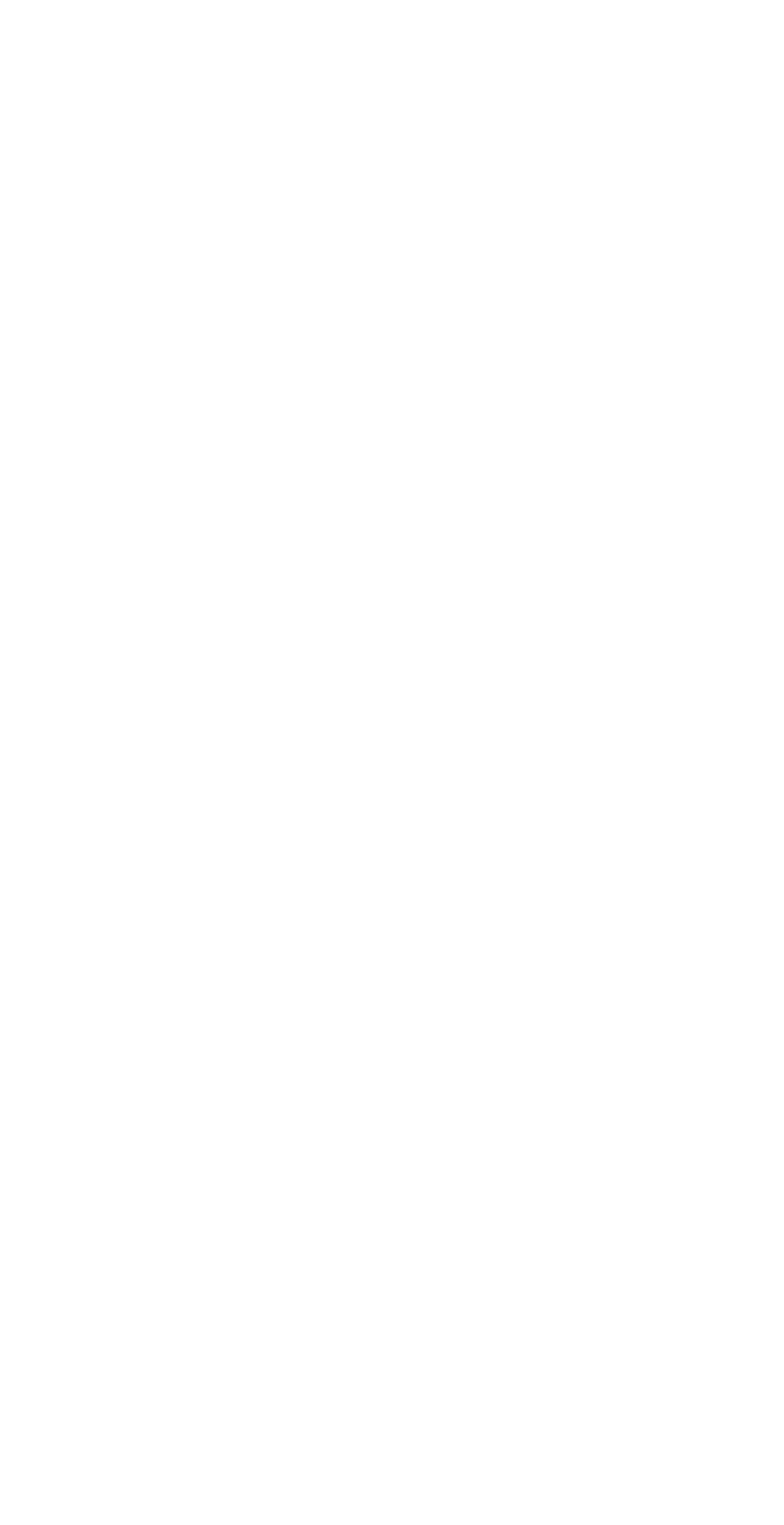




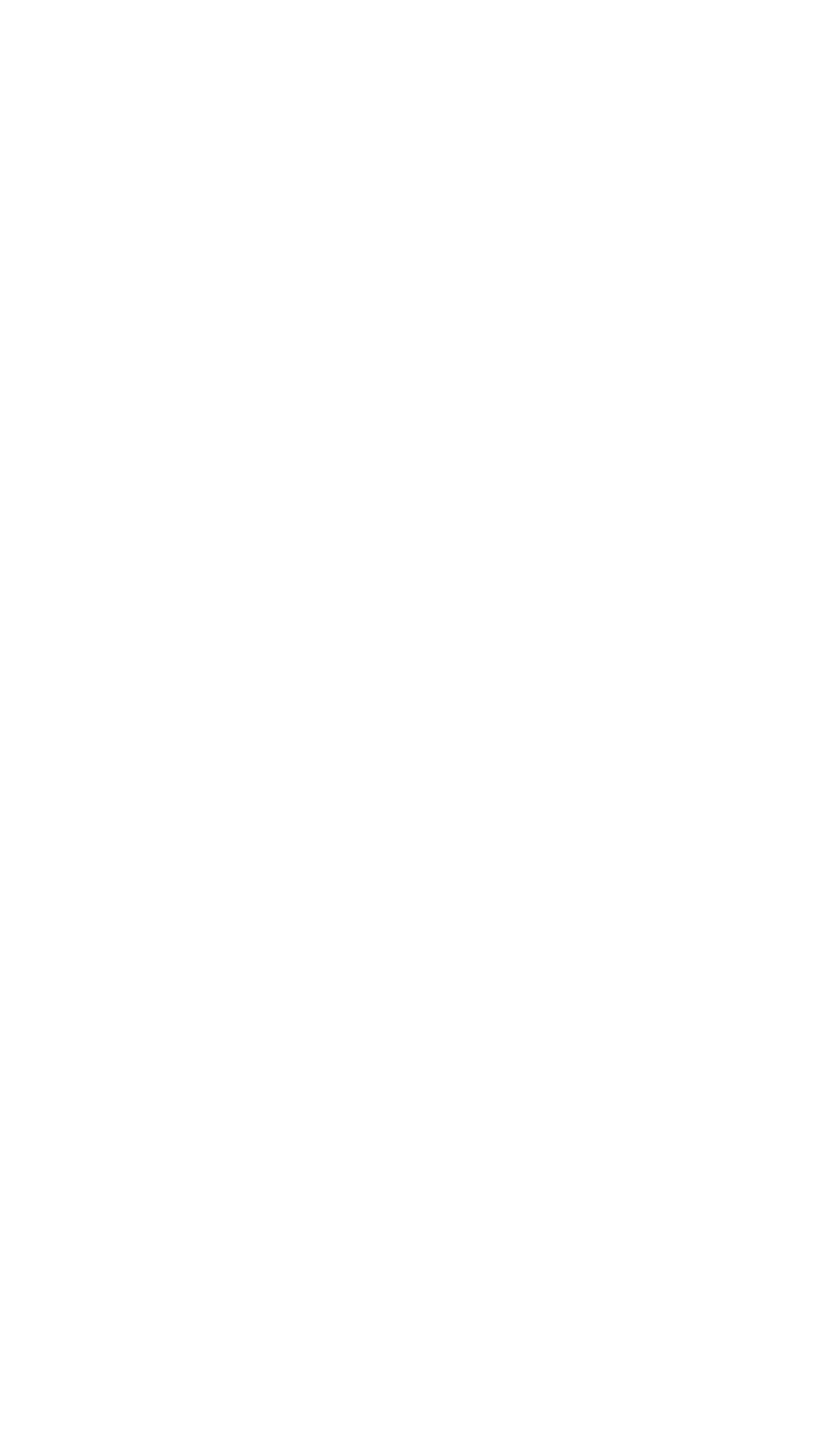


118 GROUND WATER OF WILLAMETTE VALLEY, OREG.

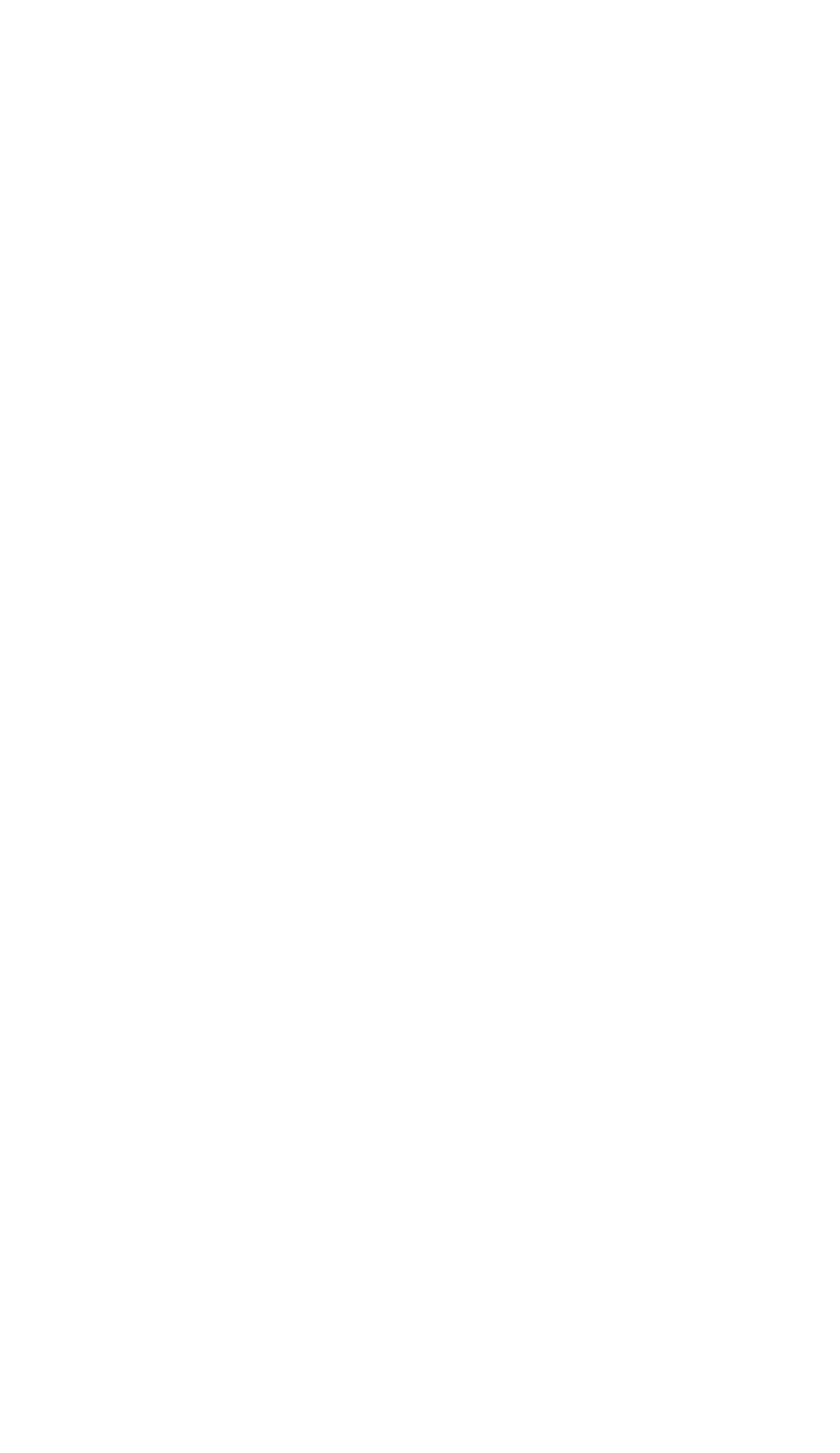




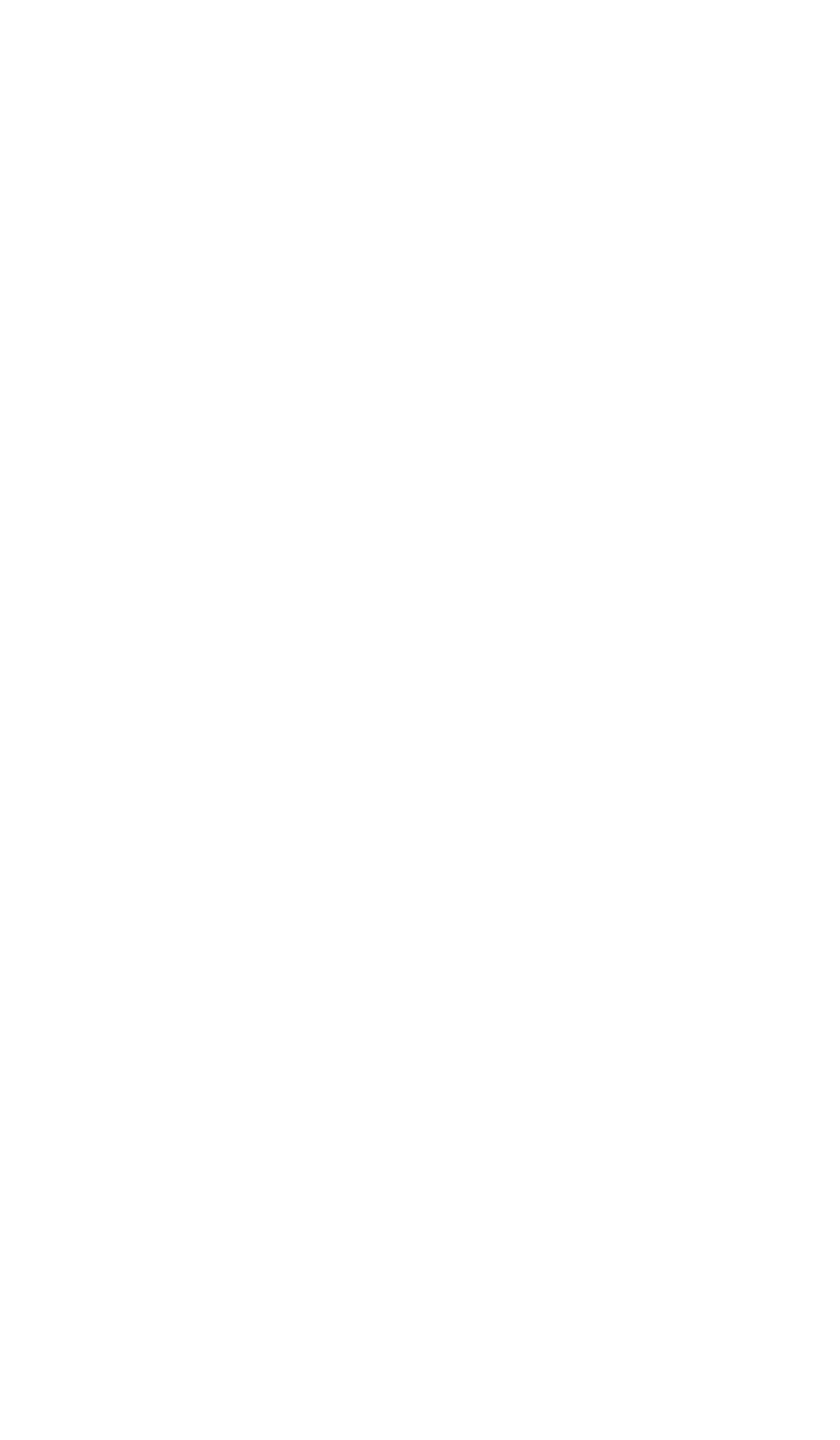




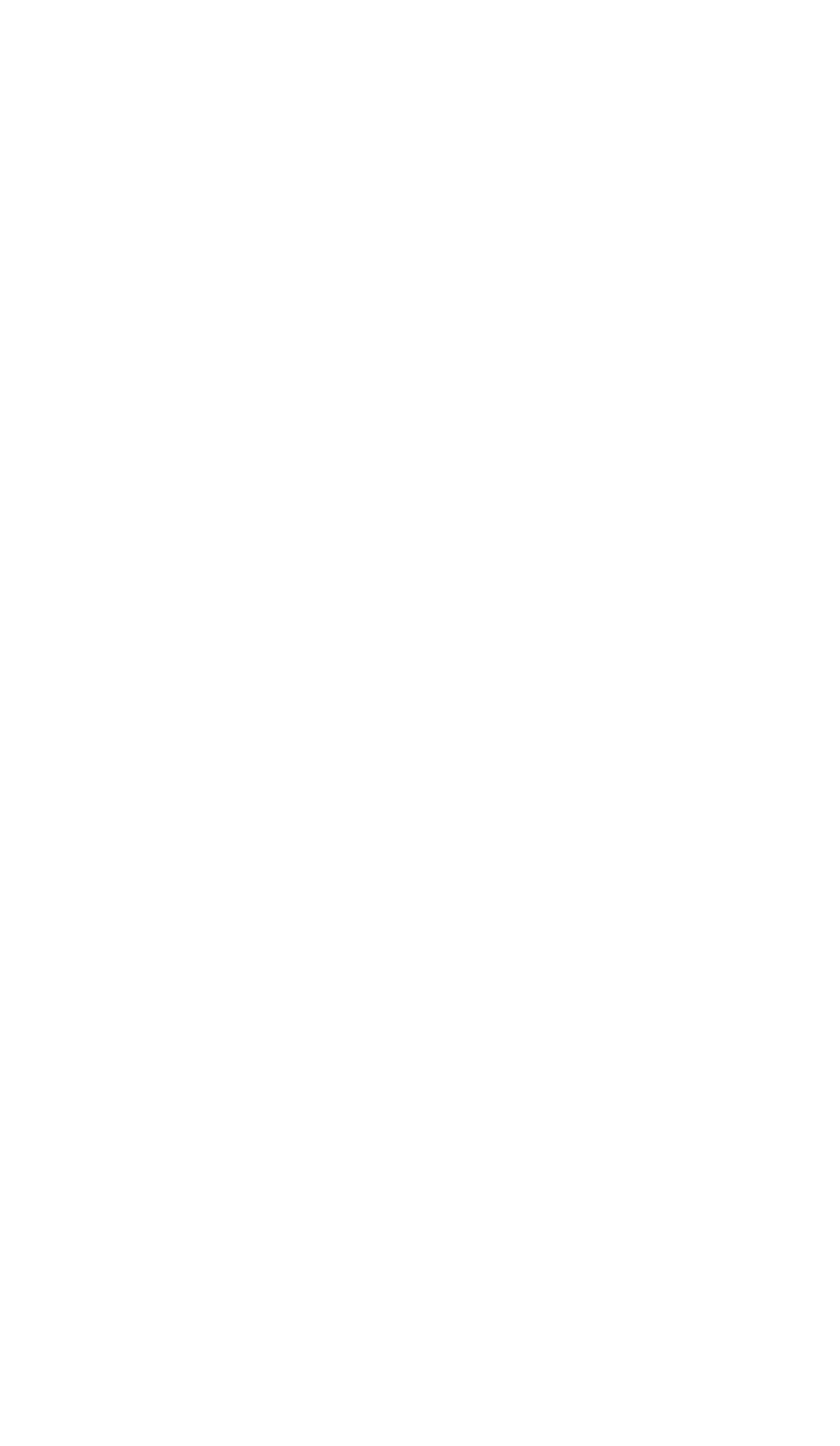


WELL RECORDS

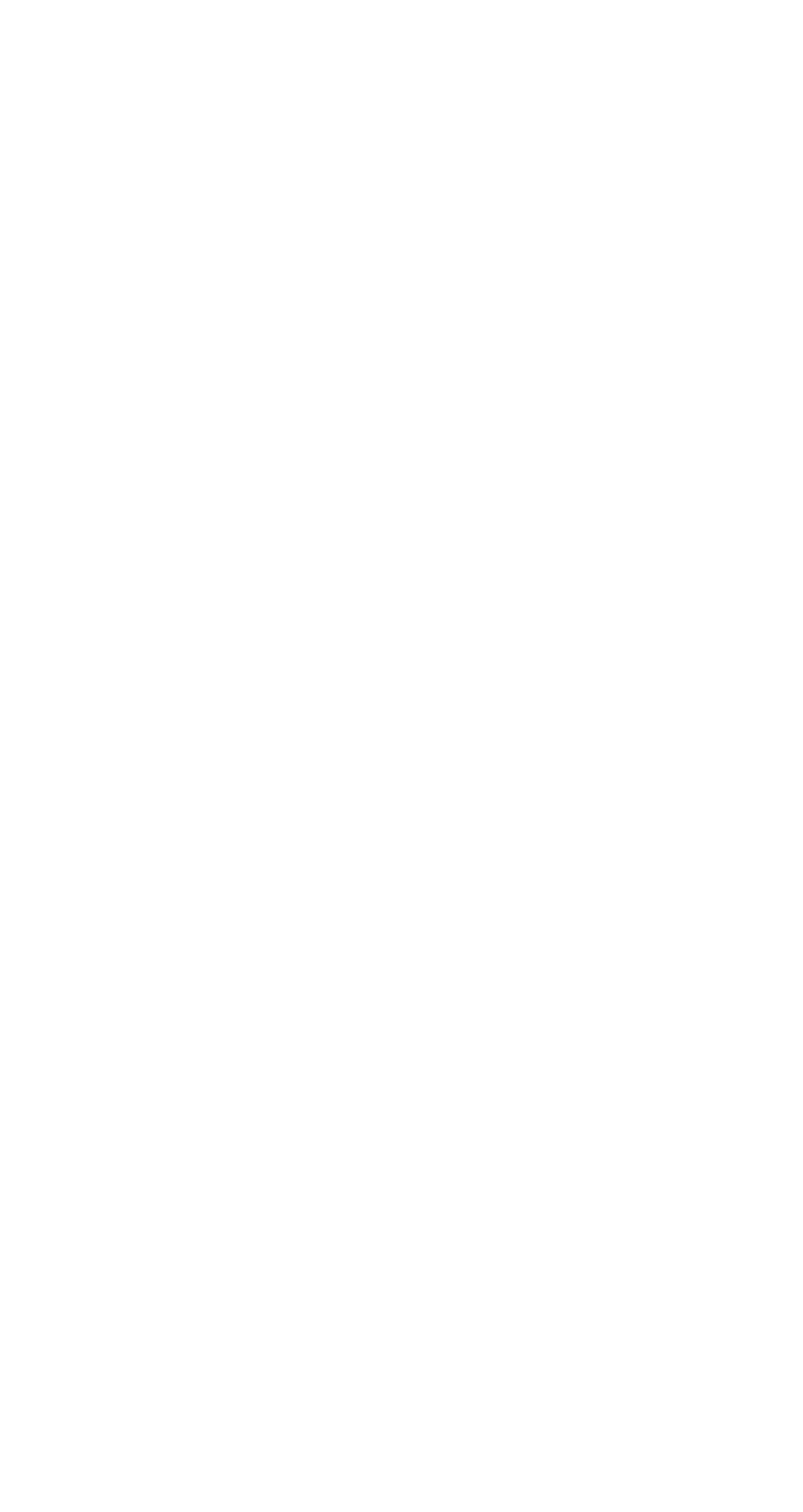




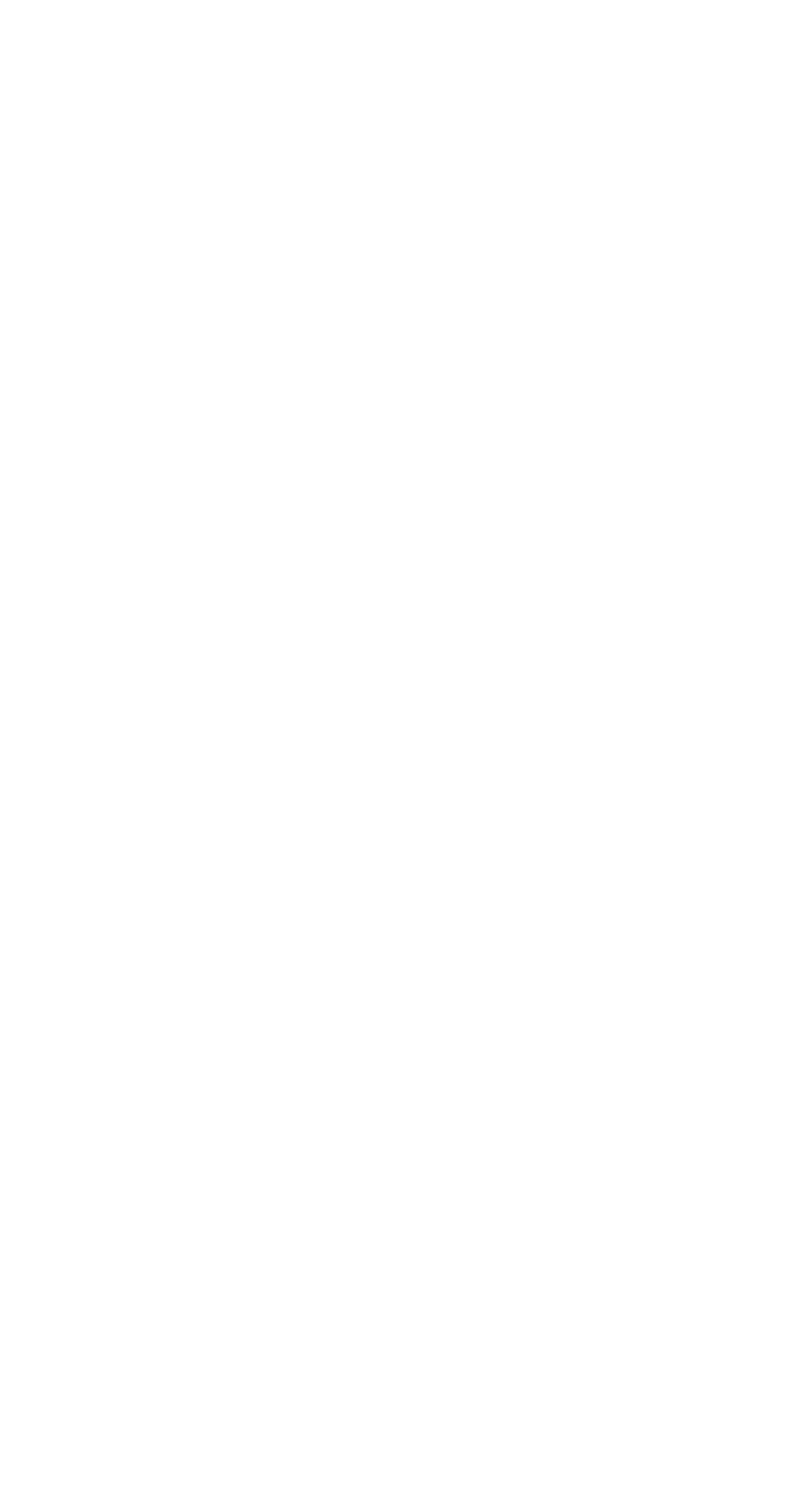




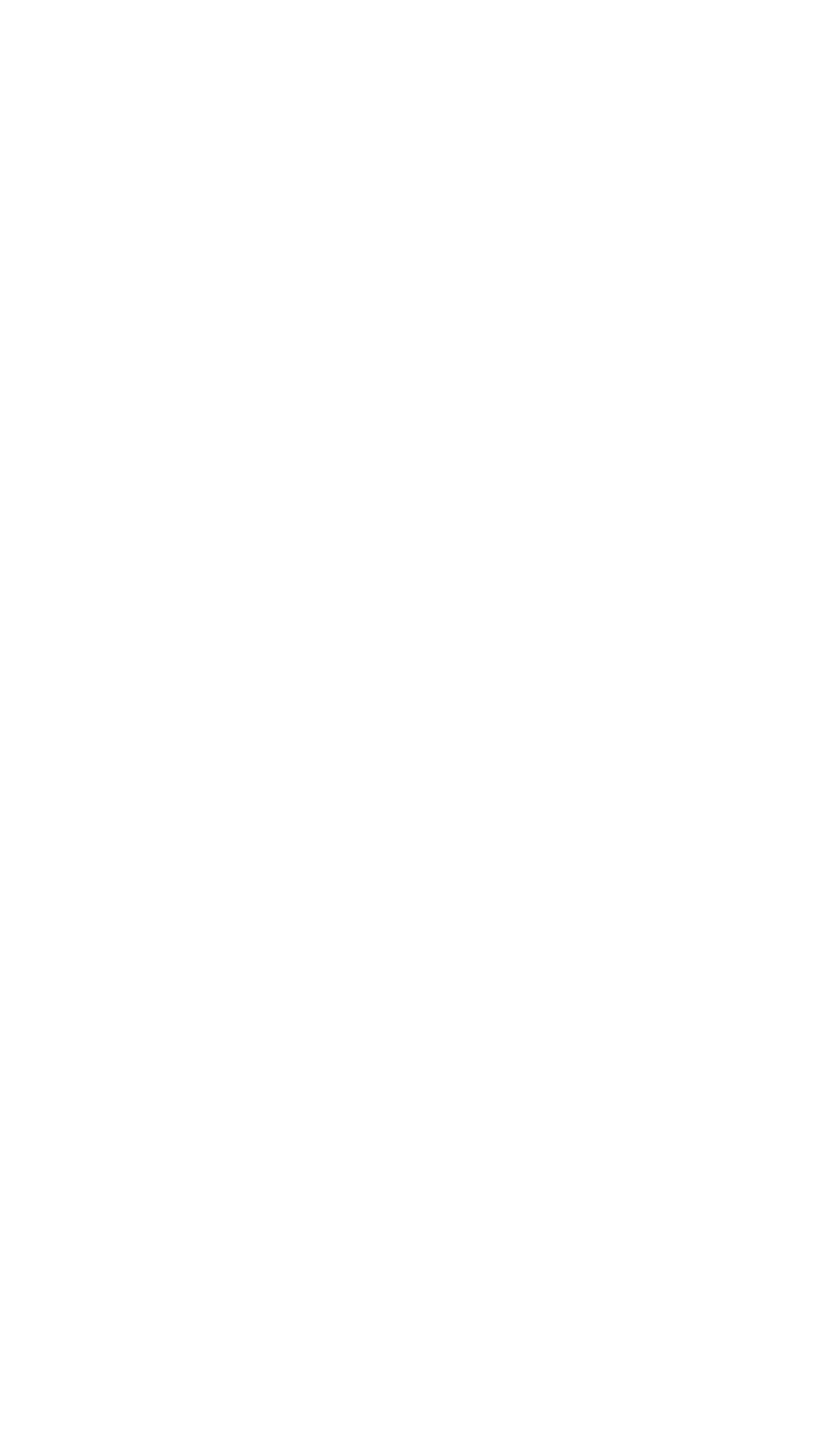


GROU'ND WATER OF WILLAMETTE VALLEY, OREG.

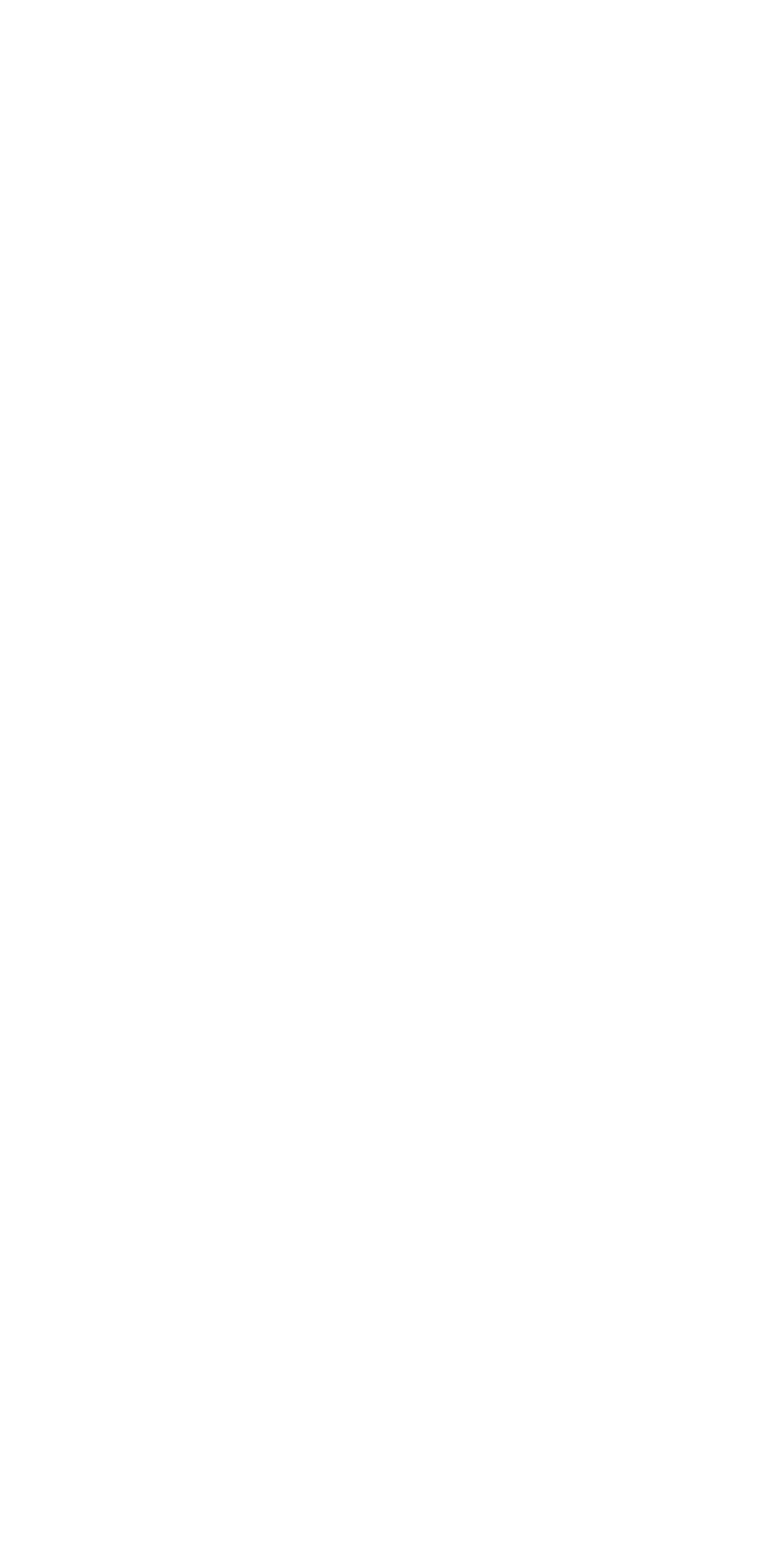




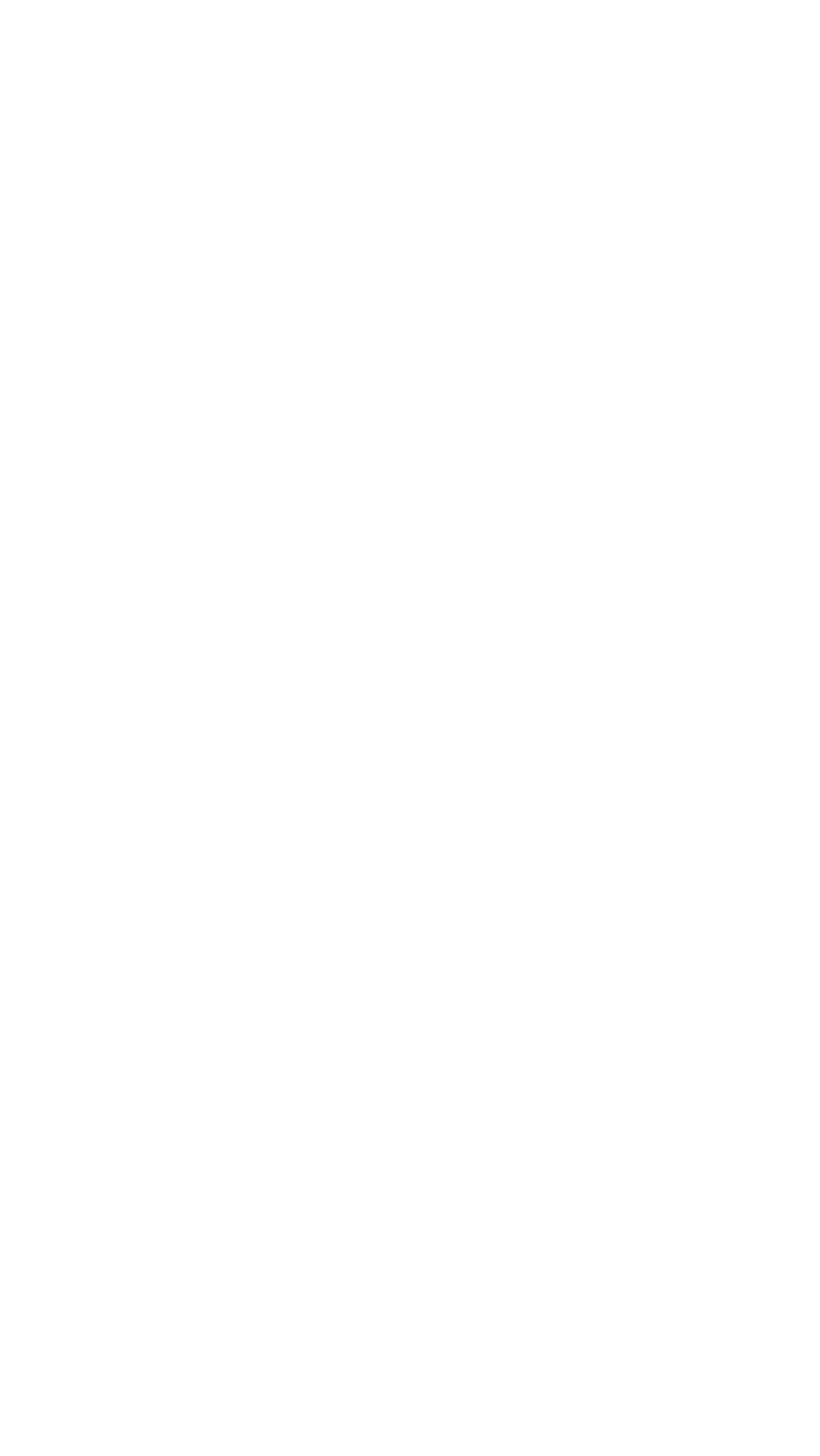




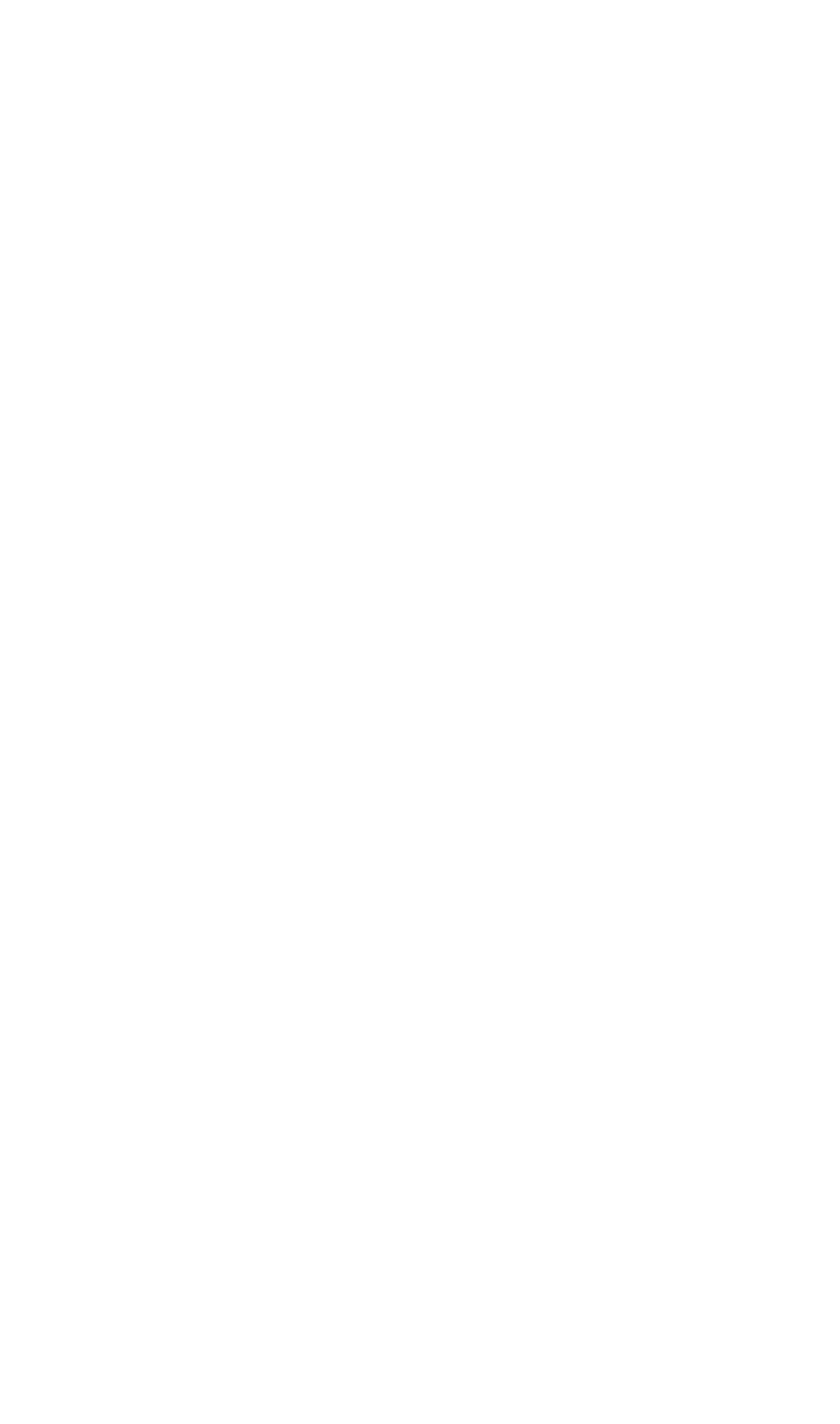




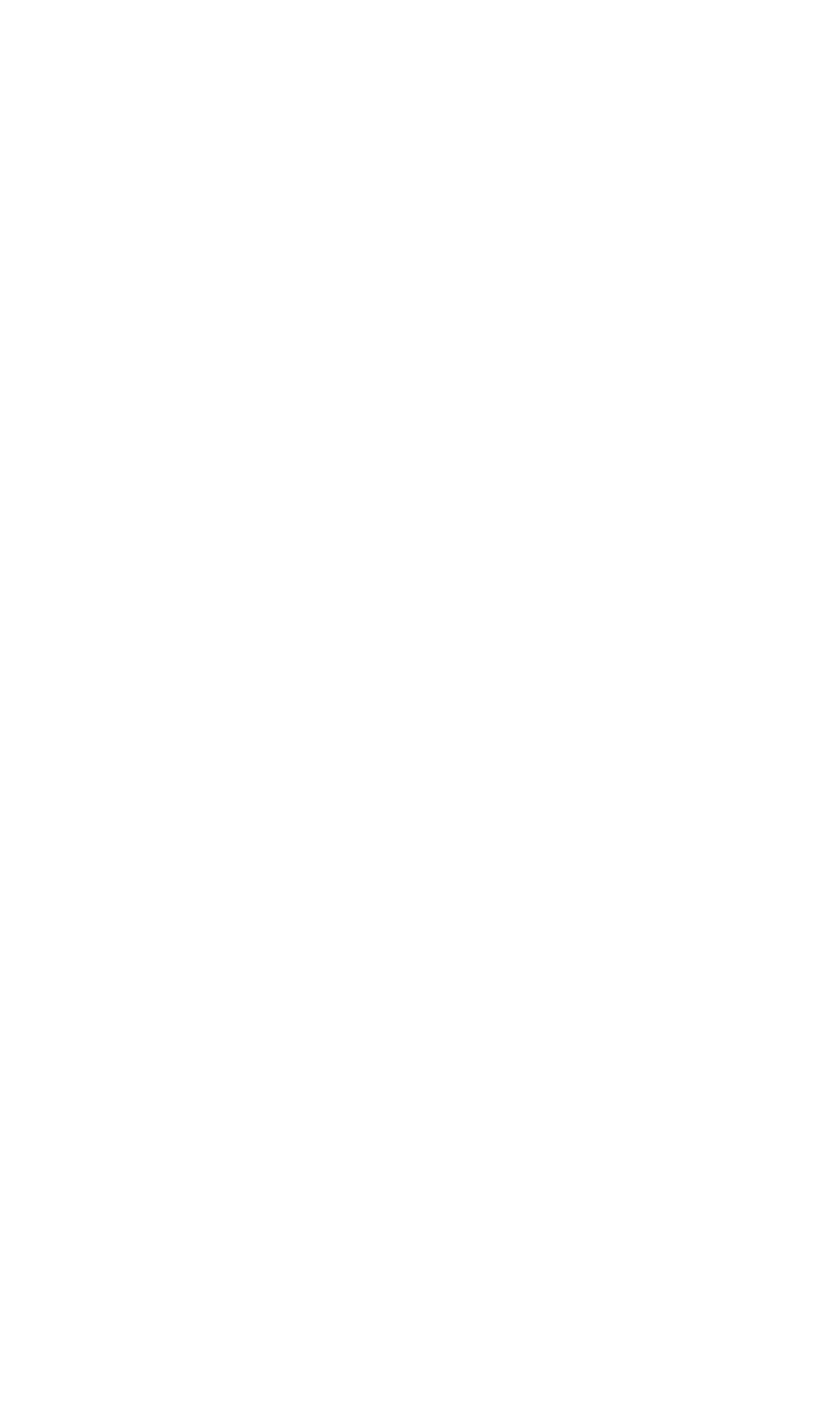


GROUND WATER OF WILLAMETTE' VALLEY, OREG.

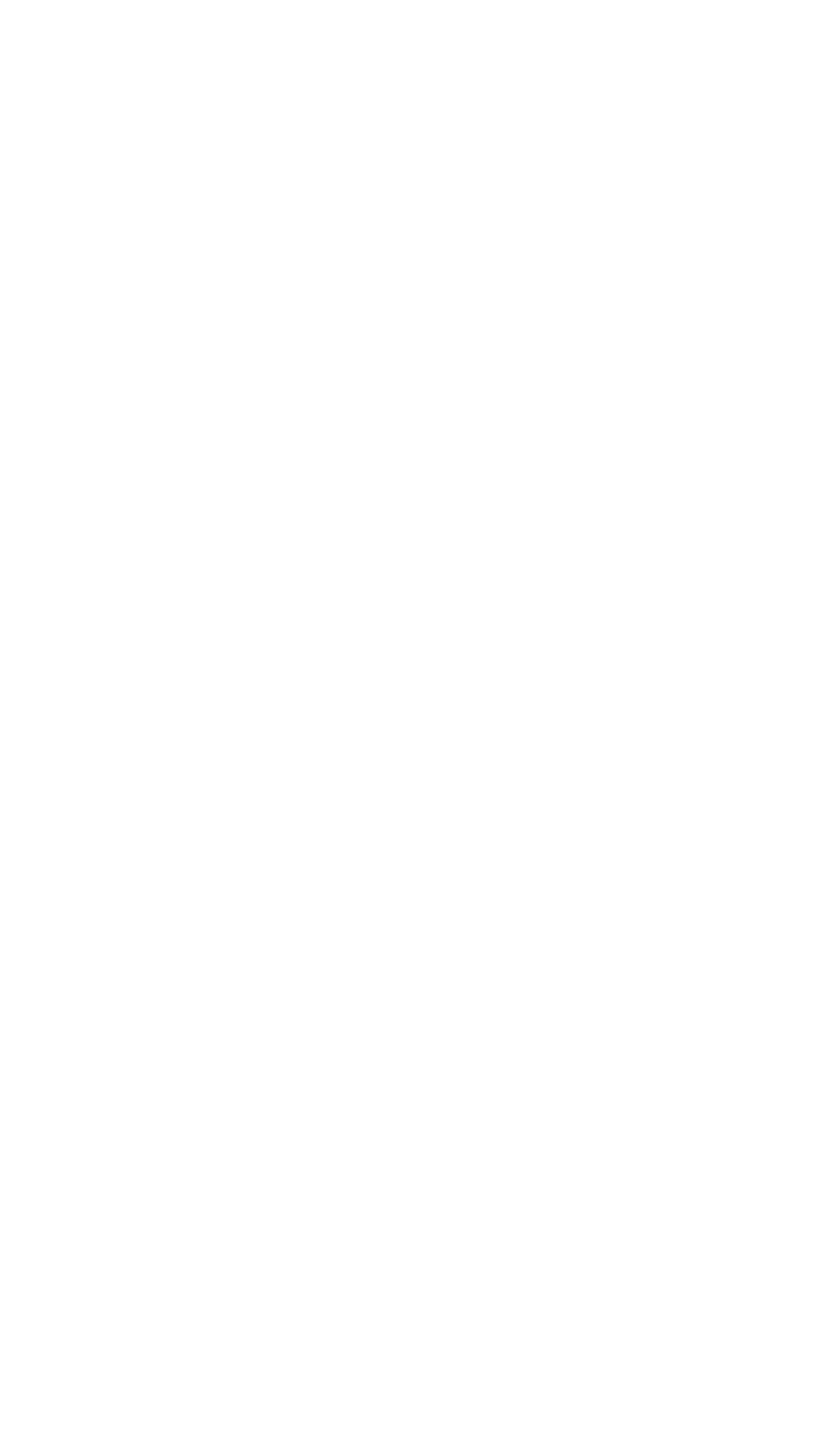


WELL RECORDS

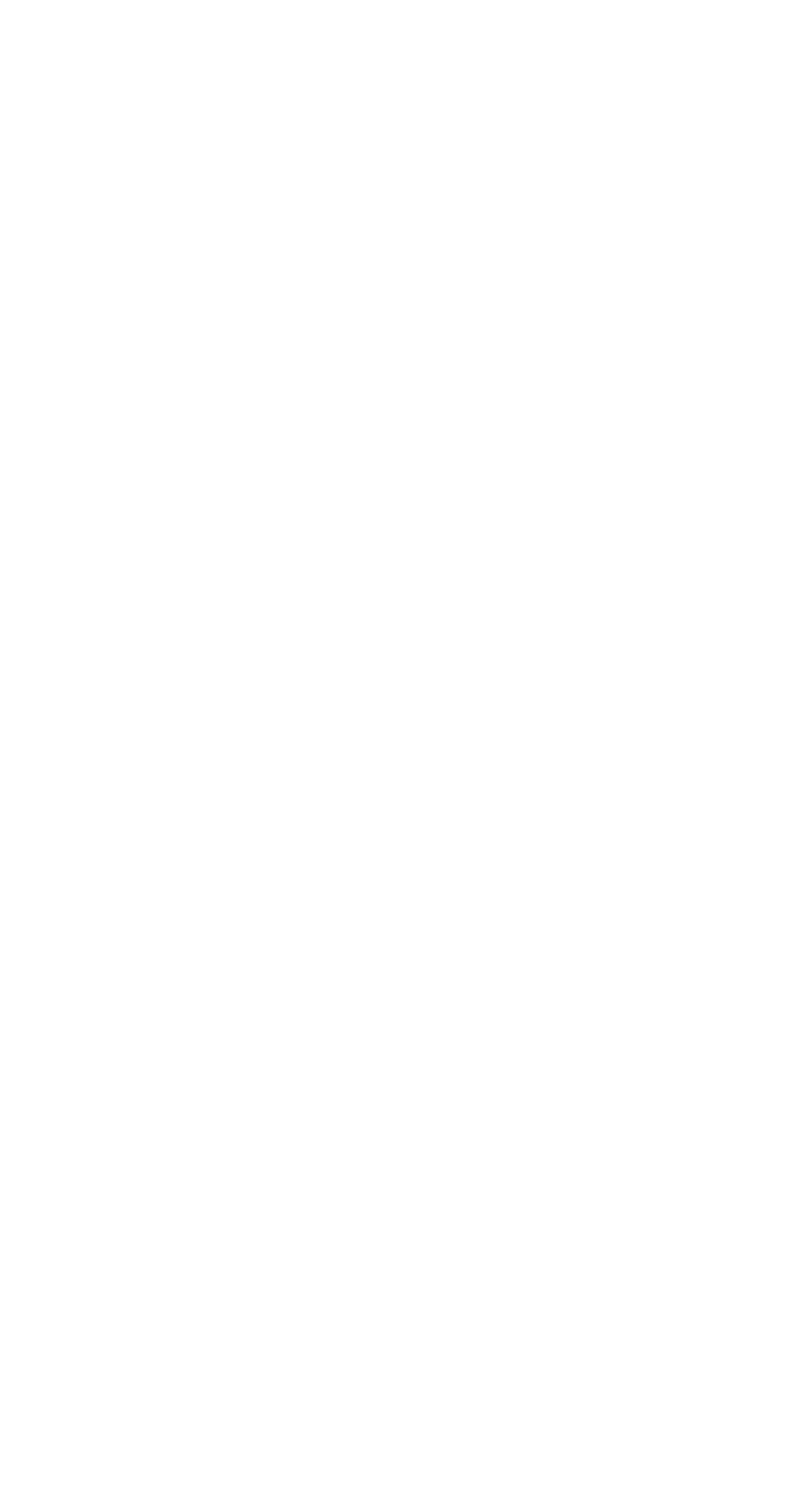




\section{TABLE 12.-Materials penetrated by typical wells}

[Based largely on drillers' records. Stratigraphic correlations, by A. M. Piper, are tentative]

\subsection{Scappoose Dike District}

[Drilled by O. E. Jannsen in 1928]

Clay and gravel

Sand and gravel $\ldots \ldots$

Sand

Sand and pea gravel

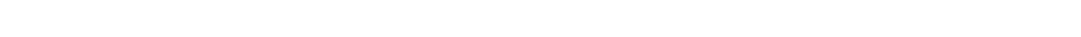

Sand and pea gravel $\ldots \ldots \ldots$ (4

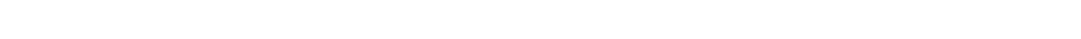

Gravel, cemented........ 17

\section{Owner unknown}

[Drilled by R. J. Strasser Drilling Co. in 1931. Deepest well on Sauvie Island]

Aluvium:

Silt and sand

Thickness

(feet)

103

Unclassified:

"Sandstone" ...

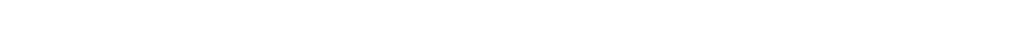

Sedimentary rocks of Tertiary age, undifferentiated:

"Shale," blue; yields "salt" water _................. 32

Sand, fine, lower half packed............. 32

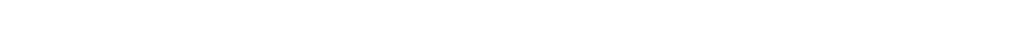

Sandstone $\ldots \ldots \ldots 37$

Rock, central part soft.

22. Masonic and Eastern Star Home

[Drilled by A..M. Jannsen Drilling Co. in 1929]

Alluvium:

Soil, clayey _...

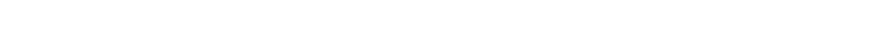

Clay, sandy

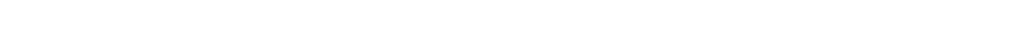

Clay, sandy

Clay, blue and gray

Fine gravel, water-bearing $\ldots \ldots \ldots 6$

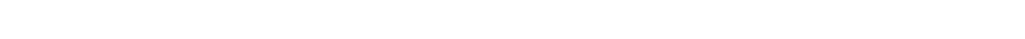

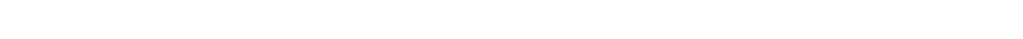

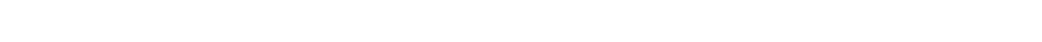

27. Terminal Ice \& Cold Storage Co.

[Drilled by O. E. Jannsen in 1914]

Alluvium:

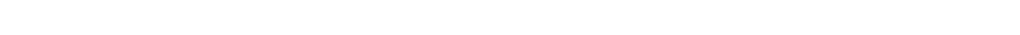

Gravel, loose._.......... $31 \quad 62$

Unclassified:

Gravel, cemented

Sand and blue clay, compact._.

1 Numbers of wells in this table correspond with the numbers used in table 11. 
TABLE 12.-Materials penetrated by typical wells-Continued

27. Terminal Ice \& Cold Storage Co.-Continued

Troutdale formation of Hodge (?):

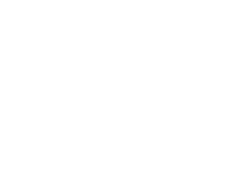

Unknown ................ 11

Gravel, cemented, and sand........ 58

Gravel, cemented, and clay ...................... 10

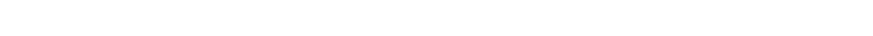

385

Yakima basalt (?):

Basalt; soft porous zones at $4161 \frac{1}{2}, 517-525$, and $540 \pm-5511 / 2$

feet; water chiefly at 478 feet but also at 465 feet ....... $166^{2} / 3$

31. Corvallis Creamery Co.

[Drilled by O. E. Jannsen Drilling Co.]

Alluvium:

\begin{tabular}{|c|c|}
\hline $\begin{array}{c}\text { Thickness } \\
\text { (feet) }\end{array}$ & $\begin{array}{c}\text { Depth } \\
\text { (feet) }\end{array}$ \\
\hline 25 & 25 \\
\hline 10 & 35 \\
\hline 19 & 54 \\
\hline 3 & 57 \\
\hline 63 & 120 \\
\hline 10 & 130 \\
\hline 30 & 160 \\
\hline 12 & 172 \\
\hline 39 & 211 \\
\hline 5 & 216 \\
\hline 38 & 254 \\
\hline 9 & 263 \\
\hline
\end{tabular}

Clay

Sand and gravel, cemented, upper part water-bearing -...

Gravel, cemented

Sand and gravel, water-bearing

Unclassified:

Gravel, cemented

Gravel, loosely cemented, water-bearing

Gravel, cemented, interstratified with clay . . . . . . . . . . . 30

Clay and gravel, water-bearing in part . . . . . . . . 12

"Shale," blue and brown, lower part sandy

"Shale," blue sandy; cemented sand _._.

263

32. Loyd Corporation, Inc.

[Drilled by A. M. Jannsen Drilling Co, in 1930-31]

Alluvium:

Sand, white, packed . . .

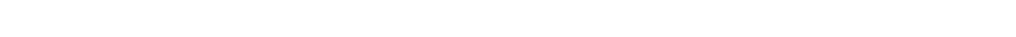

Gravel, cobbles, and boulders, cemented_............ 99145

Fine sand (?), in part clayey; cobbles in one 3-foot zone_.- $30 \quad 30 \quad 175$

Clay, sandy in part

Troutdale formation of Hodge (?):

"Sandrock," soft . . . .

Sand, fine; packed in lower part .........

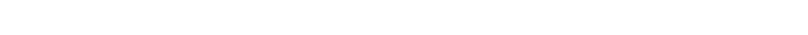

Clay, blue, and gravel.

Clay, blue.

Sand, fine.

Sand and clay

Sand, fine at top, coarsens toward bottom

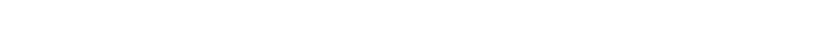

Sand _..._._.

Clay, sandy

Sand 
TABLE 12.-Materials penetrated by typical wells-Continued

33. So-called Ladd well

[Drilled about 1885 and long ago abandoned. Record from photostat copy in files of Surveys Division

Aluvium: City of Portland]

"drift" sand.

Sand, with boulders of basalt and "granite"

Boulders and gravel with layers of sand, water-bearing---- $40 \quad 40$

Gravel and sand................................. $20 \quad 200$

Boulders, cobbles, and pebbles_._.

Gravel and coarse sand, with layers of sand and "conglomerate"............ $110 \quad 330$

Sand and fine basaltic gravel_._._._._.

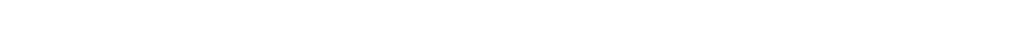

Clay, sand, and "granite" boulders in alternate layers_._- $45 \quad 405$

Troutdale formation of Hodge (?):

"Marl" and compact clay, in part sandy; fossil wood and plants.............. 31

Sand, fine and coarse, with gravel_._._._._._._. $10 \quad 730$

"Marl" and compact shale, with gravel and layers of soft fine "sandstone"

Clayey shale with layers of fine sand and plentiful fossil wood and plants.................. $150 \quad 1,000$

Fine micaceous sand, white and compact; coarse basaltic sand and fine gravel. . . . . . . . . .

Conglomerate, shale, and "marl," with iron-stained sand

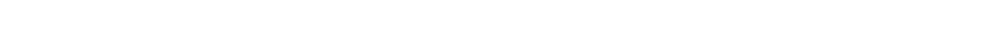

Coarse sand and gravel, basaltic _._._.

"Marl," fine and sandy; soft shale with iron-stained gravel and fossil plants............................. $50 \quad 1,300$

Yakima basalt (?):

"Granite".

44. United States Resettlement Administration, Yamhill Farms, unit 85

Aluvium:

[Drilled by $P$. Steinman in 1937]

Depth (feet)

Clay, yellow . . .

Sandy clay, blue

Sedimentary rocks of Tertiary age (?), undifferentiated:

Clay, alternately blue and yellow............... $138 \quad 233$

Clay, red, with fragmental rock; water-bearing......... $4 \quad 4 \quad 237$

Clay, yellow and red, water-bearing

"Hardpan"

"Solid rock", _... 
TABLE 12.-Materials penetrated by typical wells-Continued

53. Aaron Frank, well 2

[Drilled by A. M. Jannsen Drilling Co. in 1938. Record below 460 feet in depth from cuttings]

Unclassified:

Clay, sticky

$\begin{array}{cc}\begin{array}{c}\text { Thickness } \\ \text { (feet) }\end{array} & \begin{array}{c}\text { Depth } \\ \text { (feet) }\end{array} \\ 460 & 460 \\ 10 & 470 \\ & \\ 145 & 615 \\ 25 & 640 \\ & \\ 5 & 645 \\ 155 & 800\end{array}$

Yakima (?) basalt:

Basalt, dense, microcrystalline in part

Basalt, vesicular, slightly iron-stained in upper 3 feet.....

Sand and grit, tuffaceous (?) and iron-stained; water-

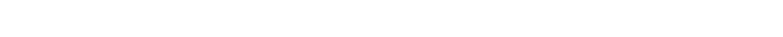

Basalt, fine-grained, dense

800

58. Northwestern Ice \& Cold Storage Co.

Fill and aluvium:

[Drilled by R. J. Strasser in 1928]

Fill and "muck",

$33 \quad 33$

Clay and "muck" - .

$79 \quad 112$

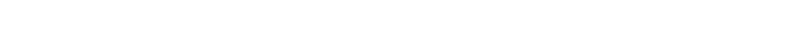

Gravel, some cobbles and boulders

Sand .

Gravel, cemented

Troutdale formation of Hodge (?):

Gravel and "shale" .......... 15

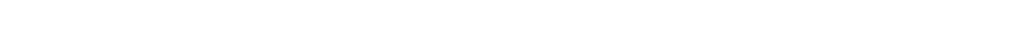

Clay, blue, with some yellow sand..._.

Sand, yellow, and clay _.

Sand, clean . .

62. Libby, McNeill \& Libby

[Drilled by R. J. Strasser in 1929. Driller's record with interpretations from representative cuttings]

Alluvium:

Fill and sandy clay

$\begin{array}{cr}\begin{array}{c}\text { Thickness } \\ \text { (feet) }\end{array} & \begin{array}{c}\text { Depth } \\ \text { (feet) }\end{array} \\ 18 & 18 \\ 7 & 25 \\ 13 & 38 \\ 24 & 62 \\ 45 & 107 \\ 3 & 110\end{array}$

Unclassified:

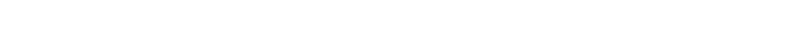

Gravel, particles less than 2 inches in diameter..........

Clay, drab; lower part sandy ... . . . . .

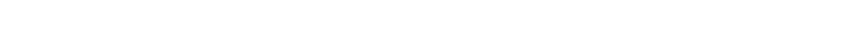

Clay, yellowish drab.......... 3

110

Gravel, cemented (tuffaceous (?) conglomerate, basaltic grit and abundant quartz grains in matrix) .............

Gravel, clay, and sand (tuffaceous (?) conglomerate, pebbles largely basaltic but some of quartzite and other rocks of distant origin; matrix contains plentiful basaltic grit, quartz grains, and some mica...........................

Troutdale formation of Hodge (?):

Clay, sandy, blue and yellow (similar to matrix of overlying conglomerate; a few pebbles, all basaltic in cuttings) ....-

Gravel, cemented, with hard sandy matrix; contains a few cobbles (pebbles basaltic; a little mica in matrix) ........

Gravel, cemented, matrix clayey 
TABLE 12.-Materials penetrated by typical wells-Continued

\section{Rockwood Water District}

[Well 1, drilled by Ziesing]

Alluvium:

Clay $\begin{array}{cc}\text { Thickness } & \text { Depth } \\ \text { (feet) } & \text { (feet) }\end{array}$

66

Sand and gravel, water-bearing

Troutdale formation of Hodge (?):

Gravel, cemented

Clay, yellow, blue, and brown

75. H. A. Roberts

[Drilled by O. E. Jannsen Drilling Co. in 1928]

Alluvium:

Clay

Gravel

"Shale," clayey at top

Sand, coarse in part, water-bearing ................ 15

Clay, greenish

Troutdale formation of Hodge (?):

"Shale," sandy

Sand, cemented

Yakima (?) basalt:

Bedrock

77. A. L. Alexander

[Drilled by O. E. Jannsen Drilling Co. in 1927]

Alluvium:

Soil and clay

Gravel, cemented

Sand

Gravel, cemented, and sand in alternate layers 4 to 10 feet thick

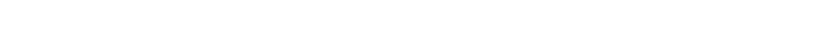

Gravel, cemented

78. F. L. Williams

[Drilled by O. E. Jannsen Drilling Co.]

Alluvium:

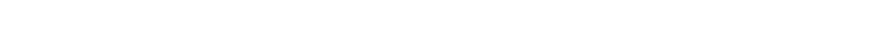

Sand and gravel ................. 8

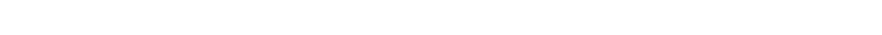

Sand

Gravel, cemented $\ldots 6$

Troutdale formation of Hodge (?):

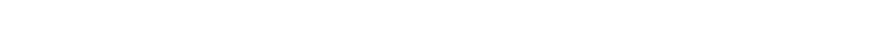

"Shale," brown and blue, grading to sand at bottom ..... $56 \quad 56 \quad 128$

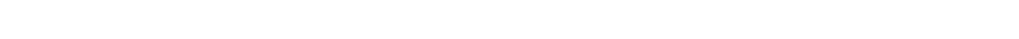

Sand, water-bearing

Sand and "shale," blue _... _. _...

"Shale," blue and green _... 
TABLE 12.-Materials penetrated by typical wells-Continued

79. Mrs. Beebe

[Drilled by O. E. Jannsen Drilling Co. in 1929]

Alluvium:

$\begin{array}{cc}\text { Thickness } & \text { Depth } \\ \text { (feet) }\end{array}$

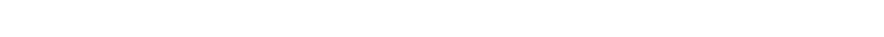

Gravel, slightly cemented; some boulders .............. 19

Gravel, cemented.................................... 17

"Sandstone," yellow .

Clay, sandy .......... 10

Troutdale formation of Hodge (?):

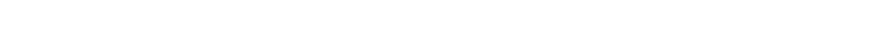

Sand, water-bearing.

102. City of Canby

[Drilled by Peter Hornig in 1912]

Alluvium:

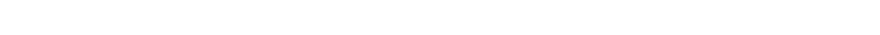

Depth (feet)

Coarse gravel and boulders, water-bearing below a depth of 40 feet. . . . . . . . . 62

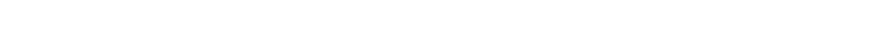

Boulders, loose . . . . . . 12

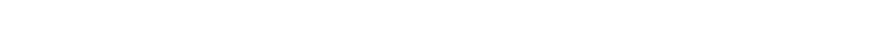

Gravel (?), loose, water-bearing .................... 2

Clay, blue, no sand or pebbles . . . .

Gravel and pebbles, loose, water-bearing .............. 2

Clay, blue.

103. City of Canby

[Drilled by Peter Hornig ln 1921]

Alluvium:

Gravel and boulders in large part .................. 87

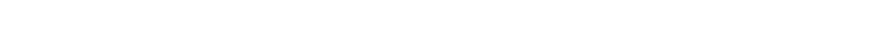

Gravel and coorse sand, water-bearing $1 \frac{1}{2}$

$\cdot 107$

Clay, blue, no sand or pebbles . . .

Sand, fine and clean.

280

281

Rocks of Tertiary age, undifferentiated:

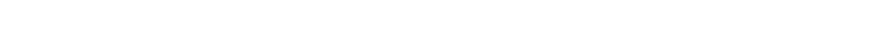

510

"Asphalt".

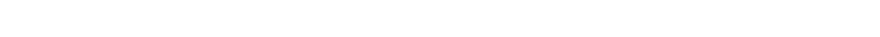

519

Fine sand, gray, and clay, blue, in alternate beds several feet thick; water-bearing ..................... 127

"Sandstone, gray, not water-bearing ............... 1

\section{J. C. Mattoon}

[Drilled by O. E. Jannsen Drilling Co.]

Soil and clay 
TABLE 12.-Materials penetrated by typicals wells-Continued

118. United States Reset tlement Administration, Yamhill Farms, unit 68

[Drilled by A. M. Jannsen Drilling Co. in 1937]

Alluvium:

Soil and "hardpan"

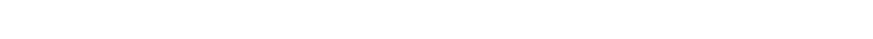

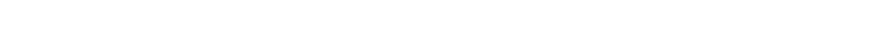

Sand, blue, and pea gravel, water-bearing ............ 2

Sand, coarse and blue, with wood and bark . . . . . . . . . 12

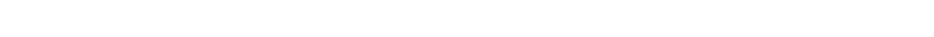

Sedimentary rocks of Tertiary age, undifferentiated:

Sandstone . . . . . . . . . . . . . . . . . . . . . . . . 6

Shale, sandy, gray, alternately hard and soft $\ldots \ldots \ldots \ldots . . .91$

$\begin{array}{cr}\begin{array}{c}\text { Thickness } \\ \text { (feet) }\end{array} & \begin{array}{r}\text { Depth } \\ \text { (feet) }\end{array} \\ 3 & 3 \\ 62 & 65 \\ 13 & 78 \\ 2 & 80 \\ 12 & 92 \\ 23 & 115 \\ & - \\ 6 & 121 \\ 91 & 212\end{array}$

139. United States Resettlement Administration, Yamhill Farms, unit 16

[Drilled by A. M. Jannsen Drilling Co. in 1937]

Alluvium:

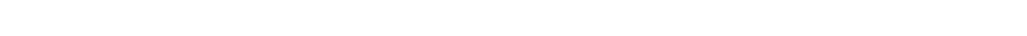

Clay, yellow . . .

Fine sand; yellow, gray, brown, and pink _........... 75

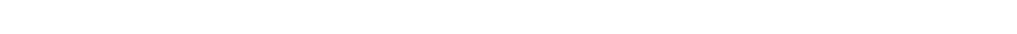

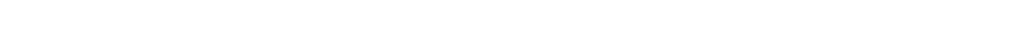

Sand and fine gravel, water-bearing ................. 1

Sedimentary rocks of Tertiary age, undifferentiated:

Shale, gray . . . .

143. United States Resettlement Administration, Yamhill Farms, unit 19

[Drilled by A. M. Jannsen Drilling Co. in 1937]

Alluvium:

Thickness (feet)'

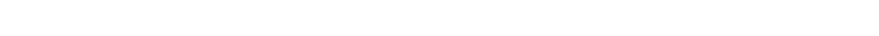

Sand, yellow and blue, hard at bottom ............ 40

Coarse sand, water-bearing . .

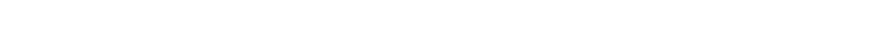

Clay, yellow, and sand . . .

Sandstone and clay . . . . . . . . . . .

Sedimentary rocks of Tertiary age, undifferentiated:

Rock, topmost foot porous and water-bearing

153. Pleasantdale School

[Drilled by H. E. Evans]

Alluvium:

Soil and clay

Boulder clay

Sand, packed, not water-bearing

Clay, blue ...

Gravel, sand, and greenish-blue clay; coarse members water-bearing

Gravel, water-bearing. 
TABLE 12.-Materials penetrated by typical wells-Continued

\section{William Gooding}

[Drilled by Peter Hornig. Record interpreted from samples of cuttings]

Alluvium:

Thickness (feet)

Soil and subsoil. 20

"Quicksand" (very fine sand and silt)

92

Sand, medium and coarse (basaltic)

Coarse sand and pebbles (basaltic)

[Drilled in 1931. Record interpreted from samples of cuttings]

Alluvium:

Soil.

Sand, dark gray (basaltic, poorly rounded grains, pebbles as much as 4 millimeters in diameter.............. 18

Sand, blue, and clay _..._........................... 20

Clay, blue (fine sand or silt with basaltic grit)

Sand, blue, and gravel; coarser toward bottom (basaltic, grains poorly rounded, 1 to 20 millimeters in diameter); water-bearing

Troutdale or Rhododendron formation of Hodge, or both (?):

Clay and sandy clay, blue (nondescript mixture of particles

. from silt to coarse sand, some of quartz and mica, many waterworn; tuffaceous?) _........................... 184

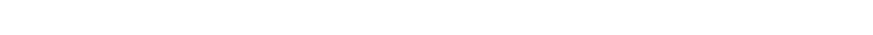

Clay, sandy, a few pebbles (from silt to medium sand; grains angular; largely basaltic though feldspar and quartz are abundant; tuffaceous?)

Sand, very fine (medium sand; grains angular to partly rounded, one-half of quartz, feldspar, muscovite, or

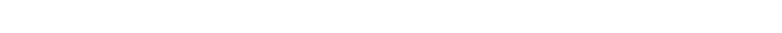

Sand, very fine, with a little clay .

Coarse sand (largest grains $2 \frac{1}{2}$ millimeters in diameter, well-rounded and assorted; include basaltic clinker, quartz, and feldspar)

Clay (silt and fine sand, nonlaminated, abundant mica, tuffaceous?) _.

Shale, soft (with fine to coarse sand, rude laminae, carbonized wood in coarser layers, moderately consolidated) ....

206. United States Resettlement Administration, Yamhill Farms, unit 6

[Drilled in 1937]

Alluvium:

Soil

Clay, yellow and blue

Sand, blue

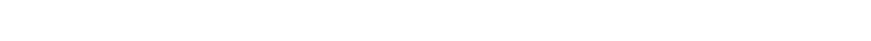

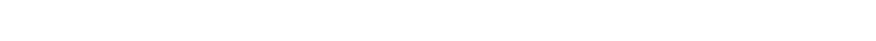

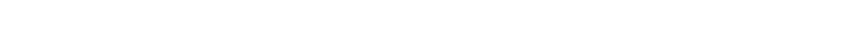

Gravel and coarse sand, water-bearing .............. 2 


\section{TABLE 12.-Materials penetrated by typical wells-Continued}

\section{United States Resettlement Administration, Yamhill Farms, unit 24}

[Drilled by Steinman Bros. Drilling Co. in 1937]

Alluvium:

Thickness Depth

(feet) (feet)

Soil

Clay, yellow

Silt .

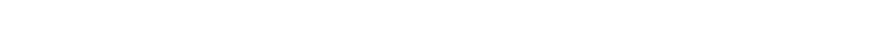

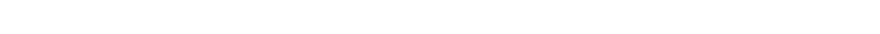

Unclassified:

Clay, blue and gray, interlaminated with silt $\ldots \ldots \ldots \ldots \ldots 115$

Clay, yellow and brown. ......................... 90

Clay, sandy, brown

Sedimentary rocks of Tertiary age:

Shale, gray, caving

\section{Agricultural Research Corporation (Sam H. Brown)}

Applied by A. M. Jannsen Drilling Co. in 1930. Record interpreted from samples of cuttings. (See physical properties of water-bearing materials, p. 31)]

Allıvium:

Thickness Depth (feet) (feet)

Loam

Clay, yellow and blue, with streaks of pea gravel at $18 \frac{1}{1}$ feet and of fine sand below 38 feet (sparse mica)

Clay grading downward into sand . . .

Sand and gravel, fine (unassorted fine sand to pebbles half an inch in diameter; largely basaltic though quartz and feldspar abundant); water-bearing

Clay; gray, blue, and brown; in part sandy and pebbly

Silt, dark bluish gray (very fine sand to medium sand; quartz grains abundant)

Sand, fine and black

Sand and fine gravel, somewhat cemented (pebbles all from basic volcanic rocks)

Gravel, coarse, and sand (grains fairly well rounded and somewhat assorted; pebbles an inch in diameter are abundant; largest are $2 \frac{1}{2}$ inches long); water-bearing ...

Clay, blue and pebbly

Sand, black (fine to coarse)

Clay, green and sticky

Sand, black and loose (medium to very coarse, fairly well assorted; feldspar and quartz abundant) $\ldots \ldots$

Clay or shale, green, streak of peat at top_._._.

Sand, black

Clay, blue, watertight

Gravel and sand, streak of clay at 227 feet, slightly cemented in part (largely basaltic; above 224 feet, poorly assorted very fine sand to pebbles; moderately assorted below 230 feet); water-bearing.

Clay, blue and sticky . . . . . . 
TABLE 12.-Materials penetrated by typical wells-Continued 247. City of Gervais

[Drilled by A. M. Jannsen Drilling Co. in 1928

Alluvium:

Soil and clay

20

(feet)

Sand, water-bearing

Clay, blue, and fine sand .......................... 61

22

Sand, black, water-bearing

Clay, blue and sticky _...

Sand, black, water-bearing .......................... 17

Gravel and coarse sand, black, water-bearing .......... 6

122

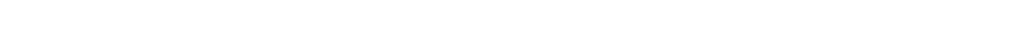

\section{Oregon State Training School}

[Drilled by George E. Scott in 1924]

Alluvium:

Thickness (feet)

Depth

(feet)

Clay

Sand, fine, dark, gravelly in part . . .

Gravel, water-bearing ................................ 8

Sand, dark $\ldots \ldots$

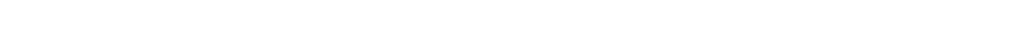

Sand, dark ....... 10

Gravel, "dirty" .

Gravel, water-bearing $\ldots \ldots 15$

Clay, blue...... 15

262. City of Woodburn

[Drilled by George E. Scott in 1925]

Alluvium:

Clay

Sand, fine, water-bearing $\ldots \ldots \ldots$

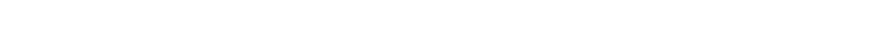

Gravel, coarse, and sand.

Clay, blue and soft_.....

Gravel, coarse, and sand; water-bearing _............ 18

$16:$

Clay, blue

263. Southern Pacific Railroad Co., Woodburn station

[Drilled by George E. Scott]

Alluvium:

Clay, yellow

Sand, black

Gravel and yellow clay .............................. 4

Gravel, yellow, and sand ........................... 28

Clay, blue, and sand _.......................... 27

Gravel, coarse, black, water-bearing 
TABLE 12.-Materials penetrated by typical wells-Continued

280. Eastern-Western Lumber Co.

[Drilled by O. E. Jannsen Drilling Co. in 1927]

Pediment deposit:

Thickness Depth

(feet)

Boulders........ 15

$15 \quad 15$

Clay _. 20

Clay, sandy _... 25

Troutdale or Rhododendron formation of Hodge, or both (?):

Clay, blue............ 100

"Shale"

Sand, water-bearing

"Shale"

"Sandstone," streak of "shale" at 265 feet, another of cemented gravel at bottom; thin water-bearing zones at

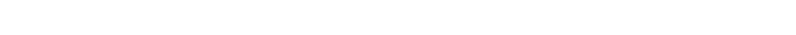

"Shale",

Sand

3321/2. Public Works Engineering Corporation

[Record interpreted [ rom samples of cuttings]

Younger and older alluvium:

Sand, gravel, and pebbles as much as $1 \frac{1}{2}$ inches in diameter; lower half free from fine and medium sand; water-bearing below depth of 21 feet

Coarse sand and pebbles, cemented with fine sand and silt; not water-bearing.

Coarse sand to pebbles half an inch in diameter, loose, waterbearing

Illahe formation of Thayer (?):

Sandstone, fine-grained and earthy, weathered yellowish drab; largely of quartz grains but some basaltic grit; yields a little water

Clay "gumbo," blue (with fine sand, largely quartz but some basaltic grit, sparse mica, tuffaceous ?); not water-bearingClay shale, blue and sandy (unassorted and unstratified, grain sizes from fine to coarse sand, tuffaceous (?)); yields a little water from several zones...................

Blue shale (with mixture of fine to medium sand and some grit, moderately indurated, basaltic grains and quartz grains about equally abundant, tuffaceous ?); not waterbearing 
TABLE 12.-Materials penetrated by typical wells-Continued 333. Oregon Linen Mills, Inc.

[Drilled by Harvey Evans]

Older alluvium:

Thickness Depth (feet) (feet)

Soil and clay

Clay and boulder "hardpan" ...

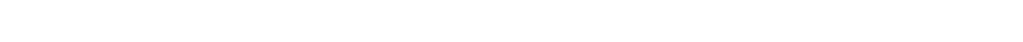

Sand and gravel, water-bearing

Clay and gravel "hardpan"

Sand and gravel, yields a little water._._.

Unclassified:

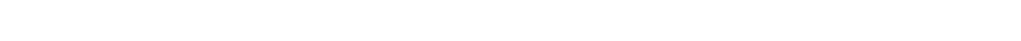

Sand and gravel, packed.

Yakima basalt (?):

"Medium blue stone," water-bearing _...... $601 / 2$

$248 \frac{1}{2}$

334. Public Works Engineering Corporation

[Drilled by Ferris \& Gardner. Record interpreted from samples of cuttings]

Older alluvium:

Thickness (feet)

Clay, water at base 20

Pebbly clay (basaltic pebbles) 3

Gravel, loose (basaltic, coarse sand to pebbles an inch in diameter), water-bearing ...... 5

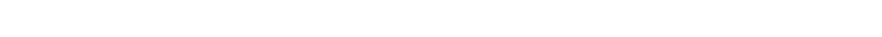

Gravel, coarse (basaltic pebbles, fine matrix in several zones),

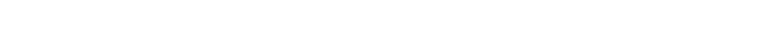

Unclassified:

$45 \quad 95$

Clay, gravel, and boulders (bloulder clay ?); not water-bearing . . . . . . .

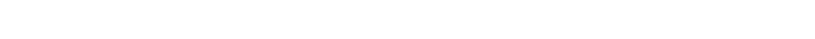

Clay, gravel, and boulders (samples show pronounced iron stain below 230 feet) ..........

Yakima (?) basalt:

Rock (weathered basalt)

336. A. W. Stryker

Alluvium:

[Drilled by West in 1929]

Thickness Depth

$45 \quad 45$

Soil and clay, blue in lower part.......... 45

Sand and gravel, water-bearing ................. 10

Gravel, cemented................................. 15

70

Sand, loose, and gravel; water-bearing _._. _ _._._. 25

95

Gravel, cemented with clay ................... 20

Gravel, loose, water-bearing 
TABLF 12.-Materials penetrated by typical wells-Continued

338. Oregon State Hospital, well 3

[Drilled by O. E. Jannsen Drilling Co. in 1925]

Alluvium:

Clay and sand .................................. 35

$35 \quad 35$

Gravel, cemented.................................... 22

Gravel and cobbles, some clay in lower half; not water-

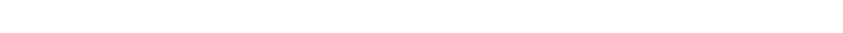

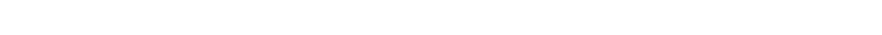

Gravel and sand, with clay matrix..._._._.......... 21

Gravel and sand, water-bearing._................... $16 \quad 111$

Clay and gravel.................................... 11/2 $1121 / 2$

3381/2. Oregon State Penitentiary

[Drilled by A. M. Jannsen Drilling Co.]

Alluvium:

Soil and clay

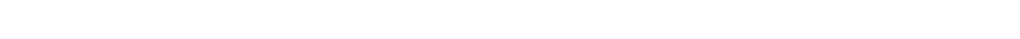

Sand and gravel, yields a little water................ $26 \quad 56$

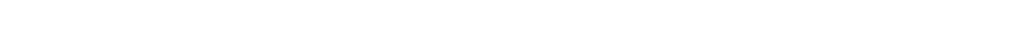

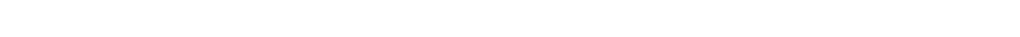

Gravel, cemented

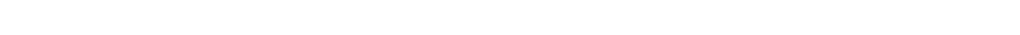

Gravel, water-bearing

Gravel, cemented............................... $20 \quad 122$

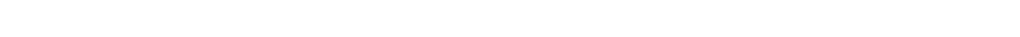

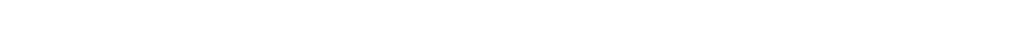

Rocks of Tertiary age, undifferentiated:

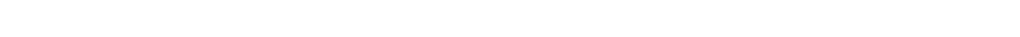

385. Cottage Farm, Oregon State Hospital

[Drilled by O. E. Jannsen Drilling Co. in 1925]

Unclassified:

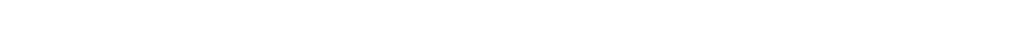

Clay and boulders............................ 13

Illahe formation of Thayer:

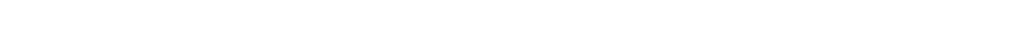

Shale and sandstone, interbedded. 236

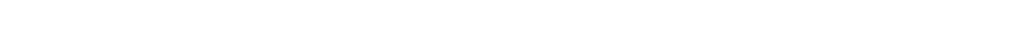

Sandstone, yields a little water at about 450 feet_....... $190 \quad 515$

Shale, with a few thin layers of sandstone............ $490 \quad 1,005$

435. Conser

Alluvium:

[Drilled by C. E. Gordonier]

Soil

Clay, yellow . . .

Thickness
(feet) $\quad \begin{gathered}\text { Depth } \\ \text { (feet) }\end{gathered}$

33

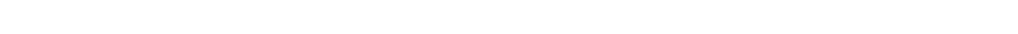

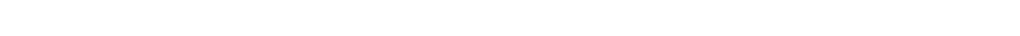

Sedimentary rocks of Tertiary age, undifferentiated:

Sandstone, blue . . .

Sandstone, yields "salt" water . . .

Sandstone, blue; water of poor quality at base $\ldots \ldots \ldots \ldots$ 
TABLE 12.-Materials penetrated by typical wells-Continued

526. Bingenheimer

Alluvium:

[Drilled by H. E. Evans]

Soil

Clay and cobbles ................ 17

Thickness Depth

$3 \quad 3$

Gravel (?), water-bearing $\ldots \ldots \ldots$

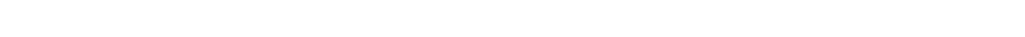

Rocks of Tertiary age, undifferentiated:

Clay, blue and red

Coarse gravel and cobbles, no sand; water-bearing _...... $17 \quad 193$

\section{Junction City}

Alluvium:

[Drilled in 1938]

\begin{tabular}{|c|c|}
\hline $\begin{array}{c}\text { Thickness } \\
(\text { feet })\end{array}$ & $\begin{array}{c}\text { Depth } \\
\text { (feet) }\end{array}$ \\
\hline 2 & 2 \\
\hline 6 & 8 \\
\hline 7 & 15 \\
\hline 16 & 31 \\
\hline 7 & 38 \\
\hline 22 & 60 \\
\hline 8 & 68 \\
\hline 132 & 200 \\
\hline 33 & 233 \\
\hline 47 & 280 \\
\hline 70 & 350 \\
\hline 17 & 367 \\
\hline 63 & 430 \\
\hline 10 & 440 \\
\hline 10 & 450 \\
\hline
\end{tabular}

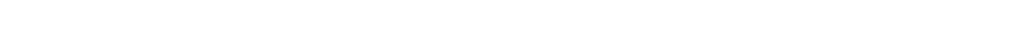

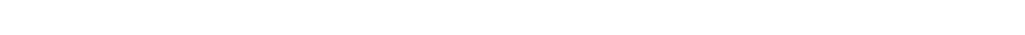

Clay and gravel $\ldots$

Gravel and sand, clayey at top and bottom, water-bearing - $16 \quad 31$

Clay, brown and blue .......................... 78

Sand, coarse, and soft brown clay, water-bearing _....... $22 \quad 60$

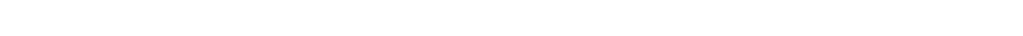

Sand, fine gravel, and blue-gray clay, water-bearing _..... $132 \quad 200$

Sedimentary rocks of Tertiary age, undifferentiated:

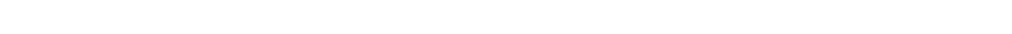

Shale, blue-gray and brown $\ldots \ldots \ldots$

Sandstone, blue-gray, soft; yields a little water _......... $70 \quad 350$

"Cement gravel" (agglomerate or conglomerate ?) _....... $17 \quad 367$

Shale, brownish-gray, lower part sandy _._._._._._._. $63 \quad 430$

"Conglomerate," very light gray, hard ............. $10 \quad 440$

Shale, brownish-gray .................. 10

701. Bethel School

Alluvium:

[Drilled by A. F. Moody]

Thickness Depth

Soil_...

Gravel.

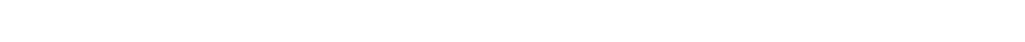

Sand and fine gravel._._.

Gravel, cemented:-...

Sand

Gravel, cemented; water-bearing ................. 2 106

722. M. E. Furrow

[Drilled by A. F. Moody]

Rocks of Tertiary age, undifferentiated:

Basalt.

"Sandrock," brown, very hard $\ldots$

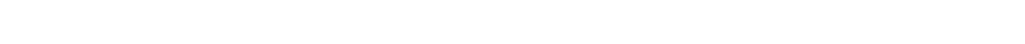

Rock, white and very soft (pumice ?) _._._._._._. $25 \quad 347$

Sandstone, water-bearing . 


\section{TABLE 13.-Ground-water levels in observation wells, 1928-30 and 1935-96 1}

87. Frank H. Newland. SW1/4SE1// sec. 32, T. 3 S., R. 4 W. Domestic well, dug $31 / 2$ feet in diameter and 39 feet deep, brick curb. Measuring points: Top of pump-house sill, 157.88 feet above mean sea level; and top of concrete wall, 0.7 foot above land surface and 157.98 feet ${ }^{2}$ above sea level.

\begin{tabular}{|c|c|c|c|c|c|c|c|}
\hline Date & $\begin{array}{l}\text { Water } \\
\text { level } \\
\text { (feet) }\end{array}$ & Date & $\begin{array}{l}\text { Water } \\
\text { level } \\
\text { (feet) }\end{array}$ & Date & $\begin{array}{l}\text { Water } \\
\text { level } \\
\text { (feet) }\end{array}$ & Date & $\begin{array}{l}\text { Water } \\
\text { level } \\
\text { (feet) }\end{array}$ \\
\hline 1928 & & 1936 & & 1936 & & 1936 & \\
\hline Sept. 25 & 138.5 & Mar. 30 & 152.42 & June 1 . & 148. 94 & Aug. 3. & 143.89 \\
\hline 1936 & & $\begin{array}{l}\text { Apr. }{ }_{-} \\
\text {Apr. } 13\end{array}$ & $\begin{array}{l}150.86 \\
149.43\end{array}$ & June 15 & $\begin{array}{l}147.81 \\
148.26\end{array}$ & $\begin{array}{l}\text { Aug. 10. } \\
\text { Aug. } 17 .-\end{array}$ & $\begin{array}{l}142.07 \\
141.92\end{array}$ \\
\hline & & Apr. 20 & 149.39 & June 22 & 148. 21 & Aug. 24 & 141.71 \\
\hline Feb. 19 & 150.68 & Apr. 27.- & 149.70 & June 29.-- & 146.70 & Aug. 31 & 141.69 \\
\hline Feb. 24 & 153.51 & May 4 & 149.79 & July $6 \ldots$ & 146.65 & Sept. 8 & 139.67 \\
\hline $\begin{array}{l}\text { Mar. } 2 \\
\text { Mar. } 9\end{array}$ & 153.59 & May $11 \ldots$ & 149.44 & July 13 - & 145. 66 & Sept. 14_.......... & 140.46 \\
\hline Mar. 16 & 150.77 & May 25 & $\begin{array}{l}149.41 \\
148.92\end{array}$ & July $27 \ldots$ & $\begin{array}{l}144.00 \\
144.39\end{array}$ & Sept. 28 & $\begin{array}{l}101.00 \\
137.62\end{array}$ \\
\hline Mar. 23 & 150.93 & & & & & & \\
\hline
\end{tabular}

1 Ground-water levels in 1928-30, by U. S. Geological Survey; in 1935-36, by U. S. Engineer Department.

2 Leveling by U. S. Engineer Department.

100. Pietro Presutti. SW1/4NW1/4 sec. 30, T. 2 S., R. 1 E. Domestic well, drilled 6 inches in diameter and 145 feet deep, standard steel casing. Substitute for well 101 , which was destroyed between 1930 and 1935. Measuring point is bottom of plugged hole in base of pump, 0.4 foot above land surface and 159.28 feet ${ }^{2}$ above mean sea level.

\begin{tabular}{|c|c|c|c|c|c|c|c|}
\hline Date & $\begin{array}{l}\text { Water } \\
\text { level } \\
\text { (feet) }\end{array}$ & Date & $\begin{array}{l}\text { Water } \\
\text { level } \\
\text { (feet) }\end{array}$ & Date & $\begin{array}{l}\text { Water } \\
\text { level } \\
\text { (feet) }\end{array}$ & Date & $\begin{array}{l}\text { Water } \\
\text { level } \\
\text { (feet) }\end{array}$ \\
\hline 1935 & & 1936 & & 1936 & & 1936 & \\
\hline $\begin{array}{l}\text { Oct. } 18 \\
\text { Oct. } 23 \\
\text { Oct. } 28 \\
\text { Nov. } 4 \\
\text { Nov. } 11 \\
\text { Nov. } 18 \\
\text { Dec. } 2 \\
\text { Dec. } 9 \\
\text { Dec. } 16 \\
\text { Dec. } 23 \\
\text { Dec. } 30 \\
\quad 1936 \\
\text { Jan. } 6 \\
\text { Jan. } 13\end{array}$ & \begin{tabular}{|l}
110.20 \\
110.28 \\
109.54 \\
110.19 \\
110.60 \\
110.58 \\
109.60 \\
109.79 \\
106.09 \\
110.68 \\
110.93 \\
\\
\\
113.28 \\
3154.44
\end{tabular} & $\begin{array}{l}\text { Jan. } 20 \\
\text { Jan. } 27 \\
\text { Feb. } 3 \\
\text { Feb. } 10 \\
\text { Feb. } 17 \\
\text { Feb. } 24 \\
\text { Mar. } 2 \\
\text { Mar. } 9 \\
\text { Mar. 16 } \\
\text { Mar. 23 } \\
\text { Mar. } 30 \\
\text { Apr. } 6 \\
\text { Apr. } 13 \\
\end{array}$ & $\begin{array}{r}3155.11 \\
108.93 \\
109.07 \\
109.13 \\
109.13 \\
109.63 \\
109.96 \\
108.83 \\
109.13 \\
109.00 \\
108.96 \\
109.01 \\
109.56\end{array}$ & $\begin{array}{l}\text { Apr. 21 } \\
\text { Apr. 27 } \\
\text { May 4 } \\
\text { May 11 } \\
\text { May 18 } \\
\text { May 25 } \\
\text { June 1. } \\
\text { June } 8 \\
\text { June 15 } \\
\text { June 22 } \\
\text { June 29 } \\
\text { July } 8\end{array}$ & $\begin{array}{r}109.90 \\
108.38 \\
108.06 \\
109.12 \\
4104.71 \\
108.72 \\
107.51 \\
108.46 \\
108.46 \\
108.15 \\
107.64 \\
107.81\end{array}$ & \begin{tabular}{|} 
July 13 \\
July 20 \\
July 28 \\
Aug. 3 \\
Aug. 10 \\
Aug. 17. \\
Aug. 24 \\
Aug. 31 \\
Sept. 8 \\
Sept. 14 \\
Sept. 21 \\
Sept. 28 \\
\end{tabular} & $\begin{array}{l}107.99 \\
107.73 \\
107.21 \\
108.14 \\
108.18 \\
107.67 \\
107.70 \\
107.53 \\
102.39 \\
106.08 \\
106.00 \\
105.81\end{array}$ \\
\hline
\end{tabular}

2 Leveling by U. S. Engineer Department.

3 Water probably entering well above static level.

4 Water level depressed by inordinately large withdrawals.

101. Isaac A. Miley estate. NW1/4SW1/4 sec. 30, T. 3 S., R. 1 E. Domestic well, bored 10 inches in diameter and 57 feet deep. Measuring point is top of casing, 0.4 foot above land surface and 157.5 feet above sea level (altimeter).

\begin{tabular}{|c|c|c|c|c|c|c|c|}
\hline Date & $\begin{array}{l}\text { Water } \\
\text { level } \\
\text { (feet) }\end{array}$ & Date & $\begin{array}{l}\text { Water } \\
\text { level } \\
\text { (feet) }\end{array}$ & Date & $\begin{array}{l}\text { Water } \\
\text { level } \\
\text { (feet) }\end{array}$ & Date & $\begin{array}{l}\text { Water } \\
\text { level } \\
\text { (feet) }\end{array}$ \\
\hline 1928 & & 1929 & & 1929 & & 1930 & \\
\hline $\begin{array}{l}\text { Sept. } 27 \\
\text { Oct. } 10 \\
\text { Dec. } 17\end{array}$ & $\begin{array}{l}108.2 \\
106.3 \\
126.7\end{array}$ & $\begin{array}{l}\text { Feb. } 3 \\
\text { Mar. } 23 \\
\text { May } 12 \\
\text { June } 7\end{array}$ & $\begin{array}{l}133.4 \\
132.45 \\
120.85 \\
107.10\end{array}$ & $\begin{array}{l}\text { July } 3 \\
\text { Aug. } 5 \\
\text { Sept. } 24 \\
\text { Oct. } 27 \\
\text { Nov. } 29\end{array}$ & $\begin{array}{l}105.90 \\
105.45 \\
102.05 \\
106.65 \\
106.45\end{array}$ & $\begin{array}{l}\text { Feb. } 2 \\
\text { Mar. } 2 \\
\text { Mar. } 30 \\
\text { Apr. 27 } \\
\text { July 22 }\end{array}$ & $\begin{array}{l}106.70 \\
105.60 \\
105.50 \\
105.25 \\
103.22\end{array}$ \\
\hline
\end{tabular}


TABLE 13.-Ground-water levels in observation wells, 1928-80 and 1985-36-Con.

126. O. B. Belieu. NW1/4NE1/4sec. 12, T. $4 \mathrm{~S}$., R. 4 W. Domestic and stock well, dug 4 feet in diameter and 27.7 feet deep, brick curb. Measuring point is top of 3 -inch plank deck, through bored hole 10 inches southeast of pump, 170.14 feet 2 above mean sea level.

\begin{tabular}{|c|c|c|c|c|c|c|c|}
\hline Date & $\begin{array}{l}\mathrm{W}^{\dagger} \text { ater } \\
\text { level } \\
\text { (feet) }\end{array}$ & Date & $\begin{array}{l}\text { Water } \\
\text { level } \\
\text { (feet) }\end{array}$ & Date & $\begin{array}{l}\text { Water } \\
\text { level } \\
\text { (feet) }\end{array}$ & Date & $\begin{array}{l}\text { Water } \\
\text { level } \\
\text { (feet) }\end{array}$ \\
\hline 1935 & & 1936 & & 1936 & & 1936 & \\
\hline $\begin{array}{l}\text { Oct. } 31 \\
\text { Nov. } 4 \\
\text { Nov. } 11 \\
\text { Nov. } 18 \\
\text { Nov. } 26 \\
\text { Dec. } 2 \\
\text { Dec. } 9 \\
\text { Dec. } 16 \\
\text { Dec. } 23 \\
\text { Dec. } 30 \\
\qquad 1936 \\
\text { Jan. } 4 \\
\text { Jan. } 6\end{array}$ & $\begin{array}{l}148.53 \\
146.97 \\
146.18 \\
146.26 \\
146.74 \\
146.72 \\
145.71 \\
146.12 \\
146.11 \\
147.86 \\
\\
152.95 \\
150.01\end{array}$ & $\begin{array}{l}\text { Jan. } 13 \\
\text { Jan. } 18 \\
\text { Jan. 20 } \\
\text { Jan. 27 } \\
\text { Feb. } 3 \\
\text { Feb. } 10 \\
\text { Feb. } 19 \\
\text { Feb. } 24 \\
\text { Mar. } 2 \\
\text { Mar. } 9 \\
\text { Mar. 16 } \\
\text { Mar. 23 } \\
\text { Mar. 27 }\end{array}$ & $\begin{aligned} 168.40 \\
167.90 \\
167.92 \\
164.81 \\
164.61 \\
5160.63 \\
5160.93 \\
5160.97 \\
167.28 \\
166.63 \\
166.65 \\
5160.04 \\
5160.81\end{aligned}$ & $\begin{array}{l}\text { Mar. } 31 \\
\text { Apr. } 6 \\
\text { Apr. 20 } \\
\text { Apr. } 27 \\
\text { May 4 } \\
\text { May } 11 \\
\text { May } 18 \\
\text { May } 25 \\
\text { June } 1 \\
\text { June } 8 \\
\text { June } 15 \\
\text { June } 22 \\
\text { June } 29\end{array}$ & $\begin{array}{r}163.96 \\
5160.87 \\
159.33 \\
158.70 \\
159.35 \\
157.67 \\
156.77 \\
156.56 \\
156.62 \\
155.35 \\
154.41 \\
154.38 \\
152.68\end{array}$ & $\begin{array}{l}\text { July } 6 \\
\text { July } 13 \\
\text { July } 20 \\
\text { July } 27 \\
\text { Aug. } 3 \\
\text { Aug. } 10 \\
\text { Aug. } 17 \\
\text { Aug. } 24 \\
\text { Aug. } 31 \\
\text { Sept. } 8 \\
\text { Sept. } 14 \\
\text { Sept. } 21 \\
\text { Sept. } 28\end{array}$ & $\begin{array}{l}151.97 \\
151.18 \\
147.86 \\
147.46 \\
145.53 \\
146.16 \\
145.93 \\
145.66 \\
145.05 \\
144.11 \\
144.05 \\
146.23 \\
146.12\end{array}$ \\
\hline
\end{tabular}

2 Leveling by U. S. Engineer Department.

5 Pump operating in well.

133. J. J. Kneeland, renter; Fred Allison, owner (formerly owned by A. J. Mott). SW1/4SW1/4 sec. 24, T. 4 S., R. 4 W. Domestic and stock well, bored 12 inches in diameter and 41 feet deep, in shed at northeast corner of residence. Copper nail with washer stamped 4-4-5 in wood pump base Measuring point is top of concrete-tile casing, 0.4 foot above land surface and 152 feet above mean sea level (hand level).

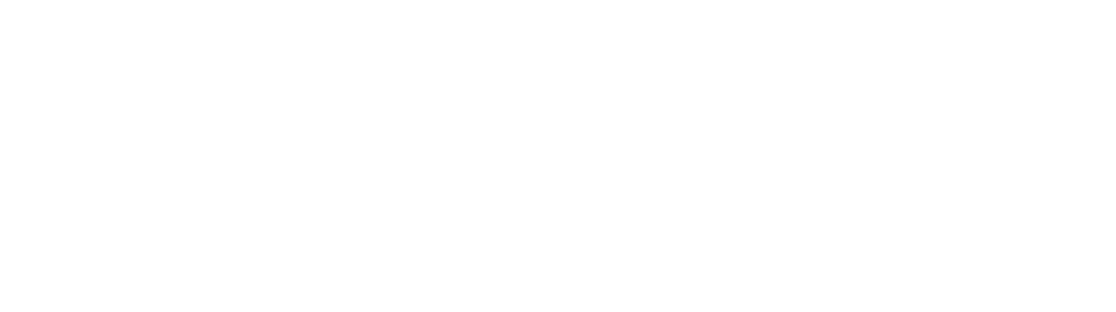

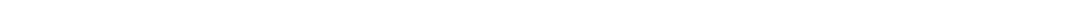
27 feet deep, concrete-tile casing. Substitute for well 133, which was inaccessible for water-level measurements in 1935-36. Measuring point is top of concrete collar of casing, 2.0 feet above land surface and 157.69 feet ${ }^{2}$ above mean sea level.

\begin{tabular}{|c|c|c|c|c|c|c|c|}
\hline Date & $\begin{array}{l}\text { Water } \\
\text { level } \\
\text { (feet) }\end{array}$ & Date & $\begin{array}{l}\text { Water } \\
\text { level } \\
\text { (feet) }\end{array}$ & Date & $\begin{array}{l}\text { Water } \\
\text { level } \\
\text { (feet) }\end{array}$ & Date & $\begin{array}{l}\text { Water } \\
\text { level } \\
\text { (feet) }\end{array}$ \\
\hline 1936 & & 1936 & & 1936 & & 1936 & \\
\hline $\begin{array}{l}\text { Feb. } 3 \\
\text { Feb. } 10 \\
\text { Feb. } 19 \\
\text { Feb. } 24 \\
\text { Mar. } 2 \\
\text { Mar. } 9 \\
\text { Mar. } 16 \\
\text { Mar. } 23 \\
\text { Mar. } 30\end{array}$ & $\begin{array}{l}152.52 \\
152.61 \\
152.56 \\
154.05 \\
154.50 \\
153.11 \\
153.01 \\
152.87 \\
153.96\end{array}$ & $\begin{array}{l}\text { Apr. } 6 \\
\text { Apr. } 13 \\
\text { Apr. } 20 \\
\text { Apr. } 27 \\
\text { May } 4 \\
\text { May } 11 \\
\text { May 18 } \\
\text { May } 25 \\
\text { June } 1\end{array}$ & $\begin{array}{l}153.14 \\
152.80 \\
152.71 \\
151.64 \\
151.00 \\
150.83 \\
150.51 \\
150.15 \\
150.10\end{array}$ & $\begin{array}{l}\text { June } 8 \\
\text { June } 15 \\
\text { June } 22 \\
\text { June } 29 \\
\text { July } 6 \\
\text { July } 13 \\
\text { July } 20 \\
\text { July } 27 \\
\text { Aug. } 3\end{array}$ & $\begin{array}{l}148.60 \\
148.28 \\
147.69 \\
147.50 \\
147.43 \\
145.37 \\
145.12 \\
145.03 \\
144.62\end{array}$ & $\begin{array}{l}\text { Aug. } 10 \\
\text { Aug. } 17 \\
\text { Aug. } 24 \\
\text { Aug. } 31 \\
\text { Sept. } 8 \\
\text { Sept. } 14 \\
\text { Sept. } 21 \\
\text { Sept. } 28 \\
\end{array}$ & $\begin{array}{l}142.65 \\
141.80 \\
141.47 \\
141.28 \\
140.66 \\
140.43 \\
139.99 \\
138.77\end{array}$ \\
\hline
\end{tabular}

2 Leveling by U. S. Engineer Department. 
TABLE 13.-Ground-water levels in observation wells, 1928-30 and 1935-36-Con.

136. W. J. Odell. SW $\frac{1}{4}$ SEx sec. 29, T. 4 S, R. 4 W. Domestic and stock well, dug 4 feet in diameter and 30.6 feet deep, brick curb. Measuring point is top of brick curb, 0.6 foot above land surface and 141.88 feet 2 above mean sea level.

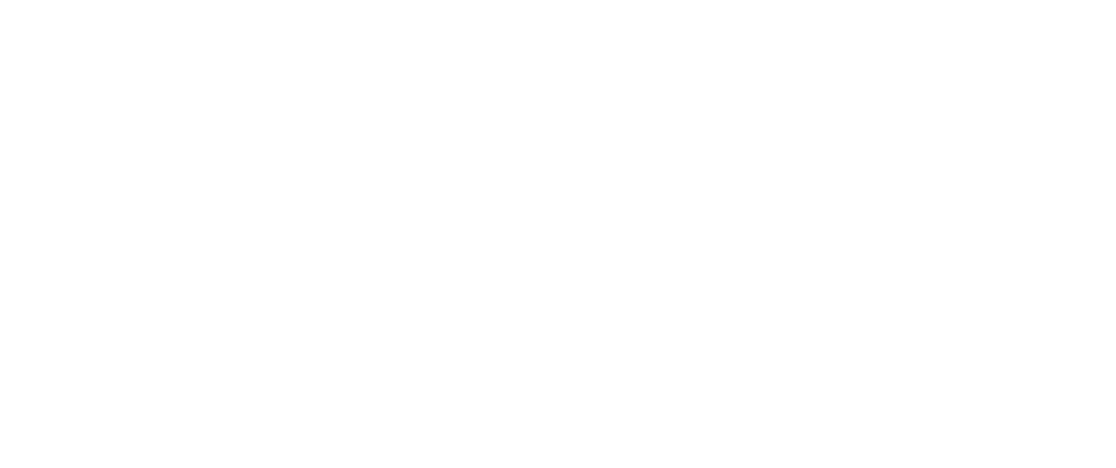

2 Leveling by U. S. Engineer Department.

- Water level depressed by inordinately large withdrawals.

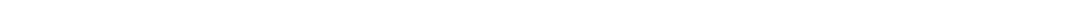
in diameter and 23 feet deep, brick curb. Measuring points: Top of 2-inch plank deck, through bored hole 10 inches north of pump, 1.2 teet above land surface and 163.94 feet ${ }^{2}$ above mean sea level; and beginning July 6, 1936, 0.6 foot above plank deck.

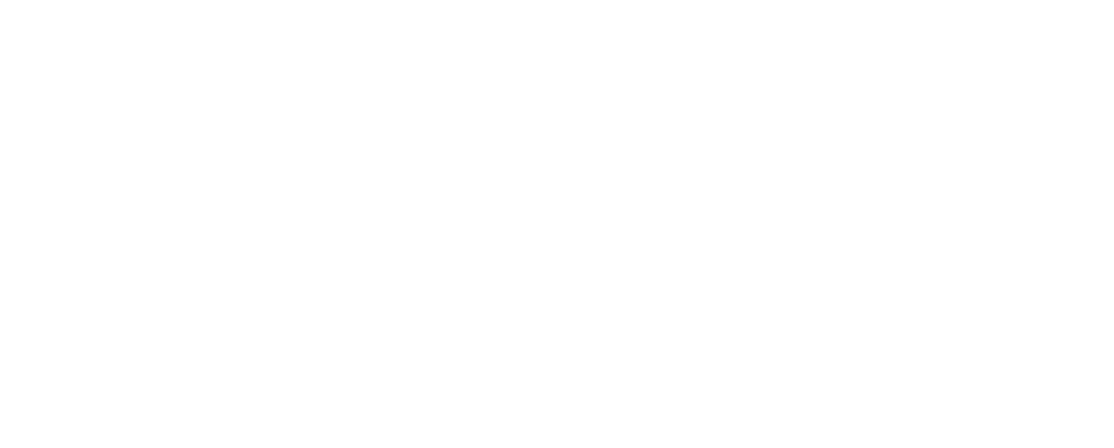

2 Leveling by U. S. Engineer Department.

158. W. J. Gering, formerly Basil Gering. NE $\frac{1}{4} \mathrm{NW}_{4}^{\frac{1}{4}} \mathrm{sec} .4$, T. 4 S., R. 2 W. Domestic and stock well, dug 30 inches in diameter and 23 feet deep, 30 feet southwest of tank tower. Measuring point is top of concrete-tile casing, east side, 1.8 feet above land surface and 125.37 feet ${ }^{2}$ above mean sea level.

\begin{tabular}{|c|c|c|c|c|c|c|c|}
\hline Date & $\begin{array}{l}\text { Water } \\
\text { level } \\
\text { (feet) }\end{array}$ & Date & $\begin{array}{l}\text { Water } \\
\text { level } \\
\text { (feet) }\end{array}$ & Date & $\begin{array}{l}\text { Water } \\
\text { level } \\
\text { (feet) }\end{array}$ & Date & $\begin{array}{l}\text { Water } \\
\text { level } \\
\text { (feet) }\end{array}$ \\
\hline 1928 & & 1935 & & 1936 & & 1936 & \\
\hline Sept. 26 & 106.6 & Oct. 3 & 106. 92 & Jan. 27 & 111.29 & June 1.. & 109.86 \\
\hline 1929 & & Oct. 12 & 106. 95 & Feb. 3 & 111.10 & June 8 & $\begin{array}{l}109.56 \\
109.80\end{array}$ \\
\hline & & $\begin{array}{l}\text { Uct. } 15 \\
\text { Oct. } 23 \text {. }\end{array}$ & $\begin{array}{l}106.54 \\
106.58\end{array}$ & Feb. 19 & $\begin{array}{l}110.90 \\
111.00\end{array}$ & June 22 - & $\begin{array}{l}109.80 \\
109.39\end{array}$ \\
\hline July 3 . & 107.92 & Oct. 28. & 106.57 & Feb. 24 & 111.18 & June 29 . & 109.15 \\
\hline Aug. 5 & 106.62 & Nov. 4 & 106. 54 & Mar. 2 & 112.61 & July 6 . & 108. 90 \\
\hline Sept. 24 & 104.43 & Nov. 11 & 106.57 & Mar. 9 & 111. 91 & July 13 & 108.38 \\
\hline Oct 27 & 10490 & Nov 18 & 106. 55 & Mar 16 & 11203 & July 20 & 108.68 \\
\hline Nov. 29 & $\begin{array}{l}104.67 \\
\end{array}$ & Dec. 2 & 106. 30 & Mar. 23. & 110.95 & July 27 & 108. 36 \\
\hline Dec. 30 & 105.12 & Dec. 9 . & 106.78 & Mar. $30^{-}$ & 112.47 & Aug. 3. & 108.08 \\
\hline \multirow{3}{*}{1930} & & 1)ec. 16 & 107.63 & Apr. 6 & 112.06 & Aug. 10 & 107. 89 \\
\hline & & Dec. 23 & 107. 69 & Apr. 13 & 111.50 & Aug. 17 & 107.56 \\
\hline & & Dec. 30 & 108. 59 & A pr. 20 & 110,92 & Aug. 27 . & 107.55 \\
\hline Feb. 2 & 107.07 & 1936 & & Apr. 27 & 111. 08 & Aug. 31 & 107.40 \\
\hline Mar. & 108. 72 & & & & 110.95 & Sept. 8 & 107.07 \\
\hline Mar. 30 & 108.87 & Jan. 6 & 109.65 & May 11 & 110.50 & Sept. 14 & 107.15 \\
\hline Apr. 27 & 108.72 & Jan. 13 & 112. 62 & May 18 & 110.91 & Sept. 21 & 106.90 \\
\hline July 25 & 106.47 & Jan. 20 & 111.85 & May 25 & 110.46 & Sept. 28 & 106.86 \\
\hline
\end{tabular}

\footnotetext{
2 Leveling by U. S. Engineer Department.
} 
TABLE 13.-Ground-water levels in observation wells, 1928-30 and 1935-36-Con.

161. Clara E. Case. SE $\frac{1}{4} \mathrm{NW}_{\frac{1}{4}}$ sec. 13, T. 4 S., R. 2 W. Domestic well, dug 28 feet deep, brick curb. Measuring point is pump-house floor at south edge of trap door, about 2 feet above land surface and 177.76 feet ${ }^{2}$ above mean sea level.

\begin{tabular}{|c|c|c|c|c|c|c|c|}
\hline Date & $\begin{array}{l}\text { Water } \\
\text { level } \\
\text { (feet) }\end{array}$ & Date & $\begin{array}{l}\text { Water } \\
\text { level } \\
\text { (feet) }\end{array}$ & Date & $\begin{array}{l}\text { Water } \\
\text { level } \\
\text { (feet) }\end{array}$ & Date & $\begin{array}{l}\text { Water } \\
\text { level } \\
\text { (feet) }\end{array}$ \\
\hline 1935 & & 1936 & & 1936 & & 1036 & \\
\hline Oet. 9 & 156.30 & Jan. 6 & 154. 71 & Apr. 6. & 160.80 & July 6 & 156.93 \\
\hline Oct. 15 & 156. 21 & Jan. 13 & 157. 59 & Apr. 13 & 160.84 & July 13 & 156.79 \\
\hline Oct. 23 . & 156.11 & Jan. 20 & 158.49 & Apr. 20 & 160.79 & July 20 & 157.32 \\
\hline Oct. 28 & 156. 28 & Jan. 27 & 157.78 & Apr. 27. & 159.47 & July 27. & 157.04 \\
\hline Nov. 4 & 155.07 & Feb. 3 & 157.74 & May 4 & 159. 24 & Aug. 3 & 156. 88 \\
\hline Nov. 11 & 155.22 & Feb. 10 & 157.18 & May 11 & 159.23 & Aug. 10 & 156. 79 \\
\hline Nov. 18 & 154.74 & Feb. 19 & 161.82 & May 18 & 159. 10 & Aug. 17- & 156.37 \\
\hline Dec. 3 & 155. 07 & Feb. 24 & 159.66 & May 25 & 159.06 & Aug. 24 & 156. 28 \\
\hline Dec. 9. & 154.09 & Mar. 2 & 160.17 & June 1 . & 158.81 & Aug. 31 & 156.05 \\
\hline Dec. 16 . & 155,12 & Mar. 9 & 160.26 & June 8 & 158. 64 & Sept. 8 & 155.83 \\
\hline Dec. 23 & 154.08 & Mar. 16 & 159.96 & June 15 & 158. 37 & Sept. 14 & 156.77 \\
\hline Dec. 30 & 153.67 & Mar. 30 & 161.36 & June 29 & 157.85 & Sept. 28 & 158.00 \\
\hline
\end{tabular}

${ }^{2}$ Leveling by U. S. Engineer Department.

164. Wm. Gooding. SW $1 \mathrm{NE}_{\frac{1}{2}} \mathrm{sec}, 20, \mathrm{~T}, 4 \mathrm{~S}, \mathrm{R}, 2 \mathrm{~W}$. Domestic and stock well, drilled 6 inches in diameter and 147 feet deep, 75 feet northeast of dwelling. Casing stamped 4-2-1 on east side. Measuring point is top of casing, 0.8 foot above land surface and 171 feet above mean sea level (altimeter). On september 26,1928 , the water level was 151.9 feet; on October $20,1928,151.2$ feet; and on December 17, 1928, 152 feet.

165. Ann M. Kaufman. NWisE: sec. 21, T. 4 S., R. 2 W. Domestic well, dug 29 feet deep, brick curb. Measuring point is top of 2 -inch plank deck, about 0.8 foot above land surface and 170.72 feet ${ }^{2}$ above mean sea level.

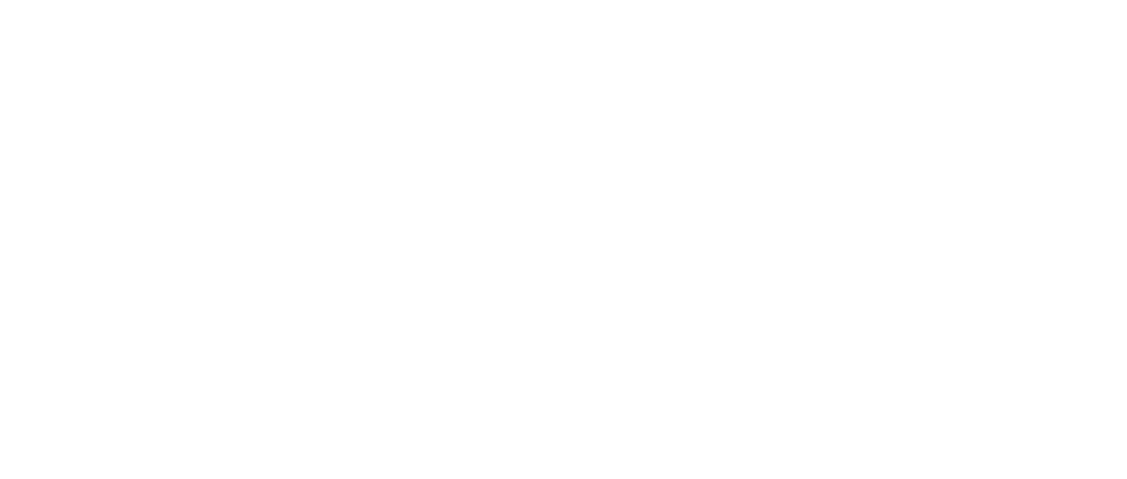

Leveling by U. S. Engineer Department. 
TABLE 13.-Ground-water levels in observation wells, 1928-30 and 1935-36-Con.

169. Theodore Boutin. NW1/4SW1/4 sec. 31, T. 4 S., R. 2 W. Domestic and stock well, drilled 10 inches in diameter and 74 feet deep, in pump shed 60 feet southwest of deep petroleum test well. Measuring point is top of 10-inch steel casing, level with land surface and 171.08 feet 2 above mean sea level.

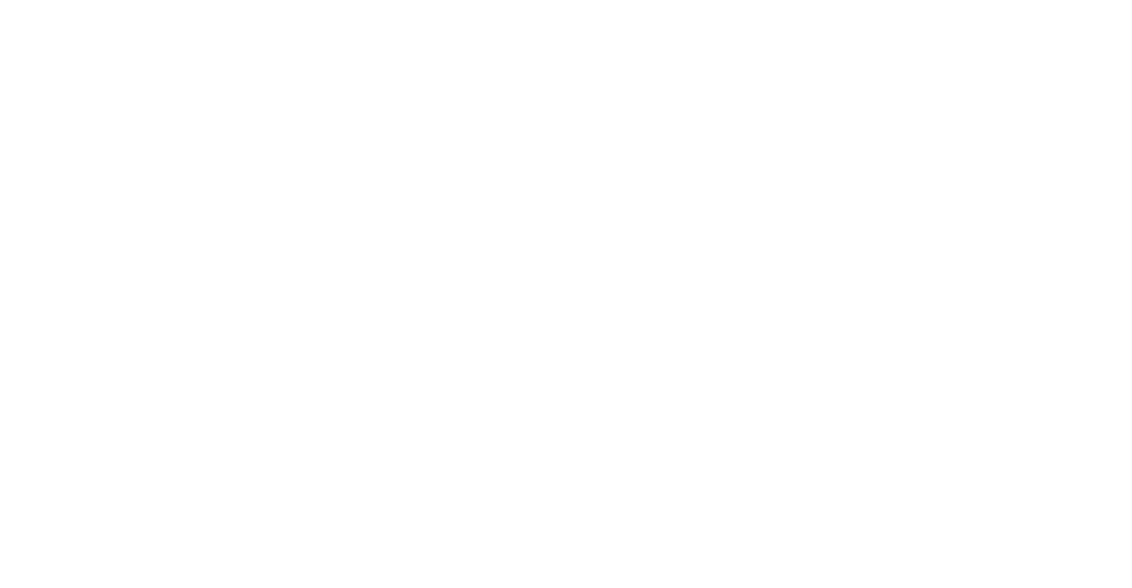

- 2 Leveling by U. S. Engineer Department.

- Pump operating in well a short time prior to measurement.

171. Johnson School. SE1/4SE1/4 sec. 34, T. 4 S., R. 2 W. Domestic well, dug 18 inches in diameter and 20 feet deep, in school yard. Copper nail with washer stamped 4-2-7 in west side of plank well cover. Measuring point is top of concrete-tile casing at copper nail with washer, 1.8 feet above land surface and 184.66 feet? above mean sea level.

\begin{tabular}{|c|c|c|c|c|c|c|c|}
\hline Date & $\begin{array}{l}\text { Water } \\
\text { level } \\
\text { (feet) }\end{array}$ & Date & $\begin{array}{l}\text { Water } \\
\text { level } \\
\text { (feet) }\end{array}$ & Date & $\begin{array}{l}\text { Water } \\
\text { level } \\
\text { (feet) }\end{array}$ & Date & $\begin{array}{l}\text { Water } \\
\text { level } \\
\text { (feet) }\end{array}$ \\
\hline 1928 & & 1930 & & 1936 & & 1936 & \\
\hline Sept. 26 & 156.8 & Mar. 2 & 171.21 & $\operatorname{Jan} 6$ & 158.06 & May 25 & 164.59 \\
\hline Oct. 20 . & 156.2 & Mar. 30 & 169.91 & Jan. 13 & 172. 06 & June 1 - & 163. 66 \\
\hline Dec. 17 & 159.4 & Apr. 27 & 167.11 & Jan. 20 & 171.98 & June 8 & 162.84 \\
\hline & & July 22 & 159.35 & Jan. 27. & 171.01 & June 15 & 161.93 \\
\hline 1929 & & & 155. 33 & Feb. 3 & 171.13 & June 22 . & 160.95 \\
\hline Feb. 3 & 171.8 & 1935 & $\begin{array}{l}155.29 \\
155.31\end{array}$ & $\begin{array}{l}\text { Feb. } 10 \\
\text { Feb. } 19\end{array}$ & $\begin{array}{l}169.77 \\
169.21\end{array}$ & $\begin{array}{l}\text { June } 29 . \\
\text { July } 6 \text {. }\end{array}$ & $\begin{array}{l}160.73 \\
159.75\end{array}$ \\
\hline Mar. 23 & 169. 21 & Oct. 3 . & 155. 39 & Feb. 24 & 171.90 & July 13. & 159. 35 \\
\hline May 12 & 168. 26 & Oct. 10 & 155. 09 & Mar. 2 & 171. 29 & July 20 . & 158. 77 \\
\hline June 7 & 164.56 & Oet. 15 & 154.86 & Mar. 9 & 170.48 & July 27 & 158.59 \\
\hline July 3 & 161.1 & Oct. 23 & & Mar. 16 & 170.15 & Aug. 3 & 157. 94 \\
\hline Aug. 5 & 158.11 & Oct. 28 . & & Mar. 23 & 169.73 & Aug. 10 & 157. 80 \\
\hline Sept. 24 & 156. 36 & Nov. 4 & & Mar. 30 & 171.25 & Aug. 17 & 157.32 \\
\hline Oct. 27 . & 155. 71 & Nov. 11 & 155.01 & Apr. 6 & 170.48 & Aug. 24 & 157.14 \\
\hline Nov. 29 & 155. 11 & Nov, 18 & 155.09 & Apr. 13 & 169.60 & Aug. 31 & 156.83 \\
\hline Dec. 30 . & 162.66 & Dec. 2 & 155.44 & Apr. 20 & 168.23 & Sept. 8 & 156.62 \\
\hline & & Dec. 9 & 155.05 & Apr. 27 & 167.72 & Sept. 14 & 156.80 \\
\hline 1930 & & Dec. 16 & 155.99 & May 4 & 166. 29 & Sept. 21 & 156.43 \\
\hline Feb. 2 & 171.26 & $\begin{array}{l}\text { Dec. } 23 \text { - } \\
\text { Dec. } 30\end{array}$ & $\begin{array}{l}156.42 \\
159.05\end{array}$ & $\begin{array}{l}\text { May } 11 \\
\text { May } 18\end{array}$ & $\begin{array}{l}166.02 \\
166.00\end{array}$ & Sept. 28 & 156.25 \\
\hline & & & & & & & \\
\hline
\end{tabular}

Leveling by U. s. Engineer Department. 
TABLE 13.-Ground-water levels in observation wells, 1928-30 and 1935-36-Con.

172. J. W. Keil (formerly D. A. Keil). NE1/4NW1/4 sec. 2, T. 4 S., R. 1 W. Domestic well, bored 10 inches in diameter and 25.5 feet deep, at rear porch at northeast corner of dwelling, concrete-tile and stovepipe casings. Stamped 4-1-2 on pump discharge pipe just below spigot. Measuring points: Prior to May 1929, top of stovepipe casing, 0.7 foot above land surface and 187.4 feet above mean sea level; June 7 and July 3,1939 , top of casing (cut-oft), 187.3 feet above mean sea level; beginning Aug. 5, 1929, top of pumpbase flange marked with white paint, 1.0 foot above land surface and 187.69 feet $^{2}$ above mean sea level.

\begin{tabular}{|c|c|c|c|c|c|c|c|}
\hline Date & $\begin{array}{l}\text { Water } \\
\text { level } \\
\text { (feet) }\end{array}$ & Date & $\begin{array}{l}\text { Water } \\
\text { level } \\
\text { (feet) }\end{array}$ & Date & $\begin{array}{l}\text { Water } \\
\text { level } \\
\text { (feet) }\end{array}$ & Date & $\begin{array}{l}\text { Water } \\
\text { level } \\
\text { (feet) }\end{array}$ \\
\hline 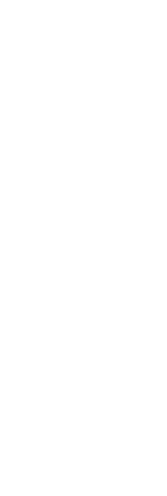 & $\begin{array}{l}175.4 \\
175.0 \\
179.1 \\
\\
185.0 \\
183.29 \\
184.44 \\
181.74 \\
178.14 \\
175.99 \\
174.14 \\
174.24 \\
184.44\end{array}$ & \begin{tabular}{l}
\multicolumn{1}{|c|}{1930} \\
Apr. 27 \\
July 22 \\
Oct. 3 \\
Oct. 10 \\
Oct. 15 \\
Oct. 23 \\
Oct. 28 \\
Nov. 4 \\
Nov. 11 \\
Nov. 18 \\
Dec. 2 \\
Dec. 9 \\
Dec. 16 \\
Dec. 23 \\
Dec. 30 \\
J 1936 \\
Jan. 6 \\
\end{tabular} & $\begin{array}{l}183.49 \\
175.97 \\
\\
174.21 \\
173.63 \\
173.11 \\
171.89 \\
171.94 \\
172.02 \\
172.31 \\
172.39 \\
174.07 \\
174.11 \\
175.63 \\
175.80 \\
181.65 \\
\end{array}$ & \begin{tabular}{l}
\multicolumn{1}{|c}{1936} \\
Jan. 10 \\
Jan. 13 \\
Jan. 20 \\
Jan. 24 \\
Jan. 27 \\
Feb. 3 \\
Feb. 10 \\
Feb. 17 \\
Feb. 24 \\
Feb. 28 \\
Mar. 2 \\
Mar. 9 \\
Mar. 16 \\
Mar. 23 \\
Mar. 30 \\
Apr. 6 \\
Apr. 13 \\
Apr. 20 \\
Apr. 27 \\
May 4 \\
May 11
\end{tabular} & $\begin{array}{l}185.00 \\
185.51 \\
185.40 \\
184.53 \\
184.35 \\
184.48 \\
184.51 \\
183.45 \\
184.15 \\
184.34 \\
185.75 \\
185.52 \\
185.11 \\
185.31 \\
184.31 \\
184.17 \\
183.92 \\
184.07 \\
183.91 \\
183.72 \\
181.50\end{array}$ & $\begin{array}{l}\quad 1936 \\
\text { May } 18 \\
\text { May } 25 \\
\text { June } 1 \\
\text { June } 8 \\
\text { June } 15 \\
\text { June } 22 \\
\text { June } 29 \\
\text { July } 8 \\
\text { July } 13 \\
\text { July } 20 \\
\text { July } 28 \\
\text { Aug. } 3 \\
\text { Aug. 10 } \\
\text { Aug. } 17 \\
\text { Aug. } 24 \\
\text { Aug. } 31 \\
\text { Sept. } 8 \\
\text { Sept. } 14 \\
\text { Sept. } 21 \\
\text { Sept. } 26\end{array}$ & $\begin{array}{l}180.83 \\
181.83 \\
181.65 \\
178.89 \\
179.53 \\
177.48 \\
176.85 \\
176.65 \\
174.66 \\
174.32 \\
173.98 \\
174.75 \\
174.87 \\
171.14 \\
174.30 \\
175.15 \\
175.00 \\
171.32 \\
170.87 \\
170.69\end{array}$ \\
\hline
\end{tabular}

Leveling by U. S. Engineer Department.

174. A. W. Geisey. NE 34 SW1/4 sec. 9, T. 4 S., R. 1 W. Stock well, dug 7 feet in diameter and 25 feet deep, concrete curb. Measuring point is top of plank deck, through crack, 1.0 foot above land surface and 191.21 feet ${ }^{2}$ above mean sea level.

\begin{tabular}{|c|c|c|c|c|c|c|c|}
\hline Date & $\begin{array}{l}\text { Water } \\
\text { level } \\
\text { (feet) }\end{array}$ & Date & $\begin{array}{l}\text { Water } \\
\text { level } \\
\text { (feet) }\end{array}$ & Date & $\begin{array}{l}\text { Water } \\
\text { level } \\
\text { (feet) }\end{array}$ & Date & $\begin{array}{l}\text { Water } \\
\text { level } \\
\text { (feet) }\end{array}$ \\
\hline 1935 & & 1936 & & 1936 & & 1936 & \\
\hline $\begin{array}{l}\text { Oct. } 9 \\
\text { Oct. } 15 \\
\text { Oct. } 23 \\
\text { Oct. } 28 \\
\text { Nov. } 4 \\
\text { Nov. } 11 \\
\text { Nov. } 18 \\
\text { Dec. } 3 \\
\text { Dec. } 9 \\
\text { Dec. } 16 \\
\text { Dec. } 23 \\
\text { Dec. } 30\end{array}$ & $\begin{array}{l}174.31 \\
171.74 \\
175.63 \\
174.12 \\
173.78 \\
173.94 \\
173.76 \\
173.61 \\
174.03 \\
176.51 \\
176.26 \\
181.71\end{array}$ & $\begin{array}{l}\text { Jan. } 6 \\
\text { Jan. } 13 \\
\text { Jan. } 20 \\
\text { Jan. } 27 \\
\text { Feb. } 3 \\
\text { Feb. } 10 \\
\text { Feb. } 17 \\
\text { Feb. } 24 \\
\text { Mar. } 2 \\
\text { Mar. } 9 \\
\text { Mar. } 16 \\
\text { Mar. } 23 \\
\text { Mar. } 30 \\
\end{array}$ & $\begin{array}{l}188.63 \\
188.82 \\
189.72 \\
187.52 \\
187.09 \\
186.14 \\
185.86 \\
188.36 \\
187.93 \\
188.05 \\
186.79 \\
186.56 \\
188.09\end{array}$ & $\begin{array}{l}\text { Apr. } 6 \\
\text { Apr. } 13 \\
\text { Apr. } 20 \\
\text { Apr. } 27 \\
\text { May } 4 \\
\text { May 11 } \\
\text { May 18 } \\
\text { May } 25 \\
\text { June } 1 \\
\text { June } 8 \\
\text { June } 15 \\
\text { June } 22 \\
\text { June } 29 \\
\text { Jun }\end{array}$ & $\begin{array}{l}187.96 \\
187.67 \\
185.51 \\
184.93 \\
184.54 \\
183.95 \\
182.78 \\
183.00 \\
181.89 \\
181.19 \\
180.13 \\
178.97 \\
178.17\end{array}$ & $\begin{array}{l}\text { July } 8 \\
\text { July 13 } 13 \\
\text { July 20 } 28 \\
\text { July } 28 \\
\text { Aug. } 3 \\
\text { Aug. 10 } \\
\text { Aug. 17 } \\
\text { Aug. 24 } \\
\text { Aug. 31. } \\
\text { Sept. 8 } \\
\text { Sept. 21 } \\
\text { Sept. 28 } \\
\end{array}$ & $\begin{array}{l}177.23 \\
176.23 \\
176.69 \\
176.23 \\
176.13 \\
176.24 \\
175.26 \\
174.48 \\
175.29 \\
175.09 \\
174.96 \\
174.82\end{array}$ \\
\hline
\end{tabular}

${ }^{2}$ Leveling by U. S. Engineer Department. 
TABLE 13.-Ground-water levels in observation: wells, 1928-30 and 1935-36-Con.

184. Harry Cochran. SW1/4SE1/4 sec. 4, T. 4 S., R. 1. E. Domestic well, dug 4 feet square and 67 feet deep, timber curb. Measuring point is top of plank curb. at middle of west side, 1.5 feet above land surface and 177.80 feet $^{2}$ above mean sea level.

\begin{tabular}{|c|c|c|c|c|c|c|c|}
\hline Date & $\begin{array}{l}\text { Water } \\
\text { level } \\
\text { (feet) }\end{array}$ & Date & $\begin{array}{l}\text { Water } \\
\text { level } \\
\text { (feet) }\end{array}$ & Date & $\begin{array}{l}\text { Water } \\
\text { level } \\
\text { (feet) }\end{array}$ & Date & $\begin{array}{l}\text { Water } \\
\text { level } \\
\text { (feet) }\end{array}$ \\
\hline 1935 & & 1936 & & 1936 & & 1936 & \\
\hline $\begin{array}{l}\text { Oct. } 9 \\
\text { Oct. } 15 \\
\text { Oct. } 23 \\
\text { Oct. } 28 \\
\text { Nov. } 4 \\
\text { Nov. } 11 \\
\text { Nov. } 18 \\
\text { Dec. } 2 \\
\text { Dec. } 9 \\
\text { Dec. } 16 \\
\text { Dec. } 23 \\
\text { Dec. } 30\end{array}$ & $\begin{array}{l}112.14 \\
112.00 \\
112.82 \\
112.85 \\
111.35 \\
111.30 \\
111.25 \\
111.00 \\
111.10 \\
111.10 \\
111.30 \\
111.30\end{array}$ & $\begin{array}{l}\text { Jan. } 6 \\
\text { Jan. } 13 \\
\text { Jan. } 20 \\
\text { Jan. } 27 \\
\text { Feb. } 3 \\
\text { Feb. } 10 \\
\text { Feb. } 17 \\
\text { Feb. } 24 \\
\text { Mar. } 2 \\
\text { Mar. } 9 \\
\text { Mar. } 16 \\
\text { Mar. } 23 \\
\text { Mar. } 30\end{array}$ & $\begin{array}{l}111.30 \\
111.30 \\
113.30 \\
114.55 \\
114.98 \\
115.15 \\
115.50 \\
115.95 \\
115.51 \\
115.55 \\
116.06 \\
115.77 \\
116.07\end{array}$ & $\begin{array}{l}\text { Apr. 6. } \\
\text { Apr. } 13 \\
\text { Apr. 20 } \\
\text { Apr. 27 } \\
\text { May } 4 \\
\text { May } 11 \\
\text { May } 18 \\
\text { May } 25 \\
\text { June } 1 \\
\text { June } 22 \\
\text { June } 29 \\
\text { July } 8\end{array}$ & $\begin{array}{l}116.67 \\
116.82 \\
115.69 \\
115.48 \\
115.43 \\
114.18 \\
115.05 \\
114.85 \\
114.60 \\
113.95 \\
113.65 \\
113.52\end{array}$ & $\begin{array}{l}\text { July } 13 \\
\text { July } 20 \\
\text { July } 28 \\
\text { Aug. } 3 \\
\text { Aug. 10 } \\
\text { Aug. 17 } \\
\text { Aug. 24 } \\
\text { Aug. } 31 \\
\text { Sept. } 8 \\
\text { Sept. 14 } \\
\text { Sept. 21 } \\
\text { Sept. 28 }\end{array}$ & $\begin{array}{l}113.40 \\
112.58 \\
112.48 \\
112.38 \\
112.13 \\
111.93 \\
111.81 \\
111.76 \\
111.61 \\
111.38 \\
111.33 \\
111.28\end{array}$ \\
\hline
\end{tabular}

${ }^{2}$ Lereling by U. S. Engineer Department.

193. Roy Wisecarver. SW 14 NE1/4 sec. 1 , T. 5 S., R. 5 W. Domestic and stock well, dug 4 feet in diameter and 39 feet deep, brick curb. Measuring point is concrete floor of pump house, 0.3 foot above land sirface and 148.43 feet ${ }^{2}$ above mean sea level.

\begin{tabular}{|c|c|c|c|c|c|c|c|}
\hline Date & $\begin{array}{l}\text { Water } \\
\text { level } \\
\text { (feet) }\end{array}$ & Date & $\begin{array}{l}\text { Water } \\
\text { level } \\
\text { (feet) }\end{array}$ & Date & $\begin{array}{l}\text { Water } \\
\text { level } \\
\text { (feet) }\end{array}$ & Date & $\begin{array}{l}\text { Water } \\
\text { level } \\
\text { (feet) }\end{array}$ \\
\hline 1936 & & 1936 & & 1936 & & 1936 & \\
\hline $\begin{array}{l}\text { Mar. 2 } \\
\text { Mar. } 9 \\
\text { Mar. 16 } \\
\text { Mar. 23 } \\
\text { Mar. } 30 \\
\text { Apr. } 6 \\
\text { Apr. } 13 \\
\text { Apr. } 20\end{array}$ & $\begin{array}{l}146.06 \\
144.31 \\
144.06 \\
142.50 \\
144.67 \\
143.02 \\
142.97 \\
140.03\end{array}$ & $\begin{array}{l}\text { Apr. 24 } \\
\text { Apr. } 27 \\
\text { May } 4 \\
\text { May } 11 \\
\text { May } 18 \\
\text { May } 25 \\
\text { June } 1 \\
\text { June } 8\end{array}$ & $\begin{array}{l}139.93 \\
139.00 \\
137.71 \\
135.82 \\
136.43 \\
136.58 \\
136.71 \\
135.49\end{array}$ & $\begin{array}{l}\text { June } 15 \\
\text { June } 22 \\
\text { June } 29 \\
\text { July } 6 \\
\text { July } 13 \\
\text { July } 20 \\
\text { July } 27 \\
\text { Aug. } 3\end{array}$ & $\begin{array}{l}135.09 \\
134.59 \\
134.16 \\
134.10 \\
133.12 \\
131.94 \\
131.86 \\
130.62\end{array}$ & $\begin{array}{l}\text { Aug. 10 } \\
\text { Aug. 17 } \\
\text { Aug. 24 } \\
\text { Aug. } 31 \\
\text { Sept. } 8 \\
\text { Sept. } 14 \\
\text { Sept. } 21 \\
\text { Sept. } 28\end{array}$ & $\begin{array}{r}130.11 \\
129.84 \\
129.70 \\
129.15 \\
+126.43 \\
126.92 \\
125.27 \\
125.57\end{array}$ \\
\hline
\end{tabular}

${ }^{2}$ Leveling by U. S. Engineer Department.

4 Water level depressed by inordinately large withdrawals.

196. George Fuller. NW1/4NE1/4 sec. 13, T. 5 S., R. 5. W. Domestic and stock well, drilled 6 inches in diameter and 64 feet deep, in pump house 45 feet northwest of residence. Copper nail with washer stamped 5-5-2 in wood clamp that supports suction pipe. Measuring point is top of casing, 0.5 foot above land surface and 151.59 feet ${ }^{2}$ above mean sea level.

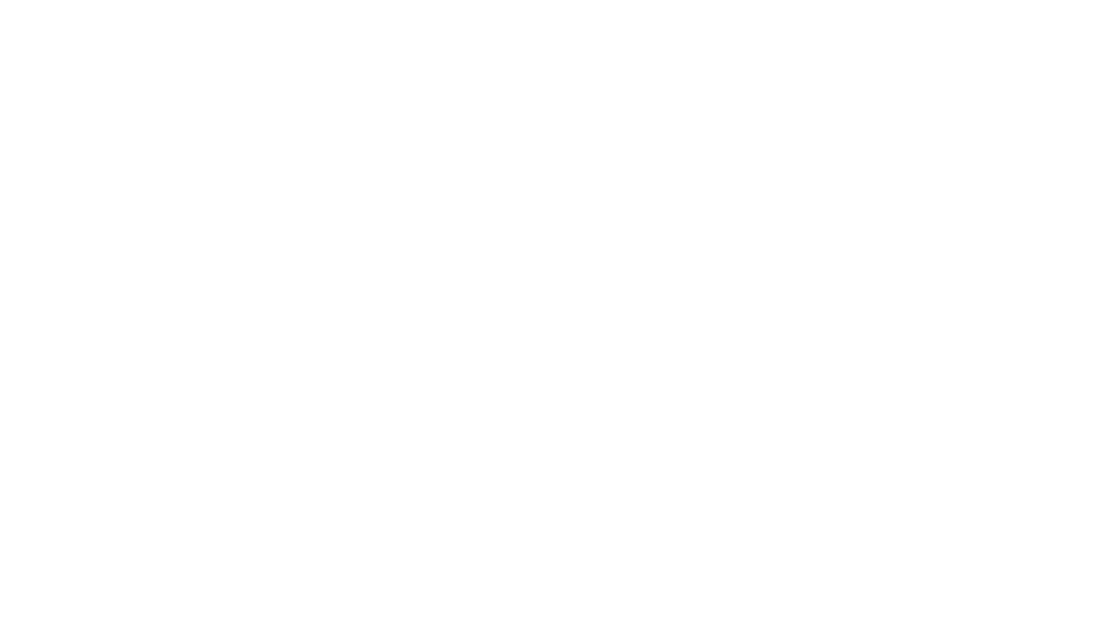

${ }^{2}$ Leveling by U. S. Engineer Department.

4 Water level depressed by inordinately large withdrawals.

$\checkmark$ Pump operating in well. 
TABLE 13.-Ground-water levels in observation wells, 1928-30 and 1935-36-Con.

203. William Piller. NE1/4NE1/4 sec. 32, T. 5 S., R. 5 W. Stock well, dug $41 / 2$ feet in diameter and 32 feet deep, masonry curb. Copper nail with washer stamped 5-5-1 in plank deck at north side of pump. Measuring point is top of 2-inch plank deck, 1.0 foot above land surface and 209.74 feet above mean sea level.

\begin{tabular}{|c|c|c|c|c|c|c|c|}
\hline Date & $\begin{array}{l}\text { Water } \\
\text { level } \\
\text { (feet) }\end{array}$ & Date & $\begin{array}{l}\text { Water } \\
\text { level } \\
\text { (feet) }\end{array}$ & Date & $\begin{array}{l}\text { Water } \\
\text { level } \\
\text { (feet) }\end{array}$ & Date & $\begin{array}{l}\text { Water } \\
\text { level } \\
\text { (feet) }\end{array}$ \\
\hline 1928 & \multirow{4}{*}{189.5} & 1936 & & 1936 & \multirow{9}{*}{$\begin{array}{r}197.65 \\
197.70 \\
193.90 \\
193.48 \\
193.39 \\
193.05 \\
189.34 \\
5188.72 \\
188.36\end{array}$} & 1936 & \multirow{9}{*}{$\begin{array}{r}187.33 \\
186.31 \\
186.17 \\
184.77 \\
185.05 \\
184.58 \\
184.42 \\
5184.27 \\
184.27\end{array}$} \\
\hline Sept. 24 & & Mar. 23 & 203. 35 & May 25 & & Aug. 3 & \\
\hline 1936 & & $\begin{array}{l}\text { Mar. } 30 \\
\text { Apr. } 6\end{array}$ & $\begin{array}{l}202.83 \\
203.57\end{array}$ & $\begin{array}{l}\text { June } 1 \\
\text { June } 15\end{array}$ & & Aug. 10 & \\
\hline & & Apr. 13 & 202.17 & June 22 & & Aug. 24 & \\
\hline Feb. 19 & 203.63 & Apr. 20 . & 202.14 & June 29 & & Aug. 31 & \\
\hline Feb. 24 & 206.72 & Apr. 27 & 200.39 & July 6. & & Sept. 8 - & \\
\hline Mar. 2 & 206.53 & May 4 & 198.02 & July 13 & & Sept. 14 & \\
\hline Mar. 9 & 204.70 & May 11. & 199.01 & July 20 . & & Sept. 21 & \\
\hline Mar. 16 & 204.78 & May 18 & 197.93 & July 27 & & Sept. 28 & \\
\hline
\end{tabular}

5 Pump operating in well.

210. Glenn Patty. NE 14 NE 14 sec. 10, T. 5 S., R. 4 W. Domestic and stock well dug, 3 feet in diameter and 38.6 feet deep, brick curb. Measuring point is top of brick curb, at west side, 2.2 feet above land surface and 158.62 feet 2 above mean sea level.

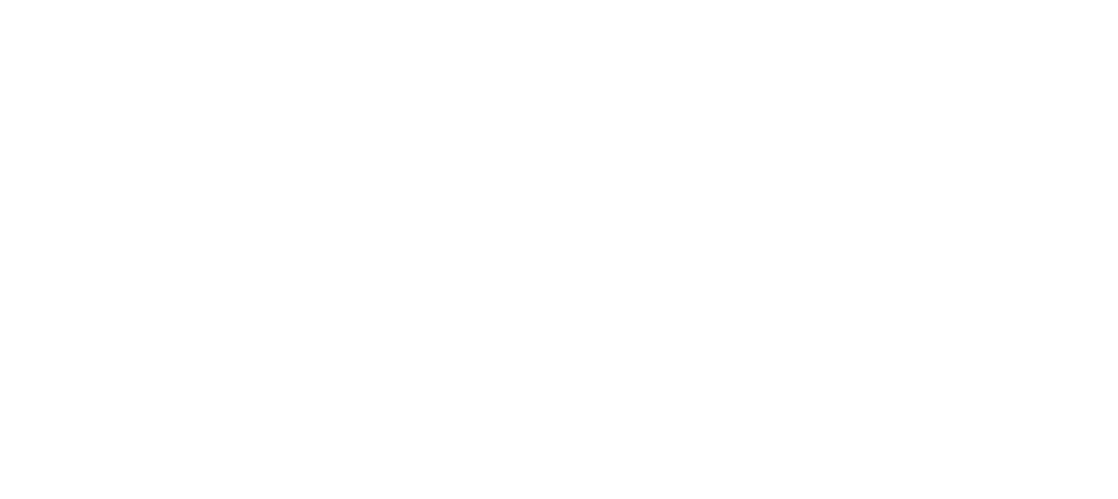

2 Leveling by U.S. Engineer Department.

225. Eunice Hibbs. NW1/4SE 14 sec. 9 , T. 5 S., R. 3 W. Domestic well, dug 4 feet in diameter and 32 feet deep, brick curb with concrete collar. Measuring point is top of 2-inch plank deck, through bored hole 8 inches northeast of pump, 0.7 foot above land surface and 163.53 feet ${ }^{2}$ above mean sea level.

\begin{tabular}{|c|c|c|c|c|c|c|c|}
\hline Date & $\begin{array}{l}\text { Water } \\
\text { level } \\
\text { (feet) }\end{array}$ & Date & $\begin{array}{l}\text { Water } \\
\text { level } \\
\text { (feet) }\end{array}$ & Date & $\begin{array}{l}\text { Water } \\
\text { level } \\
\text { (feet) }\end{array}$ & Date & $\begin{array}{l}\text { Water } \\
\text { level } \\
\text { (feet) }\end{array}$ \\
\hline 1935 & & 1936 & & 1936 & & 1936 & \\
\hline $\begin{array}{l}\text { Oct. } 31 \\
\text { Nov. } 5 \\
\text { Nov. } 11 \\
\text { Nov. } 18 \\
\text { Nov. } 26 \\
\text { Dec. } 3 \\
\text { Dec. } 9 \\
\text { Dec. } 16 \\
\text { Dec. } 23 \\
\text { Dec. } 30 \\
\quad 1936 \\
\text { Jan. } 6\end{array}$ & $\begin{array}{l}140.57 \\
140.93 \\
140.38 \\
140.18 \\
140.22 \\
141.85 \\
142.17 \\
142.26 \\
140.83 \\
144.17 \\
\\
145.32\end{array}$ & $\begin{array}{l}\text { Jan. 13 } \\
\text { Jan. 20 } \\
\text { Jan. 27 } \\
\text { Feb. 3 } \\
\text { Feb. 10 } \\
\text { Feb. 19 } \\
\text { Feb. 24 } \\
\text { Mar. 2 } \\
\text { Mar. 9 } \\
\text { Mar. 16 } \\
\text { Mar. 23 } \\
\text { Mar. 30 } \\
\text { Apr. 6. }\end{array}$ & $\begin{array}{l}160.47 \\
160.87 \\
157.46 \\
159.43 \\
160.22 \\
160.26 \\
161.45 \\
161.01 \\
160.46 \\
160.26 \\
159.39 \\
160.46 \\
160.40\end{array}$ & $\begin{array}{l}\text { Apr. } 13 \\
\text { Apr. } 20 \\
\text { Apr. } 27 \\
\text { May } 4 \\
\text { May } 11 \\
\text { May } 18 \\
\text { May } 25 \\
\text { June } 1 \\
\text { June } 8 \\
\text { June } 15 \\
\text { June } 22 \\
\text { June } 29 \\
\text { July } 6\end{array}$ & $\begin{array}{l}158.89 \\
158.64 \\
156.85 \\
155.78 \\
155.76 \\
153.74 \\
152.79 \\
151.49 \\
150.50 \\
150.36 \\
149.01 \\
147.60 \\
147.15\end{array}$ & $\begin{array}{l}\text { July } 13 \\
\text { July } 20 \\
\text { July } 27 \\
\text { Aug. } 3 \\
\text { Aug. } 10 \\
\text { Aug. } 17 \\
\text { Aug. } 24 \\
\text { Aug. } 31 \\
\text { Sept. } 8 \\
\text { Sept. } 14 \\
\text { Sept. } 21 \\
\text { Sept. } 28 \\
\end{array}$ & $\begin{array}{l}146.48 \\
146.12 \\
145.76 \\
145.04 \\
143.89 \\
143.80 \\
142.87 \\
142.46 \\
142.27 \\
141.96 \\
141.73 \\
141.40\end{array}$ \\
\hline
\end{tabular}

${ }^{2}$ Leveling by U. S. Engineer Department. 
TABLE 13.-Ground-water levels in observation wells, 1928-30 and 1935-36-Con.

228. O. Anderson (formerly S. F. Parker). SE $1 / 4$ SW 144 sec. 13 , T. 5 S., R. 3 W. Domestic well, dug 36 inches in diameter and 29.5 feet deep, in yard at northeast corner of residence. Measuring points: Top of box curb at center of west side, copper nail with washer, 1.8 feet above land surface and 177.4 feet above mean sea level; and in 1935-36, top of 2-inch plank platform, 0.8 foot above land surface and 176.40 feet 2 above mean sea level.

\begin{tabular}{|c|c|c|c|c|c|c|c|}
\hline Date & $\begin{array}{l}\text { Water } \\
\text { level } \\
\text { (feet) }\end{array}$ & Date & $\begin{array}{l}\text { Water } \\
\text { level } \\
\text { (feet) }\end{array}$ & Date & $\begin{array}{l}\text { Water } \\
\text { level } \\
\text { (feet) }\end{array}$ & Date & $\begin{array}{l}\text { Water } \\
\text { level } \\
\text { (feet) }\end{array}$ \\
\hline 1928 & & 1930 & & 1936 & & $1936^{\circ}$ & \\
\hline Sept. 20 & 149.9 & Mar. 30 & 156.25 & $\operatorname{Jan} .6$ & 153.88 & May 25 & 153.11 \\
\hline Oct. 20 & 149.4 & Apr. 27 & 153.60 & Jan. 27 & 173.32 & June $\mathbf{1}$. & 152.50 \\
\hline Dec. 16 & 148.5 & July 22 & 148.74 & Feb. 3 & 173.15 & June 8 & 152. 50 \\
\hline 1929 & & 1935 & & Feb. 19 & 161.05 & June 22 & 151.11 \\
\hline Fel & & Oet & & Feb. 24 & 173.34 & June $29 \ldots$ & 150.83 \\
\hline Mar & $\begin{array}{l}156.4 \\
154.2\end{array}$ & $\begin{array}{l}\text { Uct. } 3- \\
\text { Oct. } 11\end{array}$ & $\begin{array}{l}149.85 \\
149.75\end{array}$ & $\begin{array}{l}\text { Mar. } 2 \\
\text { Mar. } 9\end{array}$ & $\begin{array}{l}173.21 \\
170.94\end{array}$ & $\begin{array}{l}\text { July } 6 \\
\text { July } 13\end{array}$ & $\begin{array}{l}151.20 \\
150.61\end{array}$ \\
\hline May 12 & 156.2 & Oct. 15 & 149.82 & Mar. 16 & 171.08 & July 20 & 150.87 \\
\hline Jun & 152.60 & Oct. 23 & 149.90 & Mar. 23. & 164. & July 27 . & 150.51 \\
\hline July 3 - & 151.15 & Oct. 28 & 149.63 & Mar. 27 . & 165.35 & Aug. 3 . & 150.67 \\
\hline $\mathrm{Au}$ & 150.05 & Nov. 4 & 149.17 & Mar. 30 & 166.83 & Aug. 10 & 150.45 \\
\hline Sep & 149. & Nov. 11 & 149.58 & A pr. 6 & 163.82 & Aug. 17. & 150.48 \\
\hline Oct. 27 & 148. 90 & Nov. 18 & 149.21 & Apr. 13 & 161.34 & Aug. 24 & 150.46 \\
\hline Nov. 29 & 147. 30 & Dec. 2 & 148.87 & A pr. 20 & 161.29 & Ang. 31 & 150.13 \\
\hline Dec. 30 & 146.70 & Dec. 9 & 148.92 & Apr. 27 . & 156.70 & Sept. 8 & 149. 75 \\
\hline 1930 & & Dec & 148. 71 & May 4 & 155.06 & Sept. 14 & 149.85 \\
\hline Feb. 2 & 148.40 & $\begin{array}{l}\text { Dec. } 23 \\
\text { Dec. } 30 .\end{array}$ & $\begin{array}{l}148.41 \\
149.06\end{array}$ & $\begin{array}{l}\text { May } 18 . . \\
\text { May } 18 \ldots\end{array}$ & $\begin{array}{l}154.25 \\
153.50\end{array}$ & $\begin{array}{l}\text { Sept. } 21 \\
\text { Sept. } 28\end{array}$ & $\begin{array}{l}149.90 \\
149.77\end{array}$ \\
\hline
\end{tabular}

2 Leveling by U. s. Engineer Department.

235. Cora M. Nash. NE1/4NE 1/4 sec. 32, T. 5 S., R. 3 W. Unused well, dug $31 / 2$ feet in diameter and 27.4 feet deep, brick curb. Measuring point is top of concrete well cover, through 1 -inch hole 10 inches north of pump, 0.5 foot above land surface and 161.63 feet ${ }^{2}$ above mean sea level.

\begin{tabular}{|c|c|c|c|c|c|c|c|}
\hline Date & $\begin{array}{l}\text { Water } \\
\text { level } \\
\text { (feet) }\end{array}$ & Date & $\begin{array}{l}\text { Water } \\
\text { level } \\
\text { (feet) }\end{array}$ & Date & $\begin{array}{c}\text { Water } \\
\text { level } \\
\text { (feet) }\end{array}$ & Date & $\begin{array}{l}\text { Water } \\
\text { level } \\
\text { (feet) }\end{array}$ \\
\hline 1935 & & 1936 & & 1936 & & 1936 & \\
\hline $\begin{array}{l}\text { Oct. } 31 \\
\text { Nov. } 5\end{array}$ & $\begin{array}{l}142.95 \\
142.93\end{array}$ & Jan. 13 & 160.46 & Apr. 14 & 158.89 & July 15 & 150.84 \\
\hline Nov. 11 & 142. 66 & Jan. 20 & 160.00 & Apr. 21 . & 159. 00 & July 21 & 150.77 \\
\hline Nov. 18 & 142.78 & Jan. 27 & 157. 92 & Apr. 28. & 157.96 & July 28. & 150.47 \\
\hline Nov. 26 & 142.61 & Feb. 3 & 159. 54 & May 5 & 157.54 & Aug. 4 - & 150.15 \\
\hline Dec. 3 - & 142.48 & Feb. 10 & 159.01 & May 12 & 157.53 & Aug. 11 . & 7147.77 \\
\hline Dec. 9 - & 142. 52 & Feb. 19 & 158. 65 & May 18 & 157.51 & Aug. 18 & 147.54 \\
\hline Dec. 16 & 142.44 & Feb. 25 & 160.44 & May 25 & 156. 26 & Aug. 25 & 146.63 \\
\hline Dec. 23 & 148.48 & Mar. 2 & 159.74 & June 1 . & 156.34 & Sept. 1 & 145.92 \\
\hline Dec. 28 & 148.50 & Mar. 9. & 158.48 & June 8 & 154.04 & Sept. 9 & 145.54 \\
\hline Dec. 30 & 158. 39 & Mar. 17 & 159.85 & June 16 & 153.52 & Sept. 1 & 145. 26 \\
\hline 1936 & & Mar. 23 & 159.05 & June 23 & 152.36 & Sept. 22 $\ldots \ldots$ & 144. 20 \\
\hline Jan. 6_ - & 158.67 & Apr. 7 . & 159.95 & July 7 . & $\begin{array}{l}102.21 \\
151.54\end{array}$ & Depu. 29. & \\
\hline
\end{tabular}

2 Leveling by U. S. Engineer Department.

7 Adjacent land being irrigated. 
TABLE 13.-Ground-water levels in observation wells, 1928-30 and 1935-36-Con.

243. Ernest Andres. SW $1 / 4$ NE1/4 sec. 20, T. 5 S., R. 2 W. Domestic and stock well, dug 30 inches in diameter and 23 feet deep, concrete-tile casing. Measuring point is top of plank deck, through hole for discharge pipe, 0.5 foot above land surface and 177.16 feet ${ }^{2}$ above mean sea level.

\begin{tabular}{|c|c|c|c|c|c|c|c|}
\hline Date & $\begin{array}{l}\text { Water } \\
\text { level } \\
\text { (feet) }\end{array}$ & Date & $\begin{array}{l}\text { Water } \\
\text { level } \\
\text { (feet) }\end{array}$ & Date & $\begin{array}{l}\text { Water } \\
\text { level } \\
\text { (feet) }\end{array}$ & Date & $\begin{array}{l}\text { Water } \\
\text { level } \\
\text { (feet) }\end{array}$ \\
\hline 1935 & & 1936 & & 1936 & & 1936 & \\
\hline $\begin{array}{l}\text { Oct. } 9 \\
\text { Oct. } 15 \\
\text { Oct. } 24 \\
\text { Oct. } 28 \\
\text { Nov. } 4 \\
\text { Nov. } 11 \\
\text { Nov. } 18 \\
\text { Dec. } 2 \\
\text { Dec. } 9 \\
\text { Dec. } 16 \\
\text { Dec. } 23 \\
\text { Dec. } 30 \\
\end{array}$ & $\begin{array}{l}155.75 \\
156.91 \\
156.86 \\
156.86 \\
156.78 \\
156.66 \\
156.51 \\
156.58 \\
156.94 \\
158.31 \\
158.08 \\
159.87\end{array}$ & $\begin{array}{l}\text { Jan. } 6 \\
\text { Jan. } 27 \\
\text { Feb. } 3 \\
\text { Feb. 10 } \\
\text { Feb. } 19 \\
\text { Feb. } 24 \\
\text { Mar. } 2 \\
\text { Mar. } 9 \\
\text { Mar. } 16 \\
\text { Mar. } 23 \\
\text { Mar. } 30 \\
\text { Apr. } 6\end{array}$ & $\begin{array}{l}166.44 \\
171.14 \\
171.19 \\
169.42 \\
170.50 \\
175.28 \\
174.92 \\
174.15 \\
173.25 \\
172.99 \\
174.18 \\
173.42\end{array}$ & $\begin{array}{l}\text { Apr. } 13 \\
\text { Apr. } 20 \\
\text { Apr. } 27 \\
\text { May } 4 \\
\text { May } 11 \\
\text { May } 18 \\
\text { May 25 } \\
\text { June } 1 \\
\text { June } 8 \\
\text { June } 15 \\
\text { June } 22 \\
\text { June } 29\end{array}$ & $\begin{array}{l}172.85 \\
172.79 \\
171.31 \\
170.41 \\
169.97 \\
169.69 \\
168.18 \\
167.30 \\
165.90 \\
165.18 \\
164.20 \\
164.09\end{array}$ & $\begin{array}{l}\text { July } 6 \\
\text { July } 13 \\
\text { July } 20 \\
\text { July } 27 \\
\text { Aug. } 3 \\
\text { Aug. } 10 \\
\text { Aug. } 17 \\
\text { Aug. } 24 \\
\text { Aug. } 31 \\
\text { Sept. } 8 \\
\text { Sept. } 14 \\
\text { Sept. } 21 \\
\text { Sept. } 28 \\
\end{array}$ & $\begin{array}{l}162.76 \\
161.88 \\
161.31 \\
161.22 \\
160.23 \\
159.97 \\
158.20 \\
158.09 \\
157.70 \\
157.69 \\
158.80 \\
157.55 \\
157.33\end{array}$ \\
\hline
\end{tabular}

2 Leveling by U. s. Engineer Department.

245. Agricultural Research Corporation (Sam H. Brown). NW1/4SW1/4 sec. 25, T. 5 S., R. 2 W. Irrigation well, drilled; 18 -inch double stoveripe easing to depth of 155 feet, perforated from 117 to 147 feet; 6 -inch standard casing from 150 to 252 feet, perforated from 215 to 245 feet. In pump house northeast of residence. Measuring points: Drilline platform, about 0.5 foot above land surface, and 181 feet above mean sea level (by Oregon Agricultural Experiment Station); beginning April 10, 1930, upper face of pump-base flange at drilled hole, 1.0 foot above land surface and 181.31 feet ${ }^{2}$ above mean sea level.

\begin{tabular}{|c|c|c|c|c|c|}
\hline Date & $\begin{array}{l}\text { Water } \\
\text { leve! } \\
\text { (feet) }\end{array}$ & Date & $\begin{array}{l}\text { Water } \\
\text { level } \\
\text { (feet) }\end{array}$ & Date & $\begin{array}{l}\text { Water } \\
\text { level } \\
\text { (feet) }\end{array}$ \\
\hline 1930 & & 1930 & & 1936 & \\
\hline Mar. 5 & 158.1 & Aug. 28 & 13155.5 & Mar. 30........... & 166.14 \\
\hline Mar. $10 \ldots$ & 162.6 & Aug. 29 . & 13155.5 & Apr. 6 & 166. 20 \\
\hline Mar. 11- & 9162.2 & & & A pr. 13 & 166.11 \\
\hline Mar. $13,1: 30$ p. m & 163.4 & 1935 & & Apr. 20 & 166.37 \\
\hline Mar. 13,5 p. m & 10117.2 & Oct. 10 & 158.01 & Apr. 27 & 164.79 \\
\hline Mar. 14,8 a. $\mathrm{m}$ & 162.9 & Oct. 16 & 157.86 & May 4. & 164.43 \\
\hline Mar. $14,1: 30$ p. m. & 11111.0 & Oct. 24 & 157.79 & May 11. & 163.92 \\
\hline 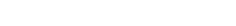 & 164.7 & Oct. 28 & 157.79 & May 18 & 163.67 \\
\hline Apr. $10,3: 35$ p. m. & 161. 0 & Nov. 4 & 157.51 & May 25 . & 163.2 \\
\hline Apr. 10,4 p. m & 12124.65 & Nov. 11 & 157.55 & June 1 . & 162.7 \\
\hline Apr. $10,4: 45$ p. m & 12122.9 & Nov. 18 & 157.46 & June 8. & 162.52 \\
\hline Apr. $10,4: 55$ p. $\mathrm{m}$ & 12120.85 & Dec. 3. & 157.41 & June $15 \ldots$ & 162.42 \\
\hline Apr. $10,5: 17$ p. m. & 12117.7 & Dec. 9 & 157.47 & June 22 & 161.28 \\
\hline Apr. $10,5: 45$ p. $\mathrm{m}$ & 12115.8 & Dec. 17 & 157.74 & June 29 . & 160.09 \\
\hline Apr. $10,6: 04$ F. m & 12114.7 & Dec. 23 . & 158.04 & Julv 8 & 160.75 \\
\hline Apr. $10,6: 15$ p. $\mathrm{m}$ & 12114.1 & Dec. 31 & 158. 36 & July 13 & 160. 71 \\
\hline Apr. $10,6: 20$ p. m & 12114.0 & & & July 20 . & 159.99 \\
\hline Apr. 11 & 157. 9 & 1936 & & July 28 & 159.8 \\
\hline Aug. $8,1 \mathrm{p}$ & 13148.3 & Jan. $6 \ldots . . . .$. & 160.15 & Aug. 3 & 159. 2 \\
\hline Aug. $8 \ldots$ & 6 116.3 & Jan. $13 \ldots$ & 162.17 & Aug. 10 & 159.07 \\
\hline Aug. 12 . & 156. 2 & Jan. 20 & 162.79 & Aug. 17 & 158.54 \\
\hline Aug. 14 & 13155.20 & $\operatorname{Jan} .27$ & 164. 36 & Aug. 24 & 158.42 \\
\hline Aug. 15 & 13155.20 & Feb. 3 & 166. 11 & Aug. 31 & 158.39 \\
\hline Aug. 21, 10: & 157.5 & Feb. 10 & 166.39 & Ser.t. 8 & 158. 24 \\
\hline Aug. $21,11:$ & 8 119.3 & Feb. 17 . & 165. 73 & Sept. 14 & 158.11 \\
\hline Aug. $21,1: 35$ p. $\mathrm{m}_{\ldots}$ & 6112.8 & Feb. 24 & 166.08 & Septl 21 & 157.98 \\
\hline Aug. $21,2: 45$ p. m & 5112.1 & Mar. 2.. & 165.88 & Sept. $28 \ldots$ & 157. 78 \\
\hline Aug. $27,1: 20$ p. m.... & 157.83 & Mar. 9 & 166.09 & & \\
\hline Aug. $27,4: 15$ p. $\mathrm{m}_{-}$ & 120.87 & Mar. 16 & 165.53 & & \\
\hline Aug. 27, & 6 119.71 & Mar. 23... & 165.61 & & \\
\hline
\end{tabular}

2 Leveling by U. S. Engineer Department.

5 Pump operating in well.

8 Outer 18-inch casing not perforated; inner 6-inch casing perforated and cut off at depth of 150 feet on

March 4.

8 Outer 18-inch casing perforated March 5-6, well being developed by intermittent pumping beginning March 8.

10 Pumping level after withdrawal at rate of 1.8 second-feet for $31 / 2$ hours.

11 Pumping level after withdrawal at rate of 1.95 second-feet for 516 hours.

12 Water level during pumping test from $3: 35$ to $6: 20 \mathrm{p}$. m.; withdrawal began at 2.09 second-feet but declined gradually to 1.82 second-feet.

13 Pump idle but water level depressed by pumping within 24 hours or less. 
TABLE 13.-Ground-water levels in observation wells, 1928-30 and 1935-36-Con.

250. Joseph Kuhn. NW1/4SE1/4 sec. 26 , T. 5 S., R. 2 W. Domestic well, dug $41 / 2$ feet square and 19 feet deep, brick curb. Measuring point is edge of 2 -inch plank, 0.5 foot above land surface and 181.47 feet 2 above mean sea level.

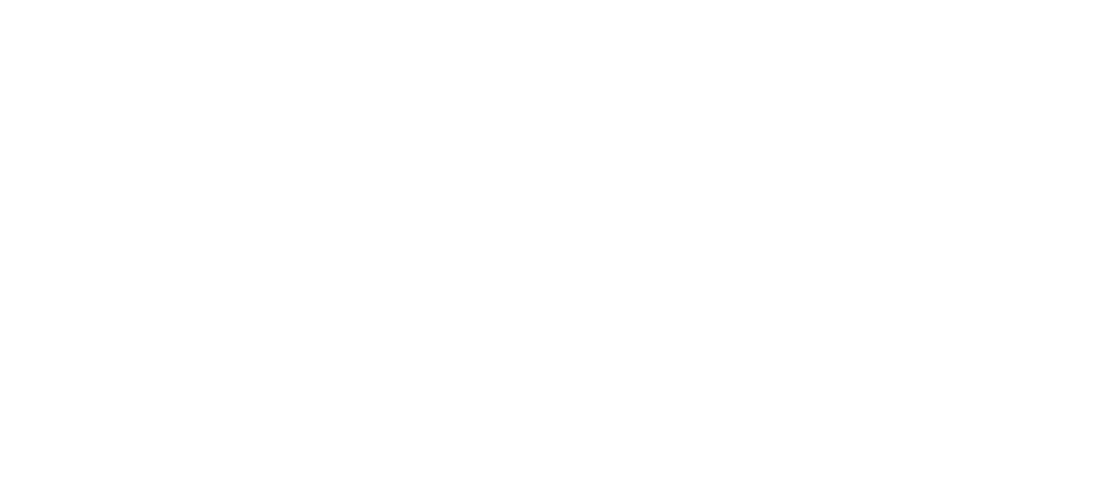

2 Leveling by U. S. Engineer Department.

254. J. P. Landen. SE1/4 NE1/4 sec. 8, T. 5 S., R. 1. W. Domestic well, dug 30 inches in diameter and 18 feet deep, culvert-pipe casing with concrete collar. Measuring point is top of concrete collar at south side, 2.8 feet above land surface and 183.95 feet ${ }^{2}$ above mean sea level.

\begin{tabular}{|c|c|c|c|c|c|c|c|}
\hline Date & $\begin{array}{l}\text { Water } \\
\text { level } \\
\text { (feet) }\end{array}$ & Date & $\begin{array}{l}\text { Water } \\
\text { level } \\
\text { (feet) }\end{array}$ & Date & $\begin{array}{l}\text { Water } \\
\text { level } \\
\text { (feet) }\end{array}$ & Date & $\begin{array}{l}\text { Water } \\
\text { level } \\
\text { (feet) }\end{array}$ \\
\hline 1935 & & 1936 & & 1936 & & 1936 & \\
\hline $\begin{array}{l}\text { Oct. } 10 \\
\text { Oct. } 15 \\
\text { Oct. } 24 \\
\text { Oct. } 28 \\
\text { Nov. } 4 \\
\text { Nov. } 11 \\
\text { Nov. } 18 \\
\text { Dec. } 3 \\
\text { Dec. } 9 \\
\text { Dec. } 16 \\
\text { Dec. } 23 \\
\text { Dec. } 30\end{array}$ & $\begin{array}{l}168.25 \\
168.48 \\
168.55 \\
168.23 \\
168.27 \\
168.13 \\
168.21 \\
167.88 \\
167.97 \\
168.16 \\
168.01 \\
168.34\end{array}$ & $\begin{array}{l}\text { Jan. } 6 \\
\text { Jan. } 13 . \\
\text { Jan. } 20 \\
\text { Jan. } 27 \\
\text { Feb. } 3 \\
\text { Feb. } 7 \\
\text { Feb. 10 } \\
\text { Feb. } 17 \\
\text { Feb. 24 } \\
\text { Feb. 28 } \\
\text { Mar. 2 } \\
\text { Mar } 9 \\
\text { Mar 16 } \\
\text { Mar. 23 } \\
\end{array}$ & $\begin{array}{l}171.47 \\
173.67 \\
175.28 \\
174.10 \\
174.47 \\
174.84 \\
175.37 \\
175.67 \\
175.53 \\
177.56 \\
177.74 \\
177.81 \\
177.95 \\
178.68\end{array}$ & $\begin{array}{l}\text { Mar. 30 } \\
\text { Apr. } 6 \\
\text { Apr. } 13 \\
\text { Apr. } 20 \\
\text { Apr. } 27 \\
\text { May } 4 \\
\text { May 11 } \\
\text { May } 18 \\
\text { May 25 } \\
\text { June } 1 \\
\text { June } 8 \\
\text { June } 15 \\
\text { June } 22 \\
\text { June } 29\end{array}$ & $\begin{array}{l}177.95 \\
177.73 \\
177.33 \\
176.37 \\
175.73 \\
175.02 \\
174.90 \\
174.60 \\
173.73 \\
172.64 \\
172.26 \\
172.15 \\
171.39 \\
171.66\end{array}$ & $\begin{array}{l}\text { July } 8 \\
\text { July } 13 \\
\text { July } 20 \\
\text { July } 28 \\
\text { Aug. } 3 \\
\text { Aug. } 10 \\
\text { Aug. } 17 \\
\text { Aug. } 24 \\
\text { Aug. } 31 \\
\text { Sept. } 8 \\
\text { Sept. } 14 \\
\text { Sept. } 21 \\
\text { Sept. } 28\end{array}$ & $\begin{array}{l}170.27 \\
170.78 \\
170.51 \\
170.32 \\
170.13 \\
169.25 \\
169.85 \\
169.64 \\
169.43 \\
169.30 \\
169.63 \\
169.33 \\
169.20\end{array}$ \\
\hline
\end{tabular}

${ }^{2}$ Leveling by U. S. Engineer Department.

258. Prudential Insurance Co. (formerly B. F. Shrock). NE1/4NW1/4 sec. 13, T. 5 S., R. 1 W. Unused well, dug 36 inches in diameter and 29 feet deep, at northwest corner of residence. Well receives run-sf f:om roof of residence, so that water level is not reliable during rainy season. Measuring points: Top of brick curb, south side, 0.5 foot above land surface and 196.42 feet above mean sea level; beginning Oct.jber 10,1935 , top face of concrete sidewalk over well, through hand hole, 196.02 feet $^{2}$ above mean sea level.

\begin{tabular}{|c|c|c|c|c|c|c|c|}
\hline Date & $\begin{array}{l}\text { Water } \\
\text { level } \\
\text { (feet) }\end{array}$ & Date & $\begin{array}{l}\text { Water } \\
\text { level } \\
\text { (feet) }\end{array}$ & Date & $\begin{array}{l}\text { Water } \\
\text { level } \\
\text { (feet) }\end{array}$ & Date & $\begin{array}{l}\text { Water } \\
\text { level } \\
\text { (feet) }\end{array}$ \\
\hline 1928 & & 1929 & & 1935 & & 1935 & \\
\hline Sept. 26 & 168.5 & Sept. 24 & 168.6 & Oct. 3 - & 169.01 & Dec. 31 & 169.45 \\
\hline Oct. 20 & 167.97 & Oct. $26_{-}$ & 168.17 & Oct. 10 & 168. 62 & 1936 & \\
\hline Dec. 17 & 168.7 & $\begin{array}{l}\text { Nov. } 29 \\
\text { Dec. } 30\end{array}$ & $\begin{array}{l}167.32 \\
169.32\end{array}$ & $\begin{array}{l}\text { Uct. } \\
\text { Oct. } 24\end{array}$ & $\begin{array}{l}168.53 \\
168.52\end{array}$ & 1936 & \\
\hline 1929 & & 1930 & & $\begin{array}{l}\text { Oct. } 28 \\
\text { Nov. } 4\end{array}$ & $\begin{array}{l}169.00 \\
168.02\end{array}$ & $\begin{array}{l}\text { Jan. } 6 \\
\text { Jan. 13 }\end{array}$ & $\begin{array}{l}177.13 \\
182.03\end{array}$ \\
\hline Feb. 3 . & 173.3 & & & Nov. 11 & 169.74 & Jan. 20 & 176.63 \\
\hline Mar. 23 & 172.07 & Feb. 2 & 172.37 & Nov. 18 & 168.12 & Jan. 27. & 172.34 \\
\hline May 12 & 176.1 & Mar. 2 & 176.17 & Dec. 3 . & 166.82 & & \\
\hline June 7 & 173.7 & Mar. 30 & 175.77 & Dec. 9 & 167.94 & & \\
\hline July 3 - & 171.4 & Apr. 27 & 173.62 & Dec. 17 & 167.72 & & \\
\hline Aug. 5 & 169.9 & July 25 & 170.57 & Dec. 23 . & 167.56 & & \\
\hline
\end{tabular}

2 Leveling by U. S. Engineer Department. 
TABLE 13.-Ground-water levels in observation wells, 1928-30 and 1985-36-Con.

259. Prudential Insurance Co. (formerly B. F. Shrock). NE1/4NW1/4 sec. 13, T. 5 S., R. 1 W. Domestic and stock well, drilled 4 inches in diameter and 137 feet deep, in pump house 15 feet west of well 258. Measuring point is top of 4 -inch casing, 0.5 foot above land surface and 196.24 feet 2 above mean sea level.

\begin{tabular}{|c|c|c|c|c|c|c|c|}
\hline Date & $\begin{array}{l}\text { Water } \\
\text { level } \\
\text { (feet) }\end{array}$ & Date & $\begin{array}{l}\text { Water } \\
\text { level } \\
\text { (feet) }\end{array}$ & Date & $\begin{array}{l}\text { Water } \\
\text { level } \\
\text { (feet) }\end{array}$ & Date & $\begin{array}{l}\text { Water } \\
\text { level } \\
\text { (feet) }\end{array}$ \\
\hline 1929 & & 1935 & & 1935 & & 1936 & \\
\hline $\begin{array}{l}\text { Sept. } 24 \\
\text { Oct. } 26 \\
\text { Nov. } 29 \\
\text { Dec. } 30 \\
\qquad 1930 \\
\text { Feb. } 2 \\
\text { Mar. } 2 \\
\text { Mar. } 30 \\
\text { Apr. } 27 \\
\text { July } 25\end{array}$ & $\begin{array}{c}157.5 \\
6157.04 \\
155.74 \\
159.44\end{array}$ & $\begin{array}{l}\text { Oct. } 3 \\
\text { Oct. } 10 \\
\text { Oct. } 16 \\
\text { Oct. } 24 \\
\text { Oct. } 28 \\
\text { Nov. } 4 \\
\text { Nov. } 11 \\
\text { Nov. } 18 \\
\text { Dec. } 3 \\
\text { Dec. } 9 \\
\text { Dec. } 17\end{array}$ & $\begin{array}{l}157.55 \\
157.46 \\
157.41 \\
157.44 \\
156.74 \\
157.44 \\
157.16 \\
157.24 \\
156.62 \\
157.64 \\
157.44\end{array}$ & $\begin{array}{l}\text { Dec. } 23 \\
\text { Dec. } 31 \\
\qquad 1936 \\
\text { Jan. } 6 \\
\text { Jan. } 13 \\
\text { Feb. } 10 \\
\text { Mar. } 9 \\
\text { Mar. } 16 \\
\text { Apr. } 13 \\
\end{array}$ & $\begin{array}{l}157.18 \\
157.56 \\
\\
159.87 \\
164.87 \\
160.29 \\
158.82 \\
162.77 \\
162.44\end{array}$ & $\begin{array}{l}\text { May } 25 \\
\text { July } 13 \\
\text { Aug. } 23 \\
\text { Sept. } 14 \\
\text { Sept. } 21 \\
\text { Sept. } 28 \\
\end{array}$ & $\begin{array}{l}160.79 \\
160.04 \\
158.26 \\
156.86 \\
156.84 \\
156.62\end{array}$ \\
\hline
\end{tabular}

2 Leveling by U. S. Engineer Department.

o Pump operating in well a short time prior to measurement.

260. Prudential Insurance Co. NE1/4NW1/4 sec. 13, T. 5 S., R. 1 W. Unused well, dug 4 feet in diameter and 27 feet deep, brick curb. Beginning Jan. 20, 1936, a substitute for well 258, which is ab out 650 feet to the south. Measuring point is top of brick curb, level with land surface and 193.87 feet 2 above mean sea level

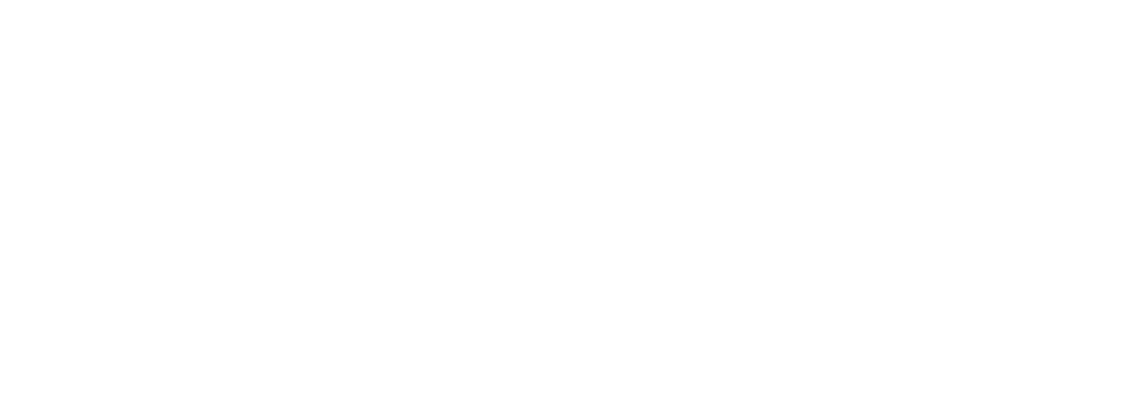

2 Leveling by U. S. Engineer Department.

283. I. O. O. F. Hall. SE1/4NE1/4 sec. 8 , T. 6 S., R. 5 W. Domestic well, dug 36 inches in diameter and 27 feet deep, brick curb. Measuring points: Top of box curb, 3.1 feet above land surface and 184.10 feet above mean sea level; beginning February 1936, top of 2-inch plank cover, through bored hole at center, 1.0 foot above land surface and 181.94 feet $^{2}$ above mean sea level.

\begin{tabular}{|c|c|c|c|c|c|c|c|}
\hline Date & $\begin{array}{l}\text { Water } \\
\text { level } \\
\text { (feet) }\end{array}$ & Date & $\begin{array}{l}\text { Water } \\
\text { level } \\
\text { (feet) }\end{array}$ & Date & $\begin{array}{l}\text { Water } \\
\text { level } \\
\text { (feet) }\end{array}$ & Date & $\begin{array}{l}\text { Water } \\
\text { level } \\
\text { (feet) }\end{array}$ \\
\hline 1928 & \multirow{3}{*}{163.8} & 1936 & & 1936 & \multirow{8}{*}{$\begin{array}{l}177.03 \\
176.21 \\
174.91 \\
174.18 \\
172.75 \\
172.11 \\
170.61 \\
170.06 \\
169.87\end{array}$} & 1936 & \multirow{8}{*}{$\begin{array}{l}168.10 \\
167.74 \\
167.25 \\
167.21 \\
166.97 \\
164.71 \\
164.88 \\
164.02 \\
163.87\end{array}$} \\
\hline Sept. 24... & & Mar. 30 & 179.15 & June 1 & & Aug. 3 . & \\
\hline 1936 & & $\begin{array}{l}\text { Apr. } 6- \\
\text { Apr. } 13 .\end{array}$ & $\begin{array}{l}179.37 \\
178.65\end{array}$ & $\begin{array}{l}\text { June } 8 \\
\text { June } 15\end{array}$ & & $\begin{array}{l}\text { Aug. 10 } \\
\text { Aug. 17 }\end{array}$ & \\
\hline & & Apr. 20 & 178.24 & June 22. & & Aug. 24 & \\
\hline $\begin{array}{l}\text { Feb. 24 } \\
\text { Mar. 2 }\end{array}$ & $\begin{array}{l}179.40 \\
179.10\end{array}$ & $\begin{array}{l}\text { Apr. 27. } \\
\text { May } 4 .\end{array}$ & $\begin{array}{l}178.01 \\
177.76\end{array}$ & $\begin{array}{l}\text { June } 29 \ldots \\
\text { July } 6 \ldots\end{array}$ & & $\begin{array}{l}\text { Aug. } 31 \ldots \\
\text { Sept. } 8\end{array}$ & \\
\hline Mar. 9 & 178.94 & May 11 & 177.70 & July 13 & & Sept. 14 & \\
\hline Mar. 16 & 179.75 & May 18 & 177.43 & July 20 - & & Sept. 21 & \\
\hline Mar. 23. & 178.80 & May 25. & 177.70 & July 27. & & Sept. 28 & \\
\hline
\end{tabular}

2 Leveling by U. S. Engineer Department. 
TABLE 13.-Ground-water levels in observation wells, 1928-30 and 1935-36-Con.

284. Frank M. McCann. SW1 $/ 4$ NW1/4 sec. 11, T. 6 S., R. 5 W. Stock well, bored 6 inches in diameter and 29 feet deep, concrete-tile casing. Measuring points: Top of casing, 1.9 feet above land surface and 187.18 feet above mean sea level; beginning February 1936, top of casing (cut-off), 0.8 foot above land surface and 186.39 feet 2 above mean sea level.

\begin{tabular}{|c|c|c|c|c|c|c|c|}
\hline Date & $\begin{array}{l}\text { Water } \\
\text { level } \\
\text { (feet) }\end{array}$ & Date & $\begin{array}{l}\text { Water } \\
\text { level } \\
\text { (feet) }\end{array}$ & Date & $\begin{array}{l}\text { Water } \\
\text { level } \\
\text { (feet) }\end{array}$ & Date & $\begin{array}{l}\text { Water } \\
\text { level } \\
\text { (feet) }\end{array}$ \\
\hline 1928 & \multirow{3}{*}{169.8} & 1936 & \multirow{8}{*}{$\begin{array}{l}184.08 \\
184.17 \\
182.95 \\
182.91 \\
182.17 \\
181.87 \\
180.15 \\
181.07 \\
180.51\end{array}$} & 1936 & \multirow{8}{*}{$\begin{array}{l}180.82 \\
179.36 \\
179.16 \\
178.26 \\
177.47 \\
177.02 \\
175.61 \\
175.03 \\
174.90\end{array}$} & \multirow{8}{*}{$\begin{array}{l}\qquad 1936 \\
\text { Aug. } 3 \\
\text { Aug. } 10 \\
\text { Aug. 17. } \\
\text { Aug. } 24 \\
\text { Aug. } 31 \\
\text { Sept. } 8 \\
\text { Sept. 14 } \\
\text { Sept. 21 } \\
\text { Sept. } 28 \\
\end{array}$} & \multirow{8}{*}{$\begin{array}{l}174.45 \\
172.87 \\
172.76 \\
172.32 \\
172.00 \\
169.32 \\
169.18 \\
169.62 \\
169.30\end{array}$} \\
\hline Sept. 24. & & Mar. 30 & & June 1 & & & \\
\hline 1936 & & $\begin{array}{l}\text { Apr. } \\
\text { Apr. } 13\end{array}$ & & June 15. & & & \\
\hline & & Apr. 20 & & June 22 -- & & & \\
\hline Feb. 19 & 177.75 & Apr. 27 & & June $29 \ldots$ & & & \\
\hline $\begin{array}{l}\text { Feb. 24. - } \\
\text { Mar. 2 }\end{array}$ & $\begin{array}{l}185.34 \\
185.82\end{array}$ & May 4 & & July $6 \ldots$ & & & \\
\hline Mar. 9 & $\begin{array}{l}183.82 \\
183.99\end{array}$ & May $18 .$. & & July $20 .-$ & & & \\
\hline $\begin{array}{l}\text { Mar. } 16 \\
\text { Mar. } 23\end{array}$ & $\begin{array}{l}183.97 \\
183.35\end{array}$ & May $25 .$. & & July 27 & & & \\
\hline
\end{tabular}

2 Leveling by U. S. Engineer Department.

291. Robert Cole. NE1/4SE1/4 sec. 11, T. 6 S., R. 3 W. Domestic and stock well, dug 3 feet in diameter and 22.5 feet deep, brick curb. Measuring point is top of 4 -inch plank deck, through bored hole, 0.9 foot above land surface and 122.76 feet ${ }^{2}$ above mean sea level.

\begin{tabular}{|c|c|c|c|c|c|c|c|}
\hline Date & $\begin{array}{l}\text { Water } \\
\text { level } \\
\text { (feet) }\end{array}$ & Date & $\begin{array}{l}\text { Water } \\
\text { level } \\
\text { (feet) }\end{array}$ & Date & $\begin{array}{l}\text { Water } \\
\text { level } \\
\text { (feet) }\end{array}$ & Date & $\begin{array}{l}\text { Water } \\
\text { level } \\
\text { (feet) }\end{array}$ \\
\hline 1935 & & 1936 & & 1936 & & 1936 & \\
\hline Nov. 5 & 100.69 & Jan. 21 & 106.44 & Apr. 14 & 102.18 & July 14 & 100.57 \\
\hline Nov. 12 & 99.56 & Jan. 28 & $\begin{array}{l}104.76 \\
104.74\end{array}$ & Apr. 20 & 101.57 & July 21 & 100.50 \\
\hline Nov. 19 & 100.08 & Feb. 4 & 103.97 & Apr. 28 & 101.50 & July 28 & 100.33 \\
\hline Dec. 3 & 99.58 & Feb. 11 & 103.38 & May 5 & 100.83 & Aug. 4 & 100.29 \\
\hline Dec. 10 & 99.78 & Feb. 19 & 102.74 & May 12 & 100.49 & Aug. 11. & 100.22 \\
\hline Dec. 17 - & 99.78 & Feb. 24 & 103.14 & May 19 & 101.18 & Aug. 18 . & 100.21 \\
\hline Dec. 24 & 99.97 & Mar. 2 & 103.17 & May 26 & 101.11 & Aug. 24 & 99.82 \\
\hline Dec. 31 & 100.52 & Mar. 9 & 102.90 & June 2 & 100.52 & Sept. 1 & 99.89 \\
\hline 1936 & & $\begin{array}{l}\text { Mar. } 16 \\
\text { Mar. } 24\end{array}$ & $\begin{array}{l}102.58 \\
103.43\end{array}$ & $\begin{array}{l}\text { June } 9 \\
\text { June } 15\end{array}$ & $\begin{array}{l}100.97 \\
101.50\end{array}$ & $\begin{array}{l}\text { Sept. } 9 \\
\text { Sept. } 15\end{array}$ & $\begin{array}{l}100.75 \\
100.68\end{array}$ \\
\hline & & Mar. 31 & 102. 09 & June 22 & 100.95 & Sept. 22 & 100.57 \\
\hline Jan. 7. - & 101.04 & Apr. 7 & 102.50 & July 9 & 100.64 & Sept. 29 & 99.97 \\
\hline
\end{tabular}

2 Leveling by U. S. Engineer Department.

293. Nick Valiech. SE14NW14 sec. 23 , T. 6 S., R. 3 W. Unused well, dug 3 feet in diameter and 75.3 feet deep. Measuring point is top of concrete deck, at east side of $5 \frac{1}{2}$-inch hole, 0.4 foot above land surface and 181.41 feet 2 above mean sea level.

\begin{tabular}{|c|c|c|c|c|c|c|c|}
\hline Date & $\begin{array}{l}\text { Water } \\
\text { level } \\
\text { (feet) }\end{array}$ & Date & $\begin{array}{l}\text { Water } \\
\text { level } \\
\text { (feet) }\end{array}$ & Date & $\begin{array}{l}\text { Water } \\
\text { level } \\
\text { (feet) }\end{array}$ & Date & $\begin{array}{l}\text { Water } \\
\text { level } \\
\text { (feet) }\end{array}$ \\
\hline 1935 & & 1936 & & 1936 & & 1936 & \\
\hline $\begin{array}{l}\text { Nov. } 5 \\
\text { Nov. } 12 \\
\text { Nov. } 19 \\
\text { Dec. } 3 \\
\text { Dec. } 10 \\
\text { Dec. } 17 \\
\text { Dec. } 24 \\
\text { Dec. } 31 \\
\quad 1936 \\
\text { Jan. } 7 \\
\text { Jan. } 14 \\
\end{array}$ & $\begin{array}{l}110.76 \\
110.74 \\
110.69 \\
110.51 \\
110.69 \\
110.81 \\
111.03 \\
111.30\end{array}$ & $\begin{array}{l}\text { Jan. } 21 \\
\text { Jan. } 28 \\
\text { Feb. } 4 \\
\text { Feb. } 11 \\
\text { Feb. } 19 \\
\text { Feb. } 24 \\
\text { Mar. } 3 \\
\text { Mar. } 9 \\
\text { Mar. } 16 \\
\text { Mar. } 24 \\
\text { Mar. } 31 \\
\text { Apr. } 7 \\
\text { Apr. } 14\end{array}$ & $\begin{array}{l}117.56 \\
113.89 \\
112.86 \\
113.51 \\
114.01 \\
112.47 \\
113.75 \\
113.16 \\
111.99 \\
111.68 \\
112.08 \\
112.26 \\
111.44\end{array}$ & $\begin{array}{l}\text { Apr. } 20 \\
\text { Apr. } 28 \\
\text { May } 5 \\
\text { May } 12 \\
\text { May } 19 \\
\text { May } 26 \\
\text { June } 2 \\
\text { June } 9 \\
\text { June } 16 \\
\text { June } 23 \\
\text { June } 30 \\
\text { July } 9 \\
\text { July } 14\end{array}$ & $\begin{array}{l}111.26 \\
111.12 \\
110.30 \\
110.19 \\
111.94 \\
111.06 \\
110.28 \\
110.91 \\
110.93 \\
109.90 \\
109.72 \\
110.41 \\
110.51\end{array}$ & $\begin{array}{l}\text { July } 21 \\
\text { July } 28 \\
\text { Aug. } 4 \\
\text { Aug. 11 } \\
\text { Aug. } 18 \\
\text { Aug. } 25 \\
\text { Sept. 1 } \\
\text { Sept. } 9 \\
\text { Sept. } 15 \\
\text { Sept. } 22 \\
\text { Sept. } 29 \\
\end{array}$ & $\begin{array}{l}110.69 \\
110.71 \\
110.69 \\
110.71 \\
110.61 \\
110.54 \\
109.32 \\
109.41 \\
109.27 \\
109.21 \\
108.03\end{array}$ \\
\hline
\end{tabular}

2 Leveling by U. S. Engineer Department. 
TABLE 13.-Ground-water levels in observation wells, 1928-30 and 1985-36-Con.

295. R. J. Hackett. NE1/4SW1/4 sec. 29 , T. 6 S., R. 3 W. Domestic and stock well, dug 5 feet in diameter and 41.6 feet deep, brick curb. Measuring point is top of 2-inch plank deck, about 2 feet south and 10 inches west of pump, 1.2 feet above land surface and 174.08 feet ${ }^{2}$ above mean sea level.

\begin{tabular}{|c|c|c|c|c|c|c|c|}
\hline Date & $\begin{array}{l}\text { Water } \\
\text { level } \\
\text { (feet) }\end{array}$ & Date & $\begin{array}{l}\text { Water } \\
\text { level } \\
\text { (feet) }\end{array}$ & Date & $\begin{array}{l}\text { Water } \\
\text { level } \\
\text { (feet) }\end{array}$ & Date & $\begin{array}{l}\text { Water } \\
\text { level } \\
\text { (feet) }\end{array}$ \\
\hline 1935 & & 1936 & & 1936 & & 1936 & \\
\hline $\begin{array}{l}\text { Oct. } 31 \\
\text { Nov. } 5 \\
\text { Nov. } 11 \\
\text { Nov. } 18 \\
\text { Nov. } 26 \\
\text { Dec. } 3 \\
\text { Dec. } 9 \\
\text { Dec. } 16 \\
\text { Dec. } 23 \\
\text { Dec. } 28 \\
\qquad 1936 \\
\text { Jan. } 4\end{array}$ & $\begin{array}{l}144.58 \\
143.83 \\
142.53 \\
144.95 \\
143.74 \\
145.03 \\
146.20 \\
145.04 \\
146.18 \\
146.41\end{array}$ & $\begin{array}{l}\text { Jan. } 6 \\
\text { Jan. } 13 \\
\text { Jan. } 18 \\
\text { Jan. } 27 \\
\text { Feb. } 3 \\
\text { Feb. } 10 \\
\text { Feb. 20 } \\
\text { Feb. } 25 \\
\text { Mar. } 3 \\
\text { Mar. } 10 \\
\text { Mar. 17 } \\
\text { Mar. 24 } \\
\text { Mar. 31 } \\
\text { Apr. } 7\end{array}$ & $\begin{array}{l}149.55 \\
170.61 \\
167.95 \\
158.53 \\
158.90 \\
161.52 \\
162.05 \\
167.94 \\
164.89 \\
164.92 \\
157.14 \\
157.01 \\
165.08 \\
165.32\end{array}$ & $\begin{array}{l}\text { Apr. } 14 \\
\text { Apr. 21 } \\
\text { Apr. } 24 \\
\text { Apr. 28 } \\
\text { May } 5 \\
\text { May } 12 \\
\text { May } 19 \\
\text { May } 26 \\
\text { June } 1 \\
\text { June } 9 \\
\text { June } 16 \\
\text { June } 23 \\
\text { June } 30 \\
\text { July } 7\end{array}$ & $\begin{array}{l}165.34 \\
5158.63 \\
5158.61 \\
5154.84 \\
153.38 \\
153.40 \\
151.94 \\
150.44 \\
151.49 \\
148.26 \\
147.20 \\
148.30 \\
145.69 \\
145.10\end{array}$ & $\begin{array}{l}\text { July } 14 \\
\text { July } 21 \\
\text { July } 28 \\
\text { Aug. } 4 \\
\text { Aug. } 11 \\
\text { Aug. } 18 \\
\text { Aug. } 25 \\
\text { Sept. } 1 \\
\text { Sept. } 9 \\
\text { Sept. } 15 \\
\text { Sept. } 22 \\
\text { Sept. } 29\end{array}$ & $\begin{array}{l}144.88 \\
144.49 \\
142.99 \\
142.26 \\
144.46 \\
3142.99 \\
145.59 \\
145.05 \\
143.97 \\
141.22 \\
144.48 \\
143.15\end{array}$ \\
\hline
\end{tabular}

2 Leveling by U. S. Engineer Department.

5 Pump operating in well.

297. Gideon E. Stolz. SE1/4 SE1/4 sec. 33, T. $6 \mathrm{~S}$., R. $3 \mathrm{~W}$. Irrigation well, drilled 8 inches in diameter and 57 feet deep, in shed west of residence, standard steel casing and concrete-lined pit. Measuring points: Top of casing in pit, 119.56 feet above mean sea level; beginning November 12, 1935, top of timber, through 1-inch bored hole at north edge of pump base, 1.0 foot above land surface and 134.14 feet above mean sea level. Reference bench mark, top of concrete pit curb at northwest corner, 13.70 feet above top of casing and 133.02 feet above mean sea level. Water-stage recorder operated on well from August 3, 1929, to July $24,1930$.

\begin{tabular}{|c|c|c|c|c|c|c|c|}
\hline Date & $\begin{array}{l}\text { Water } \\
\text { level } \\
\text { (feet) }\end{array}$ & Date & $\begin{array}{l}\text { Water } \\
\text { level } \\
\text { (feet) }\end{array}$ & Date & $\begin{array}{l}\text { Water } \\
\text { level } \\
\text { (feet) }\end{array}$ & Date & $\begin{array}{l}\text { Water } \\
\text { level } \\
\text { (feet) }\end{array}$ \\
\hline 1929 & & 1930 & & 1936 & & 1936 & \\
\hline July 3. & 109.26 & Mar. 30 & 111.31 & Jan. 7 & 109.52 & June 2. & 110.91 \\
\hline Aug. 3 & 107.26 & Apr. 27 & 109. 71 & Jan. 14 & 113.01 & June 9 & 110.61 \\
\hline Sept. 3 & 106.01 & May 29 & 109.16 & Jan. 21 & 115. 16 & June 16 & 110. 10 \\
\hline Sept. 24 & 105.47 & July 24 & 106.75 & Jan. 28 & 114.01 & June 23 & 109.58 \\
\hline Oet. 23 . & 105.07 & & & Feb. 4 & 114. 17 & June 30 & 109.40 \\
\hline Oct. 26 & 105.06 & 1935 & & Feb. 11 & 113. 49 & July 9 & 108.95 \\
\hline Nov. 29 & 104.81 & & & Feb. 19 & 113. 70 & July 14 & 108.55 \\
\hline Dec. 13 & 104.93 & Oct. 4 & 104.74 & Feb. 24 & 113. 32 & July 21 . & 109. 17 \\
\hline Dec. 21 . & 107.71 & Oct. 11 & 104.89 & Mar. 3 & 114.39 & July 28 & 107. 76 \\
\hline Dec. 30 & 109.26 & Oct. 16 & 104.86 & Mar. 9 & 114.55 & Aug. 4 & 107.87 \\
\hline & & Oct. 24 . & 104.91 & Mar. 16 & 113. 78 & Aug. 11 & 107.55 \\
\hline 1930 & & Oct. 29 & 104.98 & Mar. 24 & 113.53 & Aug. 18 & 106. 74 \\
\hline & & Nov. 5 & 105.39 & Mar. 31. & 112.82 & Aug. 25 & 106. 67 \\
\hline Jan. 12. & 109.16 & Nov. 12 & 105.46 & Apr. 7 & 112.93 & Sept. 1 & 106.26 \\
\hline Jan. 25 & 108.66 & Nov. 19 & 106.54 & Apr. 14 & 113. 27 & Sept. 9 & 106.24 \\
\hline Feb. 2 & 108.71 & Dec. 3 & 106.42 & Apr. 21 & 113.02 & Sept. 15 & 106.14 \\
\hline Feb. 8 & 101.39 & Dec. 10 . & 106.15 & Apr. 28 & 112.17 & Sept. 22 & 106.00 \\
\hline Feb. 16 & 112.11 & Dec. 17 . & 106.84 & May 5 & 112.41 & Sept. 29 & 105.78 \\
\hline Feb. 23 & 112.86 & Dec. 24 & 106.97 & May 12 & 4110.81 & & \\
\hline Mar. 2 & 113. 22 & Dec. 31.. & 106.92 & May 19. & 112.17 & & \\
\hline Mar. 19 & 111.79 & & & May 26........ & 4111.38 & & \\
\hline
\end{tabular}

- Water level depressed by inordinately large withdrawals. 
TABLE 13.-Ground-water levels in observation wells, 1928-30 and 1935-36-Con.

302. R. J. Galagher (formerly J. E. Boyce). SE1/4NE1/4 sec. 17, T. 6 S., R. 2 W. Domestic well, dug 24 inches in diameter and 21 feet deep, concrete-tile casing. Measuring point is top of casing, at east side, 0.3 foot below land surface and $\mathbf{1 7 8 . 8 4}$ feet " above mean sea level.

\begin{tabular}{|c|c|c|c|c|c|c|c|}
\hline Date & $\begin{array}{l}\text { Water } \\
\text { level } \\
\text { (feet) }\end{array}$ & Date & $\begin{array}{l}\text { Water } \\
\text { level } \\
\text { (feet) }\end{array}$ & Date & $\begin{array}{l}\text { Water } \\
\text { level } \\
\text { (feet) }\end{array}$ & Date & $\begin{array}{l}\text { Water } \\
\text { level } \\
\text { (feet) }\end{array}$ \\
\hline 1935 & & 1936 & & 1936 & & 1936 & \\
\hline $\begin{array}{l}\text { Oct. } 10 \\
\text { Oct. } 16 \\
\text { Oct. } 24 \\
\text { Oct. } 29 \\
\text { Nov. } 5 \\
\text { Nov. } 12 \\
\text { Nov. } 19 \\
\text { Dec. } 3 \\
\text { Dec. } 9 \\
\text { Dec. } 17 \\
\text { Dec. } 23 \\
\text { Dec. } 31\end{array}$ & $\begin{array}{l}163.19 \\
164.37 \\
163.18 \\
164.22 \\
162.45 \\
162.69 \\
162.60 \\
161.59 \\
164.57 \\
162.52 \\
162.62 \\
163.26\end{array}$ & $\begin{array}{l}\text { Jan. } 6 \\
\text { Jan. 13 } \\
\text { Jan. } 20 \\
\text { Jan. 24 } \\
\text { Jan. 27 } \\
\text { Feb. } 3 \\
\text { Feb. 10 } \\
\text { Feb. 17 } \\
\text { Feb. 24 } \\
\text { Mar. 2 } \\
\text { Mar. } 9 \\
\text { Mar. } 16\end{array}$ & $\begin{array}{l}166.87 \\
177.51 \\
177.27 \\
176.76 \\
174.17 \\
173.26 \\
173.21 \\
173.09 \\
177.61 \\
177.34 \\
176.46 \\
176.09\end{array}$ & $\begin{array}{l}\text { Mar. } 23 \\
\text { Mar. } 30 \\
\text { Apr. } 6 \\
\text { Apr. } 14 \\
\text { Apr. } 20 \\
\text { Apr. } 27 \\
\text { May } 4 \\
\text { May } 11 . \\
\text { May } 18 \\
\text { May } 25 \\
\text { June } 1 \\
\text { June } 8 \\
\text { June } 15 \\
\end{array}$ & \begin{tabular}{|r}
176.41 \\
177.03 \\
175.63 \\
176.63 \\
175.52 \\
172.00 \\
170.90 \\
172.19 \\
169.28 \\
5168.17 \\
167.30 \\
165.89 \\
6163.74
\end{tabular} & \begin{tabular}{|} 
June 22 \\
July 8 \\
July 13 \\
July 28 \\
Aug. 3 \\
Aug. 10. \\
Aug. 17 \\
Aug. 24 \\
Aug. 31 \\
Sept. 8 \\
Sept. 14 \\
Sept. 21 \\
Sept. 28
\end{tabular} & $\begin{array}{l}166.74 \\
165.38 \\
165.02 \\
165.24 \\
164.24 \\
164.01 \\
164.41 \\
164.21 \\
163.80 \\
163.63 \\
163.52 \\
163.23 \\
163.02\end{array}$ \\
\hline
\end{tabular}

2 Leveling by U. S. Engineer Department.

Sump operating in well.

303. Carl Aspinwall. NE1/4SW1/4 sec. 17, T. 6 S., R. 2 W. Domestic well, dug 36 inches in diameter and 16 feet deep, in yard 45 feet west of southwest corner of post office and store. Copper nail with washer stamped 6-2-4 in plank well cover. Measuring point is top of plank well cover, at copper nail with washer, 180.5 feet above mean sea level (hand level).

\begin{tabular}{|c|c|c|c|c|c|c|c|}
\hline Date & $\begin{array}{l}\text { Water } \\
\text { level } \\
\text { (feet) }\end{array}$ & Date & $\begin{array}{l}\text { Water } \\
\text { level } \\
\text { (feet) }\end{array}$ & Date & $\begin{array}{l}\text { Water } \\
\text { level } \\
\text { (feet) }\end{array}$ & Date & $\begin{array}{l}\text { Water } \\
\text { level } \\
\text { (feet) }\end{array}$ \\
\hline 1928 & & 1928 & & 1929 & & 1929 & \\
\hline $\begin{array}{l}\text { Bept. } 21 \\
\text { Oct. } 20\end{array}$ & $\begin{array}{l}166.9 \\
166.4\end{array}$ & Dec. $17 \ldots$ & 169.1 & $\begin{array}{l}\text { Feb. } 3 \\
\text { Mar. } 23 \\
\text { May } 12\end{array}$ & $\begin{array}{r}179.0 \\
178.5 \\
177.45\end{array}$ & $\begin{array}{l}\text { June } 7 \ldots \\
\text { July } 3 \ldots\end{array}$ & $\begin{array}{l}173.70 \\
169.48\end{array}$ \\
\hline
\end{tabular}

305. Nels C. Johnson. NW1/4SE1/4 sec. 24, T. $6 \mathrm{~S} .$, R. 2 W. Stock well, dug 3 feet in diameter and 20 feet deep, brick curb. Measuring point is top of 2 -inch wood deck, through bored hole, 0.5 foot above land surface and 201.66 feet 2 above mean sea level.

\begin{tabular}{|c|c|c|c|c|c|c|c|}
\hline Date & $\begin{array}{l}\text { Water } \\
\text { level } \\
\text { (feet) }\end{array}$ & Date & $\begin{array}{l}\text { Water } \\
\text { level } \\
\text { (feet) }\end{array}$ & Date & $\begin{array}{l}\text { Water } \\
\text { level } \\
\text { (feet) }\end{array}$ & Date & $\begin{array}{l}\text { Water } \\
\text { level } \\
\text { (feet) }\end{array}$ \\
\hline 1935 & & 1936 & & 1936 & & 1936 & \\
\hline $\begin{array}{l}\text { Oct. } 10 \\
\text { Oct. } 16 \\
\text { Oct. } 24 \\
\text { Oct. } 29 \\
\text { Nov. } 5 \\
\text { Nov. } 12 \\
\text { Nov. } 19 \\
\text { Dec. } 3 \\
\text { Dec. } 10 \\
\text { Dec. } 23 \\
\text { Dec. } 28 \\
\text { Dec. } 31\end{array}$ & $\begin{array}{l}184.16 \\
184.21 \\
184.04 \\
183.81 \\
183.82 \\
183.96 \\
184.08 \\
183.52 \\
186.44 \\
185.91 \\
186.39 \\
188.36\end{array}$ & $\begin{array}{l}\text { Jan. } 7 \\
\text { Jan. 14 } \\
\text { Jan. } 21 \\
\text { Jan. } 28 \\
\text { Feb. } 4 \\
\text { Feb. 11 } \\
\text { Feb. 19 } \\
\text { Feb. 24 } \\
\text { Mar. } 3 \\
\text { Mar. } 9 \\
\text { Mar. } 16 \\
\text { Mar. 23 } \\
\text { Mar. } 30 \\
\end{array}$ & $\begin{array}{l}195.78 \\
200.28 \\
200.03 \\
198.56 \\
198.71 \\
198.81 \\
198.12 \\
200.13 \\
199.36 \\
199.05 \\
199.14 \\
198.97 \\
200.08\end{array}$ & $\begin{array}{l}\text { Apr. } 6 \\
\text { Apr. } 13 \\
\text { Appr. 20 } \\
\text { Apr. 27 } \\
\text { May } 4 \\
\text { May } 11 \\
\text { May } 18 \\
\text { May } 25 \\
\text { June } 1 \\
\text { June } 8 \\
\text { June } 15 \\
\text { June } 22 \\
\text { June } 29 \\
\end{array}$ & $\begin{array}{l}201.66 \\
198.66 \\
198.08 \\
197.68 \\
197.28 \\
196.55 \\
196.09 \\
195.62 \\
194.44 \\
194.27 \\
193.64 \\
192.21 \\
191.47\end{array}$ & $\begin{array}{l}\text { July } 8 \\
\text { July } 13 \\
\text { July } 20 \\
\text { July } 28 \\
\text { Aug. } 3 \\
\text { Aug. } 10 \\
\text { Aug. 17 } \\
\text { Aug. } 24 \\
\text { Aug. } 31 \\
\text { Sept. } 8 \\
\text { Sept. 14 } \\
\text { Sept } 21 \\
\text { Sept. } 28 \\
\end{array}$ & $\begin{array}{l}188.85 \\
188.09 \\
187.38 \\
187.26 \\
186.63 \\
186.27 \\
185.63 \\
185.29 \\
185.07 \\
184.99 \\
185.22 \\
184.47 \\
184,25\end{array}$ \\
\hline
\end{tabular}

Leveling by U. s. Engineer Department. 
TABLE 13.-Ground-water levels in observation wells, 1928-30 and 1995-36-Con.

318. Fred Lucht. NW $1 / 4$ SW $1 / 4$ sec. 1, T. 6 S., R. 1 E. Stock well, dug 42 inches in diameter and 21 feet deep, in yard 60 feet west of northwest corner of barn. Copper nail with washer stamped 6-1-1 in plank well cover. Measuring point is top of plank well cover at copper nail with washer, 1.0 foot above land surface and 261.38 feet above mean sea level.

\begin{tabular}{|c|c|c|c|c|c|c|c|}
\hline Date & $\begin{array}{l}\text { Water } \\
\text { level } \\
\text { (feet) }\end{array}$ & Date & $\begin{array}{l}\text { Water } \\
\text { level } \\
\text { (feet) }\end{array}$ & Date & $\begin{array}{l}\text { Water } \\
\text { level } \\
\text { (feet) }\end{array}$ & Date & $\begin{array}{l}\text { Water } \\
\text { level } \\
\text { (feet) }\end{array}$ \\
\hline 1928 & \multirow{5}{*}{$\begin{array}{l}248.8 \\
248.53\end{array}$} & 1935 & & 1936 & \multirow{19}{*}{$\begin{array}{l}259.35 \\
258.61 \\
258.40 \\
257.76 \\
258.21 \\
258.06 \\
258.21 \\
260.03 \\
259.62 \\
259.29 \\
258.31 \\
258.53 \\
257.77 \\
259.73 \\
259.79 \\
257.54 \\
256.66 \\
256.23 \\
255.41 \\
255.18\end{array}$} & 1936 & \multirow{19}{*}{$\begin{array}{r}254.91 \\
254.32 \\
253.14 \\
253.90 \\
254.58 \\
252.89 \\
252.34 \\
251.57 \\
251.35 \\
251.04 \\
250.53 \\
249.93 \\
249.70 \\
249.04 \\
248.65 \\
247.10 \\
4246.79 \\
247.88 \\
247.76 \\
247.33\end{array}$} \\
\hline Sept. 21 & & Oct. 3 . & 245.42 & Jan. 24 & & May 18 & \\
\hline Oct. 20 & & Oct. 11 & 247.06 & Jan. 27 & & May 25 & \\
\hline 1929 & & $\begin{array}{l}\text { Oct. } 16 \\
\text { Oct. } 24\end{array}$ & $\begin{array}{l}247.70 \\
246.18\end{array}$ & $\begin{array}{l}\text { Jan. } 31 \\
\text { Feb. } 3\end{array}$ & & $\begin{array}{l}\text { June } 1- \\
\text { June } 8\end{array}$ & \\
\hline & & Oct. 28 & 246.33 & Feb. 7 & & June 15 & \\
\hline July 3 . & 252.23 & Nov. 4 & 245.11 & Feb. 10 & & June 22 & \\
\hline Aug. 5 & 249.93 & Nov. 11 & 245.08 & Feb. 17 & & June 29 & \\
\hline Sept. 24 & 247.28 & Nov. 18 & 249.74 & Feb. 24 & & July 8 & \\
\hline Oct. 26 & 245.78 & Dec. 3 & 246.57 & Feb. 28 & & July 13. & \\
\hline Nov. 29 & 245. 13 & Dec. 9 & 252.34 & Mar. 2 & & July 20 . & \\
\hline Dec. 30 & 258. 18 & Dec. 17 & 253.70 & Mar. 9 & & July 28. & \\
\hline \multirow{2}{*}{1930} & \multirow{8}{*}{$\begin{array}{l}260.13 \\
259.13 \\
257.78 \\
256.53 \\
250.69\end{array}$} & Dec. 23 & $\begin{array}{l}252.26 \\
252.97\end{array}$ & Mar. 16 & & Aug. 3 & \\
\hline & & Dec. 31 . & 258.20 & Mar. 30 & & Aug. 17 & \\
\hline Feb. 2 & & & & Apr. 6 . & & Aug. 24 & \\
\hline & & 19 & & Apr. 13 & & Aug. 31. & \\
\hline Mar. 30 & & Jan. 6 & 260.10 & Apr. 20 & & Sept. 8 & \\
\hline $\mathrm{Apr}$ & & Jan. 10 & 259.50 & Ap & & Sept. 14 & \\
\hline July 25 & & Jan. 13 & 260.06 & May 4 & & Sept. 21. & \\
\hline & & Jan. 20 & 259.58 & May 11 & & Sept & \\
\hline
\end{tabular}

- Water level depressed by inordinately large withdrawals.

321. J. J. Neufeld. SW1/4NW14 sec. 8, T. 7 S., R. 4 W. Domestic and stock well, dug 4 feet in diameter and 13 feet deep, brick curb. Measuring point is top of cover of well housing, at northwest side, 2.6 feet above land surface and 186.54 feet ${ }^{2}$ above mean sea level.

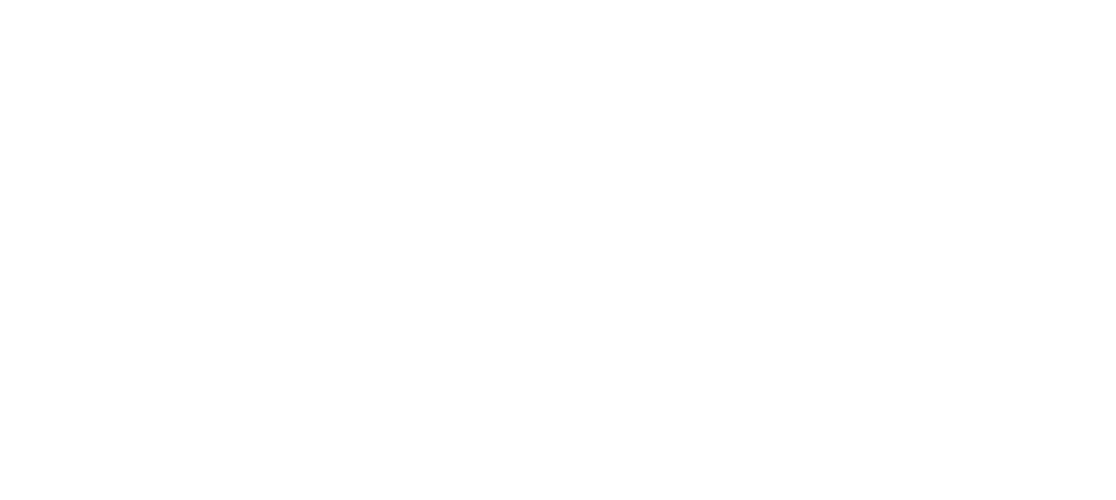

Leveling by U. S. Engineer Department.

14 Well dry. 
TABLE 13.-Ground-water levels in observation wells, 1928-30 and 1935-36-Con.

323. Wm. C. Palmer. NE1/4SW14 sec. 19, T. $7 \mathrm{~S} .$, R. $4 \mathrm{~W}$. Stock well, bored 4 inches in diameter and 21.1 feet deep, concrete-tile casing. About 235 feet north of well 322, in which the ground-water level was measured once in 1928 and again in 1935 . Measuring point is top of casing, level with land surface and 186.77 feet 2 above mean sea level.

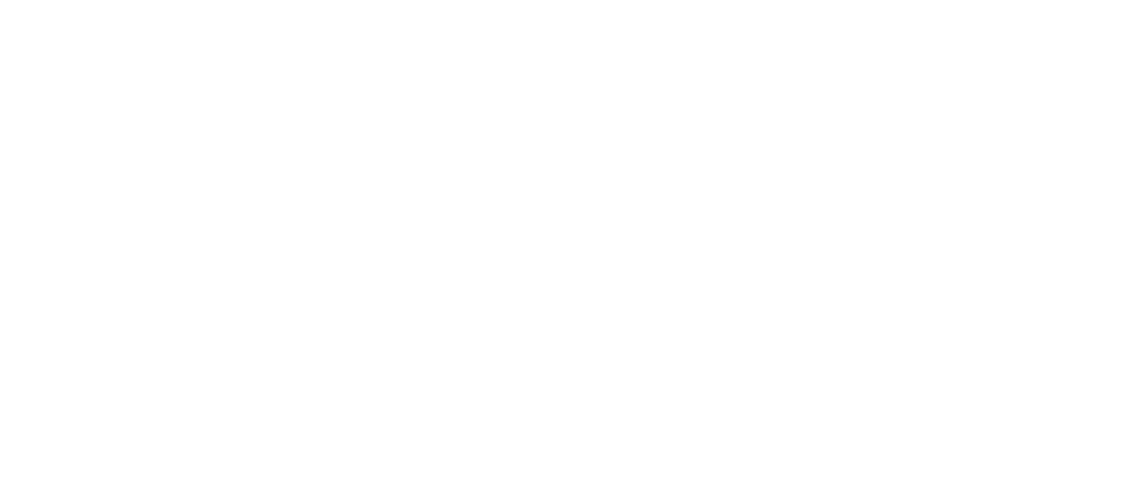

${ }^{2}$ Leveling by U. S. Engineer Department.

5 Pump operating in well.

327. Lauren Stettler. NW1/4SE1/4 sec. 1, T. 7 S., R. 3 W. Domestic and stock well, dug 3 feet in diameter and 19.6 feet deep, brick curb. Measuring point is top of brick curb, at east side, 1.0 foot above land surface and 152.85 feet ${ }^{2}$ above mean sea level.

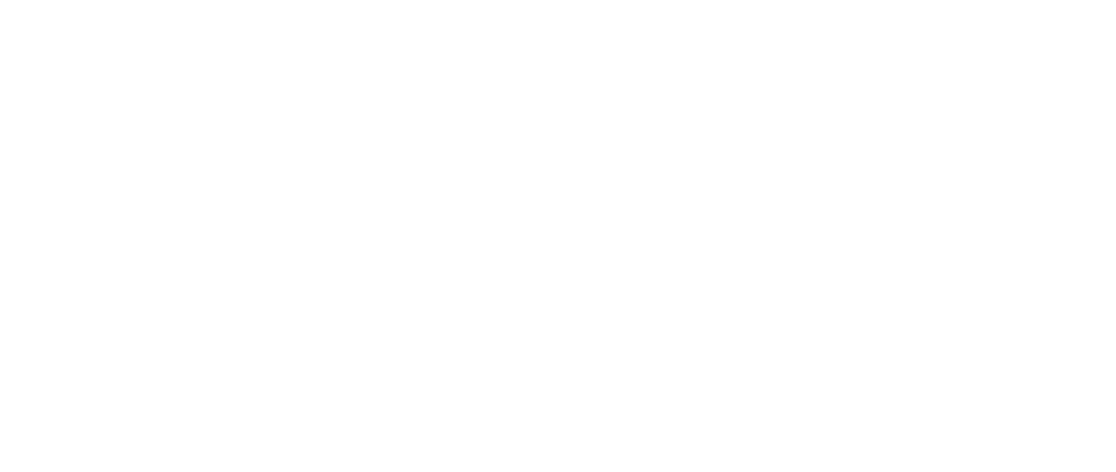

2 Leveling by U. S. Engineer Department.

4 Water level depressed by inordinately large withdrawals.

328. John C. Jackson. NE1/4NW1/4 sec. 13, T. $7 \mathrm{~S}$., R. $3 \mathrm{~W}$. Unused well, dug $31 / 2$ feet in diameter and 35.5 feet deep, brick curb. Measuring point is top of brick curb, at south side, level with land surface and 167.30 feet 2 above sea level.

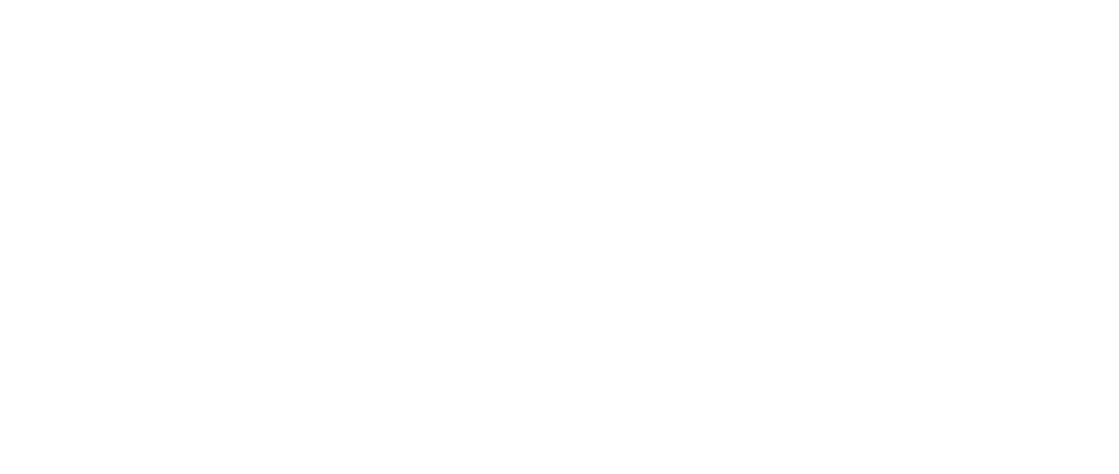

2 Leveling by U, s. Engineer Department. 
TABLE 13.-Ground-water levels in observation wells, 1928-80 and 1985-36-Con.

346. E. O. Armann. NE1/4SW1/4 sec. 35, T. 7 S., R. 3 W. Domestic well, drilled 6 inches in diameter and 28.0 feet deep, tile casing. Measuring point is top of casing, at south side, level with land surface and 179.40 feet 2 above mean sea level.

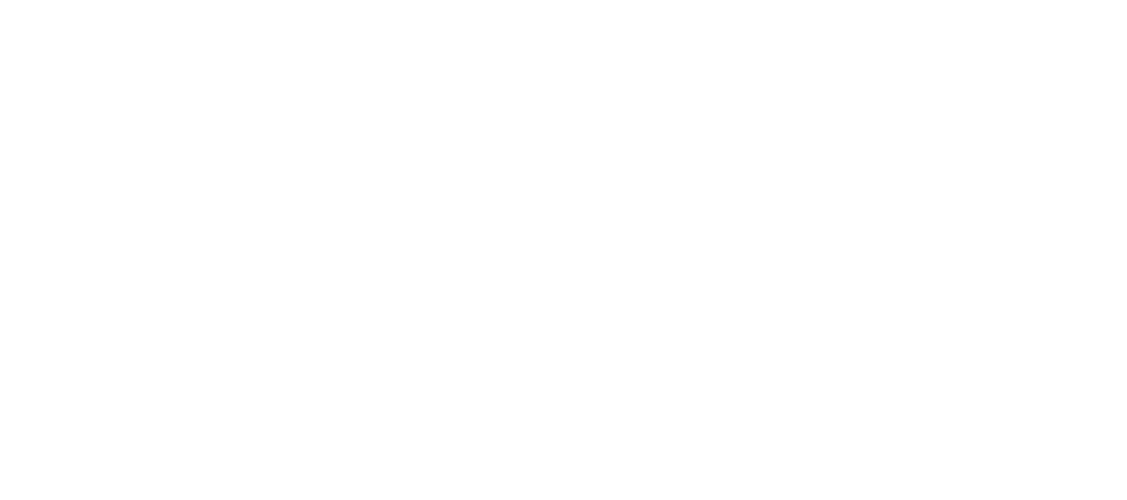

2 Leveling by U. S. Engineer Department.

1 Water level depressed by inordinately large withdrawals.

347. D. A. Steffen. SE1/4SW1/4 sec. 1, T. 7 S., R. 2 W. Domestic well, dug 6 feet in diameter and 33 feet deep, brick curb. Measuring point is top of pump platform, 0.5 foot above land surface and 199.41 feet 2 above sea level.

\begin{tabular}{|c|c|c|c|c|c|c|c|}
\hline Date & $\begin{array}{l}\text { Water } \\
\text { level } \\
\text { (feet) }\end{array}$ & Date & $\begin{array}{l}\text { Water } \\
\text { level } \\
\text { (feet) }\end{array}$ & Date & $\begin{array}{l}\text { Water } \\
\text { level } \\
\text { (feet) }\end{array}$ & Date & $\begin{array}{l}\text { Water } \\
\text { level } \\
\text { (feet) }\end{array}$ \\
\hline 1935 & & 1935 & & 1936 & & 1936 & \\
\hline $\begin{array}{l}\text { Oct. } 10 \\
\text { Oct. } 16 \\
\text { Oct. } 24 \\
\text { Oct. } 29 \\
\text { Nov. } 5 \\
\text { Nov. } 19 \\
\text { Nov. } 17\end{array}$ & $\begin{array}{l}178.51 \\
177.06 \\
176.61 \\
177.09 \\
177.11 \\
176.71 \\
178.56\end{array}$ & $\begin{array}{l}\text { Dec. } 23 \\
\text { Dec. } 31 \ldots \\
\qquad 1936 \\
\text { Jan. } 7 \ldots \ldots \\
\text { Jan. } 14\end{array}$ & $\begin{array}{l}178.70 \\
178.83 \\
\\
\\
191.50 \\
197.17\end{array}$ & $\begin{array}{l}\text { Jan. } 21 \\
\text { Feb. } 4 \\
\text { Feb. } 11 \\
\text { Feb. } 19 \\
\text { Feb. } 25 \\
\text { Mar. } 2 \\
\text { Mar. } 9\end{array}$ & $\begin{array}{l}196.52 \\
195.09 \\
194.79 \\
194.63 \\
196.94 \\
196.50 \\
196.38\end{array}$ & $\begin{array}{l}\text { Mar. 16 } \\
\text { Mar. } 23\end{array}$ & $\begin{array}{l}195.97 \\
(15)\end{array}$ \\
\hline
\end{tabular}

${ }^{2}$ Leveling by U. S. Engineer Department.

15 Discontinued as observation well.

350. Oscar Meyer (formerly R. J. Janz). SE1/4SE 14 sec. 12, 'T. 7 S., R. 2 W. Domestic well, dug 8 feet in diameter and 46.2 feet deep, dry masonry curb. Measuring points: Top of porch floor at northwest corner of trap door, 1.5 feet above land surface and 225.67 feet ${ }^{2}$ above mean sea level; 224.70 feet 2 above mean sea level.

\begin{tabular}{|c|c|c|c|c|c|c|c|}
\hline Date & $\begin{array}{l}\text { Water } \\
\text { level } \\
\text { (foet) }\end{array}$ & Date & $\begin{array}{l}\text { Water } \\
\text { level } \\
\text { (feet) }\end{array}$ & Date & $\begin{array}{l}\text { Water } \\
\text { level } \\
\text { (feet) }\end{array}$ & Date & $\begin{array}{l}\text { Water } \\
\text { level } \\
\text { (feet) }\end{array}$ \\
\hline 1928 & & 1036 & & 1936 & & 1936 & \\
\hline Sept. 13. & 182.9 & Jan. 21 & 219.04 & Apr. 13 & 211. 39 & July 13 & 184. 29 \\
\hline 1935 & & $\begin{array}{l}\text { Jan. } 28 \\
\text { Fcb. } 4\end{array}$ & $\begin{array}{r}216.12 \\
4212.32\end{array}$ & $\begin{array}{l}\text { Apr. 20- } \\
\text { Apr. } 27 .\end{array}$ & $\begin{array}{l}210: 40 \\
210.15\end{array}$ & $\begin{array}{l}\text { July 28.. } \\
\text { Aug. } 3 . . .\end{array}$ & $\begin{array}{l}183.65 \\
181.81\end{array}$ \\
\hline & & Feb. 11 & 212. 90 & May 4 & 206.19 & Aug. 10. & 181.48 \\
\hline $\begin{array}{l}\text { Dec. } 16 \\
\text { Dec. } 23\end{array}$ & $\begin{array}{l}179.32 \\
179.29\end{array}$ & $\begin{array}{l}\text { Feb. } 19 \\
\text { Feb. } 25\end{array}$ & $\begin{array}{l}213.47 \\
219.59\end{array}$ & $\begin{array}{l}\text { May } 11 \\
\text { May } 18\end{array}$ & $\begin{array}{l}205.63 \\
202.09\end{array}$ & $\begin{array}{l}\text { Aug. 17 } \\
\text { Aug. 31 }\end{array}$ & $\begin{array}{l}181.98 \\
181.08\end{array}$ \\
\hline Dec. 31 & 179. 53 & Mar. 3 & 218. 39 & June 1. & 196. 21 & Sept. 8 & 181.15 \\
\hline 1936 & & $\begin{array}{l}\text { Mar. } 9 \\
\text { Mar. } 16\end{array}$ & $\begin{array}{l}217.82 \\
214.14\end{array}$ & $\begin{array}{l}\text { June 8 } \\
\text { June } 15 .-\end{array}$ & $\begin{array}{l}196.09 \\
193.63\end{array}$ & $\begin{array}{l}\text { Sept. 14 } \\
\text { Sept. 21-. }\end{array}$ & $\begin{array}{l}181.17 \\
180.89\end{array}$ \\
\hline $\begin{array}{l}\text { Jan. } 7 \\
\text { Jan. } 14 \ldots\end{array}$ & $\begin{array}{l}199.59 \\
220.92\end{array}$ & $\begin{array}{l}\text { Mar. } 23 \\
\text { Mar. } 30 \\
\text { Apr. } 6\end{array}$ & $\begin{array}{l}214.20 \\
215.10 \\
215.10\end{array}$ & $\begin{array}{l}\text { June 22.- } \\
\text { June 29.- } \\
\text { July 8. }\end{array}$ & $\begin{array}{l}192.81 \\
191.83 \\
185.39\end{array}$ & Sept. 28 & 179.63 \\
\hline
\end{tabular}

2 Leveling by U. S. Engineer Department.

4 Water level depressed inordinately by large withdrawals. 
TABLE 13.-Ground-water levels in observation wells, 1928-30 and 1935-36-Con.

351. Cole (formerly Paul Zirkel). NW1/4NW1/4 sec. 15, T. $7 \mathrm{~S} ., \mathrm{R} .2 \mathrm{~W}$. Domestic and stock well, dug 4 feet square and 24 feet deep with brick curb, bored to total depth of about 32 feet. Measuring point is top of 2-inch plank deck, through bored hole, 0.3 foot above land surface and 191.60 feet ${ }^{2}$ above mean sea level.

\begin{tabular}{|c|c|c|c|c|c|c|c|}
\hline Date & $\begin{array}{l}\text { Water } \\
\text { level } \\
\text { (feet) }\end{array}$ & Date & $\begin{array}{l}\text { Water } \\
\text { level } \\
\text { (feet) }\end{array}$ & Date & $\begin{array}{l}\text { Water } \\
\text { level } \\
\text { (feet) }\end{array}$ & Date & $\begin{array}{l}\text { Water } \\
\text { level } \\
\text { (feet) }\end{array}$ \\
\hline 1928 & & 1935 & & 1936 & & 1936 & \\
\hline $\begin{array}{l}\text { Sept. } 14 \\
1929 \\
\text { July } 3 \\
\text { Aug. } 5 \\
\text { Sept. } 24 \\
\text { Oct. } 26 \\
\text { Nov. } 29 \\
\text { Dec. } 30 \\
\qquad 1930 \\
\text { Feb. } 2 \\
\text { Mar. } 2 \\
\text { Mar. } 30 \\
\text { Apr. } 27 \\
\text { July } 25 \\
\end{array}$ & $\begin{array}{l}170.9 \\
169.65 \\
168.68 \\
168.20 \\
167.95 \\
168.75\end{array}$ & $\begin{array}{l}\text { Oct. } 4 \\
\text { Oct. } 11 \\
\text { Oct. } 16 \\
\text { Oct. } 24 \\
\text { Oct. } 29 \\
\text { Nov. } 5 \\
\text { Nov. } 12 \\
\text { Nov. } 19 \\
\text { Dec. } 3 \\
\text { Dec. } 10 \\
\text { Dec. } 17 \\
\text { Dec. } 23 \\
\text { Dec. } 31 \\
\qquad 1936 \\
\text { Jan. } 7 \\
\text { Jan. } 14\end{array}$ & $\begin{array}{l}169.03 \\
168.96 \\
168.94 \\
168.46 \\
168.52 \\
168.33 \\
168.20 \\
168.23 \\
168.16 \\
168.22 \\
168.15 \\
168.81 \\
169.24\end{array}$ & $\begin{array}{l}\text { Jan. } 21 \\
\text { Jan. } 28 \\
\text { Feb. } 4 \\
\text { Feb. } 11 \\
\text { Feb. } 19 \\
\text { Feb. 25 } \\
\text { Mar. } 2 \\
\text { Mar. } 9 \\
\text { Mar. } 16 \\
\text { Mar. } 23 \\
\text { Mar. } 30 \\
\text { Apr. } 6 \\
\text { Apr. } 13 \\
\text { Apr. } 20 \\
\text { Apr. } 27 \\
\text { May } 4 \\
\text { May } 11 \\
\text { May } 18 \\
\end{array}$ & $\begin{array}{l}171.12 \\
171.75 \\
171.83 \\
171.28 \\
172.02 \\
172.72 \\
173.18 \\
173.37 \\
173.62 \\
173.02 \\
173.69 \\
173.60 \\
173.44 \\
173.48 \\
173.30 \\
173.30 \\
172.12 \\
172.13\end{array}$ & $\begin{array}{l}\text { May } 25 \\
\text { June } 1 \\
\text { June } 8 \\
\text { June } 15 \\
\text { June } 22 \\
\text { June } 29 \\
\text { July } 8 \\
\text { July } 13 \\
\text { July } 20 \\
\text { July } 28 \\
\text { Aug. } 3 \\
\text { Aug. } 10 \\
\text { Aug. } 17 \\
\text { Aug. } 24 \\
\text { Aug. } 31 \\
\text { Sept. } 8 \\
\text { Sept. } 14 \\
\text { Sept. 21 } \\
\text { Sept. } 28 \\
\text { Sep. }\end{array}$ & $\begin{array}{l}171.62 \\
171.00 \\
170.91 \\
171.85 \\
171.65 \\
170.48 \\
170.86 \\
170.61 \\
168.70 \\
170.15 \\
170.17 \\
169.85 \\
169.67 \\
169.59 \\
168.48 \\
169.14 \\
169.02 \\
169.09 \\
168.99\end{array}$ \\
\hline
\end{tabular}

2 Leveling by U. S. Engineer Department.

352. Fred Scharf. NE1/4NW1/4 sec. 18, T, 7 S., R. 2 W. Stock well, bored 6 inches in diameter and 38 feet decp, in yard north west of residence. Copper nail with washer stamped 7-2-4 in well cover. Measuring point, top of wooden well cover at copper nail with washer, 1.0 foot above land surface and 182 feet above mean sea level (altimeter).

\begin{tabular}{|c|c|c|c|}
\hline Date & $\begin{array}{l}\text { Water } \\
\text { level } \\
\text { (feet) }\end{array}$ & Date & $\begin{array}{l}\text { Water } \\
\text { level } \\
\text { (feet) }\end{array}$ \\
\hline $\begin{array}{l}\text { Sept. } 14 \\
\text { Oct. } 20 \\
\text { Dec. } 16\end{array}$ & $\begin{array}{l}164.5 \\
162.6 \\
172.2\end{array}$ & 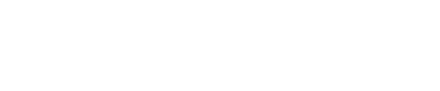 & $\begin{array}{l}179.2 \\
179.7 \\
176.6\end{array}$ \\
\hline
\end{tabular}

355. J. Grant. SW1/4SE1/4 sec. 28, T. 7 S., R. 2 W. Domestic well, dug 3 feet in diameter and 35 feet deep, brick curb. Measuring point, top of 2 -inch plank deck, through bored hole, 0.6 foot above land surface and 229.70 feet 2 above mean sea level.

\begin{tabular}{|c|c|c|c|c|c|c|c|}
\hline Date & $\begin{array}{l}\text { Water } \\
\text { level } \\
\text { (feet) }\end{array}$ & Date & $\begin{array}{l}\text { Water } \\
\text { level } \\
\text { (feet) }\end{array}$ & Date & $\begin{array}{l}\text { Water } \\
\text { level } \\
\text { (feet) }\end{array}$ & Date & $\begin{array}{l}\text { Water } \\
\text { level } \\
\text { (feet) }\end{array}$ \\
\hline 1935 & & 1936 & & 1936 & & 1936 & \\
\hline $\begin{array}{l}\text { Oct. } 10 \\
\text { Oct. } 16 \\
\text { Oct. } 24 \\
\text { Oct. } 29 \\
\text { Nov. } 5 \\
\text { Nov. } 12 \\
\text { Nov. } 19 \\
\text { Dec. } 3 \\
\text { Dec. } 10 \\
\text { Dec. } 17 \\
\text { Dec. } 24 \\
\text { Dec. } 31\end{array}$ & $\begin{array}{l}198.10 \\
198.31 \\
198.15 \\
197.21 \\
196.92 \\
197.20 \\
197.75 \\
199.01 \\
20000 \\
200.60 \\
200.73 \\
201.22\end{array}$ & $\begin{array}{l}\text { Jan. } 7 \\
\text { Jan. } 14 \\
\text { Jan. } 21 \\
\text { Jan. } 28 \\
\text { Feb. } 4 \\
\text { Feb. 11 } \\
\text { Feb. } 19 \\
\text { Feb. 25 } \\
\text { Mar. } 3 \\
\text { Mar. 10 } \\
\text { Mar. 17 } \\
\text { Mar. 24 } \\
\text { Mar. } 31 \\
\end{array}$ & $\begin{array}{l}212.92 \\
224.18 \\
223.98 \\
218.91 \\
218.06 \\
217.82 \\
219.15 \\
220.68 \\
222.25 \\
222.44 \\
220.40 \\
219.98 \\
223.95\end{array}$ & $\begin{array}{l}\text { Apr. } 7 \\
\text { Apr. } 14 \\
\text { Apr. } 21 \\
\text { Apr. } 28 \\
\text { May } 5 \\
\text { May } 12 \\
\text { May } 19 \\
\text { May 26 } \\
\text { June } 2 \\
\text { June } 9 \\
\text { June } 16 \\
\text { June } 23 \\
\text { June } 30\end{array}$ & $\begin{array}{l}224.00 \\
220.84 \\
219.45 \\
218.88 \\
218.93 \\
218.37 \\
217.98 \\
218.48 \\
217.52 \\
216.74 \\
216.98 \\
214.95 \\
214.13\end{array}$ & $\begin{array}{l}\text { July } 9 \\
\text { July } 14 \\
\text { July } 21 \\
\text { July } 28 \\
\text { Aug. } 4 \\
\text { Aug. } 11 \\
\text { Aug. 18 } \\
\text { Aug. 25 } \\
\text { Sept. } 1 \\
\text { Sept. } 9 \\
\text { Sept. } 15 \\
\text { Sept. } 22 \\
\text { Sept. } 29 \\
\end{array}$ & $\begin{array}{l}213.82 \\
208.51 \\
206.55 \\
205.72 \\
205.86 \\
205.51 \\
202.85 \\
203.30 \\
202.24 \\
202.31 \\
202.23 \\
202.11 \\
201.95\end{array}$ \\
\hline
\end{tabular}

'Leveling by U. S. Engineer Department. 
TABLE 13.-Ground-water levels in observation wells, 1928-30 and 1935-36-Con.

365. Clow. NW $1 / 4$ NW 14 sec. 7, T. 8 S., R. 4 W. Stock well, dug 3 feet in diameter and 15.3 feet deep brick curb. Measuring point, top of 2 -inch plank deck at west side of pump pipe, 0.6 foot above land surface and 208.4 feet 2 above mean sea level.

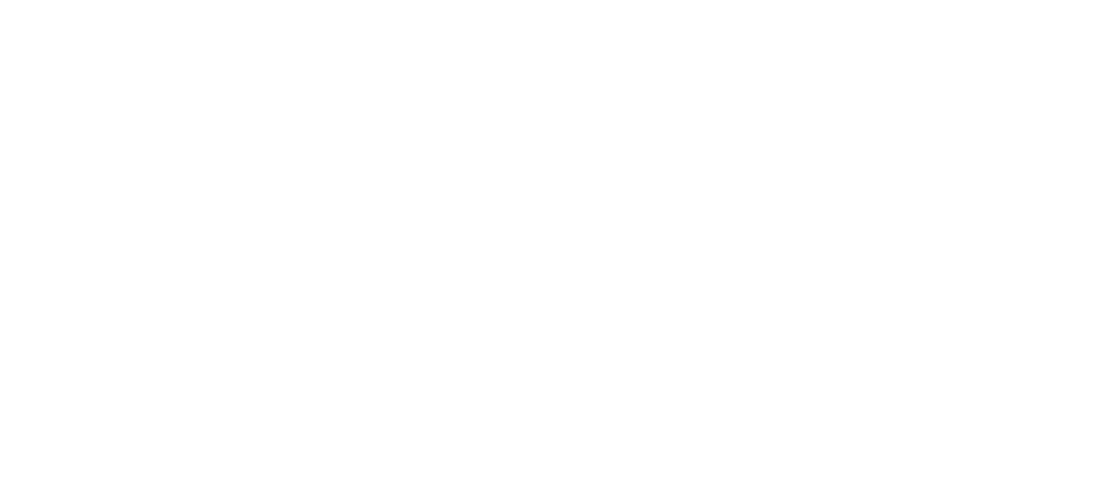

2 Leveling by U. S. Engineer Department.

366. Park Franks. NE1/4NE1/4 sec. 9, T. 8 S., R. 4 W. Stock well, dug 6 inches in diameter and 30 feet deep, in pasture 60 feet east of gate. Destroyed in March 1929. Measuring point is top of tile casing, 0.4 foot above land surface and 165.5 feet above mean sea level (hand level).

\begin{tabular}{|c|c|c|c|}
\hline Date & $\begin{array}{l}\text { Water } \\
\text { level } \\
\text { (feet) }\end{array}$ & Date & $\begin{array}{l}\text { Water } \\
\text { level } \\
\text { (feet) }\end{array}$ \\
\hline $\begin{array}{l}\text { Sept. } 11 \\
\text { Oct. } 22 \\
\text { Dec. } 1628 \\
\end{array}$ & $\begin{array}{l}140.6 \\
140.05 \\
145.70\end{array}$ & Feb. 3 1929 & 148.85 \\
\hline
\end{tabular}

367. E. Lauderback. NE1/4NE1/4 sec. 9, T. 8 S., R. 4 W. Domestic and stock well, bored 4 inches in diameter and 27 feet deep, stovepipe casing. Measuring point is top of casing, 2.0 feet above land surface and 167.34 feet ${ }^{2}$ above mean sea level.

\begin{tabular}{|c|c|c|c|c|c|c|c|}
\hline Date & $\begin{array}{l}\text { Water } \\
\text { level } \\
\text { (feet) }\end{array}$ & Date & $\begin{array}{l}\text { Water } \\
\text { level } \\
\text { (feet) }\end{array}$ & Date & $\begin{array}{l}\text { Water } \\
\text { level } \\
\text { (feet) }\end{array}$ & Date & $\begin{array}{l}\text { Water } \\
\text { level } \\
\text { (feet) }\end{array}$ \\
\hline 1935 & & 1936 & & 1936 & & 1936 & \\
\hline Oct. 28 & 140.30 & Jan. 8 . & 145.07 & Apr. 7 . & 147.92 & July 7 . & 141.81 \\
\hline Nov. 6 . & $\begin{array}{l}140.30 \\
140.14\end{array}$ & Jan. 14 & $\begin{array}{l}140.07 \\
145.55\end{array}$ & Apr. 14 & $\begin{array}{l}44.92 \\
147.96\end{array}$ & July 14 & $\begin{array}{l}1+1.01 \\
141.58\end{array}$ \\
\hline Nov. 12 & 140.14 & Jan. 21 & 147.90 & A pr. 21 & 147.21 & July 21 & 142.40 \\
\hline Nov. 19 & 139. 96 & Jan. 28 & 147.48 & A pr. 28 & $\begin{array}{l}5 \\
6\end{array}$ & July 28 & 4140.78 \\
\hline Nov. 27 & 139. 95 & Feb. 4 & 146. 53 & May 5 & 145.27 & Aug. 4 & 140.72 \\
\hline Dec. 3 . & 140.06 & Feb. 11 & 146.75 & May 12 & 144.49 & Aug. 11 & 140.63 \\
\hline Dec. 10 & 139.92 & Fob. 20 & 117.83 & May 19 & 144.40 & Aug. 18 & 144.90 \\
\hline Dec. 17 & 139.91 & Feb. 25- & 150.32 & May 26. & 143. 58 & Aug. 25- & 141. 94 \\
\hline Dec. 24 & 138.44 & Mar. 3 & 150.07 & June 2 & 142.96 & Sept. 1 & 112.18 \\
\hline \multirow[t]{4}{*}{ Dec. 31} & 144. 23 & Mar. 10 & 149.14 & June 9. & 141. 46 & Sept. 9 & 141.34 \\
\hline & & Mar. 17 & 147.87 & June 16 & 142.15 & Sept. 15_ & 141.40 \\
\hline & & Mar. 24 & 147. 11 & June 2 & 142.02 & Sept. 22 & 141.96 \\
\hline & & Mar. 31. & 148.12 & June 30 & 142.86 & Sept. 29 & 141.93 \\
\hline
\end{tabular}

2 Leveling by U. S. Engineer Department.

Water level depressed by inordinately large withdra wals.

5 Pump, operating in well. 
TABLE 13.-Ground-water levels in observation wells, 1928-30 and 1935-36-Con.

370. O. L. Foster. NE1/4SW1/4 sec. 21, T. $8 \mathrm{~S} .$, R. 4 W. Unused well, drilled 3 inches in diameter and 33.5 feet deep in yard south of residence. Casing stamped 8-4-2 on east side. Measuring point is top of casing at highest point, 0.9 foot above land surface and 160.5 feet above mean sea level (altimeter). On September 11, 1928, the water level was 133.55 feet; July 11, 1929, 135.50 feet; August 3, 1929, 134.51 feet.

374. J. W. Kelley estate. NW 1/4 NE $1 / 4$ sec. 29 T. 8 S., R. 4 W. Unused well, dug 3 feet in diameter and 27.5 feet deep, brick curb. Measuring point is top of iron bar spanning well, at south side, level with land surface and 174.03 feet ${ }^{2}$ above mean sea level.

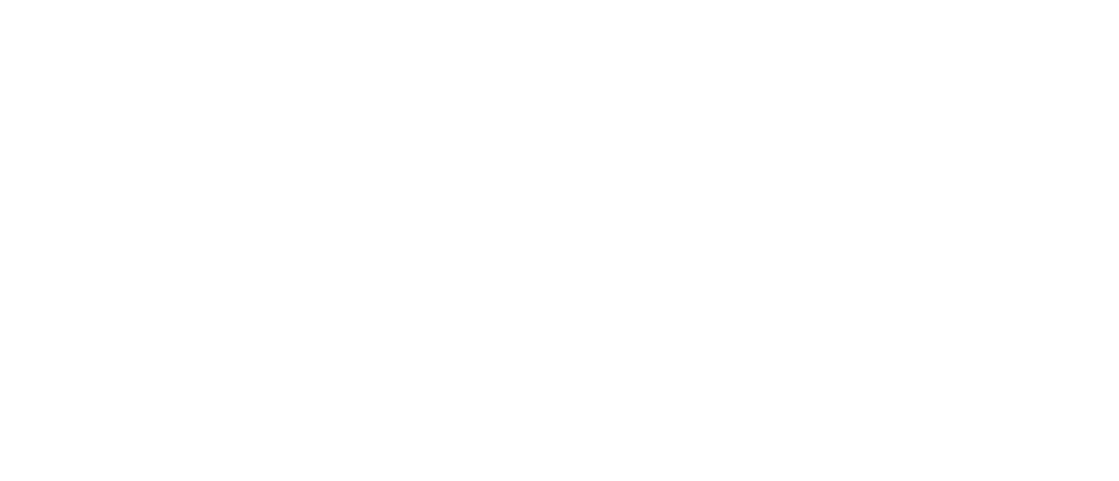

2 Leveling by U. S. Engineer Department.

377. P. O. Burbank. NW1/4NW1/4 sec. 31 , T. 8 S., R. 4 W. Domestic well, dug $31 / 2$ feet in diameter and 25.5 feet deep, brick curb with concrete collar. Measuring point is top of 2-by 4-inch timber frame, north side of well housing, 7.8 feet a bove land surface and 218.68 feet 2 a bove mean sea level.

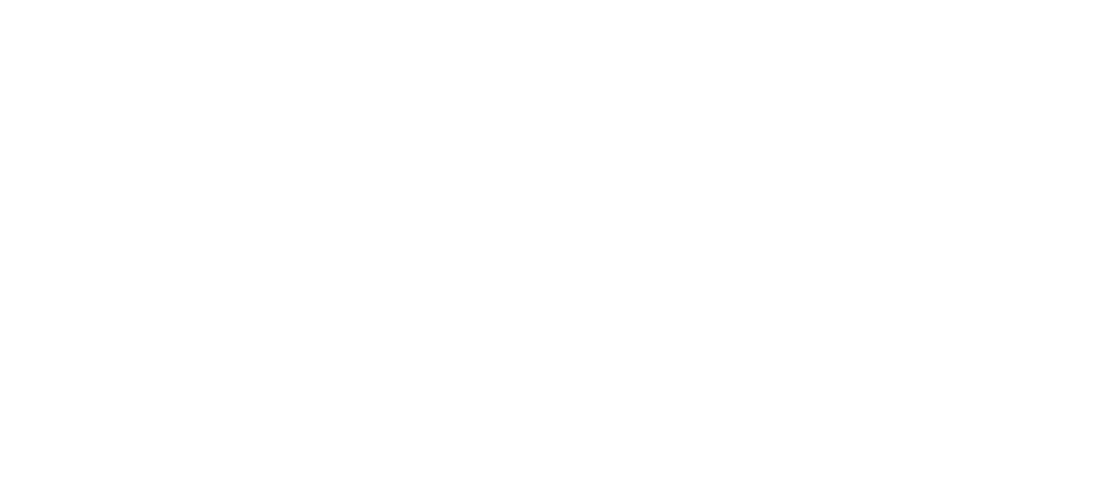

' Leveling by U. S. Engineer Department. 
TABLE 13.-Ground-water levels in observation wells, 1928-30 and 1935-36-Con.

394. Sloper Bros. SE1/4NE1/4 sec. 11, T. 9 S., R. 4 W. Unused well, drilled 8 inches in diameterand 32 feet deep, standard steel casing, in field 750 feet northeast of American Bottom School. Copper nail with washer stamped 9-4-4 in wood casing cap. Measuring point is top of casing flange, 1.0 foot above land surface and 157.93 feet 2 above mean sea level.

\begin{tabular}{|c|c|c|c|c|c|c|c|}
\hline Date & $\begin{array}{l}\text { Water } \\
\text { level } \\
\text { (feet) }\end{array}$ & Date & $\begin{array}{l}\text { Water } \\
\text { level } \\
\text { (feet) }\end{array}$ & Date & $\begin{array}{l}\text { Water } \\
\text { level } \\
\text { (feet) }\end{array}$ & Date & $\begin{array}{l}\text { Water } \\
\text { level } \\
\text { (feet) }\end{array}$ \\
\hline 1928 & \multirow{5}{*}{141.4} & 1935 & \multirow{19}{*}{$\begin{array}{l}140.39 \\
140.37 \\
140.50 \\
140.54 \\
140.45 \\
140.33 \\
140.38 \\
140.91 \\
141.15 \\
141.09 \\
141.06 \\
141.76 \\
141.73 \\
142.19\end{array}$} & 1936 & & 1936 & \multirow{19}{*}{$\begin{array}{l}145.91 \\
145.95 \\
147.00 \\
144.57 \\
144.72 \\
143.95 \\
143.54 \\
143.10 \\
142.84 \\
142.34 \\
141.95 \\
141.67 \\
141.45 \\
141.17 \\
140.00 \\
140.82 \\
140.52 \\
140.61 \\
140.44\end{array}$} \\
\hline \multirow[t]{4}{*}{ Sept. $15 \ldots$} & & Oct. 5. & & Jan. 8. & 143.40 & May 26 & \\
\hline & & Oct. 12 & & Jan. 21 & 153.08 & June $2 \ldots$ & \\
\hline & & Oct. 18 & & Jan. 28 & 151. 49 & June 9 & \\
\hline & & Oet. $21_{-}$ & & Feb. 4 & 149.21 & June 16 & \\
\hline Sept. 26. & 141. 18 & Oct. 28 & & Feb. 11 & 149.64 & June 23 & \\
\hline Oct. 27 & 140.88 & Nov. 6 & & Feb. 20 . & 148.82 & June 30 & \\
\hline Nov. 29 & 140.68 & Nov. 12 . & & Feb. 25 & 153.42 & July 7 & \\
\hline Dec. 30 & 147.23 & Nov. 19 & & Mar. 3 & 150.04 & July 14 & \\
\hline 1030 & & Nov. $27 \ldots$ & & Mar. 10 & 149.40 & July $21 \ldots \ldots$ & \\
\hline 1930 & & $\begin{array}{l}\text { Dec. } 3 \\
\text { Dec. } 10\end{array}$ & & $\begin{array}{l}\text { Mar. } 17 \\
\text { Mar. } 24\end{array}$ & $\begin{array}{l}147.96 \\
147.17\end{array}$ & $\begin{array}{l}\text { July } 28 \\
\text { Aug. } 4\end{array}$ & \\
\hline Feb. 2 . & 146.08 & Dec. 17 & & Mar. 31 & 147.22 & Aug. 11 & \\
\hline Mar. 2 & 150.38 & Dec. 24 & & Apr. 7 & 147.39 & Aug. 18. & \\
\hline Mar. 30. & 146.48 & Dec. 31 & & Apr. 14 & 146.54 & Aug. 25 & \\
\hline Apr. 27 & 144.83 & & & Apr. 21 & 146.51 & Sept. 1 & \\
\hline May 27 & 144.68 & & & A pr, $28 \ldots$ & 146.50 & Sept. 9 & \\
\hline July 23.. & 142.30 & & & May 5 & 146.09 & Sept. $15 \ldots$ & \\
\hline & & & & May $12 \ldots$ & 146.17 & Sept. 22 & \\
\hline & & & & May 19 & 146.09 & Sept. $29 \ldots$ & \\
\hline
\end{tabular}

${ }^{2}$ Leveling by U. S. Engineer Department.

396. Susan Nash. SW1/4SW1/4 sec. 23 , T. 9 S., R. 4 W. Domestic well, dug 30.9 feet deep. Measuring point is top of well deck, through bored hole at east side, 1.0 foot above land surface and 239.68 feet 2 above mean sea level.

\begin{tabular}{|c|c|c|c|c|c|c|c|}
\hline Date & $\begin{array}{l}\text { Water } \\
\text { level } \\
\text { (feet) }\end{array}$ & Date & $\begin{array}{l}\text { Water } \\
\text { level } \\
\text { (feet) }\end{array}$ & Date & $\begin{array}{l}\text { Water } \\
\text { level } \\
\text { (feet) }\end{array}$ & Date & $\begin{array}{l}\text { Water } \\
\text { level } \\
\text { (feet) }\end{array}$ \\
\hline \begin{tabular}{l}
\multicolumn{1}{c}{1935} \\
Oct. 25 \\
Oct. 28 \\
Nov. 6 \\
Nov. 12 \\
Nov. 19 \\
Nov. 27 \\
Dec. 3 \\
Dec. 10 \\
Dec. 17 \\
Dec. 24 \\
Dec. 31
\end{tabular} & $\begin{array}{l}213.78 \\
214.03 \\
214.12 \\
213.73 \\
213.66 \\
212.87 \\
212.49 \\
213.16 \\
212.77 \\
213.41 \\
221.28\end{array}$ & \begin{tabular}{l}
\multicolumn{1}{l}{1936} \\
Jan. 8 \\
Jan. 14 \\
Jan. 21 \\
Jan. 28 \\
Feb. 4 \\
Feb. 11 \\
Feb. 20 \\
Feb. 25 \\
Mar. 3 \\
Mar. 10 \\
Mar. 17 \\
Mar. 24 \\
Mar. 31
\end{tabular} & $\begin{array}{l}222.59 \\
229.11 \\
237.79 \\
237.11 \\
236.52 \\
236.62 \\
236.42 \\
238.40 \\
237.39 \\
236.97 \\
236.46 \\
236.07 \\
237.47\end{array}$ & $\begin{array}{l}1936 \\
\text { Apr. } 7 \\
\text { A pr. } 14 \ldots \\
\text { Apr. } 21 \\
\text { A pr. } 28 \\
\text { May } 5 \\
\text { May } 12 \\
\text { May } 19 \\
\text { May } 26 \ldots \\
\text { June } 2 \\
\text { June } 9 \\
\text { June } 16 \ldots \\
\text { June } 23 \\
\text { June } 30\end{array}$ & $\begin{array}{l}237.50 \\
236.36 \\
235.29 \\
234.61 \\
234.24 \\
234.71 \\
233.44 \\
233.51 \\
233.60 \\
231.69 \\
230.95 \\
230.37 \\
228.37\end{array}$ & \begin{tabular}{l}
\multicolumn{1}{c}{1936} \\
July 7 \\
July 14 \\
July 21 \\
July 28 \\
Aug. 4 \\
Aug. 11 \\
Aug. 18 \\
Aug. 25 \\
Sept. 1 \\
Sept. 9 \\
Sept. 15 \\
Sept. 22 \\
Sept. 29
\end{tabular} & $\begin{array}{r}227.09 \\
4224.65 \\
224.40 \\
+220.50 \\
220.39 \\
+218.29 \\
218.16 \\
217.48 \\
217.26 \\
215.77 \\
215.84 \\
215.56 \\
215.21\end{array}$ \\
\hline
\end{tabular}

2 Leveling by U. S. Engineer Department

4 Water level depressed by inordinately large withdrawals.

404. Addie Harman. NW1/4SE1/4 sec. 27 , T. 9 S., R. 4 W. Domestic and stock, well dug 3 feet in diameter and 30.8 feet deep, concrete-tile casing. Measuring point is top of 2-inch plank deck, through bored hole near southeast side of hand pump, 0.5 foot above land surface and 216.76 feet 2 above mean sea level.

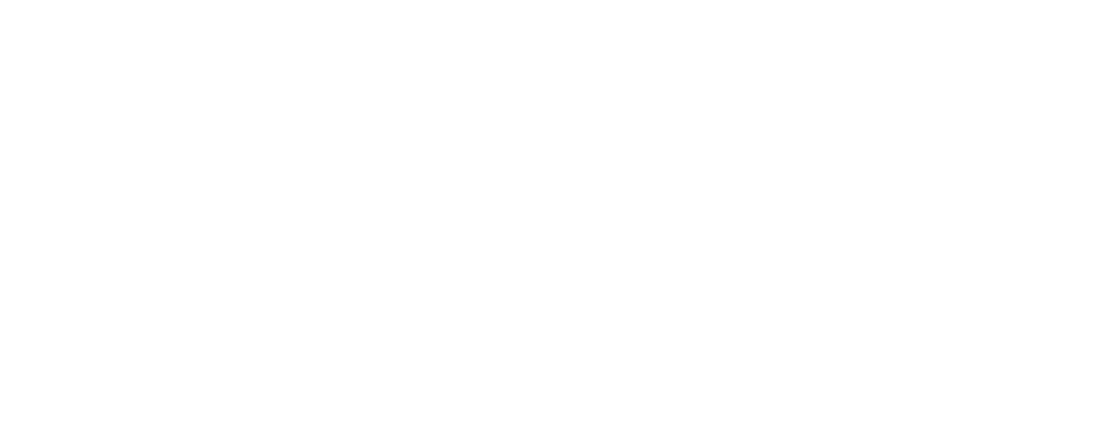

2 Leveling by U. S. Engineer Department.

4 Water level depressed by inordinately large withdrawals. 
TABLE 13.-Ground-water levels in observation wells, 1928-30 and 1985-36-Con.

405. Alfred Flickinger. NE1/4SW1/4 sec. 31, T. 9 S., R. 4 W. Domestic and stock well, bored 4 mches in diameter and 28.9 feet deep, concrete-tile casing. Measuring point is top of 2 -inch plank cover, level with land surface and 207.61 feet 2 above mean sea level.

\begin{tabular}{|c|c|c|c|c|c|c|c|}
\hline Date & $\begin{array}{l}\text { Water } \\
\text { level } \\
\text { (feet) }\end{array}$ & Date & $\begin{array}{l}\text { Water } \\
\text { level } \\
\text { (feet) }\end{array}$ & Date & $\begin{array}{l}\text { Water } \\
\text { level } \\
\text { (feet) }\end{array}$ & Date & $\begin{array}{l}\text { Water } \\
\text { level } \\
\text { (feet) }\end{array}$ \\
\hline $\begin{array}{l}\quad 1935 \\
\text { Oct. } 30 \\
\text { Nov. } 6 \\
\text { Nov. } 12 \\
\text { Nov. } 19 \\
\text { Nov. } 29 \\
\text { Dec. } 4 \\
\text { Dec. } 11 \\
\text { Dec. } 18 \\
\text { Dec. } 24 \\
\text { Dec. } 31\end{array}$ & $\begin{array}{l}194.58 \\
194.86 \\
195.01 \\
195.09 \\
194.91 \\
195.10 \\
198.57 \\
199.48 \\
199.22 \\
201.14\end{array}$ & $\begin{array}{l}\text { Jan. } 1936 \\
\text { Jan. } 14 \\
\text { Jan. } 21 \\
\text { Jan. } 28 \\
\text { Feb. 4 } \\
\text { Feb. } 11 \\
\text { Feb. } 20 \\
\text { Feb. } 26 \\
\text { Mar. } 3 \\
\text { Mar. } 10 \\
\text { Mar. } 17 \\
\text { Mar. } 24 \\
\text { Apr. 1 }\end{array}$ & $\begin{array}{l}202.32 \\
204.97 \\
206.08 \\
205.79 \\
205.54 \\
204.64 \\
204.02 \\
207.21 \\
207.18 \\
206.32 \\
205.13 \\
205.75 \\
206.00\end{array}$ & $\begin{array}{l}\quad 1936 \\
\text { Apr. } 8 \\
\text { Apr. } 15 \\
\text { Apr. } 22 \\
\text { Apr. } 29 \\
\text { May } 6 \\
\text { May } 12 \\
\text { May } 20 \\
\text { May } 27 \\
\text { June } 3 \\
\text { June } 10 \\
\text { June } 17 \\
\text { June 24 } \\
\text { July } 1 \\
\end{array}$ & $\begin{array}{l}205.98 \\
205.80 \\
205.83 \\
205.80 \\
205.04 \\
205.01 \\
205.03 \\
204.55 \\
204.43 \\
204.37 \\
203.54 \\
203.47 \\
201.77\end{array}$ & $\begin{array}{l}\quad 1936 \\
\text { July } 8 \\
\text { July } 15 \\
\text { July } 22 \\
\text { July } 29 \\
\text { Aug. } 5 \\
\text { Aug. } 12 \\
\text { Aug. } 19 \\
\text { Aug. } 26 \\
\text { Sept. } 2 \\
\text { Sept. } 10 \\
\text { Sept. } 16 \\
\text { Sept. } 23 \\
\text { Sept. } 30\end{array}$ & $\begin{array}{l}202.06 \\
201.20 \\
200.29 \\
199.64 \\
199.60 \\
198.42 \\
198.37 \\
196.21 \\
195.98 \\
195.83 \\
195.54 \\
193.52 \\
193.34\end{array}$ \\
\hline
\end{tabular}

2 Leveling by U. s. Engineer Department.

409. C. A. Bear. NE1/4NW1/4 sec. 4, T. 9 S., R. 2 W. Domestic well, driven $11 / 4$ inches in diameter and 29 feet deep, standard pipe casing. Measuring point is top of $1 \frac{1}{4}$-inch standpipe, 3.0 feet above land surface and 308.14 feet 2 above mean sea level.

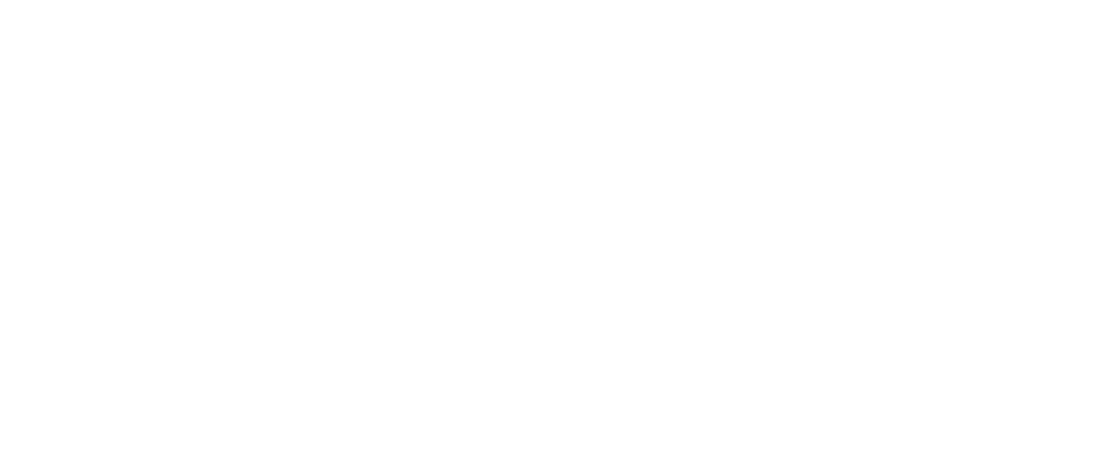

2 Leveling by U. S. Engineer Department.

412. George K. Miller. SE $1 / 4$ NE $1 / 4$ sec. 26, T. 9 S., R. 2 W. Domestic well, driven $11 / 2$ inches in diameter and 13.3 feet deep, standard pipe casing. Measuring point is top of $11 / 2$-inch standpipe, 2.8 feet above land surface and 350.63 feet 2 above mean sea level.

\begin{tabular}{|c|c|c|c|c|c|c|c|}
\hline Date & $\begin{array}{l}\text { Water } \\
\text { level } \\
\text { (feet) }\end{array}$ & Date & $\begin{array}{l}\text { Water } \\
\text { level } \\
\text { (feet) }\end{array}$ & Date & $\begin{array}{l}\text { Water } \\
\text { level } \\
\text { (feet) }\end{array}$ & Date & $\begin{array}{l}\text { Water } \\
\text { level } \\
\text { (feet) }\end{array}$ \\
\hline 1935 & & 1935 & & 1936 & & 1936 & \\
\hline $\begin{array}{l}\text { Oct. } 10 \\
\text { Oct. } 16 \\
\text { Oct. } 24 \\
\text { Oct. } 28 \\
\text { Nov. } 5 \\
\text { Nov. } 12 \\
\end{array}$ & $\begin{array}{l}337.93 \\
338.11 \\
338.05 \\
338.05 \\
338.13 \\
338.11\end{array}$ & $\begin{array}{l}\text { Nov. } 19 \\
\text { Dec. } 4 \\
\text { Dec. } 10 \\
\text { Dec. } 18 \\
\text { Dec. } 24 \\
\text { Dec. } 31\end{array}$ & $\begin{array}{l}338.27 \\
338.44 \\
338,38 \\
338.44 \\
338.76 \\
339.06\end{array}$ & $\begin{array}{l}\text { Jan. } 7 \\
\text { Jan. } 14 \\
\text { Jan. } 21 \\
\text { Jan. } 28 \\
\text { Feb. 4 }\end{array}$ & $\begin{array}{l}341.38 \\
343.73 \\
343.35 \\
342.47 \\
341.83\end{array}$ & $\begin{array}{l}\text { Feb. } 11 \\
\text { Feb. } 19 \\
\text { Feb. } 25 \\
\text { Mar. } 3 \\
\text { Mar. } 10\end{array}$ & $\begin{array}{l}341.47 \\
341.28 \\
343.86 \\
340.85 \\
339.67\end{array}$ \\
\hline
\end{tabular}

2 Leveling by U. S. Engineer Department. 
TABLE 13.-Ground-water levels in observation wells, 1928-30 and 1935-36-Con.

414. Government Land Bank (Cliff Donner, lessor). NW1/4NE1/4 sec. 34 . T. 9 S., R. 2 W. Stock well, dug 8 feet square and 18.8 feet deep. Established as observation well in lieu of well 412 . Measuring point is top of 2 -inch plank deck, through crack near draft pipe, 0.4 foot above land surface and 325.14 feet $^{2}$ above mean sea level.

\begin{tabular}{|c|c|c|c|c|c|c|c|}
\hline Date & $\begin{array}{c}\text { Water } \\
\text { level } \\
\text { (feet) }\end{array}$ & Date & $\begin{array}{l}\text { Water } \\
\text { level } \\
\text { (feet) }\end{array}$ & Date & $\begin{array}{l}\text { Water } \\
\text { level } \\
\text { (feet) }\end{array}$ & Date & $\begin{array}{l}\text { Water } \\
\text { level } \\
\text { (feet) }\end{array}$ \\
\hline 1936 & & 1936 & & 1936 & & 1936 & \\
\hline Feb. 19 & 315. 19 & Apr. 21 & 314.40 & June 16 & 311.59 & Aug. 11 & 309.86 \\
\hline Feb. 25 & 318.14 & Apr, 28 & 313.36 & June 23 & 311.39 & Aug. 18 & 309.10 \\
\hline Mar. 3 & 317.75 & May 5 & 312.81 & June 30 & 311.05 & Aug. 25 & 308.96 \\
\hline Mar. 10 & 316.24 & May 12 & 312.60 & July 9. & 311.20 & Sept. 1 & 309.02 \\
\hline Mar. 17 & 315.24 & May 19 & 312.33 & July I4. & 310.90 & Sept. 9 & 310.32 \\
\hline Mar. 24 & 314.64 & May 26 & 312.42 & July 21 . & 310.71 & Sept. 15 & 310.17 \\
\hline Mar. 3 & 315.00 & June 2. & 311.76 & July 28 & 310.34 & Sept. 22 & 309.81 \\
\hline Apr. 7 & 315.04 & June 9 & 311.90 & Aug. 4. & 310.20 & Sept. 29 & 310.23 \\
\hline Apr. 1 & 314.72 & & & & & & \\
\hline
\end{tabular}

${ }^{2}$ Leveling by U. S. Engineer Department.

415. Mrs. G. M. H. Brewer. NE1/4NE1/4 sec. 7, T. 9 S., R. 1 W. Unused well, dug 16 feet deep, backfilled around standpipe. Measuring point is top of pump coupling on standpipe, 3.6 feet above land surface and $399.9 \mathrm{I}$ feet $^{2}$ above mean sea level.

\begin{tabular}{|c|c|c|c|c|c|c|c|}
\hline Date & $\begin{array}{l}\text { Water } \\
\text { level } \\
\text { (feet) }\end{array}$ & Drate & $\begin{array}{l}\text { Water } \\
\text { level } \\
\text { (feet) }\end{array}$ & Date & $\begin{array}{l}\text { Water } \\
\text { level } \\
\text { (feet) }\end{array}$ & Date & $\begin{array}{l}\text { Water } \\
\text { level } \\
\text { (feet) }\end{array}$ \\
\hline 1928 & & 1933 & \multirow{11}{*}{$\begin{array}{l}394.32 \\
393.22 \\
392.64 \\
392.75 \\
393.69 \\
394.26 \\
393.66 \\
393.59 \\
393.17 \\
393.83 \\
393.49 \\
393.72\end{array}$} & 1936 & \multirow{11}{*}{$\begin{array}{l}392.55 \\
391.98 \\
391.91 \\
391.64 \\
392.03 \\
392.35 \\
392.23 \\
391.69 \\
391.25 \\
391.75 \\
391.67 \\
390.89\end{array}$} & 1936 & \multirow{11}{*}{$\begin{array}{l}390.97 \\
390.74 \\
390.43 \\
390.74 \\
390.41 \\
390.97 \\
391.09 \\
390.87 \\
390.94 \\
390.84 \\
391.05 \\
390.88\end{array}$} \\
\hline Aug. 31. & 392. 16 & Jan. 21.. & & Apr. 14 & & July 14 & \\
\hline Oct. 20 & 389.2 & Jan. 28 & & Apr. 21 & & July 21 & \\
\hline 1935 & & Feb. I1 & & $\begin{array}{l}\text { Apr. } 28 \\
\text { May } 5 .\end{array}$ & & $\begin{array}{l}\text { July } 28 \\
\text { Aug. } 4\end{array}$ & \\
\hline & & Feb. 19 & & May 12 & & Aug. 11 & \\
\hline Dec. 16 & 392.62 & Feb. 25 & & May 19 & & Aug. 18. & \\
\hline Dec. 24 & 392.24 & Mar. 3. & & May 26.- & & Aug. 25. & \\
\hline Dec. 31 & 393.84 & Mar. 10 & & June 2 . & & Sept. 1 & \\
\hline 1936 & & $\begin{array}{l}\text { Mar. 17-- } \\
\text { Mar. 24 }\end{array}$ & & $\begin{array}{l}\text { June } 9 . . \\
\text { June } 16 .\end{array}$ & & $\begin{array}{l}\text { Sept. } 9 \\
\text { Sept. } 15\end{array}$ & \\
\hline $\operatorname{Tan} 7$ & 394.85 & Mar. 31 & & June $23 \ldots \ldots$ & & Sept. 22 & \\
\hline Jan. 14 & 394.71 & & & & & & \\
\hline
\end{tabular}

${ }^{2}$ Leveling by U. S. Engineer Department.

421. Fenry Hoefer. SE1/4NW1/4 sec. 12, T, $10 \mathrm{~S}, \mathrm{R}, 4 \mathrm{~W}$. Unused well, dug 24 inches in diameter and 24.5 feet deep, concrete-tile casing, in yard southwest of residence. Measuring point is top of casing, 1.7 feet above land surface and 187.44 feet 2 above mean sea level.

\begin{tabular}{|c|c|c|c|c|c|c|c|}
\hline Date & $\begin{array}{l}\text { Water } \\
\text { level } \\
\text { (feet) }\end{array}$ & Date & $\begin{array}{l}\text { Water } \\
\text { level } \\
\text { (feet) }\end{array}$ & Date & $\begin{array}{l}\text { Water } \\
\text { level } \\
\text { (feet) }\end{array}$ & Date & $\begin{array}{l}\text { Water } \\
\text { level } \\
\text { (feet) }\end{array}$ \\
\hline 1928 & & 1930 & & 1936 & & 1936 & \\
\hline Aug. 1.- & 165.64 & Apr. 27. & 167.24 & Jan. 10 & 175.84 & June 2 & 166.94 \\
\hline Sept. 7 & 164.49 & May 29. & 166.79 & Jan. 14 & 17S. 12 & June 9 & 166.81 \\
\hline Oct. $20_{-}$ & 163.8 & July 24 & 165.55 & Jan. 21 & 171.79 & June 16 & 166.73 \\
\hline Dec. 17 & 166. 2 & & & Jan. 24 & 171. 09 & June 23 & 166.62 \\
\hline & & 1935 & & Jan. 28 & 170. 09 & June 30 & 166. 33 \\
\hline 1929 & & Oct 4 & 163 & $\begin{array}{l}\text { Jan. } 31 \\
\text { Feb } 4\end{array}$ & $\begin{array}{l}171.59 \\
168.71\end{array}$ & July 9 & $\begin{array}{l}166.30 \\
166.29\end{array}$ \\
\hline Feb. 23 & 168.0 & Oct. 11 . & 163.24 & Feb. 12 & 168.89 & July 21 & 166. 79 \\
\hline Mar. 19 & 167.3 & Oct. 17 & 163.24 & Feb. 20 & 170.03 & July 28 & 165.63 \\
\hline May 12 & 167.7 & Oct. 25 & I 63.24 & Feb. 25 & 170.32 & A ug. 4 & 165.34 \\
\hline June $7 \ldots$ & 167.14 & Oct. 28 & 163.24 & Mar. 3 & 170.57 & Aug. 11 & 164.64 \\
\hline July $3 \ldots$ & 166.64 & Nov. 5 & 163.04 & Mar. 10 & 171.12 & Aug. 18 & 165.09 \\
\hline Aug. 3 & 165.79 & Nov. 12 & 163.14 & Mar. 17 & 171.78 & Aug. 25..... & 164.80 \\
\hline Sept. 24 & 164.19 & Nov. 20. & 163.14 & Mar. 24 & 167.89 & Sept. 1 & 164.73 \\
\hline Oct. 26 & 163. 64 & Dec. 4 & 163.44 & Mar. 31 & 168.04 & Sept. 9. & 164.48 \\
\hline Nov. 28 & 163. 29 & Dec. 10 & 162.54 & Apr. 7 & 167.69 & Sept. 15 & 164. 69 \\
\hline Dec. 29 & 167.74 & Dec. 18 & 162.69 & Apr. 14 & 167.79 & Sept. 22 & 163. 91 \\
\hline 1930 & & & 164 & $\begin{array}{l}\text { Apr. } 21 \\
\text { Apr. } 28 \ldots\end{array}$ & $\begin{array}{l}167.33 \\
167.49\end{array}$ & sept. & 163.08 \\
\hline & & 1936 & & May 5 & 167.39 & & \\
\hline Feb & 167.94 & & & May 12 & I $66.6 \mathrm{I}$ & & \\
\hline Mar. 2 & 169. 04 & Jan. 1 & 166. 29 & May 19 & 167.42 & & \\
\hline Mar. 30 & 166.79 & Jan. 7 & 172.34 & May 26 & 167.71 & & \\
\hline
\end{tabular}

${ }^{2}$ Leveling by U. S. Engineer Department. 
TABLE 13.-Ground-water levels in observation wells, 1928-30 and 1935-38-Con.

422. A. E. Holmes. NE1/4NE1/4 sec. 15, T. $10 \mathrm{~S}$, R. 4 W. Stock well, driven 23 feet deep. Measuring point is top of draft pipe (after unscrewing pump, nipple, and coupling), 2.5 feet above land surface and 189.34 feet $^{2}$ above mean sea level.

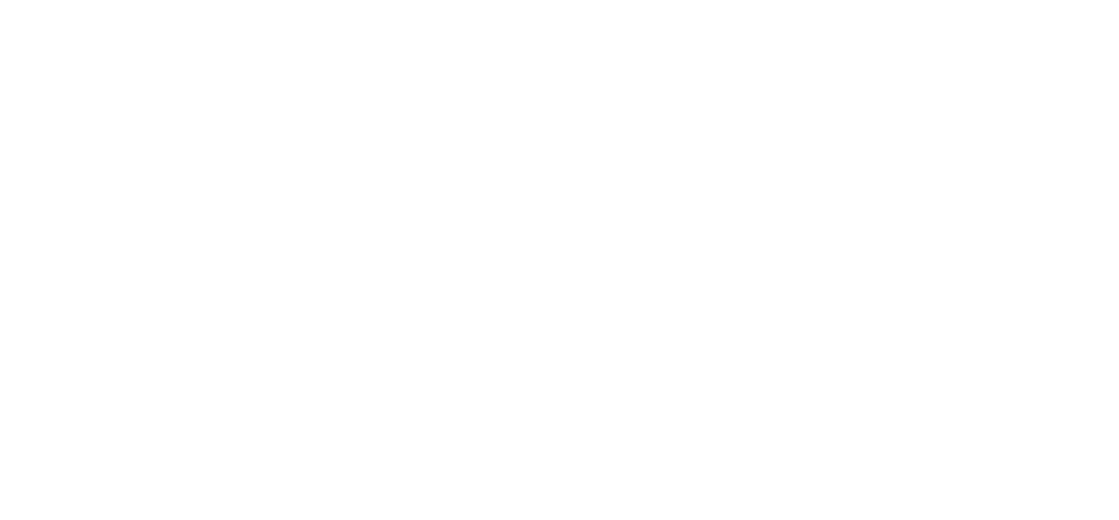

2 Leveling by U. S. Engineer Department.

425. Southern Pacific Railroad (Wellsdale). NW1/4SW1/4 sec. 17, T. 10 S., R. 4 W. Unused well, dug 6 feet in diameter and 28.5 feet deep, octagonal timber curb. Measuring point is top of 3 -inch plank deck, 1.3 feet a bove land surface and 217.15 feet $^{2}$ a bove mean sea level.

\begin{tabular}{|c|c|c|c|c|c|c|c|}
\hline Date & $\begin{array}{l}\text { Water } \\
\text { level } \\
\text { (feet) }\end{array}$ & Date & $\begin{array}{l}\text { Water } \\
\text { level } \\
\text { (feet) }\end{array}$ & Date & $\begin{array}{l}\text { Water } \\
\text { level } \\
\text { (feet) }\end{array}$ & Date & $\begin{array}{l}\text { Water } \\
\text { level } \\
\text { (feet) }\end{array}$ \\
\hline 1935 & & 1936 & & 1936 & & 1936 & \\
\hline Oct. 24 & 198.18 & Jan. 14 & 214.94 & Apr. 15 & 213.88 & July 15 . & 208.48 \\
\hline Oct. 29 & 198.06 & Jan. 21 & 215.01 & Apr. 22 & 212.66 & July 22 & 207.82 \\
\hline Nov. 6 & 197.95 & Jan. 28. & 213.98 & Apr. 29 & 212.58 & July 29. & 206.69 \\
\hline Nov. 12 . & 198. 09 & Feb. 4 & 214.68 & May 6.- & 212.28 & Aug. 5 & 206. 32 \\
\hline Nov. 19 & 198. 23 & Feb. 11 & 213.88 & May 13 & 212.31 & Aug. 12 & 204.77 \\
\hline Nov. 27 & 198.10 & Feb. 20 & 213.52 & May 20. & 212. 63 & Aug. 19 & 204.43 \\
\hline Dec. 4 & 197.98 & Feb. 26 & 214.79 & May 27. & 211. 87 & Aug. 26 & 203.08 \\
\hline Dec. 10 & 198.81 & Mar. 3 & $\begin{array}{l}213.98 \\
213.98\end{array}$ & June 3 & $\begin{array}{l}211.39 \\
219\end{array}$ & Sept. 2 & 202.93 \\
\hline Dec. 17 & 200.12 & Mar. 10 & 213.94 & June 10 & 210.97 & Sept. 10. & 201.68 \\
\hline Dec. 24 & 200.36 & Mar. 17_ & 213.72 & June 17 . & 210.61 & Sept. 15 . & 201.36 \\
\hline Dec. 31 & 211. 39 & Mar. 24 & 213.33 & June 24 & 210.16 & Sept. 23 & 200.83 \\
\hline 1936 & & Apr. 1. & 213.29 & July 1-- - & 209.65 & Sept. 30 & 200.66 \\
\hline Jan. 8 . & 214.49 & Apr. 8. & 213. 33 & July 8... & 209.13 & & \\
\hline
\end{tabular}

${ }^{2}$ Leveling by U. S. Engineer Department.

426. L. C. Williamson. SE $1 / 4$ NE $1 / 4$ sec. 18 , T. 10 S.. R. 4 W. Stock well, dug 4 feet in diameter and 28.8 feet deep, brick curb. Measuring point is top of brick curb, at south side, 0.3 foot above land surface and 218.44 feet 2 a bove mean sea level.

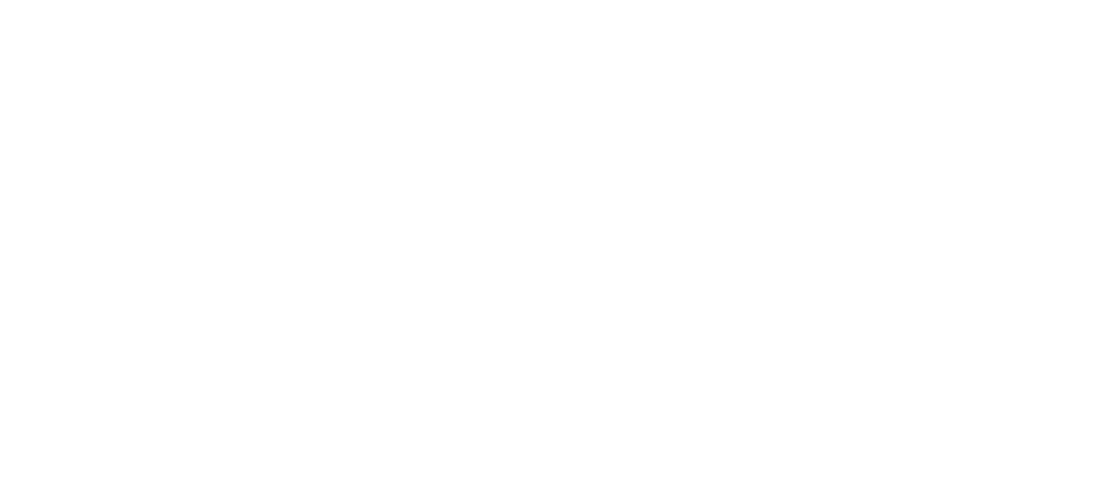

${ }^{2}$ Leveling by U. S. Engineer Department.

s Pump operating in well. 
TABLE 13.-Ground-water levels in observation wells, 1928-30 and 1935-36-Con.

433. C. B. Harnich. NE $1 / 4$ NE1/4 sec. 8, T. $10 \mathrm{~S} .$, R. 3 W. Stock well, driven $11 / 2$ in ches in diameter and 17.7 feet deep. Measuring point is bottom of pump flange, 3.0 feet a bove land surface and 199.52 feet ${ }^{2}$ above mean sea level.

\begin{tabular}{|c|c|c|c|c|c|c|c|}
\hline Date & $\begin{array}{l}\text { Water } \\
\text { level } \\
\text { (feet) }\end{array}$ & Date & $\begin{array}{l}\text { Water } \\
\text { level } \\
\text { (feet) }\end{array}$ & Date & $\begin{array}{l}\text { Water } \\
\text { level } \\
\text { (feet) }\end{array}$ & Date & $\begin{array}{l}\text { Water } \\
\text { level } \\
\text { (feet) }\end{array}$ \\
\hline 1936 & & 1936 & & 1936 & & 1936 & \\
\hline $\begin{array}{l}\text { Apr. } 27 \\
\text { May } 5 \\
\text { May } 12 \\
\text { May } 19 \\
\text { May } 26 \\
\text { June } 2\end{array}$ & $\begin{array}{l}184.24 \\
185.28 \\
184.35 \\
184.88 \\
184.32 \\
183.55\end{array}$ & $\begin{array}{l}\text { June } 9 \\
\text { June } 16 \\
\text { June } 23 \\
\text { June } 30 \\
\text { July } 9 \\
\text { July } 14\end{array}$ & $\begin{array}{l}184.91 \\
184.84 \\
184.78 \\
184.25 \\
184.33 \\
184.23\end{array}$ & $\begin{array}{l}\text { July } 21 . \\
\text { July } 28 \\
\text { Aug. } 4 \\
\text { Aug. } 11 \\
\text { Aug. } 18 \\
\text { Aug. } 25\end{array}$ & $\begin{array}{l}\mathbf{1 8 3 . 7 8} \\
183.67 \\
183.79 \\
182.69 \\
183.32 \\
183.44\end{array}$ & $\begin{array}{l}\text { Sept. } 1 \\
\text { Sept. } 9 \\
\text { Sept. } 15 \\
\text { Sept. } 22\end{array}$ & $\begin{array}{l}183.33 \\
182.83 \\
182.73 \\
182.33\end{array}$ \\
\hline
\end{tabular}

${ }^{2}$ Leveling by U. S. Engineer Department.

438. J. G. Gourley. NW1/4SE1/4 sec. 21 , T. $10 \mathrm{~S}$., R. 3 W. Stock well, dug 6 feet in diameter and 17.9 feet deep, dry-masonry curb. Measuring point is top of curb, at base of pump, 0.3 foot above land surface and 242.53 feet ${ }^{2}$ a bove mean sea level.

\begin{tabular}{|c|c|c|c|c|c|c|c|}
\hline Date & $\begin{array}{l}\text { Water } \\
\text { level } \\
\text { (feet) }\end{array}$ & Date & $\begin{array}{l}\text { Water } \\
\text { level } \\
\text { (feet) }\end{array}$ & Date & $\begin{array}{l}\text { Water } \\
\text { level } \\
\text { (feet) }\end{array}$ & Date & $\begin{array}{l}\text { Water } \\
\text { level } \\
\text { (feet) }\end{array}$ \\
\hline 1935 & & 1936 & & 1936 & & 1936 & \\
\hline Oct. 11 & 225.51 & Jan. 7 & 240.84 & Mar. 24 & 239.12 & June 30 . & 232.53 \\
\hline Oct. 17 - & 225.94 & Jan. $10_{-}$ & 241.01 & Mar. 31 & 240.18 & July 9 . & 230.99 \\
\hline Oct. 29 & 226. 06 & Jan. 14 & 241.14 & Apr. 7 & 239.85 & July 21 . & 230.19 \\
\hline Nov. 5 & 225.85 & Jan. 21. & 241.08 & Apr. 14 & 239.27 & July 28 & 229.86 \\
\hline Nov. 12 & 225.93 & Jan. 24 & 240.41 & Apr. 21 . & 237.98 & Aug. 4 & 229.13 \\
\hline Nov. 20 & 226.18 & Jan. 28. & 238.49 & A pr. 28 & 236.74 & Aug. 11 . & 228.93 \\
\hline Dec. 4 . & 226.20 & Jan. 31. & 239. 71 & May 5 & 236.42 & Aug. 18. & 228.80 \\
\hline Dec. 10 & 226.79 & Feb. 4 & 236. 49 & May 12 & 238.56 & Aug. 25 & 227.47 \\
\hline Dec. 18 . & 227.44 & Feb. 12 & 236.21 & May 19 & 239.00 & Sept. 1 & 227.28 \\
\hline Dec. 24 & 229.15 & Feb. 20 & 235.25 & May 26 & 237.76 & Sept. 9 & 226.80 \\
\hline 1936 & & $\begin{array}{l}\text { Feb. } 25 \\
\text { Mar. } 3\end{array}$ & $\begin{array}{l}240.78 \\
240.18\end{array}$ & $\begin{array}{l}\text { June 2- } \\
\text { June } 9\end{array}$ & $\begin{array}{l}236.78 \\
235,67\end{array}$ & $\begin{array}{l}\text { Sept. } 15 \\
\text { Sept. } 22\end{array}$ & $\begin{array}{l}226.93 \\
226.50\end{array}$ \\
\hline & & Mar. 10 & 239.41 & June 16 & 235.58 & Sept. 29 & 226.04 \\
\hline Jan. $1 \ldots$ & 230.06 & Mar. 17 & 239.01 & June 23 & 234.15 & & \\
\hline
\end{tabular}

2 Leveling by U. S. Engineer Department.

448. J. E. Watkins (formerly D. Watkins). NW1/4NE1/4 sec. 17, T. 10 S., R. 2 W. Unused well, dug 36 inches in diameter and 27 feet deep, in rear of residence. Measuring points: Top of brick curb, level with land surface and 276.31 feet ${ }^{2}$ above mean sea level; beginning December 10 , 1935, bottom of board spanning well, level with top edge of new concrete and steel collar, 276.67 feet 2 above mean sea level.

\begin{tabular}{|c|c|c|c|c|c|c|c|}
\hline Date & $\begin{array}{l}\text { Water } \\
\text { level } \\
\text { (feet) }\end{array}$ & Date & $\begin{array}{l}\text { Water } \\
\text { level } \\
\text { (feet) }\end{array}$ & Date & $\begin{array}{l}\text { Water } \\
\text { level } \\
\text { (feet) }\end{array}$ & Date & $\begin{array}{l}\text { Water } \\
\text { level } \\
\text { (feet) }\end{array}$ \\
\hline 1928 & & 1930 & & 1936 & & 1936 & \\
\hline Aug. 30 & 251.6 & Apr. 27 & 252.96 & Jan. 14 & 259. 52 & June 16 & 252.37 \\
\hline Oct. 20 . & 251.6 & May 29 & 252.96 & Jan. 21 & 255,67 & June 23 & 252.22 \\
\hline Dec. 16 & 252.5 & July 24 & 251.56 & Jan. 28 & 254.87 & June 30 & 251.95 \\
\hline 1929 & & 1935 & & Feb. 12 & 254.02 & July 14 & 251.87 \\
\hline Feb. 3 & 255.9 & Oct. 4 & 250.59 & $\begin{array}{l}\text { Feb. } 19 \\
\text { Feb. } 25\end{array}$ & $\begin{array}{l}253.67 \\
254.65\end{array}$ & $\begin{array}{l}\text { July } 21 \text { - } \\
\text { July } 28 .\end{array}$ & $\begin{array}{l}252.61 \\
251.30\end{array}$ \\
\hline Mar. 19 & 254.2 & Oct. 11 & 250.66 & Mar. 3 & 253.02 & Aug. 4 & 251.27 \\
\hline May 12 & 254.0 & Oct. 17 & 250.63 & Mar. 10 & 252.57 & Aug. 11 & 251.17 \\
\hline June 7 & 253.11 & Oct. 25 & 251,29 & Mar. 17 & 252.77 & Aug. 18 . & 250.37 \\
\hline July 23 & 252.76 & Oct. 28 & 251.26 & Mar. 24 & 251.47 & Aug. 25 . & 250.37 \\
\hline Aug. 3 & 251.76 & Nov. 12 & 251.11 & Mar. 31 & 253.48 & Sept. 1 & 250.32 \\
\hline Sept. 24 & 251. 21 & NoF. 19 & 251.01 & Apr. 7.- & 253.44 & Sept. 9 & 255.89 \\
\hline Oct. 26 & 251.11 & Dec. 4 & 250.91 & Apr. 14 & 253.03 & Sept. 15 & 255.87 \\
\hline Nov. 28 & 250.96 & Dec. 10 & 251.97 & Apr. 21 & 252.78 & Sept. 19 & 251.91 \\
\hline Dec. 30 & 253.86 & Dec. 18 & 251.95 & Apr. 28 & 252,71 & Sept. 22 & 250.88 \\
\hline & & Dec. 24 & 251.73 & May 5 & 252.67 & Sept. 29 & 250.86 \\
\hline 1930 & & & & May 12 & 253.35 & & \\
\hline Feb. 2 . & 253.81 & & & $\begin{array}{l}\text { May } 19 \\
\text { May } 26\end{array}$ & $\begin{array}{l}253.22 \\
253.27\end{array}$ & & \\
\hline Mar. & 255.31 & Jan. 1 & 253.50 & June 2 & 252.57 & & \\
\hline Mar. 3 & 253.51 & Jan. 7. & 255. 30 & June 9 & 252.47 & & \\
\hline
\end{tabular}

${ }^{2}$ Leveling by U. S. Engineer Department. 
TABLE 13.-Ground-water levels in observation wells, 1928-30 and 1935-36-Con.

453. Joe Wesley (Jan Brunt Sawmill, renter). NE1/4SW1/4 sec. 18. T. 10 S., R. 1 W. Unused well, dug 6 feet square and 11.7 feet deep. Measuring point is top at southeast corner of curb, about 1 foot above land surface and 315.26 feet ${ }^{2}$ above mean sea level.

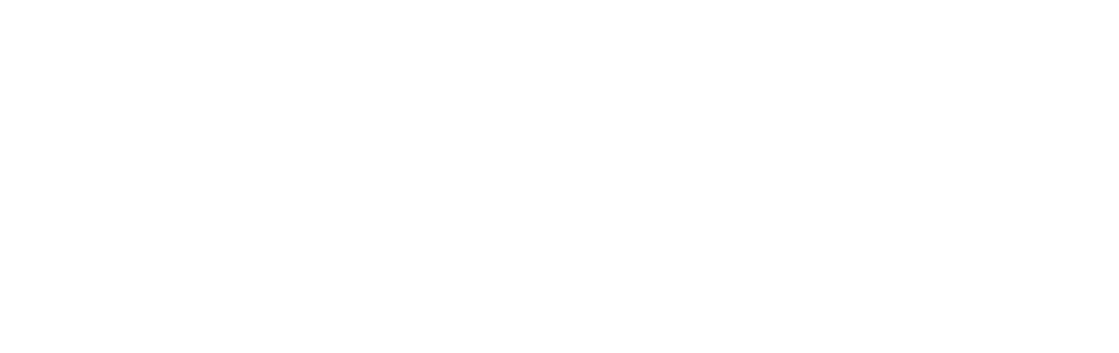

Leveling by U. s. Engineer Department.

456. Ida Locke estate (E. W. Elliott, renter). SW1/4NE1/4 sec. 13, T. $11 \mathrm{~S}$., R. 5 W. Stock well, dug $31 / 2$ feet in diameter and 25 feet deep, brick curb. Measuring point is top of 2 -inch plank deck, through opening for draft pipe, 240.34 feet 2 above mean sea level.

\begin{tabular}{|c|c|c|c|c|c|c|c|}
\hline Date & $\begin{array}{l}\text { Water } \\
\text { level } \\
\text { (feet) }\end{array}$ & Date & $\begin{array}{l}\text { Water } \\
\text { level } \\
\text { (feet) }\end{array}$ & Date & $\begin{array}{l}\text { Water } \\
\text { level } \\
\text { (feet) }\end{array}$ & Date & $\begin{array}{l}\text { Water } \\
\text { level } \\
\text { (feet) }\end{array}$ \\
\hline 1935 & & 1936 & & 1936 & & 1936 & \\
\hline Oct. 24 & 219.12 & Jan. 21 & 238. 49 & May 6 & 235.90 & Aug. 26 . & 225.81 \\
\hline Oet. 29 & 219.53 & Jan. 28 & 238.49 & May 13 & 235.85 & Sept. 2 & 225.26 \\
\hline Nov. 6. & 218.09 & Feb. 4 & 238.35 & May 20 . & 235.80 & Sept. 10 & 224.27 \\
\hline Nov. 12 & 218.67 & Feb. 11 & 237.63 & May 27 & 232. 11 & Sept. 15 & 224.02 \\
\hline Nov. 19 & 218.19 & Feb. 20 & 237.70 & June 3 & 232.30 & Sept. 23 & 224.30 \\
\hline Nov. 29 & 219.47 & Feb. 26 & 238.17 & June 10 & 231.91 & Sept. 30 & 223.91 \\
\hline Dec. 4 & 219.95 & Mar. 3 & 238.31 & June 17 . & 231. 32 & & \\
\hline Dec. 11 & 219.91 & Mar. 10 & 237.87 & June 24 & 231. 37 & & \\
\hline Dec. 18 & 219.75 & Mar. 17.. & 238.01 & July $1 .$. & 230.18 & & \\
\hline Dec. 24 & 219.77 & Mar. 24 & 235.05 & July 8 & 229.90 & & \\
\hline Dec. 31 & 223.06 & Mar. 27 & 235.16 & July 15 & 229.67 & & \\
\hline 1936 & & Apr. 1 & 236.13 & July $23 \ldots$ & 228.56 & & \\
\hline & & $\begin{array}{l}\text { Apr. } 8 \\
\text { Apr. } 15\end{array}$ & $\begin{array}{l}236.01 \\
234.59\end{array}$ & $\begin{array}{l}\text { July } 29 \ldots \\
\text { Aug. } 5 \ldots\end{array}$ & $\begin{array}{l}228.26 \\
227.98\end{array}$ & & \\
\hline Jan. 8 & 223.80 & Apr. 22 & 234.62 & Aug. 12 & 227.77 & & \\
\hline Jan. 14 & 227.37 & Apr. 29 & 234.49 & Aug. 19 & 227.28 & & \\
\hline
\end{tabular}

I Leveling by U. S. Engineer Department.

459. W. J. Moyer (J. M. Pitts, renter). SE1/4SE1/4 sec. 27, T. 11 S., R. 5 W. Domestic well, driven 40.4 feet deep. Measuring point is lower valve seat of pump, 4.7 feet above land surface and 236.28 feet 2 above mean sea level.

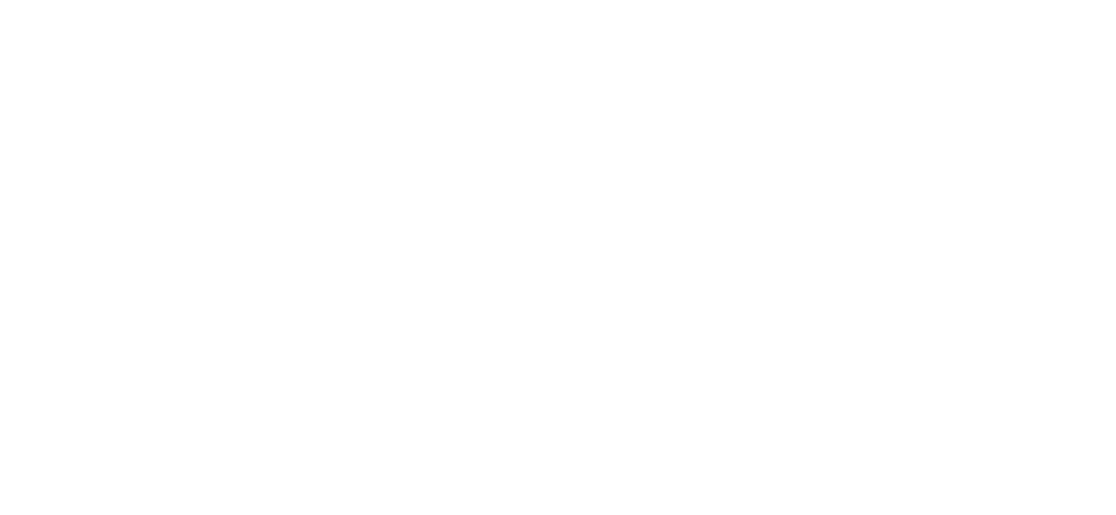

2 Leveling by U. S. Engineer Department. 
TABLE 13.-Ground-water levels in observation wells, 1928-30 and 1935-36-Con.

463. Oregon Agricultural Experiment Station, East Farm. SW $14 \mathrm{SE} 1 / 4$ sec. 36, T. $11 \mathrm{~S}$. . R. 5 W. Irrigation well, drilled 8 inches in diameter and 42 feet deep, about 6 feet east of well 464 and in the northeast corner of a common pit. Both wells pumped by joint suction. Measuring points: July 26 and Oct. 21 , 1928 , top face of flange at elbow in suction pipe, 197.53 feet above mean sea level; beginning Oct. 26, 1928, top of casing (level with zero of staff gage on east wall of pit east of well), 21.1 feet below land surface and 197.17 feet above mean sea level; beginning January 22, 1936, top of 6 - by 6 -inch timber girt bolted to top of concrete pit curb, 220.97 feet above mean sea level.

\begin{tabular}{|c|c|c|c|c|c|}
\hline Date & $\begin{array}{l}\text { Water } \\
\text { level } \\
\text { (feet) }\end{array}$ & Date & $\begin{array}{c}\text { Water } \\
\text { level } \\
\text { (feet) }\end{array}$ & Date & $\begin{array}{l}\text { Water } \\
\text { level } \\
\text { (feet) }\end{array}$ \\
\hline 1928 & & 1929 & & 29 & \\
\hline July 26. & 196.21 & Feb. 14 & 199.62 & Apr. 21 & 202. 67 \\
\hline Oct. 21 . & 195.43 & Feb. 15 & 199.57 & Apr. 22 & 202.82 \\
\hline Oct. 26 & 195.2 & Feb. 16 & 199.57 & Apr. 23 & 202.97 \\
\hline Oct. 27 & 195. 2 & Feb. 16 & 199.32 & Apr. 24 & 202. 97 \\
\hline Oct. 30 & 195. 22 & Feb. 17. & 198.97 & Apr. 25 & 202.92 \\
\hline Nov. 4 & 195. 32 & Feb. 18 & 198.72 & Apr. 26 & 202.77 \\
\hline Nov. 10 & 195.47 & Feb. 19 & 198.67 & Apr. 27- & 202.62 \\
\hline Nov. 18 & 195.57 & Feb. 20 & 198. 77 & Apr. 28 & 202.47 \\
\hline Nov. 24. & 195. 67 & Feb. 21 & 198.77 & Apr. 29 & 202.37 \\
\hline Dec. 1 & 195. 82 & Feb. 22 & 198.77 & Apr. 30 & 202. 27 \\
\hline Dee. 8 & 195.93 & Feb. 23 & 198. 77 & May 1. & 202.07 \\
\hline Dec. 13 & 19602 & Feb. 24 & 198.77 & May 2 & 201.92 \\
\hline Dec. 15 & 196. $6^{\prime \prime}$ & Feb. 25 & 198.77 & May 3 & 201.72 \\
\hline Dec. 18 & 196.72 & Feb. 26 & 198.87 & May 4 & 201.62 \\
\hline Dec. 19 & 196.77 & Feb. 27 & 198. 97 & May 5 & 201.57 \\
\hline Dec. 22- & 196.87 & Feb. 28 & 198.92 & May 6 & 201.47 \\
\hline Dec. 25 & 196. 97 & Mar. 1. & 198.87 & May 7 & 201.32 \\
\hline Dec. 27 & 197.17 & Mar. 2 & 198.87 & May 8 & 201.17 \\
\hline Dec. 28 & $197.4 i$ & Mar. 3 & 198.82 & May 9 & 201.07 \\
\hline Dec. 29. & 198. C2 & Mar. 4 & 198. 77 & May 10 & 200.72 \\
\hline Dec. 30 & 198.97 & Mar. 5 & 198.72 & May 11 & 200.62 \\
\hline Dec. 31 & 200.92 & Mar. 6 & 198.72 & May 12 & 200.63 \\
\hline & & $\begin{array}{l}\text { Mar. 7-- } \\
\text { Mar. 8... }\end{array}$ & $\begin{array}{l}\text { 199. } 27 \\
\text { 199.37 }\end{array}$ & $\begin{array}{l}\text { May } 13- \\
\text { May } 14\end{array}$ & $\begin{array}{l}200.47 \\
200.47\end{array}$ \\
\hline Jan. 1 & 200.92 & Mar. 9 & 199. 37 & May 15. & 200.47 \\
\hline an. 2 & 201.77 & Mar. 10 & 199.17 & May 16 & 200.47 \\
\hline Jan. 3 & 202.37 & Mar. 11 & 199. 22 & May 17 & 200.42 \\
\hline $\mathrm{Ja}$ & 202.47 & .12 & 199.52 & 18 & 200.42 \\
\hline Jan. 5 & 202. 67 & Mar. 13 & 199.67 & May 19 & 200.37 \\
\hline $\operatorname{Jan} .6$ & 202.97 & Mar, 14 & 199.77 & 20 & 200.32 \\
\hline Jan & 202. 97 & 15. & 199.77 & 21 & 200.27 \\
\hline Jan. 8 & 202.82 & Mar. 16 & 199. 72 & May 22 & 200.27 \\
\hline Jan. 9 & 202.07 & $\mathrm{Mr}$ & 199.62 & May 23 & 200.27 \\
\hline & 202.02 & & 198. 97 & 24 & 200.27 \\
\hline Jan. 11 & 198. 92 & 19. & 198.87 & 25 & 200.22 \\
\hline Jan. 12 & 198.67 & 20. & 198.87 & 26 & 200.17 \\
\hline & 198. $6 i$ & & 198.97 & & 200.12 \\
\hline Jan. 14 & 198. 67 & .22 & 199. 77 & 28 & 200.07 \\
\hline Jan. 15 & 198. 67 & 23 & 200.87 & 29 & 199.97 \\
\hline & 198. 67 & & 202.37 & & 199.87 \\
\hline Jan. 1 & 198. 67 & Mar. 25 & 202.07 & May 31 & 199.47 \\
\hline Jan. 18 & 198.67 & Mar. 26. & 201.87 & June 5 & 199.10 \\
\hline & 198. 67 & & 201.67 & & 199. 2 \\
\hline Jan. 20 & 198.77 & Mar. 27 & 201.27 & June 7 . & 199.32 \\
\hline Jan. 21 & 199.07 & Mar. 28 & 201.52 & June 8 - & 199.4 \\
\hline & $198.9 \overrightarrow{7}$ & & 201.37 & & 199. 52 \\
\hline Jan, 23 & 198.7 & Mar. 30 & 201.47 & June 10 & 199.5 \\
\hline Jan. 24 & 198.67 & Mar. 31. & 201.92 & June 11 & 199.4 \\
\hline Jar & 198.67 & & 201. 32 & 12 & 199.32 \\
\hline $\operatorname{Jan} .26$ & 198.67 & Apr. 2 & 201.17 & June 13 & 199.3 \\
\hline Jan. 27 & 198. 92 & Apr. 3 & 201.42 & Jime 14 & 199.22 \\
\hline & 199.67 & & 200.82 & Jume 15 & 199.2 \\
\hline Jan. 29 & 200.67 & 4 & 200.72 & June 16 & 199. 2 \\
\hline Jan. 30 & 200.67 & 5 & 200.42 & Jume 17 & 199.4 \\
\hline & 200.67 & & 200.22 & Jujle 18 & 199.47 \\
\hline & 200.77 & & 200.07 & June 19 & 199.42 \\
\hline Fel & 200.77 & A & 199.92 & June 20 & 199.4 \\
\hline Fe & 201.02 & & 199.87 & June 21 & 199.3 \\
\hline & 201.77 & 10. & 199.87 & June 22 & 199.2 \\
\hline Feb. 5 - & 201.92 & 11 & 199.87 & June 23 & 199.1 \\
\hline Fel & 202.02 & & 199.87 & June 24 & 199.0 \\
\hline $\mathrm{Fel}$ & 202.02 & & 199.87 & June 25. & 198.9 \\
\hline Fe & 202.02 & Apr. 14.. & 200.12 & June $26,7: 45$ a. $\mathrm{m}$ & 198.8 \\
\hline Feb. 8 - & 201.92 & 15 & 200.37 & June $26,3: 50 \mathrm{p} . \mathrm{m}$ & 5195.8 \\
\hline Feb & 201.67 & 16. & 200.77 & June $27,8: 00$ a. $\mathrm{m}$ & 198.7 \\
\hline Feb. 10 & 201.17 & 17. & 202.57 & June $27,5: 00$ p. m & $\begin{array}{lll}5 & 195.7\end{array}$ \\
\hline $\mathrm{Fe}$ & 200.52 & 8 & 202.87 & June $28,7: 45$ a. $\mathrm{m}$ & 198.6 \\
\hline $\mathrm{Fe}$ & 199.97 & Apl & 202.87 & June $28,5: 00$ p. m- & 5195.7 \\
\hline Feb. $13 \ldots$ & 199.72 & Apr. 20 & 202.77 & June $29,7: 45$ a. $\mathrm{m}$. & 198.5 \\
\hline
\end{tabular}

- Pump operating in well. 
TABLE 13.-Ground-water levels in observation wells, 1928-30 and 1935-36-Con.

\begin{tabular}{|c|c|c|c|c|c|}
\hline Date & $\begin{array}{l}\text { Water } \\
\text { level } \\
\text { (feet) }\end{array}$ & Date & $\begin{array}{l}\text { Water } \\
\text { level } \\
\text { (feet) }\end{array}$ & Date & $\begin{array}{l}\text { Water } \\
\text { level } \\
\text { (feet) }\end{array}$ \\
\hline 1929 & & 1929 & & 1929 & \\
\hline June $29,5: 00 \mathrm{p}, \mathrm{m}$ & 5195.6 & Aug. 22 . & 195.67 & Nov. 5 & 194.93 \\
\hline June 30 & 198.4 & Aug. 23 & 195. 63 & Nov. 6 & 194. 93 \\
\hline July $1 . .$. & 198. 3 & Aug. 24. & 195.61 & Nov. 7 & 194. 93 \\
\hline July $2,8: 10$ a. m & 198. 1 & Aug. 25 & 195.57 & Nov. 8 & 194.92 \\
\hline July $2,5: 10$ p. m- & 5195.2 & Aug. 26 & 195.53 & Nov. 9 & 194.92 \\
\hline July $3,8: 00$ a. m. & 198.0 & Aug. 27 . & 195.49 & Nov. 10 & 194.92 \\
\hline July $3,5: 10$ p. m. & 8193.9 & Aug. 28. & 195.47 & Nov. 11. & 194.93 \\
\hline July 4 & 197.9 & Aug. 29 & 195.45 & Nov. 12 & 194.94 \\
\hline July $5,7: 45$ a. m- & 197.7 & Aug. 30 - & 195. 43 & Nov. 13. & 194.95 \\
\hline July $5,5: 00$ p. m. & 5193.7 & Aug. 31 & 195.41 & Nov. 14 & 194.94 \\
\hline July $6,7: 55$ a. m & 197.6 & Sept. 1.. & 195. 39 & Nov. 15 & 194. 93 \\
\hline July $6,5: 15$ & 5193.7 & Sept. 2 & 195.37 & Nov. 16 & 194. 92 \\
\hline July 7 & 197.4 & Sept. 3 & 195. 35 & Nov. 17 & 194.91 \\
\hline July $8,7: 30$ a. m. & 197.3 & Sept. 4 & 195. 35 & Nov. 18 & 194.91 \\
\hline July $8,4: 00 \mathrm{p.m}$ & 5193.6 & Sept. 5 & 195.34 & Nov. 19 & 194.90 \\
\hline July $9,7: 30$ & 197.3 & Sept. 6. & 195.32 & Nov. 20 & 194. 89 \\
\hline July $9,5: 30 \mathrm{I}$ & 5193.6 & Sept. 7 & 195. 30 & Nov. 21 & 194.88 \\
\hline July $10,8: 00$ & 197.2 & Sept. 8 & 195. 29 & Nov. 22 & 194.87 \\
\hline July $10,5: 00$ & 5193.3 & Sept. 9 & 195. 25 & Nov. 23. & 194.87 \\
\hline July $11,7: 30 \mathrm{a} . \mathrm{m}$ & 197.2 & Sept. 10 & 195.23 & .24 & 194.87 \\
\hline July $11,5: 00$ & 5193.3 & Sept. 11 & 195. 23 & Nov. 25. & 194.87 \\
\hline July $12 \ldots$ & 197.2 & Sept. 12 & 195. 22 & Nov. 26 & 194. 89 \\
\hline & 197.1 & 13 & 195. 22 & 27 & 194. 85 \\
\hline Julv 14 & 197.1 & Sept. 14 & 195.22 & Nov. 27 , & 194.87 \\
\hline 5. & 197.0 & 15 & 195.21 & Nov. 28 & 194.85 \\
\hline & 197.0 & 16. & 195.21 & 29 & 194.83 \\
\hline $\mathrm{Jul}$ & 196.9 & Selt. 17 & 195. 20 & Nov. 30. & 194.82 \\
\hline & 196.9 & 18 & 19 & 1. & 194. 83 \\
\hline & 196.9 & 19. & 195. 19 & I & 194.82 \\
\hline July $20 \ldots$ & 197.0 & Sept. 20 & 195. 19 & Dec. 3 & 194.82 \\
\hline $\mathrm{Ju}$ & 196.9 & 21 & 18 & 4 & 194.82 \\
\hline & 196.8 & 22 & 19 & 5 & 194.81 \\
\hline $7: 10$ & 196.8 & Sept. 23. & 195. 20 & Dec. 6 & 194.82 \\
\hline $23,3: 30$ & 5193.2 & Sept. 23,6 & 195.18 & De & 194.83 \\
\hline $\begin{array}{l}4,7: 4 \\
4\end{array}$ & 196.8 & 24 & 19 & & 194.83 \\
\hline $24,4: 00$ & $\begin{array}{l}5193.2 \\
5193\end{array}$ & Sept. 25 & 195.19 & Dec. 9 & 194.84 \\
\hline 25 & 196.7 & 26 & 195. 21 & 10 & 194.84 \\
\hline & 196.7 & & 19 & 11 & 195.00 \\
\hline & 196.6 & .28 & 195.19 & 12 & 195.17 \\
\hline 8 & 196.5 & 29 & 19 & 13 & 195.45 \\
\hline & 196.4 & 30 & 19 & 14 & 195.97 \\
\hline Ju & 196.4 & $.1 \ldots$ & 195.17 & 15 & 196. 69 \\
\hline & 196. & & 19 & De & 198. 07 \\
\hline & 196. & & 19 & & 198.72 \\
\hline Aug. $2,8: 15$ a.m & 196. 36 & 4 & 195. 12 & Dec. 18 & 199.37 \\
\hline Aug. $2,5: 30$ p. m.. & 196.3 & Oet. 5 & 19511 & Dec. 19 & 199.97 \\
\hline $\mathrm{Au}$ & 196. 22 & & 19510 & & 206.72 \\
\hline 4 & 196.18 & 7. & 19509 & Dec. 21 . & 210.49 \\
\hline 5 & 196.10 & & 195.09 & Dec. 22 & 209.27 \\
\hline $6,7: 50$ & 196. 02 & & 19 & & 208.97 \\
\hline Aug. $6,12: 0$ & $\begin{array}{l}5192.87 \\
\end{array}$ & Oct. 10 & 195.11 & Dec. 24 & 208.82 \\
\hline Aug. $7,8: 10$ & 196.02 & 11 & 195.11 & Dec. 25 . & 208.59 \\
\hline 7,12 & ${ }^{5} 192.76$ & & 195.15 & & 208.17 \\
\hline $8,7: 4$ & 196.00 & 13 & 195.15 & De & 207.29 \\
\hline $8,5: 00$ & 5192.33 & 14 & 19512 & $\mathrm{De}$ & 207.42 \\
\hline Al & 195.97 & 15 & 19 & & 207.27 \\
\hline m. & 5192.22 & Oet. 16 . & & & 207.32 \\
\hline $10,7: 50$ & 195. 90 & Oct. 17 . & 195.07 & Dec. 31, & 204.47 \\
\hline $10,12: 0$ & 5192 & & 195. 07 & Dec. $31,12: 00$ & 206.17 \\
\hline & 195,8 & & 195. 0 ค & & \\
\hline $12,7: 5$ & 195.87 & 20 & 195,05 & 1930 & \\
\hline 12,12 & 5192.57 & & 19. & & \\
\hline $\mathrm{Au}$ & 195.83 & $\mathrm{Oc}$ & 195. 05 & Jan. 1. & 205.64 \\
\hline 13,12 & $\begin{array}{l}6192.47 \\
\end{array}$ & & & & 205.57 \\
\hline $14,7: 5$ & 195. 77 & & 195. 03 & $\mathrm{Ja}$ & 203. 27 \\
\hline $14,3: 15 \mathrm{p} . \mathrm{m}$ & 5192.37 & Oct. $25,12 \cdot 45 \mathrm{p} \mathrm{m}$ & 195. 02 & & 202.77 \\
\hline $15,7: 55$ a. m. & 195. 76 & Oct. $25,1: 40 \mathrm{p} . \mathrm{m}$ & 195.12 & Jan. 5 & 202.62 \\
\hline $15,5: 10$ p. $\mathrm{m}$ & 5 192.22 & Oct. 26 & 195.02 & Jan. 6 & 202.57 \\
\hline Aug. $16,7: 50$ a. $\mathrm{m}$ & 195.75 & & 195 & Jar & 202.47 \\
\hline Aug. 16, 5 & $\begin{array}{l}5 \\
5 \\
192.22\end{array}$ & Oet. 28 & 195. 00 & Jan. 8 & 202.42 \\
\hline a. $\mathrm{m}_{\text {- }}$ & 195. 70 & Oct. 29 & 194.97 & Jan. 9 & 202.17 \\
\hline Aug. $17,5: 10$ p. m & $\begin{array}{ll}5 & 192.32\end{array}$ & Oct. 30 & 194.94 & Jan. 10 & 201.97 \\
\hline Aug. $18,7: 45$ a. m- & 195. 70 & Oet. 31 . & 194.91 & Jan. 11 & 201. 53 \\
\hline Aug. $18,5: 15$ p. m & $\begin{array}{l}5192.27 \\
5\end{array}$ & Nov. 1 & 194.91 & Jan. 12. & 201.77 \\
\hline Aug. 19 & 195. 76 & Nov. 2 & 194.92 & Jan. 13 & 200.99 \\
\hline Aus & 195.71 & Nov. 3 & 194.92 & Jan. 14 & 201. 01 \\
\hline Aug. 2 & 195.69 & Nov. 4 & 194.92 & Jan. 15 & 200.61 \\
\hline
\end{tabular}

${ }^{5}$ Pump operating in well. 
TABLE 13.-Ground-water levels in observation wells, 1928-30 and 1935-36-Con.

\begin{tabular}{|c|c|c|c|c|c|}
\hline Date & $\begin{array}{l}\text { Water } \\
\text { level } \\
\text { (feet) }\end{array}$ & Date & $\begin{array}{l}\text { Water } \\
\text { level } \\
\text { (feet) }\end{array}$ & Date & $\begin{array}{l}\text { Water } \\
\text { level } \\
\text { (feet) }\end{array}$ \\
\hline 1930 & & 1930 & & 1930 & \\
\hline 16. & 200.57 & Apr. 1 & 199.42 & June 14, 8:05 a. m....... & 197. \\
\hline Jan. 17 . & 200.53 & -....... & 32 & m............. & 5192.2 \\
\hline Jan. 18 & 200.27 & & 199.22 & e 15 & 197.8 \\
\hline & 200.19 & - & 199.12 & June 16, 8:1 & $\begin{array}{r}197.7 \\
5192.2\end{array}$ \\
\hline 20 . & 200.15 & $5 \ldots \ldots+\ldots$ & 199.07 & June $16,4: 30$ p. m...... & $\begin{array}{r}6192.2 \\
197.5\end{array}$ \\
\hline & 200.08 & $6 \ldots$ & 199.02 & June 17 & $\begin{array}{l}197.5 \\
197.5\end{array}$ \\
\hline$\cdots$ & 91 & 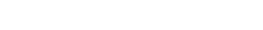 & 02 & June $18,7:$ & $\begin{aligned} & 197.5 \\
& 193.1\end{aligned}$ \\
\hline 23 & 89 & 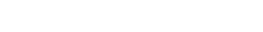 & $\begin{array}{l}97 \\
77\end{array}$ & p. m..... & $\begin{array}{r}\text { 5 } 193.1 \\
197.4\end{array}$ \\
\hline-- & .77 & 10 & $\begin{array}{l}198.77 \\
198.82\end{array}$ & a. $\mathrm{m} \ldots \ldots$ & 519 \\
\hline$\cdots-$ & 69 & 11 & $\begin{array}{l}198.82 \\
198.72\end{array}$ & a. m............ & 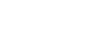 \\
\hline $\begin{array}{l}\text { Jan. } 26 \\
\text { Jan. } 27\end{array}$ & $\begin{array}{l}57 \\
49\end{array}$ & Apr. 12 & 62 & $\begin{array}{l}\text { June } 20,7: 05 \text { a. } \mathrm{m} \\
\text { June } 20,12 \mathrm{~m}\end{array}$ & 5192.9 \\
\hline Jan. 28 & 199.39 & Apr. 13 & 62 & 21 & 197.1 \\
\hline 1. 29. & 199.27 & $-\ldots-1$ & 198.47 & 22 & 197.1 \\
\hline-- & 199.67 & 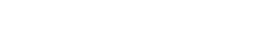 & 198.41 & June $23,7: 40$ a. $\mathrm{m}$. & 197.0 \\
\hline 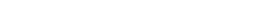 & .93 & 16 & 42 & June 23,5 p. m & 5192.2 \\
\hline$: 45$ p. m. & .87 & r. $17 \ldots$ & 198.47 & June 24 & 196.9 \\
\hline p. $m_{\ldots} \ldots$ & 82 & Apr. $18 \ldots$ & 198.42 & June 25. & 196.8 \\
\hline -.......... & .77 & Apr. 19 & 198.52 & June 26 & 196.8 \\
\hline Feb. 3 & .71 & Apr. 20 & 198. 57 & June 27. & 196.8 \\
\hline Feb. 4 & 203.51 & Apr. 21 & 198.37 & June 28. & 196.7 \\
\hline Feb. 5 & 203.57 & Apr. 22 & 27 & Jun & 196.7 \\
\hline .6 & 203.67 & .23 & 37 & June 30 & 196. 6 \\
\hline $.7--$ & 203.81 & 24 & .52 & & \\
\hline 8 & 203.87 & 25 & 52 & 1935 & \\
\hline 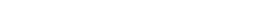 & 204.13 & $26,1: 25 \mathrm{p}, \mathrm{m}$ & 53 & $-\ldots \ldots-\ldots$ & 195. 2 \\
\hline$-n-\cdots$ & .37 & p. m..... & 47 & Oct. 12 & 195. \\
\hline 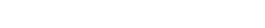 & 2 & Apr. 27 & 42 & Oet. 18 & 195. \\
\hline 12 & 20 & 28 & 42 & Oct. 21 & 195.4 \\
\hline 13 & 20 & 29. & 62 & Oct. 30 & 195. \\
\hline 14 & 20 & .30 & 67 & Nov. 7 & 19 \\
\hline 15. & 205. 6 & y 1 . & 82 & v. 13 & 18 \\
\hline$\ldots \ldots$ & 20 & ay 2 & 77 & Nov. 20 & 19 \\
\hline$\ldots \ldots \ldots+\ldots$ & 20 & ay $3 \ldots$ & 77 & $-\therefore--.-1--.-1$ & 19 \\
\hline 18 & 20 & y $4 \ldots \ldots+\ldots$ & .72 & $\ldots \ldots \ldots$ & 195.1 \\
\hline+19 & 20 & $5 \ldots$ & 72 & 11 & 19 \\
\hline$\ldots$ & 20 & $6 \ldots \ldots$ & 18 & Dec. 18 & 19 \\
\hline 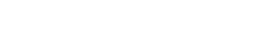 & 20 & $7-$ & 18 & Dec. $26 \ldots$ & 197.4 \\
\hline 3. & $\begin{array}{l}20 \\
20\end{array}$ & $\begin{array}{l}8 \\
9\end{array}$ & & 1936 & \\
\hline 23 & $\begin{array}{l}20 \\
20\end{array}$ & 10 & & Jan. 2 & \\
\hline $\begin{array}{l}24 \\
25\end{array}$ & 20 & 11. & & $\begin{array}{l}\text { Jan. } 2 \\
\text { Jan. } 9\end{array}$ & \\
\hline $\begin{array}{l}25 \\
26\end{array}$ & 20 & 12 & & Jan. 15 & 20 \\
\hline $\begin{array}{l}26 \\
27 \\
27\end{array}$ & 204.6 & 13 & & Jan. 22 & 201. \\
\hline .28 & 204.4 & 14 & 18 & Jan. 29 & $\begin{array}{l}2 \\
2\end{array}$ \\
\hline $45 \mathrm{p}, \mathrm{m}$ & 204.17 & 15 & $\begin{array}{l}\text { 198. } 32 \\
\text { 198. } 32\end{array}$ & Feb. 5 & 201. \\
\hline $1,12: 50$ p. m. & 204.04 & 16. & 198.27 & Feb. 12 & 200. \\
\hline a & $2 \cap 3.67$ & 17 & 19 & Feb. 21 & 200.8 \\
\hline . 3- & 203.57 & ay 18 . & 19 & Feb. 26 & 203. \\
\hline$\cdots$ & 203.47 & ay 19. & 19 & Mar. 4 & 204.6 \\
\hline$\ldots-\ldots$ & 87 & 20 & 19 & 11 & $204 . \mathrm{C}$ \\
\hline$\ldots$ & .37 & 21. & & .18 & 201.6 \\
\hline - & 202.17 & & 18 & Mar. 25 & 200.6 \\
\hline 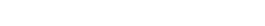 & 201.92 & 33. & 19 & T. 1 & 2004 \\
\hline 9 & f.7 & 24. & 19 & 8 & 201.2 \\
\hline M & 20 & 25 & 19 & . & 202. \\
\hline & 20 & 26 & 19 & .22 & 200. \\
\hline - & 201.07 & 1 & & 29 & 200. \\
\hline - n- & 20 & ay $28,11: 35$ a. $\mathrm{m}$ & 19 & May 6 & 200. \\
\hline$-\ldots$ & 200.77 & Lay $28,5: 35 \mathrm{p}, \mathrm{m}_{\ldots} \ldots$ & 19 & May 13 & 200. \\
\hline 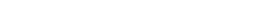 & 20 & ay $29 \ldots$ & & May 20 & 200. \\
\hline 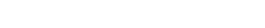 & 20 & 30 & & 27 & 20 \\
\hline$\ldots$ & 20 & 31 & & J & 199. \\
\hline$\ldots$. & 20 & 1. & & 10 & 199. \\
\hline $\mathrm{M}$ & 19 & 2. & & - n & \\
\hline $\mathrm{M}$ & 199. & 烈 & & $\ldots \ldots$ & \\
\hline $\mathbf{M}$ & 199.67 & 1 & & & 197 \\
\hline & 19 & & 19 & 15 & 4192. \\
\hline 23 & 199. 72 & 6 & 11 & July 22 & 4190. \\
\hline-- & 200.02 & e 7 & 198. 09 & Aug. 5 & 196. 2 \\
\hline$-\ldots$ & 200.17 & $\mathrm{~J}$ & 19 & Aug. $12 \ldots$ & 195. \\
\hline$--\ldots+n-1$ & 17 & 3 & & 19 & 192. \\
\hline 27 - & & & & 2 & 7180. \\
\hline Mar. 28 & 19 & 9 a. $\mathrm{m}$ & & t. 10 & 7178 \\
\hline$M$ & & $6 \mathrm{p} . \mathrm{m}_{\ldots}$ & 5 19 & & \\
\hline 30 & & $J 1$ & 19 & $S$ & 519 \\
\hline Mar. $31 \ldots$ & 199.57 & June 13 & 197.97 & 30 & 418 \\
\hline
\end{tabular}

Water level depressed by inordinately large withdrawals.

5 Pump operating in well.

7 Adjacent land being irrigated. 
TABLE 13.-Ground-water levels in observation wells, 1928-30 and 1935-36 1-Con.

472. Hector Brothers estate, G. H. Hector, director. NE1/4SW1/4 sec. 4, T $11 \mathrm{~S} .$, R. 4 W. Unused well, dug and driven, $3 \frac{1}{2}$ feet in diameter to depth of 31 feet with brick curb; total depth 84 feet. Measuring point is top of 3-inch plank deck, south of pump, 0.8 foot above land surface and 224.83 feet $^{2}$ above mean sea level.

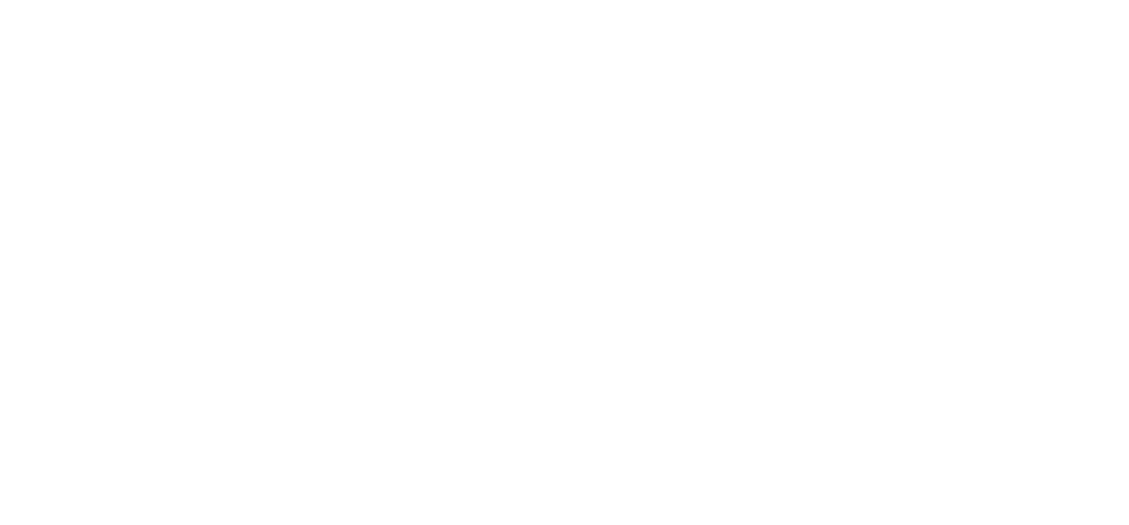

${ }^{2}$ Leveling by U. S. Engineer Department.

5 Pump operating in well.

486. Linn County Farm. SW1/4SW1/4 sec. 26 , T. 11 S., R. 4 W. Stock well, dug 6 feet in diameter and 27.3 feet deep, brick curb, in lot 75 feet south of barn. Copper nail with washer stamped 11-4-8 in well platform. Measuring point is top of 2 -inch plank deck at copper nail with washer, 0.6 foot above land surface and 221.83 feet $^{2}$ above mean sea level.

\begin{tabular}{|c|c|c|c|c|c|c|c|}
\hline Date & $\begin{array}{l}\text { Water } \\
\text { level } \\
\text { (feet) }\end{array}$ & Date & $\begin{array}{l}\text { Water } \\
\text { level } \\
\text { (feet) }\end{array}$ & Date & $\begin{array}{l}\text { Water } \\
\text { level } \\
\text { (feet) }\end{array}$ & Date & $\begin{array}{l}\text { Water } \\
\text { level } \\
\text { (feet) }\end{array}$ \\
\hline 1928 & & 1935 & & 1936 & & 1936 & \\
\hline $\begin{array}{l}\text { July } 28 \\
\qquad 1929 \\
\text { June } 6 \\
\text { July } 4 \\
\text { Aug. } 2 \\
\text { Sept. } 23 \\
\text { Oct. } 25 \\
\text { Nov. } 27 \\
\text { Dec. } 31 \\
\qquad 1930 \\
\text { Feb. } 1 \\
\text { Mar. } 1 \\
\text { Mar. } 29 \\
\text { Apr. } 26 \\
\text { May } 28 \\
\text { July } 23\end{array}$ & $\begin{array}{l} \\
215.03 \\
210.43 \\
204.7 \\
201.9 \\
200.73 \\
200.53 \\
219.38 \\
\\
\\
217.23 \\
220.08 \\
216.38 \\
216.88 \\
215.31 \\
209.82\end{array}$ & $\begin{array}{l}\text { Oct. } 4 \\
\text { Oct. } 11 \\
\text { Oct. } 17 \\
\text { Oct. } 30 \\
\text { Nov. } 6 \\
\text { Nov. } 13 \\
\text { Nov. } 20 \\
\text { Dec. } 4 \\
\text { Dec. } 11 \\
\text { Dec. } 18 \\
\text { Dec. } 26 \\
\\
\\
\text { Jan. } 19 \\
\text { Jan. } 8 \\
\text { Jan. } 15 \\
\text { Jan. } 22 \\
\text { Jan. } 29 \\
\text { Feb. } 5\end{array}$ & $\begin{array}{l}200.37 \\
200.76 \\
202.18 \\
201.99 \\
199.45 \\
200.61 \\
200.75 \\
201.46 \\
202.59 \\
203.28 \\
202.59 \\
\\
\\
204.42 \\
216.16 \\
220.14 \\
218.15 \\
216.93 \\
221.83\end{array}$ & $\begin{array}{l}\text { Feb. 12 } \\
\text { Feb. 20 } \\
\text { Feb. 26 } \\
\text { Mar. } 4 \\
\text { Mar. 11 } \\
\text { Mar. } 18 \\
\text { Mar. } 24 \\
\text { Apr. } 1 \\
\text { Apr. } 8 \\
\text { Apr. } 15 \\
\text { Apr. } 22 \\
\text { May } 6 \\
\text { May } 13 \\
\text { May } 19 \\
\text { May } 27 \\
\text { June } 3 \\
\text { June } 10 \\
\text { June } 17 \\
\text { June } 24 \\
\text { July } 1 .\end{array}$ & $\begin{array}{l}215.17 \\
212.34 \\
215.96 \\
220.21 \\
218.90 \\
218.40 \\
214.20 \\
220.01 \\
220.04 \\
216.11 \\
212.65 \\
220.06 \\
217.87 \\
218.90 \\
213.23 \\
215.30 \\
215.06 \\
214.44 \\
213.19 \\
209.39\end{array}$ & $\begin{array}{l}\text { July } 10 \\
\text { July } 15 \\
\text { July } 22 \\
\text { July } 29 \\
\text { Aug. } 5 \\
\text { Aug. } 12 \\
\text { Aug. } 19 \\
\text { Aug. } 26 \\
\text { Sept. } 2 \\
\text { Sept. } 10 \\
\text { Sept. } 16 \\
\text { Sept. } 23 \\
\text { Sept. } 30\end{array}$ & $\begin{array}{r}206.81 \\
206.03 \\
203.34 \\
202.48 \\
200.77 \\
5196.41 \\
201.78 \\
200.99 \\
200.79 \\
200.69 \\
200.63 \\
200.29 \\
200.02\end{array}$ \\
\hline
\end{tabular}

2 Leveling by U. S. Engineer Department.

- Pump operating in well.

491. O. H. Ehrlich. SW1/4SW1/4 sec. 35, T. 11 S., R. 4 W. Stock well, driven $11 / 4$ inches in diameter and 31 feet deep, in barnyard 150 feet south of residence. Copper nail with washer stamped 11-4-15 in plank level with land surface. Measuring point is top of coupling between casing and pump, 2.9 feet above land surface and 227.9 feet above mean sea level (interpolated).

\begin{tabular}{|c|c|c|c|c|c|}
\hline Date & $\begin{array}{l}\text { Water } \\
\text { level } \\
\text { (feet) }\end{array}$ & Date & $\begin{array}{l}\text { Water } \\
\text { level } \\
\text { (feet) }\end{array}$ & Date & $\begin{array}{l}\text { Water } \\
\text { level } \\
\text { (feet) }\end{array}$ \\
\hline \begin{tabular}{l}
\multicolumn{1}{c}{1928} \\
Aug. 13 \\
Oct. 21 \\
Dec. 16
\end{tabular} & $\begin{array}{l}208.0 \\
207.3 \\
213.8\end{array}$ & $\begin{array}{l}\text { Feb. } 2 \\
\text { Mar. 27 }\end{array}$ & $\begin{array}{l}216.2 \\
214.8\end{array}$ & \begin{tabular}{|c} 
\\
May 9 \\
June 6
\end{tabular} & $\begin{array}{l}214.5 \\
211.7\end{array}$ \\
\hline
\end{tabular}


TABLE 13.-Ground-water levels in observation wells, 1928-30 and 1935-36-Con.

492. MeFarland School. SE1/4NE1/4 sec. 36, T. 11 S., R. 4 W. Domestic well, driven $11 / 2$ inches in diameter and 26 feet deep. Measuring points: In 1928, concrete pump platform, level with land surface and about 238.9 feet above mean sea level; in 1935-36, top of pump-gasket flange, 2.7 feet above land surface and 239.62 feet 2 above mean sea level.

\begin{tabular}{|c|c|c|c|c|c|c|c|}
\hline Date & $\begin{array}{l}\text { Water } \\
\text { level } \\
\text { (feet) }\end{array}$ & Date & $\begin{array}{l}\text { Water } \\
\text { level } \\
\text { (feet) }\end{array}$ & Date & $\begin{array}{l}\text { Water } \\
\text { level } \\
\text { (feet) }\end{array}$ & Date & $\begin{array}{l}\text { Water } \\
\text { level } \\
\text { (feet) }\end{array}$ \\
\hline 1928 & \multirow{3}{*}{225.9} & 1936 & & 1936 & \multirow{8}{*}{$\begin{array}{l}230.00 \\
229.85 \\
229.44 \\
228.37 \\
228.32 \\
226.84 \\
226.30 \\
225.97 \\
225.76\end{array}$} & 1936 & \multirow{8}{*}{$\begin{array}{l}224.15 \\
224.00 \\
223.17 \\
222.84 \\
222.74 \\
222.60 \\
222.70 \\
222.25 \\
222.03\end{array}$} \\
\hline Aug. 2 & & Apr. 1 . & 233. 29 & June 3 & & Aug. 5 & \\
\hline 1936 & & $\begin{array}{l}\text { Apr. } 8 \\
\text { Apr. } 15\end{array}$ & $\begin{array}{l}233.17 \\
231.80\end{array}$ & $\begin{array}{l}\text { June } 10 \\
\text { June } 17\end{array}$ & & $\begin{array}{l}\text { Aug. } 12 \\
\text { Aug. } 19\end{array}$ & \\
\hline Feb 27 & 23322 & Apr. 22 & 232.05 & June 24 & & Aug. 26 & \\
\hline Mar. 4 & $\begin{array}{l}235.22 \\
233.19\end{array}$ & Apr. 29 & $\begin{array}{l}231.99 \\
231.88\end{array}$ & $\begin{array}{l}\text { July } 1 \\
\text { July } 10--\end{array}$ & & $\begin{array}{l}\text { Sept. } 2 \\
\text { Sept. } 10\end{array}$ & \\
\hline Mar. 12 & 232.87 & May 13 . & 231.55 & July 15 & & Sept. 16 & \\
\hline Mar. 18 & 232.26 & May 20 & 231. 71 & July 22. & & Sept. 23 & \\
\hline Mar. & 232.68 & May 27 & 231.70 & July 29 & & Sept. 30 & \\
\hline
\end{tabular}

2 Leveling by U. S. Engineer Department.

493. Maude Wells. NE1/4NE1/4 sec. 2, T. 11 S., R. 3 W. Stock well, dug 8 feet deep. Measuring point is top of timber cover, through bored hole, 0.8 foot above land surface and 233.35 feet 2 above mean sea level.

\begin{tabular}{|c|c|c|c|c|c|c|c|}
\hline Date & $\begin{array}{l}\text { Water } \\
\text { level } \\
\text { (feet) }\end{array}$ & Date & $\begin{array}{l}\text { Water } \\
\text { level } \\
\text { (feet) }\end{array}$ & Date & $\begin{array}{l}\text { Water } \\
\text { level } \\
\text { (feet) }\end{array}$ & Date & $\begin{array}{l}\text { Water } \\
\text { level } \\
\text { (feet) }\end{array}$ \\
\hline 1935 & & $193 \hat{6}$ & & 1936 & & 1936 & \\
\hline $\begin{array}{l}\text { Oct. } 11 \\
\text { Oct. } 17 \\
\text { Oct. } 30 \\
\text { Nov. } 6 \\
\text { Nov. } 13 \\
\text { Nov. } 20 \\
\text { Dec. } 4 \\
\text { Dec. } 10 \\
\text { Dec. } 18 \\
\text { Dec. } 26 \\
\text { Dec. } 28 \\
\qquad 1936 \\
\text { Jan. } 1 \\
\text { Jan. } 3\end{array}$ & $\begin{array}{l}227.05 \\
226.28 \\
227.32 \\
227.07 \\
227.73 \\
227.87 \\
227.96 \\
228.97 \\
229.05 \\
231.18 \\
231.68\end{array}$ & $\begin{array}{l}\text { Jan. } 7 \\
\text { Jan. 10 } \\
\text { Jan. 14 } \\
\text { Jan. 21 } \\
\text { Jan. 24 } \\
\text { Jan. 28 } \\
\text { Jan. } 31 \\
\text { Feb. } 4 \\
\text { Feb. } 12 \\
\text { Feb. 20 } \\
\text { Feb. 25 } \\
\text { Mar. } 4 \\
\text { Mar. 10 } \\
\text { Mar. 18 } \\
\text { Mar. 25 } \\
\text { Mar. 31 }\end{array}$ & $\begin{array}{l}232.47 \\
232.57 \\
232.47 \\
232.10 \\
232.20 \\
231.87 \\
231.37 \\
232.14 \\
232.33 \\
231.57 \\
232.54 \\
232.05 \\
231.70 \\
232.10 \\
231.93 \\
231.97\end{array}$ & $\begin{array}{l}\text { Apr. } 7 \\
\text { Apr. } 14 \\
\text { Apr. 22 } \\
\text { Apr. 28 } \\
\text { May } 5 \\
\text { May } 12 \\
\text { May } 19 \\
\text { May } 26 \\
\text { June } 2 \\
\text { June } 9 \\
\text { June } 16 \\
\text { June } 23 \\
\text { June } 30 \\
\text { July } 9 \\
\text { July } 14 \\
\text { July } 21 \\
\end{array}$ & $\begin{array}{l}232.45 \\
231.64 \\
231.53 \\
231.65 \\
231.97 \\
231.08 \\
231.88 \\
231.85 \\
231.33 \\
231.22 \\
231.16 \\
230.61 \\
229.78 \\
229.38 \\
229.56 \\
228.97\end{array}$ & $\begin{array}{l}\text { July } 28 \\
\text { Aug. } 4 \\
\text { Aug. } 11 \\
\text { Aug. 18 } \\
\text { Aug. 25 } \\
\text { Sept. 1 } \\
\text { Sept. } 9 \\
\text { Sept. } 15 \\
\text { Sept. 22 } \\
\text { Sept. } 29\end{array}$ & $\begin{array}{l}228.57 \\
228.33 \\
228.21 \\
227.90 \\
227.85 \\
227.94 \\
227.82 \\
227.80 \\
227.42 \\
227.07\end{array}$ \\
\hline
\end{tabular}

2 Leveling by U. S. Engineer Department.

502. Sophie Wilson. SE $1 / 4$ SW $1 / 4$ sec. 7, T. 11 S., R. 3 W. Unused well, dug 24 feet deep, backfilled around 10-inch concrete-tile casing. Measuring point is top of cover on box curb, beside draft pipe, 2.9 feet above land surface and 227.90 feet 2 above mean sea level.

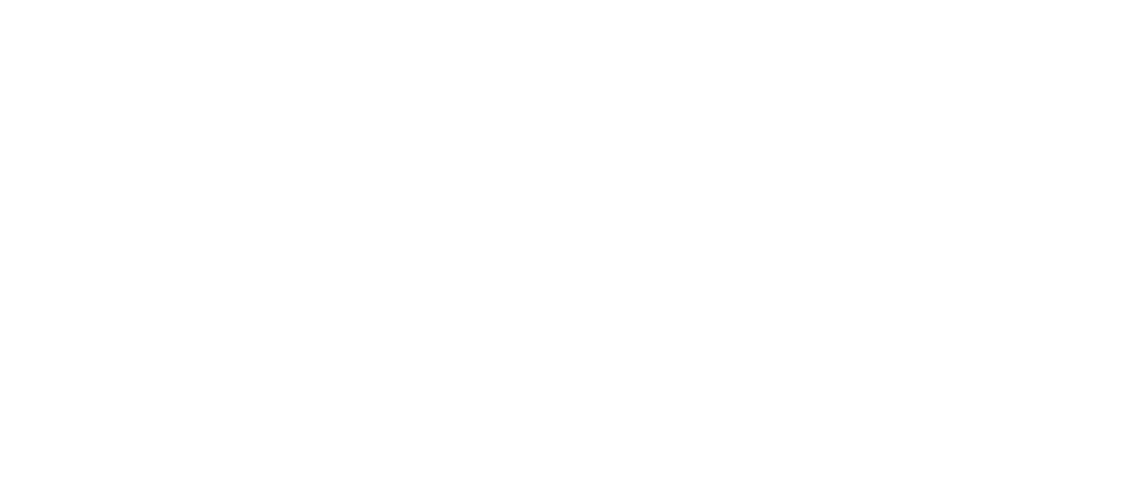

Leveling by U. S. Engineer Department. 
TABLE 13.-Ground-water levels in observation wells, 1928-30 and 1995-36-Con.

513. Leslie Cade. NE1/4NW 1/4 sec. 23, T. $11 \mathrm{~S} ., \mathrm{R}, 3 \mathrm{~W}$. Domestic well, dug 4 feet square and 22 feet deep, brick curb. Measuring point is top of brick curb, at north side, 258.81 feet $^{2}$ above mean sea level.

\begin{tabular}{|c|c|c|c|c|c|c|c|}
\hline Date & $\begin{array}{l}\text { Water } \\
\text { level } \\
\text { (feet) }\end{array}$ & Date & $\begin{array}{l}\text { Water } \\
\text { level } \\
\text { (feet) }\end{array}$ & Date & $\begin{array}{l}\text { Water } \\
\text { level } \\
\text { (feet) }\end{array}$ & Date & $\begin{array}{l}\text { Water } \\
\text { level } \\
\text { (feet) }\end{array}$ \\
\hline 1935 & & 1936 & & 1936 & & 1936 & \\
\hline $\begin{array}{l}\text { Oct. } 11 \\
\text { Oct. 17 } \\
\text { Oct. } 30 \\
\text { Nov. } 6 \\
\text { Nov. } 13 \\
\text { Nov. } 20 \\
\text { Dec. } 4 \\
\text { Dec. } 11 \\
\text { Dec. } 18 \\
\text { Dec. } 26 \\
\qquad 1936 \\
\text { Jan. } 1 \\
\text { Jan. } 8\end{array}$ & $\begin{array}{l}241.92 \\
241.99 \\
241.90 \\
242.01 \\
242.13 \\
242.28 \\
242.17 \\
242.74 \\
246.57 \\
247.47\end{array}$ & $\begin{array}{l}\text { Jan. } 15 \\
\text { Jan. 22 } \\
\text { Jan. 29 } \\
\text { Feb. } 5 \\
\text { Feb. } 12 \\
\text { Feb. 20 } \\
\text { Feb. } 26 \\
\text { Mar. } 4 \\
\text { Mar. } 11 \\
\text { Mar. } 18 \\
\text { Mar. 25 } \\
\text { Apr. } 1 \\
\text { Apr. } 8 \\
\text { Apr. } 15 \\
\text { Apr. 22 }\end{array}$ & $\begin{array}{l}255.52 \\
255.09 \\
254.84 \\
254.03 \\
255.07 \\
254.66 \\
255.35 \\
254.94 \\
254.74 \\
254.36 \\
254.19 \\
254.93 \\
255.04 \\
254.51 \\
254.20\end{array}$ & $\begin{array}{l}\text { Apr. } 29 \\
\text { May } 6 \\
\text { May } 13 \\
\text { May } 20 \\
\text { May } 27 \\
\text { June } 3 \\
\text { June } 10 \\
\text { June } 17 \\
\text { June } 24 \\
\text { July } 1 \\
\text { July } 10 \\
\text { July } 15 \\
\text { July } 22 \\
\text { July } 29 \\
\text { Aug. } 5 \\
\end{array}$ & $\begin{array}{l}253.57 \\
254.88 \\
253.84 \\
254.39 \\
253.30 \\
253.35 \\
252.91 \\
252.39 \\
252.21 \\
251.71 \\
251.07 \\
250.49 \\
249.88 \\
249.49 \\
248.43\end{array}$ & $\begin{array}{l}\text { Aug. } 12 \\
\text { Aug. 19 } \\
\text { Aug. } 26 \\
\text { Sept. } 2 \\
\text { Sept. } 10 \\
\text { Sept. } 23 \\
\text { Sept. } 30 \\
\end{array}$ & $\begin{array}{l}248.00 \\
248.00 \\
246.54 \\
246.38 \\
246.19 \\
244.38 \\
244.23\end{array}$ \\
\hline
\end{tabular}

${ }^{2}$ Leveling by U. S. Engineer Department.

518. H. O. Wilson. NW $1 / 4$ NE $1 / 4$ sec. 21, T. $11 \mathrm{~S}$, R. 2 W. Irrigation well, dug 8 feet square and 15.5 feet deep. Measuring points: Top of east 6 -by 8-inch timber pump support, near southeast corner of well, a bout 1 foot above land surface and 283.23 feet ${ }^{2}$ above mean sea level; beginning July 10, 1936, new point 281.88 feet 2 above mean sea level.

\begin{tabular}{|c|c|c|c|c|c|c|c|}
\hline Date & $\begin{array}{l}\text { Water } \\
\text { Ievel } \\
\text { (feet) }\end{array}$ & Date & $\begin{array}{l}\text { Water } \\
\text { level } \\
\text { (feet) }\end{array}$ & Date & $\begin{array}{l}\text { Water } \\
\text { level } \\
\text { (feet) }\end{array}$ & Date & $\begin{array}{l}\text { Water } \\
\text { level } \\
\text { (feet) }\end{array}$ \\
\hline 1935 & & 1936 & & 1936 & & 1936 & \\
\hline $\begin{array}{l}\text { Oct. } 11 \\
\text { Oct. } 17 \\
\text { Oct. } 30 \\
\text { Nov. } 6 \\
\text { Nov. } 13 \\
\text { Nov. } 20 \\
\text { Dec. } 4 \\
\text { Dec. } 11 \\
\text { Dec. } 18 \\
\text { Dec. } 26 \\
\quad 1936 \\
\text { Jan. } 1\end{array}$ & $\begin{array}{l}274.98 \\
275.11 \\
275.08 \\
275.03 \\
275.28 \\
275.45 \\
275.55 \\
275.79 \\
276.38 \\
276.30\end{array}$ & $\begin{array}{l}\text { Jan. } 7 \\
\text { Jan. } 15 \\
\text { Jan. } 22 \\
\text { Jan. } 29 \\
\text { Feb. } 5 \\
\text { Feb. 12 } \\
\text { Feb. 20 } \\
\text { Feb. 26 } \\
\text { Mar. 4 } \\
\text { Mar. 11 } \\
\text { Mar. 18 } \\
\text { Mar. 25 } \\
\text { Apr. 15. } \\
\text { A pr. }\end{array}$ & $\begin{array}{l}277.91 \\
279.28 \\
279.13 \\
277.64 \\
277.25 \\
277.30 \\
277.23 \\
278.01 \\
277.86 \\
277.66 \\
277.28 \\
277.10 \\
277.39 \\
277.39\end{array}$ & $\begin{array}{l}\text { Apr. 15 } \\
\text { Apr. 22 } \\
\text { Apr. 29 } \\
\text { Maay } 6 \\
\text { May 13 } \\
\text { May } 20 \\
\text { May } 27 \\
\text { June } 3 \\
\text { June } 10 \\
\text { June } 17 \\
\text { June } 24 \\
\text { July } 10 \\
\text { July } 15 \\
\text { July } 22 \\
\end{array}$ & $\begin{array}{l}277.23 \\
277.11 \\
276.99 \\
277.69 \\
276.88 \\
276.85 \\
276.88 \\
276.84 \\
276.76 \\
276.67 \\
276.79 \\
276.33 \\
276.30 \\
275.98\end{array}$ & $\begin{array}{l}\text { Jaly } 29 \\
\text { Aug. } 5 \\
\text { Aug. } 12 \\
\text { Aug. } 19 \\
\text { Aug. } 26 \\
\text { Sept. } 2 \\
\text { Sept. } 10 \\
\text { Sept } 16 \\
\text { Sept. } 23\end{array}$ & $\begin{array}{l}275.60 \\
275.88 \\
275.78 \\
275.67 \\
275.63 \\
275.63 \\
275.46 \\
275.48 \\
275.44\end{array}$ \\
\hline
\end{tabular}

Leveling by U.S. Engineer Department.

527. G.W. Cummings. SW1/4SW1/4 sec. 12, T.12 S. R. $6 \mathrm{~W}$. Unused well, driven 3 inches in diameter and 27.8 feet deep. Measuring point is top of 3 -inch casing, 1.5 feet above land surface and 263.86 feet 2 above mean sea level.

\begin{tabular}{|c|c|c|c|c|c|c|c|}
\hline Date & $\begin{array}{l}\text { Water } \\
\text { level } \\
\text { (feet) }\end{array}$ & Date & $\begin{array}{l}\text { Water } \\
\text { level } \\
\text { (feet) }\end{array}$ & Date & $\begin{array}{l}\text { Water } \\
\text { level } \\
\text { (feet) }\end{array}$ & Date & $\begin{array}{l}\text { Water } \\
\text { level } \\
\text { (feet) }\end{array}$ \\
\hline 1935 & & 1936 & & 1936 & & 1936 & \\
\hline Oct. 18 & 254.39 & Jan. 4 & 258.82 & Apr. 8 & 259.59 & July 15 & 255.76 \\
\hline Oct. 23 & 254.35 & Jan. 9 & 256.86 & Apr. 15 & 257.15 & July 22 & 255.44 \\
\hline Oct. 29 & 254.46 & Jan. 15 & 257.64 & Apr. 22. & 257.20 & July 29 & 255. 36 \\
\hline Nov. 7 . & 254.41 & Jan. 21 & 256.95 & Apr. 29 & 256.78 & Aug. 5- & 255. 24 \\
\hline Nov. 13 & 254.41 & Jan. 29 & 256.89 & May 6 & 256.59 & Aug. 12 & 255.03 \\
\hline Nov. 2 & 254.58 & Feb. 5 & 256.0 & May 13 & 256.82 & Aug. 19 & 254.95 \\
\hline Nov. 2 & 254.74 & Feb. 12 & 257.30 & May 20 & 256.73 & Aug. 26. & 254.72 \\
\hline Dec. 11 & 255.25 & Feb. 21 & 257.88 & May 27 & 256.78 & Sept. 2 & 254. 59 \\
\hline Dec. 18 & 254.84 & Feb. 26 & 259.22 & June 3 & 257.57 & Sept. 10 & 254.40 \\
\hline Dec. 26 & 254.88 & Mar. 4 & 257. 49 & June 10 & 256.43 & Sept. 16. & 254.38 \\
\hline 1936 & & $\begin{array}{l}\text { Mar. } 11 \\
\text { Mar. } 18\end{array}$ & $\begin{array}{l}257.54 \\
257.38\end{array}$ & $\begin{array}{l}\text { June 17 - } \\
\text { June 24.- }\end{array}$ & $\begin{array}{l}256.22 \\
256.13\end{array}$ & $\begin{array}{l}\text { Sept. } 23 \\
\text { Sept. } 30\end{array}$ & $\begin{array}{l}254.35 \\
254.23\end{array}$ \\
\hline & & Mar. 25. & 257.17 & July $1 \ldots$ & 256.03 & & \\
\hline Jan. $2 \ldots$ & 255.62 & Apr. 1.. & 259.57 & July 8. & 255.92 & & \\
\hline
\end{tabular}

${ }^{2}$ Leveling by U. S. Engineer Department. 
TABLE 13.-Ground-water levels in observation wells, 1928-30 and 1935-36-Con-

528. Independence School, district 18. NW1/4SE1/4 sec. 25, T. 12 S., R. 6 W. Domestic well, dug 31.2 feet deep, backfilled around 6 -inch conrete-tile casing. Measuring point is top of casing, 0.5 foot above land surface and 314.36 feet ${ }^{2}$ a bove mean sea level.

\begin{tabular}{|c|c|c|c|c|c|c|c|}
\hline Date & $\begin{array}{l}\text { Water } \\
\text { level } \\
\text { (feet) }\end{array}$ & Date & $\begin{array}{l}\text { Water } \\
\text { level } \\
\text { (feet) }\end{array}$ & Date & $\begin{array}{l}\text { Water } \\
\text { level } \\
\text { (feet) }\end{array}$ & Date & $\begin{array}{l}\text { Water } \\
\text { level } \\
\text { (feet) }\end{array}$ \\
\hline 1935 & & 1936 & & 1936 & & 1936 & \\
\hline Oct. 18 & 298.17 & Jan. 9 & 303.89 & Apr. 16 & 309.64 & July 22 . & 302.63 \\
\hline Oct. 23 & 298. 75 & Jan. 15 & 312.74 & Apr. 22 & 309.59 & July 29 & 301.64 \\
\hline Oct. 29 & 298.31 & Jan. 16 & 311.09 & Apr. 29 & 307.60 & Aug. 5 & 301.15 \\
\hline Nov. 7 - & 298. 50 & Jan. 29 & 308.72 & May 6 & 306.90 & Aug. 12 & 301.06 \\
\hline Nov. 13 & 298.89 & Feb. 5 & 308.05 & May 13. & 306.51 & Aug. 19. & 301.23 \\
\hline Nov. 20 & 299.04 & Feb. 12 & 308.43 & May 20. & 306.07 & Aug. 26 . & 300.88 \\
\hline Dec. 4 & 299.38 & Feb. 21 & 309. 30 & May 28 & 306.16 & Sept. 2 & 300.74 \\
\hline Dec. 11 & 299.27 & Feb. 26 & 310.17 & June 3 .. & 307.48 & Sept. 10 & 300.43 \\
\hline Dec. 18 & 300.73 & Mar. 4 & 311.55 & June 10 & 304.52 & Sept. 16 & 300.47 \\
\hline Dec. 26 & 300.67 & Mar. 11 & 312.18 & June 17 & 304.07 & Sept. 23 & 299.12 \\
\hline 1936 & & Mar. 18 & 309. 28 & June 24 & 303.99 & Sept. $30 \ldots$ & 298.99 \\
\hline 1930 & & Mar. ${ }^{25}$ & 309.73 & July 1 - & 303.14 & & \\
\hline Jan. 2 & 302.27 & Apr. 8 & 311. 30 & July 15 & $\begin{array}{l}505.00 \\
302.85\end{array}$ & & \\
\hline
\end{tabular}

ILeveling by U. S. Engineer Department.

540. Roy Rickard. NW1/4NW1/4 sec. 26, T. 12 S., R. 5 W. Domestic well, driven 11/4 inches in diameter and 32 feet deep. Measuring points; Top of 11/4-inch casing (after unscrewing cap), about 2 feet below surface and 236.05 feet 2 above mean sea level: beginning March 11, 1936, top of standpipe, level with land surface and 237.82 feet 2 above mean sea level.

\begin{tabular}{|c|c|c|c|c|c|c|c|}
\hline Date & $\begin{array}{l}\text { Water } \\
\text { level } \\
\text { (feet) }\end{array}$ & Date & $\begin{array}{l}\text { Water } \\
\text { level } \\
\text { (feet) }\end{array}$ & Date & $\begin{array}{l}\text { Water } \\
\text { level } \\
\text { (feet) }\end{array}$ & Date & $\begin{array}{l}\text { Water } \\
\text { level } \\
\text { (feet) }\end{array}$ \\
\hline 1928 & & 1935 & & 1936 & & 1936 & \\
\hline & & & & Feb. 26 & 217.23 & June 3.- & 211.51 \\
\hline Ang. 14 & 210.4 & Dec. 26. & 208. 39 & Mar. 11 & 217.80 & June 10 & 213. 39 \\
\hline 1935 & & . $\quad 1936$ & & Ma & $\begin{array}{l}215.25 \\
211.36\end{array}$ & June 24 . & $\begin{array}{l}214.43 \\
213.31\end{array}$ \\
\hline Oct 18 & 208.55 & $\operatorname{Tan} 2$ & 210.62 & Apr. 1 & $\begin{array}{l}212.32 \\
212.38\end{array}$ & July $1 \ldots$ & $\begin{array}{l}212.60 \\
212.43\end{array}$ \\
\hline Oct. 23 & $\begin{array}{l}200.05 \\
208.53\end{array}$ & Jan. 9 & 212.91 & Apr. 15 & 212.15 & July 15 & $\begin{array}{r}212.40 \\
5210.75\end{array}$ \\
\hline Oct. 28 & 208. 35 & Jan. 15 & 228.62 & Apr. 22 & 212.01 & July 22. & 212.14 \\
\hline Nov. 7 & 208. 50 & Jan. 22 & 221.61 & Apr. 29 & 212.23 & Aug. 5 & 208.90 \\
\hline No & 207.65 & Jan. 29 & 212.46 & May 6. & 210.98 & Aug. 12 & 208.85 \\
\hline & 208.40 & Feb. 5 & 212.24 & May 13 & 211.71 & Aug. 19 & 208.83 \\
\hline Dec. 11 & 215.14 & Feb. 12 & 212.86 & May 20 & 211.30 & Sept. 2 & 210.21 \\
\hline De & 209.41 & Feb. 2 & 214.54 & May 27 & 211.39 & Sept. 16 & 209.86 \\
\hline
\end{tabular}

${ }^{2}$ Leveling by U. S. Fingineer Department.

5 Pump operating in well.

548. Trip \& Murphy. NW1/4NE1/4 sec. 22, T. 12 s., R. 4 W. Unused well, driven $11 / 2$ inches in diameter and 27 feet deep. Measuring point is lower valve seat of pump, 3.8 feet above land surface and 244.37 feet? above mean sea level.

\begin{tabular}{|c|c|c|c|c|c|c|c|}
\hline Date & $\begin{array}{l}\text { Water } \\
\text { level } \\
\text { (feet) }\end{array}$ & Date & $\begin{array}{l}\text { Water } \\
\text { level } \\
\text { (feet) }\end{array}$ & Date & $\begin{array}{l}\text { Water } \\
\text { level } \\
\text { (feet) }\end{array}$ & Date & $\begin{array}{l}\text { Water } \\
\text { level } \\
\text { (feet) }\end{array}$ \\
\hline 1935 & & 1936 & & 1936 & & 1936 & \\
\hline Oct. 15. & 227.47 & Jan. 8 & 233.35 & Apr. 22 & 236.64 & July 29 & 232.08 \\
\hline Oct. 22 . & 227.35 & Jan. 22 & $\begin{array}{l}205.50 \\
235.19\end{array}$ & Apr. 29 & $\begin{array}{l}230.04 \\
236.26\end{array}$ & Aug. 5 & 231.52 \\
\hline Oct. 30 & 227.37 & Jan. 29 & 233.13 & May 6 & 235.76 & Aug. 12 & 231. 32 \\
\hline Nov. 6 & 227.57 & Feb. 5 & 232.69 & May 13 & 234.40 & Aug. 19 & 231.72 \\
\hline Nov. 13 & 227.17 & Feb. 12 & 235.88 & May 20. & 235.21 & Aug. 26 . & 230.07 \\
\hline Nov. 20 & 227.22 & Feb. 20 & 234.85 & May 27. & 235. 22 & Sept. 2 & 229.94 \\
\hline Dec. 4 . & 226. 29 & Feb. 26 & 237.69 & June 3. & 234.29 & Sept. 10 & 229.85 \\
\hline Dec. 11 & 227.60 & Mar. 4 & 237.65 & June 10 & 234.60 & Sept. 16 & 230.69 \\
\hline Dec. 18 & 228.89 & Mar. 11 & 236.65 & June 17. & 234.76 & Sept. 23 & 230.50 \\
\hline Dec. 26 & 229.21 & Mar. 18 & 236.68 & June 24 & 233.85 & Sept. 30 & 230.35 \\
\hline 1936 & & $\begin{array}{l}\text { Mar. } 25 \\
\text { A'pr. } 1 .\end{array}$ & $\begin{array}{l}236.58 \\
236.99\end{array}$ & $\begin{array}{l}\text { July } 1 . . \\
\text { July } 10 \ldots\end{array}$ & $\begin{array}{l}233.04 \\
232.68\end{array}$ & & \\
\hline & & Apr. 8 & 237.80 & July 1 & 233.17 & & \\
\hline Jan. 1. & 230.47 & Apr. 15.- & 237.01 & July 22 & 232.53 & & \\
\hline
\end{tabular}

Leveling by U. S. Engineer Department. 
TABLe 13.-Ground-water levels in observation wells, 1928-30 and 1935-36-Con.

553. J. H. Swatzka. SE14SE1/4 sec. 9, T. 12 S., R. 3 W. Domestic well, dug 4 feet in diameter and 18.7 feet deep, brick curb. Measuring points: Top of 2 -inch plank deck, through bored hole, 0.2 foot above land surface and 272.99 feet above mean sea level; September 6,1928 to May 28, 1930, top of instrument shelf, 274.04 feet above mean sea level. Water stage recorder operated on well from September 6,1928 , to May 28 1930.

\begin{tabular}{|c|c|c|c|c|c|c|c|}
\hline Date & $\begin{array}{l}\text { Water } \\
\text { level } \\
\text { (feet) }\end{array}$ & Date & $\begin{array}{l}\text { Water } \\
\text { Ievel } \\
\text { (feet) }\end{array}$ & Date & $\begin{array}{l}\text { Water } \\
\text { level } \\
\text { (feet) }\end{array}$ & Date & $\begin{array}{l}\text { Water } \\
\text { level } \\
\text { (feet) }\end{array}$ \\
\hline 1928 & & 1929 & & 1935 & & 1936 & \\
\hline July 28 & 263.4 & Nov. 27 & 256.49 & Nov. 6 & 256.71 & Apr. 1 & 270.47 \\
\hline Sept. 3 & 260.26 & Dec. 31 & 270.12 & Nov. 12 & 256. 79 & Apr. 8 & 270.31 \\
\hline Sept. 6 & 260.01 & & & Nov. 20 & 256.99 & Apr. 15 & 269.59 \\
\hline Sept. 15 & 259.47 & 1930 & & Dec. 4 & 257.25 & Apr. 22 & 268.95 \\
\hline Oct. 8 & 258.23 & & & Dec. 11 & 258.77 & A pr. 29. & 268.55 \\
\hline Oct. 21 & 257.72 & Jan. 5 & 270.64 & Dec. 18 & 261.86 & May 6 & 268. 61 \\
\hline Dec. 16 & 268.14 & Jan. 11 & 269.99 & Dec. 26 . & 262.72 & May 13 & 268.97 \\
\hline 1929 & & $\begin{array}{l}\text { Jan. } 18 \\
\text { Jan. } 26 .\end{array}$ & $\begin{array}{l}269.34 \\
268.87\end{array}$ & Dec. 28_- & 263.67 & $\begin{array}{l}\text { May } 20 . \\
\text { May } 27 .\end{array}$ & $\begin{array}{l}268.91 \\
268.49\end{array}$ \\
\hline & & Feb. 1 & 271.14 & 1986 & & June 3 & 267.91 \\
\hline Jan. 6 & 270.54 & Feb. 9 & .271 .02 & Jan. 1. & 264.82 & June 10 & 267.52 \\
\hline Jar & 270.84 & Feb. 16 & 270.71 & Jan. 3 & 265.03 & e 17 & 267.19 \\
\hline Feb. 2 & 271.14 & Fet & 271.29 & Jan. 8 & & e 24 & 266.69 \\
\hline Feb. 16. & 269.46 & Mar. 1 & 270.55 & Jan. 10 & 270.99 & July 1 & 266.04 \\
\hline Mar. 7 & 269.69 & Mar. 19 & 269.31 & Jan. 15 & 271.01 & July 10 & 265. 26 \\
\hline Mar. 31 & 269.93 & Mar. 29 & 269.82 & Jan. 22 & 270.47 & July 15 & 264.80 \\
\hline Apr. 18 & 270.55 & Apr. 6 & 269.09 & Jan. 24 & 269.41 & July 22 & 265.25 \\
\hline M & 269.22 & $\mathrm{Ap}$ & 269.71 & Jan. 29 & 270.01 & July 29. & 263.57 \\
\hline May 13 & 267.86 & May 28 & 268.34 & Jan. 31 & 270.37 & Aug. 5 & 262.87 \\
\hline June 3 & 267.38 & July 23 . & 264.23 & Feb. 5 & 269.64 & Aug. 12 & 262. 64 \\
\hline & 265. 92 & & & Feb. 12 & 269.71 & Aug. 19 & 263. 05 \\
\hline Jul & 265.18 & 1935 & & Feb. 20 & 269.00 & Aug. 26 & 260.88 \\
\hline $\mathrm{Au}$ & 263.80 & & & Feb. 26 & 270.03 & Sept. 2 & 260.72 \\
\hline & 260.53 & Oct. & 257. 39 & Mar. 4 & 270.51 & Sept. 10 & 260.84 \\
\hline & 260.25 & Oct. 12 & 257.18 & Mar. 11 & 269.99 & Sept. 16 & 259.19 \\
\hline Sept. 23 & 258. 91 & Oct. 17 & 257.54 & Mar. 18 & 269.79 & Sept. 23.. & 258.74 \\
\hline Oct. 25 & 257. 36 & Oct. 30. & 256.84 & Mar. 25. & 269.35 & Sept. 30 & 258. 62 \\
\hline
\end{tabular}

557. Scott Churchill. SE1/4SW1/4 sec. 34, T. 12 S., R. 3 W. Domestic well, dug 23 feet deep, brick curb. Measuring point is top of 2 -inch plank deck, alongside draft pipe, 1.3 feet above land surface and 268.04 feet $j$ above mean sea level.

\begin{tabular}{|c|c|c|c|c|c|c|c|}
\hline Date & $\begin{array}{l}\text { Water } \\
\text { level } \\
\text { (feet) }\end{array}$ & Date & $\begin{array}{l}\text { Water } \\
\text { level } \\
\text { (feet) }\end{array}$ & Date & $\begin{array}{l}\text { Water } \\
\text { level } \\
\text { (feet) }\end{array}$ & Date & $\begin{array}{l}\text { Water } \\
\text { level } \\
\text { (feet) }\end{array}$ \\
\hline 1935 & & 1936 & & 1936 & & 1936 & \\
\hline Oct. 15 & 248. 47 & Feb. 20 & 264.19 & Apr. 29.. & 262.35 & July 22 & 253.06 \\
\hline 1936 & & & & May 13 & $\begin{array}{l}262.86 \\
263.36\end{array}$ & Aug. 5 & $\begin{array}{l}251.32 \\
251.44\end{array}$ \\
\hline Jan. 8 & 265.31 & Mar. 18 & 263. 74 & May 27 & 5262.40 & Aug. 26 & 251.29 \\
\hline 15 & 265.75 & Mar. 25 & 263. & June 3 & 262 & Sept. 2 & 251.75 \\
\hline Jan. 22 & 265 & A pr. 1 & 264 . & June 10 & 6260 & Sept. 10 & 5250.62 \\
\hline Jan. 29 & 264.66 & $\mathrm{Ap}$ & 264 . & June 17 & 260 . & Sept. 16 & 250.81 \\
\hline Feb. 5 & 264.19 & Apr. 15 & 263.36 & July 10 & 255 & Sept. 23. & 249.36 \\
\hline Feb. 1 & 263.82 & Apr. 22 & 263.22 & July 15 & 255.10 & Sept. 30 & 249.11 \\
\hline
\end{tabular}

2 Leveling by U. S. Engineer Department.

5 Pump operating in well.

- Pump operating in well a short time prior to measurement.

560. Cecil Ensley, renter. SW14NW14 sec. 8, T. 12 S., R. 2 W. Domestic and stock well, drilled 4 inches in diameter and 48,2 feet deep, steel casing. Measuring point is top of steel casing, 0.6 foot above land surface and 330.1 feet 2 above mean sea level.

\begin{tabular}{|c|c|c|c|c|c|c|c|}
\hline Date & $\begin{array}{l}\text { Water } \\
\text { ievei } \\
\text { (feet) }\end{array}$ & Date & $\begin{array}{l}\text { Water } \\
\text { level } \\
\text { (feet) }\end{array}$ & Date & $\begin{array}{l}\text { Water } \\
\text { level } \\
\text { (feet) }\end{array}$ & Date & $\begin{array}{l}\text { Water } \\
\text { level } \\
\text { (feet) }\end{array}$ \\
\hline 1936 & & 1936 & & 1936 & & 1936 & \\
\hline $\begin{array}{l}\text { Apr. } 22 \\
\text { Apr. } 29 . \\
\text { May } 6 \ldots \\
\text { May } 13 \\
\text { May } 20 \\
\text { May } 27 \\
\end{array}$ & $\begin{array}{l}323.50 \\
325.40 \\
325.57 \\
325.68 \\
325.72 \\
325.52\end{array}$ & $\begin{array}{l}\text { June } 3 \ldots \ldots \ldots \\
\text { June } 10 \ldots \ldots \\
\text { June } 17 \ldots \ldots \\
\text { June } 24 \ldots \ldots \\
\text { July } 1 . \ldots \ldots \\
\text { July } 10 \ldots \ldots\end{array}$ & $\begin{array}{l}325.31 \\
325.71 \\
324.96 \\
324.60 \\
323.84 \\
323.22\end{array}$ & $\begin{array}{l}\text { July } 15 \ldots \ldots \ldots \\
\text { July } 22 \ldots \\
\text { July } 29 \\
\text { Aug. } 5 \\
\text { Aug. 12. } \\
\text { Aug. 19.......... }\end{array}$ & $\begin{array}{l}323.76 \\
322.72 \\
323.16 \\
322.96 \\
322.71 \\
323.08\end{array}$ & $\begin{array}{l}\text { Aug. } 26 \\
\text { Sept. } 2 \\
\text { Sept. } 10 \\
\text { Sept. } 16 \\
\text { Sept. } 23 \\
\text { Sept. } 30\end{array}$ & $\begin{array}{l}322.31 \\
322.20 \\
321.97 \\
322.13 \\
32206 \\
321.85\end{array}$ \\
\hline
\end{tabular}

\footnotetext{
${ }^{2}$ Leveling by U. S. Engineer Department.
} 
TABLE 13.-Ground-water levels in observation wells, 1928-30 and 1935-36-Con.

568. Ray Fisher. NE1/4NW1/4 sec. 14, T. $12 \mathrm{~S}$., R. 2 W. Irrigation well, dug 8 feet square and 19 feet deep. Measuring points: Top of pump-house sill at northwest corner of well, marked by copper nail with washer, 352.35 feet 2 above mean sea level; beginning October 4, 1935, top of 6-by-6-inch timber pump support at painted arrow, about 1 foot above land surface and 353.15 feet ${ }^{2}$ above mean sea level.

\begin{tabular}{|c|c|c|c|c|c|c|c|}
\hline Date & $\begin{array}{l}\text { Water } \\
\text { leve] } \\
\text { (feet) }\end{array}$ & Date & $\begin{array}{l}\text { Water } \\
\text { level } \\
\text { (feet) }\end{array}$ & Date & $\begin{array}{l}\text { Water } \\
\text { level } \\
\text { (feet) }\end{array}$ & Date & $\begin{array}{l}\text { Water } \\
\text { level } \\
\text { (feet) }\end{array}$ \\
\hline 1928 & \multirow{5}{*}{$\begin{array}{l}338.4 \\
339.4 \\
340.4\end{array}$} & 1930 & \multirow{7}{*}{$\begin{array}{l}341.85 \\
341.30 \\
340.30 \\
340.55 \\
338.51\end{array}$} & 1936 & \multirow{18}{*}{$\begin{array}{l}339.98 \\
346.09 \\
345.79 \\
343.20 \\
341.62 \\
342.01 \\
341.30 \\
341.47 \\
341.60 \\
341.57 \\
341.00 \\
340.50 \\
340.36 \\
340.38 \\
340.63 \\
340.40 \\
340.34 \\
340.13 \\
340.15 \\
339.23\end{array}$} & 1936 & \multirow{18}{*}{$\begin{array}{r}339.01 \\
340.01 \\
339.63 \\
339.47 \\
339.33 \\
339.33 \\
339.90 \\
338.58 \\
337.91 \\
338.15 \\
337.94 \\
337.81 \\
337.80 \\
337.46 \\
337.41 \\
5336.30 \\
336.42 \\
337.51 \\
337.31\end{array}$} \\
\hline Aug. 17. & & Mar. 1.. & & Jan. 1 . & & \multirow{17}{*}{$\begin{array}{l}\text { May } 20 \\
\text { May } 27 \\
\text { June } 3 \\
\text { June } 10 \\
\text { June } 17 \\
\text { June } 24 \\
\text { July } 9 \\
\text { July } 15 \\
\text { July } 22 \\
\text { July } 29 \\
\text { Aug. } 5 \\
\text { Aug. } 12 \\
\text { Aug. } 19 \\
\text { Aug. } 26 \\
\text { Sept. } 2 \\
\text { Sept. } 10 \\
\text { Sept. } 16 \\
\text { Sept. } 23 \\
\text { Sept. } 30\end{array}$} & \\
\hline Oct. 21 & & Mar. 29 & & Jan. 8 & & & \\
\hline Dec. 16. & & Apr. 26 & & Jan. 15 & & & \\
\hline 1929 & & $\begin{array}{l}\text { May } 28- \\
\text { Juiy } 23 .\end{array}$ & & Jan. 29 & & & \\
\hline Feb. 2 & 340.2 & & & $\begin{array}{l}\text { Feb. } 5 \\
\text { Feb. } 12\end{array}$ & & & \\
\hline Mar. 27 & 341.80 & 1935 & & Feb. 20 & & & \\
\hline $\begin{array}{l}\text { May 9. } \\
\text { June } 6\end{array}$ & 341.35 & & & & & & \\
\hline & 340.45 & Oct. 4 & 336.90 & Mar. 4 & & & \\
\hline & 340.05 & Oct. 12 & 336.89 & Mar. 11 & & & \\
\hline & 338. & Oct. 17 & 337.36 & Mar. 18 & & & \\
\hline 23 & 337.40 & Oct. $30 \ldots$ & 337.70 & Mar. 25 & & & \\
\hline Oct. 25 & 337.60 & Nov. 6 & 337.68 & Apr. 1 & & & \\
\hline 27 & 337.45 & Nov. 13 & 337.92 & Apr. 8. & & & \\
\hline Dec. 31 & 342.55 & Nov. 20 & 338.01 & Apr. 15 & & & \\
\hline 1930 & & $\begin{array}{l}\text { Dec. } 4 \\
\text { Dec. } 11\end{array}$ & $\begin{array}{l}338.57 \\
338.77\end{array}$ & $\begin{array}{l}\text { Apr. } 22 \\
\text { A pr. } 29\end{array}$ & & & \\
\hline & & Dec. 18 & 339.82 & May 6. & & & \\
\hline Feb. $1 \ldots$ & 341.40 & Dec. 26 . & 339.43 & May 13 & & & \\
\hline
\end{tabular}

2 Leveling by U. S. Engineer Department.

5 Pump operating in well.

7 Adjacent land being irrigated.

571. James R. Barnes. SW $1 / 4$ NW $1 / 4$ sec. 8, T. 13 S., R. 5 W. Domestic well, dug 23 feet deep, backfilled around 12 -inch concrete-tile casing. Measuring point is top of casing, 0.9 foot above land surface and 252.01 feet ${ }^{2}$ above mean sea level.

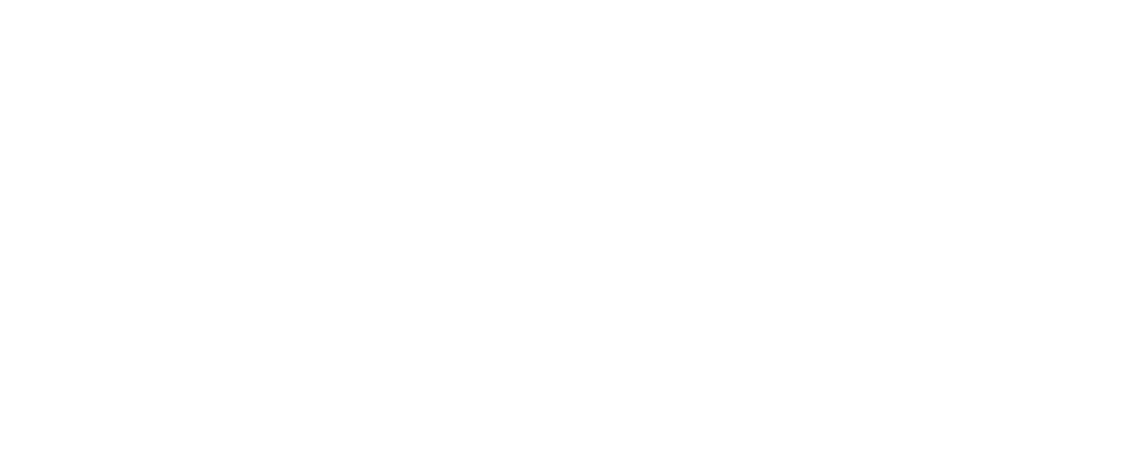

2 Leveling by U. S. Engineer Department.

- Pump operating in well a short time prior to measurement. 
TABLE 13.-Ground-water levels in observation wells, 1928-30 and 1935-36-Con.

574. L. D. Porter. SE1/4SE1/4 sec. 10, T. 13 S., R. 5 W. Stock well, dug 5 feet in diameter and 20.7 feet deep, brick curb. Measuring point is top of plank deck, at west side, 0.3 foot a bove land surface and 253.80 feet 2 a bove mean sea level.

\begin{tabular}{|c|c|c|c|c|c|c|c|}
\hline Date & $\begin{array}{l}\text { Water } \\
\text { level } \\
\text { (feet) }\end{array}$ & Date & $\begin{array}{l}\text { Water } \\
\text { level } \\
\text { (feet) }\end{array}$ & Date & $\begin{array}{l}\text { Water } \\
\text { level } \\
\text { (feet) }\end{array}$ & - Date & $\begin{array}{l}\text { Water } \\
\text { level } \\
\text { (feet) }\end{array}$ \\
\hline 1935 & & 1936 & & 1936 & & 1936 & \\
\hline Oct. 18 & 235.48 & Jan. 22 . & 251.37 & May 13 & s 241.79 & Aug. 1 & (4) \\
\hline Oct. 23 . & 237.83 & Jan. 29 & 250.70 & May 20. & 239.84 & Aug. 5 . & 235.12 \\
\hline Oct. 29 . & 237.70 & Feb. 5 & 250.42 & May 27 & 239.89 & Aug. 8 . & (4) \\
\hline Nov. 7 & 237.48 & Feb. 12 & 251.28 & June 3 & 239.33 & Aug. 12 & (4) \\
\hline Nov. $13 \ldots$ & 237.38 & Feb. 21 & 252.50 & June 10 & 5 236.61 & Aug. 15 & (4) \\
\hline Nov. 20 & 237.26 & Feb. 26 & 251.98 & June 17 & 238.78 & Aug. 19 & (4) \\
\hline Nov. $29 \ldots$ & 236.38 & Mar. 4 & 251.18 & June 24 & $\left(\begin{array}{ll}4 & 14\end{array}\right)$ & Aug. 26 & (4) \\
\hline Dec. 4 & 236.58 & Mar. 11 & 250.79 & June 26 . & $(414)$ & Aug. 29 & (4) \\
\hline Dec. 11 & 240.01 & Mar. 18 & 250.54 & July 1 & 236.28 & Sept. 2 & 237.93 \\
\hline Dec. 18 & 242.34 & Mar. 25. & 250.36 & July 3 & $\left(\begin{array}{lll}4 & 14\end{array}\right)$ & Sept. 10. & 236.89 \\
\hline Dec. 26 & 242.37 & Apr. 1 $\ldots$ & 251.10 & July 8 & 237.02 & Sept. 16-- & 237.52 \\
\hline 1936 & & Apr. 8 & $\begin{array}{l}250.89 \\
250.49\end{array}$ & July 11 & $\begin{array}{l}236.27 \\
(414)\end{array}$ & Sept. 19.- & 237. 71 \\
\hline Jan. 2. & 252.34 & Apr. 22 & 5244.41 & July 18 & $\left(\begin{array}{lll}4 & 14\end{array}\right)$ & Sept. 26 & 237.98 \\
\hline $\operatorname{Jan}, 9$ & 252.73 & Apr. 24 & 5244.45 & July 22 & $(4)$ & Sept. 30 & 237.83 \\
\hline Jan. 15 & 251.94 & Apr. 29 & 242.28 & July 25. & (4) & & \\
\hline Jan. 18 & 251.94 & May $6 \ldots$ & 239.13 & July $29 \ldots$ & 234.5 & & \\
\hline
\end{tabular}

2 Leveling by U. S. Engineer Department.

4 Water level depressed by inordinately large withdrawals.

- Pump operating in well.

14 Well dry.

575. L. J. Porter. SE $1 / 4$ SE 144 sec. 10, T. 13 S., R. 5 W., across highway from well 574 . Unused well, dug 5 feet in diameter and 20.5 feet deep, dry-masonry curb. Measuring point is top of 2-inch plank deck, 0.6 foot above land surface and 253.52 feet ${ }^{2}$ above mean sea level.

\begin{tabular}{|c|c|c|c|c|c|c|c|}
\hline Date & $\begin{array}{l}\text { Water } \\
\text { level } \\
\text { (feet) }\end{array}$ & Date & $\begin{array}{l}\text { Water } \\
\text { level } \\
\text { (feet) }\end{array}$ & Date & $\begin{array}{l}\text { Water } \\
\text { level } \\
\text { (feet) }\end{array}$ & Date & $\begin{array}{l}\text { Water } \\
\text { level } \\
\text { (feet) }\end{array}$ \\
\hline 1935 & & 1936 & & 1936 & & 1936 & \\
\hline Oct. 18 & 237.72 & Jan. 9 & 252.09 & Apr. 8 & 251.04 & July 8 . & 240.56 \\
\hline Oct. 24 . & 235.76 & Jan. 15 & $\begin{array}{l}202.09 \\
252.66\end{array}$ & Apr. 15 & 249.59 & July 15 & 240.04 \\
\hline Oct. 29 . & 235.65 & Jan. 22 & 251.43 & Apr. 22 & 247.45 & July 22 & 240.62 \\
\hline Nov. 7. & 235.47 & Jan. 29 & 250.95 & Apr. 24 & 247.46 & July $29_{-}$ & 240.33 \\
\hline Nov. 13 & 235.40 & Feb. 5 & 250.91 & Apr. 20 & 245.78 & Aug. 5 & 240.05 \\
\hline Nov. 20 & 235.37 & Feb. 12 & 252.09 & May 6 & 244.89 & Aug. 12 & 240.03 \\
\hline Nov. 29 & 236.80 & Feb. 21 & 252.76 & May 13 & 5243.99 & Aug. 19 & 239.60 \\
\hline Dec. 4 & 236.83 & Feb. 26 . & 252.19 & May 20 & 243.22 & Aug. 26 & 239. 52 \\
\hline Dec. 11 & 240.41 & Mar. 4 & 251.05 & May 27 & 243.21 & Sept. 2 & 238.32 \\
\hline Dec. 18 & 239.60 & Mar. 11 & 250.83 & June 3 & 241.83 & Sept. 10 & 237.45 \\
\hline Dec. 26 & 240.53 & Mar. 18 & 250.68 & June 10 & 241.60 & Sept. 16 & 238.90 \\
\hline 1936 & & $\begin{array}{l}\text { Mar. 25- } \\
\text { Mar. } 27\end{array}$ & $\begin{array}{l}248.40 \\
248.12\end{array}$ & $\begin{array}{l}\text { June } 17 \\
\text { June } 24\end{array}$ & $\begin{array}{l}241.42 \\
241.25\end{array}$ & $\begin{array}{l}\text { Sept. } 23 \\
\text { Sept. } 30\end{array}$ & $\begin{array}{l}238.62 \\
238.56\end{array}$ \\
\hline Jan. 2 & 251.65 & Apr. 1 . & 251.71 & July 1 & 241.09 & & \\
\hline
\end{tabular}

${ }^{2}$ Leveling by U. S. Engineer Department.

5 Pump operating in well.

579 Harold Freeman. SE1/4 SW1/4 sec 34 , T 13 S., R. 5 W. Domestic well, driven 27.3 feet deep. Measuring point is lower valve seat of pump, 2.8 feet above land surface and 267.08 feet ${ }^{2}$ above mean sea level.

\begin{tabular}{|c|c|c|c|c|c|c|c|}
\hline Date & $\begin{array}{l}\text { Water } \\
\text { level } \\
\text { (feet) }\end{array}$ & Date & $\begin{array}{l}\text { Water } \\
\text { Ievel } \\
\text { (feet) }\end{array}$ & Date & $\begin{array}{l}\text { Water } \\
\text { level } \\
\text { (feet) }\end{array}$ & Date & $\begin{array}{l}\text { Water } \\
\text { level } \\
\text { (feet) }\end{array}$ \\
\hline 1935 & & 1936 & & 1936 & & 1936 & \\
\hline Oct. 18 & 248.36 & Feb. 12 & 256.26 & Apr. 29 & 254.57 & July 22 & 250.81 \\
\hline Oct. 24 & 248.29 & Feb. 2l & 257.00 & May 6 & 254 & July 29. & 251. 28 \\
\hline Oct. 30 & 248.23 & Feb. 26 & 257.11 & May 13 & 254.27 & Aug. 5 & 250.74 \\
\hline Nov. 7 . & 248.11 & Mar. 4 & 258.11 & May 20 & 254.55 & Aug. 12 & 249.85 \\
\hline & & & 257.05 & May 27 & 253.69 & Aug. 19 & 249.39 \\
\hline 1936 & & Mar. 18 & 258.81 & June 3 & 253.77 & Aug. 26 & 249.69 \\
\hline Jan 9 & & Mar. 25 & 255.41 & June 10 & 252.74 & Sept. 2 - & 249.39 \\
\hline Jan. 15 & $\begin{array}{l}256.84 \\
258.91\end{array}$ & Mar. 27 & $\begin{array}{l}255.57 \\
255.26\end{array}$ & June 17 & $\begin{array}{l}252.19 \\
252.15\end{array}$ & $\begin{array}{l}\text { Sept. } 10 \\
\text { Sept. } 16\end{array}$ & $\begin{array}{l}249.20 \\
249.16\end{array}$ \\
\hline Jan, 22 . & 257.22 & Apr. 8 & 255.35 & July 1. & 251.81 & Sept. 23 & 248.81 \\
\hline Jan. 29 & 255.18 & Apr. 15 & 254. & July 8 & 251.59 & Sept. 30 & 248.45 \\
\hline Feb. 5 & 254.27 & Apr. 22 & 252.87 & Julv 15 & 250.90 & & \\
\hline
\end{tabular}

${ }^{2}$ Leveling by U. S. Engineer Department. 
TABLE 13.-Ground-water levels in observation wells, 1928-30 and 1935-36-Con.

581. Clingman Bros. SE14 NW1/4 sec. 16, T. 13 S., R. 4 W. Stock well, dug 36 inches in diameter and 28 feet deep, in barnyard 20 feet southeast of pump house. Measuring points: August 13, 1928 and beginning October 4, 1935, top of brick casing level with land surface, 258.49 feet ${ }^{2}$ above mean sea level; Oct. 21, 1928 , to July 23,1930 , top of plank well cover at copper nail with washer, 0.5 foot above land surface and 259.0 feet above mean sea level.

\begin{tabular}{|c|c|c|c|c|c|c|c|}
\hline Date & $\begin{array}{l}\text { Water } \\
\text { level } \\
\text { (feet) }\end{array}$ & Date & $\begin{array}{l}\text { Water } \\
\text { level } \\
\text { (feet) }\end{array}$ & Date & $\begin{array}{l}\text { Water } \\
\text { level } \\
\text { (feet) }\end{array}$ & Date & $\begin{array}{l}\text { Water } \\
\text { level } \\
\text { (feet) }\end{array}$ \\
\hline $\begin{array}{l}\quad 1928 \\
\text { Aug. } 13 \\
\text { Oct. } 21 \\
\text { Dec. } 16 \\
\\
\text { Feb. } 2 \\
\text { Mar. } 27 \\
\text { May } 9 \\
\text { June } 6 \\
\text { July } 4 \\
\text { Aug. } 2 \\
\text { Sept. } 23 \\
\text { Oct. } 25 \\
\text { Nov. } 27 \\
\text { Dec. } 30 \\
\qquad 1930 \\
\text { Feb. } 1 \\
\text { Mar. } 1 \\
\text { Mar. } 29\end{array}$ & $\begin{array}{l}255.4 \\
251.90 \\
251.00 \\
248.65 \\
246.50 \\
244.35 \\
241.48 \\
240.45 \\
239.90 \\
252.45 \\
\\
\\
254.25 \\
253.90 \\
251.30\end{array}$ & 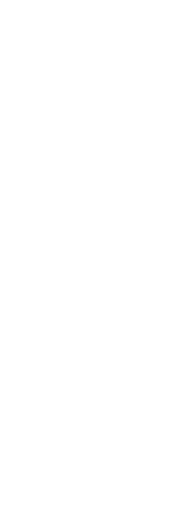 & $\begin{array}{l}250.40 \\
247.73 \\
244.15 \\
\\
240.27 \\
240.30 \\
239.92 \\
239.84 \\
239.70 \\
239.70 \\
239.79 \\
239.70 \\
243.10 \\
240.68 \\
241.01 \\
\\
243.08 \\
253.11\end{array}$ & 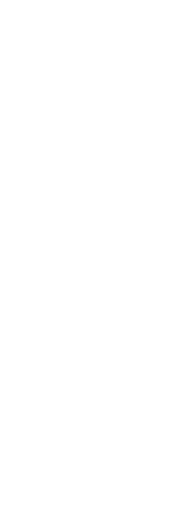 & $\begin{array}{l}256.23 \\
255.11 \\
253.87 \\
253.71 \\
252.83 \\
251.64 \\
253.46 \\
255.09 \\
253.94 \\
252.65 \\
253.11 \\
252.36 \\
252.30 \\
251.85 \\
250.32 \\
250.28 \\
248.77 \\
248.21 \\
248.43 \\
247.47 \\
246.71 \\
246.22\end{array}$ & \begin{tabular}{l}
\multicolumn{1}{c}{1936} \\
June 17 \\
June 24 \\
July 1 \\
July 10 \\
July 15 \\
July 22 \\
July 29 \\
Aug. 5 \\
Aug. 12 \\
Aug. 19 \\
Aug. 26 \\
Sept. 2 \\
Sept. 10 \\
Sept. 16 \\
Sept. 23 \\
Sept. 30
\end{tabular} & $\begin{array}{l}245.97 \\
245.59 \\
245.11 \\
244.70 \\
244.34 \\
243.78 \\
243.39 \\
242.94 \\
242.55 \\
242.20 \\
241.91 \\
241.89 \\
241.81 \\
241.77 \\
241.38 \\
241.14\end{array}$ \\
\hline
\end{tabular}

2 Leveling by U. S. Engineer Department.

589. Ida Metzger. SW14 NW1/4 sec. 30, T. 13 S., R. 3 W. Stock well, driven 24.8 feet deep. Measuring point is top of open standpipe at side of well, 1.5 feet above land surface and 274.53 feet ${ }^{2}$ above mean sea level.

\begin{tabular}{|c|c|c|c|c|c|c|c|}
\hline Date & $\begin{array}{l}\text { Water } \\
\text { level } \\
\text { (feet) }\end{array}$ & Date & $\begin{array}{l}\text { Water } \\
\text { level } \\
\text { (feet) }\end{array}$ & Dato & $\begin{array}{l}\text { Water } \\
\text { level } \\
\text { (feet) }\end{array}$ & Date & $\begin{array}{l}\text { Water } \\
\text { level } \\
\text { (feet) }\end{array}$ \\
\hline 1935 & & 1936 & & 1936 & & 1936 & \\
\hline $\begin{array}{l}\text { Oct. } 15 \\
\text { Oct. } 22 \\
\text { Oct. } 30 \\
\text { Nov. } 6 \\
\text { Nov. } 13 \\
\text { Nov. } 20 \\
\text { Dec. } 4 \\
\text { Dec. } 11 \\
\text { Dec. } 18 \\
\text { Dec. } 26 \\
\qquad 1936 \\
\text { Jan. } 1\end{array}$ & $\begin{array}{l}257.33 \\
257.30 \\
257.19 \\
257.08 \\
257.11 \\
257.15 \\
257.11 \\
258.67 \\
261.36 \\
262.11\end{array}$ & $\begin{array}{l}\text { Jan. } 8 \\
\text { Jan. } 10 \\
\text { Jan. } 15 \\
\text { Jan. } 22 \\
\text { Jan. } 24 \\
\text { Jan. } 29 \\
\text { Jan. } 31 \\
\text { Feb. } 5 \\
\text { Feb. } 13 \\
\text { Feb. } 20 \\
\text { Feb. } 26 \\
\text { Mar. } 4 \\
\text { Mar. } 11 \\
\text { Mar. } 25\end{array}$ & $\begin{array}{l}265.73 \\
266.45 \\
266.81 \\
267.05 \\
266.26 \\
266.01 \\
267.08 \\
265.72 \\
263.67 \\
264.07 \\
267.76 \\
265.51 \\
267.91 \\
267.95\end{array}$ & $\begin{array}{l}\text { Apr. 1. } \\
\text { Apr. } 8 \\
\text { Apr. 15 } \\
\text { Apr. 22 } \\
\text { Apr. 29 } \\
\text { May } 6 \\
\text { May } 13 \\
\text { May } 20 \\
\text { May } 27 \\
\text { June } 3 \\
\text { June } 10 \\
\text { June } 17 \\
\text { June } 24 \\
\text { July } 1\end{array}$ & $\begin{array}{l}268.16 \\
268.07 \\
268.00 \\
267.51 \\
267.31 \\
266.87 \\
266.01 \\
266.46 \\
267.25 \\
266.91 \\
266.45 \\
265.97 \\
266.03 \\
265.46\end{array}$ & $\begin{array}{l}\text { July } 10 \\
\text { July } 15 \\
\text { July } 22 \\
\text { July } 29 \\
\text { Aug. } 26 \\
\text { Sept. } 2 \\
\text { Sept. } 10 \\
\text { Sept. } 16 \\
\text { Sept. } 23 \\
\text { Sept. } 30\end{array}$ & $\begin{array}{l}265.20 \\
264.03 \\
263.96 \\
263.56 \\
260.64 \\
260.64 \\
260.62 \\
260.36 \\
260.14 \\
259.96\end{array}$ \\
\hline
\end{tabular}

2 Leveling by U. s. Engineer Department. 
TABLE 13.-Ground-water levels in observation wells, 1928-30 and 1935-36-Con.

590. Keeney School, district 51. SW $1 / 4$ SW1/4 sec. 34, T. 13 S., R. 3 W. Domestic well, driven $11 / 4$ inches in diameter and 18 feet deep, at east side of school near entrance. Casing stamped 13-3-5 just below pump base under pump handle. Measuring point is lower valve seat of pump, 2.5 feet above concrete pump platform and land surface and 287.5 feet ${ }^{2}$ above mean sea level.

\begin{tabular}{|c|c|c|c|c|c|c|c|}
\hline Date & $\begin{array}{l}\text { Water } \\
\text { level } \\
\text { (feet) }\end{array}$ & Date & $\begin{array}{l}\text { Water } \\
\text { level } \\
\text { (feet) }\end{array}$ & Date & $\begin{array}{l}\text { Water } \\
\text { level } \\
\text { (feet) }\end{array}$ & Date & $\begin{array}{l}\text { Water } \\
\text { level } \\
\text { (feet) }\end{array}$ \\
\hline 1928 & \multirow{3}{*}{278.0} & 1935 & \multirow{10}{*}{$\begin{array}{l}277.20 \\
277.15 \\
277.26 \\
277.50 \\
278.78 \\
279.91 \\
281.22 \\
281.82 \\
281.70\end{array}$} & 1936 & \multirow{17}{*}{$\begin{array}{c}. \\
281.93 \\
282.81 \\
281.98 \\
281.25 \\
280.76 \\
282.83 \\
280.68 \\
282.62 \\
281.77 \\
281.82 \\
282.33 \\
282.12 \\
282.20 \\
281.36 \\
281.26 \\
280.86 \\
281.46 \\
281.22 \\
281.32\end{array}$} & \multirow{17}{*}{$\begin{array}{l}\quad 1936 \\
\text { June } 3 \\
\text { June } 10 \\
\text { June } 17 \\
\text { June } 24 \\
\text { July } 1 \\
\text { July } 10 \\
\text { July } 15 \\
\text { July } 22 \\
\text { July } 29 \\
\text { Aug. } 5 \\
\text { Aug. } 12 \\
\text { Aug. } 19 \\
\text { Aug. } 26 \\
\text { Sept. } 2 \\
\text { Sept. } 10 \\
\text { Sept. } 16 \\
\text { Sept. } 23 \\
\text { Sept. } 30\end{array}$} & \multirow{17}{*}{$\begin{array}{l}280.72 \\
280.48 \\
280.71 \\
280.18 \\
279.83 \\
279.65 \\
279.44 \\
279.04 \\
278.71 \\
278.39 \\
278.13 \\
278.53 \\
277.66 \\
277.39 \\
277.30 \\
277.28 \\
277.02 \\
276.86\end{array}$} \\
\hline Aug. 16 & & Oet. 30 & & Jan. 29 & & & \\
\hline 1929 & & $\begin{array}{l}6 \\
13 \\
\end{array}$ & & $\begin{array}{l}\text { Jan. } 31 \\
\text { Feb. } 5\end{array}$ & & & \\
\hline Oct. 25 & 276.35 & Nov. 21 & & Feb. 13 & & & \\
\hline Nov. 27. & 276.10 & Dec. 5 & & Feb. 20 & & & \\
\hline Dec. 31 & 281.85 & De & & $\mathrm{Fel}$ & & & \\
\hline 1930 & & Dec. 18 & & Mar. 4 & & & \\
\hline Feb. 1 & 282.50 & Dec. 28 & & & & & \\
\hline $\mathbf{M}$ & 282.45 & & & $\mathbf{M}$ & & & \\
\hline Mar. 29 & 282.25 & 1936 & & Apr. 1 & & & \\
\hline 26. & 282.55 & & & & & & \\
\hline May 28 & 281.12 & Jan. 2 & 282.38 & Apr. 15 & & & \\
\hline July 23 & 278. 79 & Jan. 3. & 282.70 & Apr. 22 & & & \\
\hline 1935 & & Jan. 8 & & Apr. 29 & & & \\
\hline & & Jan. $10-$ & $\begin{array}{l}284.25 \\
282.99\end{array}$ & May 6 & & & \\
\hline 12 & 27 & Jan. 22 & 282.73 & May 20 & & & \\
\hline Oct. 17 . & 276.48 & Jan. 24 & 282.34 & May 27 & & & \\
\hline
\end{tabular}

2 Leveling by U. s. Engineer Department.

594. Central School, district 50. SW1/4W1/4 sec. 3, T. 14 S., R. 5 W. Unused well, 3 inches in diameter and 26 feet deep, 20 feet south of school. Stamped 14-5-1 on iron pump brace. Measuring point is lower valve seat of pump, 2.15 feet above concrete pump platform and 268.2 feet above mean sea level (interpolated).

\begin{tabular}{|c|c|c|c|}
\hline Date & $\begin{array}{l}\text { Water } \\
\text { level } \\
\text { (feet) }\end{array}$ & Date & $\begin{array}{l}\text { Water } \\
\text { level } \\
\text { (feet) }\end{array}$ \\
\hline $\begin{array}{l}\text { Aug. } 14 \\
\text { Oct. } 22 \\
\text { Dec. } 1628 \\
\end{array}$ & $\begin{array}{l}250.8 \\
250.5 \\
252.1\end{array}$ & Feb. 2 & 257.4 \\
\hline
\end{tabular}

596. Mrs. Thomas Farvey (formerly W. E. Thomas). SE1/4SE1/4 sec. 10, T. 14 S., R. 5 W. Stock well, driven $11 / 4$ inches in diameter and 19 feet deep, in southeast corner of field 0.3 mile east of residence. Casing stamped 14-5-5 on south side just below pump base. Measuring point is lower valve seat of pump, 2.9 feet above land surface and 270.39 feet ${ }^{2}$ above mean sea level.

\begin{tabular}{|c|c|c|c|c|c|c|c|}
\hline Date & $\begin{array}{l}\text { Water } \\
\text { level } \\
\text { (feet) }\end{array}$ & Date & $\begin{array}{l}\text { Water } \\
\text { level } \\
\text { (feet) }\end{array}$ & Date & $\begin{array}{l}\text { Water } \\
\text { leve] } \\
\text { (feet) }\end{array}$ & Date & $\begin{array}{l}\text { Water } \\
\text { level } \\
\text { (feet) }\end{array}$ \\
\hline 1929 & & 1935 & & 1936 & & 1936 & \\
\hline July 4 & 254.4 & Oct. 12 . & 252.11 & Feb. 12 & 260.60 & June 24 & 254.36 \\
\hline Aug. 2 & 253. & Oct. 18 & 252.35 & Feb. 21 & 261.15 & July 1 . & 254.05 \\
\hline Sept. 23 & 252.24 & Oct. 21 . & 252.07 & Feb. 26 & 267.70 & July 8 & 253.52 \\
\hline Oct. 26 & 251.94 & Nov. 7 & 251.99 & Mar. 4 & 261. 51 & July 15 & 254.13 \\
\hline Nov. 28 & 251.74 & Nov. 13 & 251.99 & Mar. 11 & 260.73 & July 22 & 254.05 \\
\hline 1930 & & $\begin{array}{l}\text { Nov. } 20 \\
\text { Nov } 29\end{array}$ & 252. 11 & $\begin{array}{l}\text { Mar. } 18 \\
\text { Mar. } 25\end{array}$ & $\begin{array}{l}260.10 \\
258.63\end{array}$ & $\begin{array}{l}\text { July } 29 \\
\text { Aug. } 5\end{array}$ & $\begin{array}{l}253.70 \\
253.43\end{array}$ \\
\hline & & Dec. 4 & $\begin{array}{l}252.89 \\
252.86\end{array}$ & Apr. 1 & 258. 28 & Aug. 12 & 253.07 \\
\hline Jan. 2 . & 260.14 & Dec. 11 . & 253. 75 & Apr. 8 & 256.46 & Aug. 19 . & ${ }^{4} 252.65$ \\
\hline Jan. 31 & 257.64 & Dec. 18 & 252.92 & Apr. 15 & 254.60 & Aug. 26 . & 25305 \\
\hline $\mathrm{Feb} .28$ & 261.09 & Dec. 26 & 254. 96 & Apr. 22 & 257.12 & Sept. 2 & 252.80 \\
\hline Mar. 28 & 257. 69 & & & Apr. 29 & 257.09 & Sept. 10 & 252.32 \\
\hline Apr. 25 & 255.99 & 1936 & & May 6 & 257.60 & Sept. 16 & 252.39 \\
\hline May 28 & 255.03 & & & May 1 & 258.06 & Sept. 23 & 252.05 \\
\hline July 23 . & 253.26 & Jan. 2 & 257.82 & May 20. & 258.31 & Sept. 30. & 251.58 \\
\hline 1935 & & $\begin{array}{l}\operatorname{Jan} .9 \\
\operatorname{Jan} 22\end{array}$ & $\begin{array}{l}260.25 \\
262.26\end{array}$ & $\begin{array}{l}\text { May 27 } \\
\text { June } 3\end{array}$ & $\begin{array}{l}255.60 \\
255.81\end{array}$ & & \\
\hline & & & 258.75 & June 10 & 255.21 & & \\
\hline Oct. 5 & 252.17 & Feb. 5_ & 260.25 & June 17 & 254. 98 & & \\
\hline
\end{tabular}

2 Leveling by U. S. Engineer Department.

4 Water level depressed by inordinately large withdrawals. 
TABLE 13.-Ground-water levels in observation wells, 1928-30 and 1985-36-Con.

602. W. N. Carpenter. SW $1 / 4$ NE1/4 sec. 33, T. 14 S., R. 5 W. Well drilled 3 inches in diameter and 61.3 feet deep, steel casing. Measuring point is top of 3 -inch steel casing, 1.2 feet above land surface and 287.58 feet 2 above mean sea level.

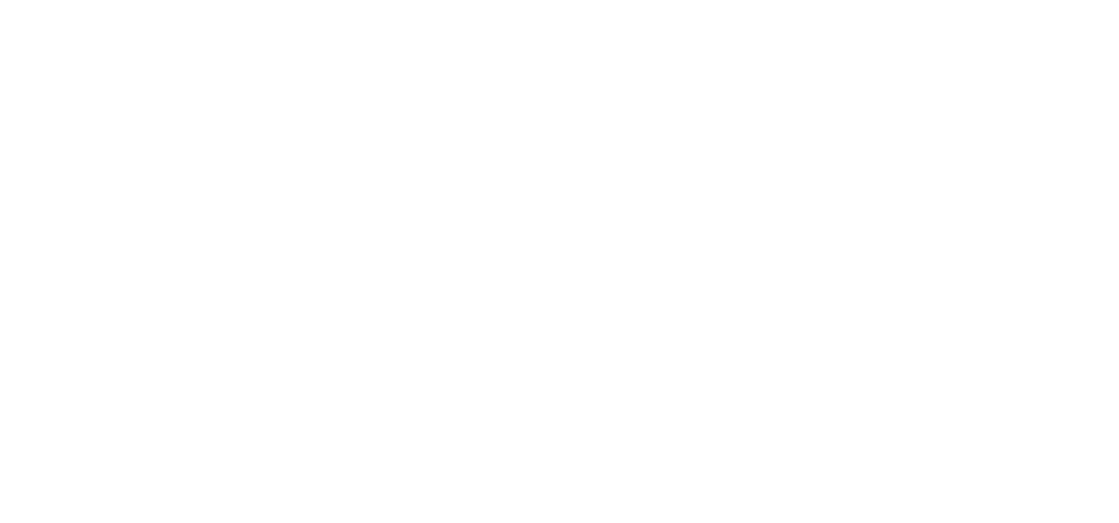

Leveling by U. S. Engineer Department.

603. R. L. George. SE $1 / 4$ NW $1 / 4$ sec. 34, T. 14 S., R. 5 W. Stock well, driven 24 feet deep. Measuring point is lower valve seat of pump, 2.4 feet above land surface and 287.65 feet 2 above mean sea level.

\begin{tabular}{|c|c|c|c|c|c|c|c|}
\hline Date & $\begin{array}{l}\text { Water } \\
\text { level } \\
\text { (feet) }\end{array}$ & Date & $\begin{array}{l}\text { Water } \\
\text { level } \\
\text { (feet) }\end{array}$ & Date & $\begin{array}{l}\text { Water } \\
\text { level } \\
\text { (feet) }\end{array}$ & Date & $\begin{array}{l}\text { Water } \\
\text { level } \\
\text { (feet) }\end{array}$ \\
\hline 1935 & & 1936 & & 1936 & & 1936 & \\
\hline $\begin{array}{l}\text { Oct. } 17 \\
\text { Oct. } 24 \\
\text { Oct. } 30 \\
\text { Nov. } 7 \\
\text { Nov. } 13 \\
\text { Nov. } 20 \\
\text { Nov. } 30 \\
\text { Dec. } 5 \\
\text { Dec. } 12 \\
\text { Dec. } 19 \\
\text { Dec. } 27 \\
\quad 1936 \\
\text { Jan. } 3\end{array}$ & $\begin{array}{l}272.28 \\
272.21 \\
272.16 \\
272.07 \\
271.97 \\
272.10 \\
272.11 \\
272.62 \\
272.97 \\
273.01 \\
273.04\end{array}$ & $\begin{array}{l}\text { Jan. } 10 \\
\text { Jan. } 16 \\
\text { Jan. } 23 \\
\text { Jan. } 30 \\
\text { Feb. } 6 \\
\text { Feb. } 13 \\
\text { Feb. } 22 \\
\text { Feb. } 27 \\
\text { Mar. } 5 \\
\text { Mar. } 12 \\
\text { Mar. } 19 \\
\text { Mar. } 26 \\
\text { Apr. } 2 \\
\text { Apr. } 9 \\
\text { Apr. } 16 \\
\end{array}$ & $\begin{array}{l}277.06 \\
285.45 \\
284.62 \\
279.68 \\
278.51 \\
277.69 \\
276.80 \\
281.84 \\
281.02 \\
280.79 \\
278.51 \\
276.97 \\
278.48 \\
278.59 \\
276.34\end{array}$ & $\begin{array}{l}\text { Apr. } 23 \\
\text { Apr. } 29 \\
\text { May } 6 \\
\text { May } 14 \\
\text { May } 21 \\
\text { May } 28 \\
\text { June } 4 \\
\text { June } 12 \\
\text { June } 18 \\
\text { June } 25 \\
\text { July } 2 \\
\text { July } 9 \\
\text { July } 15 \\
\text { July } 23 \\
\text { July } 30 \\
\end{array}$ & $\begin{array}{l}276.38 \\
276.62 \\
276.83 \\
276.37 \\
276.54 \\
276.58 \\
275.97 \\
276.21 \\
276.24 \\
275.82 \\
275.06 \\
274.87 \\
274.62 \\
274.36 \\
274.18\end{array}$ & $\begin{array}{l}\text { Aug. } 6 \\
\text { Aug. } 13 \\
\text { Aug. } 20 \\
\text { Aug. } 27 \\
\text { Sept. } 3 \\
\text { Sept. 11 } \\
\text { Sept. 17 } \\
\text { Sent. } 24 \\
\text { Oct. } 1\end{array}$ & $\begin{array}{l}273.97 \\
273.88 \\
273.59 \\
273.18 \\
272.82 \\
272.63 \\
273.06 \\
272.98 \\
272.72\end{array}$ \\
\hline
\end{tabular}

2 Leveling by U. S. Engineer Department.

604. M. L. Hewitt. SE14SW14 sec. 36, T. 14 S., R. 5 W. Stock well, driven 17.2 feet deep. Measuring point is lower valve seat of pump, 2.0 feet above land surface and 286.88 feet ${ }^{2}$ above mean sea level.

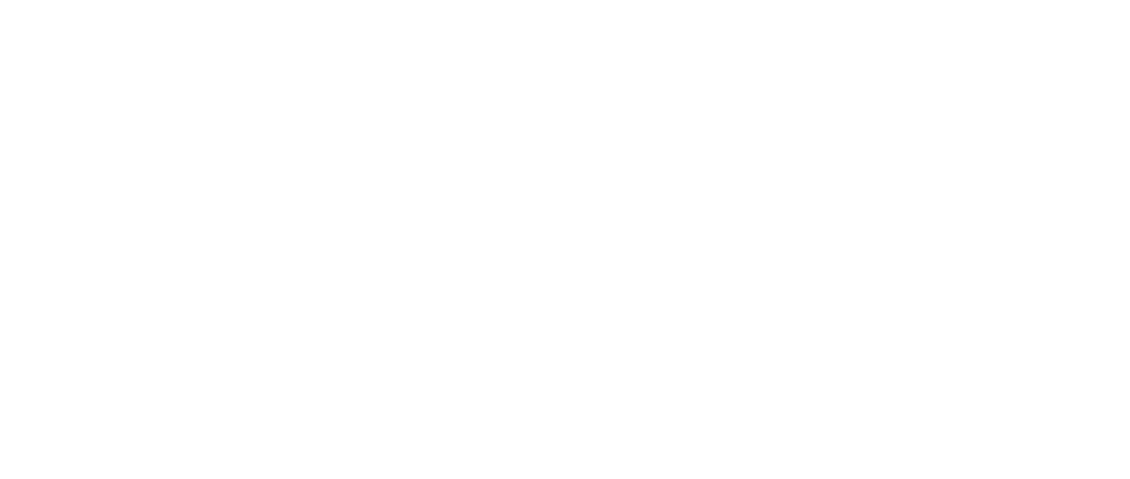

2Leveling by U. S. Engineer Department. 
TABLe 13.-Ground-water levels in observation wells, 1928-30 and 1935-36-Con.

606. Citizens Light \& Water Co. (of Halsey). SE1/4NE1/4 sec. 1, T. 14 S., R. 4 W. Public-supply well, dug 10 feet in diameter and 28 feet deep, extended by two 4 -inch drilled holes to a total depth 72 feet, in pump house north of tank tower. Copper nail with washer stamped 14-4-6 in east end of door sill. Measuring point is base of 8- by 8-inch beam at copper nail with washer, about 7.5 feet below land surface and 274.5 feet above mean sea level (interpolated).

\begin{tabular}{|c|c|c|c|}
\hline Date & $\begin{array}{l}\text { Water } \\
\text { level } \\
\text { (feet) }\end{array}$ & Date & $\begin{array}{l}\text { Water } \\
\text { level } \\
\text { (feet) }\end{array}$ \\
\hline $\begin{array}{l}\text { Aug. } 16 \ldots 28 \\
\text { Oct. } 21 \\
\text { Dec. } 16 \\
\end{array}$ & $\begin{array}{l}264.65 \\
263.3 \\
267.79\end{array}$ & $\begin{array}{l}\text { Feb. } 2 \\
\text { Mar. } 27 \\
\text { May } 929 \\
\end{array}$ & $\begin{array}{l}270.9 \\
271.25 \\
272.3\end{array}$ \\
\hline
\end{tabular}

607. J. H. Suytor. NE1/4NW1/4 sec. 5, T. 14 S., R. 4 W. Domestic well, driven $11 / 2$ inches in diameter and 23.4 feet deep. Measuring point is lower valve seat of pump, 3.5 feet above land surface and 273.0 feet ${ }^{2}$ above mean sea level.

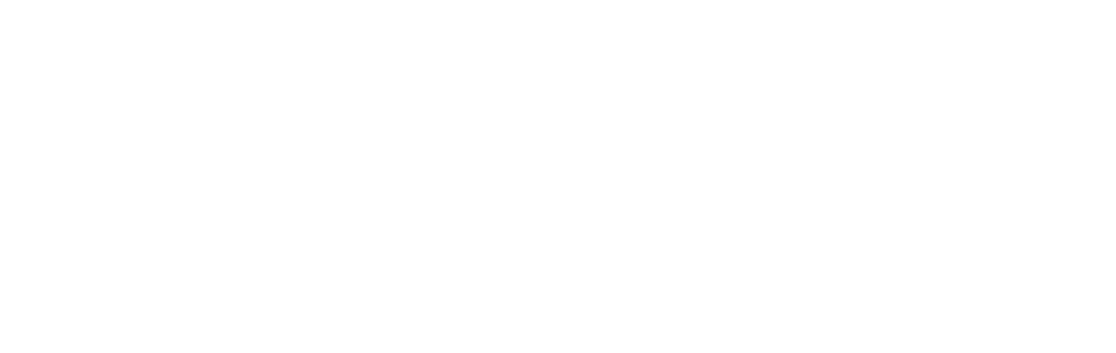

2 Leveling by U. S. Engineer Department.

608. L. O. Kroft. SE $1 / 4$ NW1/4 sec. 5, T. 14 S., R. 4 W. Unused well, drilled 6 inches in diameter and 50 feet deep, steel casing. Measuring point is top of $11 / 2$-inch standpipe, 0.2 foot above land surface and 271.87 feet ${ }^{2}$ above mean sea level.

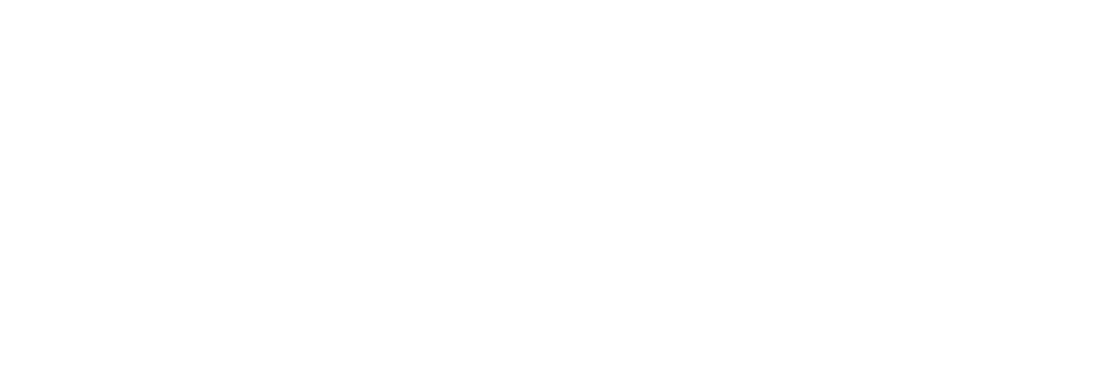

${ }^{2}$ Leveling by U. S. Engineer Department. 
TABLE 13.-Ground-water levels in observation wells, 1928-30 and 1935-36-Con.

613. B. E. Cogswell. SE1/4NW1/4 sec. 26 , T. 14 S., R. 4 W. Stoek well, dug 4 feet in diameter and 15.5 feet deep, backfilled around 6 -inch tile casing, in barnyard. Copper nail with washer stamped 14-4-7 in 6- by 10-inch timber at north side of well. Measuring point is top of tile casing, painted white on west side, level with land surface and 294.56 feet ${ }^{2}$ above mean sea level.

\begin{tabular}{|c|c|c|c|c|c|c|c|}
\hline Date & $\begin{array}{l}\text { Water } \\
\text { level } \\
\text { (feet) }\end{array}$ & Date & $\begin{array}{l}\text { Water } \\
\text { level } \\
\text { (feet) }\end{array}$ & Date & $\begin{array}{l}\text { Water } \\
\text { level } \\
\text { (feet) }\end{array}$ & Date & $\begin{array}{l}\text { Water } \\
\text { level } \\
\text { (feet) }\end{array}$ \\
\hline 1928 & & 1930 & & 1936 & & 1936 & \\
\hline Aug. 18 & 283.96 & Mar. 29 & 286.16 & Jan. 8 & 286.68 & May 21 & 285.46 \\
\hline Oct. 21 . & 283.26 & Apr. 26 & 287.16 & Jan. 10 & 287.43 & May 28 & 285.43 \\
\hline Dec. 16 . & 284.81 & $\begin{array}{l}\text { May } 28 \\
\text { July } 23 .\end{array}$ & $\begin{array}{l}280.02 \\
284.04\end{array}$ & Jan. 16 & 288.38 & June 4 & 285.09 \\
\hline 1929 & & 1935 & & Jan. 24 & 285.94 & June 18 & 284.25 \\
\hline Feb. 2 & 292.46 & Oct. 4 & 282.65 & Feb. $6 \ldots$ & $\begin{array}{l}280.91 \\
286.09\end{array}$ & July 2 & 284.41 \\
\hline Mar. 27 & 286.26 & Oet. 12 & 282.72 & Feb. 13 & 285,69 & July 11 & 284.32 \\
\hline May 9. & 286.36 & Oct. 17 - & 282.88 & Feb. 21- & 291.23 & July 16 & ${ }^{4} 282.49$ \\
\hline Jun & 285.56 & Oet. 31 - & 282.68 & Feb. 27 & 287.73 & July 23 & $\begin{array}{r}4282.58 \\
4\end{array}$ \\
\hline Jul & 284.66 & Nov. 7 & 282.56 & Mar. 5 & 290.29 & July 30 & 282. 79 \\
\hline Aug & 283.51 & Nov. 14 & 282.66 & Mar. 12 & 286.17 & Aug. 6 & 282.56 \\
\hline Sept. 23 & 283.21 & No & 282.78 & Mar. 19 & 286.75 & Aug. 12 & 282.42 \\
\hline Oet. 26 . & 282.91 & Dec. 5 . & 282.72 & Mar. 25 & 285.57 & Aug. 20 & 282.35 \\
\hline Nov. 2 & 283. 11 & Dec. 12 & 285.24 & Apr. 2. & 286.24 & Aug. 27. & 282.35 \\
\hline Dee. 31 & 285.76 & Dec. 19 & 284. 06 & Apr. 9 & 284. 59 & Sept. 3 & 282.23 \\
\hline & & Dec. 27 & 284.51 & Apr 16 & 285.75 & Sept. 11 & 282. 10 \\
\hline 1930 & & Dec. 28 & 285.74 & Apr, 23 & 285.49 & Sept. 17 & 282.06 \\
\hline & & 1936 & & $\begin{array}{l}\text { Apr. } 30 \\
\text { May } 7\end{array}$ & $\begin{array}{r}285.49 \\
285.12\end{array}$ & Sept. 24_... & $\begin{array}{l}281.70 \\
281.53\end{array}$ \\
\hline & 287.31 & Jan. 2.. & 285.21 & May 14 & 285.65 & & \\
\hline
\end{tabular}

2 Leveling by U. S. Engineer Department.

4 Water level depressed by inordinately large withdrawals.

615. F. J. George. SW1/4SE1/4 sec. 28 , T. 14 S., R. 4 W. Unused driven well, 17 feet deep. Measuring point is lower valve seat of pump, 1.4 feet ${ }^{2}$ bove land surface and 294.6 feet $^{2}$ above mean sea level.

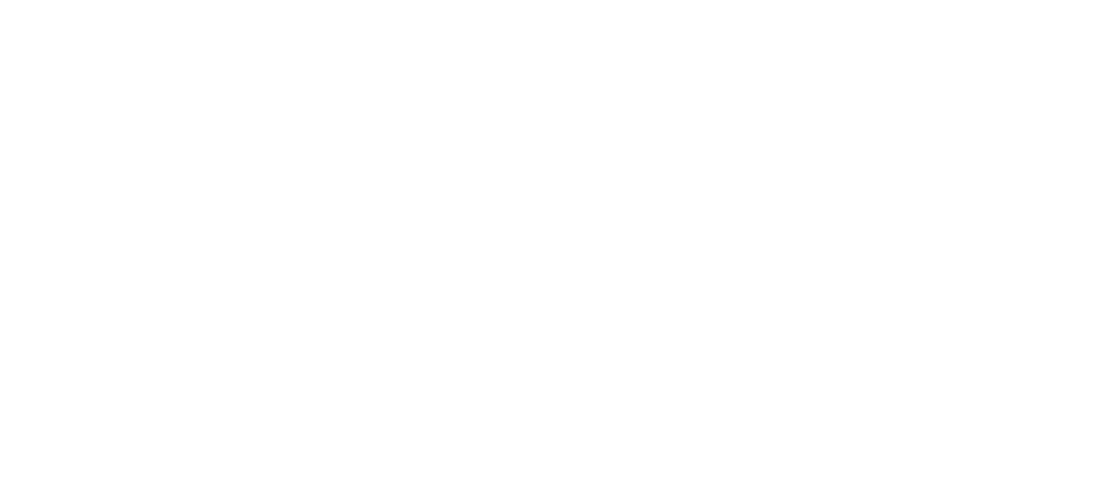

2 Leveling by U. S. Engineer Department.

616. Mrs. R. Toedtemeier. SW1/4W1/4 sec. 15, T. 14 S., R. 4 W. Unused well, dug 4 feet in diameterand 24 feet deep, in field 0.25 mile north of residence. Copper nail with washer stamped 14-3-5 in top of curb. Measuring point is lower valve sea tof pump, 1.7 feet above land surface and 308.7 feet a bove mean sea level (interpolated).

\begin{tabular}{|c|c|c|c|}
\hline Date & $\begin{array}{l}\text { Water } \\
\text { level } \\
\text { (feet) }\end{array}$ & Date & $\begin{array}{l}\text { Water } \\
\text { level } \\
\text { (feet) }\end{array}$ \\
\hline $\begin{array}{l}\text { Aug. } 23 \\
\text { Aus }\end{array}$ & $\begin{array}{l}290.3 \\
290.1 \\
291.1\end{array}$ & 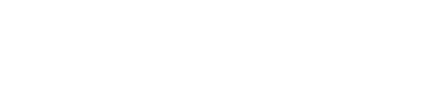 & $\begin{array}{l}302.3 \\
293.2 \\
290.2\end{array}$ \\
\hline
\end{tabular}


TABLE 13.-Ground-water levels in observation wells, 1928-30 and 1985-36-Con.

618. Charity Grange. SE1/4NE1/4 sec. 28, T. 14 S., R. 3 W. Unused well, driven $11 / 2$ inches in diameter and 35.5 feet deep. Measuring point is lower valve seat of pump, 2.5 feet above land surface and 314.4 feet $^{2}$ above mean sea level.

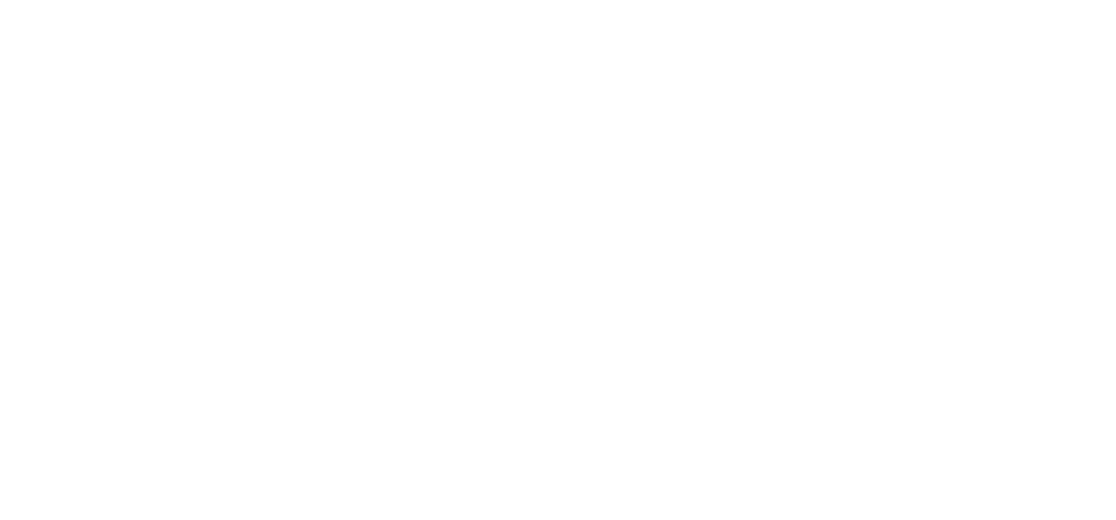

2 Leveling by U. S. Engineer Department.

621. Rosa D. Bierley (former owner, Mrs. E. Holloway). NW1/4NW1/4 sec. 7, T. 14 S., R. 2 W. Domestic well, bored 4 inches in diameter and 76 feet deep, steel lcasing. Measuring point is top of 4-inch steel casing, 0.2 foot above land surface and 332.3 feet ${ }^{2}$ above mean sea level.

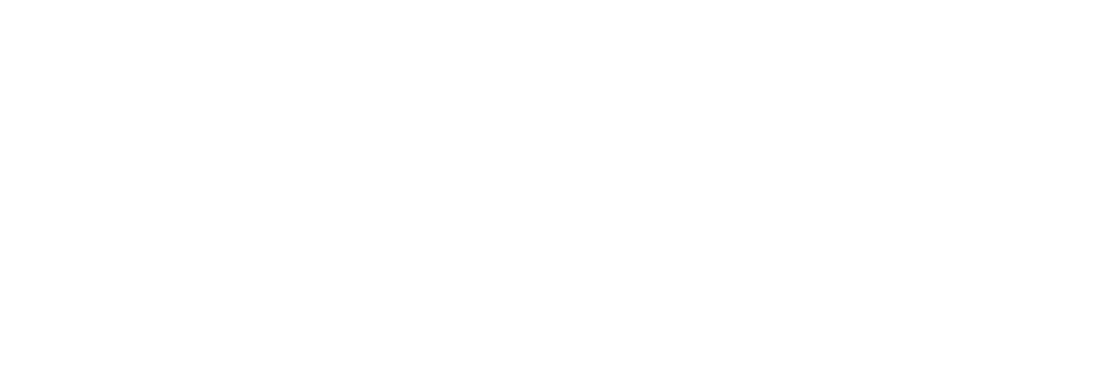

${ }^{2}$ Leveling by U. S. Engineer Department.

630. C. M. Spone. SE1/4SE $1 / 4$ sec. 9, T. 15 S., R. 4 W. Domestic well, driven $1 \frac{1}{2}$ inches in diameterand 20.6 feet deep. Measuring point is lower valve seat of pump, 1.9 feet above land surface and 309.18 feet $^{2}$ above mean sea level.

\begin{tabular}{|c|c|c|c|c|c|c|c|}
\hline Date & $\begin{array}{l}\text { Water } \\
\text { level } \\
\text { (feet) }\end{array}$ & Date & $\begin{array}{l}\text { Water } \\
\text { level } \\
\text { (feet) }\end{array}$ & Date & $\begin{array}{l}\text { Water } \\
\text { level } \\
\text { (feet) }\end{array}$ & Date & $\begin{array}{l}\text { Water } \\
\text { level } \\
\text { (feet) }\end{array}$ \\
\hline 1935 & & 1936 & & 1936 & & 1936 & \\
\hline Oet. 17 & 292.88 & Jall. 9 & 296.59 & Apr. 16 & 296.80 & July 23 & 294.46 \\
\hline Oct. 22 & 292.78 & Jan. 16 & 299.31 & Apr. 23. & 297.03 & July 30 . & 294.18 \\
\hline Oct. 31 & 292.76 & Jan. 23 & 298.20 & Apr. $30_{-}$ & 296.68 & Aug. 6 & 294.31 \\
\hline Nov. 7 & 292.66 & Jan. 30 & 297.34 & May 7 & 296.40 & Aug. 13 & 293.79 \\
\hline Nov. 14 & 293.01 & Feb. 6 & 296.28 & May 14 & 297.14 & Aug. 20 & 294. 38 \\
\hline Nov. 21 & 293.10 & Feb. 13 & 296.05 & May 21 . & 295.61 & Aug. 27. & 294.22 \\
\hline Dec. 5 & 293.11 & Feb. 21 & 296.99 & May 28 & 295.21 & Sept. 3 & 294.16 \\
\hline Dec. 12 & 293.37 & Feb. 27. & 298.75 & June 4 & 295.04 & Sept. 11 & 293.43 \\
\hline Dec. 19 & 293.71 & Mar. 5 . & 299.31 & June 11 & 295.86 & Sept. 17. & 294.00 \\
\hline Dec. 27 & 293.62 & Mar. 12 & 297.43 & June 18 & 296.17 & Sept. 24 & 293.81 \\
\hline 1936 & & $\begin{array}{l}\text { Mar. } 19 \ldots \\
\text { Mar. } 26\end{array}$ & $\begin{array}{l}297.88 \\
296.61\end{array}$ & June 25 & $\begin{array}{r}295.36 \\
295.14\end{array}$ & )et. $1 \ldots$ & 293.59 \\
\hline & & Apr. 2 & $\begin{array}{l}290.01 \\
296.99\end{array}$ & July 11. & 294.79 & & \\
\hline Jan. 2- & 294.27 & Apr. 9 & 297.24 & July 16 . & 294.61 & & \\
\hline
\end{tabular}

${ }^{2}$ Leveling by U. S. Engineer Department. 
TABLE 13.-Ground-water levels in observation wells, 1928-30 and 1935-36-Con.

631. H. F. Meersdorf. NE1/4NE1/4 sec. 16, T. 15 S., R. 4 W. Public-supply well (Harrisburg), dug 10 feet in diameterand 30 feet deep, extended by several 3 -inch driven wells to a total depth of 60 feet. Copper nail with washer stamped 15-4-2 in well curb. Measuring point is top of plank well curb at copper nail with washer, 1.0 foot below land surface and 307 feet above mean sea level (interpolated).

\begin{tabular}{|c|c|c|c|}
\hline Date & $\begin{array}{l}\text { Water } \\
\text { level } \\
\text { (feet) }\end{array}$ & Date & $\begin{array}{l}\text { Water } \\
\text { level } \\
\text { (feet) }\end{array}$ \\
\hline $\begin{array}{l}\text { Aug. } 1928 \\
\text { Oct. } 21 \\
\text { Dec. } 16, \ldots\end{array}$ & $\begin{array}{l}289.6 \\
289.6 \\
290.4\end{array}$ & 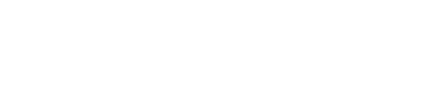 & $\begin{array}{l}294.8 \\
293.35 \\
292.85\end{array}$ \\
\hline
\end{tabular}

633. Mrs. Ingraham. SE $1 / 4 \mathrm{NW} 1 / 4$ sec. $20, \mathrm{~T} .15 \mathrm{~S}$., R. $4 \mathrm{~W}$. Unused well, driven 22.1 feet deep. Measuring point is lower valve seat of pump, 2.8 feet above land surface and 315.72 feet 2 a bove mean sea level.

\begin{tabular}{|c|c|c|c|c|c|c|c|}
\hline Date & $\begin{array}{l}\text { Water } \\
\text { leveI } \\
\text { (feet) }\end{array}$ & Date & $\begin{array}{l}\text { Water } \\
\text { Ievel } \\
\text { (feet) }\end{array}$ & Date & $\begin{array}{l}\text { Water } \\
\text { level } \\
\text { (feet) }\end{array}$ & Date & $\begin{array}{l}\text { Water } \\
\text { Ievel } \\
\text { (feet) }\end{array}$ \\
\hline 1935 & & 1936 & & 1936 & & 1936 & \\
\hline $\begin{array}{l}\text { Oct. } 17 \\
\text { Oct. } 24 \\
\text { Oct. } 30 \\
\text { Nov. } 7 \\
\text { Nov. } 13 \\
\text { Nov. } 20 \\
\text { Nov. } 30 \\
\text { Dec. } 5 \\
\text { Dec. } 12 \\
\text { Dec. } 19 \\
\text { Dec. } 27\end{array}$ & $\begin{array}{l}294.56 \\
294.65 \\
294.53 \\
294.52 \\
294.57 \\
294.79 \\
296.04 \\
294.81 \\
295.89 \\
295.55 \\
295.73\end{array}$ & $\begin{array}{l}\text { Jan. } 3 \\
\text { Jan. } 10 \\
\text { Jan. } 16 \\
\text { Jan. } 22 \\
\text { Jan. } 30 \\
\text { Feb. } 6 \\
\text { Feb. } 13 \\
\text { Feb. 27 } \\
\text { Mar. } 5 \\
\text { Mar. 12 } \\
\text { Mar. 19 } \\
\text { Mar. 26 } \\
\text { Apr. 2 }\end{array}$ & $\begin{array}{l}296.91 \\
301.08 \\
305.58 \\
304.75 \\
302.73 \\
298.65 \\
298.09 \\
300.56 \\
300.13 \\
299.71 \\
298.21 \\
298.90 \\
297.91\end{array}$ & $\begin{array}{l}\text { Apr. } 9 \\
\text { Apr. 16 } \\
\text { Apr. 23 } \\
\text { Apr. 30 } \\
\text { May } 7 \\
\text { May 14 } \\
\text { May 21 } \\
\text { May 28 } \\
\text { June } 3 \\
\text { June } 11 \\
\text { June 18 } \\
\text { June 25 } \\
\text { July 2 }\end{array}$ & $\begin{array}{l}297.68 \\
297.70 \\
297.78 \\
297.66 \\
297.74 \\
298.21 \\
297.28 \\
296.76 \\
296.58 \\
296.46 \\
296.31 \\
296.12 \\
295.83\end{array}$ & $\begin{array}{l}\text { July } 9 \\
\text { July } 16 \\
\text { July } 23 \\
\text { July } 30 \\
\text { Aug. } 6 \\
\text { Aug. } 13 \\
\text { Aug. } 20 \\
\text { Aug. } 27 \\
\text { Sept. } 3 \\
\text { Sept. } 11 \\
\text { Sept. 17 } \\
\text { Sept. } 24 \\
\text { Oct. 1. } \\
\end{array}$ & $\begin{array}{l}295.65 \\
295.46 \\
294.79 \\
293.90 \\
293.79 \\
295.08 \\
294.83 \\
294.78 \\
294.66 \\
294.40 \\
294.95 \\
294.86 \\
294.81\end{array}$ \\
\hline
\end{tabular}

2 Leveling by U. S. Engineer Department.

636. Junction City: NW1/4SW1/4 sec. 32 , T. 15 S., R. 4 W. Fire-protection well, dug 8 feet in diameter and 20 feet deep, in northwest angle of Seventh and Holly Streets. Manhole rim stamped 15-4-10 on south side. Measuring point is top of iron manhole rim at south side, level with land surface and 323.4 feet ${ }^{2}$ above mean sea level.

\begin{tabular}{|c|c|c|c|c|c|c|c|}
\hline Date & $\begin{array}{l}\text { Water } \\
\text { level } \\
\text { (feet) }\end{array}$ & Date & $\begin{array}{l}\text { Water } \\
\text { level } \\
\text { (feet) }\end{array}$ & Date & $\begin{array}{l}\text { Water } \\
\text { level } \\
\text { (feet) }\end{array}$ & Date & $\begin{array}{l}\text { Water } \\
\text { level } \\
\text { (feet) }\end{array}$ \\
\hline 1928 & \multirow{4}{*}{314.5} & 1935 & \multirow{13}{*}{$\begin{array}{l}312.76 \\
312.71 \\
312.92 \\
312.86 \\
312.55 \\
312.59 \\
312.88 \\
312.85 \\
312.80 \\
312.63 \\
313.64 \\
313.87 \\
314.00\end{array}$} & 1936 & \multirow{19}{*}{$\begin{array}{l}318.56 \\
317.83 \\
317.69 \\
317.97 \\
319.90 \\
318.93 \\
318.01 \\
317.61 \\
317.19 \\
318.32 \\
317.71 \\
317.71 \\
317.44 \\
317.14 \\
317.11 \\
317.36 \\
317.54 \\
317.44 \\
316.76\end{array}$} & 1936 & \multirow{19}{*}{$\begin{array}{l}316.57 \\
316.57 \\
316.40 \\
316.26 \\
315.97 \\
315.58 \\
315.44 \\
315.07 \\
314.75 \\
314.59 \\
314.41 \\
314.03 \\
313.95 \\
313.79 \\
313.71 \\
313.45 \\
313.42 \\
313.41\end{array}$} \\
\hline Aug. 23 & & Oct. 5 & & Jan. 22 & & June 4 & \\
\hline 1929 & & $\begin{array}{l}\text { Oct. } 12 \\
\text { Oct. } 18\end{array}$ & & $\begin{array}{l}\text { Jan. } 30 \\
\text { Feb. } 6 .\end{array}$ & & $\begin{array}{l}\text { June } 11 . \\
\text { June } 18\end{array}$ & \\
\hline & & Oct. 21 & & Feb. 13 & & June 25 & \\
\hline July 4 & 316.3 & Oct. 30 & & Feb. 22 & & July 2 & \\
\hline Aug. 2 & 315.2 & Nov. 7 & & Feb. 27 & & July 9 & \\
\hline Sept. 23 & 313.65 & Nov. 13 & & Mar. 5 & & July 16 & \\
\hline Oet. 26 & 313.1 & Nov. 20 & & Mar. 12 & & July 23 & \\
\hline Nov. 28 & 312.75 & Nov. 29 & & Mar. 19 & & July 30 & \\
\hline \multirow{3}{*}{1930} & & Dec. 5 & & Mar. 26 & & Aug. 6 & \\
\hline & & Dec. 12 & & Apr. 2 & & Aug. 13 & \\
\hline & & Dec. 19 & & Apr. 9. & & Aug. 20 & \\
\hline Jan. 2 & 318.15 & Dec. 27 & & A pr. 16 & & Aug. 27 & \\
\hline Jan. 31 & 319.4 & & & Apr. 23 & & Sept. $3_{-}$ & \\
\hline Feb. 28 & 318.8 & 1936 & & Apr. 30 & & Sept. 11 & \\
\hline Mar. 28. & 317.85 & & & May 7 & & Sept. 17 & \\
\hline Apr. 25 & 317.65 & Jan. 3 & 315.91 & May 14 & & Sept. 24 & \\
\hline May 23 & 317. & Jan. 10 & 317.89 & May 21 & & Oet. 1 & \\
\hline July 23 & 315.08 & Jan. 16 & 319.69 & May 28 & & & \\
\hline
\end{tabular}

Leveling by U. S. Engineer Department. 
TABLE 13.-Ground-water levels in observation wells, 1928-30 and 1935-36-Con.

643. Doug. Wasson. SE1/4NE1/4 sec. 11, T. 15 S., R. 3 W. Unused well, driven 115 inches in diameter and 15 feet deep. Measuring point is lower valve seat of pump, 3.4 feet above land surface and 379.47 feet 2 above a.ean sea level.

\begin{tabular}{|c|c|c|c|c|c|c|c|}
\hline Date & $\begin{array}{l}\text { Water } \\
\text { level } \\
\text { (feet) }\end{array}$ & Date & $\begin{array}{l}\text { Water } \\
\text { level } \\
\text { (feet) }\end{array}$ & Date & $\begin{array}{l}\text { Water } \\
\text { level } \\
\text { (feet) }\end{array}$ & Date & $\begin{array}{l}\text { Water } \\
\text { level } \\
\text { (feet) }\end{array}$ \\
\hline 1935 & & 1936 & & 1936 & & 1936 & \\
\hline $\begin{array}{l}\text { Oct. } 16 \\
\text { Oct. } 22 \\
\text { Oct. } 31 \\
\text { Nov. } 7 \\
\text { Nov. } 14 \\
\text { Nov. } 21 \\
\text { Dec. } 5 \\
\text { Dec. } 12 \\
\text { Dec. } 19 \\
\text { Dec. } 27 \\
\text { Dec. } 28\end{array}$ & $\begin{array}{l}363.55 \\
363.41 \\
363.43 \\
363.37 \\
363.62 \\
363.73 \\
364.08 \\
366.85 \\
365.68 \\
366.22 \\
367.41\end{array}$ & $\begin{array}{l}\text { Jan. } 2 \\
\text { Jan. } 3 \\
\text { Jan. } 9 \\
\text { Jan. } 16 \\
\text { Jan. } 23 \\
\text { Jan. } 30 \\
\text { Feb. } 6 \\
\text { Feb. } 13 \\
\text { Feb. } 21 \\
\text { Feb. } 27 \\
\text { Mar. } 5 \\
\text { Mar. } 12 \\
\text { Mar. } 19 \\
\text { Mar. 26. }\end{array}$ & $\begin{array}{l}369.62 \\
369.82 \\
369.68 \\
371.42 \\
369.99 \\
368.13 \\
367.83 \\
368.63 \\
371.98 \\
370.68 \\
372.44 \\
369.79 \\
370.88 \\
369.83\end{array}$ & 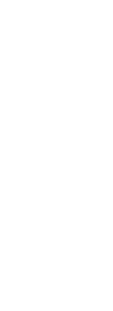 & $\begin{array}{r}370.54 \\
370.90 \\
369.77 \\
369.00 \\
368.85 \\
368.40 \\
367.70 \\
5368.23 \\
367.51 \\
366.89 \\
368.20 \\
368.50 \\
368.17 \\
367.85\end{array}$ & $\begin{array}{l}\text { July } 11 \\
\text { July } 16 \\
\text { July } 23 \\
\text { July } 30 \\
\text { Aug. } 6 \\
\text { Aug. } 13 \\
\text { Aug. } 20 \\
\text { Aug. } 27 \\
\text { Sept. } 3 \\
\text { Sept. } 11 \\
\text { Sept. } 17 \\
\text { Sept. } 24 \\
\text { Oet. } 1\end{array}$ & $\begin{array}{r}367.32 \\
366.94 \\
366.64 \\
366.21 \\
365.30 \\
4363.50 \\
364.53 \\
362.47 \\
364.73 \\
364.98 \\
365.34 \\
365.10 \\
363.37\end{array}$ \\
\hline
\end{tabular}

2 Leveling by U. S. Engineer Department.

Water level depressed by inordinately large withdrawals.

- Pump operating in well.

645. L. Hostetler, tenant. NW 1/4NE $1 / 4$ sec. 19, T. 15 S., R. 3 W. Stock well, dug 4 feet in diameter and 13 feet deep, at north side of barnyard. Copper nail with washer stamped 15-3-2 in wood platform. Measuring points: Top of wood pump phatform at copper nail with washer, about 0.2 foot above land surface and about 326.1 feet ${ }^{2}$ above mean sea level; beginning October 5,1935 , bottom of platform, level with top of brick curb and land surface and 325.9 feet 2 above mean sea level.

\begin{tabular}{|c|c|c|c|c|c|c|c|}
\hline Date & $\begin{array}{l}\text { Water } \\
\text { level } \\
\text { (feet) }\end{array}$ & Date & $\begin{array}{l}\text { Water } \\
\text { level } \\
\text { (feet) }\end{array}$ & Date & $\begin{array}{l}\text { Wat } \backslash r \\
\text { level } \\
\text { (feet) }\end{array}$ & Date & $\begin{array}{l}\text { Water } \\
\text { level } \\
\text { (feet) }\end{array}$ \\
\hline 1928 & & 1935 & & 1936 & & 1936 & \\
\hline Aug. 18 & 315.0 & Oct. 5 & 313.80 & Jan. 23 & 325.15 & June 4 & 323. 30 \\
\hline 1929 & & $\begin{array}{l}\text { Oct. } 12 \\
\text { Oct. } 17-\end{array}$ & $\begin{array}{l}313.80 \\
314.10\end{array}$ & $\begin{array}{l}\text { Jan. } 30 \\
\text { Feb. } 6\end{array}$ & $\begin{array}{l}324.69 \\
324.23\end{array}$ & $\begin{array}{l}\text { June } 11 . \\
\text { June } 18 .\end{array}$ & $\begin{array}{l}322.74 \\
322.09\end{array}$ \\
\hline Julv. & 215 & Oct. 31 & 314. 68 & Feb. 13 & $\begin{array}{l}324.68 \\
325.68\end{array}$ & June 25 & 321.71 \\
\hline $\begin{array}{l}\text { Jury } 4 \\
\text { Aug. } 2\end{array}$ & $\begin{array}{l}315.5 \\
314,4\end{array}$ & $\begin{array}{l}\text { Nov. } 7- \\
\text { Nov. } 14\end{array}$ & $\begin{array}{l}314.62 \\
314.72\end{array}$ & $\begin{array}{l}\text { Feb. } 21 \\
\text { Feb. } 27\end{array}$ & $\begin{array}{l}325.68 \\
325.45\end{array}$ & $\begin{array}{l}\text { July } 2 \\
\text { July } 11\end{array}$ & $\begin{array}{r}320.13 \\
+317.88\end{array}$ \\
\hline Sept. 23 & $\begin{array}{l}313.6 \\
313\end{array}$ & Nov. 21 & 314.70 & Mar. 5 & 325.34 & July 16 & $\begin{array}{r}4316.46 \\
\end{array}$ \\
\hline Oct. 26 & (14) & Dec. 5 & 316.22 & Mar. 12 & 324.57 & July 23 & $\begin{array}{l}431596 \\
\end{array}$ \\
\hline Nov. 28 & (14) & Dec. 12 & 321.07 & Mar. 19 & 324.37 & July 30 . & $\begin{array}{r}414.90 \\
\end{array}$ \\
\hline Dec. 31 - & 325.00 & Dec. 19 & 319. 71 & Mar. 26. & 324.23 & Aug. 6 & $\begin{array}{r}414.62 \\
+39.62\end{array}$ \\
\hline 1930 & & Dec. 27 & 324.22 & Apr. 2- & $\begin{array}{l}323.87 \\
323.49\end{array}$ & Aug. 13 & $\begin{array}{l}4314.63 \\
4314.75\end{array}$ \\
\hline & & & & Apr. 16 & 324.26 & Aug. 27. & 4315.80 \\
\hline Feb. 1 . & 325.40 & 1936 & & Apr. 23 & 323. 69 & Sept. 3 & 316.10 \\
\hline En. & 324.80 & & & & 324.20 & Sept. 11 & $\begin{array}{l}010.10 \\
316.36\end{array}$ \\
\hline Mar. 29 & 324.10 & Jan. 2 . & 325.56 & May 7 & 324.86 & Sept. 17 & 316.45 \\
\hline Apr. 26 & 324.85 & Jan. 3 & 325.60 & May 14 & 324.64 & Sept. 24 & 316.30 \\
\hline May & 320.50 & Jan. 9 & 325.57 & May 21 . & 324.23 & Oet. 1 & 315. 54 \\
\hline July 23 & 314.28 & Jan. 16. & 325.68 & May 28 & 323.40 & & \\
\hline
\end{tabular}

2 Ieveling by U. S. Engineer Department.

* Water level depressed by inordinately large withdrawals.

14 Well dry. 
TABLE 13.-Ground-water levels in observation wells, 1928-30 and 1935-36-Con.

649. J. W. Cleft. NE $34 \mathrm{SW} 1 / 4$ sec. 10, T. 16 S., R. 5 W. Stock well, driven 20.3 feet deep. Measuring points: Lower valve seat of pump, 1.0 foot above land surface and 329.06 feet ${ }^{2}$ above mean sea level; begin. ning November $30,1935,1.75$ feet below valve seat and 327.31 feet 2 above mean sea level.

\begin{tabular}{|c|c|c|c|c|c|c|c|}
\hline Date & $\begin{array}{l}\text { Water } \\
\text { level } \\
\text { (feet) }\end{array}$ & Date & $\begin{array}{l}\text { Water } \\
\text { level } \\
\text { (feet) }\end{array}$ & Date & $\begin{array}{l}\text { Water } \\
\text { level } \\
\text { (feet) }\end{array}$ & Date & $\begin{array}{l}\text { Water } \\
\text { level } \\
\text { (feet) }\end{array}$ \\
\hline 1935 & & 1936 & & 1936 & & 1936 & \\
\hline $\begin{array}{l}\text { Oct. } 17 \\
\text { Oct. } 24 \\
\text { Oct. } 30 \\
\text { Nov. } 7 \\
\text { Nov. } 13 \\
\text { Nov. } 20 \\
\text { Nov. } 30 \\
\text { Dee. } 5 \\
\text { Dec. } 12 \\
\text { Dec. } 19 \\
\text { Dec. } 27 \\
\end{array}$ & $\begin{array}{l}322.17 \\
321.56 \\
322.41 \\
322.46 \\
322.21 \\
323.19 \\
322.74 \\
322.67 \\
324.82 \\
324.88 \\
324.93\end{array}$ & $\begin{array}{l}\text { Jan. } 3 \\
\text { Jan. } 10 \\
\text { Jan. } 16 \\
\text { Jan. } 23 \\
\text { Jan. } 30 \\
\text { Feb. } 6 \\
\text { Feb. 13 } \\
\text { Feb. 22 } \\
\text { Feb. } 27 \\
\text { Mar. } 5 \\
\text { Mar. } 12 \\
\text { Mar. } 19 \\
\text { Mar. } 26 \\
\text { Apr. } 2\end{array}$ & $\begin{array}{l}326.47 \\
326.33 \\
326.45 \\
326.33 \\
324.09 \\
323.07 \\
326.29 \\
326.87 \\
327.38 \\
326.62 \\
325.49 \\
326.03 \\
326.03 \\
326.00\end{array}$ & $\begin{array}{l}\text { Apr. } 9 \\
\text { Apr. } 16 \\
\text { Apr. } 23 \\
\text { Apr. } 30 \\
\text { May } 7 \\
\text { May } 14 \\
\text { May } 21 \\
\text { May 28... } \\
\text { June } 4 \\
\text { June } 11 \\
\text { June } 18 \\
\text { June } 25 \\
\text { July } 2 \\
\text { July } 9\end{array}$ & $\begin{array}{l}326.04 \\
326.17 \\
325.34 \\
325.77 \\
326.07 \\
326.02 \\
326.00 \\
327.63 \\
327.57 \\
327.54 \\
327.64 \\
327.44 \\
327.40 \\
327.22\end{array}$ & $\begin{array}{l}\text { July } 16 \\
\text { July } 23 \\
\text { July } 30 \\
\text { Aug. } 6 \\
\text { Aug. } 13 \\
\text { Aug. } 20 \\
\text { Aug. } 27 \\
\text { Sept. } 3 \\
\text { Sept. } 11 \\
\text { Sept. 17 } \\
\text { Sept. } 24 \\
\text { Oct. } 1 . \\
\end{array}$ & $\begin{array}{l}325.81 \\
325.54 \\
326.34 \\
324.53 \\
322.62 \\
322.23 \\
322.94 \\
322.83 \\
322.57 \\
322.41 \\
322.23 \\
322.07\end{array}$ \\
\hline
\end{tabular}

2 Leveling by U. S. Engineer Department.

650. Owner unknown. SE1/4SW1/4 sec. 23, T. 16 S., R. 5 W. Stock well, driven $11 / 4$ inches in diameter and about 22 feet deep; at east end of barn. Casing stamped 16-5-3 on west side below pump. Measuring point is lower valve seat of pump, 2.9 feet above land surface and 342.9 feet above'mean sea level (interpolated).

\begin{tabular}{|c|c|c|c|c|c|}
\hline Date & $\begin{array}{l}\text { Water } \\
\text { level } \\
\text { (feet) }\end{array}$ & Date & $\begin{array}{l}\text { Water } \\
\text { level } \\
\text { (feet) }\end{array}$ & Date & $\begin{array}{l}\text { Water } \\
\text { level } \\
\text { (feet) }\end{array}$ \\
\hline \begin{tabular}{l}
\multicolumn{1}{c}{1928} \\
Aug. 24 \\
Oct. 22 \\
Dec. 16 \\
\end{tabular} & $\begin{array}{l}328.7 \\
327.6 \\
333.7\end{array}$ & $\begin{array}{l}\text { Feb. } 2929 \\
\text { Mar. } 27 \\
\text { May } 9\end{array}$ & $\begin{array}{l}335.7 \\
336.7 \\
334.5\end{array}$ & \begin{tabular}{|c|}
\multicolumn{2}{|c}{1929} \\
June 6 \\
July 4
\end{tabular} & $\begin{array}{l}333.3 \\
332.15\end{array}$ \\
\hline
\end{tabular}

653. R. W. Harrington. NE1/4NE1/4 sec. 14, T, 16 S., R. 4 W. Irrigation well, dug $4 \frac{1}{2}$ feet by 6 feet across and 10.5 feet deep, at west side of driveway 150 feet south of residence. Copper nail with washer stamped 16-4-1 in well curb at south west corner. Measuring point is top of wood curb, at copper nail with washer, level with land surface and 346 feet above mean sea level (interpolated).

\begin{tabular}{|c|c|c|c|c|c|c|c|}
\hline Date & $\begin{array}{l}\text { Water } \\
\text { level } \\
\text { (feet) }\end{array}$ & Date & $\begin{array}{l}\text { Water } \\
\text { level } \\
\text { (feet) }\end{array}$ & Date & $\begin{array}{l}\text { Water } \\
\text { level } \\
\text { (feet) }\end{array}$ & Date & $\begin{array}{l}\text { Water } \\
\text { level } \\
\text { (feet) }\end{array}$ \\
\hline 1928 & & & 341.25 & 1929 & & 1929 & \\
\hline Aug. 22 & 340.9 & Dec. $160_{-}^{-}$ & & Mar. 31. & 343.1 & June 6 & 342.55 \\
\hline Oct. 21 - & 339.8 & Feb. 2. & 343.5 & May 9 & 342.8 & July 3 & 341.55 \\
\hline
\end{tabular}

661. Celia Belleville. SW1/4NW1/4 sec. 30, T. 16 S., R. 4 W. Stock well, driven 14.2 feet deep. Measuring point is top of steel drive pipe (no pump), 2.5 feet above land surface and 348.78 feet $^{2}$ above mean sea level.

\begin{tabular}{|c|c|c|c|c|c|c|c|}
\hline Date & $\begin{array}{l}\text { Water } \\
\text { level } \\
\text { (feet) }\end{array}$ & Date & $\begin{array}{l}\text { Water } \\
\text { level } \\
\text { (feet) }\end{array}$ & Date & $\begin{array}{l}\text { Water } \\
\text { level } \\
\text { (feet) }\end{array}$ & Date & $\begin{array}{l}\text { Water } \\
\text { level } \\
\text { (feet) }\end{array}$ \\
\hline 1935 & & $\operatorname{Jan} 3^{1936}$ & 345.24 & 1936 & & 1936 & \\
\hline Oct. 17 - & 338.63 & Jan. 10 & 345.21 & Mar. 26 & 343.92 & June 11 & 342,25 \\
\hline Oct. 24 & 338.74 & Jan. 16 . & 345.17 & Apr. 2 & 344.22 & June 18 & 342.05 \\
\hline Oct. 30 & 338.76 & Jan. 23 & 345.64 & Apr. 9 & 344.12 & June 25 & 341.87 \\
\hline Nov. 8 & 338.86 & Jan. 30 & 344.21 & A.pr. 16 & 343.43 & July 2 & 341.75 \\
\hline Nov. 13 & 339.33 & Feb. 6 . & 344.74 & Apr. 23 & 343.15 & July 9 & 341.44 \\
\hline Nov. 20 & 340.36 & Feb. 13 & 344.66 & Apr. 30 & 343.20 & July 16 & 341.06 \\
\hline Nov. 30 & 341.29 & Feb. 22 & 346.64 & May 7 & 343.64 & July 23 & 340.82 \\
\hline Dec. 5 . & 341.31 & Feb. 27 & 344.90 & May 14 & 343. 52 & $\mathrm{JuJy} 30$ & (4) (14) \\
\hline Dec. 12 & 343.71 & Mar. 5 & 343.97 & May 21. & 343.55 & Aug. 6 & 340.70 \\
\hline Dec. 19 & 343.67 & Mar. 12 & 344.10 & May 28. & 342.61 & Aug. 13 & 340.95 \\
\hline Dec. 27 & 343.71 & Mar. 19 & 343.97 & June 4 & 342.65 & Aug. 20 . & 340.87 \\
\hline
\end{tabular}

2 Leveling by U. S. Engineer Department.

4 Water level depressed by inordinately large withdrawals.

14 Well dry. 
TABLE 13.-Ground-water levels in observation wells, 1928-30 and 1935-36-Con.

663. A. G. Pirtle. SW1/4NE1/4 sec. 17, T: $16 \mathrm{~S} .$, R. 3 W. Domestic well, driven $11 / 2$ inches in diameter and 20.5 feet deep. Measuring point is lower valve seat of pump, 4.2 feet above land surface and 373.8 feet above mean sea level. Record continued after March 1936 in well 664.

\begin{tabular}{|c|c|c|c|c|c|c|c|}
\hline Date & $\begin{array}{c}\text { Water } \\
\text { level } \\
\text { (feet) }\end{array}$ & Date & $\begin{array}{l}\text { Water } \\
\text { level } \\
\text { (feet) }\end{array}$ & Date & $\begin{array}{l}\text { Water } \\
\text { level } \\
\text { (feet) }\end{array}$ & Date & $\begin{array}{l}\text { Water } \\
\text { level } \\
\text { (feet) }\end{array}$ \\
\hline 1935 & & 1935 & & 1936 & & 1936 & \\
\hline Oct. 16 & 360.07 & Nov. 21 & 362.12 & $\begin{array}{l}\text { Jan. } 2 \\
\text { Jan. } 9\end{array}$ & $\begin{array}{l}368.45 \\
367.82\end{array}$ & $\begin{array}{l}\text { Feb. } 13 \\
\text { Feb. } 21\end{array}$ & $\begin{array}{l}367.47 \\
368.18\end{array}$ \\
\hline Oct. 22 & 360.10 & Dec. 5 & 361.92 & Jan. 16 & 368.17 & Mar. 5. & 368.63 \\
\hline Oet. 31 & 360.10 & Dec. 12 & 367.05 & Jan. 23 & 367.58 & Mar, 12 & 367.87 \\
\hline Nov. 7 - & 359.99 & Dec. 19 & 367.02 & Jan. 30 & 367.36 & Mar. 19 & 367.32 \\
\hline Nov. 14 & 362.01 & Dec. 27 & 367.56 & Feb. 6 - & 366.62 & & \\
\hline
\end{tabular}

664. Millwood Stock Farm. SW1/4SE1/4 sec. 17, T. 16 S., R. 3 W. Unused well, driven 11, inches in diameter and 15.1 feet deep. Measuring point is lower valve seat of pump, painted red, 3.2 feet above land surface and 379.59 feet ${ }^{2}$ above mean sea level.

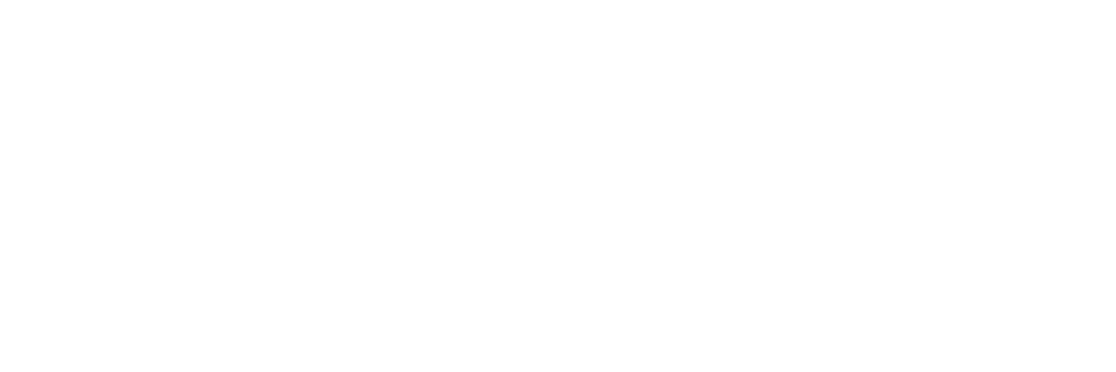

2 Leveling by the U. S. Engineer Department.

680. Leo Sidwell. SW1/4NE1/4 sec. 32, T. $16 \mathrm{~S}$, R. $3 \mathrm{~W}$. Irrigation well, dug in field 600 feet west of residence; in 1928-30, 6 feet square and 18.5 feet deep; in 1935-36, back-filled around 36-inch concrete-tile casing. Measuring points: Top of timber well curb, northwest corner, at copper nail with washer, 389.20 feet above mean sea level; from Aug. 8, 1928, to July 4, 1929, top of instrument shelf, at bored hole, 390.48 feet above mean sea level; beginning October 5, 1935, inner face at top of concrete-tile casing, marked by painted arrow, level with land surface and 388.98 feet 2 above mean sea level. Water-stage recorder operated on well from September 5,1928 , to July 4,1929 .

\begin{tabular}{|c|c|c|c|c|c|c|c|}
\hline Date & $\begin{array}{l}\text { Water } \\
\text { level } \\
\text { (feet) }\end{array}$ & Date & $\begin{array}{l}\text { Water } \\
\text { level } \\
\text { (feet) }\end{array}$ & Date & $\begin{array}{l}\text { Water } \\
\text { level } \\
\text { (feet) }\end{array}$ & Date & $\begin{array}{l}\text { Water } \\
\text { level } \\
\text { (feet) }\end{array}$ \\
\hline 1928 & & 1930 & & 1936 & & 1936 & \\
\hline Aug. 21 & 376.75 & Feb. 9 & 379.95 & Jan. 9 & 381.23 & June 18 & 376.98 \\
\hline Sept. 5 & 376.69 & Mar. 1 & 381.18 & Jan. 16 & 382.45 & June 25 & 377.72 \\
\hline Sept. 6 & 376.67 & Mar. $29_{-}$ & 379.73 & Jan. 23 & 381. 24 & July 2 . & 377.43 \\
\hline Sept. 15 & 376.66 & Apr. 26 & 379.23 & Jan. 30 & 381.75 & July 11 & 377.43 \\
\hline Oct. 8 & 376.62 & May 28. & 378.98 & Feb. 6 & 379.40 & July 16 . & 377.36 \\
\hline Oct. 21 & 376.60 & July 23 & 378.26 & Feb. 13 & 37980 & July 23 & 377.27 \\
\hline Dee. 16 & 376.88 & 1935 & & Feb. 21 & 379. 29 & July $30_{-}$ & 377.08 \\
\hline 1929 & & Oct. 5 & 376.38 & $\begin{array}{l}\text { Feb. } 28 \\
\text { Mar. } 5\end{array}$ & $\begin{array}{l}350.02 \\
379.89\end{array}$ & $\begin{array}{l}\text { Aug. } 6 \\
\text { Aug. } 13\end{array}$ & $\begin{array}{l}376.98 \\
376.88\end{array}$ \\
\hline & & Oct. 12 & 376.38 & Mar. 12 & 380.21 & Aug. 20 & 376.83 \\
\hline Jan. 6 & 379.08 & Oct. 17 & 376.66 & Mar. 19 & 379.67 & Aug. 27 & 376. 72 \\
\hline Feb. 2 & 378.60 & Oct. 31 & 376.00 & Mar. 26 & 379.71 & Sept. 3 & 376.66 \\
\hline Feb. 16 & 378.80 & Nov. 7 & 375.98 & Apr. 2 & 380.04 & Sept. 11 & 376.43 \\
\hline Mar. 7 & 378.81 & Nov. 14 & 376.46 & Apr. 9 & 378.35 & Sept. 17 & 376.40 \\
\hline Mar. 31 & 379.20 & Nov. 21 & 376.48 & Apr. 16 & 378.16 & Sept. 21 & 376.17 \\
\hline Apr. 18 & 378. 52 & Dec. 5 & 376.60 & A pr. 23 & 378.28 & Oct. $1 \ldots$ & 376.55 \\
\hline May 9. & 379.13 & Dec. 12 & 376.76 & Apr. 30 & 378.20 & & \\
\hline June 3. & 378.25 & Dec. 19 & 377.20 & May 7 & 378.16 & & \\
\hline July 4 & 377.67 & Dec. 27 & 377.26 & May 14 & 378.77 & & \\
\hline Sept. 23 & 377.85 & & & May 21 & 377.86 & & \\
\hline Oct. 26 & 377.73 & 1936 & & May 28 & 377.46 & & \\
\hline Nov. 18 & 377.73 & Jan. 2 & 377.46 & June 4 & 377. 11 & & \\
\hline Dee. 31 & 380.88 & Jan. $3_{-}$ & $377.9 t$ & June 11 & 377.06 & & \\
\hline
\end{tabular}

${ }^{2}$ Leveling by U. S. Engineer Department. 
TABLE 13.-Ground-water levels in observation wells, 1928-30 and 1935-36-Con.

693. Charles Frisbee, renter. SW1/4 NW1/4 sec, 3, T, 17 S., R. 4 W. Domestic and stock well, dug 19 feet deep, steel casing, 8 feet south of southeast corner of barn. Casing stamped 17-4-1-2 on south side. Measurjng point is top of 3 -inch casing, 2.6 feet above land surface and 376.77 feet ${ }^{2}$ above mean sea level.

\begin{tabular}{|c|c|c|c|c|c|c|c|}
\hline Date & $\begin{array}{l}\text { Water } \\
\text { level } \\
\text { (feet) }\end{array}$ & Date & $\begin{array}{l}\text { Water } \\
\text { level } \\
\text { (feet) }\end{array}$ & Date & $\begin{array}{l}\text { Water } \\
\text { level } \\
\text { (feet) }\end{array}$ & Date & $\begin{array}{l}\text { Water } \\
\text { level } \\
\text { (feet) }\end{array}$ \\
\hline 1929 & & 1936 & & 1936 & & 1936 & \\
\hline Sept. 23 & 362.47 & Oct. $25 \ldots \ldots$ & 361.53 & Feb. 6 & 369.39 & June 11 & 366.70 \\
\hline Oct. 26 . & 362.02 & Oct. 30 & 361.42 & Feb. 13 & 369.64 & June 18 & 366.45 \\
\hline Nov, 28 & 361.67 & Nov. 8 & 361.37 & Feb. 22 & 368. 69 & June $25 \ldots$ & 366.28 \\
\hline & & Nov. $13 \ldots$ & 361.35 & Feb. $27 \ldots$ & 369.39 & July $2 \ldots \ldots$ & 365.99 \\
\hline 1930 & & Nov. 20 & 361. 61 & Mar. 5........ & 368.79 & July $9 \ldots \ldots$ & 365.78 \\
\hline & & Nov. 30 & 362.09 & Mar. 12 & 368.70 & July $16 \ldots \ldots$ & 365.19 \\
\hline Jan. 2 & 367.77 & Dec. 5 & 362.13 & Mar. $19 \ldots$ & 368.16 & July 23. & 364.88 \\
\hline Feb. 28 . & 368.67 & Dec. 12 & 363.06 & Mar. 26... & 368.09 & July 30 & 364.31 \\
\hline Mar. 28 & 368.17 & Dec. 19 & 363.09 & Apr. $2 \ldots$ & 368.11 & Aug. 6 & 364.14 \\
\hline Apr. 25. & 367.92 & Dec. 27 . & 363.14 & Apr. 9 & 368.13 & Aug. 13. & 363.55 \\
\hline May 28 & 367.14 & & & Apr. $16 \ldots$ & 367.59 & Aug, 20 $\ldots \ldots$ & 363.06 \\
\hline July 23 & 364.56 & 1936 & & Apr. $23 \ldots$ & 367.58 & Aug. $27 \ldots \ldots$ & 363.09 \\
\hline 1935 & & Jan. 3 & 366.74 & $\begin{array}{l}\text { Apr. } 30 \\
\text { May } 7\end{array}$ & $\begin{array}{l}367.46 \\
367.73\end{array}$ & $\begin{array}{l}\text { Sept. } 3 \\
\text { Sept. } 11\end{array}$ & $\begin{array}{l}363.08 \\
362.94\end{array}$ \\
\hline & & Jan. 10 & 368.05 & May 14 & 367.45 & Sept. 17 & 362.49 \\
\hline Oct. 5. & $361.6 \%$ & Jan. 16 & 369.06 & Mav 21 & 367.31 & Sept. $24 \ldots \ldots$ & 362.30 \\
\hline Oct. 11 & 361.69 & Jan. 23 & 369.05 & May 28. & 367.19 & Oct. $1 \ldots$ & 361.88 \\
\hline Oct. 18 & 362.03 & Jan. 30 & 368. 13 & June 4 & 367.07 & & \\
\hline
\end{tabular}

2 Leveling by U. S. Engineer Department.

694. A. M. Rickman. SW1/4SW1/4 sec. 3, T. 17 S., R. 4 W. Irrigation well, dug 5 feet in diameter and 15 feet deep; destroyed in September 1929. Measuring point is top of plank spanning well, level with land. surface and 374 feet above mean sea level (interpolated).

\begin{tabular}{|c|c|c|c|c|c|c|c|}
\hline Date & $\begin{array}{l}\text { Water } \\
\text { level } \\
\text { (feet) }\end{array}$ & Date & $\begin{array}{l}\text { Water } \\
\text { level } \\
\text { (feet) }\end{array}$ & Date & $\begin{array}{l}\text { Water } \\
\text { level } \\
\text { (feet) }\end{array}$ & Date & $\begin{array}{l}\text { Water } \\
\text { level } \\
\text { (feet) }\end{array}$ \\
\hline 1928 & & 1928 & & 1929 & & 1929 & \\
\hline $\begin{array}{l}\text { Aug. } 24 \\
\text { Oct. } 21\end{array}$ & $\begin{array}{l}364.9 \\
364.00\end{array}$ & Dec. $16 \ldots$ & 367.9 & $\begin{array}{l}\text { Feb. } 2 \\
\text { Mar. } 27 \\
\text { May } 9\end{array}$ & $\begin{array}{l}371.8 \\
369.90 \\
369.95\end{array}$ & $\begin{array}{l}\text { June } 7 \\
\text { July 4 } \\
\text { Aug. } 2\end{array}$ & $\begin{array}{l}368.52 \\
367.05 \\
365.50\end{array}$ \\
\hline
\end{tabular}

699. John Peterson. SW 1/4SW1/4 sec. 20, T. 17 S., R. 4 W. Well driven 34.2 feet deep. Measuring point is lower valve seat of pump, 2.7 feet above land surface and 380.1 feet 2 above mean sea level.

\begin{tabular}{|c|c|c|c|c|c|c|c|}
\hline Date & $\begin{array}{l}\text { Water } \\
\text { level } \\
\text { (feet) }\end{array}$ & Date & $\begin{array}{l}\text { Water } \\
\text { level } \\
\text { (feet) }\end{array}$ & Date & $\begin{array}{l}\text { Water } \\
\text { level } \\
\text { (feet) }\end{array}$ & Date & $\begin{array}{l}\text { Water } \\
\text { level } \\
\text { (feet) }\end{array}$ \\
\hline 1935 & & 1936 & & 19.6 & & 1936 & \\
\hline $\begin{array}{l}\text { Oct. } 17 \\
\text { Oct. } 24 \\
\text { Oct. } 30 \\
\text { Nov. } 8 \\
\text { Nov. } 14 \\
\text { Nov. } 20 \\
\text { Nov. } 30 \\
\text { Dec. } 5 \\
\text { Dec. } 12 \\
\text { Dec. } 19 \\
\text { Dec. } 27 \\
\qquad 1936 \\
\text { Jan. } 3\end{array}$ & $\begin{array}{l}369.68 \\
369.74 \\
369.67 \\
369.75 \\
370.07 \\
370.55 \\
371.29 \\
371.34 \\
371.93 \\
373.62 \\
373.57\end{array}$ & $\begin{array}{l}\text { Jan. } 10 \\
\text { Jan. } 16 \\
\text { Jan. } 23 \\
\text { Jan. } 30 \\
\text { Feb. } 6 \\
\text { Feb. } 13 \\
\text { Feb. } 22 \\
\text { Feb. } 27 \\
\text { Mar. } 5 \\
\text { Mar. } 12 \\
\text { Mar. } 19 \\
\text { Mar. } 26 \\
\text { Apr. } 2 \\
\text { Apr. } 9 \\
\text { Apr. } 16\end{array}$ & $\begin{array}{l}375.25 \\
375.28 \\
375.89 \\
374.69 \\
374.52 \\
374.49 \\
377.07 \\
375.71 \\
375.49 \\
375.57 \\
375.48 \\
375.51 \\
375.19 \\
375.21 \\
374.24\end{array}$ & $\begin{array}{l}\text { Apr. } 33 \ldots \\
\text { Apr. } 30 \ldots \\
\text { May } 7 \\
\text { May } 14 \ldots \\
\text { May } 21 \\
\text { May } 28 \ldots \\
\text { June } 4 \\
\text { Jine } 11 \\
\text { June } 18 \\
\text { June } 25 \\
\text { July } 2 \\
\text { July } 9 \\
\text { July } 16 \ldots \\
\text { July } 23 \\
\text { July } 30\end{array}$ & $\begin{array}{l}374.04 \\
374.06 \\
374.05 \\
374.15 \\
374.56 \\
374.62 \\
374.67 \\
373.91 \\
373.07 \\
372.84 \\
373.04 \\
372.61 \\
372.46 \\
372.17 \\
371.92\end{array}$ & $\begin{array}{l}\text { Aug. 6. } \\
\text { Aug. 13. } \\
\text { Aug. } 20 \\
\text { Aug. } 27 \\
\text { Sept. } 3 \\
\text { Sept. } 11 \\
\text { Sept. } 17 \\
\text { Sept. } 24 \\
\text { Oct. } 1\end{array}$ & $\begin{array}{l}371.81 \\
371.64 \\
370.84 \\
370.62 \\
370.53 \\
370.24 \\
370.02 \\
370.03 \\
369.88\end{array}$ \\
\hline
\end{tabular}

2 Leveling by U. S. Engineer Department. 
TABLE 13.-Ground-water levels in observation wells, 1928-30 and 1935-36-Con.

702. Clara Blais. SE $1 / 4 \mathrm{NW} 1 / 4$ sec. 26, T. $17 \mathrm{~S}$., R. 4 W. Well driven 17.7 feet deep. Measuring point is lower valve seat of pump, 2.0 feet above land surface and 402.32 feet ${ }^{2}$ above mean sea level.

\begin{tabular}{|c|c|c|c|c|c|c|c|}
\hline Date & $\begin{array}{l}\text { Water } \\
\text { lpvel } \\
\text { (feet) }\end{array}$ & Date & $\begin{array}{l}\text { Water } \\
\text { level } \\
\text { (feet) }\end{array}$ & Date & $\begin{array}{l}\text { Water } \\
\text { level } \\
\text { (feet) }\end{array}$ & Date & $\begin{array}{l}\text { Water } \\
\text { level } \\
\text { (feet) }\end{array}$ \\
\hline 1935 & & 1936 & & 1936 & & 1936 & \\
\hline Oct. 17 & 398.79 & Jan. 10 & 394.11 & Apr. 16. & 396.39 & July 23 & 392.25 . \\
\hline Oct. 24 & 388.68 & Jan. 15 & $\begin{array}{l}094.11 \\
398.21\end{array}$ & Apr. 23 & 395.96 & July 30 & 391.65 \\
\hline Oct. 30 & 388.63 & Jan. 23 & 398,56 & Apr. 30 & 396.01 & Aug. 6 & 391. 44 \\
\hline Nov. 8 & 388.72 & Jan. 30 & 393.69 & May 7. & 396. 04 & Aug. 13 & 391.06 \\
\hline Nov. $14 \ldots$ & 398.64 & Feb. 6 & 397. 01 & May 14 & 394.70 & Aug. 20 & 390.91 \\
\hline Nov. 20 & 338.93 & Feb. 13 & 397.53 & May $21 \ldots$ & 395.73 & Aug. 27 & 389.33 \\
\hline Nov. $30 \ldots$ & 399.05 & Feb. 22 & 397.75 & May 28 & 395.84 & Sept. 3 & 389.24 \\
\hline Пec. 5 & 339.15 & Feb. 27 & 397.50 & June 4 & 395.91 & Sept. 11. & 388.61 \\
\hline Dec. 12 & 389.99 & Mar. 5 & 396.98 & June $11 \ldots$ & 394.81 & Sept. 17 & 388.31 \\
\hline Dec. 19 & 389.84 & Mar. 12 & 397.29 & June $18 \ldots$ & 394.83 & Sept. 24 & $387.93^{\circ}$ \\
\hline Dec. 27 & 389.91 & Mar. 19. & 400.41 & June $25 \ldots$ & 394,69 & Oct. $1 \ldots$ & 387.78 \\
\hline 1936 & & Mar. 26 & 397. 19 & July 2 & 394.41 & & \\
\hline Jan. 3 & 393.00 & Apr. 9 & 396. 48 & July 16 & 392.50 & & \\
\hline
\end{tabular}

${ }^{2}$ Leveling by U. S. Engineer Department.

707. George T. Morrill. NE1/4NE1/4 sec. 20, T. 17 S., R. 3 W. Domestic well, driven 11/2 inches in diam-eter and 16.7 feet deep. Measuring point is lower valve seat of pump, 4.7 feet above land surface and 427.24 feet ${ }^{2}$ above mean sea level.

\begin{tabular}{|c|c|c|c|c|c|c|c|}
\hline Date & $\begin{array}{l}\text { Water } \\
\text { level } \\
\text { (feet) }\end{array}$ & Date & $\begin{array}{l}\text { Water } \\
\text { leve] } \\
\text { (feet) }\end{array}$ & Date & $\begin{array}{l}\text { Water } \\
\text { level } \\
\text { (feet) }\end{array}$ & Date & $\begin{array}{l}\text { Water } \\
\text { level } \\
\text { (feet) }\end{array}$ \\
\hline 1935 & & 1933 & & 1936 & & 1936 & \\
\hline Oct. 15 . & 408,29 & Jan. 9 & 410.06 & Apr. 16 . & 413.96 & July 23 & 410. $92^{\prime}$ \\
\hline Oct. 22 & 409.14 & Jan. 16 & 411.47 & Apr. 23 & 412.72 & July 30 . & 410.20 , \\
\hline Oet. 31 & 40९. 09 & Jan. 23 & 411. 97 & A pr. 30 & 413. 51 & Aug. 6 & 410.12 \\
\hline Nov. 7 & 407.99 & Jan. 30 & 412. 18 & May 7 & 413.64 & Aug. 13 . & 409.60 \\
\hline Nov. 14 & 407.88 & Feb. 6 & 412.32 & May 14 & 413.77 & Aug. 20 . & 409.96 \\
\hline Nov. 21 & 407.96 & Feb. 13 & 411.83 & May 21 & 413.59 & Aug. 27 & 409.69 \\
\hline Dec. 5 & 409. 08 & Feb. 21 & 413. 16 & May 28. & 413.66 & Sept. 3 & 409.62 \\
\hline Dec. 12 & 408.56 & Feb. 28 & 413. 54 & June 4 & 413. 16 & Sept. 11 & 409.46 \\
\hline Dec. 19 & 408. 03 & Mar. 5 & 414. 18 & June 11 & 413.47 & Sept. 17 & 409.70 \\
\hline Dec, 27 & 408.21 & Mar. 12 & 411. 39 & June 18 & 412.65 & Sept. 24 & 409.48 \\
\hline 1936 & & Mar. 19 & 414. 14 & June 25 & 412. 39 & Oct. 1 & $409.10^{\circ}$ \\
\hline 1936 & & Mar. 26 & 413. 60 & July 2 & 412.05 & & \\
\hline Jan. 2 . & 408.66 & Apr. $9-$ & $\begin{array}{l}414.11 \\
413.87\end{array}$ & July 16 . & 411.07 & & \\
\hline
\end{tabular}

${ }^{2}$ Leveling by U. S. Engineer Department.

712. Chase Gardens. SW $1 / 4$ SE1/4 sec. 28, T. 17 S., R. 3 W. Irrigation well, dug 4 feet square and 20 feet deep, in field 750 feet west of greenhouses. Measuring point is top of tee in discharge pipe (second tee above. pump), 1.0 foot below land surface and 430 feet above mean sea level (interpolated). Well was inaccessible * for water-level measurements in 1935-36.

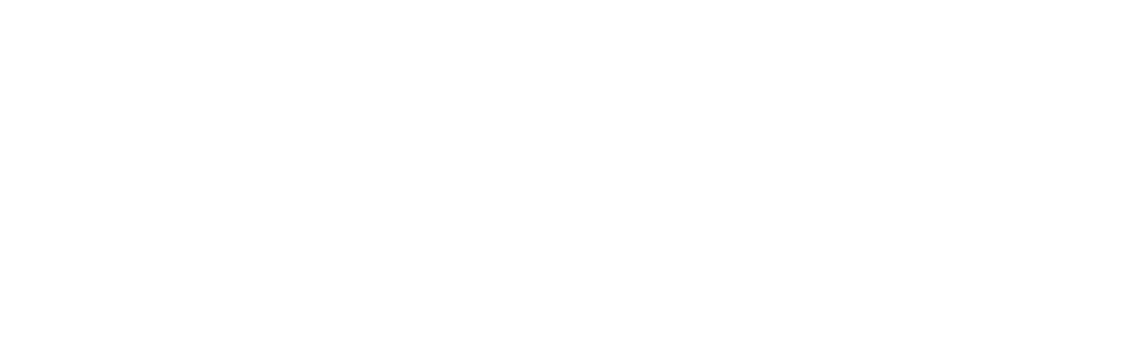




\section{INDEX}

Page

Abstract

Acknowledgments for aid

Agricultural Research Corporation, section of well of

Albany, climate at . ground water near

$14,16-18$ $44,47,48$

Alexander, A. L., section of well of ........ 134 Alluvium in the area. . ........... 27-32, pls. 1-2,4-7 Analyses of water from representative wells . _ 60-63

Beebe, Mrs., section of well of

Bethel School well, section of -

Bibliography

Bingenheimer well, section of

Brownsville, ground water near

Canby, section of well of.

Canby fan, water supply of

Cascade formation, occurrence and waterbearing properties of

Cascade Range, features of

Central lowland, subdivisions of, in relation to ground water

41-54, pls.1-2, 7,10

Chemical character of the ground water in the area

Climate of the area $12-19$

Coburg, ground water near

Collier Glacier, view of.

Columbia River, alluvial plain of, water sup-

ply of .... 45-46

Consolidated and semiconsolidated rocks, general character of _.... 19-20, pls. 1-2

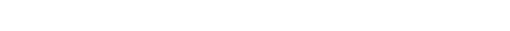

water-bearing properties of _. . ........... 24-27

Corvallis, climate at . . . . . . . . . . 14-18

ground water near................... 48

Corvallis Creamery Co. well, section of ..... 131

Cottage Farm, Oregon State Hospital, section of well of . . . 142

Dayton Prairie, water supply of ... . . . . . . 51-53

Eastern-Western Lumber Co., section of well of . ........... 140

Elmira, ground water near . ............. 46-47

Eugene, climate at .................... 14, 16-18

ground water near.

Eugene formation, occurrence and waterbearing properties of $\ldots \ldots \ldots, 22,26$

Field work in the area

Fishel, V. C., analyses by ................ 31

Fisher formation, occurrence and water-

bearing properties of $\ldots \ldots \ldots \ldots .22,26$

Flowing wells, occurrence of ........ $55-56$, pl. 1

Foster. M. D., analyses by ........... . . 60-63

Frank, Aaron, well 2, section of
Pagse

French Prairie, water supply of . .
Furrow, M. E., section of well of . $\ldots \ldots \ldots$

Geologic maps of the Willamette Valley ... pls. 1-2

(in pocket)

Geology and hydrology of the area.......... 19-41,

pls. 1-2, 4-9

Gervais, city of, section of well of .......... 139

Gooding, William, section of well of $\ldots . . . \ldots . .137$

Ground water, chemical character of ..... 27. 56-64 dependability of supply of ............ 40-41

fluctuations of level of . . . . . 34-39, 144-192

for irrigation, suitability of _......... 59,64

in relation to rainfall and streams. $39-40$, pls. 1-2

previous reports on

Harrisburg, ground water near. ......... 46

Illahe formation, occurrence and water-bearing properties of .... 23

lndependence, alluvium at .............. 48

ground water near.

Index map of Oregon showing areas covered by ground-water reports.......... 3

Investigation, purpess and scope of _....... 4-5

Irrigation, water suitable for ......... 59,64

Junction City, ground water near......... 46

wat $r$ supply of ................... 46

Junction City well, section of ......... 143

Keasey shale, occurrence and water-bearing

properties of

Ladd well, section of . .

Lebanon alluvial fan, water supply of ..... 45

Libby, McNeill \& Libby well, section of . .- 133-

Lloyd Corporation, Inc., section of well of _... 131

Main valley plain, Brownsville-Albany segment........... 47

Canby fan segment .................... 45

Coburg-Harrisburg səgment............ 46

Corrallis segment._................ 48

Dayton Prairie segment_............. 5153

Elmira segment ......... $46-47$

Eugene-Junction City segment_.......... 46

French Prairie segment . . . . . . . . . . . . . 49-51

Lebanon fan segment _................ 45

minor segments near Albany............. 48

Monmouth-Independence segment..... 48

Spring:eld delta segment ............... 43-44

Stayton-Mill Creek tasin _.......... $48-49$

Tualatin Valley segment $\ldots \ldots \ldots$

Mark, J. C., section of well of . . ........... 13i

Masonic and Eastern Star Home well, section

of . . . 130

Materials penetrated by tyfical wells . . . . . 130-143 
Mattoon, J. C., section of well of _........ $\quad \begin{array}{r}\text { Page } \\ 135\end{array}$

Mill Creek basin, ground water.

Molalla pediment, features of . . _ _ _ _ _. $33-34$ water supply of .

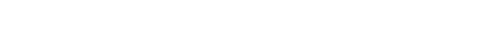

Northwestern Ice \& Cold Storage Co., section of well of .

Oregon Coast Range, features of ... . ........ Oregon Linen Mills, Inc., section of well of .... Oregon State Hospital, well 3, section of ......

Cregon State Hospital, Cottage Farm, section of well of

Oregon State Penitentiary, section of well of.. Oregon State Training School, section of well of

Pittsburg Bluff sandstone, occurrence and water-bearing properties of . ..... 22, 26

Pleasantdale School, section of well of . . .... 136

Portland, climate at....................... 13-18

Portland "delta," features of .

water supply of

Precipitation on the area

Public Works Engineering Corporation, section of well of ................ 140, 141

Purpose and scope of the investigation. ...... 4-5

Rainfall. See Precipitation.

Rhododendron formation, occurrence and water-bearing properties of . . . . . 23, 24

Roberts, H. A., section of well of . ........ 134

Rockwood Water District well, section of .... 134

Salem, climate at 14-18 ground water near.

Salty to brackish water in consolidated sedi-

mentary rocks
Scappoose Dike District well, section of Sedimentary rocks equivalent to Tyee and . Burpee formations, occurrence and water-bearing properties of . . . 22, 26-27

Snowfall. See Precipitation.

Southern Pacific Railroad Co., Woodburn station, section of well of ......... 139 Springfield delta, water supply of . . . . $43-44$, pl. 7
Stayton, ground water near.............. 48 Stayton lavas, occurrence and water-bearing properties of ................... 23, 24 Stayton-Mill Creek basin, water supply of .... 48-49 Stratigraphic sections of the rocks in the area . 22-23, Structure in the area ....................... 20-21 Stryker, A. W., section of well of . Surface features of the area.......... 8-12, pls. 1,2

Temperature in the area.................. 14

Terminal Ice \& Cold Storage Co. well, section of

Terrace deposit in the area...... . 32-34, pls. 1-2, 8 Topography of the area .............. 8-12, pls. 1, 2 Troutdale formation, occurrence and waterbearing properties of .............. 23, 24

Tualatin Valley, water supply of ......... 53

Unconsolidated deposits, general character of.. 2734 , pls. 1-2, 4-8 water in ..................... 34-41, pls. 1-2, 9 United States Resettlement Administration, Yamhill Farms, unit 6, section of well of ........................ 137 unit 16 , section of well of unit 19, section of well of ............... 136 unit 24 , section of well of unit 68 , section of well of ............... 136 unit 85, section of well of ................. 132

Water. See Ground water.

Water-bearing materials, physical properties of .................... 31

Water table, form and fluctuations of ..... 34-39, pls. $1-2,9$ Wells, flowing ........... materials penetrated by . . ............. 130-143 records of ............. $66-129$ water level in Williams, F. L. section of well of ........ 134 Willamette River, alluvial pıain of ..... 10-12, 42-53 Woodburn, city of, section of well of ........ 139

Yakima basalt, occurrence and water-bearing properties of $22,24-26$ 